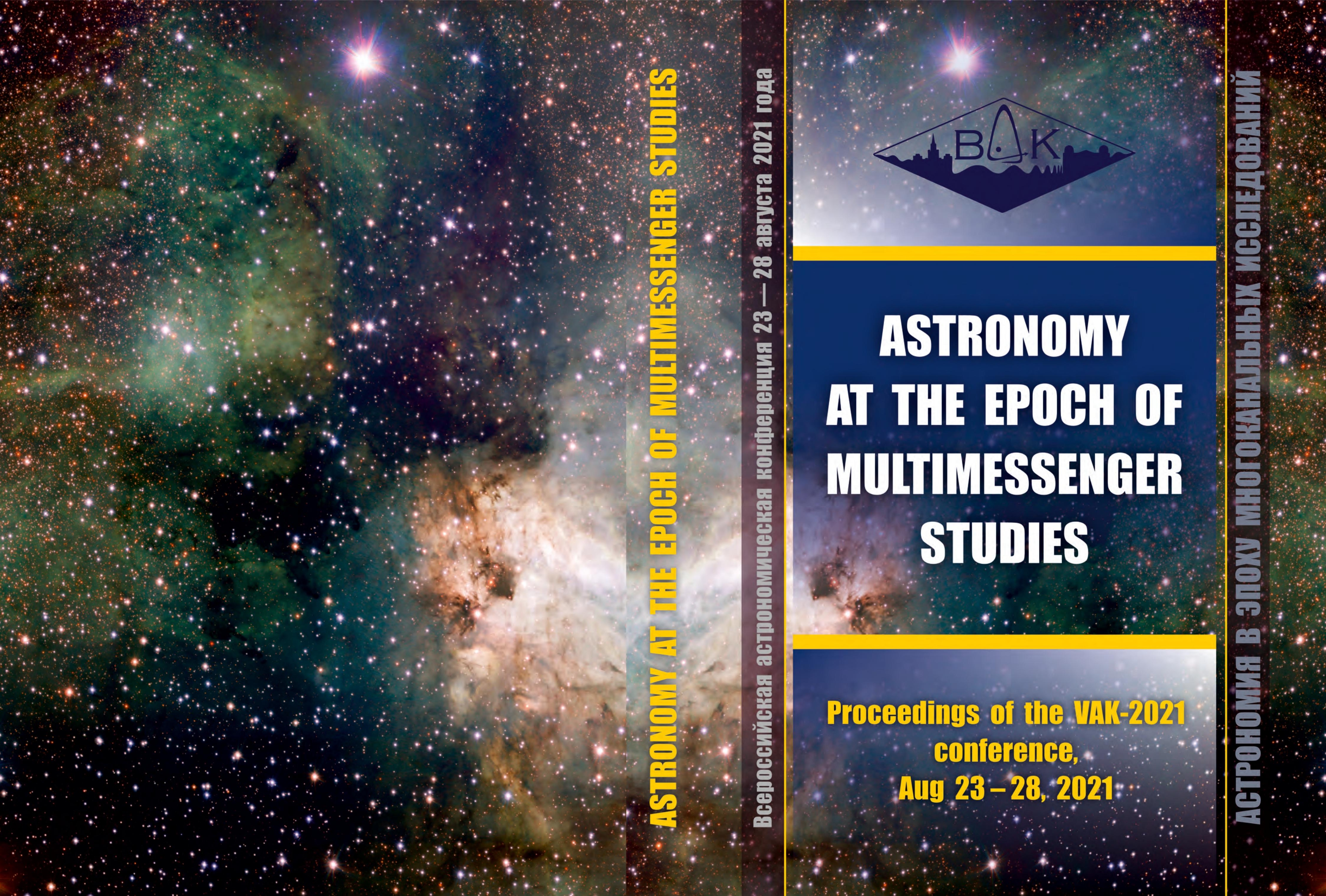


STERNBERG ASTRONOMICAL INSTITUTE

MOSCOW STATE UNIVERSITY

INSTITUTE OF ASTRONOMY

RUSSIAN ACADEMY OF SCIENCES

\section{ASTRONOMY AT THE EPOCH OF MULTIMESSENGER STUDIES}

Proceedings of the VAK-2021 conference, Aug 23-28, 2021

Edited by A.M. Cherepashchuk et al.

Moscow

Janus-K

2022 
UDK 52

BBK 22.6

Astronomy at the epoch of multimessenger studies. Proceedings of the VAK-2021 conference, Aug 23-28, 2021 - Moscow, Janus-K, 2022 (486 pp.)

ISBN 978-5-8037-0848-3

\section{Edited by}

Cherepashchuk A.M., Emelyanov N.V., Fedorova A.A., Gayazov I.S., Ipatov A.V., Ivanchik A.V., Malkov O.Yu., Obridko V.N., Rastorguev A.S., Samus N.N., Shematovich V.I., Shustov B.M., Silchenko O.K., Zasov A.V.

This volume contains papers based on talks presented at the Russian Astronomical Conference VAK-2021 and comprises a wide range of astronomical and astrophysical subjects.

(c) SAI MSU, INASAN, 2022

(c) Authors, 2022 


\section{Public Lecture}




\title{
Breakthrough projects of the scientific program of the Millimetron Observatory (“Spectrum-M” project)
}

\author{
I.D. Novikov
}

Astro-Space Center of P.N. Lebedev Physical Institute, 4/32, Profsoyusnaya str., Moscow, 117997, Russia The Niels Bohr International Academy, The Niels Bohr Institute, Blegdamsvej 17, DK-2100, Copenhagen, Denmark National Research Center Kurchatov Institute, 1, Akademika Kurchatova pl., Moscow, 123182, Russia

The article presents the scientific program of the Spectrum-M project. Three most important — "breakthrough" projects have been identified: 1) Study of physical processes at the early stage of the expansion of the Universe, 2) Investigation of processes near the horizon of a black hole and the search for possible manifestations of wormholes in the Universe, 3) Investigation of observational manifestations of the emergence life in the Universe. Examples of "breakthrough" projects are described and some related second-level projects are indicated. The article uses the materials from our other publications.

Keywords: Millimetron, early Universe, black holes, wormholes, emergence of life

DOI: 10.51194/VAK2021.2022.1.1.001

\section{Introduction}

The Millimetron space observatory of the Spectrum-M project is a space telescope a large far infrared scientific mission of Russian Space agency. It is aimed at solving the most pressing problems of modern astrophysics.

How did the Spectrum-M project come about and why is it needed?

In recent decades, astrophysics has seen a real scientific breakthrough in our knowledge of the Universe. It was mainly associated with the creation of unique space projects such as COBE, WMAP, Hubble, Planck, Chandra, Fermi-LAT, Radioastron and others. Our understanding of physical processes in space has deepened and expanded significantly. The astrophysics has reached the limit beyond which new physics begins. It becomes necessary to investigate the processes associated with the mystery of the origin of the Universe. The tasks are set to elucidate the structure of space-time, to determine the nature of the forces acting in space. These ambitious tasks require completely new efforts, the creation of new projects. One of these projects is Spectrum-M, Millimetron.

This project is a logical continuation of the Radioastron (Spectrum-R) project, which successfully completed its work in space several years ago. The initiator, creator and leader of both projects was Nikolai Semenovich Kardashev, who recently left us. We continue his work.

The AstroSpace center of Lebedev Physical Institute is the head of the international mission Millimetron, in which six more Russian institutes participate.

\section{Technical characteristics of Millimetron}

First of all, let's say about the technical parameters of the Millimetron. It is a 10-meter space telescope cooled to helium temperatures. The Millimetron will be launched into the orbit in the vicinity of the $L_{2}$ libration point of the Sun-Earth system at a distance of about 1.5 million kilometers from the Earth. It will operate in dual operation modes: single dish and space-Earth VLBI. The angular resolution for VLBI mode is of the order of $10^{-8}$ arc seconds.

\section{Structure of scientific program}

We now turn to the scientific program of Millimetron. We believe that the most expedient principle for constructing a research program at such a space observatory as Millimetron is the following: it is necessary to single out several (for example, three) fundamental problems of astrophysics, the solution of which will constitute a real breakthrough in our knowledge and which can be solved using the instruments of the Millimetron class. It is

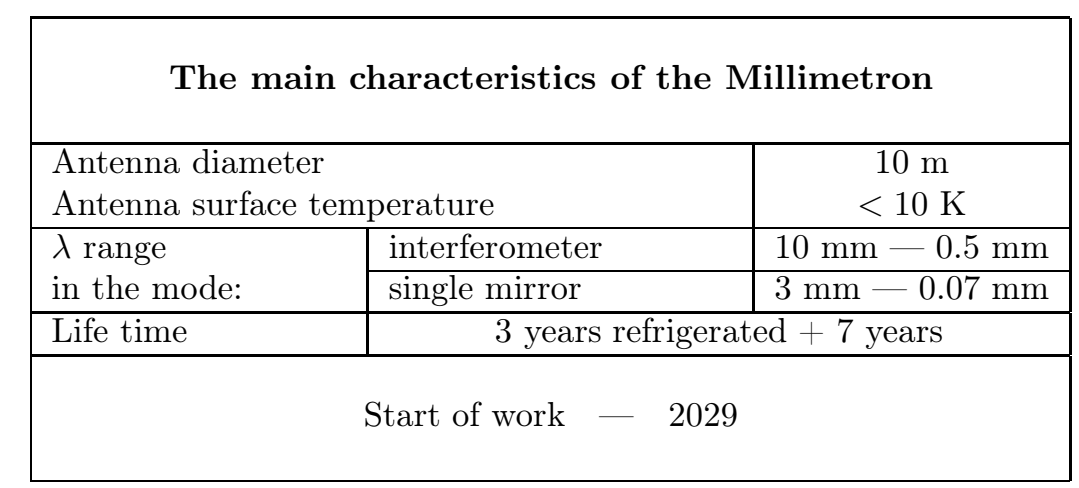


necessary to concentrate all efforts, first of all, on their solution, subordinating the rest of the projects to the solution and development of these basic ones. Thus, the scientific program should be hierarchical. There are three main directions. Each is based on a key (breakthrough) project, around which the projects of the next levels are developed, thematically related to the "breakthrough" one.

The following problems were chosen as breakthrough directions:

1. Processes near the beginning of the expansion of the Universe

2. Processes near the horizon of a black hole and the wormhole problem

3. The origin of life and its distribution in the Universe

\section{Examples of key projects}

Our program is published completely in $[1,2]$. Its description takes dozens of pages. Here we will focus on two or three examples.

The first problem involves the investigations of unknown processes in the early stages of the cosmological expansion of the Universe. Such processes are accompanied by the release of energy. Energy is transferred to CMB photons and distorts the spectrum of electromagnetic radiation left over from the origin of the Universe. Observations at the Millimetron are aimed at detecting these distortions. Their interpretation will make it possible to judge the processes that generated them. These processes are hidden from us by impenetrable hot plasma and it is impossible to study them in other ways. Near the cosmological singularity, when the dimensions of the Universe were a million times smaller than the modern ones, the distortions arising from the injection of energy into the plasma are called $\mu$-distortions. They can carry information about the existence of primordial black holes, particles with a lifetime of $10^{9}-10^{10}$ seconds, about very small inhomogeneities in the spatial distribution of plasma and about possible other unknown mysterious processes. In epochs more far from singularity appear so-called $Y$ distortions which are essentially a manifestation of the Sunyaev-Zeldovich effect. These last deviations also occur in the epoch relatively close to us, in the period of secondary ionization of cosmic plasma. We are especially interested in $\mu$-distortions. Their minimum amplitude is very small, see Fig. 1 . But even these distortions with a minimum amplitude can be attempted to be detected by the observations specially organized at the Millimetron. Detection of the distortions in the frequency spectrum of the relic radiation will open up a completely new channel of information about the structure and evolution of the Universe that is still inaccessible to us. This project will run in single mirror mode.

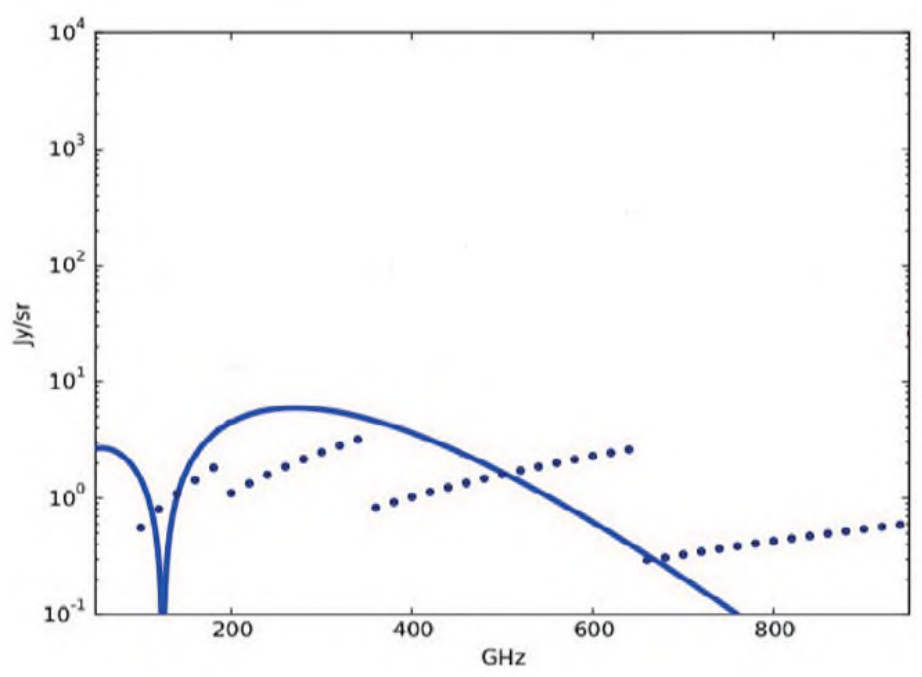

Figure 1: The solid line is the minimum amplitude of the $\mu$-distortions of the relict electromagnetic radiation. The dots indicate the possible sensitivities of the Millimetron measurements at different frequencies of electromagnetic radiation.

The second breakthrough problem is to study the properties of curved space-time in strong gravitational fields. It will be performed by the Millimetron in the space VLBI mode. This includes the study of the distribution of emission in the vicinity of supermassive black holes (SMBHs with masses from one million to several billion solar mass) in our Galaxy (Sagittarius A* source), in the M87 galaxy and in a number of other galaxies with supermassive objects, which show their presence by a number of indirect signs. The main task of the Millimetron in the space VLBI mode will be to obtain the "direct" images of the SMBHs, that is, bright halos around shadows, 

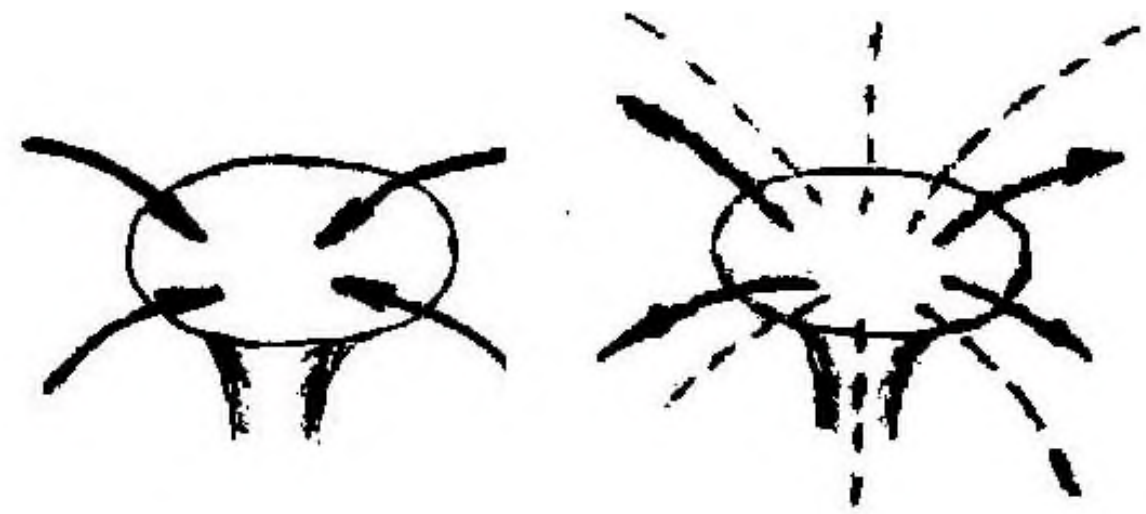

Figure 2: A sketch illustrating differences between magneto-hydrodynamics of flows and configuration of magnetic field around a black hole (left) and wormhole (right): in case of a BH only inflows can exist, whereas around a WH outflows are possible. A distinctive difference between magnetic field around $\mathrm{BH}$ and $\mathrm{WH}$ is that BHs cannot have its own magnetic field, it can exist only in the accretion flow on to the $\mathrm{BH}$. On the contrary, WHs can have their own magnetic field with a monopole topology with magnetic lines directed radially as $\sim r^{-2}$.

i.e. dark spots that appear around the black hole images when the light rays are curved in a strong gravitational field and the black holes are illuminated by any light source (for example, a luminous accretion disk). Another task of the Millimetron observatory in the space VLBI mode will be to search for observational manifestations of even more exotic objects predicted by general relativity, namely wormholes, that is, the tunnels in space-time that can connect the different regions of our Universe or even our Universe with other universes.

As said more than a hundred years ago, immediately after the creation of the General theory of Relativity, in its framework, the celestial bodies and physical phenomena in space were predicted so unusual and strange that the possibility of their real existence was categorically denied by the most prominent physicists and astronomers of that time, including A. Einstein. These are, for example, black holes and dark energy. Now both are open. They play a critical role in our understanding of he world around us.

Another prediction of General Relativity immediately after its creation was wormholes. They represent two holes in space, connected by a corridor - a wormhole. The holes can be as far apart as desired. The wormholes have not been found yet. It has been hypothesized that the cores of some galaxies may be not black holes, but the entrances to wormholes. How can this be verified? The most important criterion is the special structure of the magnetic field at the entrances, which is characteristic only for wormholes. The field must be monopole. The plasma flow at the entrances should also be special: in the case of BH only inflows can exist, whereas around a WH outflows are possible, see Fig. 2.

The most obvious and reliable observational manifestation of wormholes if a possibility for them to have their magnetic field with monopole structure varying radially as $\sim r^{-2}$. Polarization measurements is the instrument ro recognize and study characteristics of magnetic field in the vicinity of supermassive relativistic objects, either black holes or wormholes. MSO will be equipped with adequate polarimetric receivers.

One of the key projects of the Millimetron scientific program includes observations of magnetic fields and plasma flows near the nuclei of the nearest galaxies. This will require the unique angular resolution of the Millimetron space interferometer and the sensitivity of the instruments.

The possible discovery of signs of the existence of wormholes in such observations would radically change our ideas about the structure of the space-time of the Universe.

Another breakthrough problem of modern science is the problem of the origin of life in the Universe. It includes the detection of complex organic and prebiological molecules in protoplanetary disks and planetary atmospheres, which could give impetus to the development of life, including the intelligent life.

The Millimetron observatory will investigate the conditions for the occurrence of life in the protoplanetary disks of distant stars. Its task will be to determine the evolutionary sequence of physical processes in protoplanetary disks, favorable from the emergence of life point of view. It is known that the factor determining the very possibility of the existence of life is the presence of water, only if it is available on the planet or even on a protoplanet the beginning of biological evolution becomes possible. An important circumstance is that the water is located in that place of the protoplanetary disk, where the amount of energy from the central star is mostly favorable from the point of view of the existence of life. These areas around the central star are called habitable zones. Around the solar type stars they are located approximately at the distance from 0.5 to $2-3$ astronomical units. The task of the Millimetron observatory will be to study how, under what conditions, the formation and inflow of water in the regions close to the habitable zones occurs in the protoplanetary disks. This is one of the mysteries in questions about the possibility of the emergence of life in other worlds. It has not been fully resolved even for the 
Earth. Millimetron's spectral instruments have characteristics and sensitivity sufficient for observing the processes of water production and migration in the regions of protostar formation and in protoplanetary disks.

\section{Next level projects}

As mentioned above, around each key project, the projects of subsequent levels develop, which are thematically related to it, expanding and complementing the main one. So, among second level projects related to cosmology we note:

1. An alternative method for measuring the Hubble constant $H_{0}$;

2. Investigation of the Sunyaev-Zeldovich effect;

3. Studying the properties of dusty infrared galaxies

and others.

Some second-tier projects related to the second "breakthrough" project:

1. Multifrequency polarization observations of the immediate vicinity of the sources Sgr A*, M87;

2. Study of the nature of turbulence in an accretion flow

and others.

Second-level projects related to the third "breakthrough" project:

1. Filamentous structure of the interstellar medium, giant molecular clouds and star-forming regions in the immediate vicinity of the Sun;

2. Observational manifestations of the formation of terrestrial planets;

3. Exploring the water reservoirs in the Solar system

and others.

\section{Conclusions}

The scientific program of the Millimetron continues to be developed by its scientific team under active discussions with our colleagues at home and abroad. For these purposes, six Millimetron Science Working Groups were organized in 2020.

As a conclusion, we present an assessment of an early version of our scientific program, which we discussed with Prof. Kip Thorne:

"It is obvious that all four projects in your document "Some of the key projects of the Millimetron mission" are very important, and in fact very exciting...

Those methods and feasibility are very good. I look forward eagerly to the results they will bring."

$$
\text { Kip Thorne. }
$$

Many astronomers in our country and abroad took part in creation of this scientific program. We thank all our colleagues.

\section{References}

1. I. D. Novikov, S. F. Likhachev, Y. A. Shchekinov, A. S. Andrianov, et al., Physics Uspekhi, 64, 386, 2021.

2. T. d. Graauw, I. D. Novikov, S. F. Likhachev, Y. A. Shchekinov, et al., Millimetron Space Observatory, 2021. 
Plenary Talks 


\section{Period Variations of Cepheids and Stellar Evolution}

L.N. Berdnikov ${ }^{1}$, N.N. Samus ${ }^{1,2}$

berdnik@sai.msu.ru

${ }^{1}$ P.K. Sternberg Astronomical Institute, M.V. Lomonosov Moscow University, Moscow 119234, Russia,

${ }^{2}$ Institute of Astronomy, Russian Acad. Sci., 48, Pyatnitskaya Str., Moscow 119017, Russia

Classical Cepheids are pulsating supergiants in the main instability strip. In the course of evolution, they move across the instability strip, and their periods vary. Studying their period variations for a time span of several decades, it is possible to determine the rate of secular period increase or decrease, identify the number of instability strip crossing, and thus improve the period-luminosity relation. Special tests are used to determine whether the observed period variation is real or caused by random errors.

Keywords: variable stars: Cepheids, stars: photometry

DOI: 10.51194 /VAK2021.2022.1.1.002

\section{Introduction}

Classical Cepheids (called simply Cepheids in the following) are pulsating yellow supergiants. During their pulsations, their size and surface temperature vary, causing brightness variations with the same period as the period of pulsations.

$\eta$ Aql and $\delta$ Cep, the first Cepheids with the periods respectively $7.177^{d}$ and $5.366^{d}$, were discovered in 1783 and 1784. Their $V$-band light curves are shown in Fig. 1.

The General Catalogue of Variable Stars, GCVS [1], contains about 700 Cepheids. Different photometric surveys of the recent time have revealed many new Cepheids not yet in the GCVS. For example, [2] base their study on 2431 Cepheids.

Periods of Cepheids known in our Galaxy are in the range between 1 and 68 days; Cepheids with periods exceeding 100 days are known in other galaxies.

\section{Cepheid Period Variations}

Precursors of Cepheids are B stars. After the end of core hydrogen burning, the stars leave the main sequence, move to the right in the Hertsprung-Russell diagram and cross the instability strip. Entering the strip, a star unavoidably begins to pulsate and becomes a Cepheid. Figure 2 displays evolutionaty tracks for stars of different masses. These tracks can cross the instability strip (its boundaries plotted as dashed lines) once or three times, depending on the star's initial mass; it is very important that every next crossing occurs at a higher luminosity. Straight solid lines in Fig. 2 are lines of constant periods, the higher the line, the longer is the period. Their slope differs from that of the tracks, and thus, in the case of crossing the instability strip from left to right, the period increases, while it decreases in the case of crossing from right to left. Consequently, the O-C diagram will be a parabola with upward branches for increasing periods (the first and third crossings of the instability strip) and with downward branches for decreasing periods (the second crossing of the instability strip). As an example, Fig. 3 shows $\mathrm{O}-\mathrm{C}$ diagrams for the second and third crossings.

Thus, according to the theory, $\mathrm{O}-\mathrm{C}$ diagrams should be parabolas for all Cepheids.

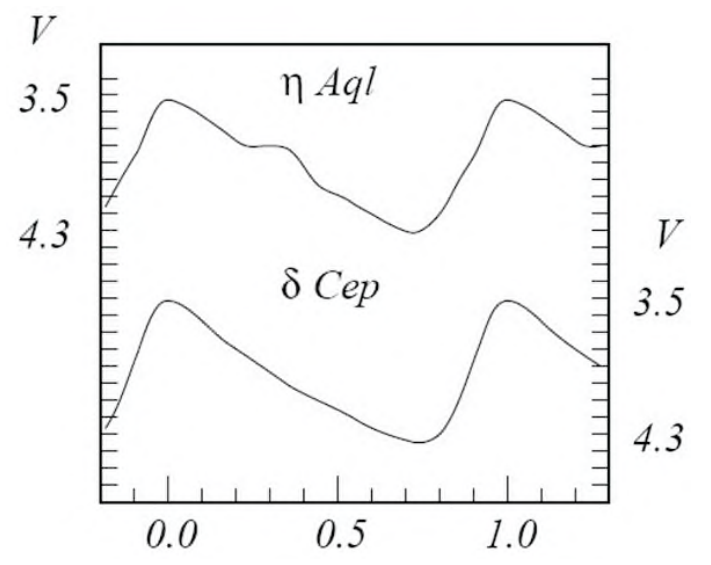

Figure 1: Light curves of $\eta \mathrm{Aql}$ and $\delta$ Cep. 


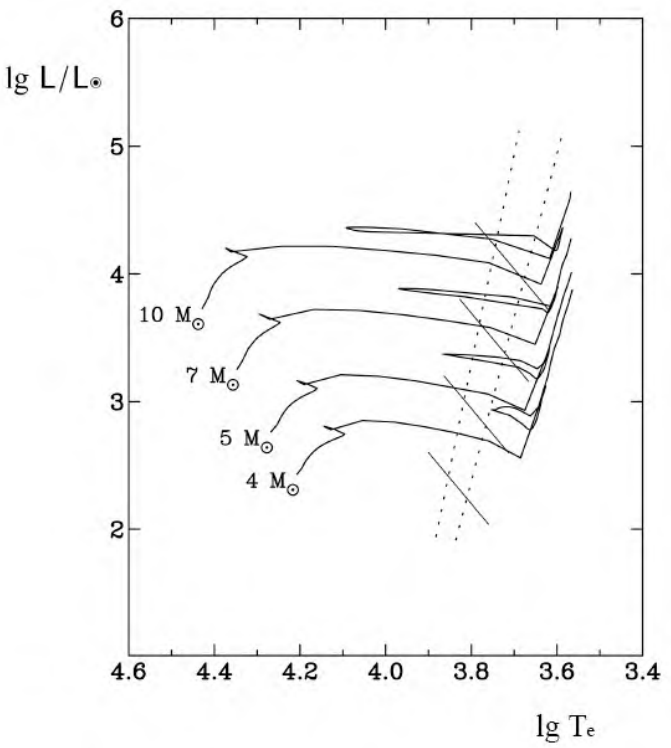

Figure 2: Evolutionary tracks and Cepheid instability strips.

Having detected parabolas in the $\mathrm{O}-\mathrm{C}$ diagrams, we become able to calculate the rate of observed evolutionary period variations. Its comparison with model computations for different crossings of the instability strip permits us to identify the number of the crossing; since each consequent crossing occurs at a higher luminosity, the period-luminosity relations for each crossing will differ. I.e., studies of Cepheid period variations give the prospect (provided we accumulate sufficient statistics) to derive individual period-luminosity relations for each of the crossings, in turn, enabling us to determine Cepheid distances more accurately.

Besides, studies of Cepheid period variations are very important for astrophysics, they provide virtually the only direct observations usable for checking results of the stellar evolution and pulsation theories.

Periods of Cepheids were studied by many authors but, till 1930s, they dealt only with improvements of actual periods for particular stars. Only [3] mentioned the secular period increase of X Pup having detected a parabola in the $\mathrm{O}-\mathrm{C}$ diagram based on the star's very first observations accumulated during $\sim 30$ years. By luck, his result turns out to be close to the correct one (Fig. 4) based on the whole time interval of observations we have now (130 years): were his studies based on a time interval of the same duration around JD 2430000, he would have derived a parabola with downward branches, i.e. a period decrease. This shows that secular period variations should be based on as long time intervals as possible.

The first systematic study of periods was published for 32 Cepheids by [4]; later, [5] published the results of his studies of 42 Cepheids. The main conclusions were that period changes were abrupt and that the longer the Cepheid's period, the stronger it varied.
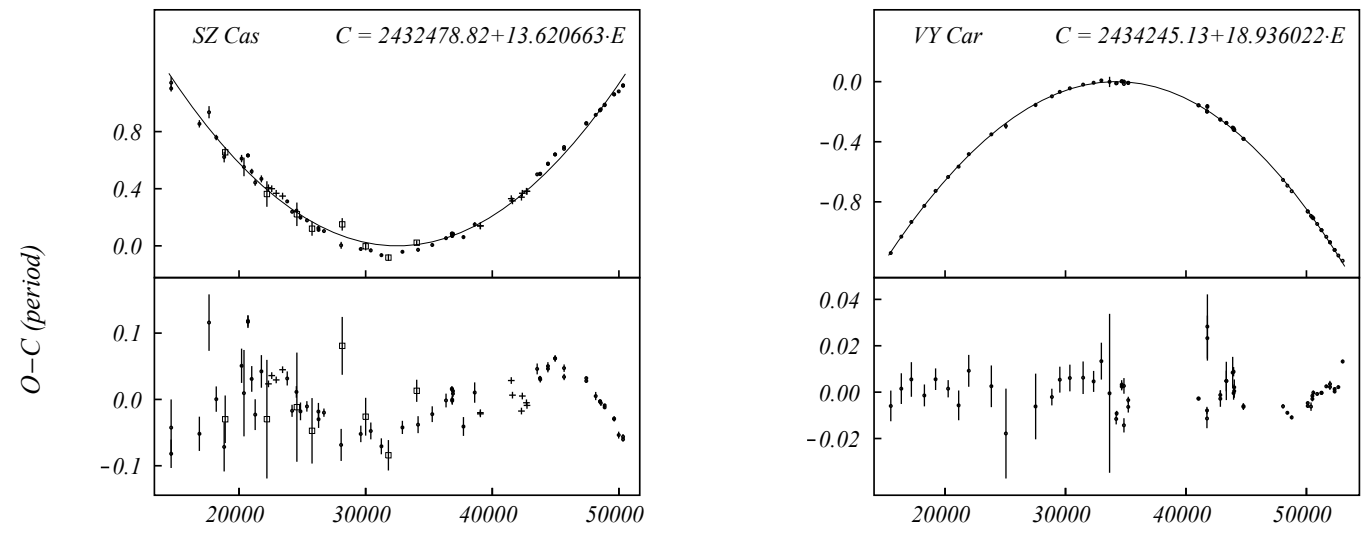

$J D 2400000+$

Figure 3: O-C diagrams for SZ Cas (the second crossing of the instability strip) and VY Car (the third crossing). 


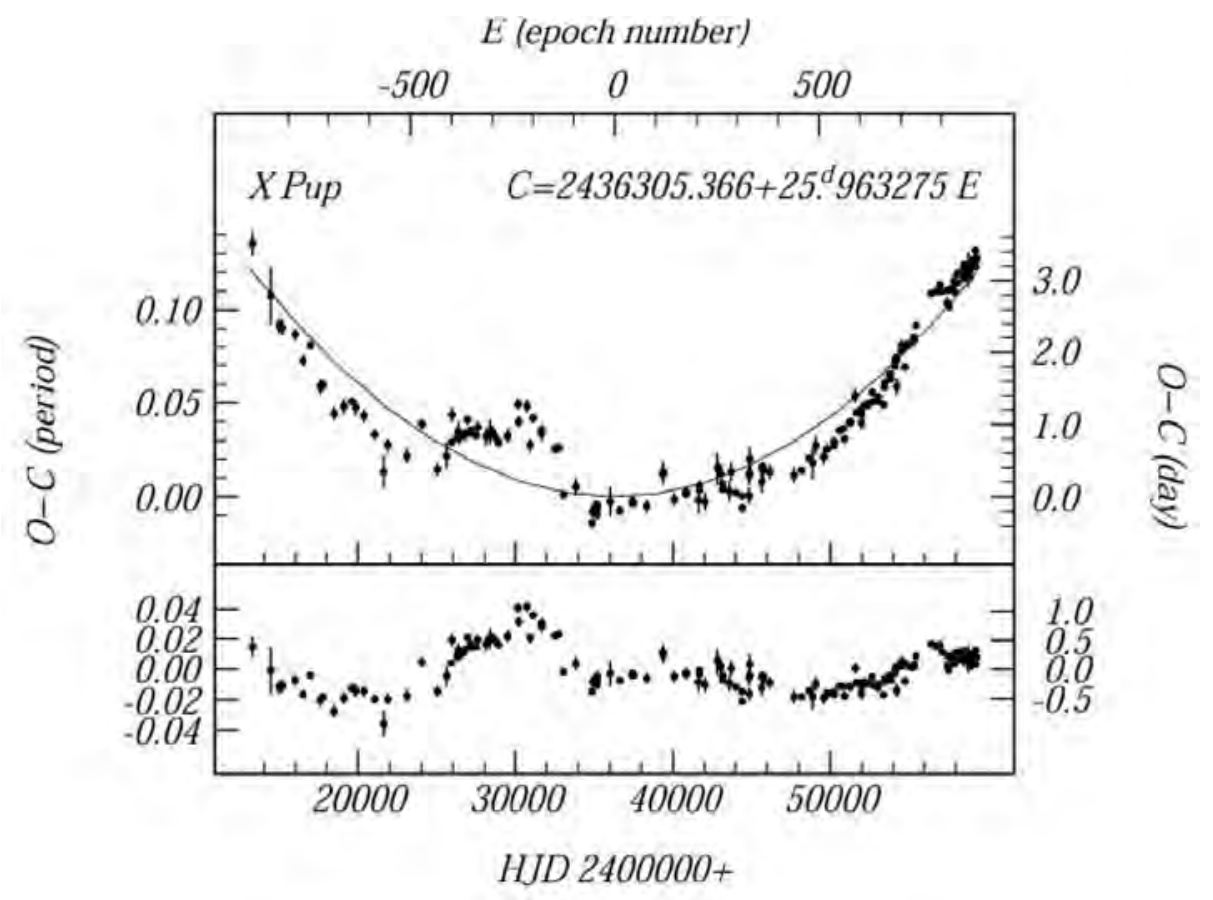

Figure 4: $\mathrm{O}-\mathrm{C}$ diagram for X Pup.

The next step in the studies of Cepheid period variations was made by Laszlo Szabados [6, 7, 8, 9, 10]. He reduced observations for $\sim 160$ bright Cepheids, hence long-known ones with many observations, and found parabolas in the $\mathrm{O}-\mathrm{C}$ diagrams for 35 of them.

For further progress, we need to improve the accuracy of derived $\mathrm{O}-\mathrm{C}$ residuals, to expand the time range of the $\mathrm{O}-\mathrm{C}$ diagrams, and to increase the number of studied Cepheids.

\section{Techniques and Results}

The most accurate method to determine $\mathrm{O}-\mathrm{C}$ residuals is that by [11]. The idea of the method is that the light curve under consideration is fitted, by least squares, to the true (standard) light curve, represented with the curve based on the most accurate, e.g. photoelectric, observations and having the zero phase of the brightness maximum. The shift providing the best fit between the observations and the standard curve gives the $\mathrm{O}-\mathrm{C}$ residual. Preliminarily, of course, the observations must be reduced to the photometric system of the standard light curve. An advantage of Hertsprung's method is that it also permits to calculate uncertainties of the $\mathrm{O}-\mathrm{C}$ residuals; its disadvantage is the large volume of computations, which was probably the reason why Hertzsprung's method was not widely used at the times of computations without computers.

We suggested a computer implementation of Hertzsprung's method [12].In the process, we write, for each observation, a conditional equation of the form:

$$
M_{i}=M_{\mathrm{av}}+A \times F\left(f_{i}, d\right),
$$

where $M_{i}$ is the observed brightness; $M_{\mathrm{av}}$, the mean brightness; $A$ is the variation semi-amplitude; $F$ is the standard light curve given in the tabular form and normalized by amplitude in the $[-1,1]$ range; $d$ is the phase shift that determines the $\mathrm{O}-\mathrm{C}$ residual. Such an approximation differs from the sine or cosine approximation only in the aspect that we use a function defined in the tabular form instead of a trigonometric function. We solve the set of non-linear equations by least squares with respect to any selection of the unknowns: $M_{\text {av }}, A, P$, and $d$.

The longer the time interval covered with the $\mathrm{O}-\mathrm{C}$ diagram, the more reliable are the results we obtain. There are two possible ways to extend the time interval: to proceed forward in time or to proceed backward in time. The first way is unlimited but very slow: to increase the time interval by 100 years, we will have to wait for 100 years... The second way is much faster, but it is limited: we can estimate a Cepheid's brightness on old plates covering 100 years within a week, but we cannot move backwards to times earlier than 1880s. Actually, we should obtain new photometric observations of Cepheids and also use brightness estimates from photographic plates of different collections, the Harvard plate stacks (USA) being the oldest (some of the plates were taken in 1880s) and the largest ( $\sim 500000$ plates $)$ collection.

Expanding the list of studied Cepheids means adding faint objects, and we encounter problems of data deficiency. Many of these stars were observed very seldom; sometimes there are no observations in the literature. 
Since late 1990s, the whole sky is regularly covered with different photometric surveys, but photometry from earlier years can be obtained only from old photographic plates.

According to the stellar evolution theory, the higher the Cepheid's mass (and hence the longer its period), the more rapidly it evolves and the faster are its period variations; for long-period Cepheids, evolutionary period variations should be detectable for time intervals of several decades (Fig. 3). However, practical cases are more difficult. We see from Figs. 3 and 4 that the parabolas are not smooth: they are overlapped with cyclic variations of the $\mathrm{O}-\mathrm{C}$ residuals. If amplitudes of cyclic variations are small compared to evolutionary variations, the parabolas are easy to detect (Fig. 5).
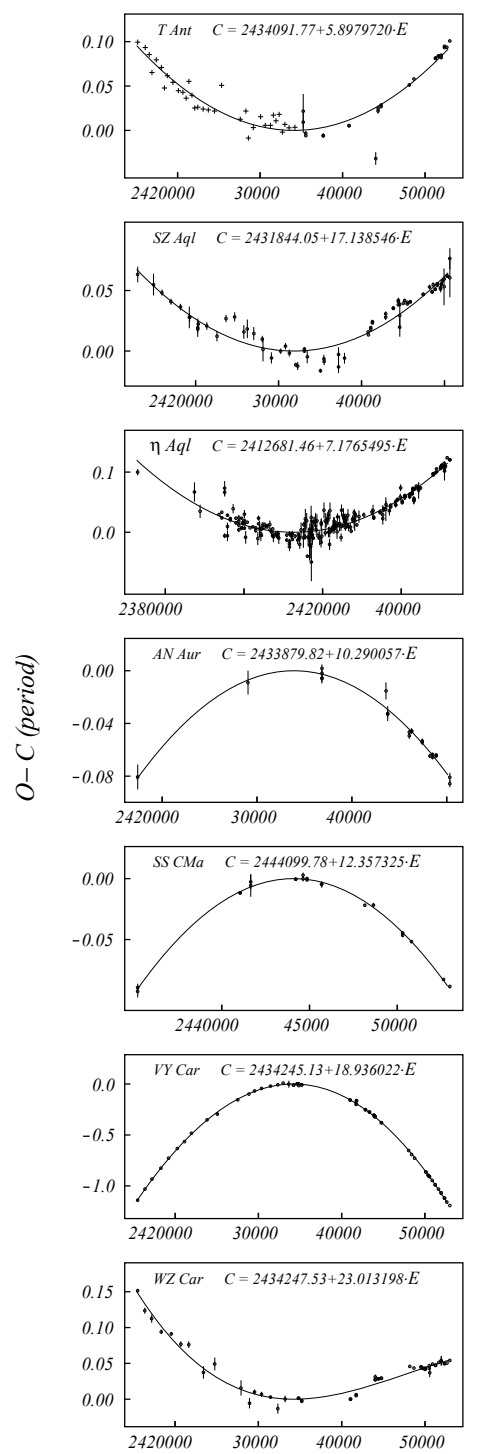
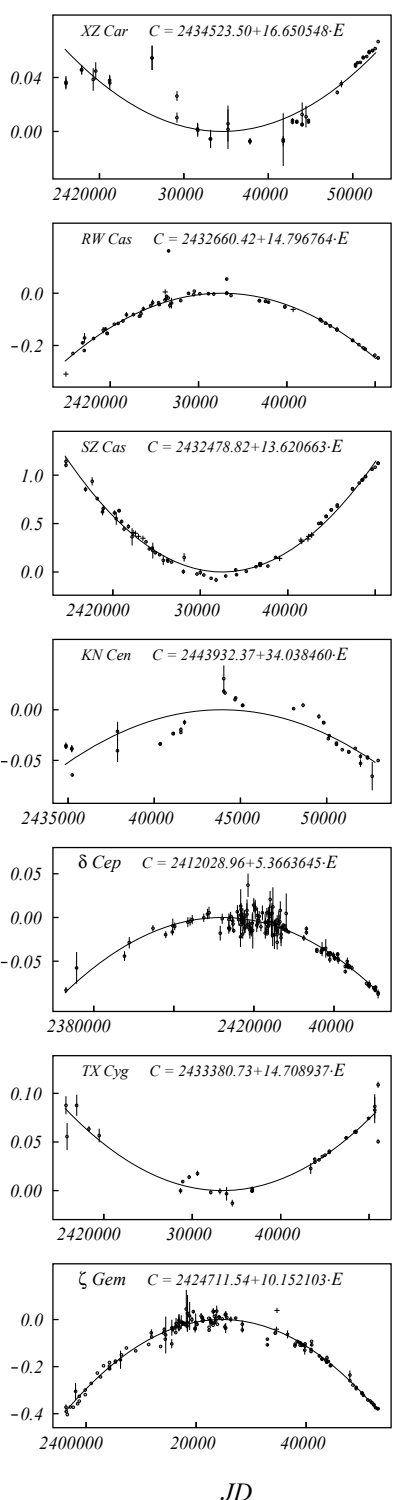
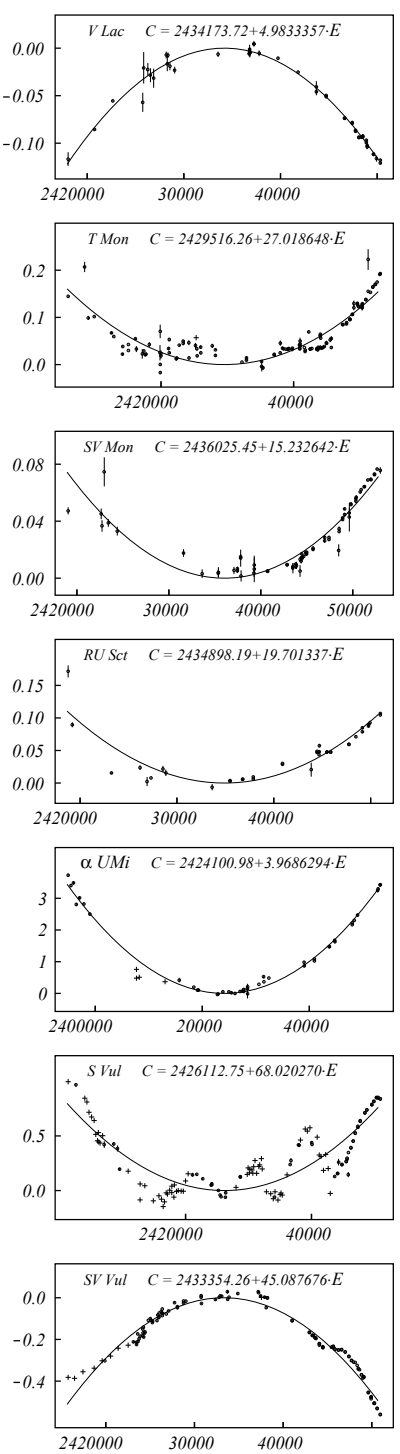

Figure 5: $\mathrm{O}-\mathrm{C}$ diagrams clearly showing parabolas.

If the cyclic-variation amplitude is comparable to that of the evolutionary variation, the parabola is traced uncertainly (Fig. 6). In the cases of large cyclic variations of the $\mathrm{O}-\mathrm{C}$ residuals, and especially if only a single wave is observed, we do not see the parabola at all. Most probably, the cyclic variations result from frequent period jumps due to random fluctuations of the pulsation period.

We computed rates of period variations for 202 Cepheids with parabolas in their $\mathrm{O}-\mathrm{C}$ diagramsand and found a good agreement between observations and theory (Fig. 7; [13]).

To confirm that the detected period variations are real, we should demonstrate that random fluctuations of the pulsation period, if present, do not dominate the $\mathrm{O}-\mathrm{C}$ diagram. We search for random fluctuations using the method suggested by [14]. 

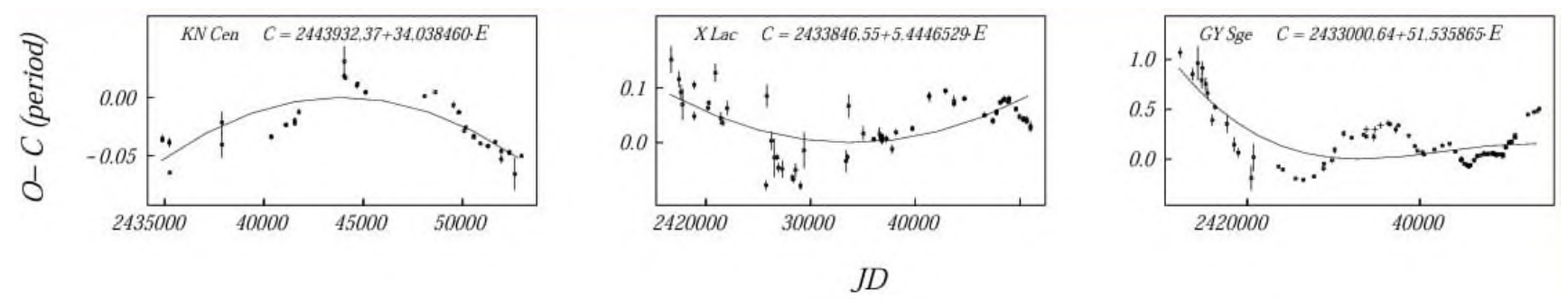

Figure 6: $\mathrm{O}-\mathrm{C}$ diagrams with uncertain parabolas.

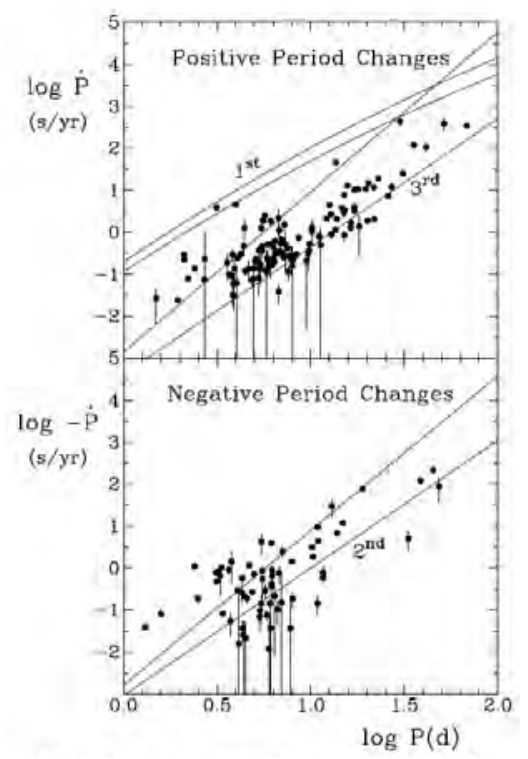

Figure 7: Rates of period variations compared to theory.

Eddington \& Plakidis assumed that some of variations in the $\mathrm{O}-\mathrm{C}$ diagram were due to random fluctuations $(\varepsilon)$ of the period from cycle to cycle. To reveal them, they computed absolute values of all delays $u(x)=|a(r+x)-a(r)|$ for maxima separated with $x$ cycles. The mean values $\langle u(x)>$ of all accumulated delays, according to Eddington $\&$ Plakidis, should be related to the random period fluctuations $\varepsilon$ as:

$$
<u(x)>^{2}=2 \alpha^{2}+x \varepsilon^{2},
$$

where $\alpha$ describes the amount of random errors in the measured times of maximum brightness. Thus, the plot of $<u(x)\rangle^{2}$ versus $x$ should be a straight line with the slope $\varepsilon^{2}$. Strictly speaking, the Eddington \& Plakidis method should be applied to reductions of $\mathrm{O}-\mathrm{C}$ residuals for consecutive individual brightness maxima; for long-period Cepheids, we may use brightness maxima determined from the mean light curve covering a time interval of several periods. Figure 8 shows the Eddington \& Plakidis test for the long-period Cepheid S Vul.

The space telescope SMEI (CORIOLIS project) obtained numerous continuous observations for the brightest short-period Cepheids. These data made it possible to derive many consecutive individual times of brightness maxima; we used them to search for random period fluctuations of these Cepheids [15]. The relative random period fluctuations, $\varepsilon / P$, are by two orders of magnitude lower for short-period Cepheids than for long-period ones, confirming the conclusions from [5].

\section{Conclusions}

Our results confirm the effectiveness of period-variation studies for identification of the instability-strip crossing number and for deriving a more accurate color-luminosity relation for classical Cepheids, with the prospects to improve the distance scale in the Universe. 

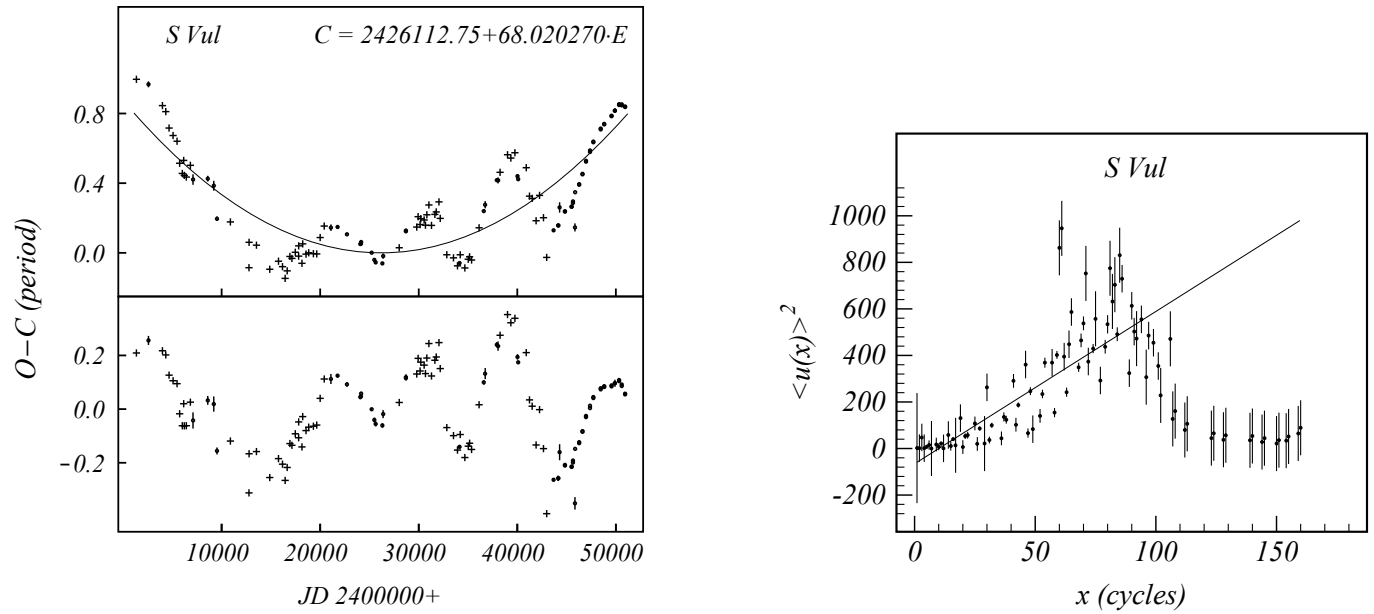

Figure 8: The Eddington \& Plakidis test for $\mathrm{S}$ Vul. $\varepsilon=2.55 \pm 0.70, \varepsilon / P=0.0375$.
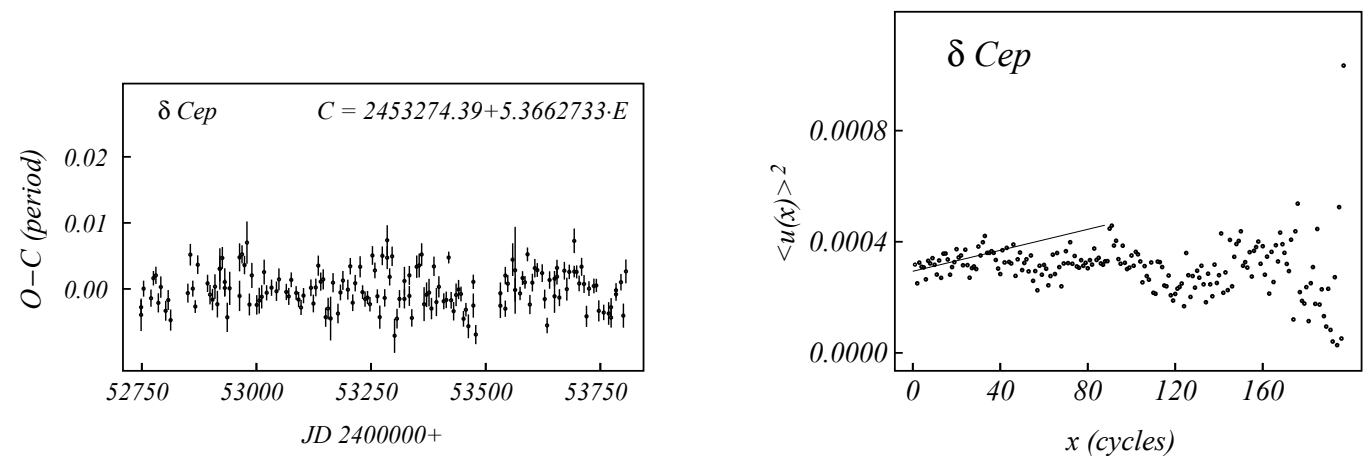

Figure 9: The Eddington \& Plakidis test for $\delta$ Cep from CMEI data. $\varepsilon=0.0014 \pm 0.0007, \varepsilon / P=0.00026$.

\section{References}

1. N. N. Samus', E. V. Kazarovets, O. V. Durlevich, N. N. Kireeva, and E. N. Pastukhova, Astronomy Reports, 61, 80, 2017.

2. D. M. Skowron, J. Skowron, P. Mróz, A. Udalski, et al., Acta Astronom., 69, 305, 2019.

3. L. V. Robinson, Harvard College Observatory Bulletin, 872, 11, 1930.

4. B. W. Kukarkin and N. Florja, Zeitschrift für Astrophys., 4, 247, 1932.

5. P. P. Parenago, Peremennye Zvezdy, 11, 236, 1956.

6. L. Szabados, Commmunications of the Konkoly Observatory Hungary, 70, 1, 1977.

7. L. Szabados, Commmunications of the Konkoly Observatory Hungary, 76, 1, 1980.

8. L. Szabados, Commmunications of the Konkoly Observatory Hungary, 77, 1, 1981.

9. L. Szabados, Commmunications of the Konkoly Observatory Hungary, 94, 1, 1989.

10. L. Szabados, A. Derekas, C. Kiss, and P. Klagyivik, MNRAS, 426, 3154, 2012.

11. E. Hertzsprung, Astronomische Nachrichten, 210, 17, 1919.

12. L. N. Berdnikov, Soviet Astronomy Letters, 18, 207, 1992.

13. D. G. Turner, M. Abdel-Sabour Abdel-Latif, and L. N. Berdnikov, PASP, 118, 410, 2006.

14. A. S. Eddington and S. Plakidis, MNRAS, 90, 65, 1929.

15. L. N. Berdnikov and I. R. Stevens, in International Conference on Space Information Technology, 26-27 November 2009, Beijing, China, 866 (2006). 


\section{On some recent advances in celestial mechanics}

B. Kondratyev ${ }^{1}$ and N. Emelyanov ${ }^{2}$

bwork@boris-kondratyev.ru; emelia@sai.msu.ru, WWW home page: http://lnfm1.sai.msu.ru/emelia/

${ }^{1}$ Lomonosov Moscow State University, Faculty of Physics, Russia

${ }^{2}$ Lomonosov Moscow State University, Sternberg State Astronomical Institute, Russia

The article is based on the plenary report at the VAK 2021. It describes the development of ideas and reviews some recent achievements in modern celestial mechanics. We proceed from the fact that the classical definition of this science given in textbooks and encyclopedias does not fully reflect the content of modern celestial mechanics. A broader and more capacious term is "dynamic astronomy". This science is complex and includes not only classical but also relativistic celestial mechanics, as well as the theory of equilibrium figures and computer simulations; besides, we should not forget about qualitative methods, the peak of which is the creation of KAM theory. Here we trace the development of a chain of ideas from Keplerian orbits to osculating Lagrange ellipses, which leads eventually to Gaussian rings and two models (R-disk and R-toroid) based on precessing Gaussian rings. The review also includes a presentation of results obtained in recent years by research groups in the Russian Federation.

Keywords: motion of celestial bodies, celestial mechanics, dynamic astronomy, perturbation theory

DOI: $10.51194 /$ VAK2021.2022.1.1.003

\section{About the subject of celestial mechanics}

Celestial mechanics is a collective science; many scientists have contributed to its development; the names of Kepler, Newton, Euler, Lagrange, and Laplace speak for themselves. In the history of this science there were bright moments: the rediscovery of the "lost" Ceres made famous Gauss, Le Verrier "at the tip of his pen" discovered the planet Neptune (Galle 1846). Successes, of course, were then (the spectacular discovery of Trojans in Jupiter's orbit), there are them now (KAM theory, the most precession calculations of the gravitational maneuvers of the Vega and Viking vehicles, the Nobel Prize for the discovery in 1995 of a planet around the main sequence star). But it is important to remember — it was celestial mechanics that became the first science and the touchstone for the human mind, where failure to solve the problem was tantamount to loss of faith in the human mind.
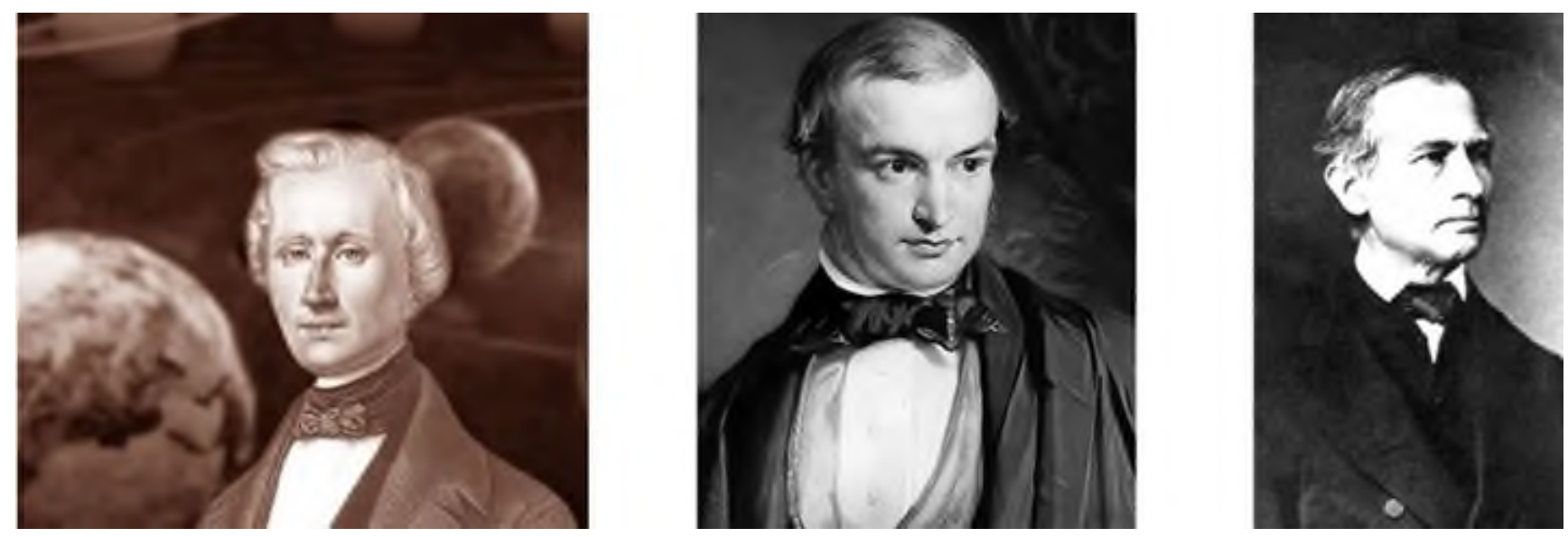

Figure 1: From left to right: Urban Le Verrier (1811-1877), John Adams (1819-1892), Johann Galle (1812-1910).

Celestial mechanics (more broadly, "dynamic astronomy") studies almost everything that moves and rotates in the cosmos: from dust particles to comets and asteroids, from AEC, planets, and their satellites to stars and galaxies. The development of celestial mechanics has gone through the practice of a variety of applications, so the range of problems in this science is exceptionally broad.

Traditionally, Celestial Mechanics is defined as the field of astronomy, where Newton's law of inverse squares and methods of classical mechanics are used to study the motion of bodies. But this definition does not fully reflect the content of modern celestial mechanics.

First, modern celestial mechanics is based not only on the law of universal gravitation, it also takes into account other kinds of forces, for example: tidal forces derived from gravity (they are inverse to the distance cube and are associated with energy dissipation and phase volume compression), electromagnetic forces on spacecraft and charged dust particles, reactive forces, radiation pressure, drag forces, etc. But gravity, of course, commands the parade.

Secondly, for more than a hundred years, relativistic celestial mechanics have been successfully developing, where the laws of GR (general relativity) are applied. Le Verrier's research revealed the abnormal effect of apsidal 
precession in Mercury, and explained it to the GR. Proof of the reality of the discrepancy $43^{\prime \prime}$ in the century in the movement of the apside line at Mercury — is also the success of celestial mechanics. The transition from GR to Newtonian theory of gravity corresponds to the field of small values of the relation $\varphi / c^{2}$. In our time, relativistic effects are studied in the movement of many celestial bodies, including the Moon, planets and satellites. And this is justified, because, for example, the amount of geodetic precession for some Jupiter satellites is comparable to their precession in the Newtonian approximation.

Thirdly, celestial mechanics also includes the theory of equilibrium figures (TEF), because the law of universal gravitation makes it possible to study not only the motion of planets around the Sun, but also the shape of the planets themselves. Nowadays, the study of the figures of equilibrium and the internal structure of celestial bodies is an actively developing direction in celestial mechanics.

Fourth, computer simulation methods play an important role in celestial mechanics. Their development is the key to new discoveries in astronomy. Numerical methods make it possible to predict the motion of a celestial body with almost unlimited accuracy, but do not give a qualitative picture of the motion.

Fifth, in the works of Poincare there appeared qualitative methods for the study of differential equations. Let us note the contribution of Poincaré:

- to the problem of divergence of series in celestial mechanics. Now the noise around the divergence of the rows has subsided, but at one time it was a big fly in the ointment. The terms with small denominators ruin the series, and Poincaré showed how to integrate differential equations with divergent series!

- in the study of periodic and almost periodic solutions. Unable to integrate the differential equation (and there are most of such equations in celestial mechanics), Poincaré proposed a small parameter method for finding periodic solutions; his idea: if there are many closed periodic orbits, it is possible to establish how the curves corresponding to real nonperiodic motions will be located between them.

- Poincaré discovered integral invariants and applied them to prove the recurrence theorem; now invariants are applied both in atomic theory and in stellar dynamics.

- New bright colors in the study of dynamical systems were introduced by George Birkhoff, who proved the ergodic theorem and the existence of two fixed points of the ring corresponding to periodic solutions. Now ergodicity has become a basic concept in the theory of dynamical systems. However, the Poincaré recurrence theorem still does not guarantee ergodicity if the phase space of the system is not uniform.

- Poincaré and A.M. Lyapunov constructed a theory of periodic solutions, and Kolmogorov, Arnold and Moser - almost periodic solutions. Qualitative methods have led to the creation of the KAM theory. Its essence: if the Hamiltonian of the system is represented in the form $H=H_{0}+\Delta H(\Delta H$ is the small perturbation and frequencies $\omega$ in $H_{0}$ are incommensurable), then the perturbed motion will almost always be bounded by the N-torus.

\section{From kinematics to dynamics}

The science of planetary motion has come a long way from astrological manipulations of priests to a witty (and effective!) kinematic method (remember the eccentrics and epicycles of Hipparchus, Eudoxus and Ptolemy). And although the revolution in astronomy, made by Copernicus and his followers (Bruno, Galileo, Kepler), did not go beyond the kinematic approach, the foundation was laid: Kepler's ellipses are still a test for the title of Homo sapiens!

With the emergence of the dynamic method in the works of Newton and Euler, the art of creating models based on differential equations of motion appeared and began to be honed in celestial mechanics. Celestial mechanics became the focus of scientists' attention, new tasks stimulated interest in comprehending the Universe.

\section{Some basic problems of celestial mechanics}

An interesting picture: the inverse square law, which lies at the foundation of dynamic astronomy, is beautiful, elegant and makes it easy to formulate many problems, but in the course of solving them, this ease evaporates somewhere. The fact is that there are very few problems with an analytical solution in celestial mechanics even now. Let's briefly dwell on them.

\subsection{Two-body problem and Keplerian ellipses}

The foundation of classical celestial mechanics is the two-body problem. It is remarkable in that it has an additional integral of motion (the Laplace-Runge-Kutta vector). At one time, it was the need to solve the Kepler equation accurate to the $1^{\prime \prime}$ arc stimulated the development of numerical methods. And now, through the study of Keplerian 
orbits, the formation of any specialist in the exact sciences passes. In the N-dimensional space, the two-body problem is still being investigated, since it allows one to check some results in the cosmology of the early Universe. Mathematically, the two-body problem has been solved, but in the astronomical aspect, a wide scope for creativity remains: these are problems about the topology of orbits, problems for bodies with variable masses, particle scattering, problems with Gaussian rings.

The inverse problem of two bodies is the Bertrand's problem of finding trajectories for a given force (in another version, it is the problem of finding a potential for a given family of orbits).

By the way, the following problem has not yet been solved: find all the potentials $\varphi(x, y)$ in which, at least in some continuous region of the plane $(x, y)$, all orbits are closed (ellipses do not count!).

\subsection{Osculating Lagrange ellipses}

By the beginning of the 19th century, the relay race from Kepler's ellipses passed to osculating Lagrange ellipses, and on their basis, through the efforts of many scientists (Lagrange, Delaunay, Encke, Hansen, Hill, Cowell), the perturbation theory appeared. It became possible to find answers to the most difficult questions of celestial mechanics when Kepler's laws become only approximate. In fact, perturbation theory is the idea that you can study something unknown starting from a known value. One of its main techniques: an ellipse is taken as the initial (intermediate, or reference) orbit, and the discrepancies between the real and reference orbits are laid out in a row. But these series do not always work (due to the presence of members with small denominators). In this situation, Hansen ingeniously proposed an idea: to take not an ellipse for the reference orbit, but a more complex curve: then the series for the deviations of the real orbit from the accepted reference orbit will not contain inconvenient terms.

\subsection{Gaussian rings}

Another direction, the Gaussian rings, originates from Kepler's ellipses. In 1818, Gauss proposed to average the motion of the mass $m$ over the ellipse of the orbit to calculate the secular perturbations; a ring (Gaussian) is formed with a density the greater, the longer the body is in motion on the corresponding arc $d s$.
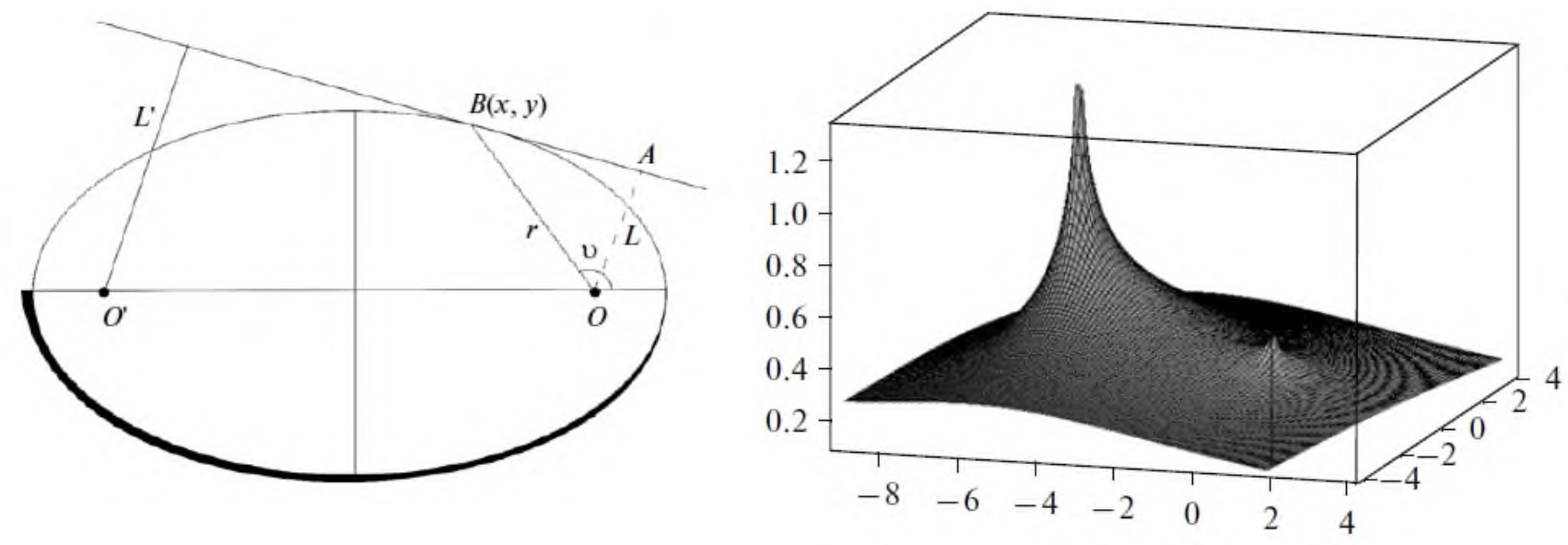

Figure 2: Geometric diagram (left) and potential surface (right) for a Gaussian ring.

Gauss himself did not find the potential of the ring, but now this potential is known in an analytical form $[1,2,3]:$

$$
\begin{gathered}
\Phi_{\text {ring }}=\frac{2 G m}{\pi \sqrt{\lambda-\nu}}\left\{K(k)+\frac{e a\left(x_{1}+e a\right)}{a^{2}+\nu}[\Pi(n, k)-K(k)]\right\}, \\
n=\frac{a^{2}+\nu}{\nu-\lambda}, \quad k=\sqrt{\frac{\mu-\nu}{\lambda-\nu}},
\end{gathered}
$$

where $m$ is the mass of the ring, $a, b$ its semiaxis, $e$ is the eccentricity. $K(k)$ and $\Pi(n, k)$ are the complete elliptic Legendre integrals of the first and third kind.

\subsection{Systems of Gauss rings. Mutual energy of rings}

With the development of the averaging method, systems of Gauss rings began to be studied [4]. 
In Sternberg State Astronomical Institute has developed a new approach to the study of long-period and secular perturbations in some problems of celestial mechanics. It relies not on the perturbing Lagrange function, but on the mutual potential energy of the Gaussian rings. For coplanar rings with a common focus, this mutual energy is [5]

$$
W_{m u t}=-\frac{G m_{1} m_{2}}{\pi a_{1}}\left[W_{0}+W_{1} e_{1}+W_{2} e_{2}+W_{11} e_{1}^{2}+W_{22} e_{2}^{2}+W_{12} e_{1} e_{2}\right] .
$$

We emphasize that the mutual energy of two elliptical Gaussian rings was also found in the more general case, when the rings have a small mutual inclination [6]. Comparison with the traditional method of expansion of the perturbing Lagrange function $R$ showed the adequacy of the new approach: instead of averaging the expression obtained in a complex way for the $R$ it is methodologically simpler to immediately calculate the mutual energy of the Gaussian rings $W_{m u t}$.

\subsection{Two-dimensional generalization of the Gaussian ring (R-disk model)}

According to the laws of mechanics, due to external disturbances and the effects of the GR (general relativity), an apsidal precession of the Gaussian ring occurs. As a result, with azimuthal averaging, an R-disk is obtained [7] (in this article, the lower bound of the disk is determined by the conditions for the survival of stars from the tidal influence of the black hole, and the upper limit depends on the parameters of the stellar orbits).
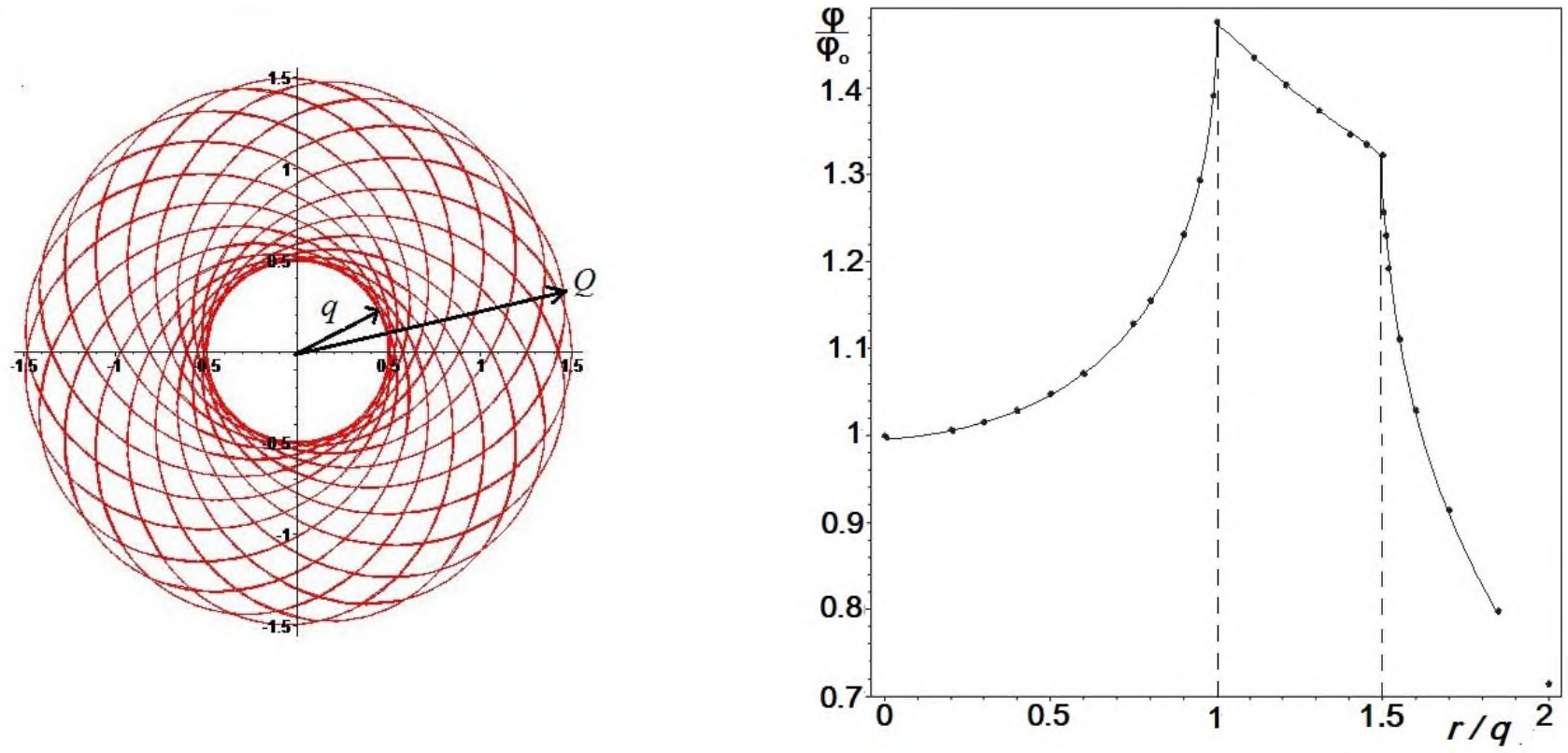

Figure 3: R-disk (at left) and R-disk potential (at right).

\subsection{D generalization of the precessing Gaussian ring (R-toroid model)}

A new analytical model (R-toroid) for studying secular perturbations in celestial mechanics has been built [8]. The model is based on a triple averaging of the motion of a material point and is reduced to a chain of transformations: 1D Gaussian ring - 2D R-ring — 3D R-toroid.

The shape, structure and gravitational potential of the R-toroid are studied, and an expression for the mutual energy of the R-toroid and the outer Gaussian ring is obtained. This made it possible to derive a system of equations for the secular evolution of osculating orbits in the gravitational field of the R-toroid (and, additionally, of the central precessing star). Besides, on the basis of a system of two R-toroids, a method has been developed for studying the secular (apsidal and nodal) precession of orbits in circumbinary systems consisting of a binary star and outer exoplanet [9].

Models of interacting and precessing Gauss rings give a new interpretation of problems in perturbation theory and make it possible to simplify complex calculations of the classical method of the perturbing Lagrange function.

\subsection{Problem of two fixed centers}

In the "storehouse" of celestial mechanics is the problem of two fixed centers, which was posed and solved by Euler. The original version of this problem was studied by J. Vinti and Soviet scientists (G.N. Duboshin, E.P. Aksenov, 

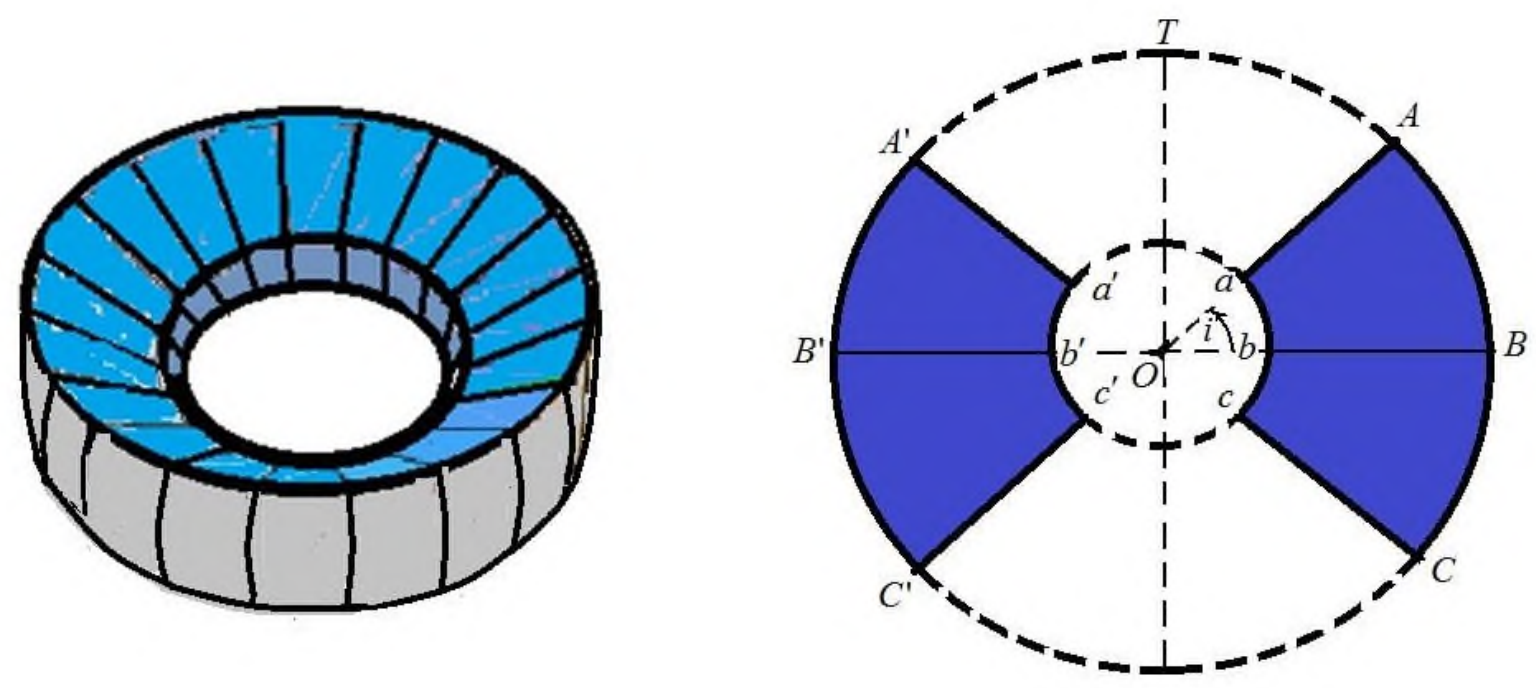

Figure 4: R-toroid, on the right - its meridional section.

E.A. Grebenikov, V.G. Demin), who managed to squeeze the problem of the motion of artificial satellites in the Earth's field into "Procrustean bed" of the problem of two stationary centers with imaginary point masses. And here a more complex curve than the Keplerian ellipse is taken as an intermediate orbit for the satellite.

Comment. Of interest is the passage to the limit for modeling the potential of two imaginary masses in the asymptotic limit from the potential of a rod with an imaginary density distribution.

\subsection{The many-body problem}

\subsubsection{Relevance of the problem}

It is important to emphasize: the many-body problem in celestial mechanics is not a waste material, but a classic that does not go out of fashion! This problem finds applications in all areas of physics, including astrophysics, nuclear physics and particle physics.

Lagrange, Laplace and Poincaré began to reveal its inner wealth: here the analytical methods of mechanics were improved, the theory of perturbations was born and flourished; here, under a veil of mathematical uncertainty, the concept of chaos in dynamical systems appeared and the mighty determinism of Laplace was shaken. In celestial mechanics, regardless of quantum mechanics (the Heisenberg uncertainty relation in 1927), it was understood that to describe the position of a particle, due to the extreme sensitivity of trajectories to initial conditions, it is necessary to proceed to determining the probability of finding a particle in space. And in this fundamental question, physics and celestial mechanics are on parallel rails! Example: the collision of an asteroid with the Earth is also a probabilistic event, since the parameters of an asteroid orbit always contain some uncertainty.

\subsubsection{Three-body problem}

In general, the three-body problem is not integrable, but for some particular cases, analytical solutions are known. Many results were obtained by computer methods, among them there are surprises (the figure- 8 solution to the three-body problem $+600(!)$ other intricate trajectories similar to it) [10].

\subsection{Particular problems in the general problem of many bodies}

\subsubsection{Equation reduction problem}

The search for new productive variants of dynamic reduction is urgent. Recall that reduction is the process of reducing the number of equations of motion with allowance for the integrals of motion and conserved quantities. Note the following version of the reduction [11]: since any configuration of three points defines a triangle, then all the variables of the problem can be represented as a set of geometric (shape and size of the triangle) and rotational (orientation of the triangle) variables. 

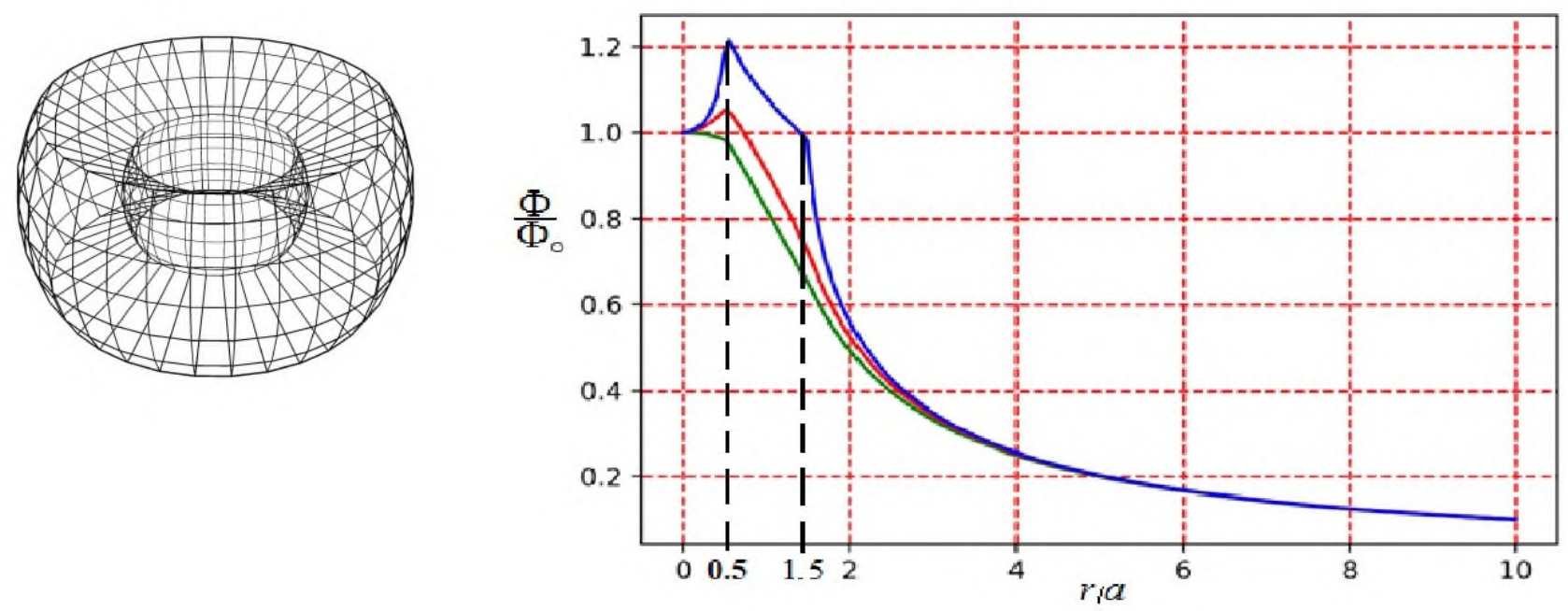

Figure 5: Figure of the R-toroid (at left) and the dependence of the normalized potential of the R-toroid from in the equatorial plane (at right).

\subsubsection{Restricted Three-Body Problem}

In practice, the restricted problem of three bodies is most often considered (when the mass of the third body is small in comparison with the two main "primaries"). Its options are: — limited circular, elliptical ..., hyperbolic ... Further, these three options are scattered in a wide fan into many subtasks (where all parameters of the system vary: relative distances, the shape of bodies, light pressure is taken into account, the mass of one or all bodies may depend on time, etc.).

\subsubsection{Libration points}

Solutions in the form of libration points in the three-body problem. Three Euler (unstable) collinear points $L_{1}$, $L_{2}, L_{3}$ were found first, then two triangular Lagrangian points $L_{4}$ and $L_{5}$ with stable motion in their vicinity were discovered. Numerous variants of tasks for the study of libration points are being considered now. In astrodynamics, it is planned to use all five libration points for the exploration of the Solar system by space vehicles. Of particular interest are two families of asteroids near the libration points $L_{4}$ and $L_{5}$ in the orbit of Jupiter. Discovered Trojan asteroids, whose orbits jump between $L_{4}$ and $L_{5}$.

\subsubsection{Central configurations}

This is a large complex of tasks in which the accelerations of all bodies are directed to one center. There are many options for research, and the study of "round dances" in $N$ dimensional space is a favorite topic among mathematicians.

\subsubsection{Sitnikov problem}

We also note the problem of K.A. Sitnikov (W. Macmillan), where two bodies of primaries have circular or elliptical orbits, and the third body of zero mass moves along the normal.

\subsubsection{Hill's Problem}

Hill's problem is especially in demand (where the motion of a small body in the vicinity of a planet with a large disturbing influence of the Sun and other planets is considered). Many variants of the Hill's problem are being actively studied now. In particular, the theory of the motion of the Moon is essentially the Hill's problem. It is the Brown-Hill series that have become a reliable tool for the most important task of calculating the ephemeris of the Moon.

\subsubsection{First integrals of motion}

There are 10 first integrals of motion - six integrals expressing the law of motion of the center of mass of the system; three integrals for the law of conservation of angular momenta of bodies, and an integral of the total 

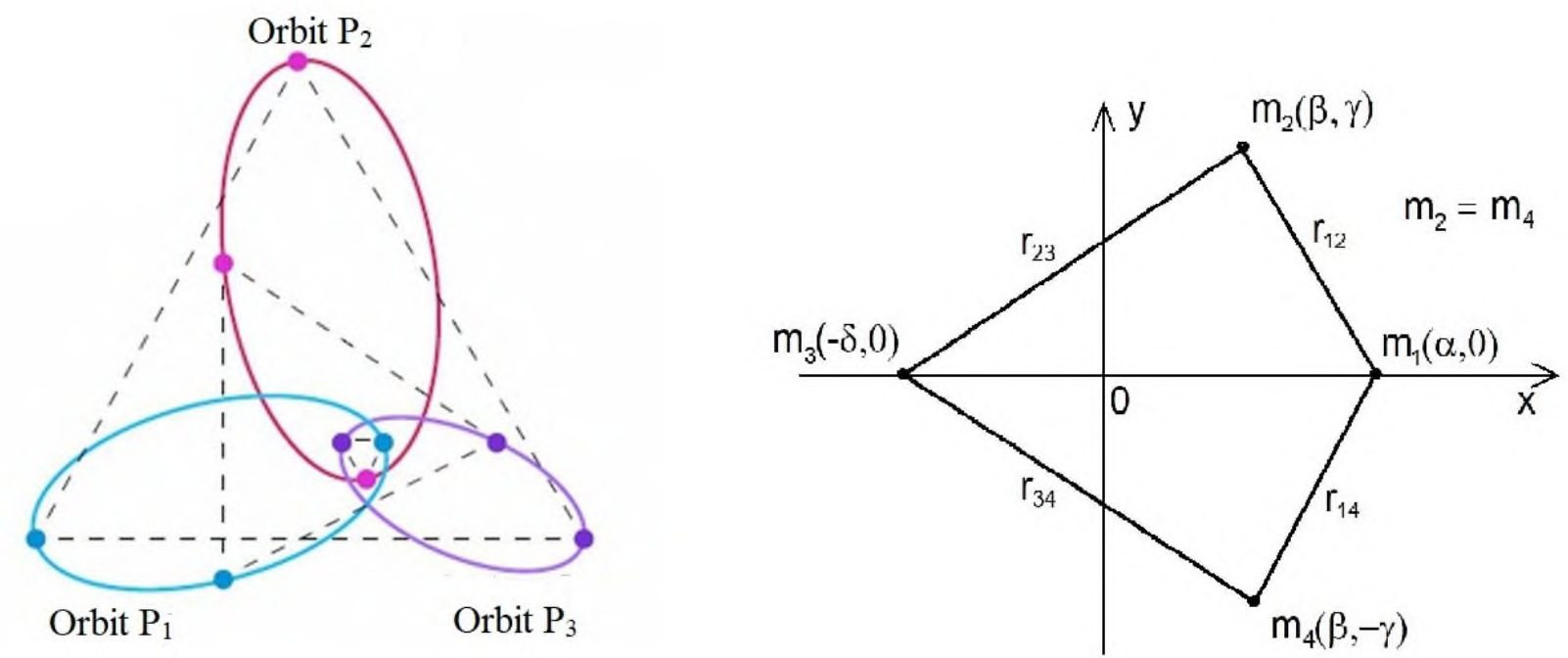

Figure 6: Scheme of the three-body problem (at left) and example of four-body central configuration (at right).

mechanical energy of the system. Alas, for the general case of the three-body problem, there are no other algebraic (Bruns) or integrals of motion expressed in terms of known functions (Poincaré).

\subsection{Theory of equilibrium figures}

Within the framework of the theory of equilibrium figures (TEF), the shape, structure and oscillations (and not only small, but also nonlinear), as well as the stability of celestial bodies are studied. This branch of science borders on geophysics, astrophysics and the dynamics of stellar systems. Equilibrium figure models are built for asteroids, planets and satellites; here is a wide range of problems for stars and galaxies. The history of the TEF is filled with bright discoveries and permeated with deep analytical research by Clairaut and Laplace, Dirichlet and Riemann, Poincaré, Lyapunov and Chandrasekhar.

\subsubsection{Maclaurin spheroids, Jacobi triaxial ellipsoids, Roche figures}

Maclaurin spheroids, Jacobi triaxial ellipsoids, Roche figures are widely used in astronomy; they are supplemented by the Clairaut equation (the flattening of the isodensite towards the center decreases!). While still young, Poincaré and Lyapunov discover the wonderful of non-ellipsoidal equilibrium figures. Here, pear-shaped figures (the 3rd harmonic on the surface of the ellipsoid), playing the role of a bridge between Jacobi ellipsoids and binary stars, attracted close attention. Through the division of the "pear", Poincaré and Darwin hoped to solve the main mystery of cosmogony: to understand how the Moon, planets with satellites, and multiple stars were formed. The special charm of this hypothesis was given by the fact that the decision on the possible division of the "pear" was hidden under a veil of mathematical uncertainty.

This crisis situation was dealt with by A.M. Lyapunov, who proved that all "pears" are age old and dynamically unstable. A heated controversy ensued. In our days, computer calculations have already confirmed: the sequence of "pears" ends not with a division, but with a figure with a "spout" (a critical point), from where, as from a watering can, the liquid flows out [12].

\subsubsection{Some current trends in TEF}

Further progress in the theory of equilibrium figures is related to consideration of the internal velocity field.

Here is the famous formulation of the Dirichlet problem [13,14,15]: do the laws of mechanics allow such motion of liquid gravitating ellipsoids that the velocity field in them would be linear on coordinates. It turned out that they do! This allowed us to reconsider the entire theory. This is where B. Rieman and S. Chandrasekhar wotked here, a mathematical apparatus was created for a set of problems about nonlinear vibrations and equilibrium figures of liquid ellipsoids, cylinders and disks. In addition to gravitation in these models, the magnetic field can also be taken into account. 

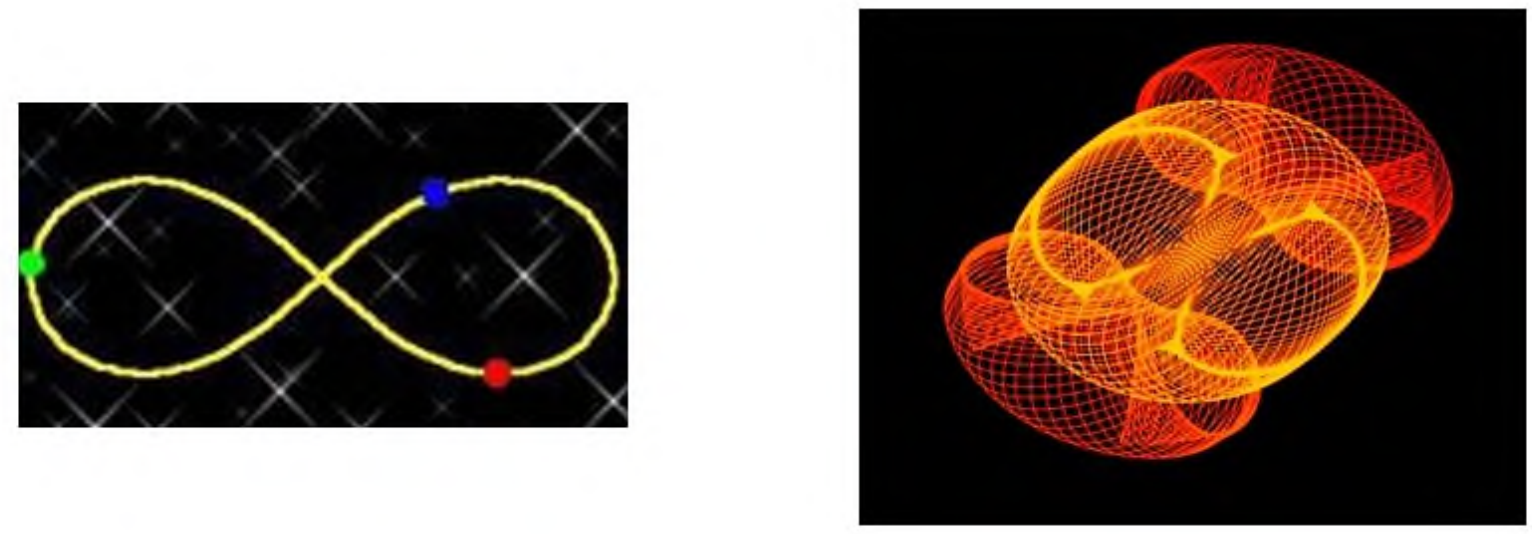

Figure 7: The Moore Eight and Related Trajectories in the 3-Body Problem.
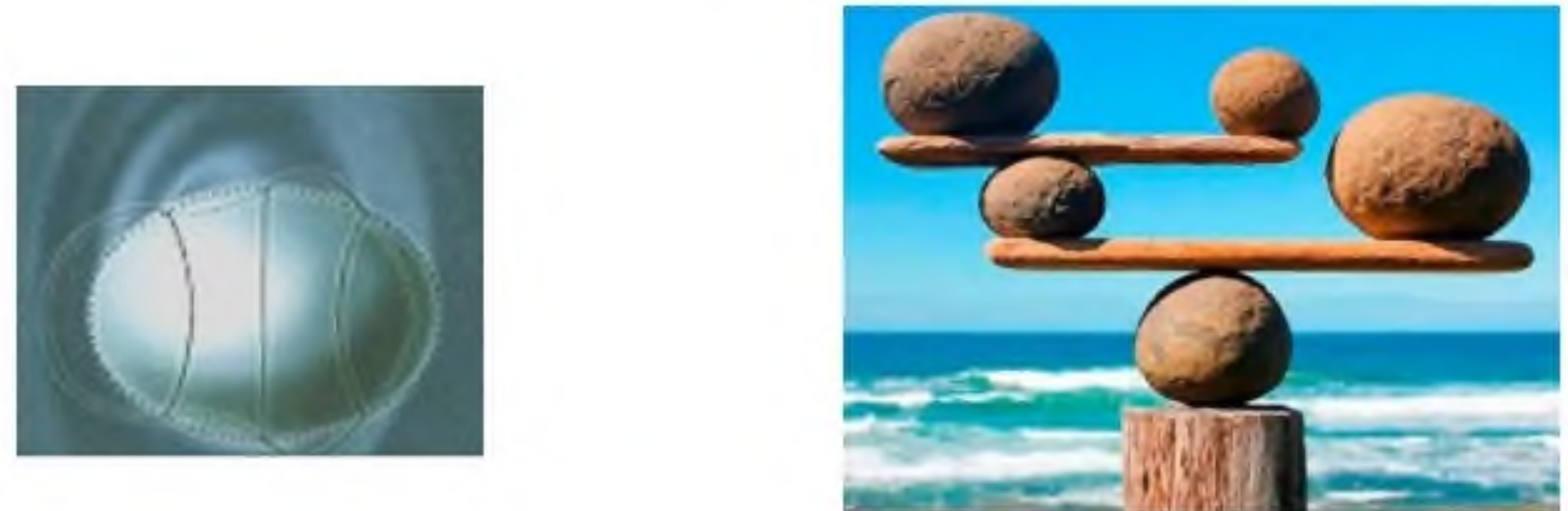

Figure 8: To the theory of equilibrium figures.

\section{Generalization of the Dirichlet Problem to collisionless Stellar Systems}

Today [15], a generalization of the Dirichlet problem for liquid figures to the dynamics of stellar systems has been obtained: a theory has been constructed that describes nonlinear oscillations, equilibrium and stability of self-consistent phase ellipsoidal models of collisionless stellar systems with a quadratic potential. All six phase models of stellar systems have been constructed and it has been proved that they exhaust all possible cases of such ellipsoidal models.

In the theory of inhomogeneous planets, the method of ellipsoidal surfaces is only a first approximation. More precisely, the surface of the equilibrium figure is described by the formula

$$
r(R, \theta)=R\left(1+s_{2}(R) P_{2}(\cos \theta)+s_{4}(R) P_{4}(\cos \theta)+\ldots\right),
$$

where $s_{2}(R)$ is describes ellipsoidal surfaces, $s_{4}(R)$ is the 4 th order correction. In this direction scientists [16] continue to study the figures of equilibrium of celestial bodies.

Non-impact mass separation mechanism In 2016, a new, non-impact mass separation mechanism was developed for two-component equilibrium figures (rocky core + ice shell), which makes it possible to explain the origin of the ring around the dwarf planet Haumea [17].

Hot topic: study of equilibrium figures of binary systems, for example, asteroids. These tasks require a good knowledge and creative development of potential theory.

Note. Let us pay attention to the fact that between the phase models of collisionless stellar systems (where the integrals of motion refer to each star separately) and the celestial-mechanical problem of gravitating bodies (where the integrals of motion refer to the entire system as a whole), there is a wide and hazy gap. In this direction, one can expect active action by theoreticians. 

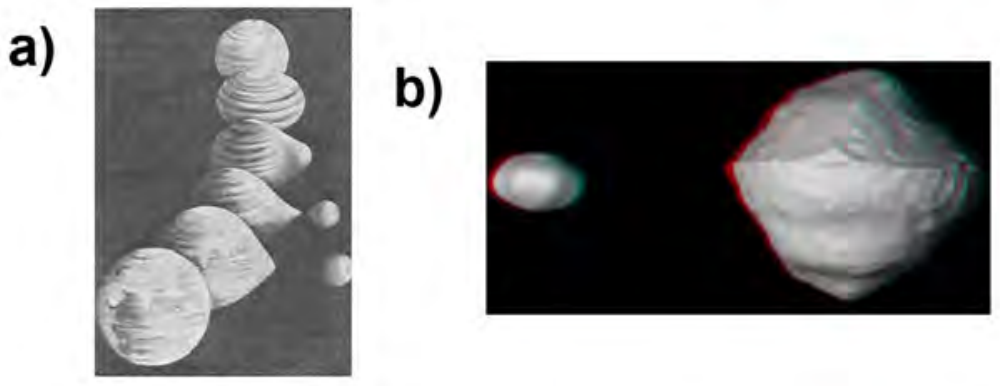

c)

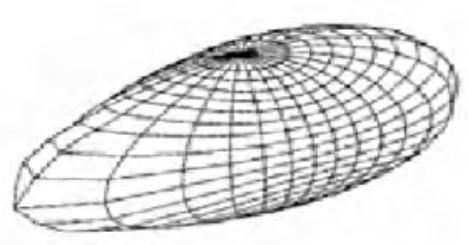

Figure 9: a) To the Darwin-Poincaré hypothesis. b) Double asteroid. c) Final pear-shaped figure.

\section{Needs of modern celestial mechanics}

\subsection{General considerations}

In theoretical terms, celestial mechanics needs further development of the theory of nonlinear dynamical systems. Studies have shown that in typical dynamical systems there is no complete integrability, but there is no complete chaos either: synthesis develops when islands of integrability are surrounded by chaos.

\subsection{The problem of correct application diverging series}

Separately, there is the problem of the correctness of the application of divergent series for the analysis of the stability of the solar system over long periods of time in tens and hundreds of millions of years. Specific numerical calculations for the Solar system for such intervals were shown [18, 19]:

- the movement of the giant planets Jupiter, Saturn, Uranus and Neptune remains predictably stable;

- in the movement of minor planets and asteroids, the level of chaos becomes much more noticeable. Mercury in a billion years can say goodbye to us. But for the Earth, due to the presence of a large satellite of the Moon, the situation with the orbit and climate is stable.

- the origin of the reverse very slow rotation of Venus remains unclear.

\subsection{Methods of computer modeling}

Methods of computer modeling have played a valuable role in the analysis of the problem of three (and more) bodies, in tracing the series of previously unknown equilibrium figures, in the study of the interaction of planets with a disk. The study of the orbits of the satellites of Mars Phobos and Deimos showed that it is possible that in an early era these two satellites represented a single celestial body. However, the debate on this issue continues. There is a hope that with the help of high-speed computers it will be possible to find out what the Solar system will be like in many millions of years. The relationship between the form of potentials and the form of chaotic orbits has not been fully clarified. In this regard, a complete inventory of resonances and the study of their influence on the motion of bodies in the solar system is relevant. The development of the topic is the study of three-body resonances in the asteroid belt.

\subsection{The KAM theory}

The hallmark of celestial mechanics is the KAM theory. The basic ideas of the theory of canonical systems and tori in phase space can be found in Morbidelli's book "Modern Celestial Mechanics" [20].

In recent years, the exploration of the Solar system and extrasolar planetary systems (exoplanets) has progressed at a rapid pace, and the constant influx of new data poses new challenges. The advances in observational astronomy place new demands on the accuracy of analytical theory and point to the importance of developing new models for studying the dynamics and evolution of planetary systems.

Methods of a purely mathematical nature, such as the theory of potential and the theory of equilibrium figures, also play an important role in modern celestial mechanics. 


\section{A new direction in potential theory: Equigravitating Bodies Theory}

\subsection{New direction in potential theory: The theory of equigravitating bodies}

Even Newton was pleasantly surprised by the possibility of replacing the outer field of the gravitating ball with a field of material point mass. But the ball is only a special case of the triaxial ellipsoid, and Maclaurin and Laplace make an important step along this path: "Homogeneous confocal ellipsoids of equal mass create identical gravitational fields in outer space." Next steps [15]: a new direction has been created - the theory of equigravitating bodies with real or imaginary density. The new mathematical apparatus allows solving a wide range of problems; developed, in particular, many original methods for finding the gravitational energy of bodies of complex shapes.

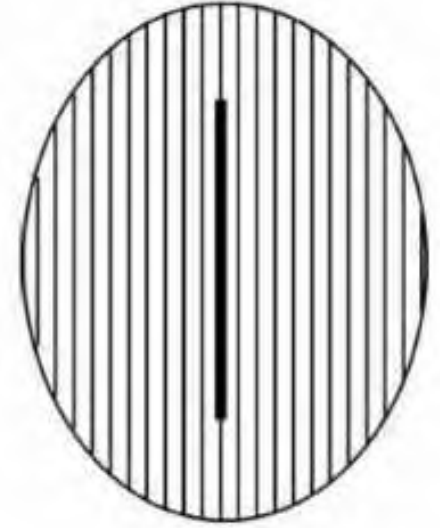

a

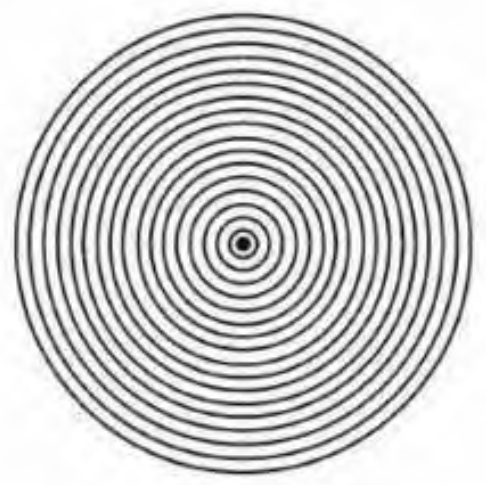

б

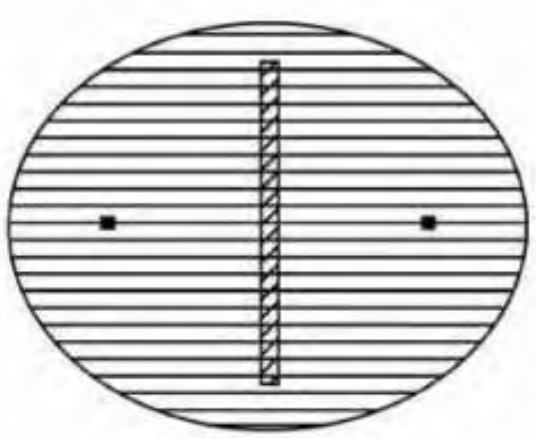

6

Figure 10: Representation of external gravitational fields: a) a prolate spheroid (by inhomogeneous material rod), b) a ball (by material point), c) an oblate spheroid (by inhomogeneous rod with a purely imaginary density distribution).
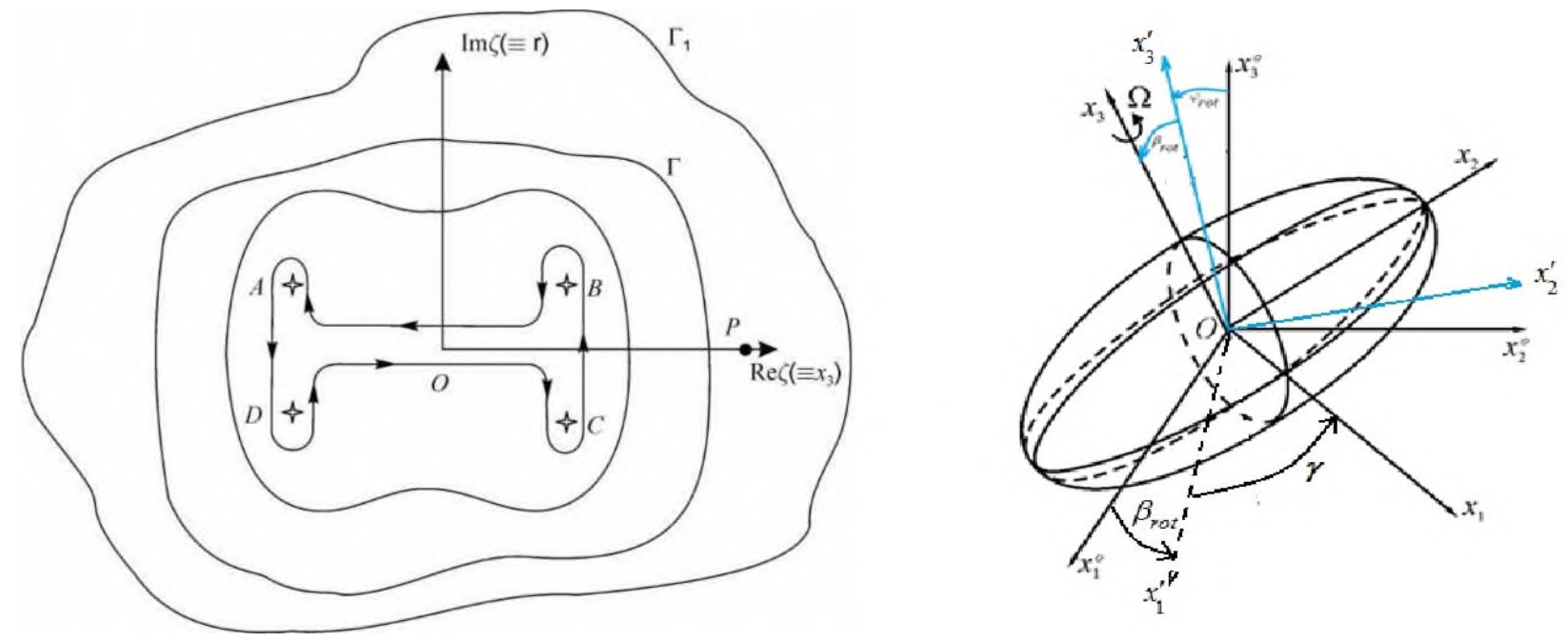

Figure 11: Left: on finding equigravitating rods using the Cauchy integral method. Right: new three angles system $\varphi_{\text {rot }}, \beta_{\text {rot }}, \gamma$.

Next Steps: A New Direction Created — Theory equigravitating bodies with real or imaginary density. The new mathematical apparatus makes it possible to solve a wide range of tasks; developed in particular many original methods for finding the gravitational energies of bodies of complex shape.

Explanation to Fig 10. The two figures on the left are a consequence of the theorem Maclaurin-Laplace (external attraction of an prolate spheroid will not change upon its confocal transformation to one-dimensional heterogeneous rod. This focal rod will be equigravitating the original prolate spheroid. When going to the ball 
the bar will turn into a point). But when the ball is deformed into a compressed spheroid, this point is again transformed into a rod, however, this rod will have an apparent density [15].

$$
\mu(\zeta)=\frac{3 M}{4 i \sqrt{a_{1}^{2}-a_{3}^{2}}}\left(1+\frac{\zeta^{2}}{a_{1}^{2}-a_{3}^{2}}\right)
$$

Such a segment of "length" $2 i \sqrt{a_{1}^{2}-a_{3}^{2}}$ has the same total mass and the same external potential as a homogeneous oblate spheroid.

The problem of equigravitating bodies has been developed in three directions [1, 15].

The first direction: development of the theory of equigravitating rods. Such rods can have both real and imaginary density distributions. For some bodies, there are "skeletons" of rods, or combinations of rods and material points. Each axisymmetric body has such a set of equigravitating elements. Rigorous mathematical methods have been developed for finding equigravitating rods (for example, using the generalized Cauchy integral).

The second direction: the representation of external gravitational fields of bodies with an equatorial plane of symmetry using special equigravitating disks.

Thirdly, the method of special confocal transformations has been developed. This leads to the expansion of the theory: it turns out that any elementary ellipsoidal shells and layered inhomogeneous ellipsoids connected by special confocal transformations are equigravitating.

\subsection{The theory of equilibrium figures}

In the theory of equilibrium figures, a new approach has been developed for solving the problem of restoring the spatial shape of a body along its limb. In this method, instead of Euler angles, a system of 3 angles $\left(\varphi_{\text {rot }}, \beta_{\text {rot }}, \gamma\right)$ is introduced, specially adapted for solving the inverse problem [21]. The kinematic formulas for the rotation of a rigid body at new angles are:

$$
\begin{gathered}
\Omega_{1}=\varphi_{r o t} \cos \gamma \cos \beta_{r o t}-\beta_{r o t} \sin \gamma, \\
\Omega_{2}=\varphi_{r o t} \sin \gamma \cos \beta_{r o t}+\beta_{r o t} \sin \gamma, \\
\Omega_{3}=\varphi_{r o t} \sin \beta_{r o t}+\gamma .
\end{gathered}
$$

\subsection{A new approach to ice and rock figures}

A new approach to ice and rock figures has been developed. A non-impact mechanism has been proposed to explain the mystery of the origin of the dense ring around the dwarf planet Haumea [17].

\subsection{Determination of the spatial shape of the body}

The inventory of resonances in Solar system could provide new applications for this theory. To solve the problem of restoring the spatial shape of the body by his limb a method is constructed. In this method instead of the Euler angles the system of 3 angles $\varphi_{r o t}, \beta_{r o t}, \gamma$ is introduced specially adapted to solve the inverse problem [21].
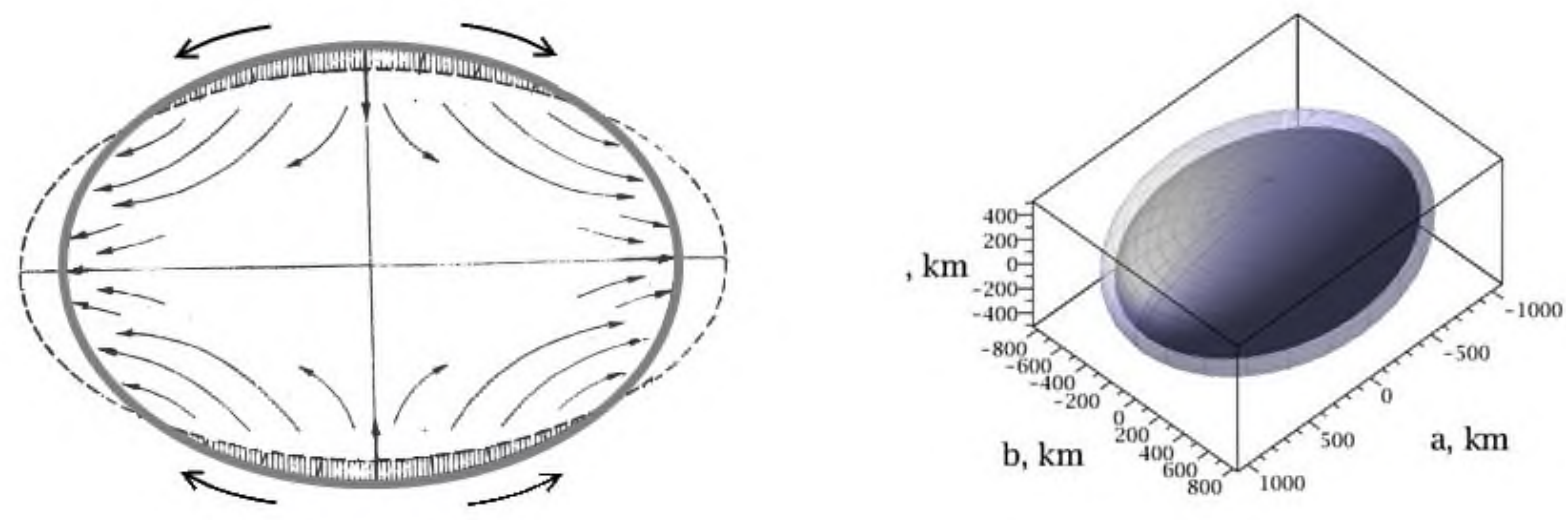

Figure 12: Left: Deformation diagram of the stone core. Right: Equilibrium figure of Haumea and its effect on the ice shell.

Explanation to the Fig. 12. The inhomogeneous model of the planet Haumea consists of a rocky core and a thick ice shell. Its boundary surface and the surface of the nucleus cannot simultaneously be level, small deviations 
from local equilibrium appear, and the mechanism of deformation of the nucleus and shell begins to operate. This leads to the elongation of the core, and when a certain limit is reached, the ice does not withstand the stresses, cracks and gradually accumulates at the sharp ends of the rapidly rotating planet. Then the excess ice masses are separated from the planet.

\section{Practical celestial mechanics}

The peculiarity of practical celestial mechanics is focus on gaining benefits and improving comfort for people's lives today and in the near future. In addition, for a comfortable life, we needs to know the space environment, in which we live. Actions are also aimed at solving the eternal problem of humanity: expansion of the habitat.

The following practical tasks are being solved:

- Satellite assisted navigation on land, at sea and in the air.

- Exploring the Earth from Space.

- Organization and implementation of space missions to other bodies of the solar system.

- Extraction of useful substances from the Moon, planets, asteroids and their satellites.

All these tasks are solved by building models of motion of the solar system bodies. Recently, experts are increasingly calling these models as ephemeris. Ephemeris are the end result of constructions in celestial mechanics because they are based on all our knowledge, including all available observations. Ephemerides are a means of solving problems of practical celestial mechanics, because they are based on all our knowledge. All methods the knowledge are used to construct motion models and ephemeris. This is outlined in the previous sections of this article.

The procedure is as follows: build a model, best suited to the actual movement of celestial bodies, then we apply the model to solve the problems. Real movement is given in the form of astrometric observations.

Since the construction of models of the motion of bodies of the solar system is a very complex and cumbersome process, then this is done, as a rule, in large scientific centers large teams of specialists with a wide international cooperation.

Contribution of Russian specialists to practical celestial mechanics is mentioned in the next section of this article.

\section{Contribution of Russian researchers to the success of celestial mechanics}

In research on celestial mechanics in the Russian Federation, one can highlight several areas.

\subsection{New dynamic models of the motion of celestial bodies}

The emergence of new high-precision and all-wave observations requires the construction of new dynamic models motion of celestial bodies. Here it should be noted the work of the MAO RAS, ISS RAS, Institute of Applied Mathematics. M.V. Keldysh RAS.

The work on the study of relativistic effects (geodesic precession and nutation) in the rotation of celestial bodies is being successfully developed at the Main Astronomical Observatory of the Russian Academy of Sciences $[22,23,24]$.

Radar observations carried out at the IAA RAS jointly with the observatory Goldstone made it possible to clarify the orbits, sizes and rotation periods of asteroids. Echo signals from 12 asteroids and the Moon were registered and processed. The lunar radio ranging method has been implemented which made it possible to clarify parameters of the model of the orbital and rotational motion of the Moon.

The IAA RAS also considers the problem of changing the shape of the cometary nucleus under the influence of sublimation of matter from the surface of the comet [25]. An analytical solution to the shape change equation is obtained for the case of a homogeneous rotating nucleus. It is shown that in the course of the evolution of the form the cometary nucleus takes on elongated shape along the axis of rotation. The dynamic features of asteroid 1I 'Oumuamua - the first interstellar celestial a body that has penetrated into the solar system and approached the Earth are investigated. It is shown that the highly elongated shape of the asteroid can be explained by collisions with high-speed dust particles in the interstellar space [26].

Ballistic Center of the Institute of Applied Mathematics M.V. Keldysh RAS has done a great work to ensure the successful launch of the spacecraft Spectr-RG into a halo orbit in the vicinity of the L2 point of the Earth-Sun system. This is the first flight to a halo orbit in the practice of Russian cosmonautics. [27].

The IAA RAS continues to improve the dynamic models of motion of the planets of the solar system. Complex structure of the solar system including planets and many small bodies makes it necessary to account the gravitational influence of all objects when constructing high-precision ephemeris of the planets of the solar system 
and the Moon [28]. New, more accurate observations of comets require taking into account the displacement of the photocenter, caused by the release of gas and dust from the surfaces of comets.

\subsection{Models of motion and ephemeris of planets and satellites}

A new version of the planetary ephemeris EPM-2021 has been created at the IAA RAS. Ephemeris of the planets and the Moon are available at a new level of accuracy in a special server on web pages.

An ephemeris server for all satellites of the planets and asteroid satellites has been created at SAI MSU [29]. The server also makes it possible to calculate the ephemeris of the planets, the sun and the moon. You can choose to use any of the 10 planetary motion models developed in JPL (USA), IMCCE (France), IPA RAS, including U. Le Verrier's model developed in the 19th century. Ephemeris of all the satellites of the larger planets built on the basis of the original models developed at the SAI [30]. As for the ephemeris of asteroid satellites then the models of the motion of all 64 satellites of the asteroids [31] are built. This is all that can be done based on the astrometric observations. Ephemeris server of asteroid satellites has no analogues in the world. This is the original development of [31].

\subsection{Development of analytical and semi-analytical methods for studying the dynamics of celestial bodies}

A method was constructed in SAI MSU for solving the inverse geometric problem of reconstructing the shape of a triaxial ellipsoid from its $2 \mathrm{D}$ projection onto the plane of the sky and photometric data. The method is based on a rotation matrix with three angles, different from Euler angles and maximally adapted to restore the shape of the ellipsoid. A system of eight equations has been compiled to solve the problem about the shape and dynamics of the dwarf planet Haumea. Arguments are obtained in favor of the fact that the Haumea ring has tilt to the equator [21].

A method has been developed for calculating harmonics for the azimuthally averagedv external potential of rapidly rotating layered inhomogeneous ellipsoids. This made it possible to calculate the period of nodal precession for Haumea ring [32].

On the basis of Gauss rings, SAI MSU has developed a new method of studying long-period and secular disturbances in celestial mechanics. The method relies on the mutual potential energy of Gaussian rings. The adequacy of the new approach was established, which made it possible to clarify parameters of the precession of the orbits of Jupiter and Saturn within two-planetary problem [5].

To study secular perturbations in celestial mechanics, two analytical models of R-disk and R-toroid. R-toroid model is based on triple averaging of the material point motion and boils down to a chain of transformations: 1D Gaussian ring - 2D R-ring - 3D R-toroid. The shape, structure and gravitational potential of the R-toroid are studied. An expression is obtained for the mutual energy of the R-toroid and the Gaussian ring. A system of equations for the secular evolution of osculating orbits is derived in the field of the R-toroid and the central precessing stars [8]. In the next step, combining two R-toroids apsidal and nodal precession of orbits in circumbinary exoplanet systems is being studied [9].

At the Ural Federal University, a theory of the secular evolution of the four-planetary system was developed [33].

In the SAI MSU work is in progress on geodynamics and creating space complex for measuring the gravitational field of the Earth and pulsar timescale [34].

At St. Petersburg State University new results have been obtained in the classical problem three bodies which allows to describe the main features of the movement small natural and artificial bodies at large intervals time inaccessible to numerical integration. Introduced the concept surface of the minimum speed, which is a modification zero velocity surfaces (Hill surfaces) playing a key role in describing the range of possible movements [35].

A qualitative study of possible modes of motion of small celestial bodies with retrograde motion in the vicinity of orbits of the large planet has been carried out in the Institute of Applied Mathematics of RAS [36]. A special form of development of the perturbing function in the doubly averaged restricted elliptic three-body problem up to the fourth degree including relatively small parameter is obtained $[37,38,39,40,41,42]$. The dynamics of Trojan asteroids has been studied. It is clarified why some of them move from the libration point L4 to L5 and back [43].

An important task is to study the dynamic features of exoplanets. Researches were carried out at Institute of cosmic research of RAS, SAI MSU, Vernadsky Institute of Geochemistry and Analytical Chemistry of RAS, St.-Petersburg State University. These efforts made it possible to analyze the most important phenomena that determine the dynamic structure of planetary systems. The effect of resonances and migration on dynamic stability systems were studied. The dynamic features of planetary systems double stars were considered $[9,44]$. 
In the Institute of astronomy of RAS long-period variations in the oscillatory process of the earth pole were investigated [45]. Problems of cosmogony on the generation of free planets and comets by planetary systems were studied [46].

\subsection{The problem of asteroid-comet hazard and space debris}

The Institute of astronomy of RAS has developed an unique space project SODA (Daytime Asteroid Detection System), designed to detect dangerous space bodies approaching the Earth from the direction of the Sun. Such bodies are impossible to observe with both ground-based and near-space flying telescopes. Placing two spacecraft in the vicinity of a point on orbits with different phases allows one to improve significantly (up to several kilometers) the accuracy of determining the orbit of a dangerous body and coordinates of the point of entry of the body into the atmosphere [47].

At the Ural Federal University, the dynamic evolution of space debris in the vicinity of the global navigation systems satellite orbits is investigated [48]. In addition, at this university, based on analysis of Kolshevnikov's metrics, a search was made for young (less than 2 million years old) pairs and groups of asteroids in close orbits. They are interesting also because the physical processes have not yet significantly changed the properties of asteroid surfaces, and from the point of view of dynamics, the system still stores information about its origin [49, 50, 51].

IAA RAS has developed a quick method for assessing the likelihood of collisions of small bodies with the Earth, allowing to identify quickly and with high reliabilityb celestial bodies dangerous for the Earth. It is a linear method for estimating the likelihood of collisions of near-Earth asteroids with the Earth. The method uses a special curvilinear coordinate system associated with the nominal orbit of the asteroid [52].

In St.-Petersbourg university and MAO RAS an efficient algorithm for calculating interorbital distance parameter MOID (Minimum orbit intersection distance) is created to detect possible asteroid collisions with each other and planets [53, 54]. Estimates of the rate of clearance of Hill's spheres are obtained for contact double celestial bodies from dust particles due to the action of various physical and dynamic factors, depending on the mass of the bodies, the Lyapunov times are estimated and the speed of clearing in chaotic planetary zones [55]. IAA RAS has developed a methodology allowing to identify families of asteroids [56].

\subsection{Special mention should be made of the work of the Research Institute of Tomsk University}

1. New theory of collocation methods for numerical integration of orbits. Development of new nonlinearity indicators as a measure of the shifts of parametric estimates in confidence problems evaluating [57, 58, 59].

2. A method has been developed for determining the overlap of visibility zones of auroral images of two spacecrafts [60].

3 , Investigations of resonant structures of near-planetary orbital spaces by numerical modeling. Orbital and secular resonance maps in the near-Earth space [61].

4. Study of secular resonances. Asteroid movement $[62,63,64]$.

\subsection{It should be specially noted the work of the team of the Kazan Federal University}

Actively working team ( Nefedyev Y. A., Petrova N.K., Zagidullin A. A., Andreev A.O., Churkin K.O. et al.). List of works for 2018-2021 contans more than 100 titles. Therefore we give here only a brief listing of the topics of work:

1. Evolution of the orbits of comets and asteroids [65], [66], [67], [68], [69, 70, 71].

2. Further study of the physical libration of the Moon. Comparative analysis of different approaches to this problem $[72,73,74,75,76,77]$.

3. Parameters of the numerical and analytical ephemeris of the Moon [78].

4. Investigation of the physical surfaces and gravitational fields of the Moon and planets [79, 80].

\section{References}

1. B. P. Kondratyev, 2007.

2. B. P. Kondratyev, Solar System Research, 46, 352, 2012.

3. V. A. Antonov, I. I. Nikiforov, and K. V. Kholchevnikov, 2008.

4. M. A. Vashkovyak and S. N. Vashkovyak, Solar System Research, 46, 69, 2012.

5. B. P. Kondratyev and V. S. Kornoukhov, Journal of Technical Physics, 64, 1395, 2019.

6. B. P. Kondratyev and V. S. Kornoukhov, Astronomy Reports, 64, 434, 2020.

7. B. P. Kondratyev, MNRAS, 442, 1755, 2014.

8. B. P. Kondratyev and V. S. Kornoukhov, Astronomy Reports, 65, 412, 2021.

9. B. P. Kondratyev and V. S. Kornoukhov, Astronomy Reports, 65, 588, 2021. 
10. C. Moore, Phys. Rev. Lett., 70, 3675, 1993.

11. B. Kol, arXiv e-prints, arXiv:2107.12372, 2021.

12. Y. Eriguchi, I. Hachisu, and D. Sugimoto, Progress of Theoretical Physics, 67, 1068, 1982.

13. S. Chandrasekhar, Ellipsoidal figures of equilibrium (1969).

14. B. P. Kondrat'ev, Dinamika ellipsoidal'nykh gravitiruiushchikh figur (1989).

15. B. P. Kondratyev, 2003.

16. V. Zharkov, 2013.

17. B. P. Kondratyev, Ap\&SS, 361, 169, 2016.

18. G. J. Sussman and J. Wisdom, Science, 257, 56, 1992.

19. J. Laskar, A\&A, 287, L9, 1994.

20. A. Morbidelli, Modern celestial mechanics : aspects of solar system dynamics (2002).

21. B. P. Kondratyev and V. S. Kornoukhov, MNRAS, 478, 3159, 2018.

22. V. V. Pashkevich and G. I. Eroshkin, Artificial Satellites, 53, 89, 2018.

23. V. V. Pashkevich and A. V. Vershkov, Solar System Research, 53, 431, 2020.

24. V. V. Pashkevich and A. N. Vershkov, Artificial Satellites, 55, 118, 2020.

25. Y. Chernetenko and Y. Medvedev, MNRAS, 493, 5499, 2020.

26. D. E. Vavilov and Y. D. Medvedev, MNRAS, 484, L75, 2019.

27. R. Sunyaev, V. Arefiev, V. Babyshkin, A. Bogomolov, et al., arXiv e-prints, arXiv:2104.13267, 2021.

28. E. V. Pitjeva and N. P. Pitjev, Astronomy Letters, 44, 554, 2018.

29. N. V. Emel'Yanov and J. E. Arlot, A\&A, 487, 759, 2008.

30. N. V. Emel'Yanov and A. A. Kanter, Solar System Research, 39, 112, 2005.

31. N. V. Emelyanov and A. E. Drozdov, Icarus, 355, 114160, 2021.

32. B. P. Kondratyev and V. S. Kornoukhov, Ap\&SS, 366, 56, 2021.

33. A. Perminov and E. Kuznetsov, Ap\&SS, 365, 144, 2020.

34. V. K. Milyukov, A. V. Burdanov, A. S. Zhamkov, V. E. Zharov, O. A. Ivlev, I. M. Nesterin, and V. K. Sysoev, Solar System Research, 54, 610, 2020.

35. K. V. Kholshevnikov and V. Titov, Solar System Research, 53, 473-47, 2020.

36. V. V. Sidorenko, AJ, 160, 257, 2020.

37. M. A. Vashkov'yak, Solar System Research, 52, 69, 2018.

38. M. A. Vashkov'yak, Solar System Research, 52, 359, 2018.

39. M. A. Vashkov'yak, Solar System Research, 53, 116, 2019.

40. M. A. Vashkov'yak, Solar System Research, 54, 329, 2020.

41. D. E. Vavilov and Y. D. Medvedev, MNRAS, 484, L75, 2019.

42. M. A. Vashkov'yak, Solar System Research, 54, 49, 2020.

43. V. V. Sidorenko, Celestial Mechanics and Dynamical Astronomy, 130, 67, 2018.

44. M. Y. Marov and I. I. Shevchenko, Ekzoplanety. Ekzoplanetologiya (Institute komp'uternykh issledovaniy) (2017).

45. V. V. Perepelkin, L. V. Rykhlova, and A. S. Filippova, Astronomy Reports, 63, 238, 2019.

46. A. V. Tutukov, G. N. Dremova, and V. V. Dremov, Astronomy Reports, 64, 936, 2020.

47. I. D. Kovalenko, B. M. Shustov, and N. A. Eismont, Acta Astronautica, 148, 205, 2018.

48. E. D. Kuznetsov and E. A. Avvakumova, Acta Astronautica, 158, 140, 2019.

49. E. Kuznetsov and V. Safronova, P\&SS, 157, 22, 2018.

50. E. D. Kuznetsov, D. V. Glamazda, G. T. Kaiser, V. V. Krushinsky, et al., in 81st Annual Meeting of the Meteoritical Society, LPI Contributions, volume 81, 6014 (2018).

51. E. D. Kuznetsov, A. E. Rosaev, E. Plavalova, V. S. Safronova, and M. A. Vasileva, Solar System Research, 54, $236,2020$.

52. D. E. Vavilov, MNRAS, 492, 4546, 2020.

53. R. V. Baluev and D. V. Mikryukov, Astronomy and Computing, 27, 11, 2019.

54. D. V. Mikryukov and R. V. Baluev, Celestial Mechanics and Dynamical Astronomy, 131, 28, 2019.

55. A. V. Mel'nikov, Solar System Research, 54, 432, 2020.

56. T. A. Vinogradova, MNRAS, 484, 3755, 2019.

57. G. O. Ryabova, V. A. Avdyushev, and I. P. Williams, MNRAS, 485, 3378, 2019.

58. V. Avdyushev, 63, 131, 2020.

59. V. A. Avdyushev, O. M. Syusina, and V. A. Tamarov, Solar System Research, 55, 71, 2021.

60. M. A. Banshchikova, V. A. Avdyushev, and A. K. Kuzmin, Cosmic Research, 58, 357, 2020.

61. I. V. Tomilova, T. V. Bordovitsyna, and D. S. Krasavin, Solar System Research, 52, 450, 2018.

62. T. Y. Galushina, L. E. Bykova, O. N. Letner, and A. P. Baturin, Astronomy and Computing, 29, $100301,2019$.

63. O. N. Letner and T. Y. Galushina, PESSS, 181, 104818, 2020.

64. T. Y. Galushina, O. N. Letner, and E. N. Niganova, PESS, 202, 105232, 2021. 
65. A. O. Andreev, V. S. Usanin, and Y. A. Nefedyev, in 81st Annual Meeting of the Meteoritical Society, LPI Contributions, volume 81, 6155 (2018).

66. A. O. Andreev, V. S. Usanin, and Y. A. Nefedyev, in 81st Annual Meeting of the Meteoritical Society, LPI Contributions, volume 81, 6157 (2018).

67. M. V. Sergienko, M. G. Sokolova, A. O. Andreev, and Y. A. Nefedyev, in 81st Annual Meeting of the Meteoritical Society, LPI Contributions, volume 81, 6162 (2018).

68. M. V. Sergienko, M. G. Sokolova, A. O. Andreev, and Y. A. Nefedyev, in 81st Annual Meeting of the Meteoritical Society, LPI Contributions, volume 81, 6165 (2018).

69. Y. A. Nefedyev, M. G. Sokolova, A. O. Andreev, M. V. Sergienko, and N. Y. Demina, in 81st Annual Meeting of the Meteoritical Society, LPI Contributions, volume 81, 6188 (2018).

70. Y. A. Nefedyev, A. O. Andreev, and N. K. Demina, in 81st Annual Meeting of the Meteoritical Society, LPI Contributions, volume 81, 6192 (2018).

71. N. Y. Demina, A. O. Andreev, Y. A. Nefedyev, S. A. Demin, and L. A. Nefediev, in Journal of Physics Conference Series, Journal of Physics Conference Series, volume 1038, 012019 (2018).

72. Y. A. Nefedyev, A. O. Andreev, N. K. Petrova, N. Y. Demina, and A. A. Zagidullin, Astronomy Reports, 62, 1016, 2018.

73. N. K. Petrova, Y. A. Nefedyev, A. A. Zagidullin, and A. O. Andreev, Astronomy Reports, 62, $1021,2018$.

74. A. O. Andreev, Y. A. Nefedyev, and N. Y. Demina, 225, 11, 2018.

75. K. Churkin, Y. A. Nefedyev, and A. O. Andreev, 225, 47, 2018.

76. N. K. Petrova, Y. A. Nefedyev, A. O. Andreev, and A. A. Zagidullin, Astronomy Reports, 64, $1078,2020$.

77. A. A. Zagidullin, V. S. Usanin, N. K. Petrova, Y. A. Nefedyev, A. O. Andreev, and T. V. Gudkova, Astronomy Reports, 64, 1093, 2020.

78. A. Zagidullin, V. Usanin, N. Petrova, Y. Nefedyev, and A. Andreev, Proceedings of 20th International Multidisciplinary Scientific GeoConference SGEM, 6.1, 703, 2020.

79. C. D. L. Morena, A. O. Andreev, Y. A. Nefedyev, E. N. Akhmedshina, and L. A. Nefediev, Journal of Physics: Conference Series, 1697, 012019, 2020, URL https://doi.org/10.1088/1742-6596/1697/1/012019.

80. A. O. Andreev, E. N. Akhmedshina, L. A. Nefediev, Y. A. Nefedyev, and N. Y. Demina, Astronomy Reports, 65, 435, 2021. 


\title{
Young stars
}

\author{
S.A. Lamzin \\ lamzin@sai.msu.ru
}

Sternberg Astronomical Institute of Lomonosov Moscow State University, Moscow, Universitetskij pr. 13, Russia

A brief overview of the current state of investigation of classical T Tauri and Ae/Be Herbig stars is presented. We describe the application of magnetospheric disk accretion theory to interpretation of activity of these objects. The connection between the accretion and matter outflow from the vicinity of these stars in the form of jets and disk wind is discussed. We also consider the variability of young stars caused by circumstellar dust and the role of episodic accretion in the process of star formation.

Keywords: stars: variables: T Tauri, Herbig Ae/Be - accretion, accretion discs - stars: winds, outflows.

DOI: $10.51194 /$ VAK2021.2022.1.1.004

\section{Introduction}

Stars are formed from interstellar matter mostly inside giant molecular clouds - condensations of interstellar gas and dust with a characteristic size of several tens of parsecs and with a mass of $\sim 10^{4}-10^{6} \mathrm{M}_{\odot}$. Molecular clouds have an inhomogeneous structure, and gravitationally bound condensations with a mass of $\sim 100 \mathrm{M}_{\odot}$, called pre-stellar cores, are the "maternity homes" of stars. Their lifetime is $\sim 10^{5} \mathrm{yr}$, after which they usially begin to contract, breaking up into separate fragments of the stellar mass — protostellar clouds — see e.g. the book [1] for details.

Initially, protostellar clouds are compressed almost in the free fall mode, but after $\sim 10^{4}$ yr, the inner regions become opaque to their own radiation and therefore quickly heat up. The pressure gradient of the gas in these regions stops the contraction, and the protostellar cloud turns into a protostar - an object consisting of a hydrostatic equilibrium core with a mass of $\sim 1 \%$ of the mass of the protostellar cloud on which the matter of the outer layers accrets. The core temperature is $\sim 1000-3000 \mathrm{~K}$, and it emits in the optical and near-infrared bands, but this radiation does not reach an external observer, since it is completely absorbed by dust particles in the envelope and re-emitted longward $\lambda \sim 20 \mu \mathrm{m}$ - these are the so-called Class 0 young stellar objects (YSOs).

Protostellar clouds have a non-zero angular momentum $J$, so as the cloud contracts, centrifugal forces make the cloud flattened in the equatorial direction. As a result, a disc-shaped envelope is formed, inside which the matter, due to the viscosity between the layers, spirally falls on the forming hydrostatic core. If $J$ exceeds a certain critical value, then centrifugal forces break off the cloud into two or more parts, each of which will continue to contract independently - most binary or multiple stellar systems apparently are formed in this way.

Over time, the mass and temperature of the core increase, whereas the mass of the shell decreases. From an observational point of view, this is manifested in the fact that the protostar becomes a source at increasingly shorter wavelengths - at this stage, protostars are called Class I YSO. At some point, infalling envelope becomes transparent along the line of site for optical radiation of the hydrostatic core, which in the first approximation looks like a main sequence star. For the observer the protostar turns into a young star, such as the larger inclination of protostar's rotation axis to the line of site the latter it occurs.

On average, the protostellar stage lasts $\sim 0.1-0.3 \mathrm{Myr}$, and by its end in the accretion disk usually remains $\leq 1 \%$ of the mass of the protostellar cloud, but over $90 \%$ of its angular momentum. Young stars are slowly contracting, and their central regions are heating up. For objects with a mass of $M>0.08 \mathrm{M}_{\odot}$, after $\sim 40\left(M / M_{\odot}\right)^{-2} \mathrm{Myr}$, central temperature reaches a value sufficient for the intensity of nuclear reactions, during which hydrogen transforms into helium, to support luminocity of the star. From this moment, the young star becomes a main sequence (MS) star.

Depending on the mass $M$, young stars are divided into T Tauri type $\left(M<2-3 \mathrm{M}_{\odot}\right)$ and Ae/Be Herbig (HAe/Be) stars. Gas of the disk, surrounding young stars, partially accrets onto the star, and partially evaporates, while the dust settles to the disk's equatorial plane, what ultimately leads to the planet formation. In a few million years, the gas from the (protoplanetary) disk almost completely disappears, accretion stops, and a planetary system and/or 'construction debris' (dust particles, asteroids, comets) remains around the young star.

Magnetic field plays fundamental role in the process of star formation: it affects the redistribution of angular momentum inside the protostellar cloud, the process of its fragmentation and disk accretion on the hydrostatic core [2]. Already in the prestellar cores, the field strength is $\sim 10-100 \mu \mathrm{G}$ [3], which implies that the magnetic pressure in these objects is comparable to thermal and turbulent ones.

\section{Magnitospheric accertion of young stars}

From observational point of view TTSs are subgiants of F5-M8 spectral type with emissiion lines and excess continuum emission in UV band which veil absorption spectrum of the star [4]. $\mathrm{H}_{\alpha}$ is the most strong line in the spectra of TTSs, and the larger equivalent width $\mathrm{EW}_{\mathrm{H} \alpha}$ of the line the more rich emission spectrum of the star 
and the larger veiling. Due to this reason $\mathrm{EW}_{\mathrm{H} \alpha}$ is used as an activity indicator of TTSs. It was found that activity (large cold spots, variable emission in Hydrogen and Ca II lines, hard X-ray and non-thermal radio emission) of TTSs with $\mathrm{EW}_{\mathrm{H} \alpha} \lesssim 5 \AA$ - so called weak line TTSs or WTTSs - is due to the presence of chromospheres and coronae more or less similar to solar, but much more powerful. This seems quite natural, since WTTSs have strong surface magnetic field $(B \sim 1-3 \mathrm{kG})$ and fast axial rotation $\left(P \sim 2-10^{\mathrm{d}}\right)$. At the same time WTTSs are usially surrounded by debris (non-accretion) disks, that are responsible for observed excess emission in mid IR band.
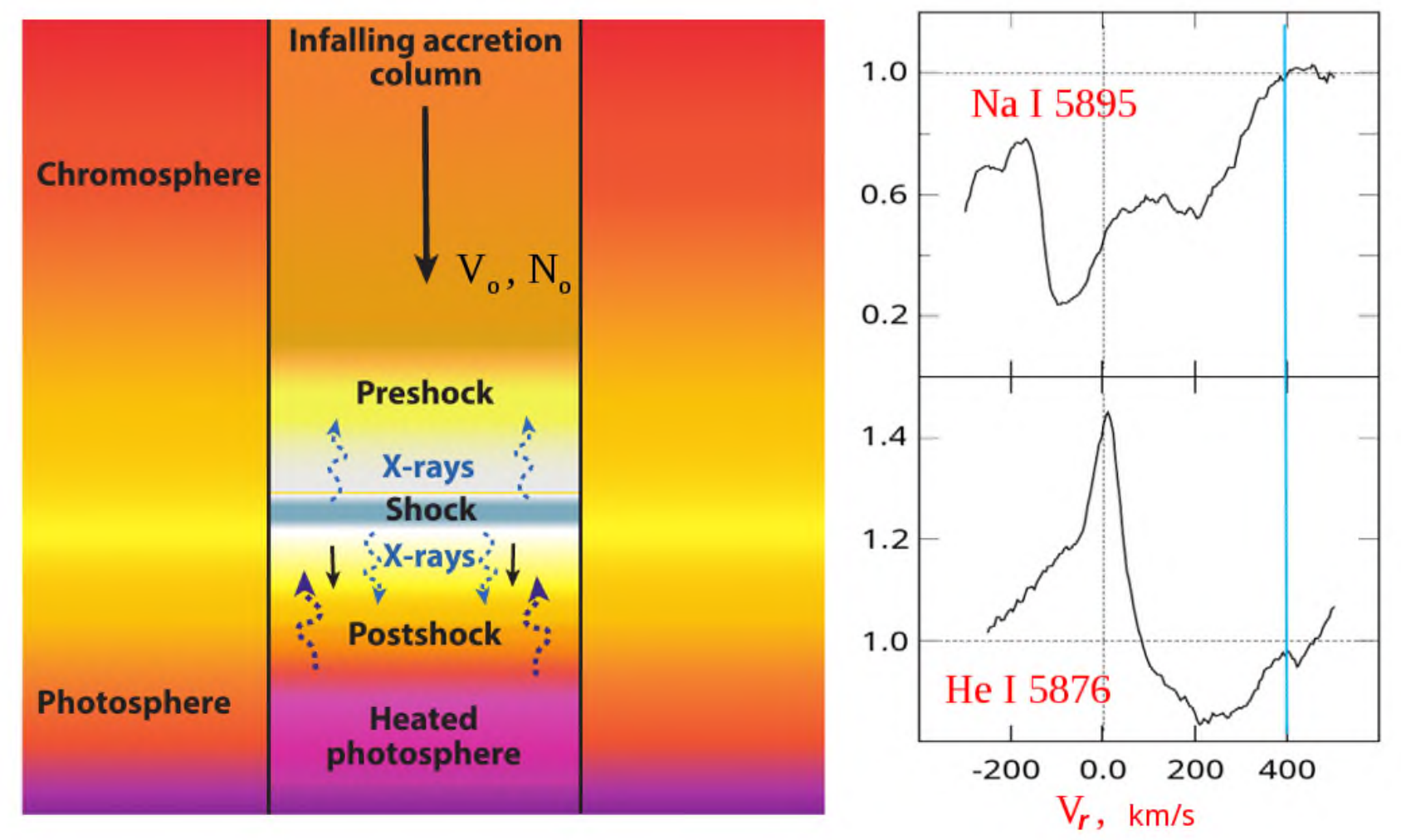

Figure 1: Left panel - schematic structure of accretion shock [5]. Right panel - profiles of some lines in the spectrum of CTTS RW Aur A [6].

Classical TTSs (CTTSs), i.e. TTSs with $\mathrm{EW}_{\mathrm{H} \alpha}>5 \AA$, as well as Herbig Ae/Be stars [7] have asymmetric profiles of emission lines in their spectra and excess continuum emission longward $\lambda \approx 2 \mu$ m. Activity of these stars is connected with magnitospheric accretion of protoplanetary disk's matter [8]. More precisely, the magnetic field of the star disrupts inner regions of the disk, in some way disk's material becomes frozen in the magnetospheric field lines and slides along them toward the stellar surface, eventually being accelerated up to velocities $V_{0} \sim 300 \mathrm{~km} \mathrm{~s}$. The gas is then decelerated in an accretion shock (AS), inside which the gas is heated to $T \approx 2 \times 10^{6} \mathrm{~K} \approx 0.2 \mathrm{keV}$. Half of the resulting X-ray quanta from the AS's cooling zone irradiate the star, creating a hot spot on its surface - see Fig. 1. Narrow components of emission lines and veiling continuum are formed in the spot. The second half of X-ray quanta irradiate infalling gas, creating pre-shock H II zone, where redshifted components of emission lines are originated $[9,10]$.

This scenario has strong observational support: detailed images of the protoplanetary disks were obtained [11], large scale magnetic fields $(B \sim 1-3 \mathrm{kG}$ for CTTSs and $B \sim 100 \mathrm{G}$ for HAeBe stars) were found [12], redshifted absorption and/or emission components are observed in spectra of CTTSs and HAeBe stars (see right panel of Fig. 1).

3D MHD numerical calculations of magnetospheric accretion onto young stars have revealed many non-trivial features of the process. For example it appeared that if the angle between magnetic and rotation axes of the star is $\lesssim 15^{\circ}$, then accretion flow is divided to several unstable 'tongues' that produce chaotic hotspots on the stellar surface and irregular light curves - see [13] for details. What is more [14] and [15] found that the postshock thickness should oscillate due to a radiative shock instability, with a typical time $<100 \mathrm{~s}$. Such oscillations have not been observed, possibly due to inhomogeneous character of magnitospheric flow. On the other hand I would like to mention the so-called 'disk locking' effect, predicted by [16] and confirmed in numerical calculations, thanks to which angular velocity of accreting young stars is not very high. This is due to the fact that some stellar magnetic field lines couple to the disk outside of corotation radius what prevents stellar spinup. 
Calculations of spectrum of the AS and the magnetospheric flow are in general agreement with observations $[17,18]$. Howevere still there are a number of serious disagreements: for example, calculations failed reproduce observed intencities and profiles of e.g. He II 1640, 4686 as well as C IV 1548, 1550 lines in spectra of CTTSs $[19,20]$. We associate special hopes for solving these problems with the WSO-UV observatory [21], because there are many strong resonant lines of ions, atoms and molecules in the UV band [22].

Chromospheric-coronal activity is also important in the case of CTTSs: for example a number of emission lines as well as X-ray emission with an energy above $1 \mathrm{keV}$ are definitely not connected with the process of magnitospheric accretion - see e.g. [23]. The origin and significance of these processes in the case of HAeBe stars is not so clear, but it is worth recalling that these objects also have strong enough magnetic field and high X-ray luminocity [24].

\section{Matter outflow from young stars}

Some emission lines (e.g. $\mathrm{H}_{\beta}$, , HeI 10830, Na I D) in the spectra of CTTSs [25] and HAe/Be stars [26] have blueshifted absorption components, which indicated the outflow of matter from these objects with terminal velocity up to $500 \mathrm{~km} \mathrm{~s}^{-1}$. It has now become clear that these absorption features are associated with the magnetospheric wind - the matter outflow from the region where the accretion disk interacts with the magnetosphere of the star. The size of the wind's lunching region is of order of several stellar radii, i.e. $\sim 10^{11} \mathrm{~cm}$. At the base of the wind gas density and temperature are $\sim 10^{11} \mathrm{~cm}^{-3}$ and $10^{4}-10^{5} \mathrm{~K}$, respectively - see e.g. [5, 27] and references therein.

[28] found that profiles of forbidden lines in low resolution spectra of young stars (e.g. [O I] 6300, [S II] 6717, 6730) have two component structure. It appeared that so called low velocity component of the profiles is formed in the wind blowing from the surface of the accretion disk over a large range of disc radii (from a few stellar radii to tens au) with terminal velocities of order of keplerian velocity in the lunching region. The reasons of the disk wind are photoevaporation of protoplanetary disk's atmosphere heated by UV and X-ray radiation of the central star and/or magneto-centrifugal mechanism [29] - see e.g. [30] and references therein. Note that MHD wind remove disc's angular momentum and thus drive accretion, however, the effectiveness of this process is still debated [31].

The second, high velocity component of the forbidden lines profiles is originated in the jets - extended (up to $3 \mathrm{pc}$ ) highly collimated bipolar gas flows: jet's opening angle drops from $20^{\circ}-30^{\circ}$ initially to a few degrees at $10-20 \mathrm{AU}$ from the central star [32]. The jets consist of a chain of compact $(r \sim 100 \mathrm{au})$ nebulae $\left(N_{e} \sim 10^{2}-10^{6}\right.$ $\left.\mathrm{cm}^{-3}, T \sim 10^{4} \mathrm{~K}\right)$, called Herbig-Haro $(\mathrm{HH})$ objects, between which there is a less dense gas. Mass loss connected with the jets of young stars is $10^{-9}-10^{-11} \mathrm{M}_{\odot} \mathrm{yr}^{-1}$, which is $\sim 0.1$ of accretion rate $\dot{M}_{\text {acc }}$. HH-objects move across the sky at a characteristic speed of $V \approx 300 \mathrm{~km} \mathrm{~s}^{-1}$, gradually changing their shape over a period of a few years. ${ }^{1}$ On the other hand spectra of HH-objects are that of shocked gas, corresponding to pre-shock velocity of $\Delta V \approx 10-30 \mathrm{~km} \mathrm{~s}^{-1}$ only. It means that HH-objects are internal shocks in the gas moving with velocity $V$, due to variability of injection velocity with amplitude $\Delta V[33]$.

Usually, chains of HH-objects form a straight line passing through the central star and perpendicular to the inner regions of the disk, and each object in the jet corresponds to an HH-object similar in parameters and distance from the star in the counterjet. But there are exceptions to this rule, e.g. in RW Aur A, the gas velocity and the number of HH-objects in the jet and counterjet differ by about twice [34]. It is not yet clear whether this effect is due to the difference in the conditions in the circumstellar medium on both sides of the accretion disk or the asymmetry is associated with the operation of the 'central engine' [35]. No one doubts now that the collimation of gas outflow from the immediate vicinity of young stars into jet(s) is due to MHD processes and is closely related to the process of magnetospheric accretion - see e.g [36] and references therein. But magnitospheric accretion, apparently, is a necessary, but insufficient condition for jet formation. For example, CTTS BP Tau has strong magnetic field as well as all the signs of magnetospheric accretion and strong gas outflow [37], but has no jet.

\section{Eruptive variables FUors and EXors}

Almost a half century ago [38] identified two groups of young stars with strong, long-lived optical outbursts. FU Orionis type stars (FUors) are TTSs which increased their brightness up to $\Delta m_{\mathrm{pg}} \approx 6^{m}$ for $\Delta t \sim 1$ yr and then for several decades returned to the initial level. EX Lupi type stars (EXors) exhibit shorter and repetitive outbursts with lower amplitude - see Fig. 2. At the moment, several dozen FUors and EXors are known, but it is still an open question whether they represent two distinct classes and occur in the same evolutionary phases - see [39] and references therein.

Although there is no doubt that the flares of FUors and EXors are caused by an increase of the disk's accretion rate - up to $10^{-4} \mathrm{M}_{\odot} \mathrm{yr}^{-1}$ [41], - the reason of this phenomenon is an open question. The following mechanisms responsible for episodic accretion rate bursts were proposed: thermo-viscous instabilities in the inner disk, tidal

\footnotetext{
${ }^{1}$ See e.g. http://sparky.rice.edu/movies/hh111jetc.gif
} 

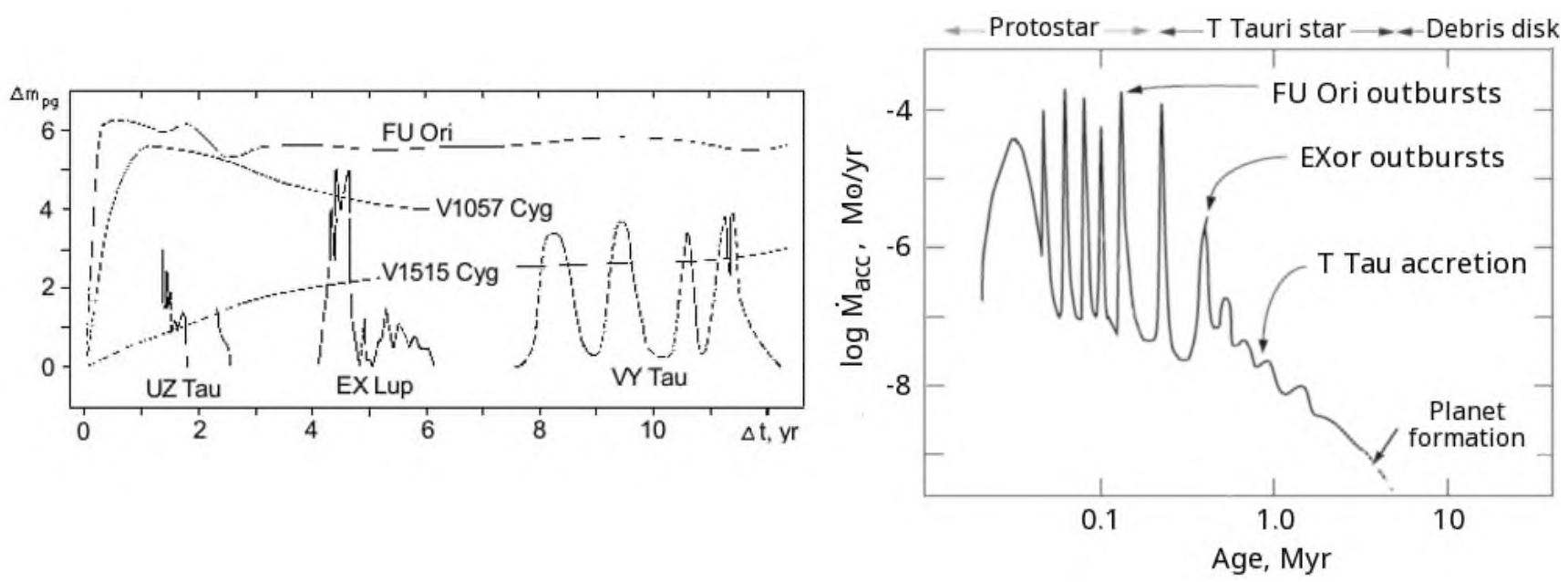

Figure 2: Left panel - lightcurves of some FUORs and EXors [38]. Right panel - schematic dependence of the accretion rate on time in the process of star formation [40].

effects from close encounters in binary systems or stellar clusters, the magnitorotational instability, accretion of dense gaseous clumps in a gravitationally unstable disk, outbursts due to planet-disk mass exchange. ${ }^{2}$

The phenomenon of eruptive variables is important for solving fundamental problems of star formation. In particular, it appeared that luminocity of protostars (Class I YSOs) is much lower than one can expect, assuming that accretion rate during the first $10^{5} \mathrm{yr}$ of star formation is nearly constant, as simple theoretical models predicted. [42] have demonstrated that episodic accretion is a possible resolution to the 'luminosity problem'. Fig. 2 schematically shows how the accretion rate changes during the transformation of a protostar into a young star in the frame of episodic accretion paradigm. Note that possible advection during high accretion rate episods can significantly change evolution tracks of YSOs at ages $t \lesssim 1$ Myr [43].

\section{Variability caused by circumstellar dust}

Photometric variability of young stars can be caused not only by 'physical' reasons (accretion process or/and chromospheric activity) but also by variable obscuration of the central star by circumstellar dust. In particular UX Ori type stars (UXORs) demonstrate aperiodic algol-like minima with amplitudes up to $\Delta V \sim 2-3^{m}$ and duration from a few days to a few weeks. The brightness variations of these (predominantly Herbig Ae) stars are accompanied by changes in color, linear polarization and spectra [44, 45]. Long-term monitoring from space observatories (CoRot, Kepler, TESS) revealed many so called dippers - a class of young variable star exhibiting day-long dimmings with depths of up to a few tenths of magnitude.

The role of the 'dust screen' responsible for observed eclipses of young stars can play 'humps' (azimuthal inhomogeneities) of the protoplanetary disk seen nearly edge on. For example in the case of AA Tau the (quasy)periodical eclipses are produced by dust of the disk warped by stellar magnetic field [46]. It appeared that about 20 per cent of young stars have this kind of variability [47].

Dusty disk wind also can lead to the eclipse of the central star as in the case of RW Aur A. This star has experienced two unusual dimming events. The first one occurred in 2010-2011 $\left(\Delta t \sim 150^{\mathrm{d}}, \Delta V \approx 2^{m}\right)$, and the second, even deeper, dimming started in the summer of 2014 and has continued up to the present - see [48] and references therein. During the second dimming the brightness of the star decreased by $\Delta V>5^{m}$ and the polarization degree of the star at this period has reached $30 \%$ in the $I$ band [49].

[50] found that dipper disc inclinations are consistent with an isotropic distribution and thus the dipper phenomenon is unrelated to the outer $(r>10 \mathrm{au})$ disc resolved by ALMA. This once again indicate that the nature of eclipsing dust clouds can be very different.

The passage of dusty cloud through the disk of a star should lead to a distortion of the line's profiles in its spectrum [51, 52]. These distortions arise due to partial obscuration of the rotating stellar surface, which results in the weakening of some parts of the profiles, corresponding to the radial velocities of the obscured regions the analog of well known Rossiter-McLaughlin effect in eclipsing binaries. This approach not only opens up an opportunity to study the structure of dust clouds on a scale smaller than the radius of the star, but also allows to conclude whether the dust clouds are connected with the disk (movement perpendicular to the axis of rotation of the star) or with the dust wind (movement parallel to the axis of rotation of the star) - compare animated gif pictures on http://deneb.sai.msu.ru/angle0.gif and http://deneb.sai.msu.ru/angle90.gif.

\footnotetext{
${ }^{2}$ See http://uxors-2019.crao.ru/images/presentation/vorobyov.pdf for details.
} 
Note finally, that variability of the brightness and spectral line profiles caused by dust eclipses requires to be careful about attempts to use asteroseismology methods to study young stars.

\section{Conclusion}

Studying the nature of activity of young stars is important not only for investigation of star and planet formation process, but also from the viewpoint of understanding the physics of disk accretion and acompanied matter outflow. First, in the case of young stars the accretion flow can be traced in spectral lines that allows to study its kinematics, and second, young stars are relatively close to us - down to $50 \mathrm{pc}$.

\section{References}

1. P. H. Bodenheimer, Principles of Star Formation (2011).

2. R. E. Pudritz and T. P. Ray, Frontiers in Astronomy and Space Sciences, 6, 54, 2019.

3. F. Nakamura, S. Kameno, T. Kusune, I. Mizuno, K. Dobashi, T. Shimoikura, and K. Taniguchi, PASJ, 71, 117, 2019.

4. A. H. Joy, ApJ, 102, 168, 1945.

5. L. Hartmann, G. Herczeg, and N. Calvet, ARA\&A, 54, 135, 2016.

6. P. P. Petrov, G. F. Gahm, J. F. Gameiro, R. Duemmler, I. V. Ilyin, T. Laakkonen, M. T. V. T. Lago, and I. Tuominen, $A \mathscr{G} A, \mathbf{3 6 9}, 993,2001$.

7. G. H. Herbig, ApJS, 4, 337, 1960.

8. C. Bertout, G. Basri, and J. Bouvier, ApJ, 330, 350, 1988.

9. S. A. Lamzin, $A \mathscr{E} A, \mathbf{2 9 5}, \mathrm{L} 20,1995$.

10. S. A. Lamzin, Astronomy Reports, 42, 322, 1998.

11. L. M. Pérez, M. Benisty, S. M. Andrews, A. Isella, et al., ApJL, 869, L50, 2018.

12. J. F. Donati and J. D. Landstreet, ARAधA, 47, 333, 2009.

13. M. M. Romanova, A. V. Koldoba, G. V. Ustyugova, A. A. Blinova, D. Lai, and R. V. E. Lovelace, $M N R A S$, 506, 372, 2021.

14. A. V. Koldoba, G. V. Ustyugova, M. M. Romanova, and R. V. E. Lovelace, MNRAS, 388, $357,2008$.

15. G. G. Sacco, C. Argiroffi, S. Orlando, A. Maggio, G. Peres, and F. Reale, A\& A, 491, L17, 2008.

16. A. Königl, ApJL, 370, L39, 1991.

17. R. Kurosawa, M. M. Romanova, and T. J. Harries, MNRAS, 416, 2623, 2011.

18. A. V. Dodin, S. A. Lamzin, and T. M. Sitnova, Astronomy Letters, 39, 315, 2013.

19. S. A. Lamzin, Astronomy Reports, 47, 540, 2003.

20. A. Dodin, MNRAS, 475, 4367, 2018.

21. A. A. Boyarchuk, B. M. Shustov, I. S. Savanov, M. E. Sachkov, et al., Astronomy Reports, 60, $1,2016$.

22. K. France, R. Schindhelm, G. J. Herczeg, A. Brown, et al., ApJ, 756, 171, 2012.

23. A. Dodin, S. Lamzin, P. Petrov, B. Safonov, M. Takami, and A. Tatarnikov, MNRAS, 497, 4322, 2020.

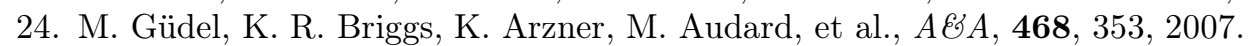

25. L. V. Kuhi, ApJ, 140, 1409, 1964.

26. U. Finkenzeller and R. Mundt, A\&A Sup., 55, 109, 1984.

27. F. López-Martínez and A. I. Gómez de Castro, MNRAS, 442, 2951, 2014.

28. J. Kwan and E. Tademaru, ApJ, 454, 382, 1995.

29. R. D. Blandford and D. G. Payne, MNRAS, 199, 883, 1982.

30. M. L. Weber, B. Ercolano, G. Picogna, L. Hartmann, and P. J. Rodenkirch, MNRAS, 496, $223,2020$.

31. P. J. Rodenkirch, H. Klahr, C. Fendt, and C. P. Dullemond, A\&SA, 633, A21, 2020.

32. S. Cabrit, in J. Bouvier and I. Appenzeller, eds., Star-Disk Interaction in Young Stars, volume 243, $203-214$ (2007).

33. A. C. Raga, J. Canto, L. Binette, and N. Calvet, ApJ, 364, 601, 1990.

34. S. Y. Melnikov, J. Eislöffel, F. Bacciotti, J. Woitas, and T. P. Ray, A\&A, 506, 763, 2009.

35. L. N. Berdnikov, M. A. Burlak, O. V. Vozyakova, A. V. Dodin, S. A. Lamzin, and A. M. Tatarnikov, Astrophysical Bulletin, 72, 277, 2017.

36. S. Cabrit, Jets from Young Stars: The Need for MHD Collimation and Acceleration Processes, volume 723,21 (2007).

37. L. Errico, S. A. Lamzin, and A. A. Vittone, $A \& A$, 377, 557, 2001.

38. G. H. Herbig, ApJ, 217, 693, 1977.

39. M. Audard, P. Âbrahám, M. M. Dunham, J. D. Green, et al., in H. Beuther, R. S. Klessen, C. P. Dullemond, and T. Henning, eds., Protostars and Planets VI, 387 (2014).

40. L. Hartmann, Accretion Processes in Star Formation: Second Edition (2009).

41. L. Hartmann and S. J. Kenyon, ARA\&A, 34, 207, 1996.

42. S. J. Kenyon and L. Hartmann, ApJS, 101, 117, 1995. 
43. V. G. Elbakyan, E. I. Vorobyov, C. Rab, D. M. A. Meyer, M. Güdel, T. Hosokawa, and H. Yorke, MNRAS, 484, 146, 2019.

44. V. P. Grinin, Soviet Astronomy Letters, 14, 27, 1988.

45. V. P. Grinin, O. V. Kozlova, A. Natta, I. Ilyin, I. Tuominen, A. N. Rostopchina, and D. N. Shakhovskoy, $A \mathscr{E} A, \mathbf{3 7 9}, 482,2001$.

46. J. Bouvier, A. Chelli, S. Allain, L. Carrasco, et al., A\&A, 349, 619, 1999.

47. A. M. Cody, J. Stauffer, A. Baglin, G. Micela, et al., AJ, 147, 82, 2014.

48. J. E. Rodriguez, R. Loomis, S. Cabrit, T. J. Haworth, et al., ApJ, 859, 150, 2018.

49. A. Dodin, K. Grankin, S. Lamzin, A. Nadjip, et al., MNRAS, 482, 5524, 2019.

50. M. Ansdell, E. Gaidos, C. Hedges, M. Tazzari, et al., MNRAS, 492, 572, 2020.

51. V. P. Grinin and I. S. Potravnov, Astrophysics, 56, 1, 2013.

52. A. V. Dodin and E. A. Suslina, MNRAS, 503, 5704, 2021. 


\title{
Planets - a modern view
}

\author{
M.Ya. Marov ${ }^{1}$, I.I. Shevchenko 2,3 \\ marov@geokhi.ru \\ ${ }^{1}$ V.I. Vernadsky Institute of Geochemistry and Analytical Chemistry, Russian Academy of Sciences, Kosygina str. 19, \\ Moscow, 119991, Russia \\ ${ }^{2}$ Saint Petersburg State University, Universitetskaya naberezhnaya, 7/9, Saint Petersburg, 199034, Russia \\ ${ }^{3}$ Institute of Applied Astronomy, Russian Academy of Sciences, Nab. Kutuzova 10, Saint Petersburg, 191187, Russia
}

The modern view of planets goes far beyond the usual concept of the planets as bodies of the Solar system. The discovery of exoplanets has immeasurably expanded the understanding of the architecture and properties of planetary systems. Major advances have been made in the study of the planets and minor bodies of the Solar system. However, no answers have been received to fundamental questions about the causes of various paths of evolution and formation of planetary natural complexes. To give answers to these questions, research on exoplanets is called upon, of which more than 5000 have been discovered to date. Exoplanet studies provide an approach to solving the key problems of stellar-planetary cosmogony the genesis and evolution of planets as byproduct of star formation. The most urgent problems concern the formation of planetary systems around stars of various spectral classes; the nature of hot Jupiters; the reasons for the predominance of super-Earths and sub-Neptunes, which are absent in the Solar system; stability of planetary systems of binary stars, including circumbinary systems. Of particular interest are terrestrial planets with orbits in zones of "potential habitability", studies of which open a new page in astrobiology.

Keywords: planets, exoplanets, Solar system

DOI: 10.51194/VAK2021.2022.1.1.005

\section{Introduction}

The modern view of planets goes far beyond the usual concept of the planets as bodies of the Solar system. The discovery of exoplanets marked the beginning of a new grandiose stage in the development of planetary science, expanding the understanding of the diversity and features of planetary systems of the stars other than the Sun. The accumulation of data on the nature of the planets of the Solar system, their physical and geological-geochemical characteristics, as well as on the nature and properties of minor bodies, progressively continues. The progress of the planetary research is provided by space probes, space telescopes, and instruments and methods of the ground-based astronomy. Taken together, the results of experimental and theoretical studies of the Solar system, exoplanetary systems and circumstellar protoplanetary disks provide a powerful impetus to the development of stellar-planetary cosmogony.

The classical planet (that residing in the Solar system), according to the IAU definition (XXVI General Assembly of the IAU, Prague, 2006) is defined as follows: it revolves around the central star (the Sun); its mass (self-gravity) is sufficient for overcoming solid-body forces and thus for acquisition of hydrostatic-equilibrium form; it clears the vicinity of its orbit. Dwarf planet does not clear the vicinity of the orbit, but is not a planetary satellite; this class includes Ceres, Pluto and a number of other trans-Neptunian bodies in the Kuiper belt. Minor bodies are satellites of planets, asteroids, comets, meteoroids, interplanetary dust.

In exoplanetary studies, by planets one generally implies discovered bodies with a mass of $M<0.013 M_{\text {Sun }}$ (i.e., less than $\sim 13$ Jovian masses); objects with masses $\left(0.013 M_{\text {Sun }}<M<0.08 M_{\text {Sun }}\right)$ are defined brown dwarfs.

Unlike stars, whose observational statistics is immense and, e.g., the evolutionary paths of which are well studied and described in the HR diagram, until recently the only example of a planetary system was known, namely, the Solar system. The discovery of exoplanets fundamentally changed the situation and made it possible to form a new modern view of the planets.

The first exoplanets were discovered in the early 1990s by Alexander Wolszczan and Dale Frail. These were planets of the pulsar PSR B1257-12, a neutron star with a mass 1.4 times that of the Sun [1]. Later on, planets of some other pulsars were identified; it should be noted, however, that discoveries of planets of pulsars are generally rare due to the supposedly small number of planets hosted by "residual" stars that have experienced a supernova stage. An exoplanet of a main sequence star was first discovered in 1995 by Michel Mayor and Didier Queloz; this was a "hot Jupiter" orbiting 51 Pegasi [2]. For this achievement, Michel Mayor and Didier Queloz were awarded the 2019 Nobel Prize in Physics with the formulation "for the discovery of an exoplanet orbiting a Sun-like star."

\section{The Solar system: key features}

The structure of the Solar system is formed by 8 major planets ( 4 terrestrial planets in orbits within $0.4-1.5$ AU from the Sun, and 4 giant planets of the Jupiter group within 5-30 AU), dwarf planets, minor bodies (satellites, asteroids, TNOs, comets, interplanetary dust). The main belt of asteroids (2.7-3.2 AU), trans-Neptunian Kuiper belt (30-100 AU, $\sim 10^{8}$ bodies), Oort cometary cloud $\left(10^{3}-10^{5} \mathrm{AU}, \sim 10^{12}\right.$ bodies) are the main reservoirs of minor bodies. Systems of planetary satellites contain in total $\sim 200$ satellites, including large ones (some of them comparable in size with Mercury) and small ones (with physical radius less than $\sim 100 \mathrm{~km}$, some of them are only 
a few km in size). The terrestrial planets have only three satellites: the Moon of the Earth and Phobos and Deimos of Mars. Traces of active geological and impact processes on the terrestrial planets, asteroids, nuclei of comets, and satellites of giant planets are often remarkable. There exist certain relationships between the composition and properties of atmospheres and geological and geochemical processes, evolution and Solar influence (see review in $[3,4]$ ). The potential habitability zone encompasses the radial interval 0.9-1.4 AU with respect to the Sun.

The most significant successes in the planetary research of the Moon, Mars, Mercury, Jupiter, Saturn, Pluto, the Kuiper belt objects, some comets such as $67 \mathrm{P} /$ Churyumov-Gerasimenko, during the last decades were due to space missions. The Moon is gaining an increasing attention since the discovery of water ice and volatiles deposits in its polar regions. The most remarkable results were achieved with NASA's LCROSS and LRO, the Chinese lunar rover Yutu, return of Lunar soil - Chang'e 5, to mention a few. Plans for Mars remain in the focus of attention among all the plans for planets in-depth study, based on the results of several recent successful space missions (NASA's probes Mars Surveyor, Mars Odyssey, rovers Spirit, Opportunity, Curiosity, Perseverance, Chinese mission Zhurong). NASA's Messenger spacecraft successfully operated at Mercury for several years, and the ESA's BepiColombo spacecraft was launched, and now en route to the planet. New data on Jupiter and Saturn, their moons and rings (NASA's Galileo and Juno missions, ESA's Cassini-Huygens mission for studies of Saturn and Titan). ESA's Rosetta-Philae mission investigated the coma and the surface of the nucleus of comet 67P/Churyumov-Gerasimenko, and NASA's New Horizons mission explored Pluto and some other bodies in the Kuiper belt.

The Russian planetary program includes, apart from installation of individual instruments on American and European probes [5], the Luna 25-28 program (from 2022 to 2030) and the Russian-European ExoMars (20162022).

The "Return to the Moon" is of fundamental importance not only for the knowledge of Moon's origin and its geological and geochemical properties, but also for the reconstruction of the main evolutionary processes of the entire large family of bodies of the Solar system. Earth's neighbours, Mars and Venus, which have experienced other (compared to the Earth) evolutionary paths, remain in the focus of attention from the point of view of comparative planetology. The discovery of water on Mars, together with geological and petrographic research, and the study of atmospheric chemistry, including variations in methane content, contribute to the study of ancient climate and the possibility of life.

From the standpoint of comparative planetary science, the emerging return of interest in the study of Venus is of paramount importance [6]. Research of giant planets and their moons, comets, asteroids, objects of the Kuiper belt make a great contribution to solving the problems of planetary cosmogony and cosmochemistry, especially in what concerns the properties of minor bodies as relics of the processes of the Solar system formation. However, despite a number of major successes, there are no "breakthrough" achievements that would give answers to fundamental questions about the genesis, various ways of evolution and the formation of natural complexes on planets.

\section{Exoplanetary systems}

Exoplanets are a new widest class of objects that provide an approach to solving the problems of stellar-planetary cosmogony and cosmochemistry, answering key questions of genesis and evolution. Discoveries of exoplanets form the basis of modern views on the structure, dynamics, formation and evolution of planetary systems and on the place of the Solar system among them.

Exoplanets are mostly hosted by stars of late spectral classes and high metallicity. Planetary systems of binary stars, including circumbinary systems, are attracting great attention. Of particular interest are terrestrial planets and super-Earths with orbits in zones of "potential habitability", where conditions favourable for life are possible.

By August 2021, about 5000 exoplanets have been discovered. They are contained in more than 3500 planetary systems, $\sim 800$ of which are multiplanetary (with two or more identified planets). Methods of discovery are distinguished as indirect ones (Doppler spectrometry - the RV method; transits, microlensing) and direct ones (differential spectrophotometry during transits, coronography, polarimetry, astrometry). Observational selection effects result, first of all, in that largest planets close to parent stars are discovered. This factor aggravates the predominance of exotic type of planets - "hot Jupiters" - among the first discoveries made by the RV method.

In searches of planets of Proxima Centauri, a red dwarf star with a mass of $M=0.12 M_{\text {Sun }}$ and a surface temperature $T \sim 3000 \mathrm{~K}$, situated at a distance of 4.3 light years from the Sun, a planet with a mass of $1.27 \sin i$ (in terrestrial units) was discovered by the RV method in 2016 at the European Southern Observatory. The radius of the planet's orbit is only $0.05 \mathrm{AU}$, which is 8 times less than the radius of Mercury's orbit, and the orbital period is 11 days [7]. The orbit is inside the zone of potential habitability: on the surface of the planet, water may theoretically exist in the liquid state. However, owing to a high level of hard radiation, the origin of life is hardly possible. Some four years before the discovery of Proxima Centauri b, a discovery of a planet orbiting the star Alpha Centauri B was reported; however, this turned out to be an artifact. 
The most distant exoplanets in orbits around stars are those belonging to SWEEPS-04 and SWEEPS-11 systems. They were discovered in the framework of the SWEEPS survey (Sagittarius Window Eclipsing Extrasolar Planet Search). The observations of planetary transits in a field saturated with stars in the direction of the bulge of the Galaxy resulted in the discovery of these two transiting planets, which are at a distance of $8.5 \mathrm{kpc}$ from the Sun. However note that observations of microlensing events reveal existence of planetary mass objects (suspected as being free-floating planets) at distances as large as megaparsec.

The mass distribution of planets and the mass-radius and density-mass relationships are of primary interest. The maximum in the mass distribution is at masses of the order of Jupiter's (including "hot Jupiters"). However, the number of discovered planets with masses from mini-Neptunes to super-earths is constantly growing.

There exists a correlation between the metallicity of a star and the presence of planets in its system: stars with low metallicity generally have no planetary systems observed; whereas for metallicities higher than that of the Sun, the probability of the presence of planets increases sharply. The correlation is most pronounced for the main sequence stars with giant planets - sub-Neptunes and hot Jupiters [8]. The birth of stars in molecular clouds with high metallicity increases the probability of a planetary system formation [9]. With an increase in metallicity, the content of the heavy component rises, including rocks, forming large cores, on which volatiles condense; therefore, the disk gas accretes and gas-ice giant planets are formed.

The Solar system planets are similar in physical properties to some types of exoplanets. Probably, the internal structure of exoplanets as a whole corresponds to the terrestrial and giant planet models. The differences may depend on the metallicity of the parent star and the composition of the protoplanetary disk. Surface temperature, composition and properties of the atmosphere are determined by the radial distance, lithosphere-atmospheric interaction and evolution. However, only the upper gas envelopes are accessible to observations.

\section{Planetary dynamics}

Unlike the planets of the Solar system, the observed exoplanets frequently have large orbital eccentricities; in addition, in many exosystems, giant planets have orbits close to their parent stars (hot Jupiters and Neptunes). There are no super-Earths in the Solar system. However, there are multiplanet systems similar to the Solar one, for example, Gliese 581, $47 \mathrm{UMa}, \mu$ Arae (HD 160691).

The long-term stability and the resonance structure of planetary systems are broadly studied nowadays. Cosmogonic processes, instabilities, resonances and migration determine the configurations of planetary systems. Captures in orbital resonances occur during the migration of protoplanets in the gas-dust disk.

Analytical and numerical-experimental criteria for the stability of planetary systems have been developed. Analytical methods are based on the adaptation of Hill's criterion and the Chirikov resonance overlap criterion (see review in $[10,11]$. The clearing of planetary chaotic zones in planetesimal disks is described by these criteria; see $[12,13,14]$.The stability analysis makes it possible to impose stringent constraints on the orbital parameters of multiplanet systems [15].

Resonances in exoplanetary systems are ubiquitous. In the Solar system, only approximate orbital commensurabilities (Jupiter-Saturn $\sim 5 / 2$, Saturn-Uranus $\sim 3 / 1$, Uranus-Neptune $\sim 2 / 1$ ) are present, apart from the NeptunePluto $3 / 2$ resonance. The Lyapunov time (the characteristic timescale of the motion predictability) estimate for the Solar System turns out to be three orders of magnitude less than its age. The multiplet of subresonances of the Jupiter-Saturn-Uranus three-body resonance can be a cause for this long-term chaos [16]. An example of short-term dynamical chaos in a planetary systems is provide by Kepler-36 [17].

About a third of discovered exoplanets belong to multiplanet systems, i.e., the systems containing two or more planets [18]. 800 multiplanet systems are known to date. Well-known examples are Kepler-11, Kepler90, Trappist-1. Orbital resonances are widespread in them; an extreme example is Kepler-223, a closely-packed completely resonant system.

Planetary systems of binary and multiple stars are of especial interest, due to their general instability, as suspected in earlier studies. They were believed to be the primary source of free-floating planets (FFPs).

Most planets in binary star systems orbit around one of the stars. In planetary dynamics in such systems the Lidov-Kozai effect may often play a prominent role; see review in [19]. The circumbinary planets orbit around both stars. Such planetary systems have also been discovered: HW Vir, NN Ser, UZ For, DP Leo, FS Aur, SZ Her, Kepler-16, 34, 35, 38 and 47. The Kepler-47 circumbinary system is multiplanet (it contains two planets). The dynamics of such planetary systems is often at the edge of stability (see $[20,10]$ ). Many planets of binary star systems may escape into interstellar space, forming "rogue planets" (orphan planets, FFPs).

\section{Formation of planetary systems}

The discovery of exoplanets and data on the structure and composition of circumstellar protoplanetary disks ensured tremendous progress in stellar-planetary cosmogony. The most significant results were obtained by observations with a high angular resolution of structure and properties of disks of MS stars by the ALMA radio telescope system. The formation of planetary systems is due to physicochemical and dynamical processes in the 
gas and dust disks that form together with the stars in the process of evolution (see reviews in [21, 3]). The evolution of protoplanetary disks includes three main stages: - the formation of dust clusters and primary solids as a result of hydrodynamic (flow) and Jeans (gravitational) instabilities; - growth of primary dust grains to pebble size solids and eventually to the size of planetesimals and the formation of planetary embryos in collisions; - oligarchic growth of planetary embryos due to dynamic processes (tides, migration, resonances). In the course of disk matter drifting towards the parent star, the fragmentation of solids at the snow line plays an important role, in what concerns primary bodies in the inner zone [22]. In the inverse motion, during the first 3-5 million years, processes at the snow line could be responsible for giant gas-icy planets growth [23]. At the stage of planet birth, secondary debris disks can be formed as a result of shock processes.

The most likely scenario for the evolution of a protoplanetary disk in a circumbinary system is the formation of a planetary embryo in the outer regions of the disk, where conditions for accretion are favorable, with its subsequent migration inside the system, to the boundary of the inner disk cavity generated by the central binary. The cavity roughly corresponds in size to the region of chaos for orbits around the binary system. Formation in situ (for planets such as Kepler-16b, 34b, and 35b) is less likely due to high planetesimal collision rates and relatively low concentration $[24,25,26]$.

The "Hot Jupiters problem" is broadly studied. Migration and tidal effects play a fundamentally important role in the formation and dynamics of planetary systems, and these effects provide an approach to solving the problem of hot Jupiters. These planets are likely to form at a distance from the star and drift from there due to the mechanism of migration closer to the star. The migration can be caused by the formation of a gap in the disk and/or deceleration in the residual gas, with the possible occurrence of resonances. The problem of survival of hot Jupiters with an extended atmosphere and the reason for the migration stop may be related to the presence of tidal effects. Scenarios similar to the Grand Tack scenario for the early Solar System are possible: Jupiter's motion toward the Sun, then stopping by Saturn and drifting in the opposite direction [27, 28, 29].

Do there exist systems closely resembling the Solar one? Such systems would provide an outer look on our system evolution stages. HR 8799 is remarkable, being a "young" structural analogue of the Solar system. It has four planets discovered from infrared images obtained by coronography. The architecture of the HR 8799 system is similar to that of ours: in HR 8799, the orbits of four giant planets are surrounded by a warm dust belt similar to the asteroid belt in our system, and from outside these four planets are surrounded by a cold belt similar to the Kuiper belt. The inner part of the system (interior to the "asteroid belt") contains a zone of potential habitability. According to [30], "the HR 8799 system is a younger, longer and more massive version of the Solar system". The "asteroid belt" is limited by the resonances $4 / 1$ and $2 / 1$ with the inner giant planet, as in the Solar system.

"Exomoons" of observed exoplanets are intensely searched for, with first successes. The Kepler-1625 system, according to the analysis of the transit light curve, contains a binary planet — "Jupiter" with a Neptune-like satellite [31].

\section{Potential habitability}

Life is highly adaptable. It can persist under significant changes in temperature, pressure, radiation dozes, acidity $(\mathrm{pH})$ of the environment. Suitable temperature at the planet's surface represents a critical condition for potential habitability of planets in the vicinity of the parent star (see, e.g., reviews in [32]).

The zone of potential habitability is an area around a star, where water in the liquid phase and the stability of climatic conditions necessary for life is possible on the surface of a terrestrial planet. The radial location and extent of this zone may change over time in the process of planetary system evolution.

The potential habitability zones are much more compact for red dwarfs. An intensely studied system is TRAPPIST-1, which consists of 7 planets orbiting around a red dwarf, with three of the planets being located in the potential habitability zone.

Of particular interest are remarkable potential habitability properties of circumbinary planet classes, including that of planets of contact-binary stars $[33,34]$.

\section{Conclusions}

Planetary sciences experience an epoch of dramatic evolution. Ground-based astronomy and space exploration broadened up tremendously our knowledge on nature of the Solar system bodies, while discovery of exoplanets brought invaluable information about diversity, architecture and properties of planetary systems around stars of various classes. Altogether, this forms a new modern view on planets and an important milestone in astronomy.

Exoplanet studies provide an approach to solving the key problems of stellar-planetary cosmogony — the genesis and evolution of planets. The most urgent problems are the formation of planetary systems in stars of various spectral classes, the nature of hot Jupiters, the reasons for the predominance of super-Earths and sub-Neptunes (which are absent in the Solar system), stability of planetary systems of binary stars, including circumbinary systems. Of particular importance are studies of the terrestrial planets with orbits in zones of "potential habitability", which open a new page in astrobiology. 


\section{Acknowledgments}

This work was supported by a grant 075-15-2020-780 (N13.1902.21.0039) "Theoretical and experimental studies of the formation and evolution of extrasolar planetary systems and characteristics of exoplanets" of the Ministry of Science and Higher Education of the Russian Federation.

\section{References}

1. A. Wolszczan and D. A. Frail, Nature, 355, 145, 1992.

2. M. Mayor and D. Queloz, Nature, 378, 355, 1995.

3. M. Marov, The Formation and Evolution of the Solar System. In: Oxford Research Encyclopedia of Planetary Science, Oxford University Press (2018).

4. M. Y. Marov, Cosmos: From the Solar System into Depth of the Universe, Fizmatlit, 3rd Ed. (2021).

5. I. G. Mitrofanov, A. B. Sanin, W. V. Boynton, G. Chin, et al., Science, 330, 483, 2010.

6. M. Y. Marov and D. H. Grinspoon, In: The planet Venus, New Haven and London, Yale University Press (1998).

7. G. Anglada-Escudé, P. J. Amado, J. Barnes, Z. M. Berdiñas, et al., Nature, 536, 437, 2016.

8. E. A. Petigura, G. W. Marcy, J. N. Winn, L. M. Weiss, et al., AJ, 155, 89, 2018.

9. D. A. Fischer and J. Valenti, ApJ, 622, 1102, 2005.

10. I. I. Shevchenko, Dynamical Chaos in Planetary Systems, Springer Nature (2020).

11. M. Y. Marov and I. I. Shevchenko, Physics Uspekhi, 63, 837, 2020.

12. T. V. Demidova and I. I. Shevchenko, ApJ, 805, 38, 2015.

13. T. V. Demidova and I. I. Shevchenko, MNRAS, 463, L22, 2016.

14. I. I. Shevchenko, AJ, 160, 212, 2020.

15. K. V. Kholshevnikov and E. D. Kuznetsov, Celestial Mechanics and Dynamical Astronomy, 109, $201,2011$.

16. N. Murray and M. Holman, Science, 283, 1877, 1999.

17. K. M. Deck, M. J. Holman, E. Agol, J. A. Carter, J. J. Lissauer, D. Ragozzine, and J. N. Winn, ApJL, 755, L21, 2012.

18. H. Rein, MNRAS, 427, L21, 2012.

19. I. I. Shevchenko, The Lidov-Kozai Effect - Applications in Exoplanet Research and Dynamical Astronomy, Springer Nature (2017).

20. I. I. Shevchenko, ApJ, 799, 8, 2015.

21. M. Y. Marov, The Fundamentals of Modern Astrophysics. A Survey of Cosmos from the Home Planet to Space Frontiers, Springer (2015).

22. M. Y. Marov, A. V. Rusol, and A. B. Makalkin, Solar System Research, 55, 238, 2021.

23. M. Lambrechts and A. Johansen, $A \mathscr{E} A, \mathbf{5 4 4}, \mathrm{A} 32,2012$.

24. A. Pierens and R. P. Nelson, A\&A, 472, 993, 2007.

25. S. Meschiari, ApJ, 752, 71, 2012.

26. S.-J. Paardekooper, Z. M. Leinhardt, P. Thébault, and C. Baruteau, ApJL, 754, L16, 2012.

27. K. J. Walsh, A. Morbidelli, S. N. Raymond, D. P. O'Brien, and A. M. Mandell, Nature, 475, $206,2011$.

28. S. A. Jacobson and A. Morbidelli, Philosophical Transactions of the Royal Society of London Series A, 372, 0174, 2014.

29. D. P. O'Brien, K. J. Walsh, A. Morbidelli, S. N. Raymond, and A. M. Mandell, Icarus, 239, $74,2014$.

30. V. Faramaz and et. al, ApJ, 693, 2009.

31. A. Teachey and D. M. Kipping, Science Advances, 4, eaav1784, 2018.

32. M. Perryman, The Exoplanet Handbook, Cambridge, Cambridge University Press (2018).

33. I. I. Shevchenko, $A J, \mathbf{1 5 3}, 273,2017$.

34. I. I. Shevchenko, International Journal of Astrobiology, 19, 500, 2020. 


\title{
A brief review on vertical electric currents in flaring active regions at the Sun
}

\author{
I. Zimovets, I. Sharykin \\ ivanzim@iki.rssi.ru \\ Space Research Institute of the Russian Academy of Sciences (IKI RAS), Profsoyuznaya str. 84/32, Moscow, 117997, \\ Russia
}

\begin{abstract}
A brief overview of vertical electric currents in flare-producing active regions (ARs) of the Sun is presented. The following issues are touched upon: the importance of studying electric currents in the solar atmosphere, ways of measuring them, some observational characteristics, spatial relations with flare sources, dynamics during flares and eruptions, connection between observations and some models.
\end{abstract}

Keywords: solar active regions, magnetic field, electric currents, solar flares

DOI: 10.51194 /VAK2021.2022.1.1.006

\section{Introduction}

The Sun is an active star. The solar activity is due to the processes of the generation of a magnetic field in its depths, the emergence of the magnetic field on the surface, and its dissipation in the solar atmosphere. Dissipation of magnetic energy means its transformation into other types of energy, e.g., into thermal energy of plasma and kinetic energy of accelerated (nonthermal) particles, into mechanical energy of movements of macroscopic plasma volumes, including various flows, ejections/eruptions and waves, into energy of electromagnetic radiation in various ranges of the spectrum, etc. The strongest magnetic fields are concentrated in active regions (ARs) and are visible on the surface of the Sun (the photosphere) in the form of sunspots with a characteristic spatial scale of several Mm. Also, apparently, there are numerous small-scale (fraction of Mm and less) magnetic elements with high field strengths of the order of $\mathrm{kG}$ outside ARs (e.g., [1, 2]). However, since the most powerful and bright phenomena of solar activity, such as solar flares and coronal mass ejections (CMEs), occur mainly in ARs, we are discussing magnetic field and electric currents in ARs in the present review.

Solar activity is electromagnetic in nature. When discussing its various manifestations, one usually operates with such a physical quantity as a magnetic field, B, and do not operate directly with an electric current density, $\mathbf{j}$. This is probably due, firstly, to the fact that many phenomena of solar activity are described within the framework of the magnetohydrodynamics (MHD) approximation, in the system of equations of which the electric current density is not present in an explicit form, and the current density is considered as a secondary physical quantity expressed in terms of a magnetic field from the Ampere-Maxwell law (e.g., [3]):

$$
\nabla \times \mathbf{B} \approx \frac{4 \pi}{c} \mathbf{j}
$$

in the differential form or

$$
\oint_{\partial S} \mathbf{B} \cdot \mathbf{d} \mathbf{l} \approx \frac{4 \pi}{c} \int_{S} \mathbf{j} \cdot \mathbf{d} \mathbf{S}
$$

in the integral form. Here, $c$ is the speed of light, and we neglected by an electric field, which is usually considered to be small in the solar atmosphere. Second, the magnetic field can be directly determined from observations, although at present this is routinely carried out only for the photosphere, and in the higher layers of the solar atmosphere, the determination of the magnetic field is still a very serious problem. Obviously, from measurements of the magnetic field vector at only one height (e.g., photosphere), it is possible to calculate only one vertical (radial) component of the electric field density vector at this height, $j_{z}$ (or $j_{r}$ ). The first such measurements were made about sixty years ago at the Crimean Astrophysical Observatory in the USSR [4]. There were also carried out unique measurements of the magnetic field vector at several altitudes in the solar atmosphere based on observations in various magnetosensitive lines and calculations of the horizontal component of the current, $j_{h}$ (e.g., [5]).

To calculate the vertical currents, both differential and integral expressions of the Ampere-Maxwell law (1) and (2) are used. The advantage of the differential approach is the ability to investigate a finer structure and obtain higher values of the current density in local areas. The advantages of the integral approach include the use of more data points and the lack of numerical differentiation, which reduces the error in current measurements (see [6]). The modern difficulties associated with determining the magnetic field in the photosphere and calculating the vertical current are discussed, e.g., by [7], and interested readers are referred to this review.

The purpose of the present short article is to give a brief overview of the works on the observation of vertical electric currents, $j_{r}$, in ARs of the Sun and their connection with solar flares. Other reviews on similar topics were also recently provided by [8] and [9]. 


\section{Why study electric currents in ARs?}

The study of electric currents in ARs of the Sun is important for a number of reasons. Some of them are listed below.

1. According to the potential minimum-energy theorem (e.g., [3]), the magnetic energy of a potential field in a bounded region is the smallest for given boundary conditions. The potential field contains no electric currents. This means that any magnetic field with currents will have an excess of magnetic energy relative to the energy of the potential field with the same boundary conditions. In other words, it will have free magnetic energy, which dissipates in ARs during the phenomena of solar activity. Indeed, the results of extrapolations in the potential and nonlinear force-free field (NLFFF, at which $\mathbf{j} \| \mathbf{B}$ ) approximations show that in the ARs the energy of the potential field is lower than the energy of the NLFFF and the free magnetic energy $\left(E_{\text {free }}=E_{\text {nlfff }}-E_{\text {pot }}\right)$ decreases during flares (e.g., $[10,11])$. It follows from this that the free magnetic energy in ARs is associated with the electric currents flowing in them, and their study is important for understanding the processes of free magnetic energy dissipation that occur during such phenomena as solar flares and eruptions.

2. In many ARs, especially in which flares and eruptions occur, the configuration of magnetic fields in the solar atmosphere observed in different spectral ranges, e.g., in $\mathrm{H} \alpha$, EUV and soft X-rays, is not well described in the potential (current-free) approximation (e.g., [12, 13, 14, 15]). A much more adequate description is achieved when electric currents are taken into account, in particular, within the NLFFF approximation (see [11]). Numerous observations show that flares and eruptions occur in non-potential magnetic structures, such as highly-sheared magnetic arcades, sigmoidal structures, and twisted magnetic flux-ropes, which could not exist without the presence of electric currents in them (e.g., [3]). Some analytical models of such magnetic structures, e.g., the well-known model of the twisted magnetic flux tube by [16], are described in terms of electric current.

3. The idea (and observational confirmations) of the presence of electric currents in ARs led to the development of the concept of coronal magnetic loops as equivalent electric (RLC) circuits, proposed originally by [17]. This concept continues to develop and with its help various phenomena and processes in the solar (and stellar) atmosphere are trying to be explained, such as flares, quasi-periodic pulsations, acceleration of charged particles, spicules, corona heating, etc. (e.g., [18, 19, 20]).

4. The presence of electric currents in coronal loops can affect various processes. Among them, we can note, e.g., the processes associated with the propagation and dissipation of MHD waves in coronal loops (see, e.g., recent reviews by $[21,22])$. Observational manifestations of MHD waves in loops may differ depending on the distribution of currents, which means that they must be taken into account when applying the methods of coronal seismology to determine the physical parameters in the loops.

5. Discussion of flare processes in terms of electric currents can be useful for comparing them with similar phenomena of energy release in cosmic plasma, such as magnetospheric substorms. For substorms, a large number of local direct measurements are performed that are inaccessible to remote observers of solar flares, and the obtained information could be used for a more detailed understanding of some physical processes in flares, e.g., those associated with dissipation of currents in the partially-ionized plasma, interaction of energetic particles with waves, small-scale details of magnetic reconnection, etc. (e.g., see [23, 24, 25]).

\section{Some properties of $j_{r}$ observed in flaring ARs}

Roughly speaking, vertical electric currents in AR can be divided into two groups - global and local. By global currents, we mean current structures on the scale of the entire AR. For example, in ARs consisting of two main sunspots, the head and the tail ones, a global current structure on the solar surface can be distinguished in the form of two large areas corresponding to the leading and tailing parts of the AR. The central portions of these regions correspond to the two main sunspots. Such currents have been found in early works by [26, 27], and their presence has recently been confirmed by [28]. The authors of these works call such currents 'distributed' currents. Such a global current system can be associated, in particular, with a large coronal magnetic structure connecting two magnetic vortex in the photosphere around oppositely twisted sunspots. The characteristic linear size of the area covered by the global current is $\sim 100 \mathrm{Mm}$. The magnitude of the total distributed currents in the ARs is $I_{\text {distr }} \sim 10^{12}-10^{13} \mathrm{~A}$, while the total unsigned current in the ARs is three orders of magnitude higher, $I_{t o t} \sim 10^{15}-10^{16} \mathrm{~A}$. [28] also found some connections between flare activity of the ARs and time variations of the distributed currents, namely the ARs with low/high flare activity show small/high variations of distributed currents in the range of $\pm 20 \times 10^{12} \mathrm{~A}$ and $(30-95) \times 10^{12} \mathrm{~A}$, respectively. Estimated magnetic energy stored in the distributed currents is $\sim 10^{32}-10^{33} \mathrm{erg}$, which is comparable with energy of a large solar flare.

There is no precise definition of local currents in the ARs. We will call local currents any currents other than the global (distributed) ones discussed above. Local currents can be located both in the immediate vicinity of sunspots, i.e. in umbra and penumbra (e.g., [30, 31]), and outside them. The characteristic observed linear dimensions of the elements of local currents are $\sim 1-10 \mathrm{Mm}$. They could probably be much smaller, but so far we do not have instruments with a higher resolution. Local currents are often concentrated around the magnetic 

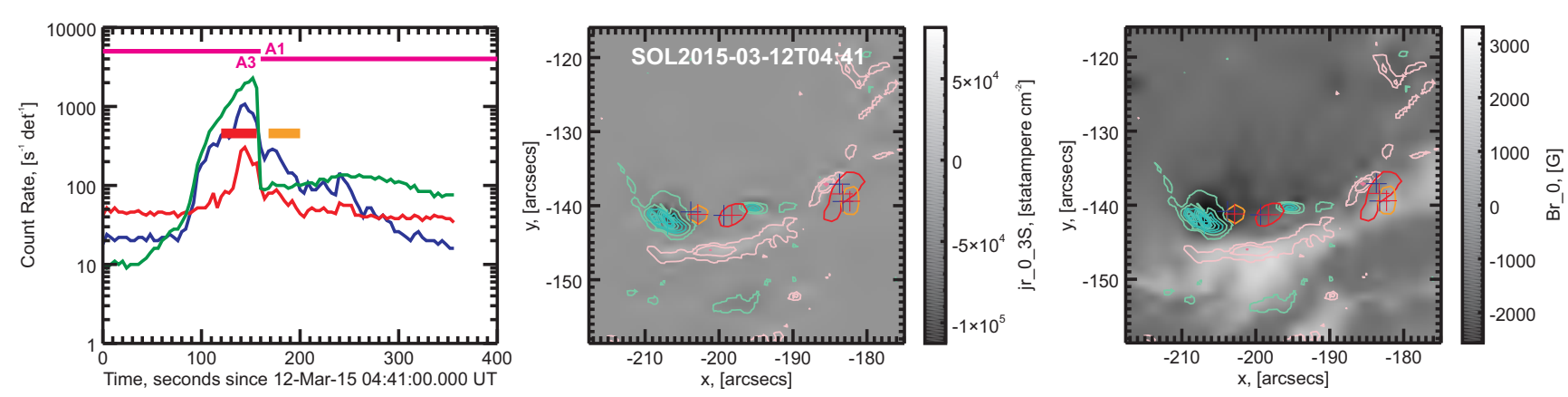

Figure 1: Count rates of X-ray emission (6-12 keV - green, 25-50 keV — blue, 50-100 keV — red) (left), maps of radial (vertical) electric current, $j_{r}$ (middle), and radial magnetic field, $B_{r}$ (right), on the photosphere for the M3.2 solar flare SOL2015-03-12T04:41. Iso-contours of $j_{r}$ at levels of $\pm 1,2, \ldots 8 \times j_{r}^{\text {thr }}$ (pink - positive, cyan negative), where the threshold value $j_{r}^{t h r} \approx 10^{4}$ statampere $\mathrm{cm}^{-2}$ or $\approx 33 \mathrm{~mA} \mathrm{~m}{ }^{-2}$. The $50-100 \mathrm{keV}$ hard X-ray sources at a level of $90 \%$ of their maximum intensity are shown in $j_{r}$ and $B_{r}$ maps by red and orange iso-contours, corresponding to the two time intervals shown by the same colors in the left panel. (Figure is taken from [29].)

polarity inversion line (PIL), where regions with a significant shear of the tangential magnetic component are observed. In such cases, on opposite sides of the PIL, elongated current islands or ribbons of opposite signs can be observed, and in the main (biggest and strongest) current ribbons $j_{z} B_{z}>0$, and they represent the direct currents, for the positive magnetic helicity (see Fig. 1). In some rare cases, around direct current ribbons, one can find areas with currents of opposite signs, for which $j_{z} B_{z}<0$ - these are called return currents. They are usually weaker than direct currents (they are not seen in Fig. 1) and it is more difficult to determine them from observations (e.g., [9] and references therein).

A number of attempts have been made to determine from observations whether the currents in the ARs are neutralized or not, which is motivated by the known discussion started by [32, 33] and [34, 35]. In most of the observational works carried out to date, it has been shown that the currents in the ARs are not neutralized (e.g., $[36,37,38,39])$. Moreover, [39] found good correlation between the characteristics of the non-neutralized currents and the flare index,

$$
\mathrm{FI}=\left(100 S^{(X)}+10 S^{(M)}+1.0 S^{(C)}+0.1 S^{(B)}\right) / \Delta t
$$

(see [40]), where $S^{(X, M, C, B)}$ is the sum of flare magnitudes within a cirtain class and $\Delta t$ is the time interval in days. Modern numerical 3D MHD simulations do show that emergence of an initially neutralized magnetic flux rope to the solar atmosphere or a shear/twist of magnetic bipolar structure is accompanied with formation of non-neutralized currents on the surface of the Sun around the PIL ([41, 42]; see also [9]).

In addition to the current ribbons around one or a few main PILs, the ARs contain many small magnetic elements, with which numerous less extended and compact islands of vertical currents are associated. They could be generated, e.g., at the boundaries of convective cells due to converging plasma motions (e.g., [20]) or as a result of the action of a turbulent dynamo. It turns out that the probability density function of the vertical current density modulus (PDF $\left(\left|j_{r}\right|\right)$ ) obtained from the vector magnetograms of the Helioseismic and Magnetic Imager (HMI; [43]) onboard the Solar Dynamics Observatory in flaring ARs can be described in the first approximation by a model consisting of a folded normal distribution at low values $\left(\left|j_{r}\right| \lesssim 9 \cdot 10^{3}\right.$ statampere $\mathrm{cm}^{-2}$ or $\left.\lesssim 30 \mathrm{~mA} \mathrm{~m}^{-2}\right)$ and a falling power law function at higher values [44]. It was argued that the folded normal distribution at low values can represent noise in the data, while the power-law tail may reflect the nature of the processes of generation of magnetic field and electric currents in ARs. These results should be checked against data from other instruments and also tried to understand based on realistic 3D MHD simulations. For different ARs, the exponential index has an average value $\langle\delta\rangle=-(3.72 \pm 0.78)$. The authors did not find its correlation with the flare class and Hale magnetic class of the AR in the studied sample of events. Interestingly, [45] showed that the distribution function of coronal current density in one AR (obtained with the NLFFF extrapolation) can be represented by a double power law function and its breaking point shows time variations toward the onset of an X-class flare. This issue requires further consideration.

\section{Spatial relation between $j_{r}$ and flare sources}

Starting with the works of A.B. Severnyi and his colleagues in the 1960s, many efforts were made to study the spatial relationship of flare emission sources in different spectral ranges and vertical electric currents, $j_{r}$, in the photosphere. A list of the main works known to us is given in Table 1. Early works, using ground-based observations in the optical range with an angular precision up to $3^{\prime \prime}-6^{\prime \prime}$, showed that in many flares a large percentage (up to $\approx 80 \%$ ) of $\mathrm{H} \alpha$ emission sources are located close (within $6^{\prime \prime}$ ) to local maxima $j_{r}^{\max }$ or overlap with them. These 
Table 1: Some observational studies of the spatial relationship between solar flare emission sources (FS) and vertical electric currents, $j_{r}$, in the photosphere.

\begin{tabular}{lccr}
\hline Work & $\begin{array}{c}\text { Wavelength } \\
\text { range }\end{array}$ & $\begin{array}{c}\text { Number } \\
\text { of Flares }\end{array}$ & Spatial relatioship \\
\hline$[48]$ & $\mathrm{H} \alpha$ & 30 & $\approx 80 \%$ FS in $j_{r}^{\max }$ \\
{$[5]$} & $\mathrm{H} \alpha$ & $\approx 10$ & $\approx 80 \%$ FS in $j_{r}^{\max }$ \\
{$[49]$} & $\mathrm{H} \alpha$ & 1 & FS in $j_{r}^{\max }$ \\
{$[50]$} & $\mathrm{H} \alpha$ & 1 & FS in periphery of $j_{r}^{\max }$ \\
{$[27]$} & $\mathrm{H} \alpha$ & Many & Majority of FS in strong $j_{r}$ \\
{$[51]$} & $\mathrm{H} \alpha$ & 4 & FS in periphery of $j_{r}^{\max }$ \\
{$[52]$} & $\mathrm{H} \alpha, \mathrm{HXR}$ & 1 & FS near but not in $j_{r}^{\max }$ \\
{$[53]$} & $\mathrm{H} \alpha$ & 3 & FS in periphery of $j_{r}^{\max }$ \\
{$[54]$} & $\mathrm{H} \alpha$ & 2 & FS in periphery of $j_{r}^{\max }$ \\
{$[55]$} & $\mathrm{H} \beta$ & 107 & FS near but not in $j_{r}^{\max }$ \\
{$[56]$} & $\mathrm{H} \beta$ & 1 & FS close to $j_{r}^{\max }$ \\
{$[57]$} & $\mathrm{H} \beta$ & 26 & $\approx 65 \%$ FS not in $j_{r}^{\max }$ \\
{$[58]$} & $\mathrm{HXR}$ & 6 & FS in periphery of $j_{r}^{\max }$ \\
{$[59]$} & $\mathrm{H} \beta$ & 1 & Some FS not in $j_{r}^{\max }$ \\
{$[60]$} & $\mathrm{H} \alpha, \mathrm{H} \beta$ & 79 & $29 \% / 50 \%$ FS in $0.9 / 0.8 \times j_{r}^{\max }$ \\
{$[61]$} & $\mathrm{EUV}$ & 1 & FS ribbons in $j_{r}$ ribbons \\
{$[62]$} & $\mathrm{H} \alpha$ & 1 & Flare ribbon in $j_{r}$ ribbon \\
{$[63]$} & $\mathrm{HXR}$ & 1 & Some FS in $j_{r}$ ribbons \\
{$[64]$} & $\mathrm{Optics}$ & 1 & Sunquake near $j_{r}^{\max }$ \\
{$[65]$} & $\mathrm{HXR}$ & 1 & Weak/strong FS in $/$ out $j_{r}^{\max }$ \\
{$[66]$} & $\mathrm{EUV}$ & 1 & FS ribbons in $j_{r}$ ribbons \\
{$[29]$} & $\mathrm{HXR}$ & 48 & FS mainly near $j_{r}$, not in $j_{r}^{\max }$ \\
\hline
\end{tabular}

observations motivated the development of models of flares with interruption of field aligned currents in magnetic loops, the first of which, apparently, was the model by [17]. However, later, starting in the 1990s, a number of detailed observations were carried out, in which it was shown that, although flare sources tend to be located near the regions of increased $j_{r}$, but outside their local maxima. This has been demonstrated not only for optical sources in the $\mathrm{H} \alpha$ and $\mathrm{H} \beta$ lines, as before, but also for hard X-ray sources. Observations of flare sources in the hard X-ray range have the advantage that they directly correspond to the sites of interaction of accelerated electrons with dense plasma in feet of the flare loops. Whereas optical sources show regions of heated plasma in dense layers of the solar atmosphere, the heating of which can be caused not only by accelerated electrons, but also by thermal conduction and enhanced coronal pressure (e.g., [46]).

Recently, [29], on the basis of a sample of 48 flares of various X-ray classes from $\mathrm{C}$ to X, confirmed that the flare sources of $50-100 \mathrm{keV}$ hard X-rays tend to be localized not in local maxima of vertical currents, but at their periphery (see an example shown in Fig. 1). No significant correlation was found between the hard Xray intensity of the sources and various vertical current characteristics under the sources, which contradicts the current interruption models. In particular, in the model of [47], the magnitude of the vertical electric field generated at the current break strongly depends on the current $\left(E_{r} \propto I_{r}^{3}\right)$ and can exceed the Dreicer field. It would be expected that the greater the electric field parallel to the guide magnetic field, the more efficient the acceleration of electrons and the greater the intensity of hard X-ray emission of the sources. However, this is not observed. The current interruption models assume a drop (at least small, due to high inductance) in current in the loop due to its dissipation during a flare, which is also not supported by observations. Moreover, in a number of events, an increase in $j_{r}$ during a flare is detected (see the following section).

\section{Temporal evolution of $j_{r}$ in flare regions}

Not much research on the dynamics of vertical currents in ARs during flares was done before the launch of the SDO. In one of such works performed by [67], two flares were studied, for which different dynamics of $j_{r}$ were established - in one of them the total current decreased during the flare, and in the other it increased. The decrease in the current in the first flare was interpreted as dissipation as a result of reconnection, and the increase in the second - as the emergence of a new flux with current from under the photosphere, which is consistent with earlier observation by [12]. Later, Wang et al. [68] showed the increase in the total unsigned current near the PIL 
during the flare precursors associated with the emergence of the current-carrying magnetic flux, which could act as a possible trigger of the following flare.

Detailed analysis of observations of the SDO/HMI data with a time step of 12 min allowed [61, 66] to detect an increase in the vertical current by a factor of $2-3$ in the flare ribbons around the PIL during two flares. [63] demonstrated that the regions of local increase in vertical currents correspond to some sources of hard X-rays that appear near the PIL during a flare. [69] using the SDO/HMI data with a step of 135 s, showed an increase in currents around the PIL during three successive sub-flares in one flaring AR. Interestingly, in the vicinity of the feet of the eruptive magnetic flux rope, a drop in current is observed in some eruptive events [70, 71]. An increase in the current in the flare ribbons around the PIL is interpreted by the modern MHD models as a result of an increase in electric currents in coronal quasi-separatrix layers due to magnetic reconnection and transformation of the magnetic configuration during a flare $[61,66]$. Whereas the drop in the current at the feet of the flux rope with a constant twist along the length can be associated with its stretching during eruption ([71]; see also [9] and references therein).

\section{Conclusion}

We have tried to review very briefly the observations of vertical electric currents in solar flaring ARs and, in general terms, relate the observations to some models. In our opinion, the observations better correspond to the models of the storage of free magnetic energy assotiated with currents flowing in the sheared/twisted structures above the PIL and its release due to reconnection than with the current interruption models. However, this topic still remains open. We hope that soon new physically significant information will be obtained on the processes of generation and dissipation of currents in ARs, on the three-dimensional configuration of currents, its temporal evolution during flares and eruptions.

This work was supported by a grant from the Russian Science Foundation (project No. 17-72-20134).

\section{References}

1. J. O. Stenflo, AछA, 529, A42, 2011.

2. V. I. Abramenko and V. B. Yurchyshyn, MNRAS, 497, 5405, 2020.

3. E. Priest, Magnetohydrodynamics of the Sun (Cambridge, UK: Cambridge University Press) (2014).

4. A. B. Severnyi, Astron. Zhurn., 42, 217, 1965.

5. A. Zvereva and A. B. Severnyi, Izv. KrAO, 41-42, 97, 1970.

6. Y. A. Fursyak, Geomagnetism and Aeronomy, 58, 1129, 2018.

7. G. Barnes and K. D. Leka, in A. Keiling, O. Marghitu, and M. Wheatland, eds., Electric Currents in Geospace and Beyond, volume 235, 81-91 (2018).

8. G. D. Fleishman and A. A. Pevtsov, in A. Keiling, O. Marghitu, and M. Wheatland, eds., Electric Currents in Geospace and Beyond, volume 235, 43-65 (2018).

9. B. Schmieder and G. Aulanier, in A. Keiling, O. Marghitu, and M. Wheatland, eds., Electric Currents in Geospace and Beyond, volume 235, 391-406 (2018).

10. C. J. Schrijver, M. L. DeRosa, T. Metcalf, G. Barnes, et al., ApJ, 675, 1637, 2008.

11. T. Wiegelmann and T. Sakurai, Living Reviews in Solar Physics, 9, 5, 2012.

12. K. D. Leka, R. C. Canfield, A. N. McClymont, and L. van Driel-Gesztelyi, ApJ, 462, 547, 1996.

13. C. J. Schrijver, M. L. De Rosa, A. M. Title, and T. R. Metcalf, ApJ, 628, 501, 2005.

14. V. Sadykov and I. Zimovets, in K. N. Nagendra, J. O. Stenflo, Z. Q. Qu, and M. Sampoorna, eds., Solar Polarization 7, Astronomical Society of the Pacific Conference Series, volume 489, 59 (2014).

15. C. J. Schrijver, ApJ, 820, 103, 2016.

16. V. S. Titov and P. Démoulin, A\&A, 351, 707, 1999.

17. H. Alfvén and P. Carlqvist, Solar Phys., 1, 220, 1967.

18. V. V. Zaitsev and A. V. Stepanov, Physics Uspekhi, 51, 1123, 2008.

19. V. V. Zaitsev and A. V. Stepanov, Solar Phys., 290, 3559, 2015.

20. V. V. Zaitsev, A. V. Stepanov, and P. V. Kronshtadtov, Solar Phys., 295, 166, 2020.

21. B. Li, P. Antolin, M. Z. Guo, A. A. Kuznetsov, D. J. Pascoe, T. Van Doorsselaere, and S. Vasheghani Farahani, Space Sci. Rev., 216, 136, 2020.

22. V. M. Nakariakov, S. A. Anfinogentov, P. Antolin, R. Jain, et al., Space Sci. Rev., 217, 73, 2021.

23. G. Haerendel, ApJ, 749, 166, 2012.

24. S.-I. Akasofu and L.-C. Lee, Journal of Atmospheric and Solar-Terrestrial Physics, 186, $104,2019$.

25. A. Artemyev, I. Zimovets, I. Sharykin, Y. Nishimura, et al., arXiv e-prints, arXiv:2105.03772, 2021.

26. V. I. Abramenko and S. I. Gopasyuk, Bulletin Crimean Astrophysical Observatory, 76, $163,1987$.

27. V. I. Abramenko, S. I. Gopasiuk, and M. B. Ogir', Solar Phys., 134, 287, 1991.

28. Y. A. Fursyak, A. S. Kutsenko, and V. I. Abramenko, Solar Phys., 295, 19, 2020.

29. I. V. Zimovets, I. N. Sharykin, and W. Q. Gan, ApJ, 891, 138, 2020. 
30. V. M. Grigoryev and L. V. Ermakova, Solar Phys., 207, 309, 2002.

31. S. Gosain, P. Démoulin, and M. López Fuentes, ApJ, 793, 15, 2014.

32. D. B. Melrose, ApJ, 381, 306, 1991.

33. D. B. Melrose, ApJ, 451, 391, 1995.

34. E. N. Parker, ApJ, 471, 489, 1996.

35. E. N. Parker, ApJ, 471, 485, 1996.

36. M. S. Wheatland, ApJ, 532, 616, 2000.

37. D. A. Falconer, R. L. Moore, and G. A. Gary, ApJ, 569, 1016, 2002.

38. M. K. Georgoulis, V. S. Titov, and Z. Mikić, ApJ, 761, 61, 2012.

39. I. Kontogiannis, M. K. Georgoulis, S.-H. Park, and J. A. Guerra, Solar Phys., 292, 159, 2017.

40. V. I. Abramenko, ApJ, 629, 1141, 2005.

41. T. Török, J. E. Leake, V. S. Titov, V. Archontis, et al., ApJL, 782, L10, 2014.

42. K. Dalmasse, G. Aulanier, P. Démoulin, B. Kliem, T. Török, and E. Pariat, ApJ, 810, 17, 2015.

43. P. H. Scherrer, J. Schou, R. I. Bush, A. G. Kosovichev, et al., Solar Phys., 275, 207, 2012.

44. I. V. Zimovets, A. B. Nechaeva, I. N. Sharykin, and W. Q. Gan, Astrophysics, 63, 408, 2020.

45. J. Kang, T. Magara, S. Inoue, Y. Kubo, and N. Nishizuka, PASJ, 68, 101, 2016.

46. R. C. Canfield, T. A. Gunkler, and P. J. Ricchiazzi, ApJ, 282, 296, 1984.

47. V. V. Zaitsev, P. V. Kronshtadtov, and A. V. Stepanov, Solar Phys., 291, 3451, 2016.

48. G. E. Moreton and A. B. Severny, Solar Phys., 3, 282, 1968.

49. Y. Lin and V. Gaizauskas, Solar Phys., 109, 81, 1987.

50. V. A. Romanov and T. T. Tsap, Sov. Astron., 34, 656, 1990.

51. R. C. Canfield, J. F. de La Beaujardiere, and K. D. Leka, Philosophical Transactions of the Royal Society of London Series A, 336, 381, 1991.

52. R. C. Canfield, H. S. Hudson, K. D. Leka, D. L. Mickey, et al., PASJ, 44, L111, 1992.

53. K. D. Leka, R. C. Canfield, A. N. McClymont, J. F. de La Beaujardiere, Y. Fan, and F. Tang, ApJ, 411, 370, 1993.

54. J. F. de La Beaujardiere, R. C. Canfield, and K. D. Leka, ApJ, 411, 378, 1993.

55. T. Wang, A. Xu, and H. Zhang, Solar Phys., 155, 99, 1994.

56. H. Zhang and T. Wang, Solar Phys., 151, 129, 1994.

57. H. Zhang, A\&A, 304, 541, 1995.

58. J. Li, T. R. Metcalf, R. C. Canfield, J.-P. Wülser, and T. Kosugi, ApJ, 482, 490, 1997.

59. H. Zhang, $A \& A, \mathbf{3 2 4}, 713,1997$.

60. H.-s. Ji, M.-t. Song, Y.-a. Zhang, and S.-m. Song, Chinese Astron. and Astrophys., 27, 79, 2003.

61. M. Janvier, G. Aulanier, V. Bommier, B. Schmieder, P. Démoulin, and E. Pariat, ApJ, 788, $60,2014$.

62. I. N. Sharykin and A. G. Kosovichev, ApJL, 788, L18, 2014.

63. S. Musset, N. Vilmer, and V. Bommier, $A \mathscr{E} A$, 580, A106, 2015.

64. I. N. Sharykin and A. G. Kosovichev, ApJ, 808, 72, 2015.

65. I. N. Sharykin, A. G. Kosovichev, and I. V. Zimovets, ApJ, 807, 102, 2015.

66. M. Janvier, A. Savcheva, E. Pariat, S. Tassev, et al., A\&A, 591, A141, 2016.

67. B. Tan, H. Ji, G. Huang, T. Zhou, Q. Song, and Y. Huang, Solar Phys., 239, 137, 2006.

68. H. Wang, C. Liu, K. Ahn, Y. Xu, et al., Nature Astronomy, 1, 0085, 2017.

69. I. N. Sharykin, I. V. Zimovets, and I. I. Myshyakov, ApJ, 893, 159, 2020.

70. X. Cheng and M. D. Ding, ApJS, 225, 16, 2016.

71. K. Barczynski, G. Aulanier, M. Janvier, B. Schmieder, and S. Masson, ApJ, 895, 18, 2020. 
Ground-based and Space-based Tools and Methods of Astronomy 


\section{Adaptation system of the Millimetron main mirror}

M.Yu. Arkhipov, E.S. Golubev, A.O. Lyakhovets, A.V. Smirnov, S.D. Fedorchuk

markhipov@asc.rssi.ru

P. N. Lebedev Physical Institute of the Russian Academy of Sciences, Russia

The paper describes the design of the main mirror of the Millimetron space observatory and its adjustment system. It also presents the results of modeling the compensation of deviations caused by various factors (temperature, opening errors, etc.) to an individual panel and to the entire mirror. The authors provide the results of experimental adaptation of an individual panel and development of a precision cryogenic actuator for the system adjustment.

Keywords: adaptive telescope, cryogenic actuator, Millimetron space observatory

DOI: $10.51194 /$ VAK2021.2022.1.1.007

The Millimetron space observatory that is being created in Russia is designed to operate in the mm and NIR wavelength ranges $[1,2,3]$. Its main mirror has a diameter of $10 \mathrm{~m}$ and consists of a fixed central part and 24 petals folding to be placed under the fairing of the launch vehicle. The operating range specifies extremely high requirements to the main mirror, in particular, the accuracy of the shape of the reflecting surface $(R M S \leq 10 \mu \mathrm{m})$ and the operating temperature $(\leq 10 \mathrm{~K})$. Analysis of the error budget and experience with the creation of the RadioAstron space observatory (its mirror having a diameter of $10 \mathrm{~m}$ and consisting of 27 petals) showed the need to align the elements of the main mirror after opening in space and cooling to operating temperatures.

The central part of the main mirror consists of 24 independent sectors, and each of the 24 petals includes 3 panels. Each panel is mounted on a support frame using 3 actuator nodes (Figure 1) of the adjustment system. The actuator node consists of a precision actuator and two flexible hinges. This provides 3 degrees of freedom for the panel alignment - 2 rotations and one linear movement.

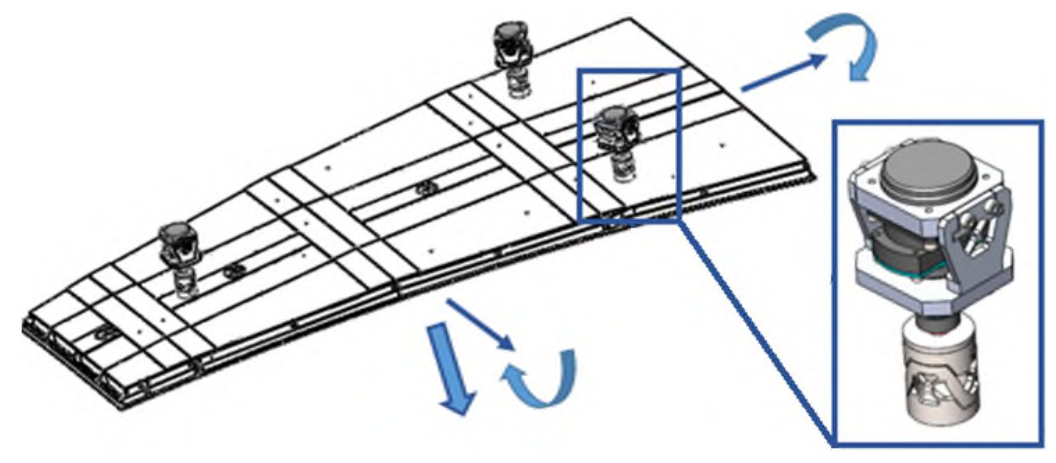

Figure 1: Main mirror panel and actuator nodes.

The main requirements for the adaptation system are to ensure the panel movement with a resolution of $\leq 1 \mu \mathrm{m}$ to align the main mirror with the required accuracy. Also, the actuator nodes must withstand overloads while the observatory is being put into orbit and have a rigidity that provides the panel with a natural oscillation frequency of at least $8.5 \mathrm{~Hz}$.

The developed mathematical apparatus and software (AstroMagick) make it possible to simulate the adjustment of both individual elements of the mirror and the entire mirror as a whole. Table 1 shows the results of a simulation to compensate for various deflections of petal panels. Figure 2 shows a field of deviations caused by cooling the observatory structure to operating temperatures (down to $\leq 10 \mathrm{~K}$ on the mirror structure) before and after adjustment.

As part of the experimental work, we developed a prototype cryovacuum actuator with the following characteristics:

- resolution: $0.64 \mu \mathrm{m}$

- displacement range $\pm 6 \mathrm{~mm}$

- developed force $60 \mathrm{~N}$

- load capacity (axial and transverse) - $300 \mathrm{~N}$

- operating temperature range $4-300 \mathrm{~K}$

A series of cryogenic tests confirmed its characteristics [4].

A prototype was developed to study the kinematics of a large-size panel (minimal displacement, repeatability at large displacements, positioning accuracy), the effect of flexible hinges on distortions of the reflecting surface. 
Table 1: Possible panel deflection and actuator displacements.

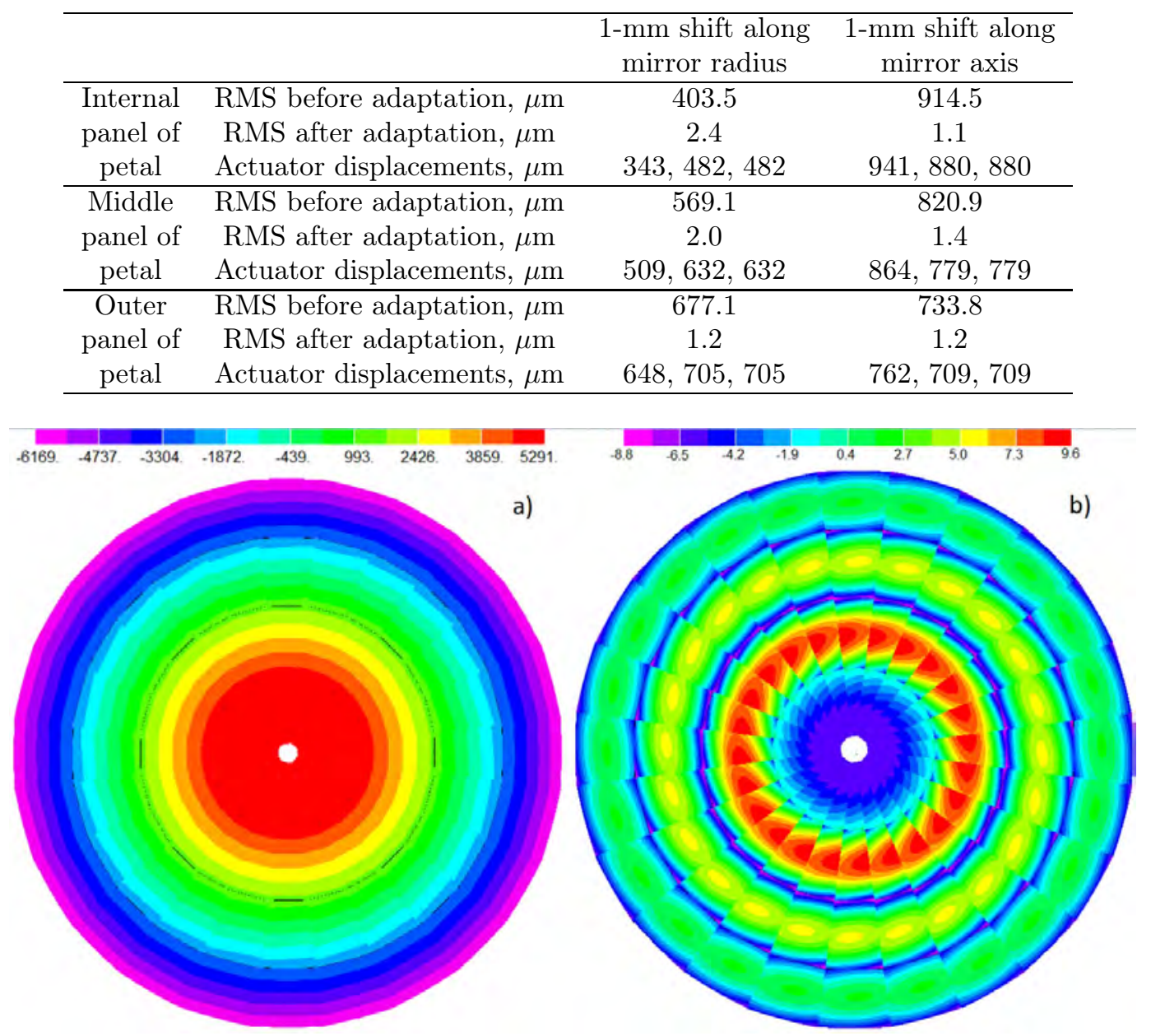

Figure 2: Deviations upon cooling to $10 \mathrm{~K}$, a) before adjustment (RMS=3601 $\mu \mathrm{m})$ and b) after adjustment $(\mathrm{RMS}=4$ $\mu \mathrm{m})$.

The prototype consists of a full-size $(1050 \times 1450 \mathrm{~mm})$ middle panel of the petal mounted on a rigid frame on three actuator nodes, one of which includes an actuator (Figure 3b). The panel is a three-layer structure made of high-modulus CFRP M55J with a cyanate ester resin. The panel thickness is $30 \mathrm{~mm}$, its weight is $8.7 \mathrm{~kg}$ (which corresponds to a surface density of $5.7 \mathrm{~kg} / \mathrm{m}^{2}$ ).

To conduct the experiment (Figure 3(c)), the panel assembly with the actuating nodes and the frame was installed on the table of a Zeiss Prismo Ultra coordinate measuring machine (CMM) with the Zeiss VAST Gold measuring head (measurement repeatability $0.4 \mu \mathrm{m}$ ). The CMM was used as the main measuring tool both for measuring the panel surface distortions and for point measurements of panel displacements under the action of the actuator.

To measure the displacements of the panel as a rigid body during the operation of the actuator, 4 dial indicators were additionally used (measurement limit: $1000 \mu \mathrm{m}$, sensitivity: $1 \mu \mathrm{m}$ ).

The following results were obtained:

- resolution of the adaptation system: $0.70 \mu \mathrm{m}$,

- repeatability upon displacement of $\pm 873 \mu \mathrm{m}$ is $\leq 1.2 \mu \mathrm{m}$,

- positioning accuracy $\leq 3.0 \mu \mathrm{m}$,

- distortions after mounting the panel on the actuator nodes (difference in panel measurements with free support and after mounting) $R M S=0.96 \mu \mathrm{m}$,

- additional panel distortions upon a 1000- $\mu \mathrm{m}$ actuator displacement: $R M S=0.84 \mu \mathrm{m}$.

The result is shown in Figure 4(a) (RMS: $0.96 \mu \mathrm{m}$ ). The difference between the point clouds of the reflecting surface before and after a 1000- $\mu \mathrm{m}$ displacement is shown in Figure 4(b) (RMS: 0.84 $\mu \mathrm{m})$. 


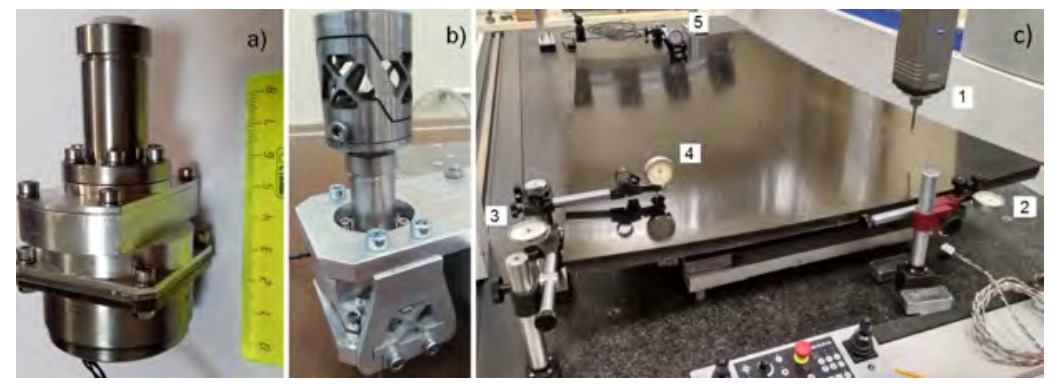

Figure 3: Cryovacuum actuator (a), actuator assembly with flexible hinges (b), experimental setup: 1 — CMM tactile probe, $2,3,4,5$ - micrometers (c).

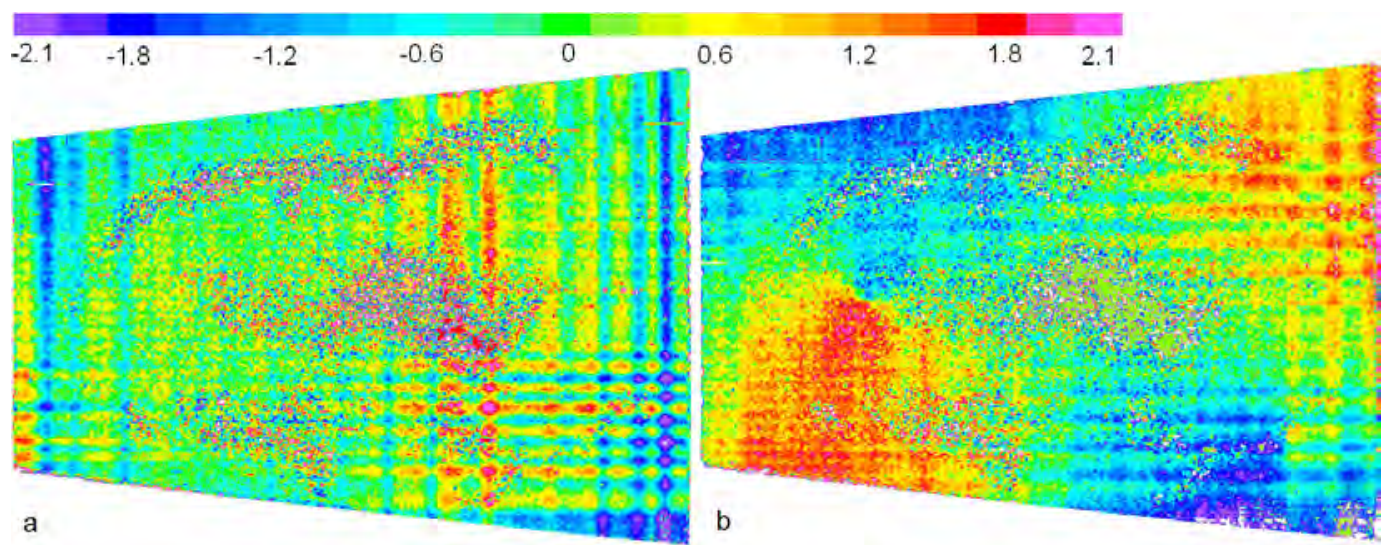

Figure 4: Influence of the elastic joints on the panel's reflecting surface, $\mu \mathrm{m}$ : a) panel mounted on the frame, b) panel moved by $1000 \mu \mathrm{m}$ during adaptation.

\section{References}

1. N. S. Kardashev, I. D. Novikov, V. N. Lukash, S. V. Pilipenko, et al., Physics Uspekhi, 57, 1199-1228, 2014.

2. A. V. Smirnov, A. M. Baryshev, S. V. Pilipenko, N. V. Myshonkova, et al., in M. C. Clampin, G. G. Fazio, H. A. MacEwen, and J. M. O. Jr., eds., Space Telescopes and Instrumentation 2012: Optical, Infrared, and Millimeter Wave, SPIE, volume 8442, 1456 - 1464 (2012).

3. MillimetronTeam, https://millimetron.ru, 2021.

4. A. Yusov, S. Kozlov, E. Ustinova, M. Arkhipov, et al., Cryogenics, 118, 103346, 2021. 


\title{
High-speed camera based on a large-format low-noise CMOS imager
}

\author{
V.I. Ardilanov, V.A. Murzin, I.V. Afanasyeva, N.G. Ivaschenko, M.A. Pritychenko, S.N. Dodonov \\ valery@sao.ru \\ WWW home page: http://www.sao.ru/hq/adlab/ \\ Special Astrophysical Observatory of RAS, Nizhnij Arkhyz, Karachaevo-Cherkesia 369167, Russia
}

The paper presents a high-speed astronomical camera based on a large-format $(4096 \times 40969 \mu \mathrm{m}$ pixels $)$ front-illuminated CMOS imager with a read-out noise of $4.4 e^{-}$. The maximum frame rate of the camera is 24 fps. The specific requirements that are taken into account when building a controller architecture are formulated. A brief description of the CMOS camera structure is introduced and the photoelectric characteristics obtained from the results of laboratory tests of the camera are presented. Test observations at the Zeiss-1000 telescope of the SAO RAS were successfully carried out in July 2021.

Keywords: CMOS camera, high performance, residual bulk image

DOI: 10.51194 /VAK2021.2022.1.1.008

\section{Introduction}

A CMOS imagers technology is now widely developed in all scope of application. There are dozens of companies that produce CMOS imagers. But those that could simultaneously meet all specific requirements of the astronomical application — namely: large-format high-speed CMOS imagers with high quantum efficiency and low read-out noise - have only begun to appear on the market in recent years. The most modern samples of such imagers are almost equal to the CCD imagers in the read-out noise quantify.

Today, Teledyne E2V (UK) and GPixel Inc. (China) are leaders in the development of this kind of imagers. CMOS imagers for the astronomical applications such as CIS113 from Teledyne E2V, GSense4040 and GSense6060BSI from GPixel are now commercially available.

The GSense series imagers have a significantly lower price and are easier to control in comparison with the CIS113 imager, so they were preferred by us.

\section{Camera overview}

When choosing the architecture and design of the camera controller, the following basic requirements were formulated and taken into account:

- videochannel design with the maximum achievable read-out speed;

- providing low read-out noise;

- compact design of the controller;

- full programmability and telemetric control of camera modes;

- wide operating temperature range.

The architecture of the controller and the interaction of its functional parts are described by [1].

The 3-D model of the camera construction equipped with GSense4040 imager is shown in Fig. 1. The camera includes the optical head and the frame containing electronics.

The optical head is a sealed chamber filled with an inert gas. The top flange of the head has threaded holes for the lens adapter. On the sides of the head there are threaded holes for attaching the camera to the optical system. The imager mounting unit is located inside the optical head. The unit design allows to arrange the imager plane relative to the plane of the front flange of the optical head mechanically wiht high precision, immediately before sealing the head.

The controller framework consists of two sealed housings with electronics located inside. The communication connectors are located on the back wall. The camera air cooling system performs heat exchange between the air received from the environment and the radiators of thermoelectric modules and electronics housings. For improved heat transfer, a liquid cooling system can also be connected to the camera.

The acquisition software for the camera should effectively use all the imager capabilities [2]. Realization of the software was carried out according to the method described by [3]. 


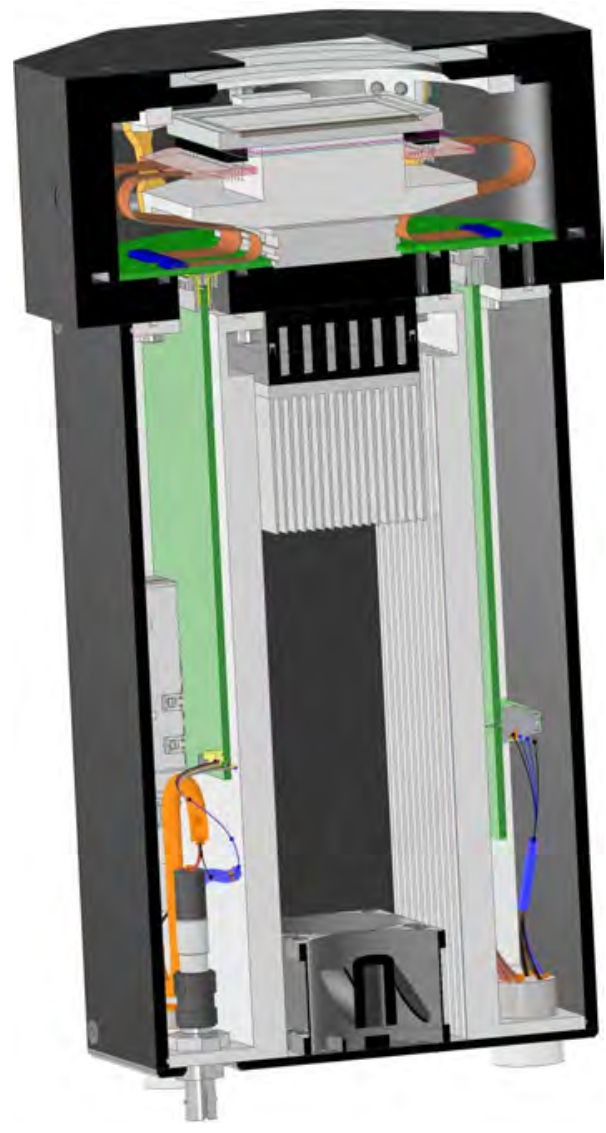

Figure 1: 3-D model of the camera design

\section{Results and conclusion}

Below are the photoelectric characteristics (for high (HG) and low (LG) gain outputs) of the GSense4040CMTbased camera obtained during laboratory tests (see also [4]) and telescope observations:

- Dark current at $-25^{\circ} \mathrm{C}$

- Read-out noise $0.054 e^{-/ s / p i x e l @ L G ~}$

- Dynamic range

- Built-in zero long term stability

Observations were carried out using the MAGIC reducer [5].

$4.4 e^{-} @ H G$

$750 @ H G, 2200 @ L G$

$0.05 A D U(\mathrm{p}-\mathrm{p})$

The analysis of the imager behavior when observing a bright object in long exposure modes with a high accumulated charge has specific features. Subsequent exposure frames contain a residual image of the previous bright subject. In this case, the residual charge behaves as a dark current. Its value and time dependence are shown in Fig. 2. [6].

Such a feature appears in front-illuminated imagers and is not typical for imagers with back-side illumination

A camera based on a high-speed CMOS imager was developed, optimized specifically for work in the field of astronomical research. The passport characteristics of the imager are confirmed. Such systems could be used both as parts of fast telescopes in the panoramic sky survey tasks and also to obtain images of rapidly changing astronomical phenomena. By using a smaller format CMOS imager with a higher frame rate (up to 5000 fps) and with up to 400 channels, it is possible to create a camera to control an adaptive optics system.

\section{References}

1. I. V. Afanasieva, V. A. Murzin, V. I. Ardilanov, N. G. Ivaschenko, M. A. Pritychenko, and A. N. Borisenko, in L. M. Zeleniy and B. M. Shustov, eds., Space Debris: Fundamental and Practical Aspects of Threat (Space Research Institute of the Russian Academy of Sciences), 52-57 (2019).

2. I. V. Afanasieva, Astroph. Bull., 70, 232, 2015.

3. I. Afanasieva, F. Novikov, and L. Fedorchenko, SPIIRAS Proceedings, 19, 481, 2020, in Russ. 


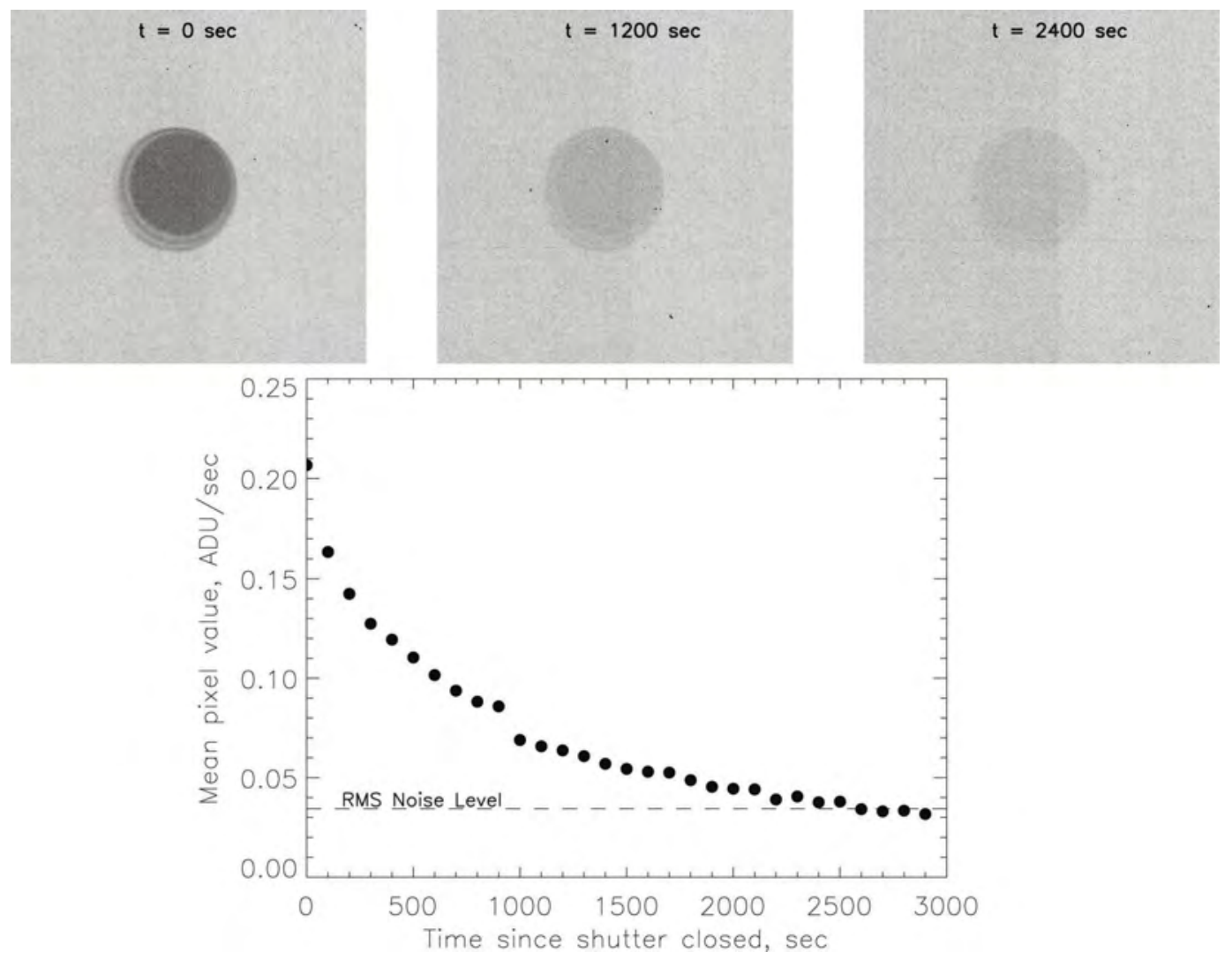

Figure 2: GSense4040CMT residual bulk image effect

4. V. I. Ardilanov, V. A. Murzin, I. V. Afanasieva, N. G. Ivaschenko, and M. A. Pritychenko, in I. I. Romanyuk, I. A. Yakunin, A. F. Valeev, and D. O. Kudryavtsev, eds., Ground-Based Astronomy in Russia. 21st Century, 115-118 (2020).

5. V. L. Afanasiev, V. R. Amirkhanyan, R. I. Uklein, A. E. Perepelitsyn, E. A. Malygin, E. S. Shablovinskaya, and I. V. Afanasieva, Astronomische Nachrichten, Proceeding, in press, 2021.

6. J. Janesick, T. Elliott, J. Andrews, J. Tower, P. Bell, A. Teruya, J. Kimbrough, and J. Bishop, in P. M. Bell and G. P. Grim, eds., Target Diagnostics Physics and Engineering for Inertial Confinement Fusion III (SPIE), volume 9211,921106 (2014). 


\title{
Design of Cryogenic Ultra-High Vacuum Setup for Simulation of Chemical Processes in the Star and Planet Formation Regions
}

\author{
G.S. Fedoseev, V.V. Krushinsky, M.G. Medvedev, K.A. Stepanova, A.I. Vasyunin \\ fedoseev@urfu.ru \\ Research Laboratory for Astrochemistry, Ural Federal University, Kuibysheva St. 48, 620026 Ekaterinburg, Russia
}

\section{DOI: $10.51194 /$ VAK2021.2022.1.1.009}

Modern astrochemical observations, carried out both with the help of the astronomical facilities in the radio and infrared wevelength ranges or in the specialized laboratories, show that complex organic molecules (COMs) form in star-forming regions long before stars and planets are shaped. COMs, defined in the context of astronomical observation as molecules of 6 or more atoms, are observed in the gas phase in diffuse and translucent clouds, cold dark clouds, prestellar cores and protoplanetary disks. Special interest is drawn to the formation of various prebiotics, i.e., COMs that can participate in the formation of sugars, amino acids, and other molecules necessary for the origin of life. It is believed that the key role in COMs formation is played by cosmic dust $[1,2]$.

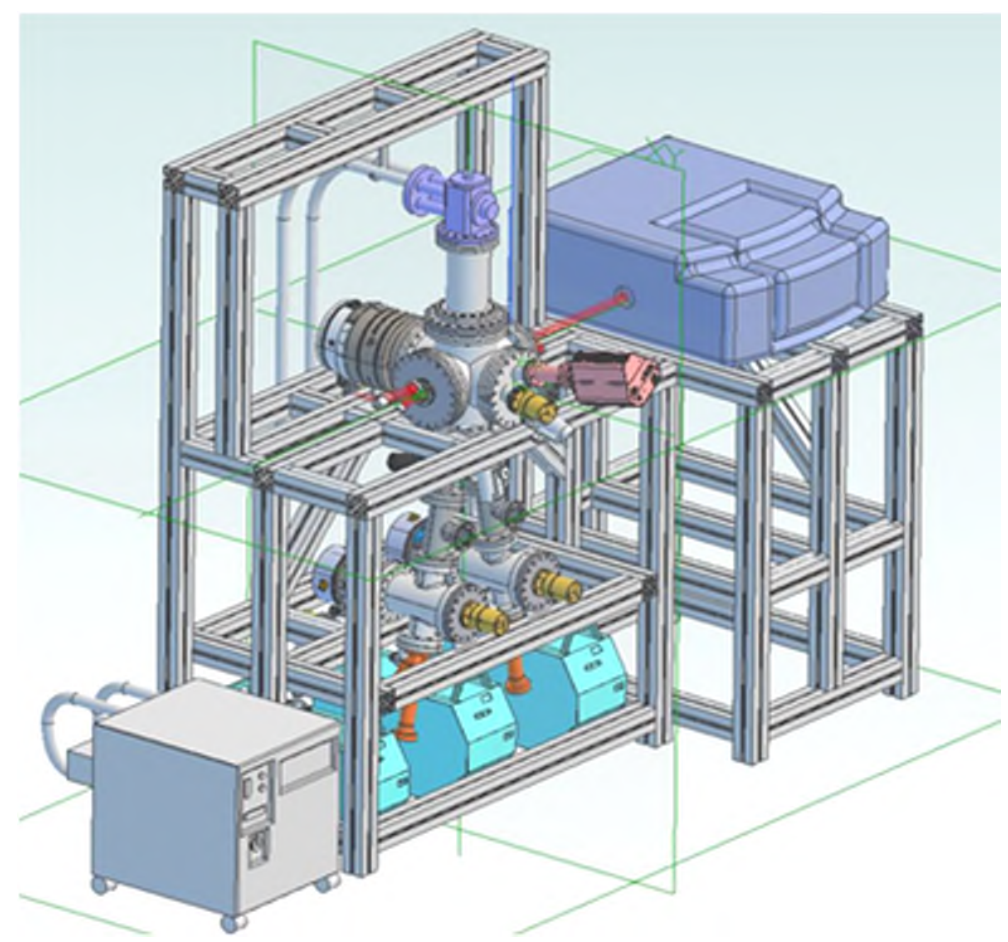

In view of the increasing interest to the formation of COMs in star-forming regions, an ultra-high vacuum cryogenic setup simulating physical and chemical processes occurring on the dust surfaces in the regions of star formation is designed at the Research Laboratory for Astrochemistry at the Ural Federal University. The obtained chemical constants are planned to be used for the accurate numerical astrochemical simulations, using MONACO code already adopted by the Research Laboratory for Astrochemistry and macro- and microscopic Monte-Carlo codes which are currently under development (see VAK2021 contributions by Sokolova V.A., Medvedev M.G., and Satonkin N.A.). The setup includes an IR spectrometer allowing for the acquisition of the IR absorption spectra of the grown ices and monitoring of their chemical evolution. The resolution and spectral range of the obtained spectra correspond to the parameters of the spectrometers on board of the James Webb Space Telescope, allowing for the direct comparison of astronomical observations with the results of the experiments.

The work is funded via the State Assignment Contract FEUZ-2020-0038.

\section{References}

1. E. Herbst and E. F. van Dishoeck, ARAछ A, 47, 427, 2009.

2. B. A. McGuire, ApJS, 239, 17, 2018. 


\section{Multi-object spectroscopy in the observational complex of the RTT150}

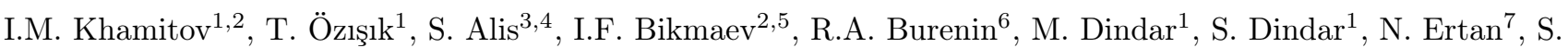
Fişek $^{3,4}$, M.V. Glushkov², T. Güver ${ }^{3,4}$, Y. Kılıç ${ }^{1}$, O. Okuyan ${ }^{1}$, S. Süvari ${ }^{1}$, Ali Tat ${ }^{1}$, F.K. Yelkenci ${ }^{3,4}$ irek_khamitov@hotmail.com

${ }^{1}$ TÜBITAK National Observatory, Antalya, Turkey,

${ }^{2}$ Kazan Federal University, Kazan, Russia,

${ }^{3}$ Department of Astronomy and Space Sciences, Faculty of Science, Istanbul University, Istanbul, Turkey,

${ }^{4}$ Istanbul University Observatory Research and Application Center, Istanbul, Turkey,

${ }^{5}$ Tatarstan Academy of Sciences, Kazan, Russia,

${ }^{6}$ Space Research Institute of the Russian Academy of Sciences (IKI), Moscow, Russia,

${ }^{7}$ Elpaş Engineering, Istanbul, Turkey

The multi-object spectroscopy technique was implemented on the Russian-Turkish 1.5m optical telescope (aka. RTT150) by modifying the slit wheel of its main instrument TFOSC (TÜBİTAK Faint Object Spectrograph and Camera). Remotely controlled rotating holders of the multi object masks were installed in the two nests of the slit wheel. The accuracy of the mask unit's rotation is the order of 1 arcmin, in which the image plane for 2 sources located at a separation of 5 arcmin corresponds to a positional accuracy of 0.1 arcsec. To investigate the quality of the MOS technique in RTT150, the well-known merging galaxy cluster Abell 1914 was observed spectroscopically. The dispersion of the differences between known and RTT150 redshift estimations is 0.0006, which is good enough for cosmological studies.

Keywords: Multi-object spectroscopy, galaxy clusters: individual: Abell 1914, optical observations, astrophysics: instrumentation

DOI: $10.51194 /$ VAK2021.2022.1.1.010

\section{Necessity of MOS at RTT150}

The main scientific goal of the Russian-Turkish 1.5m optical telescope, RTT150, is ground based follow-up observations of the X-ray sources of the joint Russian-German astrophysical space observatory Spektr-RG (SRG). For that purpose, one important task is the optical identification of high-energy sources discovered by the all-sky surveys of the Pavlinsky ART-XC X-ray telescope. The mean positional uncertainty of the ART-XC X-ray sources is about 50 arcsec, it means that in a region at low galactic latitude the number of optical sources to be observed spectroscopically with RTT150 can reach up to several dozens. In addition, nearly 20 thousand of galaxy clusters have been detected recently from the SRG eRosita telescope all-sky survey [1]. Only less than $50 \%$ of these clusters were previously known. At the end of the SRG mission it is expected to be discovered $\sim 100000$ galaxy clusters. A significant number of eRosita galaxy clusters can be observed at the RTT150. It is well-known that the projected distribution of most of the brightest members around the clusters is within a few arcmin. Thus, to increase the efficiency of the observing time in spectroscopy for the moderately crowded fields of sizes up to several arcmin, we developed and implemented the facility for multi-object spectroscopy (MOS) at the RTT150.

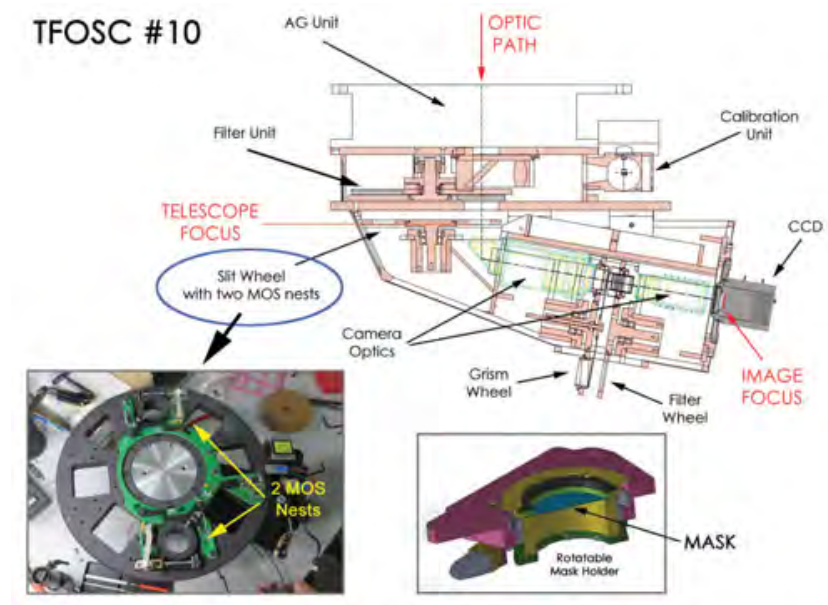

Figure 1: The schematic drawing of the TFOSC shown with the modified aperture wheel with the mask holder. 


\section{Modification of the multifunctional detector of RTT150}

\subsection{TFOSC}

Available instrumentation at the RTT150 telescope makes it possible to perform comprehensive studies of the astrophysical objects including high-precision positional, photometric, and spectroscopic measurements, as well as polarimetric measurements of the linear polarization. The main instrument of the telescope, which implements all these observational methods, is an instrument from the FOSC series (\#10) - TFOSC (TÜBİTAK Faint Object Spectrograph and Camera) produced by the Copenhagen University. The schematic drawing of TFOSC is shown in Fig. 1. The TFOSC system consists of two filter wheels before the telescope focal plane (Filter Unit), a wheel with a set of different slits and masks located in the focal plane (Slit Wheel), and a set of calibration lamps (Calibration Unit). Two additional filter wheels consisting of broad and narrow-band filters and other dispersion elements are located in the collimated part of the instrument (Grism and Filter wheels).

\subsection{Aperture wheel modification}

It is obvious that to observe all selected sources in moderately crowded and relatively small fields with less masks, it is necessary to distribute the slits with non-overlapping dispersion and to include as many sources as possible per mask. This condition implies that slit shape has to be pinhole. We have developed a remotely controlled rotating mask holder in the wheel nest with a replaceable head including a twist lock fixing. On the Fig.1 (lower left corner) the MOS mask holders are shown. All TFOSC wheels including the aperture wheel have the ability to rotate 360 degrees. Since there is only one part is moving in the MOS mask holder the other is not, transmission of power and control signals is a challenge. We solved this problem by installing concentric grooves isolated from each other on the aperture wheel itself in an unused area close to the rotation axis and fixed runners on a special mast in the aperture wheel adapter. The rotation of the mask holder is carried out by a worm gear coupled with a tiny stepper motor. Control system are based on a Teensy USB Development Board. The system has a mask rotation accuracy of 1 arcmin, which corresponds to a positional accuracy on 0.1 arcsec for simultaneous spectroscopy of the two sources in the TFOSC image plane located at a separation of 5 arcmin.

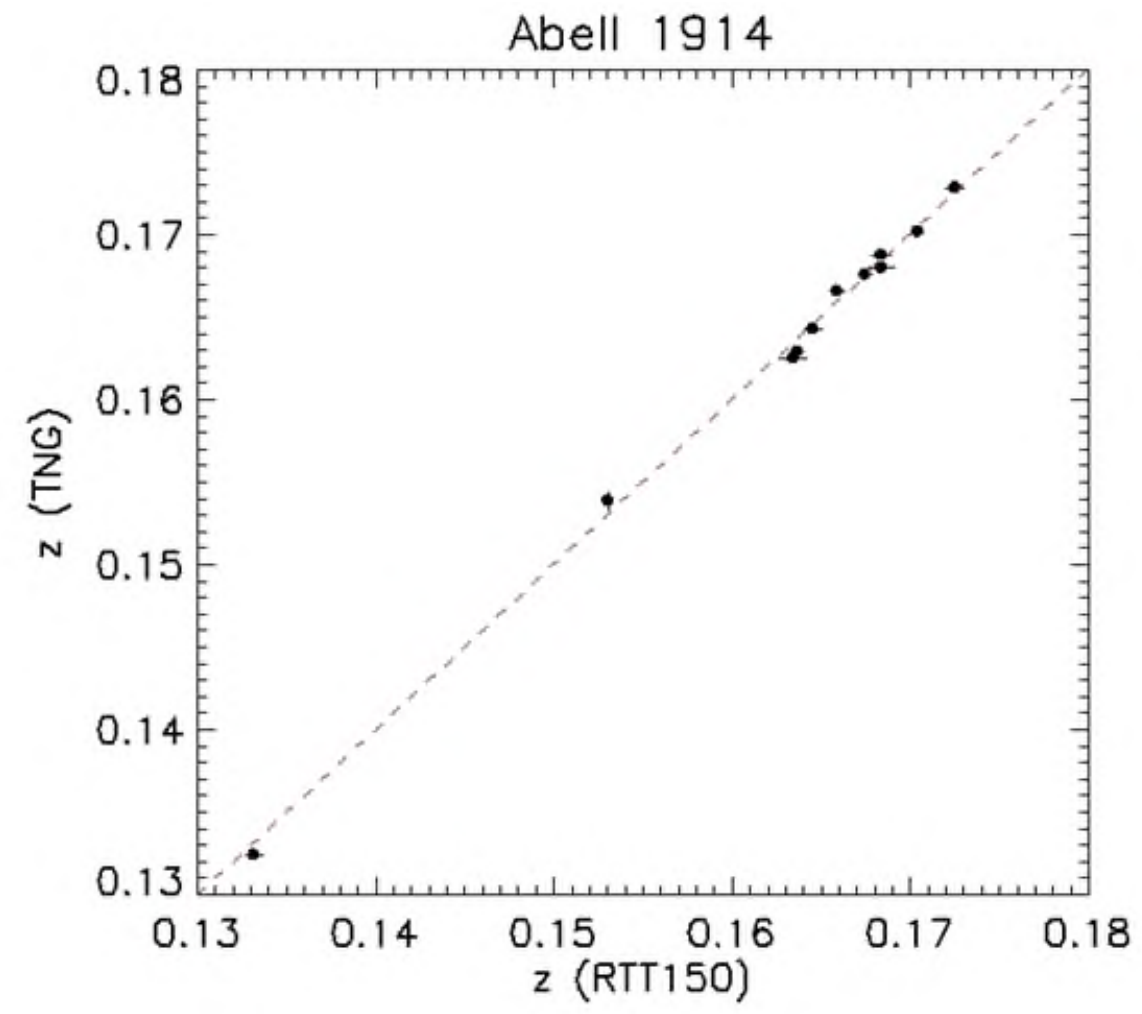

Figure 2: The correlation between TNG and RTT150 redshift measurements of the sources in the field of Abell 1914. 


\section{Test observation}

To investigate the quality of the MOS technique that we implemented at the RTT150, we observed the wellknown merging galaxy cluster Abell 1914. We used multi-objects mask with pinhole apertures. Since there is a sky background in the aperture besides the light from the source, additional holes for the blank sky were added to the mask. The details of the mask and the extraction of individual spectra are described in detail in [2]. The low resolution $(R \sim 600)$ spectra from 40 pinholes covering spectral range from 4000 to 8500 were obtained using TFOSC-MOS in 11 Mart 2018 with an exposure of 1800s.

Redshifts of the galaxies were estimated by cross-correlating the target spectrum with a template spectrum of an elliptical galaxy. The redshifts of some galaxies were spectroscopically measured previously from the MOS spectra obtained at the $3.58 \mathrm{~m}$ TNG telescope with 3600s exposure [3]. The correlation of the two sets of redshift measurements of the member galaxies of the Abell 1914 galaxy cluster and background galaxies in this field with brightness from $16^{m}$ up to $19^{m}$ is shown on Fig.2 (the dotted line shows the line of equality, $y=x$ ). The dispersion of differences between the TNG and the RTT150 redshift estimations is 0.0006 , which is good enough for cosmological studies like identification and confirmation of galaxy clusters.

Acknowledgements. We thank TÜBİTAK National Observatory, IKI, KFU, and AST for the partial support in using RTT150 (Russian-Turkish 1.5-m telescope in Antalya). This work was partially supported by the subsidy 0671-2020-0052 allocated to the Kazan Federal University for the State assignment in the sphere of scientific activities.

\section{References}

1. R. Sunyaev, V. Arefiev, V. Babyshkin, A. Bogomolov, et al., arXiv e-prints, arXiv:2104.13267, 2021.

2. I. M. Khamitov, I. F. Bikmaev, R. A. Burenin, M. V. Glushkov, S. S. Melnikov, and A. R. Lyapin, Astronomy Letters, 46, 1, 2020.

3. R. Barrena, M. Girardi, and W. Boschin, MNRAS, 430, 3453, 2013. 


\title{
RadioAstron project: calibration of the space radio telescope in flight in 2011-2019
}

\author{
Shakhvorostova $^{1}$, K. Belousov ${ }^{1}$, A. Kovalenko ${ }^{6}$, N. Nizhelsky ${ }^{7}$, G. Zhekanis $^{7}$, P. Tsubulev ${ }^{7}$ \\ ykovalev@asc.rssi.ru \\ ${ }^{1}$ Lebedev Physical Institute of RAS, Leninsky prospect, 53, Moscow, 119991 \\ ${ }^{2}$ Radio Physics Research Institute, Nizny Novgorod, 603950 \\ ${ }^{3}$ Max Planck Institute for Radio Astronomy, Bonn, Germany \\ ${ }^{4}$ Moscow Institute of Physics and Technology, Dolgoprudny, 141700 \\ ${ }^{5}$ ASTRON Netherlands Institute for Radio Astronomy, Dwingeloo, The Netherlands \\ ${ }^{6}$ Pushino Radio Astronomy Observatory of the LPI RAS, Pushino, 142290 \\ ${ }^{7}$ Special Astrophysical Observatory of RAS, Nizhny Arkhyz, 369167
}

Yu.A. Kovalev ${ }^{1}$, V. Vasil'kov ${ }^{1}$, A. Ermakov ${ }^{1}$, E. Vinyaikin ${ }^{2}$, M. Lisakov ${ }^{3}$, M. Popov ${ }^{1}$, V. Soglasnov ${ }^{1}$, M. Larionov $^{1}$, N. Nikolaev ${ }^{1}$, E. Mironova ${ }^{1}$, M. Burgin ${ }^{1}$, Yu.Yu. Kovalev ${ }^{1,4,3}$, P. Voitsik ${ }^{1}$, A. Kutjkin ${ }^{5}$, A. Alakoz ${ }^{1}$, N.

We present results of amplitude calibration of the RadioAstron Space Radio Telescope (SRT) at wavelenghts of 1.35, 6.2, 18, and $92 \mathrm{~cm}$. Flux density calibrated internal noise signals and SEFDs are found to be stable within the errors at the level of $3-13 \%$. We have used the flux density scale by Baars et al. (1977). The known issue of the variability of calibrators was resolved performing comparison with two other flux density scales. We have resolved the issue by averaging SRT noise diode signals measured relative to Cassiopeia A and Crab Nebula.

DOI: 10.51194/VAK2021.2022.1.1.011

\section{Introduction}

Juli 18, 2021 marks the 10th anniversary of launching the space radio telescope (SRT) of the RadioAstron project. It successfully operated from July 18, 2011 to January 9, 2019, significantly exceeding the planned active flight duration. On January, 9, 2019, communication with the spacecraft was interrupted and attempts to restore it were unsuccessful. The flight of the SRT is continued but without communication. This report summarizes the main results of flux density calibrations of the SRT. Detailed description of the Radioastron project and first results see in [1] and [2].

\section{Results}

1. According to the data of alignment (radiometric) sessions and calibrations relative to the primary calibrators Cassiopeia A and Crab Nebula, it was shown that the system equivalent noise temperature $\left(T_{\text {sys }}\right.$, in Kelvin) and the System Equivalent Flux Density (SEFD, $F_{\text {sys }}$, in Jansky) of the SRT in flight are stable with an accuracy not worse than about 3-4\% in 2011-2018. At the same time, the channel-averaged $T_{\text {sys }}$ or SEFD values measured by different processing tools (interactive and automated) for a full 7.5 years (in 2011-2018) and for a 4-year interval (in 2015-2018) practically coincide within 2-3\% at wavelengths of $6.2 \mathrm{~cm}$ (for channel 2) and $18 \mathrm{~cm}$ (both channels), and $8-10 \%$ at wavelengths of $92 \mathrm{~cm}$ (both channels) and $6.2 \mathrm{~cm}$ (channel 1 ).

2. It has been obtained from observations with VLBI at $1.35 \mathrm{~cm}$, that $T_{\text {sys }}$ and SEFD has drift of about $4-5 \%$ per a year. This result is apparent. It is caused by the detected drift of noise signal generator power, relative to which $T_{\text {sys }}$ and SEFD were measured in VLBI sessions. Taking this drift into account in the VLBI SEFD calibrations made it possible to compensate for the detected error. The $T_{\text {sys }}$ and SEFD values in the alignment observations with the SRT did not have this features, since they were measured relative to the astronomical calibrators directly, and not indirectly, through the noise signal generator, as in the VLBI sessions.

3. Modeling and analysis of telemetry from temperature sensors show that standard changes in the physical temperature of the elements of the antenna-feeder tract (such as the Focal Container, the Antenna Feed Unit, the Polarizer and the Cold Plate with the Low Noise Amplifier Units), despite the standard losses in them, can lead to noticeable variability of the system noise. The reason for this variability is the change in the temperature conditions of the spacecraft on the feeder elements with losses due to changes in the angle between the SRT axis and the direction to the Sun near the boundaries of the working angles. This variability had practically no effect on the results and operation of the SRT.

4. A new automated system for processing calibration measurements has been developed by a post-graduate student (see [3]). It is applied to 4-year monitoring of SRT calibrations in 2015-2018 [4]. This made it possible to use all 24 internal noise signals from special diodes as secondary calibration standards, as well as to perform a verification of astronomical calibrators and accurate flux density scales using the SRT data. This made it also possible to identify and take into account an unexpected problem. The main primary standard astronomical flux density scale turned out to be significantly variable. This scale was built according to Cassiopeia A about 40 years ago and has been widely used in radio astronomy all these years. 
We have compared 3 following accurate flux density scales: first, the scale [5], second, the scale [6], third, the scale $[7,8]$. The practically equivalence of them can be obtained at $6.2,18$ and $92 \mathrm{~cm}$ if the average calibration of noise signals between Cassiopeia A and Crab Nebula are used: $3-4 \%$ at 6.2 and $18 \mathrm{~cm}, 9-12 \%$ at $92 \mathrm{~cm}$.

So, we have the variable flux density scale, proposed in 1977, and two scales, proposed in 2017 and 2014 , corrected the first. These 3 scales practically coincide (with differences from $3 \%$ to $12 \%$ ), if only we apply the averaging of results on these astronomical calibrators. As a rule, the VLBI calibrations during the SRT flight were performed relative to the noise signal averaged over these two objects. On this reason, it may be not necessary to correct the results.

\section{Summary}

The amplitude calibration of the SRT in flight was performed mainly relative to the strong flux density calibrators Cassiopeia A and Crab Nebula within the generally accepted astronomical flux density scale by [5]. However, their recently found variability [6] had to be taken into account. We have used three flux density scales to verify SRT calibration results. The verification became possible due to the constancy of both, the effective area of the SRT and the equivalent noise temperature for most of the main and reserve noise diode signals. A similar approach can be utilized for ground radio telescopes also if changes of noise diode signal and effective area are independently monitored and corrected.

\section{References}

1. N. S. Kardashev, V. V. Khartov, V. V. Abramov, V. Y. Avdeev, et al., Astronomy Reports, 57, $153,2013$.

2. Y. A. Kovalev, V. I. Vasil'kov, M. V. Popov, V. A. Soglasnov, et al., Cosmic Research, 52, $393,2014$.

3. A. N. Ermakov and Y. A. Kovalev, Transaction of IAA RAS, 54, 21, 2020.

4. Y. A. Kovalev, V. I. Vasil'kov, A. N. Ermakov, V. E. N., et al., Transaction of IAA RAS, 54, $32,2020$.

5. J. W. M. Baars, R. Genzel, I. I. K. Pauliny-Toth, and A. Witzel, A\&A, 61, 99, 1977.

6. R. A. Perley and B. J. Butler, ApJS, 230, 7, 2017.

7. E. N. Vinyaikin, Astronomy Reports, 51, 570, 2007.

8. E. N. Vinyaikin, Astronomy Reports, 58, 626, 2014. 


\title{
Method of processing data obtained during RATAN-600 West sector surveys with static antenna
}

\author{
A.A. Kudryashova, N.N. Bursov \\ akudrysova@yandex.ru \\ Special Astrophysical Observatory of RAS
}

In this paper we present results of designing of the methods of processing data obtained at the RATAN-600 West sector static antenna during the all-year continuous survey at the declination of the source GRS 1915+105. Shapes of antennae beams were investigated as well as oblique transitions of point sources across them.

Keywords: telescopes; Methods: observational; Antenna Beam

DOI: 10.51194 /VAK2021.2022.1.1.012

\section{Introduction}

Methods of carrying out surveys on RATAN-600 were used in earlier works [1, 2]. Detailed investigations about antenna beam pattern features is presented in the works [3, 4]. RATAN-600 is a radio telescope with a horizontal system. Sources are observed because of rotation the Earth. West sector of RATAN-600 observes East sky, where sources occur rising. The important detail is sources cross antenna beam not parallel to its horizontal line. It means that shape and size of point radio sources are changed. The angle q of sources tracks and horizontal line is calculated from formula:

$$
\sin (q)=\cos (\varphi) \sin (A z) / \cos (\delta)
$$

where azimuth $A z=270^{\circ}, \varphi=43^{\circ} 49^{\prime} 52^{\prime \prime}$ is latitude of RATAN-600 and $\delta$ is declination of sources.

\section{Beam pattern}

During the survey at the declination of source GRS $1915+105$, four receivers with four channels were used. They form four antenna beams, as shown in the graphic Fig. 1. Calculations were made with help of the program $b p 1$ [5]. RATAN-600 West sector system's focus is located between the second and the third receivers. It means that every beam is deformed because of aberrations: the second and the third beams are closer to focus (angular distance 63 $\operatorname{arcsec}$ ), the first and the fourth beams are farther (angular distances are 63 arcsec and 190 arcsec). Fig. 2 show calculated shape of the first and second beams (the third and the fourth are mirror symmetric). Shapes of sources in data obtained with the first and the fourth receivers are deformed significantly.

Both factors: sources tracks inclined to horizontal axis of antenna beam and deformed shapes of beams, should be taken into account. Sources with similar right ascension and different declination cross the maximum of the

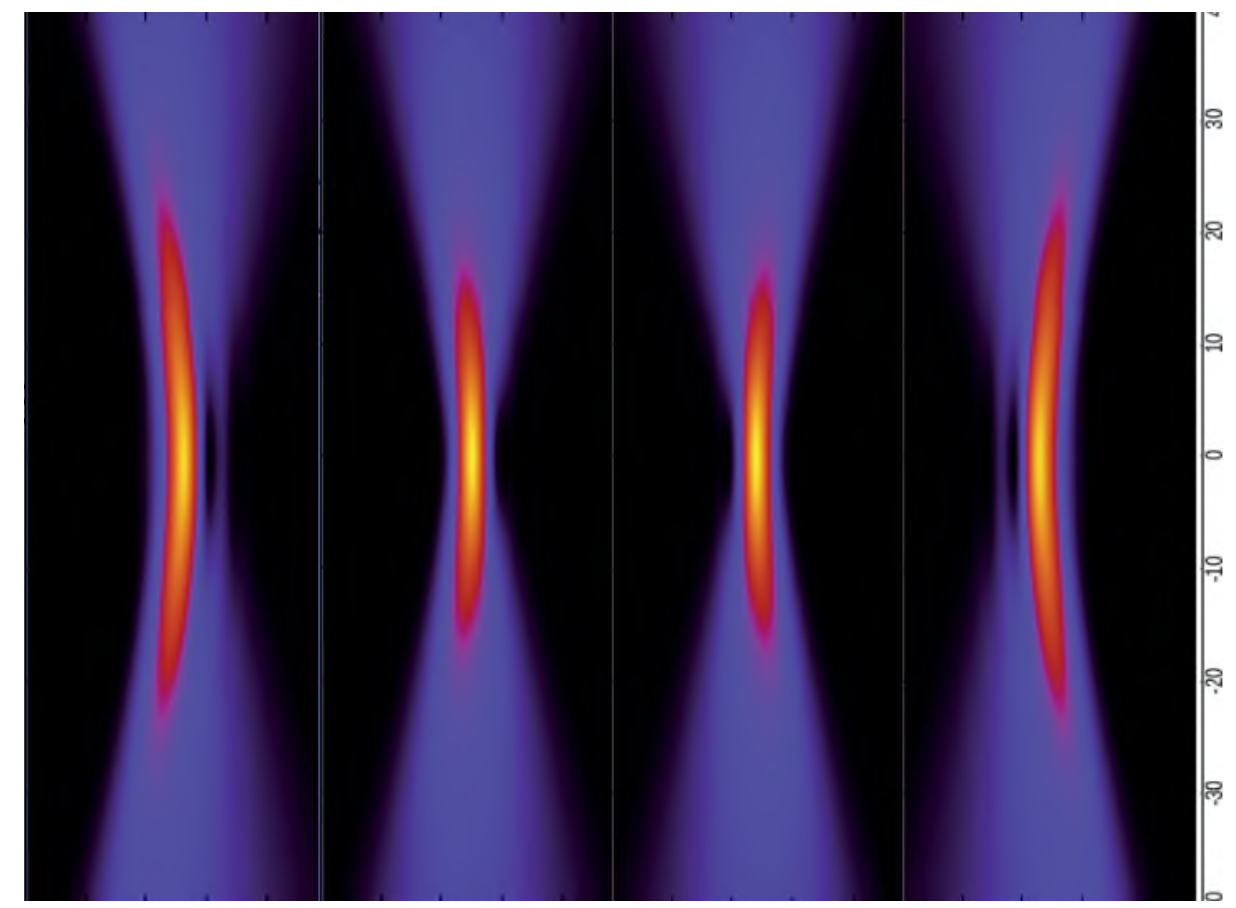

Figure 1: Calculated levels of antenna beams. Yellow is the largest level of gain. 


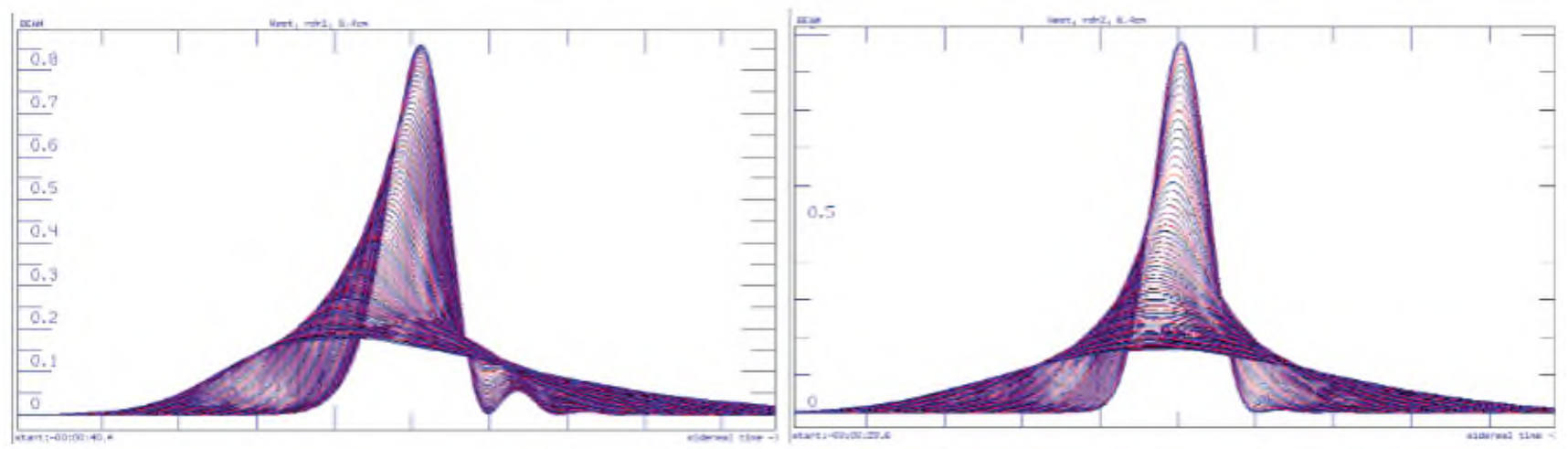

Figure 2: Beam pattern shape for receiver with horn in 190 arcsec angular distance (left) and receiver with horn in 63 arcsec angular distance (right)

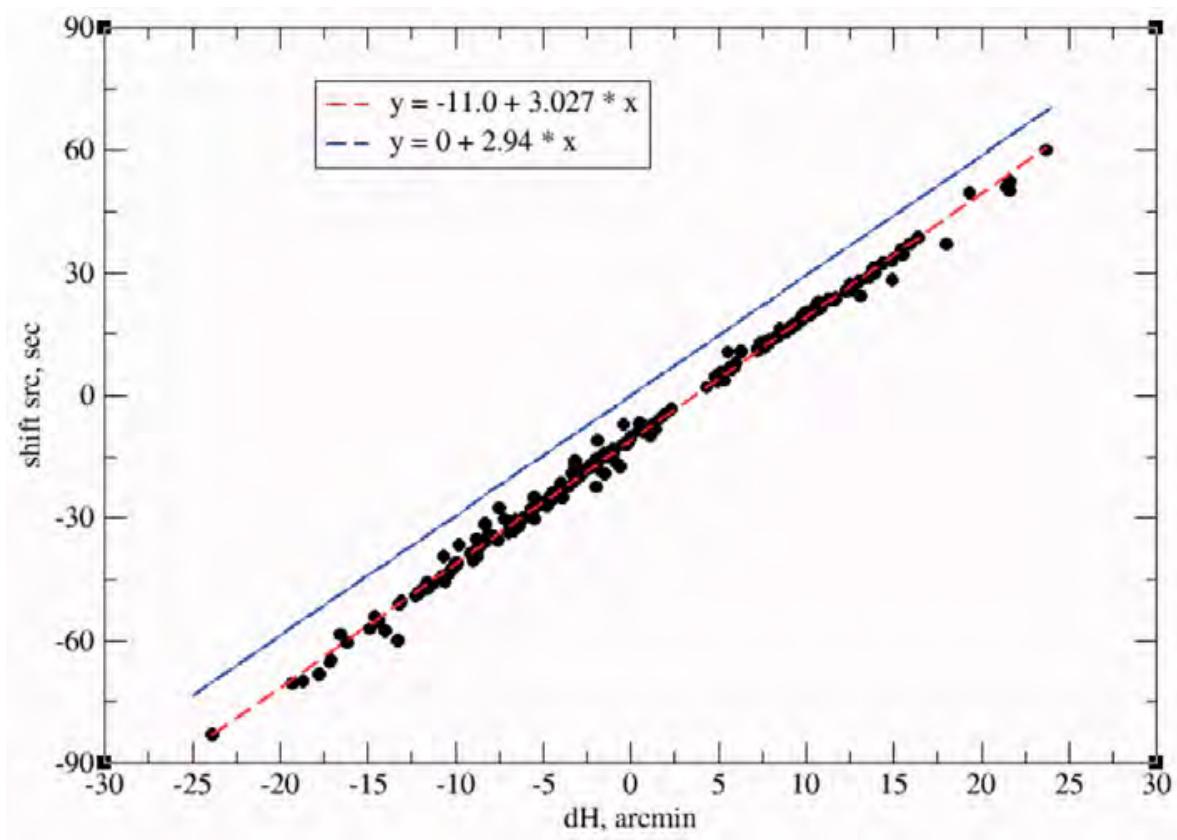

Figure 3: Shift of time sources cross antenna beam maximum. Blue line — calculated, black dots — observed.

antenna beam non-simultaneously. A shift in time was calculated and measured in the observational data. The results are depicted in Fig. 3.

\section{Conclusion}

The parameters of the static antenna beam in the survey at the declination of the source GRS $1915+105$ on RATAN-600 West sector in azimuth $=270^{\circ}$ were investigated. New method, taking into account all necessary corrections of data processing, is created. The method was used in forming the list of sources observed in the survey.

\section{References}

1. I. N. Pariiski, N. N. Bursov, N. M. Lipovka, N. S. Soboleva, and A. V. Temirova, A\&A Sup., 87, 1, 1991.

2. N. N. Bursov, Y. N. Pariiskii, E. K. Maiorova, M. G. Mingaliev, A. B. Berlin, N. A. Nizhel'Skii, I. A. Glushkova, and T. A. Semenova, Astronomy Reports, 51, 197, 2007.

3. E. K. Majorova, Bulletin of the Special Astrophysics Observatory, 53, 78, 2002.

4. E. K. Majorova and N. N. Bursov, Astrophysical Bulletin, 62, 378, 2007.

5. A. N. Korzhavin, Astrofiz. Issled. (Izv. SAO), 11, 170, 1979. 


\section{AstroMagick: software for controlling and adapting the surface of the main mirror of the Millimetron space observatory}

A.O. Lyakhovets, A.S. Andrianov, M.Yu. Arkhipov, E.S. Golubev

lyakhovets@asc.rssi.ru

P. N. Lebedev Physical Institute of the Russian Academy of Sciences, Russia

The paper provides an overview of the capabilities and algorithms of the AstroMagick software package developed at the FIAN Astro Space Center. AstroMagick is designed to control the surface and refine algorithms for adapting the main mirror of the Millimetron space observatory. AstroMagick is used in modeling, developing, manufacturing, testing, mounting and adapting the parts of Millimetron main mirror.

Keywords: best-fit paraboloid, non-linear optimization, adaptive telescope, Millimetron space observatory

DOI: $10.51194 /$ VAK2021.2022.1.1.013

\section{Goals and objectives}

The main mirror (MM) of the Millimetron Space Observatory is a paraboloid of rotation with a nominal focus of $2400 \mathrm{~mm}$ [1]. It consists of 96 carbon fiber panels of 4 standard sizes [2]. The panels are manufactured using astrositall molds.

Millimetron research tasks require the following surface accuracies: molds $1 \mu \mathrm{m}$ RMS from paraboloid; panels 3-6 $\mu \mathrm{m}$ RMS from paraboloid; whole main mirror: 6-10 $\mu \mathrm{m}$.

To meet the requirements it is necessary to:

- control the accuracy of mirror elements (manufacturing, testing);

- find the optimal position of the panel on the mold (manufacturing);

— verify finite element panel models (development);

- create realistic surface models for optical simulation (development, adaptation);

- find optimal panel displacements within the MM (mounting, adaptation);

- find optimal panel deformations using regulators and actuators (adaptation);

- optimize the MM as a whole: minimize wavefront errors (adaptation).

All the above tasks are solved using AstroMagick.

\section{Implementation}

The input data for AstroMagic are point clouds or 3D-models of surfaces close to off-axis parts of a rotational paraboloid and obtained by measurements (Zeiss CMM, ATOS, laser radar) or modeling.

Data file formats: ASC, 3DS, OBJ, STL, ANSYS/NASTRAN, NX, Zemax Grid Sag, AstroMagick internal surface formats. High calculation speed allowed integrating relevant AstroMagick components into ANSYS.

\section{Fitting a point cloud to a paraboloid}

Calculating of the best-fit paraboloid (BFP) is used: in primary processing of measurement data; when finding optimal panel position on mold and within MM; to combine several measurements; to optimize the wavefront.

The fitting to BFP can usually be achieved by minimizing distances to the paraboloid along the optical axis of the mirror (formulas (13),(14) [3] or (7),(16) [4]). However, this approach only works for nearly flat surfaces, and is not suitable for the Millimetron MM.

AstroMagick minimizes the normal distances to paraboloid. This leads in a non-linear optimization. The authors use the Levenberg-Marquardt algorithm with analytical derivatives with respect to fitting parameters.

The distance to a paraboloid of rotation is calculated by solving a cubic equation. The authors showed that this equation almost always has one root for the Millimetron mirror. Therefore, for the sake of calculation speed, AstroMagick uses a closed form solution - the Cardano formula.

Optimization of the point cloud position can be weighted and can also be performed with restrictions on movements. Concurrently with position optimization, a search for the optimal focus of the paraboloid can be performed. The total number of optimization parameters is $4-6$.

Of cases with weighted points, noteworthy is a global optimization of the MM wavefront. In AstroMagick, it is achieved by setting appropriate weights in a routine minimization and by correct final calculation of RMS.

The most important case of limiting possible movements is movement "in place": 1 shift perpendicular to the middle of the surface and 2 rotations around axes tangent to the surface. Such movements leave the surface projection in the same place of the paraboloid, and they correspond to movements induced by panel-support clamp actuators.

The operating time on a single CPU core ranges from fractions of a second or a few seconds for $10^{4}$ to $5 \cdot 10^{5}$ points to 3 minutes for $2 \cdot 10^{7}$ points. 
The results of best-fitting to a paraboloid in the simulation of thermal deformations are presented in [2].

\section{Constructing a surface on a quasi-regular grid}

Such a surface makes it possible to eliminate spatial irregularity of the measured points, interpolate sparse measurements (CMM, radar) to intermediate values, and also makes it easy to compare and combine two or more measurements of one part.

Based on a measured cloud of surface points, AstroMagick constructs a surface on a locally square grid with a given step, preserving the angles and distances on small areas of the surface. The surface is presented by deviations from a given paraboloid at grid nodes (this is how all the above deviation maps are constructed).

When constructing a surface for sparse clouds measured with a CMM or a laser radar, RBF interpolation of deviations with Gaussian functions is performed.

An additon, when constructing the surface, the measurement bounds are determined, in particular, holes in the measurements are excluded.

\section{Combining multiple measurements and creating a mean surface}

To improve measurement accuracy and to measure large parts not fitting into the Zeiss CMM workspace, it is necessary to perform several measurements of the element and combine them in the best way.

There are various known general methods for solving the problem of combining two point clouds. And there are several approaches to solving the problem of combining many clouds at a time. For our measurements, however, all known methods are either not enough accurate or too complicated.

AstroMagick uses the closeness of measurements to the analytical surface, a parabaloid of rotation. As a result, the distances between surfaces constructed from measurements in the neighbourhood of the same paraboloid (the same focus) are very accurately approximated by distances along normals to the paraboloid. Thus, we eliminate a most difficult and error prone portion in the known algorithms. To find the optimal movements of point clouds, the sum of squared distances between each pair of surfaces at each grid node is then minimized using the LevenbergMarquardt algorithm with analytical derivatives.

To construct a mean surface, the measured point clouds are moved as found for optimal combination, after which they just merge. The surface is constructed from the combined point cloud, with a RBF interpolation with smoothing done in case of sparse measurements (Zeiss CMM, radar) - the minimized function contains the term "square of the second derivative".

\section{Surface differences}

In the position of best combination of measurements, the differences between the surfaces can be easily calculated just as it is done when combining.

The differences between the surface of each measurement and the mean surface of all measurements are used to: control the quality of measurements and reject obvious errors in measurement; filter lined noise occurring in some measurements on CMM; assess the accuracy to the obtained mean surface.

For example, the mean surface based on 20 measurements of the central mirror mold before it was reground at the factory differs from any of these measurements $\leq 1 \mu \mathrm{m}$ RMS: the differences are .83 .99 .78 .84 .55 .72 .78

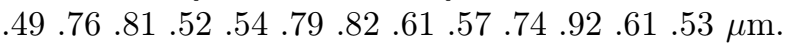

Another application of measurement differences is for testing FE-models of matrices and panels.

\section{Optimal deformations of panels}

Given the presence of regulators or deforming actuators, there arises the problem of determining their optimal loads for achieving the target shape of the panel.

The known methods for solving such a problem have the following limitations and simplications: optimization is aimed at deviations from the target shape along the optical axis of the mirror; the whole element subject to optimization is stationary. Thus, only 1D deformation fields are used, and the problem is reduced to a linear one and is solved using the SVD method [5].

In the case of the Millimetron mirror panels, deviations along the optical axis give too rough approximation. In addition, the Millimetron panels under optimization are either not yet mounted in the mirror (the case of regulators), or they are mounted on the support actuators that allow them to be moved "in place" (the case of deforming actuators).

Therefore, AstroMagick does a simultaneous search for optimal deformation coefficients and optimal panel movement, and minimizes deviations between the respective panel points and target surface points defined as the projections of panel points in the initial position to the target surface. 3D deformation fields are used as input data, the Davenport q-method is applied at each step of the Levenberg-Marquardt algorithm to find the best movement. 
For actuators, the output of this algorithm is provided in the report "Adaptation system of the Millimetron main mirror" presented at this Conference; for regulators — in [6].

\section{References}

1. A. V. Smirnov, A. M. Baryshev, S. V. Pilipenko, N. V. Myshonkova, et al., in M. C. Clampin, G. G. Fazio, H. A. MacEwen, and J. M. O. Jr., eds., Space Telescopes and Instrumentation 2012: Optical, Infrared, and Millimeter Wave, SPIE, volume 8442, 1456 - 1464 (2012).

2. E. S. Golubev, E. K. Kotsur, M. Y. Arkhipov, A. V. Smirnov, A. O. Lyakhovec, and V. N. Pyshnov, in R. Navarro and R. Geyl, eds., Advances in Optical and Mechanical Technologies for Telescopes and Instrumentation IV, SPIE, volume 11451, 135 - 147 (2020).

3. N. N. Goldobin, Vestnik SibGAU, 1, 106, 2013.

4. Z. Li, Z. Xie, J. Wang, and Y. Lei, IOP Conference Series: Materials Science and Engineering, 397, 012047, 2018.

5. H. R. Hiddleston, D. D. Lyman, and E. L. Schafer, in M. A. Ealey, ed., Active and Adaptive Optical Systems, SPIE, volume 1542, 20 - 33 (1991).

6. M. Arkhipov, A. Lyakhovets, E. Golubev, E. Kotsur, and A. M. Baryshev, Journal of Astronomical Telescopes, Instruments, and Systems, 7, 1, 2021. 


\title{
Corrections of the operational orbit of the "Spectr-RG" spacecraft in order to ensure the specified conditions of radio visibility from Russian ground stations
}

\author{
E. Mikhailov \\ MikhailovEA@laspace.ru \\ Lavochkin Association, Khimki, Moscow Region, 141400, Russia
}

"Spectr-RG" launch postponement lead to problems with radio visibility from Russian control stations. The issue can be solved by changing operational orbit out-of-ecliptic amplitude with correction impulses mostly perpendicular to the ecliptic. Two correction strategies have been developed. One of these strategies was chosen for implementation.

Keywords: out-of-ecliptic plane amplitude changing maneuvers, collinear libration points, "Spectr-RG" spacecraft

DOI: 10.51194 /VAK2021.2022.1.1.014

One of the main requirements for the nominal trajectory of the "Spectr-RG" (SRG) spacecraft is to ensure the conditions for daily radio visibility of the spacecraft from the involved ground control stations Bear Lakes and Ussuriysk. Bear Lakes is the northernmost of these two and so has the worst conditions of daily radio visibility. Therefore, the visibility of the spacecraft from this station was taken into account, first of all, when choosing the launch date.

The nominal launch dates (June 21-22 and July 12-13) were chosen in accordance with the technology for ensuring the launch of the spacecraft [1, 2]. For the first launch date Bear Lakes ground station daily radio visibility has gaps up to 25 days, starting from the second year of the flight (see Fig. 1). As for the Ussuriysk station, it's daily intervals exceed 5.5 hours throughout the spacecraft's lifetime. So the goal of controlling the spacecraft and obtaining scientific information from the board can be reached.

The launch was postponed and took place on July 13 for technical reasons. This influenced badly to radio visibility conditions. So gaps in radio visibility from Bear Lakes increased and lasted up to one and a half months, while the minimum daily intervals from Ussuriysk in 2022-2023 changed to no more than an hour (Fig. 2).

The worst conditions for radio visibility of SRG from the involved ground control stations occur when it reaches the extreme point of its orbit towards the South Pole of the world in the vicinity of the summer or winter solstice. Therefore, it is necessary to decrease the amplitude of the orbit in the negative direction along the axis $Z_{L 2}$ to improve radio visibility conditions.

\section{Methodology}

The methodology of implementation of so-called "big corrections" was chosen based on familiarization with literature $[3,4]$. It indicates that the optimal moment for a realization of the correction to decrease the amplitude of the orbit in the negative direction along the axis $Z_{L 2}$ is the moment when the spacecraft crosses the plane of

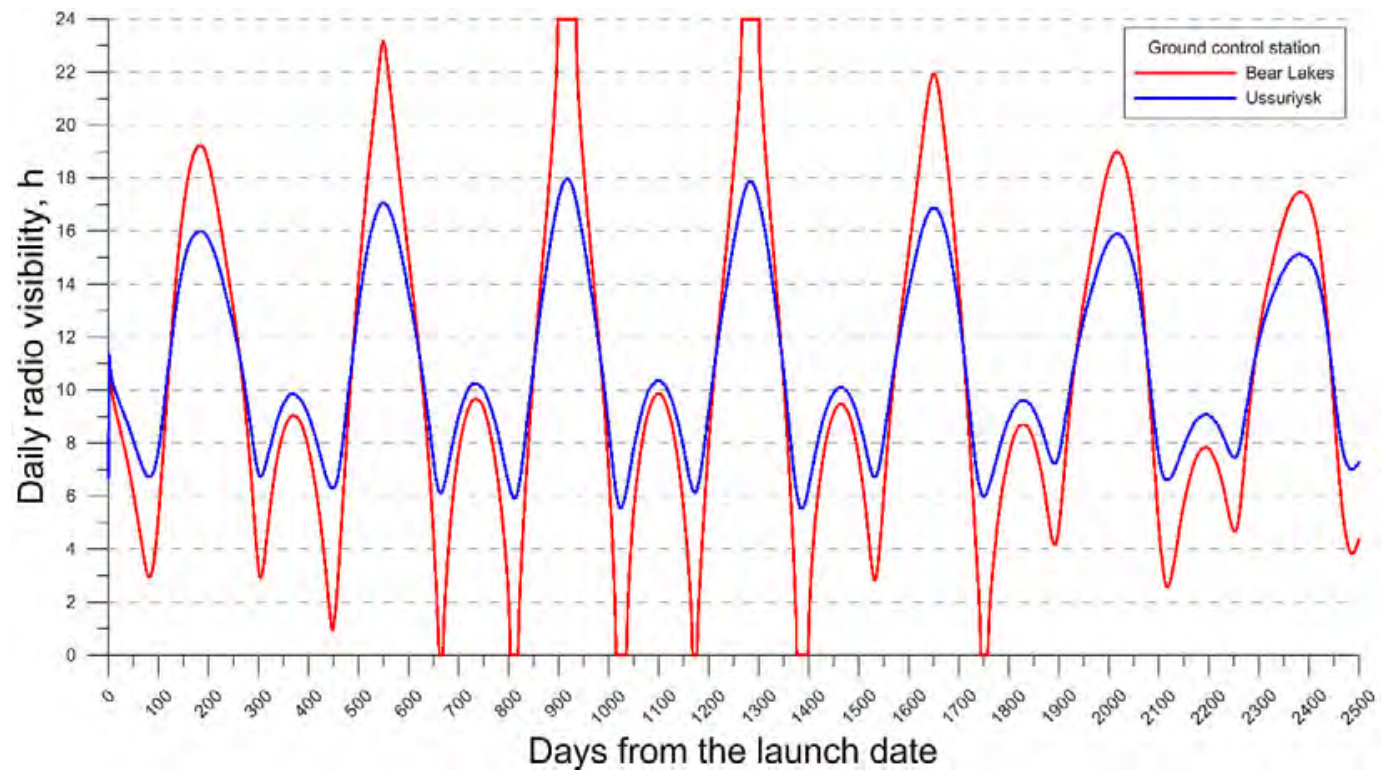

Figure 1: Daily radio visibility from Bear Lakes (red line) and Ussuriysk (blue line) stations. Launch date: June $21,2019$. 


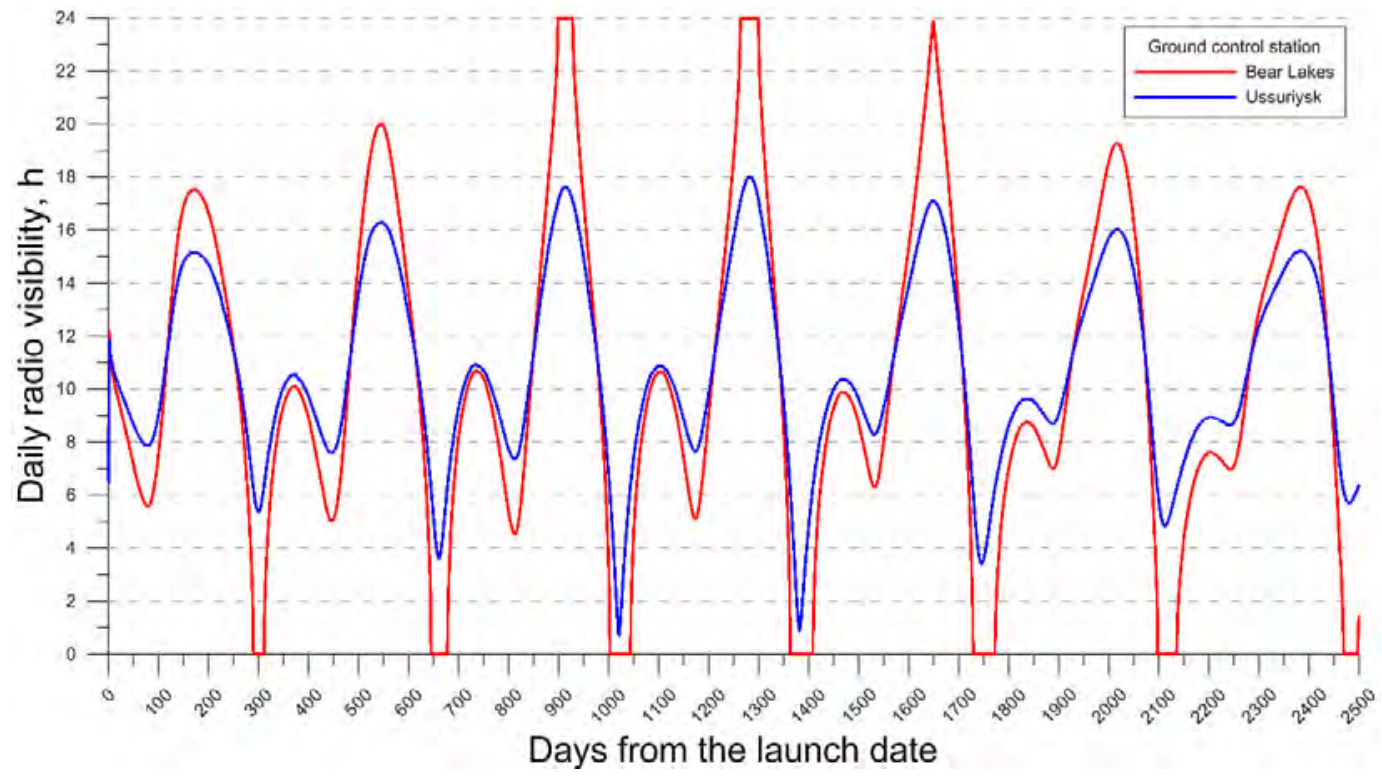

Figure 2: Daily radio visibility from Bear Lakes (red line) and Ussuriysk (blue line) stations. Launch date: July $13,2019$.

Table 1: Strategies of the impulses

\begin{tabular}{ccccc}
\hline \multicolumn{4}{c}{ Strategy No. 1 } & \multicolumn{2}{c}{ Strategy No. 2 } \\
\hline Date & Impulse, $\mathrm{m} / \mathrm{s}$ & Date & Impulse, $\mathrm{m} / \mathrm{s}$ & Main direction \\
\hline 23.11 .2020 & 6 & 23.11 .2020 & 12 & $-Z_{L 2}$ \\
28.02 .2021 & 6 & 28.02 .2021 & 12 & $+Z_{L 2}$ \\
22.05 .2021 & 6 & 22.05 .2021 & 12 & $-Z_{L 2}$ \\
02.09 .2021 & 6 & 01.09 .2021 & 12 & $+Z_{L 2}$ \\
23.11 .2021 & 6 & 23.11 .2021 & 12 & $-Z_{L 2}$ \\
02.03 .2022 & 6 & - & - & $+Z_{L 2}$ \\
22.05 .2022 & 6 & - & - & $-Z_{L 2}$ \\
02.09 .2022 & 6 & - & - & $-Z_{L 2}$ \\
23.11 .2022 & 6 & - & - & $+Z_{L 2}$ \\
02.03 .2023 & 6 & - & & -
\end{tabular}

the ecliptic. Based on simulation results, following two strategies of big corrections were proposed: 10 impulses at $6 \mathrm{~m} / \mathrm{s}$, or 5 at $12 \mathrm{~m} / \mathrm{s}$. The preliminary dates for the impulses are given in the table below. In the right column the main thrust direction of along the axis $Z_{L 2}$. These impulses should be additionally refined in order to increase the duration of the spacecraft stay in the vicinity of the libration point $L_{2}$ after the correction.

The first strategy was chosen by the decision of the main operational control group of the SRG mission.

Daily radio visibility intervals for Bear Lakes and Ussuriysk are shown on Fig. 3. Unfortunately, it was not possible to exclude the gaps. The conditions of radio visibility from Ussuriysk have significantly improved and the minimum daily interval has become about 4 hours. This already meets the requirements of the ground control complex to ensure the regular control operations.

Some specialists expressed their concern for possible scientific equipment degradation because of long engine operating intervals. This could significantly reduce the scientific data quality. That's why a test correction was implemented on October 5, 2020 with characteristic velocity of $3 \mathrm{~m} / \mathrm{s}$. It should be noted that the correction was carried out at a non-optimal (from the explained methodology point of view) moment. No negative effects were found. 


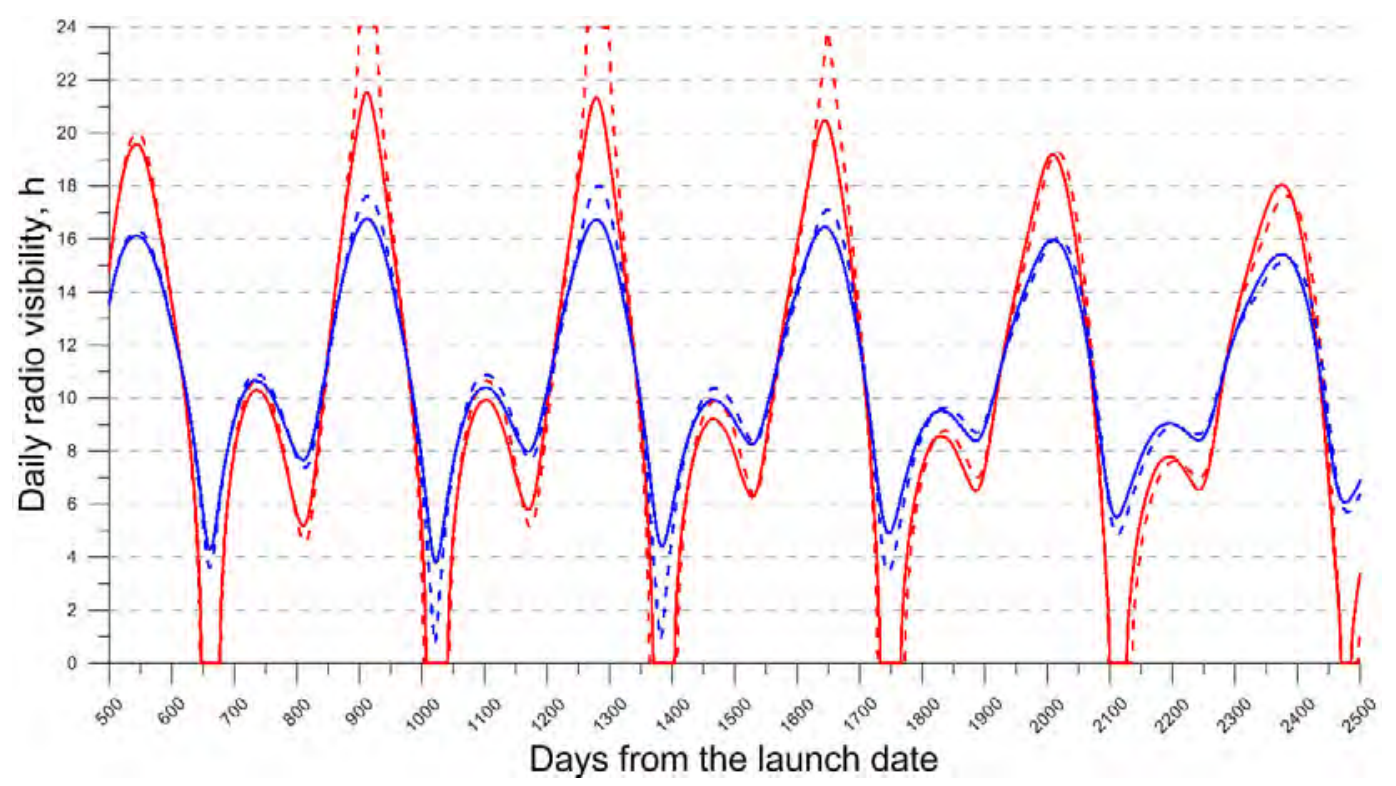

Figure 3: Daily visibility duration from Bear Lakes (red lines) and Ussuriysk (blue lines) stations: dashed lines initial visibilities, solid lines — visibility after the planned manoeuvrers of the orbit improvement.

\section{Results}

The methodology of decreasing the amplitude of the orbit SRG spacecraft in the negative direction along the axis $Z_{L 2}$ has been developed and correction schedule with 10 impulses at $6 \mathrm{~m} / \mathrm{s}$ each was accepted.

The test correction at $3 \mathrm{~m} / \mathrm{s}$ was implemented on October 5, 2020. No influence of the propulsion system on the scientific payload was found.

At the moment, three of the planned corrections have already been successfully completed: November 23 , 2020, February 28, 2021 and May 23, 2021.

\section{References}

1. I. S. Il'in, G. S. Zaslavsky, S. M. Lavrenov, V. V. Sazonov, V. A. Stepanyantz, A. G. Tuchin, D. A. Tuchin, and V. S. Yaroshevsky, Cosmic Research, 52, 437, 2014.

2. P. V. Mzhelskiy and E. A. Mikhailov, Preprint of Keldysh Inst. of Applied Math, 9, 2018.

3. V. E. Canalias, Contributions to Libration Orbit Mission Design using Hyperbolic Invariant Manifolds, Ph.D. thesis, Universitat Politècnica de Catalunya, 2007.

4. M. Hechler and J. Cobos, in G. Gómez, M. W. Lo, and J. J. Masdemont, eds., Libration Point Orbits and Applications. Proceedings of the Conference, Aiguablava, Spain, 115-135 (2002). 


\title{
Project Lyra not on ISS
}

\author{
M.E. Prokhorov, A.I. Zakharov, O.Yu. Stekolshchikov, M.S. Tuchin \\ mike.prokhorov@gmail.com \\ Sternberg Astronomical Institute of Moscow State University, Univeritetsky prospect 13, 119991 Moscow, Russia
}

The aim of the Lyra-B project was to carry out a high-precision multicolor multiple photometric survey of high and moderate brightness stars across the entire sky. It was assumed that the survey Lyra-B will be carried out from the ISS. Due to the shutdown of the ISS in 2025, this project probably will not happen. The paper discusses the possibilities and feasibility of performing the survey on an autonomous spacecraft or on board the future Russian space station ROSS.

Keywords: stellar photometry, high precision photometry, stellar catalogues, sky survey

DOI: $10.51194 /$ VAK2021.2022.1.1.015

To carry out various fundamental research and applied developments in the field of space exploration, astronomy and astrophysics, deep, high-precision and homogeneous stellar catalogues of various types are required: astrometric, photometric, spectral, etc.

The problem of high-precision astrometric catalogs was solved by performing space experiments Hipparcos (in the early 90s) and Gaia (now). The situation with the available photometric catalogs of stars is much worse. (Note that astrometric catalogs "degrade" over time (i.e., lose their accuracy) due to errors in the proper motions of stars, but photometric catalogs are practically "eternal".)

Consider the requirements for an "ideal" photometric catalog of stars. The catalog should be highly accurateno worse than $0.003^{m}$ bright stars and no worse than $0.01^{m}$ for the rest, multicolor - no less than $5-6$ photometric bands in the visible, ultraviolet and near-IR ranges, deep - completeness till to $15-17^{m}$, better to $20-21^{m}$. The survey should cover the entire celestial sphere, or at least a significant part of sky (at least 50\%). Observations of stars in different photometric bands should be simultaneous or quasi-simultaneous, and each star should be observed many times during the survey.

Until now, there have been no such surveys (and catalogs). The most accurate photometry of stars today is contained in the Hipparcos catalog. The error of its photometric measurements is $0.001^{m}$. But this catalog contains only 118218 stars, it full up to $9^{m}$, and its photometric measurements were performed in one wide panchromatic Hp band (in "white light").

The closest to it in accuracy is the WBVR-catalog of bright stars of the northern sky, created at the Alma-Ata observatory of SAI. This catalog contains only 15000 stars of the northern sky (with declination $\delta>-14^{\circ}$ ), it is full up to $7^{m}$. Its photometric measurements were performed in four bands of the Johnson photometric system. The measurement error behind the atmosphere is $0.003^{m}$.

The next in accuracy is the Tycho- 2 catalog. It contains 2.5 million stars measured in two photometric bands $B t$ and $V t$ with errors of about $0.008^{m}$. The catalog is full up to $12^{m}$ and covers the entire starry sky.

The SDSS (Sloan Digital Sky Survey) catalog is comparable with Tycho-2 in accuracy, but significantly larger in volume. It contains 500 million stars, measured in five bands of the ugriz photometric system. The measurement error is $0.008^{m}-0.016^{m}$, depending on the photometric band and the brightness of the star. The measurements do not contain bright stars - the SDSS catalog includes only stars from $16^{m}$ to $22.5^{m}$. Because of this, the SDSS photometric measurements coincide with the photometry of brighter stars from the catalogs listed above. The survey does not cover the entire sky (in SDSS excludes the region along the plane of the Galaxy, where the high density of stars prevent to observations of distant galaxies). Repeated observations were not included in the survey. Despite the listed limitations, today it is the largest multicolor photometric catalog of stars in the visible range with an accuracy better $0.1^{m}$.

There are other photometric catalogs that are also not ideal. Their characteristics are shown in Table 1.

The source of high-precision photometry of all-sky stars in three very wide bands $G, G_{B P}, G_{R P}$ is the Gaia space experiment. Whether the final release of Gaia catalog will include multicolor photometry and its accuracy is not clear today. Another source of high-precision photometry of stars could be the CSS-OS space survey, which is planned to be carried out at the China Space Station.

To create a high-precision photometric catalog of stars, SAI MSU proposed the Lyra-B space experiment on board the Russian Segment of ISS. For this, a 0.5-m telescope was to be installed on the ISS, which would measure the brightness of the stars of entire sky up to $15^{\mathrm{m}}$ in 10 photometric bands [1, 2]. The development of the Lyra-B experiment was slowly moving forward; in 2019, the stage of development of design documentation for the experiment was completed. However, now that the time for the completion of the ISS operation has been determined - 2024 year — it has become clear that left time is not enough to complete the preparation and execute of the experiment.

Since carrying out a stellar photometric survey remains actual, we are considering the possibility of "reformatting" this project. There are two possibilities for this: survey on a stand-alone satellite or on the future Russian manned space station ROSS. 
Table 1: Presentday stellar photometrical catalogues

\begin{tabular}{lcccll}
\hline \multicolumn{1}{c}{ Survey } & Precision & Stars & $m_{\lim }$ & Bands & Comments \\
\hline Hipparcos & $0.001^{m}$ & 118218 & $9^{m}$ & $H p(1)$ & \\
WBVR-SAI & $0.003^{m}$ & $\sim 15000$ & $7^{m}$ & $W B V R(4)$ & $\delta>-14^{\circ}$ \\
Tycho-2 & $0.008^{m}$ & $2.5 \cdot 10^{6}$ & $12^{m}$ & $B_{T}, V_{T}(2)$ & \\
2MASS & $0.04^{m}$ & $470 \cdot 10^{6}$ & $5^{m}-16^{m}$ & $J H K(3)$ & \\
Infra-red SDSS & $0.008^{m}-0.016^{m}$ & $500 \cdot 10^{6}$ & $16^{m}-22.5^{m}$ & ugriz (5) & Not all sky \\
USNO A2.0 & $0.3^{m}$ & $600 \cdot 10^{6}$ & $20^{m}$ & $B, R(2)$ & From photoplates \\
USNO B1.0 & $0.3^{m}$ & $10^{9}$ & $21^{m}$ & $B, R(2)$ & From photoplates \\
Gaia DR2 & $<0.001^{m}$ & $1.6 \cdot 10^{9}$ & $21^{m}$ & $G, G_{B P}, G_{R P}(3)$ & \\
\hline
\end{tabular}

For a survey from satellite the following three possibilities appear most obvious. The first possibility is to survey from a satellite in a solar-synchronous orbit. In orbital orientation of satellite, a very convenient configuration is possible, in which the telescope is always directed to the local zenith region, the service module and antennas are directed to the Earth, and the solar batteries are maximally deployed to the Sun.

Observations are carried out continuously in scanning mode due to orbital motion, and sky coverage occurs due to orbit precession. One-time coverage of the full sky occurs in six months.

The benefits of the orbit is the ability to fix the telescope motionlessly to the spacecraft. At the same time, scientific equipment and radiators of the cooling system will always be in the shadow of the Sun. A telescope with a larger diameter may be installed on the satellite than on the ISS. The disadvantages of the orbit is fact that the transmission of information to the Earth can occur only when the satellite is flying over the stations for receiving information. The interval between such passages can reach 2-3 orbital revolutions. Because of this, a large information storage device must be installed on board, and the system for transmitting information to Earth must have a high speed.

The second possibility involves a satellite in a sun-synchronous orbit and data transmission through a system of geostationary repeaters. The design of the spacecraft is the same as in the first case, but data transmission is carried out continuously, which does not require a large amount of onboard memory and high-speed data transmitters.

The third possibility is a satellite in geosynchronous orbit. From it, you can continuously transmit data to one or two dedicated ground stations for receiving information. However, a satellite in geosychronous orbit will have a more complex design. It will have many moving parts, since when the satellite turns around the Earth, the antennas must always be in the direction towards the Earth, the solar batteries must be constantly turned towards the Sun, and the telescope must conduct observations. In order to cover the entire sky with observations during continuous scanning, the telescope on a geostationary spacecraft must rotate uniformly around the transverse axis, and its axis of rotation must coincide with the local vertical (direction to the center of the Earth). If the period of rotation of the telescope is equal to the orbital period (i.e. 1 sidereal day), then it is possible to achieve that the angle between the axis of sight of the telescope and the direction to the Sun is always greater than $60^{\circ}$.

Another possibility is to perform this survey from the ROSS space station. In this case, the scientific equipment for the survey will be much more similar to that developed for the ISS during the Lyra-B project, but noticeable changes will still be required. Unfortunately, the date for launching ROSS is very uncertain today.

\section{References}

1. A. I. Zakharov, A. V. Mironov, M. E. Prokhorov, A. V. Biryukov, O. Y. Stekolśhchikov, and M. S. Tuchin, Astronomy Reports, 57, 195, 2013.

2. S. V. Zhmailov and M. E. Prokhorov, Astronomy Reports, 64, 34, 2020. 


\section{System of Observation of Day-time Asteroids (SODA)}

A. Shugarov, B. Shustov, V. Shmagin, A. Buslaeva

shugarov@inasan.ru

Institute of Astronomy, Russian Academy of Sciences, 119017 Pyatnitskaya str, Moscow, Russia

The project SODA (System of Observation of Day-time Asteroids) designed for exhaustive detection of decameter $(>5-10$ $\mathrm{m})$ meteoroids approaching the Earth from the day-time sky. The system consists of two Spacecraft (SC) placed into orbits in the vicinity of the Lagrange point L1 of the Sun-Earth system. Each SC is equipped with one to three small aperture $(25-30 \mathrm{~cm})$ telescopes that are to observe near space in a conical region around the Earth. For objects on collisional orbits the SODA ensures a warning time of $10-20$ hours and the coordinates of the atmospheric entry point with up to $10 \times 100$ $\mathrm{km}$ accuracy.

Keywords: Near Earth Objects, asteroid hazard problem

DOI: 10.51194/VAK2021.2022.1.1.016

\section{The SODA project}

It is known that the Chelyabinsk meteoroid that collided with the Earth on the 15th February 2013 was detected by neither any ground-based nor near-Earth space telescopes before it entered the Earth's atmosphere. No groundbased NEO discovery systems are able to detect meteoroids approaching the Earth from day sky. In our previous papers [1] we suggested that the only realistic way to overcome this problem is to use a system of space born telescopes located relatively far from the Earth. We named the system SODA (System of Observation of Day-time Asteroids).

The top-level SODA project goals are:

- to detect almost all bodies larger than $10 \mathrm{~m}$ entering the near-Earth space (i.e. approaching the Earth at a distance of less than 10E6 km) from the Sun direction;

- to quickly identify a body of special interest (e.g. an asteroid at collisional orbit) and to characterize it, i.e. perform body mass estimation;

- in the case of a collisional orbit, the system should ensure a warning time of about 10 hours and determine coordinates of the atmospheric entry point.

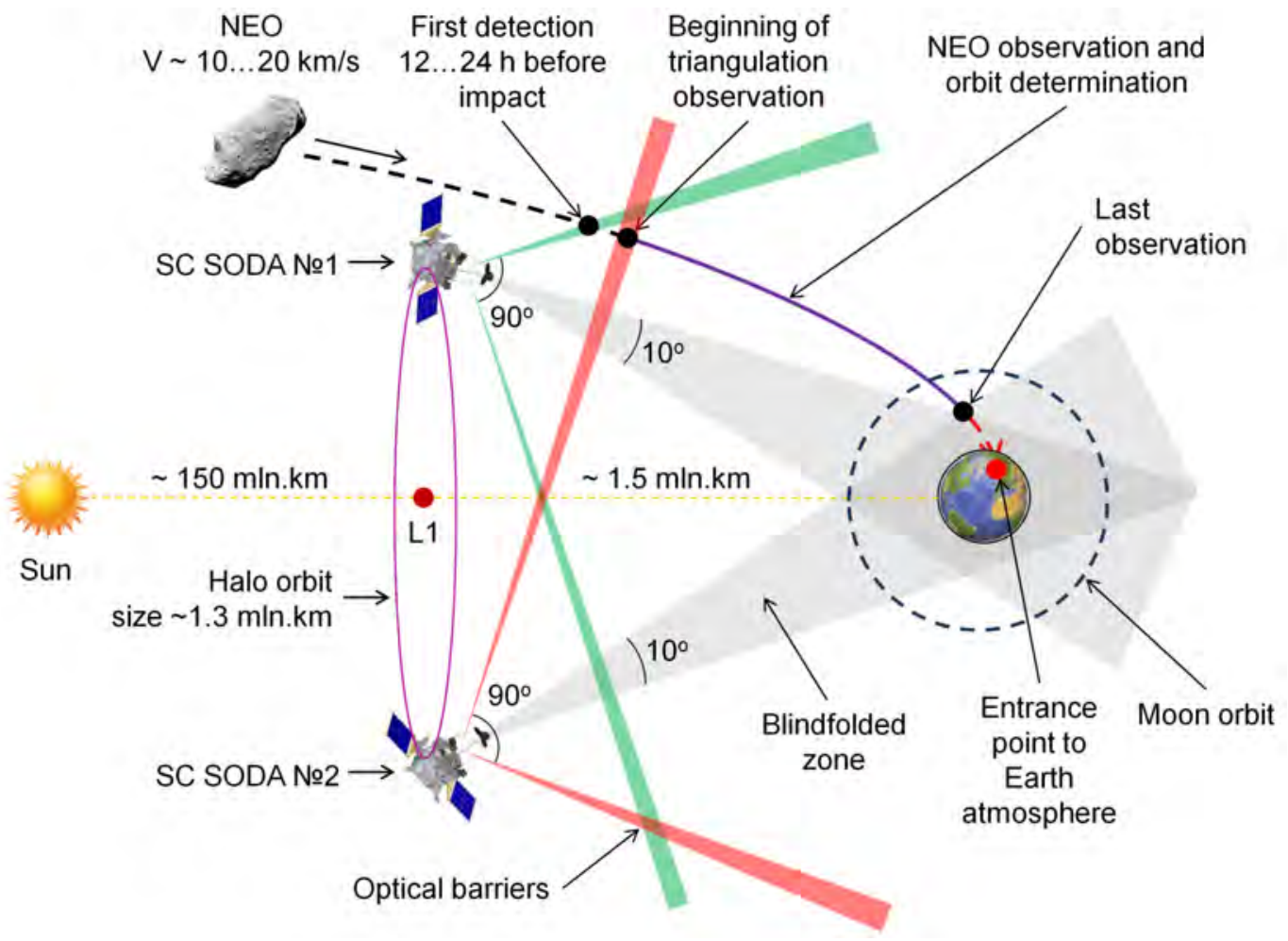

Figure 1: Scheme of observation by SODA project, projection on the ecliptic plane. 
L1 is an appropriate point to observe NEOs coming from the Sun, because of an optimal phase angle and absence of background. In [1] we described astronomical issues related to this project. The concept of the SODA system consists of one or two (in optimal configuration) spacecraft (SC) placed into orbits in the vicinity of the Lagrange point L1 of the Sun-Earth system [2]. The main advantages of two SC option are increased accuracy of orbit determination since the triangulation method can be used, prevention of the possibility of missing bodies at trajectories close to SC (less than 0.4 million $\mathrm{km}$ ), improved system reliability. The SC is equipped with one to three small aperture $(25-30 \mathrm{~cm})$ telescopes to observe near space in a conical region around the Earth.

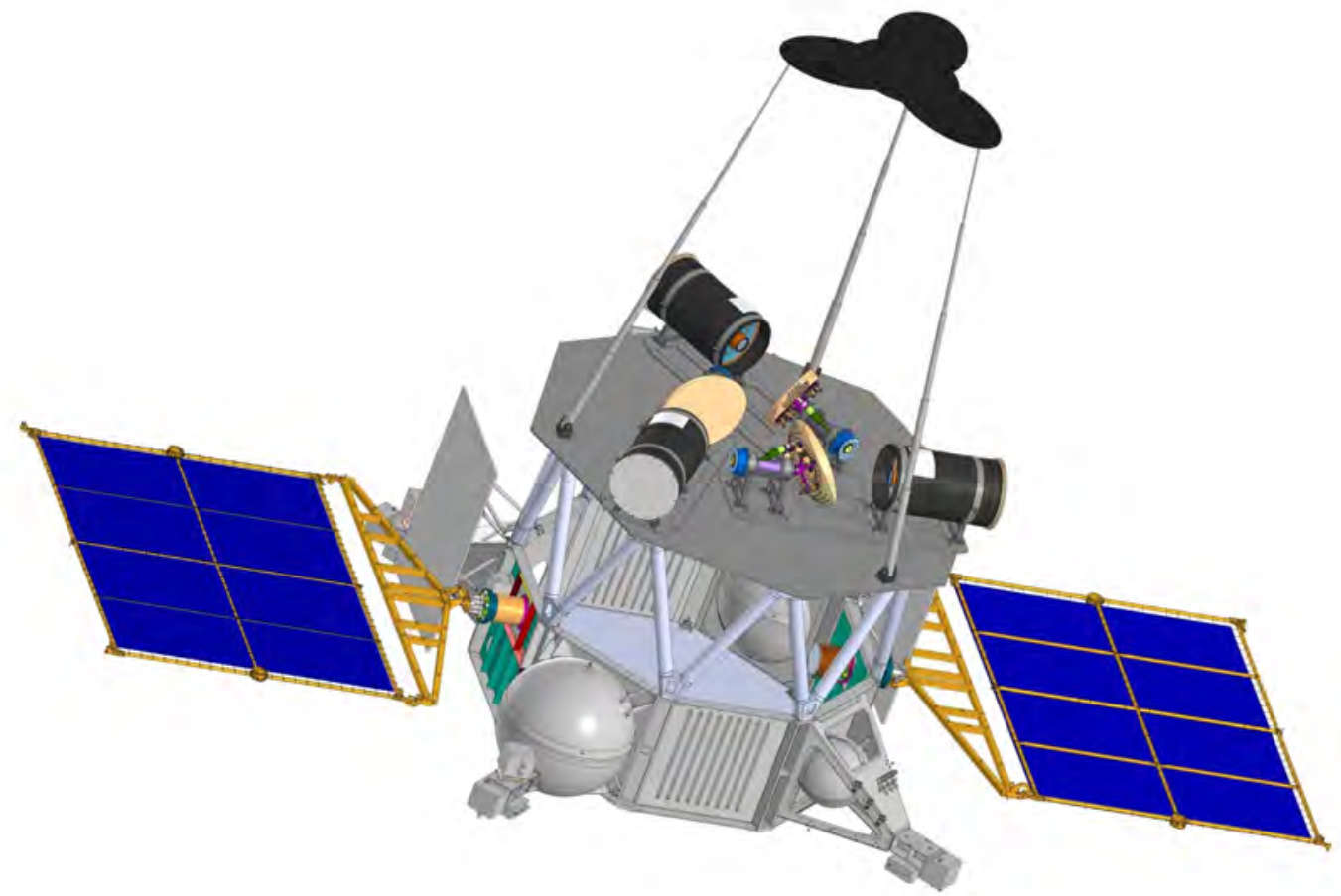

Figure 2: SODA spacecraft concept with "Navigator" space bus.

For the last two years additional research was carried out to make the SODA concept more mature.

One of the challenges of the SODA project is very frequent repointing of the telescope (every few seconds) to construct the optical barrier. We suggest to put a full-aperture slewing mirror in front of each telescope for quick repointing. This makes it possible for a very flexible observation program, tight duty-cycle and good redundancy. The telescope and slewing mirror are schematically shown in Figure 3.

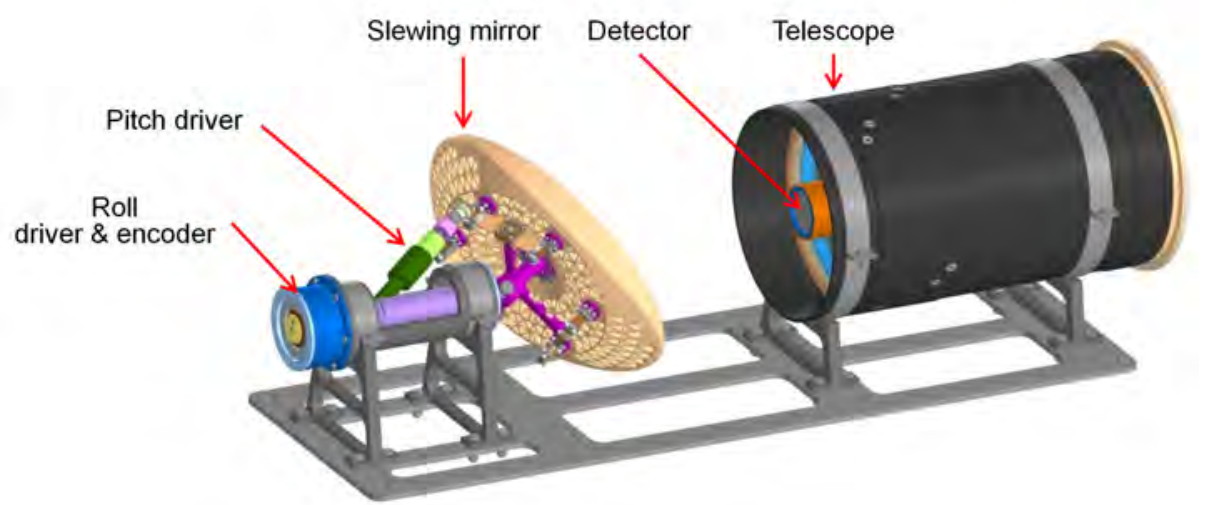

Figure 3: The concept of SODA's telescope with a slewing mirror.

Recent progress in manufacturing of CMOS detectors with a small pixel size makes it possible to design a more compact optical system without impacting the efficiency. The new optical system (30 cm aperture, F:1.5, $3.75 \mathrm{deg}$ field of view) based on the Sonnefeld camera optical design has been proposed. In combination with the pre-aperture slewing mirror $(480 \times 340 \mathrm{~mm})$ it provides a $50 \times 120$ degree area of observation with a single 
telescope. SODA SC should have has at least two of these telescopes on board, but for the redundancy and for higher astrometric accuracy, 3 or 4 telescopes options can be considered.

The scientific potential of the mission can be enhanced by including instruments for monitoring the activity of the Sun or to observe the Earth, similar to the DSCOVR space mission. A combination of SODA and ground-based survey telescopes (e.g. ATLAS) is a way to provide an efficient all-sky system for the detection of decameter size NEOs.

The proposed trajectory design for the SODA mission allows the insertion of $2 \mathrm{SC}$ (up to $500 \mathrm{~kg}$ each) into orbit around the L1 point with a required phase delay using Soyuz-2.1b medium class launcher with the Fregat-SB upper stage [3]. It takes about 100 days to reach an operational orbit.

SODA uses existing technologies and can be considered as a low risk and moderate-cost space project with valuable output for science and humanity. A request for SODA Phase A funding has been submitted to ROSCOSMOS.

International collaboration is welcome as well as cooperation with other ground-based projects focused on the detection of $10 \mathrm{~m}$ class NEOs.

\section{References}

1. B. M. Shustov, A. S. Shugarov, S. A. Naroenkov, and M. E. Prokhorov, Astronomy Reports, 59, 983, 2015.

2. A. Shugarov, B. Shustov, and S. Naroenkov, Open Astronomy, 27, 132, 2018.

3. I. D. Kovalenko, B. M. Shustov, and N. A. Eismont, Acta Astronautica, 148, 205, 2018. 


\section{Main qualification campaign results of WSO-UV mission UV CCD detectors}

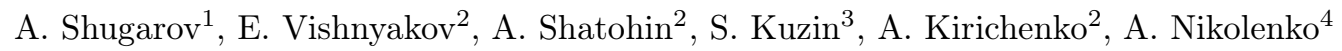
shugarov@inasan.ru

${ }^{1}$ Institute of Astronomy of the Russian Academy of Sciences, 48 Pyatnitskaya Street, Moscow 119017, Russia

${ }^{2}$ P.N. Lebedev Physical Institute of the Russian Academy of Sciences, 53 Leninskiy Prospekt, Moscow 119991, Russia

${ }^{3}$ Federal Scientific Research Centre Crystallography and Photonics of the Russian Academy of Sciences, 59 Leninskiy Prospekt, Moscow 119333, Russia

${ }^{4}$ Budker Institute of Nuclear Physics, Russian Academy of Sciences, 11 Ac. Lavrentiev Prospekt, Novosibirsk 630090, Russia

World Space Observatory Ultraviolet (WSO-UV) is a major Russian-led international collaboration to develop a large Spaceborne $1.7 \mathrm{~m}$ Ritchey-Chretien telescope to study the Universe in ultraviolet wavelengths. Three channels of the WSO-UV WUVS spectrograph operate in 115-310 $\mathrm{nm}$ range. The detectors of each channel have an almost identical custom UVoptimized CCD inside a vacuum enclosure and low noise drive electronics. The main challenges of the WUVS detectors are to achieve the best possible quantum efficiency in the FUV-NUV range, provide low readout noise $\left(<3 \mathrm{e}^{-}\right.$at $\left.50 \mathrm{kHz}\right)$ and low dark current $\left(<12 \mathrm{e}^{-} / \mathrm{pixel} /\right.$ hour$)$, operate with integral exposures of up to 10 hours and provide good photometric accuracy. This paper presents a brief summary of the WUVS Engineering Qualification Model (EQM) detectors' qualification campaign.

Keywords: WSO-UV, CCD, quantum efficiency, vacuum ultraviolet

DOI: 10.51194/VAK2021.2022.1.1.017

\section{WUVS spectrograph CCD detectors}

The WSO-UV project aims to study the Universe in the 115-310 nm wavelengths. WUVS (WSO-UV UltraViolet Spectrographs) consists of two high-resolution spectrographs $(\mathrm{R}=50000)$ covering the Far-UV range of $115-176 \mathrm{~nm}$ and the Near-UV range of $174-310 \mathrm{~nm}$, and a long-slit spectrograph $(\mathrm{R}=1000)$ covering the wavelength range of 115-305 nm [1].

The WUVS detectors should provide high sensitivity, high dynamic range and low dark current. For costsaving reasons the design of all three detectors is identical, except for minor changes such as anti-reflection coating on the CCD and the selection of active output amplifiers.

The companies, Teledyne e2v and STFC RAL Space, designed and manufactured the WUVS detector components.

Teledyne $\mathrm{e} 2 \mathrm{v}$ has designed three variants of a custom CCD272-64 sensor with different gradient UV antireflective coatings, each optimized for a specific WUVS channel dispersion [2]. The custom designed vacuum Enclosure prevents contamination and maintains the CCD at the operating temperature of $-100^{\circ} \mathrm{C}$, while the temperature of the WUVS optical bench is $+20^{\circ} \mathrm{C}[3]$.

STFC RAL Space has developed the Camera Electronics Boxes (CEBs). Digital correlated double sampling technology provides extremely low readout noise and also enables flexibility to optimize readout noise against pixel frequency for several normal and binned pixel readout modes.

The Critical Design Reviews of the CEB and Enclosure were held in 2016 and 2019 respectively. All EQM parts were delivered to Russia where three WUVS EQM detectors were assembled and tested (Fig. 1).

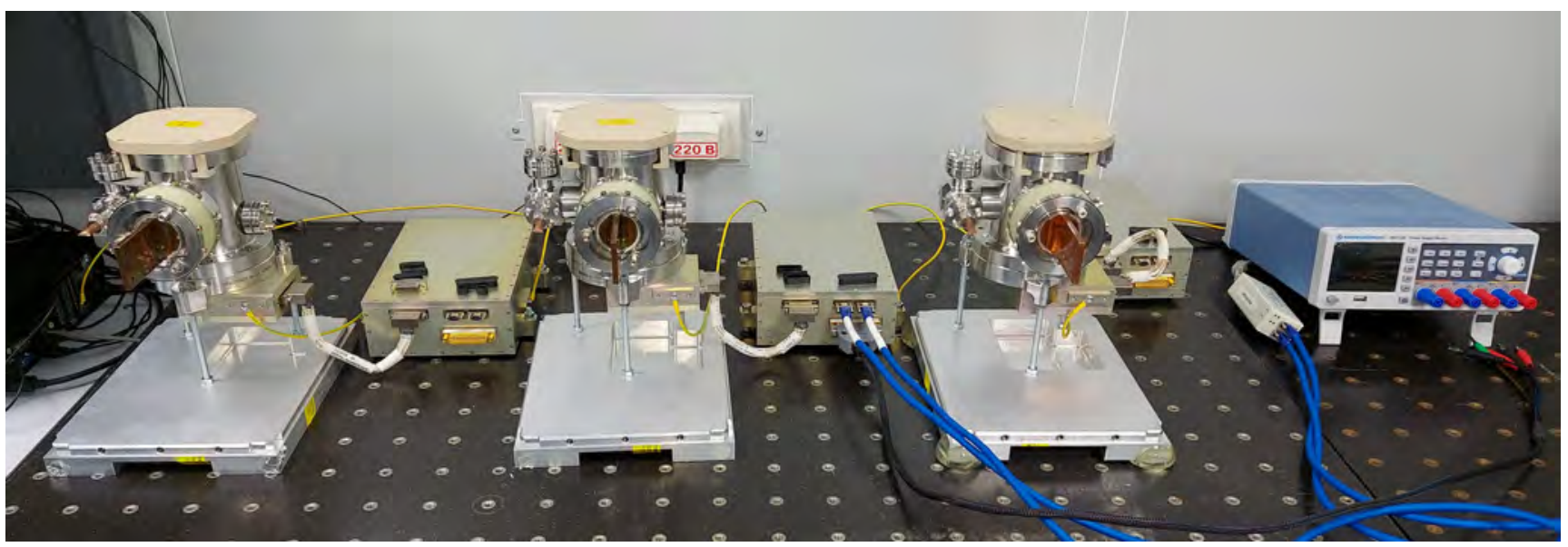

Figure 1: Fully assembled WUVS EQM (Engeneering Qualification Model) detectors. 


\section{Main qualification campaign results}

The WUVS detector components (CEB and Enclosure) successfully passed the qualification campaign at RAL Space and Teledyne e2v, key parameters were verified.

According to the RAL Space factory test the FM CEB readout noise is $2.6 \mathrm{e}^{-} \mathrm{RMS}$ at $50 \mathrm{kHz}$ and about $3 \mathrm{e}^{-} \mathrm{RMS}$ at $100 \mathrm{kHz}$, linearity is better than $0.2 \%$ and cross talk between the channels is less than $29 \mathrm{ppm}$.

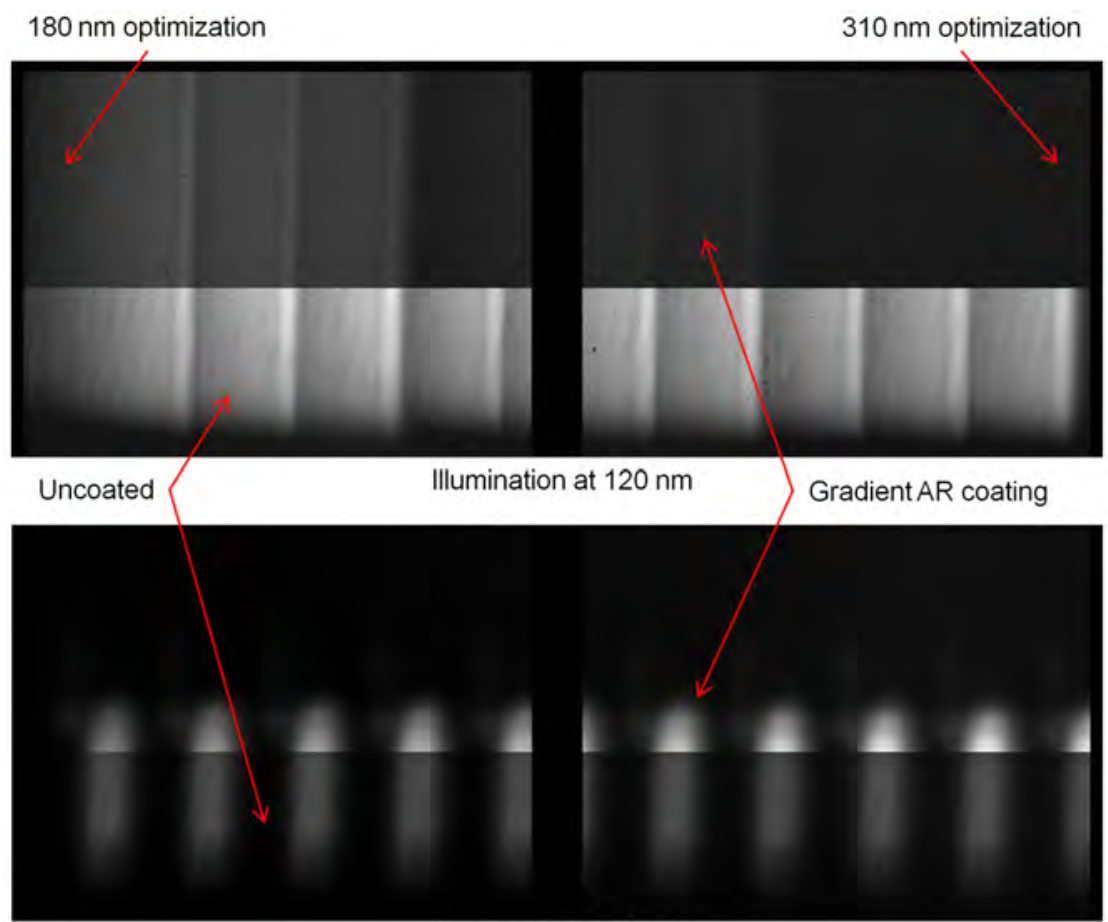

Illumination at $300 \mathrm{~nm}$

Figure 2: EQM LSS CCD test images in FUV (top) and NUV (bottom).

In 2019 at the Budker Institute of Nuclear Physics, the WUVS detector cooling system was tested and quantum efficiency measurement was carried out at the beamline "Kosmos" using synchrotron radiation from the VEPP-4M storage ring [4]. The LSS channel detector was tested in a complete configuration, including EQM Enclosure, CCD, protective $\mathrm{MgF}_{2}$ window and drive electronics. The LSS device has both uncoated and gradient anti-reflection coated regions (Fig. 2). The thickness of the anti-reflection coating is optimized for $180 \mathrm{~nm}$ on the left side of CCD and for $310 \mathrm{~nm}$ on the right side, in accordance with the LSS channel dispersion. The quantum efficiency measurements (Fig. 3) show good compliance with the theoretical predictions made by Teledyne e2v.

The Enclosure cooling system verification confirms the temperature difference between CCD and Enclosure's "Cold finger" of $5^{\circ} \mathrm{C}$.

\section{Conclusion and acknowledgments.}

We see a great potential to improve CCD responsivity in UV using specially designed anti-reflection coating. For future UV missions, a new special FUV anti-reflection coating should be designed.

In 2019 4x CEB FM (Camera Electronics Box Flight Model) units were successfully delivered to Russia and passed incoming inspection. The Enclosure Flight Models are expected to be delivered at the end of 2022. WUVS detector Flight Models are scheduled to be assembled and tested at LPI at the beginning of 2023.

We express our gratitude to the Teledyne e2v and RAL Space companies for the production of the custom CCD272-64, custom Enclosure and low readout noise electronics for the WSO-UV project. Additional thanks to the BINP team for the provision of the facilities for CCD characterization in UV.

\section{References}

1. M. Sachkov, B. Shustov, and A. I. Gómez de Castro, in J.-W. A. den Herder, S. Nikzad, and K. Nakazawa, eds., Space Telescopes and Instrumentation 2018: Ultraviolet to Gamma Ray, Society of Photo-Optical Instrumentation Engineers (SPIE) Conference Series, volume 10699, 106993G (2018).

2. A. Shugarov, I. Savanov, M. Sachkov, P. Jerram, et al., Ap\&SS, 354, 169, 2014. 

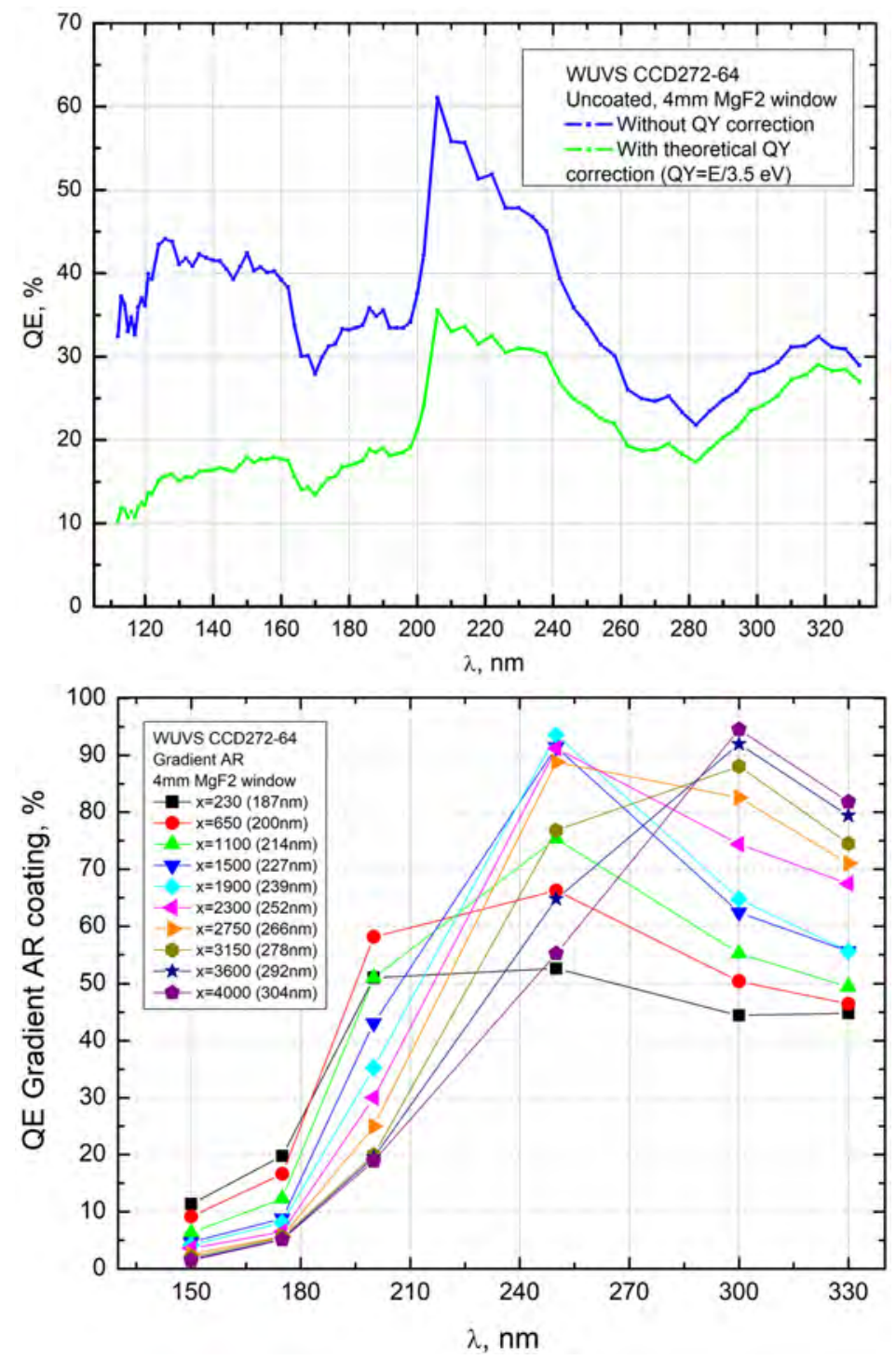

Figure 3: EQM LSS Enclosure quantum efficiency measurement results for uncoated (top) and gradient antireflection coated (bottom) regions of CCD.

3. C. Hayes-Thakore, S. Spark, P. Pool, A. Walker, M. Clapp, N. Waltham, and A. Shugarov, in R. Meynart, S. P. Neeck, and H. Shimoda, eds., Sensors, Systems, and Next-Generation Satellites XIX, Society of Photo-Optical Instrumentation Engineers (SPIE) Conference Series, volume 9639, 96390U (2015).

4. A. Nikolenko, S. Avakyan, I. Afanasyev, N. Voronin, N. Kovalenko, A. Legkodymov, V. Lyakh, and V. Pindyurin, Journal of Surface Investigation. X-ray, Synchrotron and Neutron Techniques, 6, 2012. 


\section{Astrometry and Celestial Mechanics}




\title{
Comparison of the dynamics of Jupiter's coorbital asteroids and the dynamics of bodies in debris disks
}

\author{
T. Abdulmyanov \\ abdulmyanov.tagir@yandex.ru \\ Kazan State Power Engineering University, Kazan, Russia
}

The mechanisms of fragmentation of the equatorial dust disk around young stars and the formation of planetary systems are considered. Using the model of librational motions of co-orbital asteroids of Jupiter, a model of viscous motion of gas, dust particles and small bodies in dust disks is constructed. The dynamic viscosity is obtained from the Navier-Stokes equation for three cases of characteristic flow orbits.It is shown that the librational orbits of Trojans at the early stages of the evolution of planetary systems could be transit orbits for dust particles during the formation of celestial bodies of small and large sizes and masses.

Keywords: dynamic viscosity, protoplanetary disk, debris disk, asteroids belt

DOI: 10.51194/VAK2021.2022.1.1.018

\section{Basic equations of the model of viscous dynamics of gas and dust particles in equatorial disks}

Comparison of the distributions of the polar coordinates of Jupiter's coorbital asteroids shows that larger asteroids are concentrated near the stable $L_{4}$ and $L_{5}$ Lagrange points, while smaller ones are scattered along libration orbits. Taking this into account, a model of viscous dynamics of dust particles of the disk was constructed for the initial stage of the formation of bodies in gas-dust disks. In this case, the dynamic viscosity $\eta$ characterizes the "jamming" of dust particles and forming bodies at the Lagrange points $L_{4}$ and $L_{5}$. Using the system of equations of gas dynamics, the dynamic viscosity was determined for three cases of characteristic motions under the condition of stable equilibrium of the shell of the protostar $[1,2]$.

To determine the physical foundations of the dust accumulation process at the $L_{4}$ and $L_{5}$ Lagrange points, let us consider an experiment with a round metal disk (a prototype of the accretion model). In contrast to the acoustic experiments presented in articles $[3,4,2]$, the round disk was not ideally flat, and the disturbances were central. During the experiment, the bulk of large and small stones rushed to the center of the disk and formed a circle in the center, slowly rotating counterclockwise. Simultaneously with this, in small depressions of the disk along the perimeter, the enlargement of some collections and the exhaustion of others took place. The size of the stones did not matter. The collection formation process was uneven. The course of the experiment was filmed and presented in the form of microfilm. In the accretion model, instead of a metal disk, there is a central equatorial disk of gas and dust, which is in gravitational equilibrium. Perturbations of the gravitational field form acoustic waves in the gas-dust disk, similar to those that are formed on the prototype disk. Since the basic physical (acoustic) laws for a metal disk and for a gas-dust disk are the same, the processes in them will proceed in the same way.

Let us consider a protostellar cloud with differential rotation and represent it as a union of thin axisymmetric disks, in which the motion of interstellar gas and dust occurs at the initial stage without internal friction $(\eta=0)$. As the protostar shrinks and its density changes significantly, the viscosity $\eta$ will be significant and its influence must be taken into account $(\eta \neq 0)$. The equilibrium state of the dust shell was considered in the article [1] using the well-known equations of hydrodynamics $[5,6]$ :

$$
\begin{array}{r}
\frac{\partial \rho}{\partial t}+\mathbf{V} \cdot \operatorname{grad}(\rho)=-\rho \cdot \operatorname{div} \mathbf{V} \\
\frac{\partial \mathbf{V}}{\partial t}+(\mathbf{V} \nabla) \cdot \mathbf{V}=-\frac{1}{\rho} \nabla P-\nabla \Phi-2(\mathbf{\Omega} \times \mathbf{V})+ \\
+\Omega^{2}\left(x e_{x}+y e_{y}\right)+\frac{\mathbf{N}}{\rho}, \\
P=\frac{R}{\mu} \rho T, \nabla^{2} \Phi=4 \pi g \rho .
\end{array}
$$

where $\Omega=\left(g M / R_{0}^{3}\right)^{1 / 2}, g$ is the gravitational constant, $R$ is the Boltzmann constant, $M$ is the mass of the protostar, $R_{0}$ is the radius of the protostar in local equilibrium. The density $\rho$ at the initial moment of gravitational compression of the protostellar cloud is small, and in the process of evolution it will change in accordance with changes in viscosity. Further local changes in density can be determined by solving equations (1) and (2) by numerical methods. The vector $N=\left(N_{x}, N_{y}, N_{z}\right)$ in equation (2) is determined using the well-known formulas of gas hydrodynamics [7]. The dynamic viscosity $\eta$ in the vector $\mathbf{N}$ is a function of the coordinates $x, y, z: \eta(x, y, z)$. For the case of zero viscosity $\eta$, the solution to system (1-3) was obtained as the sum $\rho=\rho_{0}+\rho_{1}, P=P_{0}+P_{1}, \Phi=$ 
$\Phi_{0}+\Phi_{1}, V=V_{0}+V_{1}$, where $\rho_{0}, P_{0}, \Phi_{0}, V_{0}$ is an unperturbed solution, $\rho_{1}, P_{1}, \Phi_{1}, V_{1}$ are small perturbations [8]. In the case of nonzero viscosity $\eta$, the equation for the density perturbations $\rho_{1}$ will have the following form [8]:

$$
\frac{\partial^{2} \rho_{1}}{\partial t^{2}}=c^{2} \nabla^{2} \rho_{1}+4 \pi g \rho_{0} \rho_{1}+2 \rho_{0} \cdot \operatorname{div}(\boldsymbol{\Omega} \times \mathbf{V})+\rho_{0} \eta \cdot \operatorname{div}\left(\frac{\mathbf{N}}{\rho}\right)
$$

where $c$ is the speed of sound. The solution of the system of equations (1-3) can be obtained by numerical methods if the dynamic viscosity $\eta$ is known. If the viscosity $\eta$ is an unknown function, then the viscosity $\eta$ can be determined from the Navier-Stokes equations under additional assumptions. For example, for thin and Keplerian disks, the viscosity $\eta$ can be determined using the Maple computer analysis system. The solution to the boundary value problem for equation (4), which takes into account the rotation of the protostellar cloud, was obtained in article $[8]$.

\section{Common elements of the equations of the dynamics of a continuous medium and the equations of the n-body problem}

In a cylindrical coordinate system, the equation (2) of viscous motion will have the following form [6]:

$$
\begin{gathered}
\frac{\partial v_{r}}{\partial t}+v_{r} \frac{\partial v_{r}}{\partial r}+v_{z} \frac{\partial v_{r}}{\partial z}-\frac{\partial v_{\varphi}^{2}}{r}=-\frac{\partial \Phi}{\partial r}-\frac{1}{\rho} \frac{\partial P}{\partial r}+N_{r} \\
\frac{\partial v_{\varphi}}{\partial t}+v_{r} \frac{\partial v_{\varphi}}{\partial r}+v_{z} \frac{\partial v_{\varphi}}{\partial z}+\frac{v_{r} v_{\varphi}}{r}=N_{\varphi} .
\end{gathered}
$$

Equations (1-3) are incomparable with the equations of the $n$ body problem even in formal terms. Despite this, it is easy to find common elements in these two different models of motion, thanks to which it becomes possible to represent mathematically and physically the entire stage of the initial formation of celestial bodies. For a physical representation of the accretion process, let us consider a prototype of the accretion model. An experiment with a prototype accretion model confirms Fesenkov's hypothesis, according to which a single star forms simultaneously with its planetary system: accretion onto the core of a protostar occurs simultaneously with accretion onto the "embryo" of the planet. An experiment with a prototype of the accretion model shows: 1) the separation of dust fragments and bodies in "debris" disks occurs in the places of the minimum of the disk potential; 2) the migration of bodies occurs in the direction from smaller minima to larger minima; 3) a nucleus of small stones is formed in the center of the disc, rotating counterclockwise. Common for equations (5), (6) and equations of the theory of perturbed motion [9] is the Keplerian part of the equations: the second, third, fourth and fifth terms of equation (5), as well as the second and third terms of equation (6). This part forms the core of equations (1-3) and the largest part in the numerical integration of equations (5-6). For this reason, in numerical integration, the influence of perturbations should be considered separately.

\section{Comparison of the dynamics of coorbital asteroids of Jupiter and the dynamics of bodies in the "debris" disks}

Fig. 1a shows the distribution of polar coordinates $\left(r, \lambda^{\prime}\right)$ of 7644 co-orbital asteroids of Jupiter with absolute magnitude $H, 7 \leq H \leq 15$ according to the TMP IAU catalog${ }^{1}$ (Epoch 20200531), and Fig. 1b shows libration orbits of small celestial bodies near orbital resonance $1 / 1$ for resonance parameters $\alpha^{2}=0.6 ; 0.8 ; 1.7 ; 2.2[8]$. The distribution of polar coordinates shows that the largest asteroids are located near the $L_{4}$ and $L_{5}$ Lagrange points, while the smaller ones are scattered along librational orbits. This feature of the dynamics of Jupiter's co-orbital asteroids is a consequence of the action of the $1 / 1$ orbital resonance mechanism, and at the early stages of evolution could be a consequence of viscous dynamics in gas-dust and "debris" disks.

The results of the experiment with the prototype of the accretion model (4) show: 1) accretion onto the core of the protostar and accretion onto the "embryo" of the planet can occur simultaneously at certain frequencies of central disturbances; 2) the prototype and the accretion model (4) have a single physical (acoustic) basis and the results of the experiment with the prototype prove the adequacy of the accretion model (4); 3) the accretion of gas and dust onto the core of the protostar occurs under the action of the force of the gravitational field of the gas-dust cloud. Accretion on the "embryo" of the planet occurs, first of all, due to the action of central disturbances of the gravitational field (density waves); 4) co-orbital asteroids of Jupiter belong to the late stage of the evolution of "debris" discs.

\footnotetext{
${ }^{1}$ https://www.iau.org
} 


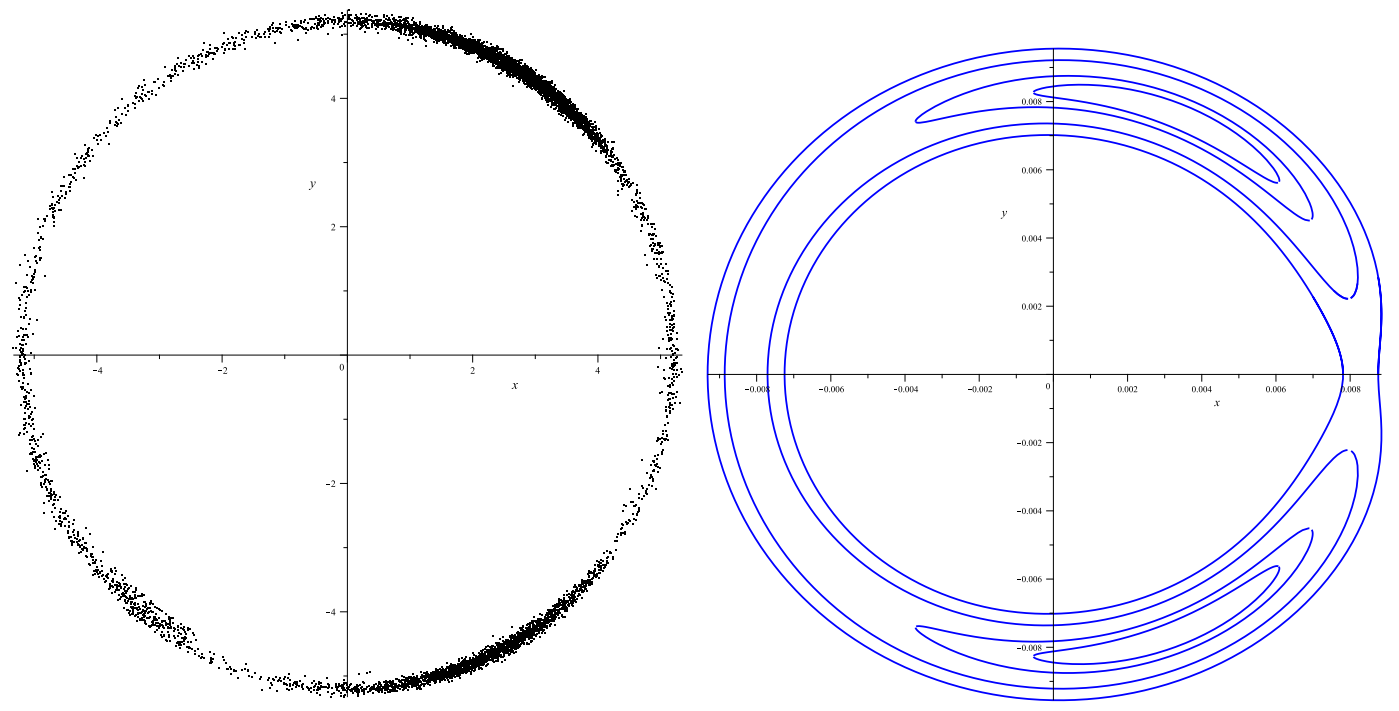

Figure 1: (a) Distribution of polar coordinates $\left(r, \lambda^{\prime}\right)$ of 7644 co-orbital asteroids of Jupiter with absolute magnitude $H, 7 \leq H \leq 15$ according to the TMP IAU catalog (Epoch 20200531); (b) librational orbits of small celestial bodies near orbital resonance $1 / 1$ for resonance parameters $\alpha^{2}=0.6 ; 0.8 ; 1.7 ; 2.2$ and orbital inclinations $i=25^{\circ}, r^{*}=$ $r-0.817$ (projections onto the equatorial plane).

\section{Conclusions}

Based on the results of numerical integration of equations (1), (5), (6), it was obtained: 1) the magnitude of disturbances from the action of acoustic (ultrasonic) waves is an order of magnitude less than their total value; 2 ) The Keplerian component is associated, first of all, with the action of the gravitational forces of the protostellar cloud and with the accretion of interstellar matter onto the core of the protostar. Perturbations that form acoustic waves of the infrasonic range form the basis of the accretion mechanism on the "embryo" of the planet; 3 ) according to the results of the experiment with the prototype of the accretion model, the main role in the formation of the "embryos" of the planets, as well as in the accretion on the "embryo" of the planet, belongs to the potential "pits" formed in the ring fragments of the equatorial disk. The form of evolution in the ring fragments of the disk and the type of librational movements of small bodies in these fragments are determined not by the type of a single resonance (orbital resonance, $\Omega$-resonance, $\omega$-resonance), but by a characteristic common for all these resonances — the depth of the potential "well". The migration of small celestial bodies occurs in the direction from zones with a shallower potential "hole" to zones with a greater depth; 4) the dynamics of bodies in "debris" disks is determined by the topology of libration orbits.

\section{References}

1. T. R. Abdulmyanov, Astrophysical Bulletin, 75, 117, 2020.

2. V. S. Korneev, Bulletin of SGUGiT. Optics, optoelectronic devices and complexes, 22, 173, 2017.

3. V. V. Meleshko and S. O. Papkov, Acoustic Bulletin, 12, 34, 2009.

4. O. A. Zhuravlev, S. Y. Komarov, and N. Y. Molevich, Mechanics and mechanical engineering, 5, $1,2003$.

5. L. D. Landau and E. M. Lifshits, Theoretical Physics. Hydrodynamics, volume VI (1986).

6. G. V. Lipunova, K. L. Malanchev, and N. I. Shakura, Accretion processes in astrophysics (in Russian) (2016).

7. B. M. Yavorskiy and A. A. Detlaf, Physics reference (in Russian) (1965).

8. T. R. Abdulmyanov, Moscow University Physics Bulletin, 74, 309, 2019.

9. G. N. Duboshin, Celestial mechanics. Basic tasks and methods (in Russian) (1975). 


\title{
On the possibility of expanding the Tisserand constant for Jupiter-family comets in a binomial series
}

\author{
T. Abdulmyanov ${ }^{1}$, M. Sokolova ${ }^{2}$, V. Usanin ${ }^{2}$ \\ abdulmyanov.tagir@yandex.ru \\ WWW home page: https://kgeu.ru/Employee/Details/18?idEmp=174 \\ ${ }^{1}$ Kazan State Power Engineering University, Kazan 420066, Russia, \\ ${ }^{2}$ Kazan (Volga region) Federal University, Kazan 420008, Russia
}

The 1st or 2nd degree binomial series expansion of the Hamiltonian of the circular restricted three-body problem expressed in terms of the Poincaré variables was used earlier to develop analytical theories of motion for the Trojan and Hilda asteroids. In the present paper we study the corresponding representation of the Tisserand constant, taking that of $6 \mathrm{P} / \mathrm{d}$ 'Arrest, a Jupiter-family comet, as a numerical example. We show that as the 6th degree series expansion, the Tisserand constant is conserved no worse than in its conventional form.

Keywords: Tisserand constant, Jupiter-family comets, 6P/d'Arrest

DOI: $10.51194 /$ VAK2021.2022.1.1.019

Developing an analytical theory of the Trojan asteroids, Garfinkel [1] wrote the Hamiltonian for the planar circular restricted problem of three bodies in a rotating heliocentric coordinate system:

$$
F=\frac{1}{2}\left(1-m_{J}\right)^{2}(G+\Gamma)^{-2}+G
$$

where $m_{J}$ is the mass parameter (assumed that for Jupiter), $G=L \sqrt{1-e^{2}}, \Gamma=L-G, L=\sqrt{\left(1-m_{J}\right) a / a_{J}}, e$ is the orbital eccentricity, $a$ is the orbital semi-major axis, and $a_{J}$ is the orbital semi-major axis of Jupiter (assumed unit of length).

Further, Zagretdinov, [2] generalized it to the spatial circular restricted three-body problem:

$$
F=\frac{1}{2}\left(1-m_{J}\right)^{2} X_{3}^{-2}\left(1+\frac{\Gamma+X_{2}}{X_{3}}\right)^{-2}+X_{3}
$$

where $X_{2}=G-H, X_{3}=H=G \cos i$, and $i$ is the orbital inclination.

Both the cited authors used the 1st degree binomial series expansions of their respective Hamiltonians:

$$
F=\frac{1}{2} G^{-2}+G-\Gamma+\Gamma\left(1-G^{-3}\right)-m_{J} G^{-2}+\frac{1}{2} m_{J}^{2}+\ldots
$$

and

$$
F=\frac{1}{2} X_{3}^{-2}+X_{3}-\Gamma-X_{2}-m_{J} X_{3}^{-2}+\ldots
$$

whereas Abdul'myanov and Zagretdinov [3] used the 2nd degree expansion of the planar Hamiltonian for the Hilda asteroids:

$$
F=\frac{1}{2 G^{2}}+G-\frac{\Gamma}{G^{3}}+\frac{3 \Gamma^{2}}{2 G^{4}}-\frac{m_{J}}{G^{2}}+\ldots
$$

In the present paper we examine the applicability of such expansions to other objects, namely, Jupiter-family comets. Evidently, higher eccentricity orbits require a higher degree expansion. In cometary astronomy, another parameter related to the total energy, the Tisserand constant, is used conventionally instead of the Hamiltonian:

$$
C=\frac{a_{J}}{a}+2 \sqrt{\frac{a\left(1-e^{2}\right)}{a_{J}}} \cos i .
$$

It is obvious that if $m_{J} \ll 1\left(1-m_{J} \approx 1\right.$ and $\left.L \approx \sqrt{a / a_{J}}\right)$, then

$$
C \approx X_{3}^{-2}\left(1+\frac{\Gamma+X_{2}}{X_{3}}\right)^{-2}+2 X_{3} \approx 2 F,
$$

and so for all $N$, we write the $N$ th degree binomial series expansion

$$
C_{N} \approx X_{3}^{-2}\left(\sum_{n=0}^{N}(n+1)\left(-\frac{\Gamma+X_{2}}{X_{3}}\right)^{n}\right)+2 X_{3} .
$$

$6 \mathrm{P} / \mathrm{d}$ 'Arrest is a usual Jupiter-family comet with a long observational history and well determined orbital elements and nongravitational parameters. Using HORIZONS Web-Interface [4], we generated heliocentric orbital 
Table 1: The statistical measures of the variation of $C_{1}, \ldots, C_{7}, C$, and of the orbital elements used in the computations

\begin{tabular}{lrrr}
\hline Parameter & Mean & Standard deviation & Relative standard deviation, $\%$ \\
\hline$a, \mathrm{AU}$ & 3.525 & 0.077 & 2.17 \\
$e$ & 0.641 & 0.021 & 3.31 \\
$i,^{\circ}$ & 12.641 & 5.497 & 43.48 \\
$C$ & 2.703 & 0.006 & 0.20 \\
$C_{1}$ & 2.056 & 0.121 & 5.90 \\
$C_{2}$ & 3.010 & 0.077 & 2.54 \\
$C_{3}$ & 2.567 & 0.051 & 1.99 \\
$C_{4}$ & 2.761 & 0.022 & 0.79 \\
$C_{5}$ & 2.679 & 0.017 & 0.64 \\
$C_{6}$ & 2.713 & 0.005 & 0.17 \\
$C_{7}$ & 2.699 & 0.008 & 0.29 \\
\hline
\end{tabular}

elements of $6 \mathrm{P} / \mathrm{d}$ 'Arrest at 40-day steps over the interval from July 3, 1678 to June 27, 2015. From this sample of the elements, we computed $C_{1}, \ldots, C_{7}$, and $C$. Fig. 1 shows the variation of $C_{1}, C_{2}, C_{6}$, and $C$ with time. Table 1 gives the statistical measures of the variation of $C_{1}, \ldots, C_{7}, C$, and for comparison also of the orbital elements used in the computations. In Fig. 2 we see the convergence of the sequence of the mean values of $C_{N}$ to the mean value of $C$ and the decrease of the sequence of the relative standard deviations (or the increase of the constancy) of $C_{N}$. The latter proceeds with fluctuations, so, ironically, the standard deviation of $C_{6}$ is less than that not only of $C_{7}$ but even of $C$ itself. Note that the presented expansions have no advantage over the conventional Tisserand constant in numerical calculations, they are intended to be a tool for developing analytical theories, and we performed our calculations only as a test example.

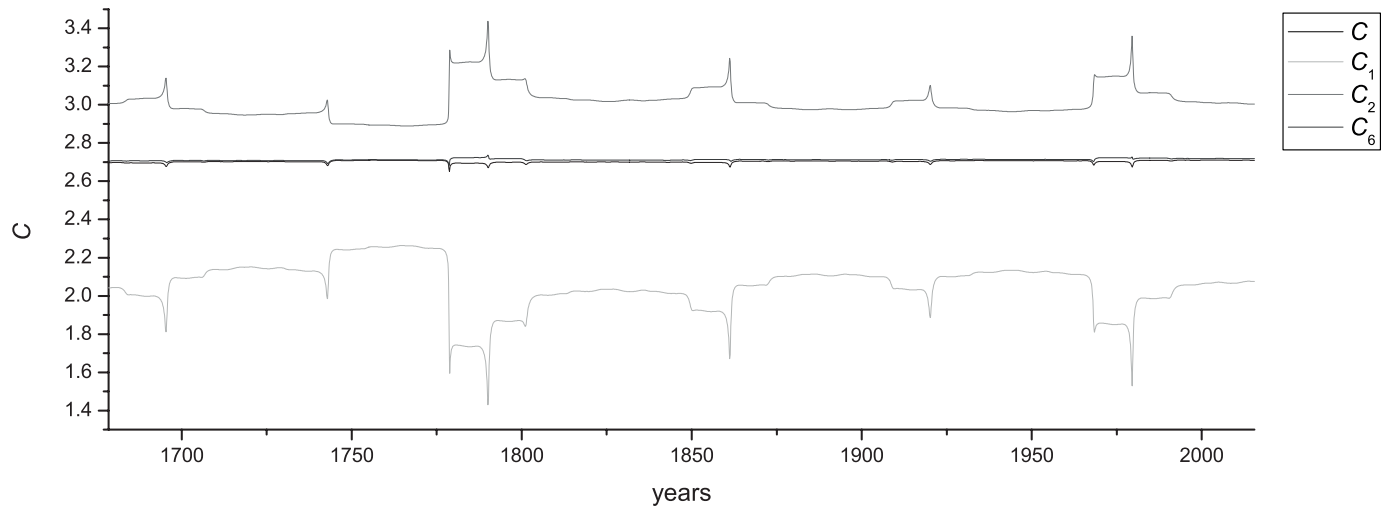

Figure 1: The variation of $C_{1}, C_{2}, C_{6}$, and $C$ with time
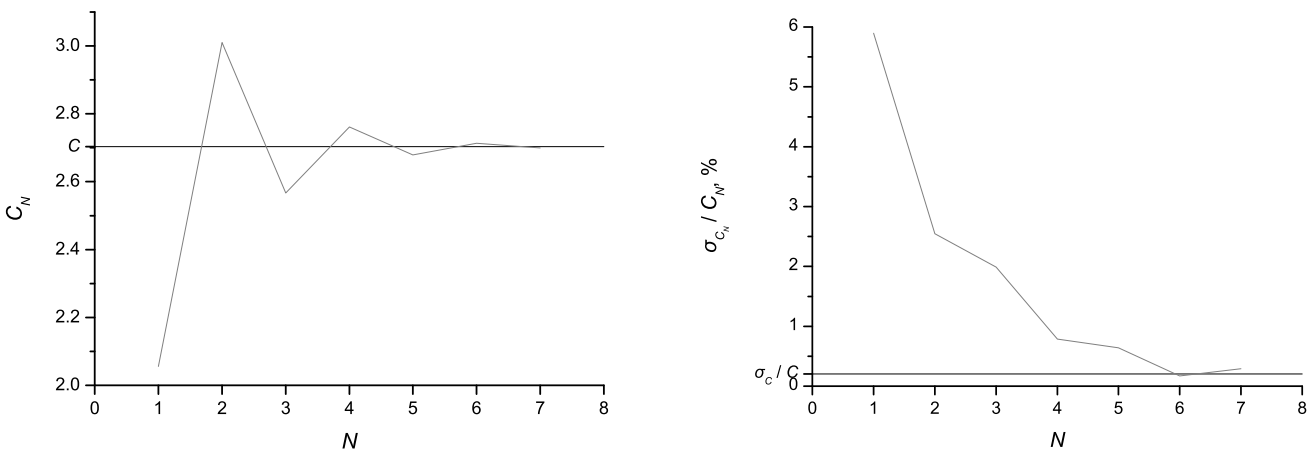

Figure 2: The sequences of the mean values (left) and of the relative standard deviations $\left(\right.$ right) of $C_{N}$ 
When the disturbing function is added to the Hamiltonian, the corresponding Tisserand constant will become more accurate, which will allow a study of orbital motion in the regime of libration.

\section{References}

1. B. Garfinkel, AJ, 82, 368, 1977.

2. R. V. Zagretdinov, Kinematics and Physics of Celestial Bodies, 2(3), 77, 1986.

3. T. R. Abdul'myanov and R. V. Zagretdinov, Kinematics and Physics of Celestial Bodies, 11(4), $26,1995$.

4. J. D. Giorgini, D. K. Yeomans, A. B. Chamberlin, P. W. Chodas, et al., Bull Amer. Astron. Soc., 28, 1158, 1996. 


\section{Libration points of uniformly rotating celestial bodies with cavities}

A. Burov ${ }^{1,2}$, V. Nikonov ${ }^{1,2}$

jtm@narod.ru; nikon_v@list.ru

${ }^{1}$ FRC CSC RAS, Vavilova st. 40, Moscow, 119333, Russia,

${ }^{2}$ HSE University, Myasnitskaya st. 20, Moscow, 101000, Russia

The problem of the existence and stability of libration points of a homogeneous gravitating uniformly rotating ball with a spherical cavity is considered. The existence and stability of relative equilibria (libration points) located outside the body are investigated. Bifurcation diagrams and areas of possible motion are constructed.

Keywords: celestial bodies with cavities, libration points, relative equilibria, motion in a non-central gravitational field, generalized gravitating dumbbell

DOI: 10.51194/VAK2021.2022.1.1.020

\section{Introduction}

The study of libration points of uniformly rotating celestial bodies probably dates back to publications $[1,2,3$, $4,5,6,7,8,9,10]$. A special class consists of the works of V.V. Beletsky and A.V. Rodnikov, in which libration points in the vicinity of a precessing dumbbell-like body formed by two balls are systematically studied. In this paper, attention is focused on the case when there is a spherical cavity in a spherical homogeneous body. The study of such a case is a natural continuation of the research undertaken in $[11,12,13,14,15,16]$. It is instructive that spherical cavities have recently been discovered in astrophysics $[17,18]$.

\section{Problem statement and basic notation}

Consider a rigid body $\mathcal{A}$ obtained from a homogeneous ball $\mathcal{B}$ with the center $B$ of radius $R_{B}$ by removing the contents of a spherical cavity $\mathcal{C}$ with the center $C$ of radius $R_{C}: \mathcal{A}=\mathcal{B} \backslash \mathcal{C}$. Suppose that the body $\mathcal{A}$ rotates with a constant angular velocity $\omega$ around an axis perpendicular to the axis $B C$ and passing through the center of mass $O$ of the body $\mathcal{A}$. Let the material point $P$ move in the gravitational field of the body $\mathcal{A}$. It is assumed that the particle does not affect the motion of the body.

Let $O x_{1} x_{2} x_{3}$ be the right reference frame, rotating together with the body. The axis $O x_{3}$ coincides with the axis of rotation, the axis $O x_{1}$ is directed along the axis of symmetry of the body, and the axis $O x_{2}$ complements these two axes to the right triple. The unit vectors of the frame $O x_{1} x_{2} x_{3}$ are denoted by $\mathbf{e}_{1}, \mathbf{e}_{2}$ and $\mathbf{e}_{3}$, respectively. The problem is to study the equilibrium of the point $P$ with respect to the frame $O x_{1} x_{2} x_{3}$ (see Fig. 1 on the left).
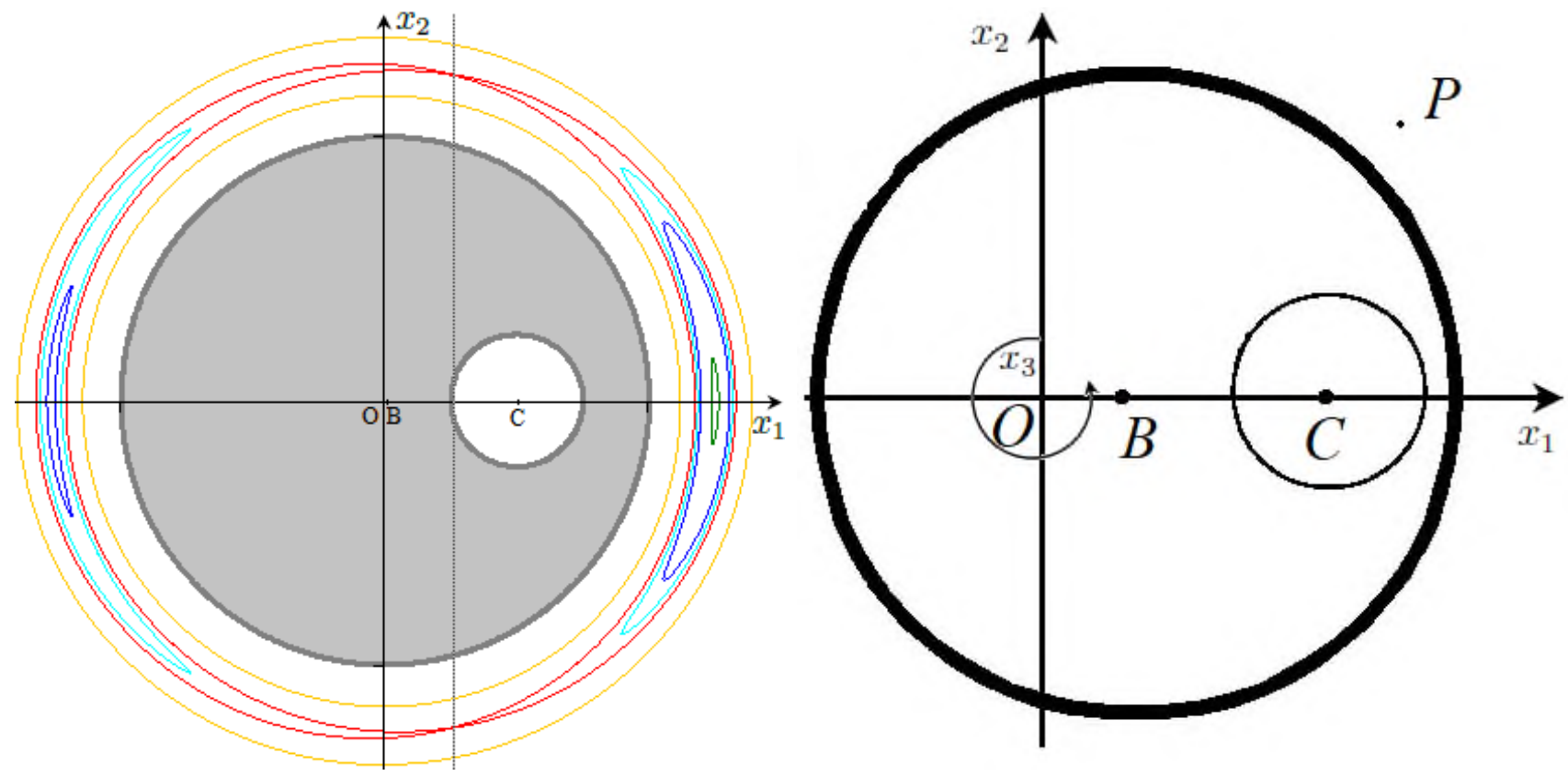

Figure 1 
Let the center of the cavity $C$ be located on the positive semi-axis $O x_{1}$, and the cavity $\mathcal{C}$ does not extend beyond the outer surface of the body. If $|B C|=d, \mu=m_{C} / m_{B}<1, b=\mu d /(1-\mu)$ and $c=d /(1-\mu)$, then

$$
\overrightarrow{O P}=\left(x_{1}, x_{2}, x_{3}\right)^{T}, \overrightarrow{O B}=(b, 0,0)^{T}, \overrightarrow{O C}=(c, 0,0)^{T}
$$

If $G$ is the constant of gravity, $\rho$ is the density of the body $\mathcal{A}$, then outside the body the intensity $\mathbf{g}_{A}$ of the field of attraction and its potential $U_{A}$ read

$$
\begin{gathered}
\mathbf{g}_{A}=-G\left(\frac{m_{B}}{r_{B}^{3}} \overrightarrow{B P}-\frac{m_{C}}{r_{C}^{3}} \overrightarrow{C P}\right), \quad U=G\left(-\frac{m_{B}}{r_{B}}+\frac{m_{C}}{r_{C}}\right) \\
r_{B}=|\overrightarrow{B P}|=\left(\left(x_{1}-b\right)^{2}+x_{2}^{2}+x_{3}^{2}\right)^{1 / 2}, \quad r_{C}=|\overrightarrow{C P}|=\left(\left(x_{1}-c\right)^{2}+x_{2}^{2}+x_{3}^{2}\right)^{1 / 2} .
\end{gathered}
$$

\section{The existence and stability of libration points}

According to [19, 20, 21, 22], critical points of the augmented potential are studied to determine relative equilibria. This potential reads

$$
W=U_{c}+U, \quad U_{c}=-\frac{\omega^{2}}{2}\left(x_{1}^{2}+x_{2}^{2}\right)
$$

where $U_{c}$ is the potential of centrifugal forces.

Introduce the parameter $\Omega \geq 0: \Omega^{2}=\omega^{2} /\left(G m_{B}\right)$. If $\Phi=r_{B}^{-3}-\mu r_{C}^{-3}, \Psi=b r_{B}^{-3}-\mu c r_{C}^{-3}$, then the equations of the critical points of the function (1) read

$$
0=\left(\Phi-\Omega^{2}\right) x_{1}-\Psi, \quad 0=\left(\Phi-\Omega^{2}\right) x_{2}, \quad 0=\Phi x_{3} .
$$

As it turned out, there are two families of libration points located at the equatorial plane in the non-degenerate case when $d \neq 0$

$$
\begin{gathered}
\Gamma_{1}: \quad x_{2}=x_{3}=0, \quad \Omega=\left(\frac{1}{x_{1}}\left(\frac{x_{1}-b}{\left|x_{1}-b\right|^{3}}-\mu \frac{x_{1}-c}{\left|x_{1}-c\right|^{3}}\right)\right)^{1 / 2} . \\
\Gamma_{2}: \quad x_{1}=\frac{b+c}{2}, \quad x_{3}=0 \\
\Omega=\left(\frac{8}{(b+c)^{2}+4 x_{2}^{2}}\left(\frac{c^{2}-b^{2}+4 x_{2}^{2}}{\left((c-b)^{2}+4 x_{2}^{2}\right)^{3 / 2}}-\mu \frac{b^{2}-c^{2}+4 x_{2}^{2}}{\left((b-c)^{2}+4 x_{2}^{2}\right)^{3 / 2}}\right)\right)^{1 / 2} .
\end{gathered}
$$

Calculations show that the degree of instability for libration points from $\Gamma_{1}$ is $\chi=2$. At the same time, the degree of instability for libration points from $\Gamma_{2}$ is $\chi=1$. The sections of the surfaces of the level of the augmented potential with the plane $x_{3}=0$ are depicted in the Fig. 1 on the right.

It is noteworthy that the observed distribution of maximum points and saddle points is opposite to the known one for a conventional dumbbell with positive masses.

\section{References}

1. V. K. Abalakin, Bullet. Inst. Theor. Astron. (in Russian), 6, 543-549, 1957.

2. Y. V. Batrakov, Bullet. Inst. Theor. Astron. (in Russian), 6, 524-542, 1957.

3. S. G. Zhuravlev, In: Collect. Sci. Work. Grad. Stud. RUDN Univ. (in Russian), 169-183, 1968.

4. S. G. Zhuravlev, Celestial Mechanics, 6, 255-267, 1972.

5. S. G. Zhuravlev, Celestial Mechanics, 8, 75-84, 1973.

6. S. G. Zhuravlev, Soviet Astronomy, 18, 792-794, 1975.

7. I. I. Kosenko, Journal of Applied Mathematics and Mechanics, 45, 18-23, 1981.

8. I. I. Kosenko, Kosmicheskie Issledovaniya, 19, 200-209, 1981.

9. I. I. Kosenko, Journal of Applied Mathematics and Mechanics, 49, 17-24, 1985.

10. I. I. Kosenko, Journal of Applied Mathematics and Mechanics, 51, 1-5, 1987.

11. V. V. Beletsky, Cosmic Research, 45, 408-416, 2007.

12. V. V. Beletsky and A. V. Rodnikov, Journal of Vibroengeneering (JVE), 10, 550-556, 2008.

13. V. V. Beletsky and A. V. Rodnikov, Cosmic Research, 46, 40-48, 2008.

14. V. V. Beletsky and A. V. Rodnikov, Rus. J. Nonlin. Dyn., 7, 569-576, 2011.

15. A. V. Rodnikov, Rus. J. Nonlin. Dyn., 9, 697-710, 2013.

16. A. V. Rodnikov, Rus. J. Nonlin. Dyn., 10, 213-222, 2014.

17. S. Bialy, C. Zucker, A. Goodman, M. M. Foley, et al., ApJL, 919, L5, 2021.

18. C. Zucker, A. Goodman, J. Alves, S. Bialy, et al., ApJ, 919, 35, 2021. 
19. E. J. Routh, Treatise on the Stability of a Given State of Motion (Cambridge: Cambridge University press) (1877).

20. E. J. Routh, The advanced part of a treatise on the dynamics of a system of rigid bodies (L.: McMillan) (1884). 21. A. V. Karapetyan, Stability of steady motions (Moscow: Editorial URSS (in Russian)) (1998).

22. V. N. Rubanovsky and V. A. Samsonov, Stability of Stationary Motions in Examples and Problems (Moscow: Nauka (in Russian)) (1988). 


\section{Generating function of Euler-Poinsot tensor's components for small celestial bodies}

A. Burov ${ }^{1,2}$, E. Nikonova ${ }^{1,2}$

jtm@narod.ru; nikonova.ekaterina.a@gmail.com

${ }^{1}$ FRC CSC RAS, Vavilova st. 40, Moscow, 119333, Russia,

${ }^{2}$ HSE University, Myasnitskaya st. 20, Moscow, 101000, Russia

The structure of the generating function of a polyhedron with triangular faces approximating the surface of a small celestial body is discussed. The derived formulas are used to calculate the components of the Euler-Poinsot tensor up to the fourth order for the asteroid (486958) Arrokoth.

Keywords: Euler-Poinsot tensor, moment of inertia of arbitrary order, generating function, inertial characteristics of asteroids, (486958) Arrokoth

DOI: $10.51194 /$ VAK2021.2022.1.1.021

\section{The generating function of a homogeneous polyhedron}

A homogeneous polyhedral body $\mathcal{B}$ is considered. It is assumed that the surface of the body consists of triangles forming a triangulation grid. This can always be achieved by appropriate triangulation of faces other than triangular ones. Such a grid generates a set $\mathcal{T}$ of oriented tetrahedra with a common vertex at some point $O$, e.g., at the center of mass, and bases coinciding the faces of the triangulation grid.

Let $T$ be a tetrahedron whose edges coming from the vertex $O$ are given by the vectors $\mathbf{a}, \mathbf{b}$, and $\mathbf{c}$. Introduce the matrix

$$
\mathcal{R}(\mathbf{a}, \mathbf{b}, \mathbf{c})=\left(\begin{array}{lll}
a_{1} & b_{1} & c_{1} \\
a_{2} & b_{2} & c_{2} \\
a_{3} & b_{3} & c_{3}
\end{array}\right)
$$

The columns of this matrix contain the coordinates of the vectors $\mathbf{a}, \mathbf{b}$, and $\mathbf{c}$ relative to the axes $O x_{1} x_{2} x_{3}$. The oriented volume of such a tetrahedron is $\operatorname{Vol}=\operatorname{det} \mathcal{R}(\mathbf{a}, \mathbf{b}, \mathbf{c})$. It turns out that for the tetrahedron under consideration, the generating function takes the form

$$
F(\mathbf{t})=V o l \cdot\left(-\frac{1}{(\mathbf{a}, \mathbf{t})(\mathbf{b}, \mathbf{t})(\mathbf{c}, \mathbf{t})}+\sum_{(\mathbf{a}, \mathbf{b}, \mathbf{c})} \frac{e^{(\mathbf{a}, \mathbf{t})}}{(\mathbf{a}, \mathbf{t})(\mathbf{a}-\mathbf{b}, \mathbf{t})(\mathbf{a}-\mathbf{c}, \mathbf{t})}\right) .
$$

Here the entry $(\mathbf{a}, \mathbf{b}, \mathbf{c})$ in the summation sign means a cyclic permutation of $\mathbf{a}, \mathbf{b}$ and $\mathbf{c}:(\mathbf{a}, \mathbf{b}, \mathbf{c}) \rightarrow(\mathbf{b}, \mathbf{c}, \mathbf{a}) \rightarrow$ $(\mathbf{a}, \mathbf{b}, \mathbf{c})$. The proof is carried out by direct calculation (cf. [1]).

Since the body $\mathcal{B}$ consists of tetrahedra $\mathcal{T}$ with a common vertex, it suffices to calculate the generating function for all of these tetrahedra $\mathcal{T}$, and then find the sum of the found functions.

\section{Inertial characteristics of the asteroid (486958) Arrokoth}

After the New Horizons interplanetary station performed a close flyby of the contact binary object (486958) Arrokoth belonging to the Kuiper Belt, it became possible to calculate its inertial characteristics. According to [2], the surface of the (486958) Arrokoth can be approximated by a polyhedron generated by 1046 vertices and 1952 triangular faces given in some coordinate system $O x_{1} x_{2} x_{3}$.

Using the generating function of the components of the Euler-Poinsot tensor calculated according to the rule presented above, first of all, the volume and position of the asteroid's center of mass $Z$ were calculated (c.f. [3]):

$$
V=\mathbf{I}_{0} / \rho=2427.722 \mathrm{~km}^{3}, \quad \overrightarrow{O Z}=\mathbf{I}_{1} /(\rho V)=(-1.709,0.125,-0.094)^{T} \mathrm{~km} .
$$

In the axes $Z x_{1} x_{2} x_{3}$, the components of the tensor $\mathbf{I}_{2}$ divided by the mass of the asteroid read (in $\left.\mathrm{km}^{2}\right)$

$$
\mathbf{I}_{2} /(\rho V)=\left(\begin{array}{rrr}
86.539 & 0.817 & 1.458 \\
0.817 & 15.116 & -0.158 \\
1.458 & -0.158 & 3.199
\end{array}\right)
$$

The unit eigenvectors of this tensor in the same axes read

$$
\mathbf{e}_{1}=\left(\begin{array}{r}
0.99978 \\
-0.01114 \\
-0.01762
\end{array}\right), \quad \mathbf{e}_{2}=\left(\begin{array}{l}
0.01139 \\
0.99983 \\
0.01442
\end{array}\right), \quad \mathbf{e}_{3}=\left(\begin{array}{r}
0.01746 \\
-0.01462 \\
0.99974
\end{array}\right)
$$


These vectors compose the frame $Z \xi_{1} \xi_{2} \xi_{3}$.

The principal central moments of inertia, divided by the mass of the asteroid, are as follows (c.f. [3]):

$$
J_{1} /(\rho V)=18.280, \quad J_{2} /(\rho V)=89.745, \quad J_{3} /(\rho V)=101.683 \quad\left(\text { in } \mathrm{km}^{2}\right) .
$$

The components of the tensors $\mathbf{I}_{3} /(\rho V)$ and $\mathbf{I}_{4} /(\rho V)$, written out in the axes $Z \xi_{1} \xi_{2} \xi_{3}$, are collected in the tables 1 and 2 .

Table 1: The components of the tensor $\mathbf{I}_{3} /(\rho V) \mathrm{km}^{3}$.

\begin{tabular}{|l|l|l|l|l|}
\hline$I_{300}=-40.326$ & $I_{021}=-0.089$ & $I_{012}=0.696$ & $I_{030}=0.166$ & $I_{102}=-9.878$ \\
\hline$I_{111}=-1.866$ & $I_{003}=0.159$ & $I_{201}=-1.340$ & $I_{210}=-1.797$ & $I_{120}=41.742$ \\
\hline
\end{tabular}

Table 2: The components of the tensor $\mathbf{I}_{4} /(\rho V) \mathrm{km}^{4}$.

\begin{tabular}{|c|c|c|c|c|}
\hline$I_{400}=12641.189$ & $I_{103}=-2.275$ & $I_{022}=30.435$ & $I_{040}=534.617$ & $I_{301}=-27.041$ \\
\hline$I_{202}=266.304$ & $I_{004}=25.269$ & $I_{130}=30.887$ & $I_{211}=-25.161$ & $I_{310}=-44.633$ \\
\hline$I_{013}=0.832$ & $I_{121}=6.822$ & $I_{031}=2.264$ & $I_{220}=1205.302$ & $I_{112}=-8.029$ \\
\hline
\end{tabular}

\section{Remark}

The gravitational potential of the asteroid Arrokoth was calculated in [3] using the modified version of the MinorGravity package ${ }^{1}$ by Dimitrios Tsoulis, see [4]. The obtained tool was used to calculate surface accelerations, slopes and escape velocity. With its help, in particular, the stability properties of relative equilibrium positions on the asteroid surface were studied. In addition, in the cited publication the existence and stability of the external libration points of the Arrokoth were investigated.

An analytical approach to calculating the potential for homogeneous polyhedra has been proposed in publications $[5,6,7,8]$.

\section{References}

1. A. A. Burov and E. A. Nikonova, Doklady Physics, 66, 139-142, 2021.

2. S. A. Stern and et. al., Science, 364, eaaw9771, 2019.

3. A. Amarante and O. C. Winter, Monthly Notices of the Royal Astronomical Society, 496, 4154-4173, 2020.

4. D. Tsoulis, Geophysics, 77, F1, 2012.

5. R. A. Werner, Celestial Mechanics and Dynamical Astronomy, 59, 253-278, 1994.

6. R. A. Werner and D. J. Scheeres, Celestial Mechanics and Dynamical Astronomy, 65, 313-344, 1996.

7. R. A. Werner, Computers \& Geosciences, 23, 1071-1077, 1997.

8. R. A. Werner, Journal of Geodesy, 91, 307-328, 2017.

\footnotetext{
${ }^{1}$ https://github.com/a-amarante/minor-gravity
} 


\section{Non-gravitational effects in the orbital motion of space debris objects}

I. Chuvashov ${ }^{1}$, P. Levkina ${ }^{2}$

ayvazovskaya@inasan.ru

${ }^{1}$ Tomsk State University, 36 Lenin Ave., Tomsk, Russia,

${ }^{2}$ Institute of Astronomy of the Russian Academy of Sciences, 48 Pyatnitskaya str., Moscow, Russia

In this work, orbital information of selected space debris objects with a high area-to-mass ratio was obtained and their dynamic evolution was researched for 20 years ahead. Improvement of the objects' orbits was carried out using a highprecision numerical model of motion, including the forces of light pressure, and positional observations obtained with the Zeiss-2000 telescope complex of the Terskol branch of INASAN. It is shown that such objects change their orbital parameters over time, become unstable and begin to pose a threat to operating objects.

The research was carried out within the state assignment of Ministry of Science and Higher Education of the Russian Federation (theme No. 0721-2020-0049).

Keywords: space debris, high area-to-mass ratio, orbital dynamics

DOI: 10.51194 /VAK2021.2022.1.1.022

\section{Numerical model of satellite motion}

The developed high-precision numerical model of the satellite motion takes into account the following perturbations: perturbations associated with the non-sphericity of the Earth (EGM2008 model); secular changes in harmonic coefficients $C_{20}, C_{30}, C_{40}$; disturbances from tidal deformations of the central body, oceanic and atmospheric tides, oceanic and solid pole tides; lunar-solar disturbances; light pressure disturbances and the influence of the Poynting-Robertson effect; relativistic effects. Accounting for these disturbing forces is recommended by the latest IERS agreement [1].

The main problem in modeling the effect of radiation pressure for space debris objects is associated with calculating the value of $A / m$, which depends in a complex way on their shape, properties of external coverings and orientation [2].

\section{Numerical model of satellite motion}

\subsection{Processing observations}

To determine the orbital parameters of the motion of space debris objects, five objects of the geostationary zone were selected (Table 1). These objects, except for 90073, have a large windage coefficient (more than 2), which indicates the complex nature of the motion of these objects, and an observation arc of about 6 months, which corresponds to about 180 revolutions around the Earth. Unlike other selected objects, object 90073 has the longest observation arc (over 10 years), but a lower windage factor. However, due to the large number of revolutions around the Earth, light pressure has a significant effect on the motion of this object. It should be noted that the observations of objects 90073 and 95608 were obtained at two observation points (Terskol peak and Sanglokh).

Table 1: Observational data of the researched objects of space debris

\begin{tabular}{lllll}
\hline Object & Number of observations & Number of stations & Observation arc & Pre-value $A / m$ \\
\hline $\mathbf{9 0 0 7 3}$ & 14219 & 2 & 10 years & 0.12 \\
$\mathbf{9 0 1 4 4}$ & 58 & 1 & 4 months & 2.13 \\
$\mathbf{9 0 4 0 4}$ & 206 & 1 & 6 months & 21.0 \\
$\mathbf{9 1 4 5 3}$ & 401 & 1 & 6 months & 13.3 \\
$\mathbf{9 5 6 0 8}$ & 201 & 2 & 6 months & 26.0 \\
\hline
\end{tabular}

Using a numerical model of the satellite motion, the orbital motion parameters were obtained for these objects (Table 2). Table 2 shows that the root mean square error of observations for object 90073 was 8754.78 arc seconds. This indicates that it is not expedient to determine the parameters of motion along such a long observational arc, even for an object with a small windage coefficient. It is better to divide long arcs into shorter ones. For other objects, the root mean square error of observations did not exceed 331 arc seconds. Also, the windage coefficient was obtained from the observations. For object 90404 , it has a fairly large value, about $29 \mathrm{~m}^{2} / \mathrm{kg}$, which explains such an anomalously overestimated error in numerical modeling. Table 2 also shows the condition number, which can be used to estimate how accurately the parameters of the motion of an object are determined.

It is known that with such values of the windage coefficient, objects will rapidly change their orbital parameters. The graphs below (Fig. 1-2) show the evolution of the orbit of the most interesting objects 90404 and 91453 for 20 years ahead. 
Table 2: Determination of the parameters of the orbit of the objects under study

\begin{tabular}{lllllll}
\hline Object & $a, \mathrm{~km}$ & $e$ & $i$, degrees & RMS error, arcsec & Condition number & $A / m, \mathrm{~m}^{2} / \mathrm{kg}$ \\
\hline $\mathbf{9 0 0 7 3}$ & 42172.654 & 0.00726 & 4.8984 & 8754.780 & $10^{6}$ & 0.12 \\
$\mathbf{9 0 1 4 4}$ & 44694.415 & 0.11779 & 15.8335 & 4.243 & $10^{9}$ & 2.9 \\
$\mathbf{9 0 4 0 4}$ & 39890.509 & 0.33492 & 22.2060 & 331.285 & $10^{9}$ & 29.0 \\
$\mathbf{9 1 4 5 3}$ & 42052.084 & 0.27333 & 10.3785 & 125.484 & $10^{9}$ & 18.7 \\
$\mathbf{9 5 6 0 8}$ & 32654.350 & 0.18114 & 2.6859 & 74.899 & $10^{9}$ & 17.6 \\
\hline
\end{tabular}

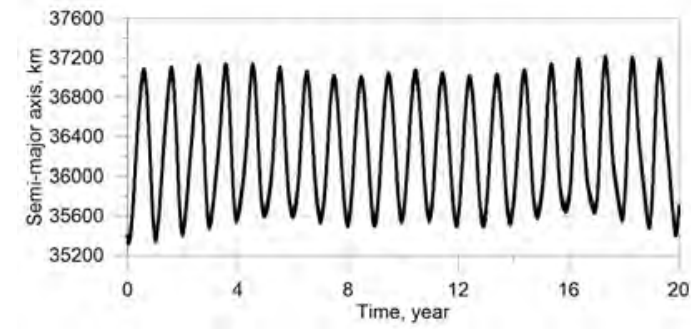

a)

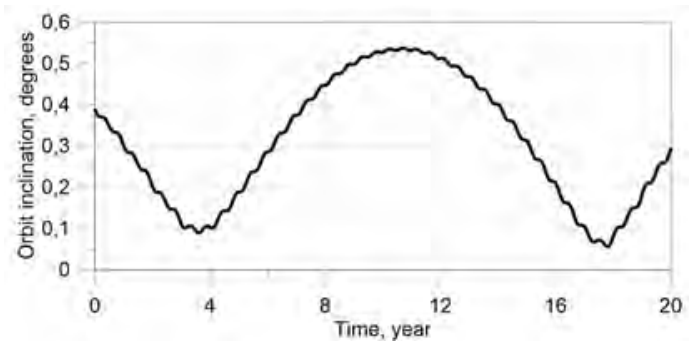

c)

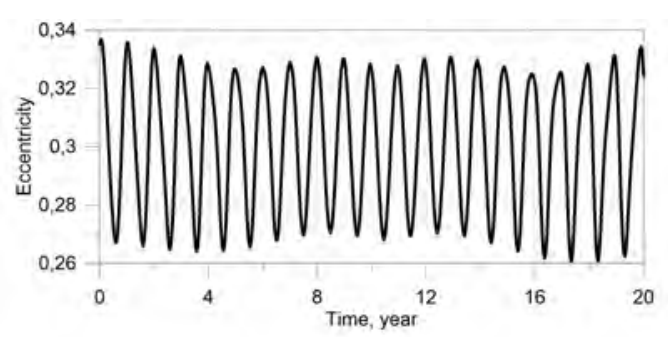

b)

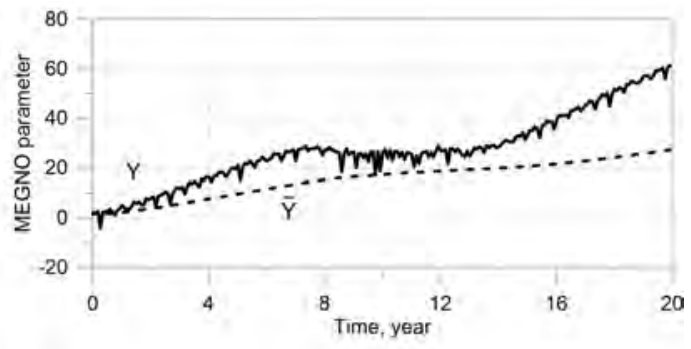

d)

Figure 1: Changes in the semi-major axis (a), eccentricity (b), orbital inclination (c) and the MEGNO parameter (d) for object 90404 over a time interval of 20 years.

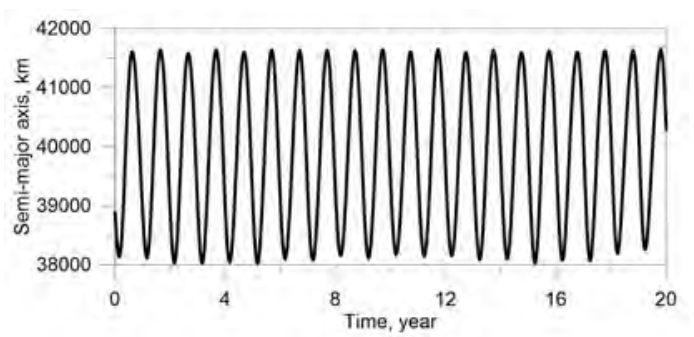

a)

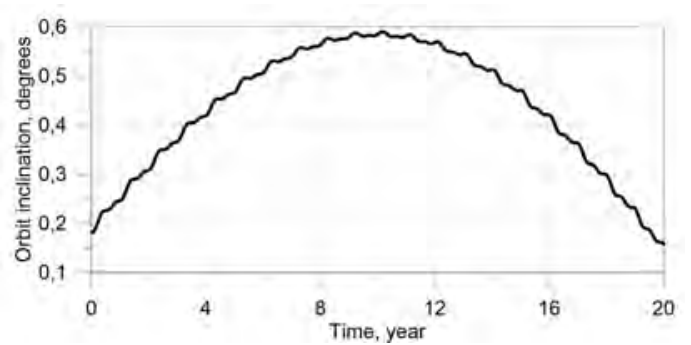

c)

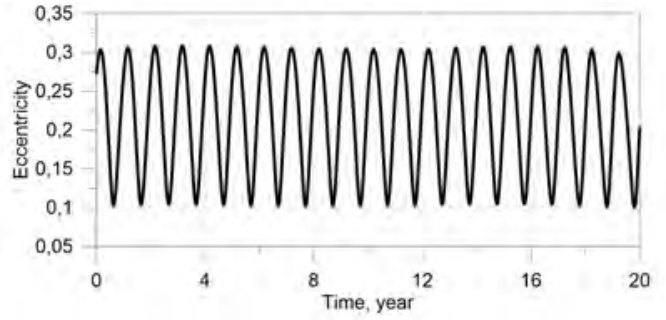

b)

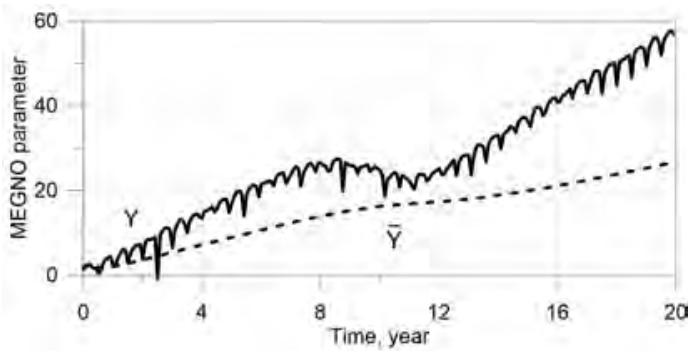

d)

Figure 2: Changes in the semi-major axis (a), eccentricity (b), orbital inclination (c) and the MEGNO parameter (d) for object 91453 over a time interval of 20 years.

It can be seen from the graphs that the orbit of these objects changes a lot under the influence of light pressure. For example, the eccentricity value changes by 0.2 values and has an oscillatory character. Consequently, 


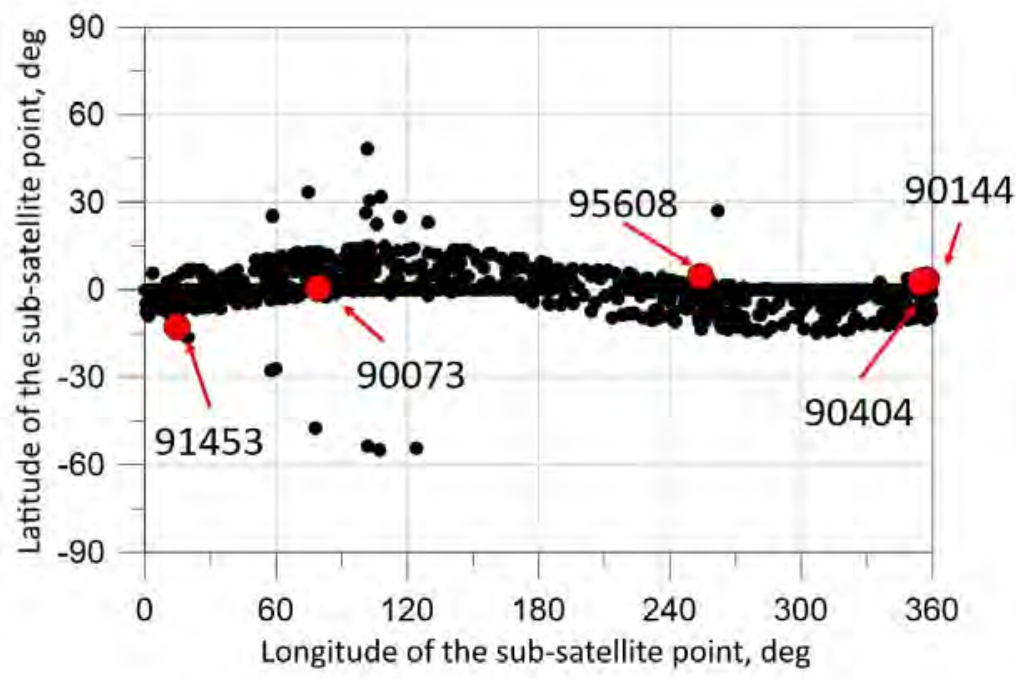

Figure 3: The position of the researched objects of space debris in the geostationary area.

Table 3: Approaches of object 90404 with objects of the geostationary area

\begin{tabular}{lllll}
\hline & Approach date & Approach object 1 & Approach object 2 & Minimum distance, $\mathrm{km}$ \\
\hline 1 & $2021.09 .16 ~ 13: 21: 03$ & 90144 & INTELSAT 2-F3 & 48.54386 \\
2 & 2021.10 .18 12:48:45 & 90144 & RADUGA 1M-1 & 99.20089 \\
3 & $2021.10 .1812: 49: 38$ & 90144 & THURAYA 3 & 97.03386 \\
4 & $2021.10 .1812: 50: 16$ & 90144 & HYLAS 2 & 64.78408 \\
5 & $2021.10 .2202: 06: 48$ & 90144 & SES 5 & 71.71232 \\
6 & $2021.10 .2904: 47: 00$ & 90144 & BEIDOU 3 & 93.64436 \\
7 & $2021.11 .0507: 15: 21$ & 90144 & EUTE 33E & 43.46432 \\
8 & $2021.11 .0820: 45: 38$ & 90144 & ARABSAT 2A & 67.18033 \\
9 & $2021.11 .1209: 58: 44$ & 90144 & ABS 6 (LMI 1) & 83.53211 \\
10 & $2022.01 .1511: 36: 26$ & 90144 & INTELSAT 903 & 71.81612 \\
11 & $2022.01 .2604: 31: 21$ & 90144 & AEHF 3 (USA 246) & 94.45914 \\
12 & $2022.01 .2917: 59: 53$ & 90144 & ASIASTAR & 91.11328 \\
13 & $2022.04 .0708: 12: 38$ & 90144 & ASTRA 1F & 82.13034 \\
14 & $2022.04 .2113: 41: 03$ & 90144 & COSMOS 2054 & 48.92043 \\
15 & $2022.08 .3023: 44: 25$ & 90144 & USA 113 & 79.73023 \\
\hline
\end{tabular}

such objects not only cross the geostationary zone, but can also pose a danger to satellites in the navigation zone. The MEGNO parameter shows that these objects are unstable and have a chaotic nature of motion.

\subsection{Assessment of the probability of collision}

In addition to studying the orbital evolution of these objects, the results of their approaches (Table 3) with operating or spent objects of the geostationary zone were obtained for one year from August 2021 to August 2022.

In Fig. 3 black dots show 970 objects of the geostationary zone (https://www.space-track.org), red circles show the positions of the researched objects of space debris.

To determine the mutual distances of the two orbits, a third-order Lagrange polynomial was constructed, the derivative of this polynomial was calculated, and the time and distance of the closest approach were determined. If the distance between the objects under study was less than $100 \mathrm{~km}$, this event was recorded: the moment in time, the minimum distance and the names of the objects.

In one year, 15 approaches were recorded. At the same time, only one object under study, 90144, approached other objects of the geostationary zone. This can be explained by the fact that this object is located in a dense cluster of other objects. 


\section{Conclusion}

In this work, the orbits of selected objects of space debris with a high windage coefficient on long orbital arcs (more than 6 months) are determined. The evolution of these objects is researched over a 20-year time interval. It is shown that all the objects under study, which have a large value of the windage coefficient, are unstable and have chaotic motion. The value of the averaged parameter MEGNO is greater than 2.

It is shown that the possibility of collision of the objects under study with other objects of the geostationary zone is minimal; at the current moment, the researched objects do not pose a threat to the existing objects of the geostationary zone.

\section{References}

1. G. Petit and B. Luzum, IERS Conventions, 179, 2010.

2. D. Vokrouhlicky, P. Farinella, and F. Mignard, Astronomy and Astrophysics, 307, 635, 1996. 


\title{
Dynamical estimation of masses of the Main asteroid belt and some individual asteroids within the EPM ephemeris using infrared data
}

\author{
M. $\operatorname{Kan}^{1}$, D. Pavlov ${ }^{2}$ \\ mo.kan@iaaras.ru; dapavlov@etu.ru \\ ${ }^{1}$ The Institute of Applied Astronomy of the RAS, St. Petersburg, Russia, \\ ${ }^{2}$ Saint Petersburg Electrotechnical University "LETI", St. Petersburg, Russia
}

The purpose of this work is to estimate the mass of the main asteroid belt and those of the individual asteroids. To solve this task we propose to use Tikhonov regularization which allows to use a priori information on the asteroids' masses and their uncertainties. Each a priori mass and its uncertainty were calculated based on the infrared diameter and density estimates of a particular asteroid. As a result, we managed to obtain mass estimates consistent with those obtained earlier, and to extend the list of asteroids whose masses are individually determined in the EPM dynamical model.

Keywords: masses estimation, infrared data, Tikhonov regularization

DOI: $10.51194 /$ VAK2021.2022.1.1.023

\section{Problem Setting and Tikhonov Regularization}

In this work we will consider the task of the parameters adjustment. Since the corrections are small enough, the problem can be set in the form of an overdetermined system of linear equations. Such problems are usually solved with the Least Squares Method.

Involving the so-called a priori data on asteroids' masses leads to obtaining more plausible results. For this purpose it is proposed to use Tikhonov regularization. Using this method, the a posteriori solution $x$ and its uncertainty $\sigma$ can be found according to the formulas:

$$
\begin{aligned}
x & =\left(A^{\mathrm{T}} P^{\mathrm{T}} P A+Q^{\mathrm{T}} Q\right)^{-1}\left(A^{\mathrm{T}} P^{\mathrm{T}} P z+Q^{\mathrm{T}} Q x_{p r}\right), \\
\sigma & =\sqrt{\operatorname{diag}\left(A^{\mathrm{T}} P^{\mathrm{T}} P A+Q^{\mathrm{T}} Q\right)^{-1}},
\end{aligned}
$$

where $z$ is the $O-C$ vector, $A$ is the partials matrix, $P^{2}$ is the weight matrix, $x_{p r}$ is the a priori solution, $Q$ is the Tikhonov matrix.

\section{A Priori Data Calculation}

To perform this task 379 asteroids were originally selected. This list consists of 301 asteroids already involved into EPM; 42 asteroids extending this list to 343 asteroids of DE and INPOP; and, finally, 36 extra asteroids which, according to [1], probably need to be taken into account individually.

17 masses out of 379 have already been pretty well determined from the spacecrafts observations and those of binary asteroids. These estimates are proposed to be used as a priori ones. However, the remaining masses calculation required preliminary diameters and densities estimation.

Numerous asteroids have their diameters estimates known from the infrared observations by the space telescopes IRAS and WISE. These estimates were obtained by [2, 3] and are catalogued by NASA: PDS SBN ${ }^{1}$. For each estimate the number of observations used is also available.

361 asteroids from the list had their diameters estimates combined assuming normal distribution of errors. Thus, for each of them we managed to obtain its a priori diameter estimate $\bar{D} \pm \Delta D$. However, for (675) Ludmilla there is no infrared diameter estimate. Therefore, for this asteroid we set $\bar{D}$ to $76.0 \mathrm{~km}^{2}, \Delta D$ to $0.35 \bar{D}$.

Asteroid densities can be determined based on their belonging to taxonomies $\mathrm{C}$ (carbonaceous), $\mathrm{S}$ (siliceous), M (metallic). 284 out of the remaining 362 asteroids have already had their densities determined by [4] similarly to the technique following.

For the remaining 78 asteroids their taxonomic classifications by $[5,6,7,8,9,10,11,12]$ are also catalogued by NASA: PDS SBN ${ }^{1}$. For the latter ones, an attempt was made to match them with the Tholen taxonomy. Finally, the class adopted by most researches was chosen as the reference one. Thus, Tholen classes were defined for 60 asteroids.

According to [4], there is a correspondence between Tholen classes and taxonomies C/S/M. However, Tholen $\mathrm{X}$-type asteroids can be mapped into none of $\mathrm{C} / \mathrm{S} / \mathrm{M}$ taxonomies. Thus, for the remaining 18 asteroids we have decided to take their classification from a series of independent studies by [13], [1], [14], [15], [16]. For 3 of them independent results contradict each other. In these cases, we have decided to take classification from the later work.

\footnotetext{
${ }^{1}$ See https://sbn.psi.edu/pds/archive/missions.html

${ }^{2}$ See https://ssd.jpl.nasa.gov/sbdb.cgi

${ }^{1}$ See https://sbn.psi.edu/pds/archive/physical.html
} 
Table 1: Correlation between Tholen and $\mathrm{C} / \mathrm{S} / \mathrm{M}$ classes and density estimates for the latter according to [4]

\begin{tabular}{rcc}
\hline Tholen taxonomic classes & Taxonomy & Density estimate, $\mathrm{g} \mathrm{cm}^{-3}$ \\
\hline $\mathrm{C}, \mathrm{D}, \mathrm{P}, \mathrm{T}, \mathrm{B}, \mathrm{G}, \mathrm{F}$ & $\mathrm{C}$ & $1.50 \pm 0.3$ \\
$\mathrm{~S}, \mathrm{~K}, \mathrm{Q}, \mathrm{V}, \mathrm{R}, \mathrm{A}, \mathrm{E}$ & $\mathrm{S}$ & $2.20 \pm 0.4$ \\
$\mathrm{M}$ & $\mathrm{M}$ & $3.84 \pm 1.2$ \\
\hline
\end{tabular}

Finally, each asteroid was provided with its density estimate based on its taxonomy according to [4] (see Table 1).

Thus, having estimated the asteroid's diameter $D=\bar{D} \pm \Delta D$ and its density $\rho=\bar{\rho} \pm \Delta \rho$ and assuming its spherical shape we can obtain its a priori mass estimate as a mass of a sphere with given diameter and density and its uncertainty as a total differential of the sphere mass function.

\section{Results}

During this work we managed to obtain asteroids masses estimates some of which were obtained for the first time. The better a priori estimates are selected, the more precise a posteriori estimates turn out to be.

Although we have obtained a few negative estimates (31 out of 379), most of the estimates obtained appeared to be in agreement with those obtained within EPM (see for example [17]) or by other authors earlier. As for the other authors' results, they were obtained either from mutual perturbations between asteroids [18, 19, 20], or from the ephemeris $[21,22,23]$.

In addition, we have estimated the mass of the main asteroid belt which amounted to $(12.257 \pm 0.139) \times$ $10^{-10} M_{\odot}$ or $(4.0817 \pm 0.0465) \times 10^{-4} M_{\oplus}$.

Our further plans include thorough analysis of the results obtained, varying the list of the asteroids included and, first of all, getting rid of negative masses obtaining by involving Bounded Variable/Non-Negative Least Squares.

\section{References}

1. P. Kuchynka, J. Laskar, A. Fienga, and H. Manche, Astronomy \& Astrophysics, 514, 2010.

2. E. Tedesco, P. V. Noah, M. Noah, and S. D. Price, IRAS Minor Planet Survey, IRAS-A-FPA-3-RDR-IMPSV6.0, NASA Planetary Data System, 2004.

3. E. T. Tedesco, M. P. Egan, and S. D. Price, MSX Infrared Minor Planet Survey, MSX-A-SPIRIT3-5-SBN0003MIMPS-V1.0, NASA Planetary Data System, 2004.

4. E. V. Pitjeva, Construction of High-precision Ephemerides of the Major Planets and Determination of Some Astronomical Constants, Ph.D. thesis, IAA RAS, 2004.

5. D. J. Tholen, Asteroid Taxonomy from Cluster Analysis of Photometry, Ph.D. thesis, University of Arizona, 1984.

6. D. J. Tholen, Asteroid taxonomic classification, 1139-1150 (1989).

7. M. A. Barucci, M. T. Capria, A. Coradini, and M. Fulchignoni, Icarus, 72, 304, 1987.

8. E. F. Tedesco, J. G. Williams, D. L. Matson, G. J. Veeder, J. C. Gradie, and L. A. Lebofsky, The Astronomical Journal, 97, 580, 1989.

9. E. S. Howell, E. Merenyi, and L. A. Lebofsky, Journal of Geophysical Research, 99, 10847, 1994.

10. S. Xu, R. P. Binzel, T. H. Burbine, and S. J. Bus, Icarus, 115, 1, 1995.

11. S. J. Bus and R. P. Binzel, Icarus, 158, 146, 2002.

12. D. Lazzaro, C. A. Angeli, J. M. Carvano, T. Mothé-Diniz, R. Duffard, and M. Florczak, Icarus, 172, 179, 2004.

13. B. E. Clark, S. J. Bus, A. S. Rivkin, M. K. Shepard, and S. Shah, The Astronomical Journal, $128,2004$.

14. S. Fornasier, B. E. Clark, and E. Dotto, Icarus, 214, 131, 2011.

15. A. Mainzer, T. Grav, J. Masiero, E. Hand, et al., The Astronomical Journal, 741, 2011.

16. M. Delbo, C. Avdellidou, and A. Morbidelli, Astronomy \& Astrophysics, 624, 2019.

17. E. Pitjeva and D. Pavlov, Epm2017 and epm2017h, https://iaaras.ru/en/dept/ephemeris/epm/2017/, 2017.

18. G. A. Krasinsky, E. V. Pitjeva, M. V. Vasilyev, and E. I. Yagudina, Icarus, 158, 98, 2002.

19. J. Baer, S. R. Chesley, and R. D. Matson, The Astronomical Journal, 141, 2011.

20. J. Baer and S. R. Chesley, The Astronomical Journal, 154, 2017.

21. W. M. Folkner, J. G. Williams, D. H. Boggs, R. S. Park, and P. Kuchynka, The Interplanetary Network Progress Report, 42-196, 2014. 
22. A. Fienga, J. Laskar, P. Kuchynka, H. Manche, G. Desvignes, M. Gastineau, I. Cognard, and G. Theureau, Celestial Mechanics and Dynamical Astronomy, 111, 363, 2011.

23. P. Kuchynka and W. M. Folkner, Icarus, 222, 243, 2013. 


\title{
Multi-wave fundamental astrometry
}

\author{
A.A. Kluykov, L.V. Rykhlova \\ kaa5774@yandex.ru \\ Institute of Astronomy, Russian Acad. Sci., 48, Pyatnitskaya Str., Moscow 119017, Russia
}

The article reviews the results in the field of creating a fundamental celestial reference system by various methods and in different parts of the electromagnetic wave spectrum.

Keywords: fundamental astrometry,fundamental catalogue, space astrometry

DOI: 10.51194/VAK2021.2022.1.1.024

\section{Introduction}

The purpose of this article is a retrospective review of the results in the field of creating a fundamental celestial coordinate system obtained by various methods and means in various regions of the electromagnetic wave spectrum.

\section{The fundamental celestial coordinate system in the optical range}

The fundamental system of celestial coordinates is realized in the form of an average equatorial coordinate system and is fixed by the data of the fundamental catalog and the proper motions of a certain number of stars for a given epoch. In 1879, Auverse created the first fundamental catalog of FC stars, which marked the beginning of a dynasty of fundamental catalogs, the characteristics of which are contained in Table 1

Table 1: Characteristics of the fundamental catalogues of stars

\begin{tabular}{|c|c|c|c|c||c|c|}
\hline Name catalogue & Number of stars & \multirow{2}{*}{ Epoch } & \multicolumn{2}{|c||}{ Accuracy of position } & \multicolumn{2}{c|}{ Accuracy of proper motion } \\
\cline { 4 - 7 } & & & $\alpha, \mathrm{s}$ & $\delta,^{\prime \prime}$ & $\alpha$ sec $\delta, \mathrm{s} /$ year & $\delta,^{\prime \prime} /$ year \\
\hline FC & 539 & J1870,0 & 0,03 & 0,5 & - & - \\
\hline FK2 (NFK) & 925 & J1870,0 & 0,03 & 0,5 & - & - \\
& & J1900,0 & 0,03 & 0,5 & - & - \\
\hline FK3 & $873+662$ & J1900,0 & 0,002 & 0,03 & 0,010 & -14 \\
& add. stars & J1950,0 & 0,005 & 0,08 & - & - \\
\hline FK4 & $1535+1111$ & J1950,0 & 0,002 & 0,03 & - & - \\
& add. stars & J1970,0 & 0,012 & 0,15 & - & - \\
\hline FK5 & $1535+3117$ & J2000,0 & 0.001 & 0.02 & & - \\
& add. stars & & & & & - \\
\hline FK6 & $878+3272$ & J2000,0 & 0,0001 & 0,01 & $0,2 \times 10^{-4}$ & $0,35 \times 10^{-3}$ \\
& add. stars & & & & & \\
\hline
\end{tabular}

It should be noted that all fundamental catalogs, except for FK6, are obtained only on the basis of groundbased astronomical observations [1, 2]. FK6 is an optimal synthesis of Hipparcos satellite data with ground-based astronomical measurements [3]. By the 70s of the twentieth century, ground-based astronomical observations of stars reached the limit of their accuracy due to the influence of the atmosphere, which blurs the images of stars, as well as the action of gravity, which leads to deformation of telescopes. As a result, the increase in the accuracy of the fundamental catalogs was insignificant.

\section{The fundamental celestial coordinate system in the radio range}

A breakthrough in improving the accuracy of positional measurements of celestial bodies was made by the method of radio interferometry with ultra-long bases (VLBI). The VLBI method occupies a special place in fundamental astrometry, since it allows you to independently construct the celestial and terrestrial reference systems and determine their mutual orientation. At the 23rd Congress of the IAU in 1997, it was adopted.The International Celestial Reference System (ICRS), the use of which began on January 1, 1998. The origin of the coordinate system ICRS is the barycenter of the Solar System. The coordinates in this system are as close as possible to the equatorial coordinates of the epoch J2000. 0. The axes of the ICRS system are fixed in space relative to quasars, whose proper motion is almost zero. The resulting coordinate system is independent of the rotation of the Earth. It replaced the fundamental celestial reference system, which was fixed by the catalog stars FK5. The practical implementation of ICRS is carried out by creating a catalog of extragalactic radio sources. Currently, three catalogues of extragalactic radio sources have been obtained $[4,5,6]$, the characteristics of which are shown in Table 2 .

It should be noted that the coordinates of the 600 quasars in the ICRF3 catalog are determined at three frequencies. 
Table 2: Characteristics of the fundamental catalogs of radio sources

\begin{tabular}{|c|c|c|c|}
\hline Parameters & ICRF1 & ICRF2 & ICRF3 \\
\hline $\begin{array}{c}\text { Number of } \\
\text { of }\end{array}$ & 608 & 3414 & 4536 \\
sources & $(212+39)$ & $(295+39+$ & $(303+4233)$ \\
\hline Radio band & & $1114+1966)$ & $\mathrm{X} / \mathrm{S}, \mathrm{X} / \mathrm{K}, \mathrm{X} / \mathrm{Ka}$ \\
\hline Freq $(\mathrm{GHz})$ & $\mathrm{X} / \mathrm{S}$ & $\mathrm{X} / \mathrm{S}$ & $8.4 / 2.3 ; 24 ; 32 / 8.4$ \\
\hline \multicolumn{3}{|c|}{} & Accuracy of position \\
\hline $\mathrm{X} / \mathrm{S}(\alpha, \mathrm{s})$ & $0.017 \times 10^{-3 \cup} 0.092072$ & $0.00273 \times 10^{-3}-0.00252791$ & $0.00202 \times 10^{-3}-0.00899265$ \\
$\left(\delta,{ }^{\prime \prime}\right)$ & $0.26 \times 10^{-3}-0.9467$ & $0.0413 \times 10^{-3}-0.0427226$ & $0.0303 \times 10^{-3}-0.2507685$ \\
\hline $\mathrm{K}(\alpha, \mathrm{s})$ & & & $0.00245 \times 10^{-3}-0.86595 \times 10^{-3}$ \\
$\left(\delta,{ }^{\prime \prime}\right)$ & & $0.0613 \times 10^{-3}-0.0118579$ \\
\hline $\mathrm{X} / \mathrm{Ka}(\alpha, \mathrm{s})$ & & $0.00291 \times 10^{-3}-0.00446018$ \\
$\left(\delta,{ }^{\prime \prime}\right)$ & & $0.0470 \times 10^{-3}-0.0735481$ \\
\hline
\end{tabular}

\section{The fundamental celestial coordinate system in the optical range obtained by methods of space astrometry}

\subsection{The project Hipparcos}

A new level of accuracy in determining the position of stars in the optical range was achieved with the help of telescopes installed on board the spacecraft. The first project in the field of space astrometry was the HIPPARCOS project, one of the main goals of which was to obtain the positions, proper motions and parallaxes of stars at the millisecond accuracy level for 100,000 stars. The main product of the HIPPARCOS mission was a catalog of the same name containing the positions, proper motions and parallaxes for 100,000 stars at the millisecond accuracy level. In addition to the HIPPARCOS catalog, the TYCHO and TYCHO-2 catalogs were obtained [7, 8], the characteristics of which are shown in Table 3. According to the IAU resolution, the HIPPARCOS catalog is the first large-scale implementation of ICRS in the optical range.

Table 3: Characteristics catalogs of the project HIPPARCOS

\begin{tabular}{|c|c|c|c|}
\hline Catalogue & HIPPARCOS & TYCHO & TYCHO-2 \\
\hline Catalog system & ICRS & ICRS & ICRS \\
\hline Average epoch of observations & J1991,25 & J1991,25 & J1991,5 \\
\hline Number of stars & 118218 & 1058332 & 2539913 \\
\hline Maximum stellar magnitude & 8 & 11,5 & 11,5 \\
\hline Accuracy of position & $(0.77 / 0.64) \times 10^{-3}(")(\alpha / \delta)$ & $0,025(")(\mathrm{J} 1991,25)$ & $0,060(")$ \\
& & $0,007(") ;$ for $\mathrm{V}<9 \mathrm{~m})$ & \\
\hline Accuracy of proper motion & $(0.88 / 0.74) \times 10^{-3}(" / \mathrm{yr})(\alpha / \delta)$ & - & $0,0025(" / \mathrm{yr})$ \\
\hline Accuracy of parallax & $\left(0.97 \times 10^{-3}(")\right.$ & - & - \\
\hline
\end{tabular}

\subsection{Project GAIA}

The successor of the HIPPARCOS project in the field of practical implementation of the fundamental celestial coordinate system ICRS in the optical range was the GAIA astrometric project of the European Space Agency. The practical implementation of the Gaia project, designed for 5 years, began in 2013 with the launch of the spacecraft in the vicinity of the second Lagrange point (L2) of the Sun-Earth system. The main task of the GAIA project is to compile a detailed map of the distribution of stars in our Galaxy. Another task of the GAIA project is the discovery of exoplanets. The number of possible candidates is estimated at 10,000 objects. The accuracy of the measuring systems located on board the GAIA spacecraft is 3-4 orders of magnitude higher than the accuracy of the measuring systems on board the HIPPARCOS spacecraft. To date, three data sets of the Gaia project have been mathematically processed $[9,10,11]$, the characteristics of which are shown in Table 4 .

The catalog of extragalactic sources obtained in the GAIA project may soon replace the HIPPARCOS catalog, which currently implements the fundamental ICRS celestial system in the optical range.

\section{The fundamental celestial coordinate system ICRS in the infrared range}

The progress of observational technology made it possible to measure very weak sources in the infrared range of electromagnetic waves. These features were implemented in the 2MASS project (The Two Micron All Sky Survey) using three-channel cameras installed on two specialized telescopes with a diameter of $1.3 \mathrm{~m}$ located in Arizona 
Table 4: Characteristics of the GAIA project data

\begin{tabular}{|c|c|c|c|}
\hline Parameters & Gaia DR3 & Gaia DR2 & Gaia DR1 \\
\hline Number of sources & 1811709771 & 1692919635 & 1142679769 \\
\hline \multirow[t]{4}{*}{ Accuracy of position, mas } & $0.01-0.02$ for $\mathrm{G}<15$ & 0.04 for $\mathrm{G}<15$ & \multirow{4}{*}{$\begin{array}{l}0.25 \alpha \\
0.23 \delta\end{array}$} \\
\hline & 0.05 for $G=17$ & 0.1 for $G=17$ & \\
\hline & 0.4 for $\mathrm{G}=20$ & 0.4 for $\mathrm{G}=20$ & \\
\hline & 1.0 for $G=21$ & 2.0 for $G=21$ & \\
\hline \multirow[t]{4}{*}{ Accuracy of proper motion, mas } & $0.02-0.03$ for $\mathrm{G}<15$ & 0.06 for $\mathrm{G}<15$ & \multirow{4}{*}{$\begin{array}{l}1.1 \alpha \\
0.9 \delta\end{array}$} \\
\hline & 0.07 for $G=17$ & 0.2 for $G=17$ & \\
\hline & 0.5 for $\mathrm{G}=20$ & 1.2 for $\mathrm{G}=20$ & \\
\hline & 1.4 for $\mathrm{G}=21$ & 3.0 for $G=21$ & \\
\hline \multirow[t]{4}{*}{ Accuracy of parallax, mas } & $0.02-0.03$ for $\mathrm{G}<15$ & 0.04 for $\mathrm{G}<15$ & \multirow{4}{*}{$\begin{array}{c}0.3 \text { for TGAS } \\
10.0 \text { for } 1140622719 \text { stars }\end{array}$} \\
\hline & 0.07 for $\mathrm{G}=17$ & 0.1 for $\mathrm{G}=17$ & \\
\hline & 0.5 for $\mathrm{G}=20$ & 1.2 for $\mathrm{G}=20$ & \\
\hline & 1.4 for $\mathrm{G}=21$ & 3.0 for $G=21$ & \\
\hline
\end{tabular}

(USA) and in Chile. The observations were carried out in the period from 1997 to 2001. The declared accuracy of determining the coordinates of objects is $100 \mathrm{~ms}$ of arc [12]. The main products of the 2MASS project are:

- catalog of 471 million point sources (Point Source Catalog);

- catalog of 1.6 million extended sources (Extended Source Catalog).

- 4,121,439 images of celestial objects covering $99.998 \%$ of the sky.

\section{Conclusion}

Having considered the results of the creation of a fundamental celestial reference system by various methods and means, it can be stated that fundamental astrometry, which for a long time was the astrometry of the optical range, has now become multi-wave fundamental astrometry.

\section{References}

1. Podobed V.V. and Nesterov V.V., General Astrometry (Nauka) (1975).

2. W. Fricke, H. Schwan, T. Lederle, U. Bastian, et al., VizieR Online Data Catalog, I/149A, 1995.

3. R. Wielen, H. Schwan, C. Dettbarn, H. Lenhardt, H. Jahreiß, and R. Jährling, Veroeffentlichungen des Astronomischen Rechen-Instituts Heidelberg, 35, 1, 1999.

4. C. Ma, E. F. Arias, T. M. Eubanks, A. L. Fey, et al., AJ, 116, 516, 1998.

5. A. L. Fey, D. Gordon, C. S. Jacobs, C. Ma, et al., AJ, 150, 58, 2015.

6. P. Charlot, C. S. Jacobs, D. Gordon, S. Lambert, et al., A\&A, 644, A159, 2020.

7. J. Vondrak, Serbian Astronomical Journal, 168, 1, 2004.

8. A. Popov and A. Tsvetkov, in Proceedings of the Journées 2003 "Systèmes deréférence spatio-temporels": Astrometry, 91-92 (2004).

9. Gaia Collaboration, A. G. A. Brown, A. Vallenari, T. Prusti, et al., A\&A, 650, C3, 2021.

10. Gaia Collaboration, A. G. A. Brown, A. Vallenari, T. Prusti, et al., A\& A, 616, A1, 2018.

11. L. Lindegren, U. Lammers, U. Bastian, J. Hernández, et al., A\&A, 595, A4, 2016.

12. M. F. Skrutskie, R. M. Cutri, R. Stiening, M. D. Weinberg, et al., AJ, 131, 1163, 2006. 


\section{Facilities of astrophysical telescopes in space debris research}

G. Kokhirova ${ }^{1}$, P. Levkina ${ }^{2}$, N. Bakhtigaraev ${ }^{2}$, U. Khamroev $^{1}$

kokhirova2004@mail.ru; ayvazovskaya@inasan.ru

${ }^{1}$ Institute of Astrophysics of the National Academy of Sciences of Tajikistan, Dushanbe, Bukhoro, 22,

${ }^{2}$ Institute of Astronomy, Russian Acad. Sci., 48, Pyatnitskaya Str., Moscow 119017, Russia

The possibilities of astrophysical telescopes for space debris research are shown. Some results of observations of space debris' fragments using the Zeiss-2000 telescope $(\mathrm{D}=2 \mathrm{~m}, \mathrm{~F}=16 \mathrm{~m})$ Terskol branch of the Institute of Astronomy of the Russian Academy of Sciences and Zeiss-1000 ( $\mathrm{D}=1 \mathrm{~m}, \mathrm{~F}=13 \mathrm{~m}$ ) of the Sanglokh observatory of the Institute of Astrophysics of the National academy of Sciences of Tajikistan are presented.

Keywords: space debris, astrometric and photometric observations, area-to-mass ratio

DOI: 10.51194 /VAK2021.2022.1.1.025

When geostationary satellites have not been transferred to disposal orbits after the termination of their service life and then they begin to move towards the nearest stable libration point and make oscillatory movements along the longitude, regularly approaching various spacecrafts, that creates a collision threat.

The theory of motion for large space objects is well developed, and their movement is well predictable. Large objects are observed by specialized survey telescopes. Small-sized fragments of space debris, unlike large ones, are highly susceptible to difficult-to-predict non-gravitational perturbations. This is especially true for objects with a high area-to-mass ratio. The evolution of the orbital eccentricity under the influence of light pressure depends on this parameter. Astrophysical telescopes provide the opportunity to obtain high-precision measurements of small-sized objects of space debris, including those with a high area-to-mass ratio. The long focal length of the telescopes causes a decrease in the influence of the sky background during observations.

\section{Zeiss-1000 of the Sanglokh observatory}

Since foundation in 1981, the Sanglokh observatory has been distinguished by very good astroclimatic conditions: $F W H M=0.54^{\prime \prime}$, transparency coefficient 0.78 . The observatory is located at an altitude of $2300 \mathrm{~m}$ above sea level, $120 \mathrm{~km}$ from Dushanbe, $\phi=38.3^{\circ} \mathrm{N}, \lambda=69.2^{\circ} \mathrm{E}$. The main instrument of the Sanglokh observatory is the Zeiss-1000 telescope with characteristics $\mathrm{D}=1 \mathrm{~m}, \mathrm{~F}=13 \mathrm{~m}$.

The advantages of the Sanglokh observatory for the space debris' investigations in the geostationary zone are as follows:

1) Excellent astroclimatic characteristics of the observatory.

2) The Sanglokh observatory is the most southern observation point in the CIS. This makes the station attractive for observing the geostationary region located above the equator.

3) The proximity of the observatory to the stable libration point of the geostationary region of $75^{\circ} \mathrm{E}$. This makes it possible to study the characteristics of many objects of space debris moving in libration mode near the point $75^{\circ} \mathrm{E}$, using observations from only one point.

Table 1 shows an example of calculating the parameters of the orbits of two objects from observations on the Zeiss-1000 telescope of the Sanglokh observatory. The small-sized object Fengyun 2D Deb moves in libration mode around the point $75^{\circ} \mathrm{E}$.

Table 1: Elements of the orbits of Sirio-1 and Fengyun 2D Deb according to observations in Sanglokh for 7 nights in August 2018

\begin{tabular}{lll}
\hline Name & Sirio-1 & Fengyun 2D Deb \\
Epoch: date, UTC & $07.08 .201819: 46: 47.216$ & $07.08 .201801: 56: 26.195$ \\
$\boldsymbol{a}(\mathbf{k m})$ & $42163.623 \pm 0.0011$ & $42156.826 \pm 0.0015$ \\
$\boldsymbol{e}$ & 0.0005596 & 0.0068171 \\
$\boldsymbol{i}\left(^{\circ}\right)$ & $13.07164\left( \pm 0.015^{\prime \prime}\right)$ & $6.65604\left( \pm 0.030^{\prime \prime}\right)$ \\
$\Omega\left(^{\circ}\right)$ & 327.59153 & 56.18293 \\
$\omega\left(^{\circ}\right)$ & 67.18407 & 333.25669 \\
$\boldsymbol{M}\left(^{\circ}\right)$ & 292.87964 & 26.39621 \\
$\boldsymbol{A} / \boldsymbol{m}\left(\mathbf{m}^{2} / \mathbf{k g}\right)$ & 0.0133 & 0.1011 \\
$\lambda\left(^{\circ}\right)$ & 74.61545 & 71.52708 \\
$\boldsymbol{m a g n i t u d e}$ & 15.1 & 17.4 \\
\hline
\end{tabular}

The observations obtained on the Zeiss-1000 telescope show very high accuracy in determining the orbits of objects in the geostationary region [1]. 


\section{Zeiss-2000 of the Terskol observatory}

The Terskol observatory of the Institute of Astronomy of the Russian Academy of Sciences is located on the Elbrus region at an altitude of $3150 \mathrm{~m}$ above sea level, $\phi=43.2^{\circ} \mathrm{S}, \lambda=42.5^{\circ} \mathrm{E}$. The main instrument of the observatory is the Zeiss-2000 telescope with characteristics $\mathrm{D}=2 \mathrm{~m}, \mathrm{~F}=16 \mathrm{~m}$ has the $21^{\text {st }}$ limiting magnitude for objects of the geostationary region. Figure 1 shows estimates of internal accuracy based on Sirio satellite observations at a 90-minute interval. These accuracy values are obtained under very good weather conditions. Usually, residuals for $R A$ and $D e c= \pm 0.1^{\prime \prime}-0.2^{\prime \prime}$.
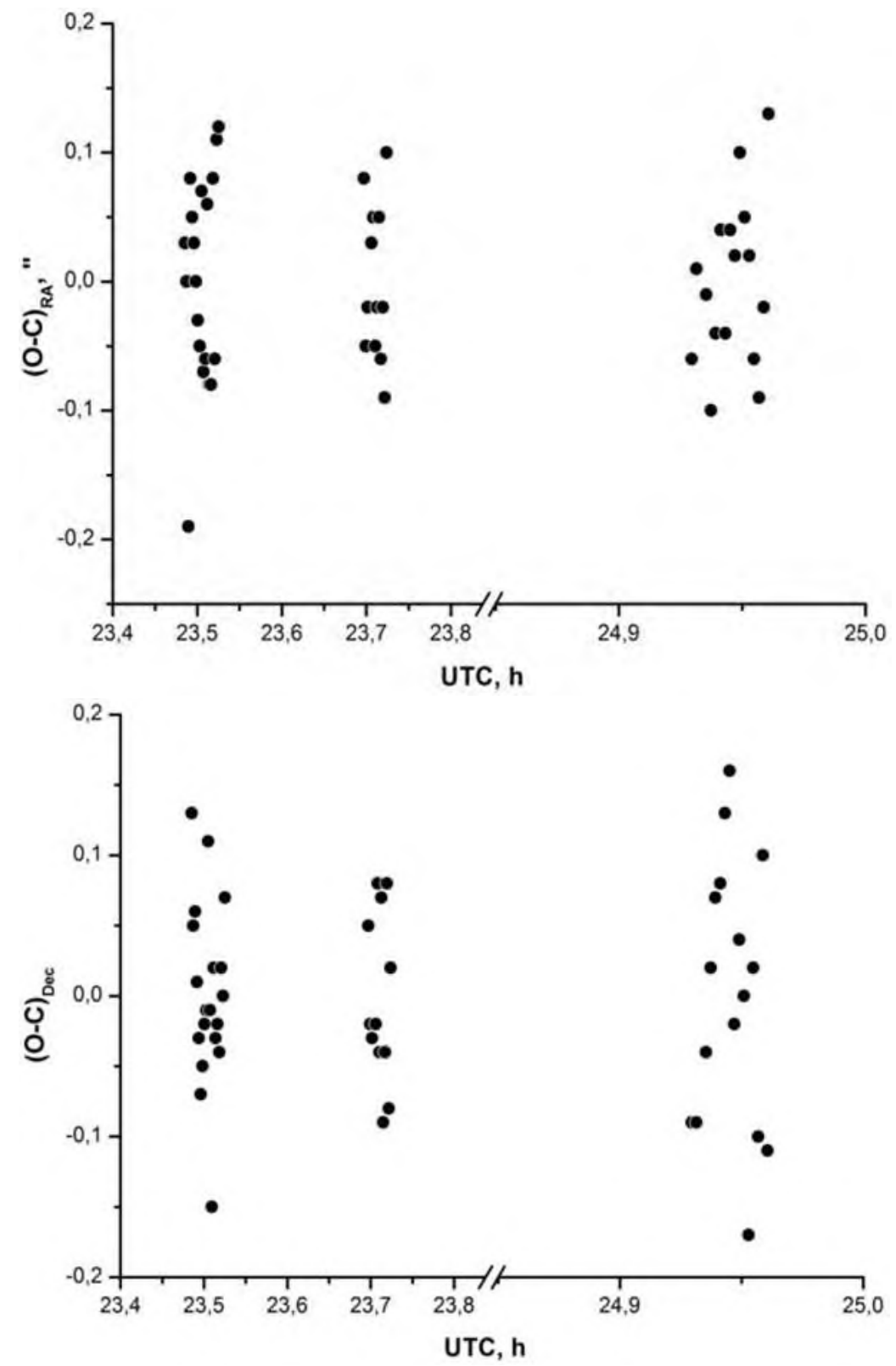

Figure 1: $(\mathrm{O}-\mathrm{C})$ according to the observations of the Sirio satellite at a 90 -minute interval. The residuals for $R A$ and $\mathrm{Del}= \pm 0.07^{\prime \prime}$.

To research of space debris objects the following tasks are carried out at the Zeiss-2000 telescope:

- Study of the small-sized objects' population of the geostationary zone.

- Study of the features of translational and rotational motion of selected space debris objects.

- Improvement of the theory of motion of some space debris objects and identification of changes in the parameters of their orbits.

- Accumulation of observational data on space debris objects with very high area-to-mass ratios (more than $10 \mathrm{~m}^{2} / \mathrm{kg}$ ) and search for patterns of changes in their orbit parameters. 


\subsection{Investigation of the small-sized objects' population of the geostationary zone}

5-6 uncorrelated new faint objects are detected in the Terskol observatory during photometric nights.

For example, a fragment of space debris with an average visible brightness of 19.8 magnitudes was detected on September 24, 2020. The fragment was observed during 64 minutes, the observations were continued on September 25. The topocentric distance of the object changed from $36,862 \mathrm{~km}$ to $37,224 \mathrm{~km}$ during observations. The accuracy of determining the positions $\left(\sigma_{\Delta \alpha \cos \delta}= \pm 0.36^{\prime \prime}, \sigma_{\delta}= \pm 0.23^{\prime \prime}\right)$ and a large observation arc were sufficient for its cataloging under the number 71113 in the dynamic database of space objects of the M.V. Keldysh Institute. The estimated size of the object is about $10 \mathrm{~cm} \mathrm{[2].}$

\subsection{Accumulation of observational data on space debris objects with very high area-to-mass ratio}

Table 2 shows the motion parameters of a space debris object with a high area-to-mass ratio based on the data from two telescopes. As seen, the elements of the orbits of the observed objects are calculated with a fairly high accuracy.

Table 2: Object 90404. The orbital parameters based on the data from two observatories

\begin{tabular}{llll}
\hline $\mathbf{9 0 4 0 4}$ & 09.07 .2019 & 31.07 .2019 & 14.09 .2019 \\
& Terskol, Zeiss-2000 & Sanglokh, Zeiss-1000 & Terskol, Zeiss-2000 \\
\hline $\boldsymbol{N}$ & $119(2$ nights $)$ & $128(4$ nights $)$ & $357(2$ nights $)$ \\
$\boldsymbol{a}(\mathbf{k m})$ & $39875.42 \pm 0.04$ & $39900.83 \pm 0.03$ & $39916.28 \pm 0.04$ \\
$\boldsymbol{e}$ & $0.308826 \pm 0.000031$ & $0.296020 \pm 0.000009$ & $0.273488 \pm 0.000002$ \\
$\boldsymbol{i}\left(^{\circ}\right)$ & $25.28184 \pm 0.00047$ & $25.12402 \pm 0.00015$ & $24.36490 \pm 0.00004$ \\
$\Omega\left(\boldsymbol{\circ}^{\circ}\right)$ & $332.88957 \pm 0.00023$ & $332.79076 \pm 0.00025$ & $332.27196 \pm 0.00013$ \\
$\boldsymbol{A} / \mathbf{m}\left(\mathbf{m}^{2} / \mathbf{k g}\right)$ & $29.18 \pm 0.02$ & $29.15 \pm 0.04$ & $29.16 \pm 0.03$ \\
\hline
\end{tabular}

\section{Conclusion}

Studies of space debris using the Zeiss-1000 and Zeiss-2000 astrophysical telescopes have shown high efficiency. Observations on the Zeiss-1000 allow calculating the parameters of the orbits of space objects with very high accuracy that is especially important for solving applied problems.

A high astrometric accuracy of observations is achieved using the described telescopes. By calculating the high accuracy orbital parameters of artificial objects, it is possible to study in detail the features of the movement of small-sized objects in the geostationary region. For example, an empirical model of area-to-mass ratio variations was constructed for the Fengyun 2D Deb object [3].

\section{References}

1. V. V. Chazov, G. I. Kokhirova, N. S. Bakhtigaraev, U. K. Khamroev, and A. S. Mullo-Abdolov, Reports AS RT, 61, 848, 2018.

2. N. S. Bakhtigaraev, P. A. Levkina, and A. V. Shein, INASAN Science Reports, 5, 358, 2020.

3. N. S. Bakhtigaraev, P. A. Levkina, and V. V. Chazov, Solar System Research, 50, 130, 2016. 


\section{Analysis of corrections to deformations of the earth surface at VLBI station "SIMEIZ"}

G. Kurbasova, A. Volvach, L. Volvach

volvach@craocrimea.ru

Crimean Astrophysical Observatory RAS, Katsively, RT-22 Crimea

\section{DOI: $10.51194 /$ VAK2021.2022.1.1.026}

The currently accepted reference framework ITRF2014 is directly supported by the network of VLBI stations. The estimated uncertainty of the ITRF2014 reference base is less than $3 \mathrm{~mm}$ in the 2010.0 epoch and less than $0.2 \mathrm{~mm} /$ year in the time evolution, which actually determines the accuracy of the displacement of stations in the VLBI network. Ignoring nonlinear station motions is a major source of errors in current reference frame implementations. Non-modeled effects (atmospheric and hydrological loads) cause periodic fluctuations in the time series of the coordinates of the observation stations.

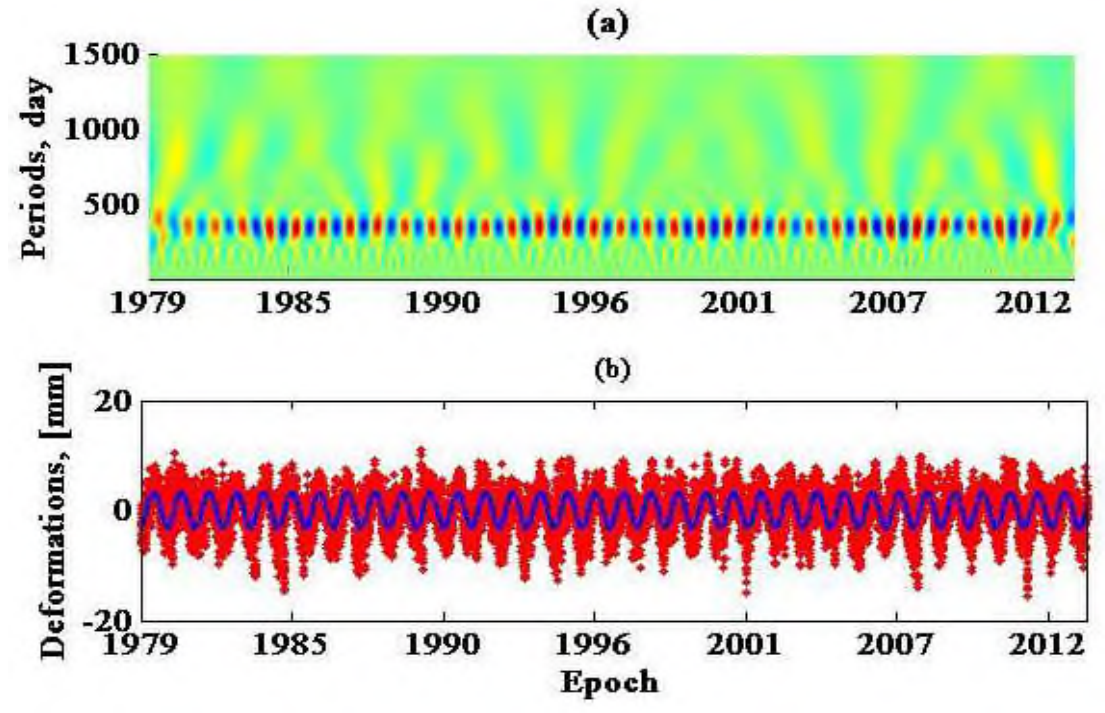

Figure 1: (a) Time-frequency wavelet analysis graph [1]; (b) Graph of data on non-modeled vertical deformations of the ground surface of the VLBI station "Simeiz" after removing the abnormal "white noise" and trend (asterisks *); curve of approximation of the seasonal component with a period of 365.3 days and an amplitude of 3.37 mm (red, solid line). The data counting interval is 0.25 days.

The Simeiz VLBI station took part in the observations of the networks of the International VLBI Service (IVS) stations. This paper presents the results of the analysis of the time series of not modeled corrections (IERS model Atmosphere [2]) to vertical deformations of the earth's surface at the Simeiz VLBI station in the time interval 1980-2014.

\section{References}

1. M. Misiti, Y. Misiti, G. Oppenheim, and J. M. Poggi, The MathWorks, Inc., 2002.

2. M. Seitz, M. Blossfeld, D. Angermann, R. Schmid, M. Gerstl, and F. Seitz, Deutsches Geodatisches Forschungsinstitut, 2016. 


\title{
Current state and future plans of the Earth's orientation parameters evaluation activities in MMC SSTF
}

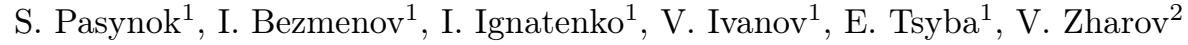 \\ pasynok@vniiftri.ru \\ ${ }^{1}$ FSUE VNIIFTRI, Moscow region, Solnechnogorsky district, Mendeleevo, \\ ${ }^{2}$ Sternberg Astronomical Institute, Moscow State University, Universitetsky pr., 13, Moscow 119234, Russia
}

Main Metrological Center of the State Service for time, frequency and EOP evaluation (MMC SSTF) regularly carries out work in the field of Earth orientation parameters (EOP) evaluation and predicting in service mode. EOP activities and improvement of the MMC SSTF hardware and software for EOP evaluation purposes in 2020 - early 2021 as well as plans for the near future are reviewed in the report.

Keywords: polar motion, UT1-UTC, EOP service, VLBI, GNSS, SLR, combination

DOI: $10.51194 /$ VAK2021.2022.1.1.027

\section{Current State}

The Earth orientation parameters (EOP) plays very important role for astronomy and geodesy. The evaluation and prediction of EOP are very important also for navigation with GNSS as part of ephemerides of satellites.

MMC SSTF contributes to world satellite measurements data by providing following regular activities [1]:

- regular satellite measurements (GNSS and SLR) at the points MDVJ and MDVS of metrological control site of the Rosstandart network, which are also are included in national (FAGS) and international networks (IGS, ILRS, EPN);

- collection and preprocessing of satellite measurement data from other sites of metrological control of the Rosstandart network located in East Siberian (Irkutsk), West Siberian (Novosibirsk), Far Eastern (Khabarovsk) and Kamchatsky (Petropavlovsk-Kamchatsky) branches of FSUE VNIIFTRI (These points are also part of the networks of national and international satellite measurement networks).

Stability of sites in ITRF is controled by it's GNSS (GPS and GLONASS) coordinates estimations at every processing step. The internal time scales of GNSS receivers are controled by comparison of PPS output of receivers and PPS hydrogen masers which generated external frequency sources for receivers. The hydrogen masers are included in Time and Frequencies Standards and therefore the direct comparison of receiver's clock with UTC(SU) is possible.

The SLR measurements in Mendeleevo and Irkutsk by laser systems named "Sazhen-TM" are providing when weather allows [2,3]. The results of measurements are regular sent in EUROLAS (EDC data base). Because off State special primary standard of unit of length also situated in VNIIFTRI, the calibration SLR distance get on unit of length from it.

Today the EOP rows are obtained in operative mode from GNSS, VLBI and SLR data processing, separately for every geodetic technics [1].

The GNSS data includes approximately 35 GNSS receivers of the various organizations and departments(RSA, RAS, ROSSTANDART and others). The realization of method of Precise Point Positioning (PPP) on base of program package BERNESE 5.0 (AIUB, Switzerland) is used for GNSS daily processing.

The ARIADNA software [4] is used for operative IVS VLBI data processing. The VLBI observations from new IAA RAS 13-meters antenna (VGOS-standards) are processing for obtaining the UT1-UTC values with the help of software package OCCAM[5], specially adapted to the daily service mode.

The ILRS SLR data including Mendeleevo (VNIIFTRI) and Irkutsk (East Siberian branch of VNIIFTRI) are processed in daily mode by the software which was developed by E. Tsyba[6].

Software for GPS and GLONASS satellites orbit/clock determination were developed in VNIIFTRI [7]. Now the this software is under modernization in order that really operative service can be started.

The software of UT1 evaluation based on Lunar Laser Ranging measurements is created in the MATLAB environment[1].

The neural networks algorithms are used for geodetic satellites orbit prediction in VNIIFTRI[8].

The software for determination defection of vertical, geoid heights and gravity anomalies from satellite altimetry data also has developed in VNIIFTRI.

In IERS (International Earth's Rotation and Reference System Service) the operative EOP evaluation and prediction is provided by the IERS Rapid Service/Prediction Centre. Now this role is assigned to USNO USA.

In State Service for time, frequency and EOP evaluation (SSTF) the operative EOP evaluation and prediction is provided by the Main Metrological Center of SSTF (MMC SSTF). Now this role is assigned to the NIO-7 of FSUE VNIIFTRI.

MMC SSTF regularly carries out work in the field of Earth orientation parameters (EOP) evaluation and predicting in service mode. The combined SSTF EOP values are formed as result of combination of satellite 
and VLBI data and EOP prediction. Combination EOP on time raws level are calculated in VNIIFTRI by the combination of the nine independent individual EOP series provided by following Russian analysis centers: MMC SSTF, IAA (Institute of Applied Astronomy), IAC (Information-Analytical Center of Russian Space Agency) and SVOEVP (Russian Space Agency). Using of 13-meters IAA RAS antennas data allows significantly to increase accuracy of UT1 combination values.

The EOP SSTF were published in the form of SSTF Bulletins and disseminate through Internet and ftp-server. The information about UT1 transmitted also in radio signals frame.

The EOP evaluation as result of the SINEX files combination continued in 2020 with the help of SINCom software package [9].

\section{Future plans}

To improve accuracy and reducing latency, the improvement of both measurement networks and data processing and analysis tools is needed.

For processing tools the improving the accuracy and reliability of evaluation of operation EOP data is planned by including in processing more measurements (measurements of other Beidou and Galileo navigation systems for GNSS processing), improving computing performance (parallelization); improving algorithms and methods for prediction of EOP, orbits and other parameters.

The improvement of measuring instruments is planned by transition to measuring by instruments of a new generation. The two new SLR instruments "Tochka" which meet the requirements SLR2000 were built in Mendeleevo (VNIIVTRI) and Irkutsk (North-Eastern branch of VNIIFTRI). These instruments have the instrumental error not exceeding units of millimeters at single measurement.

\section{Conclusion}

The current state and future plans of the Earth's orientation parameters evaluation activities in MMC SSTF are presented. More information one can find by anonymous access on addresses www.vniiftri.ru, pvz.vniiftri.ru and ftp.vniiftri.ru.

\section{References}

1. S. Pasynok, I. Bezmenov, I. Ignatenko, E. Tcyba, and V. Zharov, in Astrometry, Earth Rotation, and Reference Systems in the GAIA era, 135-140 (2020).

2. I. Y. Ignatenko, Section 8 ILRS Network of International Laser Ranging Service (ILRS) 2016-2019 Report, 8(66)-8(68), 2020.

3. V. A. Emelyanov, Section 8 ILRS Network of International Laser Ranging Service (ILRS) 2016-2019 Report, 8(47)-8(48), 2020.

4. V. E. Zharov, Osnovy radioastrometrii (In Russia) (2011).

5. O. Titov, V. Tesmer, and J. Bohm, OCCAM Version 5.0 Software. User Guide (2001).

6. E. Tcyba and O. Volkova, Proceedings of the Journees 2019, 1-4, 2020.

7. I. V. Bezmenov, Izmeritel'naya Tehknika (In Russia), volume 1 (2020).

8. E. Tcyba, O. Volkova, S. Panarin, and S. Pasynok, 2021.

9. O. Brattseva, I. Gayazov, S. Kurdubov, and V. Suvorkin, in Journées 2014 "Systèmes de référence spatiotemporels", 250-251 (2015). 


\title{
Determination of sunskirters' orbits on the example of comet 323P/SOHO
}

\author{
S. Pavlov, Yu. Medvedev \\ medvedev@iaaras.ru \\ Institute of Applied Astronomy of Russian Academy of Science, Saint-Petersburg, 191187, Russia
}

On the example of comet 323P/SOHO we demonstrate the method of orbit determination applied to sungrazing comets observed by SOHO telescope in several appearances. In this method, we determine several sets with non-gravitational acceleration parameters depending on the time interval when the corresponding non-gravitational forces were active. This approach helps us to unite all the available observations by one orbit, besides the change of non-gravitational forces over time becomes apparent. Furthermore, we analyzed the accuracy of observations made by SOHO compared to ground-based optical observations. Here we determine photo-center offset and show that the center-of-mass for comet $323 \mathrm{P} / \mathrm{SOHO}$ very likely to match the center-of-light of positional observations. We also estimated some physical parameters of the comet, in particular, the diameter of the nucleus corresponding to its absolute magnitude. The retrospective orbit evaluation was also considered.

Keywords: 323p/SOHO, sungrazers, sunskiters, Near-Sun comets, orbit determination

DOI: 10.51194 /VAK2021.2022.1.1.028

\section{SOHO comets and their observations}

Until recently, sungrazing comets were considered a rare and unusual phenomenon but with improving of observation techniques and especially after the launch of SOHO spacecraft the number of newly discovered sungrazing comets has increased dramatically. In June 2020 SOHO has opened its 4000th comet. To systematize the everincreasing data about these comets, 4 groups were identified depending on the proximity of Keplerian elements. Comets in each group are assumed to be the product of a series of consecutive repeated fragmentations of a larger body [1]. It was also proposed [2] to call comets approaching the Sun at a distance of about 5-15 Sun radii, $R_{\odot}$, "sunskirters" to distinguish them from "sungrazers", approaching the Sun closer than $5 R_{\odot}$.

However, it should be noted that all the comet observations made by the SOHO coronagraph are concentrated in around the perihelion, i.e. always almost in one point of the orbit. Therefore, in order to determine the semimajor axis, as well as the orbit, we need to process observations at least from two appearances. Sungrazers come to the Sun too close to survive after the passage of perihelion and usually represent the final phase of a such comet life [3]. Sunskirters are more lucky, and sometimes observed in more than two appearances, which allows considering transversal non-gravitational accelerations, $A_{2}$, when determining the orbit. But other components of non-gravitational forces cannot be determined due to the highly uneven distribution of observations.

\section{Comet 323P/SOHO}

Comet $323 \mathrm{P} / \mathrm{SOHO}$ is a sunskirter, approaching the Sun at a distance of about $8 R_{\odot}(0.04$ au). Its Keplerian elements differ from any of sungrazing comet groups: a relatively low inclination, about $5.4^{\circ}$, and a slightly rotated line of apsides comparing to the Marsden and Kracht groups [4]. It also has close approaches to Jupiter, which could have affected on separating of its orbit.

\subsection{Comet 323P/SOHO: Analysis of observations}

Comet $323 \mathrm{P} / \mathrm{SOHO}$ is one of the most observed sungrazing comets. It has positional observations in 6 appearances, and in last one (in 2021) it was observed from the Earth (see 1). Ground-based observations allowed to improve the initial orbital elements, however, it became possible thanks to a predetermined orbit utilizing SOHO observations.

Thus we got the opportunity to compare the accuracy of observations made by SOHO in respect to groundbased positional observations. We fitted orbits using observations from a couple of nearby appearances and compared resulting root mean square residuals, RMS. Since SOHO observations from middle appearances were presented in two pairs, we used the arithmetic mean over the two results to represent the RMS for these observations. Orbit in last appearance was fitted separately with ground-based observations. Obtained values of RMS in angular seconds are presented in the Table 1 . As one can see, the ground-based positional observations are almost 400 times more accurate than SOHO ones.

We also checked the occurrence of photo-center offsets in observations. The offsets were determined separately for observations in each appearance in a pair. Thus for middle SOHO observations the arithmetic mean was calculated. The values Offset and their errors (in $\mathrm{km}$ ) are presented in the Table 1. From here it is clear, that photo-center offsets for this comet actually are not determined, thereby we considered the center-of-mass of the comet matches center-of-light. 


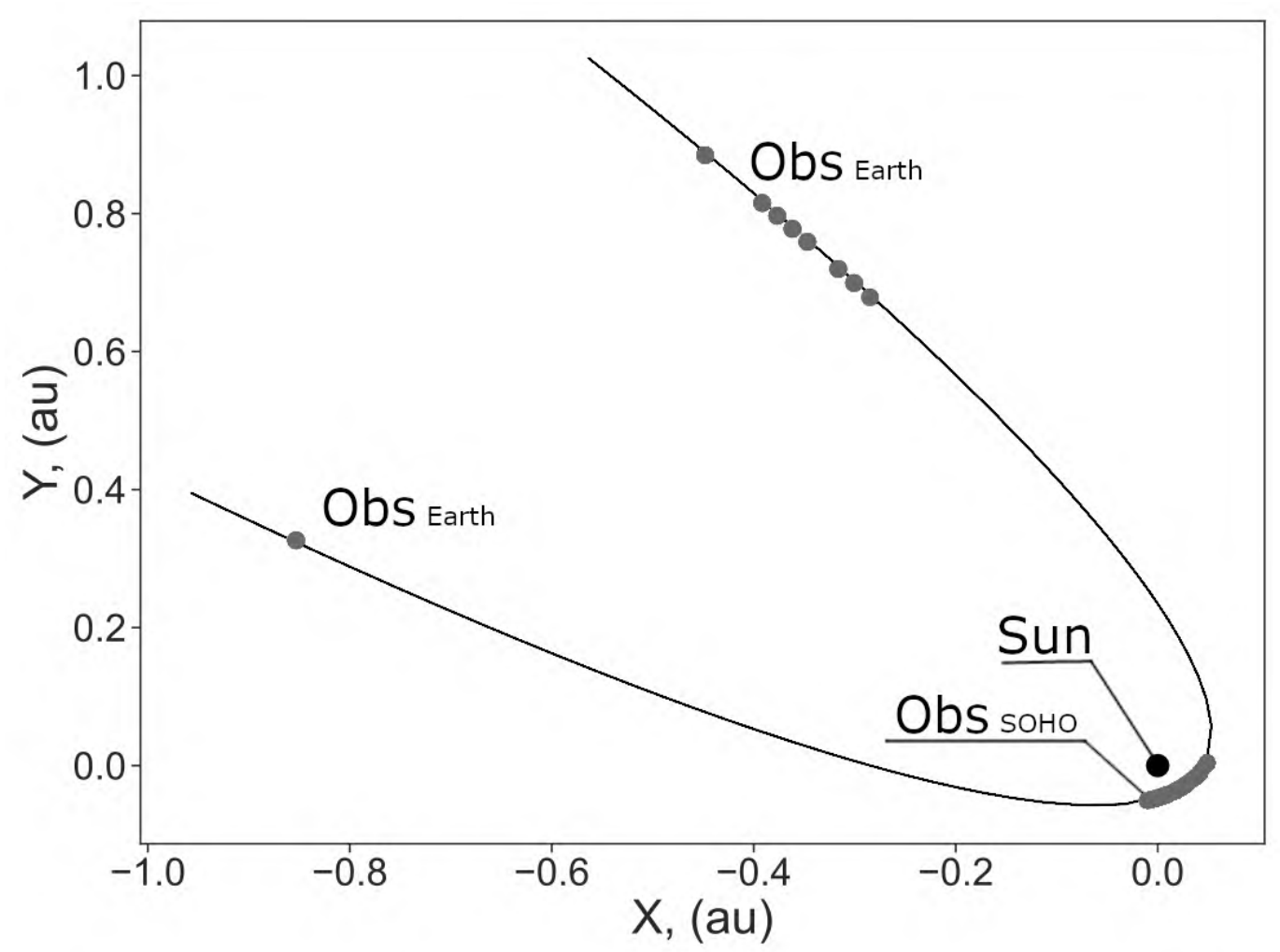

Figure 1: Distribution of 323P/SOHO observations along the orbit

Table 1: Distribution and accuracy of 323P/SOHO positional observations by appearances

\begin{tabular}{rcccccc}
\hline App. & 1999 & 2004 & 2008 & 2012 & 2016 & 2021 \\
\hline Num & 9 SOHO & 8 SOHO & 6 SOHO & 32 SOHO & 39 SOHO & 27 Earth \\
Time & {$[-0.39:-0.29]$} & {$[-0.02: 0.11]$} & {$[-0.44:-0.29]$} & {$[-0.32: 0.21]$} & {$[-0.40:-0.07]$} & {$[-27.00: 30.60]$} \\
Eq & 18 & 16 & 12 & 59 & 64 & 54 \\
RMS & 15.52 & 17.20 & 19.44 & 23.12 & 26.24 & 0.06 \\
Offset & $-1612 \pm 7800$ & $-8629 \pm 5090$ & $-1270 \pm 1135$ & $-16 \pm 1549$ & $266 \pm 616$ & $-0.06 \pm 0.13$ \\
\hline
\end{tabular}

The first line of Table 1 contains the years, when the comet passed perihelion (i.e. appearances). The second line contains the number and type of observations by appearances. The third line contains time intervals, representing when the first- and the last one observations were made with respect to the moment of perihelion. The value, Eq, in the Table 1 represents the number of least squares equations obtained for current appearance that were used for improving the orbit (it equals to twice the number of observations in the appearance if all equations were included).

\subsection{Comet 323P/SOHO: Determining the orbit over all available observations}

While determining the $323 \mathrm{P} / \mathrm{SOHO}$ orbit, we could not unite observations from all appearances using constant non-gravitational parameter $A_{2}$. Therefore we assumed that $A_{2}$ changes with time. We divided the time elapsed from the first observation to the last one into several intervals and separately determined the non-gravitational acceleration parameters on each of them.

Thus we determined coordinates, velocities and three sets of non-gravitational acceleration parameters with $A_{2}$. The Table 2 shows resultant values of $A_{2}$ and its errors by intervals. From here we can see the $A_{2}$ change over time, increasing in absolute value, which, however, may be associated with the usage of more accurate ground-based observations in the last appearance. 
Table 2: Results of orbit determination. Sets with non-gravitational transverse acceleration parameters

\begin{tabular}{cccc}
\hline Time interval & $A_{2} \pm$ error & $\left(1 \cdot 10^{-11} a u / d^{2}\right)$ \\
\hline $2012-2021$ & -2.188 & \pm & 0.019 \\
$2008-2012$ & -0.682 & \pm & 0.034 \\
$1999-2008$ & -0.634 & \pm & 0.032 \\
\hline
\end{tabular}

\subsection{Comet 323P/SOHO: Some physical parameters estimations and origin investigation}

In this section we represent the results when estimating the physical parameters of comet $323 \mathrm{P} / \mathrm{SOHO}$ and its possible retrospective dynamics. Using the energy balance equation [5], we have calculated the temperature difference on the comet's surface at aphelion and perihelion. It reached almost $1500^{\circ} \mathrm{C}$, from $-150^{\circ} \mathrm{C}$ in aphelion up to $1350^{\circ} \mathrm{C}$ in perihelion. It is less than that of sungrazers (up to $3500^{\circ} \mathrm{C}$ in perihelion) but definitely too much for a long lifetime on such an orbit.

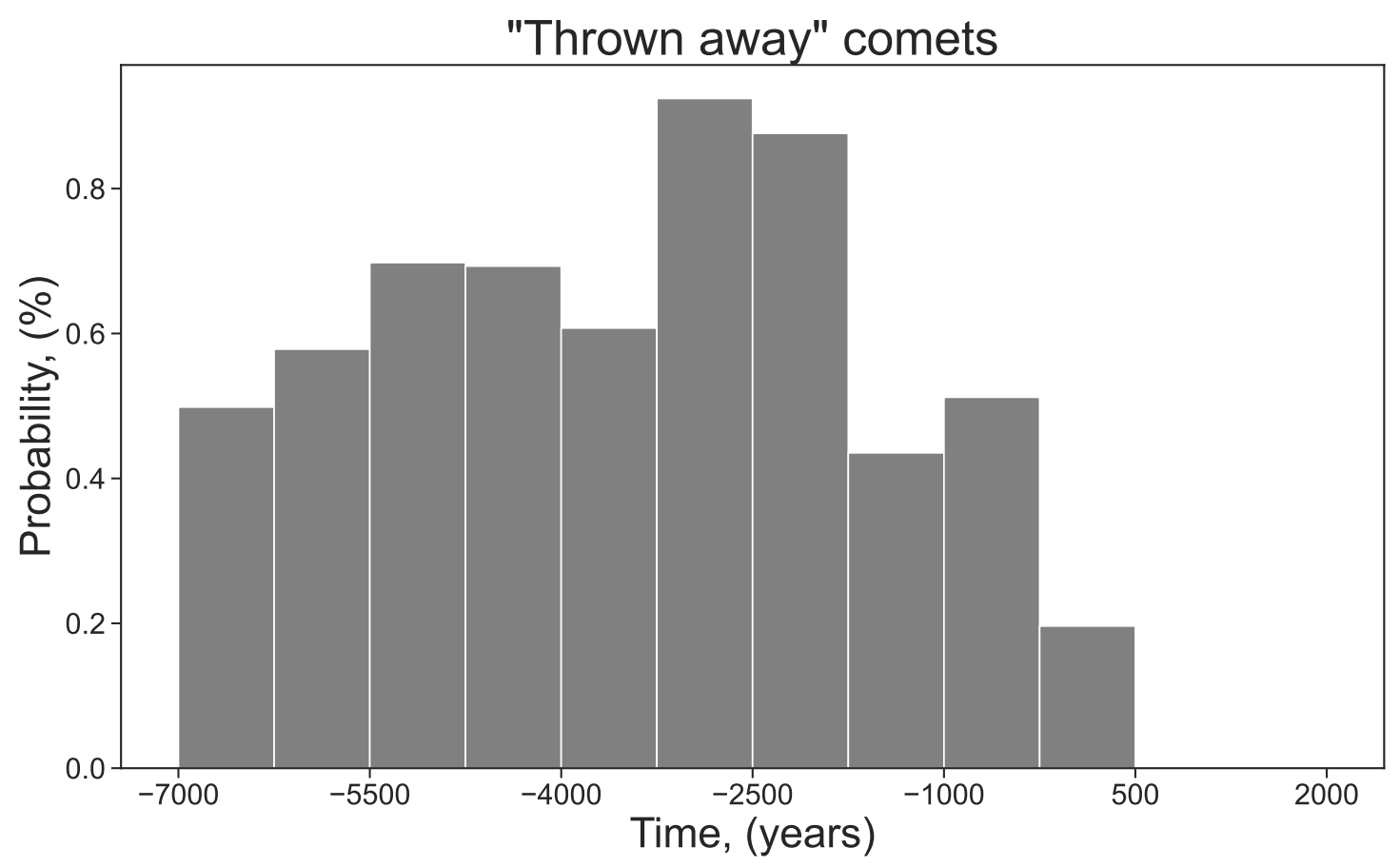

Figure 2: Probability for comet 323P/SOHO to leave the Jupiter-family by years

We estimated the comet diameter to be about $30 \mathrm{~m}$, with the absolute magnitude $H_{0}=24.3$ and an albedo of about $50 \%$. Hereafter we estimated how much the radius decreases per revolution. Calculations showed that it is about $3.5 \mathrm{~m}$ for a water ice cometary nucleus. Consequently, either this comet will evaporate in nearest years or it does not consist of water ice. Small values of non-gravitational effects and the absence of photo-center offset point in favor of the latter assumption.

We also explored the retrospective evolution of the comet. Like most Near-Sun comets belonging to the Jupiter family, it is highly chaotic. Our estimates of the Lyapunov time, obtained by the usage of MEGNO technique [6], show that it is about 40 years. Such a short period of time does not allow exploring the comet's orbit outside of observations for a longer time, when integrating the motion equations for the nominal orbit. So we had to integrate a cloud of virtual comets. We used 2550 comet copies, which were distributed according to the multivariate normal law with the correlation matrix obtained by least squares, while improving the orbits.

The results showed that some comets from the cloud approached very close to Jupiter and went into elliptical orbit with a huge semi-major axis or a hyperbolic one. As one can see 2, the chances of a close approach to Jupiter 
for examining comet are rather small during the past 1500 years. Note that this only true if non-gravitational forces will not change significantly with time.

Therefore, we computed transversal non-gravitational accelerations, which allowed the comet in nearest years approach Jupiter close enough to be thrown away. We used the following law:

$$
A_{2}(k)=k \cdot \exp \left(\frac{t_{0}-t}{t_{\text {period }}}\right)
$$

to change transversal non-gravitational acceleration parameter with time, where $t_{0}$ is time of the first observation, $t$ is current time, $t_{\text {period }}$ is a period of the comet in days and $k$ is some turning parameter. We found that if $k=-0.0013$, which means that in 1967 (the year of close approach) $A_{2}=-2.5 \cdot 10^{-8} \mathrm{au} / \mathrm{day^{2 }}$, the comet had to approach Jupiter at a distance of about $0.007 \mathrm{au}$.

\section{Conclusion}

The possibility of determining the orbits and non-gravitational effects from the SOHO observations is shown for sungrazing comet 323P/SOHO. We estimated the accuracy of SOHO observations and found out that they are about 400 times less accurate than ground-based ones for this comet.

We also found out that the center-of-mass for comet $323 \mathrm{P} / \mathrm{SOHO}$ very likely to match center-of-light.

We developed approach that allowed us to combine all the available observations of the comet by unite orbit. Several sets, containing non-gravitational acceleration parameter $A_{2}$, acting separately depending on the time interval were determined for the orbit. It allowed us to figure out how non-gravitational forces change over time. It turned out that they increase in absolute value with time for the comet.

Furthermore, some physical parameters of comet 323P/SOHO were estimated. The temperatures on comet's surface at aphelion and perihelion were calculated from energy balance. We estimated the comet diameter out from the absolute magnitude, which has to be about $30 \mathrm{~m}$ for albedo around $50 \%$.

We also investigated the retrospective evolution of the comet. We calculated the average-MEGNO parameter for the comet orbit, which showed that it is strongly chaotic, and estimated Lyapunov time, which did not exceed 40 years. Therefore, we formed a cloud of virtual comets, taking into account the covariance matrix, and traced its retrospective evolution. We found out that some comets may be "thrown away" due to a close approach with Jupiter and calculated this probability for comets from the cloud. We also calculated non-gravitational accelerations, which provide the closest approach to Jupiter (less than $0.007 \mathrm{au}$ ) in 1967.

\section{References}

1. K. Battams and M. M. Knight, Philosophical Transactions of the Royal Society A: Mathematical, Physical and Engineering Sciences, 375, 2017, URL http://dx.doi.org/10.1098/rsta.2016.0257.

2. Z. Sekanina and P. W. Chodas, ApJS, 161, 551, 2005.

3. M. M. Knight, Studies of SOHO comets, Ph.D. thesis, University of Maryland, College Park, 2008.

4. O. V. Kalinicheva, Open Astronomy, 27, 303, 2018.

5. G. H. Jones, M. M. Knight, K. Battams, D. C. Boice, et al., Space Sci. Rev., 214, 20, 2018.

6. P. Cincotta and C. Giordano, Lecture Notes in Physics, 2014. 


\title{
Estimation of the age of two young asteroid pairs with close orbits
}

\author{
V. Safronova, E. Kuznetsov \\ v.s.safronova@urfu.ru; eduard.kuznetsov@urfu.ru \\ Ural Federal University, Lenina Avenue 51, Yekaterinburg 620000, Russia
}

We investigated the probabilistic evolution of two young asteroid pairs: (87887) 2000 SS286 - (415992) 2002 AT49 and (320025) 2007 DT76 - (489464) 2007 DP16. We analyzed the low relative-velocity close encounters of objects to estimate the age of young asteroid pairs. We obtained the age estimates for asteroid pairs for a set of fixed values of the semi-major axis drift velocity due to the influence of the Yarkovsky effect.

Keywords: Kholshevnikov metrics, young pairs of asteroids, dynamic evolution, probabilistic evolution

DOI: 10.51194/VAK2021.2022.1.1.029

\section{Introduction}

The methods for estimating the closeness of Keplerian orbits were investigated by [1]. We used the Kholshevnikov metrics $\varrho_{2}$ and $\varrho_{5}$ to search for asteroid pairs with close orbits in the Main Belt. [2] selected young pairs based on the study of the dynamic evolution of asteroid pairs with close orbits along nominal trajectories. This paper presents a study of the probabilistic evolution of two young asteroid pairs: (87887) 2000 SS286 - (415992) 2002 AT49 and (320025) 2007 DT76 - (489464) 2007 DP16 was investigated to estimate their age.

\section{Method}

For each asteroid, 1000 clones of orbits were generated with initial data from the confidence region within three standard deviations in the vicinity of the nominal orbit. We obtained 1 million variants of the evolution for each pair. The simulation was carried out in the program Orbit9 [3]. The equations of motion of eight major planets, the dwarf planet Pluto and the asteroids, were jointly integrated, considering the Yarkovsky effect, the oblateness of the Sun, and relativistic effects. The integration interval was $30 \mathrm{kyr}$.

We estimated the drift rate of the semi-major axis $d a / d t$ for each asteroid to account for the influence of the Yarkovsky effect. We used the normalization by the parameters of the asteroid (101955) Bennu known with high accuracy [4]:

$$
\frac{d a}{d t}=\left(\frac{d a}{d t}\right)_{\text {Bennu }} \cdot \frac{\sqrt{a_{B}}}{\sqrt{a}} \cdot \frac{\left(1-e_{B}^{2}\right)}{1-e^{2}} \cdot \frac{D_{B}}{D} \cdot \frac{\rho_{B}}{\rho} \cdot \frac{\cos \phi}{\cos \phi_{B}} \cdot \frac{1-A}{1-A_{B}} .
$$

Here the symbols with a "B" refer to asteroid (101955) Bennu, $a$ is a semi-major axis, $e$ is a eccentricity, $D$ is a diameter, $\rho$ is a density, $\phi$ is a obliquity, and $A$ is the Bond albedo.

The obliquity $\phi$ of the asteroid's axis of rotation to the orbital plane is unknown for the considered asteroids. We consider the maximal value of modulus of semi-major axis drift $|d a / d t|_{\max }$ for $\cos \phi=1$. Therefore, in contrast to the traditional approach, when taking into account the Yarkovsky effect, we obtained age estimates for several fixed values of the semi-major axis drift rates corresponding to different obliquities of the asteroid's axis of rotation to the orbital plane:

$d a / d t=0$ at $\phi=90^{\circ}, d a / d t=1 / 2|d a / d t|_{\max }$ at $\phi=60^{\circ}, d a / d t=|d a / d t|_{\max }$ at $\phi=0^{\circ}, d a / d t=$ $-1 / 2|d a / d t|_{\max }$ at $\phi=240^{\circ}, d a / d t=-|d a / d t|_{\max }$ at $\phi=180^{\circ}$.

We analyzed the low relative-velocity close encounters of objects to estimate the age of young asteroid pairs [5]: $\Delta r<10 R_{H i l l}, \Delta v<4 V_{e s c}$. Here $R_{\text {Hill }}$ is the radius of the Hill sphere of a more massive asteroid in pair, $V_{e s c}$ is the escape velocity to a more massive asteroid, $\Delta r$ is the distance between asteroids, $\Delta v$ is the relative speed of asteroids.

\section{Results}

According to our estimates, for asteroids of the pair (87887) 2000 SS286 - (415992) 2002 AT49, the maximum values of the drift rates of the semi-major axes $|d a / d t|_{\max }$ are $1.1 \times 10^{-4} \mathrm{AU} / \mathrm{Myr}$ and $1.9 \times 10^{-4} \mathrm{AU} / \mathrm{Myr}$, respectively. The estimates of the pair's age, depending on the semi-major axis drift rates, range from $7.58 \pm 0.04$ to $8.80 \pm 0.04 \mathrm{kyr}$, which is close to the $7.4 \pm 0.3 \mathrm{kyr}$ obtained [6].

For the pair (320025) 2007 DT76 -(489464) 2007 DP16 the maximum values of the drift rates $|d a / d t|_{\max }$ are $1.1 \times 10^{-4} \mathrm{AU} / \mathrm{Myr}$ and $1.9 \times 10^{-4} \mathrm{AU} / \mathrm{Myr}$, respectively. The pair's age estimations range from $15.46 \pm 0.97$ to $24.75 \pm 2.37 \mathrm{kyr}$. [6] have estimated the age of this pair as is more than $10 \mathrm{kyr}$.

\section{Discussion and Conclusion}

The asteroid pair age estimations agree with the estimates obtained by [6]. We have obtained more stringent estimates of the range of possible ages of the studied pairs of asteroids proposed approach to estimating the age 
of asteroid pairs for a set of fixed values of the semi-major axis drift rates due to the Yarkovsky effect. To improve the pair's age estimation, obtaining the semi-major axis drift rates from observation is necessary.

Acknowledgments This work has been supported by the Russian Ministry of Science and Higher Education via the State Assignment Projects FEUZ-2020-0030 (EK) and FEUZ-2020-0038 (VS).

\section{References}

1. K. V. Kholshevnikov, G. I. Kokhirova, P. B. Babadzhanov, and U. H. Khamroev, Mon. Not. R. Astron. Soc., 25, 2275, 2016.

2. E. D. Kuznetsov, A. E. Rosaev, E. Plavalova, V. S. Safronova, and M. A. Vasileva, Solar System Research, 54, $236,2020$.

3. Orbfit Consortium, OrbFit: Software to Determine Orbits of Asteroids, Astrophysics Source Code Library, 2011.

4. F. Spoto, A. Milani, and Z. Knezevic, Icarus., 257, 275, 2015.

5. P. Pravec, P. Fatka, D. Vokrouhlický, P. Scheirich, et al., Icarus, 333, 429, 2019.

6. J. Žižka, A. Galed, D. Vokrouhlicky, P. Pravec, P. Kušnirak, and K. Hornoch, Astron. and Astrophys., 595, $2275,2016$. 


\title{
Comparison of the semi-major axis drift taking into account the Yarkovsky effect in two orbital reference frames
}

\author{
T.N. Sannikova \\ tnsannikova@craocrimea.ru
}

Crimean Astrophysical Observatory, Russian Academy of Sciences, Nauchny, 298409 Crimea

Let the asteroid move under the influence of the attraction to the Sun and the Yarkovsky force. For this problem, the long-term evolution of the semi-major axis is investigated by solving the averaged equations of motion. The drift of the semi-major axis of the object's orbit occurs under the action of the transverse or tangential component of the perturbing acceleration, but, obviously, the drift magnitude should not depend on the choice of the frame of reference. We found the numerical values of the transverse and tangential components of acceleration as average values over the orbital period based on the linear thermophysical model of the Yarkovsky force and calculated both semi-major axis drifts over 1 million years for 12 asteroids with various orbital eccentricities. Comparative analysis showed that when simulating the Yarkovsky effect by transverse acceleration, the drift estimate can be overestimated for objects with highly elliptical orbits.

Keywords: Yarkovsky effect, tangential acceleration, transverse acceleration, semi-major axis drift

DOI: $10.51194 /$ VAK2021.2022.1.1.030

The influence of the Yarkovsky effect mainly affects the change in the semi-major axis of the body's orbit. Often, when studying the evolution of the orbit taking into account the Yarkovsky effect, the perturbing acceleration is simulated by the transverse component. However, the tangential component has a more direct effect on the change in the object's velocity and, as a consequence, on the drift of the semi-major axis. This is not important for circular orbits and at small eccentricities, when the transverse and tangential components practically coincide, but this is not the case for highly elliptical orbits. We have determined the semi-major axis drifts over one million years for 12 asteroids with various orbital eccentricities in two orbital reference frames and compared them. We have obtained a significant difference in the semi-major axis drifts for objects with an orbital eccentricity greater than 0.7, although, obviously, the drift magnitude should not depend on the choice of the frame of reference.

We have considered the asteroid's motion $\mathcal{A}$ under the influence of the attraction to the Sun $\mathcal{S}$ and additional perturbing acceleration $\mathbf{P}^{\prime}$, which arises due to the Yarkovsky effect. The strength of this effect is inversely proportional to the squared distance from the Sun; therefore, we have assumed that $\mathbf{P}^{\prime}=\mathbf{P} / r^{2}$. We have introduced two orbital reference frame with a common origin $\mathcal{S}$, but with differently oriented axes. The axes for the $O_{1}$ frame are directed along the radius vector, the transversal (perpendicular to the radius vector in the osculating plane in the direction of motion), and the binormal (directed along the area vector). The axes for the $\mathrm{O}_{2}$ frame are directed along the velocity vector, along the normal to this direction in the plane of the osculating orbit, and along the binormal. We have supposed that the acceleration $\mathbf{P}^{\prime}$ be small in comparison with the main acceleration $\varkappa^{2} / r^{2}$ :

$$
\max \frac{\left|\mathbf{P}^{\prime}\right|}{\varkappa^{2} r^{-2}}=\max \frac{|\mathbf{P}|}{\varkappa^{2}}=\mu \ll 1
$$

where $\mathbf{r}=\mathcal{S} \mathcal{A}, r=|\mathbf{r}|, \varkappa^{2}$ is the product of the gravitation constant by the mass $\mathcal{S}$. And also we have assumed that components $T, N$, and $W$ of the vector $\mathbf{P}$ be constant in the $O_{1}$ frame, and be small quantities on the order of $\mu(1)$, and likewise components $\mathfrak{T}, \mathfrak{N}$, and $W$ of the vector $\mathbf{P}$ be constant in the $O_{2}$ frame, and be small quantities on the order of $\mu(1)$.

Sannikova and Kholshevnikov [1] have applied an averaging procedure to the Euler-type equations of motion and have obtained mean-element motion equations in the first order of smallness for both frames. Since the change in the semi-major axis occurs under the influence of either transverse or tangential acceleration, we have put $S=W=0, \mathfrak{N}=W=0$ and have got a particular solutions in the form of

$$
t=\frac{\varkappa^{2}}{n_{0} T}\left(\frac{\eta_{0}}{1-\eta_{0}}\right)^{3}\left(2 \ln \frac{\eta}{\eta_{0}}+\frac{1}{\eta}-\eta-\frac{1}{\eta_{0}}+\eta_{0}\right), \quad n=n_{0}\left[\frac{\eta\left(1-\eta_{0}\right)}{\eta_{0}(1-\eta)}\right]^{3}
$$

in the reference frame $\mathcal{O}_{1}$, and in the form of

$$
\begin{aligned}
t & =\frac{\pi \eta_{0}^{3} \varkappa^{2}}{4 n_{0} \mathfrak{T}} \int_{e_{0}}^{e} \frac{e}{\eta^{3}\left[\mathbf{E}(e)-\eta^{2} \mathbf{K}(e)\right]}\left(\exp \int_{e_{0}}^{e} \frac{3 x \mathbf{K}(x) d x}{2\left[\mathbf{E}(x)-\left(1-x^{2}\right) \mathbf{K}(x)\right]}\right) d e \\
n & =n_{0}\left(\frac{\eta}{\eta_{0}}\right)^{3} \exp \left[-\int_{e_{0}}^{e} \frac{3 e \mathbf{K}(e) d e}{2\left[\mathbf{E}(e)-\eta^{2} \mathbf{K}(e)\right]}\right]
\end{aligned}
$$

in $\mathcal{O}_{2}$ frame. Here, $n$ is the mean motion, $e$ is the eccentricity, $\eta=\sqrt{1-e^{2}}$; the subscript " 0 " indicates the values of the elements in the initial epoch $t=0$; and we also have used the semi-major axis $a=\varkappa^{2 / 3} n^{-2 / 3}$ and the 
Table 1: Yarkovsky acceleration components, their corresponding semi-major axis drifts and their differences in percentage

\begin{tabular}{|c|c|c|c|c|c|c|c|}
\hline Asteroid & $e$ & $\begin{array}{c}T, \\
10^{-15} \frac{\mathrm{AU}^{3}}{\mathrm{~d}^{2}}\end{array}$ & $\begin{array}{c}\mathfrak{T}, \\
10^{-15} \frac{\mathrm{AU} \mathrm{U}^{3}}{\mathrm{~d}^{2}}\end{array}$ & $\begin{array}{c}\left(\frac{|T|-|\mathfrak{T}|}{|T|}\right) \\
\times 100 \%\end{array}$ & $\begin{array}{c}(d a / d t)^{T}, \\
10^{-4} \frac{\mathrm{AU}}{\mathrm{Myr}}\end{array}$ & $\begin{array}{c}(d a / d t)^{\mathfrak{T}}, \\
10^{-4} \frac{\mathrm{AU}}{\mathrm{Myr}}\end{array}$ & $\left.\begin{array}{c}\left(\frac{\left|\frac{d a}{d t}\right|^{T}-\left|\frac{d a}{d t}\right|^{\mathfrak{2}}}{\left.\frac{\mid d a}{d t}\right|^{T}}\right. \\
\times 100 \%\end{array}\right)$ \\
\hline 2009 BD & 0.05 & -1228.14 & -1227.32 & 0.07 & -513.76 & -513.78 & -0.004 \\
Apophis & 0.19 & -37.538 & -37.187 & 0.94 & -17.24 & -17.28 & -0.232 \\
Bennu & 0.20 & -46.982 & -46.485 & 1.06 & -19.62 & -19.67 & -0.255 \\
Itokawa & 0.28 & -30.248 & -29.634 & 2.03 & -12.11 & -12.17 & -0.495 \\
Toro & 0.44 & -3.201 & -3.073 & 4.00 & -1.43 & -1.51 & -5.594 \\
Apollo & 0.56 & -6.107 & -5.548 & 9.15 & -3.12 & -3.13 & -0.321 \\
Golevka & 0.61 & -7.137 & -6.460 & 9.49 & -3.08 & -3.18 & -3.247 \\
Toutatis & 0.62 & -5.736 & -5.068 & 11.65 & -2.50 & -2.56 & -2.400 \\
1999 KW4 & 0.69 & -10.095 & -8.544 & 15.36 & -10.17 & -9.69 & 4.720 \\
1999 FK21 & 0.70 & -12.454 & -10.514 & 15.58 & -12.17 & -11.63 & 4.437 \\
Icarus & 0.83 & -6.336 & -4.661 & 26.44 & -8.20 & -7.17 & 12.561 \\
Phaethon & 0.89 & -1.930 & -1.269 & 34.25 & -3.49 & -2.84 & 18.625 \\
\hline
\end{tabular}

standard notation for complete elliptic integrals in normal trigonometric form:

$$
\mathbf{K}(e)=\int_{0}^{\pi / 2} \frac{d x}{\sqrt{1-e^{2} \sin ^{2} x}}, \quad \mathbf{E}(e)=\int_{0}^{\pi / 2} \sqrt{1-e^{2} \sin ^{2} x} d x .
$$

The first expression in systems (2), (3) is a kinematic equation that allows to find the value of $e$ for a given $t$.

To find the semi-major axis drift, we need to know the numerical values for the transverse and tangential components included in solutions (2) and (3). To calculate them, we have used expressions for the $T$ and $\mathfrak{T}$ as average values over an orbital period obtained on the basis of a linear thermophysical model of the Yarkovsky force for spherical asteroids developing by Vokrouhlický [2]. Relying on [2], Xu et al. [3] derived radial $P_{r}$, transversal $P_{t}$, and normal $P_{n}$ components of the Yarkovsky acceleration in a projection onto the axes of the $\mathcal{O}_{1}$ frame $[3$, relations (12)]. With their help, in [4] the transverse component $T$ has been deduced. We have derived $\mathfrak{T}$ in a similar way, taking into account its relationship with the radial $P_{r}$ and transverse $P_{t}$ components as $P_{\mathfrak{T}}=P_{r} \sin f+P_{t} \cos f$, where $f$ is the angle to rotate the velocity vector to align with the transversal.

The calculation of the values of the components $T$ and $\mathfrak{T}$ of the vector $\mathbf{P}$ requires knowledge such characteristics of the asteroid as its diameter, bulk density, rotation rate, obliquity of the spin axis to the orbital plane, Bond albedo, surface thermal inertia, specific heat capacity, and thermal emissivity.

We have found acceleration components and semi-major axis drifts over 1 million years for 12 asteroids, which we have chosen so that eccentricities of their orbits are, if possible, evenly distributed in the range $(0,1)$. We have collected the initial data for calculating the acceleration components from various sources.

The table 1 shows the results of calculations: the transverse $T$ and tangential $\mathfrak{T}$ components of the Yarkovsky acceleration, their corresponding the semi-major axis drifts $(d a / d t)^{T}$ and $(d a / d t)^{\mathfrak{T}}$ over 1 million years $($ Myr), as well as the difference in the values of the acceleration components and drifts in percentage.

Comparative analysis have showed that the transverse and tangential components of Yarkovsky's acceleration practically coincide at low eccentricities, as it should be according to theory. Further, the tangential component is less (in absolute value) than the transverse one for the same thermophysical parameters of the body, and the difference in percentage is the greater, the closer the eccentricity is to unity.

Semi-major axis drifts $|d a / d t|^{\mathfrak{T}}$ (modulo) and $|d a / d t|^{T}$, calculated respectively on the basis of tangential and transversal acceleration, differ by less than $1 \%$ at low eccentricities $(e<0.3)$. Their difference does not exceed $6 \%$ for $0.3<e<0.7$, but for asteroid 1566 Icarus $(e=0.827)$ the drifts differ by $12.6 \%$, and for 3200 Phaethon $(e=0.89)$ by $18.6 \%$, although it is obvious that the semi-major axis drift magnitude should not depend on the choice of the frame of reference.

Thus, if the Yarkovsky effect is simulated by transverse acceleration, then the estimate of the semi-major axis drift may be overestimated for objects with highly elliptical orbits or the simplified methods of accounting for Yarkovsky effect are not suitable in this case. 


\section{References}

1. T. N. Sannikova and K. V. Kholshevnikov, Astron. Rep., 63, 420, 2019.

2. D. Vokrouhlický, Astron. Astrophys., 344, 362, 1999.

3. Y. B. Xu, L. Y. Zhou, C. Lhotka, and W. H. Ip, Mon. Not. R. Astron. Soc., 493, 1447, 2020.

4. T. N. Sannikova, Astron. Rep., 65, 312, 2021. 


\title{
Metrics in The Space of Keplerian Orbits and in the Family of Its Quotient Spaces
}

\author{
A. Shchepalova \\ shepalovanastya@mail.ru \\ St. Petersburg State University, St. Petersburg, 199034 Russia
}

The distance functions on the set of Keplerian orbits play an important role in solving the problems of searching for the parent bodies of meteoroid streams. A special kind of function is the distances in the quotient spaces of orbits. Three metrics of this type were developed earlier. These metrics allow disregarding the longitude of the ascending node or the argument of pericenter, or both. Recently, we introduced one more quotient space, where two orbits are considered to be identical if they differ only in their longitudes of nodes and arguments of pericenters, but have the same sum of these elements (the longitude of pericenter).

Keywords: Keplerian orbit, metric, quotient space of metric space, distance between orbits

DOI: 10.51194/VAK2021.2022.1.1.031

\section{Introduction}

The investigation of the relation between meteoroid streams and asteroids, searching for the parent bodies of the streams is one of the most interesting problems of astronomy. The key in solving this is estimating the closeness of the orbits of celestial bodies, i.e., the choice of the metric on the set of orbits parametrized by some system of orbital elements. Over the past 15 years, a few metrics were proposed that transform various spaces of Keplerian orbits to metric spaces. An important role is played by the quotient spaces that allow disregarding those orbital elements that change secularly under various disturbances $[1,2,3,4,5,6]$.

Meanwhile, there are meteoroid streams for which the nodes and pericenters undergo large secular disturbances, while a change in their sum (the longitude of pericenter) is small. In this case, we need to introduce a quotient space in which the orbits with arbitrary longitudes of ascending nodes and arguments of pericenters are considered to be identical provided that their sum remains the same. Let us denote this space as $\mathbb{H}_{6}$.

\section{Spaces $\mathbb{H}_{2} \div \mathbb{H}_{5}$}

The elements of space $\mathbb{H}_{2}$ are all non-rectilinear Keplerian orbits $\mathcal{E}$. The orbit is defined by the following five Keplerian elements: $p, e, i, \Omega$ and $\omega$, which are the focal parameter, eccentricity, inclination, longitude of the ascending node, and argument of pericenter, respectively.

The elements of space $\mathbb{H}_{3}$ are the classes of orbits with fixed $p, e, i, \omega$, and arbitrary values of $\Omega$. The elements of space $\mathbb{H}_{4}$ are the classes of orbits with fixed $p, e, i, \Omega$, and arbitrary values of $\omega$. The elements of space $\mathbb{H}_{5}$ are the classes of orbits with fixed $p, e, i$, and arbitrary values of $\omega$ and $\Omega$.

Space $\mathbb{H}_{2}$ is five-dimensional, $\mathbb{H}_{3}$ and $\mathbb{H}_{4}$ are four-dimensional, and $\mathbb{H}_{5}$ is three-dimensional.

In [2], the methric $\varrho_{2}$ is introduced on space $\mathbb{H}_{2}$ such that its value on pair of orbits $\mathcal{E}_{1}$ and $\mathcal{E}_{2}$ is expressed through the Keplerian elements as follows:

$$
\begin{aligned}
& \varrho_{2}^{2}\left(\mathcal{E}_{1}, \mathcal{E}_{2}\right)=\left(1+e_{1}^{2}\right) p_{1}+\left(1+e_{2}^{2}\right) p_{2}-2 \zeta_{2}\left(\mathcal{E}_{1}, \mathcal{E}_{2}\right) \sqrt{p_{1} p_{2}}, \\
& \zeta_{2}\left(\mathcal{E}_{1}, \mathcal{E}_{2}\right)=\cos I+e_{1} e_{2} \cos P
\end{aligned}
$$

where $I$ is the mutual inclination and $P$ is the angle between the Laplace-Runge-Lenz vectors:

$$
\begin{aligned}
& \cos I=c_{1} c_{2}+s_{1} s_{2} \cos \Delta, \\
& \cos P=s_{1} s_{2} \sin \omega_{1} \sin \omega_{2}+\left(\cos \omega_{1} \cos \omega_{2}+c_{1} c_{2} \sin \omega_{1} \sin \omega_{2}\right) \cos \Delta+ \\
& +\left(c_{2} \cos \omega_{1} \sin \omega_{2}-c_{1} \sin \omega_{1} \cos \omega_{2}\right) \sin \Delta, \\
& c_{j}=\cos i_{j}, \quad s_{j}=\sin i_{j}, \quad \Delta=\Omega_{1}-\Omega_{2} .
\end{aligned}
$$

Metrics $\varrho_{3}-\varrho_{5}$ on spaces $\mathbb{H}_{3}-\mathbb{H}_{5}$ were constructed in $[2,3]$ by using the following procedure:

$$
\varrho_{3}=\min _{\omega_{1}, \omega_{2}} \varrho_{2}, \quad \varrho_{4}=\min _{\Omega_{1}, \Omega_{2}} \varrho_{2}, \quad \varrho_{5}=\min _{\Omega_{1}, \Omega_{2}, \omega_{1}, \omega_{2}} \varrho_{2} .
$$

It is easy to note that for $\varrho_{3}-\varrho_{5}$, the following formulas analogous to (1) are valid:

$$
\varrho_{k}^{2}\left(\mathcal{E}_{1}, \mathcal{E}_{2}\right)=\left(1+e_{1}^{2}\right) p_{1}+\left(1+e_{2}^{2}\right) p_{2}-2 \zeta_{k}\left(\mathcal{E}_{1}, \mathcal{E}_{2}\right) \sqrt{p_{1} p_{2}},
$$

where $\zeta_{k}\left(\mathcal{E}_{1}, \mathcal{E}_{2}\right)=\min \zeta_{2}\left(\mathcal{E}_{1}, \mathcal{E}_{2}\right)$ and the maximum is taken over the arguments corresponding to the number of metrics in (3). The exact expressions for $\zeta_{k}$ through the Keplerian elements were obtained in [3]. It appears that all three functions (3) satisfy the metric space axioms [7]-[7, 8, 9]. 


\section{Space $\mathbb{H}_{6}$}

The elements of four-dimensional quotient space $\mathbb{H}_{6}$ of space $\mathbb{H}_{2}$ are the classes of orbits with fixed $p, e, i$, and arbitrary values of $\omega, \Omega$ that satisfy condition $\omega+\Omega=\varpi$ at a fixed longitude of pericenter $\varpi$.

In [10], the minimum distance between the representatives of the equivalence classes was proposed to be a distance in quotient space $\mathbb{H}_{6}$ :

$$
\varrho_{6}=\min \varrho_{2},
$$

Here, when calculating the minimum, elements $p_{j}, e_{j}, i_{j}$ and $\varpi_{j}, j=1,2$ of both orbits are fixed, while angles $\Omega_{j}$ and $\omega_{j}$ vary, however, they remain connected by relationships $\Omega_{j}+\omega_{j}=\varpi_{j}$.

\section{Calculation of $\varrho_{6}$}

It is obvious that representation (5) holds for $\varrho_{6}$ as well:

$$
\varrho_{6}^{2}\left(\mathcal{E}_{1}, \mathcal{E}_{2}\right)=\left(1+e_{1}^{2}\right) p_{1}+\left(1+e_{2}^{2}\right) p_{2}-2 \zeta_{6}\left(\mathcal{E}_{1}, \mathcal{E}_{2}\right) \sqrt{p_{1} p_{2}}, \quad \zeta_{6}=\max \zeta_{2}
$$

However, as distinct from $\zeta_{3}-\zeta_{5}$, we were unable to express $\zeta_{6}$ through the orbit elements.

It was shown in [10] that the problem of finding the critical points of $\zeta_{2}$ on a pair of elements of space $\mathbb{H}_{6}$ is reduced to solving two equations of the following form with respect to $y$ :

$$
\begin{array}{lrl}
a_{0}+a_{1} \sin y+a_{2} \sin (y-\varpi)+a_{3} \sin (2 y-\varpi)+a_{4} \sin (2 y-2 \varpi)+a_{5} \sin (3 y-2 \varpi)=0 . & a_{1}=-e_{1} e_{2} s_{1} s_{2}\left(1+c_{1}+c_{2}-3 c_{1} c_{2}\right), \\
a_{0}=e_{1} e_{2} s_{1}^{2} s_{2}^{2} \sin \varpi, & a_{3}=-e_{1} e_{2}\left(1-c_{1}\right)\left(1-c_{2}\right)\left(1-c_{1}-c_{2}-3 c_{1} c_{2}\right), \\
a_{2}=-2 s_{1} s_{2}\left(1-c_{1} c_{2}\right)\left(2+\mu e_{1} e_{2}\right), & a_{5}=e_{1} e_{2} s_{1} s_{2}\left(1-c_{1}\right)\left(1-c_{2}\right), \\
a_{4}=s_{1}^{2} s_{2}^{2}\left(2+\mu e_{1} e_{2}\right), & & \\
\mu & = \pm 1 . &
\end{array}
$$

Let us briefly describe the algorithm of calculation of $\varrho_{6}$ presented in [10].

1. At $\mu=1$ and $\mu=-1,-\pi<y \leqslant \pi$,we find all real roots $y_{n}(\mu)$ of Eq. (11). At any $\mu$ there are at most six real roots.

2. For each root $y_{n}(\mu)$, exactly one value of $x_{n}(\mu)$ corresponds to it and it is calculated according to the following formulas:

$$
\begin{aligned}
\cos x & =\mu \frac{s_{1} s_{2}-\left(1-c_{1} c_{2}\right) \cos (y-\varpi)}{D}, \quad \sin x=\mu \frac{\left(c_{2}-c_{1}\right) \sin (y-\varpi)}{D} \\
D & =\left(1-c_{1} c_{2}\right)-s_{1} s_{2} \cos (y-\varpi), \quad \mu= \pm 1
\end{aligned}
$$

We obtain several (not more than 12) points of form $\left(x_{n}(\mu), y_{n}(\mu)\right)$.

3. For each pair $\left(x_{n}(\mu), y_{n}(\mu)\right)$, we determine $\zeta_{2}$ using formula

$$
\begin{aligned}
& \zeta_{2}(x, y)=A_{0}+A_{1} \cos (\varpi-y)+A_{2} \cos y-A_{2} \cos x+ \\
& +B_{1} \cos (\varpi-x-y)+B_{2} \cos (\varpi-2 y)+B_{3} \cos \varpi+B_{4} \cos (\varpi+x-y), \\
& A_{0}=c_{1} c_{2}, \quad A_{1}=s_{1} s_{2}, \quad 2 A_{2}=e_{1} e_{2} s_{1} s_{2}, \\
& 4 B_{1}=e_{1} e_{2}\left(1-c_{1}\right)\left(1+c_{2}\right), \quad 4 B_{2}=e_{1} e_{2}\left(1-c_{1}\right)\left(1-c_{2}\right), \\
& 4 B_{3}=e_{1} e_{2}\left(1+c_{1}\right)\left(1+c_{2}\right), \quad 4 B_{4}=e_{1} e_{2}\left(1+c_{1}\right)\left(1-c_{2}\right),
\end{aligned}
$$

4. The value of $\zeta_{6}$ is equal to the largest value from $\zeta_{2}\left(x_{n}(\mu), y_{n}(\mu)\right)$.

5. The sought value of $\varrho_{6}$ is given by formula $(6)$.

The presented algorithm is realized as a code, which is freely accessible here:

https://drive.google.com/drive/folders/1cGCTHqxqatGfReK3jXRrhhLEc8pdzJwk?usp=sharing

The code is performed in the $\mathrm{C}++$ computer language. To solve the equation numerically, the "distlink" library from [11] was used. Detailed description of this code can be found in the file Read.TXT accessible by the above link.

Let us indicate three cases in which function $\zeta_{6}$ (and thus $\varrho_{6}$ as well) can be expressed in elementary functions.

1. If at least one of the orbits is circular, then

$$
\zeta_{6}=\cos \left(i_{1}-i_{2}\right)
$$


2. If at least one of the orbits (let us denote it by number 2) lies in the main plane and describes a direct motion so that $\sin i_{2}=0, \cos i_{2}=1$, then

$$
\zeta_{6}=c_{1}+\frac{1}{2}\left(1-c_{1}\right) e_{1} e_{2}+\frac{1}{2}\left(1+c_{1}\right) e_{1} e_{2} \cos (\varpi) .
$$

Here and below, $s_{1}=\sin i_{2}, s_{2}=\sin i_{2}, c_{1}=\cos i_{1}$ and $c_{2}=\cos i_{2}$.

3. If at least one of the orbits (let us denote it by number 2) lies in the main plane but describes a backward motion so that $\sin i_{2}=0$ and $\cos i_{2}=-1$, then

$$
\zeta_{6}=-c_{1}+e_{1} e_{2}
$$

Unfortunately, function is not a full-fledged metric, it satisfies the first two axioms of a metric space; however, it does not satisfy the third axiom: the triangle inequality does not hold, at least in the case of large eccentricities. However, there are two important cases when the triangle axiom is satisfied: one of three orbits is circular and the longitudes of pericenters of all three orbits coincide. Perhaps, the inequality holds for all elliptic orbits, but this is a matter of further research.

\section{References}

1. K. Kholshevnikov, Celest. Mech. Dyn. Astron., 100, 169, 2008.

2. K. Kholshevnikov, in Space Physics: Proc. 45th Int. Stud. Sci. Conf., Yekaterinburg, Russia, Feb. 1-5, 2016, $168-185,2016$.

3. P. B. K.V. Kholshevnikov, G.I. Kokhirova and U. Khamroev, Mon. Not. R. Astron. Soc., 462, 2275-2283, 2016.

4. D. Milanov, Celestial Mech. Dyn. Astron., 130, 27, 2018.

5. E. D. Kuznetsov and V. S. Safronova, Ecological Bulletin of Research Centers of the Black Sea Economic Cooperation, 4-2, 86, 2017.

6. E. D. Kuznetsov and V. S. Safronova, Planetary and Space Science, 157, 22, 2018.

7. F. Hausdorff, American Mathematical Society, Providence, R.I., 2005, 2005.

8. Y. D. B. D. Yu. Burago and S. V. Ivanov, Graduate Studies of Mathematics, 33, 418, 2001.

9. G. A. Korn and T. M. Korn, Courier Corporation, 1152, 2013.

10. A. S. S. K. V. Kholshevnikov and M. S. Dzhazmati, Vestn. St. Petersburg Univ., Math., 53, 108, 2020.

11. R. Baluev and D. Mikryukov, Astronomy and Computing, 27, 11, 2019. 


\section{Ballistic design of flights to the planets of the Solar System}

A. Tsaregorodtsev ${ }^{1}$, D. Tuchin ${ }^{2}$

andrey.tsaregorodtsev@cosmos.msu.ru

den@kiam1.rssi.ru

${ }^{1}$ Lomonosov Moscow State University, Moscow, Russia,

${ }^{2}$ Keldysh Institute of Applied Mathematics of Russian Academy of Sciences, Moscow, Russia

\section{DOI: $10.51194 /$ VAK2021.2022.1.1.032}

One of the tasks of interplanetary flights ballistic design is to minimize fuel costs. In case of high-thrust engines usage we can model engine thrust with momentary impulses and minimize characteristic velocity of a mission. This problem requires to find optimal dates of departure from the Earth and arrival to the planet of destination. We assumed that the Sun is the only body, that gravitationally affects the motion of a spacecraft, and the orbits of the planets of the Solar System correspond to the DE430 ephemerides. Using that mathematical model, the problem of an interplanetary flight is reduced to the Lambert's problem, which is a fundamental boundary-value problem of celestial mechanics:

$$
\begin{gathered}
\ddot{\mathbf{r}}=-\mu \frac{\mathbf{r}}{r^{3}}, \\
\mathbf{r}\left(t_{1}\right)=\mathbf{r}_{1}, \mathbf{r}\left(t_{2}\right)=\mathbf{r}_{2} .
\end{gathered}
$$

Here $\mathbf{r}(t)$ is the law of spacecraft movement, $\mathbf{r}_{1}$ and $\mathbf{r}_{2}$ can be regarded as the position of the Earth at the departure time and the position of the planet of destination at the arrival time, respectively. That problem has many different methods to be solved with, but after a short analysis of some of them we chose the only one that works faster than others. It is based on Lambert's equations [1] and uses regula falsi method

$$
\begin{gathered}
x_{1}^{(0)}=x_{1}, x_{2}^{(0)}=x_{2}, \\
x_{2}^{(n+1)}=\frac{x_{1}^{(n)} F\left(x_{2}^{(n)}\right)-x_{2}^{(n)} F\left(x_{1}^{(n)}\right)}{F\left(x_{2}^{(n)}\right)-F\left(x_{1}^{(n)}\right)}, \\
x_{1}^{(n+1)}=x_{2}^{(n)}, n=1,2,3, \ldots
\end{gathered}
$$

for the solution of a non-linear equation, to which the boundary value problem is reduced. That method was used for further computational experiments.

We solved Lambert's problem on a grid of departure and arrival dates [2], and chose the optimal one among all the solutions. That algorithm allowed us to get tables of optimal flight dates. Here you can see such a table for Venus for 10 years from now:

\begin{tabular}{|l|l|l|l|l|l|}
\hline Departure & Arrival & Duration & Departure vel. & Arrival vel. & Characteristic vel. \\
\hline 26.10 .2021 & 05.04 .2022 & 161 days & $2.80 \mathrm{~km} / \mathrm{s}$ & $4.76 \mathrm{~km} / \mathrm{s}$ & $7.56 \mathrm{~km} / \mathrm{s}$ \\
\hline 27.05 .2023 & 28.10 .2023 & 154 days & $2.58 \mathrm{~km} / \mathrm{s}$ & $3.68 \mathrm{~km} / \mathrm{s}$ & $6.27 \mathrm{~km} / \mathrm{s}$ \\
\hline 06.12 .2024 & 15.05 .2025 & 160 days & $3.25 \mathrm{~km} / \mathrm{s}$ & $2.72 \mathrm{~km} / \mathrm{s}$ & $5.97 \mathrm{~km} / \mathrm{s}$ \\
\hline 09.06 .2026 & 09.12 .2026 & 183 days & $3.86 \mathrm{~km} / \mathrm{s}$ & $2.99 \mathrm{~km} / \mathrm{s}$ & $6.84 \mathrm{~km} / \mathrm{s}$ \\
\hline 11.01 .2028 & 24.07 .2028 & 195 days & $4.64 \mathrm{~km} / \mathrm{s}$ & $3.48 \mathrm{~km} / \mathrm{s}$ & $8.11 \mathrm{~km} / \mathrm{s}$ \\
\hline 25.10 .2029 & 04.04 .2030 & 161 days & $2.81 \mathrm{~km} / \mathrm{s}$ & $4.83 \mathrm{~km} / \mathrm{s}$ & $7.64 \mathrm{~km} / \mathrm{s}$ \\
\hline 24.05 .2031 & 27.10 .2031 & 156 days & $2.58 \mathrm{~km} / \mathrm{s}$ & $3.80 \mathrm{~km} / \mathrm{s}$ & $6.37 \mathrm{~km} / \mathrm{s}$ \\
\hline
\end{tabular}

And for Mars:

\begin{tabular}{|l|l|l|l|l|l|}
\hline Departure & Arrival & Duration & Departure vel. & Arrival vel. & Characteristic vel. \\
\hline 01.09 .2022 & 17.08 .2023 & 350 days & $3.84 \mathrm{~km} / \mathrm{s}$ & $2.64 \mathrm{~km} / \mathrm{s}$ & $6.48 \mathrm{~km} / \mathrm{s}$ \\
\hline 02.10 .2024 & 01.09 .2025 & 334 days & $3.35 \mathrm{~km} / \mathrm{s}$ & $2.45 \mathrm{~km} / \mathrm{s}$ & $5.80 \mathrm{~km} / \mathrm{s}$ \\
\hline 01.11 .2026 & 07.09 .2027 & 310 days & $3.04 \mathrm{~km} / \mathrm{s}$ & $2.57 \mathrm{~km} / \mathrm{s}$ & $5.61 \mathrm{~km} / \mathrm{s}$ \\
\hline 24.11 .2028 & 21.09 .2029 & 301 days & $3.01 \mathrm{~km} / \mathrm{s}$ & $2.97 \mathrm{~km} / \mathrm{s}$ & $5.99 \mathrm{~km} / \mathrm{s}$ \\
\hline 29.12 .2030 & 10.10 .2031 & 285 days & $3.21 \mathrm{~km} / \mathrm{s}$ & $3.53 \mathrm{~km} / \mathrm{s}$ & $6.74 \mathrm{~km} / \mathrm{s}$ \\
\hline
\end{tabular}

Further research will be devoted to solving the problem in a more complete model of forces.

\section{References}

1. E. Lancaster and R. Blanchard, A unified form of lambert's theorem, 1969.

2. Y. Golubev, A. Grushevskii, I. Kiseleva, V. Korianov, S. Lavrenov, A. Tuchin, and D. Tuchin, Mission design for venusian projects of the 2021-2028 years epoch. launch windows (keldysh institute of applied mathematics), 2018 . 
Stars and Interstellar Medium 


\title{
Possible Variations in the Limb-Darkening Coefficients of Eclipsed Stars at Short Time Intervals
}

\author{
M. Abubekerov, N. Gostev \\ marat@sai.msu.ru; ngostev@sai.msu.ru \\ WWW home page: http://lnfm1.sai.msu.su/ ngostev \\ Moscow State University, Sternberg Astronomical Institute, Moscow, 119234 Russia
}

\begin{abstract}
The interpretation of high-precision transit light curves of binary systems with exoplanets Kepler-5b, Kepler-6b, and Kepler$7 \mathrm{~b}$ is performed for three different epochs. It has been demonstrated that the values of the stellar limb-darkening coefficients differ significantly for each epoch, while the geometric parameters for each epoch agree well with each other within the error margin. It is shown that reliable determination of the limb-darkening coefficients requires methods that would "clear" the observed transit light curves from effects caused by surface inhomogeneity.
\end{abstract}

Keywords: transit light curves, binary systems with exoplanets, limb-darkening coefficients

DOI: $10.51194 /$ VAK2021.2022.1.1.033

\section{Problems}

Transit light curves provide important data not only on the geometrical parameters of a binary system (star radius, planet radius, orbit inclination), but also on the limb-darkening coefficients, which indirectly contain important information about the star's atmosphere. Thus, the adequacy of the classical limb darkening model to observational data is often questioned, in particular, due to various inhomogeneous structures on the star's surface. As part of this problem, the authors obtained empirical values of the limb-darkening coefficients of the parent stars of wellstudied binary systems with exoplanets Kepler-5b, Kepler-6b, and Kepler-7b for different epochs. The empirical values of the limb-darkening coefficients were obtained based on the transit light curves of the binary systems from [1], [2], [3]. The calculation of the limb-darkening coefficients is performed for the quadratic limb darkening law of the stellar disk.

\section{Interpretation}

The method for interpreting the observed transit light curves of a binary system with an exoplanet is based on the algorithm for high-precision calculation of brightness during the planet transit across the star's disk; the algorithm is described in a series of studies [4], [5], [6]. A model of two spherical stars in a circular orbit in the absence of reflection and ellipsoidal effects was used. The relative radius of the Roche lobe is tens of times larger than the planet's radius. Thus, the assumption that the planet is spherical is well founded. The same can be said of the optical star. In the calculation of the light curve, the quadratic limb-darkening law was used as a function of the brightness distribution over the stellar disk.

The "third light" in the model is absent. The system's total brightness is assumed to be known; in the adopted normalization, it is equal to unity. The observed brightness values are taken to be distributed normally. The standard deviations of the observed brightness values are also assumed to be known.

\section{Observational Material}

In this study, the high-precision transit light curves of binary systems with exoplanets Kepler-5b, Kepler-6b, and Kepler-7b from [1], [2], [3] was studied. The light curves were obtained at the Kepler Space Observatory from May 1 to June 14, 2009. The star systems Kepler-5b, Kepler-6b, and Kepler-7b are objects of $\sim 13^{m}$ magnitude. The light curves are obtained in the photometric filter of $r$ photometric system (ugriz). The central bandwidth $\lambda_{0}=6550$; half bandwidth $\Delta \lambda=900$. The transit light curves of each system include approximately 2100 individual brightness values, most of which lie in the extra-eclipse part of the light curve. The accuracy of the transit light curves of the binary systems Kepler-5b, Kepler-6b, and Kepler-7b in intensities was $\sigma=1.3759 \cdot 10^{-4}, \sigma=1.2874 \cdot 10^{-4}$, and $\sigma=1.0248 \cdot 10^{-4}$, respectively. The relative error (with respect to the eclipse depth) of the transit light curves is $\sim 1 \%$.

\section{Analytical Results Of The Transit Light Curves}

The authors of this work have set the task of finding out the reliability of limb-darkening coefficients based on the calculations of a high-precision transit light curve. The light curves of binary systems with exoplanets Kepler-5b, Kepler-6b, and Kepler-7b were used for this purpose. As noted above, each transit light curve included approximately 2100 individual brightness values obtained over a period of 44 days. The authors divided the 44-day light curve into three observational sets. The first set included individual brightness values obtained from the 1st day of observation to the 15th $\left(t_{1}\right)$; the second set, from the 15th day to the 30th $\left(t_{2}\right)$; and the third set, from the 30th day to the 44 th $\left(t_{3}\right)$. Each observational set included approximately 700 values of light curves. In this 
study, the parameters of the binary based on the transit light curves of these observational sets were determined. An analysis of the total transit light curve $\left(t_{4}\right)$ that included individual brightness values was performed as well. The coefficients were calculated under the assumption of a quadratic limb-darkening law. The discrepancy was minimized simultaneously in all parameters. The desired parameters were the radius of the star $r_{s}$, radius of the planet $r_{p}$, orbital inclination, linear coefficient, and quadratic coefficient of stellar limb darkening. The analytical results of individual light curve sets and the total light curve of the binary systems Kepler- 5b, Kepler-6b, and Kepler-7b are presented in Tables $1-3$. The errors of the sought parameters were found using the Monte Carlo method. The last column ("Theory") of Tables 1-3 shows the theoretical values of the limb-darkening coefficients from [7] obtained under the assumption of a quadratic limb-darkening law. The theoretical values of the limbdarkening coefficients are given for the filter (ugriz photometric system).

Table 1: Light curve analysis of the binary system with exoplanet Kepler-5b in the epochs $t_{1}, t_{2}, t_{3}, t_{4}$ and the theoretical values of the limb-darkening coefficients from [7].

\begin{tabular}{c|c|c|c|c|c} 
Parameters & $1^{d}-15^{d}\left(t_{1}\right)$ & $15^{d}-30^{d}\left(t_{2}\right)$ & $30^{d}-44^{d}\left(t_{3}\right)$ & $1^{d}-44^{d}\left(t_{4}\right)$ & teor \\
\hline$x$ & $0.256 \pm 0.26$ & $-0.270 \pm 0.42$ & $-0.343 \pm 0.33$ & $-0.0632 \pm 0.18$ & 0.279 \\
$y$ & $0.297 \pm 0.36$ & $1.041 \pm 0.66$ & $1.241 \pm 0.57$ & $0.744 \pm 0.27$ & 0.363 \\
$r_{s}$ & $0.2069 \pm 0.0046$ & $0.2129 \pm 0.0056$ & $0.2029 \pm 0.0063$ & $0.2091 \pm 0.0029$ & - \\
$r_{p}$ & $0.0172 \pm 0.00056$ & $0.0174 \pm 0.00070$ & $0.0163 \pm 0.00074$ & $0.0172 \pm 0.00036$ & - \\
$i$ & $82^{\circ} .03 \pm 0.47$ & $81^{\circ} .64 \pm 0.62$ & $82^{\circ} .65 \pm 0.74$ & $81^{\circ} .91 \pm 0.33$ & - \\
\hline$\chi^{2}$ & 1.165 & 0.980 & 0.999 & 1.049 & -
\end{tabular}

Table 2: Light curve analysis of the binary system with exoplanet Kepler-6b in the epochs $t_{1}, t_{2}, t_{3}, t_{4}$ and the theoretical values of the limb-darkening coefficients from [7].

\begin{tabular}{c|c|c|c|c|c} 
Parameters & $1^{d}-15^{d}\left(t_{1}\right)$ & $15^{d}-30^{d}\left(t_{2}\right)$ & $30^{d}-44^{d}\left(t_{3}\right)$ & $1^{d}-44^{d}\left(t_{4}\right)$ & teor \\
\hline$x$ & $0.319 \pm 0.28$ & $0.382 \pm 0.22$ & $0.748 \pm 0.23$ & $0.386 \pm 0.24$ & 0.366 \\
$y$ & $0.440 \pm 0.44$ & $0.314 \pm 0.35$ & $-0.144 \pm 0.33$ & $0.374 \pm 0.32$ & 0.314 \\
$r_{s}$ & $0.1801 \pm 0.0025$ & $0.1780 \pm 0.0026$ & $0.1949 \pm 0.0027$ & $0.1785 \pm 0.0016$ & - \\
$r_{p}$ & $0.01795 \pm 0.00040$ & $0.01769 \pm 0.00042$ & $0.02023 \pm 0.00044$ & $0.0177 \pm 0.00029$ & - \\
$i$ & $82^{\circ} .98 \pm 0.27$ & $83^{\circ} .11 \pm 0.0029$ & $81^{\circ} .64 \pm 0.30$ & $83^{\circ} .15 \pm 0.18$ & - \\
\hline$\chi^{2}$ & 1.051 & 1.015 & 1.47 & 1.073 & -
\end{tabular}

Table 3: Light curve analysis of the binary system with exoplanet Kepler-7b in the epochs $t_{1}, t_{2}, t_{3}, t_{4}$ and the theoretical values of the limb-darkening coefficients from [7].

\begin{tabular}{c|c|c|c|c|c} 
Parameters & $1^{d}-15^{d}\left(t_{1}\right)$ & $15^{d}-30^{d}\left(t_{2}\right)$ & $30^{d}-44^{d}\left(t_{3}\right)$ & $1^{d}-44^{d}\left(t_{4}\right)$ & teor \\
\hline$x$ & $0.511 \pm 0.21$ & $0.0428 \pm 0.75$ & $0.104 \pm 0.30$ & $0.226 \pm 0.15$ & 0.316 \\
$y$ & $0.0384 \pm 0.28$ & $0.657 \pm 1.0$ & $0.658 \pm 0.50$ & $0.435 \pm 0.22$ & 0.344 \\
$r_{s}$ & $0.1710 \pm 0.0028$ & $0.1734 \pm 0.0046$ & $0.1701 \pm 0.0038$ & $0.1711 \pm 0.0019$ & - \\
$r_{p}$ & $0.01453 \pm 0.00037$ & $0.01448 \pm 0.00058$ & $0.01407 \pm 0.00054$ & $0.01433 \pm 0.00025$ & - \\
$i$ & $83^{\circ} .24 \pm 0.29$ & $83^{\circ} .14 \pm 0.45$ & $83^{\circ} .46 \pm 0.44$ & $83^{\circ} .3 \pm 0.19$ & - \\
\hline$\chi^{2}$ & 0.919 & 1.0693 & 1.20 & 1.054 & -
\end{tabular}

\section{Discussion}

The above tables, which contain the limb-darkening coefficients and the geometric parameters, show that the values of the geometric parameters for different observational sets closely agree with each other. At the same time, the values of the limb-darkening coefficients differ significantly. It should also be noted that the obtained limb-darkening coefficients based on the light curves of different epochs also differ significantly from the theoretical values of [7] in the vast majority of cases. The limb-darkening coefficients are very sensitive to inhomogeneities of the transit light curve. These inhomogeneities can be associated with various physical processes on the star's surface, as well as processes in a binary system. First of all, inhomogeneities in the light curve can be caused by spots on the star's surface.

\section{Conclusions}

A significant difference in the limb-darkening coefficients indicates the presence of active physical processes on the star's surface. Determining the exact values of the limb-darkening coefficients based on the transit curve is difficult and requires special methods that compensate and consider the influence of the inhomogeneous surface of the star on the transit curve. Otherwise, the limb-darkening coefficients should be determined on an observational 
data set covering several rotation periods of a star. An observational data set containing brightness values for several rotation periods will statistically average and thereby minimize the influence of spots when determining the limb-darkening coefficients of a star. It is important to note that transit light curves do not only provide data about the geometrical parameters of a binary system (stellar radius, planetary radius, orbital inclination), yet they are also a unique source of information regarding limb-darkening of various spectral-class stars. From this point of view, a binary system with an exoplanet is a unique opportunity to acknowledge the stellar atmosphere physics and the formation of the limb-darkening effect of a stellar disk.

\section{References}

1. D. G. Koch, W. J. Borucki, J. F. Rowe, N. M. Batalha, et al., ApJL, 713, L131, 2010.

2. E. W. Dunham, W. J. Borucki, D. G. Koch, N. M. Batalha, et al., ApJL, 713, L136, 2010.

3. D. W. Latham, W. J. Borucki, D. G. Koch, T. M. Brown, et al., ApJL, 713, L140, 2010.

4. M. K. Abubekerov and N. Y. Gostev, MNRAS, 432, 2216, 2013.

5. M. K. Abubekerov and N. Y. Gostev, MNRAS, 459, 2078, 2016.

6. M. K. Abubekerov and N. Y. Gostev, A\&A, 633, A96, 2020.

7. A. Claret, $A \& A, \mathbf{4 2 8}, 1001,2004$. 


\title{
Light-curve modelling in a Roche plus stellar wind model: the massive binary WR22*
}

\author{
E. Antokhina ${ }^{1}$, I. Antokhin ${ }^{1}$, G. Lenoir-Craig ${ }^{2}$, N. St-Louis ${ }^{2}$, A. Moffat ${ }^{2}$ \\ elant@sai.msu.ru \\ ${ }^{1}$ Moscow Lomonosov State University, Sternberg State Astronomical Institute, 119992 Universitetskij prospect, 13, \\ Moscow, Russia \\ ${ }^{2}$ Dépt. de physique, Univ. de Montréal, C.P. 6128, Succ. C-V, Montréal, H3C 3J7, Canada, and Centre de Recherche en \\ Astrophysique du Québec
}

A code for the synthesis of light curves of close binaries in the Roche model is proposed which incorporates a stellar wind around one of the stars. The code makes it possible to analyze observations of binary systems containing Wolf-Rayet stars. This paper presents the results of the application of this code to the spectroscopic binary WR22, whose components are stars of spectral types WN7h and O9III-V $\left(P=80.336^{d}, e \sim 0.6\right)$. The light curves were obtained with one of the BRITE-Constellation space-based telescopes. Two solutions to the problem were found, corresponding to two possible luminosity classes of the $\mathrm{O}$ star, O9III and O9V. For each solution, the orbital inclination, the masses of the components, the temperature of the WR star, the WR mass-loss rate, and other parameters were determined. The resulting stellar parameters favour the solution for the $\mathrm{WN} 7 \mathrm{~h}+\mathrm{O} 9 \mathrm{~V}$ model

Keywords: eclipsing binaries, Wolf-Rayet stars, mass-loss

DOI: $10.51194 /$ VAK2021.2022.1.1.034

\section{Introduction}

The study of close binary systems (CBS) is of great interest for modern astrophysics, as they are one of the main and most reliable sources of information on the fundamental parameters of stars and relativistic objects. Methods for the synthesis of light curves (see, e.g., [1]) proposed in the early 1970s made it possible to analyze observations and obtain the parameters of the components of "non-classical" binary systems in which the components are tidally deformed and the temperature distribution over their surface is inhomogeneous. Synthesis methods are being constantly improved to take into account complex physical processes in the CBSs. We have developed software codes for the analysis of light curves and radial-velocity curves of CBSs of different types $([2,3,4,5]$ and others). To analyze the light curves of a CBS containing a Wolf-Rayet star, we proposed an algorithm in which the standard Roche model is extended by adding a stellar wind around the WR component [6]. The main mechanism of extinction in the wind is considered to be electron scattering, the density of the wind is being controlled by the mass continuity equation and the so-called $\beta$ law for the WR wind velocity.

This paper presents the results of the light-curve analysis of the WR22 binary, obtained with one of the satellites of BRITE-Constellation.

\section{Light-curve modelling}

The massive binary system WR22 (WN7h+O9III-V) is a member of the Carina OB1 association, its orbital period is $P=80.336^{d}$ and the orbital eccentricity is $e \sim 0.6$. Its spectrum indicates a large amount of hydrogen in the WR atmosphere $(\sim 44 \%,[7])$. The spectroscopic elements of the orbit and the mass ratio of the components are known, but the luminosity class of the O9 star is uncertain $[8,9]$.

The observed light curve includes three complete periods of the system, the photometric data were obtained in the red filter with the BRITE-Heweliusz telescope in 2017-2018. During the orbital period, only one minimum with a depth $\sim 0 .{ }^{m} 08$ is observed (the WR star is in front of the O9 star); the center of the minimum almost coincides with the moment of periastron. The shape of the minima is somewhat different for different orbital cycles (Fig. 1, left panel). The light-curve analysis was performed on the light curve containing all three orbital cycles (Fig. 1, central panel).

When computing the model light curves, we used the spectroscopic elements from [9] (Table 1). Since the light curve contains only one minimum, which, in addition, may not be due to a geometric eclipse, but instead to extinction in the WR wind, it is impossible to determine the relative stellar radii from the light-curve analysis alone. Therefore, we were forced to fix the radii of both stars. For the $\mathrm{O}$ star, these correspond to the two possible luminosity classes of the O star - V (Model 1) and III (Model 2). The corresponding O-star radii and temperatures were taken from [10] and the WR radius was taken from [7]. The model parameters and the results of the light-curve solutions in both models are presented in Table 1 and Fig. 1.

*This study is based on data collected by the BRITE-Constellation satellite mission, designed, built, launched, operated and supported by the Austrian Research Promotion Agency (FFG), the University of Vienna, the Technical University of Graz, the University of Innsbruck, the Canadian Space Agency (CSA), the University of Toronto Institute for Aerospace Studies (UTIAS), the Foundation for Polish Science \& Technology (FNiTP MNiSW), and National Science Centre (NCN). 

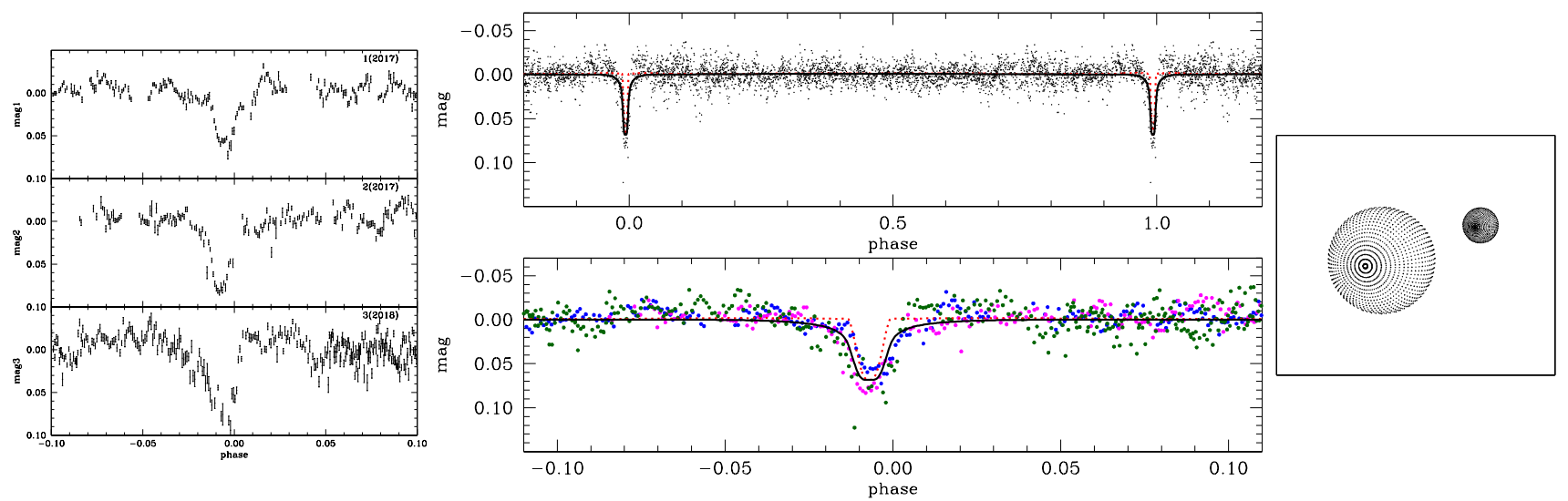

Figure 1: Left panel - observed light curve minima showing the difference between orbital cycles. The central panel shows the solution in Model 1; top - for the full orbital cycle, bottom - for the region near the minimum. The black solid line is the model light curve; the red dotted line - the model light curve when omitting to account for the WR wind extinction. Different dot colors correspond to different observed eclipses. In the right panel, the sky plane view of the system in Model 1 is shown for an orbital phase near the minimum (0.99), the WR star is in front.

The solution in Model 1 (luminosity class O9V for the O star) is preferable for physical reasons. In Model 2, the temperature of the WR star is twice as high as the temperature obtained from the results of the spectroscopic analysis by [7] while in Model 1 (O9V) our model temperature and the temperature of [7] are consistent. The eclipse in the system is a combination of the geometric and atmospheric eclipse of the O9V star by the WR star and its wind. Despite the presence of only one shallow eclipse in the observed light curve, it was possible to reliably determine the most interesting and still unknown parameters of WR22: the orbital inclination angle $i \simeq 83.5^{\circ}$, the WR mass loss rate $\dot{M}_{\mathrm{WR}} \simeq 1.9 \times 10^{-5} \mathrm{M}_{\odot} /$ year, and the component masses $M_{\mathrm{WR}} \simeq 56.4 \mathrm{M}_{\odot}, M_{\mathrm{O}} \simeq 21.0 \mathrm{M}_{\odot}$.

Table 1: Photometric solution. The major model parameters.

\begin{tabular}{|c|c|c|c|}
\hline Parameter & $\begin{array}{c}\text { Model } 1 \\
(\mathrm{WN} 7+\mathrm{O} 9 \mathrm{~V})\end{array}$ & $\begin{array}{c}\text { Model } 2 \\
\text { (WN7+O9III) }\end{array}$ & $\begin{array}{l}\text { Parameter } \\
\text { status }\end{array}$ \\
\hline$P$ [days](orbital period) & \multicolumn{2}{|c|}{$80.336^{a}$} & adopted \\
\hline$T_{0}$ (HJD) (time of periastron) & \multicolumn{2}{|c|}{$2450127.47^{a}$} & adopted \\
\hline$M_{\mathrm{O}} \sin ^{3} i\left(\mathrm{M}_{\odot}\right)\left(\right.$ mass of the $\left.\mathrm{O} \operatorname{star} \times \sin ^{3} i\right)$ & \multicolumn{2}{|c|}{$20.6^{a}$} & adopted \\
\hline$M_{\mathrm{WR}} \sin ^{3} i\left(\mathrm{M}_{\odot}\right)\left(\right.$ mass of the WR star $\left.\times \sin ^{3} i\right)$ & \multicolumn{2}{|c|}{$55.3^{a}$} & adopted \\
\hline$e$ (eccentricity) & \multicolumn{2}{|c|}{$0.598^{a}$} & adopted \\
\hline$\omega_{\mathrm{O}}\left[{ }^{\circ}\right]$ (longitude of periastron for the $\mathrm{O}$ star) & \multicolumn{2}{|c|}{$88.2^{a}$} & adopted \\
\hline$\beta$ (parameter of the $\beta$-law) & \multicolumn{2}{|c|}{1.0} & adopted \\
\hline$V_{\infty}\left[\mathrm{km} \mathrm{s}^{-1}\right]$ (terminal velocity of the WR wind) & \multicolumn{2}{|c|}{$1785^{c}$} & adopted \\
\hline$\mu_{e}($ mean electron molecular weight of the wind) & \multicolumn{2}{|c|}{$1.39^{c}$} & adopted \\
\hline$R_{\mathrm{O}}\left(\mathrm{R}_{\odot}\right)$ (radius of the $\mathrm{O}$ star) & $7.53^{b}$ & $13.38^{b}$ & adopted \\
\hline$R_{\mathrm{WR}}\left(\mathrm{R}_{\odot}\right)$ (radius of the WR star) & $22.65^{c}$ & $22.65^{c}$ & adopted \\
\hline$T_{\mathrm{O}}(K)$ ( temperature of the $\mathrm{O}$ star) & $32900^{b}$ & $31850^{b}$ & adopted \\
\hline$T_{\mathrm{WR}}(K)$ ( temperature of the WR star) & 50000 & 100000 & adjusted \\
\hline$\mu_{O}$ (Roche lobe filling factor for the O star) & 0.184 & 0.323 & computed \\
\hline$\mu_{W R}$ (Roche lobe filling factor for the WR star) & 0.340 & 0.340 & computed \\
\hline$i\left[^{\circ}\right]$ (orbital inclination) & $83.5 \pm 0.4$ & $78.8 \pm 0.4$ & adjusted \\
\hline$\tau_{0}$ (wind optical depth coefficient) & $0.0064 \pm 0.0006$ & $0.0191 \pm 0.0007$ & adjusted \\
\hline$\Delta m_{0}$ (zero point of stellar magnitudes $)$ & $-0.0007 \pm 0.0003$ & $-0.00007 \pm 0.00002$ & adjusted \\
\hline$\Delta \phi$ (phase shift of the observed light-curve) & $-0.0080 \pm 0.0004$ & $-0.0080 \pm 0.0004$ & adjusted \\
\hline$F_{\mathrm{O}} / F_{\mathrm{WR}}(\mathrm{O} / \mathrm{WR}$ flux ratio $)$ & 0.064 & 0.085 & computed \\
\hline$\dot{M}_{\mathrm{WR}}\left[10^{-5} \mathrm{M}_{\odot} /\right.$ year $]$ (WR mass-loss rate) & $1.9 \pm 0.2$ & $5.6 \pm 0.2$ & computed \\
\hline$M_{\mathrm{O}}\left(\mathrm{M}_{\odot}\right)($ mass of the $\mathrm{O}$ star $)$ & 21.0 & 21.8 & computed \\
\hline$M_{\mathrm{WR}}\left(\mathrm{M}_{\odot}\right)$ (mass of the WR star) & 56.4 & 58.6 & computed \\
\hline
\end{tabular}

a [9], b [10], c [7] 


\section{Acknowledgements}

The work of IA was supported by the RSF grant 17-12-01241 (Russia). The work of EA was supported by the Scientific Educational School of Lomonosov Moscow State University "Fundamental and applied Space Research". NSL \& AFJM are grateful to NSERC (Canada) for financial aid.

\section{References}

1. R. E. Wilson and E. J. Devinney, ApJ, 166, 605, 1971.

2. E. A. Antokhina, Sov. Astron., 32, 608, 1988.

3. E. A. Antokhina, Astronomy Reports, 40, 483, 1996.

4. E. A. Antokhina and A. M. Cherepashchuk, Astronomy Reports, 38, 367, 1994.

5. E. A. Antokhina, A. F. J. Moffat, I. I. Antokhin, J.-F. Bertrand, and R. Lamontagne, ApJ, 529, $463,2000$.

6. E. A. Antokhina, I. I. Antokhin, and A. M. Cherepashchuk, Astronomical and Astrophysical Transactions, 28, $3,2013$.

7. W. R. Hamann, G. Gräfener, A. Liermann, R. Hainich, et al., A $6 A$, 625, A57, 2019.

8. G. Rauw, J. M. Vreux, E. Gosset, D. Hutsemekers, P. Magain, and K. Rochowicz, A\& A, 306, $771,1996$.

9. J. Schweickhardt, W. Schmutz, O. Stahl, T. Szeifert, and B. Wolf, $A \& A$, 347, 127, 1999.

10. F. Martins, D. Schaerer, and D. J. Hillier, A\&A, 436, 1049, 2005. 


\section{ASASSN-19fy: The unusual dwarf nova in the period gap}

O. Antoniuk ${ }^{1}$, E. Pavlenko ${ }^{1}$, K. Antoniuk ${ }^{1}$, N. Pit ${ }^{1}$, G. Kokhirova ${ }^{2}$, F. Rakhmatullaeva ${ }^{2}$, A. Sosnovskij $^{1}$ sana duka@mail.ru

${ }^{1}$ Federal State Budget Scientific Institution "Crimean Astrophysical Observatory of RAS", Nauchny, Republic of Crimea, Russia,

${ }^{2}$ Department of astrophysics, National Academy of Science of Tajikistan, Dushanbe, Republic of Tajikistan

DOI: $10.51194 /$ VAK2021.2022.1.1.035

Dwarf novae are interacting binaries in which outbursts are caused by thermal instability in the accretion disk around the white dwarf [1]. The sub-class SU UMa type is characterized by two types of outbursts; during a brighter and longer outburst oscillations with periods slightly exceeding the orbital (superhumps) are detected. The gap in the statistical distribution of dwarf novae according to their orbital periods is distinguished between 2 and 3 hours [2] with a reduced number of dwarf novae in this interval.

During the superoutburst of ASASSN-19fy in 2020, based on photometry carried out by Crimean observatory telescopes (1.25-m telescope AZT-11 and 0.38-m telescope K-380), 1-m telescope on Sanglokh Observatory and AAVSO data, the mean period of positive superhumps was 0.09278 days. This defines the system as a long-period dwarf nova in the period gap. The transition between stages B and C of the superhumps evolution [3] was identified. It occurred on the fast extinction of the superoutburst. The superhump period increased at a rate $P=13.2 \times 10^{-4}$ at stage B. Two rebrightenings occurred at stage C.

By the superhump period ASASSN-19fy belongs to the SU UMa type dwarf nova with the highest mass ratio. However, its other characteristics (rebrightenings and long return to a quiescence) are inherent in the WZ Sge type dwarf nova, which have the smallest mass ratio. This peculiarities is probably due to the weakness of the tidal effect in accretion disk in both long-period SU UMa type and sort-period WZ Sge type stars.

\section{References}

1. B. Warner, Cataclysmic variable stars (Cambridge University Press) (1995).

2. C. Knigge, MNRAS, 373, 484, 2006.

3. T. Kato, A. Imada, M. Uemura, D. Nogami, et al., PASJ, 61, S395, 2009. 


\title{
Accretion and wind modulation in T Tauri star RY Tau: a planet at $0.2 \mathrm{AU}$ ?
}

\author{
S. Artemenko, E. Babina, K. Grankin, P. Petrov \\ artemenko@craocrimea.ru \\ Crimean Astrophysical Observatory, Republic of Crimea, 298409, Russia
}

Spectral and photometric monitoring of CTTS RY Tau in 2013-2021 revealed variations in the H $\alpha$ and Na I D lines at radial velocities of infall (accretion) and outflow (wind) with a period of about 22 days. The Na I D absorption in the infalling and outflowing gas changes in anti-phase: an increase in the density of infalling gas is accompanied by a decrease in the wind density, and vice versa. The fluctuations remain coherent for several years of observation. We assume that the observed effect may be due to processes at the disk-magnetosphere boundary in MHD propeller mode. The stability of the oscillation phase indicates the possible presence of a massive planet, causing azimuthal asymmetry of the accretion and wind flows. If the observed period (22 days) is Keplerian, then the planet is located at a distance of 0.2 AU and moves with an orbital velocity of $100 \mathrm{~km} \mathrm{~s}^{-1}$.

Keywords: Variable stars, T Tauri type stars, wind, accretion, RY Tau

DOI: 10.51194 /VAK2021.2022.1.1.036

\section{Introduction}

A young solar mass star at the age of several Myr is still surrounded by an accretion disk, where processes of planet formation are going on. Interferometric images of the protoplanetary disks in many classical T Tauri stars (CTTS) were obtained with ALMA interferometer (e.g. [1]). The streams of accretion and the wind flows in CTTS can be observed in the visual spectrum as variations in the emission line profiles broaden by the Doppler effect.

The MHD processes in the inner accretion disk and in the stellar magnetosphere were studied using numerical simulations (see review [2]). Less studied is how a planet in the inner accretion disk can influence the MHD processes of wind and accretion. A long series of spectroscopic observations of selected CTTS can potentially reveal these effects.

RY Tau is one of the brightest and well studied CTTS. It is a fast rotator, with $v \sin i \sim 52 \mathrm{~km} \mathrm{~s}^{-1}$ [3]. The star is most probably in the regime of the magnetic propeller. The extended jet of the star is wiggled, which may be caused by a warp of the inner disk, induced by a planet or a sub-stellar companion [4].

\section{Observations and results}

In 2013, at the Crimean Astrophysical Observatory, we started a series of spectral and photometric observations of CTTS RY Tau intending to reveal the pattern of the accretion and wind flows around the star. Technical details, moments of observations and light curves can be found in $[5,6]$. To date, our collection of data is based on more than 160 nights of observations. We monitor variations in the $\mathrm{H} \alpha$ and the Na I D line profiles. With the photometric backup, the equivalents width of $\mathrm{H} \alpha$ emission is converted into absolute fluxes.

Fig. 1 shows a typical example of two spectra, taken on different dates. Either the blue or red side of the $\mathrm{Na}$ I $\mathrm{D}$ absorption becomes stronger on a time scale of 10 to 20 days. The $\mathrm{H} \alpha$ line emission profile also varies in correlation with the $\mathrm{Na}$ I D lines.

Fig. 2 shows a 2-D periodogram of the $\mathrm{H} \alpha$ flux profile. There is a clear spot of high power at the radial velocity of $-100 \mathrm{~km} \mathrm{~s}^{-1}$, at the frequency of about $0.04 \mathrm{day}^{-1}$, corresponding to a period within 22-24 days.

The periodic variations in $\mathrm{H} \alpha$ flux profile can be revealed also in the analysis of the line asymmetry $(b-$ $r) /(b+r)$, where the $b$ and $r$ are equivalent widths of the blue and red halves of the line profile relative to zero radial velocity. The power spectrum of the line asymmetry reveals the most probable period of 21.6 days, with a false alarm probability FAP $<0.001$ (Fig. 3).

Remarkably, the oscillations remain coherent over the whole set of our observations. In Fig. 4 the data are split into two samples with about an equal number of observations and convolved with a period of 21.6 days. The origin of time is the same for both samples.

\section{Discussion and conclusion}

Numerical simulations of the magnetospheric accretion and wind flows reveal a rather complicated pattern, where the infall and outflow events occur repeatedly on a time scale of tens of days [7]. The magnetospheric accretion model may potentially explain the observed anti-phase variations of the blue- and red-shifted components of spectral lines. However, the model cannot explain the phase stability: in the numerical simulations the direction of the accretion (on one pole or another) changes randomly.

Our observations show a striking difference: the accretion and wind events change each other coherently, not randomly, during several years. This phase stability of the oscillations indicates a periodic process in the accretion 

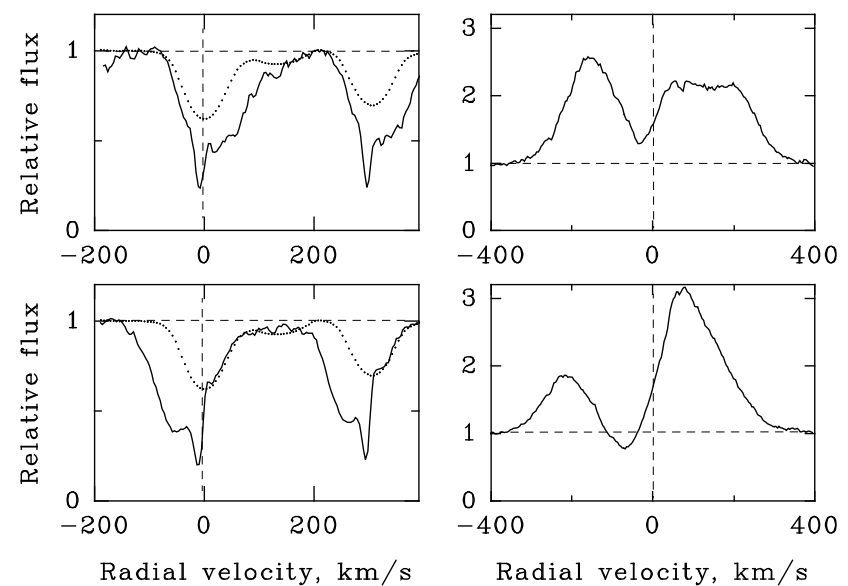

Figure 1: Example of typical variations in the $\mathrm{Na}$ I D and $\mathrm{H} \alpha$ line profiles. Upper panels: $\mathrm{HJD}=2458737.41$; lower panels: $\mathrm{HJD}=2458765.42$. The photospheric components of theNa I D absorptions, broadened by stellar rotation, are shown with the dotted lines.

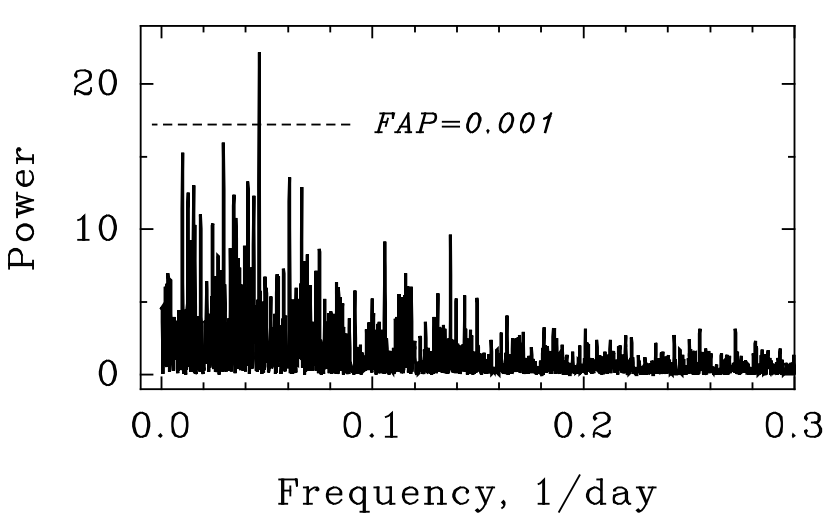

Figure 3: Power spectrum of $\mathrm{H} \alpha$ line asymmetry. The highest peak corresponds to the period of 21.6 days.

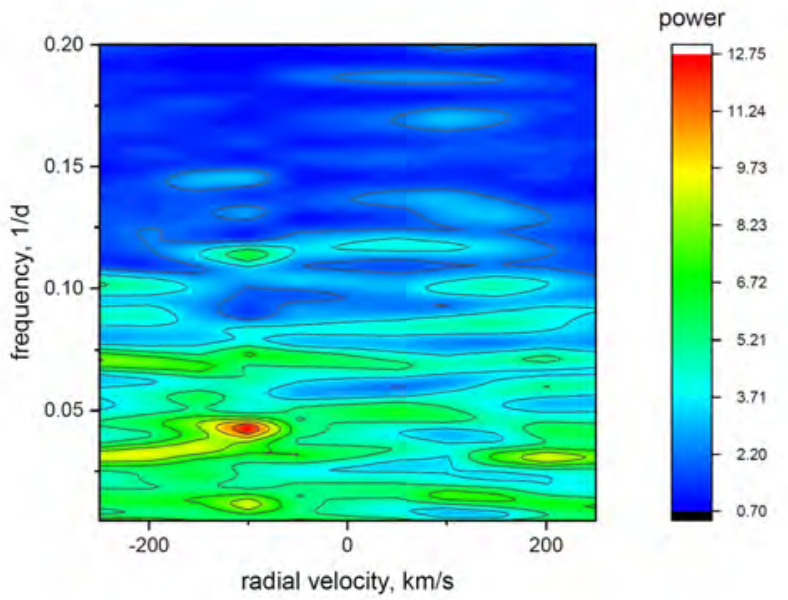

Figure 2: 2-D periodogram of the $\mathrm{H} \alpha$ flux. The maximal power is concentrated at radial velocity -100 $\mathrm{km} \mathrm{s}^{-1}$, at a frequency of about $0.04 \mathrm{day}^{-1}$.

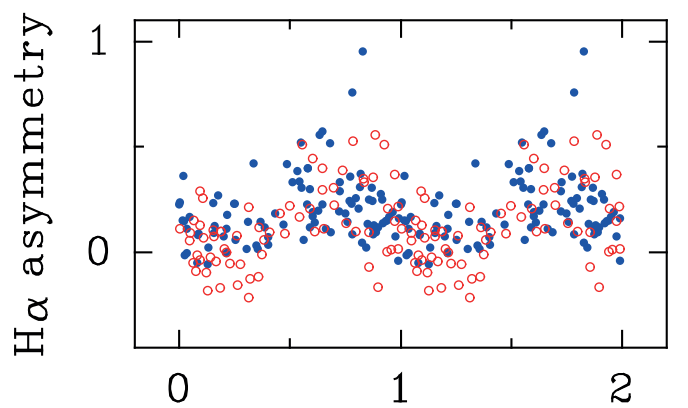

Phase

Figure 4: $\mathrm{H} \alpha$ line asymmetry convolved with a period of 21.6 days. Filled and open circles denote data from 2013-2017 and 2017-2020, respectively. The origin of time in both samples: HJD $=2456592.444$.

disk. It may be a disk warp, induced by a massive planet, which introduces an azimuthal asymmetry in the accretion and wind flows. An alternative model of a disk warp caused by an inclined magnetic dipole, like in AA Tau [8] cannot be applied in the case of RY Tau, because the observed period of $\sim 22$ days is much longer than the 3 -days period of axial rotation.

If the period of $\sim 22$ days is Keplerian, then the planet is at $\sim 0.2$ AU from the star, near the dust sublimation radius. The orbital velocity of such a planet must be $\sim 100 \mathrm{~km} \mathrm{~s}^{-1}$. It is at this radial velocity we observed oscillations in $\mathrm{H} \alpha$ profile (Fig. 2). Earlier, a planet or a sub-stellar companion of RY Tau was suspected from the resolved wiggling jet of the star [4].

We conclude that the periodic anti-phase variations of the infall and outflow signatures in RY Tau are induced by a planet at $0.2 \mathrm{AU}$ from the star. The putative planet may be confirmed by radial velocity observations: the expected amplitude is larger than $90 \mathrm{~m} \mathrm{~s}^{-1}$ if the mass of the planet is above $2 \mathrm{M}_{J}$.

Acknowledgements This work was supported by the Russian Science Foundation grant 19-72-10063.

\section{References}

1. R. Dong, J. R. Najita, and S. Brittain, ApJ, 862, 103, 2018.

2. M. M. Romanova and S. P. Owocki, SSRv, 191, 339, 2015.

3. P. Petrov, G. Zajtseva, Y. Efimov, R. Duemmler, I. V. Ilyin, I. Tuominen, and V. A. Shcherbakov, A\&A, 341, $553,1999$. 
4. A. Garufi, L. Podio, F. Bacciotti, S. Antoniucci, et al., A\&A, 628, 68, 2019.

5. P. P. Petrov, K. N. Grankin, J. F. Gameiro, S. A. Artemenko, et al., MNRAS, 483, 132, 2019.

6. P. P. Petrov, M. M. Romanova, K. N. Grankin, S. A. Artemenko, E. V. Babina, and S. Y. Gorda, MNRAS, 504, 871, 2021.

7. M. M. Romanova, A. A. Blinova, G. V. Ustyugova, A. V. Koldoba, and R. V. E. Lovelace, NewA, 62, $94,2018$.

8. J. Bouvier, S. Alencar, T. Harries, C. M. Johns-Krull, and M. M. Romanova, in Protostars and Planets $V$ (University of Arizona Press), 479-494 (2007). 


\title{
Dynamics of wind as indicator of magnetic activity of $T$ Tauri star RY Tau
}

\author{
E.V. Babina, S.A. Artemenko, K.N. Grankin, P.P. Petrov \\ helenka_truth@mail.ru \\ Crimean Astrophysical Observatory, Crimea 298409, Russia
}

We present the results of spectral and photometric monitoring of the classical Tauri star RY Tau, which has been carried out since 2013. It is shown that the relative roles of accretion and wind varied smoothly over eight years, indicating a change either in the global magnetic field of the star or in the mass accretion rate. We suppose it may be a tool to reveal a possible cycle of magnetic activity at the early stages of stellar evolution.

Keywords: T Tauri stars, magnetospheric accretion, magnetic activity

DOI: $10.51194 /$ VAK2021.2022.1.1.037

\section{Introduction}

The current understanding of the physics of classical T Tauri stars (CTTS) is based on the model of magnetospheric accretion [1]. CTTS possess relatively strong magnetic fields, up to kilo-Gauss on the surface. The observed activity of CTTS, including emission line spectrum and irregular light variability, are due to processes of mass accretion, winds outflow, and the dusty circumstellar environment.

Numerical simulations of gas flows (see review by [2] showed that the regime of accretion and winds depends on several parameters, including the mass and the angular velocity of the star, the strength of the magnetic field and the rate of mass accretion. At a given rate of accretion, the boundary between the accretion disk and the magnetosphere $\left(R_{m}\right)$ is determined by the strength of the dipole component of the field.

In turn, the position of this boundary relative to the corotation radius $\left(R_{\text {cor }}\right)$ defines the dynamics of gas flows: at $R_{m}>R_{\text {cor }}$, the wind prevails (propeller mode) with accretion episodes, while at $R_{m}<R_{\text {cor }}$ accretion prevails and with episodes of magnetospheric ejections.

Although the magnetic field at the stellar surface may be rather complex, at several stellar radii it is the dipole component that truncates the accretion disk. A change of the dipole component would result in the corresponding change of the magnetospheric radius.

We suggest that time variations in the stellar magnetic field and/or mass accretion rates can be detected by an observer as changes in the pattern of the accretion and wind flows. In order to detect these effects, we inspected a long series of spectroscopic and photometric observations of CTTS RY Tau. RY Tau is G-type CTTS at the age of about 7 Myr [3].

\section{Observations and Results}

The major part of our observations (high-resolution spectroscopy with photometric backup) was done at the Crimean Astrophysical Observatory (CrAO) in 2013-2021. Technical details, moments of observations, and light curves can be found in [4]. To date, our database includes more than 160 nights of observations.

The dynamics of gas flows can be revealed in variations of spectral lines broaden by the Doppler effect. We analyze the strongest indicators: $\mathrm{H} \alpha$ and the $\mathrm{NaI} D$ resonance doublet. The typical range of variability of these lines can be seen in Fig. 1.

The mean value of flux radiated in $\mathrm{H} \alpha$ line changed from season to season, with different dispersion of flux within each season (Fig. 2).
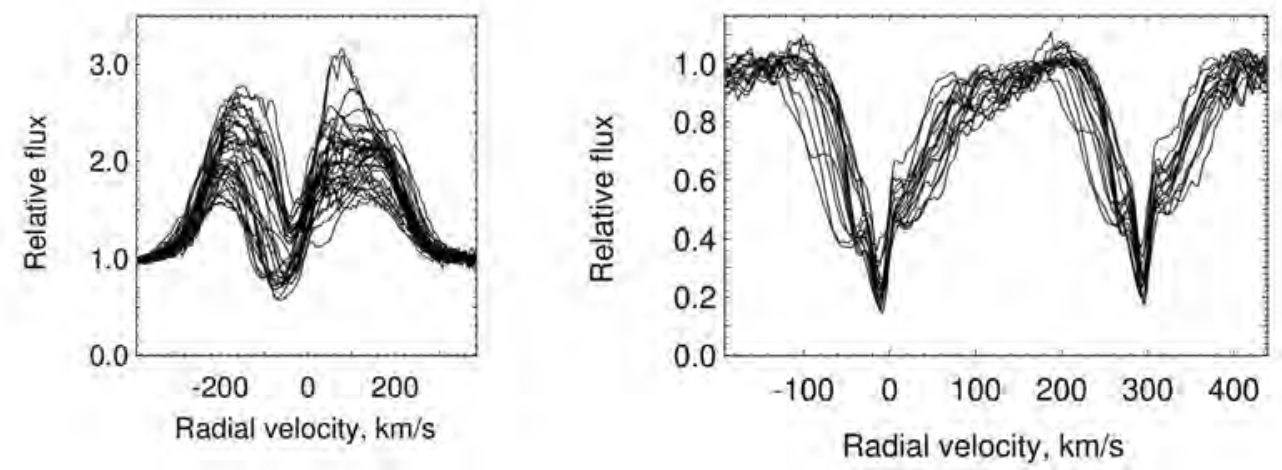

Figure 1: Sample of $\mathrm{H} \alpha$ and Na I D profiles observed in 2019-2020 season. 
In the resonance Na I D lines we analyze variation in the blue- and red-shifted absorptions at radial velocities within $\pm 200 \mathrm{~km} \mathrm{~s}^{-1}$. These variable absorptions are formed in the streams of infalling and outflowing gas on the line of sight to the star. The short time scale of variability of these absorptions is typically several days, while the largest variations occur at time scales of years.

The main result, discussed in this report, is shown in Fig. 2: the wave-like variations in the blue- and redshifted absorptions of Na I D lines, indicating how the wind and accretion flows replaced each other during the eight-year interval. Equivalent widths of the blue- and red-shifted absorptions were measured in the radial velocity ranges of $[-170 ;-20]$ and $[10 ; 160] \mathrm{km} \mathrm{s}^{-1}$ correspondingly.

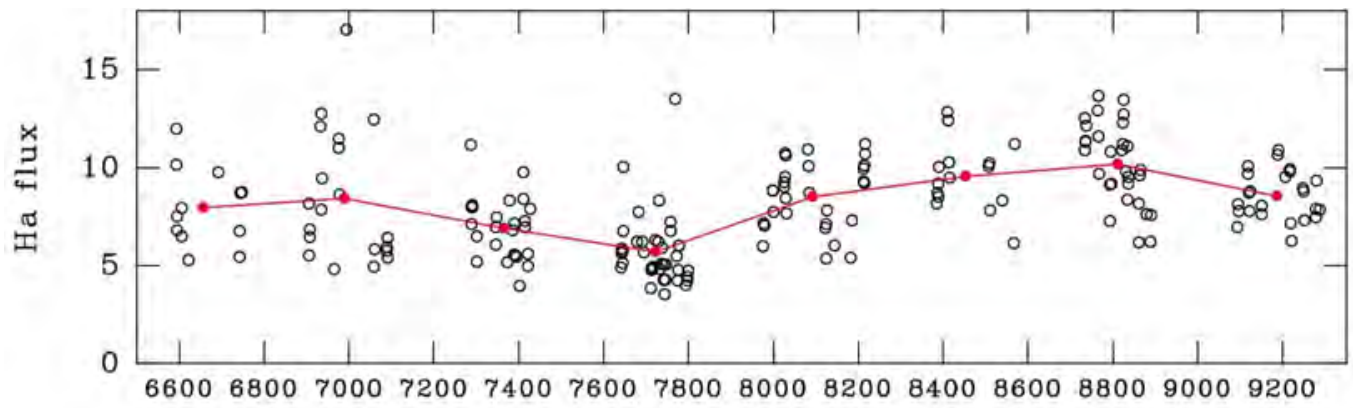

JD $245 \ldots$

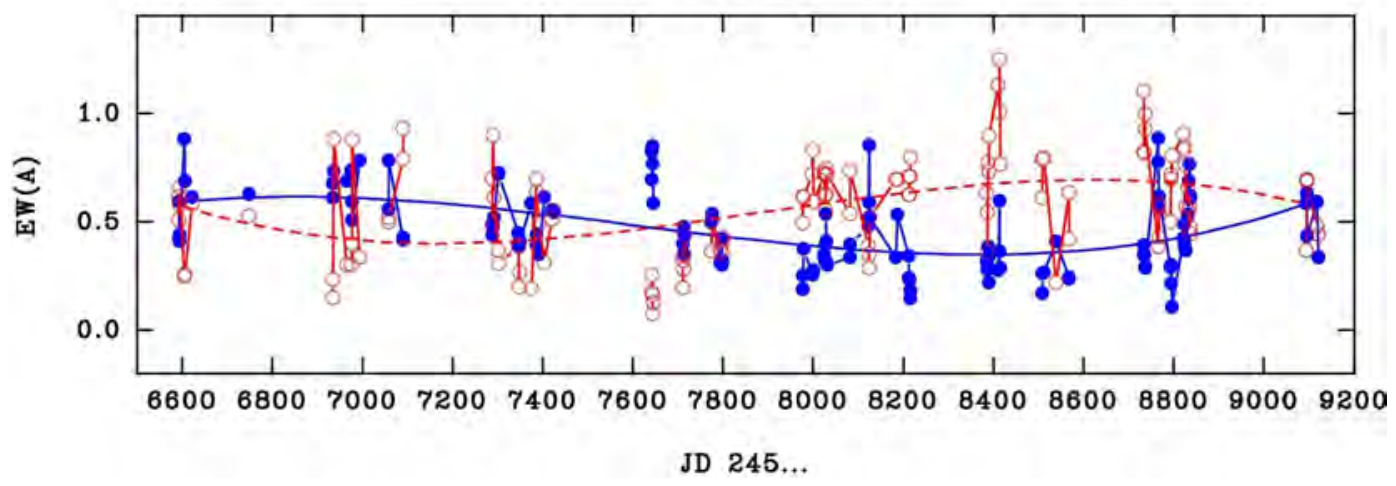

Figure 2: Upper panel: variations of the Ha flux during eight years. The flux is expressed in units $3.67 \times 10^{-13}$ erg $\mathrm{cm}^{-2} \mathrm{~s}^{-1} \AA^{-1}$. Lower panel: variations of equivalent width of the blue- and red-shifted absorptions of the Na I D2 line. Smoothed lines — polynomial approximation.

\section{Discussion and Conclusion}

The long-term wave-like variations of the accretion and wind signatures in the spectrum of RY Tau (Fig. 2) is most probably associated with changes in the magnetospheric radius of the star, $R_{m} \approx \mu^{4 / 7} /\left(G M_{*} \dot{\mathrm{M}}^{2}\right)^{1 / 7}[5]$.

In case the magnetospheric radius is larger than the radius of corotation, $R_{\text {cor }}^{3}=G M_{*} / \Omega_{*}^{2}$ the star is in the regime of magnetic propeller, while the condition $R_{m}<R_{\text {cor }}$ defines the regime of accretion [2].

When the global magnetic field of the star increases, the regime of accretion may change to the regime of wind, and vice versa. This gives a chance to detect a possible magnetic cycle at the early phase of stellar evolution. The luminosity and temperature of RY Tau places the star on the radiative track, where the radiative core occupies more than $50 \%$ of the stellar radius. One can assume that the dynamo mechanism is in operation there.

RY Tau is a fast rotator, with $R_{\text {cor }} \approx 3 R_{*}$. Provided that the typical magnetospheric radius of a CTTS is within $3-10 R_{*}$ [6], RY Tau is most probably in the propeller regime, which efficiency depends on the strength of magnetic dipole $\mu$.

Another possibility is that the disk accretion rate varies on a time scale of years, which also results in a variation of magnetospheric radius. Note, however, that magnetospheric radius depends more on the magnetic field $\mu$ than on the accretion rate $\dot{M}$. We know that the magnetic field of T Tauri stars varies on a time scale of years [7], while not much is known about variations of the disk accretion rate, except EXors and FUors outbursts.

We conclude that monitoring of the accretion and wind flows may be used as a complementary tool for investigation of magnetic activity in CTTS, where the conventional Zeeman-Doppler method cannot be applied.

Acknowledgments This work was supported by the Russian Science Foundation grant 19-72-10063. 


\section{References}

1. J. Bouvier, S. H. P. Alencar, T. J. Harries, C. M. Johns-Krull, and M. M. Romanova, in B. Reipurth, D. Jewitt, and K. Keil, eds., Protostars and Planets V, 479 (2007).

2. M. M. Romanova and S. P. Owocki, Space Sci. Rev., 191, 339, 2015.

3. N. Calvet, J. Muzerolle, C. Briceño, J. Hernández, L. Hartmann, J. L. Saucedo, and K. D. Gordon, AJ, 128, 1294, 2004.

4. P. P. Petrov, M. M. Romanova, K. N. Grankin, S. A. Artemenko, E. V. Babina, and S. Y. Gorda, MNRAS, 504, 871, 2021.

5. A. Koenigl, ApJL, 370, L39, 1991.

6. C. P. Johnstone, M. Jardine, S. G. Gregory, J. F. Donati, and G. Hussain, MNRAS, 437, 3202, 2014.

7. J. F. Donati, J. Bouvier, F. M. Walter, S. G. Gregory, et al., MNRAS, 412, 2454, 2011. 


\title{
Parameters of the microquasar SS433 based on the observations in SAI MSU
}

\author{
A.M. Cherapashchuk, A.V. Dodin, K.A. Postnov, A.A. Belinski, M.A. Burlak, N.P. Ikonnikova, T.R. \\ Irsmambetova \\ cherepashchuk@gmail.com
}

M.V. Lomonosov Moscow State University, Sternberg Astronomical Institute, 13, Universitetskij pr., 119234, Moscow, Russia

\section{DOI: $10.51194 /$ VAK2021.2022.1.1.038}

\section{Introduction}

The microquasar SS433 is a massive eclipsing X-ray binary system at an advanced stage of evolution with a precessing supercritical accretion disk and relativistic $(v / c \approx 0.26)$ jets [see reviews by [1] and [2] for all necessary references]. The object shows three kinds of periodicity: eclipsing, $P_{\text {orb }}=13^{d} .08$, precessional, $P_{\text {prec }}=162^{d} .3$, and nutational, $P_{\text {nut }}=6^{d} .29$. The optical star overfills its Roche lobe and outflows through the $L_{1}$ and $L_{2}$ points. As was shown in a recent work [3], the shape of the orbital $V$-light curve corresponds to an elliptical orbit with $e=0.05 \pm 0.01$. This is consistent with the 'slaved accretion disc' model, which traces the precession of the optical star. This model is also supported by the stability of the parameters of the kinematic model SS433 over 40 years [4]. [3] have discovered a secular increase of the orbital period with $\dot{P}_{\text {orb }} \sim 10^{-7} \mathrm{ss}^{-1}$, on this basis, the mass ratio was estimated as $q=M_{\mathrm{X}} / M_{\mathrm{V}}>0.8\left(M_{\mathrm{X}}\right.$ and $M_{\mathrm{V}}$ are masses of the relativistic object and the optical star, correspondingly), therefore, the relativistic object in the SS433 system is a black hole with a mass $M_{\mathrm{X}}>8 M_{\odot}$, which is typical for massive X-ray binaries. In this paper, we describe the observations of SS433 carried out in recent years in the SAI observatories and give refined estimates for the parameters of the kinematic model.

\section{Observations}

The observations were carried out at two SAI observatories: the Crimean station (1.25 m and $60 \mathrm{~cm}$ telescopes) and the Caucasian Mountain Observatory $(2.5 \mathrm{~m}$ and $60 \mathrm{~cm}$ telescopes). We have been performing spectral monitoring of SS433 since 1995 at the $1.25 \mathrm{~m}$ telescope, and since 2020 the $2.5 \mathrm{~m}$ telescope has been joined to this program. The BVRI-photometry of SS433 has been performed since 2015 at the $60 \mathrm{~cm}$ telescope of the Crimean station and since 2018 at the $2.5 \mathrm{~m}$ and the automatic $60 \mathrm{~cm}$ telescopes at the CMO. Photometric data during 413 nights and spectral data during 169 nights have been obtained over the past three years. Examples of CMO spectra can be found in [5]. The most dense photometric series is obtained in 2018-2020 and shown in Fig. 1.

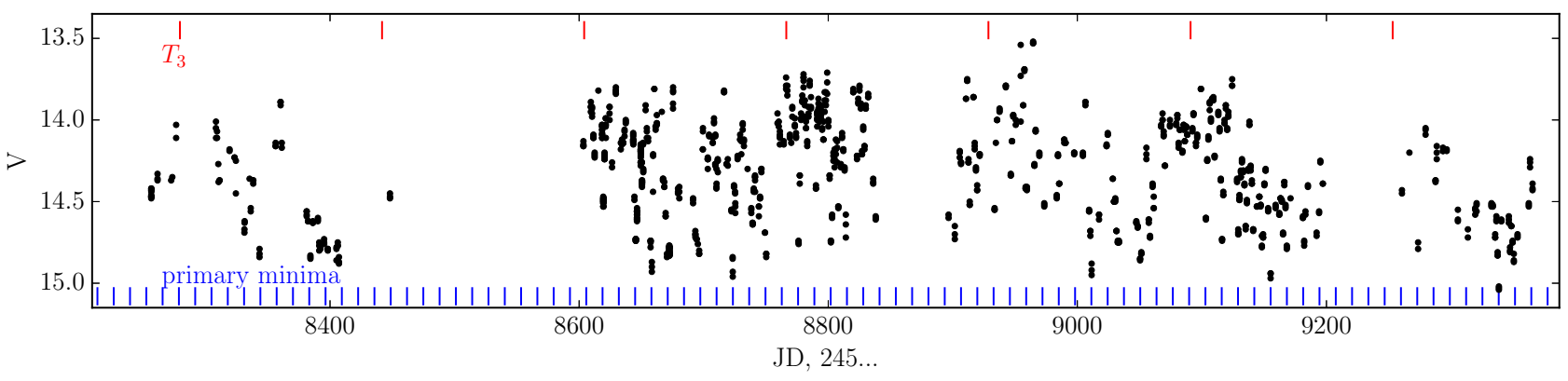

Figure 1: The V-light curve obtained at SAI telescopes in 2018-2020.

\section{Results}

New spectral observations allow us to determine the average parameters of the kinematic model over the past three years: a jet velocity $\beta=0.260 \pm 0.002$, an inclination of the accretion disk plane to the orbital plane $\theta=19^{\circ} .82 \pm 0^{\circ} .24$, an orbital inclination $i=79^{\circ} .35 \pm 0^{\circ} .15$, a precessional period $P_{\text {prec }}=161^{d} .12 \pm 0^{d} .149$, a nutation variability amplitude $z_{\text {nut }}=0.0081 \pm 0.0006$ a nutational period $P_{\text {nut }}=6^{d} .290 \pm 0^{d} .002$.

The comparison with the previous results [4] shows no systematic changes of these parameters over 43 years. The observed fluctuations of $P_{\text {prec }} \sim 2$ days are not associated with observational errors, but are caused by real the phase shifts in the precessional variability by \pm 10 days. The average jet velocity is constant over 43 years. We confirm the quasiperiodic variability of the jet velocity with an orbital period (see Fig.2). No secular changes in the equivalent width and Doppler width of the stationary emission $\mathrm{H} \alpha$, are observed, however the Doppler width shows quasiperiodicity with $P_{\text {prec }}$ phased in a such way that the minimal width achieved at $T_{3}$, i.e. when the disk 
is maximally opened to the observer that indicates the presence of a rotational component, coplanar to the disk. The precessional out-of-eclipse variability of SS433 is disturbed by irregular flares, but on average the precessional light curve is stable for 43 years (see Fig. 2). The average for 1978-2012 orbital phase curve of SS433, plotted on the basis of archived data near the $T_{3}$ moments, showed a shift of the secondary minimum relative to the middle between the two consequent primary minima (see [3]), which made it possible to estimate the eccentricity of the SS433 orbit $e=0.05 \pm 0.01$. Photometric observations over the past 3 years have revealed a noticeable delay in the moments of the primary and secondary minima relative to the linear ephemeris [6] - see Fig. 2. Applying the Herzsprung method allows us (see [3]) to detect a secular increase of the $P_{\text {orb }}$ with $\dot{P}_{\text {orb }}=10^{-7} \mathrm{~s} \mathrm{~s}^{-1}$ and derive a refined estimate for the mass ratio $q=M_{\mathrm{X}} / M_{\mathrm{V}}>0.8$.
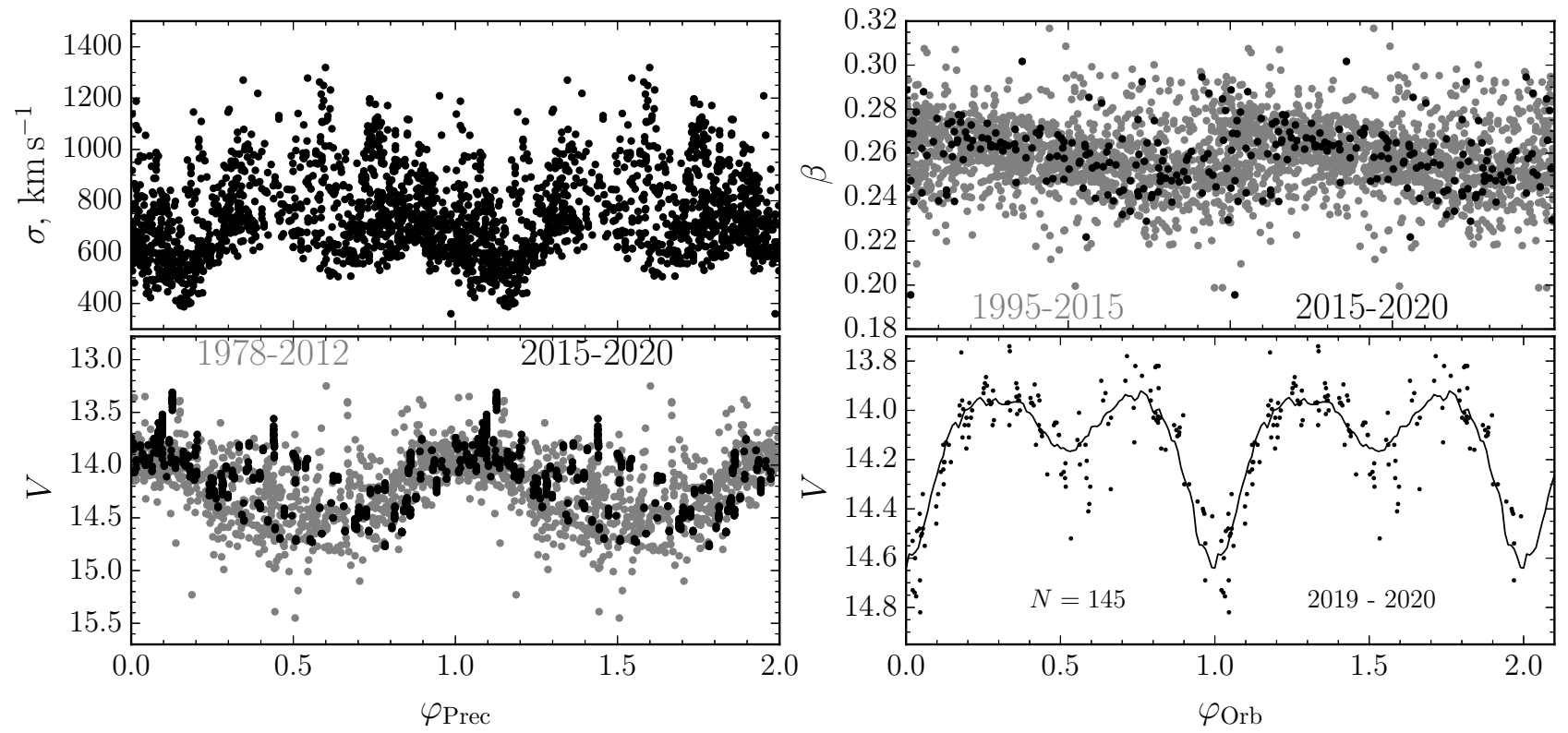

Figure 2: Left panels are for the precessional variability in the stationary $\mathrm{H} \alpha$ line width $\sigma$ and in the photometric $\mathrm{V}$ band (out of eclipses). Right panels are for orbital variability in the jet velocity and in the $\mathrm{V}$ band near the $T_{3}$ moments. The solid curve is the average phase curve over 1979-2012.

\section{Conclusion}

Systematic spectral and photometric observations of SS433, carried out at the SAI MSU observatories, allowed us to refine the parameters of SS433 and make important conclusions about the nature of this unique object [3]. The orbital ellipticity of SS433 system is consistent with the model of the slaved accretion disk tracing the rotational axis of the optical star. A high mass ratio $q>0.8$ favors to the presence of a black hole in the SS433 system, and also allows us to understand the seemingly mysterious fact that SS433, being at the stage of evolution during secondary mass exchange, evolves as a semi-detached system, without the formation of a common envelope [7].

The work is supported by the RSF grant 17-12-01241 and by the Scientific and Educational School of M.V. Lomonosov Moscow State University 'Fundamental and applied space research'. The authors acknowledge support from M.V.Lomonosov Moscow State University Program of Development.

\section{References}

1. S. Fabrika, Astrophysics and Space Physics Reviews, 12, 1, 2004.

2. A. Cherepashchuk, K. Postnov, S. Molkov, E. Antokhina, and A. Belinski, New Astron. Rev., 89, $101542,2020$.

3. A. M. Cherepashchuk, A. A. Belinski, A. V. Dodin, and K. A. Postnov, MNRAS, 507, L19, 2021.

4. A. M. Cherepashchuk, V. F. Esipov, A. V. Dodin, V. V. Davydov, and A. A. Belinskii, Astronomy Reports, 62, 747, 2018.

5. S. A. Potanin, A. A. Belinski, A. V. Dodin, S. G. Zheltoukhov, et al., Astronomy Letters, 46, 836, 2020.

6. V. Goranskij, Peremennye Zvezdy, 31, 2011.

7. E. P. J. van den Heuvel, S. F. Portegies Zwart, and S. E. de Mink, MNRAS, 471, 4256, 2017. 


\section{Parameters of Sco X-1}

A.M. Cherepashchuk, T.S. Khruzina, A.I. Bogomazov

Cherepashchuk@gmail.com

M.V. Lomonosov Moscow State University,

P.K. Sternberg Astronomical Institute, 119234, Universitetskij prospect, 13, Moscow, Russia

We modelled optical light curves of Sco X-1 obtained by the Kepler K2 mission. We calculated the ratio of the mass of the relativistic object to the mass of the optical star $q=M_{x} / M_{v}=3.6(3.5-3.8)$ and the orbital inclination $i=30^{\circ}\left(25^{\circ}-34^{\circ}\right)$.

Keywords: close binaries, neutron stars, accretion discs

DOI: $10.51194 /$ VAK2021.2022.1.1.039

A low mass persistent Z-type X-ray binary system Sco X-1 with a neutron star became the first X-ray source discovered outside the Solar system [1]. [2] suggested an idea that Sco X-1 was a low mass X-ray binary system with a low orbital inclination.

Observations of Sco X-1 by the Kepler space observatory during K2 mission [3] made it possible to construct orbital light curves for the low and high states (and the average curve). In these curves there were collected 115680 individual observations (the exposure time was $54.2 \mathrm{~s}$ ).

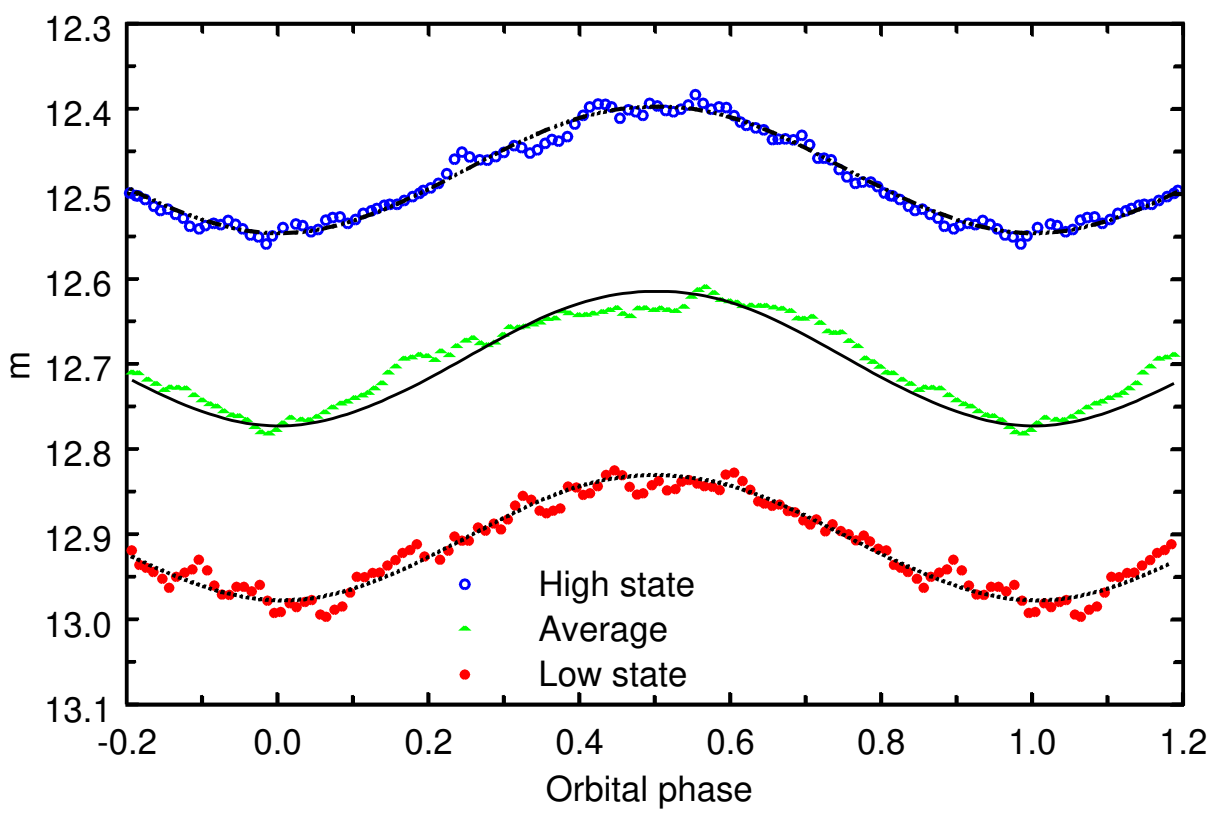

Figure 1: Observed light curves of Sco X-1 with optimal theoretical light curves.

In Fig. 1 we showed observational orbital light curves (they was calculated by us using data by [3]) of Sco X-1 with superimposed optimal theoretical curves (obtained from our modelling).

It was assumed that the donor star filled its Roche lobe and it (along with the accretion disc) was heated by the central X-ray source located in the center of the accretion disc. The temperature of elementary surfaces on the star and on the disc was defined from the sum of bolometric fluxes: $\sigma T^{4}=\sigma T_{0}^{4}+F_{x}^{b o l}$, where $T_{0}$ was the temperature of the non-disturbed surface, $F_{x}^{b o l}$ was the slanting X-ray bolometric flux. X-ray albedos of the star $\eta_{s}$ and of the disc $\eta_{d}$ were also taken into account. The opening angle of the accretion disc $\beta_{d}$ was varied from $3.2^{\circ}$ to $14^{\circ}$.

In our inverse problem of the interpretation of light curves of Sco X-1 there were six searched task parameters. In Fig. 2 we showed dependencies of residuals (minimized over all parameters except one) on parameters $q, i$ for the average light curve. It made possible to find optimal values of $q, i$ using the minimum of residuals.

In Fig. 3 we showed theoretical light curves for the donor star and accretion disc for optimal values of parameters. It can be seen that the brightness of the disc dominated in the total optical luminosity of the system, and the light curve of the optical star showed the single wave during the orbital cycle (the "reflection" effect). As the result of the inverse problem solution in the wide range of values of parameters we obtained following estimates of parameters $q, i: q=3.6(3.5-3.8), i=30^{\circ}\left(25^{\circ}-34^{\circ}\right)$. In the brackets we indicated uncertainties of values of parameters that mostly arose from the uncertainty of the physical model of Sco X-1.

Results of the solution of the inverse problem gave us a possibility to make following conclusions: 

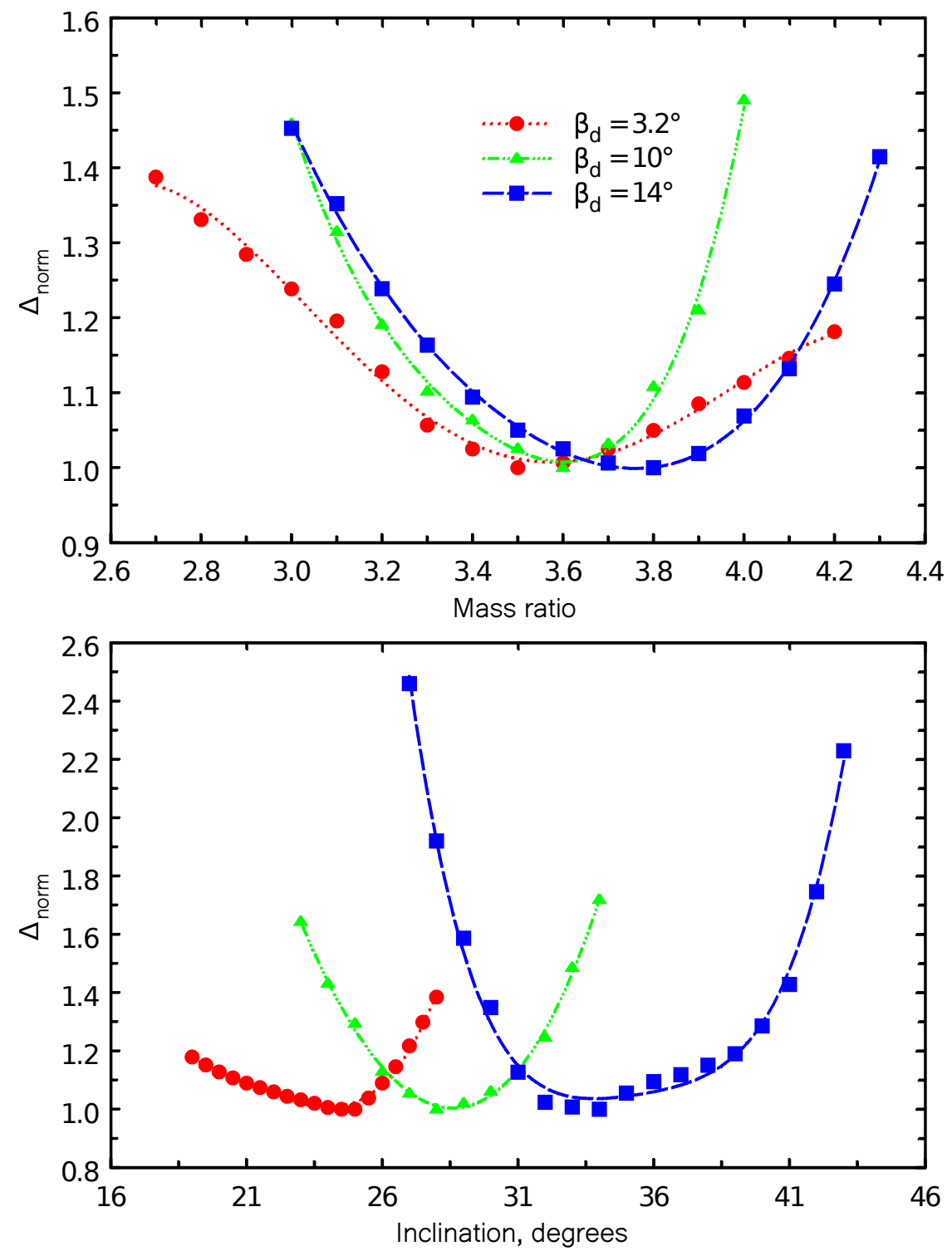

Figure 2: Dependencies of relative residuals minimized over all parameters except the mass ratio $q=M_{x} / M_{v}$ (upper panel) and inclination $i$ (lower panel) for the average light curve and different values of the disc's opening angle, $\eta_{d}=\eta_{s}=0$.

1. The transition between the low and high states of Sco X-1 corresponded to the increase of the bolometric X-ray luminosity of the central source $L_{x}$ by $2-3$ times.

2. The optical luminosity of the accretion disc dominated in the total optical luminosity of the Sco X-1 system. The contribution of the optical star did not exceed $\approx 20 \%$.

3. For our estimated value $q=0.6$ and for the mass of the neutron star $M_{x}=1.4 M_{\odot}$ the mass of the optical star was $M_{v}=0.4 M_{\odot}$. The temperature of the non-disturbed optical star was $T_{2} \approx 3000 \mathrm{~K}$, it corresponded to the spectral type M4-M5. The average radius of the star $R_{2}$ was $1.25 R_{\odot}$, its bolometric luminosity was $L_{b o l}=(2.1-4.6) \times 10^{32} \mathrm{erg} \mathrm{s}^{-1}$. So, the optical star in the Sco X-1 system possessed a significant excess of the radius and luminosity for its mass. Probably the star's mass decreased due to the mass loss in the form of the stellar wind induced by the X-ray heating [4, 5]. Another possibility to explain the anomaly of the optical star was the rapid mass loss, and due to this loss the star was not in the thermal equilibrium.

A detailed description of our results can be found in a paper by [6].

The work was supported by the Russian Science Foundation grant 17-12-01241 and by the Scientific and Educational School of M. V. Lomonosov Moscow State University "Fundamental and applied space research" (A. M. Cherepashchuk). The authors acknowledge support from M. V. Lomonosov Moscow State University Program of Development. 


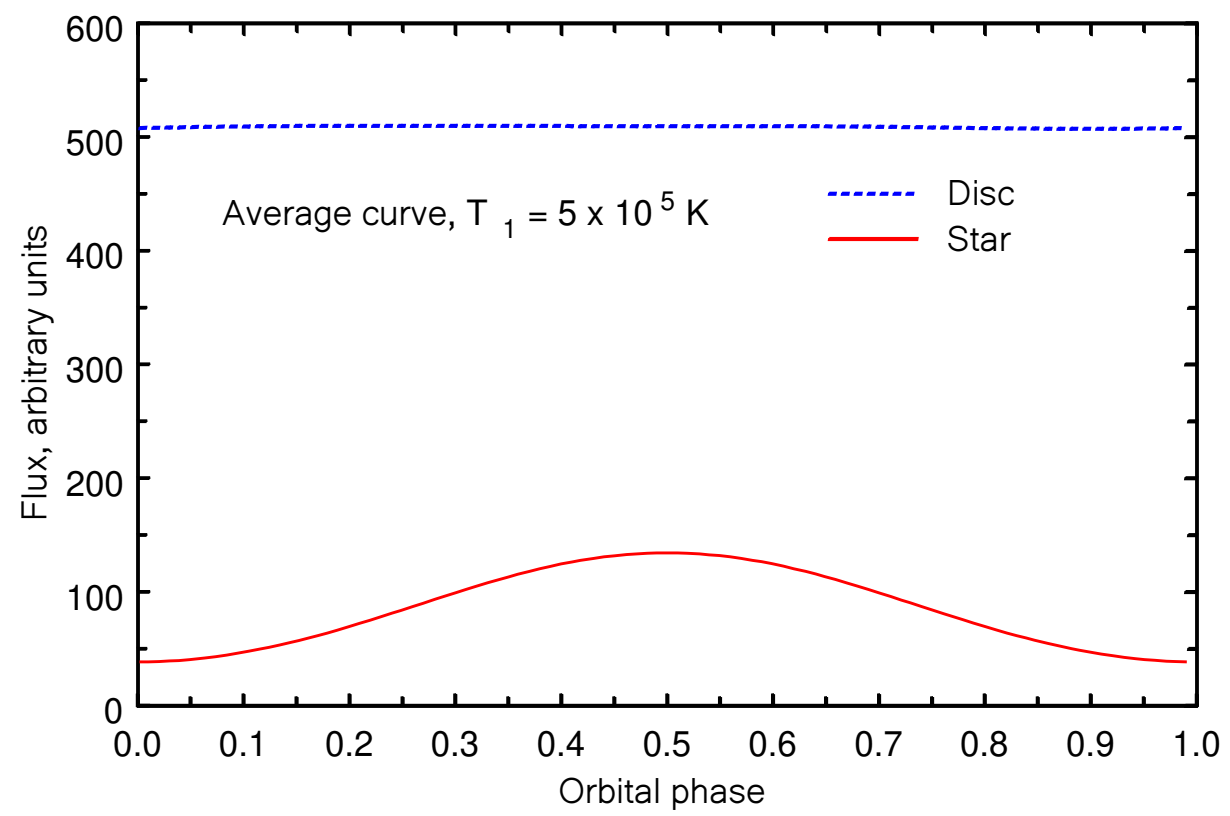

Figure 3: Light curves of the donor star and accretion disc computed as the inverse problem solution for the average light curve.

\section{References}

1. R. Giacconi, H. Gursky, F. R. Paolini, and B. B. Rossi, Phys. Rev. Lett., 9, 439, 1962.

2. J. I. Katz, $A \& A$, 39, 241, 1975.

3. R. I. Hynes, B. E. Schaefer, Z. A. Baum, C.-C. Hsu, M. L. Cherry, and S. Scaringi, MNRAS, 459, $3596,2016$.

4. M. M. Basko and R. A. Sunyaev, Ap\&SS, 23, 117, 1973.

5. J. Iben, Icko, A. V. Tutukov, and L. R. Yungelson, ApJS, 100, 233, 1995.

6. A. M. Cherepashchuk, T. S. Khruzina, and A. I. Bogomazov, MNRAS, 508, 1389, 2021. 


\title{
The mass function of pre-main sequence stars
}

\author{
O. Eretnova, A. Dudorov \\ eretnova@csu.ru \\ Chelyabinsk State University, Chelyabinsk, Russia
}

The information about 26 pre-man sequence (PMS) binary stars and 34 stars with protoplanetary disks with well determined masses from the observations is collected. We constructed the mass distribution of PMS stars and approximated it with a power law $d N \sim M^{-\gamma} d M$. The slope is $\gamma=2.16 \pm 0.17$ for stars in a mass range of $0.6 M_{\odot}<M<6.3 M_{\odot}$, which is close to the Salpeter mass function. Over the entire mass range, the mass distribution of young stars may be approximated with a lognormal law, which the most probable mass $M_{\text {prob }}=(0.78 \pm 0.12) M_{\odot}$.

Keywords: pre-man sequence stars, young stars, mass function

DOI: $10.51194 /$ VAK2021.2022.1.1.040

\section{Introduction}

The mass distribution of stars is determined by the conditions of their formation. It is presented either as the mass spectrum, $f(M)=d N / d M$, or as the mass function, $\Phi(\log M)=d N / d \log M$ [1]. If the mass spectrum is a power law, $d N \sim M^{-\gamma} d N$, then $\Phi(\log M) \sim M^{-\gamma+1}$, where $\gamma$ is the mass spectrum index (slope).

The masses are well determined from the observations for eclipsing double-line spectroscopic binaries or visual binaries and for the stars with Keplerian discs. The mass function of these stars can be constructed directly. Last decades it became possible to detect PMS binaries and the stars with protoplanetary discs in star formation regions. It is of interest to analyse the mass function of PMS stars, as they take an intermediate position in the evolutionary sequence "molecular cloud cores - initial main sequence (IMS) stars".

\section{The mass function of PMS stars}

We have collected the information about 26 young double-lined spectroscopic binaries and 34 stars with the protoplanetary disks. Among binary stars 23 systems are observed as eclipsing variables, 3 stars are both spectroscopic binaries and visual binaries. The main parameters of binary stars are published in [2]. The masses of stars with disks are determined from the Keplerian orbit of gas in their protoplanetary disks [3, 4]. We use the stars with mass uncertainties less than or equal to $10 \%$. Our sample consists 7 Ae/Be Herbig stars, 55 T Tauri stars and 25 red dwarfs. The stars are located in different star formation regions.

Figure 1 presents the histogram of the mass distribution of PMS stars on a logarithmic scale. According to Fig. 1, the largest number of stars are in a range of $\log \left(M / M_{\odot}\right) \in[-0.2,0.2]$, which corresponds to a mass of $M=(0.6-1.6) M_{\odot}$.

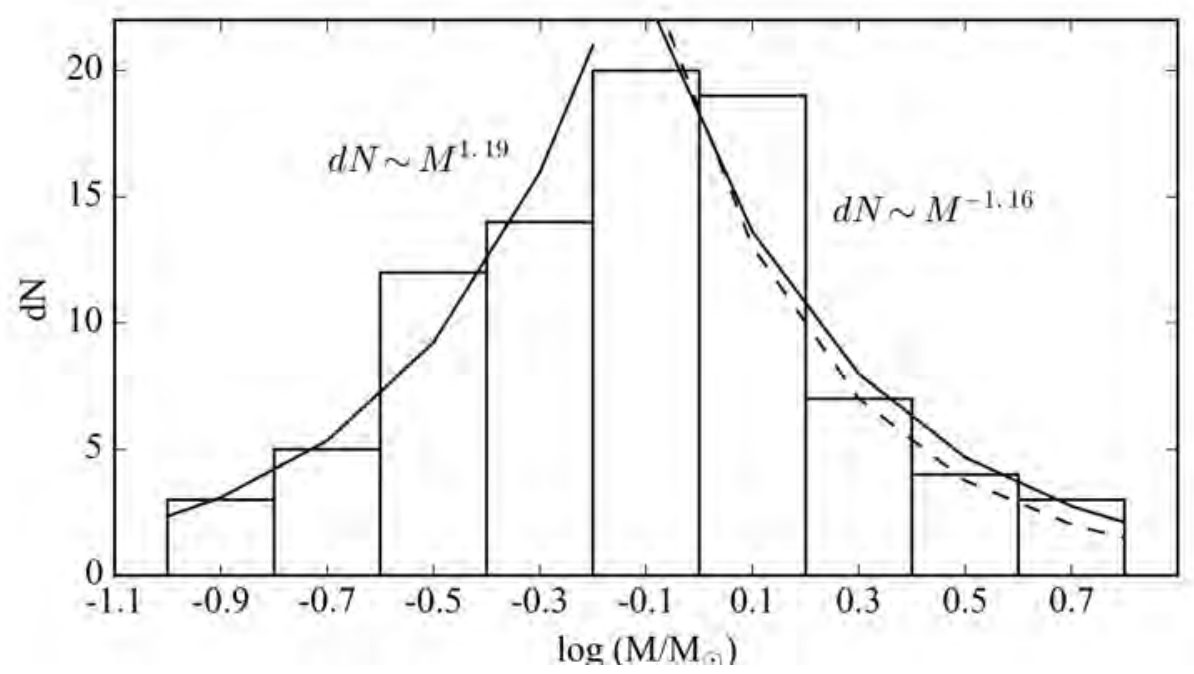

Figure 1: The mass distribution of PMS stars. The approximation of the histogram with a power law is shown by solid curves. A dashed curve shows the Salpeter IMF, $\gamma=2.35$.

The approximation of the histogram with a power law is shown by solid curves in Fig. 1: 


$$
\begin{aligned}
& \left\{\begin{array}{lll}
d N / d \log M \sim M^{-(1.16 \pm 0.17)}, & & -0.2<\log \left(M / M_{\odot}\right)<0.8, \\
d N / d \log M \sim M^{1.19 \pm 0.21}, & & -1.0<\log \left(M / M_{\odot}\right)<-0.2,
\end{array}\right. \\
& \left\{\begin{aligned}
d N / d M \sim M^{-(2.16 \pm 0.17)}, & & -0.2<\log \left(M / M_{\odot}\right)<0.8, \\
d N / d M \sim M^{0.19 \pm 0.21}, & & -1.0<\log \left(M / M_{\odot}\right)<-0.2 .
\end{aligned}\right.
\end{aligned}
$$

A dashed curve in Fig. 1 shows the stellar initial mass function (IMF) found by [5], $\gamma=2.35$.

The mass distribution of molecular cloud cores in Ophiuchus, Taurus, Perseus and Orion is studied by [6]. They found the mass spectrum index, $\gamma=(1.9-2.3)$, for cores with a mass of $1.0 M_{\odot}$ to $20 M_{\odot}$. [7] noted that the core mass function (CMF) similar to the stellar IMF, but the maximum can be shifted to higher masses.

Figure 2 presents the approximation of the mass distribution of PMS stars over the entire mass range with a lognormal law (solid curve):

$$
\Phi\left(\log M_{i}\right)=\frac{1}{\sigma \sqrt{2 \pi}} \cdot \exp \left(-\frac{\left(d \log M_{i}-\mu\right)^{2}}{2 \sigma^{2}}\right),
$$

where $\mu=-0.11 \pm 0.07, \sigma=0.36 \pm 0.06$ and the most probable mass is $M_{\text {prob }}=(0.78 \pm 0.12) M_{\odot}$ for our sample.

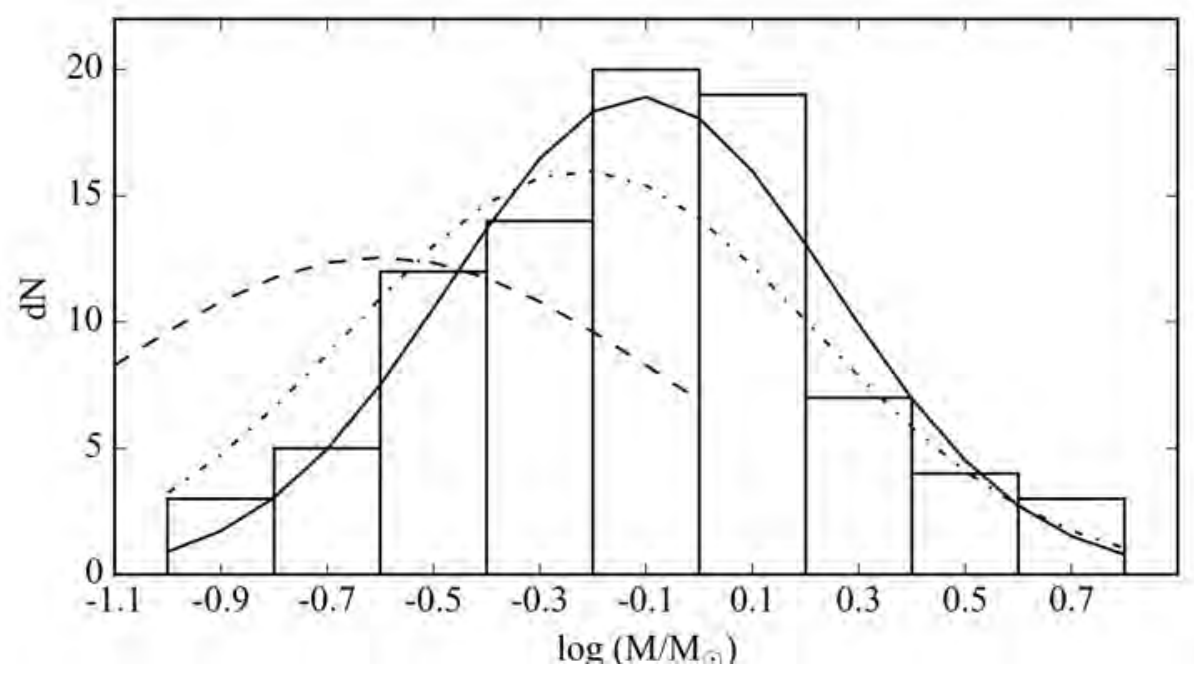

Figure 2: The mass distribution of PMS stars. The approximation of the histogram with a lognormal law is shown by a solid curve. A dashed curve shows the stellar IMF according to [8], a dash-dotted curve shows the CMF according to $[9]$.

In Fig. 2, a dashed curve shows the approximation of the stellar IMF with a lognormal law by [8], $\mu=$ $-0.60, \sigma=0.55$. [9], [10] determined the CMF for 540 molecular cloud cores in Aquila and 303 cores in Cepheus using data from the Herschel Observatory. They approximated the CMF with a lognormal law in a range of $0.1 M_{\odot}<M<10 M_{\odot}$. The most probable masses are $0.6 M_{\odot}$ for Aquila and $0.56 M_{\odot}$ for Cepheus cores. A dashdotted curve in Fig. 2 shows the CMF according to [9].

\section{Conclusions}

The data about 26 PMS binaries and 34 stars with protoplanetary disks with well determined masses from the observations were collected. Among them are 7 Ae/Be Herbig stars, 55 T Tauri stars and 25 red dwarfs.

The mass distribution was constructed and approximated it with a power law, $d N \sim M^{-\gamma} d N$, the slope is $\gamma=2.16 \pm 0.17$ for stars in a mass range of $M=(0.6-6.3) M_{\odot}$. It is close to the Salpeter IMF. The slope is $\gamma=-0.19 \pm 0.21$ in a range of $M=(0.1-0.6) M_{\odot}$.

Over the entire mass range, the mass distribution of PMS stars may be approximated with a lognormal law. The most probable mass is $M_{\text {prob }}=(0.78 \pm 0.12) M_{\odot}$. The maximum is shifted towards higher masses compared to the stellar IMF [8]. Modern data of the CMF give the most probable mass $M=(0.5-0.6) M_{\odot}[9,10]$. According to [11], the mass of the IMS star is $0.3-0.5$ of the mass of the prestellar core. Young stars occupy an intermediate position between prestellar cores and IMS stars. The value we obtained for the most probable mass for PMS stars turned out to be slightly larger than the analogous value for the cores. This can be explained by the following: the discovery of young binaries and stars with protoplanetary disks began recently and the studied sample of PMS stars is incomplete in the region of $M<1.0 M_{\odot}$; there are large uncertainties in the estimates of the masses of prestellar cores. 


\section{References}

1. J. M. Scalo, in T. Gehrels and M. S. Matthews, eds., IAU Colloq. 52: Protostars and Planets, 265 (1978).

2. A. E. Dudorov and O. V. Eretnova, Chelyabinskiy Fiziko-Matematicheskiy Zhurnal, 6, 347, 2021.

3. S. Guilloteau, M. Simon, V. Piétu, E. D. Folco, A. Dutrey, L. Prato, and E. Chapillon, A\&A, 567, A117, 2014.

4. M. Simon, S. Guilloteau, T. L. Beck, E. Chapillon, et al., ApJ, 884, 42, 2019.

5. C. F. Salpeter, ApJ, 121, 161, 1955.

6. S. I. Sadavoy, J. D. Francesco, S. Bontemps, S. T. Megeath, L. M. Rebull, E. Allgaier, and et. el, ApJ, 710, 1247, 2010.

7. S. S. R. Offner, P. C. Clark, P. Hennebelle, N. Bastian, M. R. Bate, P. F. Hopkins, E. Moraux, and A. P. Whitworth, in H. Beuther, R. Klessen, C. P. Dullemond, and T. Henning, eds., Protostars and Planets VI, 53 (2014).

8. G. Chabrier, The Initial Mass Function: From Salpeter 1955 to 2005, 41 (2005).

9. P. André, A. Men'shchikov, S. Bontemps, V. Könyves, F. Motte, N. Schneider, and et. el, A\&A, 518, L102, 2010.

10. J. D. Francesco, J. Keown, C. Fallscheer, P. André, B. Ladjelate., and et. el, ApJ, 904, 172, 2020.

11. C. F. McKee and E. C. Ostriker, ARA $\& A$, 45, 565, 2007. 


\title{
Anisotropic scattering of pulsars radio emission
}

\author{
E.N. Fadeev ${ }^{1}$, A.S. Andrianov ${ }^{1}$, M.S. Burgin ${ }^{1}$, M.V. Popov ${ }^{1}$, A.G. Rudnitskii ${ }^{1}$, T.V. Smirnova ${ }^{2}$, \\ V.A. Soglasnov ${ }^{1}$, V.A. Zuga ${ }^{1}$ \\ fadeev@asc.rssi.ru \\ ${ }^{1}$ Astro Space Center, Lebedev Physical Institute, Russian Academy of Sciences, Profsoyuznaya str 84/32, Moscow 117997, \\ Russia, \\ ${ }^{2}$ Pushchino Radio Astronomy Observatory, Astro Space Center, Lebedev Physical Institute, Russian Academy of Sciences, \\ Pushchino, Moscow oblast' 142290, Russia
}

We have made the high-resolution measurements of pulsar scattering disks with the RadioAstron. For three pulsars, B1641-45, B0833-45, and B0834+06, we measured the angular size with various directions of baseline projections and we were able to estimate the anisotropy of their scattering images. An aspect ratio of all elliptical images no more than 2:1 is consistent with the data.

Keywords: interstellar medium, pulsars scintillation

DOI: $10.51194 /$ VAK2021.2022.1.1.041

\section{Introduction}

The pulsar radiation passes through the ionized interstellar medium (IISM) that scatters it into multiple paths some of which lead to the observer. The resulting interference is the reason for the angular broadening of pulsars, the frequency and time modulation of the radio emission intensity, and some other effects. [1] discovered parabolic features in secondary spectra of many pulsars. [2] and [3] in their theoretical investigations concluded extreme elongation of pulsars' scattering disks. [4] and [5] used a one-dimensional scatter broadening function in their model of the brightness distribution of pulsar emission. To measure the anisotropy of scattering disks, it is necessary a high-resolution array. The RadioAstron makes it is possible.

\section{Theory}

Authors of [6] studied the pulsar B0329+54 with RadioAstron and showed that the modulus of the visibility function in the delay $(\tau)$ /fringe-rate $(f)$ domain, $|V(\tau, f)|$, looks like a narrow spike surrounded by a broad component. They found that spike amplitude decreased with an increase of baseline projection until the spike disappeared into the fine structure of the broad distribution. The relation of the visibility amplitude with baseline $b$ due to angular broadening is given by [7] as

$$
V=\exp \left\{-\frac{1}{2}\left[\frac{\pi}{\sqrt{2 \ln 2}} \frac{\theta_{H} b}{\lambda}\right]^{\alpha-2}\right\}
$$

where a wavelength $\lambda$ and a full width at half maximum of a Gaussian image of the scattering disk $\theta_{H}$. The parameter $\alpha$ is the spectral index of electron density fluctuations in the ISM. It could be measured with the technique proposed by [8].

Calibrating of several antennas is rarely accurate enough, so we also used the technique that had suggested by [8]. This technique implies easier relative calibrating and allows us to get the scintillation spatial scale $\rho_{\text {dif }}$ that is inversely proportional to $\theta_{H}$.

\section{Observation and Data Analysis}

The space-ground interferometer RadioAstron consisted of the high apogee satellite Spektr- $\mathrm{R}$ and a set of groundbased antennas. We observed pulsars B1641-45, B0833-45 on the central frequency $1.668 \mathrm{GHz}$, and B0834+06 on the frequency $324 \mathrm{MHz}$ with the base length up to $200000 \mathrm{~km}$. For every pulsar, the position angles of baseline projection altered in a wide range.

The interferometer resolved the scattering disk of B1641-45 not only on space-ground baselines but also on the intercontinental ones. The fitting with the equation 1 for only Australian baselines yields $\theta_{H}$ of 27 mas. Owing to the long duration of the observing session, we have got good coverage of the UV plane, making it possible to image the scattering disk using conventional ground-based VLBI methods. Fitting with a two-dimensional Gaussian yields FWHM of 20.6 mas $\times 27.5$ mas in right ascension and declination, which agrees well with the previous measurement [9].

As in the previous case, the scattering disk of B0833-45 was resolved or almost resolved on intercontinental baselines therefore we used only Australian baselines again. The dependence of visibility amplitude on baseline length is presented in Fig. 1. The left panel shows observations of 2012. The best fit with the equation 1 matches experimental data with good accuracy and gets $\theta_{H}=6.4 \pm 0.4$ mas. 

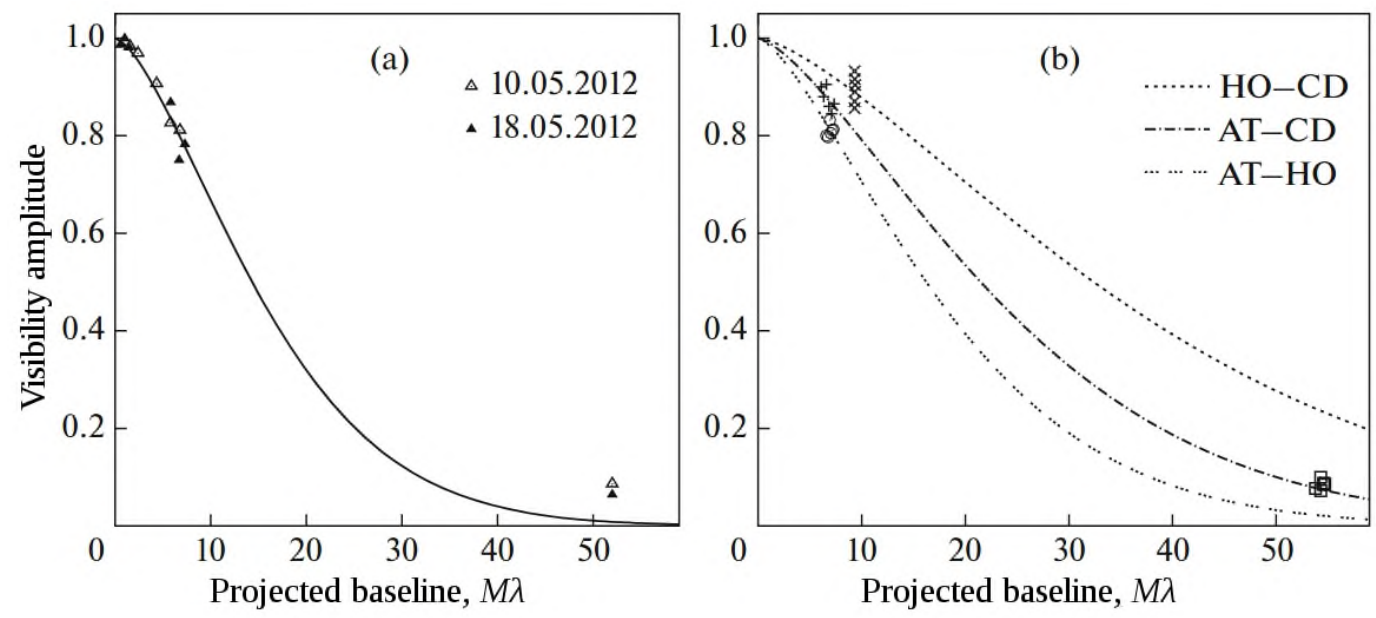

Figure 1: Visibility amplitude of the Vela pulsar versus projected baseline length in millions of wavelength for the (a) 2012 and (b) 2013 observations. The baselines larger than $50 M \lambda$ were not used for fitting in both cases.

Data for 2013 are plotted in the right panel of Fig. 1. This time we cannot obtain a single fit of all data and have to turn to the geometry of our interferometer. All Australian antennas used in observational sessions of 2012 located similar along the direction north-south. In 2013 we had only intermediate baselines with different position angles that determine pulsar's image in different sections. Derived values $\theta_{H}$ varied from 3.8 to 8.0 mas. This result corresponds to an elliptical scattering disk with an axial ratio of approximately $2: 1$ and the positional angle of the major axis $50^{\circ} \pm 20^{\circ}$. The effective pulsar velocity aligns approximately along the minor axis of the scattering disk that has to suppress the formation parabolic arcs, which we did not find in fact. [10].

In all periods of observation, we obtained distinguishable parabolic arcs in secondary spectra of pulsar B0834+06 [11] therefore we could expect a high elongated scattering disk. We measured $\rho_{\text {dif }}$ for four days of observation with different directions of space-ground baselines. We have fitted these results by an ellipse and found that the aspect ratio of the main axes is about 2.5 and the position angle of the major axis is $-8^{\circ}$. This spatial scale of scintillation corresponds to $\theta_{H}=1.7 \times 0.9$ mas with the position angle of the major axis is $-98^{\circ}$.

This result is contrary to [5] who showed that the pulsar image stretched out about 30 mas along a straight line. However, the theoretical models by [3] tells us that clearly distinguishable parabolic structures are produced by elongated scattering disks with the bright core and the wide dim envelope. The power in secondary spectra included in arc structure has just a few percent of the whole power. While Brisken's research focused on parabolic arcs, we analyzed the total power of the radio emission. Therefore we propose that we mapped only the core of the scattering disk.

\section{Conclusion}

We have made the first steps to VLBI measurement of scattering disks anisotropy that allows studying the fine structure of their brightest parts. This technique could complement the scintillometrical reconstruction of pulsar images developing rapidly in recent years.

\section{References}

1. D. R. Stinebring, M. A. McLaughlin, J. M. Cordes, K. M. Becker, J. E. E. Goodman, M. A. Kramer, J. L. Sheckard, and C. T. Smith, ApJL, 549, L97, 2001.

2. M. A. Walker, D. B. Melrose, D. R. Stinebring, and C. M. Zhang, MNRAS, 354, 43, 2004.

3. J. M. Cordes, B. J. Rickett, D. R. Stinebring, and W. A. Coles, ApJ, 637, 346, 2006.

4. F. S. Trang and B. J. Rickett, ApJ, 661, 1064, 2007.

5. W. F. Brisken, J. P. Macquart, J. J. Gao, B. J. Rickett, W. A. Coles, A. T. Deller, S. J. Tingay, and C. J. West, ApJ, 708, 232, 2010.

6. C. R. Gwinn, M. V. Popov, N. Bartel, A. S. Andrianov, et al., ApJ, 822, 96, 2016.

7. C. R. Gwinn, J. M. Cordes, N. Bartel, A. Wolszczan, and R. L. Mutel, ApJL, 334, L13, 1988.

8. V. I. Shishov, T. V. Smirnova, W. Sieber, V. M. Malofeev, et al., A $6 A$, 404, 557, 2003.

9. M. V. Popov, A. S. Andrianov, N. Bartel, C. Gwinn, et al., Astronomy Reports, 60, 792, 2016.

10. M. V. Popov, A. S. Andrianov, M. S. Burgin, V. A. Zuga, A. G. Rudnitskii, T. V. Smirnova, V. A. Soglasnov, and E. N. Fadeev, Astronomy Reports, 63, 391, 2019.

11. T. V. Smirnova, V. I. Shishov, A. S. Andrianov, M. S. Burgin, et al., MNRAS, 496, 5149, 2020. 


\title{
Two-dimensional MHD model of gas flow dynamics near a young star with a jet and a protoplanetary disk
}

\author{
V.V. Grigoryev, T.V. Demidova \\ vitaliygrigoryev@yandex.ru \\ Crimean Astrophysical Observatory RAS, p. Nauchny, Bakhchisaray, Crimea, 298409, Russia
}

Flow of matter in the immediate vicinity of a $\mathrm{T}$ Tauri type star with a constant dipole magnetic field on a two-dimensional axisymmetric computational grid is simulated. Within the framework of a nonstationary MHD approach, the interaction of a jet, a partially ionized gas of the stellar corona, and a protoplanetary disk is considered. The model takes into account the degree of gas ionization, optically thin cooling, anisotropic thermal conductivity and viscosity. Numerical modeling is performed using the free PLUTO package.

Keywords: MHD, computational astrophysics, plasma physics, protoplanetary disks

DOI: 10.51194 /VAK2021.2022.1.1.042

\section{Introduction}

The $\mathrm{T}$ Tauri type include stars of late spectral types at the initial stage of their evolution. The features in the spectra and on the light curves of such stars are associated with the movement of matter in their protoplanetary disks. They are formed from the remnants of a protostellar cloud together with a star and are the building blocks for planetary systems.

One of the most famous articles on 2D MHD modeling of gas flows around T Tauri stars is the paper [1]. However, due to a number of simplifications, this model has some non-physical features. For example, the gas above the plane of the disk is too dense, and the initial temperature of the disk is $10000 \mathrm{~K}$. Some effects are not taken into account that can potentially affect the processes occurring with gas (except viscosity): conductivity, thermal conductivity, radiation cooling. Also ignored the Gauss theorem for the magnetic field.

One of the latest works related to the study of vicinity of T Tauri stars is [2]. The authors' numerical model is implemented using the PLUTO [3] package, and the initial conditions and assumptions repeat those expressed in [1]. However, optically thin cooling is taken into account. At the same time, it was believed that the molar mass of the gas does not change. In addition, the authors removed from the calculations the region near the axis of rotation, which does not allow us to speak of jets or the formation of accretion columns at high latitudes. An important disadvantage of this model is the short time of simulation.

It should be noted that there is no detailed analysis of relaxation processes in the models under consideration in the cited works. Only the relaxation time is given. In this work, MHD simulation includes the degree of gas ionization, optically thin cooling, anisotropic thermal conductivity and viscosity. Also we discuss the relaxation process of the model.

\section{Model}

The two-dimensional model is implemented using the PLUTO package [3], which allows solving the MHD equations by the Godunov method. The model considers the basic equations of non-stationary MHD:

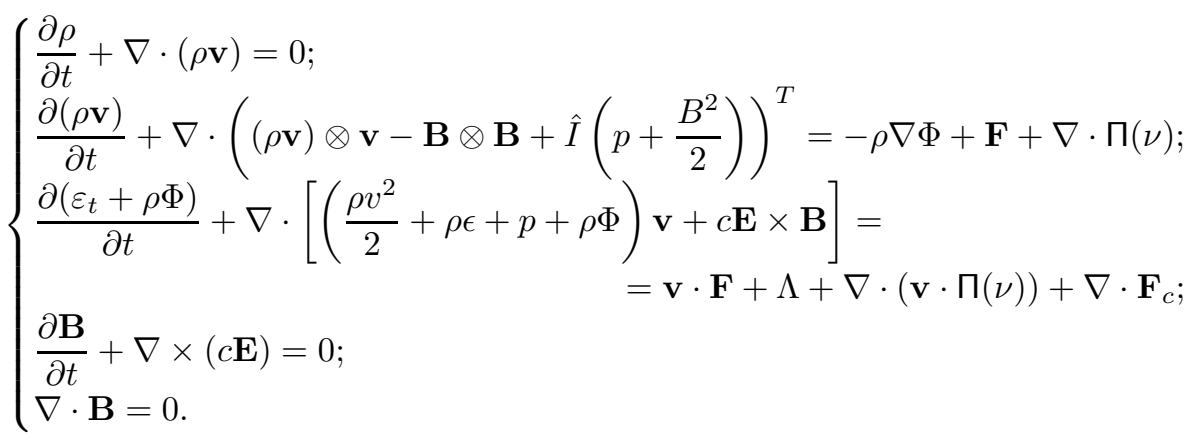

Here $\rho$ - density of the gas in the cell, $\mathbf{v}$ - its velocity, $\mathbf{B}$ - magnetic field induction, $\hat{I}$ - unit matrix, $p-$ gas pressure (equation of state is described below), $\mathbf{F}$ - external forces, $\Pi(\nu)$ - tensor of the viscosity, $\varepsilon_{t}$ - total energy density, $\Phi$ - gravitational potential, $\mathbf{E}$ - electric field strength, $\Lambda$ - optically thin cooling term, $\mathbf{F}_{c}$ heat flow, $c$ - speed of light. The last equation is solved using the Constrained transport method, which is the most accurate from available in the PLUTO arsenal. Initial conditions are the same as in [1], Type I. Constant dipole magnetic field of the star is $10^{3} \mathrm{G}$. 


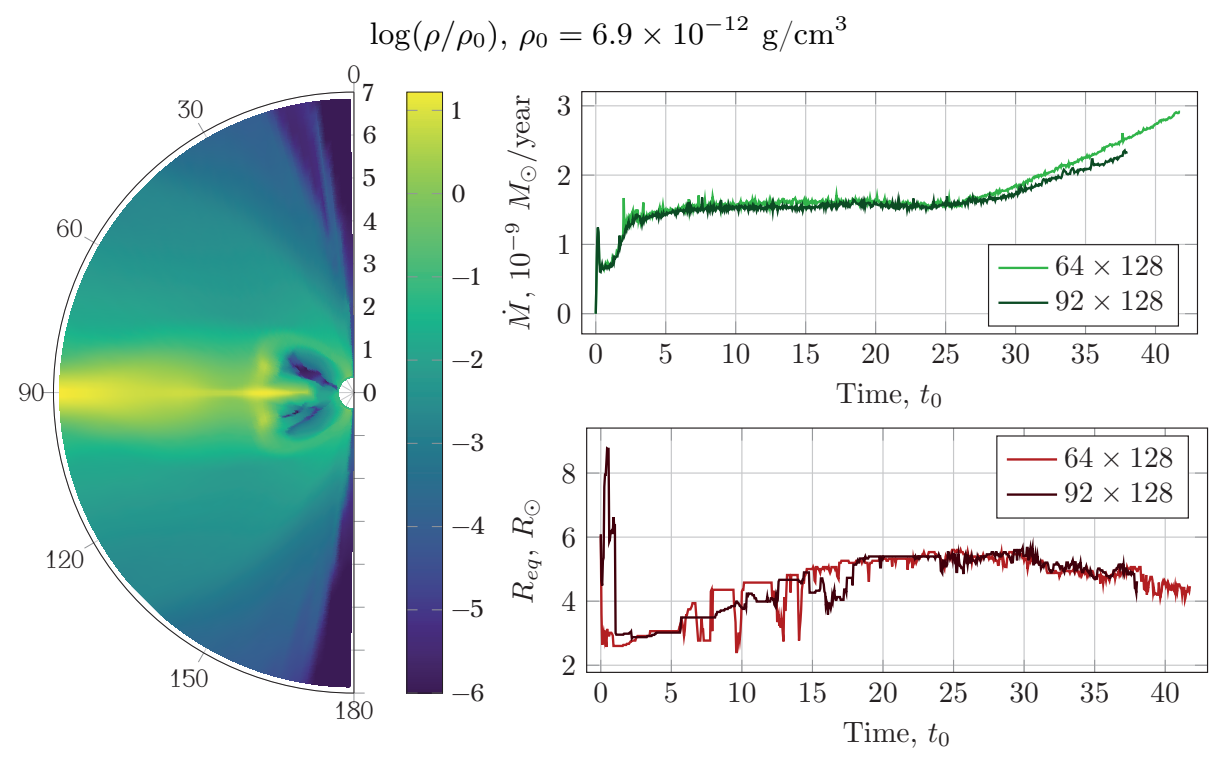

Figure 1: On the left - density distribution at $t=38 t_{0}$, top right - mass accretion onto the star surface (calculations on two grids), bottom right — the equatiorial radius of the magnetosphere (calculations on two grids) in solar radii.

The equations were solved in polar coordinates on two computational grids: the first is $(R, \theta) \in[0.35 ; 30] \times$ $(0 ; \pi), 92 \times 128$ cells, the right border is $R \approx 0.86$ a.u. and the second is $(R, \theta) \in[0.35 ; 8] \times(0 ; \pi), 64 \times 128$ cells, the right border is $R \approx 0.23$ a.u.

Outer boundary condition on $R$ is outflow, lower - outflow, but in the cells closest to the star, a stellar atmosphere (the thickness is $\sim 10^{4} \mathrm{~km}$ ) is modelled. This idea is not new and has been previously implemented, for example in [4] and [5]. The boundary conditions on $\theta$ satisfy the axisymmetry condition, but in the angle $\leq 3.5^{\circ}$ a jet is simulated, the matter of which is $10^{2}$ times less dense than the disk corona, and at the same time it flies away from the star at a speed no less than local escape velocity. Equation system (1) is closing by ideal gas equation, taking into account ionization. This is possible thanks to the SNEq module, which makes it possible to supplement the optically thin cooling, expressed by $\Lambda$.

According to $[6,7,8]$ the Rosseland mean opacity $\sim 10^{-5} \mathrm{~cm}^{2} \mathrm{~g}^{-1}$ for the temperatures and densities of our interest $\left(\sim 10^{3} \mathrm{~K}, \sim 10^{-11} \mathrm{~g} / \mathrm{cm}^{3}\right)$, which allows us to use the optical thin disk approximation.

Viscosity is included isotropically based on the Shakura-Sunyaev model [9]: $\nu=\left(\alpha_{\mathrm{ShS}}+\beta^{-1}\right) c_{s}^{2} / \Omega_{K}$, where $\alpha_{\mathrm{ShS}}=0.02$ - classical parameter of Shakura-Sunyaev, $\beta$ - plasma beta, $c_{s}$ - local sound speed, $\Omega_{K}-$ angle rotation velocity.

Anisotropic thermal conductivity is taken into account in accordance with analytical formulas [10, 11]:

$$
\left\{\begin{array}{l}
\mathbf{F}_{c}=\kappa_{\|} \mathbf{b}(\mathbf{b} \cdot \nabla T)+\kappa_{\perp}[\nabla T-\mathbf{b}(\mathbf{b} \cdot \nabla T)] \\
\kappa_{\|}=5.6 \times 10^{-7} T^{2} \sqrt{T} \quad \kappa_{\perp}=3.3 \times 10^{-16} \frac{n_{\mathrm{H}}^{2}}{B^{2} \sqrt{T}}
\end{array}\right.
$$

Here $\mathbf{b}=\mathbf{B} / B$, and $n_{\mathrm{H}}-$ hydrogen concentration.

\section{Results}

Fig. 1 (left) shows the logarithm of the density at the time $38 t_{0}\left(t_{0} \approx 2.5 \times 10^{4} \mathrm{~s}\right)$. The matter of the disk has formed two accretion columns. Initially, they were formed by the corona material during about one period of rotation $\left(2 \pi t_{0}\right)$, which is consistent with the result of [1]. These accretion columns enclose two hot bubbles that are part of the magnetosphere. Its equatorial radius is measured from the center of the star to the closest point of the magnetosphere in the plane of the disk, and it changes as shown in Fig. 1 (right bottom). The disk has a low temperature $\left(\sim 10^{3} \mathrm{~K}\right)$, which a posteriori justifies the approximation of optically thin cooling.

The vertical wall, from which the columns begin, was formed relatively recently and is moving towards the star at a low speed. Therefore, one cannot speak of reaching equilibrium at this stage of the calculations. This is also clear from the as yet unstable accretion rate (Fig. 1, right top) and non-constant so far the equatorial radius of the magnetosphere (Fig. 1, right bottom).

Calculations on two grids for the same estimated time show qualitative and quantitative similarities. Thus, we can conclude that the computational area can be reduced by refusing to consider more distant areas in order to save computing resources and reduce the computation time. 
Acknowledgment. The simulations were performed using the resources of the Joint SuperComputer Center of the Russian Academy of Sciences [12] https://www.jscc.ru/. The work on the preparation of the initial conditions and the analysis of the results of T.V.D. is supported by the Russian Science Foundation under grant 19-72-10063.

\section{References}

1. M. M. Romanova, G. V. Ustyugova, A. V. Koldoba, and R. V. E. Lovelace, ApJ, 578, 420, 2002.

2. S. Colombo, S. Orlando, G. Peres, F. Reale, C. Argiroffi, R. Bonito, L. Ibgui, and C. Stehlé, A\&A, 624, A50, 2019.

3. A. Mignone, G. Bodo, S. Massaglia, T. Matsakos, O. Tesileanu, C. Zanni, and A. Ferrari, ApJS, 170, 228, 2007.

4. V. Grigoryev and A. Krassilchtchikov, Proc-s of conf in honor of V. V. Sobolev, 305-310, 2015.

5. S. Takasao, K. Tomida, K. Iwasaki, and T. K. Suzuki, ApJ, 857, 4, 2018.

6. K. R. Bell and D. N. C. Lin, ApJ, 427, 987, 1994.

7. D. Stamatellos, A. P. Whitworth, T. Bisbas, and S. Goodwin, A\&A, 475, 37, 2007.

8. D. Forgan, K. Rice, D. Stamatellos, and A. Whitworth, MNRAS, 394, 882, 2009.

9. N. I. Shakura and R. A. Sunyaev, MNRAS, 175, 613, 1976.

10. S. A. Balbus, ApJ, 304, 787, 1986.

11. L. L. Cowie and C. F. McKee, ApJ, 211, 135, 1977.

12. G. Savin, B. Shabanov, P. Telegin, and A. Baranov, Lobachevskii J Math, 40, 1853, 2019. 


\title{
IRAS 02143+5852: W Vir Cepheid with a dust shell
}

\author{
N. Ikonnikova ${ }^{1}$, M. Burlak ${ }^{1}$, S. Shugarov ${ }^{1,2}$, A. Belinskii ${ }^{1}$, A. Fedoteva ${ }^{1}$, A. Tatarnikov ${ }^{1}$, A. Dodin ${ }^{1}$, \\ S. Potanin ${ }^{1,3}$, K. Atapin ${ }^{1}$, S. Zheltoukhov ${ }^{1}$ \\ ikon@sai.msu.su \\ ${ }^{1}$ Lomonosov Moscow State University, Sternberg Astronomical Institute, 13 Universitetskij prospekt, Moscow 119234, \\ Russia \\ ${ }^{2}$ Astronomical Institute of the Slovak Academy of Sciences, Tatranska Lomnica 05960, Slovakia \\ ${ }^{3}$ Faculty of Physics, Moscow M.V. Lomonosov State University, Leninskie gory 1, Moscow, 119991, Russia
}

We present the results of multicolor $\left(U B V R_{C} I_{C} J H K\right)$ photometry and low resolution spectroscopy of the poorly studied post-AGB candidate IRAS $02143+5852$. A Cepheid-like brightness variability with the pulsation period of about 24.8 days and the full peak-to-peak amplitude of $0.9 \mathrm{mag}$ in the $V$-band was found. The spectral type varies from F3I at maximum to F8I at minimum light. A strong $\mathrm{H} \alpha$ emission line appears on the ascending branch of the light curve. Unlike typical W Vir variables, IRAS $02143+5852$ has a significant infrared excess of radiation associated with the presence of a heavy dust envelope around the star. We computed a dust shell model to obtain the parameters of the envelope.

Keywords: Stars: variables, photometry, spectra, W Vir, post-AGB, dust shell

DOI: 10.51194/VAK2021.2022.1.1.043

\section{Introduction}

The infrared (IR) source IRAS $02143+5852$ was classified as a post-AGB star based on its location in the IRAS color-color diagram [1]. The optical counterpart of IRAS $02143+5852$ is a poorly studied star with the spectral type Ae [2] or F7Ie [3]. [4] determined the parameters of the star: $T_{\text {eff }}=7900 \mathrm{~K}, \log g=1.3$ and $[\mathrm{Fe} / \mathrm{H}]=-0.7$. More recently, the light variability of the star was discovered from the ASAS-SN data [5]. In the ASAS-SN catalog of variable stars, it has the designation ASASSN-V J021757.82 + 590552.0, a period of 50.18 days and a variability type of YSO (Young stellar object). The aim of our work was to clarify the nature of photometric variability and spectral type, as well as to determine the evolutionary status of the star.

\section{Observations}

CCD photometry of IRAS $02143+5852$ in the $U B V R_{C} I_{C}$-bands was carried out at the 60 -cm telescope RC600 of the Caucasian Mountain Observatory (CMO) of Sternberg Astronomical Institute of the Moscow State University(SAI MSU) [6] and the 60-cm telescope of the Academy of Sciences of Slovakia. As a result, more than 250 estimates of the star's brightness were obtained for the 2018-2021 interval. Also 52 brightness estimates in the $J H K$-bands were obtained at the 2.5-m telescope of the CMO SAI MSU using the ASTRONIRCAM IR-camera [7] in 2017-2021. In addition, low-resolution $(R \sim 2000)$ spectral observations were performed at the 2.5-m telescope of the CMO SAI MSU with the TDS spectrograph [8]. Twelve spectrograms were obtained in the wavelength range 3500-7500 $\AA$ in 2019-2021.

\section{Data analysis}

\subsection{Photometry}

The observational data analysis showed the star to undergo periodic brightness variations. The star demonstrates a similarity with Type II Cepheids in the morphology of light curves.

Period analysis of the $U B V R_{C} I_{C}$ curves yielded the period $P=24.885$ days that falls within the range of periods of W Vir pulsating variables. Our search for periodicity in the infrared brightness variations revealed the period $P=24.800$ days. Fig. 1 shows $U B V R_{C} I_{C}$ and $J H K$-data folded on the periods of 24.885 and 24.800 days, respectively.

So, according to the shape of the light curves, the oscillation periods and amplitudes, the star can be classified as a long-period W Vir variable. The alternating of deep and shallow minima in some photometric bands makes IRAS $02143+5852$ similar to RV Tau variables.

\subsection{Spectra}

The IRAS $02143+5852$ spectrum confirms the classification of the star as a W Vir pulsating variable. The star has weakened metal lines, and W Vir stars are known to have reduced metallicity. According to preliminary estimates, the spectral type varies from $\sim$ F3I at maximum to $\sim$ F8I at minimum light. However, the molecular CN and $\mathrm{CH}$ bands appear in the spectrum in the faint state, which implies a later spectral type. The emission line H $\alpha$ appears near minimum and persists until maximum light, then sharply weakens revealing maximum intensity in the middle of the ascending branch. The occurrence of hydrogen emission is characteristic of Type II Cepheids 

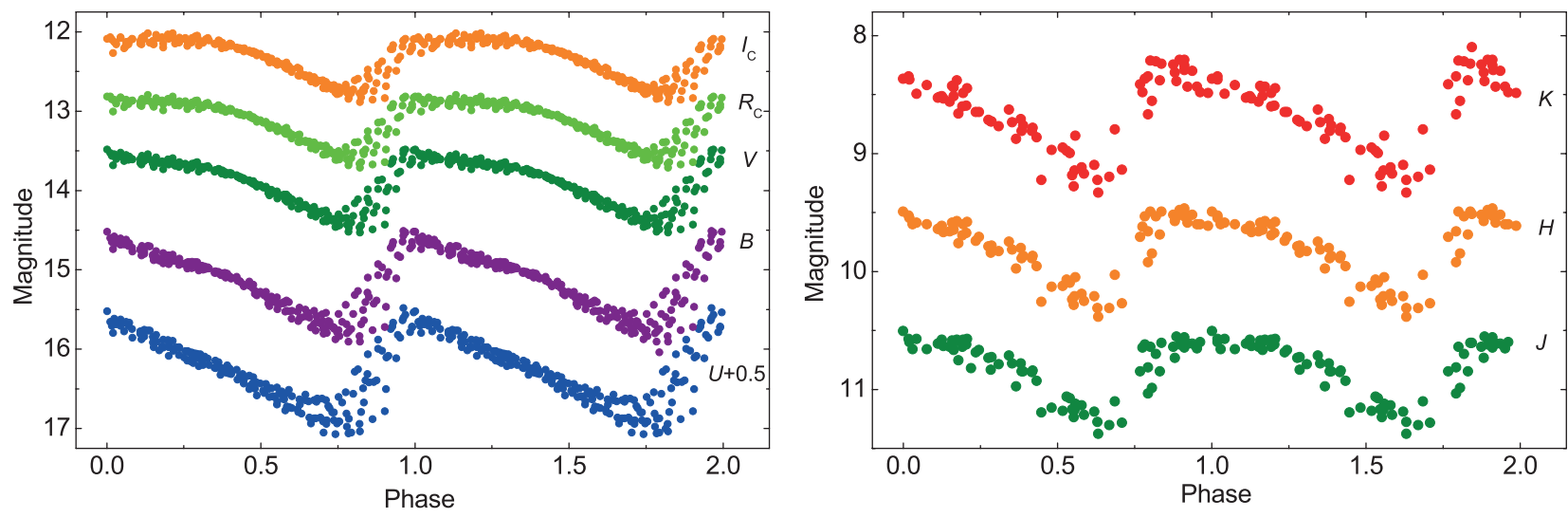

Figure 1: The $U B V R_{C} I_{C}$ light curves folded on the period of 24.885 days (left panel) and the $J H K$ light curves folded on the period of 24.800 days (right panel).

Table 1: The parameters of the dust shell model components

\begin{tabular}{ccccc}
\hline & $(1)$ & $(2)$ & $(3)$ & The whole envelope \\
\hline Inner edge, $\mathrm{AU}$ & 2.5 & 110 & 150 & 2.5 \\
\hline Outer boundary, AU & 110 & 150 & 2000 & 2000 \\
\hline Dust grain radius, $\mu \mathrm{m}$ & 0.3 & 0.3 & 5 & - \\
\hline Optical depth $\tau_{V}$ & 0.38 & 1.07 & 0.34 & 1.79 \\
\hline Dust mass, $M_{\odot}$ & $8.3 \times 10^{-9}$ & $1.4 \times 10^{-6}$ & $1.99 \times 10^{-4}$ & $2.0 \times 10^{-4}$ \\
\hline Mass-loss rate, $M_{\odot} / \mathrm{yr}$ & $2 \times 10^{-8}$ & $8 \times 10^{-6}$ & $2 \times 10^{-5}$ & - \\
\hline
\end{tabular}

and is associated with the appearance of shock waves in the stellar atmosphere during the expansion of its outer layers $[9,10,11]$.

\section{Dust shell}

The object's spectral energy distribution (SED) demonstrates a prominent IR excess in the wavelength range from $1.25 \mu \mathrm{m}$ ( $J$-band) to $160 \mu \mathrm{m}$ (AKARI). Thus it is reasonable to ascribe this excess to the heated dust. We estimated the circumstellar dust envelope parameters via simulations with the RADMC-3D code [12]. We modelled the SED using our $U B V R_{C} I_{C} J H K$-photometry at maximum light and the data available from different catalogues: WISE [13], AKARI [14], IRAS [15], MSX [16]. The SED is adequately represented as the sum of four components: a star with $T_{\text {eff }}=7400 \mathrm{~K}$ assumed from our spectral data and three dust shells. Table 1 gives the parameters of the dust shell model components.

More detailed results of this study will be published elsewhere.

\section{References}

1. A. Manchado, S. R. Pottasch, P. Garcia-Lario, C. Esteban, and A. Mampaso, A\& A, 214, $139,1989$.

2. D. M. Kelly and B. J. Hrivnak, ApJ, 629, 1040, 2005.

3. O. Suárez, P. García-Lario, A. Manchado, M. Manteiga, A. Ulla, and S. R. Pottasch, A\&A A, 458, $173,2006$.

4. R. E. Molina, Rev. Mex. Astron. y Astrof., 54, 397, 2018.

5. T. Jayasinghe, K. Z. Stanek, C. S. Kochanek, B. J. Shappee, et al., MNRAS, 486, 1907, 2019.

6. L. N. Berdnikov, A. A. Belinskii, N. I. Shatskii, M. A. Burlak, N. P. Ikonnikova, E. O. Mishin, D. V. Cheryasov, and S. V. Zhuiko, Astronomy Reports, 64, 310, 2020.

7. A. E. Nadjip, A. M. Tatarnikov, D. W. Toomey, N. I. Shatsky, A. M. Cherepashchuk, S. A. Lamzin, and A. A. Belinski, Astrophysical Bulletin, 72, 349, 2017.

8. S. A. Potanin, A. A. Belinski, A. V. Dodin, S. G. Zheltoukhov, et al., Astronomy Letters, 46, 836, 2020.

9. H. A. Abt, ApJS, 1, 63, 1954.

10. G. Wallerstein, ApJ, 130, 560, 1959.

11. C. Whitney, Annales d'Astrophysique, 19, 142, 1956.

12. C. P. Dullemond, A. Juhasz, A. Pohl, F. Sereshti, R. Shetty, T. Peters, B. Commercon, and M. Flock, RADMC3D: A multi-purpose radiative transfer tool, 2012. 
13. R. M. Cutri, E. L. Wright, T. Conrow, J. W. Fowler, et al., Explanatory Supplement to the AllWISE Data Release Products, Explanatory Supplement to the AllWISE Data Release Products, 2013.

14. H. Murakami, H. Baba, P. Barthel, D. L. Clements, et al., PASJ, 59, S369, 2007.

15. Infrared Astronomical Satellite (IRAS) Catalogs and Atlases.Volume \%: The Small Scale Structure Catalog., volume 7 .

16. M. P. Egan, S. D. Price, K. E. Kraemer, D. R. Mizuno, et al., VizieR Online Data Catalog, V/114, 2003. 


\section{Spectral survey of the massive star forming region W51E1/E2 in the 4- $\mathrm{mm}$ wavelength range}

S. Kalenskii ${ }^{1}$, P.Golysheva ${ }^{1}$, P. Bergman ${ }^{2}$, H. Olofsson ${ }^{2}$, K. Degtyarev ${ }^{3}$

kalensky@asc.rssi.ru

${ }^{1}$ Lebedev Physical Institute, Astro Space Center, Moscow, Russia

${ }^{2}$ Onsala Space Observatory, Onsala, Sweden

${ }^{3}$ Moscow Institute of Physics and Technology, Dolgoprudnyi, Russia

\section{DOI: $10.51194 /$ VAK2021.2022.1.1.044}

We present the results of a spectral survey of the massive star formation region W51 e1/e2 in the 4-mm wavelength range, performed with the 20-m radio telescope at Onsala (Sweden). This is the second survey of this region carried out at Onsala; the first was the survey in the 3 -mm wave range [1]. Emission of 85 molecules was detected at $4 \mathrm{~mm}$, beginning from diatomic and triatomic molecules, such as $\mathrm{SO}, \mathrm{SiO}, \mathrm{CCH}$, up to complex organic molecules (COMs), such as $\mathrm{CH}_{3} \mathrm{OCH}_{3}, \mathrm{CH}_{3} \mathrm{CH}_{2} \mathrm{OH}$, and $\mathrm{C}_{2} \mathrm{H}_{5} \mathrm{OOCH}$. Apart from the main isotopologues, we detected deuturated species, e.g., DCN, DNC, DCO, as well as ${ }^{13} \mathrm{C},{ }^{15} \mathrm{~N},{ }^{17} \mathrm{O}$ and ${ }^{18} \mathrm{O}$, and ${ }^{34} \mathrm{~S}$-substituded isotopologues, such as ${ }^{13} \mathrm{CH}_{3} \mathrm{OH}, \mathrm{HC}^{15} \mathrm{~N},{ }^{34} \mathrm{SO}_{2}$ etc. A significant number of the detected molecules are typical for hot regions. These include the neutral molecules $\mathrm{CH}_{3} \mathrm{OCHO}, \mathrm{CH}_{3} \mathrm{CH}_{2} \mathrm{OH}, \mathrm{CH}_{3} \mathrm{COCH}_{3}$ etc, which are currently believed to exist mainly in the gas phase in hot cores and shock-heated gas. In accordance with this point of view, for most of these molecules we obtained rotational temperatures $\gtrsim 100 \mathrm{~K}$.

Many COMs were detected using spectral line stacking, which makes it possible to detect even molecules whose individual lines are not visible under the noise. However, in spite of a significant gain in sensitivity by using this method no new for the ISM molecule was found. So far, no new molecule have been detected in the 3-4 mm wavelength range using spectral line stacking. The reasons for this failure are still unclear and will be analysed in the future.

The work was partly supported by the Ministry of Science and Higher Education of the Russian Federation by the grant No. 075-15-2021-597

\section{References}

1. S. V. Kalenskii and L. E. B. Johansson, Astronomy Reports, 54, 1084, 2010. 


\title{
Influence of the magnetic field on the formation of protostellar disks
}

\author{
N. Kargaltseva ${ }^{1,2}$, S. Khaibrakhmanov ${ }^{1,2}$, A. Dudorov $^{1,2}$, S. Zamozdra ${ }^{1}$, A.Zhilkin ${ }^{3}$ \\ kargaltsevans@mail.ru \\ ${ }^{1}$ Chelyabinsk state university, 129 Br. Kashirinykh str., Chelyabinsk 454001, Russia \\ ${ }^{2}$ Ural Federal University, 51 Lenina str., Ekaterinburg 620000, Russia \\ ${ }^{3}$ Institute of Astronomy of the Russian Academy of Sciences (INASAN), Moscow, 119017 Russia
}

We present the results of the numerical simulations of the collapse of magnetic rotating protostellar clouds with mass 10 $M_{\odot}$. The hierarchical structure of the cloud formed at the isothermal stage of the collapse is investigated for various initial ratios of the magnetic energy of the cloud to the modulus of its gravitational energy, $\varepsilon_{\mathrm{m}}$. It is shown that the size of the quasi-magnetostatic primary disk and the region of efficient magnetic braking increase with $\varepsilon_{\mathrm{m}}$. In the case $\varepsilon_{\mathrm{m}} \geq 0.6$, practically the entire cloud evolves into the state of quasi-magnetostatic equilibrium. A 'dead' zone forms inside the first hydrostatic core and in the area of the outflow formation, where Ohmic dissipation and ambipolar diffusion become essential.

Keywords: magnetic fields, magnetohydrodynamics (MHD), numerical simulations, star formation, interstellar medium

DOI: 10.51194 /VAK2021.2022.1.1.045

\section{Introduction}

Stars form in the gas-dust magnetic rotating cores of molecular clouds called protostellar clouds (PSCs). It is assumed that the self-gravitating molecular cloud cores evolve into class 0 young stellar objects (YSOs) [1]. Such objects are interpreted as protostars surrounded by dense flattened gas-dust envelopes and having bipolar outflows. Observations in the sub-millimeter range show that these flattened envelopes has radius of 200-10000 au and contain small possibly Keplerian protostellar disks with size of 5-50 au [2]. The large-scale magnetic field of the disks in class 0 YSO has a quasi-radial geometry with signs of toroidal component [3]. It is not yet possible to observe the transition from the PSC to the class 0 YSO. In this connection, numerical simulations of this evolutionary stage are of great interest.

Modern simulations are mainly concentrated on the accretion stages of the collapse of the solar mass PSC see, e.g. $[4,5]$. Special attention is paid to the magnetic braking catastrophe, i.e. too efficient magnetic braking of the rotation of PSCs with strong magnetic field [6, 7, 5]. The solution of this problem will make it possible to determine the conditions for the formation of protostellar disks. A detailed study of the initial stages of the collapse of the PSC may be the key in solving this problem.

Khaibrakhmanov et al. [8] and Kargaltseva et al. [9] modeled the isothermal collapse of the rotating magnetic PSC and have shown that an hierarchical structure of the cloud is formed, which consists of an optically thin flattened envelope containing a quasi-magnetostatic primary disk (PD) with a first hydrostatic core in the center. A fast magneto-hydro-dynamic (MHD) shock wave propagates from the boundary of the PD into the cloud envelope. The quasi-magnetostatic equilibrium is violated and an outflow forms in the vicinity of the core. The specific angular momentum is accumulated at the boundary of the PD and is then transported into the envelope by the fast MHD shock wave and outflow. In this paper, we analyse the efficiency of the magnetic braking at the isothermal stage of the collapse by simulating this stage for various initial magnetic energy of the cloud.

\section{Model and results}

We numerically simulate the collapse of a uniform spherical rotating magnetic PSC with initial mass $M_{0}=10 M_{\odot}$ and radius $R_{0}=0.1 \mathrm{pc}$. The ratios of thermal and rotational energies of the cloud to the modulus of its gravitational energy: $\varepsilon_{\mathrm{t}}=0.3$ and $\varepsilon_{\mathrm{w}}=0.01$, respectively. We performed simulations with different ratios of magnetic energy of the cloud to the modulus of its gravitational energy, $\varepsilon_{\mathrm{m}}=0.01$ (weak magnetic field ), 0.2 (moderate magnetic field) and 0.6 (strong magnetic field). The simulations are carried out using the two-dimensional MHD code Enlil [10]. Ohmic dissipation and ambipolar diffusion are taken into account in the model (see [11]), and the ionization fraction is calculated following [12].

Simulation results show that the radius $R_{\mathrm{pd}}$ of the $\mathrm{PD}$, formed at the isothermal stage of the collapse, increases with $\varepsilon_{\mathrm{m}}$. In the case of weak magnetic field, $R_{\mathrm{pd}} \approx 0.025 R_{0} \approx 500 \mathrm{au}$. Characteristic radius of PD in the case of moderate magnetic field is $\approx 0.36 R_{0} \approx 7500$ au. The dynamics of the cloud in this case was analysed in detail by [9].

The simulation results for the case of strong magnetic field are shown in Figure 1. The PD with radius of $\approx 0.37 R_{0} \approx 7600$ au forms at $t=0.6831 t_{\mathrm{fmw}}$ (Fig. 1a), where $t_{\mathrm{fmw}}$ is the characteristic time of the collapse of the rotating magnetic PSC [8]. By the time $t=0.7761 t_{\mathrm{fmw}}$, the PD grows to $R_{\mathrm{pd}} \approx 0.68 R_{0} \approx 14000 \mathrm{au}$ (Fig. 1b). The region of magnetic braking, limited by the front of the fast MHD shock wave, occupies almost half of the cloud. The front of the shock wave reaches the cloud boundary and pushes the matter out of the cloud by the time of the opaque first hydrostatic core formation, $t=0.8213 t_{\mathrm{fmw}}$ (Fig. 1c). The quasi-magnetostatic equilibrium is lost and an outflow is formed near the first core soon after that, similar to the case of moderate magnetic field. By the 
time, $t=0.8286 t_{\mathrm{fmw}}$, when the outflow from the central part reaches the cloud boundary, practically the entire cloud becomes quasi-magnetostatic (Fig. 1d).

Our simulations show that a 'dead' zone, i.e. region with low ionization fraction, $x=10^{-13}$, and efficient magnetic diffusion, forms near the equatorial plane inside the first core and in the outflow region.

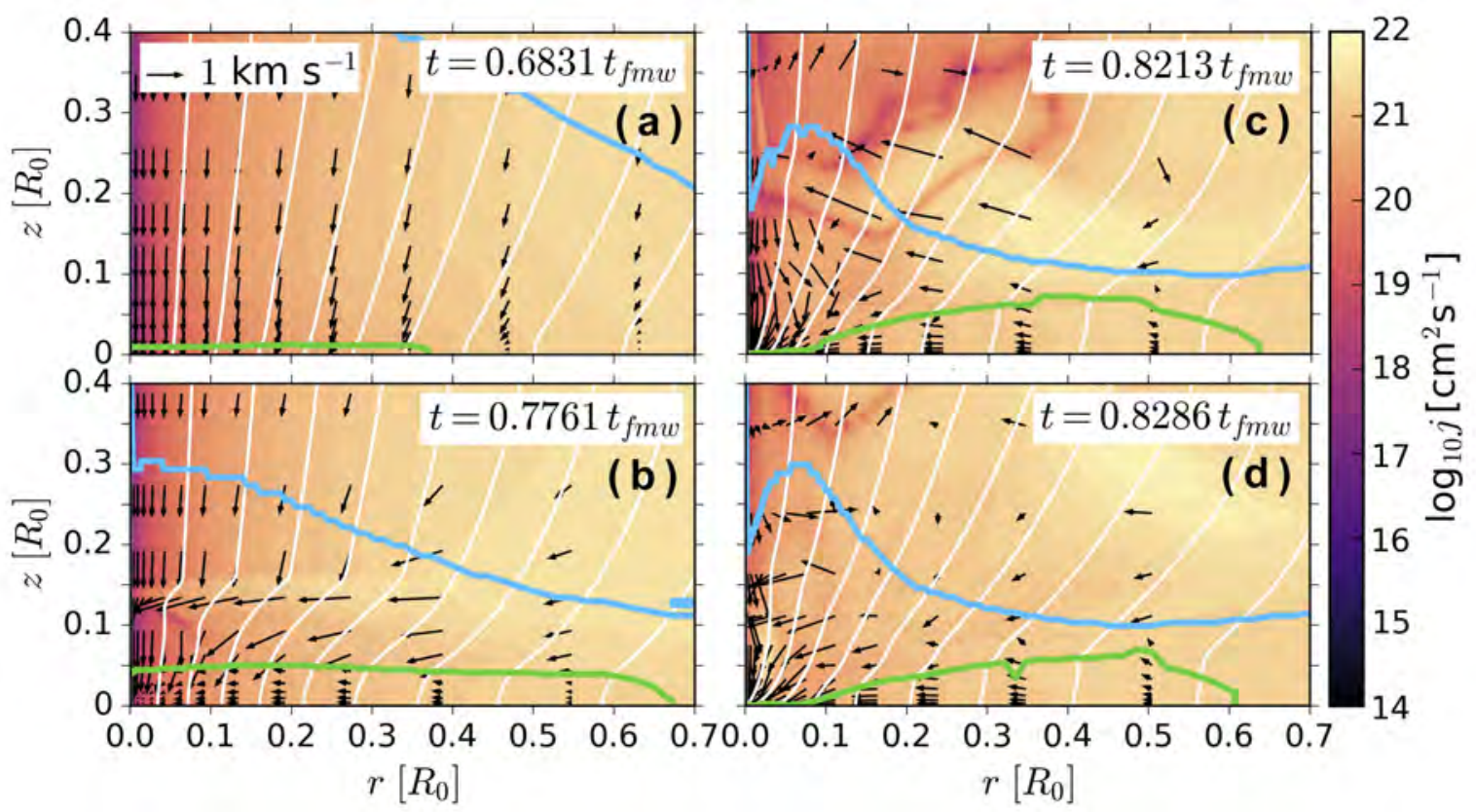

Figure 1: Distribution of angular momentum (color filling), velocity field (arrows) and poloidal magnetic field (white lines) in the simulation with $\varepsilon_{t}=0.3, \varepsilon_{m}=0.6, \varepsilon_{w}=0.01$ at (a) $t=0.6831 t_{\mathrm{fmw}}$, (b) $t=0.7761 t_{\mathrm{fmw}}$, (c) $t=0.8213 t_{\mathrm{fmw}}$, (d) $t=0.8286, t_{\mathrm{fmw}}$. The region near the primary disk (PD) is shown. The green line shows the border of the PD, the blue line is the border of the cloud.

\section{Conclusions and discussion}

We performed numerical simulations of the collapse of rotating magnetic protostellar clouds with mass $10 M_{\odot}$ up to the formation of the first hydrostatic core. The simulations were carried out for various initial magnetic energies of the cloud in order to analyse influence of the magnetic field on the properties of the PD and efficiency of the angular momentum transport.

The simulations show that the radius of the PD increases from 500 to 14000 au with $\varepsilon_{\mathrm{m}}$ increasing from 0.01 to 0.6. This radii range is consistent with the observed sizes of flattened envelopes of class 0 YSO [2].

The stronger the initial magnetic field, the larger the region of magnetic braking in the collapsing cloud. The angular momentum and mass are transported from the central part of cloud by the outflows emanating from the vicinity of the first core and by the fast MHD shock wave propagating from the PD to the envelope. In the case of strong magnetic field, the MHD shock wave comes out from the flattened envelope of the cloud by the time of the first core formation, and carry mass away from the cloud into the interstellar medium. This effect needs further investigation.

The 'dead' zone with the low ionization fraction, $x \leq 10^{-13}$, forms inside the first core and at the base of the outflow region. Further evolution of the first core and PD depends on the efficiency of the Ohmic dissipation and magnetic ambipolar diffusion in this region. We plan to investigate the role of non-ideal MHD effects in the magnetic flux evolution of the PSC in the next paper.

This work is financially supported by the Russian Science Foundation (project 19-72-10012).

\section{References}

1. P. Andre, D. Ward-Thompson, and M. Barsony, ApJ, 406, 122, 1993.

2. J. J. Tobin, P. D. Sheehan, N. Reynolds, S. T. Megeath, et al., ApJ, 905, 162, 2020.

3. C.-F. Lee, W. Kwon, K.-S. Jhan, N. Hirano, et al., ApJ, 879, 101, 2019.

4. P. Hennebelle and S. Fromang, $A \& A$, 477, 9, 2008.

5. B. Zhao, K. Tomida, P. Hennebelle, J. J. Tobin, et al., Space Sci. Rev., 216, 43, 2020.

6. A. E. Dudorov and I. V. Sazonov, Nauchnye Informatsii, 50, 98, 1982. 
7. K. Tomisaka, ApJ, 575, 306, 2002.

8. S. A. Khaibrakhmanov, A. E. Dudorov, N. S. Kargaltseva, and A. G. Zhilkin, Astronomy Reports, 65, 693, 2021.

9. N. S. Kargaltseva, S. A. Khaibrakhmanov, A. E. Dudorov, and A. G. Zhilkin, Bulletin of the Lebedev Physics Institute, 48, 268-271, 2021.

10. A. E. Dudorov, A. G. Zhilkin, and O. A. Kuznetsov, in S. M. Miyama, K. Tomisaka, and T. Hanawa, eds., Numerical Astrophysics, Astrophysics and Space Science Library, volume 240, 389 (1999).

11. A. G. Zhilkin, Y. N. Pavlyuchenkov, and S. N. Zamozdra, Astronomy Reports, 53, 590, 2009.

12. A. E. Dudorov and Y. V. Sazonov, Nauchnye Informatsii, 63, 68, 1987. 


\title{
Features of the Flow of Matter in the X-Ray Binary Her X-1
}

\author{
E. Karitskaya ${ }^{1}$, N. Bochkarev ${ }^{2}$ \\ karitsk@yandex.ru \\ ${ }^{1}$ Institute of Astronomy of the RAS, 48 Pyatnitskaya str., Moscow 119017, Russia \\ ${ }^{2}$ M.V. Lomonosov Moscow State University, P.K. Sternberg Astronomical Institute, Universitetskij prosp., Moscow \\ 119991, Russia
}

\begin{abstract}
We consider a model of discrete flow of matter in the X-ray binary Her X-1/HZHer developed by us in the late 80 s of the last century. It well explains many phenomena in this X-ray system, including peaks on optical light curves formed by the interaction of discretely incident matter with the outer parts of the accretion disk, as a result of which blobs are formed. Based on the published light curves constructed on the basis of the richest photometric data, a phase diagram $(\Phi, \Psi)$ of the identified possible peaks is constructed. It is concluded that the appearance of peaks on the orbital light curves in certain orbital phases, depending on the phase of the 35-day cycle, continues, and the mechanism of blob formation continues to work at the present time. Observations on eROSITA confirmed the existence of a corona around the accretion disk, predicted by us in the frame of this model.
\end{abstract}

Keywords: X-ray binary, Her X-1, light curves, accretion

DOI: $10.51194 /$ VAK2021.2022.1.1.046

\section{Introduction}

Recently, there has been renewed interest in the different manifestations of the "precession" cycle $P_{p r}=35^{d}$ in IMXB HZ Her/Her X-1, revealed in X-rays and described appearances "main-on" and "short-on" states. For example, [1] constructed a detailed model describing the variability of the shape of the orbital light curve in the optical range from the phase of this cycle. This model takes into account many factors, including the forced precession of the warped tilted accretion disk and the free precession of the X-ray pulsar illuminating and heating optical star. It generally describes the shapes of the light curves well as a whole without explaining the observed details - spikes (peaks) on the optical light curves, steps in the minima. In addition, the physical model proposed by the authors, despite its complexity, has not yet explained many observed factors, for example, the origin of X-ray dips (pre-eclipse and anomalous), flickering of the X-ray flux in them, bursts of linear polarization, etc. The phenomenological model for periodical mass transfer by [2], subsequently developed and supplemented by us, explains these details. The observational facts obtained subsequently confirm this model. This is what this contribution is dedicated to.

\section{Periodical mass transfer in Her $\mathrm{X}-1$}

\subsection{The model}

For the first time a periodical mass transfer empirical model for Her X-1 was described in [2]. This is the so-called "clock" mechanism of transfer of matter to the second component, obtained on the basis of long-term observations of X-ray dips. Subsequently it was developed by us $[3,4,5,6,7]$. Every $0.81^{d}$, the matter flows from the star because the X-ray pressure disappears near the internal the Lagrangian point when the star enters the shadow of a tilted precessing accretion disk. According to our estimates, the external radiation pressure $p_{x}$ is $\sim 1.5$ times higher than the internal $p$ (the sum of the gas and radiation pressure) [6]. After about $4^{h}$, the matter collides with the outer part of the disk. The gas heats up, spreads out and a blob forms on the rim of the disk. A spike is formed on the orbital light curve. Theoretical estimations of heights of these peaks $\left(\sim 0.3^{m}\right.$ in $\mathrm{B}, \mathrm{V}, \sim 0.7^{m}$ in $\mathrm{U})$ correspond to observations [3]. Blob is carried away by a circulation movement in the direction of rotation of the binary system with a period of $P_{c}=15^{h}$ and gradually relaxes. When it hits the line-of-sight, pre-eclipse and abnormal dips are formed in the X-ray range. According to [2] the floating values of the orbital phase of the moment when the X-ray is turned on are also explained by the blob hitting the line-of-sight.

In binary system Her X-1 the ratio of sizes is such that the steps up and down observed in the center of the primary minimum of the orbital light curve $\Phi=0[8,9]$ are explained by the exit or entry into the eclipse of the blob by an optical star [3]. During the observations of 1972-1975 (Shakhovskoi N. M. and Efimov Yu. S. In: Problems of Magnetic Fields in Space, Materials of International Symposium [in Russian], Izd. Krym.Astrofiz. Obs., Akad. Nauk SSSR, 1976, vol. 2, p. 133) at the moment $\Phi=0$, a burst of linear polarization with an amplitude of $\approx 0.5 \%$, FWHM $\Delta \Phi \approx 0.01$ was detected. It was explained by a partial eclipse of the blob by the optical star [3] and estimates consistent with observations were obtained. 


\subsection{The phase diagram $(\Phi, \Psi)$ of the moments of blob appearances}

Based on the described above mechanism of blob formation, we should expect their formation and therefor the appearance of peaks on the light curves in certain orbital phases $\Phi_{D}$, depending on the phase $\Psi$ of the 35 -day cycle, counted from the moment of the X-ray radiation turn-on $(\Psi=0)$ :

$$
\Phi_{D}=\Phi_{D 0}-\Psi, \Phi_{D}=\Phi_{D 0}-\Psi+0.5
$$

where $\Phi_{D 0}$ is the orbital phase of the appearance of the first blob immediately after the moment of switching on Her X-1. [2] obtained $\Phi_{D 0}=0.78$ by using long-term observations of X-ray dips. This is corresponds to the first entrance of the star to the shadow from the tilted accretion disk at $\Phi_{R}=0.68$.

This is well confirmed by the phase diagram $\Psi$ from $\Phi_{D}$ in Fig. 1 of [3]. Theoretical straight lines described by (1) are drown in the $\left(\Phi_{D}, \Psi\right)$ plane. Points corresponding to peaks observed on the light curves fit well on these lines. These peaks were taken from the light curves published by that time, mainly from [10], where the theoretical calculated orbital light curves, depending on the phase of the 35-day cycle, were compared with photometric data.

The moments of the appearance of the steps up and down observed in the center of the primary minimum of the orbital light curve $\Phi=0$ and the observed burst of linear polarization, recalculated according to the model into the corresponding phases of blob appearance also perfectly fit on these lines.

In the recent work [1] to construct optical light curves at different phases a huge photometrical material were used. They calculated light curves for 20 phases of 35-day cycle. The main features of the model include a tilted, warped and precessing accretion disk and freely precessing neutron star. Looking through these 20 light curves and comparing the observation points with the theoretical curves, we found 77 possible peaks. Fig. 1 show phase diagram $(\Phi, \Psi)$, where points correspond these peaks. Theoretical straight lines described by (1) are drown. The diagram clearly shows three sequences of points located along the theoretical lines, the middle of which is described by the left equation of (1), and the extreme ones by the right equation. The extreme sequences of points can be merged with the middle sequence by raising the lower one and lowering the upper one for a half-period of the 35-day cycle (see Fig.2). By using the least squares method, the resulting sequence of points has been fitted of an inclined line $\Psi=a-b \Phi$, where $a=0.770, \sigma_{a}=0.027, b=-0.866, \sigma_{b}=0.050$. The value of $a$ surprisingly coincides with $\Phi_{D 0}$ and $b$ within $3 \sigma_{b}$ coincides with 1.0. Thus, it can be concluded that the mechanism of blob formation continues to work at the present time.

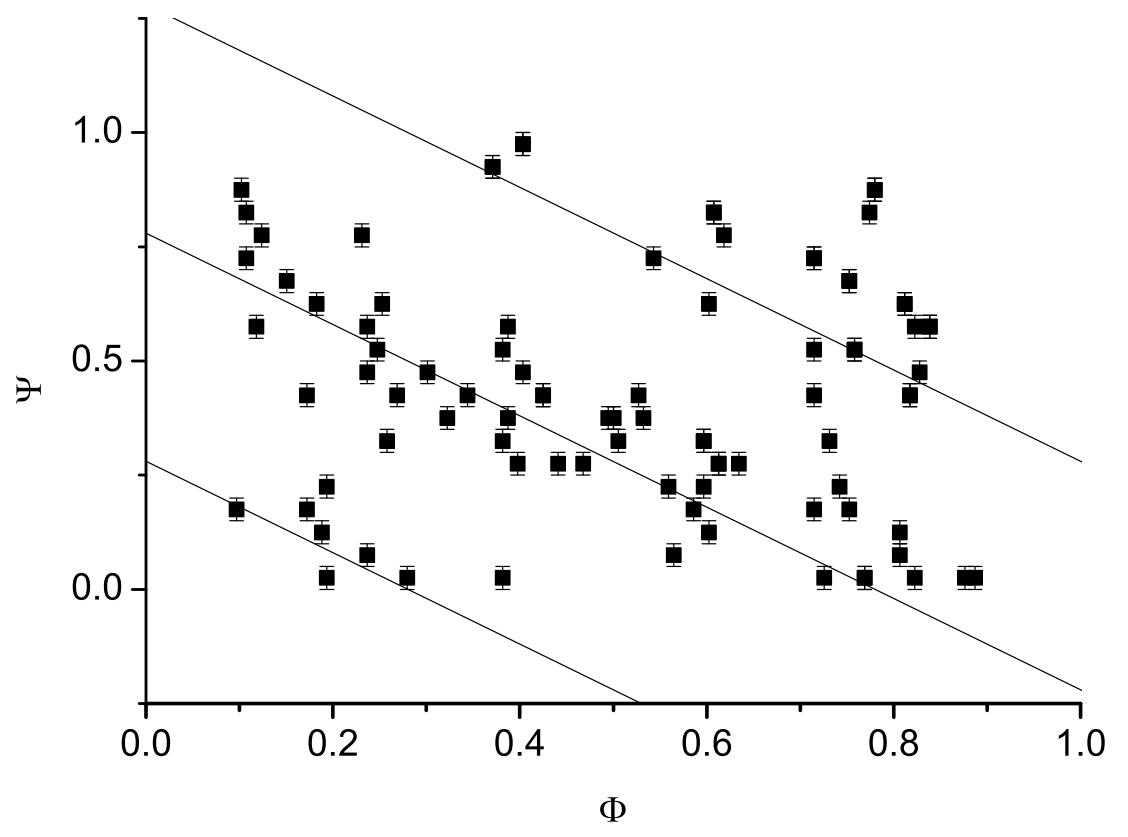

Figure 1: The phase diagram $(\Phi, \Psi)$ of the moments of blob occurrences at the disk rim, coinciding with the moments of appearance of peaks on optical orbital light curves from [1]. $\Phi$ - orbital phase, $\Psi$ - 35-day cycle phase, $\Psi=0$ corresponds to the X-ray source turn-on moment. The diagram clearly shows three sequences of points located along the theoretical lines, the middle of which is described by the left equation of (1), and the extreme ones by the right equation. 


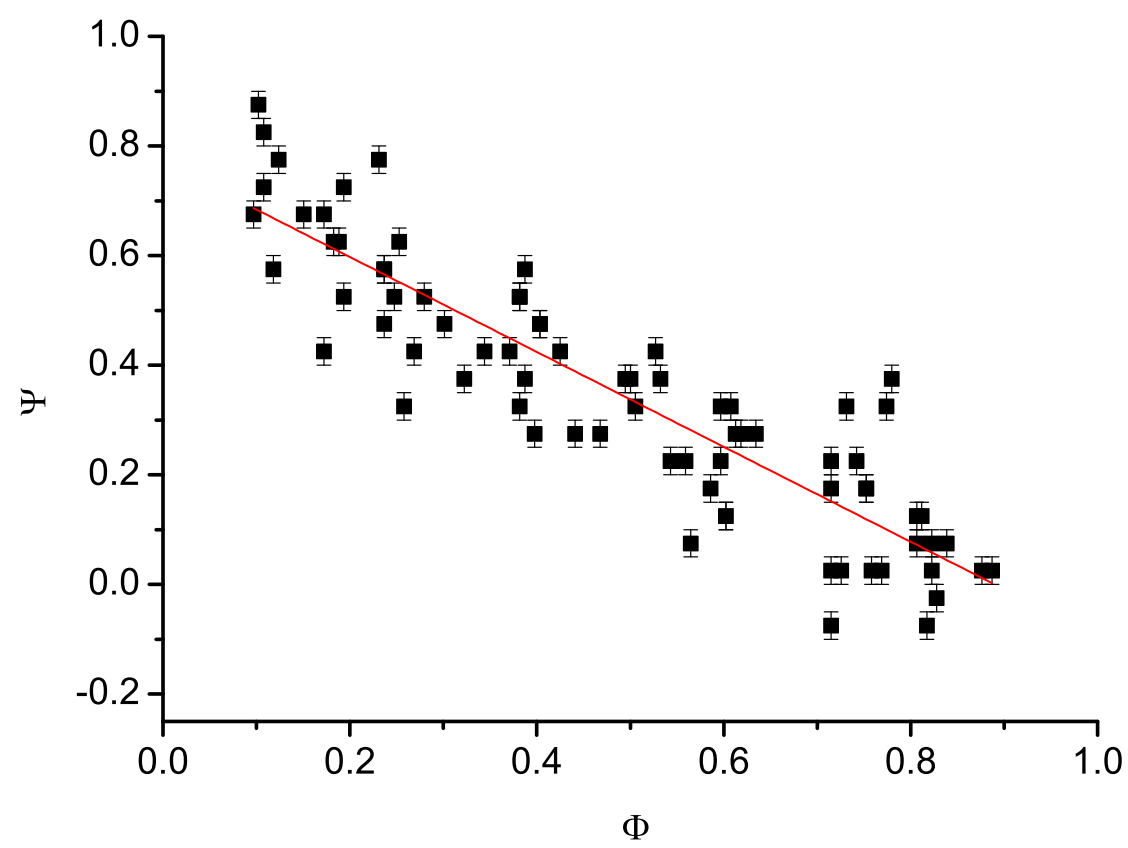

Figure 2: The phase diagram $(\Phi, \Psi)$ (see the caption of Fig.1), where the middle sequence of points from Fig.1 is shown. The extreme sequences of points have been merged with it (see text). A straight line fitting these points is drawn.

\section{A model of fragmented blobs and a hot corona around the disk. Observational evidences of corona existing}

The discovery of flickering of X-ray flux during X-ray dips at the EINSTEIN [11] and EXOSAT [12] led to a significant development of the blob model (see [4], [6], [7]). To explain this X-ray flickering during X-ray source eclipse by blob, as well as the long lifetime of the cold blob, we had to assume the existence of a hot corona around the disk. When interacting with this corona, a Rayleigh-Taylor instability develops, and after a few hours, the blob breaks up into separate drops. Small drops quickly evaporate, and large ones are held by the pressure of the corona. Each drop rotates around the disk in its Keplerian orbit. If the orbits intersect with the disk, drops fall on it. The rest of it moves on and can exist one and a half to two revolutions around the disk. The size of the drops is $\sim 3 \cdot 10^{10} \mathrm{~cm}$, the density is $\sim 4 \cdot 10^{13} \mathrm{~cm}^{-3}$, the temperature is $\sim 30000 \mathrm{~K}$. During the eclipse of the X-ray source by this fragmented blob we may expect flickering with a characteristic time of $\tau \sim 150-500 \mathrm{~s}$ which observed in dips. The following parameters of corona were obtained: density $\sim 5 \cdot 10^{11} \mathrm{~cm}^{-3}$, temperature $\sim 3 \cdot 10^{6} \mathrm{~K}$. The coronal gas rotates with the disk. A detailed calculation of the heating and ionization balance in the radiation field of a neutron star is made in [13], [14]. Observational evidence for the existence of such corona was presented and discussed in detail: the existence of an X-ray flux during 35-day "OFF" state, smooth entry and exit from the X-ray eclipse, the presence of the $\mathrm{Fe}(6-7) \mathrm{keV}$ emission line during the "OFF" states, etc. The existence of a corona with similar parameters was confirmed by the observations of a shape of an X-ray eclipse by the Rossi X-ray Timing Explorer [15].

Recently new evidences of corona around disk existing were received. SRG/eROSITA all-sky survey observed Her X-1 during the low states of the X-ray source [16] when the outer part of accretion disk eclipses the neutron star. The X-ray orbital light curves were received. The authors concluded that there are three distinct regions scattering X-ray radiation: optically thin hot corona above the irradiated hemisphere of the optical star; optically cold atmosphere of the star and an optically thin hot halo above the accretion disk. The latter is exactly the corona which was discussed.

\section{References}

1. D. A. Kolesnikov, N. I. Shakura, K. A. Postnov, I. M. Volkov, et al., MNRAS, 499, 1747, 2020.

2. L. Crosa and P. E. Boynton, ApJ, 235, 999, 1980.

3. E. A. Karitskaya, N. G. Bochkarev, and Y. N. Gnedin, Sov. Astron., 30, 592, 1986.

4. N. G. Bochkarev and E. A. Karitskaya, Astronomicheskij Tsirkulyar, 1433, 1, 1986. 
5. N. G. Bochkarev, Y. N. Gnedin, and E. A. Karitskaya, Peremennye Zvezdy, 22, 948, 1988.

6. N. G. Bochkarev and E. A. Karitskaia, Ap\&SS, 154, 189, 1989.

7. N. G. Bochkarev and E. A. Karitskaya, in Y. Kondo, R. Sistero, and R. S. Polidan, eds., Evolutionary Processes in Interacting Binary Stars, IAU Symposium, volume 151, 449 (1992).

8. N. N. Kilyachkov and V. S. Shevchenko, Soviet Astronomy Letters, 6, 378, 1980.

9. R. Kippenhahn, H. U. Schmidt, and H. C. Thomas, A $\& A$, 90, 54, 1980.

10. I. D. Howarth and B. Wilson, $M N R A S, \mathbf{2 0 2}, 347,1983$.

11. S. D. Vrtilek and J. P. Halpern, ApJ, 296, 606, 1985.

12. W. Voges, P. Kahabka, H. Oegelman, W. Pietsch, and J. Truemper, Space Sci. Rev., 40, 339, 1985.

13. N. G. Bochkarev, Sov. Astron., 33, 638, 1989.

14. N. G. Bochkarev, in C. Bertout, S. Collin-Souffrin, and J. P. Lasota, eds., IAU Colloq. 129: The 6th Institute d'Astrophysique de Paris (IAP) Meeting: Structure and Emission Properties of Accretion Disks, 385 (1991).

15. D. A. Leahy, ApJ, 800, 32, 2015.

16. N. I. Shakura, D. A. Kolesnikov, P. S. Medvedev, R. A. Sunyaev, M. R. Gilfanov, K. A. Postnov, and S. V. Molkov, A\&A A, 648, A39, 2021. 


\section{Mini-MegaTORTORA photometric survey of the northern sky}

S. Karpov ${ }^{1,2,3}$, G. Beskin ${ }^{2,3}$ on befalf of Mini-MegaTORTORA collaboration

${ }^{1}$ CEICO, Institute of Physics, Czech Academy of Sciences, Prague, Czech Republic

${ }^{2}$ Special Astrophysical Observatory, Nizhniy Arkhyz, Russia

${ }^{3}$ Kazan Federal University, Kazan, Russia

DOI: 10.51194 /VAK2021.2022.1.1.047

The Mini-MegaTORTORA (MMT-9) system is an unique multi-purpose wide-field monitoring instrument built for and owned by the Kazan Federal University, presently operated under an agreement between Kazan Federal University and Special Astrophysical Observatory, Russia. It consists of 9 channels equipped with Canon EF85/1.2 (7 cm diameter) objectives and Andor Neo sCMOS cameras. Its main tasks are detection and characterization of astrophysical transient events on a $0.1 \mathrm{~s}$ time scale, as well as systematic observations of meteors and artificial satellites of Earth. Also, during the sky monitoring, the low temporal resolution photometric sky survey is being performed.

It is based on a set of $20 \mathrm{~s}$ (60 s before May 2016) images in white light and depth down to 13.5 magnitude. Since the start of the survey in Jun 2014, more than 1.4 millions of images, covering every point of the northern sky 5000-20000 times, are acquired. These images are being processed and calibrated to Johnson V filter by deriving photometric equation for every individual image, and then statistically regressing for individual object colors assuming that their brightness variations are uncorrelated with changes of atmospheric conditions.

All the data from photometric survey are presented on a dedicated web portal at ${ }^{1}$, where both original sky images, cutouts and lightcurves are available. The images are uploaded to the portal with 1-2 days delay, while photometric data - once per several months (up to May 2021 as of now).

Acknowledgements. Mini-MegaTORTORA belongs to Kazan Federal University, and its operation is performed according to the Russian Government Program of Competitive Growth of Kazan Federal University. The work is supported by the Ministry of Education, Youth and Sports of the Czech Republic (project CoGraDS, grant number CZ.02.1.01/0.0/0.0/15_003/0000437). The work is also partially supported within the framework of the government contract of the Special Astrophysical Observatory of the Russian Academy of Sciences in its part entitled "Conducting Fundamental Research".

\footnotetext{
${ }^{1}$ See http://survey.favor2.info
} 


\title{
Dynamics of magnetic flux tubes in accretion disks of Herbig $\mathrm{Ae} / \mathrm{Be}$ stars
}

\author{
S. Khaibrakhmanov ${ }^{1,2}$, A. Dudorov $^{2,1}$ \\ khaibrakhmanov@csu.ru \\ ${ }^{1}$ Ural Federal University, 51 Lenina str, Ekaterinburg 620051, Russia \\ ${ }^{2}$ Chelyabinsk State University, 129 Br Kashirinykh str, Chelyabinsk 454001, Russia
}

\begin{abstract}
The dynamics of magnetic flux tubes (MFTs) in accretion disks of Herbig Ae/Be stars with fossil large-scale magnetic field is modeled. The model takes into account the buoyant force, turbulent and aerodynamic drag, radiative heat exchange with the surrounding gas, and the magnetic field of the disk. The structure of the disk is calculated using our MHD model, taking into account the heating of the surface layers of the disk with the intense stellar radiation. The simulations show that MFTs periodically rise from the disk with speeds of $10-12 \mathrm{~km} \mathrm{~s}^{-1}$ and form an outflowing magnetized corona of the disk. MFTs can experience magnetic oscillations under the action of the external magnetic field near the disk's surface. We argue that the oscillations can produce observed IR-variability of Herbig Ae/Be stars, which would be more intense than in the case of $\mathrm{T}$ Tauri stars, since the disks of Herbig Ae/Be stars are hotter, denser and have stronger magnetic field.
\end{abstract}

Keywords: accretion, accretion discs, MHD, ISM: magnetic fields, ISM: jets and outflows, stars: variables: T Tauri, Herbig $\mathrm{Ae} / \mathrm{Be}$

DOI: 10.51194/VAK2021.2022.1.1.048

\section{Introduction}

Accretion disks (ADs) are commonly observed around young stars. Analysis of contemporary observational data shows that accretion disks of young stars (ADYSs) evolve into protoplanetary disks (PPDs), in which conditions are favourable for planet formation.

Polarization mapping of ADs and PPDs shows that they have large-scale magnetic field with complex geometry. Robust measurements of the magnetic field strength in ADYSs are still not possible. There are indications that the magnetic field can be dynamically strong near the inner edge of the disk and that the magnetic field strength decreases with distance from the star. The observational data confirm predictions of the theory of fossil magnetic field (see [1]).

MHD modelling of ADYSs have shown that strong toroidal magnetic field is generated in the innermost region of the ADYS, where thermal ionization operates and magnetic field is frozen in gas. Runaway generation of the magnetic field in this region can be balanced by magnetic field buoyancy leading to the formation of magnetic flux tubes (MFTs), that float from the disk and carry away excess of its magnetic flux [2]. Khaibrakhmanov et al. [3] and Dudorov et al. [4] have shown that rising MFTs oscillate under certain conditions, and the oscillations can be the source of infrared (IR) variability of AD of T Tauri stars (TTSs). In this work, we further develop approach of Dudorov and Khaibrakhmanov and model the dynamics of the MFT in the AD of typical Herbig Ae/Be star (HAeBeS).

\section{Model}

We consider a toroidal MFT formed in the region of effective generation of the magnetic field. The dynamics of unit length MFT is modelled in the slender flux tube approximation. The MFT starts its motion at some radial distance $r$ from the star and height $z_{0}$ above the disk's midplane, $z=0$. The MFT moves in the $z$-direction, perpendicular to the midplane, under the action of buoyant and drag forces.

The system of equations describing the MFT dynamics takes into account turbulent and aerodynamic drag, radiative heat exchange with the external gas, magnetic pressure of the disk [3,4]. Ordinary differential equations of the model are solved with the Runge-Kutta scheme of the 4th order with step size control. Initially the MFT is in thermal equilibrium with external gas at $z_{0}=0.5 \mathrm{H}$, where $H$ is the scale height of the disk. Initial radii of the MFT, $a_{0}$, vary from 0.01 to $0.4 H$, and its magnetic field strength is specified by plasma beta $\beta_{0}=0.01-1$.

The distributions of the density, temperature and magnetic field in the disk are calculated using our MHD model of the $\mathrm{AD}[5,6]$. Vertical structure of the disk is determined from the solution of the hydrostatic equilibrium equation for polytropic dependence of the gas pressure on density. It is considered that there is an optically thin hydrostatic corona above the optically thick disk. The corona's temperature is determined by heating due to absorption of stellar radiation,

$$
T_{\mathrm{c}}=185\left(\frac{f}{0.05} \frac{L}{1 L_{\odot}}\right)^{1 / 4}\left(\frac{r}{1 \mathrm{au}}\right)^{-1 / 2} \mathrm{~K},
$$

where $f$ is the fraction of the stellar radiation flux intercepted by the disk, $L$ is the stellar luminosity. Transition from the disk to corona is characterized by exponential change in temperature over the scale $H$. 
We consider the accretion disk of Herbig Ae/Be star MWC 480 with mass $2 M_{\odot}$, radius $1.67 R_{\odot}$, luminosity $11.2 L_{\odot}$, surface magnetic field strength $1 \mathrm{kG}$, accretion rate $\dot{M}=10^{-7} M_{\odot} / \mathrm{yr}[7,8]$, and turbulence parameter $\alpha=0.01$. Ionization and magnetic diffusivity parameters are adopted from [6].

\section{Results}

The AD of the HAeBeS has higher accretion rate in comparison with the AD of the TTS. As a consequence, the former is denser and hotter than the latter at any given $r$, according to our simulations. Innermost region, where runaway growth of the magnetic field is possible due to high ionization level, is more extended in the case of the HAeBeS. For adopted parameters, this region ranges from the inner boundary of the disk, $r=0.012$ au, up to $r=1 \mathrm{au}$.

We simulated the dynamics of the MFT at three typical radial distances inside the region of thermal ionization: $r=0.012,0.15$, and $1 \mathrm{au}$. In absence of external magnetic field, the MFT evolution can be divided into three stages: acceleration inside the disk, deceleration near the surface of the disk and dissipation in the corona (see [4]). Thicker MFTs and MFTs with stronger magnetic field accelerate to higher speeds. MFTs rise from the disk with typical speeds of $10-12 \mathrm{~km} \mathrm{~s}^{-1}$.

The magnetic field of the disk causes magnetic oscillations of the MFT near the surface of the disk, $z \approx$ $2-2.5 \mathrm{H}$, where the magnetic field strengths of the MFT and the disk are nearly equal. The oscillation period increases with initial plasma beta of the MFT, while the amplitude decreases. In Figure 1, we show results of the simulations for typical $a_{0}=0.1 H, \beta_{0}=1$ at selected $r$. Figure 1 shows that the oscillation period decreases with the radial distance and ranges from few hours an the inner edge of the disk to few months at $r=1$ au.

The oscillations are accompanied by the corresponding changes in the internal MFT characteristics: density, temperature and magnetic field strength. The magnitude of temperature oscillations varies from few thousand to few hundred Kelvin, correspondingly (see bottom panels in Figure 1). The oscillations decay in time.
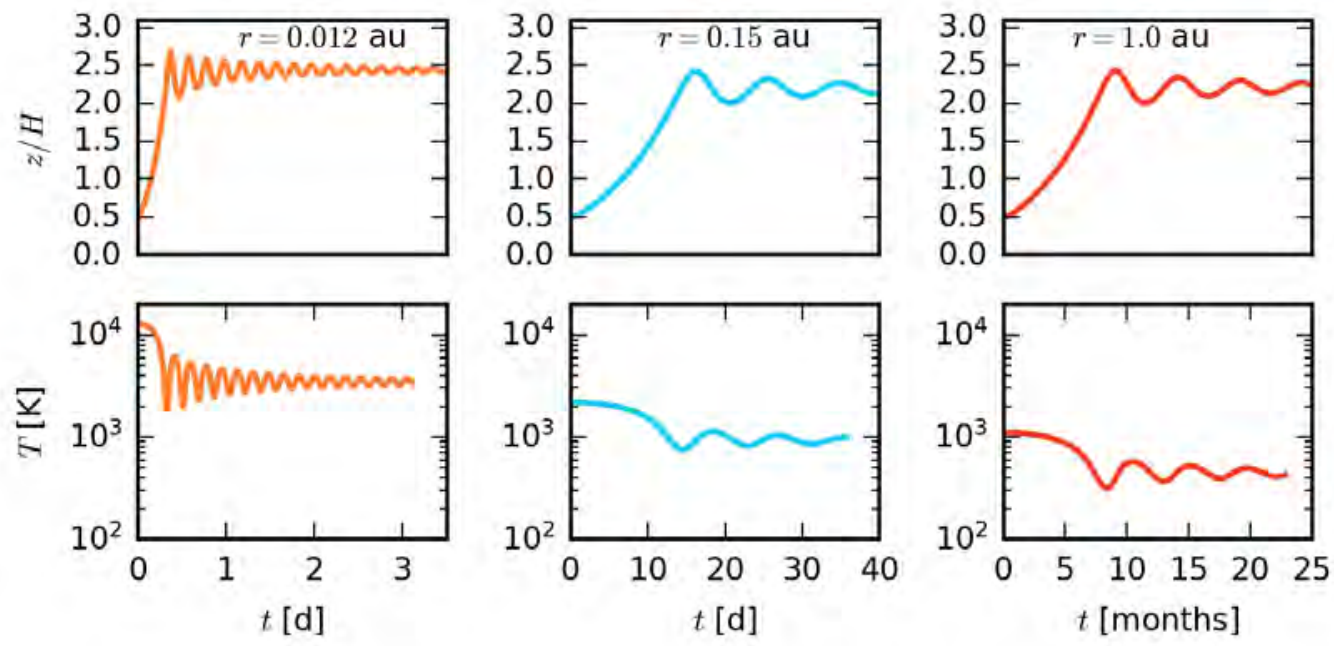

Figure 1: The dependence of the $z$-coordinate (top row) and temperature (bottom row) of the MFT on time during its vertical motion inside the disk at various radial distances $r=0.012,0.15$ and 1 au (panels from left to right). Initial radius and plasma beta of the MFT are $a_{0}=0.1 H$ and $\beta_{0}=1$, respectively.

\section{Discussion}

Our simulations show that the dynamics of the MFT in the AD of the HAeBeS is qualitatively similar to the case of typical TTS. In absence of the magnetic field, MFTs rise from the disk with typical speeds of $10-12 \mathrm{~km} \mathrm{~s}-1$ and form outflowing magnetized corona of the disk. The magnetic field of the disk causes magnetic oscillations of the MFT near the surface of the disk. We propose that the oscillations can be a source of the IR-variability and variable circumstellar extinction observed in young stars with accretion disks.

Our results show that the accretion disks of HAeBeS have more extended region of the efficient generation of the magnetic field than in the case of TTS. The temperature of their corona is higher due to more intense stellar radiation. As a consequence, temperature variations in the oscillating MFTs has larger magnitude. Therefore, the IR-variability of ADs of HAeBeSs would be more intense than in the case of TTS.

Acknowledgements. The work is supported by the Russian Science Foundation (project 19-72-10012). 


\section{References}

1. A. E. Dudorov and S. A. Khaibrakhmanov, Advances in Space Research, 55, 843, 2015.

2. S. A. Khaibrakhmanov and A. E. Dudorov, Physics of Particles and Nuclei Letters, 14, 882, 2017.

3. S. Khaibrakhmanov, A. Dudorov, and A. Sobolev, Research in Astronomy and Astrophysics, 18, $090,2018$.

4. A. E. Dudorov, S. A. Khaibrakhmanov, and A. M. Sobolev, MNRAS, 487, 5388, 2019.

5. A. E. Dudorov and S. A. Khaibrakhmanov, Ap\&SS, 352, 103, 2014.

6. S. A. Khaibrakhmanov, A. E. Dudorov, S. Y. Parfenov, and A. M. Sobolev, MNRAS, 464, 586, 2017.

7. B. Donehew and S. Brittain, AJ, 141, 46, 2011.

8. S. Hubrig, M. Schöller, I. Ilyin, C. R. Cowley, et al., A $₫ A$, 536, A45, 2011. 


\title{
Fast and superfast variability of line profiles in spectra of early type stars
}

A. Kholtygin ${ }^{1}$, A. Valeev ${ }^{2,3}$, A. Moiseeva ${ }^{2}$, I. Yakunin ${ }^{1,2}$, M. Burlak ${ }^{4}$, N. Ikonnikova ${ }^{4}$, A. Dodin $^{4}$, A. Kostenkov $^{2}$, O. Tsiopa ${ }^{5}$, V. Puzin ${ }^{6}$, M. Kurdoyakova ${ }^{1}$, I. Sokolov ${ }^{6}$ afkholtygin@gmail.com

\author{
${ }^{1}$ Saint-Petersburg University, Russia \\ ${ }^{2}$ Special Astrophysical Observatory, Russia \\ ${ }^{3}$ Crimean Astrophysical Observatory, Russia \\ ${ }^{4}$ Sternberg Astronomical Institute, Moscow State University, Russia \\ ${ }^{5}$ Main (Pulkovo) Astronomical Observatory, Russia \\ ${ }^{6}$ Institute of Astronomy, Russian Academy of Sciences, Russia
}

New results of studying the variability of line profiles in the spectra of bright OBA stars on minute and second time scales are presented. The observations were carried out in 2015-2021. Nearly 11000 spectra of 22 OBA stars were obtained. Regular variations of the line profiles with minute periods and amplitudes of $1-2 \%$ of the continuum level in spectra of the program stars are found.

Keywords: stars: variability, pulsations

DOI: $10.51194 /$ VAK2021.2022.1.1.049

\section{Observations}

The regular variability of line profiles in the spectra of OB stars has been studied in detail on time scales from hours to days $[1,2,3]$. At the same time the variations of the profiles on the minute and second scales have remained practically unstudied until recently. In the present paper new results of studying such variations are given.

Our program of looking for superfast Line Profile Variations (LPVs) started in 2016. By now 22 stars were observed. The list of targets is given in Table 1. Four telescopes were used. First of them is the 6-meter BTA telescope at the Special Astrophysical Observatory (SAO, Russia) equipped by low-resoluion SCORPIO spectrograph [4] and medium-resolution MSS spectrograph [5] with an image slicer and a circular polarization analyzer [6, 7].

Since 2019 we also used the MMCS echelle spectrometer at the Cassegrain focus of the 2-m Zeiss-2000 telescope at the Mount Terskol Observatory [8] and the 1.25-m telescope of the Crimean station of the SAI MSU [9]. The last instrument we used for our observations is the 1-m SAO telescope Zeiss-1000 equipped by the low-resoluion spectrograph UAGS [10]. The full list of our observations is given in Table 1.

\section{Line profile variations}

Analyzing the difference profiles we will use the Doppler shifts $V$ from the laboratory wavelength $\lambda_{0}$ of the line instead of the wavelength $\lambda$. The difference line profile is defined as $d(V, t)=F(V, t)-\bar{F}(V)$ where $F(V, t)$ is the normalized line flux at time $t, \bar{F}(V)$ is the normalized flux averaged over all spectra.
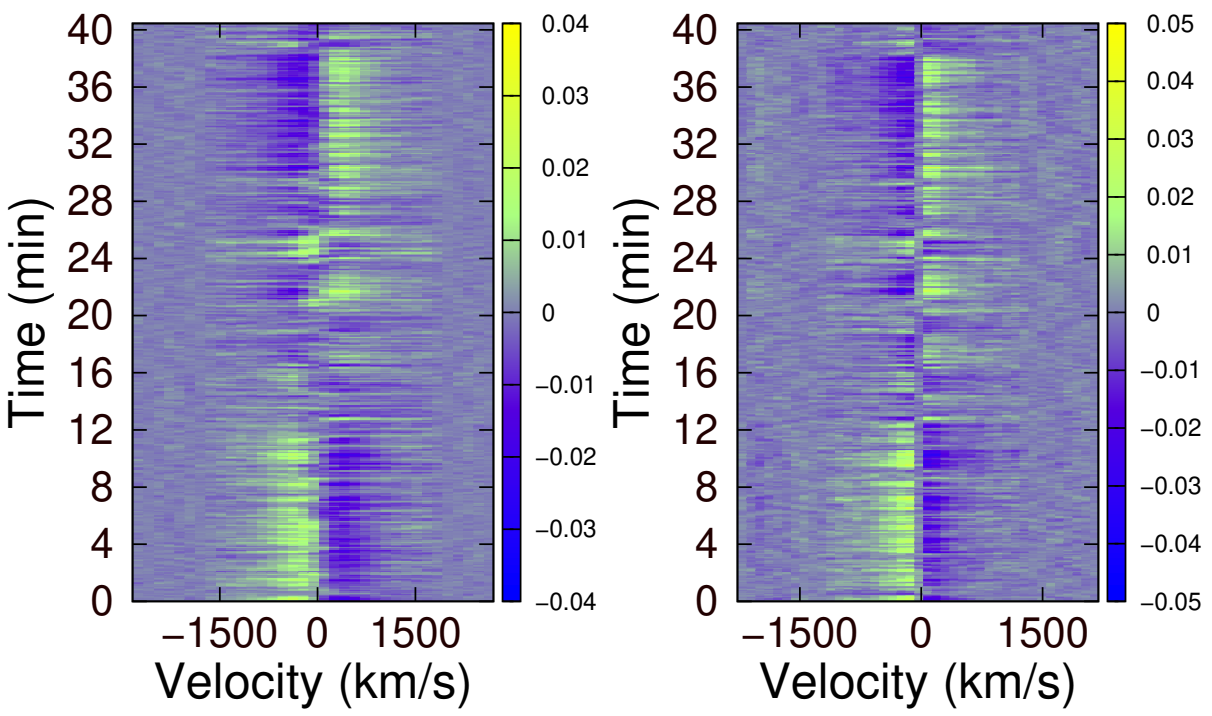

Figure 1: Dynamical spectra of $\mathrm{H}_{\beta}$ (left panel) and $\mathrm{H}_{\alpha}$ (right panel) lines for $\alpha$ And 
Table 1: Targets

\begin{tabular}{|c|c|c|c|c|c|c|c|c|}
\hline Star & Sp.Type & $\overline{\mathrm{V}}$ & $N_{\mathrm{sp}}$ & Exp, s & Telescope & Spectr. & Dates & $N_{\mathrm{sp}}^{\mathrm{tot}}$ \\
\hline \multicolumn{9}{|c|}{ O stars } \\
\hline$\xi$ Per & O7.5III & 4.06 & 35 & 300 & Zeiss-2000 & MMCS & $3-6.1 .2020$ & 35 \\
\hline 15 Mon & $\mathrm{O} 7 \mathrm{~V}+\mathrm{B} 1.5 \mathrm{~V}$ & 4.64 & 3 & 210 & - & - & $4-5.03 .2021$ & 3 \\
\hline$\lambda$ Ori A & O8IIIf & 3.66 & 24 & 420 & - & - & $18-21.01 .2019$ & 24 \\
\hline$\alpha \mathrm{Cam}$ & O9Ia & 4.29 & 5 & 220 & - & - & $4-5.03 .2021$ & 5 \\
\hline \multirow[t]{3}{*}{19 Cep } & O9Ib & 5.11 & 22 & 420 & - & - & $2019-2020^{a}$ & 637 \\
\hline & & & 390 & 2 & 6-m BTA & $\mathrm{Sco}^{b}$ & $17-18.08 .2021$ & \\
\hline & & & 225 & 33 & $1.25-\mathrm{m}$ & $\mathrm{A}-\mathrm{Sp}$ & 21.09 .2020 & \\
\hline$\delta$ Ori A & O9V+B0III & 2.41 & 49 & 90 & Zeiss-2000 & MMCS & $20-24.01 .201$ & 49 \\
\hline \multirow[t]{2}{*}{ HD 93521} & O9.5III & 7.03 & 529 & 3 & 6-m BTA & $\mathrm{Sco}^{b}$ & $19-20.01 .2015$ & 557 \\
\hline & & & 28 & 900 & - & MSS & $2020-2021^{c}$ & \\
\hline HD 34078 & $09.5 \mathrm{~V}$ & 5.96 & 27 & $300-420$ & - & - & $5-8.1 .2020$ & 27 \\
\hline HD 45314 & O9:npe & 6.64 & 20 & 600 & - & - & $5-8.1 .2020$ & 20 \\
\hline \multicolumn{9}{|c|}{ B stars } \\
\hline$\beta$ Сер & B0.5IIIs & 3.21 & 74 & $60-180$ & 6-m BTA & MSS & $2020-2021^{c}$ & 74 \\
\hline SAO 49725 & $\mathrm{~B} 0.5 \mathrm{III} / \mathrm{IVe}$ & 9.27 & 432 & 5 & - & $\mathrm{Sco}^{b}$ & $17-18.08 .2021$ & 432 \\
\hline$\gamma$ Cep & B0.5IVpe & 2.39 & 1576 & 2 & $1.25-\mathrm{m}$ & A-sp & $13-14.9 .2020$ & 1714 \\
\hline & & & 138 & 60 & 6-m BTA & MSS & $1-2.2 .2021$ & \\
\hline \multirow[t]{3}{*}{$\rho$ Leo } & B1Iab & 3.87 & 1271 & 1 & - & $\mathrm{Sco}^{b}$ & $19-21.01 .2015$ & 1614 \\
\hline & & & 80 & 90 & - & MSS & 19.02.2019 & \\
\hline & & & 263 & 10 & $1.25-\mathrm{m}$ & A-sp & $26.10-2.11 .2019$ & \\
\hline$\varepsilon$ Per & B1.5III & 2.89 & 39 & 150 & Zeiss-2000 & MMCS & 19-20.01.2019 & 39 \\
\hline V2156 Cyg & $\mathrm{B} 1.5 \mathrm{Ve}$ & 8.91 & 7 & 20 & 6-m BTA & $\mathrm{Sco}^{b}$ & 18.08.2021 & 7 \\
\hline$\gamma$ Ori & $\mathrm{B} 2 \mathrm{~V}$ & 1.64 & 42 & 50 & Zeiss-2000 & MMCS & $18-21.01 .2019$ & 42 \\
\hline$\eta \mathrm{Uma}$ & B3V & 1.86 & 10 & 50 & - & MMCS & $18-21.01 .2019$ & 10 \\
\hline \multirow[t]{2}{*}{$\alpha$ And } & B8IV & 2.06 & 2600 & 2 & $1.25-\mathrm{m}$ & A-sp & $13-14.9 .2020$ & 3309 \\
\hline & & & 709 & 10 & Zeiss-1000 & UAGS & $13-14.9 .2020$ & \\
\hline \multicolumn{9}{|c|}{ A stars } \\
\hline \multirow[t]{2}{*}{$\alpha^{2} \mathrm{CVn}$} & A0spe & 2.90 & 866 & 1 & 6-m BTA & $\mathrm{Sco}^{b}$ & $21-22.01 .2015$ & 937 \\
\hline & & & 71 & 90 & - & MSS & 5.1 .2020 & \\
\hline HD 21389 & A0Ia & 4.54 & 330 & 11 & - & $\mathrm{Sco}^{b}$ & $25-26.09 .2016$ & 330 \\
\hline$\gamma \mathrm{UMi}$ & A2III & 3.00 & 249 & 20 & - & $\mathrm{Sco}^{b}$ & $25-26.09 .2016$ & 249 \\
\hline$\alpha$ Cep & $\mathrm{A} 8 \mathrm{Vn}$ & 2.46 & 641 & 10 & Zeiss- 1000 & UAGS & $28-30.10 .2020$ & 641 \\
\hline
\end{tabular}

As an illustration we consider the first 500 spectra of a B8IV-V star $\alpha$ And obtained at the 1.25 -m telescope on 13-14 of September, 2020. Dynamical spectra $d(V, t)$ for $\mathrm{H}_{\beta}$ and $\mathrm{H}_{\alpha}$ lines are given in Fig. 1. Inspecting Fig. 1 we see the similarity of these dynamical spectra at the minute time scales. For searching the periodic components of the LPVs in the spectra of $\alpha$ And the CLEAN method by [11] was used. We find 3 components with the periods of $20.74 \pm 10.64,18.38 \pm 8.36$, and $4.93 \pm 0.60$ minutes.

Resuming our studies we can conclude that for all studied objects short time line profile variations in the interval from 5 minutes to $2-3$ hours were revealed. The shortest periods of LPVs (up to 1 minute) corresponds to OB stars, while for late B and A stars the periods are more than 10-20 minutes.

Acknowledgments: A.K, A.V., A.M., A.K., and M.K. thanks the Russian Foundation for Basic Research for the support with RFBR grant no. 19-02-00311 A. I.A. is grateful to RFBR grant 19-32-60007 for the support.

\section{References}

1. V. V. Dushin, A. F. Kholtygin, G. A. Chuntonov, and D. O. Kudryavtsev, Astrophysical Bulletin, 68, 184, 2013.

2. L. Kaper, H. F. Henrichs, A. W. Fullerton, H. Ando, et al., A\&A, 327, 281, 1997.

3. A. F. Kholtygin, D. N. Monin, A. E. Surkov, and S. N. Fabrika, Astronomy Letters, 29, 175, 2003.

4. V. L. Afanasiev and A. V. Moiseev, Astronomy Letters, 31, 194, 2005.

5. V. E. Panchuk, G. A. Chuntonov, and I. D. Naidenov, Astrophysical Bulletin, 69, 339, 2014.

6. G. A. Chountonov, in L. Mashonkina and M. Sachkov, eds., Spectroscopic methods in modern astrophysics, 336-349 (2007).

7. G. A. Chountonov, Astrophysical Bulletin, 71, 489, 2016.

8. F. A. Musaev, S. I. Barabanov, and A. V. Sergeev, INASAN Science Reports, 4, 158, 2019.

9. A. F. Kholtygin, V. B. Puzin, I. V. Sokolov, and G. M. Karataeva, Astrophysics, 63, 356, 2020.

10. V. V. Komarov, A. S. Moskvitin, V. D. Bychkov, A. N. Burenkov, et al., Astrophysical Bulletin, 75, 486, 2020.

11. D. H. Roberts, J. Lehar, and J. W. Dreher, AJ, 93, 968, 1987. 


\title{
Modeling the formation of complex organic molecules in a protoplanetary disk
}

\author{
M.Yu. Kiskin ${ }^{1}$, A.I. Vasyunin ${ }^{1}$, V.V. Akimkin ${ }^{2}$ \\ mikhail.kiskin@urfu.ru \\ ${ }^{1}$ Research Laboratory for Astrochemistry, Institute of Natural Sciences and Mathematics, Ural Federal University, \\ Ekaterinburg, 620002, Russia, \\ ${ }^{2}$ Institute of Astronomy of the Russian Academy of Sciences, Moscow, 119017, Russia
}

\section{DOI: 10.51194 /VAK2021.2022.1.1.050}

Protoplanetary disks, rich in dust and gas, are the places of planet formation. They are found almost ubiquituously around young stars. Understanding the chemical composition of disks is critical for further studies of the chemical composition of future exoplanets and smaller objects in planetary systems such as asteroids and comets.

We aim to develop a computational code capable of efficiently calculating the evolution of the chemical composition of the protoplanetary disk under the wide range of physical parameters and conditions typical of protoplanetary disks.

The evolution of the chemical composition is calculated by numerical integration of the system of differential rate equations using the Adams method. Numerical integration is implemented in MONACO Fortran code using the DVODE integrator [1, 2]. Due to large variety of physical conditions throughout the protoplanetary disk [3], the calculation of the evolution of the chemical composition at different points of the physical model grid takes different processor time, and in some cases may not be completed successfully. We expect that supplying the analytical Jacobian of a system of differential equations for a numerical integrator should increase the speed and stability of calculations, as well as their accuracy.

We define the Jacobian of the system of differential equations using the SymPy symbolic computation package for the Python. After adding the Jacobian to the two-phase mode (gas phase and grain surface), the calculation speed increased on average by 4-5 times, and the number of unsuccessfully completed calculations decreased by 150 times.

Thus, supplying the Jacobian of the system of differential equations to numerical integration increased the efficiency and speed of calculations and will allow further application of more complex chemical and physical models for the studies of the chemical evolution of protoplanetary disks.

Acknowledgements. The work is supported by the Ministry of Science and Higher Education of the Russian Federation via the State Assignment Projects FEUZ-2020-0038.

\section{References}

1. A. Vasyunin and E. Herbst, The Astrophysical Journal, 769, 34, 2013.

2. A. I. Vasyunin, P. Caselli, F. Dulieu, and I. Jiménez-Serra, The Astrophysical Journal, 842, 33, 2017.

3. V. Akimkin, S. Zhukovska, D. Wiebe, D. Semenov, Y. Pavlyuchenkov, A. Vasyunin, T. Birnstiel, and T. Henning, The Astrophysical Journal, 766, 8, 2013. 


\title{
Photometry and kinematics of star forming complexes in external galaxies
}

\author{
A. Kuzin, D. Lisitsin \\ alv.kuzin@gmail.com \\ Moscow State University, Moscow, Russia
}

We investigate spectral and photometric properties of star forming complexes (SFC) in external galaxies. The SFCs were selected in 17 nearby galaxies of spiral or irregular type, having inclinations less than $45^{\circ}$ and distances less than $15 \mathrm{Mpc}$. To identify SFCs, we developed a method based on matching sources of emission at $160 \mu \mathrm{m}$ (cold dust) and $8 \mu \mathrm{m}$ (polycyclic aromatic hydrocarbons). Using photometry in different spectral bands, correlations between SFC properties for spiral and irregular galaxies were considered. Spectral and kinematic analysis was carried out for several SFCs, and a method to detect gas motion patterns in these SFCs was suggested.

Keywords: star formation, molecular clouds, interstellar medium

DOI: 10.51194 /VAK2021.2022.1.1.051

Current models of star formation are largely based on information obtained from our Galaxy. However, now we are able to explore star formation in detail in other galaxies. One way to do it is to analyze active star forming regions (star forming complexes, SFC). This includes comparison of SFC data in different bands of electromagnetic spectrum. In the current work, we investigate photometric and kinematic characteristics of SCFs in external galaxies for purposes mentioned above.

For our study, we need galaxies, where separate SFCs can be identified. This implies the following selection criteria.

- Distance is less than $15 \mathrm{Mpc}$.

- Spiral or irregular morphological type.

- Inclination of the symmetry plane is less than $45^{\circ}$.

- Galaxy has been observed at $8 \mu \mathrm{m}$ and $160 \mu \mathrm{m}$ (data from "Spitzer" [1] and "Herschel" [2] telescopes) and in the near UV band (data from GALEX telescope [3]).

Using these criteria, we selected 17 nearby galaxies from the HyperLeda database [4]. We detected SFCs in these galaxies, matching "Spitzer" and "Herschel" data. The Spitzer images were convolved to the Herschel point spread function, using kernels from [5]. After that we pick out 20-200 complex candidates using circular apertures on each image of each galaxy. Then we superimpose different emission maps. We believe that an SCF is reliably detected if a single region on the $160 \mu \mathrm{m}$ map overlaps with a single region on the $8 \mu \mathrm{m}$ maps, and the distance between region centres, $D_{12}$, is less than the average radius of regions, $\bar{r}$.

\section{Photometry and comparison of various star formation tracers}

Flux in each aperture was calculated by summarizing intensity in each pixel. Then local background was subtracted from the total flux as described by [6].

We present the results on Fig. 1. Effects of extinction were not taken into account. It is easy to see that relation $F_{160} \sim F_{8}$ is valid for spiral galaxies and the slope is the same for all galaxies. This is not surprising since all these galaxies are nearby and probably of similar metallicity. One can also see that there is no simple relation for irregular galaxies. Similar relations can be obtained for other "dust" bands: $F_{160} \sim F_{24}, F_{24} \sim F_{8}$ and for fluxes in $\mathrm{CO}(2-1)$ spectral line (PHANGS data: [7]): $F_{\mathrm{CO}} \sim F_{\text {dust }}$.

\section{Kinematics of star formation complexes}

PHANGS data contain images in the $\mathrm{CO}(2-1)$ line with a resolution of 2 arcsec for galaxies NGC 628, NGC 4254, NGC 5236, which are included in our list. Therefore we can obtain spectra for some of our SFCs. There are two peaks on some spectra. This may indicate the existence of two spectrally (and therefore kinematically) separated regions disguised as one photometric region.

In these three galaxies we have no need to examine such two-peak regions since they all can be resolved using unconvolved "Spitzer" images. Our method of finding kinematically distinct regions can be applied if there are no high resolution images, but a spectrum of the region is available. 


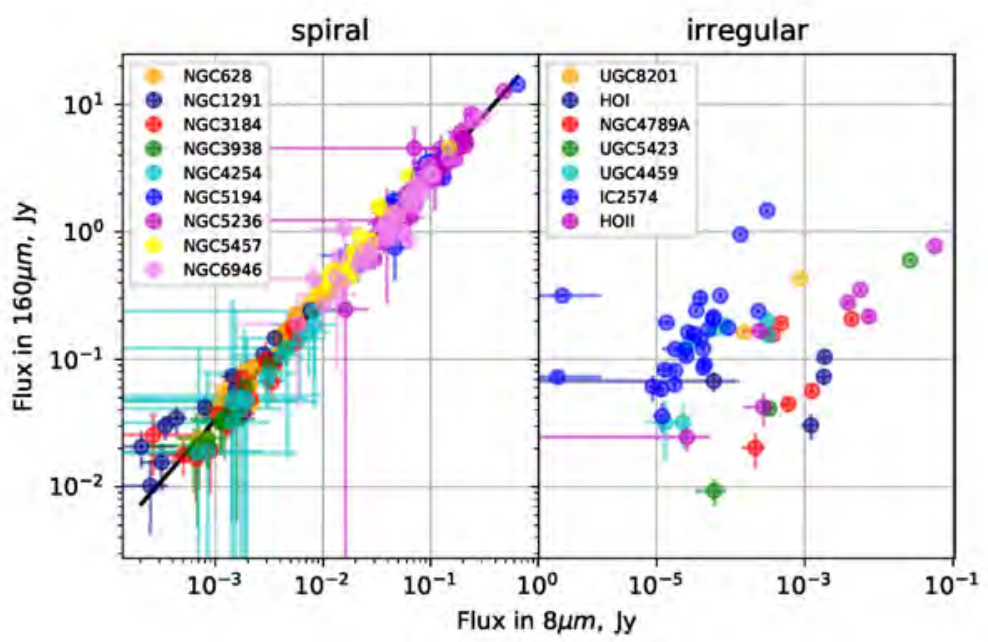

Figure 1: Relations between aperture flux in $160 \mu \mathrm{m}$ band and $8 \mu \mathrm{m}$ band for spiral and irregular galaxies. All fluxes normalized as they appear on distance $D=10 \mathrm{Mpc}$. The line on the left shows a least-square fit of slope 0.98 on a logarithmic plane

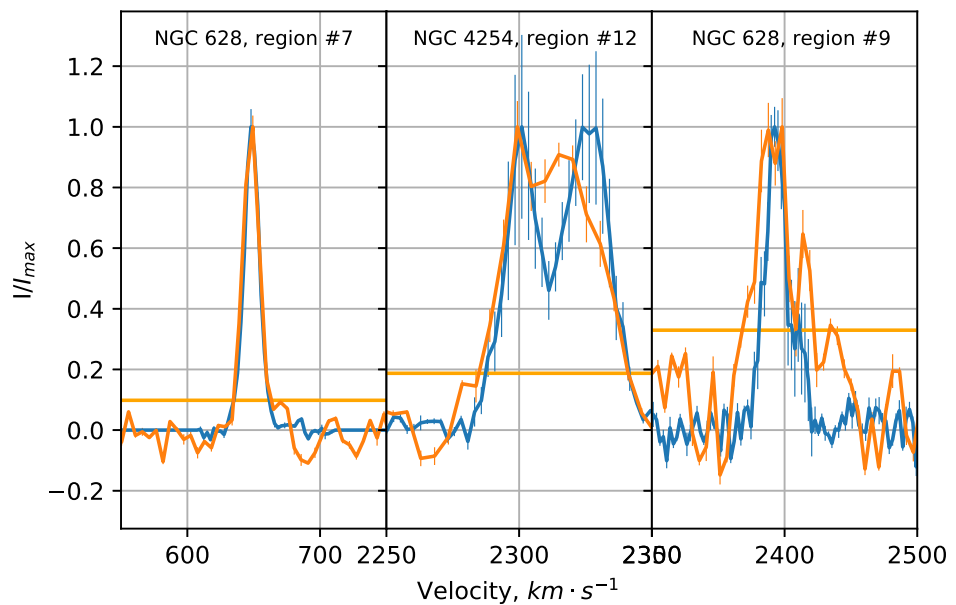

Figure 2: Spectra of three regions taken from PHANGS (blue) and HERACLES (orange) data. From left to right: one-peak region in NGC 628, two-peak region in NGC 4254, two-peak region in NGC 628. Horizontal line represents $S N R \approx 3$ in HERACLES data

\section{References}

1. M. W. Werner, T. L. Roellig, F. J. Low, G. H. Rieke, et al., ApJS, 154, 1, 2004.

2. G. L. Pilbratt, J. R. Riedinger, T. Passvogel, G. Crone, et al., A $6 A$, 518, L1, 2010.

3. A. Conti, L. Bianchi, and B. Shiao, Ap\&SS, 335, 329, 2011.

4. D. Makarov, P. Prugniel, N. Terekhova, H. Courtois, and I. Vauglin, A $6 A$, 570, A13, 2014.

5. G. Aniano, B. T. Draine, K. D. Gordon, and K. Sandstrom, PASP, 123, 1218, 2011.

6. M. S. Khramtsova, D. S. Wiebe, P. A. Boley, and Y. N. Pavlyuchenkov, MNRAS, 431, 2006, 2013.

7. A. K. Leroy, A. Hughes, D. Liu, J. Pety, et al., ApJS, 255, 19, 2021. 


\title{
Multiwavelength analysis of star-forming regions using the online database of maser sources MaserDB.net
}

\author{
D. Ladeyschikov, A. Sobolev
}

Ural Federal University, 51 Lenina st., Yekaterinburg, 620083, Russia

In this paper, we report on the possibilities of using the online database of interstellar masers (http://maserdb.net) for multiwavelength studies of star-forming regions of the Galaxy. The collected maser data were used to construct the model of water maser presence in any ATLASGAL sources with defined physical parameters. The model allows the calculation of the probability of maser detection using several independent source physical parameters with the maximum prediction power. The model may be extended to any maser species and catalog of gas-dust clump physical parameters - the only requirement is a large enough training set for maser observations.

Keywords: interstellar masers, millimeter emission, ATLASGAL clumps, generalized linear model

DOI: 10.51194/VAK2021.2022.1.1.052

\section{Introduction}

In astronomy, there is a trend to use large astronomical catalogs and databases to study different classes of astrophysical objects. The recent development includes databases of exoplanets (EXO.MAST, Exoplanets.org), The Mikulski Archive for Space Telescopes (MAST) focused on scientific data sets in the optical, ultraviolet, and near-infrared parts of the spectrum, and others. All these databases were created focusing on specific phenomena, the wavelength of detection, or the class of an astronomical object.

Interstellar masers provide us with unique information on the properties of the interstellar medium. However, the complete database of masers in star-forming regions is still missing to date. Thus it is pretty expensive to search across all observations available in the literature.

Recently, new maser surveys have appeared, significantly changing our view on the number of known maser sources. In the blind HOPS survey [1], more than 500 water masers were detected, of which 334 were detected for the first time. The methanol multi-beam (MMB) survey [2] results in more than 800 methanol maser detections at $6 \mathrm{GHz}$. 439 water masers were detected toward 1600 Bolocam clumps in observations by [3]. We present the number of known maser sources as the function of time in Figure 1. In this regard, it is important to be aware of all recent surveys and observations while studying statistics of maser emission and preparing the proposals for observations with single-dish and interferometric facilities. The MaserDB project aims to solve the problem of masers data access through various technological solutions, a user-friendly interface, and rich opportunities of the SQL-queries.

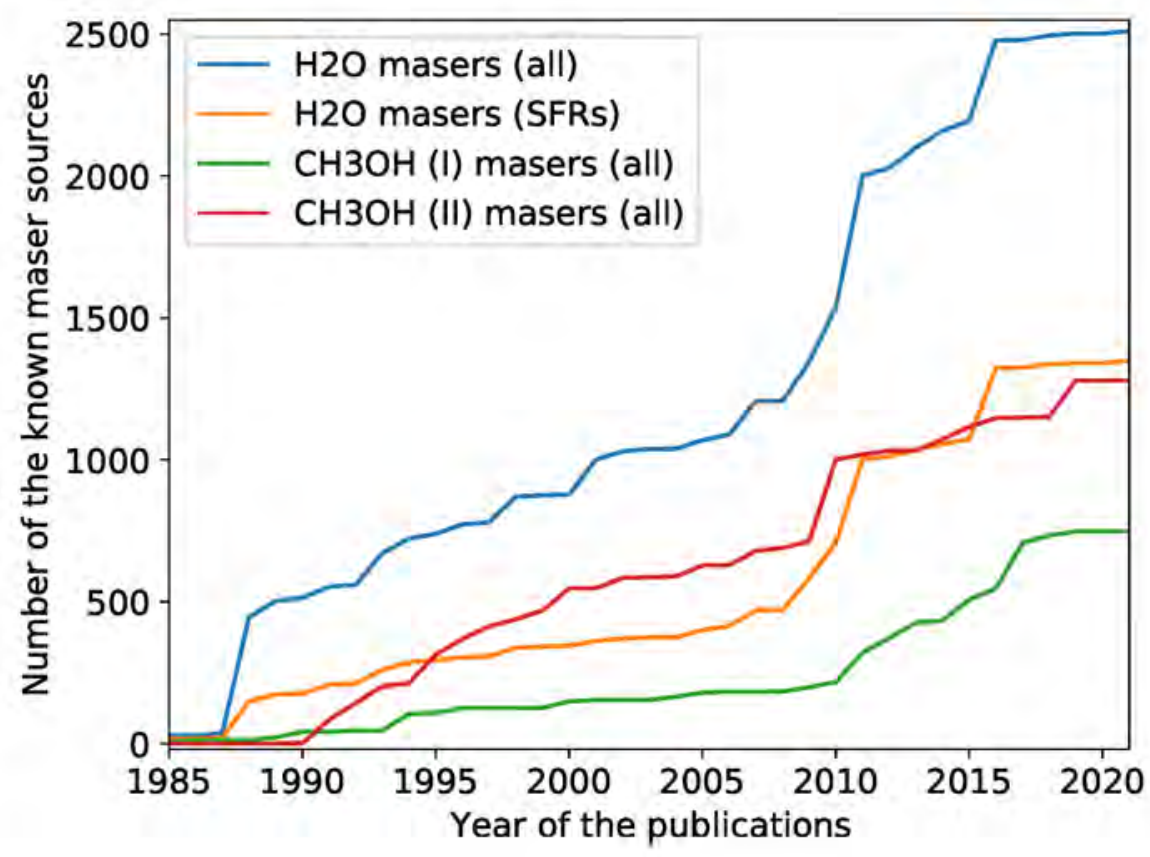

Figure 1: The number of known methanol and water maser sources as the function of the year of publication. Water masers are displayed in all sources (blue) and limited only to star-formation regions (orange). 
The maser database includes the following maser species: $\mathrm{H}_{2} \mathrm{O}, \mathrm{CH}_{3} \mathrm{OH}$ (class I and II), OH, SiO. The coverage of the water maser database is currently $93 \%$ of all available observations in the literature since 1989 . The data entry for class I and II methanol masers has been completed at the level of 95\%, and work continues on the data input for water and hydroxyl masers in the galactic star-forming regions. For $\mathrm{OH}$ and $\mathrm{SiO}$ masers, the data entry is approximately $70 \%$.

\section{Prediction of maser emission thought physical parameters of associated dust clumps}

For studying the association of maser sources with millimeter dust clumps, we used the ATLASGAL $870 \mu$ m source catalog [4]. We calculate the probability of detecting a maser emission from the physical parameters of ATLASGAL dust clumps [5]. In practice, we used only the $22 \mathrm{GHz}$ water masers as an indicator of the maser emission. However, any other maser species may be studied if a large enough training set is available.

To train the model, we used the water maser observations from [3]. Only those observations were included in the analysis that has associated ATLASGAL source within 60 arcsec match radius and has defined values of clump physical parameters. There are 822 such sources in total, and 359 water masers were detected at the $5 \sigma$ level, where $\sigma \sim 0.03 \mathrm{Jy}$.

Similar to the work of [6] we analyze the physical parameters of ATLASGAL clumps for their sensitivity to the presence or absence of a water maser using the Akaike information criterion [7]. The simplest model was obtained, which has the best power for predicting the maser presence depending on the physical parameters. We found that the probability of water maser detection may be expressed as a linear combination of the following ATLASGAL clumps physical parameters:

$$
\log \frac{p}{1-p}=-0.12 T_{\mathrm{dust}}+1.85 L_{\mathrm{bol}}^{\mathrm{log}}-0.80 M_{\mathrm{FWHM}}^{\mathrm{log}}+2.01 n_{\mathrm{H}_{2}}^{\log }
$$

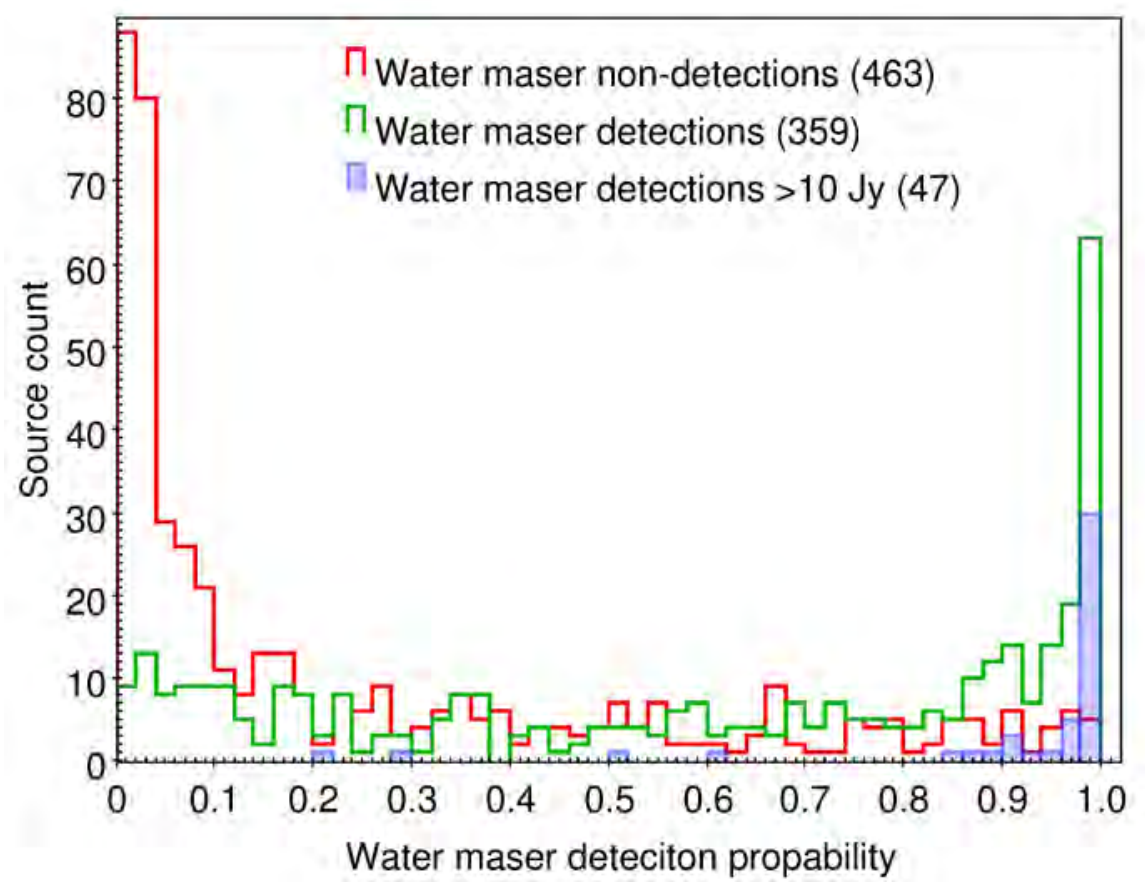

Figure 2: Histogram distribution of number of detections and non-detections of water masers as the function maser deteciton propability.

where the log superscript indicates the use of the logarithmic values of the corresponding physical parameters.

Figure 2 shows the results of applying the model to the observations of [3]. From the analysis, we conclude that the probability of detecting a maser increases with an increase in the flux density of the ATLASGAL clumps. That generally shows that water masers can be found in a wide range of physical parameters of dust clumps. Nevertheless, from the Figure 2 we conclude that at probability values less than 0.1 , the number of non-detections of water masers increases significantly. These non-detections are mainly associated with clumps that have an ATLASGAL flux density of less than $1 \mathrm{Jy}$.

We conclude that overall prediction quality is $\sim 70 \%$ for $22 \mathrm{GHz}$ maser emission brighter than $0.5 \mathrm{Jy}$ and $92 \%$ for emission brighter than $10 \mathrm{Jy}$. The same result is obtained through training the neural network on the previously 
observed sources. Thus we assume that there is no non-linear relation between source physical parameters and the presence of the maser emission.

The proposed maser detection probability may be used to search for new maser sources in objects previously non-detected or even not observed. Due to the strong temporal variability of water and methanol masers, we propose that sources with high maser detection probability with no observations or single-epoch non-detection may be detected at some of the observed epoch. Multi-epoch observations are required to increase the detection rate toward sources with high maser detection probability. Currently, the proposal has been approved to Effelsberg 100-m telescope (project code 80-21) to search for $22 \mathrm{GHz}$ water masers toward ATLASGAL sources using the source selection criteria based on maser detection probability.

\section{References}

1. A. J. Walsh, S. L. Breen, T. Britton, K. J. Brooks, et al., MNRAS, 416, 1764, 2011.

2. J. L. Caswell, G. A. Fuller, J. A. Green, A. Avison, et al., MNRAS, 404, 1029, 2010.

3. B. E. Svoboda, Y. L. Shirley, C. Battersby, E. W. Rosolowsky, et al., ApJ, 822, 59, 2016.

4. J. S. Urquhart, T. J. T. Moore, T. Csengeri, F. Wyrowski, et al., MNRAS, 443, 1555, 2014.

5. J. S. Urquhart, C. König, A. Giannetti, S. Leurini, et al., MNRAS, 473, 1059, 2018.

6. S. L. Breen, S. P. Ellingsen, and et al., MNRAS, 377, 491, 2007.

7. K. P. Burnham and D. R. Anderson, eds., Monte Carlo Insights and Extended Examples, 206-266 (2002). 


\section{Light curve features extraction from astronomical source}

A.D. Lavrukhina ${ }^{1}$, K.L. Malanchev ${ }^{2,3}$

lavrukhina.ad@gmail.com

${ }^{1}$ Lomonosov Moscow State University, Faculty of Space Research, Russia

${ }^{2}$ Lomonosov Moscow State University, Sternberg astronomical institute, Russia

${ }^{3}$ University of Illinois at Urbana-Champaign, Department of Astronomy, USA

\section{DOI: 10.51194/VAK2021.2022.1.1.053}

Astronomy is entering the era of large surveys of the variable sky such as Zwicky Transient Facility and the forthcoming Legacy Survey of Space and Time which are able to yield 10-100 thousands alerts every night. Nowadays, machine learning is often used to solve tasks of classification [1] or anomaly detection [2] in such large amounts of photometric data. Usually, each considered light curve is represented by a set of features which describe its variability properties best. In this work, we present the new library for extraction of light curve features of variable astronomic sources. The library is available in Python and Rust programming languages. ${ }^{1}$

We solved a test problem of the classification of two types of objects, Cepheid variable and dwarf nova, to validate that features describe important properties of light curves. Each light curve was represented by 25 features. The data set is consisted of 1855 light curves of Cepheids and 2394 ones of dwarf novae in the R band from ZTF DR3 [3]. To solve this problem, we used an algorithm of random forest implemented in the package scikit-learn [4]. We performed 75\%-25\% train-test split with shuffling. Three models in total were developed:

1. Random forest trained on the data represented by all 25 extracted features.

2. Random forest with the data set preprocessed using PCA (dimension was reduced to 15 components).

3. Random forest with the sequential feature selection procedure.

Four metrics were counted to evaluate model quality: precision, recall, ROC AUC and accuracy. All models showed satisfactory performance, while the third model showed the best values of the metrics: $0.962,0.968,0.992,0.966$.

Using described features apparently allows to successfully apply machine learning methods to solve tasks of classification or anomaly detection. In the future, we are going to extend the library, making up the new features appropriate for solving these and other tasks.

The reported study was funded by RFBR and CNRS according to the research project No. 21-52-15024.

\section{References}

1. D.-W. Kim, P. Protopapas, C. A. L. Bailer-Jones, Y.-I. Byun, S.-W. Chang, J.-B. Marquette, and M.-S. Shin, Astronomy \& Astrophysics, 566, A43, 2014, URL http://dx.doi.org/10.1051/0004-6361/201323252.

2. K. L. Malanchev, M. V. Pruzhinskaya, V. S. Korolev, P. D. Aleo, et al., arXiv e-prints, arXiv:2012.01419, 2020.

3. F. J. Masci, R. R. Laher, B. Rusholme, D. L. Shupe, et al., Publications of the Astronomical Society of the Pacific, 131, 018003, 2018, URL https://doi.org/10.1088/1538-3873/aae8ac.

4. F. Pedregosa, G. Varoquaux, A. Gramfort, V. Michel, et al., Journal of Machine Learning Research, 12, 2825, 2011.

\footnotetext{
${ }^{1}$ https://github.com/light-curve
} 


\title{
The specifics of pulsar radio emission
}

\author{
B. Losovsky
}

P.N. Lebedev Physical Institute of the Russian Academy of Sciences, Pushchino Radio Astronomy Observatory of the Astrocosmic Center of the Physical Institute of the Academy of Sciences, Pushchino, Russia

DOI: 10.51194 /VAK2021.2022.1.1.054

A characteristic property of pulsars is pulsed periodic radio emission, which has a high stability of periods. Despite the high stability of the emission periods of pulsars, monitoring the time of arrival of pulses (timing) shows the presence of different types of irregularities: variations of residual deviations, changes in the shape of the pulse, switching on and off of radio emission. [1] showed, that they are linked and caused by processes occurring in the pulsar's magnetosphere. The special interest causes the observations of a pulsar in the Crab Nebula, performed, in particular, at Jodrell Bank. Processing by Cadez et al. Jodrell Bank radio ephemerides of the Crab pulsar shows a relationship between the failures of the period(glitches), the braking index and the dispersion measure (See [2] Fig. 7). Variations of the dispersion measure follow the glitches with a delay 1010 day and are explained by the ionization of the nebula by streams of charged particles of the pulsar wind. Change of the braking index has to do with a pulsar-wind-driving torque. Regular observations of pulsar radio emission in the Crab Nebula at a frequency of $111 \mathrm{MHz}$ (Fig 1.), carried out by [3] at the Large Phased Array radio telescopes of the Pushchino Radio Astronomy Observatory, demonstrate a good correlation between the dispersion measure and scattering time scale variation. Plasma turbulence increases scattering time scale. Plasma instabilities occurring through magnetic field lines reconnection drive gamma-ray flares.

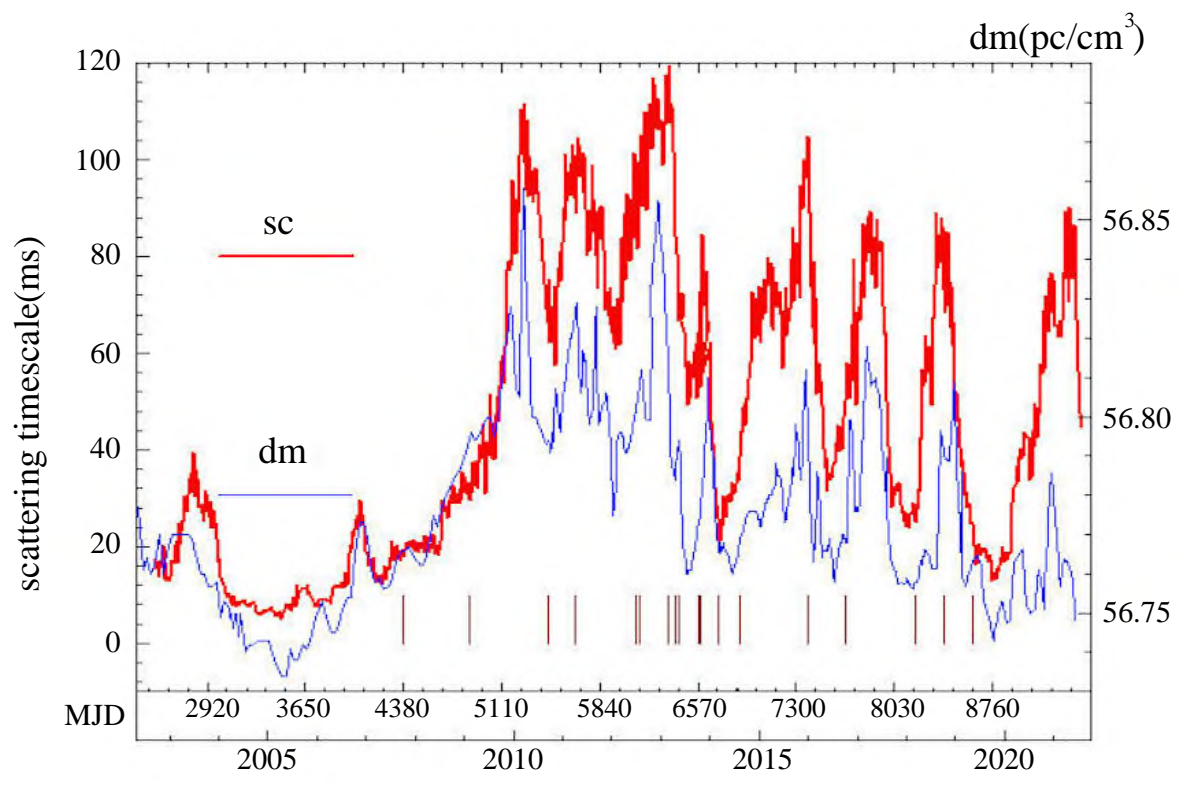

Figure 1: PSR B0531 +21 . Comparison of scattering time scale variations in $\mathrm{ms}$ (scale on the left) and the conditional dispersion measure $d m=(D M-56.7) \times 1000$ (scale on the right). The correlation coefficient is 0.8 Along the abscissa axis is the epoch of observations in modified Julian days MJD = JD-2450000 and the corresponding years. The vertical brown segments show gamma-ray flares with the energy $>100 \mathrm{MeV}$, registered by the Fermi — LAT Gamma-ray Space Telescope and AGILE satellite [4].

\section{References}

1. A. Lyne, G. Hobbs, M. Kramer, I. Stairs, and B. Stappers, Science, 329, 408, 2010.

2. A. Cadež, L. Zampieri, C. Barbieri, M. Calvani, G. Naletto, M. Barbieri, and D. Ponikvar, A\&A, 587, A99, 2016.

3. B. Y. Losovsky, D. V. Dumsky, and Y. A. Belyatsky, Astronomy Reports, 63, 830, 2019.

4. X. Huang, Q. Yuan, and Y.-Z. Fan, ApJ, 908, 65, 2021. 


\title{
Near-infrared detection of $\mathrm{H}_{2}$ flows in the core of Mon R1 association
}

\author{
T. Yu. Magakian ${ }^{1}$, A. M. Tatarnikov², T. A. Movsessian ${ }^{1}$, H. R. Andreasyan ${ }^{1}$ \\ tigmag@sci.am \\ ${ }^{1}$ Byurakan Astrophysical Observatory, Aragatsotn reg.,Armenia, \\ ${ }^{2}$ M. V. Lomonosov Moscow State University, Sternberg Astronomical Institute, 119991, Universitetskij pr. 13, Moscow, \\ Russia
}

We report the discovery of 4 new $\mathrm{H}_{2}$ jets in Mon $\mathrm{R} 1$ star-forming region on the images obtained with the 2.5-m telescope of the Caucasian Mountain Observatory of SAI MSU through the filter, centered on the $\mathrm{H}_{2}$ 1-0 S(1) emission line. This discovery confirms the nature of these flows, which existence was previously suspected using archival Spitzer GLIMPSE360 and WISE survey images. Also two infrared reflection nebulae were revealed. On the Herschel PACS survey images we found a small group of far-infrared sources, mostly new ones. Among them are the possible exciting objects of these outflows. Spectral energy distributions of new sources show their extremely red colour and the bolometric luminosities reaching $3 L \odot$ and even $10 L \odot$. Several of them should belong to Class I and even to Class 0 objects.

Keywords: open clusters and associations: individual: Mon R1, stars: pre-main-sequence; ISM: jets and outflows

DOI: 10.51194/VAK2021.2022.1.1.055

\section{Introduction}

In our previous paper [1] we described the results of a search of Herbig-Haro (HH) objects in the field, centered on the three brightest nebulae NGC 2245, NGC 2247 and IC 446 in the Mon R1 association. Many new HH flows and their probable sources were found, significantly increasing the number of YSOs in Mon R1. In the course of this work we noted several elongated nebulous knots, arranged in chains and visible mainly in $4.5 \mu \mathrm{m}$ bands both of Spitzer and WISE surveys, but not detected in $3.6 \mu \mathrm{m}$. It was natural to assume that they can represent molecular $\mathrm{H}_{2}$ flows. To check this assumption we organized their observations with $2.5 \mathrm{~m}$ telescope of Sternberg Astronomical Institute.

\section{Observations}

The narrow-band filter observations of Mon R1 association region were carried out with the 2.5-m telescope of the Caucasian Mountain Observatory of SAI MSU [2] in 2020. We obtained images of two fields in the $v=1-0 S(1)$ $\mathrm{H}_{2}$ band $\left(\lambda_{c}=2.132 \mu \mathrm{m}\right.$, bandwidth $\left.=0.046 \mu \mathrm{m}\right)$ and "Kcont" band $\left(\lambda_{c}=2.273 \mu \mathrm{m}\right.$, bandwidth $\left.=0.039 \mu \mathrm{m}\right)$ with the ASTRONIRCAM infrared camera-spectrograph [3]. The field of view and image scale were about $4.5^{\prime}$ and $0.27^{\prime \prime} /$ pixel respectively. The final images are the result of sum a number of individual images with a total exposure time of about $2000 \mathrm{~s}$.

\section{Results and Discussion}

The field of probable $\mathrm{H}_{2}$ flows was covered with two pair of images, obtained in $2.12 \mu \mathrm{m} \mathrm{1-0} \mathrm{S}(1) \mathrm{H}_{2}$ line and K-band continuum. Reality of all three suspected molecular flows was confirmed; besides, the existence of one more flow, not well discernible on GLIMPSE360 images, was revealed. However, it was not so easy to point their probable sources.

In Table 1 we present the approximate coordinates of the centers of all four flows and their projected lengths in parsecs. We assumed $715 \mathrm{pc}$ for the distance of Mon R1 association (see for the further details [1]). As one can see, visible sizes of the flows correspond to significant lengths - up to nearly half of parsec.

We analysed the far-infrared PACS 70 and 160 micron images (100 micron survey did not cover our area), obtained with Herschel space observatory. They immediately reveal the existence of a small cluster of several far-IR sources just in the center of the area of interest. Two brightest ones coincide with luminous IRAS $06297+1021$ (W) and IRAS 06297+1021 (E) objects [1]. Beyond this field only the bright HAeBe stars HD 259431, LkH $\alpha 215$ and VY Mon with their reflection nebulae, as well as IRAS $06292+1029$ source, also described in our previous paper, can be seen. It is remarkable, that none of these brightest IR sources is associated with any of newly found flows. Therefore, we prepared the list of eleven sources, visible in the PACS image (some of them are double),

Table 1: The coordinates and lengths of $\mathrm{H}_{2}$ flows in the Mon R1 field.

\begin{tabular}{lccc}
\hline No. & RA(2000) & Decl.(2000) & length (pc) \\
\hline MHO 3143 & 063240.9 & +101937 & 0.36 \\
MHO 3144 & 063223.9 & +102111 & 0.49 \\
MHO 3145 & 063239.8 & +101819 & 0.43 \\
MHO 3146 & 063223.8 & +102033 & 0.22 \\
\hline
\end{tabular}


Table 2: Luminosities of far-IR sources, detected in the field.

\begin{tabular}{cc}
\hline Objects & $L\left(L_{\odot}\right)$ \\
\hline $1-\mathrm{W}$ & 1 \\
$1-\mathrm{E}$ & 0.1 \\
2 & 31 \\
3 & 36 \\
4 & 3 \\
$5 \mathrm{a}$ & 1.1 \\
$5 \mathrm{~b}$ & 0.2 \\
6 & 0.7 \\
7 & 0.5 \\
8 & 3 \\
9 & 1.8 \\
10 & 10.4 \\
11 & 3.4 \\
\hline
\end{tabular}

and attempted to identify these far-IR sources with known objects and with the newly discovered $\mathrm{H}_{2}$ flows. Using the Vizier photometry viewer and, when available, the data from the PACS Point Source Catalog, we also built spectral energy distributions (SEDs) for the all sources, found by us. Coordinates were measured from the WISE images, excluding the cases when objects were not visible even in the WISE survey.

The SEDs of nearly all described above objects are flat from near-IR to far-IR, which points to the wide temperature range in the surrounfing dust envelopes. The exception is extremely red objects number 1 -E, 1 -W, 8 and 11.

We roughly estimated the bolometric luminosities of all these sources, integrating their SEDs and assuming $715 \mathrm{pc}$ for the distance of the complex. These values are listed in the Table 2 . The luminosities of the objects 2 (IRAS $06297+1021 \mathrm{~W}$ ), 3 (IRAS $06297+1021 \mathrm{~W}$ ) and 4 (Petr 7), for which far-IR measurements were added, should be compared with the estimates presented in our previous paper [1]. As one can expect, they slightly increased. Then, the source 10 stands out by its bolometric luminosity. It is the fourth object in this field with $L \geqslant 10 L_{\odot}$ (besides of three more luminous HAeBe stars). This star is very probable source of MHO 3143 . Among other objects we should note the sources 8 and 11. Their bolometric luminosities are near $3 L_{\odot}$; on the other hand, they are very red and are not visible in the optical range. Besides, they are the probable sources of the observed or suspected $\mathrm{H}_{2}$ outflows. All this makes the sources 8, 10 an 11, as well as nebulous 1-E and 1-W a good target for the further IR studies.

\section{Conclusion}

Recent studies $[1,4]$ found that the star formation in Mon R1 association is significantly more active than appeared before and is continuing in the present time. The field, described in the present paper, is a region with enhanced sub-mm emission on 350 and $550 \mu \mathrm{m}[5,4]$, but it did not became the target of further studies in these papers. Nevertheless, the discovery of a compact cluster of far-IR sources and a group of related to them new $\mathrm{H}_{2}$ outflows inside this area again indicates significant activity of the star formation in Mon R1. Moreover, this previously inefficiently studied area contains the group of very young Class II and Class I sources; and the source 11 can be considered even as the Class 0 object. Thus, the rate of the current low and intermediate mass star formation in Mon R1 becomes even higher.

\section{References}

1. T. A. Movsessian, T. Y. Magakian, and S. N. Dodonov, MNRAS, 500, 2440, 2021.

2. N. Shatsky, A. Belinski, A. Dodin, , et al., Ground-Based Astronomy in Russia. 21st Century, $127,2020$.

3. A. E. Nadjip, D. W. Tatarnikov, A. M.and Toomey, N. I. Shatsky, A. M. Cherepashchuk, S. A. Lamzin, and A. A. Belinski, Astrophysical Bulletin, 72, 349, 2017.

4. N. K. Bhadari, L. K. Dewangan, L. E. Pirogov, and D. K. Ojha, Ap. J., 899, 167, 2020.

5. J. Montillaud, M. Juvela, C. Vastel, J. He, et al., Astron.Astrophys., 631, A3, 2019. 


\title{
On features of the pulsar B0950+08 radiation at the frequency of 111 $\mathrm{MHz}$
}

\author{
V.M. Malofeev, I.F. Malov, O.I. Malov, D.A. Teplykh \\ teplykh@prao.ru \\ P.N.Lebedev Physical Institute of the Russian Academy of Sciences, Leninskii pr. 53, Moscow, 119991, Russia
}

Results of long time observations of the pulsar B0950+08 are given. These observations were carried out at the LPA radio telescope at the frequency of $111 \mathrm{MHz}$ from January of 2016 to May of 2019 (450 days). A strong variability in emission of this pulsar has been detected with changes of signal to noise ratios hundreds of times. In addition to the main pulse (MP) and interpulse (IP), this pulsar has "the bridge" and "the precursor" (Pr) located between MP and IP [1]. We detected the unusual IP and Pr radiation on August 1, 2017. On the base of strong 65 interpulses we found the correlations between energies of IP and Pr and between the phase of IP and the distance Pr-IP. It is shown that the observed peculiarities of this pulsar can be explained in the frame of the aligned rotator model.

Keywords: stars: pulsars: individual: $B 0950+08$

DOI: $10.51194 /$ VAK2021.2022.1.1.056

\section{Observations and results}

All observations were carried out using the meridional radio telescope LPA (Large Phase Array). Its antenna is the phased array composed of 16384 dipoles. The geometric area of this antenna is more than $70000 \mathrm{~m}^{2}$ and the effective area is $47000 \pm 2500 \mathrm{~m}^{2}$ [2]. For the processing 460 channels of the 512 channel digital receiver are used. The single channel bandwidth is $4.88 \mathrm{kHz}$, and the time resolution is $1.23 \mathrm{msec}$, the total bandwidth is $2.245 \mathrm{MHz}$. All data is stored in the server. For the processing the special program has been worked out [3]. Here we present the analysis of integrated profiles and single pulses for the pulsar B0950+08. The focus is on the unique event that has taken place on 1 August 2017.

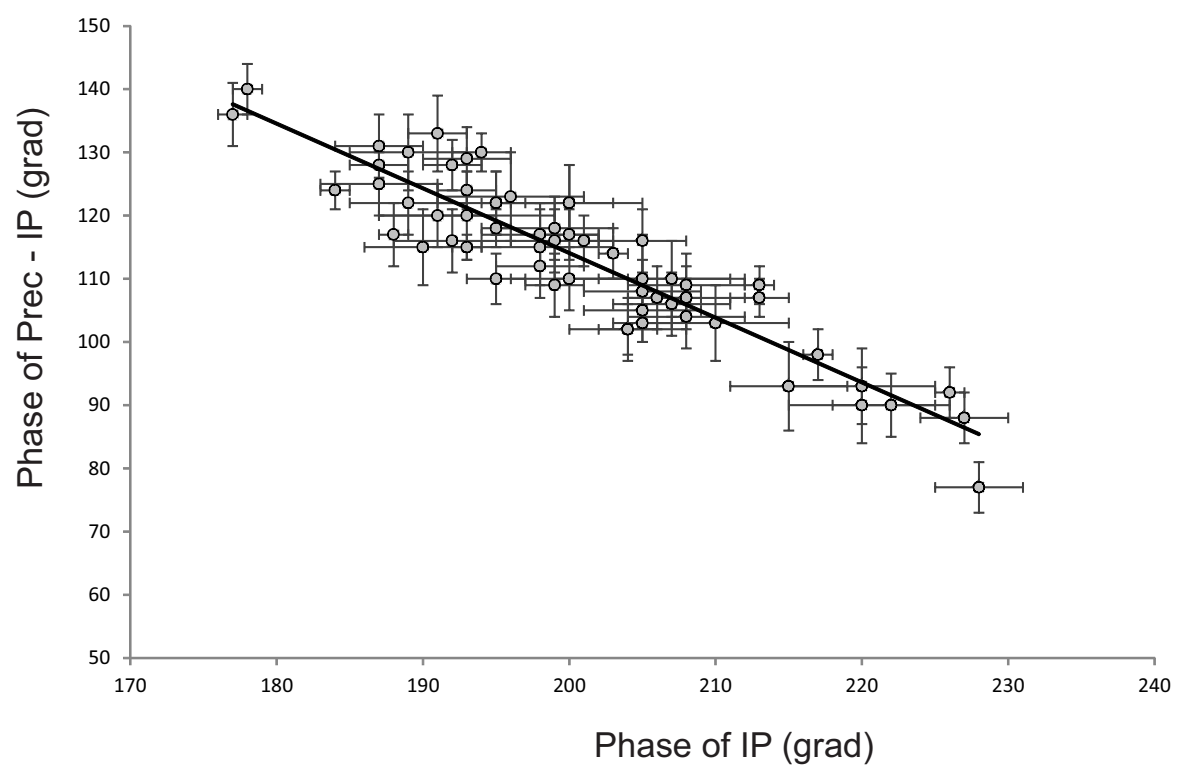

Figure 1: Relationship between phases of IP and Pr

It is evident that $\mathrm{S} / \mathrm{N}$ or flux densities of integrated pulses are extremely variable. Integrated pulses were constructed on the base of 3.2 minutes of observations (764 pulsar periods). These pulses change the intensities on the time scales from one to five days and show power bursts when $\mathrm{S} / \mathrm{N}$ grows up to the values of 2000 in individual pulses. There are also long quasi-periodic variations of flux densities with scales of several months.

We give detailed analysis of IP and its connection with Pr and MP for the first time. We analyzed 4 components of individual pulses, namely, IP, Pr, components C1 and C2 of MP. The following parameters of these components have been investigated: phases, amplitudes, durations at level 50\%, and integrated energies. We search for connections between IP and other components (Pr, MP, and separately C1 and C2). More than 20 paired relationships between parameters of different components (phases, amplitudes, durations, and energies) were analyzed. The strong inverse dependence between the phase of IP and the difference D of phases (Pr-IP) has been detected. (Fig. 1) The correlation coefficient for 62 pairs is $K=-0.92$. The probability of the random distribution in Fig. 1a $p<0.0001$. 


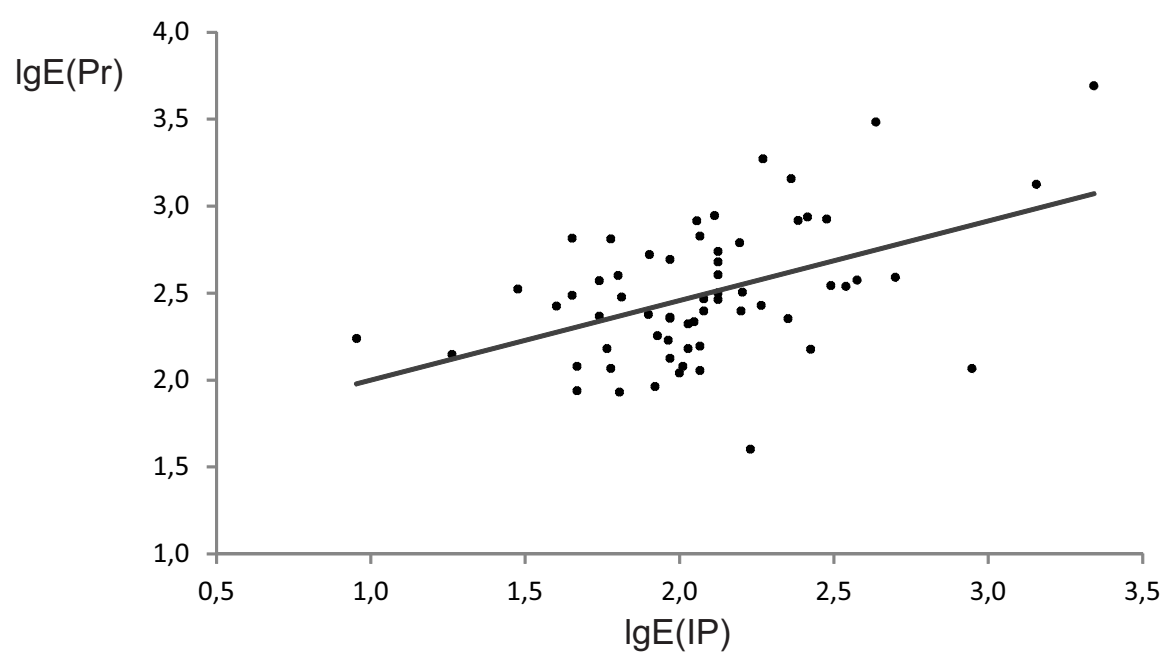

Figure 2: Relationship between energies of Pr and IP.

It is very important to know which component connects with IP. We conclude that this is Pr. Firstly, we can see that the intensity of $\mathrm{Pr}$ in the integral pulse on 01.08.2017 is much higher than during other days and is about $29 \%$ of MP. Secondly, when IP appears Pr, as a rule, is observed also (62 cases from 65 ones). In the same time we see $57 \mathrm{C} 2$ and $32 \mathrm{C} 1$ only. Third, there are positive correlations between energies of different components (Fig. 2). For 62 pairs of $\operatorname{Pr}$ and IP we obtained correlation coefficient $K=0.49$ and the probability of the random distribution $p<0.0001$.

\section{Conclusions}

A strong variability of the intensity was revealed in radiation of the pulsar showing the changes of signal to noise ratio hundreds times at different time scales.

This variability is caused mainly by peculiarities of the emission mechanism. However, long-term fluctuations with the characteristic time of several days and months can be explained by refractive interstellar scintillations (RISS) in the interstellar medium.

We detected the high correlations both between changes of intensities of Pr and IP and between phases of IP and Pr.

New peculiarities of the pulsar emission such as the correlation between parameters of IP and Pr, the rather wide distribution of IP phases and distances between IP and MP can be explained in the frame of the aligned rotator model. The empirical version of such a model of the pulsar magnetosphere is proposed. We believe that there are some spots at the surface of the central neutron star in the pulsar B0950 +08 . They determine the structure of emission cones at different frequencies. Particularly, such a model explains qualitatively the complex picture of emission at low frequencies and gives the possibility to calculate distances where the observed emission is generated.

Variations of $\mathrm{D}$, the wide distribution of this distance and the mentioned difference from $180^{\circ}$ are the strong additional arguments in favor of the alignment of this pulsar. These are mentioned here for the first time. For an orthogonal rotator D must be very near $180^{\circ}$ with minor deviations from this value. Variability in aligned objects can be caused by different intensities of separate parts of corresponding spots and by their movement on the surface of the neutron star caused by oscillations of the surface and waves spreading over it.

We estimated also the initial period of the pulsar acquired at birth $\left(P_{0} \approx 0.2 s\right)$. This means that not all pulsars are born with millisecond periods. The large age of the pulsar (6.8 millions of years) and the small angle between the magnetic moment and the rotation axis are the evidences of the evolution of pulsars to aligned rotators.

\section{References}

1. T. Hankins and J. Cordes, ApJ, 249, 241, 1981.

2. S. Tyul'bashev, V. Tyul'bashev, V. Oreshko, and S. Logvinenko, ARep, 60, 220, 2016.

3. V. Malofeev, D. Teplykh, and S. Logvinenko, ARep, 56, 35, 2012. 


\section{On giant pulses in radio pulsars}

Igor Malov

malov@prao.ru,

WWW home page: http://www.prao.ru

P.N. Lebedev Physical Institute of the Russian Academy of Sciences, Leninskii pr. 53, Moscow, 119991, Russia

Analysis of parameters for radio pulsars with detected giant pulses (GPs) is given. Several models proposed for the explanation of an appearance of GPs are discussed. These models differ by the location of regions where GPs are generated. One of them works near the neutron star surface, some others operate in the pulsar magnetosphere and even at the light cylinder. It is very probable that there are different models for different types of GPs.

Keywords: pulsars: general; gamma-rays: stars; X-rays: stars

DOI: 10.51194 /VAK2021.2022.1.1.057

\section{Introduction}

One of the unsolved problems in pulsar investigations is the problem of their giant pulses (GPs). These features are detected in 18 objects from more than 2800 radio pulsars listed in the ATNF catalog [1]. The most power GPs were detected firstly in the pulsar B0531+21 [2]. List the main peculiarities of GPs. 1) High flux densities up to thousands of those in average normal pulses. High brightness temperatures ( $10^{37} \mathrm{~K}$ and even higher) [3]. 2) Short durations (sometimes of several nanoseconds) [4]. 3) Circular polarization of both signes [3]. 4) Power-law distribution of their energies differing from the Gaussian distribution for normal pulses [5]. These peculiarities lead to the conclusion that mechanisms of their radiation are coherent and non-thermal. Table 1 contains some parameters of pulsars with GPs.

We estimated the angles $\beta$ between magnetic moments and rotation axes of pulsars considered. This parameter is important in considered further models. Let discuss some models proposed for the explanation of the GP phenomena.

\section{Models}

1. Istomin [6] proposed explain GPs in the pulsars B0531+21 and B1937+21 in the frame of the orthogonal rotator model. Magnetic fields near the light cylinder $\mathrm{B}_{\mathrm{LC}}$ are very strong in these pulsars. The peculiar structure of the magnetosphere provides conditions for the acceleration of relativistic particles up to Lorenz-factors of $10^{9}$. The two-stream instability leads to generation of radiation with the density of energy of $10^{10} \mathrm{erg} / \mathrm{sec}$ at the frequency of $10^{9} \mathrm{~Hz}$. This model can explain some features in observed GPs. However, strong magnetic fields $\mathrm{B}_{\mathrm{LC}}$ are observed only in 7 pulsars and only one of them is the nearly orthogonal rotator. Hence, for other pulsars we need use other models.

Table 1: Radio pulsars with GPs

\begin{tabular}{|c|c|c|c|c|c|c|c|c|}
\hline & PSR & $\begin{array}{c}\mathrm{P} \\
(\mathrm{sec})\end{array}$ & $\begin{array}{c}\text { Frequency } \\
(\mathrm{MHz})\end{array}$ & $\begin{array}{c}\mathrm{R}_{\text {lum }} \\
\left(\mathrm{mJy} \times \mathrm{kpc}^{2}\right)\end{array}$ & $\begin{array}{c}\mathrm{B}_{\mathrm{LC}} \\
(\mathrm{G})\end{array}$ & $\mathrm{SGP} / \mathrm{S}$ & $\begin{array}{c}\mathrm{W}_{10} \\
(\mathrm{msec})\end{array}$ & $\beta^{0}$ \\
\hline 1 & $\mathrm{~J} 0034-0721$ & 0.9429 & 40 & 55.17 & $7.02 \mathrm{E}+00$ & 400 & 104.50 & 12 \\
2 & $\mathrm{~J} 0218+4232$ & 0.0023 & 610 & 466.36 & $31.21 \mathrm{E}+05$ & & $(7.50)$ & $(5)$ \\
3 & $\mathrm{~J} 0304+1932$ & 1.3876 & 111 & 14.79 & $4.76 \mathrm{E}+00$ & 69 & 73.40 & 25 \\
4 & $\mathrm{~J} 0529-6652^{*}$ & 1.0249 & 610 & - & $3.97 \mathrm{E}+01$ & & 50.00 & 28 \\
5 & $\mathrm{~J} 0534+2200$ & 0.0331 & $40-8300$ & 2200 & $9.8 \mathrm{E}+05$ & 50000 & 4.70 & 85 \\
6 & $\mathrm{~J} 0540-6919 *$ & 0.0500 & 1390 & - & $3.62 \mathrm{E}+05$ & 5000 & $(38.80)$ & $(3)$ \\
7 & $\mathrm{~J} 0653+8051$ & 1.2140 & 111 & 35.90 & $1.40 \mathrm{E}+00$ & & 419.00 & 41 \\
8 & $\mathrm{~J} 0659+1414$ & 0.3849 & 111 & 0.54 & $7.66 \mathrm{E}+02$ & 630 & 300.00 & 19 \\
9 & $\mathrm{~J} 0814+7429$ & 1.2922 & $70-103$ & 14.74 & $2.05 \mathrm{E}+00$ & & 89.50 & 19 \\
10 & $\mathrm{~J} 0953+0755$ & 0.2530 & 111 & 27.25 & $1.41 \mathrm{E}+02$ & 490 & 200.00 & 19 \\
11 & $\mathrm{~J} 1115+5030$ & 1.6564 & 111 & 10.16 & $4.24 \mathrm{E}+00$ & 80 & 35.00 & 90 \\
12 & $\mathrm{~J} 1136+1551$ & 1.1879 & 111 & 35.52 & $1.90 \mathrm{E}+00$ & 86 & 41.80 & 40 \\
13 & $\mathrm{~J} 1239+2453$ & 1.3824 & 111 & 77.62 & $4.14 \mathrm{E}+00$ & 65 & 60.60 & 31 \\
14 & $\mathrm{~J} 1752+2359$ & 0.4091 & 111 & 32.13 & $7.11 \mathrm{E}+01$ & 320 & 11.00 & 72 \\
15 & $\mathrm{~J} 1823-3021 \mathrm{~A}$ & 0.0054 & 685 & 2342.60 & $2.52 \mathrm{E}+05$ & & 1.60 & 5 \\
16 & $\mathrm{~J} 1824-2452 \mathrm{~A}$ & 0.0030 & 1510 & 1210.00 & $7.41 \mathrm{E}+05$ & 1700 & 0.98 & 13 \\
17 & $\mathrm{~J} 1939+2134$ & 0.0016 & $111-5500$ & 2940.00 & $1.02 \mathrm{E}+06$ & 600 & 0.09 & 79 \\
18 & $\mathrm{~J} 1959+2048$ & 0.0016 & 610 & 59.86 & $3.76 \mathrm{E}+05$ & & 0.07 & 90 \\
\hline
\end{tabular}




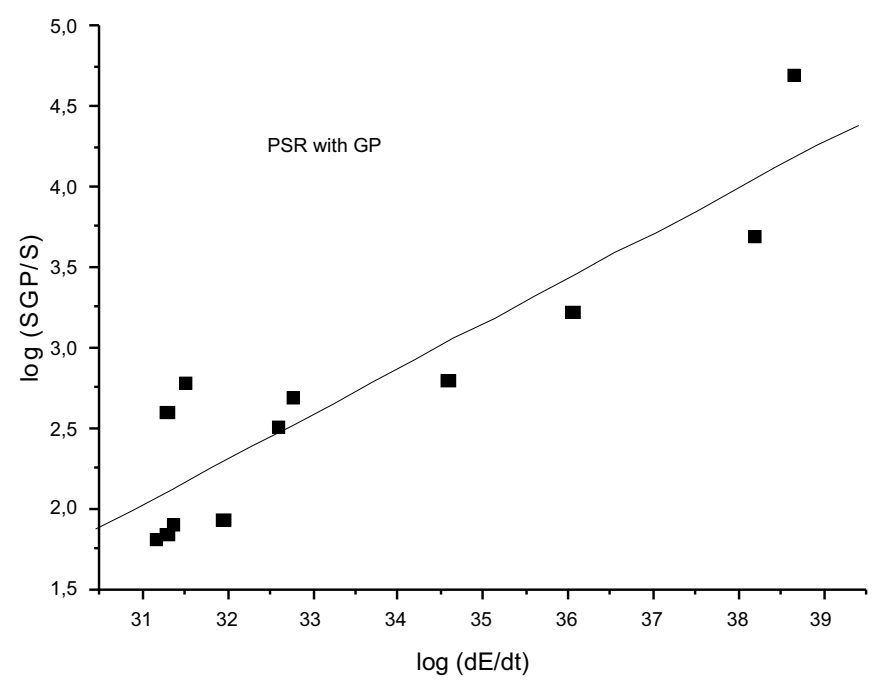

Figure 1: Distribution of integrated radio luminosities of pulsars considered.

2. Petrova [7] considered induced Compton scattering of radio emission of the secondary electrons. This process leads to the decreasing of angular sizes of the emitting beam and the noticeable increase in its intensity. This increasing is exponential and leads to the ratio of GP intensities comparing to the intensity of average pulses up to hundreds-thousands times. This mechanism can operate in pulsars with short periods and with high values of luminosities. Apart from the mentioned pulsars B0531+21 and B1937+21 Table 1 contains 3 additional objects (J0218+4232, J1823-3021A and J1959+2048) with short periods. As for their luminosities the corresponding distribution (see Fig. 1) is bimodal. We used here the formulas for the transformation of $R_{\text {lum }}$ values into integrated luminosities $L$ from [8]. Fig. 1 shows that pulsars with short periods are indeed more power emitters $(\langle\log L\rangle=$ 30.50) than long-periodic ones ( $\langle\log L\rangle=27.77)$. However, a number of pulsars can not be describe by this model, and we again must use other ideas.

3. Kontorovich [9] tried to use for this aim the vacuum gap near the neutron star surface as a specific resonator for waves and emissions inside it. The central region near the magnetic axis and two slots on the board between open and closed field lines represent waveguides providing an exit of observed radiation. Phases of GPs are determined by the localization of such waveguides. They can be in the center of an average pulse (B1112+50 [10]), at its leading (B1937+21 [5]) or trailing (B1937+21 [11]) edges.

4. We proposed to consider the drift waves at the periphery of the pulsar magnetosphere as the primary reason of GPs [12]. Along with longitudinal waves here transverse waves are excited due to the drift of plasma particles across the lines of force. These waves move slowly across the field lines and remain during a long time in the region of the cyclotron resonance. They store energy of resonance particles and then due to some non-linear processes emit this energy in the form of GPs. The estimates show that the induced scattering of waves on the plasma particles lead to energy storage during $10^{5} \mathrm{sec}$. This provide the ratio of flux densities of GP to this of the normal average pulse of $10^{5}$.

The dependence in Fig. 2 can be described by the following equation:

$$
\log \left(S_{g p} / S\right)=(0.28 \pm 0.04) \log d E / d t-6.71 \pm 1.37
$$

and the correlation coefficient $K=0.91$.

\section{Conclusions}

The analysis of some parameters of radio pulsars with GPs is given. The dependence of relative powers of GPs on the rotation energy losses is detected (Fig. 2). Some models of GPs generation are discussed. The most perspective models as we believe are models of Kontorovich and of Machabeli et al. The existence of vacuum gaps and electric fields near the surface in radio pulsars considered generally accepted today. On the other hand, the generation of drift waves at the periphery of pulsar magnetospheres is also inevitable. It is possible that there is no any unique model of GP generation and different models operate in radio pulsars.

\section{References}

1. R. N. Manchester, G. B. Hobbs, A. Teoh, and M. Hobbs, AJ, 129, 1993, 2005.

2. J. M. Cordes, N. D. R. Bhat, T. H. Hankins, M. A. McLaughlin, and J. Kern, ApJ, 612, 375, 2004. 


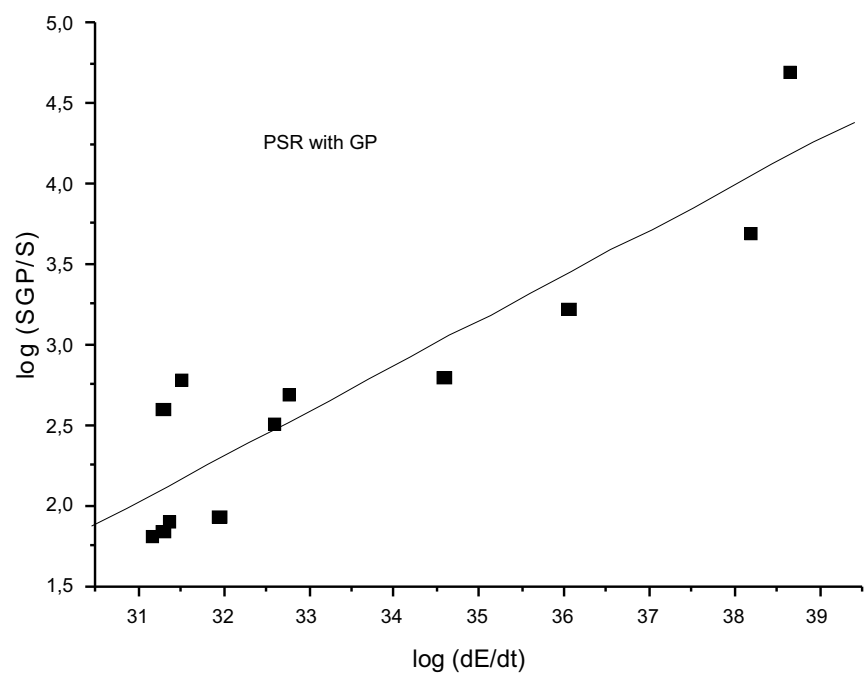

Figure 2: Dependence of relative power of GP on the rotation energy losses.

3. T. H. Hankins and J. A. Eilek, ApJ, 670, 693, 2007.

4. T. H. Hankins, J. Kern, J. C. Weatherall, and J. A. Eilek, Nature, 422, 141, 2003.

5. M. V. Popov and B. Stappers, $A \& A$, 470, 1003, 2007.

6. Y. N. Istomin, IAU Symposium, 218, 265, 2004.

7. S. A. Petrova, $A \xi B A, \mathbf{4 2 4}, 227,2004$.

8. I. F. Malov and O. I. Malov, Astron. Rep., 50, 483, 2006.

9. V. M. Kontorovich, Journal of Physical Science and Application, 5, 48, 2015.

10. A. A. Ershov and A. D. Kuzmin, Astronomy Letters, 29, 91, 2003.

11. H. S. Knight, M. Bailes, R. N. Manchester, and S. M. Ord, ApJ, 625, 951, 2005.

12. G. Machabeli, N. Chkheidze, and I. Malov, ApछSSS, 364, 40, 2019. 


\section{The epsilon Aurigae Observation. Infrared Photometry and Speckle-polarimetry}

I. Maslov ${ }^{1}$, V. Shenavrin ${ }^{2}$, B. Safonov ${ }^{2}$

imaslov@iki.rssi.ru

${ }^{1}$ Space Research Institute of the Russian Academy of Sciences (IKI), 84/32 Profsoyuznaya Str, Moscow, Russia, 117997

${ }^{2}$ Sternberg Astronomical Institute, Moscow State University Universitetsky pr., 13, Moscow, Russia, 119234

DOI: 10.51194 /VAK2021.2022.1.1.058

A long cycle of photometric observations in the infrared bands of JHKLM of the eclipsed star $\epsilon$ Aur on the 1.25-m telescope of the Crimean Astronomical Station GAISH was carried out. We found a change in the infrared color of the star with the phase of its period (Fig. 1). We explain this by the scattering of supergiant light on the dust disk surrounding the second component of the system. Speckle-polarimetric observations of $\epsilon$ Aur in the band centered at $625 \mathrm{~nm}$ with a half-width of $50 \mathrm{~nm}$ were carried out on the 2.5-m telescope of the Caucasian Mountain Observatory GAISH on November 28, 2020. The resulting polarization value: $2.0 \pm 0.2 \%$ with the positional angle of $145 \pm 5^{\circ}$. We have not detected a polarized shell.
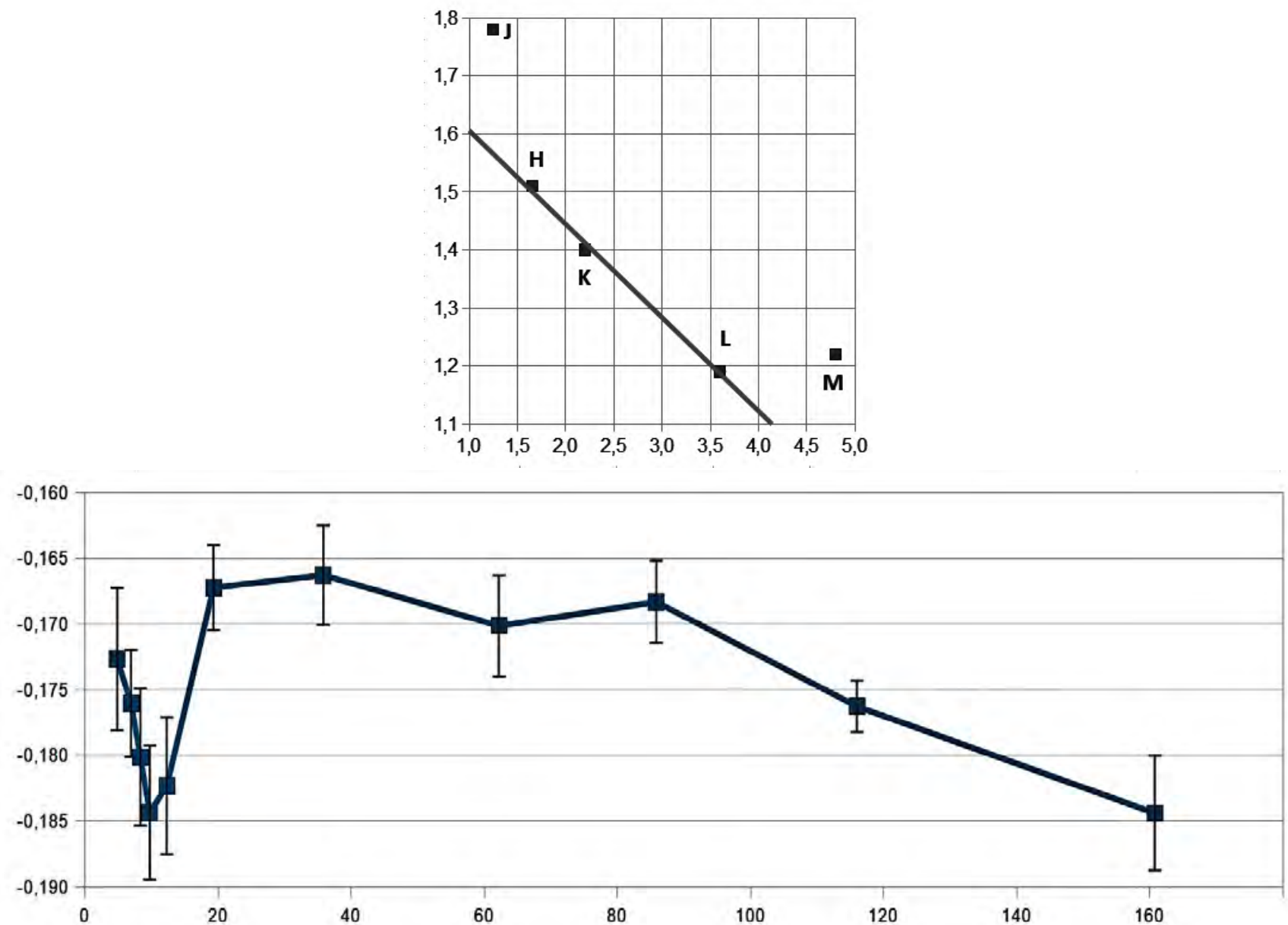

Figure 1: Top — the spectral dependence of the stellar magnitude on the wavelength ( $\mu \mathrm{m})$ for $\epsilon$ Aur and its linear approximation. Bottom — the slope of the spectrum for the HKL bands as a function of the scattering angle (degrees) on the dust disk. $0^{\circ}$ — forward scattering, $180^{\circ}$ — backward scattering. 


\title{
Wray 15-906 low mass Luminous Blue Variable on a pre-supernova stage
}

\author{
O. Maryeva ${ }^{1,2}$ V. Gvaramadze, A. Kniazev ${ }^{2,3,4}$, L. Berdnikov $^{2}$ \\ olga.maryeva@asu.cas.cz \\ ${ }^{1}$ Astronomical Institute, Czech Academy of Sciences, Ondřejov, Czech Republic \\ ${ }^{2}$ Sternberg Astronomical Institute of Moscow State University, Moscow, Russia \\ ${ }^{3}$ South African Astronomical Observatory, Cape Town, South Africa \\ ${ }^{4}$ Southern African Large Telescope Foundation, Cape Town, South Africa
}

\begin{abstract}
Evolutionary link between Red Supergiants and Luminous Blue variables is interesting, but still poorly understood. We present the results of study of the Galactic candidate luminous blue variable Wray 15-906, revealed via detection of its infrared circumstellar shell (of $\approx 2 \mathrm{pc}$ in diameter) with the Wide-field Infrared Survey Explorer (WISE) and the Herschel Space Observatory. Using the stellar atmosphere code CMFGEN and the Gaia parallax, we found that Wray 15-906 is a relatively low-luminosity, $\log \left(L / L_{\odot}\right) \approx 5.4$, star of temperature of $25 \pm 2 \mathrm{kK}$. In the framework of single star evolution, the obtained results suggest that Wray $15-906$ is a post-red supergiant star with initial mass of $\approx 25 M_{\odot}$ and that before exploding as a supernova it could transform for a short time into a WN11h star. The presence of shell with mass $2.9 \pm 0.5 M_{\odot}$ indicates that Wray 15-906 has suffered substantial mass loss in the recent past.
\end{abstract}

Keywords: stars: massive - stars: variables: S Doradus - stars: emission-line, Be - stars: evolution - stars: individual: Wray 15-906

DOI: $10.51194 /$ VAK2021.2022.1.1.059

Luminous Blue Variable (LBV) phase is a relatively short evolutionary stage in the life of massive star [1], related to transformation of stellar structure after exhaustion of hydrogen in its core. LBV stars are extremely rare and there are probably no more than a few dozen such objects in whole Galaxy. Every new star classified as bona fide LBV or just candidate LBV is important for theory of stellar evolution.

LBVs are divided into two subclasses: low luminosity LBVs and classical LBVs [2]. The former originate from stars with $M_{*} \approx 25-40 M_{\odot}$ reaching the LBV stage after being red supergiants (RSG) [2, 3]. Based on Geneva evolutionary tracks, [4] demonstrated that low luminosity LBVs could be progenitors of core-collapse supernovae (SN Type IIL/b). Thus, the low luminosity LBVs are probably a final stage of stellar evolution, rather than a transitory evolutionary phase between RSGs and Wolf-Rayet (WR) stars as was suggested earlier [3]. Classical LBVs originate from more massive stars $\left(M_{*} \approx 40-60 M_{\odot}\right)$ moving off the Main Sequence and evolving towards the WR stage via the blue supergiant and LBV phases $[3,5]$.

This note is devoted to evolutionary status of the new Galactic candidate LBV (cLBV) Wray $15-906^{1}$. The star, also known as ALS 2533 or Hen 3-729, attracted our attention after we discovered a circular shell around it using WISE data [6]. In the WISE $22 \mu \mathrm{m}$, image the nebula appears as a circular limb-brightened shell with diameter $\approx 2 \mathrm{pc}$, which is typical of circumstellar nebulae around LBVs (e.g. [7]). The star shows spectral line variability (Figure 1) and low amplitude photometric variability [8].

The basic parameters of Wray 15-906 were determined using CMFGEN atmospheric modeling code [9]. For fitting we used the medium resolution spectrum of Wray 15-906 obtained with SALT telescope on 2016 May 5. The derived parameters of Wray 15-906 allow to find position of the star in the Hertzsprung-Russell (H-R) diagram (Figure 3).

If Wray 15-906 was born single, then its initial mass could be estimated to be $25 M_{\odot}$, which is close to the minimum mass for single (non-rotating) stars to become a WR star (e.g. [10]). The surface He abundance (65 per cent by mass) derived for Wray 15-906 is typical of LBVs (e.g. [11]) and is intermediate between that of O and WR stars. This suggests that Wray 15-906 has already gone through the red supergiant phase and is currently near the end of the He-burning stage, and implies the evolutionary age of this star of $\approx 7-8$ Myr. If Wray 15-906 was born with a low rotational velocity then it would need only several $1000 \mathrm{yr}$ to finish its life with a supernova explosion [10]. The possibility that Wray 15-906 may soon explode is indirectly supported by the curious finding by [4] and [12] that single stars with $M_{\text {init }}=20-25 M_{\odot}$ have LBV-like spectra before exploding as a supernova. Although unexpected, this finding conforms with previous claims (e.g. [13]) that LBVs could be the immediate precursors of supernovae.

More details may be found in our work [8].

Acknowledgements: We dedicate this publication to the memory of our dear colleague Vasilii Gvaramadze, who passed away on September 2nd, 2021, shortly after the end of the conference. The search for evolved stellar objects by means of detection of their circumstellar nebulae in modern sky surveys has been the topic that Vasilii successfully developed for last ten years, and he assembled and inspired our diverse team with his intellectual and organizational talents. This work has not been possible without his efforts.

${ }^{1}$ The object has coordinates $\alpha=11^{\mathrm{h}} 54^{\mathrm{m}} 43.54^{\mathrm{s}}, \delta=-63^{\circ} 13^{\prime} 31^{\prime \prime} 1$ at J2000 epoch. 

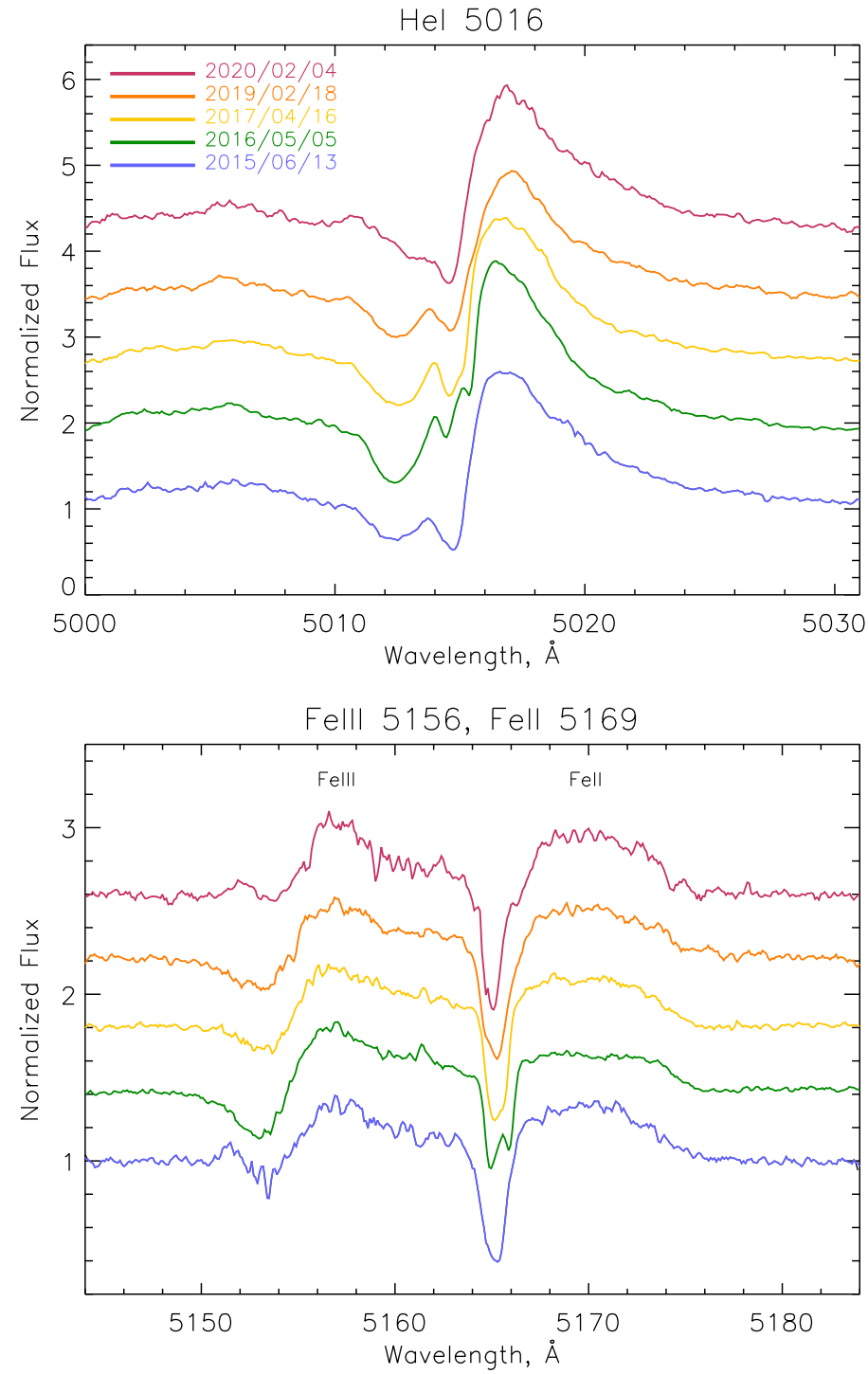

Figure 1: Variability of the absorption components in the He I $\lambda 5016$ and Fe II $\lambda 5169$ lines in the medium-resolution spectra taken in 2015-2020 on SALT telescope.

This work is based on observations obtained with the Southern African Large Telescope (SALT), programmes 2013-1-RSA OTH-014, 2013-2-RSA OTH-003, 2015-1-SCI-017, 2016-1-SCI-012, 2017-1-SCI-006 and 2018-1-MLT008, and supported by the Russian Foundation for Basic Research grant 19-02-00779.

\section{References}

1. R. M. Humphreys and K. Davidson, PASP, 106, 1025, 1994.

2. R. M. Humphreys, K. Weis, K. Davidson, and M. S. Gordon, ApJ, 825, 64, 2016.

3. G. Meynet and A. Maeder, $A \mathscr{E} A, \mathbf{4 2 9}, 581,2005$.

4. J. H. Groh, G. Meynet, and S. Ekström, $A \& \zeta A$, 550, L7, 2013.

5. G. Meynet, C. Georgy, R. Hirschi, A. Maeder, P. Massey, N. Przybilla, and M.-F. Nieva, Bulletin de la Societe Royale des Sciences de Liege, 80, 266, 2011.

6. A. Kniazev and V. Gvaramadze, in SALT Science Conference 2015 (SSC2015), 49 (2015).

7. K. Weis, Reviews in Modern Astronomy, 14, 261, 2001.

8. O. V. Maryeva, V. V. Gvaramadze, A. Y. Kniazev, and L. N. Berdnikov, MNRAS, 498, 5093, 2020.

9. D. J. Hillier and D. L. Miller, ApJ, 496, 407, 1998.

10. C. Georgy, S. Ekström, G. Meynet, P. Massey, E. M. Levesque, R. Hirschi, P. Eggenberger, and A. Maeder, A $\& A$, 542, A29, 2012. 


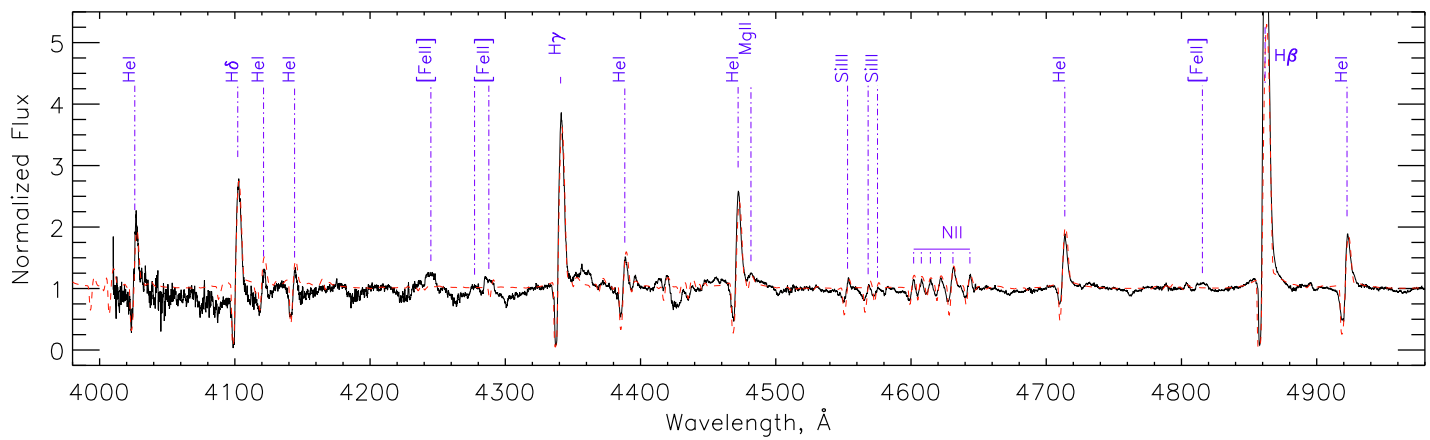

Figure 2: Normalized medium-resolution spectrum of Wray 15-906 taken on 2016 May 5 (black solid line), compared with the best-fitting CMFGEN model (red dashed line).

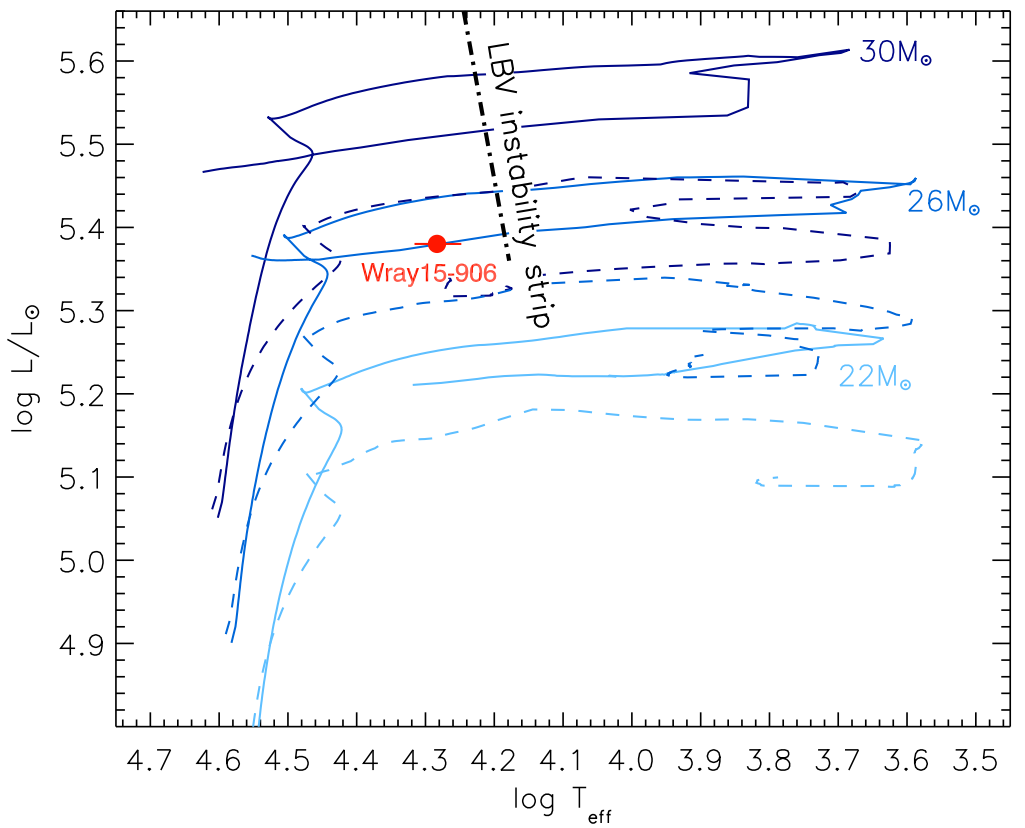

Figure 3: Position of Wray 15-906 in the Hertzsprung-Russell diagram (marked with the red circle with error bars). The horizontal lines represent the evolutionary tracks, taken from the Geneva library [14].

11. P. A. Crowther, in A. Nota and H. Lamers, eds., Luminous Blue Variables: Massive Stars in Transition, Astronomical Society of the Pacific Conference Series, volume 120, 51 (1997).

12. J. H. Groh, G. Meynet, C. Georgy, and S. Ekström, A\&AA, 558, A131, 2013.

13. R. Kotak and J. S. Vink, A\& A, 460, L5, 2006.

14. S. Ekström, C. Georgy, P. Eggenberger, G. Meynet, et al., A\&A, 537, A146, 2012. 


\section{Microscopic simulation of chemical processes on interstellar dust grains}

M.G. Medvedev, A.B. Ostrovskii, G.S. Fedoseev, A.I. Vasyunin

mikhail.medvedev@urfu.ru

Research Laboratory for Astrochemistry, Ural Federal University, Kuibysheva St. 48, 620026 Ekaterinburg, Russia

DOI: 10.51194 /VAK2021.2022.1.1.060

We develop a continuous time random walk (CTRW) Monte Carlo model for simulation of chemical processes occurring on the surface of sub-micrometer dust particles in star-forming regions. In our model, a grain of dust is a three-dimensional periodic grid structure based on a hexagonal close packing of equal spheres. To simplify the calculations, we represent each layer of the ice mantle as flat hexagonal layer with periodic boundary conditions.

Currently, the model includes four processes: adsorption, desorption, thermal diffusion, reaction between to neighbouring species.

Simulations were performed with a simple network that consist of 10 reactions and 12 species. In the network three reactions possess an activation barrier [1]. Fractional abundances of species vs. time were calculated. The modelled ice is predominantly composed of water, methanol and carbon dioxide (see Fig. 1).

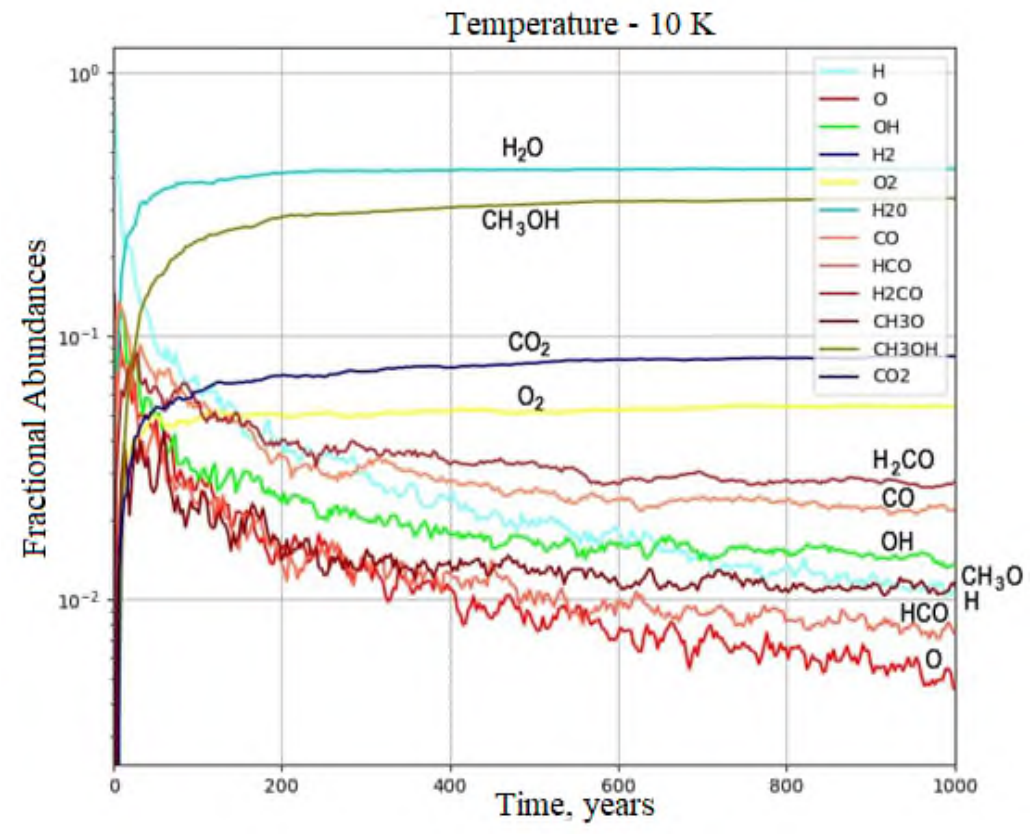

Figure 1: Fractional abundances of species vs. time

To sum up, a computational code in the Fortran language has been written. The code simulates chemical processes on the surface of a dust particle; the OpenMP library was used to implement parallel computations. The results of numerical modeling, namely the formation of $\mathrm{H}_{2} \mathrm{O}, \mathrm{CH}_{3} \mathrm{OH}, \mathrm{CO}_{2}$, are in qualitative agreement with the results of experimental and theoretical studies available from the literature [2].

The work is supported by the Ministry of Science and Higher Education of the Russian Federation via the State Assignment Project FEUZ-2020-0038.

\section{References}

1. H. M. Cuppen, E. F. van Dishoeck, E. Herbst, and A. G. G. M. Tielens, A\& A, 508, 275, 2009.

2. H. Linnartz, S. Ioppolo, and G. Fedoseev, International Reviews in Physical Chemistry, 34, $205,2015$. 


\section{Supernovae search in the Zwicky Transient Facility Data Releases}

A. Novinskaya ${ }^{1,2}$, M.V. Pruzhinskaya ${ }^{2}$, K.L. Malanchev ${ }^{2,3}$, M.V. Kornilov ${ }^{2,4}$, F. Mondon ${ }^{5}$, A.A. Volnova ${ }^{5}$, P.D. Aleo ${ }^{3}$, E.E.O. Ishida ${ }^{5}$, V.S. Korolev ${ }^{7,8}$, S. Sreejith ${ }^{5}$ alexandranovinskaya@gmail.com; pruzhinskaya@gmail.ru

${ }^{1}$ Lomonosov Moscow State University, Faculty of Physics, Leninskie Gory, 1-2, Moscow, 119991, Russia

${ }^{2}$ Lomonosov Moscow State University, Sternberg Astronomical Institute, Universitetsky pr. 13, Moscow, 119234, Russia

${ }^{3}$ Department of Astronomy, University of Illinois at Urbana-Champaign, 1002 West Green Street, Urbana, IL 61801, USA

${ }^{4}$ National Research University Higher School of Economics, 21/4 Staraya Basmannaya Ulitsa, Moscow, 105066, Russia

${ }^{5}$ Université Clermont Auvergne, CNRS/IN2P3, LPC, F-63000 Clermont-Ferrand, France

${ }^{6}$ Space Research Institute of the Russian Academy of Sciences (IKI), 84/32 Profsoyuznaya Street, Moscow, 117997, Russia

${ }^{7}$ Central Aerohydrodynamic Institute, 1 Zhukovsky st, Zhukovsky, Moscow Region, 140180, Russia

${ }^{8}$ Moscow Institute of Physics and Technology, 9 Institutskiy per., Dolgoprudny, Moscow Region, 141701, Russia

The discovery of new astrophysical phenomena, as well as the study of already known transients, are among the most expected results of the next generation of large-scale astronomical sky surveys. Prompt data processing using modern machine learning techniques allows us to quickly classify objects and provides information for their further study. For the last three years the SNAD team is working on developing a pipeline where human expertise and modern machine learning algorithms complement each other in the task of identifying unusual astronomical objects. In this paper, we applied the active anomaly detection algorithm to the Zwicky Transient Facility Data Releases. As a result, we discovered 37 supernova candidates, 21 of which are discovered for the first time.

Keywords: supernovae, transients, machine learning, anomaly detection

DOI: 10.51194 /VAK2021.2022.1.1.061

\section{Introduction}

The discovery of previously unrecorded astrophysical phenomena, as well as the study of already known transients, are among the most anticipated results of the next generation of large-scale astronomical surveys such as the Vera C. Rubin Observatory Legacy Survey of Space and Time (LSST; [1]).

In this work, we study the ability of the active anomaly detection algorithm (AAD; $[2,3])$ to learn from the human expert basing on data from the Zwicky Transient Facility (ZTF) Data Releases (DR). As a class of interest we use supernova stars.

The Zwicky Transient Facility ${ }^{1}[4]$ is a wide-field sky survey aimed at discovering and observing supernovae, kilonovae, optical afterglows of gamma-ray bursts, near-Earth objects, rare and rapidly evolving transients, as well as various classes of variable stars. To search for supernova candidates, we analyse the photometric data from the first 9.4 months of the ZTF survey, between 2018 March 17 and December 31 (58194 $\leq$ MJD $\leq 58483$ ). This period includes data from ZTF private survey, thus having a better cadence than the rest of DR $3^{2}$. However, for the further analysis of discovered supernovae, we use more complete light curves from the 4th data release.

Below we briefly describe the main steps of the work and the obtained results.

\section{Search for supernovae}

\subsection{Fields selection and data preparation}

ZTF DR3 is divided into 1020 observing fields. Since the probability of detecting a supernova is higher outside the Milky Way plane and in the regions with lower interstellar extinction, we mainly investigate the fields with high galactic latitude (see Fig. 1).

Before applying a machine learning algorithm, each ZTF object was described by 42 features extracted from the light curves in $z r$-passband. The full description of these features is given in [5].

\subsection{Active anomaly detection algorithm}

To search for supernovae in ZTF DR3, we use the active anomaly detection algorithm. Its efficiency on the example of the Photometric LSST Astronomical Time-series Classification Challenge (PLAsTiCC) and real light curves from the Open Supernova Catalog is proven by [6]. The algorithm looks for the objects (outliers) that are unique for the sample under study in a given parameter space. Having selected the object with the highest anomaly score, the algorithm presents it to a human expert for classification. The expert analyzes the object light curves, fits-images, and collects all available information from other databases and catalogues. In this work, the main instrument to perform such expertise is the SNAD ZTF web-viewer ${ }^{3}$. Based on the assembled knowledge

\footnotetext{
${ }^{1}$ http://www.ztf.caltech.edu

${ }^{2}$ https://www.ztf.caltech.edu/page/dr3

${ }^{3}$ https://ztf.snad.space/
} 


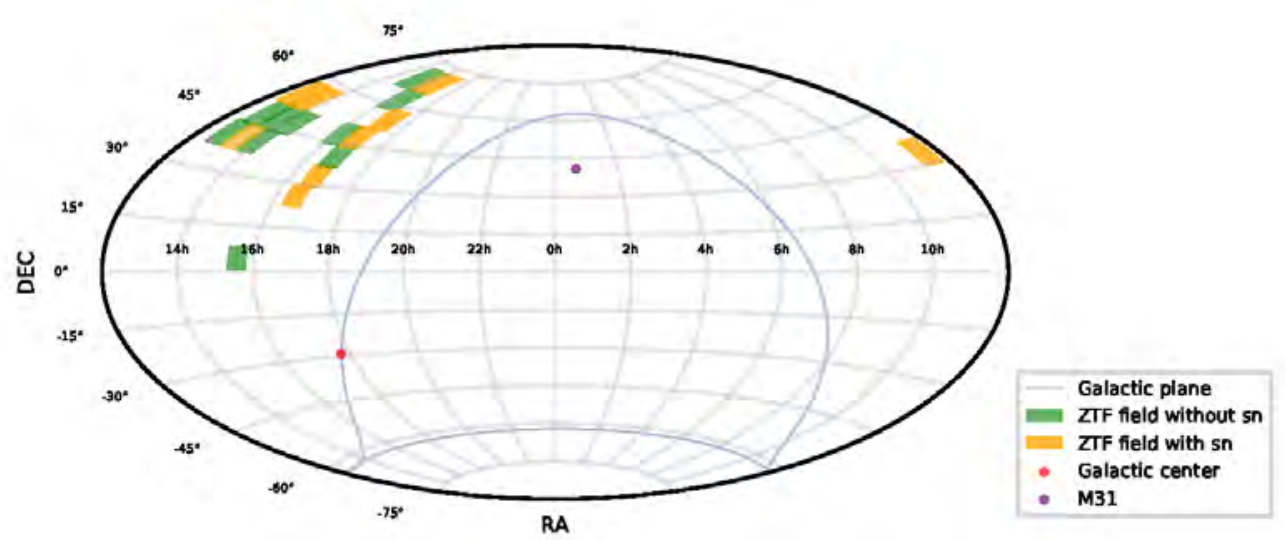

Figure 1: The location of the studied ZTF DR3 fields in the sky. Those fields, in which no supernovae are found, are marked in green, fields that contain supernova candidates are marked in yellow. Red circle denotes the center of the Galaxy, the purple circle is the M31 galaxy, and the blue curve is the galactic plane.

the expert can judge whether the object is interesting for a further study or not, and give a decision back to the algorithm. The algorithm processes the expert's answer and learns to search for the objects of expert interest. In our case, we train the AAD for supernova search.

Applying the algorithm to 19 ZTF fields, we visually inspected 570 objects $(19 \times 30$ objects in each field). Among them, we found 37 supernova candidates, 21 of which are discovered for the first time and 16 are previously mentioned in other catalogs either as supernovae with known types or as supernova candidates.

All supernova candidates found for the first time were sent to the Transient Name Server ${ }^{4}$ (TNS) and got an official TNS identifier as well as an internal SNAD name.

\section{Models and fitting}

To fit the supernova light curves we use the Nugent's templates ${ }^{5}$ of different supernova types: Ia, Ib/c, IIP, IIL, IIn, and SALT2 light-curve model [7]. As a result of fitting and visual classification of 21 objects, it is found that 18 candidates are likely supernovae of different types (6 SNe Ia, 2 SNe IIn, 10 - other types), 2 objects could be quasars, and one more object is a variable star of unknown nature of variability. An example of supernova light-curve fit with SALT2 model is shown in Fig. 2.

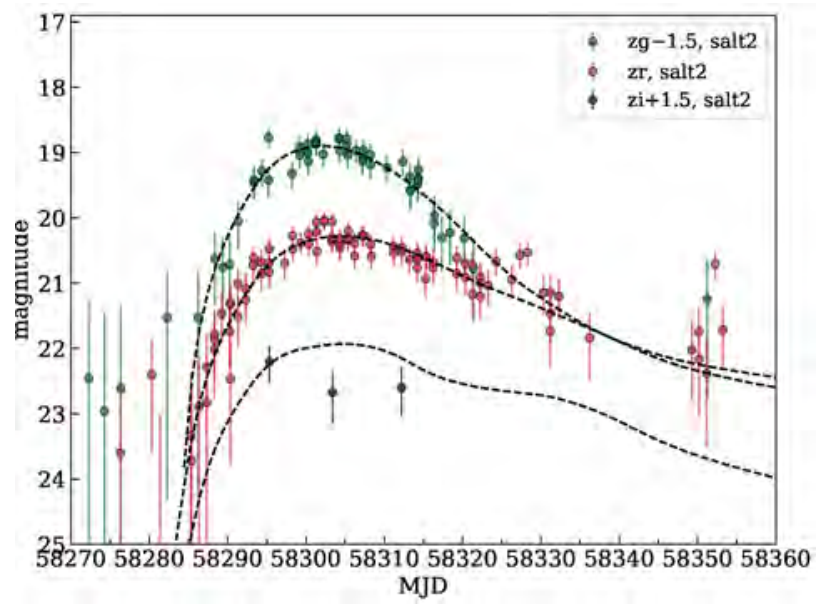

Figure 2: Results of light-curve fit of SNAD111 (AT2018lwr) by SALT2 model. Observational data correspond to OIDs: $796101100001026(z g), 796201100002136(z r), 796301100002192(z i)$.

\footnotetext{
${ }^{4}$ https://www.wis-tns.org/

${ }^{5}$ https://c3.lbl.gov/nugent/nugent templates.html
${ }^{5}$ ter.
} 


\section{Results}

We applied the active anomaly detection algorithm to 19 ZTF fields from DR3 to search for supernovae. As a result, 16 known supernovae were found and 21 supernova candidates were discovered for the first time and published in TNS. The light curves of discovered supernova candidates were also fitted with several models to determine their type. According to the fit results, out of 21 objects, only 18 belong to supernovae, 2 to quasars and 1 object is a variable star.

During the inspect of outliers, we also assigned labels to the database objects, that will be further used for other tasks.

Some of discovered in DR3 supernova are missing in ZTF alerts that stressed the importance of anomaly detection algorithms for analysing large amounts of data.

\section{Acknowledgements}

The reported study was funded by RFBR and CNRS according to the research project No21-52-15024. The authors acknowledge the support by the Interdisciplinary Scientific and Educational School of Moscow University "Fundamental and Applied Space Research".

\section{References}

1. LSST Science Collaboration, P. A. Abell, J. Allison, S. F. Anderson, et al., arXiv e-prints, arXiv:0912.0201, 2009.

2. S. Das, W.-K. Wong, A. Fern, T. G. Dietterich, and M. Amran Siddiqui, arXiv e-prints, arXiv:1708.09441, 2017.

3. S. Das, M. Rakibul Islam, N. Kannappan Jayakodi, and J. Rao Doppa, arXiv e-prints, arXiv:1809.06477, 2018.

4. F. J. Masci, R. R. Laher, B. Rusholme, D. L. Shupe, et al., PASP, 131, 018003, 2019.

5. K. L. Malanchev, M. V. Pruzhinskaya, V. S. Korolev, P. D. Aleo, et al., MNRAS, 502, 5147, 2021.

6. E. E. O. Ishida, M. V. Kornilov, K. L. Malanchev, M. V. Pruzhinskaya, et al., A\&gA, 650, A195, 2021.

7. J. Guy, M. Sullivan, A. Conley, N. Regnault, et al., Aछ̈A, 523, A7, 2010. 


\title{
Astrochemical study of a dense molecular clump of WB 673 filament
}

\author{
O.L. Ryabukhina, M.S. Kirsanova, D.S. Wiebe \\ ryabukhina@ipfran.ru
}

Institute of Astronomy of the Russian Academy of Sciences, Moscow 119017,Russia

We present results of modelling of dark interstellar cloud WB 673 with the PRESTA astrochemical model. We searched for the best agreement between the simulated and observed molecular abundances in a wide range of model physical parameters, and found it satisfactory at the model time $t \approx 5 \cdot 10^{5}$ years. The model with observed temperature and gas density distribution and a cosmic ray ionization rate of $\zeta=1.3 \cdot 10^{-17} \mathrm{~s}^{-1}$ gives a minimum discrepancy between the simulated and model abundances.

Keywords: stars: formation - ISM: clouds - ISM: molecules - ISM

DOI: $10.51194 /$ VAK2021.2022.1.1.062

\section{Introduction}

Physical conditions in interstellar clouds and time-dependent chemical evolution define distribution of molecular abundances in them and their observed properties. Therefore, astrochemical modeling might help estimating the 'chemical' age of a cloud and its evolutionary stage. In general, cold and low-density interstellar gas does not support an extensive chemistry that produces complex molecules. Chemical complexity must be stimulated, e. g. by ultraviolet emission, cosmic rays, catalytic reactions on dust grains and gas dynamics [1]. The aim of the study is to check whether a young stellar cluster, which is embedded into the dense clump WB673, affects its observed chemical abundances. The clump occupies a central position and is the most massive among the others, in extended interstellar filament WB673. Earlier we obtained column densities of $\mathrm{CO}, \mathrm{CS}, \mathrm{HCN}, \mathrm{HNC}$ and $\mathrm{N}_{2} \mathrm{H}^{+}$ molecules in the filament [2], and we use these data in the present study. Analysis of ammonia emission was also performed in order to obtain gas temperature and density in the filament (Ryabukhina et. al., in preparation). In the present study we compare the observed column densities with simulated values using the time-dependent multi-point astrochemical model PRESTA [3].

\section{Time-dependent astrochemical modelling}

The model has 1D geometry, therefore we assume that the clumps are spherically symmetric. We average the molecular column densities and physical parameters over the positional angle relative to the peak of the hydrogen column density $N_{\mathrm{H}_{2}}$. The radial profiles of the gas parameters and column densities are shown in Fig. 1. We describe the radial profile of the molecular hydrogen by a power-law function with the flat inner part:

$$
n_{\mathrm{H}_{2}}(r)=\frac{n_{0}}{\left(1-\left(r / r_{0}\right)^{2}\right)^{p / 2}},
$$

where $n_{0}$ is the density at the center of the clump, $r_{0}$ is the radius of the flat inner region, $p$ is the power-law exponent. We found $p=0.4$ from the analysis of the ammonia emission (Ryabukhina et. al., in preparation). A similar function was used to create the profile of the gas kinetic temperature. The power-law fits are shown in the top row of Fig. 1 by violet curves.

We calculated the astrochemical model varying the physical parameters in a certain range. First, we increased and decreased the $T_{\text {kin }}$ by $10 \mathrm{~K}$, bearing in mind that $T_{\text {kin }}$ in the inner part of the clump might be different from the observed value, because the ammonia lines are optically thick. The gas temperature was assumed to be equal to the dust temperature. The value of gas density $n_{\mathrm{H}_{2}}$ and the cosmic ray ionization rate were varied by an order of magnitude. The model includes 582 components and 4524 gas-phase reactions. The chemical evolution of the clump was modelled over $2 \times 10^{7}$ years.

\section{Results}

Time-dependent column densities for the model with the observed distributions of $n_{\mathrm{H}_{2}}$ and $T_{\text {kin }}$ are shown in Fig. 1. It is seen that the simulated column densities of N-bearing molecules become higher than the observed ones at $t \geq 10^{5}$ years. At the same time, the abundances of C-bearing species drop below the observed values. Freeze-out of the C-bearing molecules on the dust grains explains such behaviour, because CO molecules mostly destroy N-bearing species in the gas phase at the early evolutionary times [4].

We introduce a $\sigma_{\text {fit }}$ value to quantitatively describe the difference between the simulated $\left(N_{\text {sim }}\right)$ and observed $\left(N_{\text {obs }}\right)$ column densities for each molecule:

$$
\sigma_{\mathrm{fit}}=\frac{\sum_{r}\left(N_{\mathrm{obs}}-N_{\mathrm{sim}}\right)^{2}}{\sum_{r} N_{\mathrm{obs}}^{2}},
$$



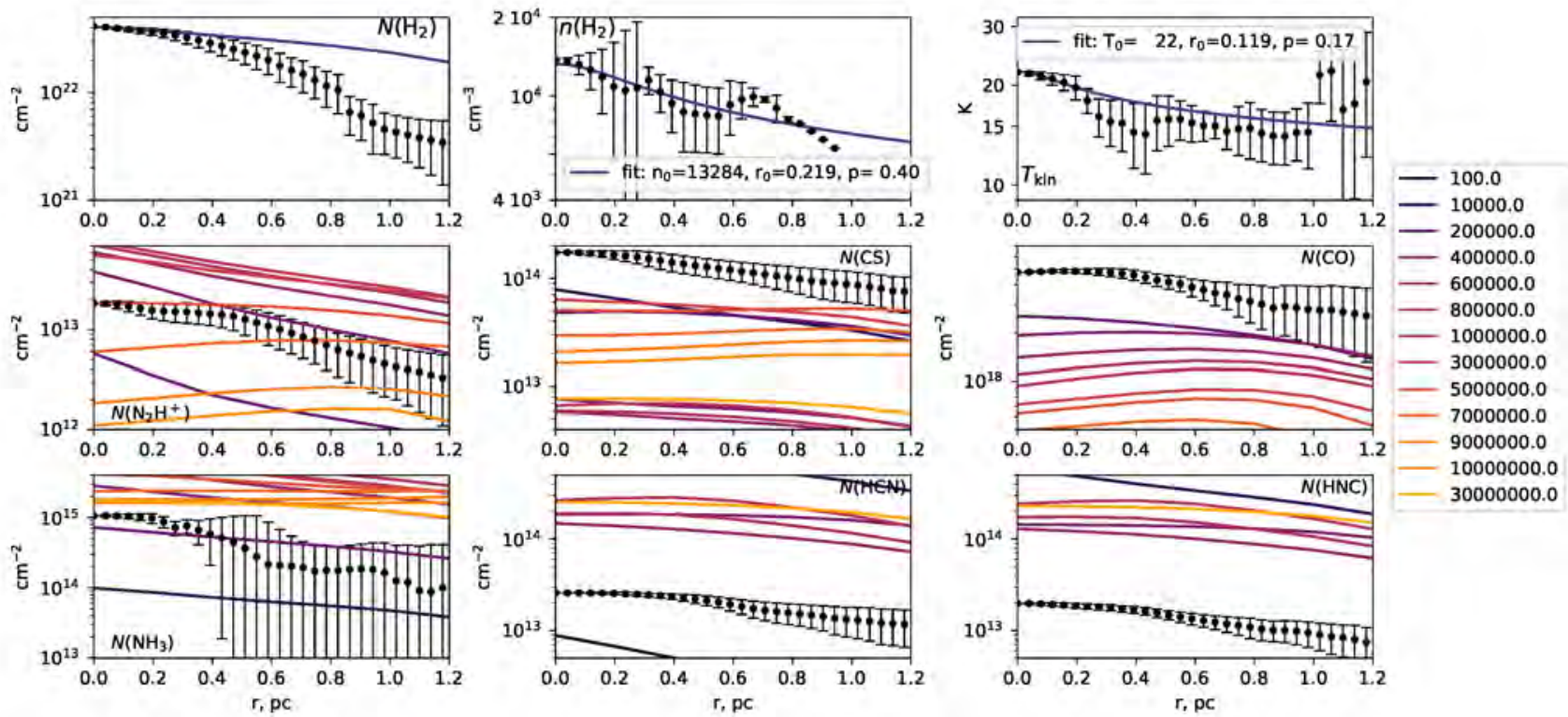

Figure 1: Radial profiles of physical conditions in the clump (top row) and column densities of molecules (middle and bottom rows) in the clump WB 673 . The observed values are shown by black dots with error bars. The colour lines shows results of the PRESTA model for different time moments, which are written below the plots.

where $r$ is the impact parameter of the radial profile. The time dependence of the total $\sigma_{\text {fit }}$, summed over the individual values for $\mathrm{CS}, \mathrm{CO}, \mathrm{NH} 3, \mathrm{~N} 2 \mathrm{H}+, \mathrm{HCN}$ and $\mathrm{HNC}$, is shown in Fig. 2. Three models (the first one with the observed physical parameters, the second one with the decreased $n\left(\mathrm{H}_{2}\right)$, the third one with the decreased $\left.T_{\text {kin }}\right)$ show the minimum total $\sigma_{\text {fit }}$ values. We reject the model with the decreased $n_{\mathrm{H}_{2}}$ because it seems to be unrealistic in the star-forming region with embedded star cluster [5]. The model with the decreased $T_{\text {kin }}$ shows almost the same values of $\sigma_{\text {fit }}$ as the model with the observed gas parameters. The model time for the minimum total $\sigma_{\text {fit }}$ value is $5 \times 10^{5}$ years in the model with the observed parameters and with the decreased $T_{\text {kin. }}$. The model with the decreased $\zeta$ value also gives minimum total $\sigma_{\text {fit }}$, however that $\zeta$ value does not correspond to the observed limits [6].

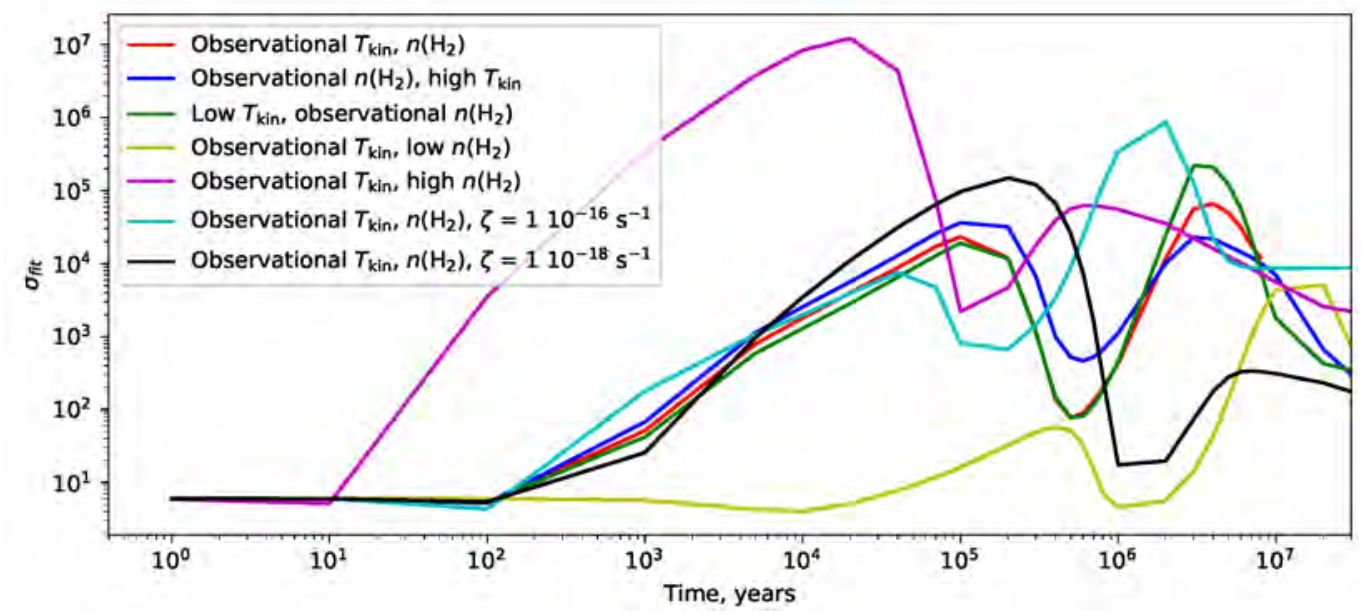

Figure 2: The total $\sigma_{\text {fit }}$ as a function of time for the considered models. The red line shows $\sigma_{\text {fit }}$ for the observed $T_{\text {kin }}, n_{\mathrm{H}_{2}}$ and the standard $\zeta=1.3 \times 10^{-17} \mathrm{~s}^{-1}$. The blue and green lines show the models with increased and decreased $T_{\text {kin }}$, respectively. The yellow and purple lines show the models with the increased and decreased $n_{\mathrm{H}_{2}}$, respectively. The turquoise and black lines show the models with the observed $n\left(\mathrm{H}_{2}\right)$ and $T_{\text {kin }}$, but with increased and decreased $\zeta$. 


\section{Conclusion}

Astrochemical modelling of WB 673 shows that the observed molecular column densities corresponds to the cold and dense physical conditions. We estimate the chemical 'age' of the clump $t \approx 5 \cdot 10^{5}$ years and conclude that the heating of the gas by the embedded young star clusters is not needed to fit the observed column densities reasonably well.

Acknowledgements The study was supported by the RFBR foundation, project number 20-32-90102.

\section{References}

1. D. A. Williams and S. Viti, Observational Molecular Astronomy (2013).

2. O. L. Ryabukhina and M. S. Kirsanova, Astronomy Reports, 64, 394, 2020.

3. O. V. Kochina, D. S. Wiebe, S. V. Kalenskii, and A. I. Vasyunin, Astronomy Reports, 57, 818, 2013.

4. E. A. Bergin and W. D. Langer, ApJ, 486, 316, 1997.

5. J. Jijina, P. C. Myers, and F. C. Adams, ApJS, 125, 161, 1999.

6. F. F. S. van der Tak and E. F. van Dishoeck, $A \mathscr{E} A$, 358, L79, 2000. 


\title{
Flares and coronal mass ejections for stars of different classes
}

\author{
A. Sadovski, A. Struminsky \\ a.sadovski@cosmos.ru \\ Space Research Institute, Moscow, Russia
}

\begin{abstract}
According to observations the number of flares on different stars almost do not change from dwarf to hot stars. The number of stellar coronal mass ejections (CME's) and their properties are estimated assuming the same statistical relations as obtained for the Sun. We made the estimates of a magnetic field strength in tubes in photospheres of O-M stars and used simple formulas with a clear physical meaning. Such approach gives results for the Sun that differ by no more than a factor of 2-3 from the observed ones. The main motivation for such approach is as follows: our knowledge of the stars' medium is at approximately the same level as our knowledge of the Sun in the 1950s. Moreover, such simple models allow a complete picture of the processes in the system to be obtained without knowing any details of the stellar activity. Basing on the obtained values and parameters of different objects we made estimates of flare energies and estimates of CME's masses and compare them with flares energies, stars luminosities, and temperatures.
\end{abstract}

Keywords: flares and coronal mass ejections, stars

DOI: 10.51194 /VAK2021.2022.1.1.063

\section{Introduction}

In last years many observations for flares across $\mathrm{H}-\mathrm{R}$ diagram were made. For example, observations by the Kepler space telescope [1] showed that the relative number of flare stars decrease from about $10 \%$ for $\mathrm{K}-\mathrm{M}$ stars to $2.5 \%$ for A-F stars. It occurs there does not seem to be a drop in stellar activity along the main sequence of $\mathrm{H}-\mathrm{R}$ diagram. Also the estimates on coronal mass ejections (CMEs) were reported (see [2] and reference therein).

The better statistic of observations as for the flares as for the CMEs was obtained for M and K stars. Mainly it is because that the rate of energy release during the flare is of the same order as the normal release of the star in the quiescent condition and CMEs are also cataloged for the same stars classes [3]. Flare energies and CME masses are strongly correlated with stellar luminosity and radius [1,2]. The activity of O-B type stars might not be excluded, but the flare power should be small in comparison with stellar luminosity.

A number of excellent reviews of stellar magnetic field across H-R diagram $[4,5,6]$ show the average surface magnetic field pressure in most cases roughly equal to the thermal pressure of surrounding gas. The equipartition magnetic field strength is the minimum possible value for the field strength dynamic flare loops. So, we used these hypothesis for our estimations.

Also the solar CMEs are one the most investigated phenomena (see review [7]) and the typical masses are $10^{14}-10^{16} \mathrm{~g}$. The observations of TESS telescope bring to the estimations of typical CME masses from $10^{18}$ till $10^{32} \mathrm{~g}$ [2]. However, in this paper the scaling factor about 100 was used. The using of it was explained by the analogy with Sun active phenomena.

We also use the Sun-star analogy to obtain the typical magnetic field from the first principles. Main motivation for such approach is as follows: our knowledge of the stars' medium is at approximately the same level as our knowledge of the Sun in the 1950s. Then we reestimate typical flare energies (the first estimations were made in $[8,9])$ and estimate CME masses for different stars' classes and try to justify the Sun-star analogy.

\section{Estimations of the flare energies and CME masses}

Equating the magnetic field on the star to the thermal plasma pressure and using the mean free path as a characteristic length we obtain

$$
\frac{B^{2}}{8 \pi}=n k T=G \frac{\rho M H}{R^{2}}, \quad H=\lambda=\frac{1}{\sigma_{T} n} .
$$

Here $n$ is the plasma density, $T$ is the temperature, $M$ and $R$ are the star mass and radius, $k$ is the Bolzmann constant, $G$ is the gravitational constant, and $\sigma_{T}$ is the Thompson cross-section. We assume that the height scale $H$ is equal to the star photosphere thickness.

For the photospheric magnetic field we obtain $B_{p h}=B_{0 \odot} R_{\odot} / R\left(M / M_{\odot}\right)$, where values with index $\odot$ are the corresponding Sun values.

So the flare energy is (here $\alpha$ and $\beta \ll 1$ are the scale parameters with values about 0.1 )

$$
E_{f l}=\beta^{2} \alpha^{3} \frac{R}{R_{\odot}} \frac{M}{M_{\odot}} \frac{R_{\odot} G m_{p} M_{\odot}}{\sigma_{T}}=2.3 \times 10^{37} \beta^{2} \alpha^{3} \frac{R}{R_{\odot}} \frac{M}{M_{\odot}} \mathrm{erg} .
$$

The maximum coronal temperature is $T_{\text {cor max }}=\left(5.77 \times 10^{6} R_{\odot} / R\right) M / M_{\odot} \mathrm{K}=\gamma T_{\text {cor }}$, where $\gamma$ is the scaling factor about 5 . So the CME mass is

$$
M_{C M E}=\frac{\alpha^{3} \beta^{2}}{\gamma} \frac{4 m_{p}}{\sigma_{T}} R^{2}=4.87 \times 10^{22} \frac{\alpha^{3} \beta^{2}}{\gamma}\left(\frac{R}{R_{\odot}}\right)^{2} \mathrm{~g} .
$$




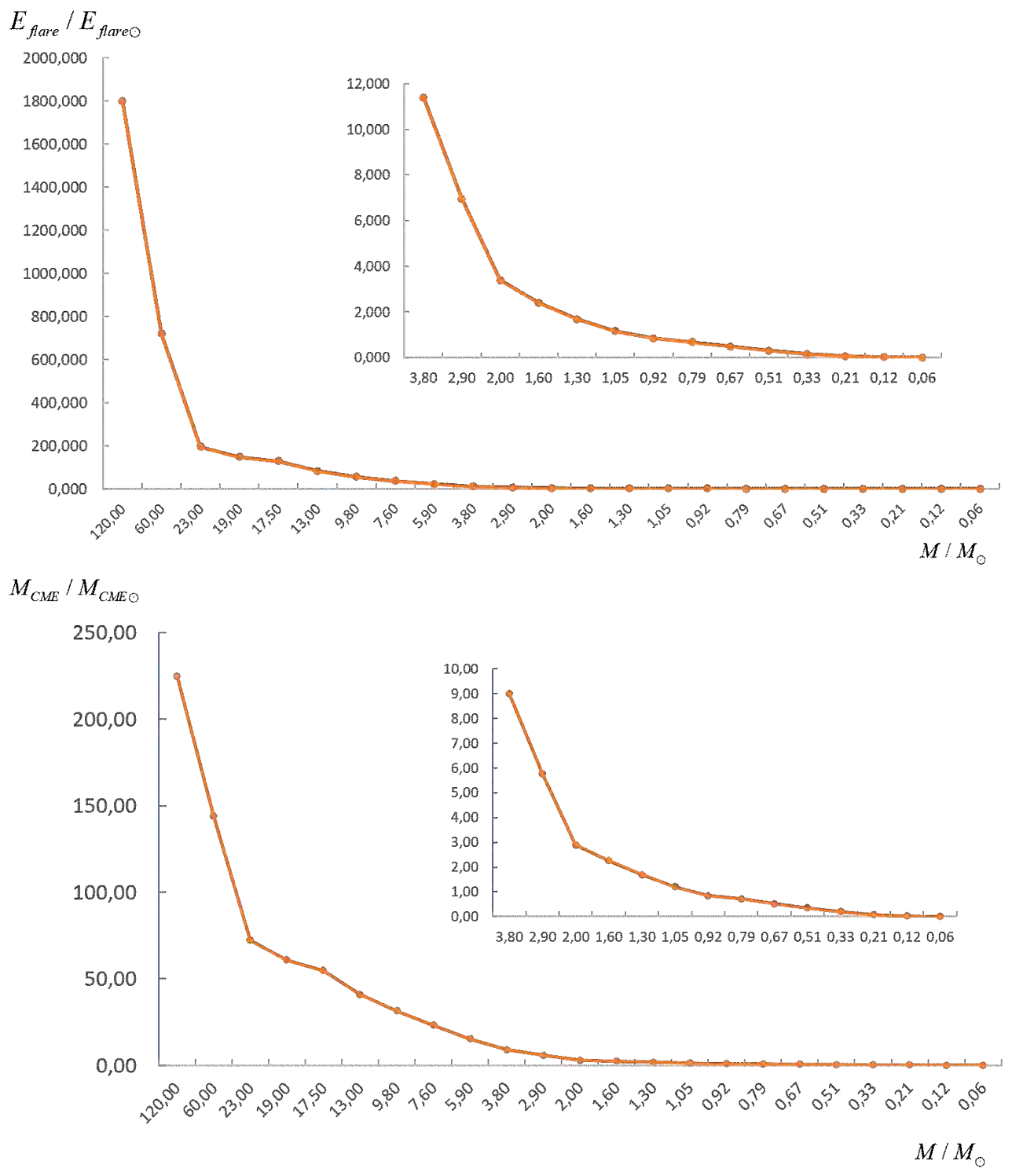

Figure 1: Flare energy (upper) and CME masses vs. stars masses. The part of curve with low mass stars is given in inset.

Table 1: Average parameters for different stars classes

\begin{tabular}{ccccccccc}
\hline Class & $M / M_{\odot}$ & $R / R_{\odot}$ & $T_{\text {star }}[K]$ & $T_{\text {cor }}[\mathrm{K}]$ & $E_{f l} / E_{f l \odot}$ & $M_{\mathrm{CME}} / M_{\mathrm{CME} \odot}$ & $L_{\text {star }} / L_{\odot}$ & $B_{p h}[G]$ \\
\hline O3 & 120.00 & 15.00 & 52500 & 46.10 & 1800 & 225.00 & 1400000 & 937 \\
B5 & 5.90 & 3.90 & 15400 & 8.72 & 23.01 & 15.21 & 830 & 799 \\
A5 & 2.00 & 1.70 & 8200 & 6.78 & 3.4 & 2.89 & 14 & 1067 \\
F5 & 1.30 & 1.30 & 6440 & 5.77 & 1.69 & 1.69 & 3.200 & 1125 \\
G0 & 1.05 & 1.10 & 6030 & 5.50 & 1.155 & 1.21 & 1.500 & 1195 \\
G5 & 0.92 & 0.92 & 5770 & 5.77 & 0.846 & 0.85 & 0.790 & 1338 \\
K0 & 0.79 & 0.85 & 5250 & 5.36 & 0.672 & 0.72 & 0.420 & 1342 \\
K5 & 0.67 & 0.72 & 4350 & 5.37 & 0.482 & 0.52 & 0.150 & 1459 \\
M0 & 0.51 & 0.60 & 3850 & 4.90 & 0.306 & 0.36 & 0.077 & 1527 \\
M5 & 0.21 & 0.27 & 3240 & 4.49 & 0.057 & 0.07 & 0.011 & 2178 \\
\hline
\end{tabular}

Table 1 and Fig. $\tilde{1}$ show the dependences of flare energy and CME masses for different stars classes. The average values were taken from [10]. 


\section{Conclusions}

The estimates of the magnetic field energy in the photospheres of O-M stars were obtained. We used these data to find the flares energy and masses of coronal mass ejections. The values obtained for the Sun coincide with the observed values on the order of magnitude, thus justifying the possibility of such consideration for other stars. The magnetic field in the magnetic force tube grows a little (less than for times) for flares from O to M stars (930 and $3100 \mathrm{Gs}$ ), but for $\mathrm{O}$ stars the flare energy appears to be more than 5 orders of magnitude higher. The masses of CME also differ about 5 oders of magnitudes for different classes.

\section{References}

1. L. A. Balona, MNRAS, 447, 2714, 2015.

2. I. S. Savanov, Astr. J. Lett., 46, 831, 2020.

3. H. Yang and J. Liu, ApJS, 241, 29, 2019.

4. S. V. Berdyugina, in K. G. Strassmeier, A. G. Kosovichev, and J. E. Beckman, eds., Cosmic Magnetic Fields: From Planets, to Stars and Galaxies, volume 259, 323-332 (2009).

5. J. F. Donati and J. D. Landstreet, $A R A \& A, 47,333,2009$.

6. J. L. Linsky and M. Schöller, Space Sci. Rev., 191, 27, 2015.

7. E. Kilpua, H. E. J. Koskinen, and T. I. Pulkkinen, Living Reviews in Solar Physics, 14, 5, 2017.

8. A. Struminsky and A. Sadovski, in Y. Y. Balega, D. O. Kudryavtsev, I. I. Romanyuk, and I. A. Yakunin, eds., Stars: From Collapse to Collapse, Astronomical Society of the Pacific Conference Series, volume 510,105 (2017).

9. A. Struminsky and A. Sadovski, in Journal of Physics Conference Series, Journal of Physics Conference Series, volume 1181, 012002 (2019).

10. K. R. Lang, Science, 257, 1148, 1992. 


\title{
New Name-lists of Variable Stars and Prospects of GCVS
}

\author{
N.N. Samus ${ }^{1,2}$, E.V. Kazarovets ${ }^{1}$, O.V. Durlevich ${ }^{2}$, N.N. Kireeva ${ }^{1}$, E.N. Pastukhova ${ }^{1}$, A.V. Khruslov ${ }^{1,2}$ \\ samus@sai.msu.ru \\ ${ }^{1}$ Institute of Astronomy, Russian Acad. Sci., 48, Pyatnitskaya Str., Moscow 119017, Russia \\ ${ }^{2}$ P.K. Sternberg Astronomical Institute, M.V. Lomonosov Moscow University, Moscow 119234, Russia
}

The revision of the NSV catalogue has been completed. As a result, a third part of all its stars have been transferred to the GCVS. We began to add variable stars in globular clusters to the GCVS. Two Name-lists published so far contain more than 1700 variable stars in 36 globular clusters; an additional Name-list (about 900 variable stars in 27 globular clusters) will be published before the end of 2021. We discuss problem cases in the Catalogue of Variable Stars in Galactic Globular Clusters revealed during our preparation of the Name-lists.

Keywords: variable stars, globular star clusters

DOI: $10.51194 /$ VAK2021.2022.1.1.064

The General Catalogue of Variable Stars (GCVS) was a project started in the USSR in 1948 on behalf of the IAU. The GCVS author team consists of scientists from two Moscow institutes, INASAN and SAI.

Since 1948, there appeared four editions of the GCVS, to the five-volume fourth edition (1985-1995). While GCVS I contained 10930 stars, GCVS IV had already 28435 variable stars of our Galaxy plus about 12000 stars in external galaxies. Now the GCVS is a purely electronic catalogue (GCVS 5.1; [1]). The number of stars in the GCVS is 57247. New objects are added via Name-lists of variable stars. They were published in the IAU Information Bulletin on Variable Stars (IBVS); recently, IBVS was discontinued, and we have started publishing new Name-lists in our electronic journal Peremennye Zvezdy/Variable Stars.

Besides catalogues of variable stars, intended to include only sufficiently well-studied objects, catalogues of suspected variable stars are published. The GCVS team prepared several suspected-variable catalogues, the latest of them being the NSV catalogue issued in 1982 and containing about 14800 objects. Later, a supplement to the NSV catalogue was prepared by [2], it contained about 11200 entries.

The GCVS is currently not the largest list of galactic variable stars. The American Association of Variable Star Observers established an electronic register of variable stars (VSX); [3]. As of August 9, 2021, it contained more than 2114000 stars. Some of its stars are extragalactic. VSX also includes stars that we would consider only suspected variables. It can contain several entries for the same object in the case of erroneous coordinates in the literature. Nevertheless, it is a very important source of information. The GCVS team has good contacts with the VSX team (administrator S. Otero).

Most recently, a full revision of the NSV catalogue has been completed. Of 3105 stars in the catalogue with lacking or erroneous finding charts, we were able to find 2809 objects. About 4850 stars (one third of all the NSV catalog) are being transferred into the GCVS; of them, 52 stars could be simply identified with stars already contained in the GCVS. 17 NSV "stars" turned out to be asteroids with available ephemerides. We could not identify 16 doubtful Novae; among them, there can be plate defects or "phantoms" of bright stars.

For reasons of tradition, the GCVS did not include variable stars in galactic globular clusters. There exist Catalogues of Variable Stars in Globular Clusters, compiled and published in Canada. Several editions were prepared by H. Sawyer Hogg (1905-1993). Later on, the catalogue was continued in the electronic form [4]. By now, it contains about 8000 variable stars.

The fact that variable stars in globular clusters are outside the GCVS contradicts the purpose of the GCVS as a general catalogue of galactic variables. The main obstacle on the way to adding globular-cluster variables to the GCVS was that Canadian catalogues used rectangular coordinates, referred to very approximate centers of globular clusters. The GCVS team took the effort to determine good-quality equatorial coordinates, by identification with modern catalogues or by measurements using existing images. The result was published for 3398 stars in 103 globular clusters [5]. Later on, equatorial coordinates were introduced in the catalogues of variable stars in globular clusters.

In 2019-2020, we started issuing Name-lists that contained GCVS names for variable stars in globular clusters, selected from Canadian catalogues as stars satisfying GCVS criteria. For these stars, we presented all variability information according to the GCVS standards. The GCVS uses the naming system based on constellations. The two lists of GCVS names for globular-cluster variables issued so far [6, 7] contain more than 1700 variable stars in 36 globular clusters (constellations Apus-Lynx). The next list we have now prepared, expected to be published before the end of 2021, will contain about 900 stars in 27 clusters (Musca-Ophiuchus). Wherever possible, we give equatorial coordinates from the Gaia DR2 and EDR3 releases.

A GCVS tradition is to improve and update information, to derive new light elements from data mining. This is not easy in the case of faint and crowded globular-cluster variable stars. Nevertheless, we always check existing possibilities. Thus, the second of the lists we have published [7] presents light elements derived by our author team in $16 \%$ of all cases. 
We noticed cases of our coordinates [5] replaced, in the Canadian catalogue, with much worse newer coordinates. In NGC 5286 (Centaurus), they were replaced with those from [8], where all declinations are wrong, on average by $\sim 6^{\prime \prime}$, while right ascensions are nearly correct. Our coordinates agree with those from Gaia well. In the Canadian catalogue, our disagreement in declinations with Zorotovic et al., as well as the agreement of our declinations with those from [9], is mentioned but not considered as a reason for using our coordinates. A similar situation is in NGC 6266 (M 62, Ophiuchus). All declinations from [10], used in the Canadian catalogue instead of those by us, are erroneous; it is mentioned that Contreras et al. are going to publish an Erratum - but such an Erratum cannot be expected after 11 years since the original publication.

For NGC 4833 (Musca), we use data from [11]. The published light curves have obviously incorrect magnitudes along the ordinate axis. The authors promised a correction but have not provided it so far.

An example of a correction in the light elements is a variable star in the cluster NGC 6402 (M 14, Ophiuchus). [12] provided access to their CCD observations. Their light elements for the RR Lyrae variable V3 with the period they determined, 0.52244d (type RRAB), give a low-quality light curve with a comparatively small amplitude, unusual asymmetry, and something like a secondary maximum. We used their own observations to derive light elements we find more probable, with the period 0.33797d (type RRC).

Our star-by-star data analysis is effort-consuming. The approach used in the VSX permits to work quicker, but will also reach limits of possibility soon. A cause of data avalanche is that a lower limit of amplitude needed for a star to be considered variable was never established. Millions of future discoveries are predicted even for groundbased observations. Space missions are able to find variations virtually for any star. For instance, preliminary results from Kepler mission [13] indicate that some $63 \%$ of all stars could be detected as variable. The prospects of traditional variable-star catalogues are pessimistic. A way out is adding variability information to big stellar catalogues, like in the catalogues of Hipparcos and Gaia missions.

N.N. Samus wishes to thank the RFBR grant 20-52-53009 for partial support.

\section{References}

1. N. N. Samus', E. V. Kazarovets, O. V. Durlevich, N. N. Kireeva, and E. N. Pastukhova, Astronomy Reports, 61, 80, 2017.

2. E. V. Kazarovets, N. N. Samus, and O. V. Durlevich, Information Bulletin on Variable Stars, 4655, $1,1998$.

3. C. L. Watson, A. A. Henden, and A. Price, Journal of the AAVSO, 35, 414, 2007.

4. C. M. Clement, A. Muzzin, Q. Dufton, T. Ponnampalam, et al., AJ, 122, 2587, 2001.

5. N. N. Samus, E. V. Kazarovets, E. N. Pastukhova, T. M. Tsvetkova, and O. V. Durlevich, PASP, 121, 1378, 2009.

6. E. V. Kazarovets, N. N. Samus, O. V. Durlevich, A. V. Khruslov, N. N. Kireeva, and E. N. Pastukhova, Information Bulletin on Variable Stars, 6261, 1, 2019.

7. N. N. Samus, E. N. Pastukhova, O. V. Durlevich, E. V. Kazarovets, and N. Kireeva, Peremennye Zvezdy, 40, 8, 2020.

8. M. Zorotovic, M. Catelan, H. A. Smith, B. J. Pritzl, et al., AJ, 139, 357, 2010.

9. R. Figuera Jaimes, D. M. Bramich, J. Skottfelt, N. Kains, et al., A\& A, 588, A128, 2016.

10. R. Contreras, M. Catelan, H. A. Smith, B. J. Pritzl, J. Borissova, and C. A. Kuehn, AJ, 140, $1766,2010$.

11. A. N. Darragh and B. W. Murphy, Journal of the Southeastern Association for Research in Astronomy, 6, 77, 2018.

12. C. Contreras Peña, M. Catelan, F. Grundahl, A. W. Stephens, and H. A. Smith, AJ, 155, $116,2018$.

13. G. Basri, L. M. Walkowicz, N. Batalha, R. L. Gilliland, et al., AJ, 141, 20, 2011. 


\title{
Three-dimensional modeling of the formation of molecular hydrogen on the surface of an interstellar dust grain by the off-lattice Monte Carlo method
}

\author{
N.A. Satonkin ${ }^{1,2}$, A.B. Ostrovsky ${ }^{1}$, K. Kalnin ${ }^{1}$, G.S. Fedoseev ${ }^{1}$, A.I. Vasyunin ${ }^{1,2}$ \\ n.a.satonkin@urfu.ru \\ ${ }^{1}$ Research Laboratory for Astrochemistry, Ural Federal University, Kuibysheva St. 48, 620026 Ekaterinburg, Russia, \\ ${ }^{2}$ Ventspils University of Applied Sciences, Inženieru 101, LV-3601, Ventspils, Latvija
}

\section{DOI: 10.51194 /VAK2021.2022.1.1.065}

The study of chemical processes on the surface of interstellar dust is important for understanding the formation and enrichment of the molecular composition in the interstellar medium. It is important to consider the morphology of dust grain to discover processes of molecular formation on it. One can implement a detailed model of dust particle with chemical reactions on it using various methods. Stochastic microscopically accurate kinetic MonteCarlo method is much slower than the rate equations-based methods, but it allows to track the positions of specific atoms and molecules on the surface of dust grains. There are two main types of this method: lattice and off-lattice. The lattice approach is faster, but the off-lattice approach is more reliable.

In this work the evolution of a dust particle is simulated and the efficiency of molecular hydrogen recombination in a range of dust temperatures is investigated using the off-lattice Monte Carlo method. Precompiled carbon grain core is used. The model implements the processes of accretion, desorption and migration of hydrogen on grain surface. Surface potential is continuous and represents the sum of potentials of all atoms on surface. Hydrogen molecule is formed when two hydrogen atoms collide on the surface of a particle and then it desorb to gas phase. We found that existence of deep potential wells in surface potential makes possible hydrogen molecule formation at the grain temperatures up to $20-25 \mathrm{~K}$. The most efficient mechanism of $\mathrm{H}_{2}$ formation is Harris-Kasemo mechanism at temperatures below $9 \mathrm{~K}$ and Langmuir-Hinshelwood mechanism dominates at temperatures above $10 \mathrm{~K}$ due to efficient thermal hopping of atomic hydrogen on grain surface.

The work is supported via the grant lzp-2018/1-0170. 


\title{
Investigation of Galactic cirri based on SDSS Stripe 82 images
}

\author{
S. Savchenko ${ }^{1,2,4}$, D. Polyakov ${ }^{1}$, A. Mosenkov ${ }^{4,1}$, A. Marchuk ${ }^{1,2}$, V. Il'in ${ }^{1,2,3}$, G. Gontcharov ${ }^{1}$, A. Smirnov $^{1,2}$, P. \\ Usachev $^{1,2,4}$ \\ s.s.savchenko@spbu.ru \\ ${ }^{1}$ Central (Pulkovo) Astronomical Observatory, Russian Academy of Sciences, Pulkovskoye chaussee 65/1, St. Petersburg \\ 196140, Russia \\ ${ }^{2}$ Saint Petersburg State University, Universitetskij pr. 28, St. Petersburg 198504, Russia \\ ${ }^{3}$ Saint Petersburg University of Aerospace Instrumentation, Bol. Morskaya ul. 67A, St. Petersburg 190000, Russia \\ ${ }^{4}$ Special Astrophysical Observatory, Russian Academy of Sciences, 369167 Nizhnij Arkhyz, Russia \\ ${ }^{5}$ Department of Physics and Astronomy, N283 ESC, Brigham Young University, Provo, UT 84602, USA
}

Galactic cirri are filamentary structures observed at high Galactic latitudes. Because of their rather large spatial scales, it is better to study them using images of wide-angle sky surveys. In this work, we use deep optical images from Sloan Digital Sky Survey (SDSS) Stripe 82 database to study Galactic cirri. We propose a semi-automatic algorithm based on artificial neural networks for data analysis. The algorithm allows one to find regions of images that contain cirri and to determine the cirri parameters, including their optical colours.

DOI: 10.51194 /VAK2021.2022.1.1.066

\section{Introduction}

The SDSS Stripe 82 survey [1] covers a 2.5 degree stripe along the Celestial Equator $\left(-1.25^{0}<\delta<1.25^{0}\right)$ with a total area of 275 square degrees. The survey images were further reduced by [2] to obtain the high quality deep co-adds in the framework of the IAC Stripe 82 Legacy Project. These images have been used by [3] to find the parameters of the Galactic cirri in optical. As was shown in their work, an analysis of cirri is a rather difficult task: the presence of numerous background objects, extended wings of bright stars, scattering of the light in the telescope optics, all impede the investigation of faint and diffuse cirri. To deal with this problems, [3] used a sophisticated image processing pipeline that requires user's manual intervention. The main goal of our work is to use the data, produced by [3], to train a new artificial network based algorithm for the cirri analysis.

\section{The algorithm}

The pipeline of the image processing for our analysis of Galactic cirri analysis consists of the following main steps:

1. Masking of the background objects: image regions that contain all the objects (except of cirri) should be covered by a binary mask;

2. Filling the masked regions: the lacunae that were created after removing of the background objects should be interpolated inwards to produce smooth and continuous cirri images;

3. Separating the cirri from the image artefacts produced by the light of the bright stars scattered in the telescope optics.

In the first step, we used the mask images prepared by [3] to train a conditional generative adversarial network (cGAN, [4]). The cGAN architecture is a variant of a generative adversarial network architecture [5], where two networks, a generator and a discriminator, compete with each other: the generator tries to create a realistic-looking image, while the discriminator has to decide if a given image is real or simulated. In the setting of our problem, the generator network for a given sky image shall generate a mask image of such a quality that the discriminator will not be able to decide if this mask was generated by the generator, or this is real mask made by [3]. To train the network, we used 500000 image-mask pairs randomly cut out from the data of [3].

The second step was done using linear interpolation of the masked regions from their bounds inwards with the aid of the GRIDDATA function of the NUMPY ${ }^{1}$ Python $^{2}$ package.

To separate the cirri on an image (the last step), a reduced (masked and interpolated) image was shown to a user. The only objects in this image are the cirri and the artefacts due to the scattered stellar light. To set the bounds of these objects, a 29-th isophote in the $r$-band was also shown on the image. The user then select those objects that he considers to be true cirri by mouse clicks. After this step, a map that contains the regions with the cirri is used for the future analysis. Fig. 1 shows the results of the main steps of the algorithm applied to one field.

To test our algorithm, we applied it to 270 fields of the IAC Stripe 82 Legacy survey (roughly a quarter of the whole survey). This provided us with a number of extracted cirri objects, which can be further analysed to infer their geometrical and photometrical parameters. As an example, the Fig. 2 shows a colour-colour diagram for the cirri and a histogram with the distribution of the cirri by the Gini value [6].

\footnotetext{
${ }^{1}$ https://numpy.org

${ }^{2}$ https://python.org
} 


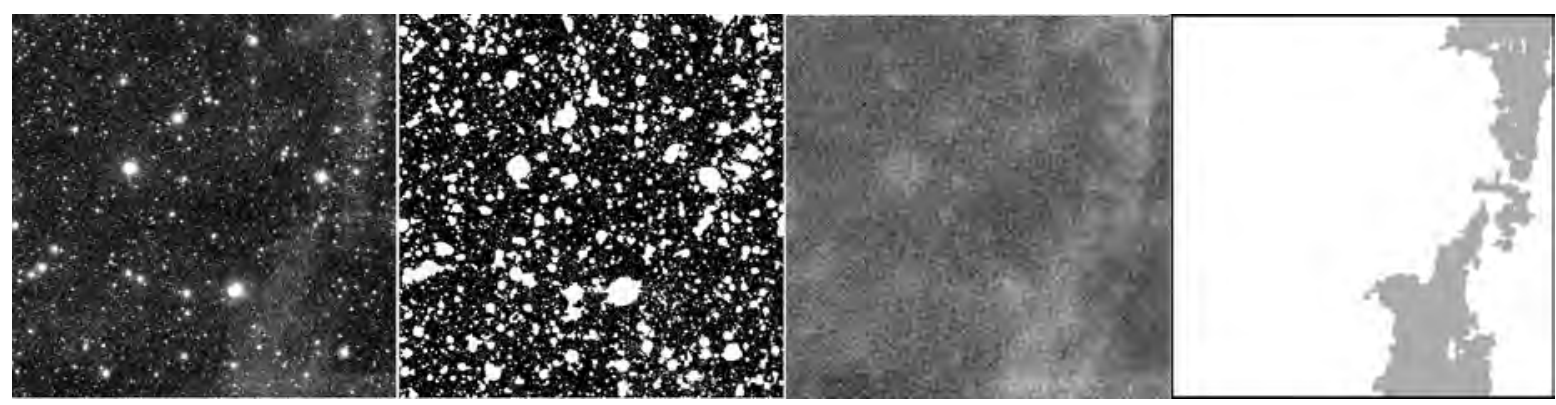

Figure 1: The results of the application of our algorithm to one field. Left panel: original field image; second panel: automatically generated map; third panel: the field image after removal of the background objects with the applied interpolation, the right panel: map of field regions that contain cirri.
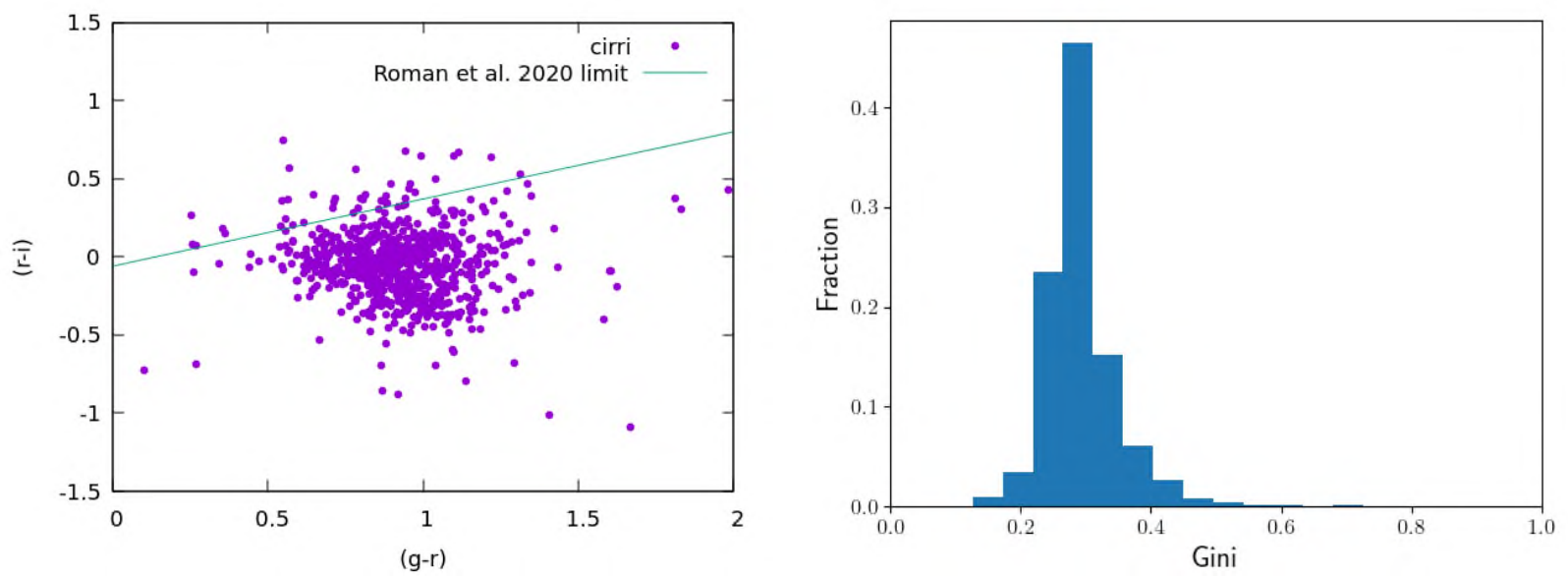

Figure 2: Examples of obtained cirri parameters: (g-r)-(r-i) colour diagram (left) and the distribution of cirri by the Gini value. The green solid line on the left panel is a colour limit for cirri from [3].

This last step of the algorithm requires a manual intervention, therefore there is still room for the automatisation of the process. To make one step further, we used the described above manually prepared fields (i.e. manually selected cirri regions) to train a neural network to detect cirri in the images and to discriminate them from the scattered stellar light.

Then we used this data to train a U-Net based [7] neural network with the Mobile NetV2 [8] feature detector as the encoder and layers from the Pix2Pix architecture [4] as the decoder. The training of this network was done on a sample of 1000000 of 128x128 randomly selected windows in $g, r$ and $i$ passbands. The application of this network to a test sample gives us a mean intersection over union (IoU) value of 0.357 in comparison with the manual cirri selection. To increase this value, the further work should be focused on a training of a deeper network architecture, and, probably, using smaller windows to obtain smoother cirri maps.

\section{Conclusions}

In this work, we have described an algorithm for the detection of Galactic cirri in the IAC Stripe 82 Legacy images. We proposed two versions of this algorithm. In the first version, the main decision of attributing the detected regions to cirri is upon the observer. The second algorithm is fully automatic with a neural network making this decision. The algorithm outputs location maps of Galactic cirri in the images. This maps can be used to infer the cirri geometrical and photometrical parameters.

Acknowledgement. We acknowledge financial support from the Russian Science Foundation (grant no. 20-7210052).

\section{References}

1. K. N. Abazajian, J. K. Adelman-McCarthy, M. A. Agüeros, S. S. Allam, et al., ApJS, 182, 543, 2009.

2. J. Fliri and I. Trujillo, MNRAS, 456, 1359, 2016. 
3. J. Román, I. Trujillo, and M. Montes, A\&A, 644, A42, 2020.

4. P. Isola, J.-Y. Zhu, T. Zhou, and A. A. Efros, arXiv e-prints, arXiv:1611.07004, 2016.

5. I. J. Goodfellow, J. Pouget-Abadie, M. Mirza, B. Xu, D. Warde-Farley, S. Ozair, A. Courville, and Y. Bengio, arXiv e-prints, arXiv:1406.2661, 2014.

6. J. M. Lotz, J. Primack, and P. Madau, AJ, 128, 163, 2004.

7. O. Ronneberger, P. Fischer, and T. Brox, arXiv e-prints, arXiv:1505.04597, 2015.

8. M. Sandler, A. Howard, M. Zhu, A. Zhmoginov, and L.-C. Chen, arXiv e-prints, arXiv:1801.04381, 2018. 


\title{
Bolometric light curves and parameters of superluminous supernova explosions
}

\author{
T. Semenikhin ${ }^{1,2}$, M. Kornilov², M. Pruzhinskaya ${ }^{2}$ \\ ofmafowo@gmail.com \\ ${ }^{1}$ Lomonosov Moscow State University, Faculty of Space Research, Moscow, Russia \\ ${ }^{2}$ Lomonosov Moscow State University, Sternberg Astronomical Institute, Moscow, Russia
}

\section{DOI: 10.51194/VAK2021.2022.1.1.067}

The goal of this work is to obtain bolometric light curves of superluminous supernovae (SLSN) using data from the Open Supernova Catalog [1] (OSC) and vector Gaussian processes (GP) ${ }^{1}$. The bolometric light curve gives us a fundamental understanding of the supernova explosion and allows us to determine the parameters of pre-supernovae.

The catalog contains photometric observations (light curves) of supernovae in different bandpasses. The multicolor light curves presented in the OSC are inhomogeneous in time. To obtain a quasi-bolometric light curve, it is necessary to sum the fluxes measured in different bands. Therefore, the existing light curves must be approximated. In this work, it is done by vector GP.

Gaussian processes assume that each point on the light curve is a time-indexed random variable. By specifying the covariance matrix, we can find the conditional mathematical expectation of the light curve at the moment of time of interest to us. When forming the sample, the following criteria were taken into account: presence of at least one spectrum, observations in one of the photometric systems (ugriz, $\left.u^{\prime} g^{\prime} r^{\prime} i^{\prime} z^{\prime}, U B V R I\right)$, presence of at least 3 bandpasses. After applying these cuts, only 27 SLSN were included in our sample out of 224 OSC SLSN. We applied the vector GP in the corresponding bandpasses to each SLSN, thereby obtaining light curves.

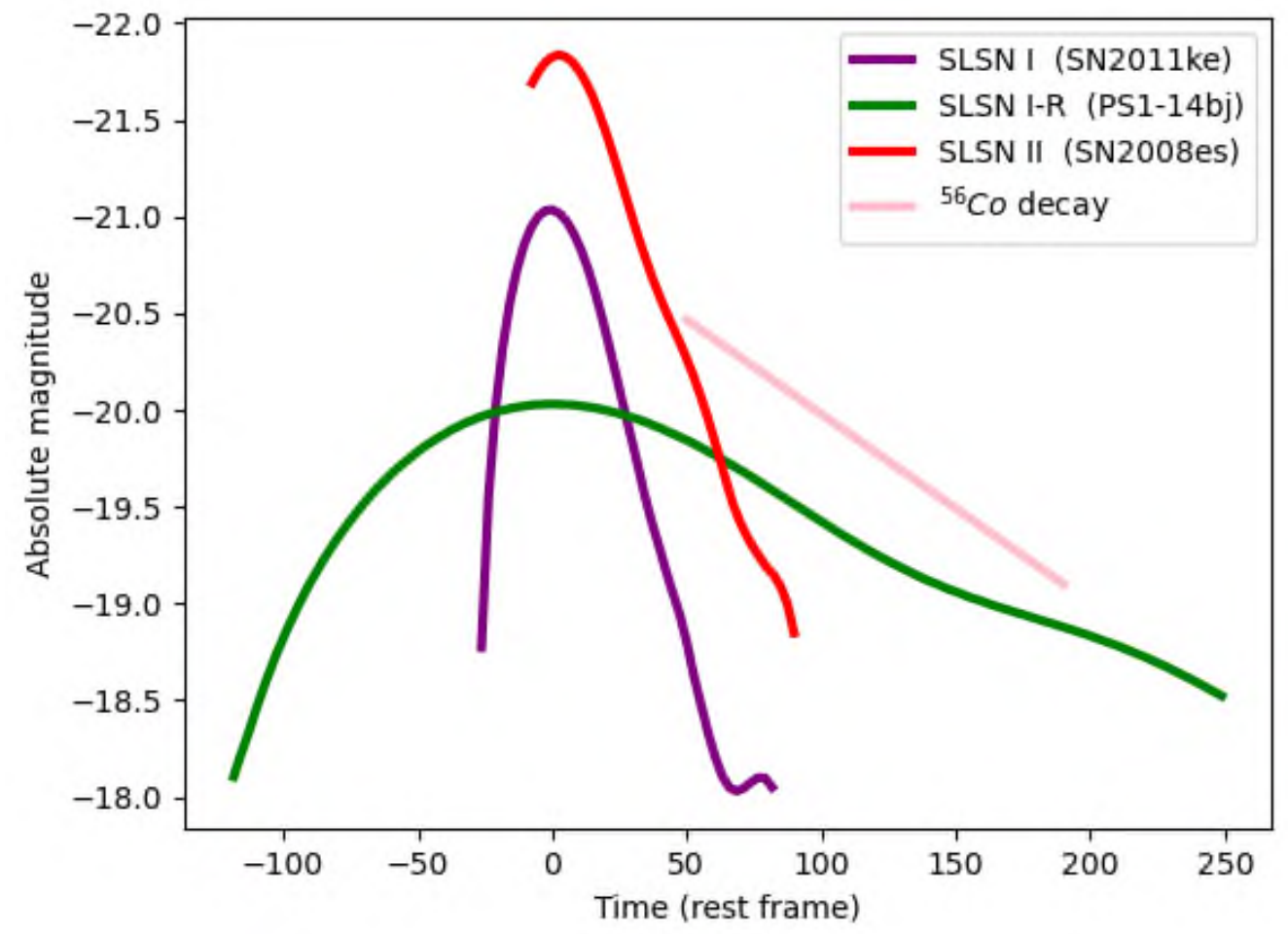

Figure 1: Bolometric light curves for various classes of superluminous supernovae.

To obtain the bolometric light curves for chosen SLSN, we used the Superbol Python library [2]. This library implements a method that assumes that at any moment in time the spectrum of a supernova can be described by a black body spectrum with a certain temperature. Using the multicolor light curves, which we approximated, we can fairly accurately estimate the temperature of a black body at a given time and calculate the bolometric light curve. Three objects from the sample were taken as a representative of each class of SLSN: SN2011ke (SLSN I), PS1-14bj (SLSN I-R), and SN2008es (SLSN II). The obtained bolometric light curves are shown in Fig. 1. Zero point in time corresponds to the maximum of the light curves. When constructing the bolometric light curves, the

\footnotetext{
${ }^{1}$ https://gp.snad.space/
} 
redshift and interstellar extinction in the Galaxy were taken into account. The incompleteness of the increasing part of SN2008es is due to the fact that there were practically no observations in the OSC before the explosion. Fig. 1 shows that SN2008es has the highest absolute magnitude, but the event itself is faster than the others. In contrast, PS1-14bj has the lowest absolute magnitude but lasts longer. Also, in order to explain the bolometric light curves of the investigated supernovae, the figure shows the curve due to radioactive decays $\left({ }^{56} \mathrm{Ni} \rightarrow{ }^{56} \mathrm{Co} \rightarrow{ }^{56} \mathrm{Fe}\right)$. It can be seen that only the PS1-14bj light curve can be explained by radioactive decays; to explain the luminosities of SN2011ke and SN2008es, other models must be invoked.

In the future, it is planned to obtain bolometric light curves for all the remaining objects from the chosen sample, as well as, using theoretical and analytical models, to extract from bolometric light curves information on the parameters of pre-supernovae and explosion parameters, which will help to understand their astrophysical nature.

The reported study was funded by RFBR and CNRS according to the research project No. 21-52-15024.

\section{References}

1. J. Guillochon, J. Parrent, L. Z. Kelley, and R. Margutti, ApJ, 835, 64, 2017.

2. M. Nicholl, Research Notes of the AAS, 2, 230, 2018. 


\section{Checking the possibility of determining the orbits of stars, orbiting the center of the Galaxy, and the estimate some relativistic effects}

N. Shakht, D. Gorshanov, I. Izmailov natalia.shakht@yandex.ru

The Central (Pulkovo) observatory of Russian Academy of Sciences

\section{DOI: 10.51194 /VAK2021.2022.1.1.068}

The results of observations of stars orbiting the central body of our Galaxy with a mass of $4.15 \times 10^{6}$ solar masses are considered. We used modern high-precision observations with the Keck and VLT telescopes. Earlier, [1], the star S02 was studied, the orbital period of which - 16 years was at that time the shortest. Currently, information has appeared about the motion of even closer stars, the so-called "squeezars" with even shorter periods. It was of interest to determine the orbit in an independent way for such stars, as well as for stars with exact positions "star - central body", but with ambiguous results obtained at different telescopes (Keck and VLT).

We have chosen star S102 . The orbit was determined by us from the combined Keck, [2] and VLT, [3] series: with dynamic elements: $P=12.3 \mathrm{yr}, a=856$ a.u., $e=0.58$, error of $\mathrm{O}-\mathrm{C}$ equals $0^{\prime \prime} .018$. The star S27 was considered, for which the observers have not received an orbit due to uncertain values of curvature and radial velocity, [4]. The new orbit was determined by us taking into account the additionally selected radius of curvature. Then orbital elements are $P=103 \mathrm{yr}, a=3535$ a.u., $e=0.99$. O-C error is $0^{\prime \prime} .003$. Using the methods developed at Pulkovo, we estimated the S4711 "squeezar" orbit with an extremely short orbital period of 7.4 years. The theoretical relativistic parameters were calculated, for S02: $\Gamma$, equaled $3.4 \times 10^{-4}$, is the ratio of the Schwarzschild radius $r_{s}$ to the distance to the periastron $r_{p}$. The Schwarzschild precession $\Delta \varphi$, equaled 6.3 arcmin.

We hope that our methods, [1] would be useful for determinations of preliminary orbits of such objects. The reported study was funded by RFBR according to the research project № 20-02-0563A.

\section{References}

1. A. A. Kisselev, Y. N. Gnedin, E. A. Grosheva, N. A. Shakht, D. L. Gorshanov, and M. Y. Piotrovich, Astronomy Reports, 51, 100, 2007 .

2. L. Meyer, A. M. Ghez, R. Schödel, S. Yelda, et al., Science, 338, 84, 2012.

3. M. Parsa, A. Eckart, B. Shahzamanian, V. Karas, M. Zajaček, J. A. Zensus, and C. Straubmeier, ApJ, 845, $22,2017$.

4. S. Gillessen, P. M. Plewa, F. Eisenhauer, R. Sari, et al., ApJ, 837, 30, 2017. 


\title{
Modeling of UX Ori Stars Eclipses
}

\author{
S. Shulman ${ }^{1}$, V. Grinin ${ }^{1,2}$ \\ sgshulman@gmail.com
}

${ }^{1}$ St. Petersburg State University, Universitetskii pr. 28, St. Petersburg, 198504, Russia

${ }^{2}$ Pulkovo Astronomical Observatory, Russian Academy of Sciences, Pulkovskoe sh. 65, St. Petersburg, 196140, Russia

Eclipses of UX Ori stars by compact gas-dust clouds and large-scale circumstellar disk perturbations are modeled. A flared disk and a disk with a puffing-up in the dust sublimation zone are considered. It is shown that these models are able to explain a number of the observed features of eclipses that cannot be obtained when a star with a flared disk is eclipsed by a compact gas-dust cloud.

Keywords: radiative transfer, stars: variables: T Tauri, Herbig Ae/Be, circumstellar matter, polarization

DOI: 10.51194/VAK2021.2022.1.1.069

\section{Introduction}

UX Ori stars are hot young stars of spectral types A-F, surrounded by protoplanetary disks. Their disks are inclined at a slight angle to the line of sight. Observations of UX Ori stars show deep sporadic eclipses with a depth of $2-4^{m}$. The linear polarization degree usually reaches $5-8 \%$ at the minima. Changes in color indices are also observed during eclipses. The eclipses last from days to several months.

Grinin [1] proposed an eclipse model of a star by a compact gas-dust cloud, which does not affect the radiation scattered by the disk. This model was studied for a flared disk and the results fit well most observations [2]. Natta et al. [3] showed that there must be a puffing-up in the dust sublimation zone to explain infrared excesses in the spectra of stars.

\section{Model}

We use the model of the UX Ori disk based on Kreplin et al. [4]. The puffing-up in the dust sublimation zone is obtained by adding Safier disk wind [5,6]. Eclipses were simulated by both compact gas-dust clouds and large-scale disk perturbations described by two Gaussians in radius and azimuth.
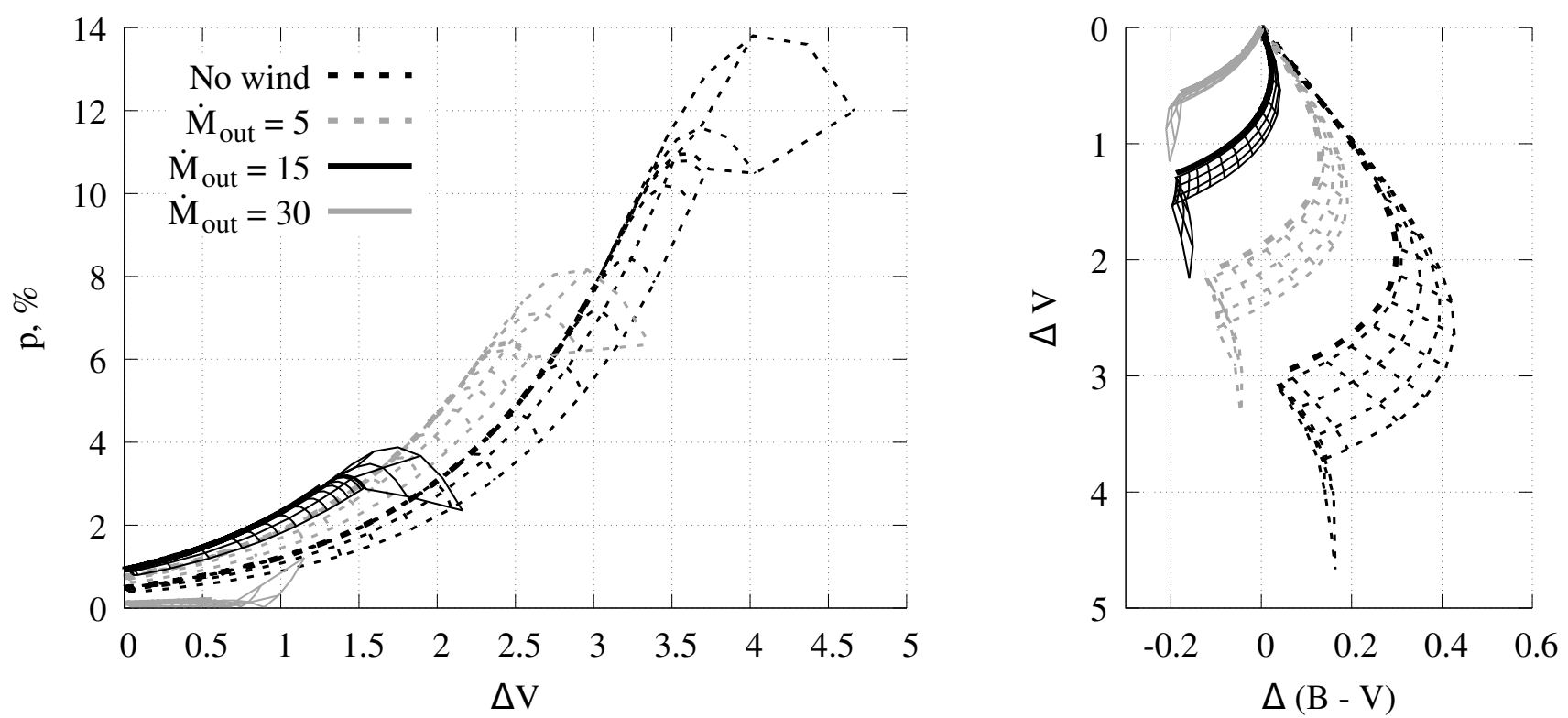

Figure 1: Changes in the linear polarization degree (left panel) and color indices (right panel) during eclipses. The thick lines show the compact cloud eclipses results. The grids demonstrate the scatter of the large-scale disk perturbation eclipse parameters depending on the perturbation parameters. The results are shown in different colors for various mass outflow rates, which determine the puffing-up. The mass outflow rate is indicated in $10^{-9} M_{\odot}$ per year.

\section{Results}

Using Shulman [7] numerical method, we conducted a simulation by considering two eclipse models and various disk models.

We found that the disk puffing-up explains several observed features of eclipses [8]: eclipses without a significant increase in the degree of linear polarization [9]; eclipses without reddening in the blue region of the spectrum [10]; strong changes (up to $90^{\circ}$ ) in the polarization positional angle when passing from one spectral band to another [11]. 
A large-scale disk perturbation also absorbs the scattered light from the inner regions of the disk. This makes it possible to obtain substantially deeper eclipses compared to the compact gas-dust cloud model. The increase in the eclipse depth can be about 1.5 times. When eclipses become deeper, the maximum polarization degree also increases. The effect depends on the wavelength and can reach 1.4 times in I band and 1.7 times in $\mathrm{U}$ band.

Both the disk puffing-up and the large-scale disk perturbation eclipse model make it possible to explain the scatter of the eclipse parameters at one fading level [12]. The results are shown in Fig. 1.
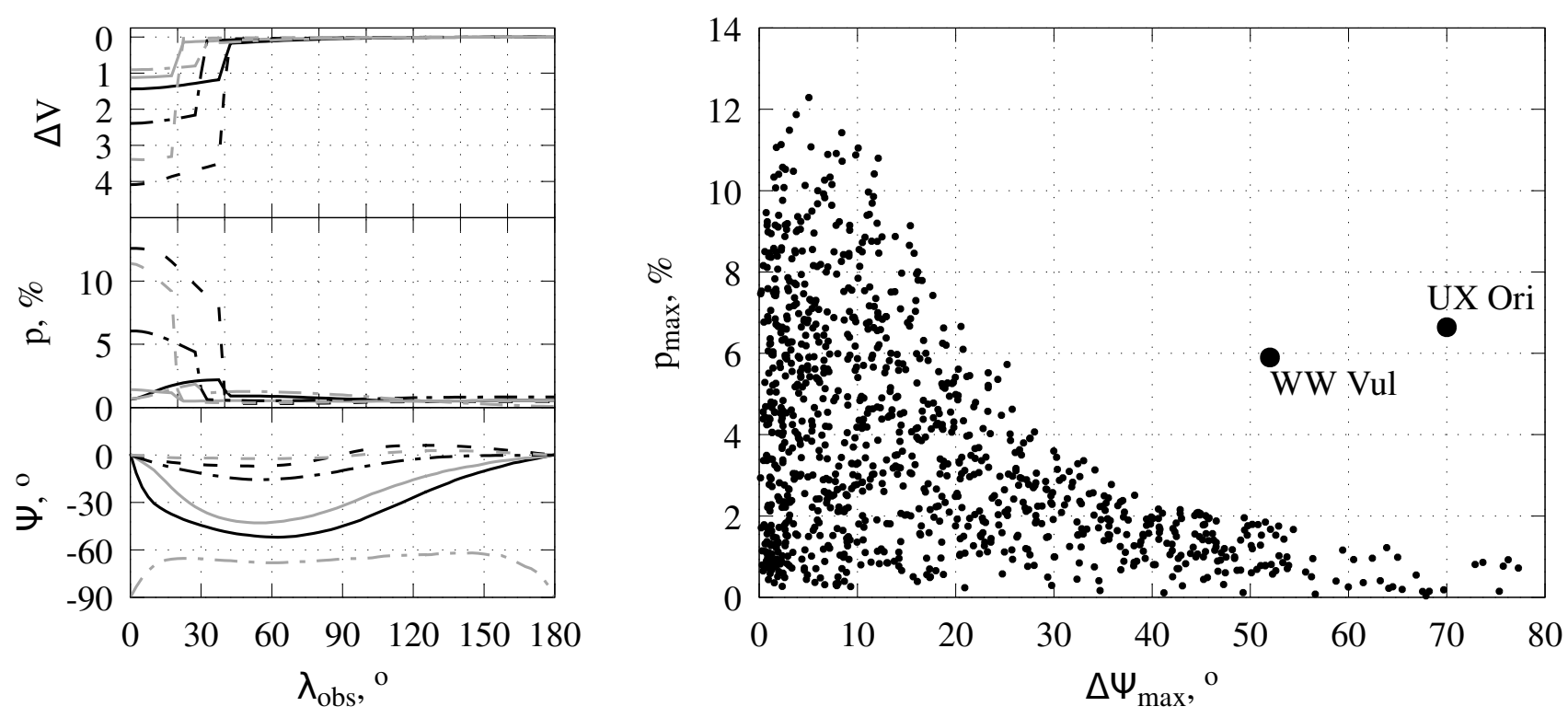

Figure 2: Change in the magnitude, linear polarization degree, and polarization position angle in the $\mathrm{V}$ band versus longitude of the observer (left panel) and the comparison of the model ratios of maximum polarization in the minima and the maximum change in the positional angle after passing through it with UX Ori and WW Vul observation (right panel).

\section{Change of eclipse parameters in time}

During the long eclipses of UX Ori [13] and WW Vul [14], not only changes in the polarization degree and color indices were observed during the eclipse, but also strong changes in the polarization position angle after passing through the minima. Such changes are impossible in the compact gas-dust cloud eclipse model. We have shown that an eclipse by a large-scale disk perturbation can lead to noticeable changes in the position angle after the minima [15]. It can reach $80^{\circ}$ in the disk models with the puffed-up inner rim.

With a weak puffing-up, we obtain deep eclipses with the small changes in the positional angle after the minima. On the contrary, a strong puffing-up decreases the amplitude of eclipse changes in magnitude and leads to significant changes in the positional angle. We went through many parameters of the disk perturbation and puffing-up and could not obtain a deep minimum with a high polarization degree followed by a heavy change in the positional angle, as it was observed for UX Ori and WW Vul. Fig. 2 demonstrates the results for various models. The time changes are obtained under the assumption that the perturbation rotates around the star together with the disk. The angle $\lambda_{\text {obs }}$ in the disk plane between the directions to the observer and the perturbation center represents time.

\section{Conclusion}

We have generalized the compact gas-dust cloud eclipse of a star surrounded by a flared disk. We considered eclipses by large-scale disk perturbations in the puffed-up disks. Such a model is able to explain a number of the observed features of eclipses. Nevertheless, for prolonged eclipses accompanied by strong changes in the polarization positional angle after the minima, it was impossible to obtain a good agreement with observations (deep eclipse followed by a large change in the positional angle) in the considered models.

Thus, we can conclude that the inner regions of the disk have a rather complex structure. Photopolarimetric observations of eclipses can be used to study the disk puffing-up in the dust sublimation zone and estimate the shape and size of the disk perturbation eclipsing the star. It may provide valuable information about the processes taking place in the circumstellar disks.

The simulation results strongly depend on the observation wavelength, therefore, the observations of long-term minima of UX Ori type stars at different wavelengths are very important for studying their circumstellar disks.

\section{References}

1. V. P. Grinin, Soviet Astronomy Letters, 14, 27, 1988. 
2. A. Natta and B. A. Whitney, A\&SA, 364, 633, 2000.

3. A. Natta, T. Prusti, R. Neri, D. Wooden, V. P. Grinin, and V. Mannings, $A \& A, \mathbf{3 7 1}, 186,2001$.

4. A. Kreplin, D. Madlener, L. Chen, G. Weigelt, S. Kraus, V. Grinin, L. Tambovtseva, and M. Kishimoto, A\&A, 590, A96, 2016.

5. P. N. Safier, ApJ, 408, 115, 1993.

6. P. N. Safier, ApJ, 408, 148, 1993.

7. S. G. Shulman, Astronomy and Computing, 24, 104, 2018.

8. S. G. Shulman and V. P. Grinin, Astronomy Letters, 45, 384, 2019.

9. A. N. Rostopchina-Shakhovskaja, V. P. Grinin, and D. N. Shakhovskoi, Astrophysics, 55, 147, 2012.

10. C. A. Grady, M. R. Perez, P. S. The, V. P. Grinin, D. de Winter, S. B. Johnson, and A. Talavera, A\&A, 302, 472, 1995.

11. A. Pereyra, J. M. Girart, A. M. Magalhães, C. V. Rodrigues, and F. X. de Araújo, A\&A A, 501, 595, 2009.

12. A. N. Rostopchina, V. P. Grinin, D. N. Shakhovskoi, P. S. Thé, and N. K. Minikulov, Astronomy Reports, 44, 365, 2000 .

13. V. P. Grinin, in P. S. The, M. R. Perez, and E. P. J. van den Heuvel, eds., The Nature and Evolutionary Status of Herbig Ae/Be Stars, Astronomical Society of the Pacific Conference Series, volume 62, 63 (1994).

14. V. P. Grinin, N. N. Kiselev, N. K. Minikulov, and G. P. Chernova, Soviet Astronomy Letters, 14, $219,1988$.

15. S. G. Shulman and V. P. Grinin, Astronomy Letters, 45, 664, 2019. 


\title{
Dust destruction at high Galactic altitude
}

\author{
E. Sivkova, D. Wiebe, M. Murga \\ sivkovae@gmail.com \\ Institute of Astronomy, Russian Acad. Sci., 48, Pyatnitskaya Str., Moscow 119017, Russia
}

We consider the destruction of dust at high galactic altitudes based on an earlier developed model of the movement of dust in the Galaxy, due to the stellar radiation pressure, gravity and gas drag. The possibility of dust sweeping is considered taking into account its collisions with the Galactic gas and between dust particles. Also, we clarify the range of grain sizes that can get into intergalactic space due to the described mechanism.

Keywords: interstellar dust, radiation pressure, dust destruction, high Galactic altitude

DOI: 10.51194/VAK2021.2022.1.1.070

\section{Introduction}

Earlier, we studied in detail the process of dust sweeping out by the stellar radiation pressure from our Galaxy [1] based on [2]. In the model the dust dynamics is determined by three factors: the stellar radiation pressure, the gravitational attraction, and the drag force of the interstellar gas. The model also includes the processes of dust destruction described in $[3,4,5]$. It was shown that medium-sized carbonaceous dust particles are most effectively swept out of the Galaxy, and the mass loss in the form of dust is $0.03 M_{\odot} /$ year. Also, our calculations show that dust grains can move with high relative velocities at the same locations.

\section{The model of the dust motion and destruction in the Galaxy}

In this paper we consider graphite and silicate dust grains. The optical properties and size distributions of dust grains were taken from $[6]^{1}$. The gas distribution is taken from [7]. We consider two destruction mechanisms: shattering (grain-grain collisions) and sputtering (gas-grain collisions). We calculate masses of fragments for collision velocities calculated with the dynamic model for different sizes and chemical compositions in the same way as [8,9]. The main sources of dust are considered to be evolved stars [10] with the scale height of $350 \mathrm{pc}[11,12]$. We assume that the dust is formed at the same rate over the entire calculated time. The mass loss rates of stars of different types diverge by more than an order of magnitude $[13,14]$. We assume that this value is $10^{-9} M_{\odot} /$ yr.

\section{Results}

Our calculations show that dust particles do not fragment as a result of sputtering, since this process requires high collision rates and concentrations of gas particles.

Here we consider only dust distribution above the central region of the Galactic disk $(r=0)$ to show the destruction effects. Fig. 1a,b present initial dust grains distributions. Fig. 1c,d present dust grains distributions calculated with the dynamical model for the estimated time $10 \mathrm{Gyr}$ without taking into account any destruction effects. It is seen that the grains of different size and chemical composition move differently and reach different altitudes. Fig. 1e,f present dust grain distributions after $10 \mathrm{Gyr}$ in the model with collisions between dust grains. These diagrams show that carbonaceous grains fragment effectively due to the shattering and dust grains with radii over than $\sim 100 \AA$ are destroyed by other grains and cannot reach high galactic altitudes due to the mechanism of sweeping out by radiation pressure force. Silicate dust grains distribution looks similar to the one without shattering taking into account for large and medium-size grains, but small silicate grains are visible up to the altitudes $\sim 2 \mathrm{kpc}$, where they are absent in the initial distribution.

\section{Conclusions}

Our calculations show that dust grains fragment as a result of shattering while being swept out of the Galaxy by the stellar radiation pressure and do not fragment as a result of sputtering.

Numerical simulations were partially carried by using the equipment of the shared research facilities of HPC computing resources at Lomonosov Moscow State University supported by the project RFMEFI62117X0011. E. Sivkova acknowledges support from Russian Science Foundation (project no. 19-72-20089).

\section{References}

1. E. E. Sivkova, D. S. Wiebe, and B. M. Shustov, Astronomy Reports, 65, 370, 2021.

2. B. M. Shustov and D. Z. Vibe, Astronomy Reports, 39, 578, 1995.

3. M. S. Murga, S. A. Khoperskov, and D. S. Wiebe, Astronomy Reports, 60, 233, 2016.

4. H. Hirashita and H. Yan, MNRAS, 394, 1061, 2009.

5. M. S. Murga, D. S. Wiebe, E. E. Sivkova, and V. V. Akimkin, $M N R A S$, 488, 965, 2019.

6. A. P. Jones, M. Köhler, N. Ysard, M. Bocchio, and L. Verstraete, A 64 A, 602, A46, 2017.

7. P. M. W. Kalberla and J. Kerp, Ann. Rev. Astron. and Astrophys., 47, 27, 2009.

8. A. P. Jones, A. G. G. M. Tielens, and D. J. Hollenbach, ApJ, 469, 740, 1996.

9. H. Hirashita and H. Yan, MNRAS, 394, 1061, 2009.

\footnotetext{
${ }^{1}$ https://www.ias.u-psud.fr/DUSTEM/
} 


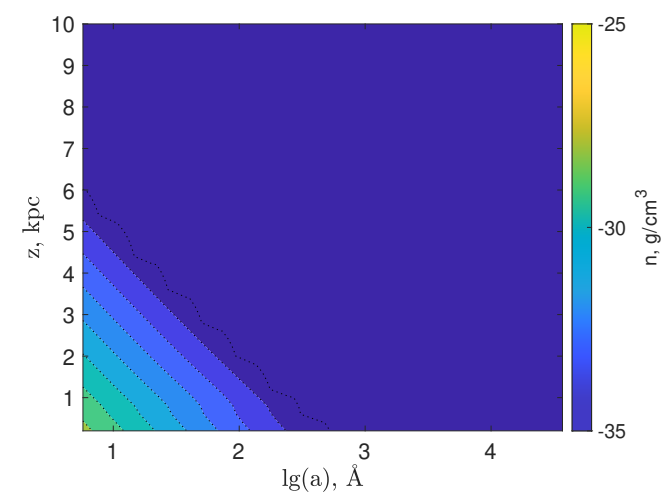

a)

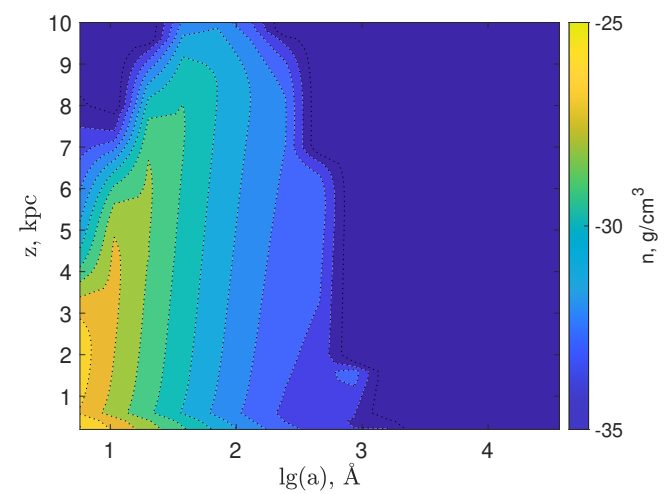

c)

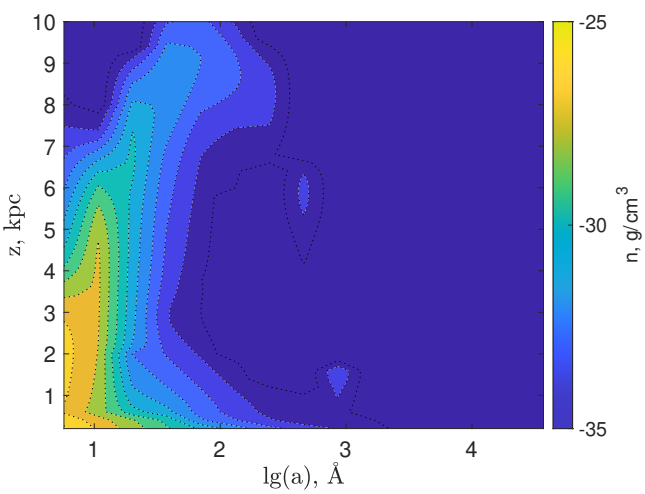

e)

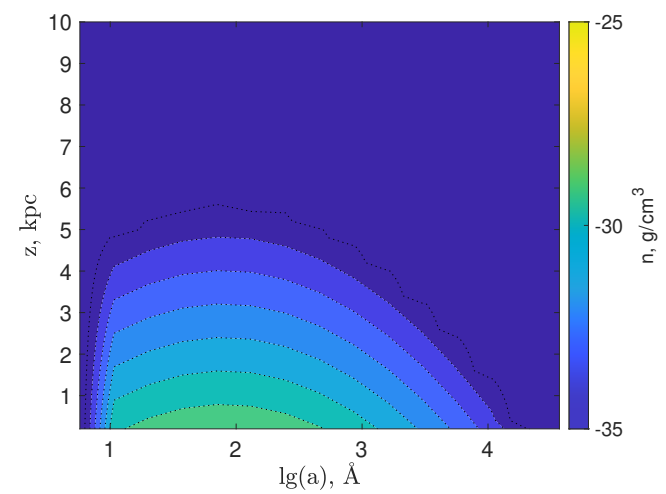

b)

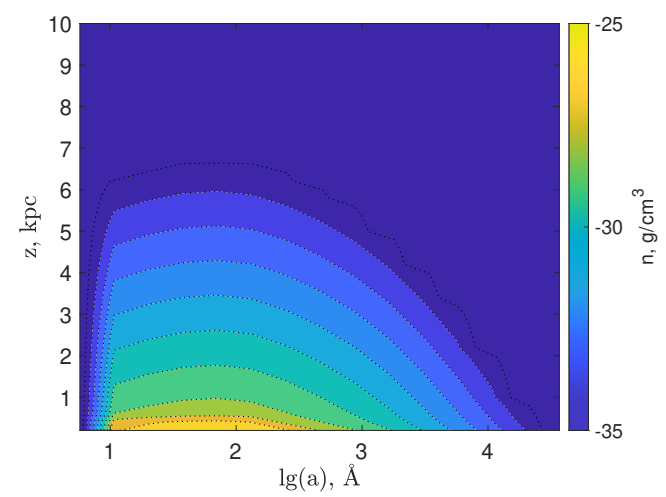

d)

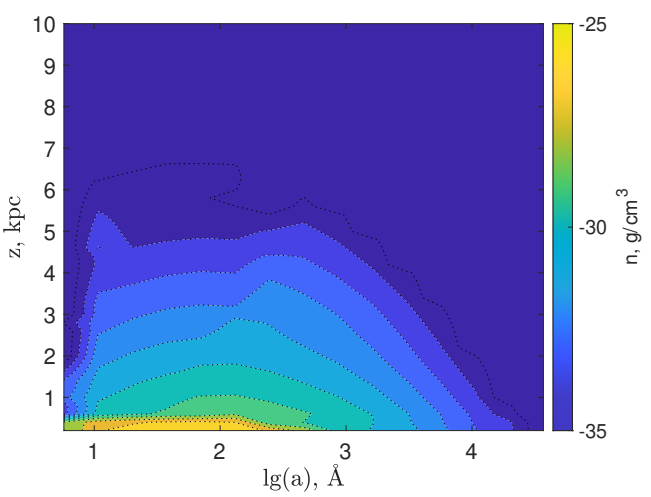

f)

Figure 1: Initial carbonaceous (a) and silicate (b) dust grains distributions. Carbonaceous (c) and silicate (d) dust grains distributions without taking into account destruction effects; carbonaceous (e) and silicate (f) dust grains distributions taking into account all kinds of collisions. Dark blue colour on the diagrams denotes all concentrations $n$ below $10^{-35} \mathrm{~g} / \mathrm{cm}^{3}$.

10. A. Gupta and S. Sahijpal, arXiv e-prints, arXiv:2004.11328, 2020.

11. T. Jackson, Ž. Ivezić, and G. R. Knapp, Monthly Not. Roy. Astron. Soc., 337, 749, 2002.

12. A. Siebert, O. Bienaymé, and C. Soubiran, Astron. and Astrophys., 399, 531, 2003.

13. M. Matsuura, M. J. Barlow, A. A. Zijlstra, P. A. Whitelock, et al., Monthly Not. Roy. Astron. Soc., 396, $918,2009$.

14. A. Nanni, M. A. T. Groenewegen, B. Aringer, S. Rubele, A. Bressan, J. T. van Loon, S. R. Goldman, and M. L. Boyer, Monthly Not. Roy. Astron. Soc., 487, 502, 2019. 


\title{
EZ Lyn: White Dwarf fast cooling after 2010 outburst
}

\author{
A.A. Sosnovskij, E.P. Pavlenko \\ Federal State Budget Scientific Institution "Crimean Astrophysical Observatory of RAS", Nauchny, 298409, Republic of \\ Crimea, Russia
}

The analysis of the white dwarf pulsations of the dwarf nova EZ Lyn (which outbursted in 2010) in 2015-2020 years based on photometry at 2.6-m Shajn telescope of the Crimean Astrophysical Observatory is presented. It revealed an increase in the period of pulsations from 234 to 756 sec. In accordance with the empirical relationship between the period of the pulsations and the temperature of the white dwarf, this means a fast cooling of the white dwarf after the 2010 outburst.

Keywords: EZ Lyn, cataclysmic variables, stars : binaries

DOI: 10.51194 /VAK2021.2022.1.1.071

\section{Introduction}

The study of accreting pulsating white dwarfs (hereafter WD) among the dwarf novae) is an extremely urgent problem aimed at determining the WDs instability strip. While this strip is well defined for the isolated WDs ( $\mathrm{T}=10800-12300$ K) [1], it is not for accreting WDs. The measurements of a few accreting WDs temperature indicate some wider range $(\mathrm{T}=10500-16000 \mathrm{~K})$. During the evolutionary cooling, isolated WDs pass through the instability strip for millennia. Accreting pulsators can leave the instability strip instigated by outburst heating and re-enter it during the subsequent cooling process, which can last ip to eight years [2]. There is an empirical relation between the WD pulsation period and its effective temperature. On average, the pulsation periods vary from 100 to 1000 sec. when the temperature decreases from "blue" to "red" edge.

In 2014, the number of accreting pulsators reached 14 objects [3], and only eight of this number had an outburst, which made it possible to measure the pulsation periods over several years during the WD cooling. The available information shows that the pulsation behavior of accreting and isolated WDs is very different. [2] published a review of pulsations and temperatures for 12 accreting WDs, which revealed some trends and some "abnormalities" in their behavior. The deviations from the "norm" (if by "normal" we mean the behavior of isolated WDs) can be attributed by pulsations disappearance and resumption on the few day scale, observed in some systems; the drift of pulsations, sometimes reaching 17 sec within five hours; and an uneven change of the pulsations period on a scale of months-years, simulating the uneven WD cooling after an outburst.

EZ Lyn as a cataclysmic variable was discovered by Szkody in its minimum [4]. The object was classified as WZ Sge eclipsing dwarf nova with rebrightenings and 0.06-d orbital period [5], [6]. It entered the instability strip twice: once after 8 months of 2006 outburst and the second time 7 months after the 2010 outburst [3]. The first passage of the instability strip lasted at least $\sim 900$ days and during this time the dominant 12.6-minute pulsations were recorded (as well as their doubled values), drifting chaotically from 732 to $768 \mathrm{sec}$. After the 2010 outburst, the pulsations resumed, however, at a completely different (short) period of 234-235 s (based on data from the 2.6-m ZTSh [7] and Hubble ST [8]. Based on spectral and photometric data and modelling, the secondary component mass was estimated at $0.042 \mathrm{M} \odot$, the mass transfer rate was $0.3-3 \cdot 10^{-12} \mathrm{M} \odot$ /year. It was found that the white dwarf of the system has cooled down by $\approx 3000$ degrees $(14250(250) \mathrm{K}$ - 11250 (50) K) from the 2010 outburst to the mid of 2018 [9]. To analyse the time series we used the Stellingwerf method implemented in the ISDA package [10]

\section{WD Pulsations}

The photometry of EZ Lyn was carried out at the 2.6-m Shajn telescope of the Crimean Astrophysical Observatory in unfiltered light. The periodograms for the longest observation series ( 5 - 9 hours) are shown in Fig.1. The orbital brightness modulation was previously removed. Periodograms for the first five years after the outburst indicate the most significant pulsations with a short period of $\sim 234-235$ sec. and amplitudes of 0.02-0.04 mag., and for the next five years (and continuing at the present time) - on the most significant pulsations with a relatively long period of 1500-1680 sec and the same amplitudes. Note that this is the doubled value of the pulsation period, which dominated after the 2006 outburst for $\sim 2.5$ years.

Examining periodograms, one can notice not only some drift an uneven increase in the period of pulsations, but also their rapid disappearance and rapid recovery (the pulsations that disappeared on December 24 recovered within a day on December 25).

\section{Discussion: uneven increase of pulsation period and WD cooling}

10-year monitoring of WD non-radial pulsations in the EZ Lyn showed that the white dwarf continues to pulsate, which means that it has been in the instability strip for at least 10 years and continues to cool. If we are guided by the empirical dependence of the white dwarf temperature on the average pulsation period for isolated white dwarfs, we get $T \sim 12050$ $\mathrm{K}$ for $P=234 \mathrm{sec}$ and $T \sim 11400 \mathrm{~K}$ for $P=756 \mathrm{sec}$. Note that if a $\sim 1500-1680 \mathrm{sec}$ period is true and 756 sec is its second harmonic, then the WD temperature should be $<10800 \mathrm{~K}$ where the "Temperature-Period" dependence is not defined. So we consider the more reliable period of pulsations to be $756 \mathrm{sec}$. The corresponding temperature estimate is consistent with independent estimates from spectral modelling [9].

So, for 10 years after the outburst, WD, has passed most of the instability strip. However, photometric monitoring did not reveal a smooth increase in the pulsation period (and, hence a smooth decrease of the WD temperature), which would 

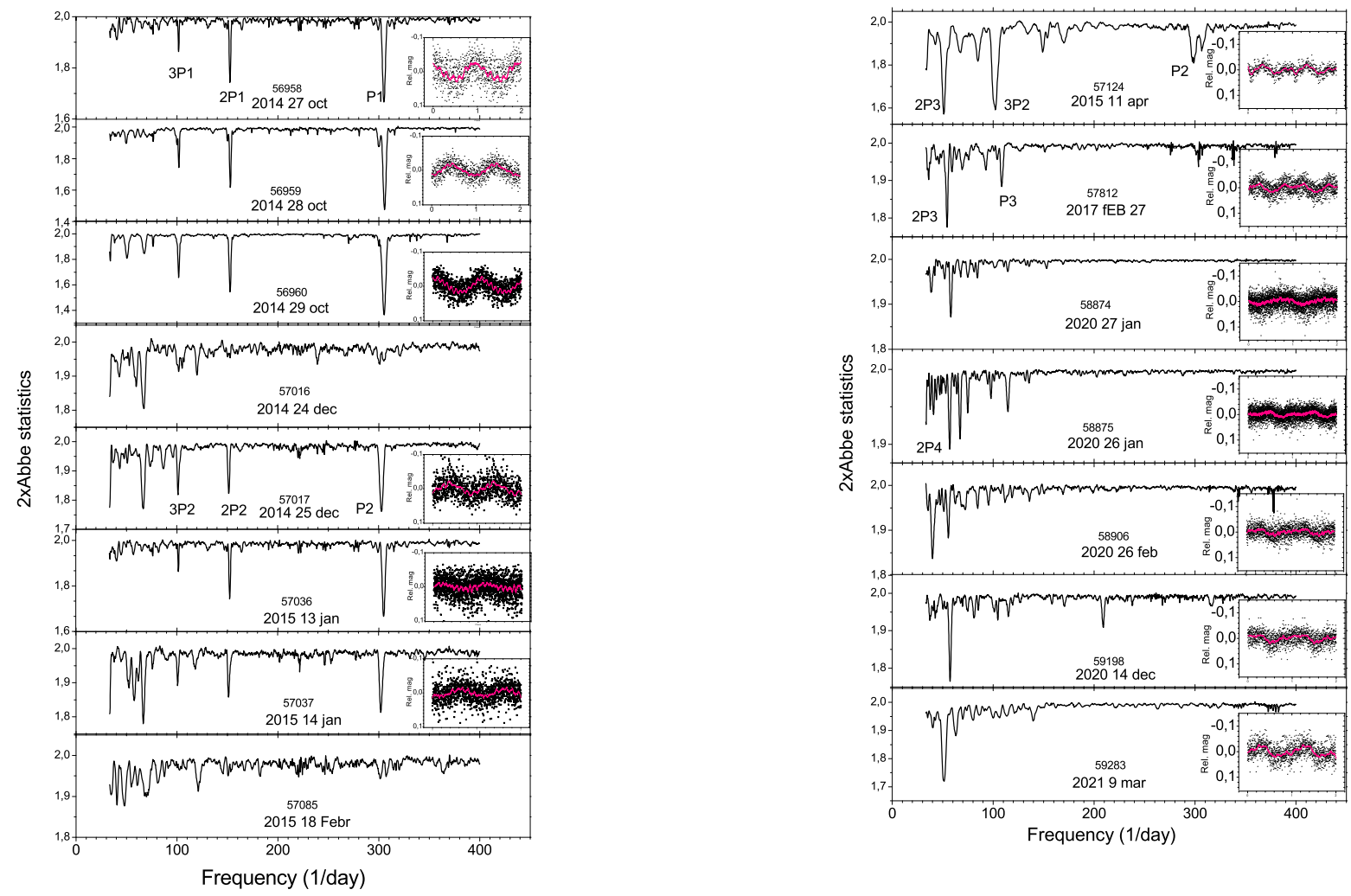

Figure 1: Periodograms for longest nights of observations (2015-2021) in chronological order. Convolutions with a most significant period are shown on the right in the sidebars.

be expected in accordance with theoretical predictions, as the white dwarf cools. Exactly the same non-monotonic cooling of a white dwarf after an outburst, as well as a change in pulsation modes, was found in the prototype of accreting white dwarfs GW Lib [2]. A more detailed investigation of the EZ Lyn pulsation evolution will be published elsewhere.

It is necessary to continue a monitoring of the EZ Lyn pulsations up to their complete disappearance to determine the instability strip of accreting pulsators and the time scale of white dwarf cooling in dwarf nova systems after outburst.

\section{Acknowledgment}

We are grateful for the excellent work and patience during these years to the ZTSh team: chief I. Drozdov, S. Petrov, F. Nemtsev, V. Dolgopolov, V. Frolov, N. Zubko.

Observations are supported by the grant of RSF (Project Number 19-72-10063).

\section{References}

1. A. S. Mukadam, M. H. Montgomery, A. Kim, D. E. Winget, S. O. Kepler, and J. C. Clemens, in R. Napiwotzki and M. R. Burleigh, eds., 15th European Workshop on White Dwarfs, Astronomical Society of the Pacific Conference Series, volume 372, 587 (2007).

2. P. Szkody, A. S. Mukadam, B. T. Gänsicke, P. Chote, et al., AJ, 152, 48, 2016.

3. E. P. Pavlenko, T. Kato, A. A. Sosnovskij, M. V. Andreev, T. Ohshima, A. S. Sklyanov, I. F. Bikmaev, and A. I. Galeev, PASJ, 66, 113, 2014.

4. P. Szkody, A. Henden, L. Mannikko, A. Mukadam, et al., AJ, 134, 185, 2007.

5. E. Pavlenko, S. Y. Shugarov, N. A. Katysheva, D. Nogami, et al., in R. Napiwotzki and M. R. Burleigh, eds., 15th European Workshop on White Dwarfs, Astronomical Society of the Pacific Conference Series, volume 372, 511 (2007).

6. T. Kato, E. P. Pavlenko, H. Maehara, K. Nakajima, et al., PASJ, 61, 601, 2009.

7. E. Pavlenko, V. Malanushenko, G. Tovmassian, S. Zharikov, et al., Mem. Soc. Astron. Ital., 83, 520, 2012.

8. P. Szkody, A. S. Mukadam, E. M. Sion, B. T. Gänsicke, A. Henden, and D. Townsley, AJ, 145, 121, 2013.

9. A. Amantayeva, S. Zharikov, K. L. Page, E. Pavlenko, A. Sosnovskij, S. Khokhlov, and M. Ibraimov, ApJ, 918, 58, 2021.

10. Y. Pel'T, Frequency analysis of astronomical time series. (1980). 


\section{Angular momentum evolution of collapsing magnetic protostellar clouds}

I.M. Sultanov ${ }^{1}$, S.A. Khaibrakhmanov ${ }^{1,2}$, A.E. Dudorov $^{1,2}$

syltahof@yandex.ru

${ }^{1}$ Chelyabinsk State University, Chelyabinsk 454001, Russia,

${ }^{2}$ Ural Federal University, Yekaterinburg 620002, Russia

DOI: $10.51194 /$ VAK2021.2022.1.1.072

The magnetic braking catastrophe of protostellar discs is one of the unsolved problems of star formation theory. Solution of this problem consits in the weakening of magnetic flux of protostellar clouds (magnetic field diffusion, turbulence), however the relative role of different mechanisms is still not clear.

We model isothermal stage of collapse of protostellar clouds with frozen-in magnetic field and analyze the effiency of magnetic braking of the cloud. We use one-and-half dimensional approach of [1], which let us study influence of magnetic field on the collapse dynamics in wide range of initial parameters without significant numerical costs.

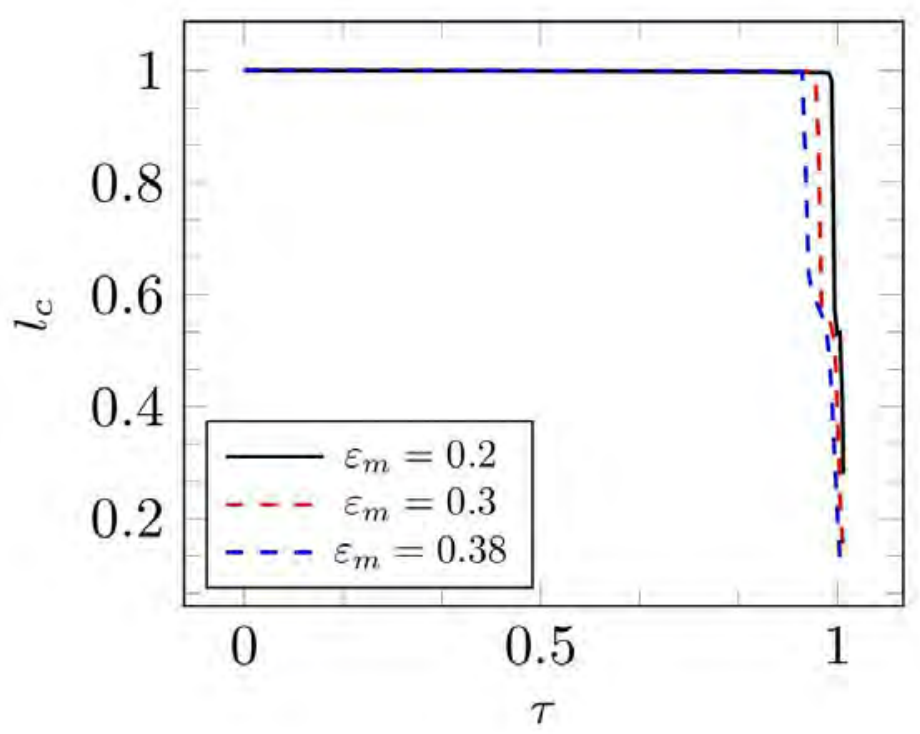

Figure 1: Dependence of the specific angular momentum in the cloud's center, $l_{c}=L_{c} / L_{0}$, on time $\tau=t / t_{\mathrm{ff}}$. Here $L_{0}$ is initial angular momentum, $t_{\mathrm{ff}}$ is free fall time.

We study the collapse of a cloud with mass $1 M_{\odot}$ and temperature $15 \mathrm{~K}$. Parameters of the simulations are various initial ratios of thermal $\varepsilon_{T}$ rotational $\varepsilon_{\omega}$ and magnetic energy $\varepsilon_{m}$ to the gravitational energy.

The dependence of specific angular momentum in the cloud's center on time for $\varepsilon_{T}=0.6, \varepsilon_{\omega}=0.01$ and different $\varepsilon_{m}$ is shown in Figure 1. Angular momentum remains constant until $\tau=0.95-0.99$ and then rapidly decreases. For $\varepsilon_{m}=0.2-0.38$ the angular momentum loss is of $72-83 \%$.

Our results show that the magnetic braking can be effective at the isothermal stage of the collapse. This effect requires further investigation.

Acknowledgements. The work of S. Khaibrakhmanov is supported by The Russian Science Foundation (project 19-72-10012).

\section{References}

1. A. E. Dudorov and Y. V. Sazonov, Nauchnye Informatsii, 49, 114, 1981. 


\title{
Observations of the gamma-ray pulsar J1836+5925 at $111 \mathrm{MHz}$
}

\author{
M. Timirkeeva, I. Malov, V. Malofeev, O. Malov \\ timirkeeva@prao.ru, malov@prao.ru,malofeev@prao.ru,jedi@prao.ru, \\ WWW home page: http://www.prao.ru \\ P.N. Lebedev Physical Institute of the Russian Academy of Sciences, Leninskii pr. 53, Moscow, 119991, Russia
}

We describe the 10-day radio observations of the gamma-ray pulsar J1836 +5925 . Observations were carried out on the Pushchino Radio Astronomical Observatory at the frequency of $111 \mathrm{MHz}$ using the Large Phased Array of the Lebedev Physical Institute.

Keywords: pulsars: general; gamma-rays: stars; X-rays: stars

DOI: $10.51194 / \mathrm{VAK} 2021.2022 .1 .1 .073$

\section{Introduction}

The pulsar was discovered during the EGRET mission aboard the NASA Compton Gamma-Ray Observatory (CGRO) by [1] and later by [2]. It is also called as GRO J1837+59, 2EG J1835+5919, 3EG J1835+5918 and GEV J18351+5921. Attempts were made to identify it in optical and radio range [3] and [4]. According to the ATNF catalog its period was set by [5]. This pulsar is called Geminga-like because it have some characteristic features of Geminga: there is hard radiation with no recorded radio emission.

In the [6] we suggested that gamma-ray pulsars with strong magnetic fields at the light cylinder and high rotational energy loss rates can could radiate also radio emission. This article is devoted to the search for radio emission of this pulsar.

\section{Radio observations}

Radio observations of the gamma-ray pulsar J1836+5925 were carried out using the meridian radio telescope LPA (Large Phase Array). Its antenna is the phased array composed of 16384 dipoles. The geometric area of this antenna is more than $70000 \mathrm{~m}^{2}$ and the effective area is $47000 \pm 2500 \mathrm{~m}^{2}$. Antenna has 64 space beams with the size of one beam $1^{\circ} \times 0.5^{\circ}$. The duration of an observing session is about 3.5/ $\cos \delta$ minutes [7]. In order to make a more sensitive search for radio pulsations from gamma-ray pulsar J1836 +5925 observations were carried out with the set durations about $5-10$ days each on more than 200 days for 4 years at the frequency of $111 \mathrm{MHz}$. For the processing 460 channels of the 512 channel digital receiver are used. The single channel bandwidth is $4.88 \mathrm{kHz}$, and the time resolution is $2.46 \mathrm{msec}$, the total bandwidth is 2.245 $\mathrm{MHz}$. All data is stored in the server. For the processing the special program has been worked out by [8]. Data processing is carried out in 2 stages. First, there is an accumulation with a pulsar period and a search for dispersion measures from 2 to $20 \mathrm{~cm}^{-3} \mathrm{pc}$. Then the search for strong individual pulses with the presence of a dynamic spectrum in the bandwidth of the receiver. At the first stage to verify the presence of weak signals and enhance the reliability of results we selected observing sessions equal to double or triple periods. To increase the reliability of identification of the pulsar signal during the observing sessions 725 (for triple period window) pulses have been accumulated. After analyzing other works ([3,4] and [5]) we proposed to concentrate our efforts in the search for radiation in radio range for dispersion measures from 2 to 20 $\mathrm{cm}^{-3}$ pc. We carried out 10-day sessions of observations of the pulsar J1836+5925 in May 2021. We removed the 1-4 May data from the consideration because they had a low quality caused by interferences. After summation of all pulses we get the weak pulsed signal with the signal-to-noise ratio (SNR) no more than 4 for several values of the dispersion measure $(\mathrm{DM})$. The value of this DM is unknown up to now. Unfortunately, we were unable to find a reliable integrated signal on a certain dispersion measure over 6 days of observations.

\section{Results}

The analysis of the received data showns that there is no any relationship between the values of SNR and the DM after summation of 725 pulses. It is necessary to consider in more detail each pulse for each dispersion measure (in range from 2 to $20 \mathrm{~cm}^{-3} \mathrm{pc}$ ) and for each day. We separate the strongest pulses (with a signal-to-noise ratio of more than 4 ) in the range of dispersion measures from 2 to $20 \mathrm{~cm}^{-3} \mathrm{pc}$ ) in the analyzed data set and plotted the following dependencies: the number of such pulses in 10 intervals of the dispersion measures (Fig. 1) and the number of strong pulses depending on the phase of their arrival in a certain time interval equal to 3 pulsar periods (see Fig. 1 and 2).

For a deeper analysis of the data and search for the radio emission, we analyzed the distribution of the dispersion measure in the strongest pulses (see Fig. 1).

After analyzing the data of Fig. 1, it became clear that there was not an expected strongly significant range of DM. We do not see an evident maximum.

In the case of presence of several strong individual pulses (observed with triple period) in one day of observations, we could expect the presence of three maxima (Fig. 2) with a phase difference of 70 points after summing up the records for 6 days. We got 2 wide ranges with "collapse" in the phases of 60-90 points. If the number of observed days increases, this collapse becomes more significant.

Thus, we can conclude that during 6 days of observations we failed to detect pulsed periodic radiation at the level of 82 mJy [9], and also individual pulses with SNR $\geq 5$ with the presence of a dynamic spectrum in the bandwidth of receiver used for our observations were not detected.

The findings are of direct practical relevance for further search of radio emission of $\mathrm{J} 1836+5925$. 


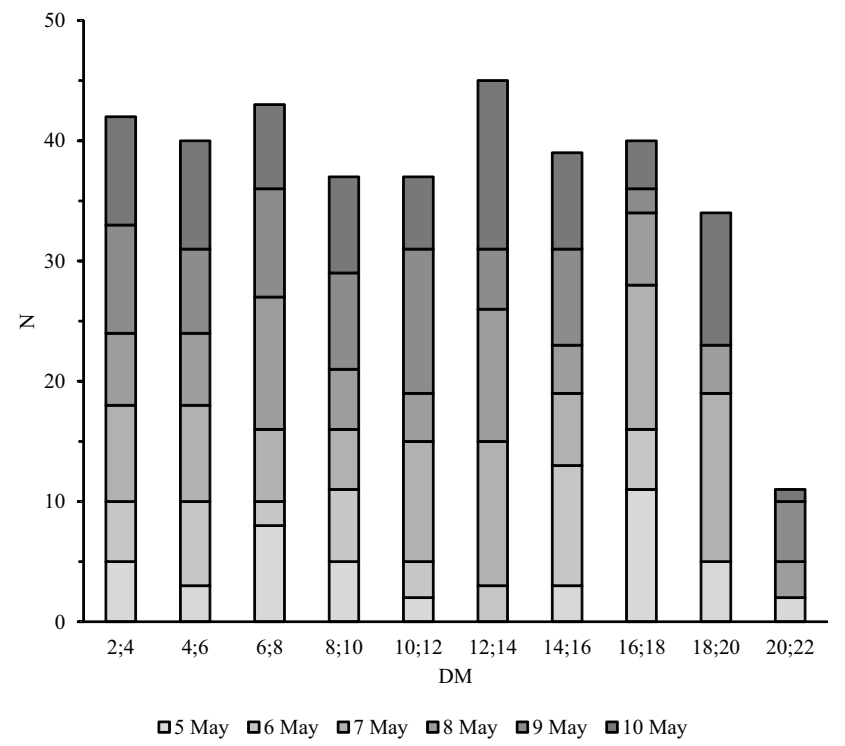

Figure 1: The distribution of the number of individual pulses with the different DM for 6-days observations in May 2021

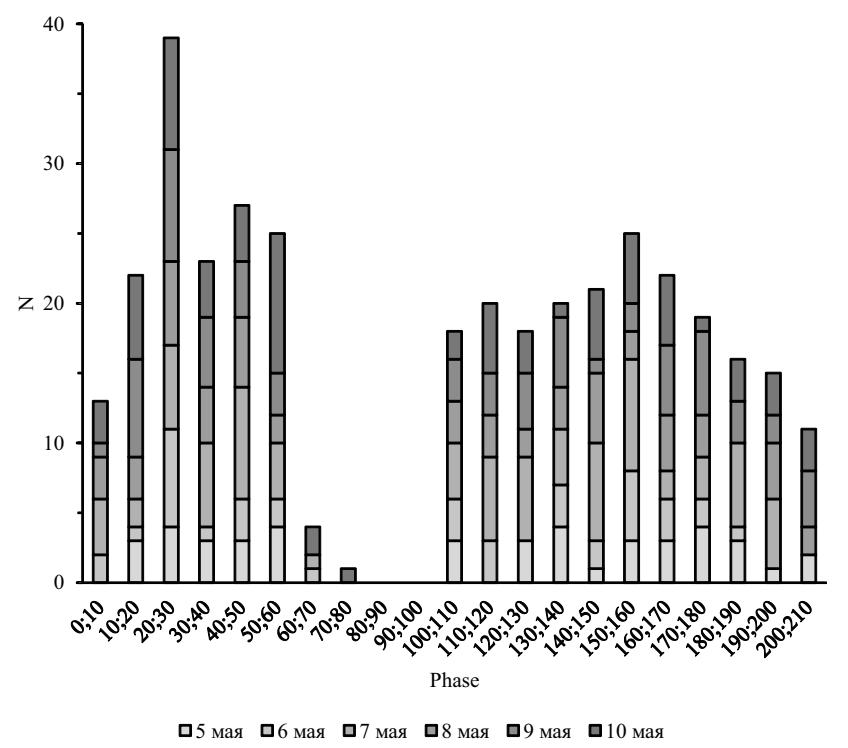

Figure 2: The distribution of phases for 6-days of observations in May 2021

\section{References}

1. Y. C. Lin, J. Chiang, J. Fierro, P. F. Michelson, P. L. Nolan, K. Brazier, and e. a. G. Kanbach, IAU Circ., 5676, 360, 1994.

2. P. L. Nolan, J. M. Fierro, Y. C. Lin, P. F. Michelson, T. D. Willis, and J. Chiang, AIP Conf. Proc., 304, $360,1994$.

3. J. P. Halpern, E. V. Gotthelf, N. Mirabal, and F. Camilo, ApJ, 573, L41, 2002.

4. J. P. Halpern, F. Camilo, and E. V. Gotthelf, ApJ, 668, 1154, 2007.

5. A. A. Abdo, M. Ackermann, M. Ajello, W. B. Atwood, L. Baldini, and J. B. et al., ApJ, 712, 1209, 2010.

6. I. F. Malov and M. A. Timirkeeva, $R A A, \mathbf{1 8}, 89,2018$.

7. S. A. Tyul'bashev, V. S. Tyul'bashev, V. V. Oreshko, and S. V. Logvinenko, Astron. Rep., 60, $220,2016$.

8. V. M. Malofeev, D. A. Teplykh, and S. V. Logvinenko, Astron. Rep., 56, 35, 2012.

9. M. A. Timirkeeva, I. F. Malov, and O. I. Malov, Ground-Based Astronomy in Russia. 21st Century, Proceedings of the All-Russian Conference held 21-25 September, 2020 in Nizhny Arkhyz, Russia. Edited by I. I. Romanyuk, I. A. Yakunin, A. F. Valeev, D. O. Kudryavtsev, 187, 451, 2020. 


\section{Planets and Planetary Systems}




\title{
Comparison of non-thermal atmospheric losses for hot exoplanets
}

\author{
A. Avtaeva ${ }^{1,2}$, V. Shematovich ${ }^{2}$ \\ ${ }^{1}$ Sternberg Astronomical Institute, Moscow State University, Moscow 119234, Russia \\ ${ }^{2}$ Institute of Astronomy of the Russian Academy of Sciences, Moscow 11901\%, Russia
}

The non-thermal atmospheric losses due to the contribution of the exothermic photochemistry to the formation of a fraction of suprathermal atomic hydrogen in the $\mathrm{H}_{2} \rightarrow \mathrm{H}$ transition region were studied and compared for two exoplanets of different types and different parent star. Exoplanet GJ 436b is a warm neptune orbiting the red dwarf GJ 436. The calculated nonthermal fluxes due to the exothermic photochemistry for both exoplanets were found in the range $(3.0-3.5) \times 10^{12} \mathrm{~cm}^{-2}$ $\mathrm{s}^{-1}$ for a moderate level of stellar activity in UV radiation.

Keywords: exoplanets, non-thermal atmospheric losses, planetary atmospheres, numerical modeling

DOI: $10.51194 /$ VAK2021.2022.1.1.074

\section{Introduction}

In recent studies (see for example, [1]) it was found, that the most frequently discovered planets are hot sub-neptunes planets located quite close to their parent stars, in orbits closer then Mercury to Sun, and placed by radius between the Earth and Neptune. The proximity of the planets to the parent stars results in the intense influence of the stellar radiation on the exoplanetary upper atmospheres. The most intense fluxes of stellar radiation are in the ranges of soft X-rays $(1-10$ $\mathrm{nm}$ ) and extreme ultraviolet (Extreme UltraViolet - EUV, $10-100 \mathrm{~nm}$ ), at such close orbital distances will significantly change the structure and composition of the upper layers of the atmospheres of these planets [2] and, in particular, lead to the formation of extended gas envelopes of hot exoplanets (see, for example, [3]) and total or partial loss of the atmosphere.

In this work, the non-thermal processes in the upper atmospheric layers of exoplanets were studied and the escape fluxes from the atmosphere due to these processes were calculated using the numeric model of a hot planetary corona described in detail in [4].

\section{Warm neptune GJ 436b}

Exoplanet GJ 436b is a warm neptune with radius $R_{p}=4.2 R_{\text {Earth }}$ and mass $M_{p}=22.2 M_{\text {Earth }}$, orbiting with a semi-major axis of $0.028 \mathrm{AU}$ around the red dwarf GJ 436. The first gas dynamic models GJ $436 \mathrm{~b}$ (all references of this planet are in [4]) had showed that molecular hydrogen does not dissociate completely in the upper atmosphere of GJ 436b and is carried away with the atmospheric outflow far from the planet. The values of atmospheric loss rate due to hydrodynamic outflow and radiation pressure were found in the range $\sim\left(5.0 \times 10^{8}-5.0 \times 10^{9}\right) \mathrm{g} \mathrm{s}^{-1}$.
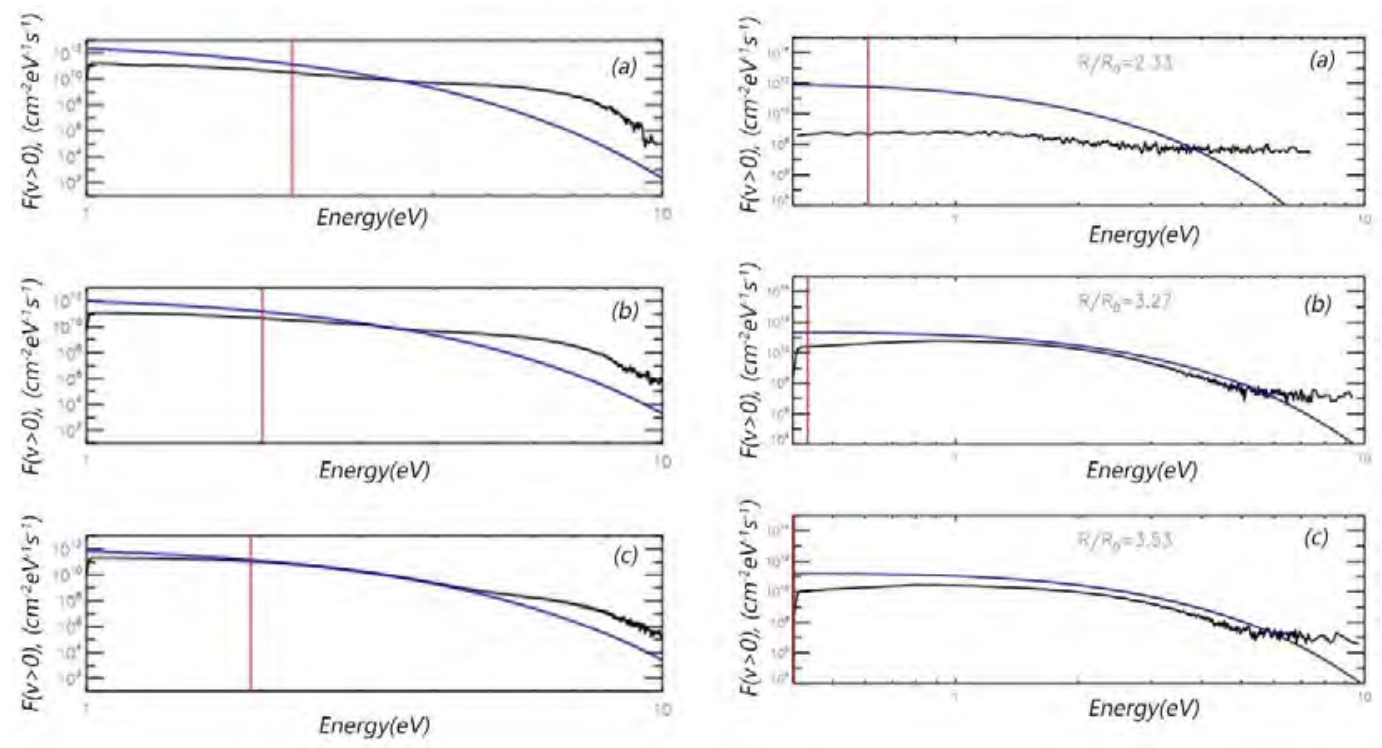

Figure 1: Calculated energy spectra of the flux of upward moving suprathermal hydrogen atoms at heights of $1.56 R_{p}(\mathrm{a}), 1.75 R_{p}$ (b), and $1.84 R_{p}$ (c) for exoplanet GJ $436 \mathrm{~b}$ (left panel) and at heights of $2.33 R_{p}$ (a), 3.27 $R_{p}$ (b) and $3.53 R_{p}$ (c) for exoplanet $\pi$ Men c (right panel). The blue lines show the fluxes of thermal $\mathrm{H}$ atoms calculated for the locally equilibrium distribution of atomic hydrogen in accordance with the temperature profile from the models ([5] for GJ 436b; [6] for $\pi$ Men c). The vertical red lines show the escape energies of hydrogen atoms at given heights. 
In the recent study it was found that the processes of $\mathrm{H}_{2}$ dissociation by stellar extreme UV radiation are an important source of suprathermal hydrogen atoms in the extended upper atmosphere of exoplanet GJ 436b, resulting in the formation of a stable fraction of suprathermal hydrogen atoms. The escape flux is estimated to be $3.0 \times 10^{12} \mathrm{~cm}^{-2} \mathrm{~s}^{-1}$ (Fig. 1 , left panel) for a moderate level of stellar activity in UV radiation in the planet-star direction. This flux escapes atmosphere through the upper boundary of the transition region (located at an altitude of $\sim 1.84 R_{p}$ ), and results in the atmospheric loss rate equal to $7.8 \times 10^{8} \mathrm{~g} \mathrm{~s}^{-1}$ due to the $\mathrm{H}_{2}$ dissociation processes. This estimate corresponds to the escaping flux range of $\sim\left(3.7 \times 10^{6}-1.1 \times 10^{9}\right) \mathrm{g} \mathrm{s}^{-1}$ obtained from observations of the possible atmospheric loss rate of exoplanet GJ $436 \mathrm{~b}$.

\section{Sub-neptune $\pi$ Men c}

Exoplanet $\pi$ Men c is a super-earth or sub-neptune with radius $R_{p}=2.06 R_{\text {Earth }}$ and mass $M_{p}=4.52 M_{\text {Earth }}$, orbiting with a semi-major axis of $0.067 \mathrm{AU}$ around the sun-like star $\pi$ Men. HST observations $[7,8]$ showed the formation of extended atmosphere of hot sub-neptune $\pi$ Men c. From estimates of the planet mass and bulk density of the hot sub-neptune $\pi$ Men c, it can be assumed that this planet could hold a significant atmosphere [7, 9]. In the recent study by [8] the results of the search for atmospheric hydrogen formed due to $\mathrm{H}_{2}$ and $\mathrm{H}_{2} \mathrm{O}$ photodissociation in the upper atmosphere of $\pi$ Men c were given using the transmission spectroscopy in the HI Ly $\alpha$ line in HST/STIS spectrograph observations. However, the absorption in this line was not detected.

Current aeronomic models $[9,8,6]$ of the upper atmosphere of the exoplanet $\pi$ Men c predict a significant rate of atmospheric loss. Calculations of the energy spectra of the upward flux of suprathermal hydrogen atoms were carried out using the numerical model [4], and are presented in right panel of Fig. 1. The calculated energy spectrum of the suprathermal $\mathrm{H}$ flux escaping from the atmosphere through the upper boundary of the transition region at a height of $\sim 3.6 R_{p}$ is shown by black line on the right bottom panel of Fig. 1. Using it we can obtain the following estimate of the non-thermal escape flux with value of $3.5 \times 10^{12} \mathrm{~cm}^{-2} \mathrm{~s}^{-1}$ in the direction of the planet-star under conditions of a moderate level of stellar activity in the considered range of UV radiation. Note that this calculated value of the non-thermal escape flux of suprathermal hydrogen atoms is comparable to the value of $3.9 \times 10^{12} \mathrm{~cm}^{-2} \mathrm{~s}^{-1}$ of the thermal flux calculated by the Jeans formula for the numerical flux of thermal hydrogen atoms (blue line on the right bottom panel of Fig. 1).

\section{Comparison of calculation results}

Let's compare the results obtained in the studies of these two planets. GJ 436b is larger and heavier planet than $\pi$ Men c, and its atmosphere is more compact (the upper boundary of the transition region is placed at $\sim 1.84 R_{p}=7.7 R_{\text {Earth }}$ ), and more pressed by gravity to the surface. Accordingly, the transition region is less extended, therefore the probability of escape from the atmosphere is higher. Whereas, in the case of exoplanet $\pi$ Men c, the transition region is more extended (the upper boundary of the transition region is placed at $\sim 3.6 R_{p}=9.4 R_{\text {Earth }}$ ), and before escaping from the atmosphere, the suprathermal atoms have to experience many collisions with the surrounding atmospheric gas, in which their excess energy is lost and, accordingly, the escape flux should decrease. But if we compare the escape flux values obtained in the calculations, it could be seen that for $\pi$ Men c the average non-thermal loss rate is equal to $9.1 \times 10^{8} \mathrm{gs}^{-1}$. In the case of warm neptune GJ 436b, the average non-thermal loss rate is equal to $7.8 \times 10^{8} \mathrm{~g} \mathrm{~s}^{-1}$. This is due to the effect of contrary circumstances - the escape probabilities are higher for neptune GJ 436b because of less extended transition region, but the XUV radiation flux is higher for $\pi$ Men c because its parent star is a sun-like star while GJ 436 is a red dwarf.

This study was supported by Project No. 075-15-2020-780 "Theoretical and experimental studies of the formation and evolution of extrasolar planetary systems and characteristics of exoplanets" of the Ministry of Science and Higher Education of the Russian Federation.

\section{References}

1. F. Fressin, G. Torres, D. Charbonneau, S. T. Bryson, et al., ApJ, 766, 81, 2013.

2. J. E. Owen, Annual Review of Earth and Planetary Sciences, 47, 67, 2019.

3. D. V. Bisikalo, P. V. Kaygorodov, and V. I. Shematovich, Exoplanets: Atmospheres of Hot Jupiters, 103 (2019).

4. A. A. Avtaeva and V. I. Shematovich, Solar System Research, 55, 150, 2021.

5. A. G. Berezutsky, I. F. Shaikhislamov, I. B. Miroshnichenko, M. S. Rumenskikh, and M. L. Khodachenko, Solar System Research, 53, 138, 2019.

6. I. F. Shaikhislamov, L. Fossati, M. L. Khodachenko, H. Lammer, A. García Muñoz, A. Youngblood, N. K. Dwivedi, and M. S. Rumenskikh, $A \mathscr{E} A$, 639, A109, 2020.

7. D. Gandolfi, O. Barragán, J. H. Livingston, M. Fridlund, et al., AछA, 619, L10, 2018.

8. A. García Muñoz, A. Youngblood, L. Fossati, D. Gandolfi, J. Cabrera, and H. Rauer, ApJL, 888, L21, 2020.

9. G. W. King, P. J. Wheatley, V. Bourrier, and D. Ehrenreich, MNRAS, 484, L49, 2019. 


\title{
The impact of star spots and other photospheric structures on exoplanetary transit timings
}

\author{
R.V. Baluev ${ }^{1}$, E.N. Sokov ${ }^{1,2}$, V.Sh. Shaidulin ${ }^{1}$, and the EXPANSION team \\ r.baluev@spbu.ru \\ ${ }^{1}$ Saint Petersburg State University, 7-9 Universitetskaya emb., Saint Petersburg 199034, Russia, \\ ${ }^{2}$ Central Astronomical Observatory at Pulkovo of RAS, Pulkovskoje sh. 65/1, Saint Petersburg 196140, Russia
}

We consider possible ways to explain the issue of excessive noisy transit timing variation (TTV) observed for several transiting exoplanets. In particular, for HD $189733 \mathrm{~b}$ we find a TTV jitter of $\sim 70 \mathrm{~s}$ that cannot be easily explained by a simple model. A possible cause can be the effect of photospheric activity structures on the derived transit timings. Based on our systematic search of 1598 transit lightcurves for 26 targets, we found 109 potential spotcrossing or facula-crossing events. However, this rate appears too low to explain the observed TTV excess, espicially for HD 189733.

Keywords: extrasolar planets, transit photometry, TTV, starspots

DOI: 10.51194 /VAK2021.2022.1.1.075

\section{Introduction}

Transit timing variations, or TTVs, appear if the Keplerian motion of a transiting exoplanet is somehow perturbed. For example, the perturbation can come from another body in the system, so the TTVs can work as an additional method of exoplanet detection [1,2]. Using this method, multiple exoplanets were already detected in Kepler mission data [3, 4, 5].

Our EXPANSION project (EXoPlanetary trANsit Search with an International Observational Network) is a groundbased TTV programme, grown on the basis of the Exoplanet Transit Database [6, 7, 8, 9, 10]. It builds up an international network of several dozens of amateur and professional telescopes, aimed to monitor exoplanetary transits. The observatories are spread over the world in the both hemispheres.

An important issue was revealed with timing analyis of these data. Some targets show an obvious TTV excess above the expected timing uncertainties, and it cannot be explained through a simple deterministic model (like polynomial trend or sinusoidal variation). For example, HD $189733 b$ reveals TTV noise roughly twice as expected, with TTV excess above $1 \mathrm{~min}$. It was hypothesized that this can be related to stellar activity, and HD 189733 is indeed known for a stronger activity $[11,12,13]$.

Our ultimate goal is to verify this hypothesis and to construct a practical way to model the associated TTV noise in HD 189733 and in other targets.

\section{Star spots as possible cause}

Star spots and star faculae are the most obvious activity manifestation capable to distort apparent exoplanetary transit times. See the detailed review in [10], where an attempt is presented of a systematic search of all statistically significant spot-crossing events in a big photometric database. Formally, each spot- or facula-crossing event triggers a bell-like anomaly in the transit lightcurve that we model by a Gaussian:

$$
m_{\mathrm{GA}}(t, K, \mu, \sigma)=K \exp \left(-\frac{(t-\mu)^{2}}{2 \sigma^{2}}\right),
$$

In our conventions, $K>0$ for facula-crossings and $K<0$ for spot-crossings.

We considered a sample of 1598 exoplanetary transits where we found 109 such Gaussian Anomalies (GAs). Their parameters are shown in Fig. 1. Although about a hundred of potential events were detected, this corresponds to just a few per cent of all transit lightcurves. No obvious correlation was revealed between the TTV excess and the number of GAs detected for a particular target. Therefore, starspots cannot offer an easy explanation of the TTV noise issue.

\section{Further work}

Since direct modeling of individual spot anomalies does not explain the TTV issue, on the next step we undertook an attempt to model the photospheric activity regions statistically, expressing the star surface brightness as a random field with multiple inhomogeneities. This new analysis is presented elsewhere [14]. But summarizing its results in brief, in this way we still cannot consistently explain the TTV scatter in HD 189733. No more than $\sim 10 \mathrm{~s}$ can be explained of the observed $70 \mathrm{~s}$ TTV jitter. This requires us to promote different hypotheses, like the effect of planetary atmosphere or complicated perturbations from other possible bodies.

The support of Ministry of Science and Higher Education of Russian Federation is acknowledged under the grant 075-15-2020-780 (N13.1902.21.0039).

\section{References}

1. M. J. Holman and N. W. Murray, Science, 307, 1288, 2005.

2. E. Agol, J. Steffen, R. Sari, and W. Clarkson, MNRAS, 359, 567, 2005.

3. E. B. Ford, J. F. Rowe, D. C. Fabrycky, J. A. Carter, et al., $A \& A$, 197, 2, 2011. 


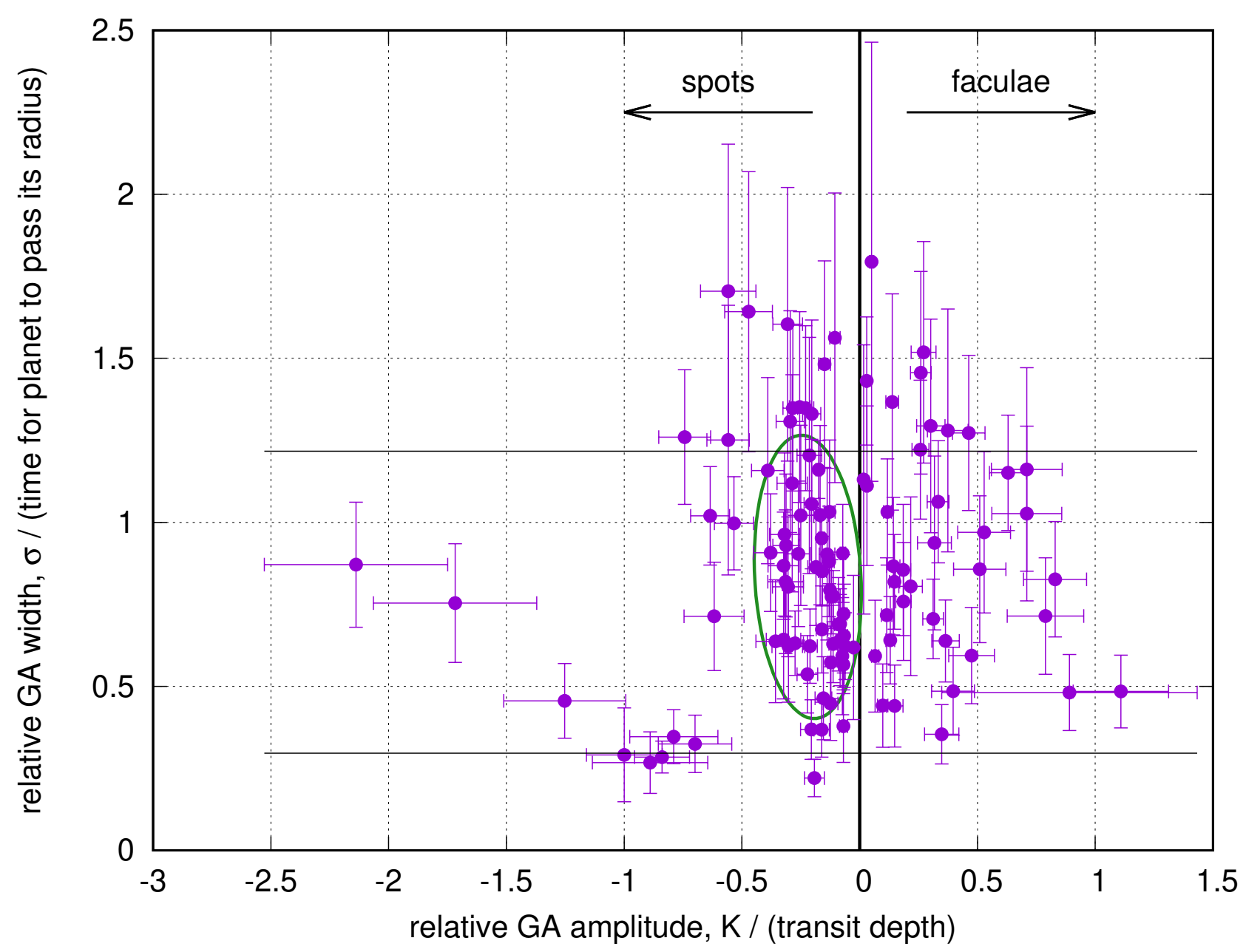

Figure 1: All detected GAs in the amplitude--width diagram. We outline a slightly inclined concentration likely reflecting physical spot-crossing events.

4. J. H. Steffen, D. C. Fabrycky, E. Agol, E. B. Ford, et al., MNRAS, 428, 1077, 2013.

5. J.-W. Xie, ApJS, 208, 22, 2013.

6. R. V. Baluev, E. N. Sokov, V. S. Shaidulin, I. A. Sokova, et al., MNRAS, 450, 3101, 2015.

7. E. N. Sokov, I. A. Sokova, V. V. Dyachenko, D. A. Rastegaev, et al., MNRAS, 480, 291, 2018.

8. R. V. Baluev, E. N. Sokov, H. R. A. Jones, V. S. Shaidulin, et al., MNRAS, 490, 1294, 2019.

9. R. V. Baluev, E. N. Sokov, S. Hoyer, C. Huitson, et al., MNRAS, 496, L11, 2020.

10. R. V. Baluev, E. N.Sokov, I. A. Sokova, V. S. Shaidulin, et al., Acta Astron., 71, 25, 2021.

11. I. Boisse, C. Moutou, A. Vidal-Madjar, F. Bouchy, et al., A\&A A, 495, 959, 2009.

12. I. Pillitteri, S. J. Wolk, J. Lopez-Santiago, H. M. Günther, S. Sciortino, O. Cohen, V. Kashyap, and J. J. Drake, ApJ, 785, 145, 2014.

13. P. W. Cauley, S. Redfield, and A. G. Jensen, $A J, \mathbf{1 5 3}, 217,2017$.

14. R. V. Baluev, MNRAS, 509, 1048, 2022. 


\title{
Active asteroids of the Main Belt as probable relics of the formation processes of giant planets
}

\author{
V.V. Busarev \\ busarev@sai.msu.ru \\ Lomonosov Moscow State University, Sternberg Astronomical Institute, University Av., 13, Moscow, 119992, Russia
}

The discovery and study of cometary activity of some bodies in the Main-Belt of asteroids (MBA) over the past decade allowed us suggest that the most common cause of this phenomenon is a significant content of water ice in the subsurface layers of primitive asteroids. Accordingly, this may be a sign of the formation of such asteroids (or their parent bodies) behind the snowline. As demonstrated analytical calculations performed more than 50 years ago [1], a sharp increase in the number and total mass of stone-ice bodies behind the snowline let possible the runaway growth of giant planets there, especially Jupiter. As follows from predominately hydrogen content of Jupiter's atmosphere, its core probably formed over a few first million years due to the accretion of smaller bodies. However, should keep in mind that this process gradually changed into its opposite. When the core of a giant planet is reaching several masses of the Earth, the capture and absorption by it of smaller bodies is replacing by their gravitational ejection far beyond the zone of formation. Thus, all giant planets might have alternately turned (starting with Jupiter and ending with the most distant Neptune in accordance with the chronology of their formation) into "transporters" of small bodies of rock-ice composition from their zones of formation to other regions of the early Solar System including the MBA, where they now could be observed as active asteroids.

Keywords: asteroids, spectrophotometry, ices, sublimation activity, formation of giant planets

DOI: $10.51194 /$ VAK2021.2022.1.1.076

\section{Introduction}

As is seen from the structure of modern Solar System and its simulation, as well as from the results of study of exoplanetary systems, gravitational resonances control not only the formation of planetary systems, but also support their subsequent stability. The same is probably true for the populations of small bodies of the Solar System, which will be discussed here.

The key importance of gravitational resonances at the formation of planetary bodies is hinted by the similarity of the structure of protoplanetary disks (PPDs) around young stars. According to the data of the mm-range ALMA radio telescope system (e. g., [2]; [3]), the ring structure of PPDs describes the distribution of gas-dust matter which characterizes possible mechanisms of interactions between growing embryos of proto-planets (e. g., [4]; [5]). But what factors contribute to the appearance of the initial heterogeneity of the PPDs' substance, which leads to resonances? Models of the early Solar system formation (e.g., [1]; [6]; [7]; [8], and references there in) show that the main factors are the gravitational and stream instabilities, as well as the accumulation of the most abundant chemical compounds (silicates, $\mathrm{H}_{2} \mathrm{O}$ ice, etc.) near the boundaries of their condensation at different distances from the central star, where the formation of planetesimals and planetary embryos are possible.

Similarity of resonant structures of PPDs and that of the MBA with Kirkwood gaps (corresponding to the mean motion resonances "asteroid/Jupiter", such as 3/1,5/2, 7/3, and 2/1) points to similar mechanisms of their formation. Probably, we now observe the resonant structure of the MBA that was established after the finishing of Jupiter's formation. However, despite of the correct (relative to the Sun) disposition of the maxima of concentrations of high-temperature asteroids, at the MBA's inner boundary, and the low-temperature asteroids at its outer boundary, the spatial proximity of these distributions (Fig. 1, [9]) looks strange. Based on this, we assumed that the heliocentric distributions of primitive and magmatic asteroid classes do not correspond to more smooth preliminary heliocentric distribution of the matter composition in the early Solar System. The distribution of asteroids by composition in the MBA might have been significantly changed under the action of small bodies invading from outside. This probably occurred due to planet-formational processes in the region of Earth-group planets and beyond the snowline. Let us consider the latter in more details.

Another universal mechanism, along with gravitational resonances at formation of planets, is the process of cleaning their feeding zones from smaller bodies. This mechanism is now known as the "gravity assist" and used to correct the trajectory and velocity of a spacecraft by means of pointing it sufficiently close to a massive celestial body, e. g. a planet. As a result, the vector and value of the spacecraft speed can be considerably changed under the gravitational influence of the planet. The mechanism was first proposed by [1], along with scanning the MBA by gravitational resonances at the growth of Jupiter, to explain removing the predominant amount of matter from the formation zone of parent bodies of asteroids (PBAs). We will show further that this mechanism probably played a key role in the formation of the structure and the distribution of matter by composition in the MBA.

\section{Active main-belt asteroids}

The cometary activity of some bodies in the MBA recorded over the last $\sim 15$ years (now known more than 30 of them, e. g., [10]) can be caused by different reasons, such as collisions and fragmentation of asteroids, sublimation of the exposures of ice on the asteroid surface, dehydration stresses, thermal fracture, electrostatic repulsion, and radiation pressure sweeping or their combinations [11]. But it is obvious, the most frequent of them is sublimation activity, which is connected with a considerable content of volatile compounds (mainly $\mathrm{H}_{2} \mathrm{O}$ ice, because of a considerably higher volatility of close to it in parameters and abundance $\mathrm{CO}_{2}$ ice) in the matter of main-belt primitive asteroids. Taking into account a probable high porosity and, respectively, a low thermal conductivity of the surface layers of such asteroids, it was shown that $\mathrm{H}_{2} \mathrm{O}$ ice could be preserved for more than 4 billion years in their interiors [12]. In turn, it may be considered as an indication of the 


\section{Heliocentric distribution of Tholen's (1989) asteroid taxonomic classes}

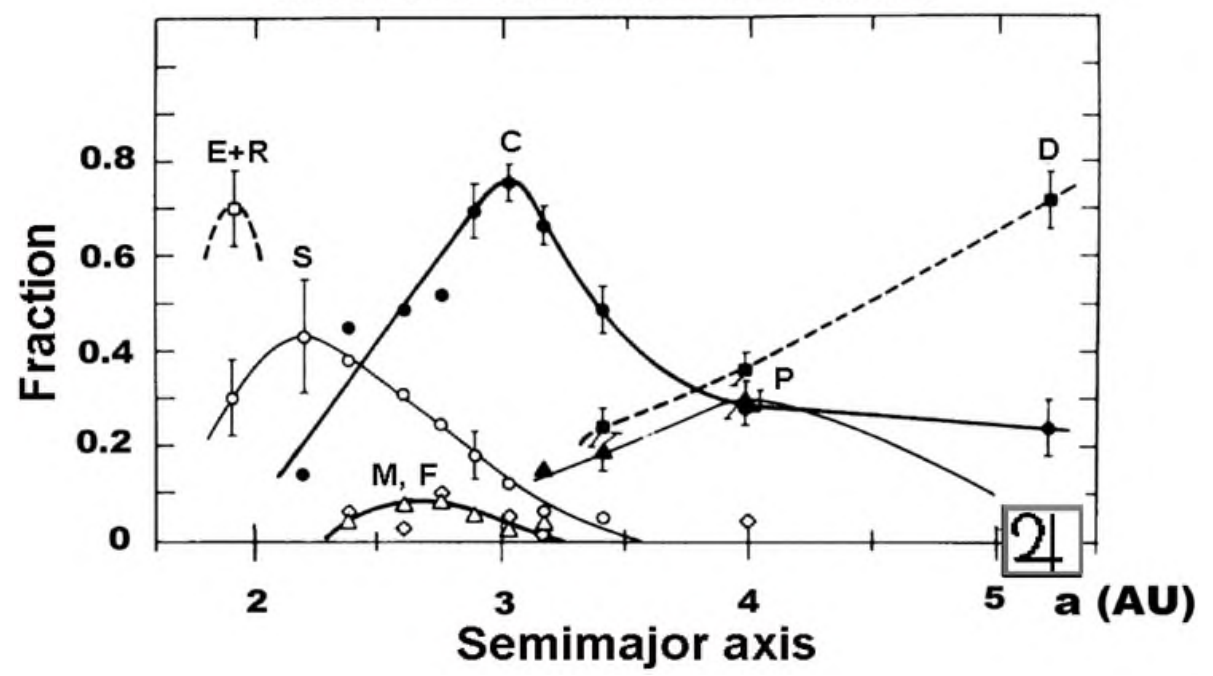

Figure 1: The heliocentric distributions of taxonomic classes of main-belt asteroids [9]. A special symbol shows heliocentric distance of Jupiter.

origin of primitive-type asteroids beyond the snowline in the early Solar system (namely, in the formation zones of Jupiter and other giant planets), which is in agreement with our previous hypothesis [13].

One of our latest results is the registration of simultaneous sublimation activity of several primitive main-belt asteroids near perihelion, which indicates the widespread nature of this phenomenon. The spectral signs of such activity are unusual light scattering maxima near $\sim 0.5$ or $\sim 0.6$ microns in asteroid reflectance spectra appearing in their dust exospheres [14, 15] (Fig. 2). The graphs show the observed and standard reflectance spectra (from the SMASSII database for comparison) of primitive main-belt asteroids 145 Adeona (Fig. 2a), 704 Interamnia (Fig. 2b), 779 Nina (Fig. 2c), and 1474 Beira (Fig. $2 \mathrm{~d})$. The latter is a Mars-crosser with large eccentricity of its orbit $(\mathrm{e}=0.488659)$ and, respectively, having the most temperature variations of the subsolar temperature $(\sim 190 \div 330 \mathrm{~K})$ among the asteroids [16].

\section{How active main-belt asteroids may be connected with the formation of giant planets}

As follows from model calculations (e. g., [18]), after the beginning of Jupiter formation and the appearance of the primary resonant structure in the early Solar system, there was probably one of the rings in the site of the MBA with its own resonant structure formed under the influence of growing proto-Jupiter in the adjacent zone. This may have accelerated the formation of several dozen of PBAs in the MBA.

[1] analytically estimated the total mass of the primary the MBA as about 100 times of its modern mass. However, depending on the initial conditions for the formation of planetesimals in the vicinity of the snowline, the mass of MBA could be significantly less [8]. It is also important to note, the present resonant structure of the MBA (and the heliocentric distribution of the matter by content) was hardly affected by a possible early (over the first $\sim 200$ kyr) joined proto-Jupiter proto-Saturn migration in the central direction (forward and back) (e. g., [19]; [20]) since it has been predominately formed in a more later time of Jupiter's growth. Thus, when Jupiter reaches a mass equal to several masses of the Earth, stone-ice bodies ejected from its zone of formation (JZBs) started to collide with PBAs in the MBA. Close approaches or collisions of large and high-speed (up to $\sim 10 \mathrm{~km} / \mathrm{s}$, according to [1]) JZBs and PBAs led probably to removal of a predominant matter of the latter from the MBA. At the same time, as known from experiments and model calculations (e. g., [21]; [22]), an outcome of a collision of two asteroids of stone (or stone-ice) composition is a considerable part of fragmented matter of the both bodies remained in the site of the collision. As a result, a large mass of ice-organic volatile matter of JZBs (or even the bodies themselves) probably remained in the MBA and could form asteroids of primitive types. The proportion of the substance delivered to the MBA is inversely proportional to the relative velocity of the colliding bodies, and is also determined by the probability of collisions. As shown in Figure 3, both these parameters of an invading body are depended on the shape of its orbit and the extent of its overlap with the space of MBA.

Importantly, due to action of the same one mechanism, not only JZBs might have been the source of primitive mainbelt asteroids, but stone-ice bodies ejected from the formation zones of Saturn (as SZBs) (e. g., see Fig. 3, orbit 5), Uranus and Neptune.

The more so, Jupiter Trojans, near the Lagrange points L4 and L5, might have a similar origin. One of the main observational confirmations of the considered scenario of Jupiter Trojans' origin is the discovering of two approximately equal in number groups of these bodies having different gradients of reflectance spectra (Fig. 4) [23].

As the authors suggested, the reason of found discrepancy is unequal content of organic matter in the matter of Trojans as a sign of their origin at different heliocentric distances [23].Then, according to our interpretation of the formation of 

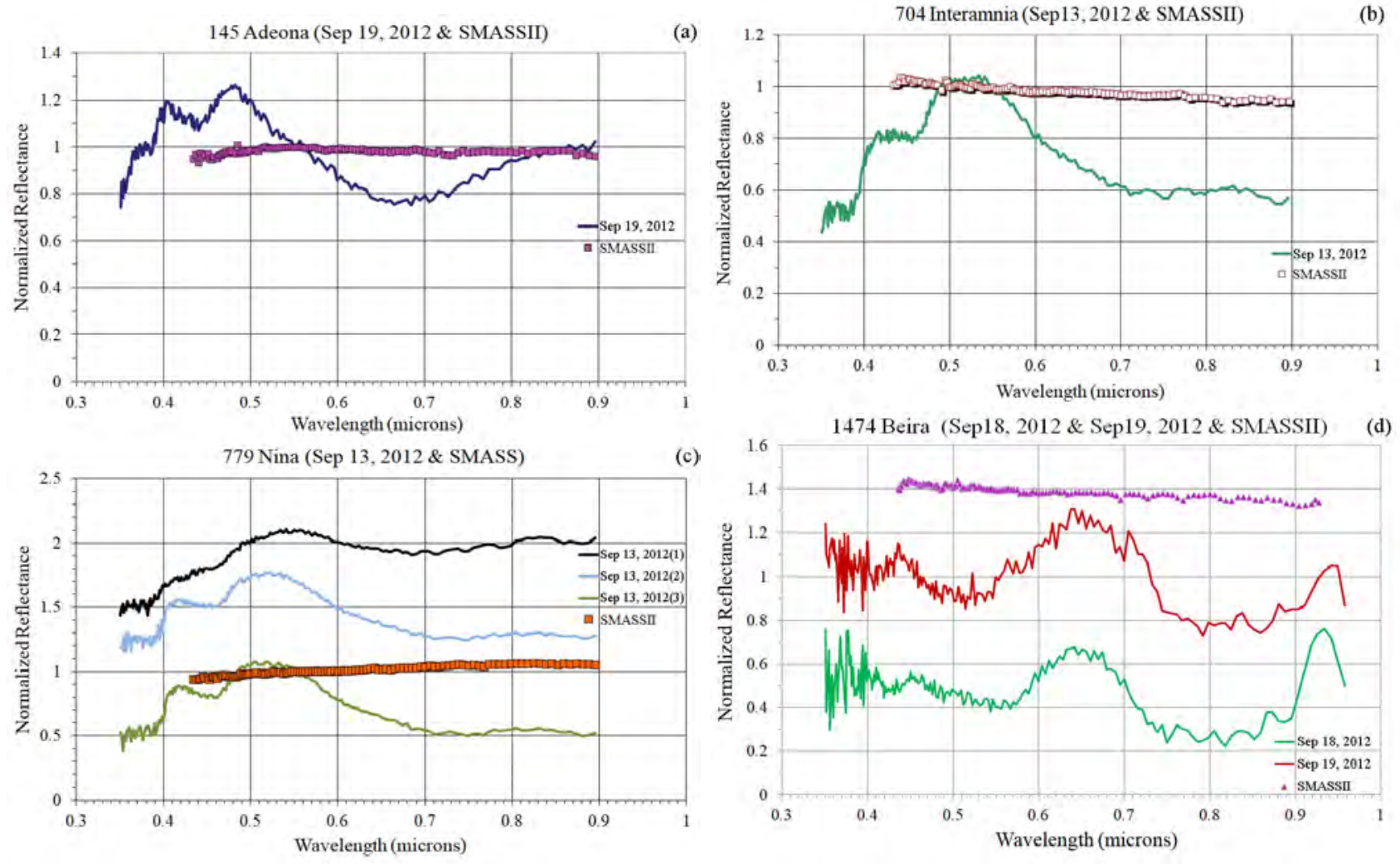

Figure 2: (a, b, c, and d). The reflectance spectra of active primitive asteroids of the MBA (normalized to the value at $0.55 \mu \mathrm{m}$ ) obtained in September 2012 [14]: (145) Adeona (a), (704) Interamnia (b), (779) Nina (c), and (1474) Beira (d). Three spectra of (779) Nina and two spectra of (1474) Beira are shifted along the vertical axis for clarity. The SMASSII reflectance spectra of the asteroids are given for comparison ([17]; https://sbnapps.psi.edu/ferret/).

Jupiter Trojans, the less red group of the bodies (Fig. 4a, dark blue curve), having lower organic content, might have originated in situ, and the more red group of the bodies (Fig. 4a, red curve) - in the formation zone of Saturn (as SZBs) ejected afterwards to the feeding zone of Jupiter.

As is seen from the comparison of Trojans' reflectance spectra with those of main-belt asteroids, the average spectrum of less red Trojans is close to spectra of main-belt asteroids of X- and C-types, and average spectrum of more red Trojans - to those of D-type asteroids (Fig. 4b). Then, in accordance with our scenario, main-belt D-type asteroids and the more red Jupiter Trojans could have a similar origin in the formation zones of Saturn, Uranus and Neptune.

As for duration of the most intense repopulation of the MBA by bodies injected from the feeding zones of giant planets, it should depend on the total period of their growth which, in turn, is determined by the lifetime of the solar protoplanetary disk. According to models of giant planets' formation (e. g., [24]; [25]; [26]; [18]; [27]; [28]; [29]), the timescale (and, respectively, the duration of the MBA's repopulation) may be from $2.5-3.5$ Myr (for Jupiter and Saturn) and up to $\sim 12$ Myr (for Uranus and Neptune).

\section{Conclusions}

Thus, as follows from the above discussion, the rock-ice bodies, that formed in Jupiter feeding zone and escaped its capture, could be intensively ejected into the formation zone of the MBA and probably form (or repopulate) the classes of primitive asteroids in MBA during the first few million years after the beginning of the formation of the Solar System. Such asteroids, primitive in composition and retained ice in their interiors, can now exhibit sublimation activity. This is a confirmation of the previously stated suggestion about the origin of C-type asteroids and carbonaceous chondrites [13]. Similar processes of ejection of rock-ice bodies by other growing giant planets in a later period (during the first ten million years) probably led to the capture of these bodies by the Jupiter resonance system and replenishment by them of the MBA, as well as the families of Jupiter Trojans.

Moreover, the formation processes of all giant planets and the ejection of rock-ice bodies in the central direction by the planets during clearing of their orbits over the first $\sim 100$ million years possibly created conditions for the delivery of a large volume of volatile compounds (predominately $\mathrm{H}_{2} \mathrm{O}$ ice) to the feeding zone of the terrestrial planets. These materials probably replenished the composition of pre-planetary bodies, from which the Earth and other planets were formed during the process of mutual collisions (e. g., [1]; [30]). 


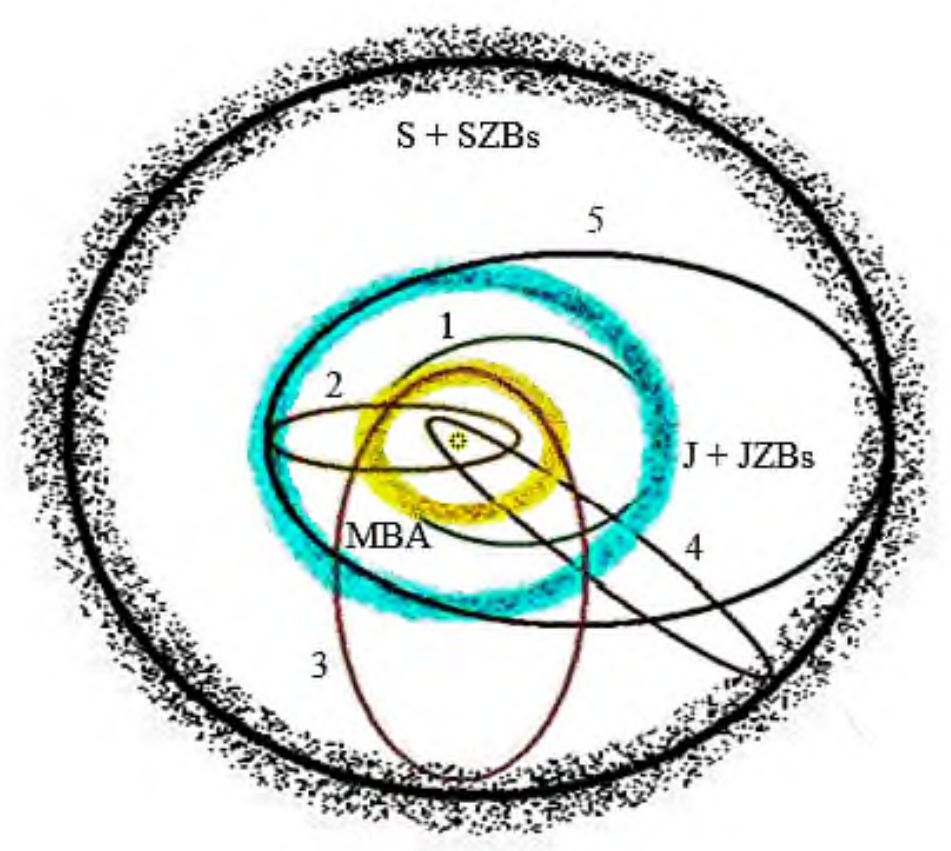

Figure 3: The formation zones of MBA (yellow), Jupiter (blue), and Saturn (black) filled with small bodies are shown in the figure. Stone-ice bodies ejected from the feeding zones of Jupiter (JZBs) and Saturn (SZBs) could move in low- and high-velocity orbits designated as "1" and "2" (for JZBs) and "3" and "4" (for SZBs). Some stone-ice bodies ejected by proto-Saturn in the central direction could move in the orbit 5 and possibly became Jupiter Trojans.
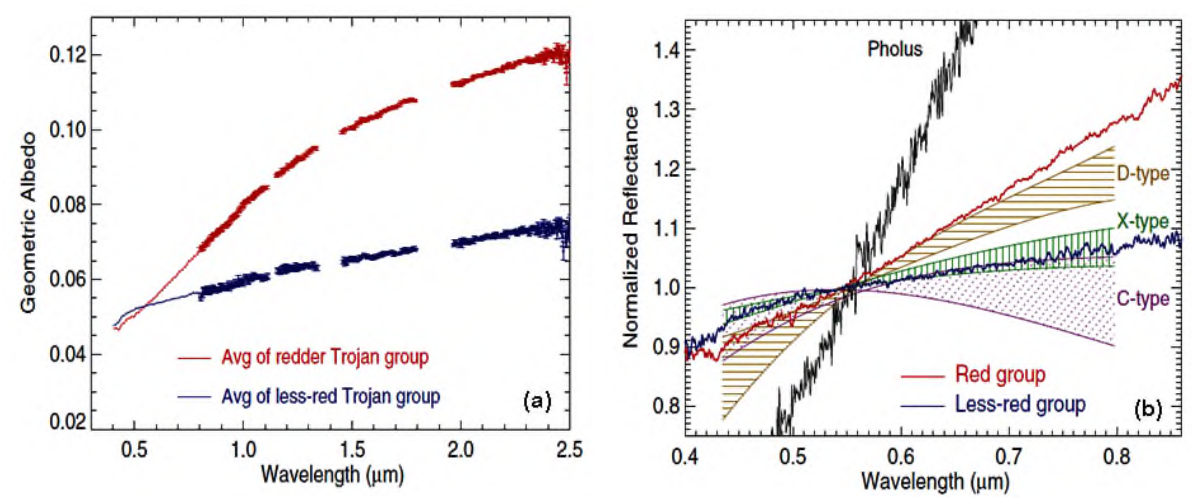

Figure 4: (a, b). (a) Averaged reflectance spectra of Jupiter Trojans in the near-IR range [23]. (b) Normalized visible-range reflectance spectra of Jupiter Trojans compared with the spectral boundaries of C-, D-, and Xtaxonomic classes of primitive main-belt asteroids and with a reflectance spectrum of Centaur 5145 Pholus [23] moving within heliocentric distances of $8.8 \div 31.9$ AU.

\section{References}

1. V. S. Safronov, Evolution of the protoplanetary cloud and the formation of the Earth and planets, Nauka (1969).

2. S. Guilloteau and A. Dutrey, Astrophysics and Space Science, 313, 95, 2008.

3. S. Guilloteau, A. Dutrey, V. Piétu, and Y. Boehler, Astronomy E Astrophysics, 529, A105, 2011.

4. P. J. Armitage, ARAEA, 49, 195, 2011.

5. G. A. Coleman and R. P. Nelson, Monthly Notices of the Royal Astronomical Society, 460, 2779, 2016.

6. J. S. Lewis, Science, 186, 440, 1974.

7. A. Makalkin and V. Dorofeeva, Solar System Research, 43, 508, 2009.

8. A. Makalkin and M. Artyushkova, Solar System Research, 51, 491, 2017.

9. D. Tholen and A. Barucci, Asteroid taxonomy in asteroids ii, eds. rp binzel, t. gehrels, ms matthews, univ. of arizona press, 1989.

10. C. O. Chandler, A. M. Curtis, M. Mommert, S. S. Sheppard, and C. A. Trujillo, Publications of the Astronomical Society of the Pacific, 130, 114502, 2018. 
11. D. Jewitt, The Astronomical Journal, 143, 66, 2012.

12. N. Schorghofer, The Astrophysical Journal, 682, 697, 2008.

13. V. Busarev, arXiv preprint arXiv:1211.3042, 2012.

14. V. Busarev, S. Barabanov, and V. Puzin, Solar System Research, 50, 281, 2016.

15. V. V. Busarev, E. V. Petrova, T. R. Irsmambetova, M. P. Shcherbina, and S. I. Barabanov, Icarus, 369, $114634,2021$.

16. V. V. Busarev, A. B. Makalkin, F. Vilas, S. I. Barabanov, and M. P. Scherbina, Icarus, 304, 83, 2018.

17. D. Lazzaro, C. Angeli, J. Carvano, T. Mothe-Diniz, R. Duffard, and M. Florczak, NASA Planetary Data System, EAR-A, 2007.

18. A. Morbidelli, A. Crida, F. Masset, and R. P. Nelson, Astronomy \& Astrophysics, 478, 929, 2008.

19. K. J. Walsh, A. Morbidelli, S. N. Raymond, D. P. O'Brien, and A. M. Mandell, Nature, 475, $206,2011$.

20. M. S. Clement, S. N. Raymond, and N. A. Kaib, The Astronomical Journal, 157, 38, 2019.

21. D. Davis, C. Chapman, R. Greenberg, S. Weidenschilling, and A. Harris, Asteroids, 528-557, 1979.

22. J.-M. Petit and P. Farinella, Celestial Mechanics and Dynamical Astronomy, 57, 1, 1993.

23. J. P. Emery, D. M. Burr, and D. P. Cruikshank, The Astronomical Journal, 141, 25, 2010.

24. J. B. Pollack, O. Hubickyj, P. Bodenheimer, J. J. Lissauer, M. Podolak, and Y. Greenzweig, icarus, 124, 62 , 1996.

25. T. Guillot, Annu. Rev. Earth Planet. Sci., 33, 493, 2005.

26. S. E. Dodson-Robinson, P. Bodenheimer, G. Laughlin, K. Willacy, N. J. Turner, and C. Beichman, The Astrophysical Journal Letters, 688, L99, 2008.

27. O. G. Benvenuto, A. Fortier, and A. Brunini, Icarus, 204, 752, 2009.

28. C. P. Bell, T. Naylor, N. Mayne, R. Jeffries, and S. Littlefair, MNRAS, 434, 806, 2013.

29. R. Frelikh and R. A. Murray-Clay, The Astronomical Journal, 154, 98, 2017.

30. B. M. Hansen, The Astrophysical Journal, 703, 1131, 2009. 


\title{
On the nongravitation effect in the motion of the main belt asteroids
}

\author{
Yu.A. Chernetenko, V.B. Kuznetsov
}

cya@iaaras.ru, vb.kuznetsov@iaaras.ru

Institute of Applied Astronomy of the Russian Academy of Sciences, Saint-Petersburg, 191187 Russia

The hypothesis is being tested about the presence of an additional nongravitational acceleration depending on the albedo of asteroids in the motion of the main belt asteroids. On the example of the asteroid family (8) Flora, we obtained the values of the transverse component $A_{2}$ of the nongravitational acceleration using observations of the asteroids. The results do not confirm the presence this kind of acceleration leading to separation of light and dark asteroids within the same family.

Keywords: asteroids, orbits, nongravitational acceleration, albedo

DOI: 10.51194/VAK2021.2022.1.1.077

\section{Introduction}

In papers [1, 2, 3], it is suggested that some asteroid families ((4) Vesta, (5) Astraea, (8) Flora, (135) Hertha, (221) Eos) manifest an additional nongravitational acceleration (NGA) in their motion, depending on the albedo of asteroids. The NGA leads to an increase with a velocity of 3-12 km/year the values of the semimajor axes of the orbits of asteroids with diameters $D<40 \mathrm{~km}$ and albedo $<0.1$. The authors conclude $[1,2,3]$ that it is this mechanism that is responsible for the separation of asteroids in families along the semimajor axes. (It should be noted that such a conclusion cannot be drawn for all asteroid families.) The research method in these papers is a comparison of the values of the semimajor axes obtained at different intervals of observations and reduced later to the same epoch. In this case, the errors of neither the semimajor axes themselves, nor their changes over time are estimated.

In this work, we set the task to evaluate the values of the NGA for 11289 numbered asteroids of the family (8) Flora [4] using their observations and to compare them with the values of their albedo and semimajor axes. The choice of this family is due to the fact that a statistically significant connection of the albedo values $p$ and the values of the semimajor axis $a$ is estimated for it, which is shown in Fig. 1. Coefficient $b$ of the linear approximation $(y=a+b x)$ is equal to $-0.21 \pm 0.06$. The same (with differente values of $b$ ) can be said about the families (4) Vesta, (25) Phocaea, (135) Hertha, (158) Koronis, (221) Eos. The albedo data were obtained from the WISE database [5].

We assumed that the magnitude of the NGA depends on the heliocentric distance as $1 / r^{2}$. The parameters of the NGA $A_{2}$ were evaluated together with the orbital parameters for each asteroid. The coordinates of the major planets were calculated using the DE440 ephemeris [6]. The positional observations of asteroids presented in the catalog of the Minor Planet Center ${ }^{1}$ were used.

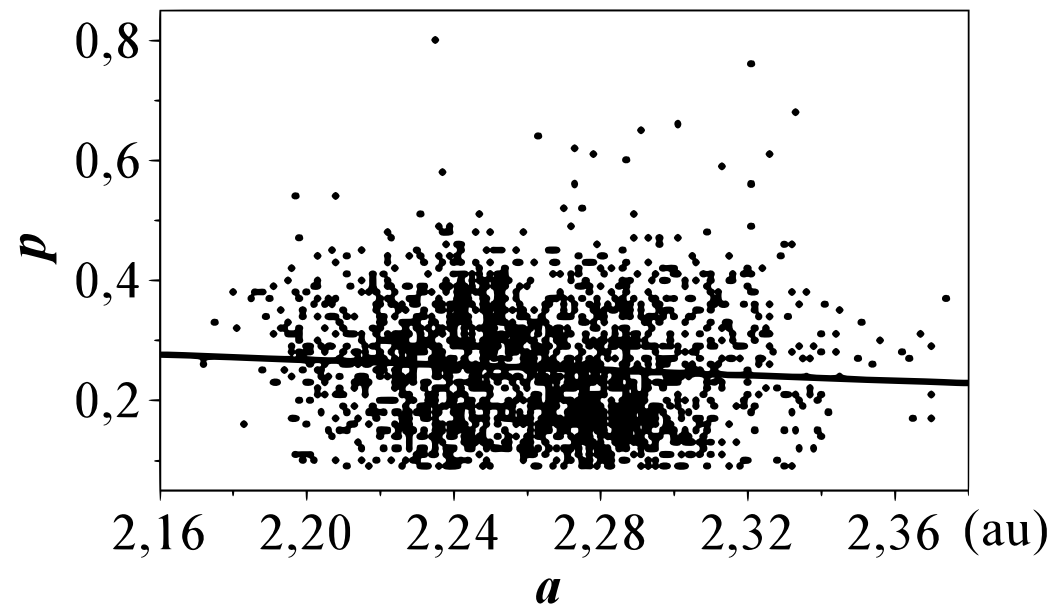

Figure 1: Albedo $p$ versus semimajor axes $a$ for asteroids of the family (8) Flora.

\section{Calculations}

The calculations for 11289 asteroids showed noticeable range of $A_{2}$ values (here and below, the values of $A_{2}$ are given in $10^{-14} \mathrm{au} \mathrm{day}^{-2}$ ): from -400 to +600 , and their errors - from 0.2 to 800 . There is some connection between the values of $A_{2}$ and their errors. For further consideration those values of $\left|A_{2}\right|$ were selected, which are three or more times more than their errors. There were 2598 such asteroids. For them, the range of $A_{2}$ values is \pm 350 , and the error values do not exceed 75. For this set of asteroids, Fig. 2 (a, b) show the values of $\left|A_{2}\right|$ and their errors versus the number of oppositions. It can be seen on Fig. 2 a sharp increase in both the errors and the values themselves when the number of oppositions is less than about 20. Most likely, this is a consequence of an unstable solution of the normal systems and/or an influence of

\footnotetext{
${ }^{1}$ https://minorplanetcenter.net/
} 

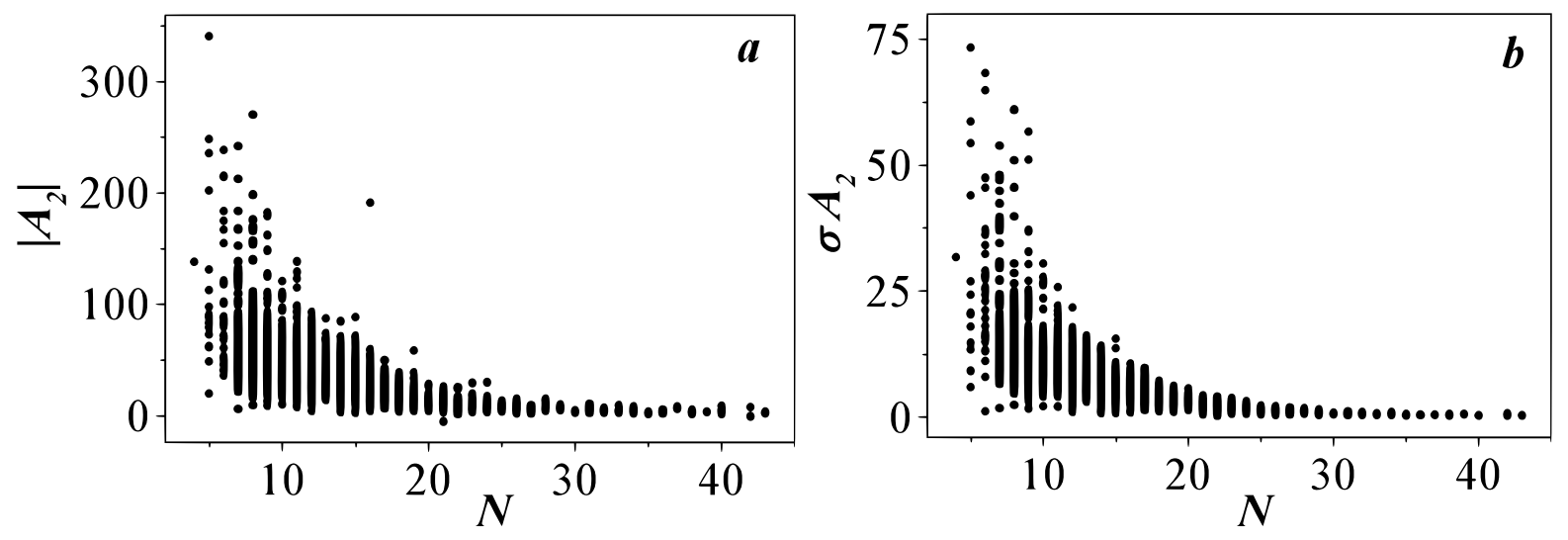

Figure 2: Values of $\left|A_{2}\right|$ (a) and $\sigma_{A_{2}}$ (b) versus the number of oppositions, $N$.
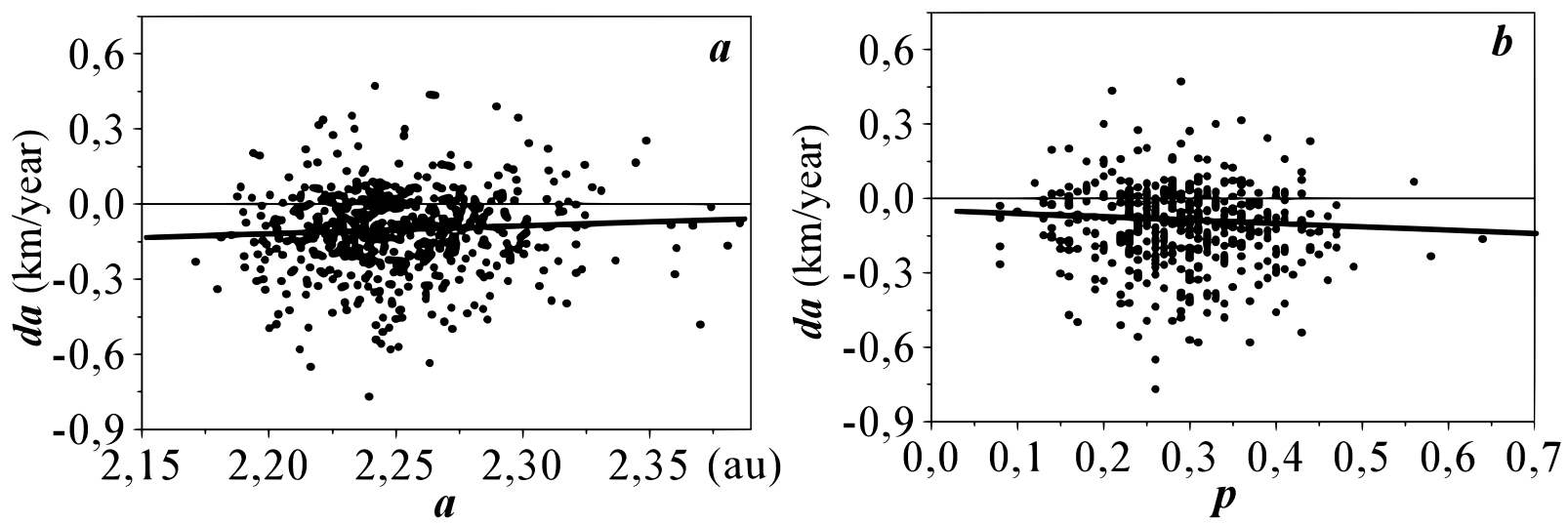

Figure 3: Values of $d a$ for asteroids with $\sigma_{A_{2}}<2.5$ versus semimajor axes $a$ (a) and albedo $p$ (b).

systematic and random observation errors. Therefore, further we considered asteroids with the number of oppositions more than 20. Only 237 asteroids from this sample have albedo values in the database [5], and for them a statistically significant connection of $A_{2}$ with albedo values is not found: coefficient $b=-2.5 \pm 3.8$.

The analysis of $A_{2}$ values was also performed in a different way. We have chosen the $A_{2}$ values for which the error $\left(\sigma_{A_{2}}\right)$ $<2.5$. This set contained 593 asteroids. Comparison of changes in the semimajor axis $d a$ with the values of the semimajor axis and albedo does not reveal a significant relationship: $b=0.33 \pm 0.21$ (Fig. 3, a), $b=-0.11 \pm 0.09$ (Fig. 3, b), The values of $|d a|$ do not exceed $0.8 \mathrm{~km} /$ year, and the average value of $d a$ is $\sim-0.1 \mathrm{~km} /$ year.

\section{Results}

The hypothesis $[1,2,3]$ is being tested about the presence of additional NGA in the motion of the main belt asteroids, which depends on the albedo of asteroids and leads to a change in the semimajor axes of their orbits by $3-12 \mathrm{~km} / \mathrm{year}$.

We obtained the values of the transverse component $A_{2}$ of the NGA using observations of the asteroids of the family (8) Flora. It is shown that both the $A_{2}$ values themselves and their errors largely depend on the considered observation interval; therefore, for confident conclusions regarding the action of the NGA, observations are needed for at least 20 oppositions. With a decrease in the number of oppositions, the amplitude of the $A_{2}$ values and their errors increases sharply due to the poor conditioning of the systems of normal equations and the possible influence of random and systematic observation errors.

Two sets of values $A_{2}$ have been reviewed which we believe to be reliable. No statistically significant dependence of the values of $A_{2}$ on the values of $p$ or on the values of $a$ was found. For considered asteroids of the family (8) Flora, the change of the semimajor axis, $|d a|$, does not exceed $0.8 \mathrm{~km} /$ year, and the average value is close to $0.1 \mathrm{~km} /$ year.

The values of $d a$ obtained by us as a result of the action of NGA are approximately an order of magnitude less than those values that are given in the papers $[1,2,3]$. We believe that the reason for such discrepancies is the lack of taking into account in these papers the errors of the orbital elements (semimajor axis) obtained at different time intervals and increasing when the systems of elements are transferred from one epoch to another. This circumstance did not allow the separation of reliable and unreliable results. Apparently, the spatial separation of light and dark asteroids marked in [1,2, 3] for the asteroid families (4) Vesta, (5) Astraea, (8) Flora, (135) Hertha, (221) Eos is caused not by the action of additional nongravitational acceleration, but by some other reasons. 


\section{References}

1. A. M. Kazantsev, Kinematics and Physics of Celestial Bodies, 23, 258, 2007.

2. A. M. Kazantsev and L. V. Kazantseva, Kinematics and Physics of Celestial Bodies, 30, 255, 2014.

3. A. M. Kazantsev and L. V. Kazantseva, Solar System Research, 51, 527, 2017.

4. T. A. Vinogradova, MNRAS, 484, 3755, 2019.

5. J. R. Masiero, A. K. Mainzer, T. Grav, J. M. Bauer, et al., ApJ, 741, 68, 2011.

6. R. S. Park, W. M. Folkner, J. G. Williams, and D. H. Boggs, AJ, 161, 105, 2021. 


\section{Ephemerides of the Neptune's satellites}

I. Dolgakov

ia.dolgakov@iaaras.ru

Institute of Applied Astronomy of the Russian Academy of Sciences,

Russia, 19118\%, St. Petersburg, Kutuzova Embankment, 10

Planetary satellite's ephemerides are valuable for spacecraft mission planing. Moreover, precise numerical theories of the natural satellites motion make it possible to improve the precision of their central planet's ephemeris using the positional observations of their satellites. This paper describes the numerical theories of the Neptune's satellites — Triton, Nereid and Proteus constructed using the modern CCD ground-based observations as well as space-based observations from the Voyager 2 flyby mission.

Keywords: Planetary systems, ephemerides, Neptune, Triton, Nereid, Proteus

DOI: $10.51194 /$ VAK2021.2022.1.1.078

\section{Model}

The model for the satellite orbits is a numerical integration of their equations of motion which are formulated in Neptunecentered ICRF cartesian coordinates.

Gravitational effects include the Neptune-satellites interactions, the perturbation of satellites from each other, and the perturbations from Sun, Jupiter, Saturn, and Uranus. The oblateness of Neptune is taken into account by decomposing its gravitational potential into spherical harmonics and retaining only the second and fourth zonal terms.

The Neptune's pole motion is modeled with equations proposed in [1]:

$$
\begin{aligned}
& \alpha=\alpha_{r}+\epsilon \sec \delta_{r} \sin \left(\Omega_{0}+\dot{\Omega} T\right)-\frac{1}{2} \epsilon^{2} \sec \delta_{r} \tan \delta_{r} \sin 2\left(\Omega_{0}+\dot{\Omega} T\right), \\
& \delta=\delta_{r}-\epsilon \cos \left(\Omega_{0}+\dot{\Omega} T\right)-\frac{1}{4} \epsilon^{2} \tan \delta_{r}\left(1-\cos 2\left(\Omega_{0}+\dot{\Omega} T\right)\right),
\end{aligned}
$$

$\alpha$ and $\delta$ are the pole right ascension and declination, $\alpha_{r}$ and $\delta_{r}$ are the right ascension and declination of the system's angular momentum, and $\epsilon$ is the angle between the pole direction and the system's angular momentum. $\dot{\Omega}$ is the precession rate, $\Omega_{0}$ is the epoch value of ascending node of Triton's orbit.

\section{Observations and methods}

The observations applied in our calculation are taken from the NSDB [2] and JPL website, which includes the precise CCD observations of both satellites captured since 1975. The observations made by the Voyager 2 spacecraft in 1988-1989 are used as well. They are obtained from the JPL website and reduced with the use of the latest Voyager 2 spacecraft ephemerides (vgr2-nep081.bsp). A total of 4285 Triton and 878 Nereid observations have been used, including the ground-based and made by the Voyager 2 spacecraft.

The orbits are integrated using the 10th-order Adams-Bashforth-Moulton method with the step of 0.01 day. The initial epoch set to 1 Jan 1990. Partials required for the parameters refinement are obtained from the integration of the variational equations. The process of construction of such equations is similar to [3], but performed by the software using the automatic differentiation technique [4].

Initial values of the dynamical parameters and the state vector are taken from [1] and the HORIZONS [5] system. The positions of the perturbing bodies are provided by the EPM2021 ephemerides.

\section{Results}

Residuals of 181 Proteus observations are 0.371 and 0.288 in the Voyager's camera pixel and line, respectively. That is comparable with values 0.353 and 0.262 obtained in [1].

As seen from Tables 1 and 2 most of Triton and Nereid residuals tend to be very close to the published values, which indicate the correctness of the current theory.

We compared obtained ephemerides with those given by the HORIZONS system during the time interval 01.01.197001.01.2018. The maximum difference between the Triton ephemerides is less than 0.05 and 0.04 arcsec in right ascension and declination, respectively. The deviations of Nereid ephemerides are larger, but they are less than 0.4 and 0.15 arcsec in right ascension and declination.

The possible reason for such deviations can be a shorter observation arc used in the current study. Also, we observed the same shift in the Tritons' declination residuals as in [6], but we were unable to reduce it during the Neptune's orbit refinement.

The dynamical parameters values were obtained as a by-product of the fit. Despite the relatively short observation arc, we managed to refine the Triton's and the system's GMs with greater accuracy, as seen in Table 3. The accuracy of the remaining parameters is slightly worse compared with [1], mainly due to the shorter observation arc. 
Table 1: Triton residuals in comparison with other solutions. Lower values are highlighted in bold.

\begin{tabular}{|c|c|c|c|c|c|c|c|c|}
\hline Dates & Type & Code & No. & $\mathrm{rms}_{x}$ & $\mathrm{rms}_{y}$ & $\mathrm{rms}_{x}$ & $\mathrm{rms}_{y}$ & source \\
\hline $1975-1977$ & phot.rel & 689 & 28 & $0.063^{\prime \prime}$ & $0.097^{\prime \prime}$ & $0.068^{\prime \prime}$ & $0.129^{\prime \prime}$ & Zhang \\
\hline 1979-1983 & phot.rel & 689 & 114 & $0.032^{\prime \prime}$ & $0.073^{\prime \prime}$ & $0.047^{\prime \prime}$ & $0.121^{\prime \prime}$ & Zhang \\
\hline 1984-1986 & phot.rel & 689 & 56 & $0.023^{\prime \prime}$ & $0.069^{\prime \prime}$ & $0.029^{\prime \prime}$ & $0.061^{\prime \prime}$ & Zhang \\
\hline 1986-1993 & phot.abs & 119 & 54 & $0.421^{\prime \prime}$ & $0.541^{\prime \prime}$ & $0.423^{\prime \prime}$ & $0.442^{\prime \prime}$ & Zhang \\
\hline 1988-1989 & Voyager 2 & & 366 & $0.197 p x$ & $0.183 \mathrm{px}$ & $0.2 \mathrm{px}$ & $0.17 p x$ & Jacobson \\
\hline 1989-1994 & CCD, rel & 874 & 432 & $0.465^{\prime \prime}$ & $0.203^{\prime \prime}$ & $0.133^{\prime \prime}$ & $0.139^{\prime \prime}$ & Zhang \\
\hline 1990-1990 & phot.abs & 188 & 5 & $0.135^{\prime \prime}$ & $0.295^{\prime \prime}$ & $0.074^{\prime \prime}$ & $0.143^{\prime \prime}$ & Zhang \\
\hline 1993-1998 & CCD, rel & 874 & 174 & $0.234^{\prime \prime}$ & $0.246^{\prime \prime}$ & $0.163^{\prime \prime}$ & $0.132^{\prime \prime}$ & Emelyanov \\
\hline 1995-1997 & $\mathrm{CCD}$, rel & 874 & 759 & $0.156^{\prime \prime}$ & $0.213^{\prime \prime}$ & $0.155^{\prime \prime}$ & $0.211^{\prime \prime}$ & Zhang \\
\hline $1996-2006$ & CCD. abs & 337 & 943 & $0.094^{\prime \prime}$ & $0.072^{\prime \prime}$ & $0.039^{\prime \prime}$ & $0.032^{\prime \prime}$ & Jacobson \\
\hline 1998-2014 & CCD. abs & 689 & 1234 & $0.136^{\prime \prime}$ & $0.153^{\prime \prime}$ & - & - & - \\
\hline 2000-2002 & CCD, abs & 874 & 66 & $0.159^{\prime \prime}$ & $0.242^{\prime \prime}$ & $0.331^{\prime \prime}$ & $0.247^{\prime \prime}$ & Zhang \\
\hline
\end{tabular}

Table 2: Nereid residuals in comparison with other solutions. Lower values are highlighted in bold.

\begin{tabular}{|c|c|c|c|c|c|c|c|c|}
\hline Dates & Type & Code & No. & $\mathrm{rms}_{x}$ & $\mathrm{rms}_{y}$ & $\mathrm{rms}_{x}$ & $\mathrm{rms}_{y}$ & source \\
\hline 1989-1989 & Voyager 2 & & 89 & $0.333 \mathrm{px}$ & $0.282 \mathrm{px}$ & $0.32 \mathrm{px}$ & $0.24 \mathrm{px}$ & Jacobson \\
\hline 1993-1998 & CCD, abs & 874 & 230 & $0.216^{\prime \prime}$ & $0.224^{\prime \prime}$ & - & - & - \\
\hline 1993-1998 & CCD, rel & 874 & 174 & $0.234^{\prime \prime}$ & $0.246^{\prime \prime}$ & $0.163^{\prime \prime}$ & $0.132^{\prime \prime}$ & Emelyanov \\
\hline 2001-2004 & CCD, abs & $568,807,415$ & 13 & $0.514^{\prime \prime}$ & $0.309^{\prime \prime}$ & - & - & - \\
\hline $2002-2008$ & CCD, abs & $415,213,644, \mathrm{D} 35$ & 190 & $0.306^{\prime \prime}$ & $0.32^{\prime \prime}$ & - & - & - \\
\hline 2006-2007 & CCD. abs & 327 & 112 & $0.21^{\prime \prime}$ & $0.19^{\prime \prime}$ & $0.208^{\prime \prime}$ & $0.188^{\prime \prime}$ & Jacobson \\
\hline 2009-2010 & CCD, abs & 415,673 & 9 & $0.895^{\prime \prime}$ & $0.306^{\prime \prime}$ & - & - & - \\
\hline 2009-2010 & CCD. abs & 689 & 6 & $0.16^{\prime \prime}$ & $0.279^{\prime \prime}$ & - & - & - \\
\hline 2011-2011 & CCD, abs & $673, \mathrm{E} 07$ & 55 & $0.444^{\prime \prime}$ & $0.162^{\prime \prime}$ & - & - & - \\
\hline
\end{tabular}

Table 3: Dynamical parameters of the satellite system.

\begin{tabular}{rrr}
\hline Parameter & Initial & Current \\
\hline$G M \mathrm{~km}^{3} \mathrm{~s}^{-2}$ & $6836527.1 \pm 10.0$ & $6836592.8 \pm 5.3$ \\
$G M_{\text {Triton }} \mathrm{km}^{3} \mathrm{~s}^{-2}$ & $1427.6 \pm 1.9$ & $1427.56 \pm 0.01$ \\
$J_{2}\left(\times 10^{-6}\right)$ & $3408.4 \pm 4.5$ & $3115.1 \pm 64.6$ \\
$\alpha_{r} \mathrm{deg}$ & $299.46 \pm 0.14$ & $298.42 \pm 0.14$ \\
$\delta_{r} \mathrm{deg}$ & $43.40 \pm 0.03$ & $44.9 \pm 0.6$ \\
\hline
\end{tabular}

\section{Conclusion}

We developed new numerical theories of the Neptunian satellites: Triton, Nereid, and Proteus. These theories are in good agreement with the published ones despite the shorter observation arc used in this study.

Increasing the observation arc is not only reducing the difference between theories but also make it possible to refine the larger set of the dynamical parameters, which is our goal in further work.

The author appreciates Dmitry Pavlov and Elena Piteva for the help with the observations processing software and valuable discussions. 


\section{References}

1. R. A. Jacobson, AJ, 137, 4322, 2009.

2. J. E. Arlot and N. V. Emelyanov, $A \mathscr{E} A, \mathbf{5 0 3}, 631,2009$.

3. C. F. Peters, $A \mathscr{E} A, \mathbf{1 0 4}, 37,1981$.

4. I. Dolgakov and D. Pavlov, Journal of Mathematical Sciences, 251, 354, 2020.

5. J. D. Giorgini, A. B. Chamberlin, and S. B. Park, Horizons On-Line Ephemeris System (https://ssd.jpl.nasa.gov/horizons.cgi), 1995.

6. H. Zhang, K. X. Shen, G. Dourneau, D. Harper, et al., MNRAS, 438, 1663, 2014. 


\section{Astronomical constraints on primordial black holes in the Solar System}

Yu. Eroshenko

eroshenko@inr.ac.ru

Institute for Nuclear Research RAS, 117312, Moscow, Russia

DOI: $10.51194 /$ VAK2021.2022.1.1.079

Some features of the trans-Neptunian objects movement have led to the hypothesis that the 9th planet or primordial black hole $(\mathrm{PBH})$ with mass $M_{\mathrm{PBH}} \sim 5-15$ Earth masses may be at the distance $r_{i} \sim 300-1000$ A.U. from the Sun [1]. The existence of PBHs in the Universe is quite possible, and the probabilities of the free-flying planet and PBH capturing from the Galaxy into the Solar system are comparable [2]. Such a PBH should perturb the orbits of the dust particles and ice bodies in the inner Oort cloud, and some of the particles can be redirected inside the Earth orbit. The dust flux near the Earth is estimates to be

$$
F \simeq \frac{9}{4 \pi^{2}} \frac{G M_{\mathrm{PBH}}^{2} M_{d} t_{\mathrm{ej}}}{M_{\odot} r_{\mathrm{E}} r_{i}^{4}}
$$

where $M_{d}$ is the (poorly known) dust mass in the volume $\sim r_{i}^{3}, t_{\mathrm{ej}}$ is the lifetime on the Earth crossing orbit due to Jupiter ejection, and $r_{\mathrm{E}}=1$ A.U. For sufficiently large particles, light pressure and Poynting-Robertson effect are not important, so such particles could be preserved in the Solar system. Numerically,

$$
F \simeq 6\left(\frac{M_{\mathrm{PBH}}}{10 M_{\mathrm{E}}}\right)^{2}\left(\frac{M_{d}}{5 M_{\mathrm{E}}}\right)\left(\frac{t_{\mathrm{ej}}}{5 \times 10^{9} \mathrm{yrs}}\right)\left(\frac{r_{i}}{300 \mathrm{~A} . \mathrm{U} .}\right)^{-4} \mathrm{mg} \mathrm{m}^{-2} \mathrm{yr}^{-1}
$$

where $M_{\mathrm{E}}$ is the Earth mass. If $M_{\mathrm{PBH}} \sim 10 M_{\mathrm{E}}$, then the dust flow (2) is comparable to what is actually observed by counting the dust particles on the Antarctic ice or by direct measurements at spacecrafts $\sim 3.0-5.6 \mathrm{mg} \mathrm{m}^{-2} \mathrm{yr}^{-1}[3]$. Therefore, the $M_{\mathrm{PBH}}$ and $M_{d}$ cannot be significantly greater than the values in (2).

\section{References}

1. K. Batygin, F. C. Adams, M. E. Brown, and J. C. Becker, Phys. Rep., 805, 1, 2019.

2. J. Scholtz and J. Unwin, Phys. Rev. Lett., 125, 051103, 2020.

3. J. Rojas, J. Duprat, C. Engrand, E. Dartois, L. Delauche, M. Godard, and et.al, Earth and Planetary Science Letters, 560, 116794, 2021. 


\title{
Search for deviations in exoplanetary infrared profiles, based on the secondary eclipse analysis
}

\author{
A.A. Fedotov, R.V. Baluev \\ Saint Petersburg State University, Faculty of Mathematics 8 Mechanics, Universitetskij pr. 28, Petrodvorets, Saint \\ Petersburg 198504, Russia
}

\begin{abstract}
Aperture photometry of Spitzer space telescope observations of HD209458 b in the $3.6 \mu$ m channel was conducted. To find deviations in the exoplanetary infrared emission, the modelling of secondary eclipse light curves was carried out. The noise from intrapixel sensitivity variations was modelled by Gaussian processes. The obtained value of the eclipse depth is $0.094 \pm 0.013 \%$ of the total flux of the system and is consistent with the results of previous work and the works of other authors.
\end{abstract}

Keywords: planets and satellites: techniques, general; stars: planetary systems; techniques: photometric, data analysis; stars: individual: HD209458

DOI: $10.51194 /$ VAK2021.2022.1.1.080

\section{Introduction}

The study of extrasolar planets undergoes rapid development. While its main focus shifts from the detection methods to exploring physical characteristics, a significant amount of knowledge can be obtained from light, reflected from or emitted by the planet. However, planet brightness is much lower than that of its host star, making direct observations difficult. The best opportunity to detect the planetary radiation is when it passes behind the host star, when the planet flux gets obscured for some time, triggering a much faster variation in the total system flux than over the rest of the phase curve. This event is known as a secondary eclipse.

The analysis of secondary eclipse light curves helps us to refine the orbit of an exoplanet, as well as to obtain some important knowledge about the planet shape and its surface brightness distribution. The most remarkable brightness inhomogeneities are expected in hot Jupiters because they (i) are more heated because of their proximity to the star and (ii) possess a large radius, providing the best planet/star flux ratio. This ratio is further increased in the infrared range, where the spectral maximum of the planetary thermal radiation is located. It is also worth noting that hot Jupiters are expected to be tidally locked with their stars. This causes the existence of a highly heated region in the planet's atmosphere (hot spot) which appears shifted from the substellar point [1]. Our work represents an attempt to detect possible deviations that such a hot spot could trigger in the infrared light curve of the secondary eclipse.

There are several articles that consider the similar problem [2, 3, 4]. We selected the well-investigated exoplanet HD209458 b as our primary target because our work is still at an early stage and we considered this planet as a good test case for the analysis. We used observations in the band of 3.6 microns of Spitzer/IRAC, while other authors considered the band of 8 microns of Spitzer/IRAC. This band was selected because it had a large number of observations of secondary eclipses (14) for the investigated planet.

\section{Observations}

For the analysis, we used basic calibrated data (BCD) of the Spitzer Space Telescope in the band of 3.6 microns, with are in the public domain ${ }^{1}$. A detailed description, of how to obtain these frames, can be found in Chapter 5 of the IRAC Instrument Handbook ${ }^{2}$.

To obtain photometric curves, we perform aperture photometry using astropy [5] and photutils [6] python packages. The implementation of the algorithm can be found at GitHub ${ }^{3}$.

It is worth noting that Spitzer was not designed for sub-mmag photometry on bright point targets. Because of this fact, there are some artefacts in BCD. For the used wavelength the most significant one is the intrapixel sensitivity variations [7]. The pointing drift during observations generates a correlated noise at the level of an individual pixel, and telescope pointing correction also generates a quasi-periodic component.

\section{Model}

An approximation of the uniformly bright disk of the planet passing behind the star uniformly and rectilinearly with the same impact parameter was used as a model of the eclipse. The ratio of the radii of the planet and the star, the moment of the centre of the eclipse, the impact parameter, and the orbital characteristics were taken from the works of other authors [8]. The ratio of the brightness of the planet and the star, as well as the offset of the eclipse centre relative to the one calculated from the orbital elements, were taken as model parameters.

In a previous work [9], the least-squares method was used for modelling. The noise component was approximated by fifth-degree polynomials. The polynomial coefficients were approximated differently for each eclipse, and the model parameters were common for all observations.

In the presented work, the Gaussian processes (GP) model was used. This method models the statistical covariance between data points. Thus, it is more flexible, because it allows a broad ranger of models to capture with few free parameters.

\footnotetext{
${ }^{1}$ https://sha.ipac.caltech.edu/applications/Spitzer/SHA/

${ }^{2}$ https://irsa.ipac.caltech.edu/data/SPITZER/docs/irac/iracinstrumenthandbook/IRAC_Instrument_Handbook.pdf

${ }^{3} \mathrm{https}: / /$ github.com/dosidicus-deprimas/HD209458b
} 
GP-method allows approximating these models without explicit functional form. However, we must introduce the kernel function, to calculate the elements of the covariance matrix. More details on the implementation of the method can be found in the article[10].

In this work, the kernel function used was

$$
k(d t)=\sigma_{w n}^{2} E+\sigma_{r n}^{2} \exp \left(-\frac{d t^{2}}{2 l^{2}}\right)+D \cos \left(\frac{2 \pi d t}{P}\right) \exp \left(-\frac{|d t|}{\tau}\right)
$$

It consisted of 3 main parts. $\sigma_{w n}^{2} E$ described the white-noise component, $\sigma_{r n}^{2} \exp \left(-\frac{d t^{2}}{2 l^{2}}\right)$ the red-noise component, and $D \cos \left(\frac{2 \pi d t}{P}\right) \exp \left(-\frac{|d t|}{\tau}\right)$ the quasiperiodic component.

The polynomial trend using third-degree polynomials was also taken into account. The noise model parameters and polynomial coefficients were selected for each eclipse separately, while the eclipse parameters were common to all observations.

\section{Results}

As a result, the values of the eclipse centre offset of $2.48 \pm 3.64$ minutes and the brightness ratio of $0.094 \pm 0.013 \%$ were obtained. These values are in agreement with the results of the previous stage of the work $(1.64 \pm 1.29$ minutes, $0.101 \pm 0.009 \%)$ and with the data obtained by other authors [4]. The work on further improvement of the model is in progress.

RVB acknowledges the support of Ministry of Science and Higher Education of Russian Federation under the grant 075-15-2020-780 (N13.1902.21.0039).

\section{References}

1. A. P. Showman and T. Guillot, $A \mathscr{E} A, \mathbf{3 8 5}, 166,2002$.

2. H. A. Knutson, D. Charbonneau, L. E. Allen, J. J. Fortney, et al., Nature, 447, 183, 2007.

3. C. Majeau, E. Agol, and N. B. Cowan, ApJL, 747, L20, 2012.

4. J. de Wit, M. Gillon, B. O. Demory, and S. Seager, A\&A, 548, A128, 2012.

5. Astropy Collaboration, T. P. Robitaille, E. J. Tollerud, P. Greenfield, et al., A\&A, 558, A33, 2013.

6. L. Bradley, B. Sipocz, T. Robitaille, E. Tollerud, et al., astropy/photutils: v0.6, 2019.

7. T. M. Evans, S. Aigrain, N. Gibson, J. K. Barstow, D. S. Amundsen, P. Tremblin, and P. Mourier, MNRAS, 451, 680, 2015 .

8. A. S. Bonomo, S. Desidera, S. Benatti, F. Borsa, et al., A\&A, 602, A107, 2017.

9. A. A. Fedotov, and G. M. K. and, in ASTRONOMY AND SPACE EXPLORATION, Ural University Press (2021), URL https://doi.org/10.15826/b978-5-7996-3229-8.52.

10. R. V. Baluev, Astronomy and Computing, 25, 221, 2018. 


\title{
Analisys of morphological features of Mercury craters based on a new database
}

\author{
E.A. Feoktistova ${ }^{1}$, Zh.F. Rodionova ${ }^{1}$, A.Yu. Zharkova ${ }^{1,2}$, A.A. Kokhanov ${ }^{2}$, I.Yu. Zavyalov ${ }^{2}$ \\ jeanna@sai.msu.ru \\ ${ }^{1}$ Sternberg Astronomical Institute, Universiteytskiy pr. 13, Moscow, Russia, 119234 \\ ${ }^{2}$ Moscow State University of Geodesy and Cartography, Gorokhovskiy per.4, Moscow, Russia, 105064
}

The new global catalog of craters for Mercury, containing morphological and morphometric information, includes data on the location (latitude, longitude), diameter, depth and such features of craters as the degree of preservation of the rampart, the presence of terraces and collapses on the inner slope, the presence of peaks, hills and ridges, cracks and chains, the nature of the bottom, lava at the bottom, the ray system, the nature of the underlying surface. The new catalog is based on measurements of the coordinates and diameters of craters carried out at Brown University (USA) for craters with a diameter of $20 \mathrm{~km}$ or more and is supplemented by craters with diameters of $10-20 \mathrm{~km}$. To describe the morphological features of craters we used the methodology developed in SAI MSU.

Keywords: Mercury, catalog, craters, morphology, morphometric parameters, statistical analysis

DOI: 10.51194/VAK2021.2022.1.1.081

\section{Mercury craters catalogs}

Two NASA spacecraft photographed Mercury: "Mariner 10" flew past Mercury three times (between on March 19741975). It was only able to map 40-45\% of Mercury's surface, taking over 2,800 photos, "Messenger" ("Mercury Surface, Space Environment, Geochemistry, and Ranging") orbited the planet Mercury between 2011 and 2015, studying Mercury's chemical composition, geology and magnetic field and collecting close to 100,000 images. Images returned by MESSENGER have higher-resolution and better illumination conditions than the Mariner 10 imagery thus allowing an improved assessment of crater morphologies and associated landforms.

Early studies comparing the morphology and scale of craters on Mercury to the Moon focused primarily on crater interior structures, such as central peaks, crater depth, rim height, rim scallops, and wall terraces (e.g., [1, 2, 3]. The first catalogues of craters of the western hemisphere of Mercury appeared in 1976-1977 [4]. In 2011, catalogues of craters appeared on the entire surface of Mercury [5,6]. Catalog authors usually use different morphological parameters in their catalogs. In the catalog created at the Geophysical Institute, University of Alaska [6, 7], in addition to the coordinates and sizes of craters, the following morphological characteristics are given:

1. Degradation State: (f)resh, (s)tandard, and (d)egraded,

2. Rim and Wall Structure: (c)ircular, (s)calloped, and (t)erraced,

3. Interior Shape: (s)imple, (f)lat-floored, (cp) central peak, (cridge) central ridge, (mp) multiple peaks, (rpc) ringed peak cluster, $(\mathrm{pb})$ protobasin, and $(\mathrm{pr})$ peak ring basin,

4. Ejecta: bright-(r)ayed deposits, (fz) forbidden zone, (b)utterfly ejecta, and (d)ark ejecta,

5. Miscellaneous attributes: (e)lliptical planform, (p)it in floor, (h)ollows deposits, (b)right interior deposits, (d)ark interior deposits, (s)hadowed interior, (csp) central-structure summit pit, (cfp) central-structure floor pit, and additional comments.

\section{New Global Mercury Crater Catalog}

The new Global catalog of Mercury craters is created jointly by SAI MSU and Moscow State University of Geodesy and Cartography using a global mosaic of images of the Mercury surface according to the MESSENGER spacecraft. To compile the catalog, we used the global mosaic of Mercury MESSENGER MDIS with a resolution of $\sim 166 \mathrm{~m} /$ pixel and several MESSENGER DEMs - the first global DEM of Mercury with a resolution of $665 \mathrm{~m} /$ pixel [8], and at the same time four DEMs in the quadrants of Mercury with a resolution of $\sim 222 \mathrm{~m} /$ pixel [9]. The location and diameters of craters are determined automatically in ArcGIS software. Currently, the catalog includes more than 25,000 craters with a diameter of $1 \mathrm{~km}$ or more. The catalog consists of two parts. The first part is a morphological catalog of craters with a diameter of 10 $\mathrm{km}$ and more and contains a morphological description, as well as depth values for 12,265 impact craters of Mercury. The second part is a morphometric catalog containing information on the morphometric parameters of craters.

\subsection{System of morphological description of impact craters}

The system for morphological description of craters, which was used to create this catalog, is an extended version of the system for the morphological description of impact craters on planets of the Solar System, developed at GAISH MSU and used earlier to create morphological catalogs of craters on the Moon [10], Mars, and Mercury. This system includes 10 morphological features of a crater: 1) clarity or degree of preservation; 2) the presence of terraces and collapses on the slopes; 3) the nature of the crater wall; 4) the presence and nature of the central uplift (peaks, hills, ridges); 5) the presence of chains of craters and cracks at the bottom;6) the nature of the crater bottom; 7) the presence of lava at the bottom; 8) the presence of a ray system; 9) the type of surface underlying the crater. 


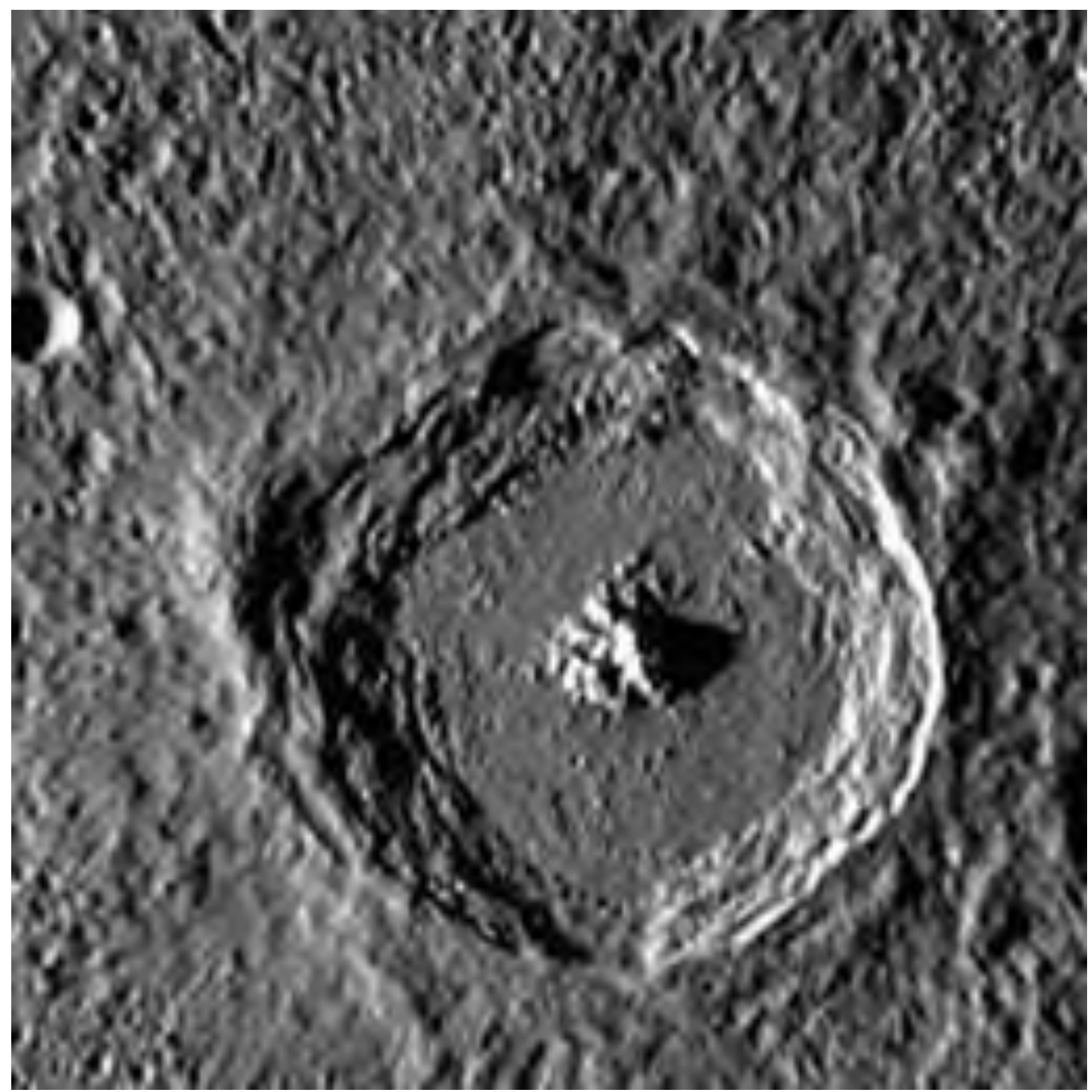

Figure 1: Alencar crater $\left(63.5^{\circ} \mathrm{S}, 103.5^{\circ} \mathrm{W}\right)$ according to the MESSENGER spacecraft.

In the New Global Mercury Crater Catalog, the morphological description of craters has been expanded. The following features were added: the presence of a dark halo around the crater, the elliptic shape of the crater, the location of the crater in the scarp area, the presence of an annular ridge, the presence of pits at the bottom of the crater. Each morphological feature includes a number of sub-features, the number of which in some cases reaches 14 . The distribution of the studied craters of Mercury by morphological features is given in Table 1. For 100\% in the table, 12,265 craters are taken.

Figure 1 shows an image of the Alencar crater $\left(D=120 \mathrm{~km}, 63.5^{\circ} \mathrm{S}, 103.5^{\circ} \mathrm{W}\right)$ obtained by the MESSENGER spacecraft. The description of the morphological characteristics of this crater in the catalog will look as follows: 2 (a fresh rim), 5 (many terraces on the slopes), 3 (a powerful outer rim), 9 (many peaks and hills at the crater floor), 0 (no chains of craters or fissures at the crater floor), 3 (rough floor), 2 (lava is present at the crater floor), 0 (no ray system), 3 (underlying surface is a transition zone between the highland and the plains).

\subsection{Morphological parameters of Mercury craters}

Statistical processing of the craters of Mercury with diameters of $10 \mathrm{~km}$ and more, included in the New Global Catalog, showed that craters of the third and fourth degrees of preservation (with a smoothed or partially destroyed wall) prevail on Mercury. The proportion of such craters reaches $\sim 70 \%$ of the total number of investigated craters $\geq 10 \mathrm{~km}$ in diameter (Table 1). Fresh craters (1st and 2nd preservation classes) make up only $\sim 18 \%$, and the oldest (ruins) 14\%. A significant number of the craters of Mercury have terraces and faults on the slopes: $65 \%$ of the craters, which is a distinctive feature of this planet. Almost $39 \%$ of craters have a central uplift, while only $11 \%$ of craters have central peaks. Central ridges are found only in $1.2 \%$ of craters, while $0.7 \%$ of craters have circular central ridges. A significant part of the central uplifts 
Table 1: Distribution of craters of Mercury by morphological features.

\begin{tabular}{|c|c|c|c|c|c|c|c|}
\hline $\begin{array}{l}\text { Morphological } \\
\text { features }\end{array}$ & $\begin{array}{l}\text { Designa- } \\
\text { tion }\end{array}$ & Semantic meaning & $\begin{array}{l}\text { \% cra- } \\
\text { ters }\end{array}$ & $\begin{array}{l}\text { Morphological } \\
\text { features }\end{array}$ & $\begin{array}{l}\text { Designa- } \\
\text { tion }\end{array}$ & Semantic meaning & $\begin{array}{l}\text { \% cra- } \\
\text { ters }\end{array}$ \\
\hline \multirow{5}{*}{ Rim degradation } & 1 & high preserved rim & 2.3 & \multirow{15}{*}{$\begin{array}{l}\text { Central uplift } \\
\text { (peaks. hills and } \\
\text { ridges) }\end{array}$} & 0 & no central uplift & 14 \\
\hline & 2 & preserved rim & 15 & & 1 & unknown & 46.4 \\
\hline & 3 & smooth rim & 37.3 & & 2 & hill & 5.3 \\
\hline & 4 & destroyed rim & 31.2 & & 3 & many hills & 21.1 \\
\hline & 5 & wholly degraded rim & 14.2 & & 4 & peak & 1.9 \\
\hline \multirow[t]{7}{*}{ Terraces and faults } & 0 & no terraces, no faults & 3.8 & & 5 & peak and hill & 0.9 \\
\hline & 1 & unknown & 31.3 & & 6 & peak and many hills & 4.4 \\
\hline & 2 & terrace & 22 & & 7 & many peaks & 0.7 \\
\hline & 3 & fault & 14.4 & & 8 & many peaks and hill & 0.04 \\
\hline & 4 & terrace and fault & 2.3 & & 9 & many peaks and hills & 3 \\
\hline & 5 & many terraces & 24.5 & & 10 & ridge & 0.4 \\
\hline & 6 & many terraces and fault & 1.7 & & 11 & ridge and hill & 0.1 \\
\hline \multirow[t]{4}{*}{$\operatorname{Rim}$} & 0 & no rim & 4.1 & & 12 & ridge and many hills & 0.6 \\
\hline & 1 & unknown & 7.3 & & 13 & ridge and peak & 0.02 \\
\hline & 2 & rim & 78 & & 14 & ridge and many peaks & 0.1 \\
\hline & 3 & massive rim & 10.6 & \multirow[t]{9}{*}{ Chains and fissures } & 0 & no chains and fissures & 5.8 \\
\hline \multirow[t]{5}{*}{ Lava on the floor } & 0 & no lava & 1.1 & & 1 & unknown & 52.2 \\
\hline & 1 & unknown & 49.8 & & 2 & chain & 18.7 \\
\hline & 2 & lava on the crater floor & 37.1 & & 3 & many chains & 22.9 \\
\hline & 3 & the whole bottom is & 12 & & 4 & fissure & 0.2 \\
\hline & & flooded with lava & & & & & \\
\hline \multirow[t]{3}{*}{ Ray system } & 0 & no ray system & 98.7 & & 5 & chain and fissure & 0.2 \\
\hline & 1 & unknown & 0.1 & & 6 & many chains & 0.1 \\
\hline & 2 & ray system & 0.8 & & 7 & $\begin{array}{l}\text { many chains } \\
\text { and fissure }\end{array}$ & 0.01 \\
\hline \multirow[t]{3}{*}{ Local terrain } & 1 & plain & 18.2 & \multirow{3}{*}{$\begin{array}{l}\text { Character of the } \\
\text { crater floor }\end{array}$} & 1 & unknown & 10.4 \\
\hline & 2 & highland & 36.5 & & 2 & flat floor & 26.4 \\
\hline & 3 & transitional zone & 45.5 & & 3 & rough floor & 63.1 \\
\hline
\end{tabular}

Table 2: Additional morphological characteristics of Mercury craters.

\begin{tabular}{ll}
\hline Morphological features & \% craters \\
\hline pit at the floor & 0.2 \\
escarp & 1 \\
dark halo & 0.2 \\
ring ridge & 0.7 \\
elliptical shape & 0.2 \\
\hline
\end{tabular}

is represented by hills: they are found in $26.5 \%$ of craters (Table 1 ). Chains of small craters can be seen on the floor and slopes of $40 \%$ of the craters. Most of the craters of Mercury have a rough floor, with about half of all craters filled with lava in whole or in part. Despite the wide distribution of scarps on the planet's surface, only $1 \%$ of the craters are intersected by these formations.

We compared the morphological characteristics of the populations of the impact craters of the Moon and Mercury. The Moon was chosen for comparison because, like Mercury, it is an atmosphereless body of the solar system and is close to it in size. We found the following differences in crater morphology on both bodies:

1. The presence of terraces and faults on the inner slopes is typical for the majority $(65 \%)$ of the craters of Mercury (on the Moon, the proportion of such craters: $7.4 \%$ )

2. At half of all craters of Mercury $(\sim 49 \%)$, the bottom is partially or completely filled with lava, which indicates a period of intense volcanism on Mercury in the past. For comparison, the share of such craters on the Moon is only $11 \%$.

3. $\sim 40 \%$ of Mercury craters have central uplifts at the bottom (hills, peaks, ridges) (on the Moon, the share of such craters is $\sim 21 \%$ )

4. $\sim 45.5 \%$ of Mercury's craters are located in the transition zone between continents and plains. For comparison, the share of such craters on the Moon is only $2.6 \%$.

\section{Conclusions}

The morphological characteristics of the craters of Mercury indicate that the craters of this planet were significantly affected by endogenous processes. The activity of intense processes on Mercury was more towers than on some other atmosphereless bodies of the solar system, for example, on the Moon.

Acknowledgments: E.A. Feoktistova, A.Yu. Zharkova, A.A. Kokhanov and Zh.F. Rodionova were supported by Russian Foundation for Basic Research (RFBR), project No 19-31-37001.

\section{References}

1. J. W. Head, Lunar and Planetary Science Conference Proceedings, 3, 2913, 1976.

2. V. R. Oberbeck, W. L. Quaide, R. E. Arvidson, and H. R. Aggarwal, JGR, 82, 1681, 1977.

3. R. J. Pike, Geomorphology of impact craters on Mercury., 165-273 (1988). 
4. Y. N. Lipskij, Z. F. Rodionova, T. P. Skobeleva, K. I. Dekhtyareva, et al., Catalogues of craters of Mercury and the Moon, Soyuzgeolfond, Moskva (1977).

5. C. I. Fassett, S. J. Kadish, J. W. Head, S. C. Solomon, and R. G. Strom, Geophys. Res. Lett., 38, L10202, 2011.

6. R. R. Herrick, L. L. Curran, and A. T. Baer, Icarus, 215, 452, 2011.

7. R. R. Herrick, E. M. Bateman, W. G. Crumpacker, and D. Bates, Journal of Geophysical Research (Planets), 123, 2089, 2018.

8. A. Y. Zharkova, I. P. Karachevtseva, A. E. Zubarev, E. S. Brusnikin, A. A. Kokhanov, and M. A. Kreslavsky, Sovremennye problemy distantsionnogo zondirovaniya Zemli iz kosmosa, 13, 265, 2016.

9. C. L. Johnson and S. A. Hauck, Journal of Geophysical Research (Planets), 121, 2349, 2016.

10. Z. F. Rodionova and E. A. Kozlova, Solar System Research, 34, 390, 2000. 


\title{
Impact flare in exosystems: optical manifestation
}

\author{
M.A. Ibrahimov \\ mansur@inasan.ru \\ Institute of Astronomy RAS, 48 Pyatnitskaya Str., 119017 Moscow, Russia
}

\begin{abstract}
A general discrepancy of statistics observed for known population of exosystems (exoplanets, exocomets and exosateroids) is noted. The most populated exosystem objects (i.e. exoasteroids) demonstrate the most poorly optic/IR manifestation. So far, only 2 events observed in IR are interpreted as an exoasteroid manifestation. In the paper, recently found unusual "red flare" on stars are also supposed to interpret as an exoasteroid manifestation but in optic. Observed red flares are suggested to be an impact flare of asteroid in some exosystem. Main optic features of observed red flares are presented and discussed.
\end{abstract}

Keywords: flare stars, asteroid impacts, optic photometry

DOI: $10.51194 /$ VAK2021.2022.1.1.082

\section{Introduction: observed population of exosystems}

Up to date known population of exosystems consist of 3 type of objects: exoplanets (about $5 \times 10^{3}$ objects), exocomets (about $5 \times 10^{2}$ events observed in 20-30 exosystems) and exoasteroids $\left(2 \times 10^{0}\right.$ objects). So far, there are IR observations only for 2 exoasteroids [[1], Wang et al., ApJL, 2019, 886, L5].

Comparing above numbers, we see that there is some obvious discrepancy in observational manifestation for different types of exosystem population. Using Solar system analogy, exoasteroids are believed to be the most populated objects in exosystems. Their expected sizes (about 100-1000 km), denses (about $5 \mathrm{~g} / \mathrm{cm}^{3}$ ) and relative velocities (about 10-30 km/sec) suggest that during impact events they could produce an energy which well exceed an energy of typical UV Cet type stars (about $10^{28}-10^{30} \mathrm{erg}$ ). If so, a question is: why so few (only 2) asteroid manifestation in exosystems had been observed so far?

\section{Red flare on stars}

Optic flare of a typical UV Cet type star demonstrates "blue" maxima (normally in $U$ band), "blue" distribution of flare amplitudes (which normally decline from blue to red/IR bands), and "blue" optical colors (so called "blueing" during the flare). However, recently a new flare events (so called "red flares" on stars or RFs) had been proposed (Ibrahimov M.A., in: Ground-Based Astronomy in Russia. 21st Century, Proceedings of the All-Russian Conference held 21-25 September, 2020 in Nizhny Arkhyz, Russia. Edited by I. I. Romanyuk, I. A. Yakunin, A. F. Valeev, D. O. Kudryavtsev, 2020, p. 288-292). RFs demonstrate right opposite characteristics to compare the flares on UV Cet type stars.

Available RF statistics includes 3 objects and 5 RFs observed on them. These are UU CrB (1 RF in 1980), FF Ori (2 RFs in 1991 and 1992), and IX Oph (2 RFs in 1992 and 1993). RFs demonstrate the following features in optic: "slow" flares with "red" maxima, "red" distribution of flare amplitudes (opposite to that observed for typical UV Cet type stars), and "red" optical colors. RFs markedly differ from typical flares observed for UV Cet type stars. Main differs are: i) RFs happened in systems hosted F-K type stars, ii) RFs appeared to be powerful flares or superflares (estimated powers ranged in $10^{36}-10^{39}$ erg), iii) RFs are "slow" flares (all well-observed RFs had a duration more than 30 min), iv) RFs flare energy distribution notably differs from that of UV Cet type flares (so called "3-redness" of RFs: red maxima, red amplitude distribution, and red colors). Also important to note that RFs seem to be recurrent flares (2 of 3 objects demonstrated recurrent flares).

\section{Red flare statistics and observed optical features}

All so far observed optical data for RFs is summarized in Table 1. Chronologically, the first RF was observed on May 21, 1980 on F8V star UU CrB [[2]]. Flare duration was more than 40 min. The inverse ("red") distribution of flare amplitudes was $\Delta I_{K}: \Delta V=0.30: 0.05$. However, the flare colors seemed to be difficult to analyse and compare with that of other RFs (observations had been done in specific photometric system using combination of Stromgren-Crawford and Kron $u v b y+I_{K}$ filters).

The next two RFs were observed in FF Ori eclipsing binary during its $U B V R$ monitor [[3], [4]]. System includes B8V + F0 IV-III stars with $P=1.8105$ day. The first RF had been observed on October 26, 1991 ( 1 observation), the second one on October 22, 1992 ( 8 observations). The both RFs happened near the primary minimum at phase interval $0.001-0.025 P$ (63 min). RFs were observed in $R$-band only. No synchronous brightness variations were found in other bands. Maximal flare amplitude detected was $\Delta R \approx 0.1^{\mathrm{m}}$. During the both RFs, $(V-R)$ color reddening was observed. At the primary minimum $(V-R) \approx 0.25^{m}$, while during RFs the average color was $(V-R) \approx 0.31^{m}$ (see Tables 3 and 5 in [4]). So, during the both flares the $(V-R)$ reddening was $0.06^{m}$.

Two more RFs were detected by [5] in IX Oph during its $U B V R$ monitor. The star is $S B 1$-type spectral binary system. Binaries was concluded from a joint analysis of IX Oph spectra obtained in 2004 using Keck/HIRES [[6]] and its 22-yr optic photometry [[7]]. Primary component is appeared to be an invisible object with a strong $U V$ radiation. Secondary one is a normal K1-3 giant. The first RF was observed on August 28, 1992 (observations lasted more than 70 minutes, three BVR and one $U$ brightness estimates were obtained). The second RF was observed on July 27, 1993 (observation was about 2 minutes, one $B V R$ - and no $U$-band brightness estimates were made). $1992 U B V R$ light curve of IX Oph is shown in Fig. 1 (left panel). Accuracies of brightness estimates were $0.01^{\smile} 0.03^{m}$ for $B V R$ and $0.05^{\smile} 0.07^{m}$ for $U$ bands. 1992 RF of IX Oph (perhaps the most spectacular ever observed RF) is also shown in the panel. RF appears itself as characteristic "jumps" in all 
Table 1: Red flare statistics and observed optical features

\begin{tabular}{ccccccc}
\hline No. & Star & Flare & Duration \\
name & date & $\begin{array}{c}\text { Red } \\
\text { maxima } \\
\Delta R\end{array}$ & $\begin{array}{c}\text { Red amplitude } \\
\text { distributions } \\
\Delta R: \Delta V: \Delta B: \Delta U\end{array}$ & $\begin{array}{c}\text { Red colors } \\
(U B):(B V):(V R) \\
(m a g)\end{array}$ & $\begin{array}{c}(m a g) \\
(m a g)\end{array}$ \\
\hline 1 & UU CrB & 1980 May 21 & 40 & $0.3\left(\Delta I_{K}\right)$ & $\Delta I_{K}: \Delta V=0.30: 0.05$ & $n o$ \\
2 & FF Ori & 1991 Oct 26 & 2 & 0.1 & $0.1: 0.0: 0.0:$ no & $0.06(V-R)$ \\
3 & FF Ori & 1992 Oct 22 & 60 & 0.1 & $0.1: 0.0: 0.0: n o$ & $0.06(V-R)$ \\
4 & IX Oph & 1992 Aug 28 & 70 & 1.8 & $1.8: 1.0: 0.2:-1.3$ & $2.12: 1.94: 1.98$ \\
5 & IX Oph & 1993 Jul 27 & 2 & 1.8 & $1.8: 0.9: 0.3: n o$ & $n o: 1.94: 2.01$ \\
\hline
\end{tabular}

bands compared to generally smooth brightness variations. $B V R$ jumps happened upward (flare), while $U$ jump happened downward (anti-flare or eclipse). RF shown in Fig. 1 is the best example to demonstrate so called "3-redness": red maximum, red distribution of amplitudes and extremely red colors (see also Table 1 for exact numbers).

Remarks to Table 1:

UU CrB [[2]]: F8V star; observed in 5 color Stromgren-Crawford and Kron uvby $+I_{K}$ system; Johnson $\Delta V$ system magnitude from original paper; estimated flare power $\approx 7 \times 10^{35} \mathrm{erg}$.

FF Ori [[3], [4]]: eclipsing binary system with B8V + F0 IV-III stars and $P=1.8105$ day; observed in $U B V R$ system; recurrent RFs with a time lag of 12 months between the flares; both flares happened in MinI at phases $0.001-0.025 P$ (63 minutes); estimated flare power $10^{36} \mathrm{erg}$.

IX Oph [[5]]: $S B 1$ spectral binary system with invisible primary and visible K1-3 III secondary components; observed in $U B V R$ system; brightness variations with amplitude $\sim 1^{m}$, possible period $\sim 150$ day, and $U V$-excess of $-0.3^{m}$ with 15-20 day long "plateau" in $U$-curve were detected by [7]; recurrent RFs with a time lag of 11 months between the flares; estimated flare power $\gtrsim 10^{39} \mathrm{erg}$.
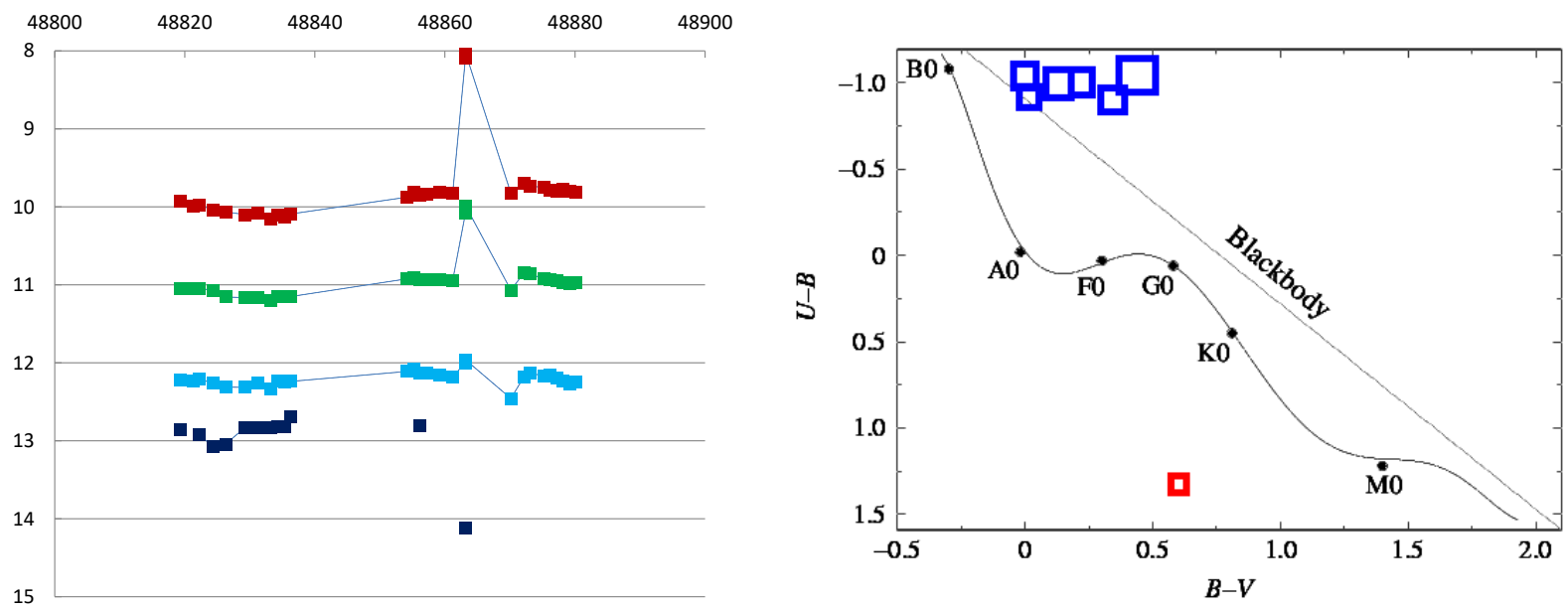

Figure 1: Left panel: 1992 light curves of IX Oph in (top to bottom) $R, V, B$, and $U$ bands. Scales in mag and $J D 2400000+$ units. Red flare is seen in all curves with its red maximum in $R$ and red amplitude distribution from flare in $R$ to anti-flare (eclipse) in $U$. Right panel: $(U-B)-(B-V)$ diagram in mag for intrinsic flare radiation. Typical UV Cet type flare radiation are plotted as blue squares, while red square is that for red flare of IX Oph.

\section{Possible interpretation: red flare as an impact flare in exosystems}

Two-color $(U-B)-(B-V)$ diagram for intrinsic flare radiation is shown in Fig. 1 (right panel). Data to plot such a radiation for flares of typical UV Cet type stars is taken from the recent monograph of Gershberg (2015) [Gershberg R.E., SolarType Stellar Activity of the Main Sequence Stars, Simferopol: Antikva Ltd., 2015, p.299] and shown as blue squares in the diagram. That for red flares observed on IX Oph calculated using [5] and plotted as red square.

Principal difference between two type of flares is clearly seen in the diagram. "Blue" flares locate above the line of nonreddened colors and this implies a star nature of UV Cet type flares. "Red" flares locate beneath the line and this could be considered as a non-star nature of RFs. There are two general mechanisms to produce non-star radiation: i) envelope/disc accretion and ii) small (exo)body impacts. The latter seems to be reasonable to use as a possible interpretation of RF nature. If so, RF features (their long duration, unusual 3-redness, large energy and recurrence) could naturally be explained 
in light of impact event happened in some exosystem. In that picture, small bodies in some exoplanet system impact to each other or/and impact to exoplanets/exomoons themselves producing (perhaps recurrently) slow and powerful flares in optic with unusual "3-redness" characteristics.

\section{Some conclusions}

If above interpretation is correct:

1. Statistics for so far observed impact events in exosystem should be enlarged from 2 to 5 systems and from 2 to 7 impact flares ( 2 of them observed in IR and 5 in optic).

2. Mentioned discrepancy in observed statistics when the most populated objects (i.e. exoasteroids) demonstrate poorly observation manifestation a bit improved (see above item) but still remains.

3. Both intensive and simultaneous optical and IR monitor of the selected promising targets are needed to verify impact nature of the red flares and to reduce mentioned discrepancy.

\section{References}

1. H. Y. A. Meng, K. Y. L. Su, G. H. Rieke, D. J. Stevenson, et al., Science, 345, 1032, 2014.

2. E. C. Olson, Information Bulletin on Variable Stars, 1825, 1980.

3. M. M. Zakirov, Information Bulletin on Variable Stars, 3925, 1993.

4. M. M. Zakirov, Astronomy Letters, 22, 593, 1996.

5. M. A. Ibrahimov, INASAN Science Reports, 4, 199, 2019.

6. G. H. Herbig, $A J, \mathbf{1 3 0}, 815,2005$.

7. M. A. Ibrahimov and K. N. Grankin, INASAN Science Reports, 4, 192, 2019. 


\section{Formation of the terrestrial planets and the Moon}

\section{S.I. Ipatov}

siipatov@hotmail.com,

WWW home page: https://siipatov.webnode.ru

Vernadsky Institute of Geochemistry and Analytical Chemistry of RAS, Moscow, Russia

The amounts of material from different parts of the zone from 0.7 to 1.5 AU from the Sun, which entered into almost formed the Earth and Venus, differed for these planets by no more than 3 times. For the TRAPPIST exoplanetary system, the ratio of the fraction of planetesimals collided with the planet, around which orbit initial orbits of planetesimals were located, to the fraction of planetesimals collided with the neighbouring planet was typically less than 4 . Embryos of the Earth and the Moon with a total mass equaled to about 0.01-0.1 Earth mass could be formed as a result of compression of a rarefied condensation. The fraction of material ejected from the Earth's embryo and acquired by the Moon's embryo could exceed by an order of magnitude the sum of the total mass of the planetesimals acquired by the Moon's embryo and of the initial mass of the Moon's embryo.

Keywords: Earth, Moon, solar system: formation

DOI: $10.51194 /$ VAK2021.2022.1.1.083

\section{Migration of planetesimals to the terrestrial planets and to TRAPPIST planets from their feeding zones}

First studies of the formation of the terrestrial planets were based on analytical estimates. Later computer simulations of the evolution of disks of gravitating bodies combined at their collisions were used for studies of the process of accumulation of the terrestrial planets (e.g. [1]). [2], [3] considered also pebble accretion at the gas stage. [1] and [4] studied mixing of planetesimals during accumulation of the terrestrial planets and estimated fractions of planetesimals, initially located at different distances from the Sun, that collided with planets. [4] considered migration of planetesimals with initial semi-major axes $a_{o}$ between $a_{\text {min }}$ and $a_{m i n}+d_{a}$, where $a_{m i n}$ varied from 0.3 to $1.5 \mathrm{AU}$ with a step of $0.2 \mathrm{AU} ; d_{a}$ equaled to $0.2 \mathrm{AU}$, exclusive for $a_{\text {min }}=1.5 \mathrm{AU}$ with $d_{a}=0.5 \mathrm{AU}$. Initial eccentricities $e_{o}$ of orbits of planetesimals equaled to 0.05. In some variants with present planets they were 0.3 . Initial inclinations were equal to $e_{o} / 2$ rad. It was obtained by [1], [2], [3] that, due to the mutual gravitational influence of planetesimals, the average eccentricity of orbits of planetesimals in the feeding zone of the terrestrial planets could exceed 0.3. In the series MeN of calculations, the migration of planetesimals was studied under the gravitational influence of all planets (from Mercury to Neptune). In the series $\mathrm{Me}_{01} \mathrm{~S}$ of calculations, I considered the embryos of the terrestrial planets with masses equal to 0.1 of the present planet masses moving in present orbits of the planets, and also Jupiter and Saturn with their present masses and orbits (Uranus and Neptune were excluded). For series $\mathrm{Me}_{03} \mathrm{~N}$, masses of the embryos of the terrestrial planets equaled to 0.3 of the present masses of the planets, and all giant planets were considered. For integration of the motion equations, the symplectic method of the Swift integration package written by [5] was used. Ejections of planetesimals into hyperbolic orbits and their collisions with the Sun were taken into account. At integration, planets were considered by [4] as material points. The probabilities of collisions of planetesimals with the terrestrial planets or their embryos were calculated based on the arrays of orbital elements of migrated planetesimals similar to Below for shortness I denote such model as model $A$.

[4] concluded that inner layers of the Earth or Venus were formed by accumulation mainly of material from the neighbourhood of the planet's orbit. The amounts of material from different parts of the zone from 0.7 to 1.5 AU from the Sun, which entered into almost formed the Earth and Venus, differed, probably, by no more than 2 or 3 times. Some authors (e.g., [6]) suppose that Mars has acquired most of its mass in not more than 5-10 Myr. Such fast growth was not obtained for accumulation starting from small planetesimals. To consider rapid formation of Mars in 5 Myr, Ipatov (2019) supposed that the Mars embryo with a mass of about 0.2 of the present mass of Mars was formed by contraction of a rarefied condensation. Similar to the supposition for Mars, [4] also suggested that Mercury's embryo could form with mass about $0.02 m_{E}$ (where $m_{E}$ is the mass of the Earth) as a result of compression of a rarefied condensation. The ratio of probabilities of planetesimals collided with the embryos of the Earth and the Moon in the considered calculations did not exceed the ratio of their masses. In some runs with $0.3 \leq a_{o} \leq 0.5 \mathrm{AU}$ and $1.1 \leq a_{o} \leq 2.0 \mathrm{AU}$ at $e_{o}=0.3$ at time interval $T=20 \mathrm{Myr}$, more than $10 \%$ of initial planetesimals could fall onto the Sun. The fraction of planetesimals ejected into hyperbolic orbits from the feeding zone of the terrestrial planets did not exceed $10 \%$. The probability of a collision of a planetesimal from the terrestrial feeding zone with Jupiter did not exceed a few percent of the probability of the collision with the Earth. The probability of the collision with Saturn was by an order of magnitude smaller than that with Jupiter.

The model considered by [4] didn't exclude planetesimals that already collided with a planet and so could not collide with another planet. If the probailities of collisions with planets were not small, then the model $A$ overestimated the number of collisions with planets. Therefore, I also considered the model $C$ which calculates moments of collisions of planetesimals with planets and excludes collided planetesimals from integration of the motion of planetesimals. The number of collisions of planetesimals with planets was smaller for the model $C$ than for the model $A$ at the same time interval. The conclusions from [4] presented above are true also for the model $C$. Accumulation of a half of masses of the Earth and Venus from the zone at 0.7-1.1 AU from the Sun could take more than 5 Myr in the model C (for the model A such time did not exceed 5 Myr).

The TRAPPIST exoplanetary system includes a star with a mass equal to 0.09 mass of the Sun and 7 planets with semi-major axes from 0.01 to $0.06 \mathrm{AU}$. Using the model $C$, I calculated migration of planetesimals located near each orbit 
of an exoplanet with initial eccentricities equaled to 0.02 or 0.15 (in different variants of calculations). During 1000 yr more than a half of initial planetesimals collided with planets. The ratio of the fraction of planetesimals collided with the planet, around which orbit initial orbits of planetesimals were located, to the fraction of planetesimals collided with a neighbouring planet was typically less than 4 .

\section{Formation of the Moon}

The giant impact model considered the formation of the Moon as a result of a collision of the Earth with an object with a mass of about the mass of Mars. [7] suggested that the embryos of the Earth and the Moon with a total mass of about 0.01-0.1 mass of the Earth formed as a result of contraction of the same rarefied condensation. The angular momentum of the condensation needed for formation of the binary system probably was acquired at a collision of two rarefied condensations. Other terrestrial planets have not such large satellites because the parent condensations for their embryos had not got the angular momentum needed for formation of the satellite system. The angular momentum of the condensation needed for formation of the Earth-Moon system could be acquired by accumulation only of small objects. However, for such accumulation of only small objects, other terrestrial planets would have large satellites.

For the fraction of iron in the initial embryo of the Moon and in the planetesimals equaled to 0.33 and the fraction of iron in the Earth's crust and in the Moon equaled to 0.05 and 0.08 , respectively, the fraction $k_{E}$ of the Earth crust in the Moon would be about 0.9 . This estimate follows from the ratio $0.05 k_{E}+0.33\left(1-k_{E}\right)=0.08$. Therefore, in order to explain the current fraction of iron in the Moon, the mass of matter ejected from the Earth embryo and fallen onto the Moon embryo should have been by an order of magnitude greater than the sum of the total mass of planetesimals falling on the Moon embryo and the initial mass of the embryo of the Moon formed from the parental condensation, if the initial Moon embryo contained the same fraction of iron as planetesimals. The considered model differs from the known multiple impact models (e.g., [8]) by that the initial embryo of the Moon in my model was formed from the same rarefied condensation, as the Earth embryo, but not from a disk of material ejected from the Earth embryo. In my opinion, the material ejected from the Earth embryo could be easier captured by the Moon embryo than it was combined to form a larger body. The model of the formation of a solid planet with a large satellite can also work for some exoplanet.

\section{Acknowledgements}

Studies of the formation of the terrestrial planets were carried out as a part of the state assignments of the Vernadsky Institute of RAS No. 0137-2019-0004. For studies of migration of planetesimals in the TRAPPIST system the author acknowledges the support of Ministry of Science and Higher Education of the Russian Federation under the grant 075-152020-780. Studies of the formation of the Moon were carried out with the support of the Russian Science Foundation grant No. 21-17-00120, https://rscf.ru/project/21-17-00120/.

\section{References}

1. S. I. Ipatov, Solar System Research, 27, 83, 1993, URL https://www.academia.edu/44448077.

2. J. M. Woo and et al., Icarus, 359, 114305, 2021.

3. J. M. Woo and et al., Icarus, 371, 114492, 2021.

4. S. I. Ipatov, Solar System Research, 53, 332, 2019, URL https://arxiv.org/abs/2003.11301.

5. H. F. Levison and M. J. Duncan, Icarus, 108, 18, 1994.

6. L. T. Elkins-Tanton, Nature, 558, 522, 2018.

7. S. I. Ipatov, Solar System Research, 52, 401, 2018, URL https://arxiv.org/abs/2003. 09925.

8. R. Rufu and O. Aharonson, Nature Geoscience, 10, 89, 2017. 


\title{
Migration of planetesimals to planets located in habitable zones in the Solar System and in the Proxima Centauri system
}

\author{
S.I. Ipatov \\ siipatov@hotmail.com, \\ WWW home page: https://siipatov.webnode.ru \\ Vernadsky Institute of Geochemistry and Analytical Chemistry of RAS, Moscow, Russia
}

\begin{abstract}
The values of the probability of a collision of a planetesimal with the Earth were typically greater for smaller distances $R$ from the Sun at $3<R<40 \mathrm{AU}$. The probability varied from about $10^{-6}$ at $R \sim 30-40 \mathrm{AU}$ to $10^{-3}-10^{-2}$ at $R$ about 3.2-3.3 AU. Though only one of several hundreds of planetesimals from the zone of exoplanet $c$ in the Proxima Centauri system reached the inner exoplanet $b$, it often collides with the planet $b$. The probability of a collision of such planetesimal with the exoplanet $b$ could be about several $10^{-4}$. A lot of icy material could be delivered to inner exoplanets $b$ and $d$ in the Proxima Centauri system.
\end{abstract}

Keywords: Earth, Moon, migration of planetersimals, Proxima Centauri system

DOI: 10.51194 /VAK2021.2022.1.1.084

\section{Migration of planetesimals to the Earth}

Earth's ocean water and its D/H ratio could be the result of mixing water from several exogenous and endogenous sources with high and low $\mathrm{D} / \mathrm{H}$ ratios. The exogenous source includes small bodies coming from the zone of outer asteroid belt and from beyond the Jupiter's orbit. Migration of planetesimals under the gravitational influence of 7 planets (from Venus to Neptune) was studied. In the JN series of calculations, initial semi-major axes $a_{o}$ of planetesimals varied from $a_{m i n}$ to $a_{m i n}+d_{a}$ at $d_{a}=2.5 \mathrm{AU}$. For different runs, $a_{\text {min }}$ varied from 2.5 to $40 \mathrm{AU}$ with a step equaled to $2.5 \mathrm{AU}$. Initial eccentricities $e_{o}$ of planetesimals equaled to 0.05 or 0.3 . Initial inclinations were equal to $e_{o} / 2 \mathrm{rad}$. The mean eccentricities equaled to 0.3 could be reached due to mutual gravitational influence of planetesimals during evolution of a disk of planetesimals in the feeding zone of the giant planets $[1,2]$. For the AST series of calculations, $a_{m i n}$ varied from 3.0 to 4.9 AU with a step equaled to $0.1 \mathrm{AU}, d_{a}=0.1 \mathrm{AU}, e_{o}$ equaled to 0.02 or 0.15 . For the same values of $a_{\text {min }}$ and $e_{o}$ several (up to 8) runs with 250 planetesimals could be made. For integration of the motion equations, the symplectic method from the Swift integration package written by [3] was used. The probabilities of collisions of planetesimals with the terrestrial planets were calculated based on the arrays of orbital elements of migrated planetesimals similar to [4] and [5].

At $a_{\text {min }}>12 \mathrm{AU}$ the probability $p_{E}$ of a collision of a planetesimal with the Earth averaged over 250 planetesimals did not exceed $10^{-5}$. It usually was $\sim 10^{-6}$ at $a_{\text {min }}>27$ AU. At $a_{m i n} \leq 10 \mathrm{AU}$ the values of $p_{E}$ averaged over 250 bodies can vary by up to a factor of a thousand, and in some calculations they exceeded $10^{-3}$. The maximum values of $p_{E} \sim 10^{-2}$ were obtained at $a_{\min }$ about 3.2-3.3 AU. Most of collisions with the Earth of bodies, originally located at a distance of 4 to $5 \mathrm{AU}$ from the Sun, occurred during the first 10 million years. For $3 \leq a_{\min } \leq 3.5 \mathrm{AU}$ and $e_{o} \leq 0.15$, individual bodies could fall onto the Earth and the Moon in a few billions years. For example, at $a_{\text {min }}=3.3 \mathrm{AU}$ and $e_{o}=0.02, p_{E}=4 \cdot 10^{-5}$ for $0.5 \leq t \leq 0.8 \mathrm{Myr}$ (the time of the late-heavy bombardment) and $p_{E}=6 \cdot 10^{-6}$ for $2 \leq t \leq 2.5 \mathrm{Myr}$. For $a_{\text {min }}=3.2 \mathrm{AU}$ and $e_{o}=0.15$, it was obtained that $p_{E}=0.015$ at $0.5 \leq t \leq 1 \mathrm{Myr}$, and $p_{E}=6 \cdot 10^{-4}$ at $1 \leq t \leq 2$ Myr. The zone of the outer asteroid belt can be one of the sources of the late heavy bombardment.

At $p_{E}=2 \cdot 10^{-6}$ and the total mass of planetesimals equaled to $100 m_{E}$ (where $m_{E}$ is the mass of the Earth), the total mass of planetesimals collided with the Earth is about the mass of the Earth's oceans. While considering thousands of planetesimals, the mean probability of a collision of a planetesimal with the Earth for the region between 5 and 10 AU could exceed $2 \cdot 10^{-6}$ by at least a factor of several. On average, for the region between 20 and 40 AU the probability could be about $10^{-6}$. Some fraction (may be $1 / 3$ ) of this material was composed of water and volatiles. Estimates of the fraction of ice in comets do not exceed $33 \%$. However, a number of authors believe that primary planetesimals may have contained more ice than present comets. The amount of water delivered from beyond the Jupiter's orbit to the Earth could exceed the mass of Earth's oceans if the total mass of planetesimals was about $200 m_{E}$. For planetesimals migrated from distances $R>3 \mathrm{AU}$, the ratio of planetesimals collided with the Earth and the Moon is about 17 . Velocities of bodies migrated from the zone of Jupiter and Saturn and collided with the Moon were mainly about $20-23 \mathrm{~km} / \mathrm{s}$. For Mars, the ratio of the mass of water in planetesimals delivered from beyond the orbit of Jupiter to a planet to the mass of the planet was approximately two to three times greater than that for the Earth. The mass of water delivered to Mercury or Venus, calculated per unit mass of the planet, was a little greater than that for the Earth. These mass fractions would result in relatively large ancient oceans on Mars and Venus.

\section{Migration of planetesimals in the Proxima Centauri system}

The Proxima Centauri system includes a star with a mass equal to 0.122 of the mass of the Sun, two confirmed planets $b$ and $c\left(a_{b}=0.04857 \mathrm{AU}, e_{b}=0.11, m_{b}=1.17 m_{E}, a_{c}=1.489 \mathrm{AU}, e_{c}=0.04, m_{c}=7 m_{E}\right)$, and one unconfirmed planet $d$. It is considered that the planet $b$ can be located in a habitable zone. I studied migration of planetesimals from the vicinity of the planet $c$. Gravitational influence of a star and planets $b$ and $c$ was taken into account. It was supposed that $i_{b}=i_{c}=0$. The densities of the exoplanets $b$ and $c$ were considered to be equal to densities of the Earth and Uranus, respectively. In different calculation variants, initial semi-major axes of planetesimals were in the range from $a_{\text {min }}$ to $a_{\text {min }}+0.1 \mathrm{AU}$, at $a_{\text {min }}$ from 1.2 to $1.7 \mathrm{AU}$ with a step of 0.1 AU. Initial eccentricities of planetesimals equaled to $e_{o}=0.02$ or $e_{o}=0.15$. 
Greater initial eccentricities could be a result of the mutual gravitational influence of planetersimals. Initial inclinations of the planetesimals equaled to $e_{o} / 2 \mathrm{rad}$. 250 planetesimals were considered in each calculation variant. The motion of planetesimals was calculated with the use of the symplectic code from [3]. In the series MP of calculations, planets were considered as material points, and the probabilities of collisions of planetesimals with the exoplanets were calculated based on the obtained arrays of orbital elements of migrated planetesimals stored with a step of 100 yr. I also calculated the probabilities of collisions of planetesimals with the unconfirmed exoplanet $d\left(a_{d}=0.02895 \mathrm{AU}, m_{d}=0.29 m_{E}, e_{d}=i_{d}=0\right)$, which was not considered in integrations. If the calculated probability of a collision of some planetesimal with an exoplanet reached 1 with time (it was obtained for a few planetesimals), then for a later time this planetesimal was not considered for calculation of the mean probability for the calculation variant. Such approach of calculations of the probabilities is good when the probabilities are small, as in this case it is not needed to consider a large number of planetesimals. However, probabilities of collisions of planetesimals with the planet $c$ were not small, especially for almost circular initial orbits of planetesimals which were close to the orbit of the planet $c$. So in the series $\mathrm{C}$ of calculations, the times of collisions of planetesimals with planets were calculated, and such collided planetesimals excluded from the integrations. For the series $\mathrm{C}$ at $e_{o}=0.02$, the ratio $k_{e-c}$ of the number of planetesimals ejected into hyperbolic orbits to the number of planetesimals collided with the planet $c$ was between 0 and 0.1 at $t<1 \mathrm{Myr}$, between 1 and 2.5 at $1<t<2.5 \mathrm{Myr}$, and was greater than 5 at $t>2.5 \mathrm{Myr}$. At $e_{o}=0.15, k_{e-c}$ was between 0.3 and 0.7 at $t<1 \mathrm{Myr}$, between 3 and 7 at $1<t<2.5 \mathrm{Myr}$, between 10 and 15 at $t>2.5 \mathrm{Myr}$, and between 1.6 and 2.5 for a whole considered time interval. At $e_{o}=0.15$, less that $10 \%$ of initial planetesimals were left in the disk after $10 \mathrm{Myr}$. In both MP and C series of calculations, only one of several hundreds of planetesimals reached the orbits of the exoplanet $b$, but among a few thousands of planetesimals there were planetesimals that collided with the planet $b$. So the probability $p_{b}$ of a collision of one planetesimal with the exoplanet $b$ (averaged over thousands planetesimals) equaled to a few $10^{-4}$, depending on $e_{o}$ and a series of calculations, and was greater than the probability of a collision with the Earth of a planetesimal from the zone of the giant planets in the Solar System. The latter probability for most calculations with 250 planetesimals was less than $10^{-5}$ per one planetesimal. The calculations of the probabilities based on the arrays of orbital elements showed that the probability of a collision of a planetesimal with the exoplanet $d$ was a little smaller than that with exoplanet $b$. Therefore, a lot of icy material could be delivered to the exoplanets $b$ and $d$.

\section{Acknowledgements}

Studies of the migration of planetesimals to the Earth were carried out as a part of the state assignments of the Vernadsky Institute of RAS No. 0137-2019-0004. For studies of migration of planetesimals in the Proxima Centauri system, the author acknowledges the support of Ministry of Science and Higher Education of the Russian Federation under the grant 075-152020-780. Studies of of collisions of planetesimals with the Moon were carried out with the support of the Russian Science Foundation grant No. 21-17-00120, https://rscf.ru/project/21-17-00120/.

\section{References}

1. S. I. Ipatov, Earth, Moon, and Planets, 39, 101, 1987, URL https://www.academia.edu/44448077.

2. S. I. Ipatov, Solar System Research, 27, 83, 1993, URL https://www.academia.edu/44448077.

3. H. F. Levison and M. J. Duncan, Icarus, 108, 18, 1994.

4. S. I. Ipatov and J. C. Mather, Annals of the New York Academy of Sciences, 1017, 46, 2004, URL https://arxiv.org/format/astro-ph/0308448.

5. S. I. Ipatov, Solar System Research, 53, 332, 2019, URL https://arxiv.org/abs/2003.11301. 


\title{
Thermal evolution of the cores of the icy satellites of the giant planets
}

\author{
E. Kronrod, V. Kronrod, O. Kuskov \\ e.kronrod@gmail.com \\ Vernadsky Institute of Geochemistry and Analytical Chemistry RAS, Moscow, Russia
}

Modern models of satellites of the giant planets include a water-ice shell and an iron silicate core with the possible presence of an inner Fe-FeS core. During missions to Jupiter and Saturn (Galileo, Cassini-Huygens), new data were obtained that impose restrictions on the structure of the ice giant satellites Ganymede, Callisto and Titan. However, questions about the composition, size, and physical properties of satellite cores and their thermal evolution are still the subject of numerous discussions. Temperature distributions in the cores of satellites largely determine the degree of hydration of their silicate component, the presence or absence of internal metal cores. The thermal evolution of the cores is determined by their size, as well as physical properties - density, thermal conductivity, heat capacity, and radiogenic heat release. In this work, we considered two geochemical models of core composition and their combinations that are maximally different in physical properties: silicate models with the composition of matter of ordinary (L / LL) chondrites and hydrosilicate models close to the composition of carbonaceous (CI) chondrites. The results of calculations of unsteady temperature regimes in the cores of ice satellites are presented, taking into account the composition, convective heat transfer, and possible processes of dehydration of hydrated silicates in the cores. Calculations of thermal evolution and dehydration processes in cores with concentrations of hydrosilicates $C_{H \text { sil }}=0-1$ and core radii $R_{\text {core }}=500-2000 \mathrm{~km}$ were performed. Based on the results of numerical experiments, it can be concluded that the initial concentration of hydrosilicates $\left(C_{H \text { sil }}\right)$ and the radius of the core $\left(R_{\text {core }}\right)$ significantly affect the thermal evolution and dehydration of the core.

Keywords: primordial rocky core, temperature, convection, thermal evolution, hydrated silicates, dehydration

DOI: $10.51194 /$ VAK2021.2022.1.1.085

\section{Introduction}

Models of the internal structure of large icy satellites [1] impose restrictions on the composition of satellite cores, and temperature distributions in satellite cores largely determine the degree of hydration of their silicate component, the presence or absence of internal metal cores. This paper presents the results of calculations of the evolutionary thermal model of the proto-cores of rocky-ice satellites taking into account the composition, convective heat transfer, and possible processes of dehydration of hydrated silicates in the depths of the iron-rocky proto-core.

\section{Models and Methods}

At the last stage of accretion, the temperature in the near-surface region of the satellite reaches the melting point of ice, as a result of which the materials of the upper regions are separated into water and an iron-rocky component, which migrates to the center of the satellite. The process of heating to a temperature sufficient for the redistribution of ice, water and rock (about $500 \mathrm{~K}$ ) takes about 500 million years [2,3]. Subsequently, the heating of the substance in the core occurs due to radioactive energy sources.

The composition of protocores is modeled by a combination of two geochemical models that are maximally different in physical properties. In the first model, silicate (Sil), the composition of the iron-rocky material corresponds to the bulk composition of the matter of ordinary (L / LL) chondrites [4, 5]. The second type of models (hydrated silicate, HSil) is used in models of the internal structure of fully differentiated satellites; the iron-rocky core can be composed of a hydrated mineral material of low density, close in composition to the substance of carbonaceous CI chondrites [3].

At a temperature of $873 \mathrm{~K}$, an endothermic dehydration reaction of hydrated silicates occurs [6], during which anhydrous olivine and water are formed from serpentine and talc. As a first approximation, we assume that the water released as a result of dehydration processes does not significantly affect the physical properties and chemical composition of the rock.

The temperature distributions in the core are found as a result of the numerical solution of the one-dimensional nonstationary heat conduction equation taking into account the conductive and convective mechanisms of heat transfer. Heat transfer in the convective zone is modeled by multiplying the thermal conductivity coefficient by a dimensionless coefficient (Nusselt number):

$$
K_{\mathrm{ef}}=N u k_{\mathrm{cond}} ; N u=1.04\left(R a / R a_{\mathrm{crit}}\right)^{1 / 3} ; R a_{\mathrm{crit}} \approx 1000
$$

where $K_{\text {ef }}, k_{\text {cond }}, N u, R a$ are effective thermal conductivity, conductive thermal conductivity, Nusselt number, Rayleigh number [2].

The heating of the core material occurs due to the energy of radioactive decay, while part of the energy is spent on the phase transition from hydrated silicates to silicates. At time $t=500 \mathrm{Ma}$, the initial conditions are assumed to be $T$ $=500 \mathrm{~K}$, and the boundary conditions are assumed to be $360 \mathrm{~K}$ [3]. The accuracy and stability of calculations by the finite-difference method was tested on an analytical solution.

The viscosity of a mixture of silicates and hydrated silicates is calculated using the isostress model for the composite. The viscosity of silicates depends on temperature and pressure and is determined similarly to [7]; the viscosity of hydrated silicates is assumed to be constant $4 \cdot 10^{19} \mathrm{~Pa}$ s over the entire range of temperatures and pressures [8]. The Rayleigh numbers in each computational zone are calculated from the volume-averaged parameters. The Rayleigh number in convective regions is determined by the expressions for the case of convection in a layer with internal sources [2]. Since the density of silicates 

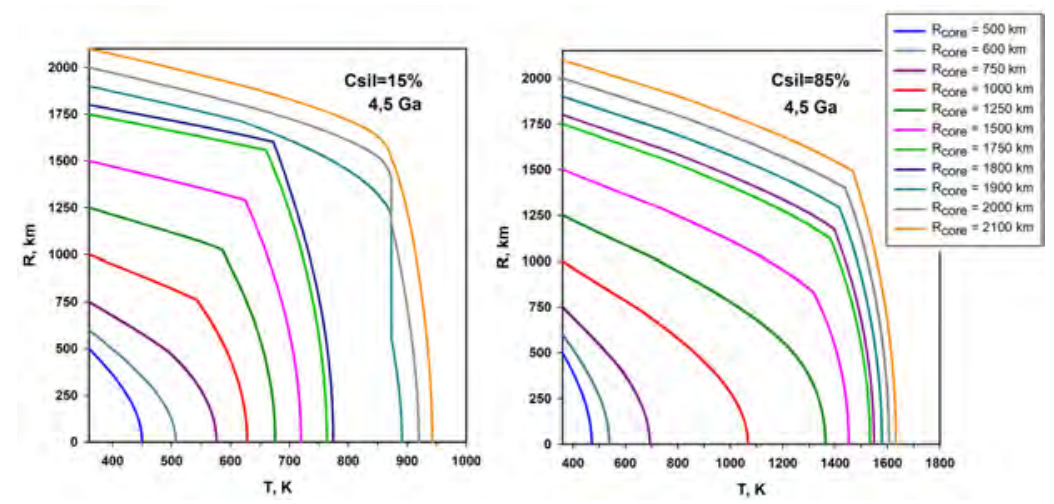

Figure 1: Temperatures at the present time (4.5 Ga from CAI) depending on the radius for different core radii and initial concentration of silicates in the core of $15 \%$ (left) and $85 \%$ (right).
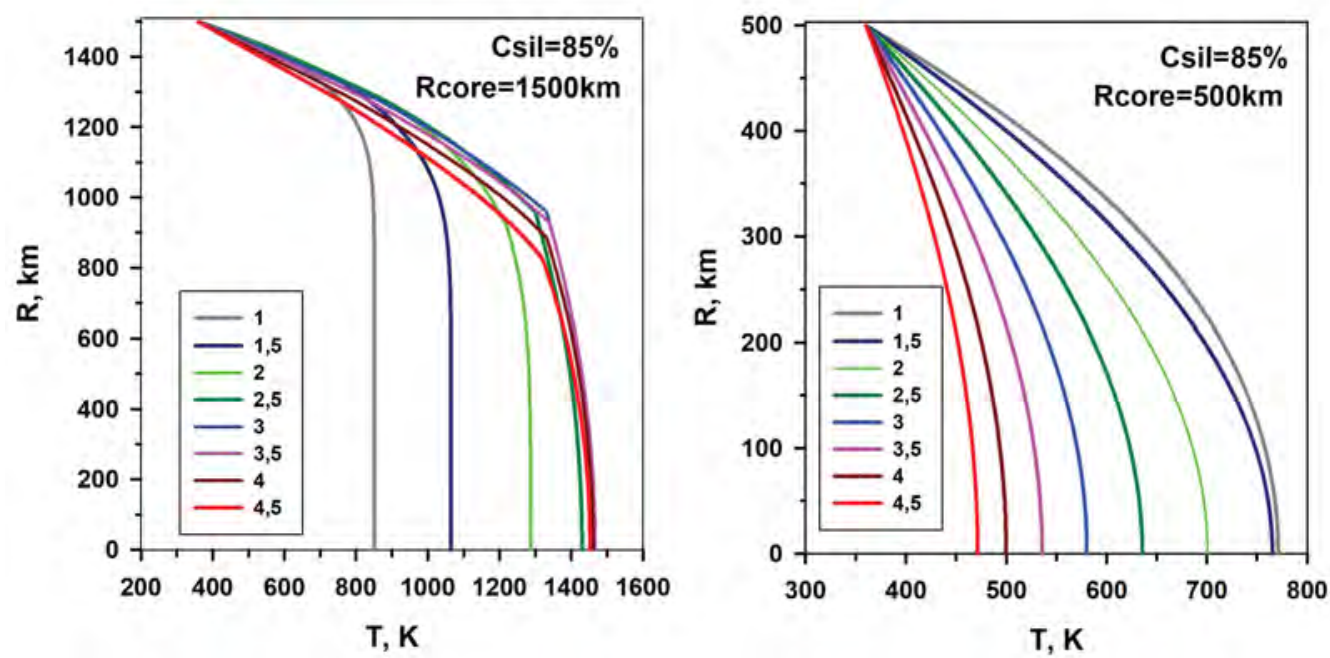

Figure 2: Change in the temperature distribution in the core in time from 1 to 4.5 Ga from the CAI for core radius $R_{\text {core }}=1500 \mathrm{~km}$ (left) and $500 \mathrm{~km}$ (right) and initial concentration of silicates $C_{\text {sil }}=85 \%$.

significantly exceeds the density of hydrated silicates, it is assumed that there is no mass transfer between the calculated zones. Changes in concentrations due to mass transfer processes within each zone are also not taken into account.

The physical properties of the composite (density, heat capacity, thermal conductivity, viscosity) were calculated at each time step.

\section{Results}

The thermal evolution of satellites with a core radius from 500 to $2100 \mathrm{~km}$ was considered. The results of calculations are shown in Fig. 1 and Fig. 2.

The core temperature significantly depends on the composition (the ratio of $C_{\mathrm{Sil}}$ and $C_{\mathrm{Hsil}}$ ) and the size of the core (Fig. 1, 2).

Small cores $(500 \mathrm{~km}$ ) heat up fast (by 1.5 billion years), after which, by 4.5 Byr from CAI formation, they cool down to temperatures lower than the initial one (about $450 \mathrm{~K}$ ). With an increase in the radius of the core, the temperature that can be attained to date increases. The final temperature also increases with increasing silicate content.

\section{Conclusion}

Based on the simulation results, it can be concluded that the initial concentration of hydrated silicates in the satellite proto-core essentially affects its thermal evolution.

\section{Acknowledgements}

The work was performed as part of the state assignment of Vernadsky Institute of Geochemistry and Analytical Chemistry. 


\section{References}

1. A. Dunaeva and V. Kronrod, Geochemistry International, 54, 27, 2016.

2. O. Grasset, C. Sotin, and F. Deschamps, Planetary and Space Science, 48, 2000.

3. J. C. Castillo-Rogez and J. I. Lunine, Geophysical Research Letters, 37, 2010.

4. O. L. Kuskov and V. A. Kronrod, Icarus, 151, 204, 2001.

5. O. L. Kuskov and V. A. Kronrod, Icarus, 177, 2005.

6. S. Wakita and M. Sekiya, Earth, Planets and Space, 63, 2011.

7. J. Kimura, T. Nakagawa, and K. Kurita, Icarus, 202, 216, 2009.

8. N. Hilairet, B. Reynard, Y. Wang, I. Daniel, S. Merkel, N. Nishiyama, and S. Petitgirard, Science, 318, 2007. 


\title{
Estimation of the age of pairs of trans-Neptunian objects in close orbits
}

\author{
E. Kuznetsov, O. Al-Shiblawi, V. Gusev, D. Ustinov \\ eduard.kuznetsov@urfu.ru
}

Ural Federal University, Lenina Avenue 51, Yekaterinburg 620000, Russia

We studied the dynamic evolution for pairs of trans-Neptunian objects in close orbits with semi-major axes of more than $30 \mathrm{au}$. Early have been found 26 pairs of trans-Neptunian objects. All pairs belong to cold Classical Kuiper belt objects. The dynamic evolution of pairs of trans-Neptunian objects in the past 10 Myr has been studied numerically based on nominal orbits. We searched for low relative-velocity close encounters between trans-Neptunian objects in pairs as well as the minima of the Kholshevnikov metrics and the convergence of the lines of nodes and apses to estimate the age of the pairs.

Keywords: trans-Neptunian objects, Classical Kuiper belt objects, pairs of trans-Neptunian objects, Kholshevnikov metrics

DOI: $10.51194 /$ VAK2021.2022.1.1.086

\section{Introduction}

[1] performed a search for statistically significant pairs and groups of dynamically correlated objects through those with a semi-major axis greater than $30 \mathrm{au}$, applying a novel technique that uses Kholshevnikov metrics [2, 3] in the space of Keplerian orbits. Found 26 pairs of trans-Neptunian objects (TNOs) in close orbits. All pairs belong to cold classical Kuiper belt objects. Among the dynamically cold population of the classical Kuiper belt, during the evolution of the protoplanetary disk and the migration of planets, conditions are implemented for the preservation of close binary or contact TNOs with components of approximately equal masses [4]. On the other hand, the evolution of wide binary trans-Neptunian objects turns out to be unstable due to frequent encounters with other TNOs, which lead to the decay of binary systems [5] and the formation of TNO pairs in close orbits.

We perform a study of the dynamical evolution of pairs of TNOs in which one of the component is binary. This paper is organized as follows. Section 2 reviews the methods which we used to search for TNOs with close orbits, research the dynamical evolution of TNOs, and estimate the age of the TNO pairs. Some pairs found by our approach are presented in Section 3. In Section 4, we discuss the results and summarize our conclusions.

\section{Method}

The dynamic evolution of TNO pairs was studied in two stages. In the first step, to find close approaches of TNOs in pairs in the past and, therefore, estimate the age of the pairs, we have performed numerical integrations of the orbits of TNOs in pairs backward in time (a time span of $10 \mathrm{Myr}$ ) with the code known as Orbit9 (the OrbFit Software Package, http://adams.dm.unipi.it/orbfit/ [6]). The numerical integrations were made taking the nominal orbits given by AstDyS database as initial conditions. The eight major planets and the dwarf planet Pluto were integrated consistently. The mean ecliptic of J2000.0 was taken as reference plane for the output. We used heliocentric coordinates.

The standard methods for determining the age of pairs of small bodies in close orbits include analysis of 1) low relativevelocity close encounters of objects (see, e.g. [7]), 2) the minimum distances between the orbits of objects (see, e.g. [8]), 3) simultaneous approaches of node lines and apse lines of objects orbits (see, e.g.[9]).

The condition of convergence of orbits does not yet guarantee the convergence of objects moving in these orbits. Therefore, to estimate the age of pairs, it is also necessary to analyze the possibility of the onset of low relative-velocity close encounters, at which the distance between objects $r_{r e l}$ is comparable to the radius of the Hill sphere $R_{H}$ of a more massive body, and the relative velocity $v_{r e l}$ is of the order of the escape velocity $V_{e s c}$ relative to a more massive body. For each close approach of TNOs in pair we determined the relative distance $r_{r e l}$ between TNOs and relative velocity $v_{r e l}$, as well as the Hill sphere radius $R_{H}$ and escape velocity $V_{e s c}$ of the primary body.

\section{Results}

We carried out a numerical simulation of the orbital evolution in the time interval of 10 Myr into the past for all selected pairs. The nominal values of the osculating elements of the TNO orbits from the AstDyS base for the epoch MJD 58800 were chosen as the initial ones. Tab. 1 shows the minimum values of the metric $\varrho_{2 \text { min }}$ and the corresponding moments $t_{\varrho}$, measured from the epoch MJD 58800.

Table 1: Minimum values of the metric $\varrho_{2}$ and the moments of their occurrence $t_{\varrho}$.

\begin{tabular}{llr}
\hline TNO pair & $\varrho_{2 \min }\left[\mathrm{au}^{1 / 2}\right]$ & $t_{\varrho}[$ years] \\
\hline (534405) 2014 TW $85-2015 \mathrm{GS}_{56}$ & 0.0028 & -3348100 \\
(88268) 2001 $\mathrm{KK}_{76}-2013 \mathrm{UL}_{17}$ & 0.015 & -6403830 \\
2002 CY & 0.019 & -1189840 \\
2013 $\mathrm{SD}_{101}-2005 \mathrm{EW}_{318}$ & 0.021 & -86000 \\
2003 $\mathrm{QL}_{91}-2015 \mathrm{VA}_{173}$ & 0.022 & -6624140 \\
\hline
\end{tabular}


The moments $t_{\varrho}$ corresponding to the minima of the metrics are not the moments of TNO pairs formation since, in addition to the approach of the orbits, the objects must also encounter. The criteria for the search for low relative-velocity close encounters were chosen taking into account the uncertainty of the density $\rho$ and albedo $p_{v}$ of the TNO: $r_{r e l}<3 R_{H}$, $v_{r e l}<4 V_{e s c}$. On the considered time interval, for none of the pairs, the conditions of low relative-velocity close encounters were fulfilled.

\section{Discussion and Conclusion}

The analysis of dynamic evolution based on nominal orbits over $10 \mathrm{Myr}$, carried out using three approaches: the search for the approaches of orbits, lines of nodes and apsides, the TNOs themselves in orbits, showed ambiguous results. As a rule, estimates of the age of pairs obtained by different methods gave significantly different estimates. This may indicate that the age of the pairs exceeds 10 Myr.

The interval of $10 \mathrm{Myr}$ is relatively short for TNO because, during this time, objects of the classical Kuiper belt make only 33 - 36 thousand periods in orbit. For comparison, young pairs in the main asteroid belt are pairs with an age of up to 2 Myr. During this time, asteroids make $400-600$ thousand the orbital periods. In the future, it is planned to increase the integration interval to $200 \mathrm{Myr}$. On such a long interval, the manifestation of stochastic properties of the TNO dynamic evolution is inevitable; therefore, the main methods used to estimate the age of pairs should be methods that estimate the distance between orbits, their nodes, and pericenters.

5. Acknowledgments. This work has been supported by the Russian Ministry of Science and Higher Education via the State Assignment Project FEUZ-2020-0038.

\section{References}

1. E. D. Kuznetsov, O. M. Al-Shiblawi, V. D. Gusev, and D. S. Ustinov, in Lunar and Planetary Science Conference, 1859, Lunar and Planetary Science Conference (2021).

2. K. V. Kholshevnikov, G. I. Kokhirova, P. B. Babadzhanov, and U. H. Khamroev, Mon. Not. R. Astron. Soc., 462, 2275, 2016.

3. K. V. Kholshevnikov, A. S. Shchepalova, and M. S. Jazmati, Vestnik St. Petersburg University: Mathematics, 53, 108, 2020 .

4. D. Nesvorný and D. Vokrouhlický, Icarus, 331, 49, 2019.

5. H. Campbell, in AAS/Division of Dynamical Astronomy Meeting, AAS/Division of Dynamical Astronomy Meeting, volume 53, 501.04 (2021).

6. Orbfit Consortium, OrbFit: Software to Determine Orbits of Asteroids, Astrophysics Source Code Library, 2011.

7. P. Pravec, P. Fatka, D. Vokrouhlický, P. Scheirich, et al., Icarus, 333, 429, 2019.

8. E. D. Kuznetsov, A. E. Rosaev, E. Plavalova, V. S. Safronova, and M. A. Vasileva, Solar System Research, 54, $236,2020$.

9. A. Rosaev and E. Plávalová, P\&SS, 140, 21, 2017. 


\section{Orbital evolution of young pairs of main belt asteroids in the vicinity of resonances and approaching planets}

E. Kuznetsov ${ }^{1}$, M. Vasileva ${ }^{1}$, A. Rosaev ${ }^{2}$, E. Plávalová ${ }^{3}$ eduard.kuznetsov@urfu.ru

${ }^{1}$ Ural Federal University, Lenina Avenue 51, Yekaterinburg 620000, Russia,

${ }^{2}$ Yaroslavl State University, Research and Educational Center "Nonlinear Dynamics", Sovetskaya Street 14, Yaroslavl 150000, Russia,

${ }^{3}$ Mathematical Institute of Slovak Academy of Sciences, Stefanikova 848/49, Bratislava 81473, Slovakia

\section{DOI: $10.51194 /$ VAK2021.2022.1.1.087}

We have investigated the orbital evolution of young pairs of main belt asteroids using numerical simulations in the Mercury6 and Orbit9 software. We took gravity attraction from the eight major planets and the Yarkovsky effect into account. The integration interval was 1 Myr.

We found four young pairs that, in addition to resonant interaction, can also have close encounters with Mars: (9068) $1993 \mathrm{OD}$ - (455327) $2002 \mathrm{OP}_{28}$, (88666) $2001 \mathrm{RP}_{79}$ - (501710) $2014 \mathrm{UY}_{23}$, (313701) $2003 \mathrm{UN}_{3}-(531260) 2012 \mathrm{KL}_{9}$, and (348452) $2005 \mathrm{RU}_{20}$ - (418312) $2008 \mathrm{FF}_{88}$. Approaches to Mars have the strongest effect on the orbital evolution of pairs (9068) $1993 \mathrm{OD}$ - (455327) $2002 \mathrm{OP}_{28}$ and (88666) $2001 \mathrm{RP}_{79}$ - (501710) $2014 \mathrm{UY}_{23}$, moving in the vicinity of the three-body resonance 10-7S-3J with Saturn and Jupiter. Approaches to Mars make it much more difficult to estimate the age of pairs.

For example, the pair (88666) $2001 \mathrm{RP}_{79}-(501710) 2014 \mathrm{UY}_{23}$, when the semi-major axes drift rate is $-5 \cdot 10^{-4}$ au $\mathrm{Myr}^{-1}$, first passes through a three-body resonance 10-7S-3J (nearly $480 \mathrm{kyr}$ ago). Then the pair has a close approach to Mars (nearly $400 \mathrm{kyr}$ ago), which increases the semi-major axes of the orbits of both asteroids. After which, the pair again passes through the three-body resonance (nearly $320 \mathrm{kyr}$ ago), remaining in close orbits. The conditions determining the result for passing through resonance and approaching Mars depend on the semi-major axes drift rate caused by the Yarkovsky effect. Because the semi-major axes drift rate is unknown for the pair (88666) $2001 \mathrm{RP}_{79}-(501710) 2014 \mathrm{UY}_{23}$, making it difficult to obtain estimates of the pair's age.

We plan to study a probabilistic evolution for the four selected pairs.

Acknowledgments. This work has been supported by the Russian Ministry of Science and Higher Education via the State Assignment Projects FEUZ-2020-0030 (EK) and FEUZ-2020-0038 (MV). 


\section{Experimental evidence of the dust particles detachment possibility in the E-field}

I. Kuznetsov ${ }^{1}$, I. Shashkova ${ }^{1}$, A. Poroykov ${ }^{2}$, A. Zakharov ${ }^{1}$, S. Bednyakov ${ }^{1}$, A. Bychkova ${ }^{1}$, G. Dolnikov ${ }^{1}$, A. Dubov ${ }^{1}$, A. Kartasheva ${ }^{1}$, A. Lyash ${ }^{1}$, A. Shehovtsova ${ }^{1,3}$

kia@iki.rssi.ru

${ }^{1}$ Space Research Institute of the Russian Academy of Sciences (IKI RAN), Moscow, Russia

${ }^{2}$ Moscow Power Engineering Institute, Moscow, Russia

${ }^{3}$ Moscow State University, Moscow, Russia

One of the complicating factors of the future robotic and human lunar missions, as well as the missions to atmosphereless bodies, is the influence of the dust. Due to the solar wind and ultraviolet irradiation influence, the regolith of the atmosphereless body can obtain significant charge and therefore can produce strong electric fields above the surface. The electric field influences the surface dust particles and can become a trigger for the charged dust particles to detach from the surface under the Coulomb force influence. This study demonstrates the possibility of dust particles' detachment under strong electric field conditions. The main goal of this work is to simulate the dynamics of charged dust particles in the experimental vacuum chamber with the influence of the electric field with predicted values.

Keywords: dust, dusty plasma, airless bodies, vacuum chamber, experimental set-up

DOI: 10.51194 /VAK2021.2022.1.1.088

\section{Introduction}

The direct observations of the lunar horizon glow that made more than 50 years ago by the Surveyor-7 automatic spacecraft [1] and Apollo-17 astronauts [2] are one of the most mysterious observations related to Moon and are still under debate. [3], [4], [5] and other authors stated that the electric potential of the lunar surface in the terminator region and in the lunar nightside can highly vary and reach as low as minus $4000 \mathrm{~V}$. [6] estimated the possible electric field on the Moon up to 3000 $\mathrm{V} / \mathrm{cm}$. Levitation mechanism explanation is based on the idea that the electrically charged surface and electrically charged dust grains repel each other. A very similar phenomenon is proposed for most of the celestial atmosphereless bodies. Due to much lower gravity force, the increasing of the dust dynamics on the asteroids $[7,8]$ and Martian satellites Phobos and Deimos $[9,10]$ is predicted. [11] estimated the electric field on asteroids in the range of $500-1500 \mathrm{~V} / \mathrm{cm}$. Alongside the theoretical works, there is an experimental simulation approach $[12,13]$ that can help to solve the dust dynamics mystery. In this work, we describe the experimental simulation approach to the airless dust particles dynamics.

\section{Airless bodies conditions}

[14] calculated the possible electric fields required for dust particle detachment from the surface for spherical particles of different sizes and for various conditions. They took 3 reference bodies for calculations - Moon, Eros, Itokawa. Assuming the bodies are seismically quiet and particles are with a density of $3.5 \mathrm{~g} / \mathrm{cm}^{3}$, [14] obtained the range of electric field $4000-50000 \mathrm{~V} / \mathrm{cm}$ required for a particle to left the surface in the case of absence of both seismic and charge acceleration influences. For the experimental set-up we have used the $\mathrm{SiO}_{2}$ particles with radius $r_{\exp }=50 \mu m$ and density of $\rho_{\exp }=2.196$ $\mathrm{g} / \mathrm{cm}^{3}$. With model by [14] one can find the required electric field for the Moon, Eros, Itokawa, and Earth gravity using the following equation:

$$
E_{\mathrm{req}} \geq\left[\frac{4}{3 \varepsilon_{0}} r_{\exp } \rho_{\exp } g+\frac{C S^{2}}{\pi \varepsilon_{0} r_{\exp }}\right]^{1 / 2}
$$

where $g=1.622 \mathrm{~m} / \mathrm{s}^{2}$ for the Moon, $g=0.0055 \mathrm{~m} / \mathrm{s}^{2}$ for Eros, $g=8.603 \times 10^{-5} \mathrm{~m} / \mathrm{s}^{2}$ for Itokawa and $g=9.81 \mathrm{~m} / \mathrm{s}^{2}$ for Earth, $\varepsilon_{0}$ is the permittivity of free space, $C=5.14 \times 10^{-2} \mathrm{~kg} / \mathrm{s}^{2}, S$ is surface cleanliness that affects cohesion defined as 0.1 and 1. The calculated results for our experiment and chosen celestial bodies are presented in Table 1.

Table 1: Calculated results of the required electric field that necessary for charged dust particles of $50 \mu \mathrm{m}$ of $\mathrm{SiO}_{2}$ to separate from the surface

\begin{tabular}{lllll}
\hline Celestial body & Gravity, $\mathrm{m} / \mathrm{s}^{2}$ & $\begin{array}{l}\text { Surface potential } \\
\text { values range, } \mathrm{V}\end{array}$ & $\begin{array}{l}\text { Electric field, } \\
\mathrm{V} / \mathrm{cm}(\mathrm{S}=0.1)\end{array}$ & $\begin{array}{l}\text { Electric field, } \\
\mathrm{V} / \mathrm{cm}(\mathrm{S}=1)\end{array}$ \\
\hline Moon & $1.622^{\mathrm{a}}$ & down to minus $4000^{\mathrm{b}}$ & $6.30 \times 10^{3}$ & $6.08 \times 10^{4}$ \\
Eros & $0.0055^{\mathrm{a}}$ & $\mathrm{NA}$ & $6.08 \times 10^{3}$ & $6.08 \times 10^{4}$ \\
Itokawa & $8.603 \times 10^{-5 \mathrm{a}}$ & $\mathrm{NA}$ & $6.08 \times 10^{3}$ & $6.08 \times 10^{4}$ \\
Experimental & 9.81 & 2.5 & $7.29 \times 10^{3}$ & $6.09 \times 10^{4}$ \\
Set-Up & & & \\
\hline
\end{tabular}

${ }^{a}[14]$

${ }^{b}[15]$ 


\section{Experimental set-up}

The idea of the experimental set-up is to place a small portion of regolith-like particles between two electrodes in a vacuum chamber and observe their movement using laser illumination through a chamber flange. The experimental schematics and description are given in Figure 1. The detailed description of the experimental set-up is in [16]. $\mathrm{The}^{\mathrm{SiO}_{2}}$ particles rest on the nonconductive plate (composite epoxy material). Its bottom side is laminated with a thin layer of copper foil connected to the high voltage potential source (up to $V_{\max }=12 k \mathrm{~V}$ ). The second electrode - the conductive grid that is connected to the electrical ground - is located at $8 \mathrm{~mm}$ above.

The analytical part consists of the laser surface to light up the moving dust particles and a set of two synchronized CMOS cameras. Recorded images of the detached dust are then processed in specially designed software that gets us resulting dynamic and electrostatic parameters of the investigated samples [16, 17]. Active processes with the particles began to occur starting from $2.5 \mathrm{kV}$ of applying potential. Dust dynamics can be seen with the naked eye (Figure 2). Statistical information about the levitating dust particles can be obtained with the help of image processing (e.g., Figure 3).

\section{Results and conclusions}

With a lot of models, one can find a number of theoretical estimations of the possible dust particle size and their dynamics. However, the experimental evidence of such processes is not common. Here we successfully observed the dust particle

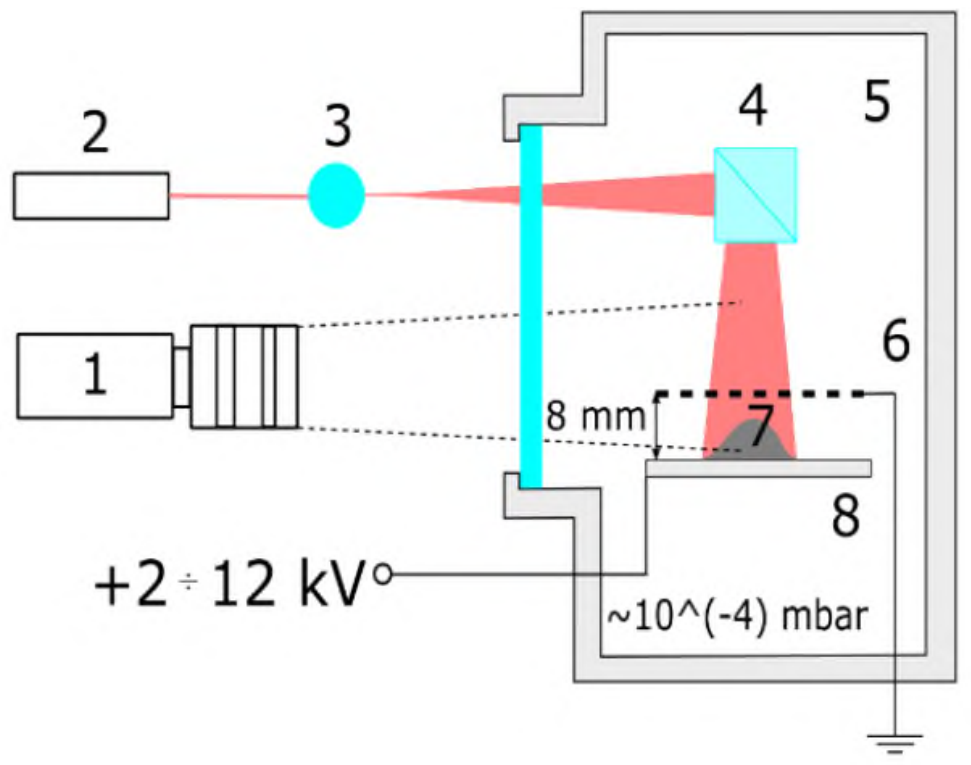

Figure 1: Picture and scheme of the experimental setup for investigation of the dusty plasma dynamics (one of the chamber flanges is transparent). 1 - CMOS camera; 2 - laser; 3, 4 - optics for laser illumination of dust particles; 5 - vacuum chamber; 6 - steel mesh; 7 - dust particles $\left(50 \mu \mathrm{m}, \mathrm{SiO}_{2}\right) ; 8$ - conductive substrate.

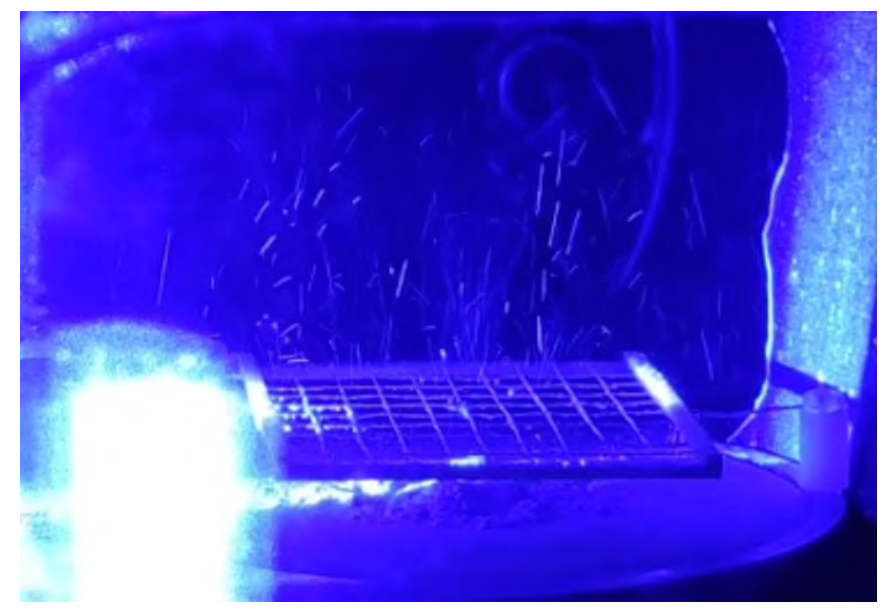

Figure 2: Dust particles movement in the experimental set-up

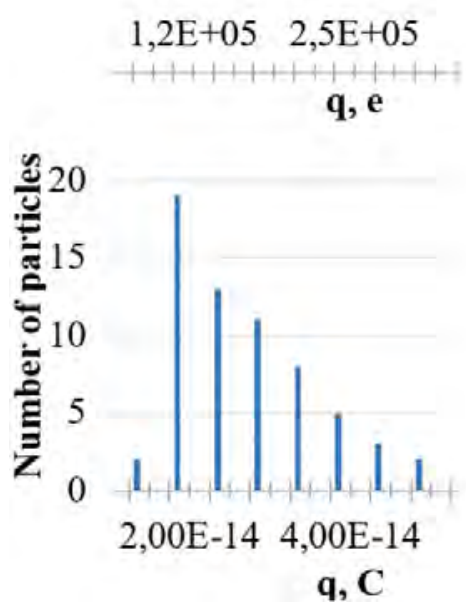

Figure 3: Histogramm of the detected dust particles charges 
dynamics with $\mathrm{SiO}_{2}, 50 \mu \mathrm{m}$ in the relatively strong electric field. Its amplitude during experiments was $E \leq 3125 \mathrm{~V} / \mathrm{cm}$ which highly corresponds with the predicted electric field [6, 11]. With this experiment, we demonstrate potential evidence of the possibility of the active dust dynamics and dust transport at the lunar and other atmosphereless celestial bodies surfaces.

\section{References}

1. J. J. Rennilson and D. R. Criswell, Moon, 10, 121, 1974.

2. H. A. Zook and J. E. McCoy, Geophysical Research Letters, 18, 2117, 1991, URL https://doi .org/10.1029/91GL02235.

3. T. J. Stubbs, R. R. Vondrak, and W. M. Farrell, Advances in Space Research, 37, 59, 2006, URL https://www.sciencedirect.com/science/article/pii/S0273117705004989, the Moon and Near-Earth Objects.

4. J. S. Halekas, G. T. Delory, R. P. Lin, T. J. Stubbs, and W. M. Farrell, Journal of Geophysical Research: Space Physics, 113, 2008, URL https://agupubs.onlinelibrary.wiley.com/doi/abs/10.1029/2008JA013194.

5. W. Farrell, T. Stubbs, R. Vondrak, G. Delory, and J. Halekas, Geophysical Research Letters, 34, L14201, 2007.

6. B. R. De and D. R. Criswell, Journal of Geophysical Research (1896-1977), 82, 999, 1977, URL https://agupubs.onlinelibrary.wiley.com/doi/abs/10.1029/JA082i007p00999.

7. P. Lee, Icarus, 124, 181, 1996.

8. L. H. Yeo, X. Wang, J. Deca, H. W. Hsu, and M. Horányi, Icarus, 366, 2021.

9. S. I. Popel, A. P. Golub', A. V. Zakharov, and L. M. Zelenyi, JETP Letters, 106, 485, 2017.

10. A. V. Krivov and D. P. Hamilton, Icarus, 128, 335, 1997.

11. N. Rennó and J. Kok, Electrical Activity and Dust Lifting on Earth, Mars, and Beyond, volume 137, 419-434 (2008).

12. X. Wang, J. Schwan, H. W. Hsu, E. Grün, and M. Horányi, Geophysical Research Letters, 43, 6103, 2016.

13. N. C. Orger, K. Toyoda, H. Masui, and M. Cho, Advances in Space Research, 63, 3270, 2019, URL https://doi.org/10.1016/j.asr.2019.01.045.

14. C. M. Hartzell and D. J. Scheeres, Planetary and Space Science, 59, 1758, 2011, URL https://www.sciencedirect.com/science/article/pii/S0032063311001644, lunar Dust, Atmosphere and Plasma: The Next Steps.

15. J. S. Halekas, G. T. Delory, R. P. Lin, T. J. Stubbs, and W. M. Farrell, Journal of Geophysical Research: Space Physics, 113, 1, 2008.

16. A. Y. Poroykov, S. A. Bednyakov, A. V. Zaharov, G. G. Dolnikov, A. N. Lyash, I. A. Shashkova, and I. A. Kuznetsov, Journal of Physics: Conference Series, 1421, 012037, 2019.

17. A. V. Zakharov, A. Y. Poroykov, S. A. Bednyakov, A. N. Lyash, I. A. Shashkova, I. A. Kuznetsov, and G. G. Dolnikov, Measurement, 171, 108831, 2021, URL https://www.sciencedirect.com/science/article/pii/S0263224120313245. 


\section{Investigation of meteors with sub-second temporal resolution in wide-field optical sky monitoring}

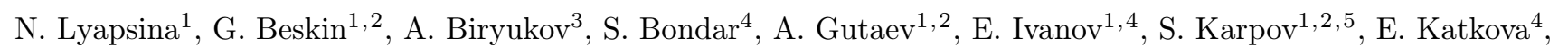
A. Perkov ${ }^{4}$, V. Sasyuk ${ }^{2}$ nadezhdavl@inbox.ru

${ }^{1}$ SAO RAS, Nizhny Arkhyz, Russia

${ }^{2}$ Kazan Federal University, Kazan, Russia

${ }^{3}$ Moscow State University, Moscow, Russia ${ }^{4}$ Research and Production Corporation "Precision Systems and Instruments", Russia

${ }^{5}$ CEICO, Institute of Physics, Czech Academy of Sciences, Prague, Czech Republic

The article presents the results of meteor observations with the wide-field monitoring system Mini-MegaTORTORA (MMT9). There are more than 300 thousand events in the MMT-9 meteor database as of May 2021. The distribution of meteor tracks in the sky during the night makes it possible to distinguish active meteor showers, as well as sporadic meteors. We present the results of double-station meteor observations with MMT-9 and FAVOR wide-angle camera, separated by a distance of $3.8 \mathrm{~km}$.

Keywords: meteor, meteor showers, wide-field monitoring

DOI: 10.51194 /VAK2021.2022.1.1.089

This article reports on a study of meteors with high temporal resolution based on the results of wide-field monitoring. To implement this idea the robotic 9-channel telescope Mini-MegaTORTORA (MMT-9) located near the 6-meter BTA telescope at SAO RAS is used. The system's field of view is $30^{\circ} \times 30^{\circ}$. Each channel's field of view is 100 square degrees. The channels are equipped with a Canon EF 85 f/1.2 lens, an Andor Neo sCMOS detector, BVR filters and a polaroid.

In addition to meteors, MMT-9 detects and analyses other optical transients of various nature - optical companions of gamma-ray bursts, gravitational-wave events, fast radio bursts, novae, supernovae and variable stars, brightness variations of quasars and active galaxies, satellites and space debris [1].
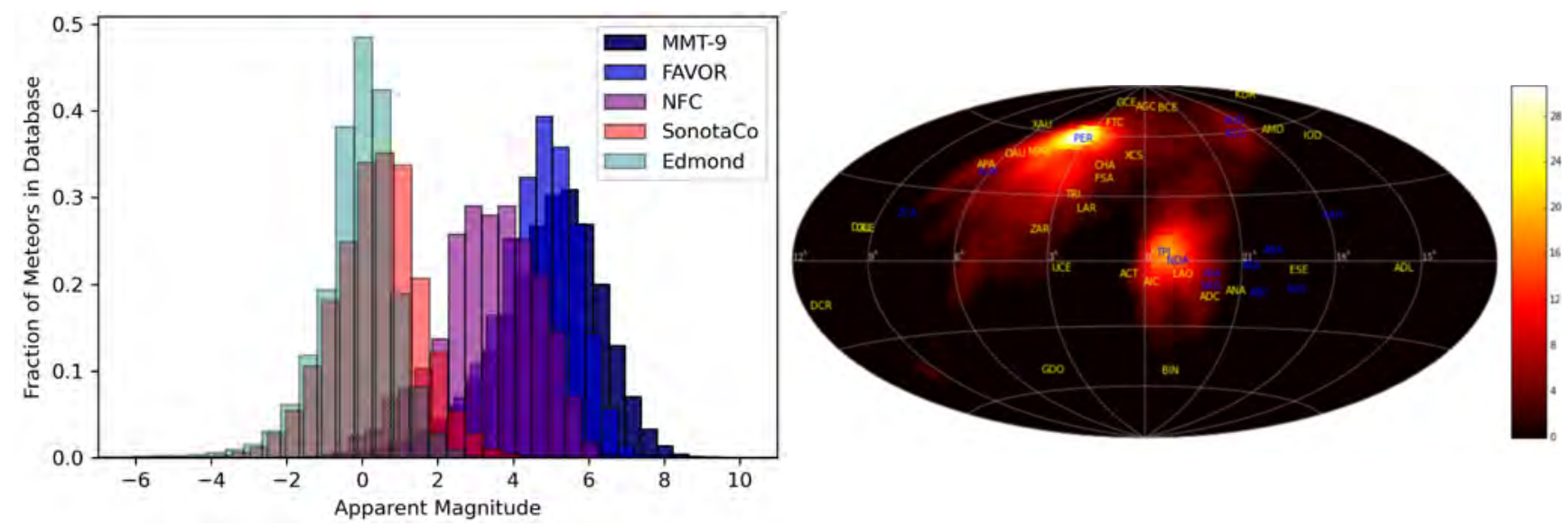

Figure 1: a. Comparison of typical meteor brightness from several experiments and public databases. b. A statistical radiant determination using meteors from Mini-MegaTORTORA database around the annual Perseid activity interval

Several hundreds of meteors are recorded by MMT-9 during a wide-field sky monitoring every observation night. They are automatically analyzed in real-time after their detection to determine their coordinates, directions, angular velocities, apparent magnitudes, etc. That information and series of original images for each meteor are collected in the MMT-9 Meteor Database. It contains data about more than 310000 meteors, collected since 2014. All this data is available online (http://mmt.sonarh.ru).

The capabilities of the MMT-9 system allow to record the faintest meteors in optical range — down to 9th magnitude. Most meteors in the Database have apparent magnitude of 5-6. It is several magnitudes fainter than EDMOND, SonotaCo and NFC Databases have (Fig. 1a).

Most meteors are observed in white light (the brightness is then calibrated to $\mathrm{V}$ magnitude), some are observed in Johnson-Cousins B, V and R photometric filters simultaneously. For such events, their colors are derived automatically [2]. Usanin et al. [3] presented the results of BVR observations of several meteor showers and sporadic meteors carried out with the Mini-MegaTORTORA system.

Additionally, it is possible to perform a statistical analysis of the activity of meteor showers with the distribution of meteor tracks in the sky observed with the MMT-9 system. We calculate statistical radiants for all meteors recorded during one night. They are determined as intersections of 90 degrees arcs of great circles along which the paths of the meteors are seen. Areas with side of 2 degrees on the celestial sphere with intersections are marked. Those areas which have more 
intersections have higher indexes (Fig. 1b). After that we can compare statistical radiants distribution with meteor shower radiants in catalogue of IAU Meteor Data Center $[4,5,6,7,8]$.

We carried out the double-station meteor observations using the MMT-9 system along with FAVOR camera. The latter was designed by our group and was in operation from 2004 to 2009 in Nizhny Arkhyz. The FAVOR telescope parameters are described in [9]. In 2017 it was modernized and installed in a new location at a distance of $3.8 \mathrm{~km}$ from MMT-9 [2, 10]. Some results are shown in Fig. 2. At present, double-station observations are carried out in the following mode: FAVOR is the leading instrument. It has two priorities: observing active meteor showers and searching for sporadic meteors in areas near zenith. And MMT-9 is aimed according to the coordinates of the area observed by FAVOR if there are no targets with extraordinary significance (triggers from FERMI, SWIFT telescopes, etc.). Data from the two telescopes is collected and compared using software developed specially for this task.
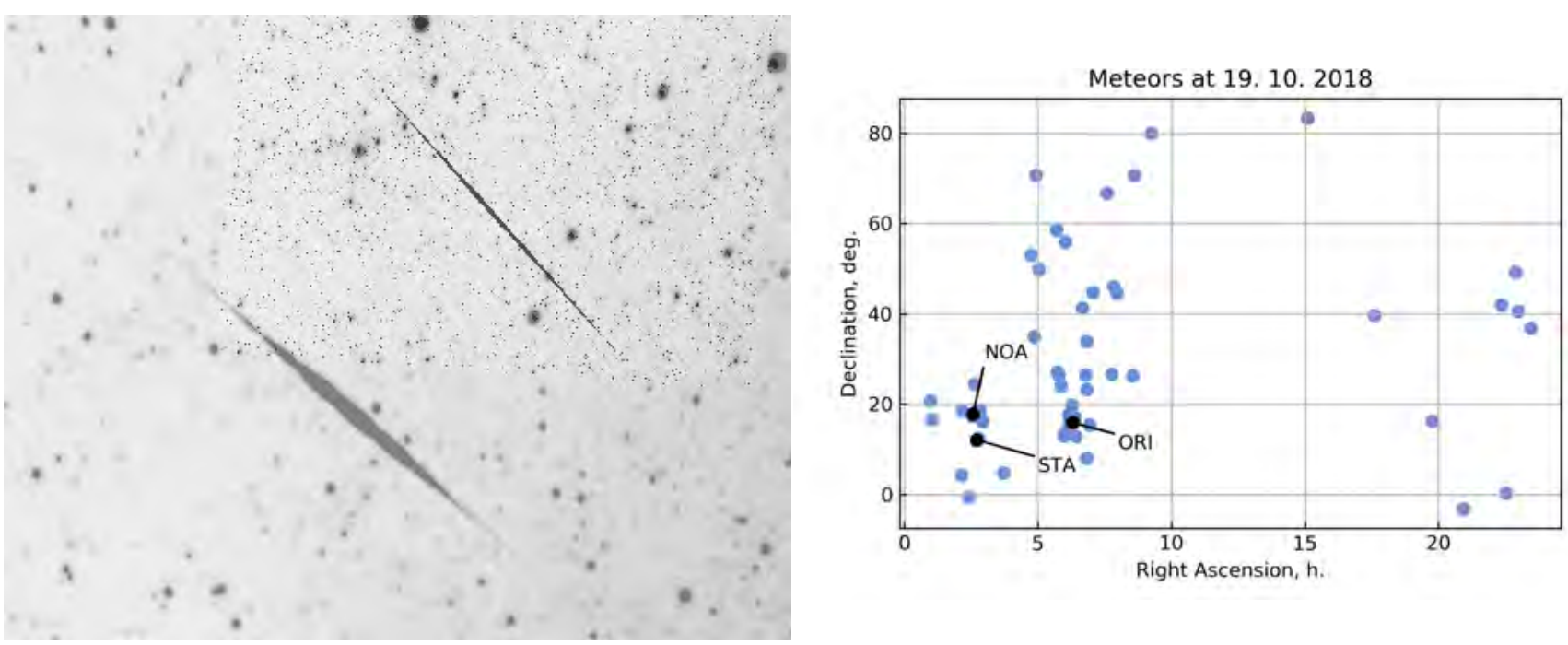

Figure 2: a. Two combined meteor images taken in double-station observations with MMT-9 (above) and FAVOR (below). b. Radiants of individual meteors calculated from double-station observations with MMT-9 and FAVOR

As the main task is to calculate the orbits of registered meteors, a complete set of algorithms and programs for their determination will be created in the near future. Also in future we plan to start double-station observations with MMT9 as the leading tool. With this mode it will be possible to provide regular multiband meteor observations and make a comparative analysis of spectral characteristics of stream and sporadic meteors.

As a result, we will obtain complete information about registered meteor events and will be able to carry out a detailed analysis in order to determine the evolution of their physical characteristics.

Acknowledgements: The work was (partially) performed as part of the government contract of the SAO RAS approved by the Ministry of Science and Higher Education of the Russian Federation.

\section{References}

1. G. Beskin, S. Karpov, A. Biryukov, S. Bondar, et al., Astrophysical Bulletin, 72, 81, 2017.

2. S. Karpov, N. Orekhova, G. Beskin, A. Biryukov, et al., in Revista Mexicana de Astronomia y Astrofisica Conference Series, volume 48, 97-98 (2016).

3. V. Usanin, Y. A. Nefedyev, and M. Sokolova, Astronomy Reports, 63, 666, 2019.

4. P. Jenniskens, T. J. Jopek, D. Janches, M. Hajduková, G. I. Kokhirova, and R. Rudawska, Planetary and Space Science, 182, 104821, 2020.

5. T. J. Jopek and Z. Kaňuchová, Planetary and Space Science, 143, 3, 2017.

6. T. Jopek, F. Rietmeijer, J. Watanabe, and I. Williams, Meteoroids 2013, 371, 2014.

7. T. Jopek and P. Jenniskens, Meteoroids: The Smallest Solar System Bodies, 7, 2011.

8. L. Neslusan, V. Porubcan, J. Svoren, and M. Jakubik, WGN, Journal of the International Meteor Organization, 48, 168,2020

9. S. Karpov, G. Beskin, A. Biryukov, S. Bondar, et al., in Nuovo Cimento, volume 28C, 747-750 (2005).

10. S. Karpov, N. Orekhova, G. Beskin, A. Biryukov, et al., in Revista Mexicana de Astronomia y Astrofisica Conference Series, volume 51, 127-130 (2019). 


\title{
On load Love numbers for Venus
}

\author{
T. Menshchikova, T. Gudkova \\ ms.tamm@mail.ru \\ Schmidt Institute of Physics of the Earth RAS, Moscow, Russia
}

Load Love coefficients are calculated for different rheological models of Venus. A static approach is used, for which two load levels are considered: the surface load (the planetary relief) and anomalous density variations located at depth. The planet is modeled as an elastic, self-gravitational body, while the density, elastic modulus and shear modulus being dependent on the radius. The calculations have been performed up to the spherical harmonic degree and order 70 , based on the accuracy of the gravity field at the moment. Several types of rheological models of Venus are considered. As a first approximation, we take an elastic model (Model A). In the second case (Model B) we assume the presence of an elastic lithosphere. Beneath the lithosphere there is a softened layer, which partly lost its elastic properties. Spreading till the core, softened layer is characterized by a reduced (to one-tenth of the initial value) shear modulus. In the third model (Model C) the shear modulus is changing gradually: one-tenth of the initial value of shear modulus just beneath the crust is increasing up to its elastic value at the core-mantle boundary.

Keywords: Venus, load Love coefficients, topography, gravity fields, rheological models

DOI: 10.51194/VAK2021.2022.1.1.090

\section{Introduction}

We are on the eve of the seismic experiment on Venus [1]. Joint mission "Venus-D" of Russian Space Agency and "LLISSE" (Long Lived Insitu Solar System Explorer) of NASA is going to deliver two landers on Venus and deploy seismometers to the surface for two months. There is little knowledge of Venus' seismic activity. The surface of Venus is characterized by a number of highstanding topographic structures (e.g., [2]). Magellan data reveal such signs of tectonic activity as extensional fractures and wrinkle ridges on the Venusian surface. Current seismic activity could be expected in the areas of Beta, Atla, Phoebe regions, which are strongly supported by upwelling mantle plumes [3]. The Venus is assumed to be geodynamically active [4], and the evidence for current volcanism is confirmed [5]. Since there is no sign of plate boundaries or their motions on Venus like on the Earth, areas with high stresses could be potential sources of quakes. Stresses' modeling needs knowledge of boundary conditions, which are determined from the gravity field data and the values of load Love numbers. For this purpose, in present study we calculate load Love numbers for different rheological models of Venus.

\section{Observational data}

The gravity and topography data provided by the Magellan mission: the spherical harmonic global topography model (SHTJV360u) [6] and gravity field model (SHGJ180u) [7] are available at the Planetary System data ${ }^{\mathrm{a}}$. We have used these data up to the spherical harmonic degree and order 70, based on the accuracy of the gravity field at the moment. An effectively equilibrium figure of Venus [8, 9], which survives from an earlier epoch, is chosen as a reference surface.

\section{Method of calculations}

The distribution of non-hydrostatic stresses in a planet can be obtained by considering the planet as an elastic spherically symmetric body under the influence of both surface (relief on the surface of the planet) and internal (buried density anomalies) loads. The load coefficients technique accounting the deformation of a planet under loads that we have used was developed in [10].

The amplitudes of loads $\left(R_{i, n, m}^{1}\right.$ and $\left.R_{i, n, m}^{2}\right)$ that serve as boundary conditions for solving the system of equations of elastic equilibrium of a gravitating planet are determined according to the topography and gravitational field data (coefficients of the Legendre polynomial expansions $C_{t n m}$ and $C_{g n m}$, respectively) and load Love numbers $\left(k_{n}\right.$ and $\left.h_{n}\right)$ (Fig. 1). Load Love numbers (load coefficients) are functions of the planet's response to loads. Load numbers $k_{n}(r)$ and $h_{n}(r)$ for the density anomaly located at a given depth $\mathrm{r}$ are obtained from the solution of the system of equations of elastic equilibrium of a gravitating planet (the equation of motion of a deformed body, the Poisson equation, Hooke's law for an isotropic medium), which can be represented as six first-order differential equations [10] and solved by the fourth-order Runge-Kutta method. The scheme of load numbers calculations is presented in Fig. 2. As noted above, the topography and gravity field coefficients are taken relative to the equilibrium figure. Load Love numbers (or load coefficients) are calculated for a given model of the planetary interior, characterized by density profile and the distribution of bulk modulus and shear modulus [11].

\section{Model}

The reaction of the planet in response to the applied load depends on the behavior of the material. The rheological properties of Venus, at present, are not precisely established. We consider several variants of models of inhomogeneous elasticity of Venus (see Fig. 3). As a first approximation, we take an elastic model (Model A). In the second case (Model B) we assume the presence of an elastic lithosphere. Beneath the lithosphere there is a softened layer, which partly lost its elastic properties. Spreading till the core, softened layer is characterized by a reduced (to one-tenth of the initial value) shear modulus. In the third model (Model C) the shear modulus is changing gradually: one-tenth of the initial value of shear modulus just beneath

${ }^{\mathrm{a}}$ http://pds-geosciences.wustl.edu 


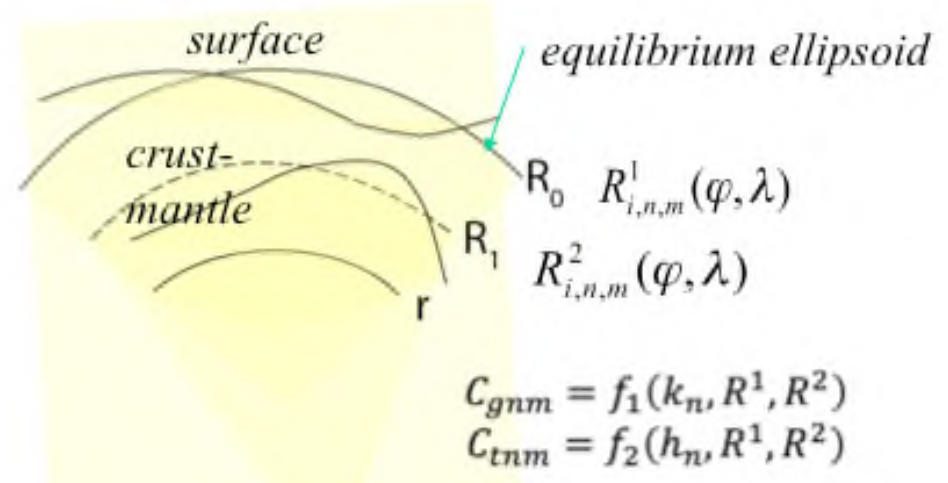

Figure 1: The loading model. The notations: $R_{0}$ — planetary radius, $R_{1}$ — crust-mantle radius, $R_{i, n, m}^{1}$ and $R_{i, n, m}^{2}$ are the amplitudes of loads; $\varphi$ and $\lambda$ are the latitude and longitude; $C_{t n m}$ and $C_{g n m}$ are coefficients of the Legendre polynomial expansions of the topography and gravity field, $k_{n}$ and $h_{n}$ are load Love numbers of order $n$ for a density anomaly buried at some depth $r$.

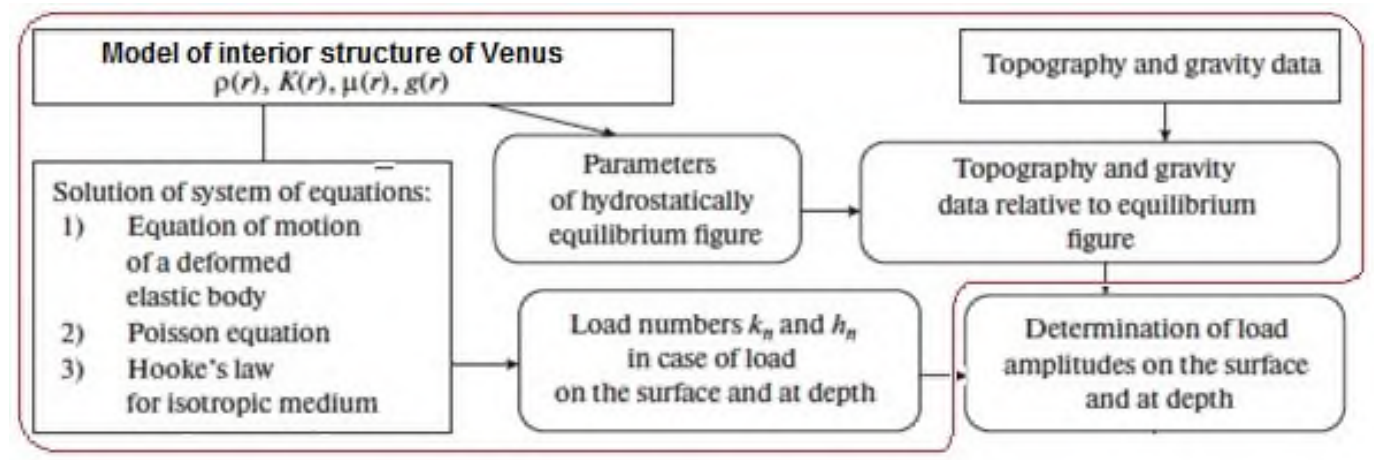

Figure 2: The scheme of load numbers calculations.

the crust is increasing up to its elastic value at the core-mantle boundary. In the absence of seismic measurements, there is no exact knowledge how thick the Venusian lithosphere is. Estimates of the crustal and lithosphere average thickness of Venus vary in wide ranges: $25-70 \mathrm{~km}$ and $100-600 \mathrm{~km}$, respectively. In this study the thickness of the lithosphere is taken from 100 to $500 \mathrm{~km}$ according to the results of Orth and Solomatov [12].

\section{Results}

Load Love numbers $k_{n}(r), h_{n}(r)$ have been calculated for different rheological models of Venus (Models A, B and C, see Fig. 3). Load numbers $k_{n}(r)$ and $h_{n}(r)$ (with opposite sign) for a test interior model are shown in Fig. 4 for degree and order $n=2-70$ and various load depths: at the surface, at the crust-mantle boundary and some depths in the mantle. The existence of density contrasts in the interiors of a planet can be related to the changes in temperature and (or) chemical composition, as well as the deformations of boundaries, such as the crust-mantle boundary and the boundaries of phase transitions.

The value of $k_{n}\left(r_{j}\right)$ determines the response of the external gravity field on the anomalous density wave with some amplitude. At $k_{n}\left(r_{j}\right)=0$ the interiors of a planet behaves as "undeformed", while there is a bounding of layers: light material is replaced by heavier one and heavy material — by lighter one. The value $\left(-k_{n}\left(r_{j}\right)\right)=1$ corresponds to full isostatic compensation, the physical reason is the effects of deformation and bounding of elastic shells of the mantle, located above softened layers. At $0<\left(-k_{n}\left(r_{j}\right)\right)<1$ there is a partial compensation of anomalous density wave. It is seen from Fig. 4 , that the response function (the load coefficients) is sensitive to a rheological structure of the planet, this fact can be used to discriminate between the rheological models of Venus.

\section{Conclusion}

In this study, load Love numbers are calculated for different rheological models of Venus, using topography and gravitational field data up to the 70th degree and order. Further these values will be used to determine the stress pattern in Venus. The 


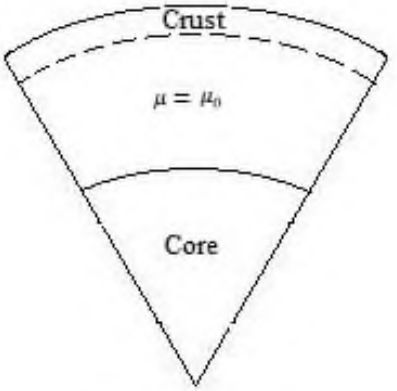

Model A

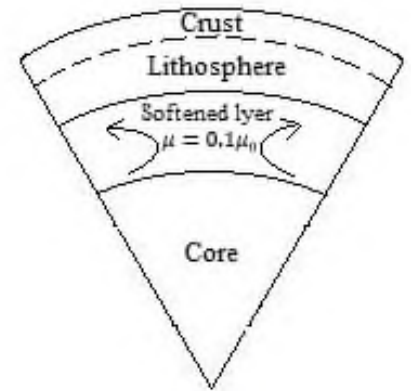

Model B

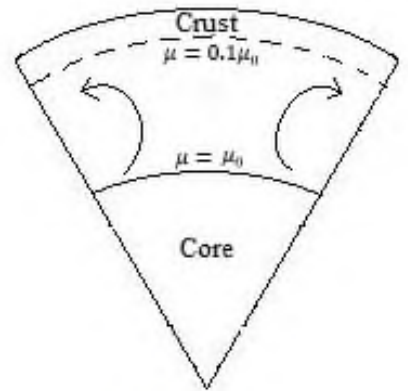

Model C

Figure 3: Rheological models of Venus: Model A - an elastic model. Model B - a model with the lithosphere of various thickness values $(100-500 \mathrm{~km})$ located on a softened layer, which partially lost its elastic properties. The softening is modeled by the reduced (to one-tenth of the initial value) shear modulus in the layer beneath the lithosphere, stretching down to the core. Model $\mathrm{C}$ - a model with a reduced shear modulus value just beneath the crust, while its value increasing up to its elastic value at the core-mantle boundary.
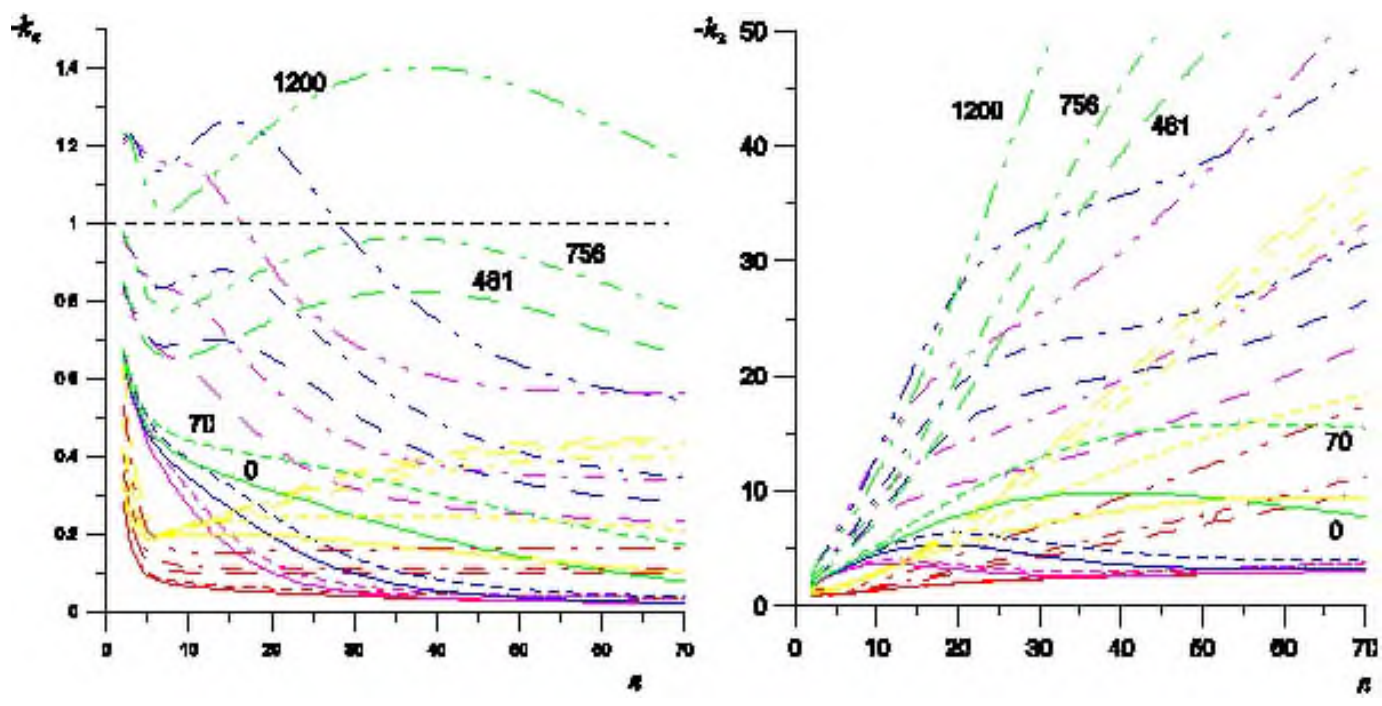

Figure 4: Load numbers $k_{n}(r)$ and $h_{n}(r)$ (with opposite sign) for the elastic Venusian model of type A (red lines); type B models with a lithospheric thickness of $100 \mathrm{~km}$ (green lines), $300 \mathrm{~km}$ (blue lines) and $500 \mathrm{~km}$ (pink lines), and type $\mathrm{C}$ models (yellow lines) as a function of the spherical harmonic number $\mathrm{n}$ for different depths of anomalous density waves: at the surface of $0 \mathrm{~km}$, at the crust-mantle depth of $70 \mathrm{~km}$, at depths of $481 \mathrm{~km}, 756 \mathrm{~km}$ and 1200 $\mathrm{km}$. The horizontal dashed line corresponds to the value $k_{n}(r)=-1$ (isostasy).

values of load Love numbers depend on the depth of the load location, the degree of harmonics $\mathrm{n}$ and the rheological model of the planet. Based on these calculations, the long-wave anomalous external gravitational field is interpreted. However, there are some uncertainties in the choice of a rheological model (lithosphere thickness and mechanical properties and dynamics of the lithosphere and the mantle), and locations of the anomalous densities.

Acknowledgments: The study was performed under a government contract of the Schmidt Institute of Physics of the Earth of the Russian Academy of Sciences.

\section{References}

1. T. Kremic, R. Ghail, M. Gilmore, G. Hunter, et al., P\&SSS, 190, 104961, 2020.

2. M. A. Ivanov and J. W. Head, P\&SSS, 59, 1559, 2011.

3. S. E. Smrekar, W. S. Kiefer, and E. R. Stofan, in S. W. Bougher, D. M. Hunten, and R. J. Phillips, eds., Venus II: Geology, Geophysics, Atmosphere, and Solar Wind Environment, 845 (1997).

4. A. T. Basilevsky, Geophys. Res. Lett., 20, 883, 1993. 
5. E. V. Shalygin, W. J. Markiewicz, A. T. Basilevsky, D. V. Titov, N. I. Ignatiev, and J. W. Head, Geophys. Res. Lett., 42, 4762, 2015.

6. N. J. Rappaport, A. S. Konopliv, A. B. Kucinskas, and P. G. Ford, Icarus, 139, 19, 1999.

7. A. S. Konopliv, W. B. Banerdt, and W. L. Sjogren, Icarus, 139, 3, 1999.

8. V. N. Zharkov, T. V. Gudkova, and A. Nikol'skii, Solar System Research, 53, 1, 2019.

9. T. I. Menshchikova, T. V. Gudkova, and V. N. Zharkov, Solar System Research, 55, 11, 2021.

10. K. Marchenkov, V. Lyubimov, and V. Zharkov, Doklady Earth Science Sections, 279, 14, 1984.

11. T. V. Gudkova and V. N. Zharkov, Solar System Research, 54, 20, 2020.

12. C. P. Orth and V. S. Solomatov, Geochemistry, Geophysics, Geosystems, 13, Q11012, 2012. 


\title{
Variations of the wind speed at the top cloud layer in equatorial latitudes according to results of long-term observations of VMC (Venus Express) and UVI (Akatsuki)
}

\author{
M.V. Patsaeva, I.V. Khatuntsev, A.V. Turin, L.V. Zasova \\ marina.pats@gmail.com \\ Space Research Institute RAS, Profsoyuznaya 84/32, Moscow, 117997 Russia
}

Wind vectors derived by processing data of UV (365 nm) images obtained by Venus Monitoring Camera (VMC) onboard Venus Express spacecraft from 2006 to 2013 and by Ultraviolet Imager (UVI) onboard Akatsuki from 2016 to 2019 were used to study mesosphere dynamics. We found that the trend of the mean zonal wind speed at South equatorial latitudes of Venus changed from upward to downward in time period from 2014 to 2015. Analysis of the longitudinal dependence of the average zonal velocity, obtained from both the VMC and the UVI data, reveals decrease in wind speed associated with Aphrodite Terra.

Keywords: Venus, mesosphere, atmosphere dynamics, topography.

DOI: 10.51194 /VAK2021.2022.1.1.091

\section{Long-term variations of the mean zonal wind speed}

Due to the presence of the UV absorber in the upper cloud layer, the cloud details have contrast of more than $30 \%$ at altitude $70 \pm 2 \mathrm{~km}$, corresponding to the upper boundary of the cloud layer. This makes it possible to determine the wind speed by their movement. Wind vectors obtained by processing data of VMC/Venus-Express [1, 2, 3] and UVI/Akatsuki images of Venus $(365 \mathrm{~nm})$ were derived by means of digital tracking technique.

In 2006 - 2013 the VMC registered increase of the mean zonal wind speed from 85 to $115 \mathrm{~m} / \mathrm{s}$ at equatorial latitudes [1]. The Akatsuki spacecraft continuing observations of Venus since 2015, makes it possible to trace the change in the average zonal wind over long time. The results obtained from UVI images from 2016 to 2019 manifest a downward trend of the mean zonal wind speed (from 103 to $84 \mathrm{~m} / \mathrm{s}$ ), opposite to the upward trend obtained from VMC images (Figure 1).

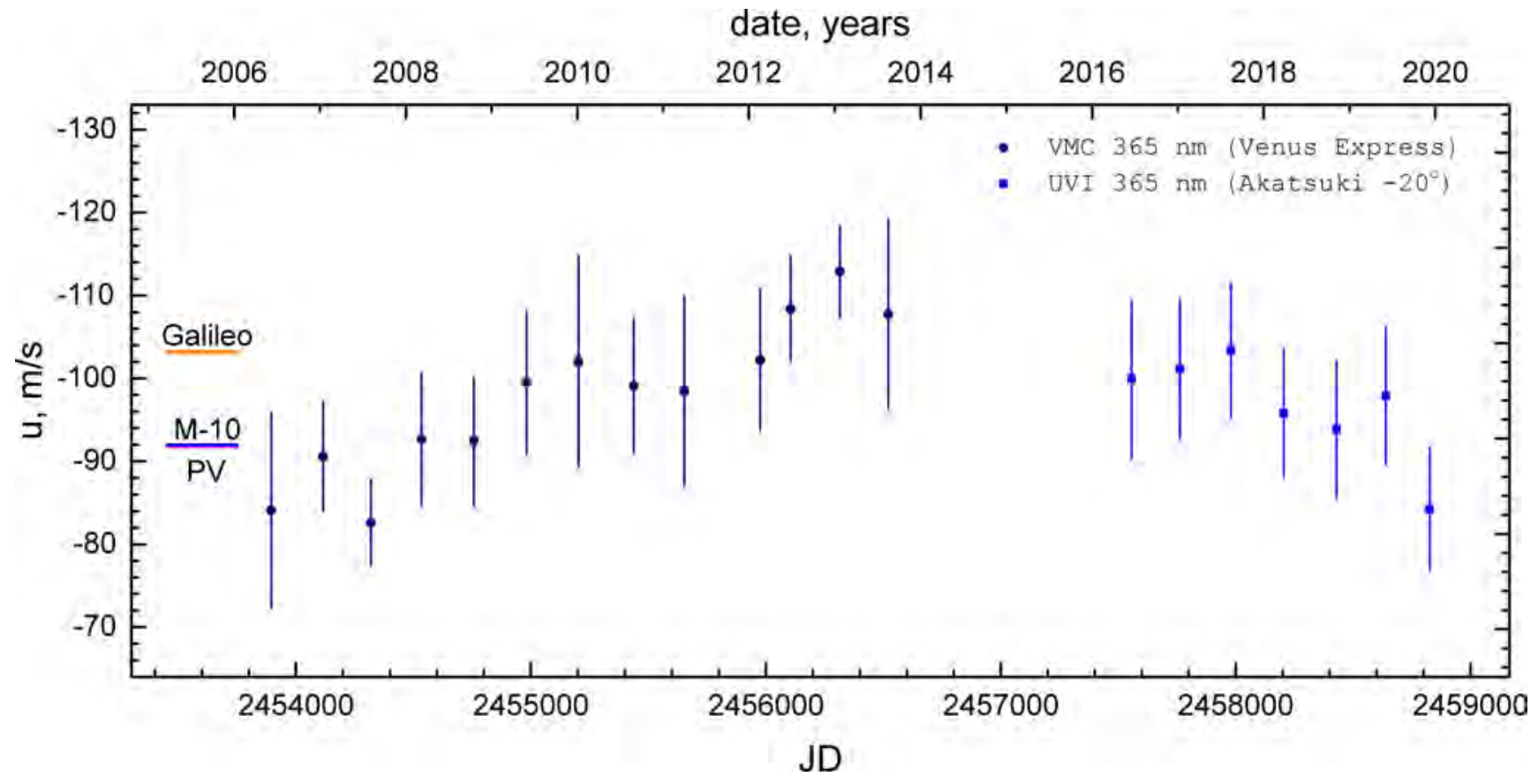

Figure 1: Variations of the mean zonal wind speed at $20 \pm 2.5^{\circ} \mathrm{S}$ over the mission time. Every point is the result of averaging over the Venusian year (224.7 days) in the latitude band $20 \pm 2.5^{\circ} \mathrm{S}$. Dark blue circles show zonal wind averaged through the Venusian year derived from VMC images, light blue squares are the same for UVI. The results from the Mariner-10 (92 m/s), Pioneer-Venus $(91.8 \pm 3 \mathrm{~m} / \mathrm{s})$ and Galileo $(103 \mathrm{~m} / \mathrm{s})$ missions for the same latitude zone are shown at the left edge of the plot for comparison.

\section{Influence of the surface on the change in the zonal speed}

Analysis of the longitudinal dependence of the average zonal speed obtained from VMC data for the entire observation period revealed a decrease in the wind speed associated with Aphrodite Terra, which is the highest region in the equatorial 
latitudes [4, 3]. At the same time, results obtained from UVI images from December, 2015 to March, 2017 discovered some wind acceleration above Aphrodite Terra and didn't reveal the influence of topography on the cloud top winds [5].
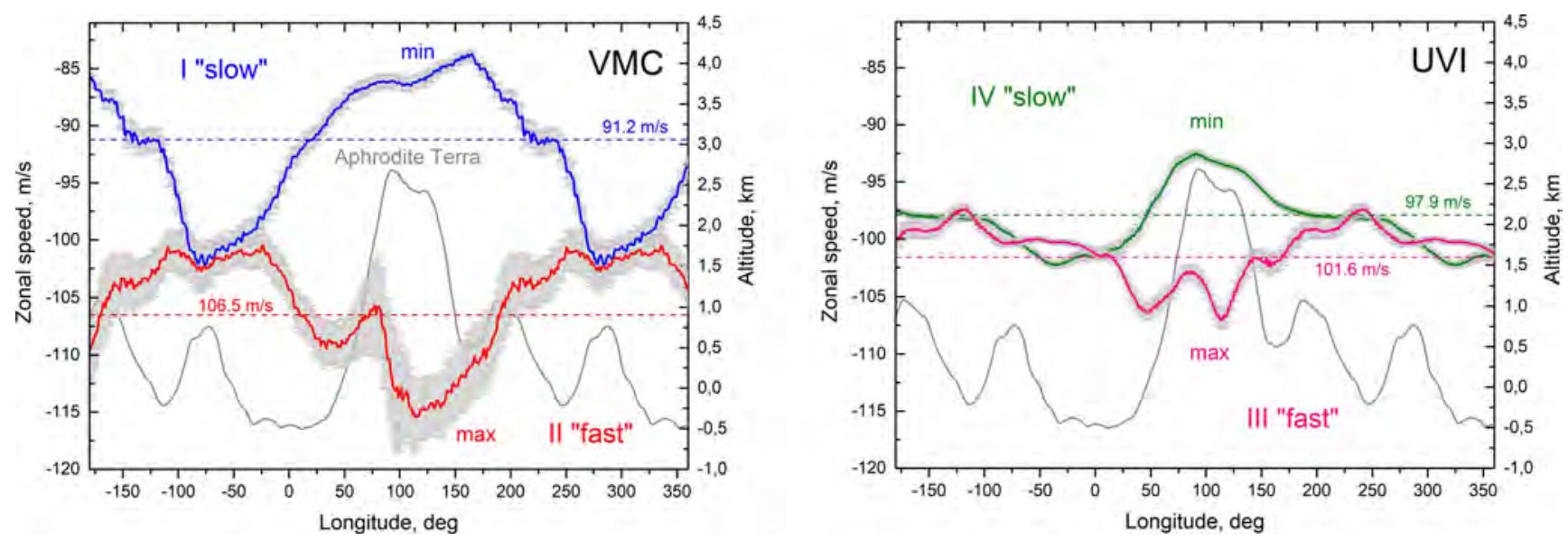

Figure 2: Mean profiles of zonal wind speed for first 4 years of VMC (blue line, left panel), last 4 years of VMC (red line, left panel), first 1.5 years of UVI (pink line, right panel), next 1.5 years of UVI (green line, right panel) averaged within latitude zone $10 \pm 5^{\circ} \mathrm{S}$. The error bars (light gray) correspond to $3^{*} \mathrm{SEM}$, where SEM is standard error of the mean. The gray line (left and right panels) shows the mean surface topography at the same latitude zone. The dotted lines correspond to the mean-longitudinal zonal speed for the time intervals I, II, III, IV.

Our analysis of the longitudinal dependence of the zonal wind speed for time-limited observation intervals shows that a maximum of the zonal wind (acceleration) over Aphrodite Terra is observed for intervals with higher average speed ("fast" intervals (II, III): the second half of the Venus Express mission and the first 1.5 years of the Akatsuki mission). The effect of "deceleration" of the zonal flow is observed over Aphrodite Terra for intervals with lower average speed ("slow" intervals (I, IV): the first half of the Venus Express mission and the second 1.5 years of the Akatsuki mission) (Figure 2).

Is the influence of the underlying surface persistent when the maximum zonal velocity is observed over Aphrodite Terra? To answer this question, we investigated the longitudinal dependence of the number of results. It was found that the largest number of results is close to the position of the minimum zonal speed for all the cases presented in Figure 2 (for "fast" intervals the zonal speed minimum is shifted relative to Aphrodite Terra). The number of results obtained is closely related to the presence of contrasting cloud structures (wave formations), whose movement determines wind vectors. According to VMC observations in UV, wave formations correlate with the highest regions of Venus [6, 7]. From this we assume that the influence of the surface persists and manifests itself as a deceleration of the zonal flow during the entire observation period.

\section{Supposed causes of the zonal speed trend}

It was suggested that the increase in the average zonal speed can be caused by decrease in the average surface height due to slow drift of the Venus Express orbit with respect to the surface topography over the course of the mission Venus Express [4]. The dashed lines in Figure 2 show longitudinally averaged zonal speeds for time intervals I, II, III, IV. The difference between "slow" and "fast" periods argues that the observed long-term variations in the zonal speed cannot be attributed to the sole surface effect. According to many years of observations, these long-term zonal speed variations correlate with changes in MgII index, i.e. temporal variations in the solar UV flux (http://www.iup.uni-bremen.de/gome/gomemgii.html). At the same time, according to [8], there is anticorrelation between the Venus UV albedo and solar activity. It turns out that the wind speed increases with decreasing albedo that may be a consequence of atmosphere warming. Nevertheless, given that the nature of the UV absorber at the upper cloud layer is still unknown, it is impossible to relate the cause-and-effect linkage between the observed phenomena.

Acknowledgments Patsaeva M. V. was supported by the Ministry of Science and Higher Education of the Russian Federation Grant 075-15-2020-780. Khatuntsev I. V., Turin A. V. and Zasova L. V. were supported by the program AAAAA18-118052890092-7 of the Ministry of Science and Higher Education of the Russian Federation. Original Venus Monitoring Camera data used in this work are available at the ESA Planetary Science Archive (PSA; ftp://psa.esac.esa.int/pub/mirror/ VENUSEXPRESS/VMC/). Original Ultraviolet Venus Imager data used in this work were provided by the Akatsuki project of JAXA [9].

\section{References}

1. I. V. Khatuntsev, M. V. Patsaeva, D. V. Titov, N. I. Ignatiev, et al., Icarus, 226, 140, 2013, URL https://doi.org/10.1016/j.icarus.2013.05.018.

2. M. V. Patsaeva, I. V. Khatuntsev, D. V. Patsaev, D. V. Titov, N. I. Ignatiev, W. J. Markiewicz, and A. V. Rodin, Planetary and Space Science, 113(08), 100, 2015, URL https://doi.org/10.1016/j.pss.2015.01.013. 
3. M. V. Patsaeva, I. V. Khatuntsev, L. V. Zasova, A. Hauchecorne, D. V. Titov, and J.-L. Bertaux, Journal of Geophysical Research: Planets, 124, 1864, 2019, URL https://doi.org/10.1029/2018JE005620.

4. J.-L. Bertaux, I. V. Khatuntsev, A. Hauchecorne, W. J. Markiewicz, et al., Journal of Geophysical Research: Planets, 121, 1087, 2016, URL https://doi.org/10.1002/2015JE004958.

5. T. Horinouchi, T. Kouyama, Y. J. Lee, S. Murakami, and K. Ogohara, Earth, Planets and Space, 70(1), 10, 2018, URL https://doi.org/10.1186/s40623 017-0775-3.

6. A. Piccialli, D. V. Titov, A. Sanchez-Lavega, J. Peralta, O. Shalygina, W. J. Markiewicz, and H. Svedhem, Icarus, 227, 94, 2014, URL https://doi.org/10.1016/j.icarus.2013.09.012.

7. D. V. Titov, W. J. Markiewicz, N. I. Ignatiev, L. Song, et al., Icarus, 217(2), 682, 2012, URL https://doi.org/10.1016/j.icarus.2011.06.020.

8. Y. J. Lee, K.-L. Jessup, S. Perez-Hoyos, D. V. Titov, et al., The Astronomical Journal, 158(3), 16, 2019, URL https://doi.org/10.3847/1538-3881/ab3120.

9. S. Murakami, K. Ogohara, M. Takagi, H. Kashimura, M. Yamada, T. Kouyama, T. Horinouchi, and T. Imamura, JAXA Data Archives and Transmission System, 2018, URL https://doi.org/10.17597/isas.darts/vco-00016. 


\section{Migration of water to the polar regions of the Moon}

S. Pugacheva, E. Feoktistova, V. Shevchenko hrulis@yandex.ru

Moscow State University, Sternberg Astronomical Institute, Moscow 119234, Russia

Keywords: Moon, water ice, polar regions

DOI: 10.51194 /VAK2021.2022.1.1.092

\section{Sources of water molecules in the lunar exosphere}

Volatile compounds, including water, can enter the lunar exosphere as a result of endogenous processes such as degassing of the interior and volcanic activity on the surface of the satellite. Exogenous sources are solar wind and collisions with the surface of meteorites, asteroids and comets. As a result of these processes, compounds such as $\mathrm{H}_{2} \mathrm{O}, \mathrm{CO}, \mathrm{CO}_{2}$, etc. can appear in the lunar exosphere.

\section{Model for calculating the migration of water molecules}

The simulation of the migration of water molecules in the lunar exosphere was carried out by the Monte Carlo method. The simulation involved 2,000,000 water molecules. The direction and starting angle of the molecule were determined randomly. The initial position and time of the beginning of the movement of molecules was chosen arbitrarily, with one limitation the movement of particles always began on the illuminated side of the moon. The area of permanently shaded areas in the region of the south pole of the Moon was taken equal to $16055 \mathrm{~km}^{2}$ [1], and in the region of the north $-12866 \mathrm{~km}^{2}$.

\section{Results}

Our calculations show that most of the water molecules (about $69 \%$ ) will be destroyed as a result of photolysis on the lunar surface or in hop. Only $0.5 \%$ of water molecules will leave the lunar exosphere as a result of exceeding the escape velocity. Cold traps in the region of the Moon's South Pole will contain $18.6 \%$ of the particles participating in the modeling. Only $11.1 \%$ of water molecules will fall into cold traps near the moon's north pole.

\section{References}

1. E. Mazarico, G. A. Neumann, D. E. Smith, M. T. Zuber, and M. H. Torrence, Icarus, 211, 1066, 2011. 


\title{
Modeling of asteroids reflectance spectra and estimation of their taxonomic types and surface composition
}

\author{
A.A. Savelova ${ }^{1}$, V.V. Busarev ${ }^{1,2}$, M.P. Shcherbina ${ }^{1}$ \\ aa.rezaeva@physics.msu.ru \\ ${ }^{1}$ Sternberg Astronomical Institute, Moscow State University, Universitetsky pr., 13, Moscow 119234, Russia \\ ${ }^{2}$ Institute of Astronomy, Russian Academy of Sciences, Pyatnitskaya str., 48, Moscow 119017, Russia
}

\section{DOI: $10.51194 /$ VAK2021.2022.1.1.093}

Nowadays estimation of surface mineralogy of the most of asteroids can be performed only by distant measurements and modeling results of these measurements. This is the reason why it is necessary to automatize this procedure.

Some time ago a program for approximation of reflectance spectra of asteroids was written [1]. It searches for the best combination of spectra of minerals or meteorites to fit the reflectance spectrum of an asteroid. Also another program was written [2], that helps to estimate a taxonomic class of asteroid using its reflectance spectrum and geometric albedo.

In December 2020 our group collected new spectral data for 10 main belt asteroids (19, 52, 102, 177, 200, 203, 250, $266,379,383)$ when they were moving near their perihelia. We applied our programs to these data. Thus our results are:

- confirmed taxonomic classes for asteroids 102 and 266,

- found meteorite approximations for asteroids 102 and 266 include only primitive type meteorites, so they agree with primitive types of these asteroids,

- estimated an unknown taxonomic class of asteroid 203 (SMASSII type C).

Spectra shapes of other 7 asteroids do not consist with their SMASSII taxonomic classes [3]. Taking into account that

- all of the asteroids were moving near perihelion where formation of a dust exosphere is possible due to sublimation of subsurface ices at the most high subsolar temperatures,

- spectral transmission of terrestrial atmosphere has not been changing during the observational time,

- surface mineral composition of the asteroids could not be changed much at the observations, because the asteroids were observed for a short time,

a conclusion is made that the spectral variations of these asteroids were probably caused by their dust exospheres formed near perihelion.

\section{References}

1. A. Rezaeva, M. Shcherbina, and V. Busarev, Abstracts of the Tenth Moscow Solar System Symposium (10M-S3), 439-440, 2019.

2. M. Shcherbina, A. Savelova, and V. Busarev, Proceedings of the All-Russian Conference "Ground-Based Astronomy in Russia. 21st Century", 196-197, 2020.

3. Jet Propulsion Laboratory, Small-body database lookup, 2021, URL https://ssd.jpl.nasa.gov/tools/, last accessed 27 September 2021. 


\title{
New reflectance spectra of Main belt asteroids: clarification of taxonomic types and composition of matter
}

\author{
M.P. Shcherbina ${ }^{1}$, V.V. Busarev ${ }^{1,2}$, S.I. Barabanov ${ }^{2}$ \\ morskayaa906@yandex.ru \\ ${ }^{1}$ Lomonosov Moscow State University, Sternberg Astronomical Institute (SAI MSU), University Av., 13, Moscow, 119992, \\ Russian Federation \\ ${ }^{2}$ Institute of Astronomy, Russian Academy of Sciences (INASAN), Pyatnitskaya St. 48, Moscow, 109017, Russian \\ Federation
}

The mineralogy of the surface matter of asteroids can be judged using a qualitative interpretation of the reflection spectra of these bodies. The features of the reflectance spectra (absorption bands) are wide enough for correct interpretation even at a low resolution of the recording device. It should be noted that the number of events of registration of sublimation activity among primitive asteroids has been increasing recently. The emergence of a temporary exosphere affects the asteroid's reflection spectrum. The reflection spectra of 12 asteroids of the Main Belt, mainly of primitive type, have been obtained and analyzed. The aim of the research was to study the influence of the temporal exosphere on the correct determination of the spectral type of the asteroid. In addition, for asteroid 203 Pompeia, the spectral type has been determined for the first time(class C).

Keywords: asteroids of the Main belt, reflectance spectra, sublimation activity, Tholen classification, Bus-Binzel classification

DOI: $10.51194 /$ VAK2021.2022.1.1.094

\section{Introduction}

Asteroids, as atmosphereless bodies, are ideal objects for spectrophotometric ground-based observations. The features of their reflection spectra (the absorption bands) are wide enough to use a low resolution $(R \sim 100)$, which makes it possible to expand the range of the CCD spectrograph. Analysis of the reflection spectra of asteroids makes it possible to qualitatively assess the predominant type of mineralogy of their matter, using the classifications of Tholen [1] and Bus-Binzel [2]. To control the correctness of such estimates, the features of the reflection spectra of asteroids are compared with the reflection spectra of the corresponding groups of meteorites. We use reflection spectra of meteorites from open databases such as RELAB (Brown University, USA) [3] and the University's of Winnipeg database (Canada) [4]. Thus, a qualitative assessment of the mineralogy of each asteroid is based on a comparison of its reflection spectra with the reflection spectra of meteorites, for which the parent bodies could presumably be asteroids of this taxonomic type [5]. But we also admit the possibility of discovering new connections between existing groups of meteorites and classes of asteroids. SAI MSU, together with the Institute of Astronomy of the Russian Academy of Sciences, participates in the international program "Astronomy in the Elbrus region" in terms of spectral observations of asteroids and comets, including those approaching the Earth, based on the use of the Zeiss-2000 telescope with a low-resolution spectrograph at the "Terskol Peak observatory" (Terskol branch of INASAN). In December 2020, we obtained and analyzed new reflection spectra of the main belt asteroids: 13 Egeria, 19 Fortuna, 52 Europe, 102 Miriam, 177 Irma, 200 Dynamene, 201 Penelopa, 203 Pompeia, 250 Bettina, 266 Aline, 379 Huenna and 383 Janina. The purpose of these observations was to study the effect of maximum temperatures on the surface matter of the listed asteroids (which have significant orbital eccentricities), including hydrosilicates and, probably, ice inclusions.

\section{The qualitative assessment of the mineralogy of asteroids}

The obtained reflectance spectra of asteroids were studied to register features (absorption bands, unusual growth in the long-wavelength region, which may indicate the presence of a temporary exosphere). The taxonomic type was assessed according to the Tholen's classification or SMASS. To confirm the correctness of the estimate, a meteorite analogue was selected. We present the results for two of the studied asteroids.

\subsection{Miriam}

The reflectance spectrum of meteorite 102 Miriam is presented in Fig. 1 Originally classified as a D-type asteroid, it was later classified as a C-asteroid, most likely due to the presence of phyllosilicates on the surface [6]. There is a weak band at $0.44 \mu \mathrm{m}$ (a sign of the presence of hydrosilicates). The object rotates extremely slowly, however, in the two spectra we obtained, there are some differences, which can be explained by the result of light scattering by an extremely rarefied dusty exosphere. The selected meteorite analogue Lewis Cliff (CM2) is in good agreement with the spectrum of the asteroid, confirming the low-temperature nature of the surface matter.

\subsection{Aline}

The reflectance spectra are shown in Figure 2. The shape of the spectrum corresponds to the reflection spectrum of class C. There is an increase in the range of $0.85-0.9 \mu \mathrm{m}$, which may be a sign of a dusty exosphere. The selected meteorite analogues that showed the best similarity to the asteroid's reflection spectrum are Elephant (CM2) and Orgueil (C1), which is an additional indication of the correctness of the asteroid's class estimate. 


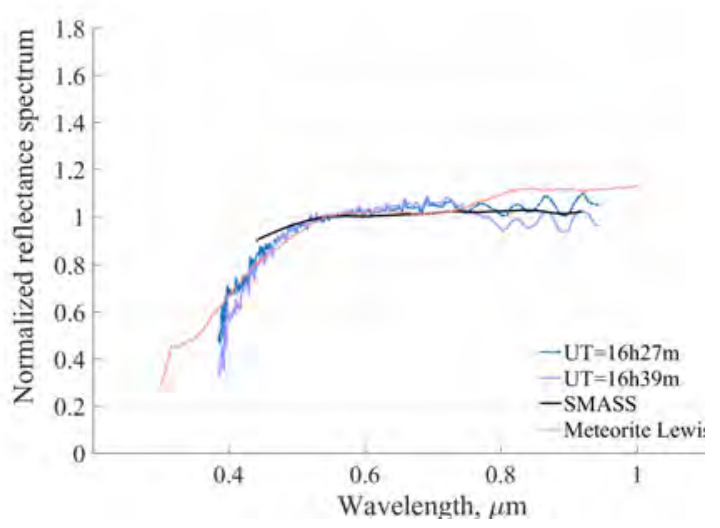

Figure 1: Reflectance spectra of asteroid 102 Miriam, reflectance spectrum according to SMASS data and reflectance spectrum of the selected analogue of the meteorite

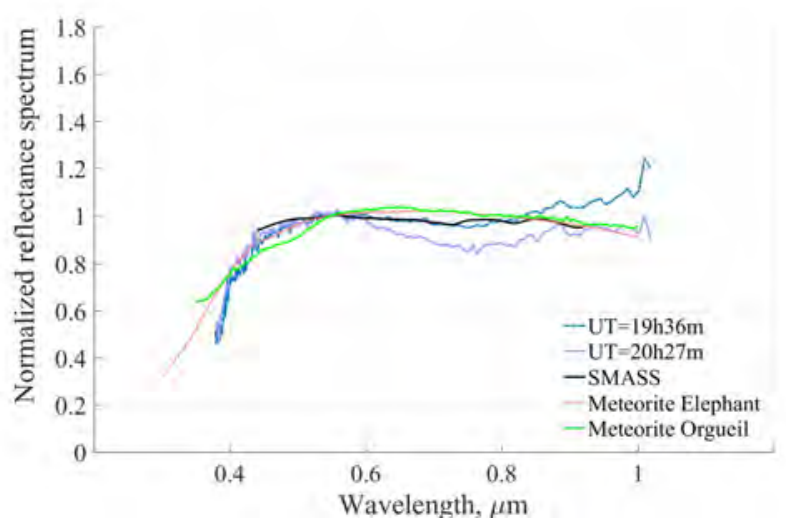

Figure 2: Reflectance spectra of asteroid 266 Aline, reflectance spectrum according to SMASS data and reflectance spectrum of the selected analogue of the meteorite

\section{Conclusion}

It should be emphasized that almost all considered asteroids (except for M-type asteroids 201 Penelope and 250 Bettina) belong to primitive taxonomic classes, that is, they have low-temperature mineralogy (typical minerals are hydrosilicates with probable inclusions of water ice). In addition, we deliberately chose the observational time so that all these bodies were near the perihelion, where their sublimation activity and the formation of a dusty exosphere are possible [7, 8, 9]. Therefore, we probably obtained the reflection spectra of these asteroids partially distorted by the influence of a rarefied dusty exosphere, consisting of submicron conglomerates of particles of ice, silicate, and combined (ice-silicates) composition. The influence of such an exosphere is in good agreement with the modelling of light scattering by the above components [9]. At the same time, as the study of the presented reflection spectra has shown, if such distortions are not considered, it is possible to assess the taxonomic types and mineralogy of the considered asteroids mainly in the short-wave part of these spectra. The estimates obtained are in good agreement with the known data on these objects, as well as with some indirect characteristics, such as the rotation period, geometric albedo, and similarity with the reflection spectra of meteorites of different groups. For 203 Pompeia, the spectral type was determined for the first time (type C). Not all asteroids were able to find a suitable meteorite analogue, and sometimes a discrepancy was observed with the mineralogical properties of the meteorite matter with similar reflection spectra. This can be caused both by the mixed composition of the surface matter of the asteroid, for example, because of collisions with bodies differing in mineralogy, and by the incompleteness of the collections of meteorite matter. Note that for most of the considered asteroids, the presence of such a temporary exosphere did not significantly affect the position of the absorption bands and the shape of the spectrum, that is, it does not interfere with the assessment of the mineralogy of the surface matter.

\section{References}

1. D. Tholen, Asteroids II, 1139-1150, 1989.

2. S. Bus and R. Binzel, Icarus, 158, 239, 2002.

3. C. Pieters and T. Hiroi, Lunar and Planetary Science Conference, 35, 2004.

4. PSF Scientific Collaboration, 2020.

5. M. J. Gaffey, 1386, 129, 2011.

6. A. Fitzsimmons, M. Dahlgren, C.-I. Lagerkvist, P. Magnusson, and I. Williams, Astronomy and Astrophysics, 282, 634, 1994.

7. V. Busarev, S. Barabanov, and V. Puzin, Solar System Research, 50, 281, 2016.

8. V. Busarev, M. Shcherbina, S. Barabanov, T. Irsmambetova, et al., Solar System Research, 53, 261, 2019.

9. V. V. Busarev, E. V. Petrova, T. R. Irsmambetova, M. P. Shcherbina, and S. I. Barabanov, Icarus, 369, $114634,2021$. 


\section{Yarkovsky effect: relationship between acceleration $A_{2}$ and semi-major axis drift velocity for Main Belt asteroids}

M. Vasileva, E. Kuznetsov

vasilyeva.maria@urfu.ru; eduard.kuznetsov@urfu.ru

Ural Federal University, Lenina Avenue 51, Yekaterinburg 620000, Russia

DOI: 10.51194 /VAK2021.2022.1.1.095

It is necessary to take into account the non-gravitational effects for a reliable study of the orbital evolution of asteroids. Such effects can be taken into account in various ways in different software packages that allow simulating the motion of bodies of the Solar system.

The semi-major axes drift rate $d a / d t$ can be estimated based on the physical and dynamic parameters of the asteroids. Also acceleration $A_{2}$ and drift rate $d a / d t$ can be obtained from the analysis of observations.

It becomes necessary to switch from the non-gravitational acceleration $A_{2}$ to the drift of the semi-major axis $d a / d t$ to solve some tasks.

We simulated the orbital evolution of model asteroids with circular ecliptic orbits with semi-major axes from 1.75 to $3.0 \mathrm{AU}$ with a step of $0.01 \mathrm{AU}$ at the values of the parameter $A_{2}=1 \cdot 10^{-15}, 1 \cdot 10^{-14}, 1 \cdot 10^{-13} \mathrm{AU} /$ day $^{2}$. The integration interval was 1 Myr. The semi-major axis drift rate $d a / d t$ for each asteroid was estimated based on the simulation results. Further, we performed an approximation of the dependence using a polynomial model of the 3rd degree.

We propose empirical relationship between the non-gravitational acceleration $A_{2}\left[\mathrm{AU} / \mathrm{day}^{2}\right]$ and semi-major axis drift rate $d a / d t[\mathrm{AU} / \mathrm{Myr}]$ for Main belt asteroids:

$$
\frac{d a}{d t}=A_{2} \cdot 10^{10} \cdot\left(k_{0}+k_{1} a+k_{2} a^{2}+k_{3} a^{3}\right),
$$

where $k_{0}=20.10, k_{1}=-17.94, k_{2}=5.353, k_{3}=-0.5338$.

Acknowledgments. This work has been supported by the Russian Ministry of Science and Higher Education via the State Assignment Projects FEUZ-2020-0038 (MV) and FEUZ-2020-0030 (EK). 


\section{Investigation of sulphurous components of rarefied atmosphere of Venus: ISCRA-V experiment at the Venera-D lander}

I. Vinogradov and Work team of the ISCRA-V experiment imant@iki.rssi.ru,

WWW page: http://iki.rssi.ru

Space Research Institute (IKI) of the Russian Academy of Sciences, 84/32 Profsoyuznaya Str, Moscow, Russia

Keywords: sulphur dioxide, atmosphere of Venus, Venera-D lander

DOI: 10.51194 /VAK2021.2022.1.1.096

To continue studies of the atmosphere of Venus [1, 2], an experiment ISCRA-V have been proposed [3] for retrieving of vertical profiles of sulphur dioxide, sulphurous and minor gas components of the atmosphere on descent trajectory of the Venera-D lander.

An active phase of the experiment ISCRA-V starts when a protective shield of the lander will be dropped off at an altitude of about $73 \mathrm{~km}$ and will be continued until it touches down the surface of Venus, and afterwards near the surface at the landing zone during the lander lifetime.

Multichannel tunable laser absorption spectrometer (MTLAS) is the core of the ISCRA-V instrument. Sequential operation of monochromatic tunable diode and quantum cascade lasers will make it possible fine study of the ambient atmosphere composition. Probing laser beams will be coupled into a multi-pass optical cell analytical volume, filled in by a sampled interchangeable portion of the surrounded atmosphere, rarefied down to 50 mbar work pressure.

Measurements are currently planned for:

- $\mathrm{SO}_{2}, \mathrm{OCS}, \mathrm{CO}, \mathrm{CO}_{2}, \mathrm{H}_{2} \mathrm{O}$ content; $\mathrm{HCl}$ or $\mathrm{PH}_{3}$ optional;

- ratios of ${ }^{13} \mathrm{C} /{ }^{12} \mathrm{C}$ for $\mathrm{CO}$ and $\mathrm{CO}_{2},{ }^{18} \mathrm{O} /{ }^{17} \mathrm{O} /{ }^{16} \mathrm{O}$ for $\mathrm{CO}_{2},{ }^{34} \mathrm{~S} /{ }^{32} \mathrm{~S}$ for OCS.

\section{References}

1. J.-L. Bertaux, T. Widemann, A. Hauchecorne, V. I. Moroz, and A. P. Ekonomov, JGR, 101, 12709, 1996.

2. L. V. Zasova, D. A. Gorinov, N. A. Eismont, I. D. Kovalenko, A. S. Abbakumov, and S. A. Bober, Solar System Research, 53, 506, 2020.

3. I. I. Vinogradov and D. A. Belyaev, Int. Conf. DLS-23, 27 Oct. 2015, GPI, Moscow, http://www.dls.gpi.ru/rus/sem/23/1.pdf. 


\section{Relativistic Astrophysics and Gravity Waves}




\title{
NGC $5474 X-1$ : the neutron star ULX in an old stellar cluster
}

\author{
K. Atapin ${ }^{1}$, A. Vinokurov ${ }^{2}$, Yu. Solovyeva ${ }^{2}$, S. Fabrika ${ }^{2}$ \\ atapin.kirill@gmail.com \\ ${ }^{1}$ Sternberg Astronomical Institute, Moscow M.V. Lomonosov State University, Universitetskij pr., 13, 119992, Moscow, \\ Russia, \\ ${ }^{2}$ Special Astrophysical Observatory, Nizhnij Arkhyz, Russia
}

We report the discovery of a cyclotron resonant scattering feature (CRSF) in the ultraluminous X-ray source (ULX) NGC5474 X-1 that makes it the third ULX with a CRSF. Cyclotron lines arise in strong magnetic fields, which requires the object to be a neutron star. The line energy of $7.5 \mathrm{keV}$ corresponds to the magnetic field strength $\sim 10^{12} \mathrm{G}$. The source shows a hard X-ray spectrum with $\Gamma \sim 1$ and strong X-ray variability which is also typical for neutron star ULXs. In the optical range, the object is interesting by the fact that it is projected onto an old stellar cluster (or located inside) with an age of about 1 Gyr.

Keywords: accretion, neutron stars, ultraluminous $X$-ray sources

DOI: $10.51194 /$ VAK2021.2022.1.1.097

\section{Introduction.}

Ultraluminous X-ray sources (ULXs) are extra-galactic accreting binary systems with apparent X-ray luminosities above the Eddington limit for a black hole with a mass of $15 M_{\odot}[1,2]$. Several years ago it became evident that at least part of them contains neutron stars. In most cases, the conclusion about the presence of a neutron star was made due to the detection of coherent pulsations of their X-ray emission; now 11 ultraluminous pulsars are known. However, in the case of M51 ULX-8 which does not show pulsations, the neutron star nature follows from the presence of a cyclotron resonance scattering feature [3] - the absorption line in the X-ray spectrum that produced by charged particles transitions between Landau levels in strong magnetic field. Also a CRSF is suspected in the spectrum of the confirmed ultraluminous pulsar NGC 300 ULX1 [4]. In this paper we present the discovery of a CRSF in one more ULX - NGC 5474 X-1. Below we will discuss its X-ray spectrum and optical observations.

\section{Data analysis and results.}

The source was observed twice with Chandra, in 2006 and 2007, with exposure times $1.7 \mathrm{ks}$ and $30 \mathrm{ks}$, respectively. Unfortunately, both observations are affected by pileup, therefore we analyzed only the longest one. The pileup damages mainly inner pixels of the source PSF where the count rate per frame is maximal. So, the common practice to deal with pileuped spectra is using of an annular aperture to exclude events from these inner pixels. Alternatively, one can extract the spectrum from full (circular) aperture but include special model component to the fitting model in order to account spectral distortions produced by pileup. Below we have tried both approaches.

We extracted the source spectra from a circular aperture of $3^{\prime \prime}$ radius and from an annulus with inner and outer radii of $1.3^{\prime \prime}$ and $3^{\prime \prime}$, respectively. The spectrum from the annulus which is supposed to contain a minimal number of damaged events shows prominent absorption feature near $7 \mathrm{keV}$ (Fig. 1, left panel). We fitted this spectrum with XSPEC using the model tbabs*gabs*powerlaw consisting of a power-law continuum with a Gaussian absorption line and accounted for interstellar absorption. The obtained model parameters are $L_{E}=7.0_{-0.9}^{+2.0}, L_{\sigma}=1.0_{-0.6}^{+1.4}$ and $\Gamma=1.01_{-0.09}^{+0.2}$, the C-statistic [5] is 168 for 187 degrees of freedom. The spectrum from the full aperture was fitted by the same model with added XSPEC model component pileup by [6]. The resultant model parameters turned out to be close to those from the annular aperture. To test the significance of the line we fitted the same spectrum by the model with excluded Gaussian component. The obtained difference in statistics of 40.1 (difference in degrees of freedom is only 3) indicates the high significance of the observed absorption line.

The source luminosity in the Chandra observations were $\sim 10^{40} \mathrm{egr} / \mathrm{s}$. Then X-1 was observed 20 times with Swift (from 2011 to 2018) which found it in a low luminosity state. In the most long Swift observation its flux decreased at least 100 times compared to the Chandra ones.

The Hubble Space Telescope (HST) observed the area of NGC 5474 X-1 four times in different filters, from 2009 to 2015. For the first time these observations was analyzed by [7], who identified a single optical object at the place of the the X-ray source. However, this optical source is slightly extended, its FWHM size $0.23^{\prime \prime} \times 0.16^{\prime \prime}$ corresponds to the linear size of $7 \times 5 \mathrm{pc}$ at the distance of the NGC 5474 galaxy. Our more careful analysis of the optical data has revealed a point source within the Chandra error circle in the first HST image in the UV band, which is not seen in subsequent observations (Fig. 1, right panel). We suppose that this point source is true counterpart of X-1 caught in an outburst.

To clarify the nature of the extended source, we performed its photometry and found that the source is clearly seen only in the visual and near IR bands while at shorted wavelengths it is either very faint or completely invisible. Three possibilities were considered: the source could be a nebula, a background galaxy at arbitrary red shift and a stellar cluster. The first one can be rejected due to the absence of an excess in the $H \alpha$ filter. Choosing between the remaining options, we fitted the source spectral energy distribution (SED) by the starburst99, LePhare and MAGPHYS codes. The most plausible result is given by the model of a stellar cluster of about $3000 \mathrm{M}_{\odot}$ with an age of $1.0_{-0.25}^{+0.15} \mathrm{Gyr}$. 

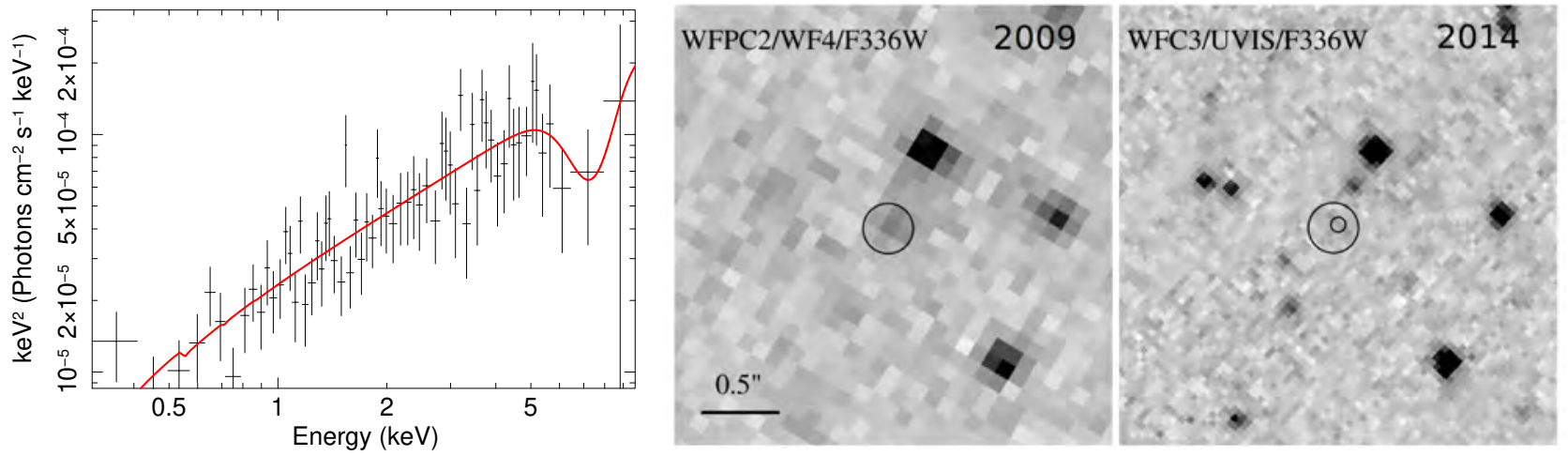

Figure 1: Left panel: Observed X-ray spectrum with clear absorption line at $7 \mathrm{keV}$ extracted from the annular aperture, and the best fitting model. Right panel: Position of the ULX optical counterpart in the HST images. Big circles indicate the corrected Chandra position of the X-ray source, small circle — the point source position measured during the outburst from the WFPC2/F336W image. It is seen that the point source disappeared in the later image.

\section{Discussion and conclusions.}

We have reliably detected a strong absorption line near $7 \mathrm{keV}$ in the X-ray spectrum of NGC 5474 X-1 which is more likely a cyclotron line already discovered in two ULXs (at $4.5 \mathrm{keV},[3]$ and at $\sim 13 \mathrm{keV},[4]$ ) and often seen in Galactic neutron stars [8]. Assuming the electron nature of the line, its energy corresponds to the magnetic field strength of $7.5 \times 10^{11} \mathrm{G}$. The residence of the source in the old stellar cluster is of great interest because most of other known sources with CRSFs are young object. However, we cannot exclude that this is just a result of an accidental projection. These doubts are supported by the presence of young stellar associations with ages of $10 \mathrm{Myr}$ within $300 \mathrm{pc}$, one of which may turn out to be parental for the ULX. Nevertheless, ULXs with neutron stars of $\sim 1$ Gyr are predicted by some population synthesis modeling (for example, [9]).

\section{Acknowledgments}

This research was supported by the Russian Foundation for Basic Research N 19-02-00432.

\section{References}

1. P. Kaaret, H. Feng, and T. P. Roberts, ARAधA, 55, 303, 2017.

2. S. N. Fabrika, K. E. Atapin, A. S. Vinokurov, and O. N. Sholukhova, Astrophysical Bulletin, 76, 6, 2021.

3. M. Brightman, F. A. Harrison, F. Fürst, M. J. Middleton, et al., Nature Astronomy, 2, 312, 2018.

4. D. J. Walton, M. Bachetti, F. Fürst, D. Barret, et al., The Astrophysical Journal, 857, L3, 2018, URL https://doi.org/10.3847/2041-8213/aabadc.

5. W. Cash, ApJ, 228, 939, 1979.

6. J. E. Davis, ApJ, 562, 575, 2001.

7. S. Avdan, A. Vinokurov, S. Fabrika, K. Atapin, et al., MNRAS, 455, L91, 2016.

8. R. Staubert, J. Trümper, E. Kendziorra, D. Klochkov, et al., A\&A, 622, A61, 2019.

9. G. Wiktorowicz, M. Sobolewska, J.-P. Lasota, and K. Belczynski, ApJ, 846, 17, 2017. 


\section{The influence of small-scale magnetic field on the heating of J0250+5854 polar cap}

D.P. Barsukov ${ }^{1,2}$, A.A. Matevosyan ${ }^{2}$, I.K. Morozov ${ }^{1}$, A.N. Popov ${ }^{1}$, M.V. Vorontsov ${ }^{1,2}$ bars.astro@mail.ioffe.ru

${ }^{1}$ Ioffe Institute, Saint Petersburg, Russia

2 Peter the Great St. Petersburg Polytechnic University, Saint Petersburg, Russia

DOI: 10.51194 /VAK2021.2022.1.1.098

Radio pulsar J0250+5854 is the slowest pulsar among rotation powered pulsars. It is an old pulsar with spin-down age $\tau=13.7 \cdot 10^{6}$ years, which rotates with period $P=23.54 \mathrm{~s}$, the strength of dipolar magnetic field at pole estimated by pulsar spin-down rate is $B_{d i p}=5.1 \cdot 10^{13} \mathrm{G}$ [1]. Such pulsars lie beyond conventional pulsar "death line" and its existence is usually explained by the presence of surface small-scale magnetic field (see, for example, [2]). The other explanation is presented by [3]. In this paper the influence of surface small-scale magnetic field on the heating of PSR J0250+5854 polar cap is considered with assumption that the pulsar is close to aligned, i.e. the inclination angle is $\chi \lesssim 30^{\circ}$. It is assumed that the polar cap is heated only by reverse positrons accelerated in pulsar diode. It is supposed that pulsar diode is located near the star surface (polar cap model) and operates in the steady state space charge-limited flow regime. The reverse positron current is calculated in the framework of two models: rapid and gradually screening. To calculate the production rate of electron-positron pairs we take into account only the curvature radiation of primary electrons and its absorption in magnetic field. It is assumed that some fraction of electron-positron pairs may be created in bound state that can later be photoionized by thermal photons from star surface. We do not take into account the influence of polarization of curvature radiation on pair generation. Also we do not taken into account positronium decay. It is shown that under this assumptions almost all electron-positron pairs are produced in bound state and the multiplicity of photoionized pairs may exceed $10-10^{2}$ per primary electron.

\section{References}

1. C. M. Tan, C. G. Bassa, S. Cooper, T. J. Dijkema, et al., ApJ, 866, 54, 2018.

2. J. A. Hibschman and J. Arons, ApJ, 554, 624, 2001.

3. E. M. Novoselov, V. S. Beskin, A. K. Galishnikova, M. M. Rashkovetskyi, and A. V. Biryukov, MNRAS, 494, 3899, 2020 . 


\title{
Radio pulsars - two old questions
}

\author{
V. Beskin ${ }^{1,2}$ \\ beskin@lpi.ru
}

${ }^{1}$ P.N.Lebedev Physical Institute, Moscow 119991, Russia

${ }^{2}$ Moscow Institute of Physics and Technology, Dolgoprudny 141701, Russia

We draw attention to two questions in the theory of radio pulsars, which have not yet found their answer. The first one concerns the inclination angles $\chi$ between magnetic and rotation axes. We show that the very existence of interpulse pulsars indicates that all possible angles are realized. The second one is the question of the break of the death line on the $P \dot{P}$-diagram. We show that this break can be easily explained by the very mechanism of secondary plasma generation.

Keywords: neutron stars, radio pulsars

DOI: 10.51194/VAK2021.2022.1.1.099

\section{Introduction}

More than 50 years have passed since the discovery of radio pulsars. However, we still do not know the mechanism of their coherent radio emission, e.g., the direction of evolution of the inclination angle $\chi$. Some questions, formulated in the early years, were not further discussed. Here we outline the solution to one of such forgotten questions connecting with the possible range of inclination angles.

The second question concerns the so-called death line, i.e., the line limiting from below the population of pulsars on the $P \dot{P}$-diagram that has a break at $P \approx 0.1 \mathrm{~s}$. As we show, this break can be easily explained by the well-known feature of secondary plasma production. This work is dedicated to the blessed memory of B.V. Komberg, with whom we discussed these issues back in the 70s.

\section{Inclination angle}

If we take almost any catalog of the inclination angles $\chi$, we find that these angles lie in a range of $0<\chi<90^{\circ}$. But this does not mean that there are no radio pulsars with the inclination angle $\chi$ in a range of $90^{\circ}<\chi<180^{\circ}$. This is simply because most of the existing methods for determining this angle do not distinguish between $\chi$ and $180^{\circ}-\chi$.

On the other hand, acute and obtuse angles between the axes correspond to two principally different physical conditions because for $\chi<90^{\circ}$, the longitudinal current flowing along open magnetic field lines (with its sign determined by the sign of the [1] charge density $\rho_{\mathrm{GJ}}=-\mathbf{\Omega B} / 2 \pi c$ ) requires the outflow of negative charges from the star surface. By contrast, for the angles $\chi>90^{\circ}$, the surface should eject positive ones. Hence, in models of plasma generation, in which the ejection of particles from the surface plays a significant role, these are two fundamentally different cases. Recall that in [2] model, particle creation is insensitive to axis orientation.

At first glance, orthogonal pulsars give an immediate answer to this question. As shown in Fig. 1, for $\chi=90^{\circ}$, an observer sees the regions of radio emission corresponding to different signs of the Goldreich-Julian charge density. However, in the case where the inclination angle $\chi$ differs from $90^{\circ}$, the line of sight can intersect the radiation patterns from two magnetic poles in the areas corresponding to the same charge density. Hence, a more detailed study is required.

We analyzed eight orthogonal interpulse pulsars presented by [3], for which inclination angles $\chi$, the angles of closest approach of the line of sight to the magnetic axis $\beta$, and the emission heights $h$ were determined. This allowed us to estimate the inclination angles of magnetic field lines to the rotation axis $\delta$ in the plasma generation regions: $\delta=\chi+\beta(R / h)^{1 / 2}$. Here $R$ is the star radius. As shown from Table 1, for most pulsars, the angles $\delta$ for the main pulse and the interpulse correspond to different signs of the charge density. The uncertainty for two pulsars is apparently associated with a significant difference between the values of $h_{\mathrm{MP}}$ and $h_{\mathrm{IP}}$ (the accuracy of which is low). For other pulsars, our conclusion depends only slightly on the values of $h$. Thus, one can conclude that plasma ejection from the surface of a neutron star does not affect the process of particle production in the polar regions.

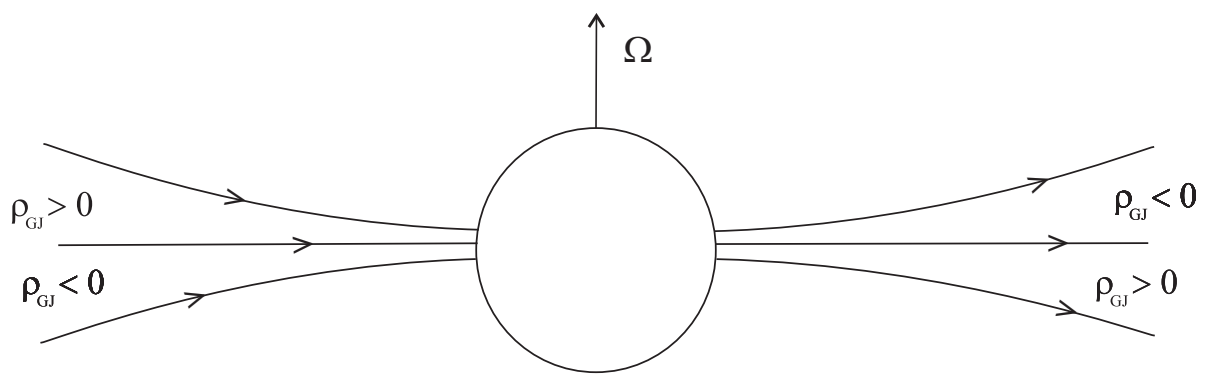

Figure 1: Observer located not on the equator sees the radio emission from two poles of the orthogonal rotator corresponding to two different signs of the charge density. 
Table 1: Interpulse pulsars data taken from [3]. All the angles are measured in degrees, and the radiation heights $h$ are in $\mathrm{km}$.

\begin{tabular}{ccrrrrrrrr}
\hline PSR & $P(s)$ & $\chi_{\mathrm{MP}}$ & $\beta_{\mathrm{MP}}$ & $\chi_{\mathrm{IP}}$ & $\beta_{\mathrm{IP}}$ & $h_{\mathrm{MP}}$ & $h_{\mathrm{IP}}$ & $\delta_{\mathrm{MP}}$ & $\delta_{\mathrm{IP}}$ \\
\hline $\mathrm{J} 0627+0705$ & 0.48 & 94.0 & -8.6 & 86.0 & -0.6 & 380 & 160 & 93 & 86 \\
$\mathrm{~J} 0905-5157$ & 0.35 & 90.4 & -3.6 & 89.5 & -2.8 & 970 & 40 & 90 & 88 \\
$\mathrm{~J} 0908-4913$ & 0.11 & 83.6 & 7.4 & 96.4 & -5.4 & 60 & 70 & 87 & 94 \\
$\mathrm{~J} 1549-4848$ & 0.29 & 87.3 & 2.3 & 92.7 & -3.2 & 130 & 110 & 88 & 92 \\
$\mathrm{~J} 1722-3712$ & 0.24 & 87.4 & -5.6 & 92.6 & -10.9 & 110 & 300 & 86 & 91 \\
$\mathrm{~J} 1739-2903$ & 0.32 & 94.6 & -2.2 & 85.4 & 7.1 & 240 & 200 & 94 & 87 \\
$\mathrm{~J} 1828-1101$ & 0.07 & 82.7 & 7.3 & 97.3 & -7.3 & 35 & 45 & 87 & 94 \\
$\mathrm{~J} 1935+2025$ & 0.08 & 93 & -13 & 87 & -7 & 200 & 30 & 90 & 83 \\
\hline
\end{tabular}

\section{Death line}

At the time of this writing, 3177 pulsars was already discovered [4]. This rather rich statistics clearly shows that the line limiting from below the population of pulsars on the $P \dot{P}$-diagram has a break at $P \approx 0.1 \mathrm{~s}$ (see Fig. 2 , left panel). Here we show that this break can be easily explained by the well-known feature of secondary plasma production.

Indeed, for pulsars with periods $P<0.1 \mathrm{~s}$, the radiation reaction becomes significant, so the energy of primary particles does not reach the values dictated by the potential drop (see, e.g., [5]). Recently, we carried out a detailed study [6] that took into account many still neglected phenomena, such as the role of high-energy $\gamma$-quanta in the synchrotron radiation spectrum, the possible wide distribution in moments of inertia, and, naturally, the effects of general relativity. In particular, the conclusion about the role of the radiation reaction for fast pulsars was confirmed.

A detailed analysis of this issue will be presented in a separate work. In the same article, we only show that even for standard values of the polar cap size and the parameters of a neutron star (and for dipole magnetic field!), taking into account the above effects significantly changes the position of the death line.

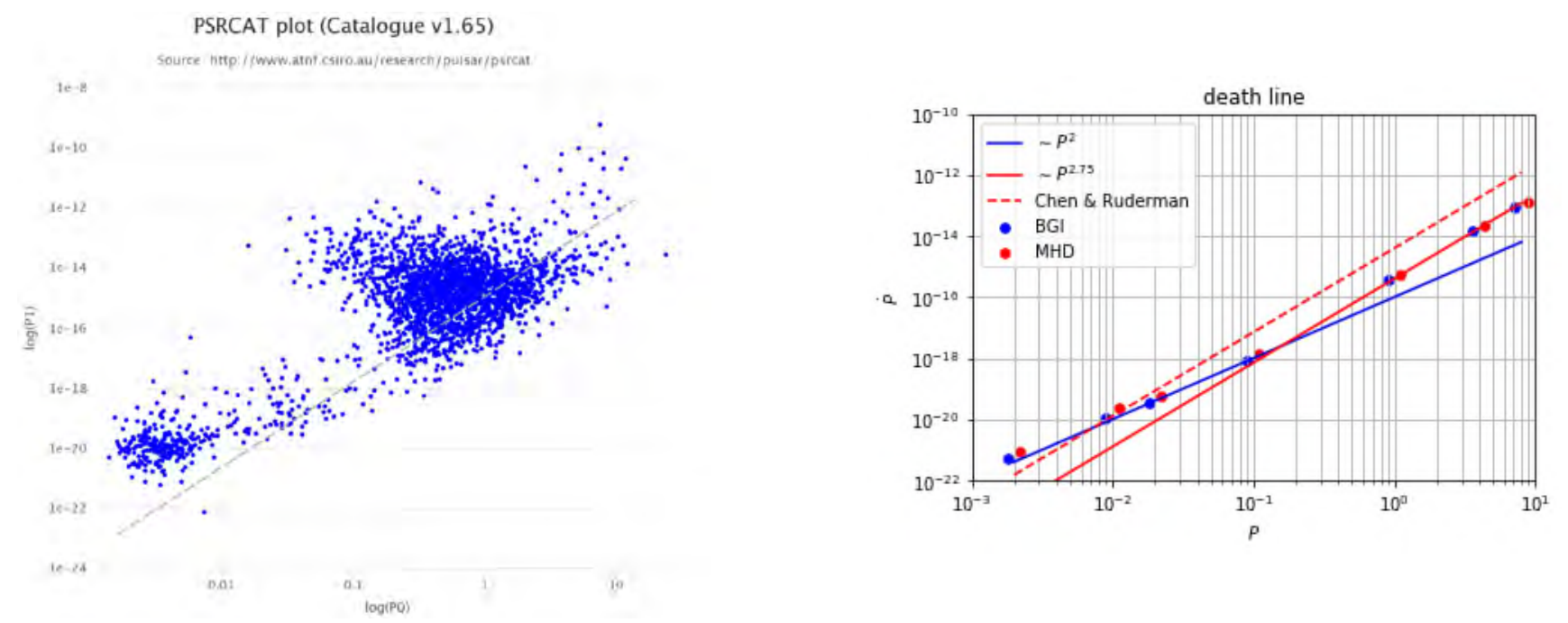

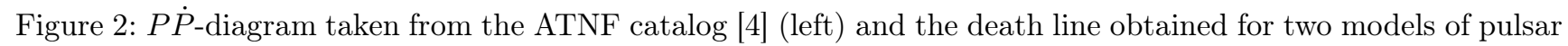
braking (right). The dashed lines correspond to the "classical" death line presented by [7].

The right panel in Fig. 2 shows the position of the death line obtained from the condition of the absence of particle production for two models of pulsar braking (see [6] for more detail). The dashed lines correspond to the classic death line [7], for which, by the way, unrealistic magneto-dipole losses were assumed. As we can see, regardless of the model of pulsar evolution, for $P>0.1 \mathrm{~s}$, the death line locates one order of magnitude lower than the classical one. On the other hand, at $P<0.1 \mathrm{~s}$, the slope becomes noticeably flatter (it corresponds to proportionality $\dot{P} \propto P^{2}$ ). Since our death line is still above many pulsars, a more detailed consideration is required, which we plan to carry out in the very near future.

I thank A. Philippov and A. Timokhin for the useful discussions. This work was supported by Russian Foundation for Basic Research, grant 20-02-00469. 


\section{References}

1. P. Goldreich and W. H. Julian, ApJ, 157, 869, 1969.

2. M. A. Ruderman and P. G. Sutherland, ApJ, 196, 51, 1975.

3. S. Johnston and M. Kramer, MNRAS, 490, 4565, 2019.

4. R. N. Manchester, G. B. Hobbs, A. Teoh, and M. Hobbs, The Astronomical Journal, 129, 1993, 2005, URL https://doi.org/10.1086/428488.

5. P. B. Jones, MNRAS, 506, L26, 2021.

6. V. S. Beskin and P. Litvinov, MNRAS, (in press), 2021.

7. K. Chen and M. Ruderman, ApJ, 402, 264, 1993. 


\title{
Building of zero geodesics in the wormhole field
}

\author{
M. Bugaev ${ }^{1}$, I. Novikov ${ }^{2,3,4}$, S. Repin ${ }^{5,2}$, A. Shelkovnikova ${ }^{5,6}$ \\ ${ }^{1}$ Moscow Institute of Physics and Technology, 9, Institutskiy per., town Dolgoprudnyi, Moscow region, 141700, Russia \\ ${ }^{2}$ Astro-Space Center of P.N. Lebedev Physical Institute, 84/32, Profsoyusnaya str., Moscow, 117997, Russia \\ ${ }^{3}$ The Niels Bohr International Academy, The Niels Bohr Institute, Blegdamsvej 17, DK-2100, Copenhagen, Denmark \\ ${ }^{4}$ National Research Center Kurchatov Institute, 1, Akademika Kurchatova pl., Moscow, 123182, Russia \\ ${ }^{5}$ Physics and Mathematics Colledge No. 2007, 9, Gorchakova str., Moscow, 117042, Russia \\ ${ }^{6}$ Moscow State Technical University, 5, 2-nd Baumanskaya str., Moscow, 105005, Russia
}

Keywords: black holes, wormholes, general relativity

DOI: $10.51194 /$ VAK2021.2022.1.1.100

The trajectories of light rays (zero geodesics) in the field of a zero-mass wormhole and in the field of a Schwarzschild black hole are constructed.

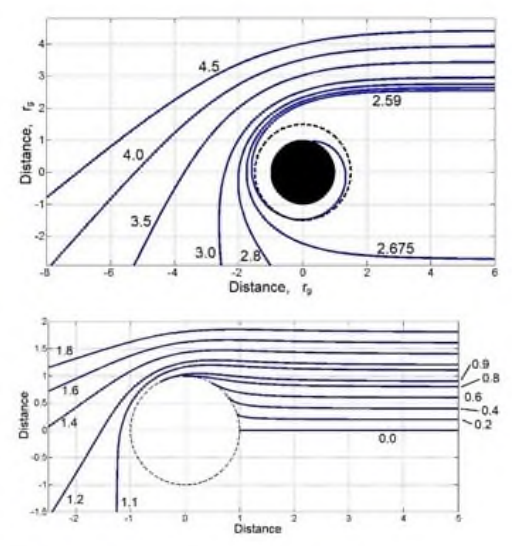

All constructed geodesics pass in outer space for both the Schwarzschild black hole and the wormhole. Geodesics are obtained by numerically solving the equations of motion of quanta (a system of ordinary differential equations).

$$
\begin{gathered}
\text { Schwarzschild metric: } \\
d s^{2}=\left(1-\frac{r_{g}}{r}\right) d t^{2}-\frac{d r^{2}}{1-\frac{r_{g}}{r}}-r^{2} d \Omega^{2} \\
\text { Morris - Thorne - Ellis - Bronnikov } \\
\text { metric: } \\
d s^{2}=d t^{2}-\frac{r^{2} d r^{2}}{r^{2}-q^{2}}-r^{2} d \Omega^{2}
\end{gathered}
$$

The coordinate $r$ is chosen so that the circumference is $2 \pi r$.
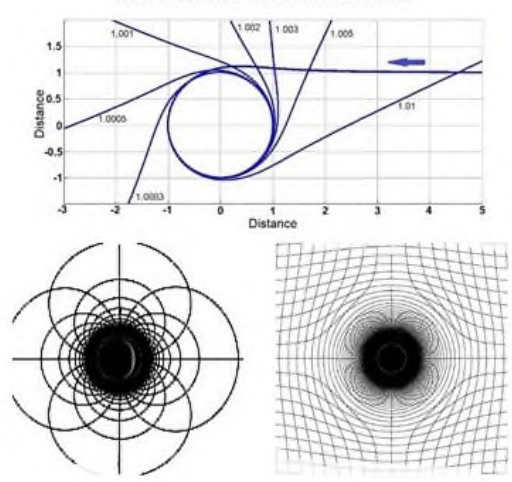

Computing a large number of zero geodesics is needed to generate the shadows of black holes and wormholes. The geodesic equations are derived from the Hamilton-Jacobi equation and solved numerically. There are geodesics that make many turns around the wormhole. The result of shading is shown below. The differences between the shadows of a black hole and a wormhole are clearly visible.
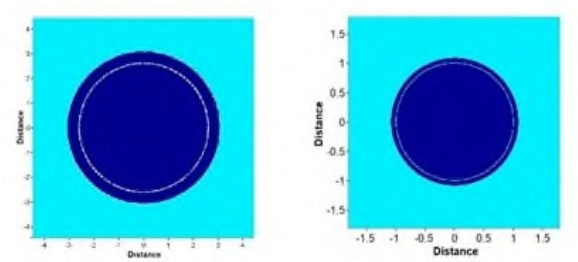

A uniform grid drawn on a screen behind a massive object as seen by a distant observer. More than $10^{6}$ geodesics were used to build the grid. 


\title{
Shadows of black holes and wormholes: similarities and differences
}

\author{
M. Bugaev ${ }^{1}$, I. Novikov ${ }^{2,3,4}$, S. Repin ${ }^{5,2}$, A. Shelkovnikova ${ }^{5,6}$ \\ ${ }^{1}$ Moscow Institute of Physics and Technology,9, Institutskiy per., town Dolgoprudnyi, Moscow region, 141700, Russia, \\ ${ }^{2}$ Astro-Space Center of P.N. Lebedev Physical Institute, 84/32, Profsoyusnaya str., Moscow, 117997, Russia, \\ ${ }^{3}$ The Niels Bohr International Academy, The Niels Bohr Institute, Blegdamsvej 17, DK-2100, Copenhagen, Denmark, \\ ${ }^{4}$ National Research Center Kurchatov Institute, 1, Akademika Kurchatova pl., Moscow, 123182, Russia, \\ ${ }^{5}$ Physics and Mathematics Colledge No. 2007, 9, Gorchakova str., Moscow, 117042, Russia, \\ ${ }^{6}$ Moscow State Technical University, 5, 2-nd Baumanskaya str., Moscow, 105005, Russia
}

The problem of bending and scattering of light rays moving in the outer space of a wormhole with zero gravitational mass is considered. The process of formation of a wormhole shadow against the background of a distant Lambert screen is investigated.

Keywords: black holes, wormholes, general relativity

DOI: $10.51194 /$ VAK2021.2022.1.1.101

\section{Introduction}

Recently, issues related to the physics of wormholes (WH) have attracted a special attention of specialists. This attention has increased after it was hypothesized that some galactic nuclei may not be the supermassive black holes (BH), but entrances to wormholes [1, 2]. There is a need to test this hypothesis [3]. We consider the simplest zero-mass Ellis-Bronnikov-MorrisThorne wormhole model $[4,5,6,7]$ and study the distortion of the motion of light rays passing in the vicinity of the entrance to a wormhole. Then we construct the shadow of a wormhole illuminated by the simplest model of a flat Lambert light screen located behind the wormhole and compare the result with the shadow of a Schwarzschild black hole illuminated by the same screen.

\section{Comparison of wormhole and black hole shadows}

Note that we consider only the rays that move outside the WH, assuming that the interior of the WH is impenetrable for light. The equations of zero geodesics in the wormhole metric can be obtained by writing down the Hamilton-Jacobi equation. After separating the variables, we obtain a system of ordinary differential equations that describes the trajectories of quanta. To construct the shadow of a wormhole, it is necessary to compute several million trajectories [8].
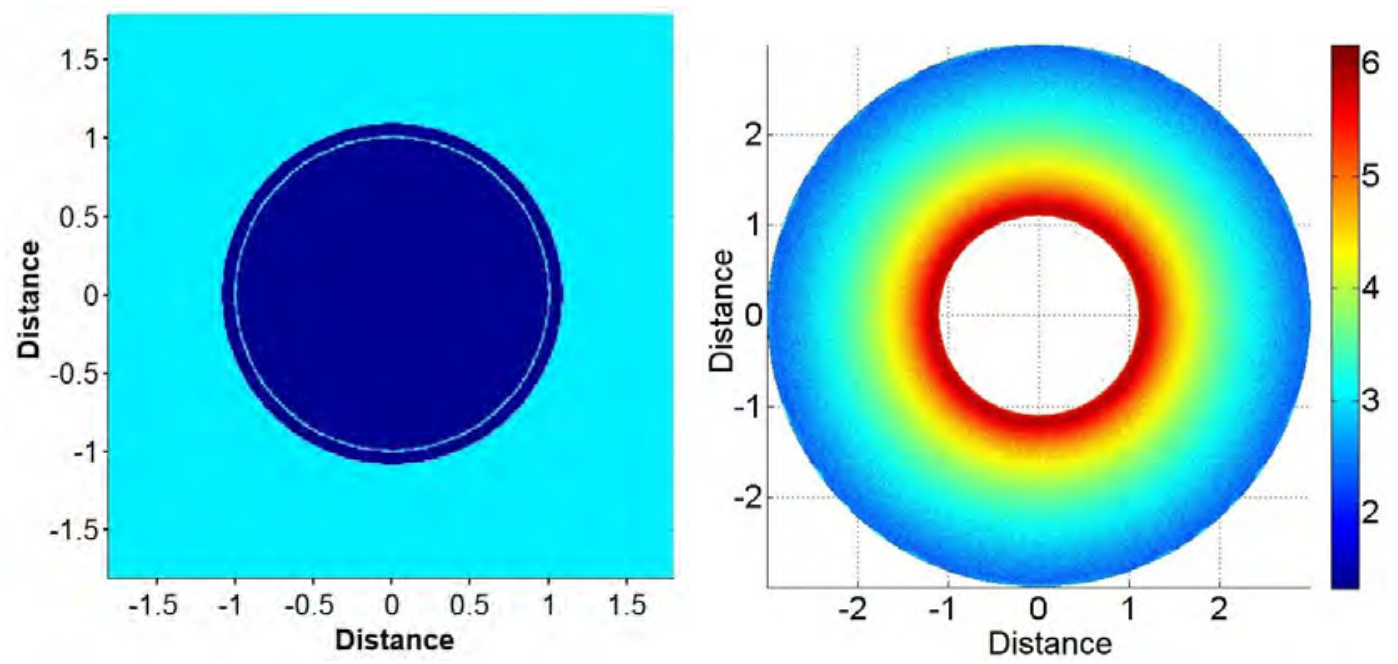

Figure 1: The shape of the WH shadow and the distribution of the radiation intensity near its edge. The radial coordinate is expressed in units of the radius of the WH throat.

The result of modeling the shadow of a wormhole is shown in Fig. 1. The left panel shows the shape of the silhouette of a wormhole against the background of a distant Lambert screen, perpendicular to the line of sight of the observer. The right panel shows the distribution of radiation intensity near the boundaries of the shadow. A thin photonic ring inside the silhouette of a shadow is formed by the quanta that have made more than one revolution around the wormhole, while remaining in our space-time.

For comparison, Fig. 2 shows the shadow of the Schwarzschild black hole against the background of the same flat screen and the distribution of the radiation intensity near its boundaries. Figures 1 and 2 clearly show the difference in the location of the photon ring for the shadows of a wormhole and a black hole.

The distribution of radiation intensity near the boundaries of the shadow is also different for a wormhole and a black hole. Figure 3 shows a graph of the intensity distribution in the equatorial section of both shadows. When approaching the 

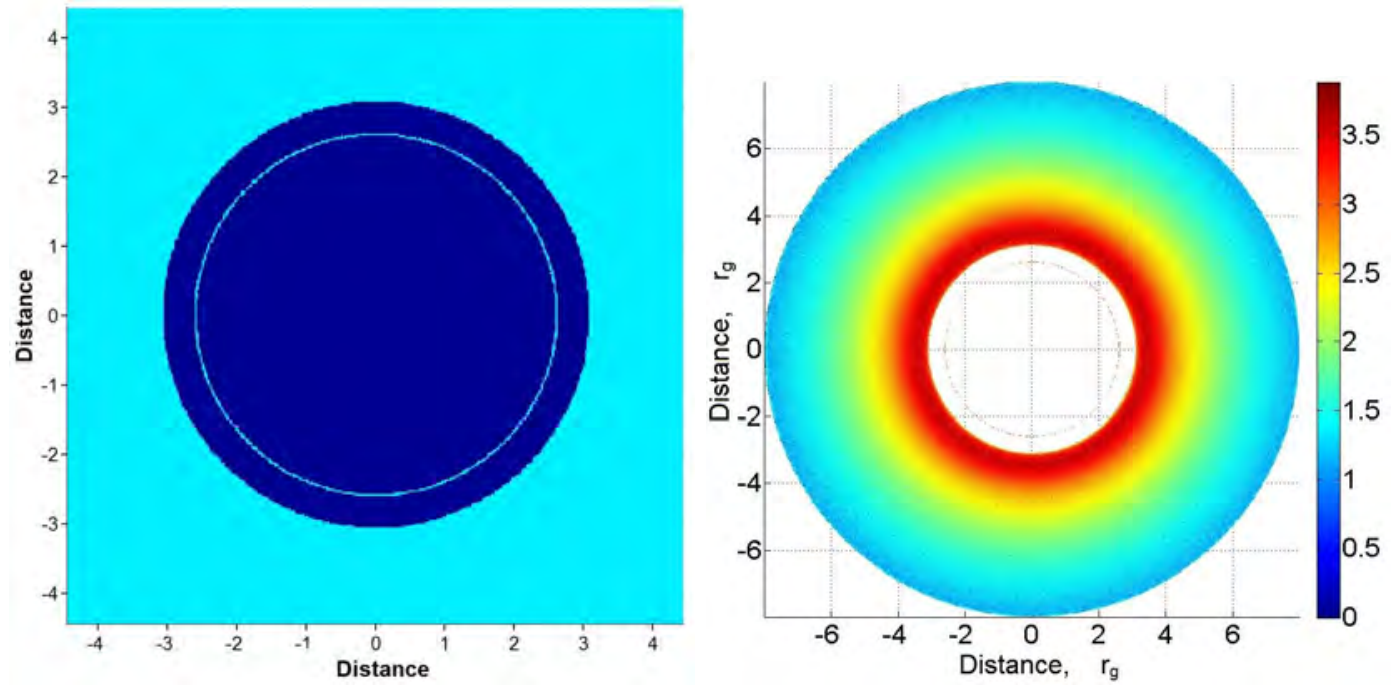

Figure 2: The shape of the shadow of the Schwarzschild black hole and the distribution of the radiation intensity near its edge. The radial coordinate is expressed in units of the gravitational radius $r_{g}=2 G m / c^{2}$.
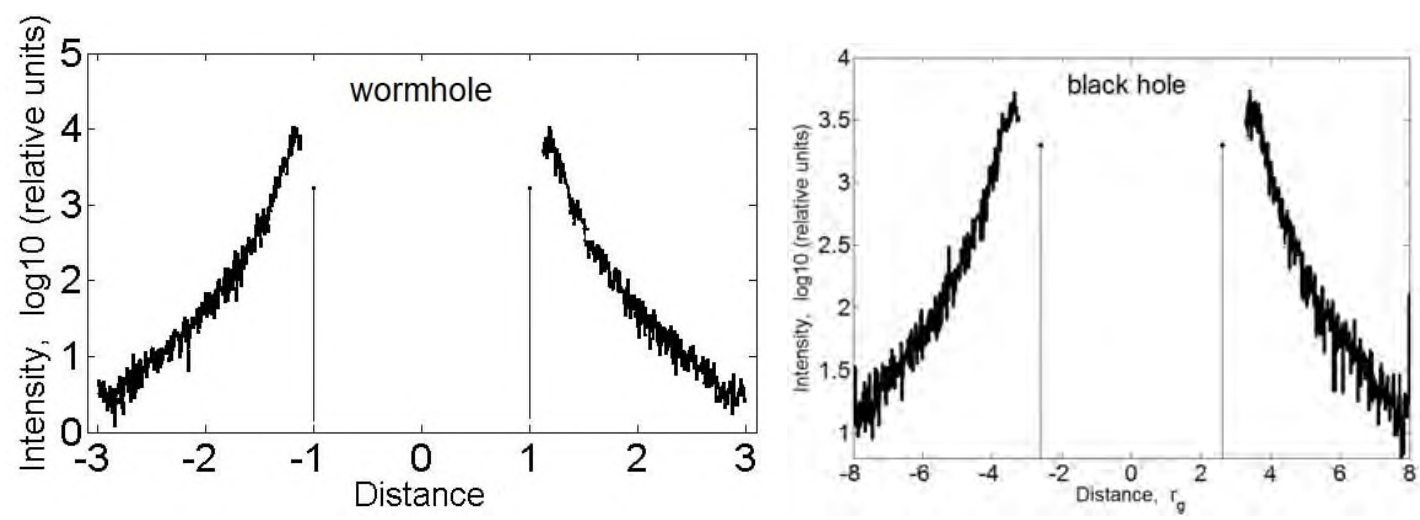

Figure 3: Distribution of radiation intensity in the equatorial section of the shadow.

border of the shadow, the intensity increases more sharply for a wormhole. This difference is directly related to the different structure of space-time near a wormhole and a black hole.

\section{Notes and comments}

The incresing of the intensity near the edge of the shadow as well as the relative position of the photon ring, makes it possible to distinguish a wormhole from a black hole.

\section{References}

1. N. S. Kardashev, I. D. Novikov, and A. A. Shatskii, Astronomy Reports, 50, 601, 2006.

2. K. K. Nandi, Y.-Z. Zhang, and A. V. Zakharov, Phys. Rev. D, 74, 024020, 2006.

3. I. D. Novikov, S. F. Likhachev, Y. A. Shchekinov, A. S. Andrianov, et al., Physics Uspekhi, 64, 386, 2021.

4. H. G. Ellis, Journal of Mathematical Physics, 14, 104, 1973.

5. K. Bronnikov, Acta Phys Pol, 84, 251, 1973.

6. M. S. Morris and K. S. Thorne, American Journal of Physics, 56, 395, 1988.

7. M. S. Morris, K. S. Thorne, and U. Yurtsever, Phys. Rev. Lett., 61, 1446, 1988.

8. M. A. Bugaev, I. D. Novikov, S. V. Repin, and A. A. Shelkovnikova, arXiv e-prints, arXiv:2106.03256, 2021. 


\title{
Rotation measure and vertical structure of accretion disks
}

\author{
M.A. Buldakov ${ }^{1}$, E.O. Vasiliev ${ }^{1,2}$, A.S. Andrianov ${ }^{1}$ \\ buldakov@phystech.edu \\ ${ }^{1}$ Lebedev Physical Institute, 84/32 Profsoyuznaya st., 117997, Moscow, Russia, \\ ${ }^{2}$ Southern Federal University, 105/42 Bolshaya Sadovaya st., 344006, Rostov on Don, Russia
}

Rotation measure (RM) can give information about properties of magnetohydrodynamic (MHD) turbulence in accretion disks. RM measured in observations is averaged by large volumes and it may have complicated alternating-sign structure. We study possible relations between RM and properties of turbulence in disks. We numerically simulate development of MRI turbulence in a local fragment of disk. Evolution and spatial distribution of $\alpha$-parameter, vertical mass flux and RM are studied for two initial magnetic field configurations and values of initial midplane plasma $\beta_{0}$ (ratio of gas pressure to magnetic pressure) in range $10^{\circ}-10^{3}$. The pairwise correlations between $\alpha$ parameter, vertical mass flux and RM are calculated. The values of correlation levels are in range 0.50-0.95. It shows that oscillations of RM roughly correspond to oscillations of $\alpha$ and vertical mass flux. The period of these oscillations is approximately equal to the one of dynamo cycle.

Keywords: accretion, accretion disks, $M H D$

DOI: 10.51194 /VAK2021.2022.1.1.102

\section{Introduction}

Rotation measure $(\mathrm{RM})$ can characterize properties and spatial distributions of magnetic fields and magnetohydrodynamic (MHD) turbulence which arise in accretion disks of various types $[1,2,3]$. The structure of magnetic fields have strong influence on evolution of turbulence and disk outflows [4,5]. Since RM is an observable quantity, it is of interest to find possible relations between RM and turbulent and outflow properties of the disk. In this paper we study spatial distributions and evolution of $\alpha$-parameter, vertical mass outflow and RM and calculate correlations between them.

\section{Numerical model and results}

The simulations of accretion disk are conducted by using stratified shearing box model of Pluto code [6]. A small local disk part corotating with global Keplerian accretion disk is considered in this model. The size of shearing box is $H \times 3.14 \cdot H \times 8 \cdot H$, where $\mathrm{H}$ is a scale height of the disk. We use an isothermal equation of state. Our simulation time is $160 \cdot T$, where $\mathrm{T}$ is a disk orbital period. We consider two initial magnetic field configurations. The first magnetic field configuration is a vertical magnetic field with zero net magnetic flux, $B_{z}=B_{z 0} \cdot \sin \left(2 \pi x / L_{x}\right)$, where $L_{x}$ is a box size in the $x$ direction. The second magnetic field configuration is a constant azimuthal magnetic field, $B_{y}=B_{y 0}$. The range of $\beta_{0}$ (initial midplane plasma $\beta$ ) is $10^{0}-10^{3}$ for the models with vertical magnetic field and $10^{1}-10^{3}$ for the models with azimuthal magnetic field.

One of the main quantities that describe turbulence in the disk is an $\alpha$-parameter, $\alpha=\frac{\int\left(\bar{T}_{M}(z)+\bar{T}_{R}(z)\right) d z}{\int \bar{\rho}(z) c_{s}{ }^{2} d z}$, where $\bar{T}_{M}=-\left(B_{x} \cdot B_{y}\right) / 4 \pi$ is the Maxwell stress, $\bar{T}_{R}=\rho \cdot v_{x} \cdot \delta v_{y}$ is the Reynolds stress, where $\rho$ is the gas density, $B$ is the magnetic field, $c_{s}$ is the speed of sound and overbar denotes horizontal averaging [7]. In this paper we present the results of calculations for the model with vertical magnetic field and $\beta_{0}=10$. The evolution of $\alpha$ for this model is shown in Figure 1 .

The properties of the turbulence are qualitatively similar for all models. In the initial phase of calculations magnetorotational instability (MRI) is growing [8] and $\alpha$-parameter reaches its maximum. Then a quasi-steady regime is established with $\alpha$ oscillating near its mean value $(\approx 0.02)$. Duration of the initial MRI growth is different in models with different magnetic field configurations: it is larger in case of azimuthal magnetic field $(\approx 25 \cdot T)$ than in case of vertical one $(\approx 10 \cdot T)$.

Accretion disks exhibit processes of mass loss by accretion and disk winds and they are connected with disk turbulence $[9,5]$. We use zero-gradient outflow boundary conditions for velocities in $z$ direction which permit fluxes of mass of the gas through vertical boundaries. Mass flux in our models is defined as $F_{b}=\left|\int_{x_{l}, y_{l}} \rho v_{z}\right|+\int_{x_{u}, y_{u}} \rho v_{z}$, where $v_{z}$ is a vertical component of velocity and summation is performed over the grid cells at lower and upper (X, Y) planes of computational domain. The evolution of $F_{b}$ for one of the models is shown in Figure 1. Mass flux shows oscillatory behaviour with large amplitudes of the oscillations in a quasi-steady regime for all models. About $30 \%$ of the initial mass of gas is lost during simulation time.

Vertical structure of the main characteristics of disk (density, magnetic field, magnetic energy) is similar in all models. Vertical structure of density is approximately constant in time with nearly Gaussian vertical profiles. The evolution of vertical profiles of magnetic field and magnetic energy correspond to dynamo process and "butterfly diagrams" [9, 10] can be seen in all models. Magnetic energy outbursts seen in butterfly diagrams occurs approximately at the same time as the local maxima of $\alpha$ and $F_{b}$.

We calculate RM along vertical line of sights in our models, i.e. $R M \sim \int_{z} B_{z} \cdot \rho d z$. The evolution of horizontally averaged RM for one of the models is shown in Figure 1. $\overline{R M}$ is oscillating around zero with quasi-periodic minima and maxima. The period of these quasi-periodic oscillations is approximately equal to the one of dynamo cycle $(\approx 10 \cdot T)$. The local maxima of $|\overline{R M}|$ roughly correspond to the maxima of $\alpha$ and $F_{b}$ (they are reached approximately at the same time). It may indicate that these quantities are correlated. We measure pairwise correlation levels (maxima of cross-correlation functions) between $\alpha, F_{b}$ and $|\overline{R M}|$ for our models. The data are smoothed for correlation analysis by means of moving average method. The results are shown in Table 1 ("Bz" and "By" in names of models correspond to the vertical and azimuthal magnetic field configurations, numbers in names of models correspond to $\beta_{0}$ values). The values of correlation levels in all models are in the interval from 0.50 to 0.95 . There is no dependence of correlation levels on magnetic field configuration and $\beta_{0}$. More detailed statistical analysis of correlations will be published elsewhere. 


\section{Summary and conclusions}

We have studied evolution and spatial distributions of RM, $\alpha$-parameter and the vertical mass flux for shearing box models with two magnetic field configurations and several $\beta_{0}$ values in wide range. The evolution of these quantities is characterized by quasi-periodic oscillations with a period about $10 \cdot T$. We found that the pairwise correlation levels between $\alpha, F_{b}$ and $|\overline{R M}|$ are in range 0.50-0.95 and the correlation levels are model-independent. Such correlations indicate that minima and maxima of $|\overline{R M}|$ oscillations roughly correspond to those of $\alpha$ and $F_{b}$. Therefore, measurements of RM can give information about structure and periods of $\alpha$ and $F_{b}$ oscillations.

It is not clear whether the period of quasi-periodic oscillations of RM is physically related to the dynamo cycle. The period of dynamo cycle is fixed in models with zero net magnetic flux, but this period depends on magnetic field strength in models with net vertical magnetic flux [9]. If there is a relation between periods of RM and dynamo process, it is possible to estimate a period of dynamo cycle by RM measurements. This question will be investigated in future paper.
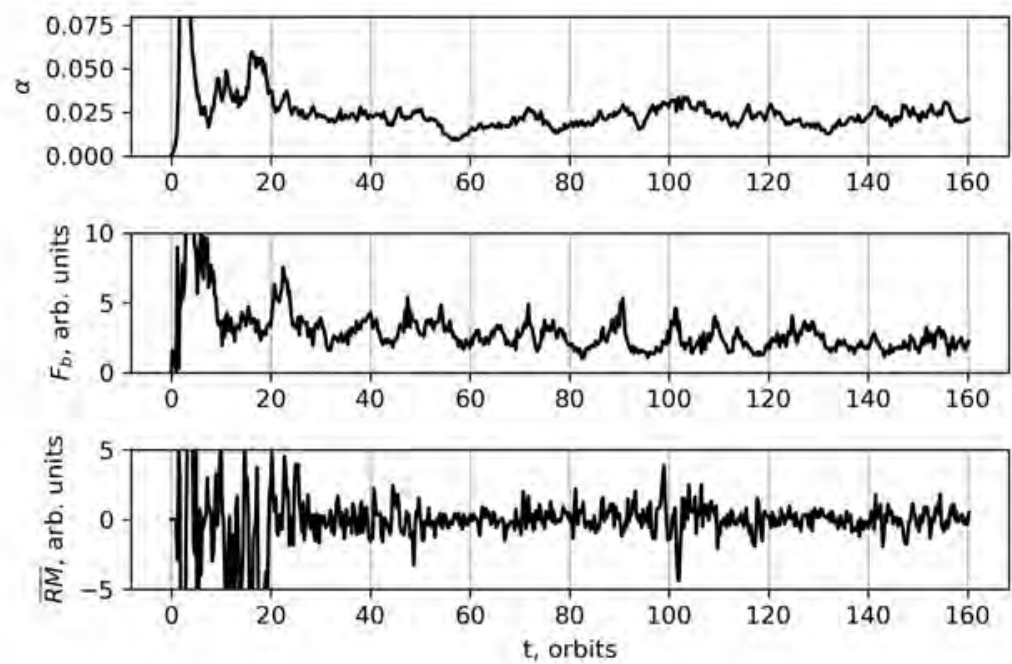

Figure 1: Evolution of $\alpha$ (top), $F_{b}$ (middle) and $\overline{R M}$ (bottom) for the model with the vertical magnetic field and $\beta_{0}=10$.

Table 1: Results of correlational analysis.

\begin{tabular}{|l|l|l|l|}
\hline \multirow{2}{*}{ Model } & \multicolumn{3}{|l|}{ Maxima of cross-correlation functions between: } \\
\cline { 2 - 4 } & $|\overline{R M}|$ and $\alpha$ & $|R M|$ and $F_{v}$ & $\alpha$ and $F_{v}$ \\
\hline SB1_Bz_10 & 0.74 & 0.73 & 0.58 \\
\hline SB1_Bz_10 & 0.83 & 0.56 & 0.44 \\
\hline SB1_Bz_10 & 0.96 & 0.88 & 0.79 \\
\hline SB1_Bz_10 & 0.90 & 0.85 & 0.73 \\
\hline SB1_By_10 & 0.84 & 0.71 & 0.78 \\
\hline SB1_By_10 & 0.90 & 0.60 & 0.58 \\
\hline SB1_By_10 & 0.85 & 0.75 & 0.53 \\
\hline
\end{tabular}

\section{References}

1. P. C. Tribble, $M N R A S, \mathbf{2 5 0}, 726,1991$.

2. J. A. Eilek, $A J, \mathbf{9 8}, 244,1989$.

3. J. A. Eilek, $A J, \mathbf{9 8}, 256,1989$.

4. B. Mishra, M. C. Begelman, P. J. Armitage, and J. B. Simon, MNRAS, 492, 1855, 2020.

5. J. Jacquemin-Ide, G. Lesur, and J. Ferreira, A $\& A$, 647, A192, 2021.

6. A. Mignone, G. Bodo, S. Massaglia, T. Matsakos, O. Tesileanu, C. Zanni, and A. Ferrari, ApJS, 170, $228,2007$.

7. N. I. Shakura and R. A. Sunyaev, $A \& A$, 500, 33, 1973.

8. S. A. Balbus and J. F. Hawley, ApJ, 376, 214, 1991.

9. X.-N. Bai and J. M. Stone, ApJ, 767, 30, 2013.

10. P. Dhang and P. Sharma, MNRAS, 482, 848, 2019. 


\section{Peculiar objects in the probable pulsar birthplaces - stellar-mass black hole candidates}

L. Chmyreva, G. Beskin

lisa.chmyreva@mail.ru

Special Astrophysical Observatory of the RAS, Nizhnij Arkhyz, Russia 369167

\section{DOI: $10.51194 /$ VAK2021.2022.1.1.103}

Evolutionary predictions for disrupted binaries with neutron stars (NS) and stellar-mass black holes (BH) result in a higher probability of finding the latter in regions of possible NS birth (binary disruption). Tracing the trajectories of NS (pulsars) back in time according to their characteristic ages [1], we located their probable birth locations, where a search for $\mathrm{BH}$ candidates was carried out. We selected sources with properties similar to those expected for isolated BH undergoing spherical accretion, including a non-thermal spectrum with no lines, $16-25^{m}$ magnitudes, variations, proper motions, and rapid flares [2, 3]. Eight candidates have shown luminosities comparable with those expected for theoretical BHs in these locations. A two-dimensional probability space, derived from the distributions for the velocities and masses of BHs that originated in disrupted binaries [4] was used to evaluate the likelihood that these candidates really are black holes. The probability that these objects are $\mathrm{BH}$ ranged from 1.5 to $5.2 \%$, and the total probability that at least one candidate is a $\mathrm{BH}$ is between 11 and $29 \%$. See our upcoming paper for full details and list of candidates.
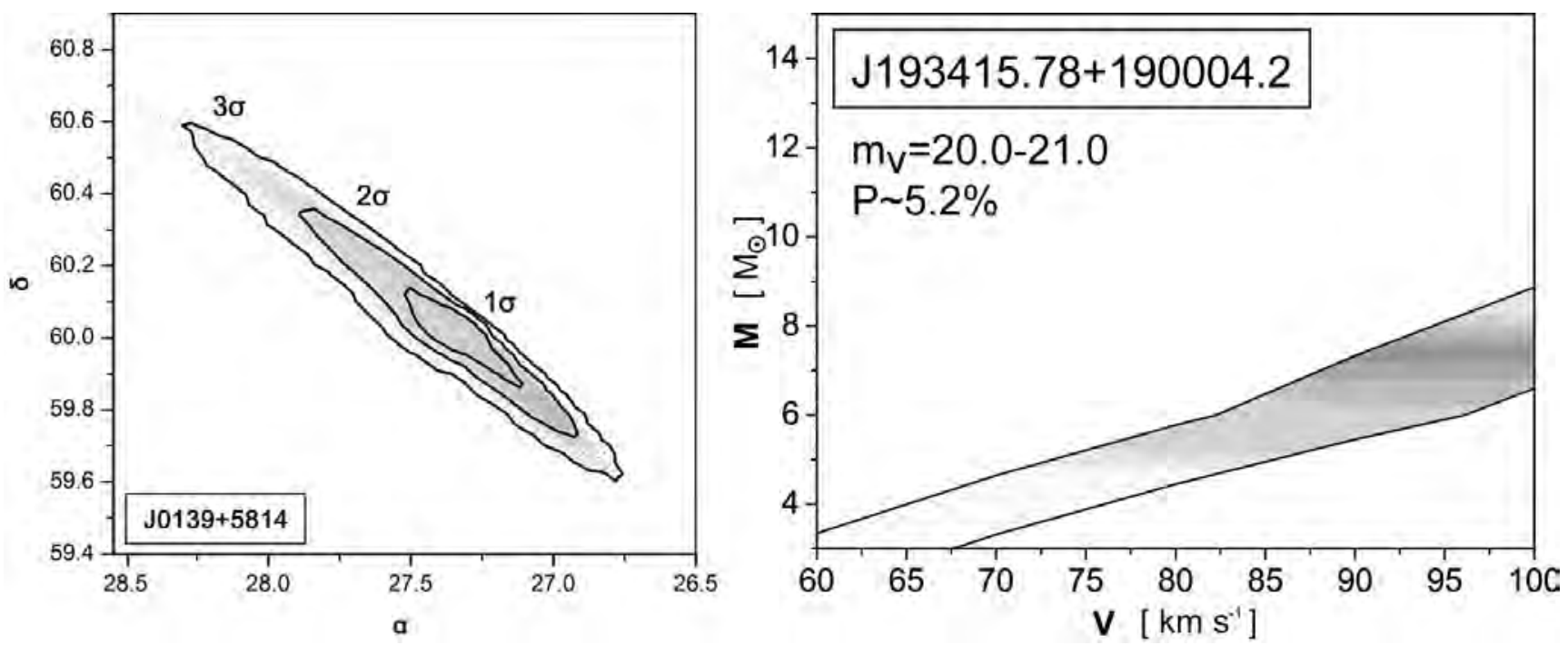

Figure 1: Left: birth region for one of the pulsars, PSR J0139+5814. Right: sample BH probability for one of the candidates. The region between the two curves shows the ranges of $M$ and $V$ that result in a luminosity similar to that expected for a BH. Greater likelihood regions are shown by darker shades of grey.

\section{References}

1. E. G. Chmyreva, G. M. Beskin, and A. V. Biryukov, Astronomy Letters, 36, 116, 2010.

2. V. F. Shvartsman, Sov. Astron., 15, 377, 1971.

3. G. M. Beskin and S. V. Karpov, A\&A, 440, 223, 2005.

4. G. Wiktorowicz, Ł. Wyrzykowski, M. Chruslinska, J. Klencki, K. A. Rybicki, and K. Belczynski, ApJ, 885, $1,2019$. 


\section{Mergers of primordial black holes in compact clusters}

Yu. Eroshenko

eroshenko@inr.ac.ru

Institute for Nuclear Research RAS, 117312, Moscow, Russia

It is shown that $\sim 10 \%$ of the total dark matter mass can be transformed into gravitational radiation by primordial black hole $(\mathrm{PBH})$ mergers inside the compact clusters. If the mass transformation occurred after recombination, but at redshifts $z \geq 10$, the generated gravitational radiation do not contribute to the current density of the Universe. This effect increases the rate of cosmological expansion in the era of recombination compared to models without the mass transfer. Accordingly, the Hubble constant, obtained from measurements in the early Universe, changes, which can provide a solution to the "Hubble tension" problem.

Keywords: primordial black holes, cosmology, Hubble tension

DOI: $10.51194 /$ VAK2021.2022.1.1.104

\section{Introduction}

One of the unsolved mysteries in cosmology is the "Hubble tension" problem [1, 2]. It lies in the fact that the current Hubble constant, determined by phenomena in the early Universe, turned out to be about $5 \%$ larger than the Hubble constant measured directly in the nowadays Universe. Phenomena in the early Universe are studied by observing fluctuations of relic radiation, whereas measurements in the contemporary Universe are based on constructing a cosmic ruler on the basics of Cepheids, Ia supernovae and other objects.

Many solutions to the "Hubble tension" problem have been proposed. In the work [3] the influence of intergalactic dust on the spectrum of relic radiation was considered, therefore the work [3] is based only on known physical phenomena. Other approaches introduce new physical entities. For example, variants with decaying dark matter or with a variable dark energy were proposed. See [4] for the review of models. A frequently used approach, which we also apply in this work, is the assumption that the value of Hubble constant was larger in the era of recombination. Since the position of the first acoustic peak is fixed by the observational data, this leads to the increase of the nowdays Hubble constant, obtained from the first acoustic peak position.

In this paper, we assume that the dark matter in the Universe consists of PBHs with masses $m \sim 10^{20}-10^{24} \mathrm{~g}$, and these PBHs are located in dense clusters. We show that, under certain cluster parameters, the collisions and mergers of PBHs lead to the transformation of $\sim 10 \%$ of the total dark matter mass into gravitational waves in the time interval from recombination epoch $(z \sim 1100)$ to the redshifts $z \sim 10$. Since in the era of recombination, the density of matter in the comoving volume was $10 \%$ higher, the Hubble constant was $5 \%$ higher (compared to models without the mass transformation into radiation). This is just necessary to solve the "Hubble tension" problem. The existence of the clusters with the required parameters is our assumption. A possible mechanism for the formation of PBHs and their clusters was proposed in $[5,6]$.

\section{Clusters of primordial black holes}

Consider a spherical cluster with mass $M$ and radius $R$, consisting of $N=M / m$ PBHs. The velocity dispersion in this cluster is $v \sim(G M / R)^{1 / 2}$. Denote $x=v^{2} / c^{2}$. In [7], assuming that the masses $m$ of all the objects (PBHs in our case) are the same, the equations of the cluster dynamical evolution were obtained,

$$
\begin{gathered}
\frac{1}{x} \frac{d x}{d t}=\frac{\left(\alpha_{2}-\alpha_{1}\right)}{t_{r}}+\frac{7}{5 t_{\mathrm{cap}}} \\
\frac{1}{M} \frac{d M}{d t}=-\frac{\alpha_{2}}{t_{r}}, \\
\frac{1}{m} \frac{d m}{d t}=\frac{1}{t_{\mathrm{cap}}}
\end{gathered}
$$

where $\alpha_{1}=8.72 \times 10^{-4}, \alpha_{2}=1.24 \times 10^{-3}$, the rate of PBH mergers $t_{\text {cap }}^{-1}=\sigma_{\text {cap }} v n / \sqrt{2}, n$ is the PBH number density, and the $\mathrm{PBH}$ merger cross-section is

$$
\sigma_{\text {cap }} \approx \frac{6 \pi G^{2} m^{2}}{c^{10 / 7} v^{18 / 7}}
$$

The time of two-body dynamic relaxation is

$$
t_{\mathrm{r}}=\left(\frac{2}{3}\right)^{1 / 2} \frac{v^{3}}{4 \pi G^{2} m^{2} n \Lambda}
$$

where $\Lambda=\ln (0.4 N) \simeq 7$. Let us denote

$$
x_{\mathrm{dis}} \equiv\left[\frac{10 \Lambda\left(\alpha_{2}-\alpha_{1}\right)}{(7 \sqrt{3})}\right]^{7 / 5} \simeq 1.8 \times 10^{-4},
$$

and introduce for convenience the notation $y \equiv\left(x / x_{\mathrm{dis}}\right)^{5 / 7}$. 
The equations (1-3) can be solved numerically by using the dimensionless variable $\tau=t c^{3} /\left(G m_{i} N_{i}^{2}\right)$, where index "i" denotes the initial values at the moment of the cluster birth. The solution shows that the cluster evolves slowly at first, and at the last stage its evolution accelerates sharply. Part of the PBHs flies out of the cluster, and the remaining cluster is compressed. For the parameters under consideration, the cluster does not collapse at the end of its evolution, but it completely disperses. A good approximation for the cluster lifetime is the following analytical estimate $\tau_{b} \sim 2.5 \times 10^{6} y_{i}^{-31 / 10}$.

The rate of $\mathrm{PBH}$ mergers in the cluster

$$
\frac{d N_{c}}{d t}=\frac{N}{t_{\text {cap }}} .
$$

The merging mass is $d M_{c}=m d N_{c}$, and the total merged mass of the PBHs in all merger cycles is

$$
\frac{M_{c}}{M_{i}}=\frac{y_{i}^{\varkappa}}{\left(1+y_{i}\right)^{\varkappa}} \int_{y_{i}}^{y} \frac{(1+y)^{\varkappa-1} d y}{y^{\varkappa}} .
$$

Its maximum value could be $M_{c} \sim M_{i} \log _{2} N_{i}$, but due to the flying of PBHs from the clusters, it turns out to be $M_{c} \sim M_{i}$.

\section{Results}

Consider for example clusters with $y_{i}=5$. These clusters were weakly relativistic already at the time of their birth, because they had $v / c \simeq 0.04$. By requiring that the cluster lifetime $t_{b}=\tau_{b} G m_{i} N_{i}^{2} / c^{3}$ is enclosed between the recombination epoch and the moment $t_{10}$ corresponding to the redshift $z=10, t_{r}<t_{b}<t_{10}$, we obtain the following condition for the initial cluster masses

$$
18\left(\frac{m_{i}}{10^{-12} M_{\odot}}\right)^{1 / 2} \leq \frac{M_{i}}{M_{\odot}} \leq 560\left(\frac{m_{i}}{10^{-12} M_{\odot}}\right)^{1 / 2} .
$$

According to (8), one gets that during the lifetime of the cluster, the total merged mass is $M_{c} \sim M_{i}$. Approximately $\sim 10 \%$ of this mass was transformed into gravitational waves during the mergers of the PBHs. From the perturbed Friedman equation

$$
(H+\delta H)^{2}=\frac{8 \pi G}{3}[\bar{\rho}(t)+e(t)]
$$

one obtains the relative change $\delta H / H \simeq(1 / 2) e(t) / \bar{\rho}(t)$, where $e(t)$ is the energy density of gravitational waves. Consequently, the transformation of $\sim 10 \%$ of the mass into gravitational waves leads to the Hubble constant shift by $5 \%$ (compared to models without the transformation). Moreover, the gravitational waves did not affect the Hubble constant in the modern era. This can explain the "Hubble tension" problem.

Note also that if the PBH clusters had an initial distribution over density, and if $\sim 10^{-4}-10^{-3}$ part of the clusters, the densest clusters, collapsed into black holes with masses (9), then the mergers of these black holes in pairs could explain also the LIGO/Virgo events.

\section{References}

1. Z. Berezhiani, A. D. Dolgov, and I. I. Tkachev, Phys. Rev. D, 92, 061303, 2015.

2. E. Di Valentino, L. A. Anchordoqui, Ö. Akarsu, and Y. e. a. Ali-Haimoud, Astroparticle Physics, $131,102605,2021$.

3. V. N. Yershov, A. A. Raikov, N. Y. Lovyagin, N. P. M. Kuin, and E. A. Popova, MNRAS, 492, 5052, 2020.

4. E. Di Valentino, O. Mena, S. Pan, L. Visinelli, et al., Classical and Quantum Gravity, 38, 153001, 2021.

5. S. G. Rubin, A. S. Sakharov, and M. Y. Khlopov, Soviet Journal of Experimental and Theoretical Physics, 92, $921,2001$.

6. M. Y. Khlopov, S. G. Rubin, and A. S. Sakharov, arXiv e-prints, astro-ph/0202505, 2002.

7. G. D. Quinlan and S. L. Shapiro, ApJ, 321, 199, 1987. 


\title{
Study of the transient X-ray pulsar XTE J1946 + 274 observed with NuSTAR
}

\author{
A.S. Gorban ${ }^{1,2}$, S.V. Molkov ${ }^{1}$, S.S. Tsygankov ${ }^{3,1}$, A.A. Lutovinov ${ }^{1,2}$ \\ gorban@iki.rssi.ru \\ ${ }^{1}$ Space Research Institute, Russian Academy of Sciences, Profsoyuznaya ul. 84/32, Moscow, 117997 Russia \\ ${ }^{2}$ Higher School of Economics, National Research University, Myasnitskaya ul. 20, Moscow, 101100 Russia \\ ${ }^{3}$ Tuorla Observatory, University of Turku, Turku, Finland
}

DOI: 10.51194 /VAK2021.2022.1.1.105

We present the results of our spectral and timing analysis of the emission from the transient X-ray pulsar XTE J1946 +274 based on observations by the NuSTAR in the broad energy range $0.3-79 \mathrm{keV}$ and Swift/XRT in the range $0.2-10 \mathrm{keV}$.

The power law model with an exponential cutoff at high energies TBABS * (GAUS + BB + POWERLAW * HIGHCUT) was applied to fit the averaged spectrum with features. The cyclotron feature at the energies of $\sim 38 \mathrm{keV}$ has confirmed to compare with [1] results and was described by the absorption component in the form of the GABS model. The energy of the cyclotron line was used to estimate the magnetic field strength on the surface of the neutron star, B $\sim 3.2 \cdot 10^{12} G$.

Phase-resolved spectroscopy has also allowed the variation in spectral parameters with neutron star rotation phase, whose period is $\sim 15.755 \mathrm{~s}$, to be studied. It was found that the iron emission line is present in all phases. The cyclotron absorption line is recorded only in five phase bins and line changes significantly (from 34 to $39 \mathrm{keV}$ ). The observed behavior of the cyclotron line parameters can be interpreted in terms of the model of the reflection of emission from a small accretion column [2]. The equivalent iron line width also change significantly with phase of the pulse. The time delay between the equivalent width and pulse profile can be explained by the reflection of neutron star emission from the outer regions of the accretion disk [3].

\section{References}

1. R. Doroshenko, A. Santangelo, V. Doroshenko, and S. Piraino, Astronomy \& Astrophysics, 600, A52, 2017.

2. J. Poutanen, A. A. Mushtukov, V. F. Suleimanov, S. S. Tsygankov, D. I. Nagirner, V. Doroshenko, and A. A. Lutovinov, The Astrophysical Journal, 777, 115, 2013.

3. A. E. Shtykovsky, A. A. Lutovinov, V. A. Arefiev, S. V. Molkov, S. S. Tsygankov, and M. G. Revnivtsev, Astronomy Letters, 43, 175, 2017. 


\title{
Heat diffusion in outer crust and X-ray superbursts of accreting neutron stars
}

\author{
A.D. Kaminker ${ }^{1}$, D.G. Yakovlev ${ }^{1}$, A.Y. Potekhin ${ }^{1}$, P. Haensel ${ }^{2}$ \\ kam.astro@mail.ioffe, \\ WWW home page: http://www.ioffe.ru/DTA/kam/kam.html \\ ${ }^{1}$ Ioffe Institute, Politekhnicheskaya 26, St Petersburg 194021, Russia, \\ ${ }^{2}$ Copernicus Astronomical Center, Bartycka 18, 00-716 Warsaw, Poland
}

A simple model is presented of heat diffusion after a superburst in deep layers of the outer crust of an accreting neutron star $\left(10^{7} \lesssim \rho \lesssim 4 \times 10^{11} \mathrm{~g} \mathrm{~cm}^{-3}\right)$. Since the warm outer crust $\left(T \sim 10^{8}-10^{9} \mathrm{~K}\right)$ has large heat capacity and acts as a heat reservoir, it is able to keep the outburst energy for months. The basic features of thermal afterburst evolution are outlined. They can be useful for interpreting observations of superbursts in low-mass X-ray binaries.

Keywords: conduction - dense matter - stars: neutron - X-rays: bursts

DOI: 10.51194 /VAK2021.2022.1.1.106

Superbursts constitute a special class of X-ray bursts (e.g., [1, 2]) demonstrated by some accreting neutron stars (NSs). These bursts are rare events. They differ from standard type Ia X-ray bursts mainly because they are more powerful and longer. Standard bursts are interpreted as explosive nuclear burning of accreted hydrogen and/or helim just under the surface of NSs, at densities $\rho \lesssim 10^{5} \mathrm{~g} \mathrm{~cm}^{-3}$. In contrast, superbursts are thought to be powered by explosive burning of carbon $\left({ }^{12} \mathrm{C}\right)$ that is produced during nuclear evolution of accreted matter. Carbon can survive in NSs to much higher densities $\rho \sim\left(10^{7}-10^{10}\right) \mathrm{g} \mathrm{cm}^{-3}$. According to the theory, a layer of carbon accumulated to the ignition depth $\rho_{\text {ign }}$ burns out in few seconds. Some fraction of the generated heat is carried away to the surface producing an observable event. Another fraction is thermally conducted inside the star, while the rest is carried away by neutrino emission from a hot burning shell.

Here we focus on heat diffusion in the NS crust. The problem has been extensively studied, mainly by direct modeling (e.g. $[3,4,5,6,7,8]$ and references therein). We combine modeling and semi-analytic consideration and outline most reliable results. More detailed discussion is given in [9] (hereafter P1).

A short explosive carbon burning produces heavy (iron-like) elements, huge heat release and temperature rise on spot; heat has no time to spread away. After that one can distinguish three main superburst stages.

Stage I is characterized by an efficient heat transport from the ignited layer to the surface by heat diffusion and/or convection, accompanied by neutrino cooling. This stage ends with the appearance of a quasi-stationary heat outflow to the surface from top of the ignited layer.

Stage II is accompanied by the strongest energy outflow from the surface; the lightcure $L(t)$ reaches maximum and starts to fade. The temperature profile within the heated layer gradually quasi-equilibrates, starting from top. Then the quasi-equilibration moves inside. Stage II ends when the equilibration reaches the boton of the ignited layer, $\rho \sim \rho_{\text {ign }}$. By that time the layer is cooler and the neutrino cooling stops. Since the heat capacity of deep outer crust is large, the thermal wave moves inside the star much slower than outside.

At the final stage III the lightcurve fades, the ignited zone becomes almost isothermal, but the heat continues to diffuse inside the star. This diffusion becomes the main mechanism which regulates temperature drop in the upper layers and at the surface. The fading of $L(t)$ imprints the information on properties of NS matter.

Superbursts can be studied with full thermal evolution codes (e.g. [7]), which can simulate (in General Relativity) creation of the carbon layer from accreted $\mathrm{H} / \mathrm{He}$ matter, subsequent explosion and evolution. However, if one wants to focus on diffusion of heat after the explosion, one can start with the burst of an assumed carbon layer and follow heat propagation within the outer NS crust (a thin layer under the surface, e.g. [10]). The space-time is locally flat there and heat conduction is described by the equation $C \partial T / \partial t-\partial(\kappa \partial T \partial z) / \partial z=Q$, where $T$ is the non-redshifted local temperature, $z$ is the proper depth from the surface, $C$ is the heat capacity per unit volume, $\kappa$ is the thermal conductivity, and $Q$ is the heat generation rate per unit volume. At densities $10^{7} \mathrm{~g} \mathrm{~cm}^{-3} \lesssim \rho \lesssim 10^{11} \mathrm{~g} \mathrm{~cm}^{-3}$ and temperatures $10^{8} \mathrm{~K} \lesssim T \lesssim 3 \times 10^{9} \mathrm{~K}$, important for superbursts, it is a good approximation to set $\rho \propto z^{3}, C \propto z^{3}$ and $\kappa \propto z^{2}$. Then the equation becomes linear in $T$, and its Green's function in the free heat propagation regime can be found in the analytic form. This gives an easily programmable (toy) solution for the temperature $T$ as a function of $z$ (or $\rho$ ) and $t$ for any source function $Q$ (see P1).

Fig. 1 shows semi-analytic toy (a) and exact (b) solutions for a star of mass $1.4 M_{\odot}$ and radius $R=12 \mathrm{~km}$. The exact solution has been obtained using the numerical code presented by [11] (see details in P1). Shown are snapshots of $T(\rho, t)$ at certain moments of non-redshifted time $t$. The exploded carbon layer extends to $\rho_{\text {ign }}=10^{8} \mathrm{~g} \mathrm{~cm}^{-3}$, the burst duration is $100 \mathrm{~s}$. The fuel calorimetry ( $5 \mathrm{keV}$ per nucleon) is intentionally taken small (for real superbursts) to avoid heating of the matter to $T \gtrsim 3 \times 10^{9} \mathrm{~K}$, which would trigger strong neutrino emission disregarded in the toy model. The total energy release for the burst in Fig. 1 is $\approx 10^{40} \mathrm{erg}$.

The temperature profiles in panels (a) and (b) qualitatively agree. Some disagreement occurs because the toy model uses simplified $C$ and $\kappa$. Note that this model cannot be directly extended to the surface, which complicates calculation of the toy lightcurves.

The inset on panel (b) shows the computed (non-redshifted) lightcurve $L(t)$. In our case, only $\approx 1 / 4$ of the released heat escapes from the surface. Stage I lasts for about $10 \mathrm{~min}$, stage II for $\sim 10 \mathrm{~h}$, and the burst fades in a few weeks. The inward thermal wave needs months to reach the bottom of the outer crust. 

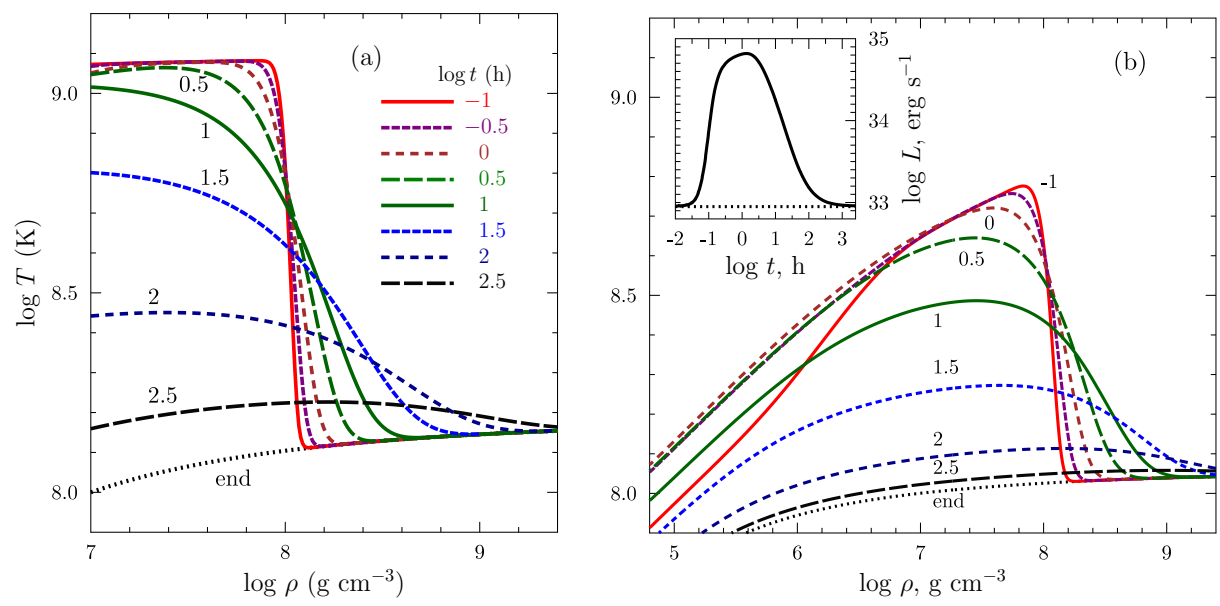

Figure 1: Snapshots of internal temperature $T(\rho)$ versus density after carbon explosion at $\rho \leq 10^{8} \mathrm{~g} \mathrm{~cm}^{-3}$ in different moments of time (labeled by $\log t[\mathrm{~h}]$ ). Calculations are performed with the semi-analytic toy model (a) and a computer code (b). The inset in panel (b) is shows the lightcurve computed by the code. The dotted lines (marked 'end') refer to a quiet NS. After Figs. 6 and 7 in P1. See text for details.

The onset of phase III is marked by the beginning of power-law-like tail of the lightcurve at time $t=t_{\mathrm{tr}}$, which can be inferred from observations of lightcurves. This time is identified as the heat propagation time from the bottom of the ignited layer $\left(\rho=\rho_{\text {ign }}\right)$ at some (neatly uniform) temperature $T \approx T_{\mathrm{tr}}$. For the star with $M=1.4 M_{\odot}$ and $R=12 \mathrm{~km}$, the dependence of $t_{\mathrm{tr}}$ on $T_{\mathrm{tr}}$ and $\rho_{\mathrm{ign}}$ has been approximated by Eq. (18) in P1. The redshifted time $t_{\mathrm{tr}}^{\mathrm{S}}$ for arbitrary $M$ and $R$ is given by Eq. (19) in P1. For instance, for the outburst of KS 1731-269 [12] one has $t_{\mathrm{tr}}^{\mathrm{S}} \approx 10 \mathrm{~h}$. If $M=1.4 M_{\odot}$ and $R=10$ $\mathrm{km}$ for $\log T_{\mathrm{tr}}[\mathrm{K}] \approx 9-9.3$, we can obtain $\log \rho_{\text {ign }}\left[\mathrm{g} \mathrm{cm}^{-3}\right] \approx 8.7$, which agrees with calculations by [4]. Taking $R=12 \mathrm{~km}$ for the same $M$ and $T_{\mathrm{tr}}$, we have noticeably smaller $\log \rho_{\mathrm{ign}}\left[\mathrm{g} \mathrm{cm}^{-3}\right] \approx 8.2$. Therefore, the ignition depth and other burst parameters do depend on $M$ and $R$.

The thermal evolution at stage III is accompanied by a power-law fading of the ignition curve and slow propagation of the inward thermal wave in the outer NS crust. The heat capacity of this crust is sufficient to absorb $\left(10^{43}-10^{44}\right)$ erg. As long as the thermal wave moves in, the surface stays thermally decoupled from the NS inteior.

\section{References}

1. J. in 't Zand, in M. Serino, M. Shidatsu, W. Iwakiri, and T. Mihara, eds., 7 years of MAXI: monitoring X-ray Transients, 121 (2017).

2. D. K. Galloway and L. Keek, in T. Belloni, M. Mendez, and C. . Zang, eds., Timing Neutron Stars: Pulsations, Oscillations and Explosions, ASSL, Springer, volume 461, 209 (2021).

3. A. Cumming and J. Macbeth, ApJ, 603, L37, 2004.

4. A. Cumming, J. Macbeth, J. J. M. in 't Zand, and D. Page, ApJ, 646, 429, 2006.

5. L. Keek and A. Heger, ApJ, 743, 189, 2011.

6. D. Altamirano, L. Keek, A. Cumming, G. R. Sivakoff, et al., MNRAS, 426, 927, 2012.

7. L. Keek, A. Heger, and J. J. M. in 't Zand, ApJ, 752, 150, 2012.

8. L. Keek, A. Cumming, Z. Wolf, D. R. Ballantyne, V. F. Suleimanov, E. Kuulkers, and T. E. Strohmayer, MNRAS, 454, 3559, 2015.

9. D. G. Yakovlev, A. D. Kaminker, A. Y. Potekhin, and P. Haensel, MNRAS, 500, 4491, 2021.

10. P. Haensel, A. Y. Potekhin, and D. G. Yakovlev, Neutron Stars. 1. Equation of State and Structure, Springer, New York (2007).

11. A. Y. Potekhin and G. Chabrier, $A \& A, 609$, A74, 2018.

12. E. Kuulkers, J. J. M. in 't Zand, M. H. van Kerkwijk, R. Cornelisse, et al., A $\& A$, 382, 503, 2002. 


\title{
Magneto-rotational evolution of neutron stars with hysteresis effect and fallback
}

\author{
A. Khokhriakova ${ }^{1,2}$, S. Popov ${ }^{1,2}$ \\ alenahohryakova@yandex.ru \\ ${ }^{1}$ Department of Physics, Lomonosov Moscow State University, Moscow, 119991 Russia, \\ ${ }^{2}$ Sternberg Astronomical Institute, Lomonosov Moscow State University, Moscow, 119234 Russia
}

In recent years, accreting neutron stars (NSs) in X-ray binary systems in supernova remnants have been discovered. They are a puzzle for the standard magneto-rotational evolution of NSs, as their age $\left(\lesssim 10^{5}\right.$ years $)$ is much less than expected duration of the preceding Ejector and Propeller stages. To explain such systems, we consider rotational evolution of NSs with fallback accretion and asymmetry in direct/backward transitions between Ejector and Propeller stages. It is shown that at certain values of the initial period and the magnetic field, a young neutron star may not enter the Ejector stage during its evolution.

Keywords: neutron stars, X-ray binaries, supernovae

DOI: $10.51194 /$ VAK2021.2022.1.1.107

\section{Evolution of neutron stars}

Recently, six accreting NSs in X-ray binary systems located in supernova remnants (SNRs) have been discovered, see [1]. They are specifically interesting objects allowing to probe early evolution of accreting NSs, as in this cases the compact objects reach the stage of accretion very rapidly, in contradiction with standard assumptions (see e.g., the book [2]). We discuss modifications in early evolution of NSs in close massive binaries.

\subsection{Hysteresis}

Transitions between evolutionary stages of NS are determined by pressure balance at critical radii. However, this equality can be reached at different radii for direct and backwards cases. ${ }^{a}$ In particular, we are interested in asymmetry in EjectorPropeller transition, first noticed by [3] and dubbed as hysteresis.

In the phase of ejection the NS slows down. This results in decrease of the wind power. Direct transition Ejector $\rightarrow$ Propeller happens when external pressure equalizes the relativistic wind pressure at gravitational capture radius $\left(R_{\mathrm{G}}\right)$. Pressure of gravitationally captured cold matter grows towards the NS with distance as $r^{-5 / 2}$, i.e. faster than the wind pressure $\left(\sim r^{-2}\right)$. So, particle generation and acceleration are stopped. Transition in the opposite direction (Propeller $\rightarrow$ Ejector) proceeds in a different way. When the propeller phase is on and accretion rate decreases, the magnetospheric radius is growing gradually approaching the light cylinder, where the pressure of external gravitationally captured matter is large. To overcome it, much larger wind power is needed than in the case of the equilibrium at $R_{\mathrm{G}}$.

Here we apply this effect to the situation when a newborn NS experiences an intense fallback, and then while accretion rate $(\dot{M})$ is decreasing switches to Propeller, but can skip an Ejector phase due to hysteresis. Thus, time before wind accretion can be much shortened.

\subsection{Fallback}

After formation of an NS, some amount of the progenitor star material expelled in the supernova explosion can fall back onto the compact object [4]. We assume that in the case of fallback the NS is an Accretor when it starts to interact with the stellar wind.

If the star is isolated, fallback can last quite long. However, in a close binary system, expelled matter mostly leaves the Roche lobe of the future compact object. Falling back it might feel joint gravity of both components. In addition, outside the Roche lobe the matter interacts with the stellar wind of the secondary component. We assume that mostly matter which does not leave the Roche lobe of the compact object can fall back onto it. Thus, intensive fallback accretion cannot last long in binary systems, and so we can neglect this stage in our modeling, assuming as initial values of parameters those that they obtain after the fallback is over.

After a short period of fallback accretion, the interaction of the NS and the companion star will be determined by the intensity of the stellar wind. Thus, the accretion rate would rapidly decrease. Correspondingly, the size of the magnetosphere changes dramatically approaching the light cylinder. At this moment the transition Propeller $\rightarrow$ Ejector can happen. However, as it is not a direct transition, the hysteresis effect might be taken into account. Such scenario was not considered in other works on this topic.

\section{Results}

We start our calculations when a brief period of fallback is over, and the external medium is determined by wind of the donor. At this moment the NS has a period $p_{0}$, magnetic field $B_{0}$, and the accretion rate (from the wind) is $\dot{M}$. If the NS does not become an Ejector at this moment, it will not enter this stage in the future (for non-decreasing rate of capture of external matter) and will start accreting quite fast if it is a Propeller.

\footnotetext{
"We call "direct" transitions in the sequence Ejector $\rightarrow$ Propeller $\rightarrow$ Accretor.
} 


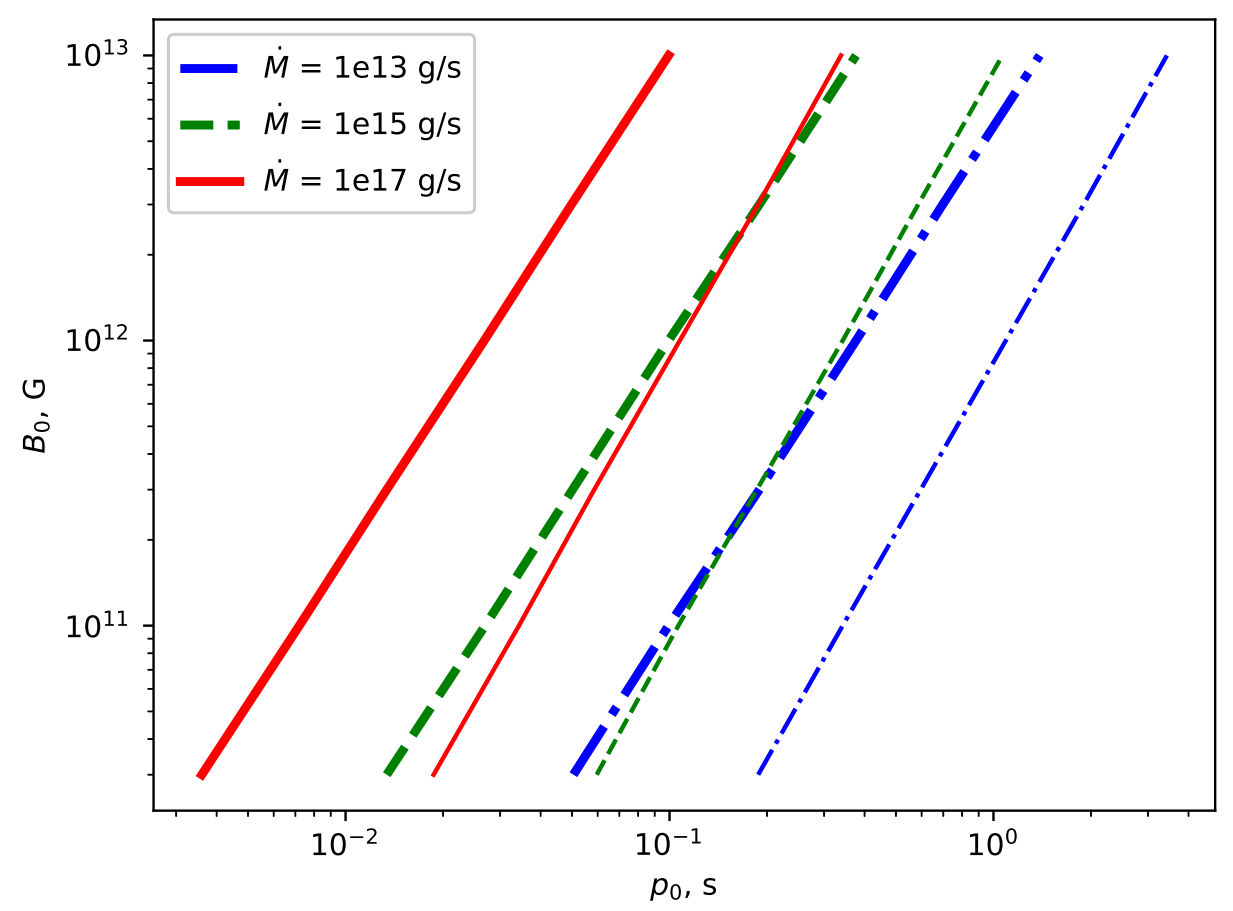

Figure 1: For three characteristic values of $\dot{M}$ we calculate critical values of $p_{0}$ and $B_{0}$. For a given $\dot{M}$, if the point is located to the left of the line, then a star is an Ejector after the intense fallback is over. If initial period is higher or magnetic field is lower than critical values, then an NS will not be in ejector phase. Thick and thin lines represent the presence and absence of the hysteresis effect, respectively.

For three characteristic values of $\dot{M}$ we calculate critical values of $p_{0}$ and $B_{0}$ (Fig. 1). For a given $\dot{M}$, if the point is located to the left of the line, then a star is "born" as an Ejector (after short initial fallback accretion). If initial period is higher or magnetic field is lower than critical values, then an NS will not be in the ejector phase and will start to accrete quite fast. We compare our results with an approach without accounting for the hysteresis effect. Bold and thin lines represent the presence and absence of the hysteresis effect, respectively. The greater the accretion rate of the stellar wind, the easier it is to avoid the ejection phase.

Calculations for different sets of parameters $\left(p_{0}, B_{0}, \dot{M}\right)$ allowed to reproduce situations when a NS reaches the stage of accretion within $10^{5}$ yrs, i.e. within the lifetime of a SNR.

\section{Conclusions}

Presence of the fallback stage can change the evolution and observational properties of neutron stars in binary systems. The hysteresis effect, proposed by Shvartsman, allows a young neutron star in a massive X-ray binaries to avoid the Ejector stage in the presence of fallback. Such NSs can start accreting in a relatively short period, sometimes shorter than the lifetime of a SNR. With magnetic fields $\sim 10^{12} \mathrm{G}$ and initial spin periods $\sim 0.1-0.2 \mathrm{~s}$ NSs can avoid the Ejector stage if the accretion rate is $\gtrsim 10^{14}-10^{15} \mathrm{~g} \mathrm{~s}^{-1}$. Some of the massive binaries in SNRs can be examples of such systems.

\section{Acknowledgements}

This work was supported by the Russian Science Foundation, grant 21-12-00141. A.K. also acknowledges fellowship from "Basis" foundation, grant 20-2-1-77-1.

\section{References}

1. Z.-p. Xing and X.-d. Li, arXiv e-prints, arXiv:2107.09325, 2021.

2. V. M. Lipunov, Astrophysics of Neutron Stars (1992).

3. V. F. Shvartsman, Sov. Astron., 14, 527, 1970.

4. R. A. Chevalier, ApJ, 346, 847, 1989. 


\title{
RATAN-600 spectra as indicators of proton jets producing neutrino in blazars
}

\author{
Yu.A. Kovalev ${ }^{1}$, Yu.Yu. Kovalev ${ }^{1,2}$, A. Popkov ${ }^{2,1}$, Y. Sotnikova ${ }^{3}$, S. Troitsky ${ }^{4}$, A. Plavin ${ }^{1}$, A. Erkenov $^{3}$ \\ ykovalev@asc.rssi.ru \\ ${ }^{1}$ Lebedev Physical Institute of RAS, Leninsky prospect, 53, Moscow, 119991, \\ ${ }^{2}$ Moscow Institute of Physics and Technology, Dolgoprudny, 141700, \\ ${ }^{3}$ Special Astrophysical Observatory of RAS, Nizhny Arkhyz, 369167, \\ ${ }^{4}$ Institute for Nuclear Research of RAS, Moscow, 117312
}

\section{DOI: $10.51194 /$ VAK2021.2022.1.1.108}

According to the data obtained with the RATAN-600 in 2020-2021, the active galaxy TXS 0506 +056 reached the absolute maximum of the radio emission flux in the previous 20 years in the cm-wave lengths. About 3 years have passed since the supposed detection of neutrino from it in September 2017. However, the question of the nature for neutrino emitters remains open. One of the scenarious under consideration assumes that the neutrino and synchrotron radio emission of the relativistic jet in this and other VLBI-bright AGNs is generated by relativistic protons, but from different parts of their energy spectrum.

In this report, such approach is applied to fit a model to $1-22 \mathrm{GHz}$ spectra obtained with the RATAN-600 over 10 previous years, and extended to the frequency range from 0.1 to $1000 \mathrm{GHz}$ using the CATS SAO RAS data base [1]. It was previously shown that most of the RATAN-600 spectra for VLBI-compact AGNs have a component that can be succesfully simulated by the radio emission of relativistic electrons or protons in a strong longitudinal magnetic field of a jet. Two-component Hedgehog jet model, suggested by [2], has been used earlier and is used in this work.

The proton or electron jet model is fitted to the average observed spectra for 7 AGNs: 3C 84 , 3C 279 , B0716+71, $\mathrm{B} 1502+10, \mathrm{~B} 1616+04$, OJ 287, 3C454.3. Protons with energies $E>100-1000 \mathrm{TeV}$ can generate neutrinos in protonproton or proton-photon interactions and with $0.001<E<10-100 \mathrm{TeV}$ - synchrotron emission. The frequency of spectra maximums is $\nu_{m} \sim 10-20 \mathrm{GHz}$. The flux is $\sim(1-10) \mathrm{Jy}$ at $\nu_{m}$. The gamma-factor is $\left(E / m c^{2}\right) \sim 300$ at $\nu_{m}$ for both protons and electrons. The viewing angle of the jet is $\sim(1-5)$ degrees. The energy density of the magnetic field is much greater than the kinetic energy of emitting particles. As a result we obtain the estimates for magnetic field $B \sim 10^{3}-10^{4}$ Gauss and the brightness temperature $T_{b} \sim 10^{13}-10^{14} \mathrm{~K}$ in the case of the proton jet. $B$ and $T_{b}$ will be 1836 times less for the electron jet (1836 is the proton to electron mass ratio).

\section{References}

1. O. V. Verkhodanov, S. A. Trushkin, V. N. Chernenkov, and H. Andernach, Baltic Astronomy, 9, 604, 2000.

2. N. S. Kardashev, Astronomicheskij Tsirkulyar, 497, 1, 1969. 


\section{X-ray morphology of pulsar wind nebulae: the effect of local medium motion}

K. Levenfish ${ }^{1}$, G. Ponomaryov ${ }^{1,2}$, A. Petrov ${ }^{1}$

ksen@astro.ioffe.ru

${ }^{1}$ Ioffe Institute, St. Petersburg, Russia

${ }^{2}$ Peter the Great St. Petersburg Polytechnic University, St. Petersburg, Russia

The effect of the relative motion of the pulsar wind nebula (PWN) and the local medium on the PWN X-ray morphology is studied using relativistic MHD modelling. It is shown that even the slow (subsonic) motion may strongly affect the X-ray appearance of PWNe and the integral flux of their emission. Accounting for the relative motion is also necessary to avoid misinterpretation of the PWN structure.

Keywords: pulsar wind nebulae, relativistic MHD simulations, PLUTO

DOI: 10.51194 /VAK2021.2022.1.1.109

\section{Introduction}

Pulsar wind nebulae (PWNe) are natural laboratories for studying relativistic magnetized plasma. In particular, by comparing the results of relativistic magnetohydrodynamic (RMHD) modeling with the PWNe observables - spectrum, dynamics, morphology. The correct interpretation of X-ray morphologies is crucial for understanding plasma processes and patterns in PWNe.

X-ray morphologies naturally fall into two groups depending on the Mach number of the PWNe motion relative to their surroundings [1]. At $M \lesssim 1$, nebulae tend to acquire a jet-torus structure with an X-ray morphology determined by the pulsar parameters - spin-down luminosity, initial pulsar wind magnetization $\sigma_{0}$, magnetic axis tilt $\alpha$, etc. At $M \gg 1$, nebulae drive a bow-shock ahead and acquire a cometary head-tail structure, in which jets and tori are usually crushed by the ram pressure of an ambient matter stream.

Slow PWNe with $M \lesssim 1$ are usually modeled under assumption of stationary or radially expanding surroundings. But in fact, moving PWNe in their rest frame usually see the oncoming free stream of surrounding matter. We argue that the influence of such a stream must be taken into account in order to correctly determine the type of PWN (single- or double-torus) and to achieve better correspondence of the simulated and actual X-ray morphology of PWNe.

\section{Results}

\subsection{Subsonic motion and PWN morphology}

The effect of the free stream on the X-ray morphology is illustrated in figure 1, in panels (1) and (3). Shown are X-ray maps of the same PWN model, of the same age, viewed at the same aspect, and interacting with the same stream; only the directions of this stream are opposite, as shown by arrows. This stream is initiated in the entire computation domain before the PWN begins to evolve. (The RMHD model is built with the PLUTO code [2], with the setup as in [3]).

Clearly, the X-ray appearance of the PWN in panels (1) and (3) is drastically different; see [3] for detail. Depending on whether the PWN is facing us upwind or leeward, it can exhibit either a Crab-like or Vela-like morphology (these PWNe are shown in panels (2) and (4)). In the former case, the prominent X-ray features are: steady torus and inner ring of nearly uniform brightness, transient wisps, and bright SE jet with "sprite" at its base. In the latter case, PWN exhibits two steady bright arcs, bright NW jet and feeble SE counter-jet. Interestingly, that in both cases, only stream-facing (windward) jets appear bright, while counter-jets are faint and embedded in a diffuse structure.

Thus, taking into account the ram pressure of the ambient matter stream is important for avoiding misinterpretation (as single- or a double-torus objects) of those PWNe that are not resolved that well as the Crab and Vela nebulae.
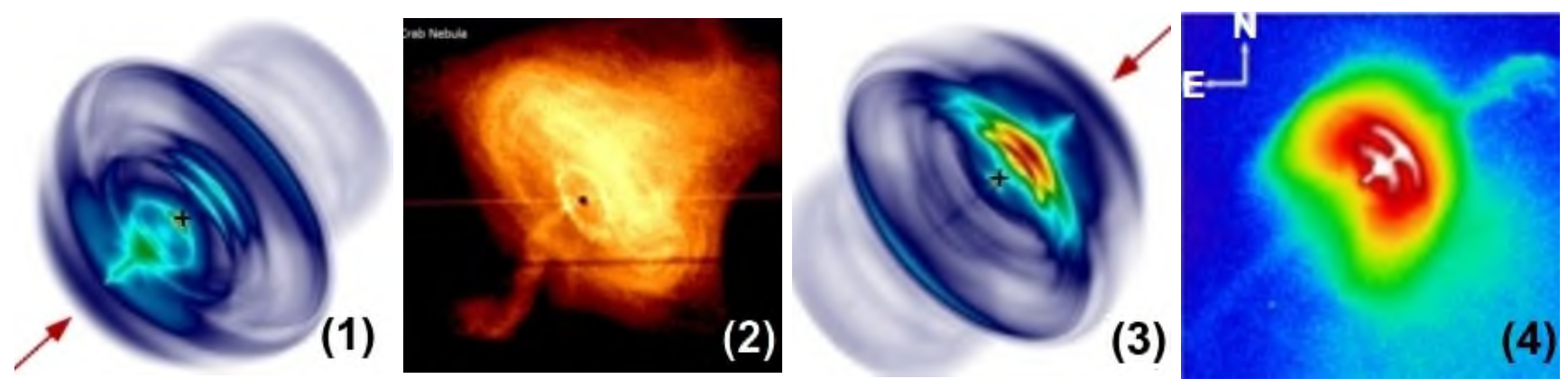

Figure 1: Panels (1), (3): synthetic maps of synchrotron X-ray radiation of the same PWN model $\left(\alpha=45^{\circ}, \sigma_{0}=\right.$ $0.03)$ of age $t=14 \mathrm{yr}$, which meets a subsonic flow of the surrounding matter having the same Mach number $(M=0.7)$ but opposite direction (shown by red arrows). The PWN model is viewed at the same aspect as the Crab [4] and Vela PWNe, whose X-ray images are shown in panels (2) and (4), respectively $\left(\theta_{\text {view }}=120^{\circ}\right.$; the position angle is $310^{\circ}$, north through east). 


\subsection{The effect of the motion on the integral flux}

The visible difference between the synthetic maps of the Crab-like and Vela-like models shown in Figure 1 translates into the difference of their integral X-ray fluxes by about (10-15)\% (Figure 2). The flux is higher when the nebula is viewed from the leeward side (the Vela-like case). Both model PWNe exhibit variations of the integral fluxes of $\sim 5 \%$ on a year time-scale, due to reverberations of the torus. Thus, taking the motion into account can also be important for studying the variability of high-energy emission from PWNe. In particular, the observed variability of the Crab nebula, which may be related to the fluctuations of the magnetic field in the PWN (e.g., [5], [6]).

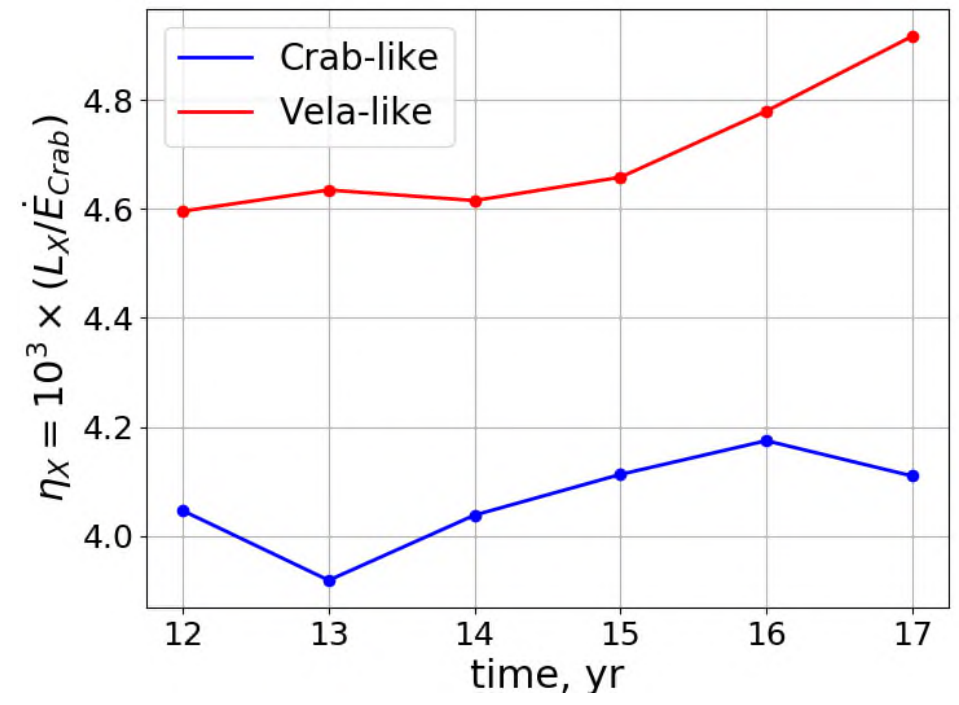

Figure 2: The evolution of integral fluxes of the Crab-like (blue curve) and Vela-like (red curve) model PWNe from Figure 1 between $t=12$ and $t=17 \mathrm{yr}$. The fluxes are expressed through X-ray efficiencies (see, e.g., [1]), assuming the distance and the spin-down luminosity of the Crab pulsar $(d \simeq 2 \mathrm{kpc}$ and $\left.\dot{E}=5 \cdot 10^{38} \mathrm{erg} \mathrm{s}^{-1}\right)$.

\subsection{Double-torus PWN: stationary versus transonic models}

Accounting for the relative motion of the X-ray PWN and the surrounding matter also facilitates the interpretation of the PWN morphology. Let us take the model of the double-torus Vela PWN, which is believed to interact with a transonic stream of $M \sim 1.3$ induced by the reverse shock of the supernova [7]. This model $\left(\alpha=80^{\circ}, \sigma_{0}=0.03\right)$ is inflated either (1) into an stationary medium ("stationary model"), or (2) into an oncoming transonic stream of $M=1.3$ ("transonic model"). In the latter case, the stream is co-aligned with the PWN axis; see [8] for detail. In Figure 3, synthetic X-ray maps for the cases (1) and (2) are compared to the Chandra X-ray map of the Vela PWN.

It is apparent that the transonic model provides a better match with the morphology of a real nebula. First, the arcs (Doppler-brightened parts of the tori) are of similar brightness, while in the stationary model the SE torus is much brighter overall. Second, the NW (windward) torus appears larger than the SE (leeward) one. In Vela, its NW arc has a larger extent than the SE arc. Third, the far side of the SE torus does not appear that bright as in the stationary model. In Vela, none of the tori has a bright far side. Fourth, between the bright arcs begins to show the windward jet of the nebula (see [9] for detail).
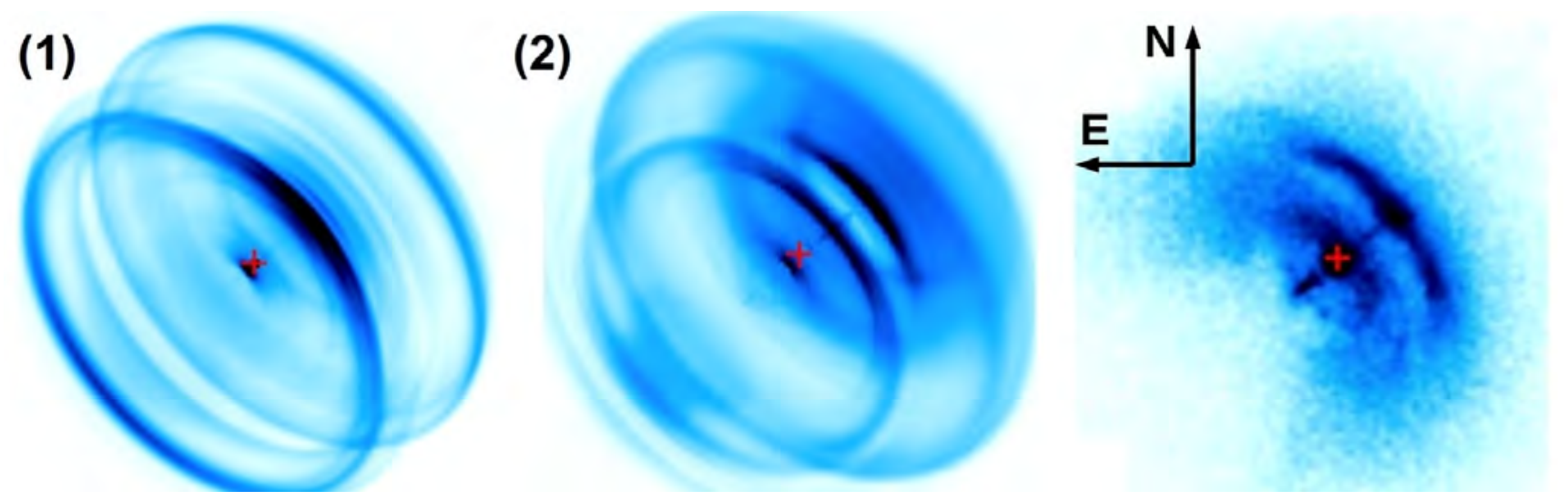

Figure 3: Left to right: (1),(2) - synthetic maps of synchrotron X-ray radiation of the double-torus PWN model $\left(\alpha=80^{\circ}, \sigma_{0}=0.03\right)$ for a a "stationary" and "transonic" $(M \sim 1.3)$ cases; $(3)$ - the Chandra X-ray map of the Vela PWN. The viewing and position angles are $\theta_{\text {view }}=120^{\circ}$ and $\Psi=310^{\circ}(\mathrm{E}$ of $\mathrm{N})$ as in the maps in Figure 1 . 


\section{Acknowledgements}

K.L. acknowledges the support from the baseline project 0040-2019-0025 of the Ioffe Institute. PLUTO simulations of PWNe were performed by G.P. \& A.P., supported by RSF grant 21-72-20020. Modelling was carried out partly at the Tornado subsystem of the supercomputer center of Peter the Great St.Petersburg Polytechnic University. The authors are grateful to the PLUTO developers.

\section{References}

1. O. Kargaltsev and G. G. Pavlov, in C. Bassa, Z. Wang, A. Cumming, and V. M. Kaspi, eds., 40 Years of Pulsars: Millisecond Pulsars, Magnetars and More, American Institute of Physics Conference Series, volume 983, 171-185 (2008).

2. A. Mignone, G. Bodo, S. Massaglia, T. Matsakos, O. Tesileanu, C. Zanni, and A. Ferrari, ApJS, 170, $228,2007$.

3. K. P. Levenfish, G. A. Ponomaryov, A. E. Petrov, A. M. Bykov, and A. M. Krassilchtchikov (2021).

4. A. M. Krassilchtchikov, A. M. Bykov, G. M. Castelletti, G. M. Dubner, O. Y. Kargaltsev, and G. G. Pavlov, in Journal of Physics Conference Series, Journal of Physics Conference Series, volume 798, 012003 (2017).

5. C. A. Wilson-Hodge, M. L. Cherry, G. L. Case, W. H. Baumgartner, et al., ApJL, 727, L40, 2011.

6. M. S. Pshirkov, B. A. Nizamov, A. M. Bykov, and Y. A. Uvarov, MNRAS, 496, 5227, 2020.

7. R. A. Chevalier and S. P. Reynolds, ApJL, 740, L26, 2011.

8. G. A. Ponomaryov, K. P. Levenfish, A. E. Petrov, and Y. A. Kropotina, in Journal of Physics Conference Series, Journal of Physics Conference Series, volume 1697, 012022 (2020).

9. G. A. Ponomaryov, K. P. Levenfish, A. E. Petrov, and Y. A. Kropotina (2021). 


\title{
Modeling outbursts of viscous accretion discs
}

\author{
G. Lipunova ${ }^{1}$, K. Malanchev ${ }^{1,2}$, A. Avakyan ${ }^{1,3}$, N. Shakura ${ }^{1,4}$, S. Tsygankov ${ }^{5,6}$, A. Tavleev $^{1}, 7$, D. Kolesnikov $^{1}$ \\ gvlipunova@sai.msu.ru \\ ${ }^{1}$ Moscow Lomonosov State University Sternberg Astronomical Institute, Moscow 119992, Universitetskiy pr., 13, Russia \\ ${ }^{2}$ Department of Astronomy, University of Illinois at Urbana-Champaign, 1002 West Green Street, Urbana, IL 61801, USA \\ ${ }^{3}$ Universität Tübingen, Institut für Astronomie und Astrophysik Tübingen, Sand 1, Tübingen, Germany \\ ${ }^{4}$ Kazan Federal University, 420008 Kazan, Russia \\ ${ }^{5}$ Department of Physics and Astronomy, FI-20014 University of Turku, Finland \\ ${ }^{6}$ Space Research Institute of the Russian Academy of Sciences, Moscow, Russia \\ ${ }^{7}$ Physics Faculty of Moscow Lomonosov State University, Moscow 119992, Russia
}

A brief review is given on the topic of viscously-evolving accretion discs around compact objects that covers the development of analytical studies and our numerical model freddi allowing comparison of theory with observations.

Keywords: accretion, accretion discs

DOI: $10.51194 /$ VAK2021.2022.1.1.110

\section{Introduction}

The most suitable sources to compare observations with predictions of the theory of viscously evolving accretion discs are X-ray novae with black holes (BHs), when emission of the disk dominates during flares, if a source is in a 'soft' state. An outburst of an X-ray novae typically lasts from a couple of tens of days to few months and can have quite different shapes. Among all the variety of outburst profiles, there are so-called FRED flares ("fast-rise exponential-decay"). They are of particular interest for theoretical study.

Although there is still a lot to be learned about spectra formation and variability, and mechanisms causing peculiarities of light curves, the general properties of FRED outbursts are reliably explained in the theory of a viscous disk.

\section{Theoretical advance}

The equation of the viscous disc evolution that governs its long-term dynamics (see, e.g., [1]

$$
\frac{\partial \Sigma}{\partial t}=\frac{1}{4 \pi} \frac{\left(G M_{\star}\right)^{2}}{h^{3}} \frac{\partial^{2} F}{\partial h^{2}}
$$

is derived from equations of conservation of mass and angular momentum (see also [2], hereinafter LP74). Since the suitable geometry for the disc is a cylindrical one, all values are integrated over the disc height: the surface density $\Sigma$ and the viscous torque $F=2 \pi r^{2} W_{r \varphi}$, where $W_{r \varphi}=\frac{3}{2} \omega_{\mathrm{K}} \nu_{\mathrm{t}} \Sigma$ is the integrated component of the viscous stress tensor. Note that the radial coordinate is substituted by the specific angular momentum $h=\sqrt{G M_{\star} r}$. This change of variable allows one to deal with a simplified form of the equation as well as to pose boundary conditions in the most suitable way.

Turbulent motions, believed to be generated by the MHD instabilities, sustain the viscosity of astrophysical accretion discs. The kinematic coefficient of viscosity $\nu_{\mathrm{t}}$ is a product of the characteristic length and velocity of turbulent motions and can be related to the $\alpha$-parameter of the standard model $[3,4]$.

A list of analytic solutions of Eq. (1) is given in Fig. 1. There are two groups of solutions (see also Fig. 2): when the kinematic coefficient of viscosity $\nu_{\mathrm{t}}$ depends only on the radius (the left column) and when it also depends on the hydrodynamic parameters (the right column; this case is of a special importance since $\alpha$-discs falls into this category). When Eq. (1) is a linear differential equation, Green functions (GF) is an effective method to solve it. LP74 in their foundational work found GF for a disc with a zero inner radius and infinite outer radius. Later, [5] and [6] found GF when the inner boundary is at the finite radius. GF for a disc with finite outer radius are given by [7] and [8]. The latter have been used to build a power density spectra of X-ray variability generated by mass accretion fluctuations over the disc around a magnetized neutron star [8].

[9] (KR98) proposed a model with constant $\nu_{\mathrm{t}}$ to explain the exponential evolution of X-ray nova FREDs; [10] (LS00) showed that in an $\alpha$-disc the accretion rate evolves not exponentially but as a power-law, although it is observed in X-rays to be very close to exponential. Figure 2 compares the solutions of the linear and non-linear Eq. (1) with similar absolute values of $\nu_{t}$. It is evident that during few viscous characteristic (exponential) times these solutions are very close.

\section{Observations vs. theory}

Analysing observed light curves of X-ray novae, one can in principle infer the value of the $\alpha$-parameter $[11,12,13,14,15]$. It turns out that self-irradiation has impact not only on the observed optical flux but also on the course of the evolution. Thus, a combined analysis of observations in X-ray and optical is necessary to build a physically-consistent model of an accretion disc. To determine reliably $\alpha$-parameter, the binary parameters (masses, inclination) are need to be known quite accurately.

Furthermore, a spectral analysis in X-ray band is desirable, since we need to separate the disc flux from other spectral contributions to derive the central accretion rate variation in order to compare it with a model. If non-thermal components in spectra are bright and evolving, jets are contributing, the analysis becomes very involved. 


\section{Linear case}

$$
\nu_{\mathrm{t}}=\nu_{0} r^{b}
$$

- Lynden-Bell \& Pringle (1974) - Green

Functions

->Pringle (1991) - circumbinary disc

- Tanaka $(2011,2012)$ - circumbinary disk with finite inner radius also Nixon \& Pringle (2020) accretion/decretion discs with finite inner radius

\section{Non-linear case}

$$
\nu_{\mathrm{t}}=\nu_{0} \Sigma_{\mathrm{o}}^{a} r^{b}
$$

- Pringle (1974), Lyubarski \& Shakura (1987)

- $\alpha$-disc with Kramers or Thomson opacity; also Fillipov (1984), Cannizzo+(1990) - Pringle (1991); Ivanov+(1991) zero accretion rate

- Rafikov (2013) general inner boundary condition ( $\alpha$-disc around SMBH binary) Lin \& Pringle (1987) Viscosity due to gravitational instability

Rafikov (2016) accretion/decretion discs : $\alpha$-disc around SMBH binary, TDE, mass-loosing stars (e.g., Be), disc around a NS in a propeller regime

\section{Linear case \\ $\nu_{\mathrm{t}}=\nu_{\mathrm{r}} r^{\mathrm{b}}$ \\ time-independent viscosity}

- King \& Ritter (1998) : $v_{t}=$ const,$b=0$ accretion decay

- Zdziarski, Kawabata \& Mineshige

(2009) : numerical solution

\author{
- Lipunova (2015) Green Functions for zero \\ inner radius \\ - Mushtukov+ (2019) GF for non-zero inner \\ radius
}

\section{- Lipunova \& Shakura (2000) - $\alpha$-disc , accretion decay}

Figure 1: Analytic solutions for freely expanding (top) and radially confined (bottom) discs.

It must be noted that analytic solutions are not applicable to discs with non-uniform type of viscosity. For example, if a disc is large, the matter in its outer part is not ionised and quite 'cold' (temperature $\lesssim(1-3) 10^{4} \mathrm{~K}$ ), and the viscous time is considerably larger there. The boundary, or transition zone, between 'hot' and 'cold' part of the disc moves during an outburst.

To numerically model the disc evolution and to take into account described components of the model, we have developed an open code freddi [16]. Its original version dealt with accretion discs around black holes [14]. Recently, accretion discs around neutron stars are incorporated in freddi and applied to an outburst of Aql X-1, a LMXB with a NS [17]. The code allows one to include a thermal wind from its surface (Avakyan et al., in press). It calculates the evolution of the disc radial structure and light curves in user-specified bands. Optical flux can be calculated taking into account irradiation of the companion star.

\section{Acknowledgments}

This work is supported by the Russian Science Foundation under grant 21-12-00141 and performed in Moscow Lomonosov State University. NS acknowledges support of the Interdisciplinary Scientific and Educational School of Moscow University "Fundamental and Applied Space Research". ST acknowledges support by the grant 14.W03.31.0021 of the Ministry of Science and Higher Education of the Russian Federation.

\section{References}

1. Y. E. Lyubarskij and N. I. Shakura, Soviet Astronomy Letters, 13, 386, 1987.

2. D. Lynden-Bell and J. E. Pringle, $M N R A S, \mathbf{1 6 8}, 603,1974$.

3. N. I. Shakura and R. A. Sunyaev, $A \xi A$, 24, 337, 1973.

4. N. Shakura, G. Lipunova, K. Malanchev, V. Zhuravlev, et al., Accretion Flows in Astrophysics, volume 454 (2018). 


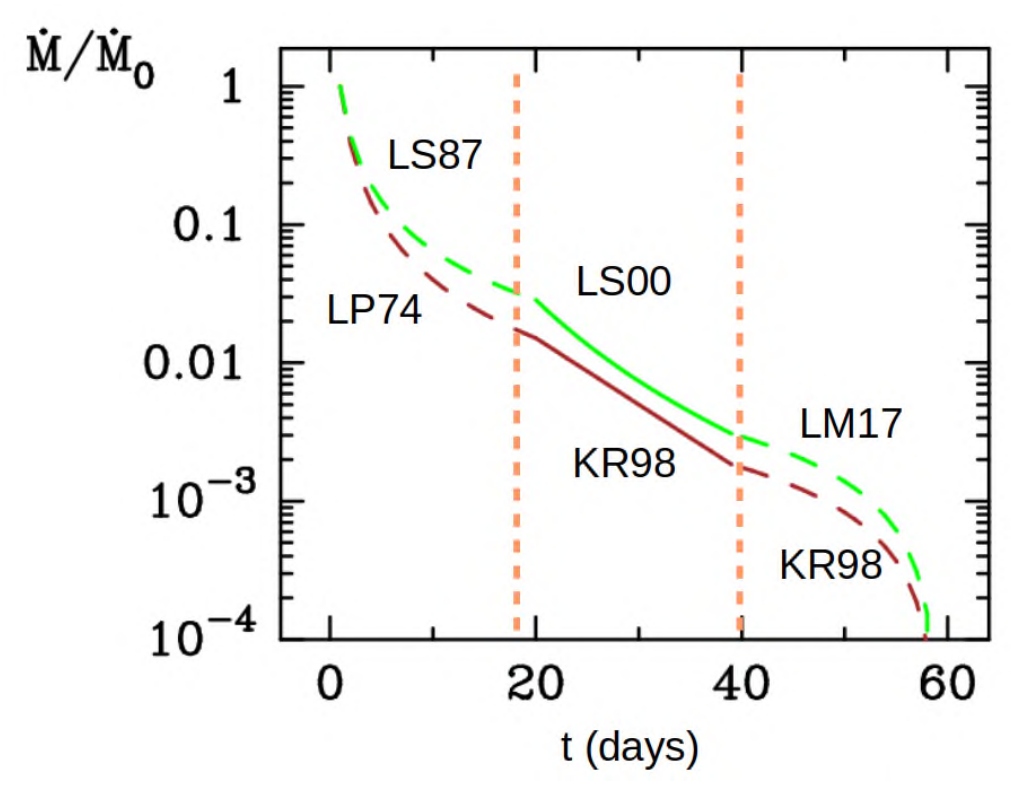

Figure 2: Analytic solutions for discs with constant $\nu_{\mathrm{t}}$ (brown line) and $\alpha$-discs (green line). In interval A, discs are freely-expanding over radius. In a binary, there is a stage when a disc 'feels' its outer boundary: interval B. Curves in interval $\mathrm{C}$ depicts accretion rate evolution when the hot part of a disc shrinks.

5. T. Tanaka, MNRAS, 410, 1007, 2011.

6. C. J. Nixon and J. E. Pringle, arXiv e-prints, arXiv:2008.07565, 2020.

7. G. V. Lipunova, $A p J$, 804, 87, 2015.

8. A. A. Mushtukov, G. V. Lipunova, A. Ingram, S. S. Tsygankov, J. Mönkkönen, and M. van der Klis, MNRAS, 486, 4061, 2019.

9. A. R. King and H. Ritter, MNRAS, 293, L42, 1998.

10. G. V. Lipunova and N. I. Shakura, $A \& A$, 356, 363, 2000.

11. J. Smak, Acta Astronom., 49, 391, 1999.

12. G. V. Lipunova and N. I. Shakura, Astronomy Reports, 46, 366, 2002.

13. V. F. Suleimanov, G. V. Lipunova, and N. I. Shakura, A\&A, 491, 267, 2008.

14. G. V. Lipunova and K. L. Malanchev, MNRAS, 468, 4735, 2017.

15. B. E. Tetarenko, J. P. Lasota, C. O. Heinke, G. Dubus, and G. R. Sivakoff, Nature, 554, 69, 2018.

16. K. L. Malanchev and G. V. Lipunova, Freddi: Fast Rise Exponential Decay accretion Disk model Implementation, 2016.

17. G. Lipunova, K. Malanchev, S. Tsygankov, N. Shakura, A. Tavleev, and D. Kolesnikov, arXiv e-prints, arXiv:2110.08076, 2021. 


\section{Uncertainties in estimations of actual luminosity in bright $\mathrm{X}$-Ray pulsars}

I.D. Markozov, A.A. Mushtukov, D.I. Nagirner

Saint Petersburg State University, Saint-Petersburg 198504, Russia

\section{DOI: 10.51194 /VAK2021.2022.1.1.111}

Estimations of X-Ray pulsar (XPR) luminosity are based on the measurement of X-Ray energy flux averaged over the observed pulse profile. This method has a number reasons of errors. In particular, the flux averaged over the pulse profile depends on the angles between the magnetic axis and the axis of rotation of the neutron star, as well as between the magnetic axis and the direction to the observer. Currently accurate determination of these angles is not possible, which leads to inevitable errors in determining the luminosity of X-ray pulsars. In this work, we analyzed the errors associated with this factor. The study was carried out for bright XPRs with an accretion column above magnetic poles of a neutron star (see e.g. [1]), which in this case is the source of radiation.

We have started with a simplest model of a pulsar with an accretion columns approximated by two one-dimensional sticks above magnetic pole of a star. Our model account for the effects of gravitational lensing and reprocessing of X-ray flux by the atmosphere of NS (see, e.g., [2], [3]) and makes it possible to obtain pulse profiles for an observer at a fixed value of the orientation angles. Then the angles were taken as random variables with a uniform distribution, thus the variance of the possible values of the observed luminosity and the probability of exceeding the true luminosity by an order of magnitude were calculated. Uniform and fractional-linear profiles of the brightness distribution over the column were taken into account. We have investigated the influence of different velocity profiles in a boundary layers of accretion column. We show that it is hard to get dramatic difference between the apparent and actual luminosity. However, there is still some uncertainty, which has to be taken into account.

\section{References}

1. M. M. Basko and R. A. Sunyaev, Monthly Notices of the Royal Astronomical Society, 175, 395, 1976, URL https://doi.org/10.1093/mnras/175.2.395.

2. J. Poutanen, A. A. Mushtukov, V. F. Suleimanov, S. S. Tsygankov, D. I. Nagirner, V. Doroshenko, and A. A. Lutovinov, ApJ, 777, 115, 2013.

3. A. A. Mushtukov, P. A. Verhagen, S. S. Tsygankov, M. van der Klis, A. A. Lutovinov, and T. I. Larchenkova, $M N R A S$, 474, 5425, 2018. 


\title{
Simulations of black hole observations with space-ground interferometers
}

\author{
E. Mikheeva, S. Repin, V.N. Lukash
}

helen@asc.rssi.ru

Astro Space Center of P.N. Lebedev Physical Institute, Profsoyuznaya 84/32, Moscow, 117991, Russia

We consider the black hole $(\mathrm{BH})$ shadow images which can be restored by data processing and image recovery procedures in future Space VLBI (Very Large Baseline Interferometry) missions. For sources SgrA*, M87* and M31* we consider three kinds of observation: the ground-based interferometer, space-ground interferometer with a satellite at low geocentric orbit, and space-ground interferometer with a satellite located in Lagrange point $L_{2}$. We report that the second case is the most preferable for the $\mathrm{BH}$ shadow observations among considered ones. The demo images for all the cases are presented.

Keywords: black hole physics, data analysis

DOI: $10.51194 /$ VAK2021.2022.1.1.112

\section{Introduction}

$\mathrm{BH}$ shadows have tiny angular sizes even for the closest ones. It means that an VLBI technique should play a key role in shadow observations. The ground-based interferometers are limited by the Earth diameter and their baselines cannot exceed $12800 \mathrm{~km}$. The idea of space-ground interferometer is very tempting and now it is under heat consideration and discussion. It can be realized in future space missions. One of them is the Millimetron Space Observatory (see [1]) with cooled 10-meter mirror operating in millimeter- and submillimeter bands, which is planned to be launched in the mid-2020s. The angular resolution of this instrument in the interferometric mode is assumed to be so high that we can clearly observe, in principle, the shadows of the BHs in many galaxies.

In the paper, we focus on the fundamental possibility of observing the shadow of a $\mathrm{BH}$ and neglect the nuances associated with the characteristics of specific observational instruments, as well as specific astronomical objects.

Main goal of the paper is to discuss the preferred satellite orbit, which should allow to obtain the shadow of a $\mathrm{BH}$ with high resolution quickly. Such a high-quality image is able to deliver the important information about the physical processes in the very vicinity of supermassive BHs, inhabiting the innermost parts of massive galaxies. This also can be applied to test the General Relativity in strong gravity field.

\section{Model of BH shadow}

We consider the spinning BH and its shadow in a simple geometry. We assume that a BH is described by the Kerr metric and its spin is close to maximal, $a=0.9981$. The spin axis is perpendicular to the view line of the distant observer. Behind the $\mathrm{BH}$ and far away there is a bright flat screen, which emits the quanta uniformly to a hemisphere (in solid angle $2 \pi$ ). If the screen plane is perpendicular to the view line of the distant observer then the BH shadow looks like the one shown in Fig. 1 (see details in [2]). To build the photon trajectories we solved the equations of motion under the General Relativity assumptions for each quantum. The system of six ordinary differential equations can be found in [3]. Ordinary differential equation solvers are included in many packages and freely distributed in Internet $\left(\right.$ see $\left.[4,5]^{\mathrm{a}}\right)$. The simulated image counts the trajectories of approximately 10 million quanta.

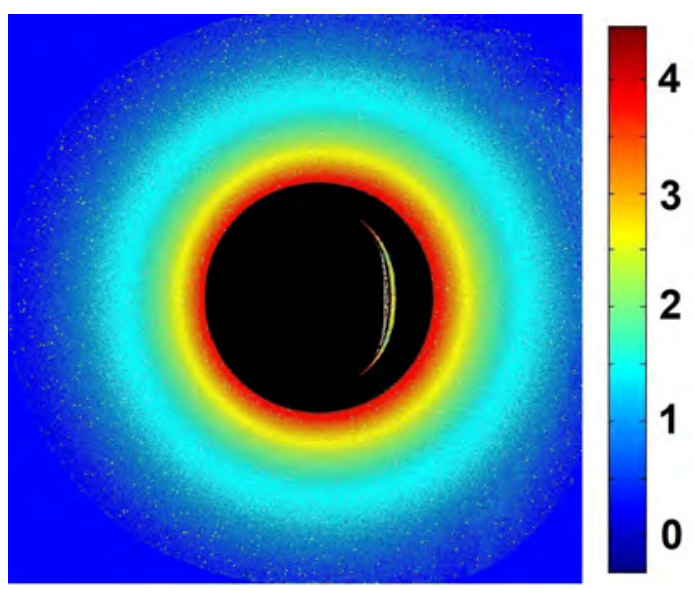

Figure 1: The shadow of a Kerr black hole (see details in the text). The intensity is presented in logarithmic scale and normalized by the brightness of the background screen.

ahttps://www.mcs.anl.gov/petsc/ 


\section{Images of Black Hole Shadows}

To reconstruct the images we used the well-established CLEAN procedure (see [6, 7]) which allows to extract the image from the Fourier coefficients on a finite number of $(u, v)$ plane points.

The result of our simulations for BH with mass and coordinates of SgrA* is presented at the Fig. 2. On the upper pannel (from left to right) one can see the coverage of the $(u, v)$ plane for ground-based interferometer, space-ground interferometer with a satellite at low geocentric orbit, and space-ground interferometer with a satellite located in Lagrange point $L_{2}$. On the bottom pannel the restored $\mathrm{BH}$ images are presented. Figures for other considered cases (M87* and M31*) and more details can be found in [8].
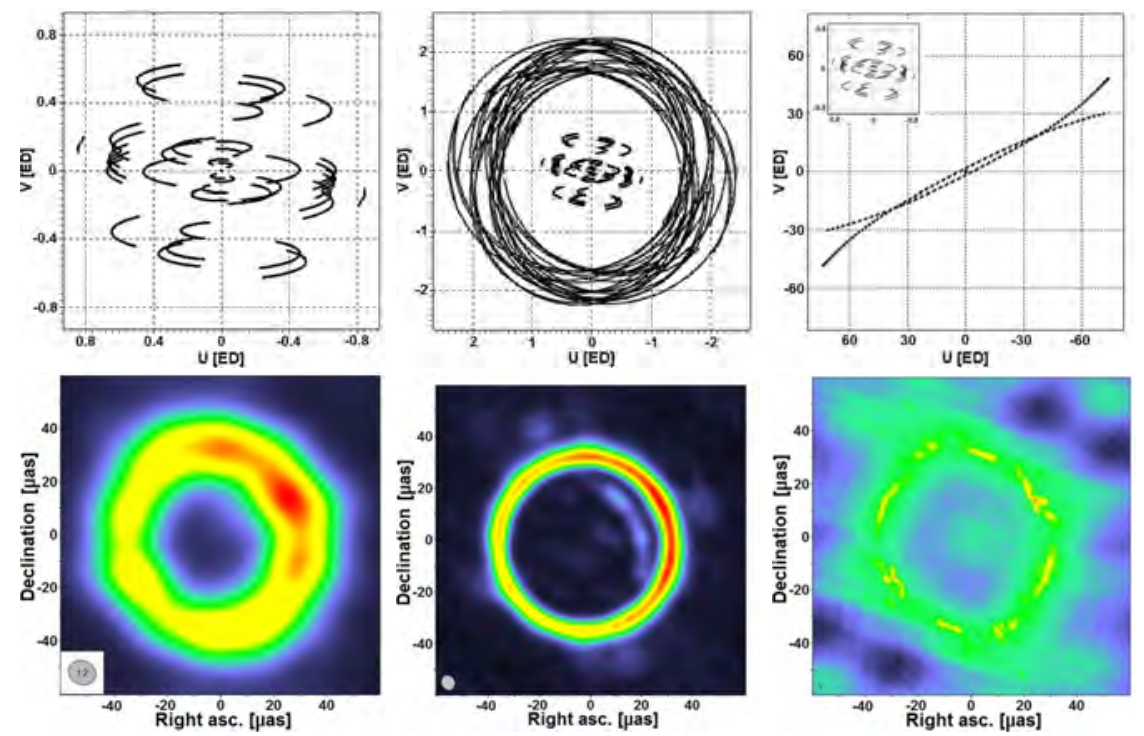

Figure 2: Top pannel. Coverage of the $(u, v)$ plane for three considered configurations. Bottom pannel. Restored shadow images of $\mathrm{BH}$ with mass and coordinates of $\mathrm{SgrA}^{*}$. The images are shown in conditional colors.

We found that the interferometer, which includes both the ground-based telescopes and a low-orbit satellite has the advantage in comparison with other reviewed cases. The key role here plays an ability to fill the $(u, v)$ plane with high density during the relatively short time (a day or week).

The ground-based arrays have an enough $(u, v)$ coverage, but the most objects cannot be observed all day long because they are below the horizon during some period.

A case of a high-orbit satellite differs from others. Indeed, its angular resolution is incredibly high. Theoretically, it is so high that we would be able to see the annuli inside the shadow area in Fig. 1 separately. However, we face here a problem with a poor $(u, v)$ plane coverage. The reliable image reconstruction is possible only with a dense enough $(u, v)$ plane coverage. One turn around the Earth of the satellite in Lagrangian point $L_{2}$ takes a full year. And even an increase in the duration of observations will not have a radical effect on the coverage of the $(u, v)$ plane. A spacecraft moving in the orbit near point $L_{2}$ every year almost repeats its track on the $(u, v)$ plane.

\section{Notes and Comments.}

This activity has been partly supported by Russian Foundation for Basic Research, grant 19-02-00199. The work was financially supported of the RAS project KP 19-270 'Questions of the origin and evolution of the Universe using methods of ground observations and space research". S.R. is very grateful to Dr. O.Sumenkova, Dr. R.Beresneva and Dr. O.Kosareva for the possibility of fruitful working on this problem.

\section{References}

1. N. S. Kardashev, I. D. Novikov, V. N. Lukash, S. V. Pilipenko, et al., Physics Uspekhi, 57, 1199-1228, 2014.

2. S. V. Repin, D. A. Kompaneets, I. D. Novikov, and V. A. Mityagina, arXiv e-prints, arXiv:1802.04667, 2018.

3. A. F. Zakharov and S. V. Repin, Astronomy Reports, 43, 705, 1999.

4. C. W. Gear and L. R. Petzold, SIAM Journal on Numerical Analysis, 21, 716, 1984.

5. S. Abhyankar, J. Brown, E. M. Constantinescu, D. Ghosh, B. F. Smith, and H. Zhang, arXiv e-prints, arXiv:1806.01437, 2018.

6. J. A. Högbom, A\&SA Sup., 15, 417, 1974.

7. U. J. Schwarz, $A \& A, \mathbf{6 5}, 345,1978$.

8. E. V. Mikheeva, S. V. Repin, and V. N. Lukash, Astronomy Reports, 64, 578, 2020. 


\title{
Magnetic fields of accretion disks and their vertical structure
}

\author{
M.V. Pashentseva ${ }^{1}$, E.A. Mikhailov ${ }^{1,2}$ \\ ${ }^{1}$ Faculty of Physics, Lomonosov Moscow State University, Moscow, Russia \\ ${ }^{2}$ P.N. Lebedev Physical Institute, Russia
}

Accretion disks formed around compact objects such as black holes, neutron stars and white dwarfs are of great interest in astrophysics. An important role in the processes of their evolution is played by magnetic fields, which can be associated, for example, with the transfer of angular momentum between different parts of objects. There are various approaches to studying these magnetic fields and explaining the mechanism of their generation. Different works showed that it can be generated by the dynamo mechanism which is based on differential rotation and alpha-effect. Previously, the no-z approximation which firstly was developed for galaxies, made it possible to obtain a number of results related to the structure of the magnetic field of the accretion disk and the nature of its evolution with time. At the same time, such phenomena as convection, which requires taking into account the vertical structure of the disk, cannot be studied in such simplified models. In addition, the half-thickness of the disk changes as the distance to the center increases. All this requires us to expand the representations used in modeling. In the case of galaxies, this approach is called the $R Z$-model. In this article, we plan to present the first results obtained using these approaches for accretion disks.

Keywords: Magnetic fields, accretion disks, dynamo theory, no-z model, RZ-model

DOI: $10.51194 /$ VAK2021.2022.1.1.113

\section{Introduction}

Magnetic fields of various astrophysical objects are of great interest from the point of view of magnetohydrodynamics and dynamo theory. One of the most interesting examples is connected with accretion disks $[1,2]$.

The equations of the dynamo are quite difficult to be solved, so different approximate models, taking into account the geometrical parameters of the object, are used. Some of them, which have been developed for the galactic disks, can be used for the accretion ones [3].

\section{No- $z$ approximation for accretion disks}

In the case of disks of galaxies, a lot of results are obtained using no- $z$ approximation [4]. It can also be used to study the magnetic fields of accretion disks.

In the framework of this approximation, we will describe the field by two main components [5].To make equations easier some dimensionless parameters are introduced: $R_{\alpha}$ (alpha-effect), $R_{\omega}$ (differential rotation) and $\lambda$ (half-thickness).

The equations for the magnetic field in the accretion disk are the following:

$$
\begin{gathered}
\frac{\partial B_{r}}{\partial t}=-R_{\alpha} B_{\varphi}\left(1-\frac{B^{2}}{B^{* 2}}\right)-\frac{\pi^{2} B_{r}}{4}+\lambda^{2}\left(\frac{\partial}{\partial r}\left(\frac{1}{r} \frac{\partial}{\partial r}\left(r B_{r}\right)\right)\right)-\frac{V_{0}}{r\left(1-\sqrt{\frac{r_{i n}}{r}}\right)}\left(\frac{\partial B_{r}}{\partial r}\right), \\
\frac{\partial B_{\varphi}}{\partial t}=-\frac{3 R_{\omega}}{2 r^{\frac{3}{2}}} B_{r}-\frac{\pi^{2} B_{\varphi}}{4}+\lambda^{2}\left(\frac{\partial}{\partial r}\left(\frac{1}{r} \frac{\partial}{\partial r}\left(r B_{\varphi}\right)\right)\right)-\frac{V_{0}}{r\left(1-\sqrt{\frac{r_{i n}}{r}}\right)}\left(\frac{\partial B_{\varphi}}{\partial r}\right) .
\end{gathered}
$$

These formulaes are written for dimensionless units: time is measured in units of typical dynamo time periods, and distances are measured in outer radius of the disk. The equations are solved for $r_{i n}<r<1$, where $r_{i n}$ is the inner radius. As for the boundary conditions we have:

$$
\left.B_{r}\right|_{r=r_{i n}}=\left.B_{r}\right|_{r=1}=\left.B_{\varphi}\right|_{r=r_{i n}}=\left.B_{\varphi}\right|_{r=1}=0 .
$$

\section{3. $R Z$ approximation for accretion disks}

Unfortunately, no-z approximation cannot be used for some objects, where we should study the vertical structure, or if the disk is quite thick. For this case we can take so-called $R Z$-approximation [6, 7], which presents the field as:

$$
\mathbf{B}=B \mathbf{e}_{\varphi}+\nabla\left(A \mathbf{e}_{\varphi}\right)
$$

The components are described by the equations:

$$
\begin{gathered}
\frac{\partial A}{\partial t}=R_{\alpha} z B\left(1-B^{2}\right)+\lambda^{2} \Delta A-V \frac{\partial A}{\partial r}, \\
\frac{\partial B}{\partial t}=R_{\omega} r\left(-\frac{3}{2} r^{\frac{5}{2}} \frac{\partial A}{\partial z}\right)+\lambda^{2} \Delta B-V \frac{\partial B}{\partial r} .
\end{gathered}
$$

The equations are solved for $-h(r)<z<h(r)$, where

$$
h(r)=h_{0} r^{9 / 8}\left(1-\sqrt{r_{i n} / r}\right)^{3 / 20} .
$$

As for the boundaries, we shall use the zero condition for $B$ and zero normal derivative condition for $A$.

If we compare these two models, we obtain interesting results (Fig.1). The field reaches different values and the radial structure is different, too. The 2D-plot is shown on Fig.2. 


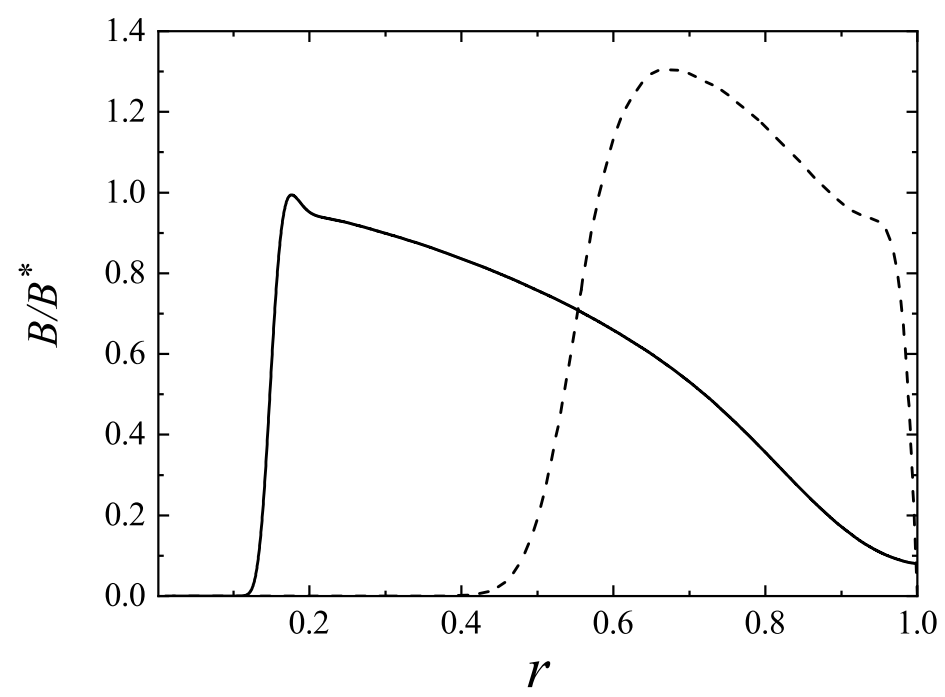

Figure 1: Comparison of two models. Dashed line shows RZ-model and solid line shows no-z model

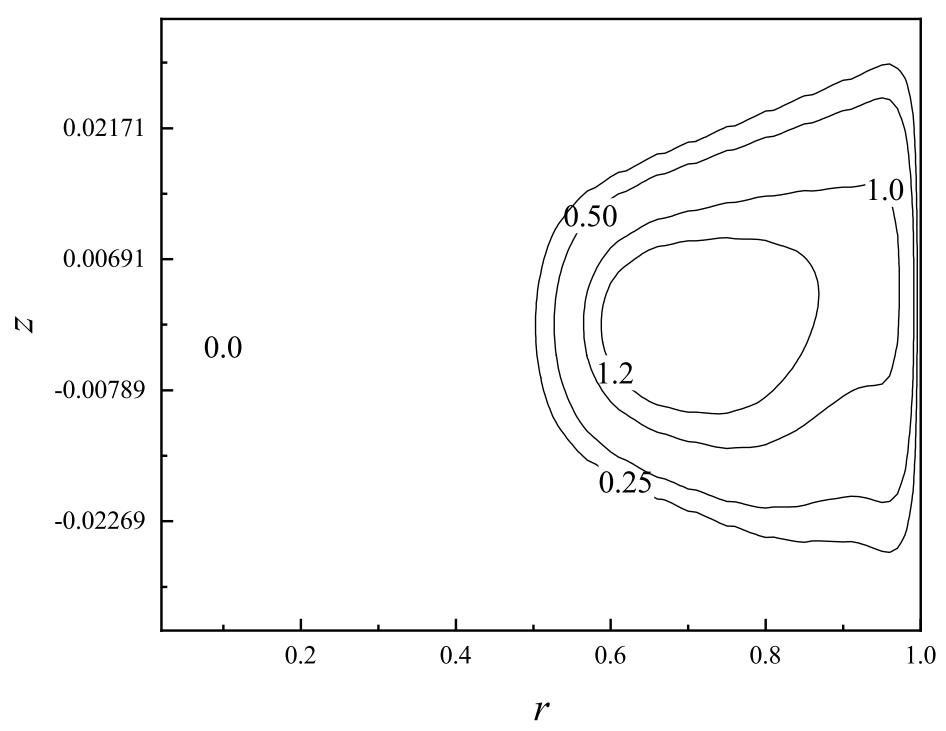

Figure 2: The dependence of the magnetic field on $r$ and $z$ coordinates for $R Z$-mode

\section{Conclusion}

We have studied the magnetic field of the accretion disks using different approaches. They are connected with no- $z$ model and $R Z$-approximation. They give qualitatively close results, but the values of the field and its structure is different.

The work of M.P. was supported by BASIS Foundation, project 21-1-1-4-4.

\section{References}

1. A. Brandenburg, A. Nordlund, R. F. Stein, and U. Torkelsson, ApJ, 446, 741, 1995.

2. A. Brandenburg and K. J. Donner, MNRAS, 288, L29, 1997.

3. D. V. Boneva, E. A. Mikhailov, M. V. Pashentseva, and D. D. Sokoloff, A $\mathscr{G A , 6 5 2 , ~ A 3 8 , ~} 2021$.

4. D. Moss, MNRAS, 275, 191, 1995.

5. N. I. Shakura and R. A. Sunyaev, A\&A, 500, 33, 1973.

6. E. A. Mikhailov, Astrophysics, 61, 147, 2018.

7. E. A. Mikhailov and V. V. Pushkarev, Research in Astronomy and Astrophysics, 21, 056, 2021. 


\title{
X-ray luminosity function of accreting neutron stars and black holes
}

\author{
K. Postnov ${ }^{1}$, A. Kuranov ${ }^{1}$, L. Yungelson ${ }^{2}$, M. Gil'fanov ${ }^{3}$ \\ kpostnov@gmail.com \\ ${ }^{1}$ Sternberg Astronomical Institute, 14 Universitetskij pr., Moscow, Russia, \\ ${ }^{2}$ Institute of Astronomy, RAS, 48 Pyatnitskaya str., Moscow, Russia, \\ ${ }^{3}$ Space Research Institute, RAS, 84/32 Profsoyuznaya str., Moscow, Russia
}

We model X-ray luminosity functions (XLF) of accreting neutron stars and black holes in $10^{35} \leq L_{X} \leq 10^{41}$ erg s ${ }^{-1}$ range in star-forming galaxies and galaxies with the initial star formation burst. XLFs are obtained by combining a fast generation of compact object+normal star population using the binary population synthesis code BSE and calculation of the subsequent detailed binary evolution by the MESA code.

Keywords: neutron stars, black holes, X-ray sources

DOI: $10.51194 /$ VAK2021.2022.1.1.114

\section{Introduction}

Stellar-mass field X-ray sources in galaxies are usually identified with binaries harbouring neutron stars (NS) or black holes $(\mathrm{BH})$ accreting matter from their companions. Here, we consider the X-ray luminosity functions (XLF) of model galaxies with an instantaneous star-formation burst as a proxy for elliptical galaxies and a model with constant star-formation rate (SFR) as a proxy for star-forming galaxies. In $\S 2$, we present our assumptions and method of computations, in $\S 3$ we show the results of computations and discuss them.

\section{Method of Computations}

The matter from the optical star in a binary system with NS or BH can be transferred to the compact object in different ways - via the Roche lobe overflow (RLOF) of the donor star or by the gravitational capture of the stellar wind from the optical companion. In the case of RLOF onto magnetized NSs, we take into account that the NS magnetic fields of about $10^{12} \lesssim B \lesssim 10^{14} \mathrm{G}$ modify the standard Shakura-Sunyaev disk accretion pattern. This can result in a super-Eddington X-ray luminosity $L_{X}$ from the accreting NS even before the Eddington accretion rate is attained [1]. This effect allows us to explain the existence of ultra-luminous X-ray sources (ULX) with NS without assuming a strong beaming of X-ray radiation, which is confirmed by the inconsistency of the strong beaming with the observed high pulsed fraction of emission from pulsating ULXs [2]. In the case of wind accretion, we include in the model the subsonic settling quasi-spherical accretion onto magnetized NS which is efficient if $\dot{M} \lesssim 4 \times 10^{16} \mathrm{~g} \mathrm{~s}^{-1}[3,4]$. Otherwise, both BH and NS experience supersonic BondiHoyle-Lyttleton accretion. We also take into account transiency of LMXB with unstable accretion disks [5].

To model the population of X-ray binaries, we applied a "hybrid" method: the evolution of binaries up to the formation of compact object (c.o.) is computed by an updated and modified rapid binary population synthesis (BPS) code BSE [6], while their further detailed evolution with mass transfer onto the c.o. is followed by the interpolation in the grid of evolutionary tracks precomputed by a regular stellar evolution code MESA (and references therein [7]).

In the BPS computations, the common envelope stage is treated using the $\alpha \lambda$ formalism $[9,10]$ with the "common envelope efficiency" $\alpha_{c e}=1$ and the binding energy parameter $\lambda$ from [11]. Among the most pressing issues in stellar evolution is the formation mechanism of NSs and BHs. It is still an unsolved 3D-problem. Ignoring the details of this process, as common in BPS, we adopt one of the 1D-approximations discussed in the literature; namely, we assume that the mass of c.o. is equal to $90 \%$ of the pre-SN CO core mass. A unique mass $1.4, M_{\odot}$ is assigned to all newborn NSs. The kick velocity received by nascent NS is assumed to follow a Maxwellian distribution with $\sigma=265$

$\mathrm{km} \mathrm{s}^{-1}$ [12]. An exception are NSs produced by the electron-capture SN (ECSN) from $(8-9), M_{\odot}$ progenitors, to which we assigned (quite arbitrarily) Maxwellian kicks with $\sigma=30 \mathrm{~km} \mathrm{~s}^{-1}$. Similar kicks reduced by the factor $M_{B H} / M_{O V}$, where $M_{O V}=2.5 M_{\odot}$ is the assumed maximum possible NS mass, are assigned to nascent BHs. A more detailed description of our assumptions and method can be found in $[13,1]$.

\section{Results and Discussion}

It is known that in the absence of a substantial emission from the central supermassive object, the X-ray luminosity of star-forming galaxies is dominated by high-mass X-ray binaries (HMXB) with initial donor masses exceeding (8-10), $M_{\odot}$, while in elliptical galaxies the binaries with the initial donor masses $\sim 1, M_{\odot}(\mathrm{LMXB})$ dominate. This allowed one to conclude that, owing to the difference in the evolutionary timescales, in the star-forming galaxies the number of X-ray sources should be proportional to the star formation rate (SFR), while in the ellipticals - to the total mass of the galaxy $M_{\star}$ (see, e.g., [8] and references therein). XLF (defined as $L_{X} \times \frac{d N}{d L_{X}}$ ) for the samples of galaxies of two types is shown in Fig. 1. The lines in this plot can be compared to the model XLF for galaxies with similar mass $\left(10^{10}, M_{\odot}\right)$, in which either constant continuous SFR or instantaneous burst of star formation were assumed (Fig. 2). Crudely, they may be taken as proxies for star-forming and elliptical galaxies, respectively.

Remarkably, the simplest assumptions on the c.o. masses and moderate kicks for BH and post-ECSN NSs allowed us to reproduce the slope of the observed XLF for HMXB close to -0.6. The broken shape of XLF for "ellipticals" reflects the "death" of systems with high and moderate mass donors soon after the star formation burst. Only stars with $\sim 1 M_{\odot}$ donors survived after $\sim 10$ Gyr of evolution. In "spirals", massive binaries producing HMXBs are born continuously and, 


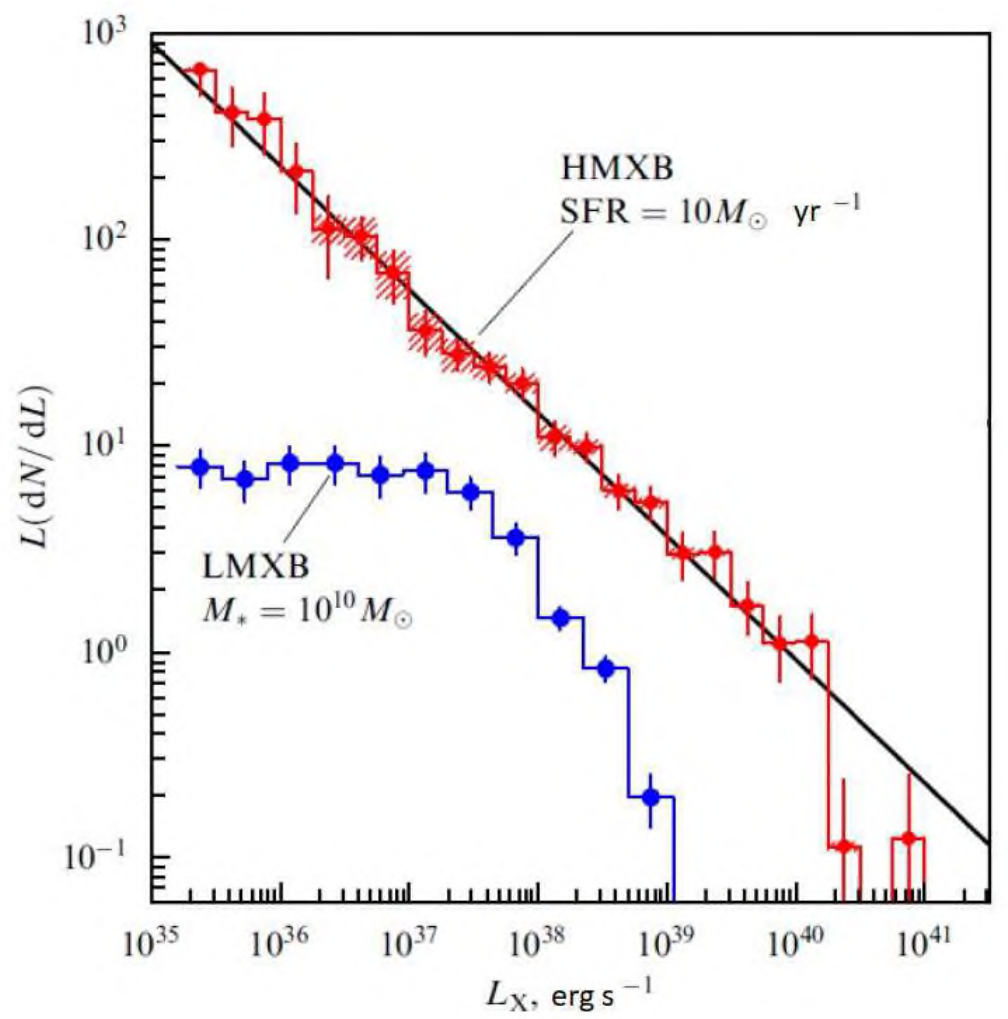

Figure 1: XLF of accreting binaries in star-forming and elliptical galaxies, HMXB and LMXB, respectively [8].
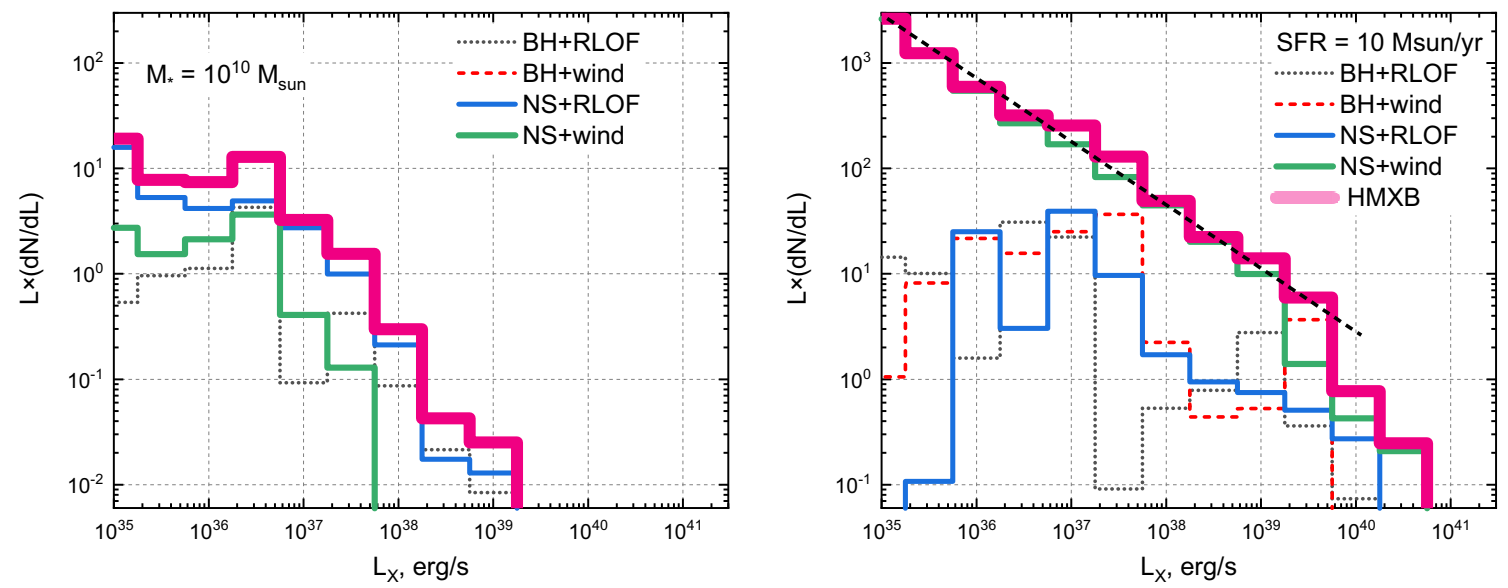

Figure 2: XLF for a $M_{\star}=10^{10}, M_{\odot}$ model galaxy with instantaneous burst of star formation (left panel) and star-forming galaxy of the same mass with constant SFR (right panel) at $\mathrm{t}=10$ Gyr. Separately is shown the contribution of sources with different c.o. and accretion patterns. Thick magenta line shows the total XLF. Dashed line indicates the slope (-0.6) of XLF for HMXB.

simultaneously, binaries with low- and moderate-mass donors born at earlier epochs replenish the population of X-ray sources. Note that in "spirals", XLF is always dominated by the systems with NS accreting from stellar wind, thanks to the permanent presence of sufficiently massive stars prior to RLOF (while the latter stage is, predominantly, unstable). In "ellipticals", at late stages, giant donor stars with strong winds have already finished their evolution. In this case, low-mass semidetached systems, in which the donors experienced RLOF on the main-sequence or shortly after TAMS and have low mass-loss rates, mainly contribute to XLF.

X-ray sources with $L_{X} \gtrsim 10^{39}$, erg s${ }^{-1}$ appropriate to the Eddington luminosity of $\gtrsim 10, M_{\odot}$ BH are classified as ULX. Figure 2 shows that in the populations of both types the objects with NS accretors should dominate. Non-detection of them in elliptical galaxies may suggest that the NS magnetic fields decayed or were buried by accretion long ago, preventing their appearance as ULX. 
The authors acknowledge support by RFBR grant 19-12-00790. L.Y. was partially supported by RFBR grant 19-0701198. The work of K.P. and A.K. is supported by the Interdisciplinary Scientific Educational School of Moscow University "Fundamental and applied space research".

\section{References}

1. A. G. Kuranov, K. A. Postnov, and L. R. Yungelson, Astronomy Letters, 46, 658, 2020.

2. A. A. Mushtukov, S. Portegies Zwart, S. S. Tsygankov, D. I. Nagirner, and J. Poutanen, MNRAS, 501, $2424,2021$.

3. N. Shakura, K. Postnov, A. Kochetkova, and L. Hjalmarsdotter, MNRAS, 420, 216, 2012.

4. N. I. Shakura, K. A. Postnov, A. Y. Kochetkova, L. Hjalmarsdotter, L. Sidoli, and A. Paizis, Astronomy Reports, 59, $645,2015$.

5. G. Dubus, J.-P. Lasota, J.-M. Hameury, and P. Charles, MNRAS, 303, 139, 1999.

6. J. R. Hurley, C. A. Tout, and O. R. Pols, MNRAS, 329, 897, 2002.

7. B. Paxton, P. Marchant, J. Schwab, E. B. Bauer, et al., ApJS, 220, 15, 2015.

8. M. R. Gil'fanov, Physics Uspekhi, 56, 714-722, 2013.

9. R. F. Webbink, ApJ, 277, 355, 1984.

10. M. de Kool, ApJ, 358, 189, 1990.

11. A. J. Loveridge, M. V. van der Sluys, and V. Kalogera, ApJ, 743, 49, 2011.

12. G. Hobbs, D. R. Lorimer, A. G. Lyne, and M. Kramer, MNRAS, 360, 974, 2005.

13. L. R. Yungelson, A. G. Kuranov, K. A. Postnov, and D. A. Kolesnikov, MNRAS, 496, L6, 2020. 


\title{
Change of the structure and physical properties of optical pulsations from transitional millisecond pulsar J1023+0038
}

\author{
artyom.tanashkin@gmail.com \\ ${ }^{1}$ Ioffe Institute, Saint Petersburg 194021, Russia \\ ${ }^{2}$ Special Astrophysical Observatory, Nizhniy Arkhyz, Russia \\ ${ }^{3}$ Kazan Federal University, Kazan, Russia \\ ${ }^{4}$ Institute of Physics, Prague, Czech Republic
}

A. Tanashkin ${ }^{1}$, G. Beskin ${ }^{2,3}$, S. Karpov ${ }^{2,3,4}$, V. Plokhotnichenko ${ }^{2}$, Yu. Shibanov ${ }^{1}$, D. Zyuzin ${ }^{1}$

In this paper we present some results of high temporal resolution multichannel optical observations of a transitional millisecond pulsar J1023+0038 with the panoramic photometer-polarimeter mounted on the 6-meter BTA telescope of Special Astrophysical Observatory. Besides the double-peaked optical pulsations with the neutron star rotation period, similar to those reported by other authors, during one of the nights we observed dramatic state change of the system. For about 230 seconds instead of typical double-peaked structure single peak with strongly increased amplitude was seen. Simultaneous observations in two channels also allowed us to detect energy redistribution in the spectrum of the system in this event. We also note chaotic orbital phase changes of the signal with maximum amplitude of about 25 seconds, corresponding to the linear shifts of about 3000 kilometers.

Keywords: stars: neutron; pulsars: individual (PSR J1023 + 0038); accretion, accretion disks

DOI: 10.51194 /VAK2021.2022.1.1.115

\section{Introduction}

Transitional pulsars constitute a subclass of millisecond pulsars in close binary systems which demonstrate switches between two distinctive regimes of interaction of a neutron star with its companion: episodes of intense accretion, during which the system is seen as a low mass X-ray binary, are followed by quiescent states observed as millisecond radio pulsars. The archetypal member of this class, J1023+0038, shows extremely rich variety of observational properties. One of the most intriguing is millisecond optical pulsations, first found by [1] and confirmed by a number of subsequent studies [2, 3]. The pulsing optical emission is characterized by double-peaked structure with variable amplitudes of the peaks, reaching about $1 \%$ in maximum. Simultaneous optical and X-ray observations revealed strong connection between optical pulsations and behaviour of the system in X-rays [3].

\section{Observations}

We observed PSR J1023+0038 during seven nights between Feb 172017 and Jan 52020 with the 6-m telescope BTA of the Special Astrophysical Observatory of the Russian Academy of Sciences (SAO RAS). The observations were carried out using the panoramic photometer-polarimeter operating in the dual-channel regime [4]. This provides the advantage of collecting photons simultaneously with the two MCP-based panoramic photon counters, the "red" one with the GaAs photocathode sensitive at the $5640 \AA$ effective wavelength, and the "blue" one with the multi-alcali photocathode sensitive at the 4170 $\AA$ effective wavelength. All the photons in a $10^{\prime \prime} \times 10^{\prime \prime}$ diaphragm around the object were detected and registered with an effective temporal resolution $1 \mu \mathrm{s}$. The overall length of the collected data is about 10 hours.

The times of arrival of every photon were converted to the Solar system barycenter. In order to search for the periodic signal one also should account for the orbital motion of the neutron star in the binary system. It was shown in previous studies that the system exhibits rather complex orbital behaviour, so we performed standard analysis (see, e.g., [5]) to find the best orbital phase of the emitting area for each data segment (see the next section).

\section{Results and discussion}

In the majority of our data we see double-peaked pulsations with variable amplitudes, similar to those reported in previous studies (see Fig. 1). However, during the Nov 152017 night we observed very strange behaviour of the object. For about 230

Table 1: Summary of the BTA observations.

\begin{tabular}{ccccc}
\hline \hline Night & Date & Start, UT & Duration, s & Orbital phases \\
\hline 1 & 17.02 .2017 & $19: 19: 16$ & 3800 & $0.31-0.54$ \\
2 & 14.11 .2017 & $02: 00: 21$ & 3200 & $0.64-0.83$ \\
3 & 15.11 .2017 & $00: 54: 19$ & 6700 & $0.46-0.91$ \\
4 & 07.04 .2018 & $21: 33: 51$ & 3800 & $0.68-0.89$ \\
5 & 31.01 .2019 & $23: 55: 27$ & 6000 & $0.54-0.89$ \\
6 & 04.01 .2020 & $00: 22: 25$ & 8700 & $0.83-0.33$ \\
7 & 05.01 .2020 & $02: 28: 36$ & 3300 & $0.32-0.38$ \\
\hline \hline
\end{tabular}




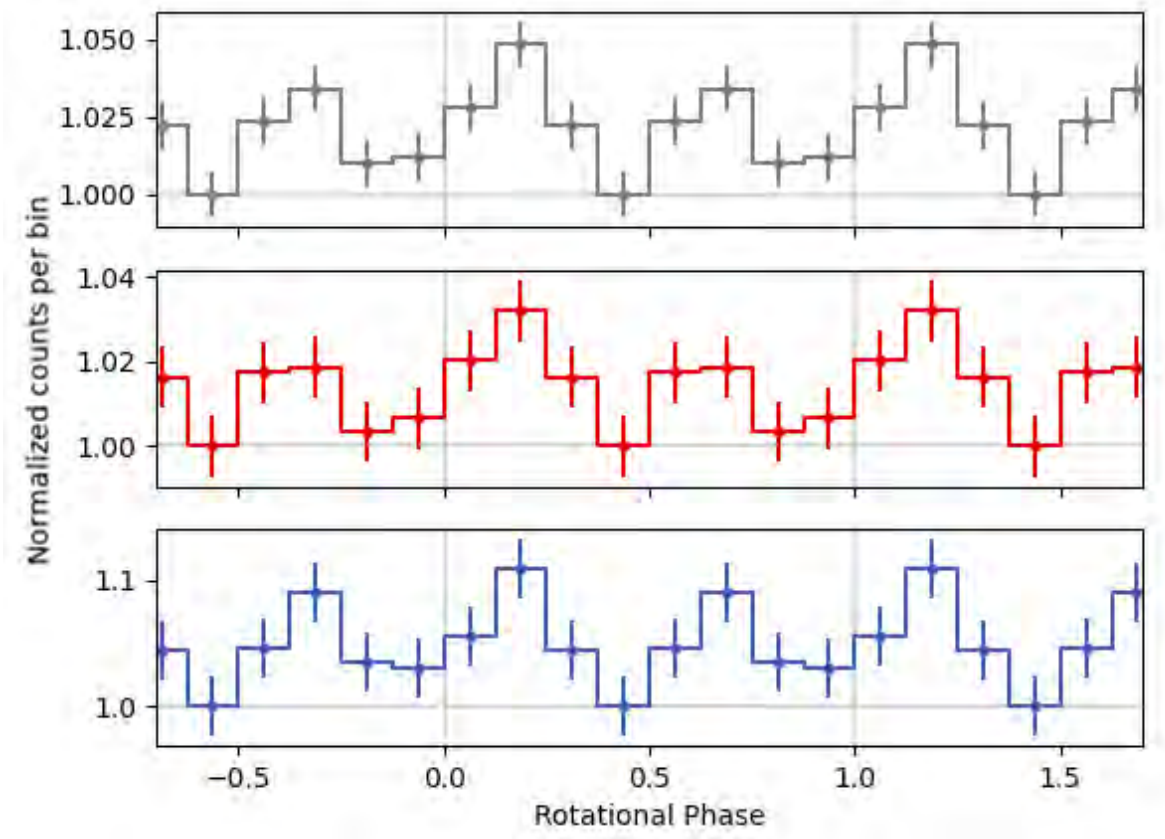

Figure 1: Typical double-peaked pulse profile observed in the major part of our data. Top, middle and bottom panels show both channels joined, only red, and only blue channels, respectively.

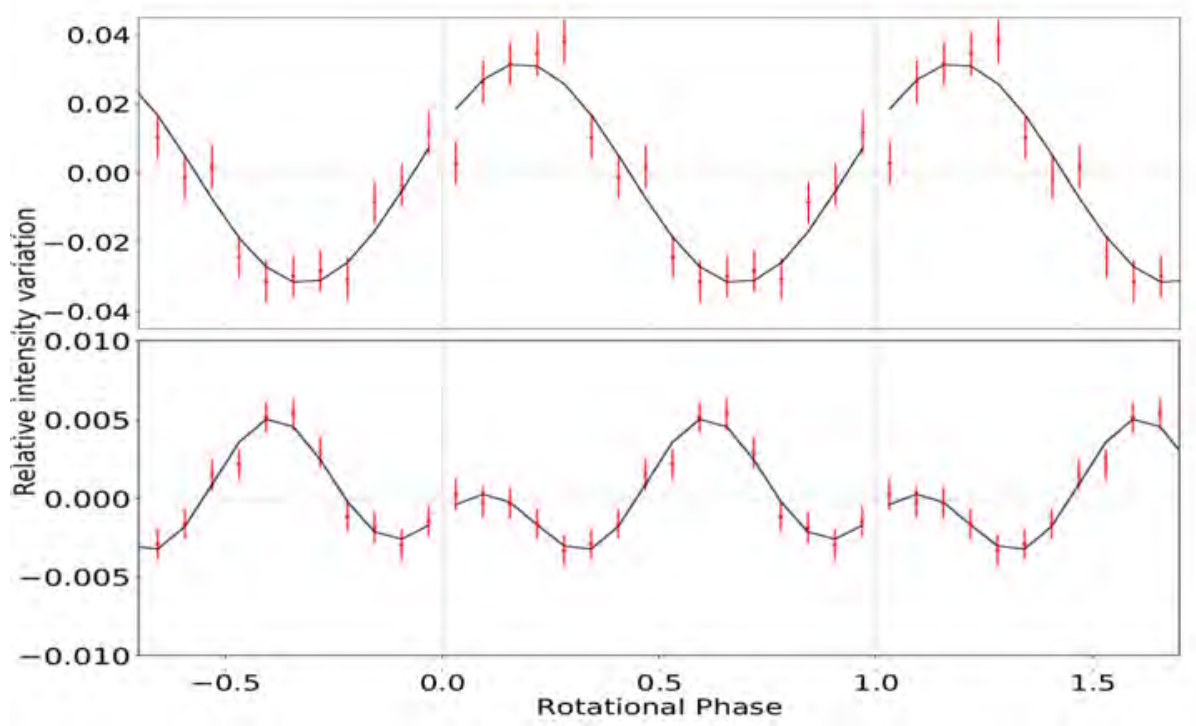

Figure 2: Pulse profiles in the "red" channel during the 230-s long event (upper panel) and the rest of the night (lower panel).

seconds the "standard" double-peaked pulsations (Fig. 2, lower panel) were replaced by much stronger single-peaked ones (Fig. 2, upper panel). The profile became nearly sinusoidal and the distribution of energy in the spectrum of the pulsing component became softer. Significant flaring activity was also seen on the light curve during the event.

Another interesting feature seen in our data is the chaotic orbital phase changes of the area responsible for the pulsing emission. This peculiarity was noted in previous studies, but the characteristic timescales were thought to be much longer than a few days. In contrast to this, we observe such changes on timescales of one day and in some cases even a few hours. Maximum orbital shifts of about 25 seconds correspond to linear displacements up to 3000 kilometers. This fact indicates that the emission area can not be bound to the neutron star; in particular, it can not be located near the light cylinder of the neutron star, as proposed by some models. More probable scenario seems to be the one in which various clouds of matter are scattered along the orbit and spontaneously generate physical conditions favorable for the pulsar wind energy to be re-emitted in the optical band. 


\section{Acknowledgements}

This work was supported within the framework of the government contract of the Special Astrophysical Observatory of the Russian Academy of Sciences in its part entitled "Conducting Fundamental Research".

\section{References}

1. F. Ambrosino, A. Papitto, L. Stella, F. Meddi, et al., Nature Astronomy, 1, 854, 2017.

2. L. Zampieri, A. Burtovoi, M. Fiori, G. Naletto, A. Spolon, C. Barbieri, A. Papitto, and F. Ambrosino, MNRAS, 485, L109, 2019.

3. A. Papitto, F. Ambrosino, L. Stella, D. F. Torres, et al., arXiv e-prints, arXiv:1904.10433, 2019.

4. V. L. Plokhotnichenko, G. M. Beskin, V. G. de Bur, S. V. Karpov, D. A. Bad'in, Z. V. Lyubetskaya, A. P. Lyubetskij, and V. V. Pavlova, Astrophysical Bulletin, 64, 308, 2009.

5. A. Jaodand, A. M. Archibald, J. W. T. Hessels, S. Bogdanov, C. R. D’Angelo, A. r. Patruno, C. Bassa, and A. T. Deller, ApJ, 830, 122, 2016. 


\title{
Modelling of vertical structure of accretion discs around neutron stars and black holes
}

\author{
A.S. Tavleev, G.V. Lipunova, K.L. Malanchev \\ tavleev.as15@physics.msu.ru
}

\begin{abstract}
M.V. Lomonosov Moscow State University, Sternberg Astronomical Institute, Universitetsky pr. 13, Moscow, 119234, Russia
\end{abstract}

\section{DOI: 10.51194 /VAK2021.2022.1.1.116}

Bursts in X-ray binaries are associated with instability in an accretion disk around a compact object (neutron star or black hole). To examine the stability of the disc, it is necessary to obtain the dependences between the physical parameters (for example, the pressure and energy flux) in the disk. These dependences are found from the solution of the equations of the vertical structure of the disc [1]:

$$
\begin{aligned}
\frac{1}{\rho} \frac{d P}{d z} & =-\omega_{\mathrm{K}}^{2} z=-\frac{G M}{r^{3}} z, \\
\frac{d \Sigma}{d z} & =-2 \rho, \\
\frac{\mathrm{d} \ln T}{\mathrm{~d} \ln P} & \equiv \nabla=\left\{\begin{array}{l}
\nabla_{\mathrm{rad}}, \nabla_{\mathrm{rad}}<\nabla_{\mathrm{ad}}, \\
\nabla_{\mathrm{conv}}, \nabla_{\mathrm{rad}}>\nabla_{\mathrm{ad}},
\end{array}\right. \\
\frac{d Q}{d z} & =\frac{3}{2} \omega_{\mathrm{K}} w_{r \varphi}=\frac{3}{2} \omega_{\mathrm{K}} \alpha P, \\
z & \in\left[0, z_{0}\right] .
\end{aligned}
$$

Here (1) is the equation of hydrostatic equilibrium, (2) is the equation for mass coordinate, (3) is the equation for temperature gradient (convective of radiative), (4) is the equation of viscous heating ( $\alpha$-prescription [2] of viscosity is used). Equations are supplemented by the equation of state and opacity law. System has one free parameter $z_{0}$, half-thickness of the disc.

Python 3 code is developed to solve system (1-4) numerically. Code allows us to change the equation of state, opacity law and chemical composition, including tabular values from MESA [3].
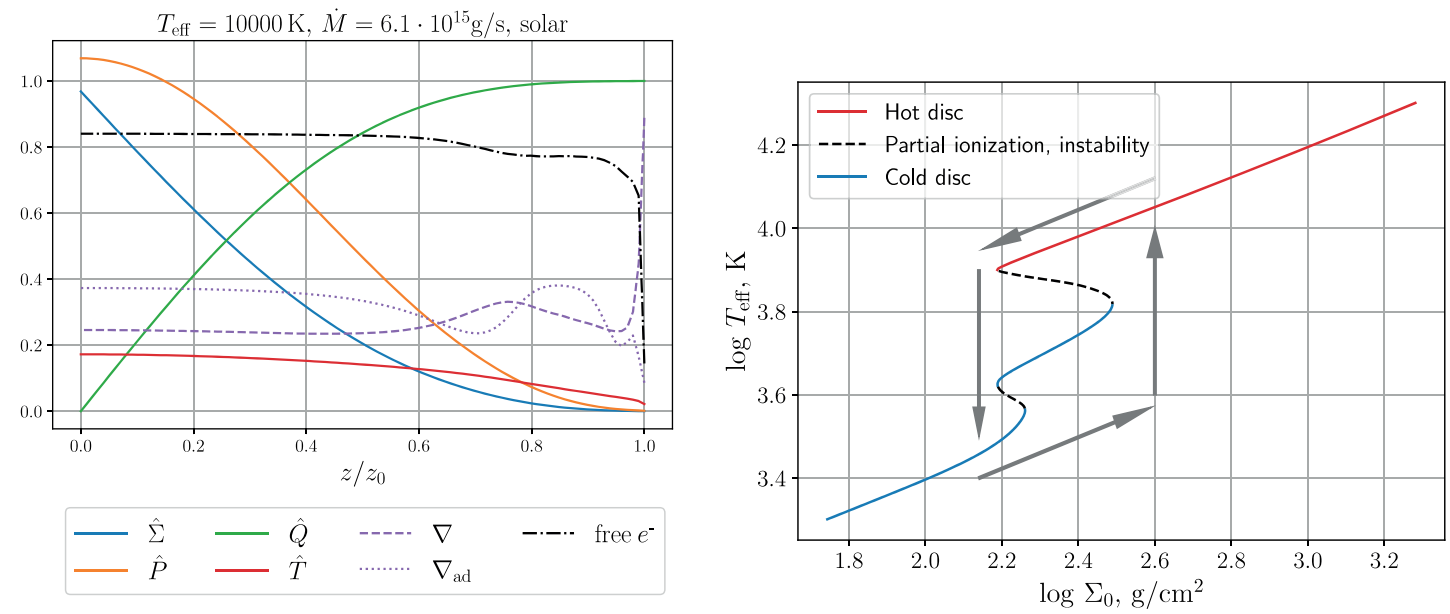

Figure 1: Shown on the left side is the vertical structure for $M=6 M_{\odot}, \alpha=0.01, r=10^{10} \mathrm{~cm}$ and tabular opacity. Also shown are temperature gradient $\nabla$, adiabatic gradient $\nabla_{\text {ad }}$ and ionization parameter. Other quantities are normalized to their characteristic values. Structure is calculated for an unstable disc $\left(T_{\text {eff }}=10000 \mathrm{~K}\right)$ with solar composition. One can see, that the unstable disc has convective regions (where $\nabla_{\text {rad }}>\nabla_{\text {ad }}$ ). On the left side shown is the stability curve (S-curve) obtained for $M=6 M_{\odot}, \alpha=0.01, r=10^{10} \mathrm{~cm}$ and tabular opacities with solar chemical composition. Also the limit cycle instability is showed by arrows. The cold disc region, the region of partial ionization (in which the instability takes place) and the region of the hot disc are marked by different styles. 
Fig. 1 shows the typical vertical structure of unstable part of the disc (where partial ionization of hydrogen occurs) and Stability curve (S-curve) of disc, where the branch with negative slope is unstable. The presence of unstable branch (due to partial ionization of hydrogen) leads to limit instability cycle in binary stars (shown in Fig. 1 by arrows).

Our calculations can improve the accuracy of the modelling of X-ray binary outbursts. Code is open-source ${ }^{\mathrm{a}}$.

\section{Acknowledgements}

This work was supported by the RSF grant 21-12-00141.

\section{References}

1. N. I. Shakura, G. V. Lipunova, K. L. Malanchev, et al., Accretion flows in astrophysics (2018).

2. N. I. Shakura and R. A. Sunyaev, $A \& 4 A, \mathbf{5 0 0}, 33,1973$.

3. B. Paxton, L. Bildsten, A. Dotter, et al., ApJS, 192, 3, 2011. 


\title{
Confirmation of intermediate-mass black holes candidates with $\mathrm{x}$-ray observations
}

\author{
V. Toptun ${ }^{1,3}$, I. Chilingarian ${ }^{1,2}$, K. Grishin ${ }^{1}$, I. Katkov ${ }^{1,4}$, I. Zolotukhin ${ }^{1}$, V. Goradzhanov ${ }^{1,3}$, \\ M. Demianenko ${ }^{1,5}$, I. Kuzmun ${ }^{3}$ \\ ${ }^{1}$ Sternberg Astronomical Institute, Lomonosov Moscow State University, Universitetsky pr. 13, Moscow 119234, Russia \\ ${ }^{2}$ Center for Astrophysics - Harvard and Smithsonian, 60 Garden Street MS09, Cambridge, MA 02138, USA \\ ${ }^{3}$ Department of Physics, M.V. Lomonosov Moscow State University, 1 Vorobyovy Gory, Moscow 119991, Russia \\ ${ }^{4}$ Center for Astro, Particle, and Planetary Physics, NYU Abu Dhabi, PO Box 129188 Abu Dhabi, UAE \\ ${ }^{5}$ Moscow Institute of Physics and Technology, Institutskiy per. 9, Dolgoprudny 141701, Russia
}

The origin of supermassive black holes $(\mathrm{SMBH})$ in galaxy centers still remains uncertain. There are two possible ways of their formation - from massive $\left(10^{5}-10^{6} M_{\odot}\right)$ and low-mass $\left(100 M_{\odot}\right)$ BH nuclei. The latter scenario should leave behind a large number of intermediate mass black holes (IMBH, $\left.10^{2}-10^{5} M_{\odot}\right)$. The largest published sample of bona-fide IMBHpowered AGN contains 10 objects confirmed in X-ray. Here we present a new sample of 15 bona-fide IMBHs, obtained by confirming the optically selected IMBH candidates by the presence of radiation from the galactic nucleus in the X-ray range, which increases the number of confirmed IMBHs at the centers of galaxies by 2.5 times. In the same way, 99 black holes with masses of $2 \cdot 10^{5}-10^{6} M_{\odot}$ were confirmed. The sources of X-ray data were publicly available catalogs, archives of data, and our own observations on XMM-Newton, Chandra and Swift. The Eddington coefficients for $30 \%$ of the objects from both samples turned out to be close to critical, from 0.5 to 1 , which is an unusually high fraction. Also for the first time for light-weight SMBH the correlations between the luminosity in the [OIII] emission line or the broad component of the $\mathrm{H} \alpha$ line and the luminosity in the X-ray range were plotted.

Keywords: supermassive black holes, active galaxies, $x$-ray observations

DOI: $10.51194 /$ VAK2021.2022.1.1.117

\section{Introduction}

Massive black holes in galaxy centres are believed to co-evolve with the spheroids of their hosts [1], growing via coalescences during galaxy mergers [2] and by gas accretion [3]. The discovery of quasars in the early Universe $(z>6.3$, only 750-900 Myr after the Big Bang) hosting super-massive black holes (SMBHs) as heavy as $10^{10} M_{\odot}[4,5]$ cannot be explained by gas accretion onto stellar mass black hole seeds $\left(<100 M_{\odot}\right)$ alone. Alternatively, the rapid inflow and subsequent direct collapse of gas clouds [6, 7] can form massive seeds $\left(\mathrm{M}>10^{5}-10^{6} M_{\odot}\right)$. The latter scenario solves the SMBH early formation puzzle butleads to a gap in the present-day black hole mass function in the IMBH regime $\left(100<M_{\mathrm{BH}}<2 \cdot 10^{5} M_{\odot}\right)$, whereas stellar mass seeds should leave behind a large number of IMBHs. Therefore, the elusive IMBH population holds a clue to the understanding of SMBH formation. Now the largest published sample of bona-fide IMBH-powered AGN contains 10 objects confirmed in X-ray.

\section{Sample description}

The work is based on a sample of 1928 galaxies with active nuclei with virial masses of black holes up to $10^{6} M_{\odot}, 305$ of which are IMBHs with masses less than $2 \cdot 10^{5} M_{\odot}$. The sample was obtained as part of the work on the article [8] by analyzing the spectra of about a million galaxies from the SDSS DR7 [9] survey. A broad component of the $H_{\alpha}$ emission line was identified in the spectra, and the virial masses of the central black holes were calculated from it's parameters. A method for selecting BHs of intermediate masses and light-weight SMBH was proposed in the work [8]. Also only galaxies with well-measured central BH masses and reliable broad component in lines were selected, which ratio of the $\mathrm{BH}$ mass to the mass error is more than 3, important lines do not overlay on the sky lines due to the redshift, and falls into the AGN or transition region on the BPT [10] diagnostic diagram.

\section{Data analysis methods}

\subsection{X-ray data collection, calibration and analysis}

To confirm the AGN nature of an object it is necessary to detect the X-ray emission, produced by the accretion disk of the black hole. X-ray data were obtained from the Chandra Source Catalog 2, 4XMM-DR10, Second Swift XRT Point Source Catalog, Second ROSAT all-sky survey, from the x-ray data archives as Chandra Data Archive and XMM-Newton science archive and our own observations at XMM-Newton, Chandra and Swift. For calibration of raw observational data and extraction of spectra was used special software: Science Analysis System (SAS) for XMM-Newton data, Chandra Interactive Analysis of Observations for Chandra and XSelect for Swift.

The next important step was extracting the spectrum of the object and fitting its parameters. Using the parameters and form of the spectrum we can once again confirm its AGN nature. Spectral data was fitted with powerlaw and photoelectric absorption models with XSPEC and Sherpa packages in Python 3. It turned out that most of our objects have a photon index over 2, that is typical for AGN. 

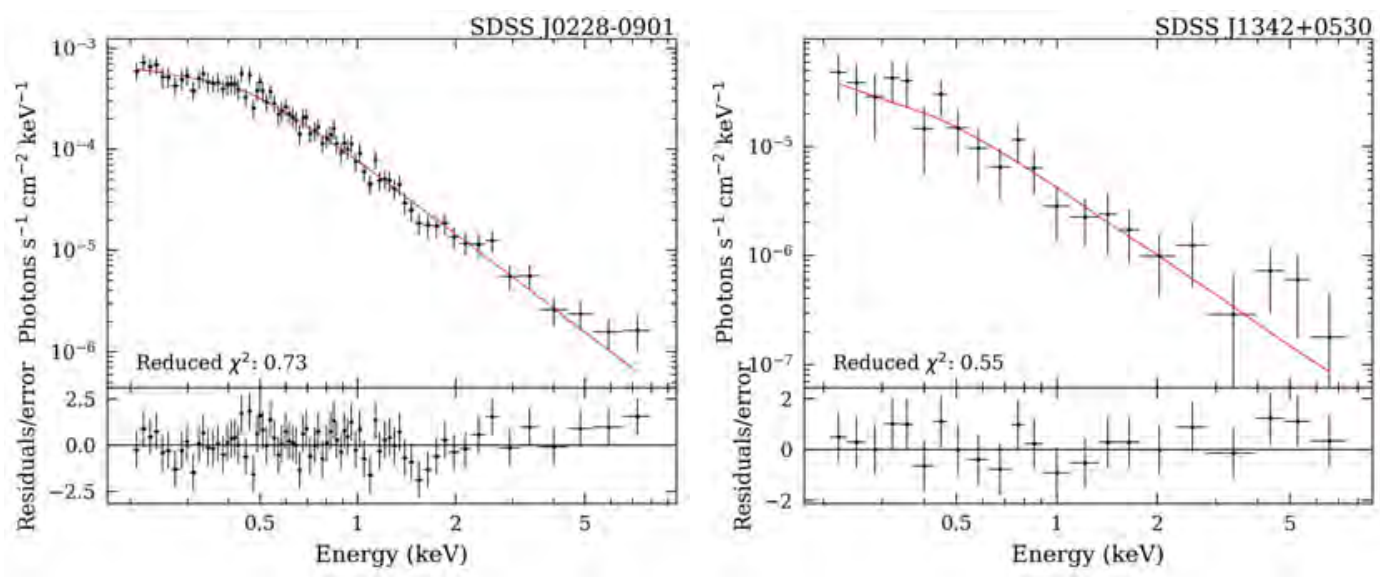

Figure 1: Examples of spectra. The model is shown with a red line.

\subsection{X-ray luminosity of the stellar population}

It is worth remembering that the object's X-ray luminosity should be higher than expected from the stellar population of the host galaxy. X-ray stellar population mainly consists of low-mass and high-mass X-ray binaries. Their contribution to luminosity can be calculated using the following relations

$$
\begin{gathered}
L_{\mathrm{HX}}^{\mathrm{gal}}=\alpha M_{*}+\beta S F R \\
\alpha=(9.05 \pm 0.37) \times 10^{28} \operatorname{ergs~s}^{-1} M_{\odot}^{-1} \\
\beta=(1.62 \pm 0.22) \times 10^{39} \operatorname{ergs~}^{-1}\left(M_{\odot} \mathrm{yr}^{-1}\right)^{-1}
\end{gathered}
$$

\subsection{Eddington luminosities}

The luminosity of the accretion disk directly depends on this accretion rate. Therefore, in order to understand how high the rate of accretion onto a black hole is, it is necessary to calculate the Eddington ratio. The masses are known from optical observations and the Eddington luminosity is easily calculated

$$
L_{\text {edd }}=\frac{4 \pi G M m_{p} c}{\sigma_{T}} \approx 10^{38} \frac{M}{M_{\odot}} \operatorname{ergs} \cdot s^{-1}
$$

The bolometric luminosity values are determined from the luminosity in the X-ray range through the bolometric correction coefficient defined as

$$
K\left(L_{X}\right)=\frac{L_{\text {bol }}}{L_{X}}=15.33\left[1+\left(\frac{\log \left(L_{X} / L_{\odot}\right.}{11.48}\right)^{16.20}\right]
$$

\section{Results and conclusions}

\subsection{New IMBHs with X-ray confirmation}

From the sample of 1928 candidates for low-mass black holes, 124 black holes with masses less than $10^{6} M_{\odot}$ were confirmed in the X-ray range, of which 24 are intermediate masses black holes with masses $M_{\mathrm{BH}}<2 \cdot 10^{5} M_{\odot}$, and their x-ray luminosities were calculated. For 55 objects from this sample, in addition to the luminosity, the parameters of their X-ray spectra were determined. Thus, confirmed intermediate-mass black holes sample was increased by a factor of 2.5 , and this number of IMBHs is a strong argument for the formation of supermassive black holes from low-mass progenitors. Most of the sources have "soft" X-ray spectra - for 39 of 55 objects the photon index is $\Gamma>2$. For a large number of objects the Eddington ratio turned out to be very high. Thus, for 39 of $123 \mathrm{BHs}$ it is more than $30 \%$. This points on very high accretion rates on many IMBH.

\subsection{Correlation between X-ray luminosity and luminosity in emission lines}

For supermassive black holes have found a correlation between the luminosity in the X-ray range and the optical emission lines [OIII] and $H_{\alpha}[11,12,13]$. We verified this correlation for light-weight SMBH and IMBH for the first time. The fluxes in lines were obtained together with the black holes masses by processing optical spectra. Correlations were approximated by linear regression

$$
\log \left(L_{\text {emis. Line }}\right)=a \cdot \log \left(L_{X}\right)+b
$$

The results of this approximation are shown in Table 1.

It turned out that for low-mass black holes the slope of the regression is much smaller than for the sample of supermassive black holes. It could be caused by a change in the geometry of the region of X-ray radiation — this and other 
Table 1: Approximation results

\begin{tabular}{|c|c|c|c|}
\hline Line & $\mathrm{a}$ & $\mathrm{b}$ & error \\
\hline$[\mathrm{OIII}]$ & $0.339 \pm 0.045$ & $25.950 \pm 1.898$ & 0.475 \\
$H_{\alpha}$ & $0.344 \pm 0.039$ & $25.680 \pm 1.642$ & 0.411 \\
\hline
\end{tabular}

possible hypotheses requires further research. However, it is already clear that these correlations are extremely useful — it can be used to estimate the X-ray luminosity for calculating the exposures necessary to accumulate a sufficient number of photons for spectral analysis.
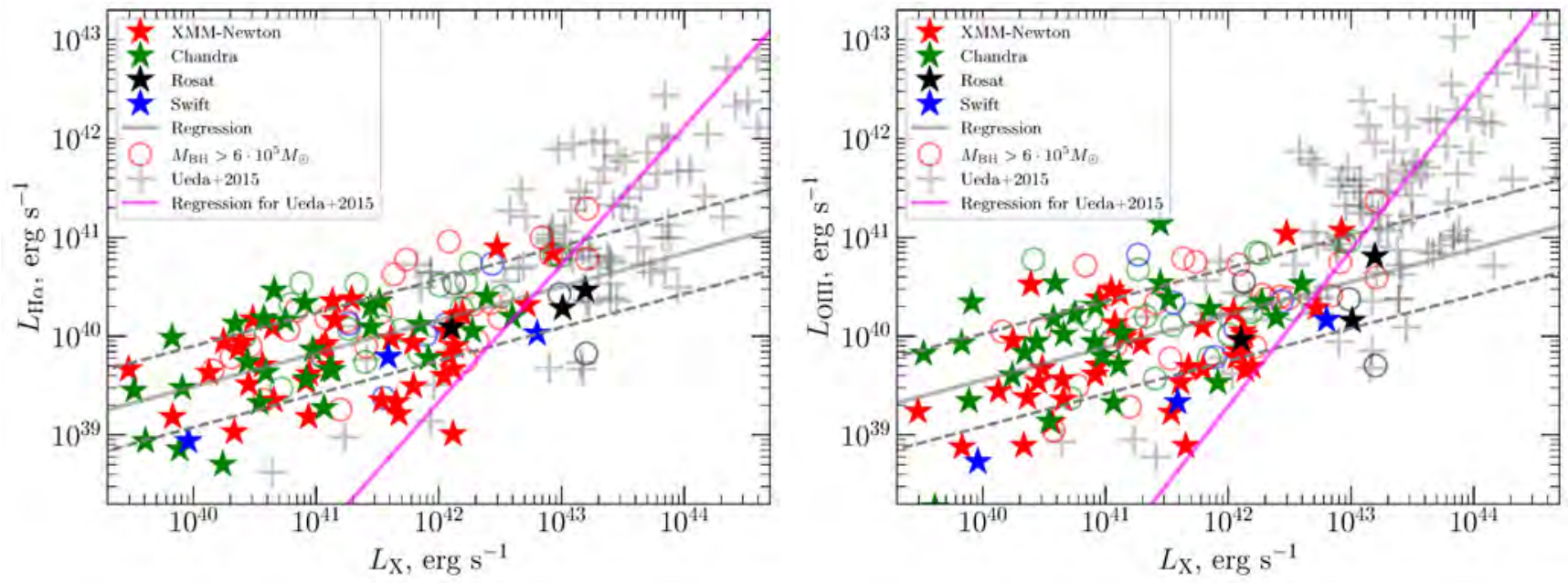

Figure 2: Correlations between the luminosity in the [OIII] emission line or the broad component of the H $\alpha$ line and the luminosity in the $\mathrm{X}$-ray range

\subsection{Conclusions about the ways of black holes formation}

The key result of this study is a significant increase in sample of IMBH, and this confirms that SMBH grew from their lower mass progenitors. Also we show that at low mass regime the number of objects emitting close to the Eddington limit is unexpectedly large. This indicates that $\mathrm{BH}$ can grow rapidly, including due to accretion of matter, not only due to merging and host galaxies bulges growth.

Acknowledgements. This project is supported by the RScF Grant 17-72-20119.

\section{References}

1. J. Kormendy and L. C. Ho, ARA $₫ A, \mathbf{5 1}, 511,2013$.

2. D. Merritt and M. Milosavljević, Living Reviews in Relativity, 8, 2005.

3. M. Volonteri, Science, 337, 544, 2012.

4. D. J. Mortlock, S. J. Warren, B. P. Venemans, M. Patel, et al., Nature, 474, 616, 2011.

5. X.-B. Wu, F. Wang, X. Fan, W. Yi, et al., Nature, 518, 512, 2015.

6. A. Loeb and F. A. Rasio, ApJ, 432, 52, 1994.

7. M. C. Begelman, M. Volonteri, and M. J. Rees, MNRAS, 370, 289, 2006.

8. I. V. Chilingarian, I. Y. Katkov, I. Y. Zolotukhin, K. A. Grishin, Y. Beletsky, K. Boutsia, and D. J. Osip, ApJ, 863, $1,2018$.

9. K. N. Abazajian, J. K. Adelman-McCarthy, M. A. Agüeros, S. S. Allam, et al., ApJS, 182, 543-558, 2009.

10. J. A. Baldwin, M. M. Phillips, and R. Terlevich, PASP, 93, 5, 1981.

11. T. M. Heckman, A. Ptak, A. Hornschemeier, and G. Kauffmann, ApJ, 634, 161, 2005.

12. Y. Ueda, Y. Hashimoto, K. Ichikawa, Y. Ishino, et al., ApJ, 815, 1, 2015.

13. C. Jin, M. Ward, and C. Done, MNRAS, 422, 3268, 2012. 
The Sun 


\title{
Observations at near-infrared wavelengths at the Tower Solar Telescope TST-2 in Crimean Astrophysical Observatory
}

\author{
O. Andreeva, V. Malashchuk \\ olga@craocrimea.ru \\ Crimean Astrophysical Observatory RAS (CrAO RAS), Nauchny, Crimea, Russia
}

DOI: $10.51194 /$ VAK2021.2022.1.1.118

Near-infrared spectroscopy is a modern instrumental method for quantitative and qualitative analysis of various objects, based on a combination of spectroscopy and statistical methods for studying multivariate dependences.

In the 1980s, works on the preparation of technical capabilities and software to observe in the infrared region of the spectrum were started at the Crimean Astrophysical Observatory under the supervision of N.N. Stepanian. An observation and image processing system was developed. These observations in CrAO are carried out with the diffraction spectrograph and Universal Spectrophotometer (USPh) at the Tower Solar Telescope TST-2. By means of USPh it is possible to take images of the Sun in different spectral lines and record spectra of solar formations. The USPh scanner allows for shifting within two coordinates of the solar image at the spectrograph slit. The photomultiplier tube FEU-83 (Russ. abbr.) or CCD camera are used as a receiver at the spectrograph exit. The rather high sensibility of these receivers allows one to use USPh for studying the Sun in the near-infrared region, particularly, in the HeI $1083 \mathrm{~nm}$ line (He I).

The He I line is the strongest triplet neutral helium line, which makes it possible to study the physical properties of the upper chromosphere and the transition layer between the chromosphere and the corona. It is in this line, which is formed in the upper chromosphere at an altitude of about $2000-3000 \mathrm{~km}$ and is excited by ultraviolet radiation, that it is possible to observe coronal holes $(\mathrm{CHs})$ from Earth. This line is the absorption line; in $\mathrm{CHs}$ it becomes weaker. The $\mathrm{CHs}$ in the He I line are brighter than the surrounding corona. Service programs enable us, within several minutes to take into account limb darkening and analyze the image to identify CHs.

Observations in the He I line have been carried out at the CrAO regularly from 1999 at the present. In 2020, the observation process was modernized. The catalog of images of the solar disk obtained before modernization with the TST-2 telescope in 1999-2018, is being formed at this time. 


\section{First flare M 1.9 AR 10365: comparing results real-scale time MHD modeling and observational data}

A. Borisenko ${ }^{1}$, I. Podgorny ${ }^{2}$, A. Podgorny ${ }^{1}$ sunw77@mail.ru

${ }^{1}$ Lebedev Physical Institute of the RAS, Moscow, Russia

${ }^{2}$ Institute of Astronomy of the RAS, Moscow, Russia

Results of MHD simulation and observations for first flare M 1.9 (05/26/2003 05:34) in multi-flare AR 10365 are presented. Keywords: solar flares, magnetic fields, solar corona,current sheet,scientific computing,parallel computing,computational magnetohydrodynamics, Syrovatskii

DOI: $10.51194 /$ VAK2021.2022.1.1.119
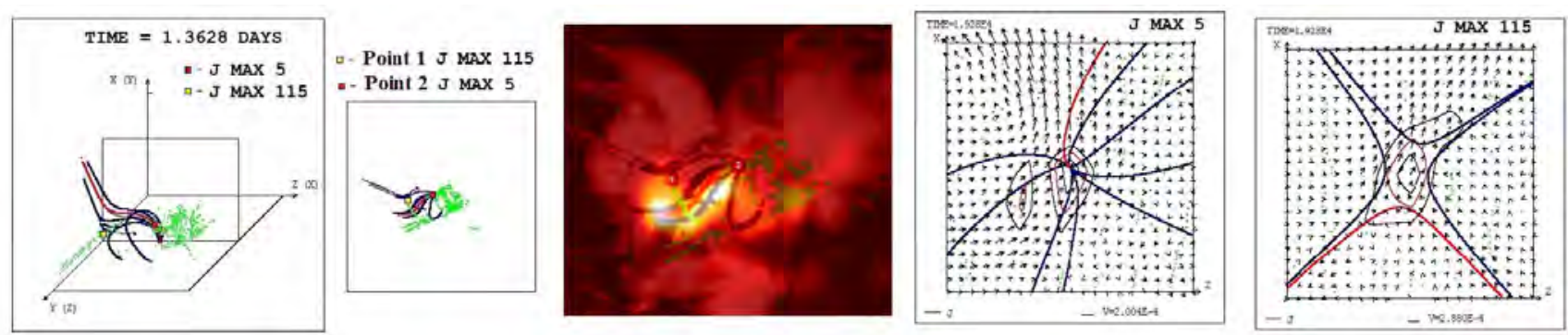

Figure 1: MHD results for first flare in AR 10365 (05/26/2003 05:34) overlays SOHO/MDI,SOHO/EIT 171A and NoRH $17 \mathrm{GHz}$ zooming to $0.6 \mathrm{arcsec} / \mathrm{pixel}$

MHD modelling above the 10365 active region using PERESVET Fortran program [1] is performed in the real scale of time. The first results show that maximum current densities appeared before the beginning of the first flare M 1.9 (05:34/06/2003) shows on Fig. 1 in the vicinity of a singular X-type magnetic field line with superimposed divergent magnetic flux at an heights of about 16(Point 1)-18(Point 2) Mm (coronal heights). Superposition of magnetic field configuration with current density maxima obtained from MHD simulations and observed NoRH 17 GHz emission confirms S.I. Syrovatskii [2] mechanism of the flare energy accumulation in the current sheet.

\section{References}

1. A. I. Podgorny and I. M. Podgorny, Geomagnetism and Aeronomy, 52, 150, 2012.

2. S. I. Syrovatskii, Journal of Experimental and Teoretical Physics, 50, 1133, 1966. 


\title{
On the polarization of velocity fluctuations in undisturbed solar wind
}

\author{
D.V. Erofeev \\ dve_08@mail.ru \\ Institute of Applied Astronomy of Russian Academy of Sciences, Ussuriisk Department, Ussuriisk 692533, \\ Gorno-Taezhnoe, Solnechnaya st., 21, Russia
}

Measurements of velocity in near-Earth heliosphere were analized in order to investigate systematical deflection from transversality of velocity fluctuations in solar wind. Deflections of two kinds have been considered. One of them is characteristic of fluctuations occured in azimuthal plain of heliosphere, while another is inherent in fluctuations occurred in meridional plain during minima of solar cycle.

Keywords: solar wind, velocity, fluctuations

DOI: 10.51194 /VAK2021.2022.1.1.120

As has been found by a number of investigators, fluctuation of magnetic field and velocity in solar wind (SW) mainly are transversal with respect to mean magnetic field (see [1] and references therein). However, there were reports about systematical deflections from transversality of magnetic and velocity fluctuations, probably did not associated with interplanetary disturbances (for example, [2]). In the present paper, we consider such deflections by using data obtained in near-Earth SW.

We use measurements of velocity $\mathbf{V}$, proton density $N_{P}$, and magnetic field $\mathbf{B}$ made in near-Earth heliosphere by WIND spacecraft in years 1995-2020. The data are averaged over $92 \mathrm{~s}$. We selected for analysis only time intervals when no significant disturbances of SW were observed. Method of investigation consists in analysis of correlation between pairs of projections of velocity vector, $\delta V_{i}$ and $\delta V_{j}$, on the axes of RTN reference system (hereafter, $\delta V_{i}$ denotes fluctuations of $\left.V_{i}\right)$. In particular, we analyse correlation coefficients $C_{i j}$ calculated in dependency of frequency $f: C_{i j}=\operatorname{Re}\left[\Gamma_{i j}(f)\right]$, where $\Gamma_{i j}(f)$ is function of coherency of $\delta V_{i}$ and $\delta V_{j}$ estimated by averaging over a series of short time intervals of 1.5 day. We also apply separation of the time intervals with respect to alfvénicity (i.e., conribution of Alfvén waves) by using normalized cross-helicity $\sigma_{c}$.

We first consider velocity fluctuation occurred in the azimuthal (RT) plain. Since mean magnetic field $\mathbf{B}_{0}$ lies in the RT plain and it is inclined at angle $\approx-45^{\circ}$ to radial direction $\mathrm{R}$, transversal fluctuation, such as Alfvén waves, must show positive correlation between $\delta V_{R}$ and $\delta V_{T}$, while for longitudinal fluctuations the correlation should be negative. Calculation indicates that correlation coefficient of $\delta V_{R}$ and $\delta V_{T}$ is negative, independently of phase of solar cycle or polarity of magnetic sector. Therefore, fluctuations of velocity are rather longitudinal than transversal with respect to mean magnetic field. However, dependency of the correlation on frequency (dashed line in Fig.1a) shows that $C_{i j}(f)$ is negative only at lowest frequencies $f<5 \cdot 10^{-5} \mathrm{~Hz}$. On the other hand, a sample which includes only time intervals with low alfvénicity $\left(\left|\sigma_{c}\right|<0.4\right)$ demonstrates negative correlation of $V_{R}$ and $V_{T}$ within a wider range of frequency extended up to $10^{-3} \mathrm{~Hz}($ thin solid line in Fig. 1a). This probably means that non-negative correlation arises due to presence of Alfvénic fluctuations. We have eliminated contribution of Alfvén waves to velocity data by going to Elsässer variable $\mathbf{E}^{+}=\mathbf{V}+\mathbf{b} \cdot \operatorname{sign}\left(\mathrm{B}_{0}\right) \cdot \alpha$, where $\mathbf{b}=\mathbf{B} / \sqrt{\mu_{0} \rho}, \rho$ is plasma density, and $\alpha=0.7$. Correlation of $\delta E_{R}^{+}$and $\delta E_{T}^{+}$calculated with no selection of the data for alfvénicity (thick line in Fig. 1a) is negative wihin a wide range of frequency extended up to $2 \cdot 10^{-3} \mathrm{~Hz}$ (corresponding period $\approx 8 \mathrm{~min}$ ), in contrast with correlation of $\delta V_{R}$ and $\delta V_{T}$ shown by dashed line.

In Fig.1, we also show two-dimensional distribution of $\delta V_{R}$ and $\delta V_{T}$ for intervals of low alfvénicity (Fig. 1b), and distribution of $\delta E_{R}^{+}$and $\delta E_{T}^{+}$(Fig. 1c). The two distributions have principal axes of maximum variance tilted to radial direction in the same manner as mean magnetic field, but at smaller angle. Estimates of tilt angles yield values of $-23^{\circ}$
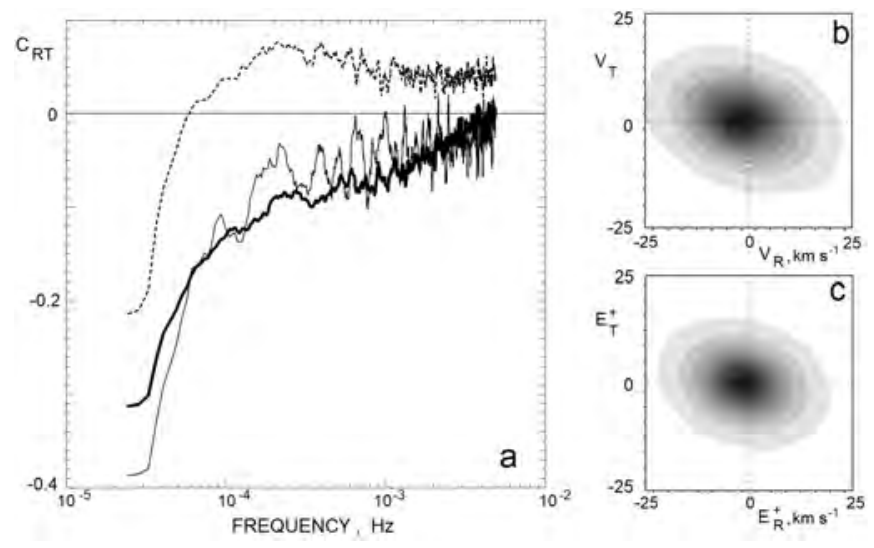

Figure 1: (a) dashed line: correlation between radial $\delta V_{R}$ and tangential $\delta V_{T}$ fluctuations of velocity as function of frequency, for slow solar wind; thin solid line: the same for streams with low alfvénicity; thick solid line: correlation between fluctuations $\delta E_{R}^{+}$and $\delta E_{T}^{+}$of Elsässer variable $\mathbf{E}^{+} ;(\mathbf{b}) 2 \mathrm{D}$ distribution of $\delta V_{R}$ and $\delta V_{T}$ for streams with low alfvénicity; (c) $2 \mathrm{D}$ distribution of $\delta E_{R}^{+}$and $\delta E_{T}^{+}$ 
and $-25^{\circ}$. Thus, variations of velocity are neither transversal nor longitudinal with respect to both mean magnetic field and direction of SW flow. Above analysis concerns data of slow SW. Analysis of measurements made in fast SW yields qualitatively the same resuls, although it indicates less tilt of maximum variance axis to radial direction of about $16^{\circ}$.

Let us consider fluctuations occurred in the meridional $(\mathrm{RN})$ plain. Note that transversal and longitudinal fluctuations both should indicate no correlation between R- and N-component of velocity (and magnetic field). As it first has been shown
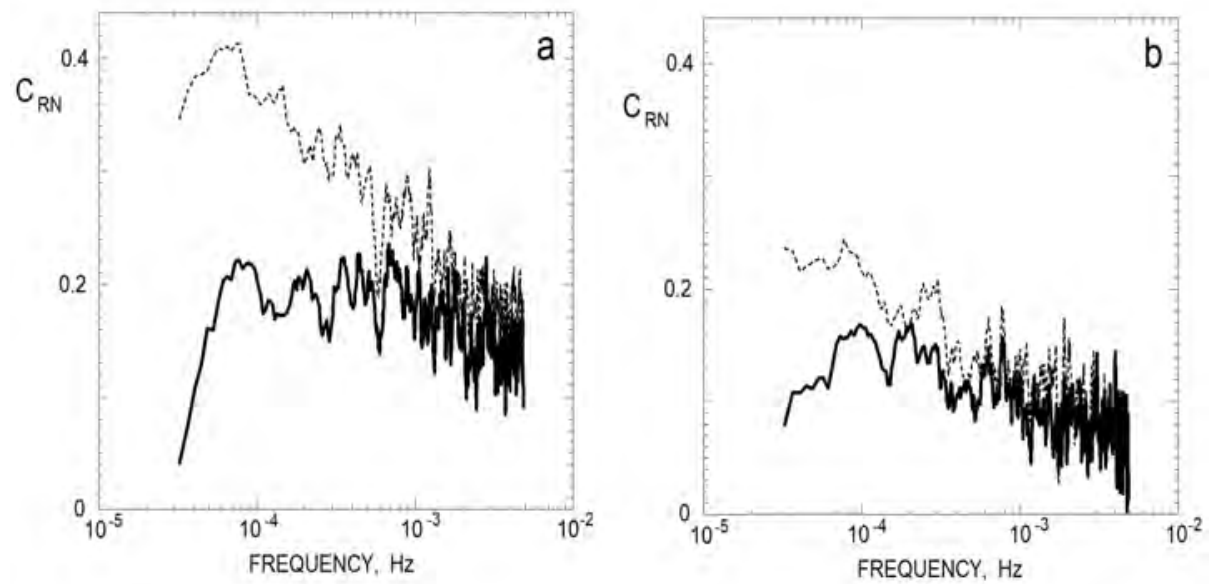

Figure 2: (a) correlation between radial (R) and normal $(\mathrm{N})$ components of magnetic field (thin dashed line) and velocity (thick solid line) as function of frequency, for data obtained during minima of solar cycle and within time intervals of low alfvénicity; (b) the same for time intervals with high alfvénicity.

by [2], components of magnetic field $\delta B_{R}$ and $\delta B_{N}$ significantly correlate during periods of low solar activity, and sign of the correlation coefficient depends on both direction of mean magnetic field $B_{0}$ (i.e., polarity of magnetic sector) and direction of polar magnetic field of the Sun, $B_{P}$. This dependency is expressed by the "sign rule": $\operatorname{sign}\left(C_{R N}\right)=\operatorname{sign}\left(B_{0}\right) \cdot \operatorname{sign}\left(B_{P}\right)$. Thus, magnetic fluctuations become non-transversal close to solar cycle minima. [3] has found that the same properties are characteristic of velocity fluctuations, but only those with periods shorter than about $5 \mathrm{hr}$.

[2] expained non-transversality of magnetic fluctuations during cycle minima by refraction of Alfvén waves due to latitudinal gradient of plasma density. Another explanation [4] consists in deformation of any transversal fluctuations, both Alfvénic or not, by action of latitudinal gradient of SW velocity. It is of interest to clear up whether deflection from transversality exclusively is characteristic of Alfvén waves or not. We calculated correlation $C_{R N}(f)$, using data obtained during three cycle minima in years 1995-1997, 2008-2010, and 2018-2020. In calculating, changes of $\operatorname{sign}\left(C_{R N}\right)$ were compensated in accordance with the "sign rule" mentioned above. Also, we separated time intervals whith low and high alfvénicity. Comparison between data of different alfvénicity (see Fig. 2) as well as other approaches (we also analysed Elsässer variable and cross-correlation between $B_{R}$ and $V_{N}$ ) show that most pronounced deflection from transversality is exhibited by fluctuations with small contribution of Alfvén waves. Thus, latitudinal gradient of velocity seems to be more suitable for explanation of non-transversality of magnetic and velocity fluctuations during cycle minima as compared to refraction of Alfvén waves.

\section{References}

1. R. Bruno and V. Carbone, Living Rev. Solar Phys., 10, 2, 2013.

2. W. Lyatsky, A. Tan, and S. Lyatskaya, Geophys. Res. Lett., 30, L2258, 2003.

3. D. Erofeev, Geomagnetism and Aeronomy, 59, 7, 2019.

4. D. Erofeev, Solar-Terrestrial Physics, 5, 42, 2019. 


\title{
Temperature characteristics of radio emission of polar coronal holes on the Sun
}

\author{
O.A. Golubchina \\ golubchina_olga@mail.ru \\ St. Petersburg Branch of Special Astrophysical Observatory, St. Petersburg, Russia
}

\section{DOI: $10.51194 /$ VAK2021.2022.1.1.121}

The solar eclipse on March 29, 2006 was observed in the "Relay" mode on the North-Eastern Sector of the RATAN600 radio telescope at wavelengths: $(1.03,1.38,2.7,6.2,13.0,30.7) \mathrm{cm}$ [1]. The open part of the optical disk of the Sun was $0.2 \%$. The center of the antenna radiation pattern was shifted +15 arc minutes to North from the center of the optical disk of the Sun. The RATAN-600 radio telescope has a knife-shaped antenna pattern (AP). The antenna temperatures of the Sun (Moon) $\left(T a^{S}, T a^{M}\right)$ model are calculated according to obtained brightness temperatures $T b^{S}, T b^{M}$, using the antenna smoothing equations for the normalized vertical AP: $T a\left(\varphi^{0}\right)=\int T b\left(\varphi^{0}\right) A\left(\varphi-\varphi^{0}\right) d \varphi[2]$. The brightness temperatures obtained with the observations of the eclipse of 29.03.2006 were compared with the temperatures according to the observations of [3] on the LPR and RATAN-600, obtained during the years of minimal solar activity (1973-1976; 1984-1987).

\section{Conclusions}

1. The observation of the solar eclipse of 29.03.2006 on RATAN-600 made it possible for the first time to determine the distribution of brightness temperatures over the North Pole of the Sun within the polar coronal hole in a wide range of centimeter wavelengths: $(1.03,1.38,2.7,6.2,13.0,30.7) \mathrm{cm}$ at a distance interval of $(1.005-2.0)$ Rs from the center of the optical disk of the Sun.

2. A sharp decrease in the brightness temperatures of the radio emission of the polar coronal hole at centimeter wavelengths $(\lambda>6 \mathrm{~cm})$ near the solar limb was detected. It confirmed the real registration of the polar coronal hole over the North Pole of the Sun.

3. The polar coronal hole is not visible at short centimeter wavelengths: $(1.03,1.38,2.7) \mathrm{cm}$.

4. The coincidence of the brightness temperatures of the polar coronal hole and large low-latitude coronal holes at close wavelengths in the Northern hemisphere indicate the identity of the temperature properties of the polar $\mathrm{CH}$ and low-latitude $\mathrm{CH}$, regardless of their location on the Sun and, consequently, from the mechanism of their organization during the period of minimum solar activity $[4,5,6,7]$.

\section{References}

1. O. A. Golubchina, Astronomy Reports, 65, 322, 2021.

2. O. A. Golubchina, A. N. Korzhavin, and S. K. Tokhchukova, Astrophysical Bulletin, 66, 488, 2011.

3. V. N. Borovik, M. S. Kurbanov, M. A. Livshits, and B. I. Ryabov, Sov. Astron., 34, 522, 1990.

4. L. A. Fisk and N. A. Schwadron, ApJ, 560, 425, 2001.

5. V. I. Abramenko, L. A. Fisk, and V. B. Yurchyshyn, ApJL, 641, L65, 2006.

6. R. H. Munro and B. V. Jackson, ApJ, 213, 874, 1977.

7. O. A. Golubchina, Geomagnetism and Aeronomy, 57, 964, 2017. 


\section{Origin and length of solar cycle}

V.A. Kotov

vkotov@craocrimea.ru

Crimean Astrophysical Observatory, Nauchny, Crimea 298409, Russian Federation

DOI: 10.51194 /VAK2021.2022.1.1.122

Current cycle 25 prolonged the list of the Wolf number extrema observed from Galileo's time: 75 epochs, from 1610 to 2021, fix the Schwabe's period as 11.07(4) years. The Hale's magnetic cycle $P_{H}$, therefore, is equal to 22.14(8) years (it agrees well with the 22.14-year duration of the cycle following from [1], analysis of the tidal resonance of Venus, Earth and Jupiter).

According to 54-year observations of the Sun-as-a-star performed in 1968-2021 by the CrAO and six other observatories, $P_{H}$-variation of the solar mean magnetic field reveals a saw-tooth shape, supporting a cosmological status of the cycle [2]. Now we present arguments in favour of a tight connection between $P_{H}$ and the Earth's orbital period $P_{E}$ :

$$
P_{E}=\left(1-\frac{3}{\pi}\right) P_{H},
$$

and note that motions of the Sun, Earth and Venus are involved in a close mutual resonance as well. Namely, the Venusian spin period (sidereal, in days),

$$
P_{V} \approx \frac{2}{3} P_{E} \approx 3^{2} P_{\odot} \approx 3^{5} P_{D}=243.000,
$$

with the observed $P_{V}=243.025$ days, $P_{\odot}=27.027(7)$ days, synodic period of the Sun's spinning, and $P_{D}$, the mean terrestrial day. Moreover, the Sun-Venus-Earth gravitational configuration repeats each $P_{V E}=243$ years, while

$$
\frac{P_{V}}{P_{D}} \approx \frac{P_{V E}}{P_{E}}=3^{5}
$$

$P_{E} \approx 3^{3} P_{\odot} / 2, P_{\odot} \approx 3^{3} P_{D}$ and $P_{D} \approx 3^{2} P_{0}$, where $P_{0} \approx 1 / 9$ days is a period of solar pulsations [3]. A hypothesis is advanced that timescales $P_{H}, P_{\odot}, P_{E}, P_{D}$ and $P_{0}$ are fundamental constants of the World at the present cosmological epoch.

\section{References}

1. N. Scafetta, Pattern Recognition in Physics, 2, 1, 2014.

2. V. A. Kotov and F. M. Sanchez, ApESSS, 362, 6, 2017.

3. V. A. Kotov and V. I. Haneychuk, Astronomische Nachrichten, 341, 595, 2020. 


\title{
Observation of solar flare intensity curves and comparing them with stellar flares
}

\author{
Yu.A. Kupryakov ${ }^{1,2}$, K.V. Bychkov², O.M. Belova ${ }^{2}$, A.B. Gorshkov², P. Heinzel ${ }^{1}$, P. Kotrč ${ }^{1}$ \\ kupry@asu.cas.cz, \\ WWW home page: http://www.asu.cas.cz/ sos/staff/kuprya/welcome.html \\ ${ }^{1}$ Astronomical Institute of the Czech Academy of Sciences, Ondřejov, Czech Republic, \\ ${ }^{2}$ Sternberg Astronomical Institute, M.V. Lomonosov Moscow State University, Moscow, Russia
}

\begin{abstract}
We present intensity curves of solar flares obtained in the $\mathrm{H} \alpha$ hydrogen line and CaII H, CaIR 8542Ålines using multichannel spectrographs Ondřejov Observatory (Czech Republic) for the period 2000-2012. The general behavior of observed intensity curves is practically the same for all flares and is consistent with temporal variations of X-ray emission. However, our results differ significantly from those obtained by other authors for selected flare stars, for example, AD Leonis. EV Lac. YZ Cmi. We tried to explain the difference in the behavior of $\mathrm{Ca}$ II and $\mathrm{H} \alpha$ luminescence by appearance of a shock wave during a flare and slow heating of the plasma.
\end{abstract}

Keywords: Flares Spectrum, chromosphere, GOES flux

DOI: $10.51194 /$ VAK2021.2022.1.1.123

\section{Observations and Analysis}

All observational data on solar flares were obtained at two spectrographs of the Astronomical Institute of the Czech Academy of Sciences (Ondřejov). These are Multichannel-Flare-Spectrograph (MFS, $230 \mathrm{~mm} / 13.5 \mathrm{~m}$ ) and HorizontalSonnen-Forschungs-Anlage (HSFA-2, $500 \mathrm{~mm} / 35 \mathrm{~m})$ ), described in [1, 2].Since the observations were carried out on two different instruments using analog and digital CCD matrices, the processing and calibration of the data differed. For the data obtained on HSFA-2 and MFS, we used the IDL software environment. We selected 25 class C, M flares with different positions on the solar disk for the period 2002-2012. The paper provides data for two flares (2012-11-24 C3.3, 2012-07-05 M1.8).

a) The 2012-11-24, C3.3 flare was observed on the MFS spectrograph with digital cameras. Its position on the disk and obtained spectra are shown in Fig. 1. After data calibration, the intensity plots were plotted in absolute units as a function of time. The graph in Fig. 2 also presents an X-ray flux (GOES 15).
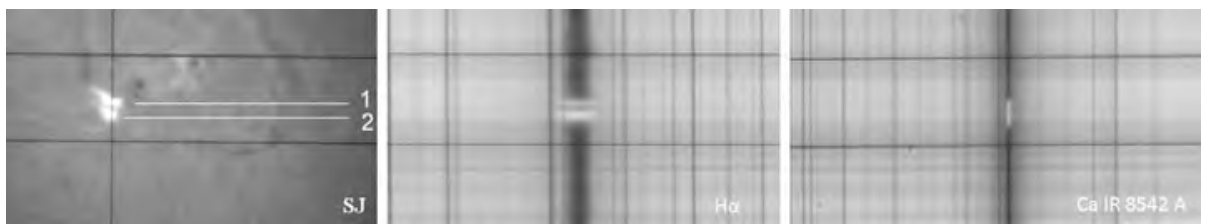

Figure 1: Flare 2012-11-24, 13:24:15 UT, from left to right SJ, H $\alpha$, Ca $8542 \AA$, NOAA 11618.

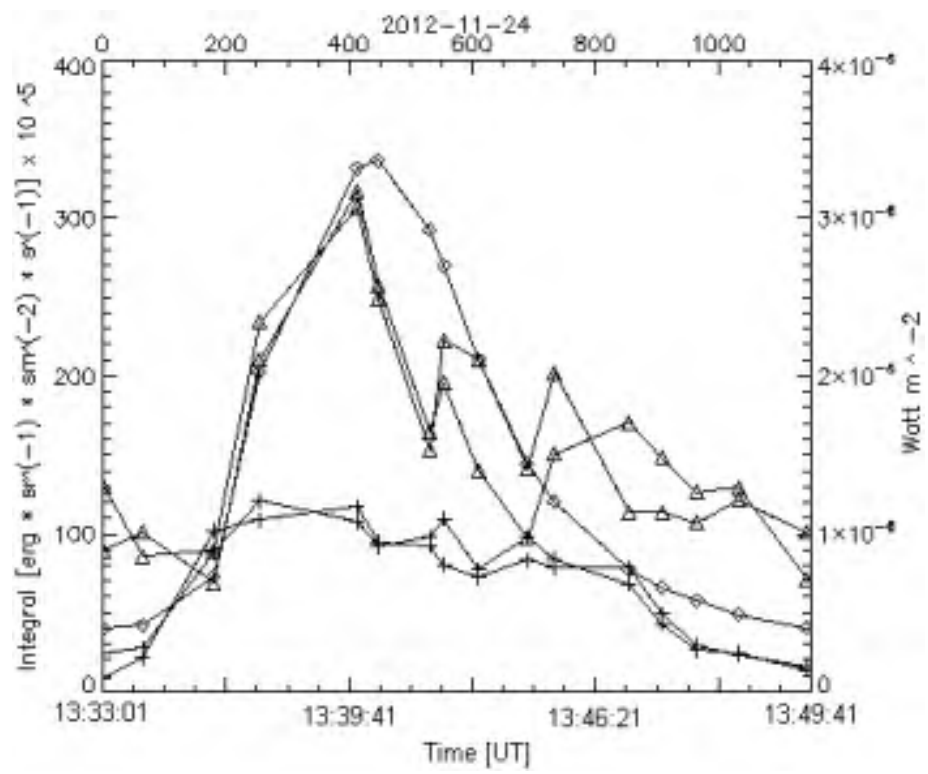

Figure 2: Graph of the intensity of two flare nuclei in the lines: $(+) \mathrm{H} \alpha$, (triangle) Ca $8542 \AA$, (rhomb) X-ray. 
b) The flare 2012-07-05, M1.8, NOAA 11515, observations on HSFA-2 (Fig. 3). For this flare, we calculated the values of the radiation flux $\mathrm{F}$ in the lines $\mathrm{H} \alpha, \mathrm{H}$ CaII (Fig. 4)

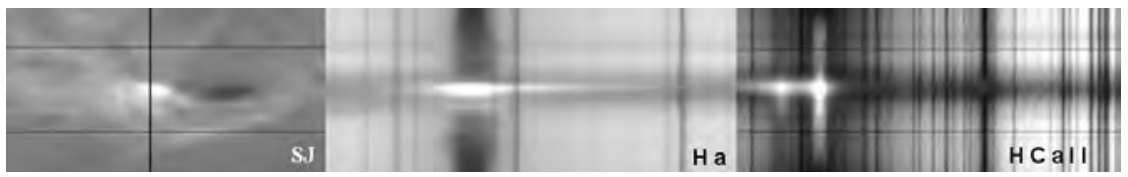

Figure 3: Flare 2012-07-05, 10:46:47 UT, from left to right SJ, H $\alpha$, H CaII, NOAA 11515.
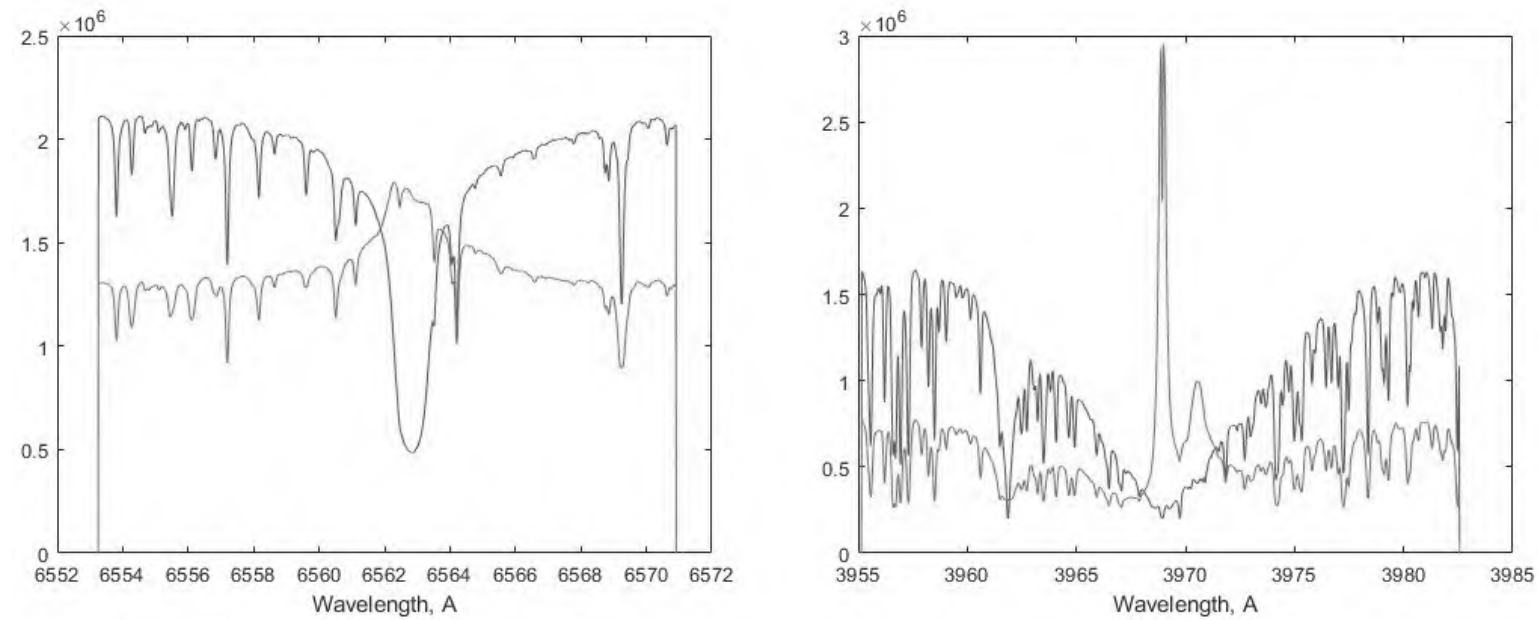

Figure 4: An example of calculating the flux F for lines $\mathrm{H} \alpha$ and $\mathrm{H}$ CaII. F H $\alpha-1.7910^{7} \mathrm{erg} \mathrm{cm}^{-2} \mathrm{~s}^{-1}$; F CaII $2.4310^{7} \mathrm{erg} \mathrm{cm}^{-2} \mathrm{~s}^{-1}$. The integration was carried out over the ranges: $\mathrm{H} \alpha-6561.88-6563.79 \AA$, H CaII 3968.09-3971.36 А.

\section{Stellar flares}

The results of solar flares analysis indicate the same change of the $\mathrm{H} \alpha$ and CaII lines in time. Their impulse and gradual phases reach the same values at the same time.

Then we looked at stellar flares. [3] provide observational data from the Apache Point Observatory in southern New Mexico. As an example, note the evolution of the CaII and $\mathrm{H} \alpha$ lines for the YZ Cmi star flare (Fig. 5) observed in 2011 Feb 24. We also considered flares AD Leonis observed in 2011 Feb 08 and EV Lac observed in 2010 Oct 11 . For these three stars, the lag of the maximum for the K CaII line relative to the $\mathrm{H} \alpha$ line is within the range of 12-24 min.

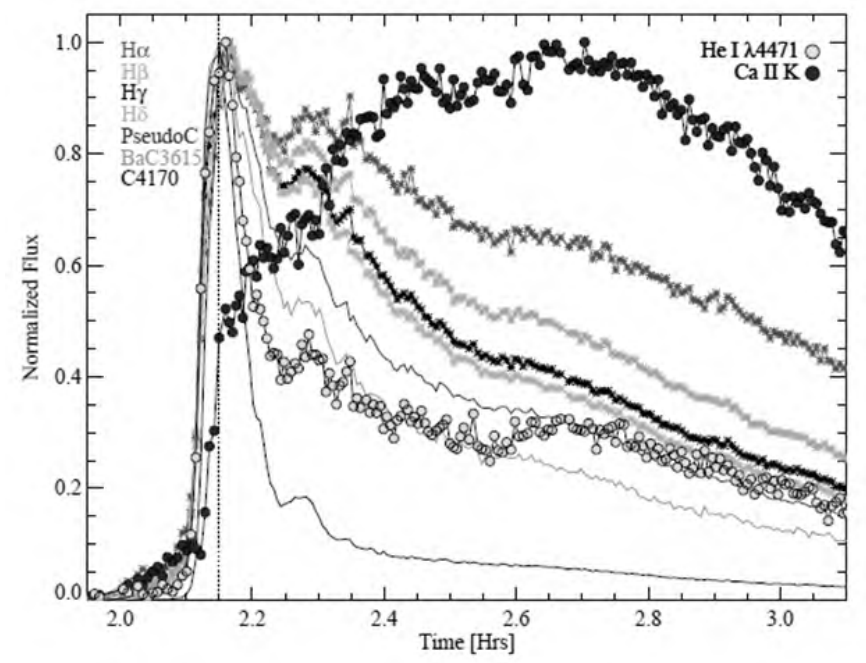

Figure 5: Radiation flux curves for YZ Cmi. 


\section{Calculation of the Plasma Heating Models}

Trying to solve the inconsistency that has arisen, we calculated two models: 1) a shock wave; 2) slow heating of the gas. In the shock wave hypothesis, we assume a sudden blow on the gas from above, resulted in a non-equilibrium situation sharp development. As a cause of the impact a stream of particles from the corona can be assumed. Note that a powerful rarefied flow produces weak X-rays. At $\mathrm{T}=5500 \mathrm{~K}$ we calculated the flux $\mathrm{F}\left[\mathrm{erg} \mathrm{cm}^{-2} \mathrm{~s}^{-1}\right]$ for the lines CaII 3945, CaIR 8542, $\mathrm{MgII}, \mathrm{H} \alpha$, Ly $\alpha$. Table 1 shows the values corresponding to lines we observed. Shock velocity values are listed at the beginning of each table. The rightmost column shows the date of the outbreak, which corresponds to calculated values.

Table 1: Calculation of the shock wave model.

\begin{tabular}{|l|l|l|l|}
\hline $\mathrm{N}_{0}\left(\mathrm{~cm}^{-3}\right), \mathrm{V}=40 \mathrm{~km} / \mathrm{s}$ & $\mathrm{F}$ CaII 3945 & F CaIR 8542 & $\mathrm{F} \mathrm{H} \alpha$ \\
\hline $1.010^{15}$ & $3.0310^{8}$ & $3.610^{7}$ & $1.1910^{8} 2012-07-05$ \\
\hline $3.010^{14}$ & $1.4410^{8}$ & $1.5310^{7}$ & $4.910^{7}$ \\
\hline $1.010^{14}$ & $6.710^{7}$ & $6.110^{6}$ & $2.010^{7}$ \\
\hline $3.010^{13}$ & $2,410^{7}$ & $1.710^{6}$ & $6.810^{6}$ \\
\hline $1.010^{13}$ & $8.810^{6}$ & $5.310^{5}$ & $2.310^{6}$ \\
\hline $\mathrm{N}_{0}\left(\mathrm{~cm}^{-3}\right), \mathrm{V}=20 \mathrm{~km} / \mathrm{s}$ & $\mathrm{F} \mathrm{CaII} 3945$ & $\mathrm{~F} \mathrm{CaIR} 8542$ & $\mathrm{~F} \mathrm{H \alpha}$ \\
\hline $3.010^{12}$ & $2.010^{6}$ & $2.010^{5}$ & $6.310^{4} 2012-11-24$ \\
\hline $1.010^{13}$ & $8.210^{6}$ & $1.410^{6}$ & $1.910^{5}$ \\
\hline $3.010^{13}$ & $2.110^{7}$ & $3.910^{6}$ & $7.410^{5}$ \\
\hline $1.010^{14}$ & $6.910^{7}$ & $1.310^{7}$ & $4.510^{6}$ \\
\hline $3.010^{14}$ & $1.610^{8}$ & $2.910^{7}$ & $2.110^{7}$ \\
\hline $1.010^{15}$ & $3.310^{8}$ & $5.610^{7}$ & $6.810^{7}$ \\
\hline
\end{tabular}

In the second model, we assume a slow heating of the gas, which is stronger in given area than in neighboring areas. The source of heating is the dissipation of MHD waves. Warming up occurs from below. In the second model (table 2), the gas is heated to lower temperatures than in the shock wave. Therefore, in the stationary case, there may be a steeper Balmer decrement.

Table 2: Calculation of the model of slow heating of gas.

\begin{tabular}{|l|l|l|l|l|l|l|}
\hline $\mathrm{T}, \mathrm{K}$ & $\mathrm{H} \alpha$ & $\mathrm{H} \beta$ & $\mathrm{H} \gamma$ & $\mathrm{H} \delta$ & $\mathrm{Ca} 3945$ & $\mathrm{Ca} 8542$ \\
\hline 7000 & $2.3110^{6}$ & $2.6910^{5}$ & $7.4510^{4}$ & $3.0810^{4}$ & $3.8510^{6}$ & $4.2810^{6}$ \\
\hline $8000\left(^{*}\right)$ & $1.2110^{7}$ & $1.4210^{6}$ & $3.9410^{5}$ & $1.6310^{5}$ & $1.1610^{7}$ & $8.9110^{6}$ \\
\hline 8700 & $5.5610^{7}$ & $6.5010^{6}$ & $1.8210^{6}$ & $7.5710^{5}$ & $1.9310^{7}$ & $1.2910^{7}$ \\
\hline
\end{tabular}

(*) Data in $\mathrm{H} \alpha$ and Ca 3945 correspond to radiation fluxes in stellar flare.

\section{Discussion and conclusion}

The results of solar flares analysis indicate the same course of $\mathrm{H} \alpha$ and CaII lines in time. Their impulse and gradual phases reach the same values at the same time. These observations contradict those of other stars, for example, of the spectral type dMe. These individual types of stars demonstrate a significant delay in the emission of CaII (H, K) lines, relative to $\mathrm{H} \alpha$ [3]. Possibly, in stars the flare causes sharp ionization followed by slow recombination. These issues have not been adequately studied yet. The authors are grateful to the Ondřejov Observatory team for the opportunity to participate in joint observations.

\section{References}

1. P. Kotrč, P. Heinzel, and M. Knížek, A. von Alvensleben (ed.), Annual Report, 1992.

2. P. Kotrč, Cent. Eur. Astrophys. Bull, 1, 2008.

3. A. F. Kowalski, S. L. Hawley, J. P. Wisniewski, R. A. Osten, E. J. Hilton, J. A. Holtzman, S. J. Schmidt, and J. R. A. Davenport, ApJS, 207, 15, 2013. 


\section{Thermal Trigger for Solar Flares}

L. Ledentsov

leonid.ledentsov@gmail.com

Sternberg Astronomical Institute, Moscow State University, Moscow 119234, Universitetsky pr., 13, Russia

We consider the thermal stability of three piecewise homogeneous models of the preflare current layer: a neutral current layer, a non-neutral current layer, and a current layer with oblique fragmentation. Solution to the problem allows the formation of an instability of thermal nature. The instability results in fragmentation of the current layer with a spatial period of $1-10 \mathrm{Mm}$ along the current direction in a wide range of coronal plasma parameters. The result can explain the observed spatial periods of energy release in solar flares.

Keywords: magnetic reconnection, instabilities, flare models

DOI: 10.51194 /VAK2021.2022.1.1.124

\section{Neutral Current Layer}

The flare energy is accumulated during the formation of a preflare current layer above the arcade of magnetic loops [1]. Separate bright loops in the flare arcade, observed in the ultraviolet range, show that the energy release in a solar flare is spatially inhomogeneous [2]. Consequently, there must be a mechanism leading to a quasiperiodic destruction of the current layer in the direction of the current [3]. We assume that such a transverse fragmentation of the preflare current layer is associated with a disturbance in its heat balance [4].

Following [5], we consider a piecewise homogeneous model of a preflare current layer, which consists of the plasma of the current layer and the surrounding plasma, separated by a tangential discontinuity (Fig. 1). The surrounding plasma is considered ideal, while inside the layer all dissipative effects are taken into account: finite thermal and electrical conductivity, viscosity, and radiative cooling. First, we assume that the current layer is thin $(b \gg a, \partial / \partial x=0)$, and there is no undisturbed magnetic field inside it. With these conditions, we solve the problem of small perturbations in the approximation of dissipative magnetohydrodynamics [6].

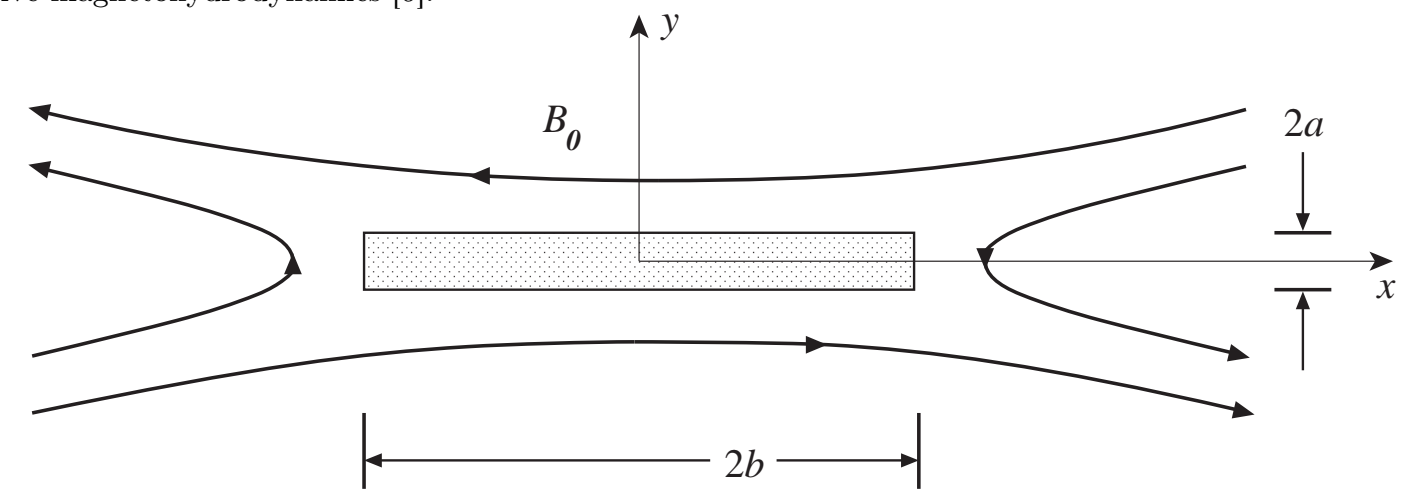

Figure 1: Neutral current layer model.

We found the instability of a thermal nature [7]. In the linear phase, the increment of the instability $\Gamma$ is inversely proportional to the characteristic time of radiation cooling of the plasma $\tau_{\lambda}$

$$
\Gamma=\frac{2}{5} \frac{\beta-\alpha}{\tau_{\lambda}}
$$

where $\alpha$ and $\beta$ are the logarithmic derivatives of the radiative cooling function $\lambda$ with respect to the temperature $T$ and number density $n$ of the current layer

$$
\alpha=\frac{\partial \ln \lambda}{\partial \ln T}, \quad \beta=\frac{\partial \ln \lambda}{\partial \ln n} .
$$

The spatial period of instability is shown in Fig. 2 and is $1-10 \mathrm{Mm}$ in the expected temperature range of the preflare current layer $\left(3 \times 10^{6}-10^{7} \mathrm{~K}\right)$.

\section{Non-Neutral Current Layer and Oblique Fragmentation}

We show that the presence of a weak guide field $\left(B_{z}\right.$ in Fig. 3a) does not change spatial period of the thermal instability in the most expected range of the parameters of the preflare current layer (Fig. 4a) and, moreover, contributes to the formation of the instability [8]. On the other hand, a strong guide magnetic field promotes spatial stabilization of the current layer.

We also show that the fragmentation transverse to the current $\left(k_{z}\right.$ in Fig. 3b) is a natural feature of the model [9]. The fragmentation tilt does not exceed a few degrees for realistic preflare parameters of the coronal plasma. As a consequence, oblique fragmentation generally does not have a strong impact on the simulation results, however, extreme changes can reach an order of magnitude (Fig. 4b). 

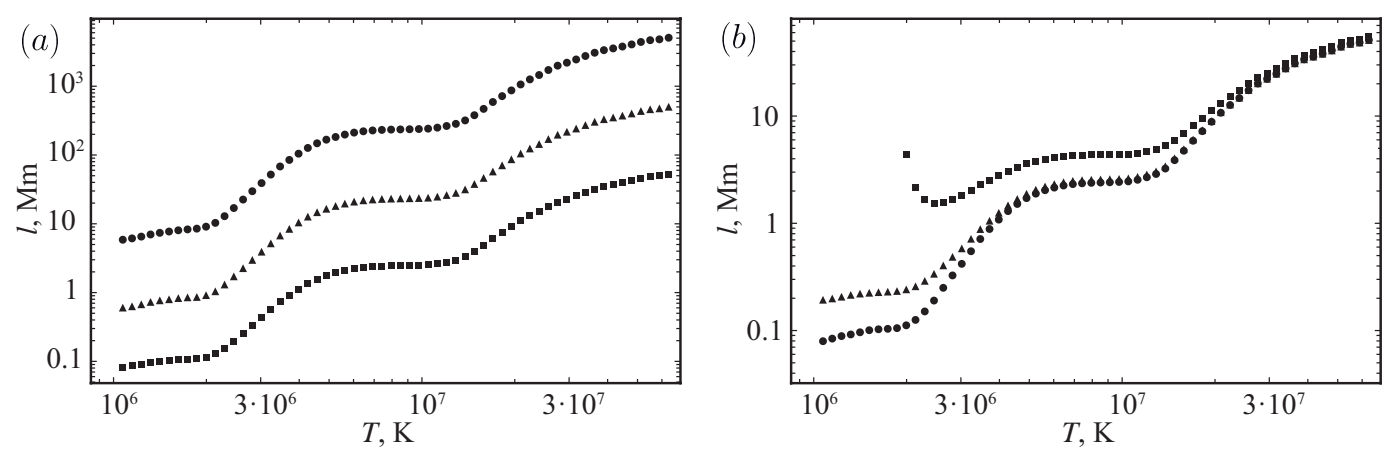

Figure 2: The spatial period of the instability depending on the temperature of the current layer. Coronal $\left(n_{0}\right)$ and current layer's $\left(n_{s}\right)$ numerical densities: $n_{0}=10^{10} \mathrm{~cm}^{-3}, n_{s} / n_{0}=10$. One of the parameters changes in each figure: (a) $n_{0}=10^{8} \mathrm{~cm}^{-3}$ (circles), $n_{0}=10^{9} \mathrm{~cm}^{-3}$ (triangles), $n_{0}=10^{10} \mathrm{~cm}^{-3}$ (squares), (b) $n_{s} / n_{0}=10$ (circles), $n_{s} / n_{0}=100$ (triangles), $n_{s} / n_{0}=1000$ (squares).
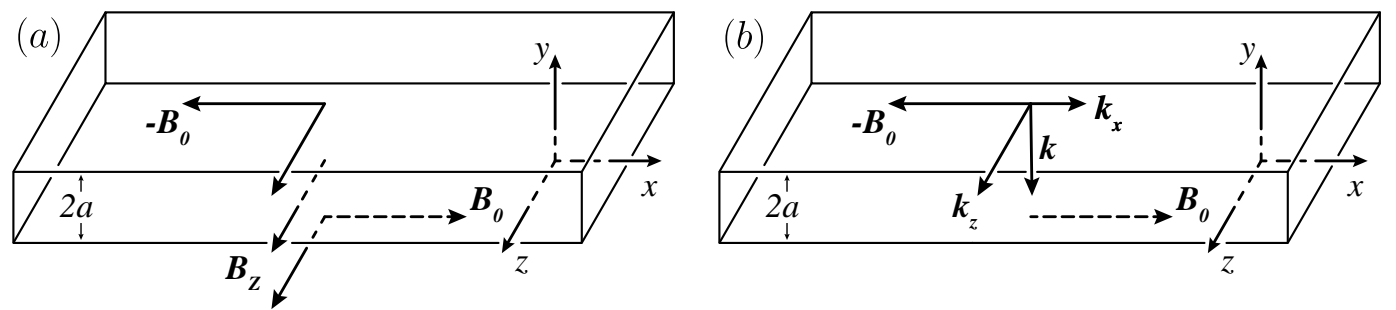

Figure 3: (a) Non-neutral current layer model. (b) Oblique fragmentation model.
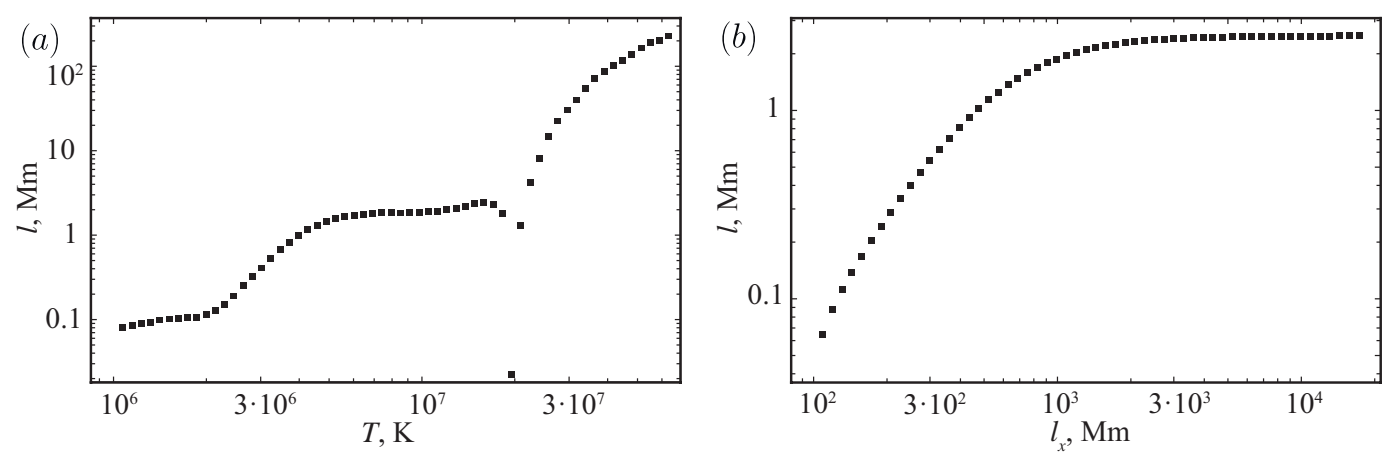

Figure 4: (a) The spatial period of the instability depending on the temperature of the current layer for numerical densities $n_{0}=10^{10} \mathrm{~cm}^{-3}$ and $n_{s} / n_{0}=10$ and guide magnetic field $B_{z}=10^{-3} \mathrm{G}$. (b) The relationship between the spatial scales of the instability $l_{x}$ and $l$.

\section{References}

1. B. V. Somov, Plasma Astrophysics. Part II: Reconnection and Flares, 2nd edn., ASSL, volume 392 (2013).

2. A. O. Benz, Liv. Rev. Solar Phys., 14, 2, 2017.

3. I. V. Zimovets, J. A. McLaughlin, A. K. Srivastava, D. Y. Kolotkov, et al., Space Sci. Rev., 217, 66, 2021.

4. G. B. Field, ApJ, 142, 531, 1965.

5. B. V. Somov and S. I. Syrovatskii, Solar Phys., 75, 237, 1982.

6. B. V. Somov, Plasma Astrophysics. Part I: Fundamentals and Practice, 2nd edn., ASSL, volume 391 (2012).

7. L. Ledentsov, Solar Phys., 296, 74, 2021.

8. L. Ledentsov, Solar Phys., 296, 93, 2021.

9. L. Ledentsov, Solar Phys., 296, 117, 2021. 


\title{
MHD wave modes of axial symmetry in coronal flux tubes with magnetically twisted layer
}

\author{
I. Lopin \\ lopin78@mail.ru
}

Institute of Applied Astronomy of the Russian Academy of Sciences, Ussuriisk department, Russia

The properties of axisymmetric MHD wave modes are examined for the structured model of coronal magnetic tube, consisting of a central cord with homogeneouse axial magnetic field, surrounded by an annulus with twisted magnetic field. The derived dispersion relation is solved numerically. A number of limiting cases are examined analytically. The two families of axisymmetric modes are found to exist in the model. The first one includes an infinite number of fast-sausage modes, modified by the twist and the second one is a set of modes with frequencies, lying in a narrow band, closed to Alfvén frequency of a twisted layer $\omega_{\mathrm{A} 0}$. The fundamental fast-sausage mode (the mode of the lowest radial order) exists as a trapped mode for the entire range of axial wavenumbers. Its phase speed is always below the Alfvén speed of a magnetically twisted layer. This mode has a weak dispersion in the range of long and intermediate wavelengths. The application of the main results of the study to coronal seismology is considered.

Keywords: Sun: helioseismology, Sun: oscillations

DOI: $10.51194 /$ VAK2021.2022.1.1.125

\section{Model, Equilibrium and Governing Equations}

We study a coronal magnetic flux tube, which is a cylindrical density enhancement of a coronal magnetized plasma. The tube consists of a central part or core with radius $r=a$, plasma density $\rho_{i}$ and uniform axial magnetic field $\mathbf{B}_{i}=B_{i} \mathbf{e}_{\mathbf{z}}$, surrounded by a cylindrical layer or annulus with outer radius $r=b$ and density $\rho_{0}$. The magnetic field in the layer has both longitudinal and azimuthal components $\mathbf{B}_{0}=B_{0 z} \mathbf{e}_{\mathbf{z}}+B_{0 \phi} \mathbf{e}_{\phi}$. Here $\mathbf{e}_{\mathbf{z}}$ and $\mathbf{e}_{\phi}$ are unit longitudinal and azimuthal vectors in cylindrical coordinate system. The core and the annulus are both embedded within a magnetic environment with plasma density $\rho_{e}$ and uniform axial magnetic field $\mathbf{B}_{e}=B_{e} \mathbf{e}_{\mathbf{z}}$. In what follows the subscripts " $i$ ", "0" and " $e$ " refer to the quantities in the core, annulus and external region respectively. We use the cold plasma $(\beta=0)$ limit, which is an appropriate choice under coronal conditions. The background equilibrium is provided by a radial force balance, given by

$$
\frac{d}{d r}\left(\frac{B_{\phi}^{2}+B_{z}^{2}}{2 \mu_{0}}\right)+\frac{B_{\phi}^{2}}{\mu_{0} r}=0
$$

Equation (1) yields that the radial gradient of the total magnetic pressure is compensated by the magnetic tension of the azimuthal component of the magnetic field. The static boundary condition at $r=a$ and $r=b$ reads that the magnetic pressure have to be continuous across the interfaces. Following [1], we model the force-free twisted magnetic field in the cylindrical layer, using the solution to Equation (1) of the form

$$
B_{z 0}^{2}=\frac{B_{0}^{2}}{1+\alpha_{0}^{2}}\left(\frac{a}{r}\right)^{2 \alpha_{0}^{2} /\left(1+\alpha_{0}^{2}\right)}, \quad B_{\phi 0}^{2}=\frac{B_{0}^{2} \alpha_{0}^{2}}{1+\alpha_{0}^{2}}\left(\frac{a}{r}\right)^{2 \alpha_{0}^{2} /\left(1+\alpha_{0}^{2}\right)},
$$

where

$$
\alpha_{0}=\frac{B_{\phi 0}}{B_{z 0}}
$$

is a twist parameter.

The set of ideal MHD equations for the zero- $\beta$ plasma motions results in the following wave equation for radial plasma displacement

$$
\frac{d}{d r}\left(\frac{D}{r C_{2}} \frac{d}{d r}\left(r \xi_{r}\right)\right)+\left(\frac{1}{D}\left[C_{3}-\frac{C_{1}^{2}}{C_{2}}\right]-r \frac{d}{d r}\left(\frac{C_{1}}{r C_{2}}\right)\right) \xi_{r}=0
$$

where

$$
\begin{gathered}
D=\rho V_{A}^{2}\left(\omega^{2}-\omega_{A}^{2}\right), \quad C_{1}=\frac{2 B_{\phi}^{2} \omega^{2}}{\mu_{0} r}, \quad C_{2}=\omega^{2}-k^{2} V_{A}^{2}, \\
C_{3}=\rho D\left(\omega^{2}-\omega_{A}^{2}+\frac{2 B_{\phi}}{\mu_{0} \rho} \frac{d}{d r}\left(\frac{B_{\phi}}{r}\right)\right)+4 \omega^{2}\left(\frac{B_{\phi}^{2}}{\mu_{0} r}\right)^{2}-\rho V_{A}^{2} \frac{4 B_{\phi}^{2}}{\mu_{0} r^{2}} \omega_{A}^{2}, \\
\omega_{A}^{2}=\frac{B_{z}^{2} k^{2}}{\mu_{0} \rho}=V_{A z}^{2} k^{2}, \quad V_{A}^{2}=V_{A z}^{2}+V_{A \phi}^{2}, \quad V_{A \phi}^{2}=\frac{B_{\phi}^{2}}{\mu_{0} \rho} .
\end{gathered}
$$

\section{Dispersion Relation}

It can be shown that equation (4) reduces to the Bessel equation in all regions and corresponding solutions are expressed in terms of Bessel functions. After some standard procedures we arrive at the following desired dispersion relation

$$
\frac{\left(R_{1}(a)-X_{i}\right)\left(R_{2}(b)-X_{e}\right)}{\left(R_{2}(a)-X_{i}\right)\left(R_{1}(b)-X_{e}\right)}=\frac{I_{\nu}\left(n_{0} b\right) K_{\nu}\left(n_{0} a\right)}{I_{\nu}\left(n_{0} a\right) K_{\nu}\left(n_{0} b\right)}
$$


where

and

$$
\begin{gathered}
X_{i}=\frac{m_{i} a J_{0}\left(m_{i} a\right)}{J_{1}\left(m_{i} a\right)}, \quad X_{e}=-\frac{n_{e} b K_{0}\left(n_{e} b\right)}{K_{1}\left(n_{e} b\right)}, \quad R_{1,2}(r)=\frac{\left(\omega^{2}-\omega_{A 0}^{2}\right)}{\left(\omega^{2}-V_{A 0}^{2} k^{2}\right)} Q_{1,2}(r)+\eta, \\
Q_{1}(r)=X_{01}+1-\nu-\frac{\eta\left(\omega^{2}+\omega_{A 0}^{2}\right)}{\left(\omega^{2}-\omega_{A 0}^{2}\right)}, \quad Q_{2}(r)=X_{02}+1-\nu-\frac{\eta\left(\omega^{2}+\omega_{A 0}^{2}\right)}{\left(\omega^{2}-\omega_{A 0}^{2}\right)},
\end{gathered}
$$

$$
X_{01}(r)=\frac{n_{0} r I_{\nu-1}\left(n_{0} r\right)}{I_{\nu}\left(n_{0} r\right)}, \quad X_{02}(r)=-\frac{n_{0} r K_{\nu-1}\left(n_{0} r\right)}{K_{\nu}\left(n_{0} r\right)} .
$$

We have solved the dispersion relation in Equation (8) numerically. The results of calculations are shown in Figure 1. The main finding indicates that there are two types of symmetric modes. The first type is a set of an infinite number of dispersive modes, identified with the fast-sausage modes in coronal magnetically twisted flux tube. The distinct observed feature is that the principal (fundamental) fast-sausage mode (the lowest radial order mode) exists in the trapped regime throughout the range of axial wavenumbers, i.e. its phase speed $V_{\mathrm{ph}}<V_{\mathrm{Ae}}$. Moreover, the phase speed of this mode is below the Alfvén speed of the magnetically twisted layer, i.e. $V_{\mathrm{ph}}<V_{\mathrm{A} 0}$ even in the long wavelength limit. The higher values of a twist parameter $\alpha_{0}$ correspond to the lower values of phase speed of the fundamental mode.
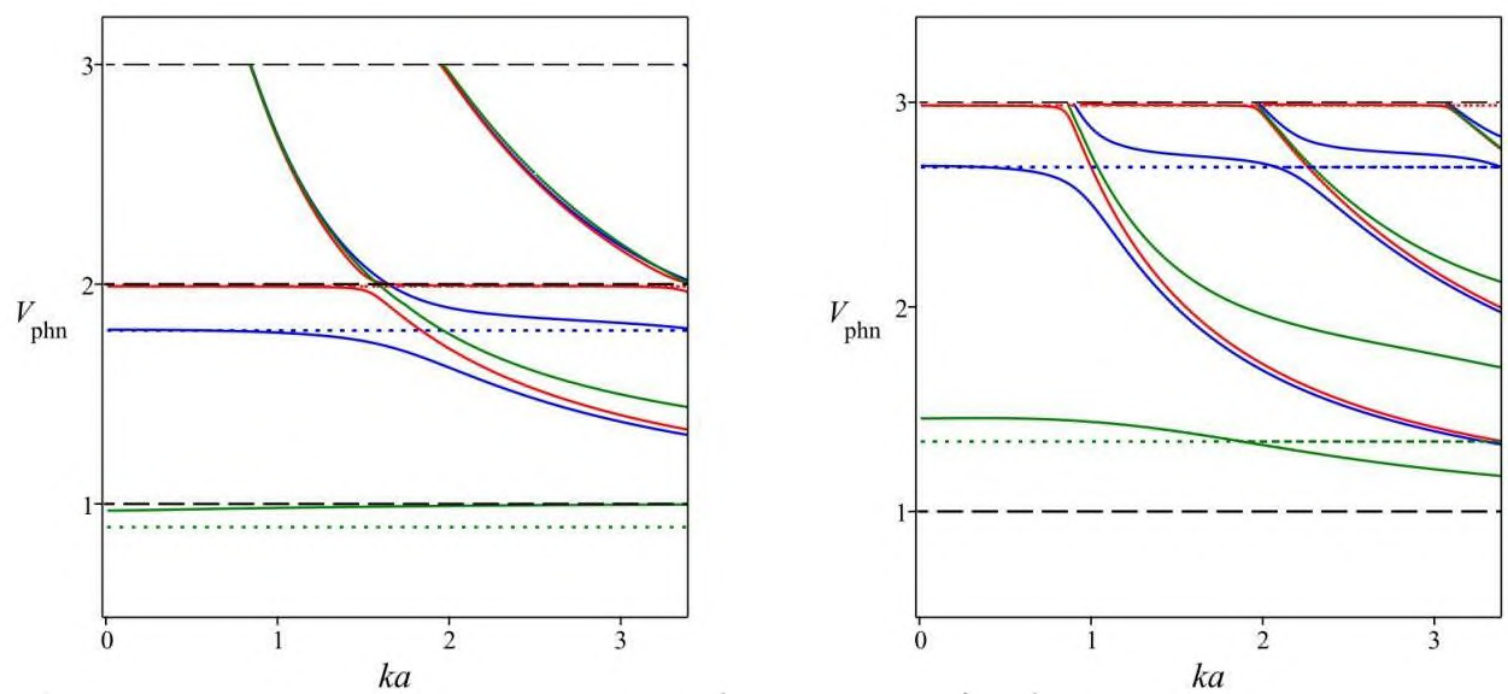

Figure 1: The dimensionless phase speed $\left(V_{\mathrm{phn}}=V_{\mathrm{ph}} / V_{\mathrm{Ai}}\right)$ dependence against the normalized longitudinal wavenumber $(\mathrm{ka})$ for axisymmetric modes in the twisted coronal tube. Red, blue and green curves are plotted for the twisted parameter $\alpha=0.1,0.5$ and 2 respectively. The dotted curves correspond to an infinite number of symmetric modes, concentrated in a narrow band about $\omega_{\mathrm{A} 0}$ of a twisted layer. The ratio of Alfvén speeds and the ratio of outer to inner radii of an annulus are taken to be $V_{\mathrm{Ae}} / V_{\mathrm{A} 0} / V_{\mathrm{Ai}}=3 / 2 / 1$ (left panel) and $V_{\mathrm{Ae}} / V_{\mathrm{A} 0} / V_{\mathrm{Ai}}=3 / 3 / 1$ (right panel), $b / a=1.1$.

The long wavelength approximation was studied analytically. It is found that the frequency of the fundamental sausage mode is given by

$$
\omega_{s}^{2}=\frac{\left(2-2 \eta^{2}+\eta^{2}\right) \ln (b / a) V_{A_{0}}^{2} \kappa^{2}+2 \omega_{A_{0}}^{2}}{2+\left(2-\eta^{2}\right) \ln (b / a)}
$$

Equation (12) indicates that its frequency lies in the range between the internal Alfvén frequency and Alfvén frequency of the twisted layer.

\section{Application to Coronal Seismology}

[2] studied multiperiodic QPPs of the microwave emission in strong X3.2 solar flare on 14 May, 2013, observed with the Nobeyama Radioheliograph (NoRH). It was found three periodicities $P_{1}=100 \mathrm{~s}, P_{2}=45 \mathrm{~s}$ and $P_{3}=15 \mathrm{~s}$. This event was associated with the flaring loop of estimated length $L \approx 40 \mathrm{Mm}$ and width $d=8 \mathrm{Mm}$. The authors interpreted two former modes in terms of the fundamental and second longitudinal harmonics of standing kink waves. Considering these modes as the fundamental and first axial harmonics of a certain MHD mode and using simple calculations we find that corresponding phase speeds are $V_{\mathrm{ph} 1} \approx 800 \mathrm{kms}^{-1}$ and $V_{\mathrm{ph} 2} \approx 880 \mathrm{kms}^{-1}$ respectively. Since the kink speed is given by relation $c_{k}=\sqrt{2} V_{\mathrm{Ai}}$ and $V_{\mathrm{ph}}=c_{k}$ in the framework of the above interpretation, we arrive at the estimate for the internal Alvén speed $V_{\mathrm{Ai}} \approx 567-624 \mathrm{~km} \mathrm{~s}^{-1}$. It seems that these values are rather small even for dense flaring loops, but not quite unrealistic. Alternatively, the former two periodicities could be interpreted with the two leading axial harmonics of the radially fundamental fast-sausage mode in twisted flaring loop. As it was shown in our work, this mode is trapped for all axial wavenumbers. Its phase speed $V_{\mathrm{ph}}<V_{\mathrm{Ae}}$. Taking the loop model with localized internal magnetic twist, we obtain 
that the calculated phase speeds are considered to be the estimates of the internal Alfvén speed of twisted flaring loop, i.e. $V_{\mathrm{Ai}} \approx 800-880 \mathrm{~km} \mathrm{~s}^{-1}$. This is an appropriate value for the dense flaring loop. The third observed mode with period 15 $\mathrm{s}$ can be interpreted as the second radial harmonic of fast-sausage mode, which phase speed is about the external Alfvén speed.

\section{References}

1. I. Lopin and I. Nagorny, ApJ, 882, 134, 2019.

2. D. Y. Kolotkov, V. M. Nakariakov, E. G. Kupriyanova, H. Ratcliffe, and K. Shibasaki, A\&A, 574, A53, 2015. 


\title{
Visualization of the microturbulence distribution on the surface of the Sun
}

\author{
S.G. Mozharovsky \\ mozharovskys@mail.ru \\ Ussurijsk department IAA RAS, Ussurijsk, Russia
}

A technique is described that can be used to obtain approximate values of microturbulent velocity $v_{\text {mi }}$ by comparing the equivalent widths of two spectral lines Fe I $\lambda 6302$ and $6301 \AA$. A map of the distribution of microturbulence over a solar surface are given according to the observational data of Hinode SOT/SP. It is shown that $v_{\mathrm{mi}}$ correlates with the line of sight (LOS) velocity gradient and that it does not correlate with the solar surface brightness and LOS velocity itself.

Keywords: Solar photosphere, Microturbulence, Hinode SOT/SP

DOI: 10.51194 /VAK2021.2022.1.1.126

\section{The ratio of equivalent widths $W_{6302} / W_{6301}$ as an approximate estimate of the mi- croturbulent velocity value $v_{\mathrm{mi}}$}

Some reasons led me to the need to study in detail the response of the spectral line equivalent width $W$ to changes in the microturbulent velocity $v_{\mathrm{mi}}$. For this model calculations were applied: a one-dimensional model of the photosphere was used, $v_{\mathrm{mi}}$ was specified as a set of values, and a set of artificial spectral lines was also taken. The lines varied the oscillator strengths $\log (g f)$ and their belonging to a chemical element. The lines wavelength, their damping parameters and the excitation potential remained unchanged. And the numerical integration of the spectral line profiles was carried out by the Runge-Kutta method. The result is a vague analogue of the procedure for constructing a curve of growth by method of numerical simulation using the photosphere model. The following curves turned out, see Fig. 1.

Let's pay attention to the fact that the distribution of temperature and pressure in the photosphere model had a complicated form, close to the real one. But the pictures, especially for $\log (R)$, turned out to be plain and smooth. For neutral iron lines (solid line) at $W=80-100 \mathrm{~m} \AA(\log (W / \lambda) \approx 4.8)$, there are $0.28 \%$ of changes in $W$ per $1 \%$ of change in $v_{\mathrm{mi}}$. The lines of heavy chemical elements $(\mathrm{Zr}, \mathrm{Ba})$ respond more strongly to the change in $v_{\mathrm{mi}}$, the lines of the light ones $(\mathrm{Si}, \mathrm{Na}, \mathrm{O})$ respond less. But the value of the response also depends on the line equivalent width $W_{1}$, i.e. $W$ at $v_{\mathrm{mi}}=1$ $\mathrm{km} \cdot \mathrm{s}^{-1}$ (or some another fixed value). Weak and strong lines change much less than lines of moderate equivalent width.

Fe I $\lambda 6302 \AA$ is a line of moderate strength, it is highly sensitive to changes in turbulent velocity, while Fe I $\lambda 6301$ $\AA$ is strong, and in the simplest case, we can assume that its equivalent width does not change with a change in $v_{\text {mi }}$. If we take the HOLMU photosphere model at different heliocentric angles $\cos (\theta)$ and carry out a numerical simulation, we will see the dependence $W_{6302} / W_{6301}\left(v_{\mathrm{mi}}\right)$ close to linear, see Figure 2, left. However, taking different models, we will see that the $W_{6302} / W_{6301}$ ratio depends not only on $v_{\mathrm{mi}}$, but also on the model (temperature distribution, iron abundance, etc.). It is advisable to check the model calculations on real data. For this we use the data of the Hinode spectropolarimeter.

The linear dependence of $W_{6302} / W_{6301}$ on $v_{\mathrm{mi}}$ allows us to interpret the map of these ratios as a map of the distribution of microturbulent velocities over the photosphere surface (see Fig. 4c).
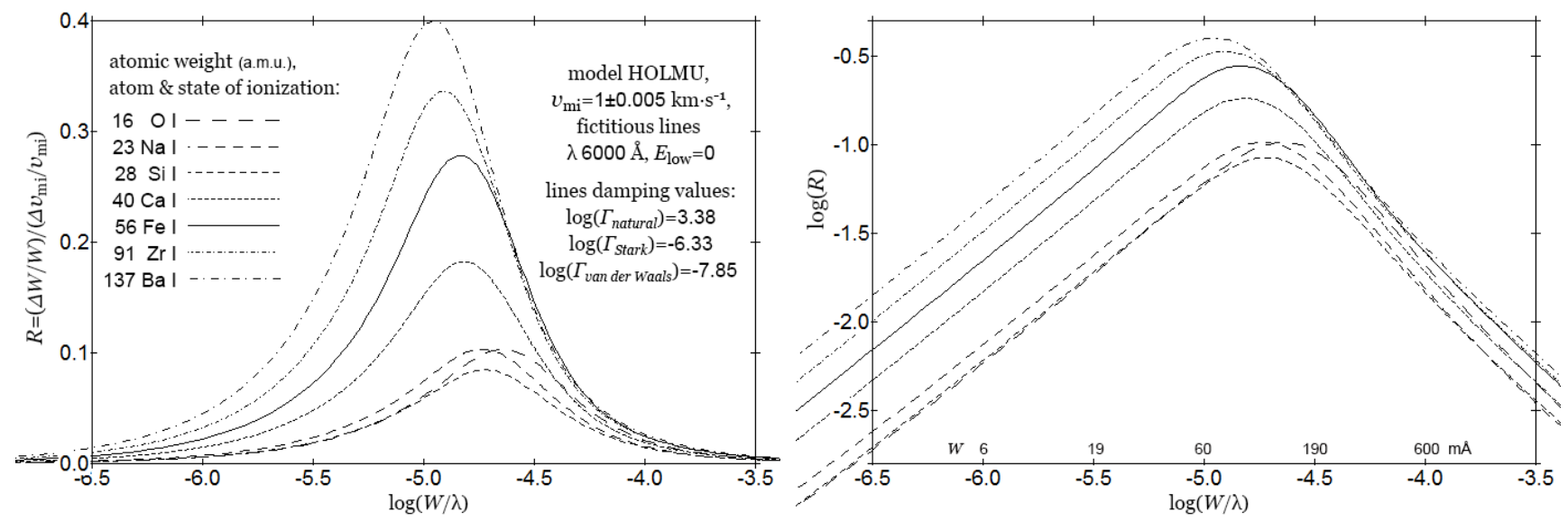

Figure 1: Left: Dimensionless value $R$, which is an estimate of the $\boldsymbol{R}$ esponce of spectral lines of different elements to changes in microturbulence and Right: its $\operatorname{logarithm} \log (R)$. Calculated for a set of artificial lines with a variable $\log (g f)$ value for the HOLMU (Holweger, Müller 1974) photosphere model . 

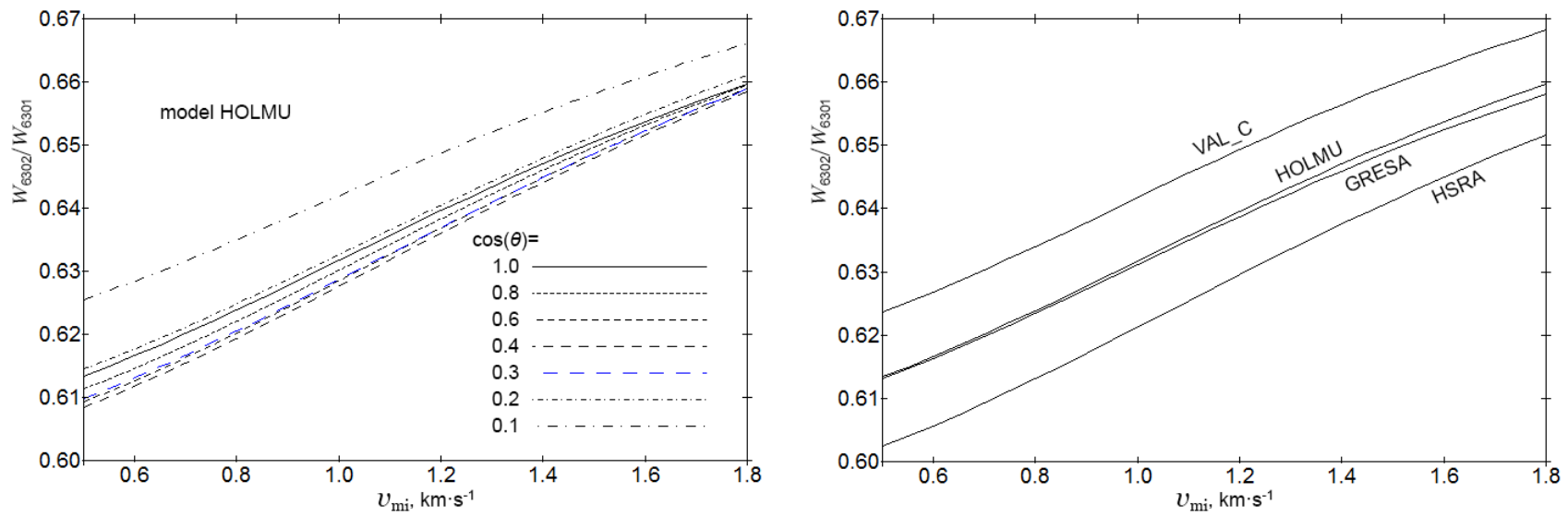

Figure 2: Dependence of the ratio $W_{6302} / W_{6301}$ on the microturbulent velocity according to calculations for onedimensional models of the photosphere. Left: for a set of heliocentric angles, Right: for four photospheric models — VAL_C [1], HOLMU [2], GRESA [3], HSRA [4].

\section{Analysis of Hinode SOT/SP statistical data}

We can check the conclusions of the numerical simulations using the data of the Hinode SOT/SP — spectropolarimeter of the solar optical telescope [5] of the Hinode satellite mission [6].
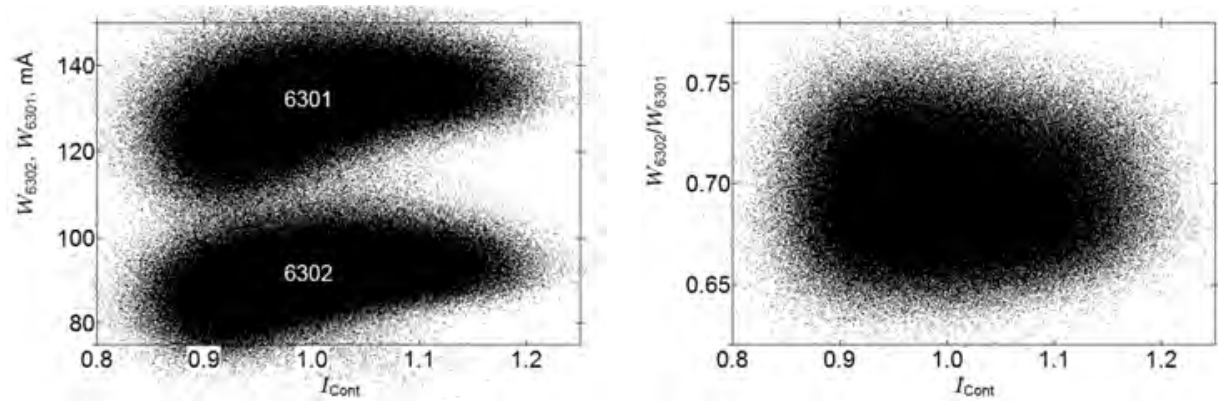

Figure 3: Left: the dependence of the equivalent widths of $W_{6302}$ and $W_{6301}$ on the intensity of the continuous spectrum $I_{\text {Cont }}$ (which indirectly shows the dependence on temperature). Right: dependence of the $W_{6302} / W_{6301}$ ratio on $I_{\text {Cont }}$. Hinode SOT/SP observation session 202001151031

Each map of the SOT/SP Hinode spectropolarimeter contains the Stokes profiles of the Fe I $\lambda 6301$ and $6302 \AA$ lines for many thousands of points on the solar surface area. This allows us to construct scatterplots for pairs of various parameters that can be extracted from Stokes profiles. We see that the equivalent widths change with temperature, but their ratio, which reflects the value of $v_{\mathrm{mi}}$, does not. Let's plot the distribution on the map, see Fig. 4c)

We can see that the characteristic size of the variability of microturbulence is approximately twice the characteristic size of granulation.

\section{Finding the relationship of $v_{\mathrm{mi}}$ with other physical values using the ACE algorithm}

The ACE (Alternating Conditional Expectations) algorithm [7] is used when there is a lot of measurement data. Suppose we have in each measurement a set of predictors $X$ and a value of the result $Y=f\left(X_{1} . . X_{N}\right)$. Then ACE automatically builds the so-called "optimal transformations" of the result $Y$ and the predictors $X$ so that the correlation between $\Psi(Y)$ and $\Phi(X)$ becomes maximum. And so the ACE draws a shape of functional relationship between variables.

As follows from the Hinode's data if we choose $Y=W_{6302} / W_{6301}$ as the result function, and $X=I_{\text {Cont }}$ or $v_{\mathrm{LOS}}$ as the predictor, then the optimal transformation $\Psi(Y)$ lies along the line $\Psi=0$. This indicates that there is no relationship between these quantities. We found such a physical parameter that correlates with the ratio $W_{6302} / W_{6301}$ and at the same time, from physical considerations, is associated with the $v_{\mathrm{mi}}$ - this is the LOS velocity gradient $\Delta v_{\mathrm{LOS}} / \Delta h-$ see Fig. 5 . How to find the LOS velocity gradient is described in [8].

\section{References}

1. J. E. Vernazza, E. H. Avrett, and R. Loeser, ApJS, 45, 635, 1981.

2. H. Holweger and E. A. Mueller, Solar Phys., 39, 19, 1974.

3. N. Grevesse and A. J. Sauval, A\&A, 347, 348, 1999. 

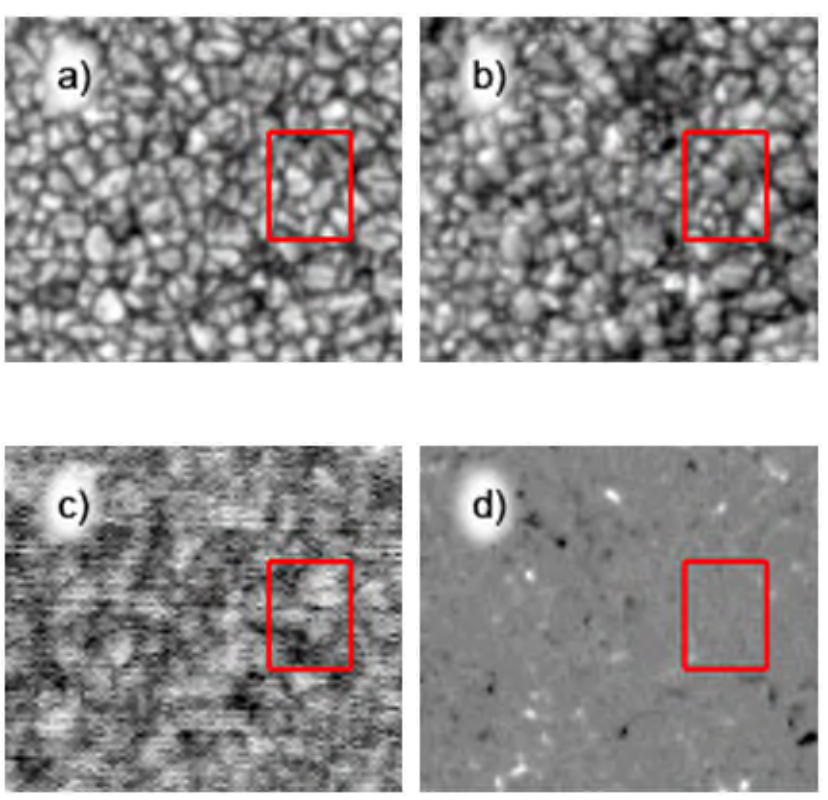

Figure 4: Fragment of the solar surface in a quiet region, obtained from Hinode SOT/SP observations. a) continuum intensity $I_{\mathrm{C}}, \mathrm{b}$ ) line of sight velocities $v_{\mathrm{LOS}}$ (dark patches correspond to downflows), c) microturbulent velocities $v_{\text {mi }}$ (light areas correspond to minimum values), d) longitudinal magnetic field values $B_{\|}$. The red frame is needed to compare the granular structures in Fig. a, b and $\mathrm{c}$.
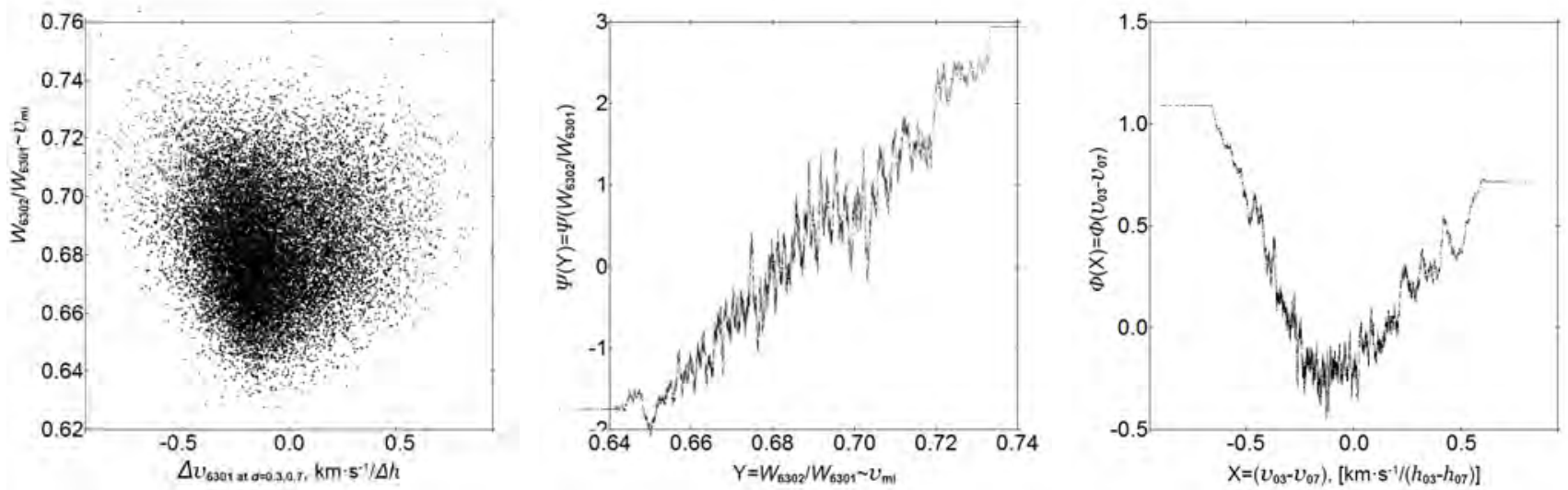

Figure 5: Left: The scatterplot $v_{\mathrm{mi}}\left(\Delta v_{\mathrm{LOS}}\right)$ for Hinode SOT/SP observation session 20191109_0334; a subset of points (18300 points) was selected for which $\left|B_{\|}\right|<10$ G. Center: Optimal transformation function $\Psi\left(W_{6302} / W_{6301}\right)$, which is close to linear. Right: the optimal transformation function $\Phi\left(\Delta v_{\mathrm{LOS}}\right)$, the shape of which is similar to the function $Y=|X|$ or $Y=X^{2}$. ACE smoothing window is 100 points.

4. O. Gingerich, R. W. Noyes, W. Kalkofen, and Y. Cuny, Solar Phys., 18, 347, 1971.

5. B. W. Lites, D. L. Akin, G. Card, T. Cruz, et al., Solar Phys., 283, 579, 2013.

6. T. Kosugi, K. Matsuzaki, T. Sakao, T. Shimizu, et al., Solar Phys., 243, 3, 2007.

7. L. Breiman and J. H. Friedman, Journal of the American Statistical Association, 80, 614, 1985, URL http://www. jstor.org/stable/2288477.

8. S. G. Mozharovsky, in Astronomy at the epoch of multimessenger studies. Proceedings of the VAK-2021 conference, Aug 23-28, 2021 Moscow (this volume), 328 (2021). 


\title{
Comparison of tree methods for estimating the magnetic field value obtained from the Hinode spectropolarimeter data
}

\author{
S.G. Mozharovsky
}

mozharovskys@mail.ru

Ussurijsk department IAA RAS, Ussurijsk, Russia

Three methods of estimating the magnetic field, which are based on the analysis of Stokes profiles of the Hinode SOT/SP data, are compared. These are field values from the Milne-Eddington (ME) inversion, the values obtained by the center of gravity (COG) method, and the values from the absolute width at half maximum (FWHM) of the Fe I $\lambda 6302 \AA$ line. It is shown that in the areas of the quiet photosphere, COG and FWHM methods give data that are related by a dependence close to linear. The data obtained as a result of the ME inversion for the quiet photosphere cannot be considered reliable.

It is also shown that most of the elements of the photosphere with a magnetic field move downward with an average velocity of $0.65 \mathrm{~km} \cdot \mathrm{s}^{-1}$. The downflow velocity depends on the brightness of the elements, as well as on the height of the photosphere layer in which the velocity is measured.

Keywords: Solar photosphere, Magnetic field, Hinode SOT/SP

DOI: $10.51194 /$ VAK2021.2022.1.1.127

\section{Introduction}

The Hinode satellite was launched in 2006. It is equipped with a spectropolarimeter, which makes it possible to obtain Stokes profiles of Fe I $\lambda$ 6301, $6302 \AA$ lines for a map (i.e., for a matrix) of points on the Sun's surface. The Hinode team from the Stokes profiles using the Milne-Eddington inversion calculates the magnetic field values and publishes them on their websites. But besides ME inversion, there are other methods for evaluating the magnetic field strength.

\subsection{Center of gravity method $C O G$}

According to [1], the positions of the centers of gravity of the $I+V$ and $I-V$ profiles (the result of subtracting their wavelengths) can be described by the formula that specifies the magnetic splitting of a spectral line in a longitudinal magnetic field:

$$
\Delta \lambda[(I+V),(I-V)]=2 k G_{\mathrm{eff}} \cdot \lambda^{2} B_{\|},
$$

where $k=4.67 \cdot 10^{-5}$ when $\lambda$ is expressed in centimeters.

Numerical simulations show that the result will not change if we take an umbra model instead of a quiet photosphere model, if we add a velocity field, and so on. Deviations of $\Delta \lambda$ from the formula (1) appear only when the anomalous dispersion is noticeable. Because of this simplicity, linearity, and robustness, some researchers use the COG method when interpreting Hinode SOT/SP data instead of ME inversion.

\subsection{Absolute widths $\mathrm{FWHM}_{B}-\mathrm{FWHM}_{B=0}$ difference method}

The magnetic field increases the absolute width of the spectral line profile, in particular the FWHM (Full Width at Half Maximum). The excess width obeys the same formula (1):

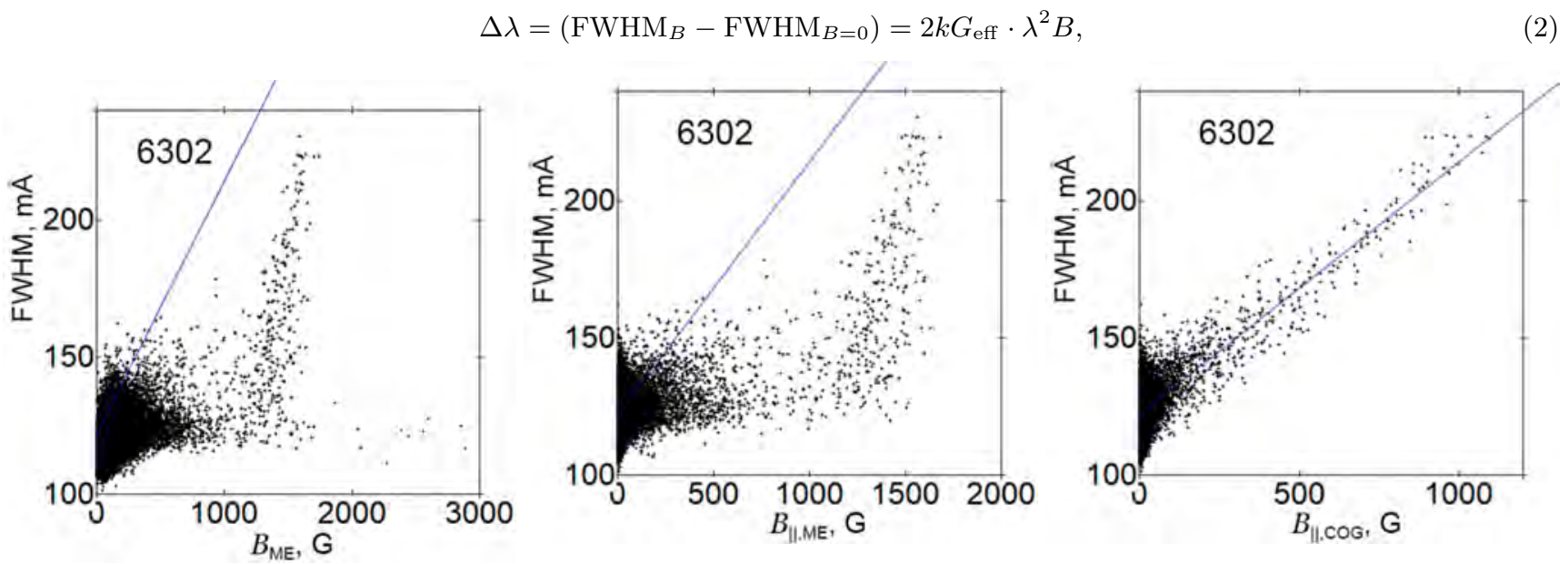

Figure 1: Line width at half maximum (FWHM) for the Fe I $\lambda 6302 \AA$ versus magnetic field strength. Left: $B_{\mathrm{ME}}$,

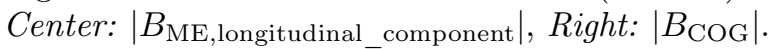



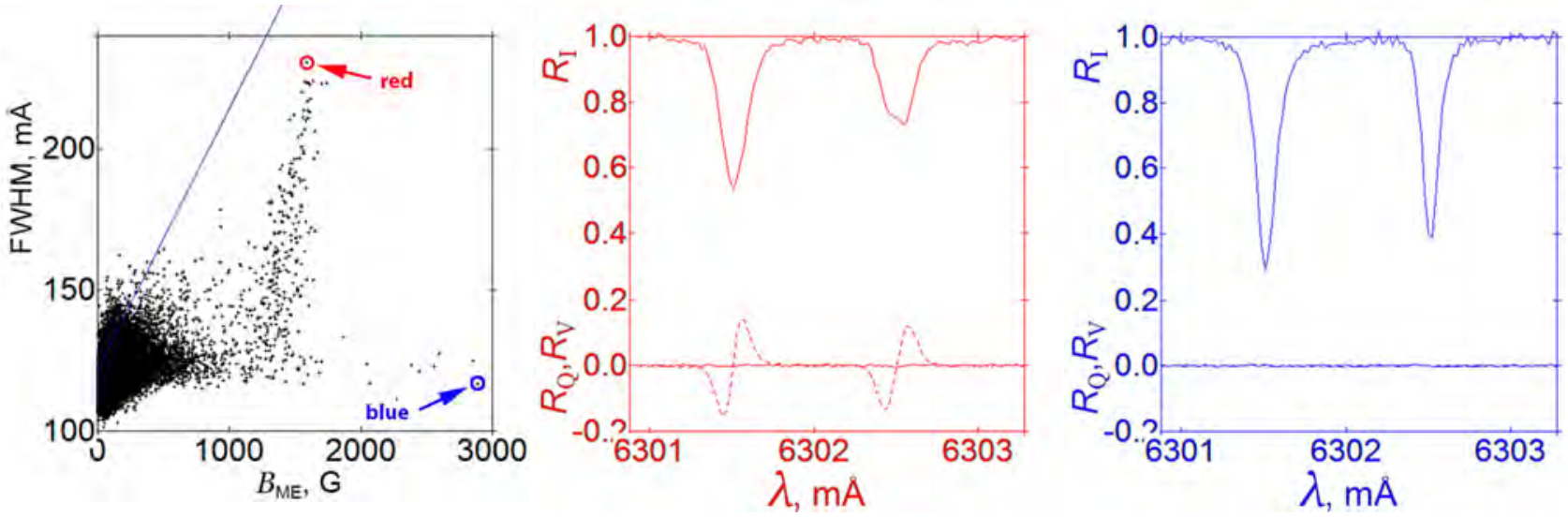

Figure 2: Stokes profiles for selected points. Left - the scatter plot. Center — the red dot: $B_{\mathrm{ME}} \approx 1600 \mathrm{G}$, $B_{\|, \mathrm{COG}} \approx 1100 \mathrm{G}, R_{\mathrm{V}}$ has significant values. Right - the blue dot: $B_{\mathrm{ME}} \approx 3000 \mathrm{G}, B_{\|, \mathrm{COG}} \approx 10 \mathrm{G}, R_{\mathrm{V}}$ and $R_{\mathrm{Q}}$ correspond to $B \approx 0$.
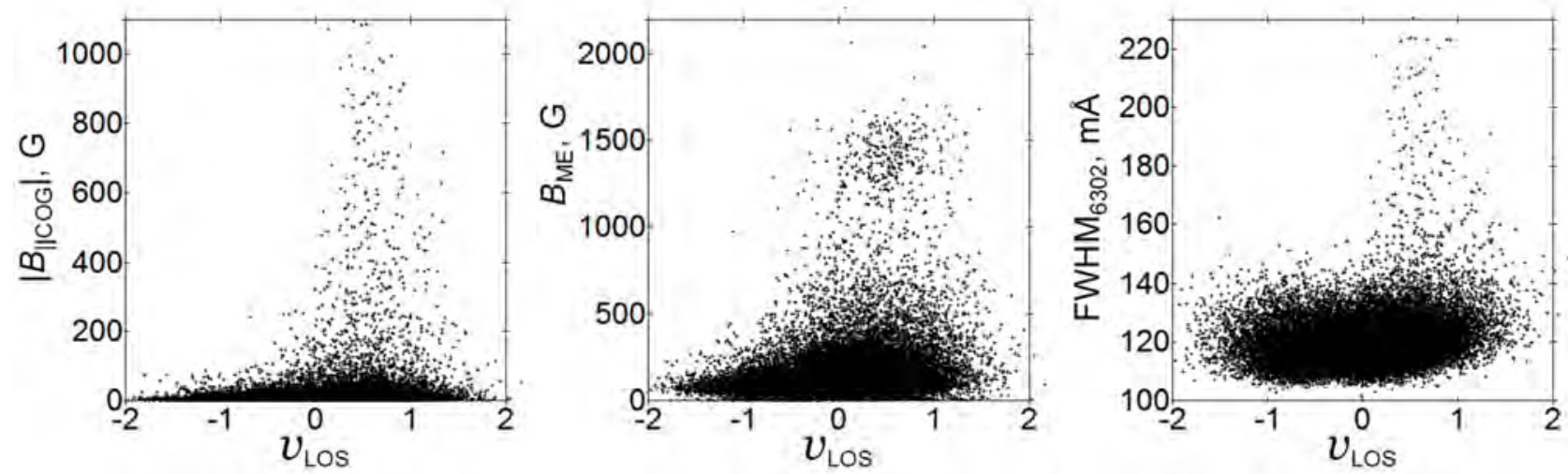

Figure 3: Dependence of the magnetic field on the line-of-sight velocity $v_{\text {LOS }}$ : Left: longitudinal field obtained by the COG method Center: field strength obtained by ME inversion Right: field estimates made from $\mathrm{FWHM}_{6302}$

This method does not apply at a single point on the Sun's surface, since we have no line width at a point with and without a magnetic field. However, Hinode maps include many thousands of points, and for averaged data, the excess FWHM width describes the field strength quite well.

\section{Comparison of different methods}

Let's compare different methods in pairs on scatter plots, see Fig. 1.

Longitudinal field strength $\left|B_{\mathrm{COG}}\right|$ describes well the changes in the $\mathrm{FWHM}_{6302}$ (Fig. 1, right). The straight blue line is the magnetic splitting depending on the field strength, it corresponds to formula (2), where $\mathrm{FWHM}_{B=0}=122 \mathrm{~m} \AA$. The ME-inversion results $\left(B_{\mathrm{ME}}\right.$ and $\left.\left|B_{\mathrm{ME}, \|}\right|\right)$ do not match with the change in the FWHM.

Let's check the results with examples of Stokes profiles for two points on the scatter plot, marked in blue and red, see Fig. 2. This confirms that to analyze the state of the magnetic field in the photosphere, it is better to use the method of centers of gravity.

\section{Velocities of the elements with a magnetic field}

As a practical application to this study, let us consider scatter plots that relate the LOS velocity (measured by the centers of gravity of the profiles of both spectral lines $6301+6302)$ and the magnetic field strength. All three scatter plots show that elements with a magnetic field predominantly sink at a velocity of about $0.6 \mathrm{~km} \cdot \mathrm{s}^{-1}$.

Next, we will leave only $B_{\mathrm{COG}}$ for analysis and turn the graph, direction $B$ is along the $\mathrm{X}$ axis, $v$ is along the $\mathrm{Y}$ axis, see Fig. 4. It can be seen from the figure that elements with a magnetic field sink at an average velocity of $0.65 \mathrm{~km} \cdot \mathrm{s}^{-1}$. The downflow velocity depends on the strength of the longitudinal field (or on its flux). In addition, elements with different field / flux values differ in brightness. Elements with a low field (or low fill factor) are darker, as well as elements with a strongest field $\approx 1000 \mathrm{G}$. Elements with a measured strength of 600-800 G may be brighter than the average non-magnetic photosphere. 

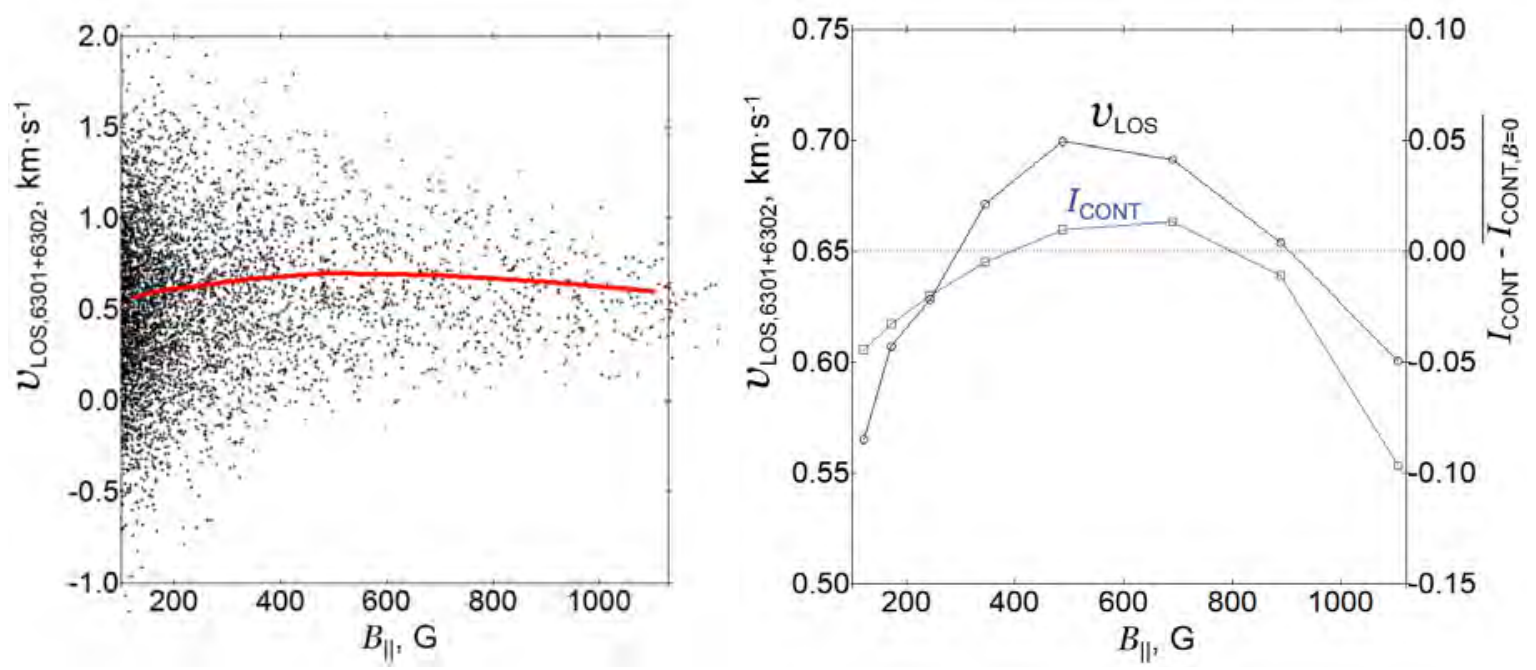

Figure 4: Dependence on the longitudinal field strength $B_{\|}$: Left: For velocity $v_{\text {LOS }}$ derived from the centers of gravity of both profiles 6302 and 6301 (scatter plot and averaged curve). Right: the same curve stretched to the scale along $\mathrm{Y}$, and curve for $\left(I_{\mathrm{CONT}}-\overline{I_{\mathrm{CONT}, B=0}}\right)$ - the intensity of the continuous spectrum. Averaged data from 8 maps taken at 10-minute intervals for the same area 2019.11.09 from 13:34 to 14:44 UT.

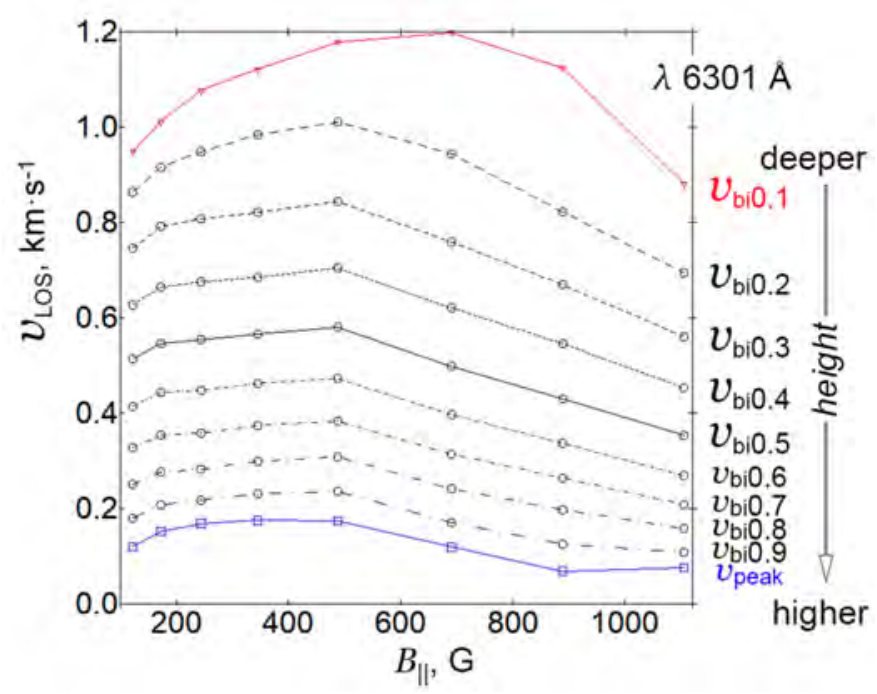

Figure 5: Dependence on the strength of the longitudinal field $B_{\|}$LOS velocities $v_{\text {LOS }}$ measured in different parts of the profile of the Fe I $\lambda 6301 \AA$ line (at different points of the bisector of this line and at its peak). The method for measuring the LOS velocities at different points of the bisector is described in [2].

Finally, we can analyze changes in the LOS velocity of magnetic elements at different heights of the photosphere, see Fig. 5. Here we see that in deep layers the velocities of magnetic elements exceed $1 \mathrm{~km} \cdot \mathrm{s}^{-1}$, while at the level of formation the Fe I $\lambda 6301 \AA$ peak, these velocities are no more than $0.2 \mathrm{~km} \cdot \mathrm{s}^{-1}$.

\section{References}

1. D. E. Rees and M. D. Semel, $A \mathscr{E} A, \mathbf{7 4}, 1,1979$.

2. S. G. Mozharovsky, in Astronomy at the epoch of multimessenger studies. Proceedings of the VAK-2021 conference, Aug 23-28, 2021 Moscow (this volume), 328 (2021). 


\section{Possibilities of velocity field analysis from Hinode SOT/SP data}

S.G. Mozharovsky

mozharovskys@mail.ru

Ussurijsk department IAA RAS, Ussurijsk, Russia

In this article the possibility of analyzing the line of sight (LOS) velocity gradients at each point of the Hinode SOT/SP maps is revealed. A technique for obtaining such gradients is described. Maps of the distribution of LOS velocity gradients over the surface of the photosphere were constructed.

DOI: $10.51194 /$ VAK2021.2022.1.1.128

\section{Bisectors construction}

Spectral line bisectors provide information on the LOS velocity gradient. From the Hinode data, we construct bisectors from a small number of points.

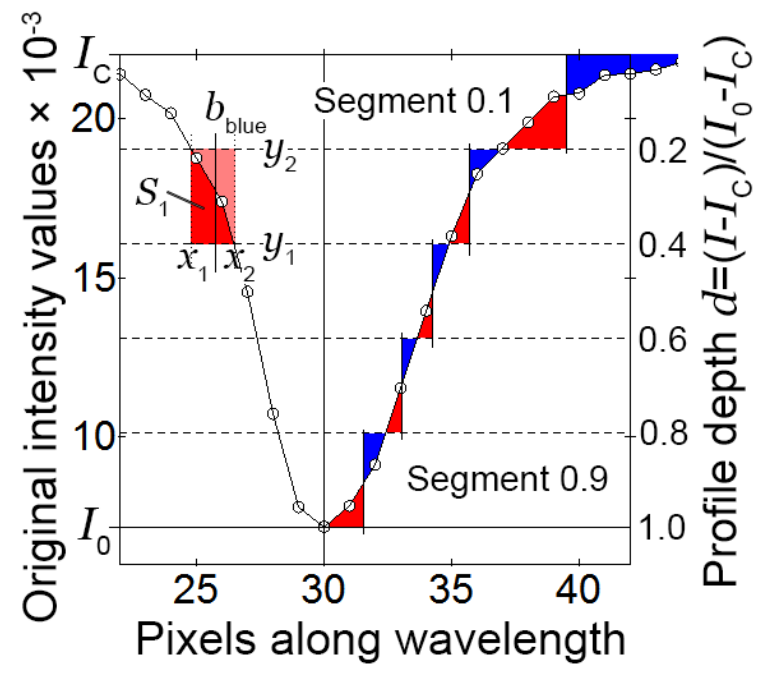

Figure 1: Algorithm for bisectors constructing. The horizontal lines divide the profile into 5 trapeziums -5 segments the same height $20 \% d$ each, ( $d$ is the line profile depth). Inside each segment we find the position of the wing $b=x_{1}+S_{1} / S \cdot \Delta x$, where $S_{1}$ is indicated on the graph, $S=\Delta x \Delta y, \Delta x=x_{2}-x_{1}$, $\Delta y=y_{2}-y_{1}$. The vertical lines along " $b$ "-position and the profile line form pairs of triangles of equal area (red and blue triangles $)$. The positions of the centers $c=\left(b_{\text {blue }}+b_{\text {red }}\right) / 2$ are the points of the bisector, and the values $w=b_{\text {red }}-b_{\text {blue }}$ are the absolute widths of the profile in the segments.

\section{LOS velocities vertical distribution in the photosphere}

If we know that the core of the line is formed higher in the atmosphere, and the wings are formed deeper, then we can obtain the distribution of line-of-sight velocities $v_{\mathrm{LOS}}$ in height in the photosphere layer.
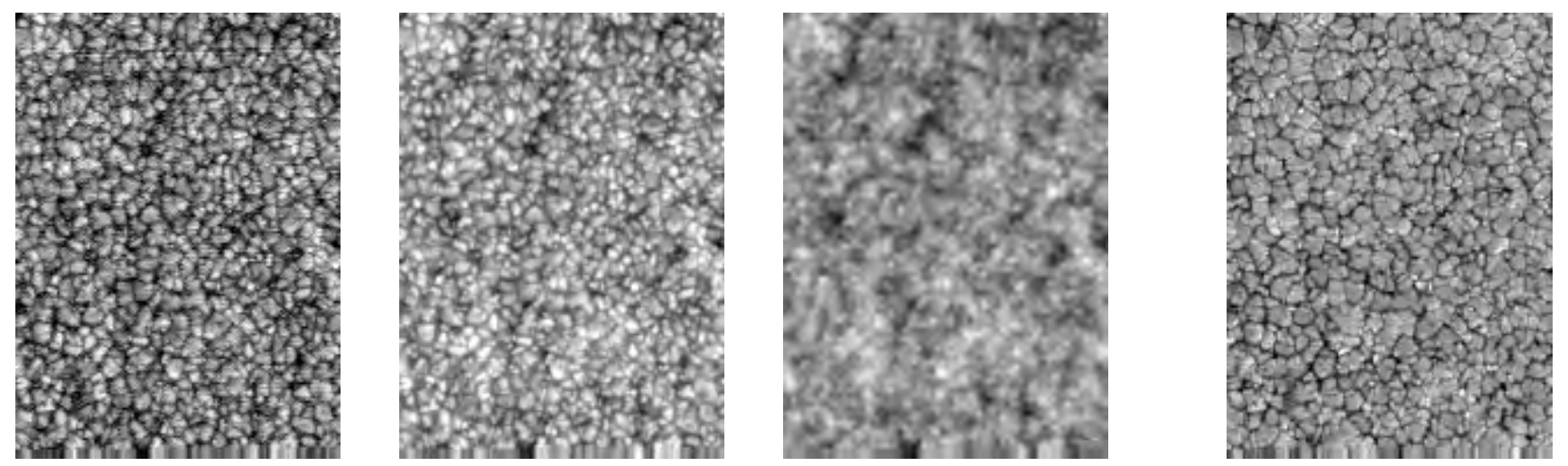

Figure 2: $v_{\text {LOS }}$ maps for the layers $d=0.1,0.5,0.9$ and gradient $v_{0.7}-v_{0.3}$ map. 


\title{
The choice of parameters and the appearance of a flare situation in MHD simulations above the active region in the real scale of time
}

\author{
A.I. Podgorny ${ }^{1}$, I.M. Podgorny ${ }^{2}$, A.V. Borisenko ${ }^{1}$, N.S. Meshalkina ${ }^{3}$ \\ podgorny@lebedev.ru, \\ WWW home page: http://sites.lebedev.ru/ru/podgorny/ \\ ${ }^{1}$ Lebedev Physical Institute RAS, Moscow, Russia \\ ${ }^{2}$ Institute of Astronomy RAS, Moscow, Russia \\ ${ }^{3}$ Institute of Solar-Terrestrial Physics SB RAS, Russia
}

The mechanism of release of the magnetic energy of the current sheet formed in vicinity of singular X-type magnetic field line explains the observed primordial release of solar flare energy in corona. The observed manifestations of the flare are explained by the electrodynamic model of a solar flare proposed by I.M. Podgorny. The study of the flare mechanism is impossible without performing MHD simulations above AR, in real scale of time, which can only be carried out thanks to parallel calculations using CUDA technology. Mainly, the configuration of the magnetic field at the maxima of the current density is the configuration of the X-type singular line, which is strongly distorted by superimposed diverging magnetic field.

DOI: $10.51194 /$ VAK2021.2022.1.1.129

\section{Introduction}

The observed primordial energy release of solar flare above the active region (AR) at an is explained by the mechanism of S.I. Syrovatskii [1], according to which the flare energy is accumulated in the magnetic field of current sheet formed in the vicinity of the X-type singular line. The primordial release of energy high in the corona is evidenced by observations of thermal X-ray emission from flares on the limb [2], and by considerations from other observations [3, 4] The flare release of the energy of the current sheet causes the observed manifestations of the flare, which are explained by the electrodynamic model of the flare proposed by I.M. Podgorny [5]. According to this model the hard X-ray beam radiation on the surface of the sun during a flare is explained by electron fluxes accelerated in field aligned currents caused by the Hall electric field in the current sheet. The model was developed based on analogies with the electrodynamic substorm model proposed earlier by the author on the basis of Intercosmos-Bulgaria-1300 satellite data [6].

The study of the flare mechanism is impossible without carrying out MHD simulations above a real AR, in which the calculation begins several days before the appearance of flares. When setting the problem, no assumptions were done about the flare mechanism.

\section{Numerical method and stabilization of instabilities arising near the boundary}

In order for the finite-difference scheme approximating the system of MHD equations to remain stable for the maximum possible time step, an absolutely implicit upwind finite-difference scheme, conservative with respect to the magnetic flux, was developed. The scheme is solved by the iteration method. The MHD simulation in the real scale time is impossible without parallel computation. The methods of parallel computations of iterations on GPU have been developed using CUDA technology [7, 8, 9]. The magnetic and usual viscosities were set in accordance with the principle of limited modeling [10]. Due to the difficulties in matching the solution in the computational domain with the values specified at the boundary, the numerical instabilities arise near the boundary. The developed methods for instability stabilizing (artificial viscosity
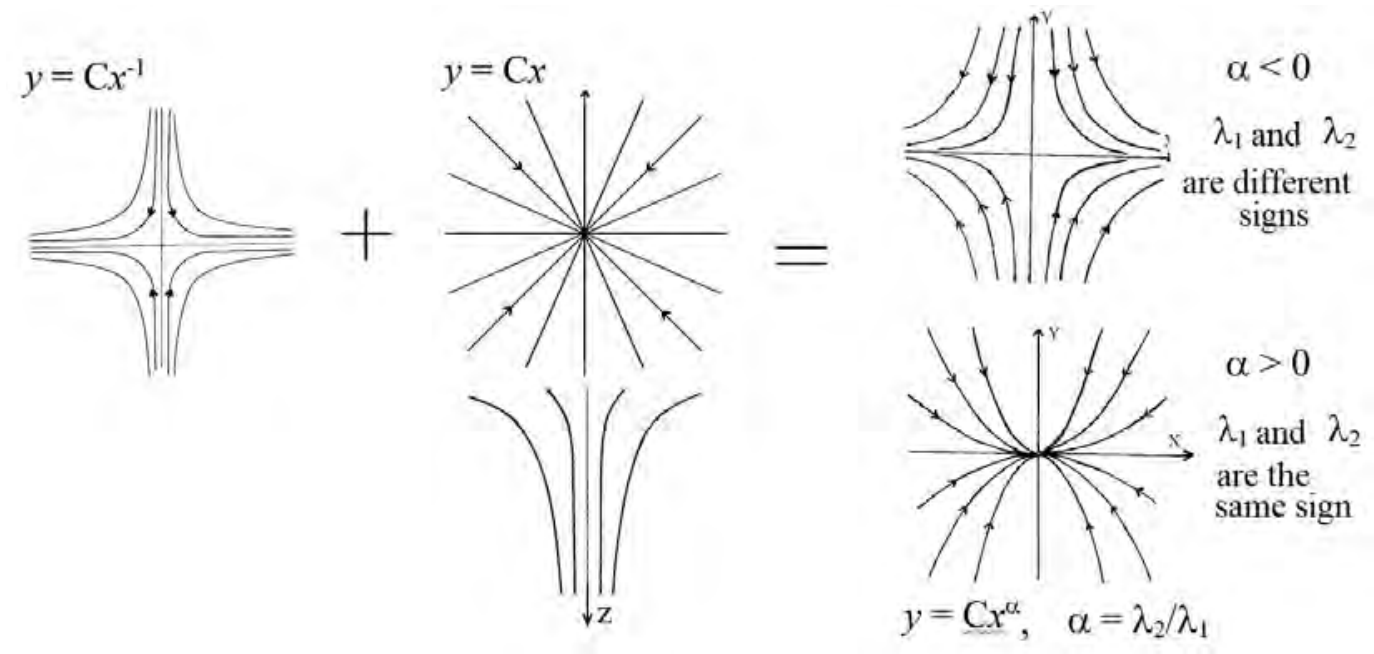

Figure 1: Overlay of X-type magnetic field and divergent magnetic field of the "mirror cell" type in the vicinity of singular line 

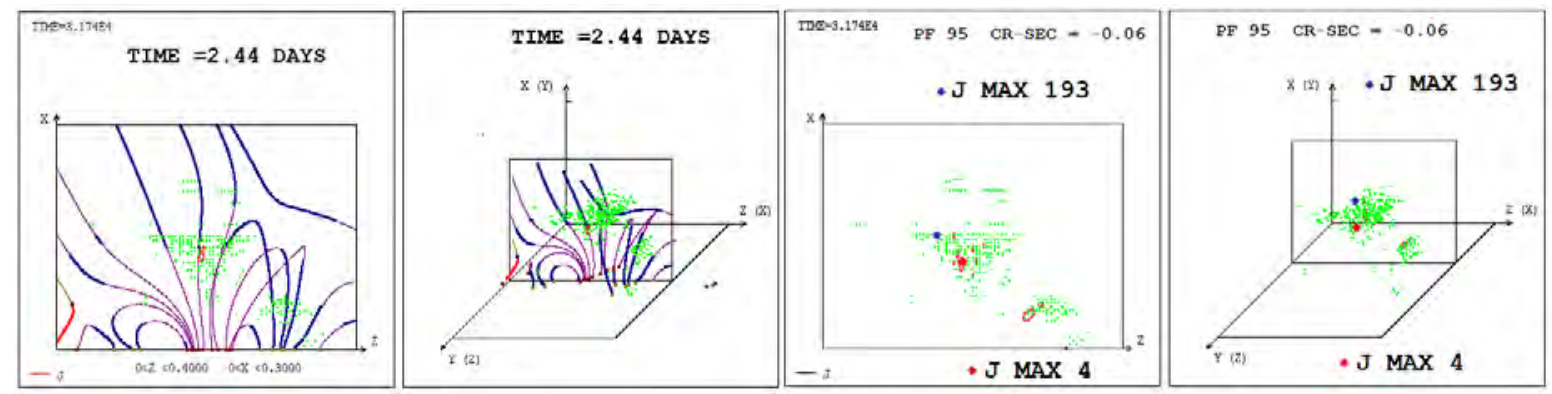

Figure 2: Three-dimensional configuration of the magnetic field in the computational domain, the projection of the magnetic lines on the central plane, and the location of the current density maxima in the region for the flare M1.4 27.05.2003 at 2:43
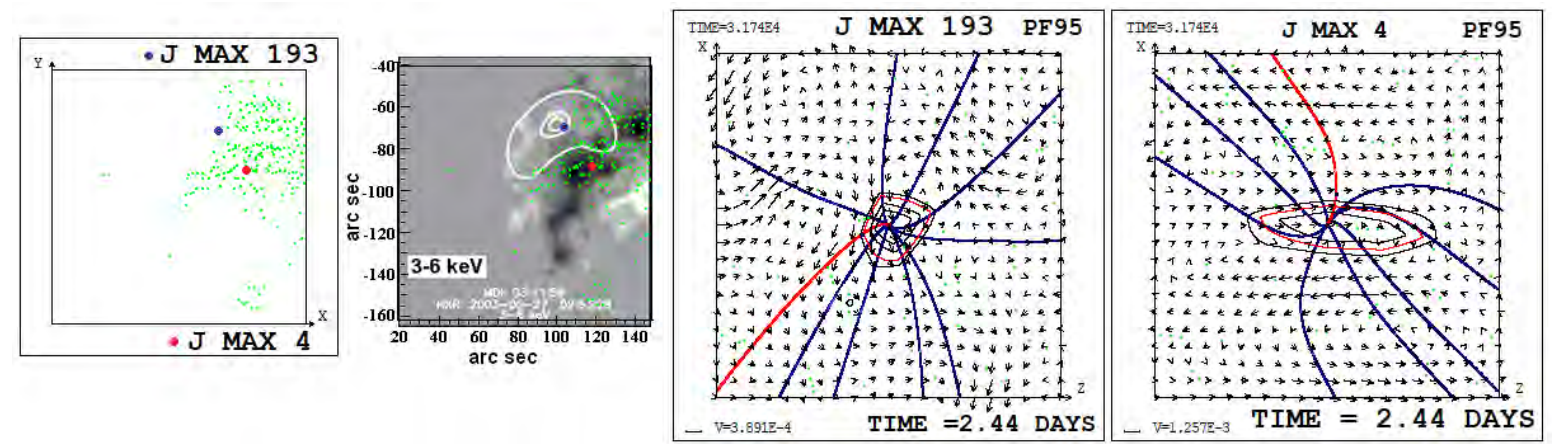

Figure 3: The location of the current density maxima in the picture plane, the distribution of soft $\mathrm{X}$ ray emission of $3-6 \mathrm{keV}$, received by the RHESSI spacecraft during the M 1.4 flare on May 27, 2003 (http://rhessidatacenter.ssl.berkeley.edu), magnetic field configurations near the 193rd and 4th current density maxima

near boundary, artificial limitation of the rate of plasma inflow into the computational domain, and others) permit perform calculation without increase of computational time for sufficiently large magnetic and usual viscosity [7]. But due to the suppression of the perturbation propagating from the photosphere by the artificial viscosity, a sufficiently intense accumulation of the flare energy does not occur in the region. Therefore, the calculation with much smaller viscosities $\left(R m=10^{9} ; R e=10^{7} ; \nu_{\text {ArtPhot }}, \nu_{m-\text { ArtPhot }}=10^{-4}\right)$ are performed, which results are presented here. To stabilize instability near the boundary for such small viscosity in addition to developed methods it is necessary to decrease the time step and number of iterations increase which decelerate computations. The work on stabilization of the instabilities is continued.

\section{MHD simulation results. Magnetic field singularities in corona for Flare M1.4 27.05.2003 at $2: 43$}

MHD simulation above AR 10365 showed the appearance of singular lines in which a diverging magnetic field is superimposed on the X-type magnetic field (Fig. 1). Even if diverging field is dominate the presence of the X-type configuration leads to formation of a powerful current sheet (Fig. 3, J Max 4).

In Fig. 2. shows the configuration of the magnetic field and the current density maxima in the computational domain of corona at the time of the M 1.4 flare on May 27, 2003 at 2:43 am, which corresponds to 2.44 days from the beginning of the calculation. The current density maxima found by the graphical search system through which singular magnetic field lines can pass are indicated by green dots. 4th and 193rd current density maxima are specially highlighted (all the maxima are numbered in decreasing order). Fig. 3 shows the location of many of the current density maxima in the picture plane close to the thermal X-ray source. The 193rd maximum of the current density is located in the central region of the source. The 4th has the most powerful current sheet among the maxima near the source of thermal X-ray radiation. The field configurations near the 193rd and 4th current density maxima (Fig. 3) show the dominance of the diverging magnetic field over the X-type field. MHD simulation showed the coincidence of the positions of some current density maxima with the position of the source of the flare thermal X-ray radiation.

\section{Conclusion}

1. To understand the physical processes causing the flare situation, simulation in the real scale of time is necessary, and the calculation should begin several days before the flare. A technique has been developed for the numerical solution of MHD equations in the solar corona in real scale of time, which is impossible without the use of parallel computations. Methods for stabilizing instabilities have been developed. 
2. The simulation performed above AR 10365 showed the formation of local maxima of the current density on singular lines of the magnetic field. The field near some singular lines has complicated structure with superimposed diverging magnetic field, but even in such configuration sufficiently powerful current sheets appear. Coincidence of current sheet positions with positions of flare emission sources support the flare mechanism based on flare energy accumulation in the magnetic field of current sheet.

\section{References}

1. S. I. Syrovatskii, Zh. Eksp. Teor. Fiz., 50, 1133, 1966.

2. R. P. Lin, S. Krucker, G. J. Hurford, and et al., Astrophys. J., 595, L69, 2003.

3. A. I. Podgorny, I. M. Podgorny, and N. S. Meshalkina, Astronomy Reports, 59, 795, 2015.

4. I. M. Podgorny and A. I. Podgorny, Astronomy Reports, 62, 696, 2018.

5. I. M. Podgorny, Y. V. Balabin, E. M. Vashenuk, and A. I. Podgorny, Astronomy Reports, 54, 645, 2010.

6. I. M. Podgorny, E. M. Dubinin, P. L. Israilevich, and N. S. Nicolaeva, GRL, 15, 1538, 1988.

7. A. I. Podgorny, I. M. Podgorny, and A. V. Borisenko, Proc. 12-th Workshop Solar Influences on the Magnetosphere, Ionosphere and Atmosphere, Primorsko, Bulgaria, June, 2020., 93, 2020.

8. A. V. Borisenko, I. M. Podgorny, and A. I. Podgorny, Geomagnetism and Aeronomy, 60, 1101, 2020a.

9. A. V. Borisenko, I. M. Podgorny, and A. I. Podgorny, Proc. 12-th Workshop Solar Influences on the Magnetosphere, Ionosphere and Atmosphere, Primorsko, Bulgaria, June, 2020., 81, $2020 \mathrm{~b}$.

10. I. M. Podgorny, Fundamentals of Cosmic Physics, 1, 1, 1978. 


\title{
Solar-terrestrial relations: solar activity and the COVID-19 pandemic
}

\author{
M. Ragulskaya ${ }^{1}$, E. Tekutskaya ${ }^{2}$ \\ ra_mary@mail.ru \\ ${ }^{1}$ Institute of Terrestrial Magnetism and Radio Wave Propagation RAS, Moscow, Russia \\ ${ }^{2}$ Kuban State University, Krasnodar, Russia
}

COVID-19 pandemic took the start at the lows of the 11-year and quasi-century solar cycle. The genogeographic characteristics of the population have become one of the significant factors determining the development of the local epidemics. The largest number of victims per 1 million inhabitants is recorded in the territories with a dominant haplogroup R1b: Italy, Spain, France, Belgium, Great Britain, and the United States. The R1a haplogroup is characterized by the rapid development of the COVID-19 pandemic with low mortality and a large number of asymptomatic patients (Russia, Germany, and Iran). The level of herd immunity achieved through vaccination also depends on the genetic makeup of the population and solar activity. Its value is highest for countries with a dominant haplogroup R1b (about $80 \%$ for haplogroup R1b versus $40 \%$ for haplogroup N). The resulting effect can be associated with the generation of reactive oxygen species and affected human adaptive capabilities.

Keywords: COVID-19, haplogroups R1b and R1a, solar activity, leukocyte antigens, reactive oxygen species

DOI: $10.51194 /$ VAK2021.2022.1.1.130

\section{Introduction}

Solar activity has a significant impact on the occurrence and development of pandemics. The main factor is the mutations of the virus occurring under the influence of solar cosmic rays at the maximum of the SA cycle. Galactic cosmic rays are the main mutations factor at the SA cycle minimum [1]. All influenza pandemics have occurred only at extremes of solar activity (SA) over the past 130 years. Pandemics occurred at the maximums of the 11-year SA cycle in the 20th century, at the phase of the maximum of the 100-year cycle. Pandemics occur both at the maximum and minimum of the 11-year SA cycle during the growth and decline phase of the 100-year SA cycle (19 and 21 centuries). Infectious disease number difference during the maximum and minimum of the 11-year SA cycle is more than 26 million people for Russia [2].

COVID-19 pandemic took the start at the lows of the 11-year and quasi-century solar cycle. A feature of the COVID-19 pandemic is significant variability in the number of deaths per 1 million population in different countries. The maximum mortality rates are observed in fairly prosperous countries with a high level of medicine, regardless of the introduction of a lockdown. The aim of the work was to find possible reasons for the paradoxical difference in mortality (more than 10 times), not related to quarantine measures and medical services. The genogeographic features of the development of local epidemics in conditions of a global minimum of solar activity were considered. The degree of oxidative damage to deoxyribonucleic acid (DNA) was studied as a molecular predictor of exogenous disorders [3]. Damage to the DNA of blood serum and lymphocytes from healthy donors was assessed after exposure to an alternating magnetic field (MF), in terms of intensity comparable to the geomagnetic field, in the frequency range from 3 to $60 \mathrm{~Hz}$ in vitro and gamma radiation by determining the content of 8-hydroxy-2-deoxyguanosine (8-OHdG) and single strand breaks (SSB) in DNA.

\section{Materials and methods}

The objects of the research were statistics of COVID-19 infections and deaths in various countries (based on the Johns Hopkins University database). Studied samples of peripheral blood collected from healthy donors (20 people), men, nonsmokers, aged 21 to 23 years. The degree of oxidative damage to DNA was assessed by the concentration levels of 8-OHdG in the blood serum. The determination was carried out by ELISA using monoclonal antibodies to 8-OHdG on a Thermo Fisher Scientific Multiskan microplate reader (Finland). The number of single-strand DNA breaks was assessed by the ratio of the fluorescence values of the control and experimental samples. The objects of the research were statistics of COVID-19 infections and deaths in various countries (based on the Johns Hopkins University database). Studied samples of peripheral blood collected from healthy donors (20 people), men, nonsmokers, aged 21 to 23 years. The degree of oxidative damage to DNA was assessed by the concentration levels of 8-OHdG in the blood serum. The determination was carried out by ELISA using monoclonal antibodies to 8-OHdG on a Thermo Fisher Scientific Multiskan microplate reader. The number of single-strand DNA breaks was assessed by the ratio of the fluorescence values of the control and experimental samples.

\section{Results}

The rapid development of the COVID-19 epidemic is observed in countries with Y-DNA haplogroup R1 (genetic markers of Y-DNA are transmitted with the Y chromosome exclusively through the paternal line, from father to son).

Moreover, in haplogroup R1b the most severe course was registered, high mortality, duration of the course of the disease, and low efficiency of quarantine measures (Fig 1a). The R1b haplogroup is dominant in northern Italy, France, Great Britain, Belgium, Armenia, the USA, and Spain. In the related haplogroup R1a, which diverged from R1b about 22 thousand years ago, the rapid development of local epidemics and the ineffectiveness of quarantine measures are also observed. However, at the same time, a small number of deaths and severe cases, a fairly rapid recovery, and a large number of asymptomatic patients are recorded. Haplogroup R1a is common in the European part of Russia, eastern Germany, Iran, India. The easiest development of local epidemics was observed in countries with a predominance of haplogroup N (Fig 1b). Countries with a similar genetic makeup of the population show the same mortality dynamics regardless of lockdown (Fig 1c, Sweden without a lockdown, and in Germany with lockdowns). 


\section{Deaths per million}

b)
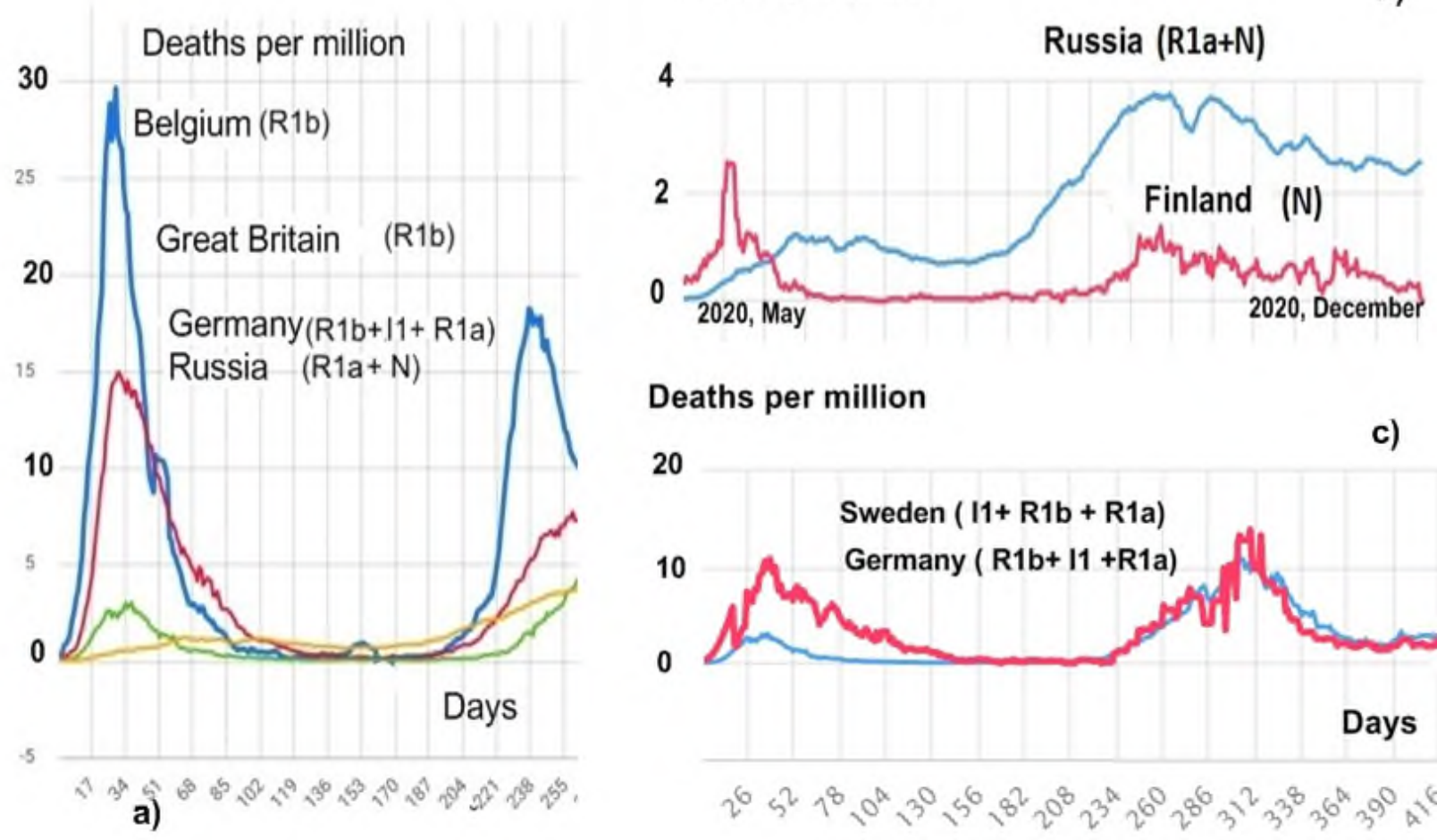

\section{Deaths per million}

20

c)

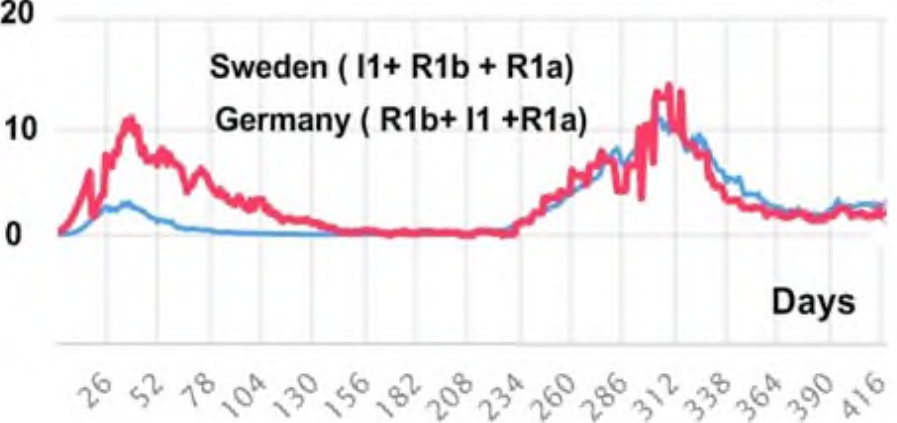

Figure 1: a) the relative number of deaths in countries with haplogroups R1b and R1a before the start of mass vaccination; b) dynamics of mortality in haplogroups $\mathrm{R} 1 \mathrm{a}$ and $\mathrm{N}$; c) coincidence of the dynamics of deaths in countries with a similar genetic composition of the population, regardless of lockdowns.

Differences in the strength of immune responses can be explained by antigen genetic variations in different haplogroups. This concept is confirmed in the article [4]. Authors showed that the haplotype and individual genetic variability affects the immune response and the ability of the population to respond to the SARS-CoV-2 virus. It was found that HLA-B * 46: 01 (corresponding to one of the branches of haplogroup R1b) had the lowest peptide binding for both SARS-CoV in 2003 and SARS-CoV-2 in 2019-2020. The authors found that the alleles HLA-B * 15: 03; HLA-A * 02: 02; HLA-C * 12: 03 (haplogroup R1a) showed the greatest ability to bind SARS-CoV-2 peptides and hence greater resistance to the virus.

Also, this effect can be associated with the generation of reactive oxygen species (ROS) and changing of the human organism adaptive capabilities after SA is affected. The content of damage to nitrogenous bases of 8-OHdG in DNA of human blood serum was determined after exposure to an alternating $\mathrm{MF}$ of $550 \pm 30 \mathrm{~A} / \mathrm{m}$ in the frequency range from 3 to $50 \mathrm{~Hz}$ for $30 \mathrm{~min}$ in vitro. There was an increase in the content of 8-OHdG in DNA by a factor of $1.5-2$ in comparison with non-irradiated samples, as well as a nonlinear change in the level of lipid peroxide in blood serum with maxima at MF frequencies of 8 and $50 \mathrm{~Hz}$. The increase in the level of oxidized modifications of nitrogenous bases 8-OHdG in blood serum DNA after treatment with MP is apparently associated with the generation of ROS [3, 5]. The protonated form of the superoxide anion radical dismutes with the formation of hydrogen peroxide as the most long-lived ROS. The rate of these reactions in natural conditions correlates with solar activity [5]. This effect can reduce the activity of antiradical defense enzymes, thereby reducing the antioxidant potential of the body.

\section{Conclusion}

Solar activity dynamics and the genetic variability of humanity increase the chances of survival of humans as a biology species during pandemics. All influenza pandemics of the 19 - 21st centuries developed only at the extremes of solar activity. This effect can be associated with the ROS generation and changing of the human organism adaptive capabilities after SA is affected. COVID-19 pandemic took the start at the lows of the 11-year and quasi-century solar cycle. The genogeographic characteristics of the population played a decisive role in the development of local epidemics in these conditions. The most severe course of COVID-19 and the highest mortality rate are characteristic of countries with the dominant haplogroup R1b. The haplogroup R1a is characterized by the rapid spread of coronavirus with a large number of asymptomatic patients. The easiest development of local epidemics was observed in countries with a predominance of haplogroup N. The level of herd immunity achieved through vaccination also depends on the genetic makeup of the population. Its value is highest for countries with a dominant haplogroup R1b (about $80 \%$ for haplogroup R1b versus $40 \%$ for haplogroup N). In conditions of a global minimum of solar activity, a twofold increase in the number of pandemics is expected in the next 30 years (every 5-6 years instead of 10-11 years) with pronounced genogeographic differences. 


\section{References}

1. V. N. Obridko, M. V. Ragulskaya, and E. G. Khramova, Journal of Atmospheric and Solar-Terrestrial Physics, 208, 105395, 2020, URL https://www.sciencedirect.com/science/article/pii/S1364682620302042.

2. M. Ragulskaya, Journal of Novel Physiotherapy and Physical Rehabilitation, 7, 031, 2020, URL https://doi.org/10.17352/2455-5487.000074.

3. E. E. Tekutskaya, M. G. Baryshev, L. R. Gusaruk, and G. P. Ilchenko, Biophysics, 65, 564, 2020, URL https://doi.org/10.1134/s0006350920040247.

4. A. Nguyen, J. K. David, S. K. Maden, M. A. Wood, B. R. Weeder, A. Nellore, and R. F. Thompson, Journal of Virology, 94, 2020, URL https://doi.org/10.1128/jvi.00510-20.

5. M. Dizdaroglu, Cancer Letters, 327, 26, 2012, URL https://doi.org/10.1016/j.canlet.2012.01.016. 


\section{Conditions of generating a toroidal magnetic field during the period of decreasing magnetic flux of the Sun}

L. Starkova

starkova@izmiran.ru

Pushkov Institute of Terrestrial Magnetism, Ionosphere and Radio Wave Propagation, RAS, Moscow, Troitsk, Russia

The conditions of generation of the Sun's magnetic field after a decrease the magnetic flux in the 23rd solar cycle are studied. It was shown that this decrease did changed the generation condition of the magnetic toroidal component in the 24 th solar cycle.

Keywords: sun, magnetic field, solar cycles

DOI: $10.51194 /$ VAK2021.2022.1.1.131

At the decline of the 23rd solar cycle the magnetic flux of the Sun significantly decreased. To determine how much the generation conditions have changed in the 24th solar cycle, an analysis of the relationship between the toroidal and poloidal components of the magnetic field was carried out. For this purpose the functional relationship between the indicators of these components - the Wolf number $\left(W^{\mathrm{a}}\right)$ and the strength of the polar magnetic field $\left(B p^{\mathrm{b}}\right)$ is obtained. It is supposed that there is a relation between the maximum values of these indicators:

$$
W=K B p^{n}
$$

Parameters (1) were determined for the situation of 22 and 23 solar cycles. We have found $K=117, n=0.76$. In the minimum of 24 solar cycle $B p=0.60 \mathrm{Gs}$, then from (1) the maximum of the Wolf number of 24 solar cycle is $W=118$. This value differs from the observed value by less than $2 \%$, which indicates that the relationship (1) in the 24 solar cycle is the same as in previous solar cycles. This means that the conditions of toroidal component generating of the Sun's magnetic field have not changed.

${ }^{\text {ahttps://www.bis.sidc.be/silso/datafiles }}$

bhttp://wso.stanford.edu/gifs/Polar.gif 


\title{
CME acceleration in impulsive (X6.9 09.08.2011) and gradual (M3.7 07.03.2011) solar flares
}

\author{
A. Struminsky ${ }^{1}$, A. Sadovski ${ }^{1}$, I. Grigorieva ${ }^{2}$ \\ astrum@iki.rssi.ru \\ ${ }^{1}$ Space Research Institute, Moscow, Russia \\ ${ }^{2}$ Central (Pulkovo) Astronomical Observatory, St. Petersburg, Russia
}

\begin{abstract}
We analyze solar events associated with flares: gradual — M3.7 on March 7, 2011 and impulsive — X6.9 on August 9, 2011. These flares were accompanied by hard X-ray (HXR), microwave (MW) and $>100 \mathrm{MeV}$ gamma radiation, fast coronal mass ejection (CME). Estimates of the magnitude and duration of acceleration for CME were obtained from the condition of stitching the assumed uniformly accelerated motion and the observed uniform motion. These estimates indicate that the CME should have been accelerated significantly longer than the estimated minimum time, at least more than 30 min. The obtained mean values and duration of CME acceleration do not contradict CME acceleration in two phases-impulsive and prolong. The largest bursts of HXR and MW radiation were observed both during the CME impulsive acceleration (X6.9 flare on August 9, 2011), and after it (M3.7 flare on March 7, 2011). This shows that the acceleration processes of charged particles in flares do not depend on the magnitude of the CME acceleration. The average CME velocity deduced from the $\mathrm{SOHO} / \mathrm{LASCO}$ observations at $20 R_{\odot}$ in the high corona $2034 \mathrm{~km} / \mathrm{s}$ on March 7, 2011 and $1506 \mathrm{~km} / \mathrm{s}$ on August 9, 2011 suggests that a residual decelerating force acting on CME on August 9, 2011 was larger.
\end{abstract}

Keywords: solar flares and coronal mass ejections, acceleration of coronal mass ejections, impulsive and gradual flares, solar proton acceleration, solar gamma radiation

DOI: 10.51194/VAK2021.2022.1.1.132

\section{Introduction}

The first results of solar $>100 \mathrm{MeV}$ gamma ray observations by Fermi/LAT were presented 10 years ago at the ICRC 2011 conference in Beijing. By now the catalogue of Long Duration Gamma Ray Flares (LDGRF) includes more than 30 such events, but the nature of long-duration gamma-ray emission remains uncertain: is it prolonged proton acceleration or is it trapping; is it the flare or the CME shock acceleration? These questions are closely related to the problems of the acceleration of relativistic electrons and protons in solar flares [1]; the origin of solar energetic particles (SEP) [2, 3], the flare-CME relationship [4,5,6], and the very definition of an eruptive flare [7]. According to [5], the term "eruptive flare" was introduced for all solar active phenomena, which lead to mass ejections resulting in a CME (with or without effects in the chromosphere) and appears to be a unique process operating in eruptive flares, with differences resulting from the configuration of the pre-event magnetic plasma (i. e. the height and density). Below we illustrate the flare-CME relationship using examples of two famous flares from the LDGRF list [8], described in [9, 8] (1) the gradual M3.7 flare SOL2011-03-07, and (2) the impulsive X6.9 flare SOL2011-08-09.

\section{Observations and their interpretations}

Figure 1 shows data overviews for the two events. We have assembled information on plasma heating (SXR observations from GOES, temperature $T$ and emission measure $E M)$, thence $d T / d t$ and $d E M / d t$; plasma motions in the flare region $(d E M / d t$, plus images obtained by SDO/AIA, frequency drifts at radio frequencies (1415-245 MHz from RSTN); electron acceleration (microwaves (15.4-2.695 GHz, also from RSTN) and HXR > $150 \mathrm{keV}$ (the INTEGRAL anticoincidence shield; CME positions from LASCO/SOHO; proton acceleration via $\gamma$-rays $>100 \mathrm{MeV}$ from Fermi/LAT, and SEPs (protons $>100 \mathrm{MeV}$ ), also from INTEGRAL. Both flares started at coronal heights with densities characteristic for plasma emission at $245 \mathrm{MHz}$. We noted the difference of about two orders of magnitude between the maximum values of $d E M / d t$ for SOL2011-03-07 [10] and for SOL2011-08-09 [11]. The impulsive flare had strong chromospheric evaporation, but the gradual not.

The gradual M3.7 flare SOL2011-03-07 was followed by $>100 \mathrm{MeV} \gamma$-ray emission during 25 min after 20:15 UT $(+28 \mathrm{~min}$ ) and over $13 \mathrm{hr}$ later (LDGRF). The impulsive X6.9 flare SOL2011-08-09 was accompanied by $>100 \mathrm{MeV} \gamma$-ray emission for only $3.3 \mathrm{~min}$ after 08:01 UT (0 min), Due to arrival of solar protons the ACS SPI count rate showed a small and slow rise after 20:17 UT (+30 min) on 07/03/2011, but a large and sharp increase at 08:11 UT $(+10 \mathrm{~min})$ on 09/08/2011. Let us assume that CME starts moving at $t_{0}$ with $V_{0}=0$ and $a=$ const from $R_{0}=1.25 R_{\odot}$ till $t$. After $t$ CME moves with $V=$ const, which is known from the LASCO observations, since $R=A+B t, V_{12}=B, a_{1}=B /\left(t-t_{0}\right)$. A stretching condition of accelerated and steady motion gives us $t=\left(2 R_{0}-2 A-B t_{0}\right) / B$. Lower indexes at $V$ means which positions of CME in the LASCO field we have used for linear extrapolation (the first and the second- $(1,2)$, or the second and the third- $(2,3))$. We note that for both events $V_{23}>V_{12}$, i. e. CME's were accelerating at least till $t_{3}$ (the third position). An estimate of acceleration rate would be $a_{2}=\left(V_{23}-V_{12}\right) /\left(t_{3}-t\right)<a_{1}$. The CME's were accelerated in two phases-impulsive $\left(a_{1}\right)$ and prolong $\left(a_{2}\right)$. Results of these calculations are presented in Table 1. Besides in both cases $V_{23}<V_{20 R_{\odot}}$, i. e. moving to $20 R_{\odot}$ the CME's were decelerating. Comparing values of CME velocities we may conclude that residual decelerating force acting on CME on March 7, 2011 was smaller. One of possible reasons is an existence of additional acceleration force during the decay flare (post eruptive) phase, which has been stronger and longer on March 7, 2011. 


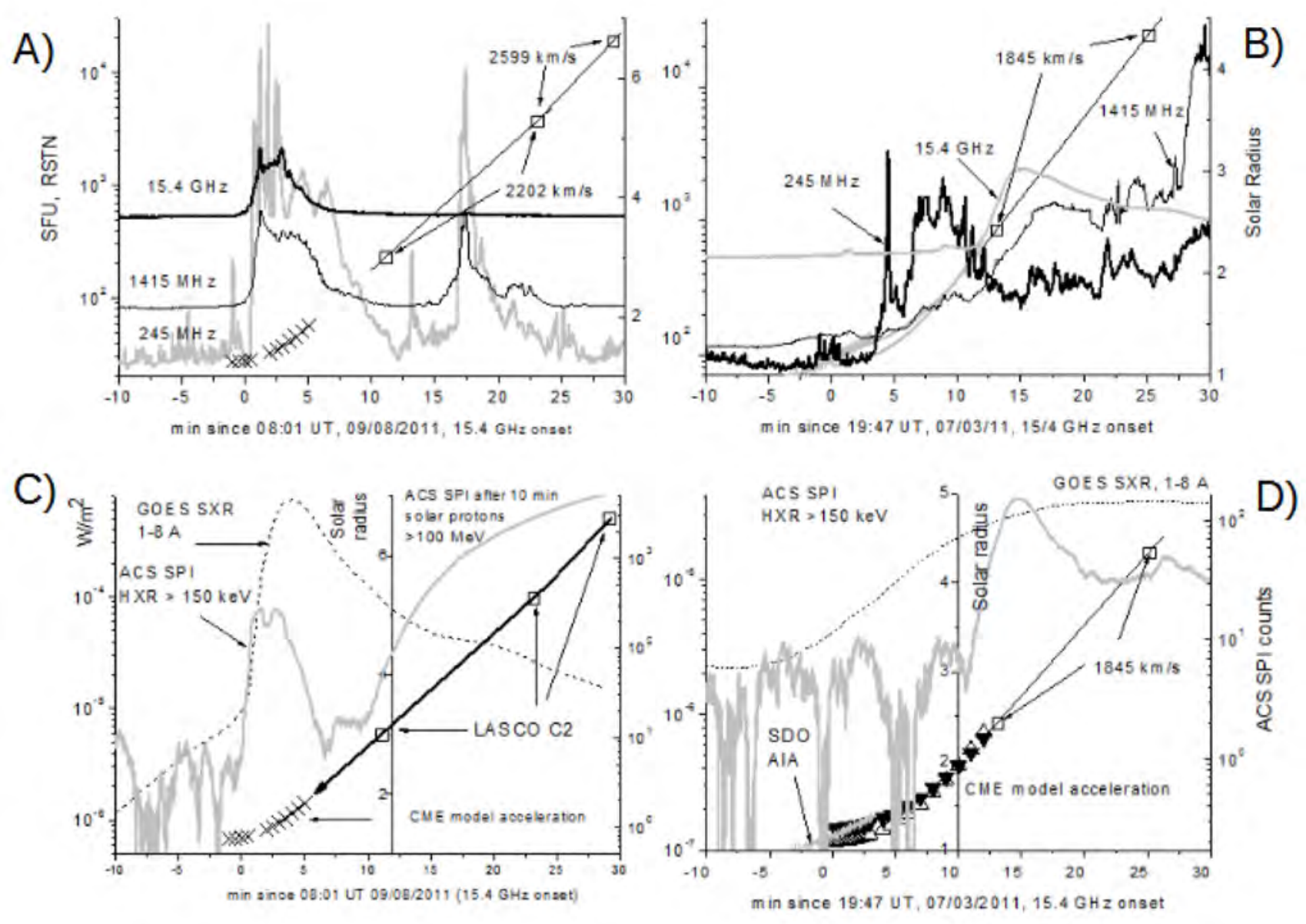

Figure 1: A, B) Radio emission on $15.4 \mathrm{GHz}, 1415 \mathrm{MHz}$ and $245 \mathrm{MHz}$ frequencies (RSTN) in comparison with CME positions (model and LASCO observations); C, D)

Table 1: Parameters of the observed uniform CME motion and the assumed uniformly accelerated CME motion (onset is considered at $15.4 \mathrm{GHz}$ )

\begin{tabular}{ccccccccccc}
\hline Event & $\begin{array}{c}t_{0} \\
\mathrm{UT}\end{array}$ & $\begin{array}{c}A \\
{[\mathrm{~s}]}\end{array}$ & $\begin{array}{c}B=V_{12} \\
{[\mathrm{~km}]}\end{array}$ & $\begin{array}{c}t \\
{[\mathrm{~km} / \mathrm{s}]}\end{array}$ & $\begin{array}{c}a_{1} \\
{[\mathrm{~s}]}\end{array}$ & $\begin{array}{c}t_{3} \\
{\left[\mathrm{~km} / \mathrm{s}^{2}\right]}\end{array}$ & $\begin{array}{c}V_{23} \\
{[\mathrm{~s}]}\end{array}$ & $\begin{array}{c}a_{2} \\
{[\mathrm{~km} / \mathrm{s}]}\end{array}$ & $\begin{array}{c}V_{20 R \odot} \\
{\left[\mathrm{km} / \mathrm{s}^{2}\right]}\end{array}$ & \begin{tabular}{c}
{$[\mathrm{km} / \mathrm{s}]$} \\
\hline $07 / 03 / 2011$
\end{tabular} \\
$19: 47$ & 0 & 244037 & 1845 & 676 & 2.7 & 1876 & 2707 & 0.7 & 2034 \\
$09 / 08 / 2011$ & $08: 01$ & -60 & 629439 & 2202 & 278 & 6.5 & 1746 & 2599 & 0.3 & 1506 \\
\hline
\end{tabular}

\section{Conclusions}

Based on these observations we conclude: 1) the CME associated with the gradual flare, SOL2011-03-07 (with weaker chromospheric effects and GOES class only M3.7) was accelerated for a longer period but at a smaller rate. The nonthermal electrons did not reach the chromosphere (coronal sources of HXR and radio emission were observed when the CME was at $R_{\odot}>2$ ); 2) the CME associated with impulsive flare (strong chromospheric effects) - X6.9 SOL2011-08-09 was accelerated with maximal rate during the period of effective chromospheric evaporation (between $T_{\max }$ and $E M_{\max }$ ); 3) maximal velocity of both CMEs is $\sim 2600 \mathrm{~km} / \mathrm{s}$ and corresponds to the Alfven velocity available in the CME acceleration region; 4) residual decelerating force was smaller for SOL2011-03-07 with stronger and longer post eruptive phase. Finally, $5)$ since the properties of the LDGRFs reflect the characteristics of the flares, but not the CMEs, the $>100 \mathrm{MeV} \gamma$-ray emission is caused by protons accelerated during the flares.

\section{References}

1. J. P. Wild, S. F. Smerd, and A. A. Weiss, ARA\& A, 1, 291, 1963.

2. E. W. Cliver, S. W. Kahler, M. Kazachenko, and M. Shimojo, ApJ, 877, 11, 2019.

3. A. B. Struminsky, Y. I. Logachev, I. Y. Grigorieva, and A. M. Sadovski, Geomagnetism and Aeronomy, 60, $1057,2020$.

4. J. T. Gosling, JGR, 98, 1993. 
5. Z. Švestka, Solar Phys., 160, 53, 1995.

6. H. Hudson, B. Haisch, and K. T. Strong, JGR, 100, 3473, 1995.

7. R. Pallavicini, S. Serio, and G. S. Vaiana, ApJ, 216, 108, 1977.

8. G. H. Share, R. J. Murphy, S. M. White, A. K. Tolbert, B. R. Dennis, R. A. Schwartz, D. F. Smart, and M. A. Shea, ApJ, 869, 182, 2018.

9. M. Ackermann, M. Ajello, A. Albert, A. Allafort, and et. al, ApJ, 787, 15, 2014.

10. A. B. Struminskii and et. al, Geomagnetism and Aeronomy, 65, 174, 2021.

11. A. B. Struminsky, I. Y. Grigorieva, Y. I. Logachev, and A. M. Sadovskii, Bulletin of the Russian Academy of Sciences, Physics, 85, 907, 2021. 


\title{
Flare energy release and avalanche ionization of plasma by runaway electrons in lower solar atmosphere
}

\author{
Yu. Tsap ${ }^{1}$, A. Stepanov ${ }^{2}$, Yu. Kopylova ${ }^{2}$ \\ yur_crao@mail.ru \\ ${ }^{1}$ Crimean Astrophysical Observatory, Nauchny 298409, Russia \\ ${ }^{2}$ Central Astronomical Observatory at Pulkovo, St. Petersburg 196140, Russia
}

The analysis of the electron acceleration by the quasi-stationary sub-Dreiser electric fields in the lower solar atmosphere has been done. It has been shown that the Dreiser electric field turned out to be several orders of magnitude larger than coronal values due to the inelastic collisions between electrons and hydrogen atoms. The ionization of hydrogen atoms gives rise to the resulting secondary electrons, which become runaway under the action of sub-Dreiser electric fields. This causes an further avalanche-like ionization of the plasma and leads to the acceleration of the large number of fast electrons up to relativistic energies at small $(\lesssim 100 \mathrm{~km})$ distances.

Keywords: solar flare, electron acceleration, partial ionization

DOI: 10.51194 /VAK2021.2022.1.1.133

\section{Introduction}

The acceleration of charged particles in solar flares is one of the most actual problems of the heliophysics. At present, many acceleration mechanisms have been proposed [1]. In this work we shall consider the electron acceleration under the action of the quasi-stationary large scale electric field $E$ since regular acceleration mechanisms are the most effective [2]. If all electrons are accelerated under the action of the super-Dreicer electric field $E>E_{D}$, where $E_{D}$ is the Dreicer electric field, the strong plasma turbulence should be developed. Therefore, we shall study only the acceleration in the sub-Dreicer electric field.

It is often considered that electrons are accelerated more effectively in the solar corona than in the solar chromosphere and photosphere due to large energy losses caused by their collisions with particles of the the dense weakly ionized plasma $[3,4,5]$. In spite of that the electron acceleration in the lower solar atmosphere by large scale sub-Dreicer electric fields allows us to solve three problems related to the coronal acceleration.

The first one deals with the origin of the white light flares. According to some estimates, almost all solar flares from C to $\mathrm{X}$ classes have a continuous component, which accounts for about $70 \%$ of the flare energy emission [6]. In order to provide the heating of the solar photosphere the energies of electrons accelerated in the corona should exceed several hundreds keV because of the collisional energy losses in the chromosphere. Meanwhile, the typical values of the lower electron energy threshold do not exceed $20 \mathrm{keV}$ [7], therefore it is necessary to assume unrealistically large fluxes of accelerated particles to effectively heat the photosphere [8].

The second problem is related to the small values of the Dreicer electric field $E_{D}$ in the solar corona. For example, the typical length of non-relativistic electron acceleration $l_{a}$ at $E=0.3 E_{D}$ would be greater than $10^{9}-10^{10} \mathrm{~cm}$ while $l_{a} \lesssim 10^{7}$ $\mathrm{cm}[9]$ in the lower solar atmosphere. This suggests that the length $l_{a}$ would exceed in the corona the characteristic size of the region of flare energy release $L$.

The third one is caused by the small plasma density in coronal loops. Simple estimates indicate that almost all thermal electrons contained in the coronal part of a magnetic loop should be accelerated in order to provide the observed hard X-ray emission of solar flares [1,2].

The first two problems were discussed in our previous works $[9,10]$. The purpose of this study is to consider the third problem on the basis of the theory proposed by [11] for the explanation of lightning in the terrestrial atmosphere.

\section{Avalanche ionization and electron acceleration by sub-Dreicer electric field}

Equation of motion for non-relativistic electrons in the weakly ionized hydrogen plasma when the $n / n_{a} \ll 1$, where $n$ and $n_{a}$ are the number densities of protons (electrons) and hydrogen atoms, respectively, can be represented as [9]

$$
\frac{d W}{d s}=e E\left(1-\frac{E_{D}}{E} \frac{W_{0}}{W}\right)\left(1+\ln \frac{W}{W_{0}}\right)
$$

where $W=m v^{2} / 2$ is the kinetic energy, $s$ is a length of acceleration, $e$ is the electron charge, and $W_{0}=(\exp / 2) I \approx 18.5 \mathrm{eV}$ ( $I=13.6 \mathrm{eV}$ is an ionization energy of hydrogen atoms).

As it follows from (1), runaway electrons can be accelerated $(d W / d s>0)$ if

$$
\frac{E}{E_{D}}>\frac{W_{0}}{W}
$$

For example, assuming $E / E_{D}=1 / 3$, we get $W>3 W_{0} \approx 56 \mathrm{eV}$. Therefore, taking into account that the thermal velocities of electrons $v_{T}=\sqrt{k T / m}$ in the lower solar atmosphere correspond to the thermal energies $W_{T}=3 / 2 m v_{T}^{2}=1-10 \mathrm{eV}$, we can conclude that even sufficiently weak sub-Dreicer electric fields $E$ can effectively accelerate electrons with the relative velocity $v_{r} / v_{T e}>\sqrt{3 W_{0} / W_{T}}=2-8$ it the tail of the Maxwellian distribution. 
The degree of ionization of a plasma is very small in the lower solar atmosphere $\left(n / n_{a}=10^{-4}-10^{-3}\right)$. This suggests that the number density of accelerated electrons $n_{r}$ is also quite small. Indeed, the number density of accelerated electrons [12]

$$
n_{r} \approx n \exp \left[1 / 2\left(-v_{r} / v_{T}\right)^{2}\right]
$$

Adopting $\left(v_{r} / \sqrt{2} v_{T}\right)^{2}=10$ and the number density of electrons for instance in the region of the temperature minimum $n \approx 10^{11} \mathrm{~cm}^{-3}$, we find $n_{r} \approx 5 \times 10^{6} \mathrm{~cm}^{-3}$ that is insufficient even for subflares.

The problem of small number of accelerated electrons can be solved if we take into account the avalanche ionization of plasma by runaway electrons. First of all, this means that the characteristic length of ionization $l_{I}$ would be less than the typical size of flare region $L=10^{8}-10^{10} \mathrm{~cm}$. In this connection let us make some estimates using results obtained by [11].

Fast electrons lose their kinetic energy basically as a result of the ionization of hydrogen atoms. The number of electrons per unit length with $W>W_{I}$, where $W_{I}$ is the energy of electrons, formed due to ionization is [13]

$$
\frac{d N_{I}}{d s}=\frac{\pi e^{4}}{m c^{2} W_{I}} n_{a}
$$

Secondary electrons can switch to a continuous acceleration mode if $W>W_{\min }$, where [11]

$$
W_{m i n}=\frac{E_{m i n}}{E} m c^{2}, \quad E_{m i n}=\frac{F_{m i n}}{e} .
$$

Note the minimum braking force $F_{\min }$ corresponds to electrons with the relativistic factor $\gamma=2-3$ [10].

Thus, the characteristic length of ionization $l_{I}$, which are approximately equal to the characteristic length of runaway electron acceleration $l_{a}[13]$, is

$$
l_{I}=\left(\frac{d N_{I}}{d s}\right)_{W_{I}=W_{\min }}^{-1}=\frac{\left(m c^{2}\right)^{2} E_{\text {min }}}{2 \pi e^{4} n_{a} E} \approx 3 \times 10^{23} n_{a} \frac{E_{\text {min }}}{E} .
$$

For $E_{\text {min }} \approx 10^{-3} E_{D}[10]$, adopting $n_{a}=10^{14}-10^{17} \mathrm{~cm}^{-3}$, we find the characteristic length of ionization $l_{I}=3 \times\left(10^{4}-10^{7}\right)$ $\mathrm{cm}$, i.e., $l_{I} \ll L$. Consequently, taking into account that the number density of accelerated electrons $n_{r}$ can be increased by orders of magnitude, we come to the conclusion that the avalanche ionization of hydrogen atoms by runaway electrons in the lower solar atmosphere is possible under the action of the sub-Dreicer electric field. The origin of large scale electric fields in solar flares we plan to consider in our next work.

This work was carried out with the partial support of the Russian Foundation for Basic Research (project no. N 20-52-26006) and the Ministry of Education and Science (No. 0831-2019-0006).

\section{References}

1. J. A. Miller, P. J. Cargill, A. G. Emslie, G. D. Holman, et al., JGR, 102, 14631, 1997.

2. Y. T. Tsap, Bulletin Crimean Astrophysical Observatory, 96, 146, 2000.

3. Y. T. Tsap, Astronomy Reports, 42, 275, 1998.

4. J. C. Brown, R. Turkmani, E. P. Kontar, A. L. MacKinnon, and L. Vlahos, A\&SA, 508, 993, 2009.

5. V. V. Zaitsev, P. V. Kronshtadtov, and A. V. Stepanov, Solar Phys., 291, 3451, 2016.

6. M. Kretzschmar, $A \notin A, \mathbf{5 3 0}, \mathrm{A} 84,2011$.

7. M. J. Aschwanden, E. P. Kontar, and N. L. S. Jeffrey, ApJ, 881, 1, 2019.

8. J. Aboudarham and J. C. Henoux, $A \mathscr{E} A$ A, 156, 73, 1986.

9. Y. T. Tsap and Y. G. Kopylova, Geomagnetism and Aeronomy, 57, 996, 2017.

10. Y. T. Tsap, A. V. Stepanov, and Y. G. Kopylova, Geomagnetism and Aeronomy, 59, 789, 2020.

11. A. V. Gurevich and K. P. Zybin, Physics Uspekhi, 44, 1119, 2001.

12. S. A. Kaplan and V. N. Tsytovich, Plasma astrophysics. (1972).

13. L. D. Landau and E. M. Lifshits, Moscow Izdatel Nauka Teoreticheskaia Fizika, 8, 1982. 


\title{
Processing and imaging of a series of monochromatic images of regions of the Sun, obtained on the multichannel CCD spectroheliograph, in the programs ImageJ and ParaView
}

\author{
F.V. Vereshchagin \\ zverted@gmail.com \\ Sternberg Astronomical Institute (SAI), Moscow State University, Moscow, 119234 Russia
}

DOI: 10.51194 /VAK2021.2022.1.1.134

The multichannel CCD spetroheliograph [1] is used in the Department of Solar Physics at the ATB-1 solar tower telescope to study nonstationary phenomena in the chromosphere of the Sun.

The processing and imaging of series of monochromatic images of regions of the Sun in the light of individual spectral lines (spectroheliograms and filtergrams) obtained on the multichannel CCD spetroheliograph is performed in freely distributed programs: the program for image analysis and processing Image J [2] and the application for data analysis and interactive visualization ParaView [3].

The imaging functions built into ParaView allowed a series of monochromatic images to be presented as a tomographic image in space, where the $\mathrm{Z}$ coordinate corresponded to wavelengths and points outside a certain range of values were hidden.

The built-in image series processing operations, the ability to add user-defined menu (by adding the free ActionBar plug-in [4]), and the built-in macro programming language in ImageJ reduced the development time of the data processing program and allowed to obtain a series of combined images consisting of both the monochromatic image itself and the averaged spectral profile with a wavelength mark corresponding to the image [1].

\section{References}

1. I. F. Nikulin and F. V. Vereshchagin, Instruments and Experimental Techniques, 62, 558, 2019.

2. C. A. Schneider, W. S. Rasband, and K. W. Eliceiri, Nat Methods, 9, 671, 2012.

3. A. H. Henderson Squillacote, Amy Squillacote, The ParaView guide, Kitware (2007).

4. J. Mutterer, Custom toolbars and mini applications with action bar, figshare, 2017. 


\section{Cyclic variations of polarity of the weak photospheric magnetic fields}

E. Vernova ${ }^{1}$, M. Tyasto $^{1}$, D. Baranov ${ }^{2}$

elenavernova96@gmail.com

${ }^{1}$ IZMIRAN, SPb Filial, St. Petersburg, Russia

${ }^{2}$ Ioffe Institute, St. Petersburg, Russia

DOI: $10.51194 /$ VAK2021.2022.1.1.135

The distribution of magnetic fields of different polarities over the solar surface is studied. Synoptic maps of the photospheric magnetic field (NSO Kitt Peak, 1978-2016) were used. In the time-latitude diagram for the weak magnetic fields $(B<5 \mathrm{G})$, oblique bands are clearly visible, indicating flows (surges) about 1 year in width with domination of positive or negative polarity drifting from the equator to the poles. The surges are observed in the period between the so-called Rush-to-the-Poles (RTTP): magnetic field flows with the width of about 2-3 years which are responsible for the inversion of the polar field and are connected with the decay of the following sunspots. The surges form a pattern of magnetic field with cyclic change of polarity (see in Fig. 1a the change of sign of the weak magnetic field at the fixed latitude $-40^{\circ}$ ).
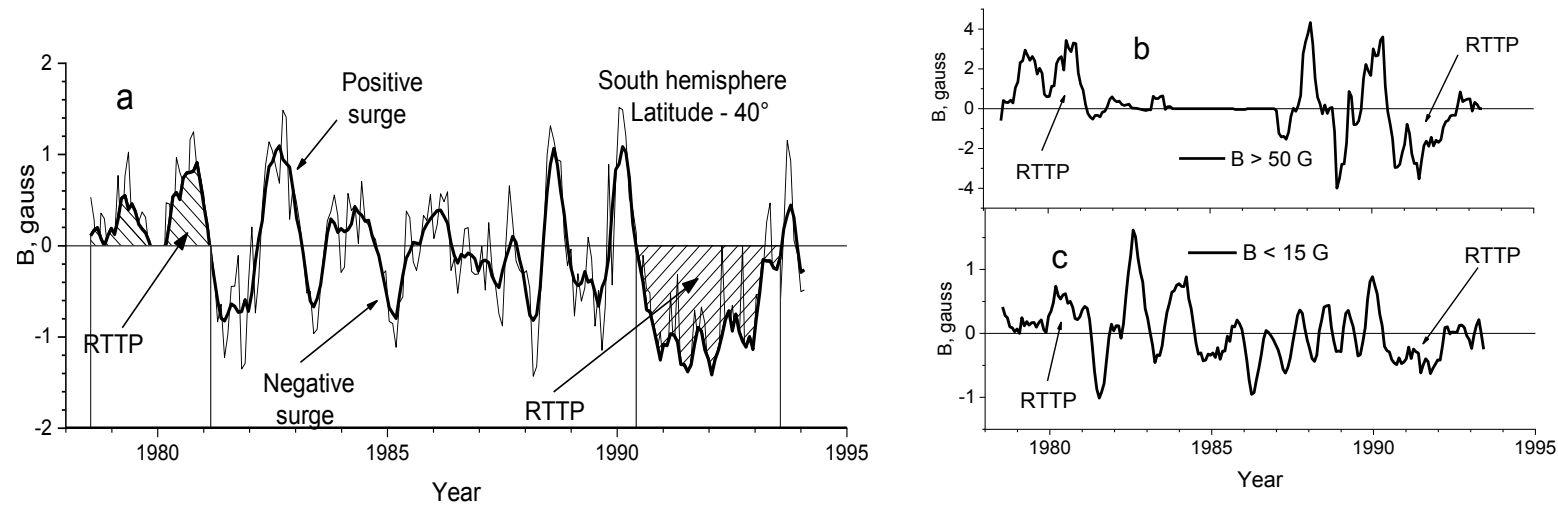

Alternation of dominating polarity was observed only in time-latitude diagrams of the weak fields. Figs. b,c show that at the latitude $-40^{\circ}$, a cyclic pattern is observed for the fields smaller than $B<15 \mathrm{G}$ (Fig. 1c). As the magnetic field strength increases, the alternation of flows of different polarities becomes less noticeable and disappears at $B>50 \mathrm{G}$ (Fig. 1b). These flows, characteristic to the weak magnetic fields, appear mostly in the hemisphere with the positive polar field and are limited in time by the period between two solar maxima. 


\title{
Activity Complexes on the Sun in Cycle 24
}

\author{
S. Yazev ${ }^{1,2}$, V. Tomozov ${ }^{2}$, E. Isaeva ${ }^{1}$ \\ syazev@gmail.com \\ ${ }^{1}$ Irkutsk State University, Karl Marks st., Irkutsk, 664003, Russian Federation, \\ ${ }^{2}$ Institute of Solar-Terrestrial Physics of SD RAS, 126A Lermontov st., Irkutsk, 664033, Russian Federation
}

We have compiled a catalog of activity complexes (AC) in cycle 24 and revealed the main parameters of AC evolution in the cycle. A total of 118 ACs were identified in cycle 24. New ACs occur mostly near the existing ACs. From cycle 21 to 24 , the north-south asymmetry in AC spatial distribution increases, and the power of AC decreases monotonically. There are coronal holes near all the ACs. About $80 \%$ of all M- and X-class X-ray flares occurred in ACs.

Keywords: activity complexes, solar flares, 25 cycle of solar activity, north-south asymmetry

DOI: 10.51194/VAK2021.2022.1.1.136

\section{Activity Complexes on the Sun}

As part of the concept being developed in Irkutsk, activity complexes (AC) on the Sun are the regions of permanent stain formation in the Carrington coordinate system existing for at least three solar rotations. Such region sized 20 by 20 heliographic degrees is called an AC core. The adjacent active region (AR) located less than 30 degrees away from the AC core and connected to the AC core by coronal loops is called an AC branch. The system that comprises an AR in the core and AR branches is called a complex of active regions (CAR) $[1,2]$. In this approach, a CAR is the AC "time slice" in a given Carrington rotation. ARs evolving in AC cores or representing AC branches comprise 50-60\% of the total package of sunspot groups. Studies have shown that active regions inside ACs tend to have greater area. Their flare activity is higher than the average for the entire population of sunspot groups. The paper focuses on studying ACs on the Sun in cycle 24.

\section{24 cycle activity complexes}

Synoptic maps of spot formation activity in the Carrington coordinate system allowed us to identify and catalogue ACs. A total of 1720 groups of sunspots were detected in cycle 24 (January 2009 to January 2019). According to the above approach, 710 objects (41\%) can be attributed to ARs inside AC cores, 323 (19\%) — to AC branches, and 687 (40\%) are outside ACs.

In cycle 24, 118 ACs were observed, including 68 in the northern and 50 in the southern hemisphere. Their main properties are as follows.

1. In solar cycles 21-23, ACs developed pulsewise: the number of simultaneously existing AC cores was growing and then decreasing, the pulse duration (minimum-to-minimum) was 16-20 rotations. In cycle 24, the pattern of AC development was different: initially, in the northern hemisphere, one could observe the rapid growth of the number of ACs with the maximum of 7 concurrent AC cores approximately the 2115 th rotation. Then there was a recession, with the number of $\mathrm{AC}$ cores fluctuating at 3-4 for a long time, up to rotation 2181 when a recession began at the end of the cycle. In the southern hemisphere, the number of ACs was gradually increasing to rotation 2144 (9 ACs) and then decreasing approximately to rotation 2181, after which low residual AC activity (0-2 AC) was detected up to the end of the cycle. There were no manifestations of the above impulses.

2. The mean coefficient for north-south asymmetry in the 24 cycle $K=(N n-N s) /(N n+N s)=0.15(-0.05$ in 23 cycle, 0 in 22 cycle, -0.08 in 21 cycle). Thus, the modulus of coefficient $\mathrm{K}$ was the highest over the last 4 cycles. Since ACs are the main contributors to the sunspot population, this fact appears to induce the north-south asymmetry of sunspot activity in cycle 24 .

3. New AC cores occur basically near the existing AC cores. A new AC core is most likely to form "next door" (20-30 degrees from the center of the existing AC core). The probability of a new AC core occurrence decreases monotonically with the distance from the existing $\mathrm{AC}$ core.

4. Coronal holes $(\mathrm{CH})$ developed near all the ACs in cycle 24. In some cases, after the disappearance of AC sunspots, a $\mathrm{CH}$ changed propagating to the previous location of the $\mathrm{AC}$ core. Low-latitude isolated $\mathrm{CHs}$ not related to polar $\mathrm{CHs}$ exhibited rigid Carrington rotation during their evolution near the AC core.

5. Among 1720 ARs in cycle 24, 234 generated M and X-class X-ray flares. Of these, 172 regions were in AC cores and branches, and only 55 were not related to ACs. The total flare index in cycle 24 was 285 , with 231 in ACs and only 54 outside ACs. The specific flare index per AR was 0.27 for ARs in AC cores, 0.12 in AC branches, and 0.08 outside ACs.

6. In AC-related ARs in cycle 24, $78 \%$ of all $\mathrm{M}$ and X-class flares were observed, $87 \%$ of all flares with long decay of $\mathrm{X}$-ray emission (LDE-events), and $74 \%$ of gamma flares.

\section{Conclusions}

Formally distinguished structures (ARs located in zones of permanent sunspot formation for at least 3 rotations in a row and named ACs) show a number of specific qualities. According to the AC catalog in cycle 24:

- variations in the number of ACs in cycles manifest the Gnevyshev-Ohl rule; 
- the intensity of ACs decays from cycle 21 to cycle 24;

- the AC north-south asymmetry grows from cycle 21 to cycle 24 ;

- the likelihood of new AC cores increases closer to the existing AC core;

- there were coronal holes in the vicinity of all the ACs;

- the vast majority (about 80\%) of M and X-class X-ray flares occurred in ACs;

- about $80 \%$ of the total flare index relates to flares that occurred in AC cores and branches.

The method the authors used for AC identification appears to be important for analyzing the brightest manifestations of solar activity and predicting powerful flares on the Sun. The work was performed at the UNU " Astrophysical Complex of MSU-ISU" (agreement 13.UNU.21.0007), the work is supported by the Russian Federation Ministry of Science and High Education (projects FZZE-2020-0017, FZZE-2020-0024), and by the project II.16.3.1 of Institute of Solar-Terrestrial Physics.

\section{References}

1. S. A. Yazev, Astron. Rep., 59, 228, 2015.

2. E. S. Isaeva, V. M. Tomozov, and S. A. Yazev, Astron. Rep., 62, 243, 2018. 


\section{First observations of the microwave fine structures with the $3-6 \mathrm{GHz}$ Siberian Radioheliograph}

D. Zhdanov

zhdanov@iszf.irk.ru

Institute of Solar-Terrestrial Physics, Irkutsk, Russia

DOI: $10.51194 /$ VAK2021.2022.1.1.137

The Siberian Radioheliograph is new modern solar instrument consist of three independent antenna arrays (3-6 GHz, 6-12 GHz, 12-24 GHz) founded on the Siberian Radioheliograph with the 48-antenna array [1]. Since January 2021, the 3-6 GHz array has been in test mode for solar imaging, the other arrays are being prepared for launch.

The paper aim is demonstrate of the first united observations of the solar microwave fine structure (FS) obtained the 3$6 \mathrm{GHz}$ antenna array of the Siberian Radioheliograph (SRH 3-6) and the Badary Broadband Microwave Spectropolarimeter $[2]$.

We found that 4 (see table) out of 20 solar events observed on BBMS was observed on SRH 3-6, from January to July (half a year) 2021.

Table 1: The observed FS events.

\begin{tabular}{cccccc}
\hline & Data & Tstart & Tstop & Powerfull & Background \\
\hline 1 & $2021-05-22$ & $02: 59: 03$ & $02: 49: 23$ & middle & yes \\
2 & $2021-05-22$ & $06: 13: 11$ & $06: 25: 25$ & middle & yes \\
3 & $2021-05-23$ & $09: 17: 41$ & $09: 21: 55$ & weak & yes \\
4 & $2021-06-01$ & $04: 27: 42$ & $04: 30: 02$ & weak & no \\
\hline
\end{tabular}

In two events of C6.1 and C6.0 classes on May 22, the fine structure was accompanied by broadband microwave burst, while in event on June 1, the fine structure observed without the microwave burst [3].

Using SRH 3-6 data for the event 2021-06-01 we determined the position of sources of fine structure at frequencies of 3.9, 4.7 and 5.6 GHz. The temporal resolution SRH 3-6 maps was $1.3 \mathrm{~s}$ to the six frequencies.

\section{References}

1. S. Lesovoi, A. Altyntsev, A. Kochanov, V. Grechnev, et al., Solar-Terrestrial Physics, 3, 3, 2017.

2. D. A. Zhdanov and V. G. Zandanov, Central European Astrophysical Bulletin, 35, 223, 2011.

3. D. A. Zhdanov and V. G. Zandanov, Solar Phys., 290, 287, 2015. 


\section{Physics of Galaxies and Cosmology}




\title{
The database for studying edge-on galaxies
}

\author{
A.V. Antipova ${ }^{1}$, D.I. Makarov ${ }^{1}$, S.S. Savchenko ${ }^{2,1}$ \\ ${ }^{1}$ Special Astrophysical Observatory, Russian Academy of Sciences, Nizhnii Arkhyz, 369167, Russia \\ ${ }^{2}$ Saint Petersburg State University, Universitetskii pr. 28, St. Petersburg, 198504, Russia
}

Galaxies oriented edge-o to the observer give one unique possible to study the vertical distribution of matter in galaxy disks. It is crucial for understanding the formation and evolution of galaxies. To date, large observational material has been accumulated on photometry and kinematics of the edge-on galaxies. In connection with the growing interest in this type of galaxies, it became necessary to systematize information, as well as provide convenient and quick access to data obtained by different authors. Here we present the edge-on galaxy database ${ }^{\mathrm{a}}$, which combines information from different catalogs and ongong projects.

Keywords: edge-on galaxies, database, galaxies

DOI: $10.51194 /$ VAK2021.2022.1.1.138

\section{Introduction}

Edge-on galaxies are practically the only objects in which a direct observation of the vertical distribution of matter in the disks is possible, which in turn opens up the possibility of studying the 3D distribution of matter in galaxies. Recently, interest in this type of galaxies has increased, as well as a rich observational material has been accumulated, which needs to be systematized, collecting all known information in one place, and to provide convenient access to it. In this regard, it became necessary to create a database.

\section{Description of the database}

The edge-on galaxy database consists of 3 previously published catalogs and a catalog created on the basis of the PanSTARRS data (the article is submitted to MNRAS).

The Revised Flat Galaxy Catalog is based on data from the Palomar Observatory Sky Survey and contains information on 4236 galaxies [1]. The Catalog of Edge-on Disk Galaxies from SDSS is based on the SDSS survey and contains data on 5747 galaxies [2]. The 2MASS-selected Flat Galaxy Catalog was created based on the 2MASS infrared sky survey and contains information on 18020 galaxies [3].

The database also contains The Catalog of the Edge-on Galaxies In Pan-STARRS1, in the creation of which our information system took part. Using an artificial neural network we selected about 27000 candidates in edge-on galaxies using public images of the Pan-STARRS1 survey. All candidates underwent visual classification, as a result, 16551 edge-on galaxies were selected. The information system was used to store the candidates, and provide an interface for the visual inspection and classification of galaxies. The developed classification system allows you to create complex polls, scale the image, view the image of the galaxy from other surveys, thanks to the use of the Aladin Lite ${ }^{\mathrm{b}}$ program.

Cross-identification between the same objects in different catalogs is carried out using a special identifier, which was provided by the HyperLeda ${ }^{\mathrm{c}}[4]$ database.

The first page ${ }^{\mathrm{d}}$ of the database provides information about the project, its goals and objectives. The "Projects" page e contains information about current projects and a table that contains the parameters obtained during the decomposition for 150 ultrathin galaxies. Interaction with catalogs takes place through the "Catalogs" page ${ }^{f}$. Search in the catalog is carried out through the name of the object or its coordinates. Data from catalogs can be visualized in the form of a table and an information map of the object.

Figures 1 and 2 show the schema of the database. For the sake of compactness, it contains only those fields that important for understanding the relationship between tables.

Figure 1 shows the following tables:

leda - the table with a unique galaxy identifier - pgc number;

egis — the list of galaxies with their identification and astrometry;

egis_phot $1 d$ - photometric parameters galaxies obtained from the analysis of one-dimensional galaxy profile according to SDSS data; data;

egis_phot3d — disk parameters obtained from modeling the surface brightness distribution of galaxies using SDSS

$r f g c$ - the list of galaxies with their identification, astrometry and photometric parameters according to the Palomar Observatory Sky Survey;

$2 m f g c$ - the list of galaxies with their identification, astrometry and photometric parameters according to the $2 \mathrm{MASS}$ infrared sky survey;

Figure 2 shows the following tables:

ps1candidate - the list of candidates;

\footnotetext{
${ }^{a}$ https://www.sao.ru/edgeon/

b https://aladin.u-strasbg.fr/

${ }^{\mathrm{c}}$ http://leda.univ-lyon1.fr

${ }^{d}$ https://www.sao.ru/edgeon/index.php

ehttps://www.sao.ru/edgeon/projects.php

f https://www.sao.ru/edgeon/catalogs.php
} 


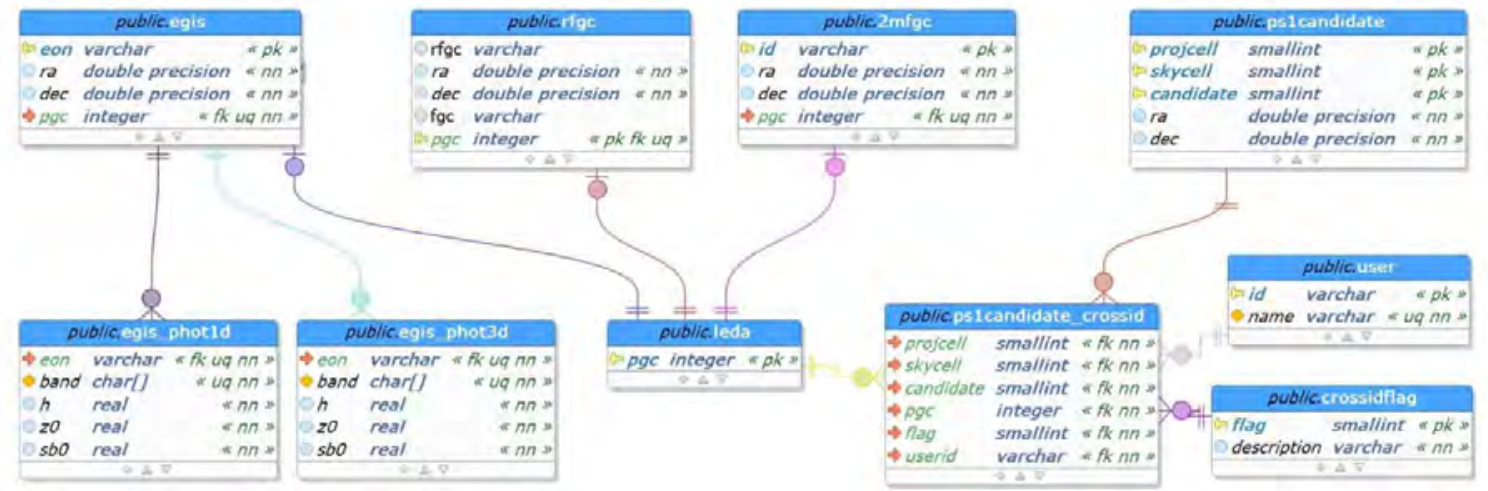

Figure 1: Database structure diagram.

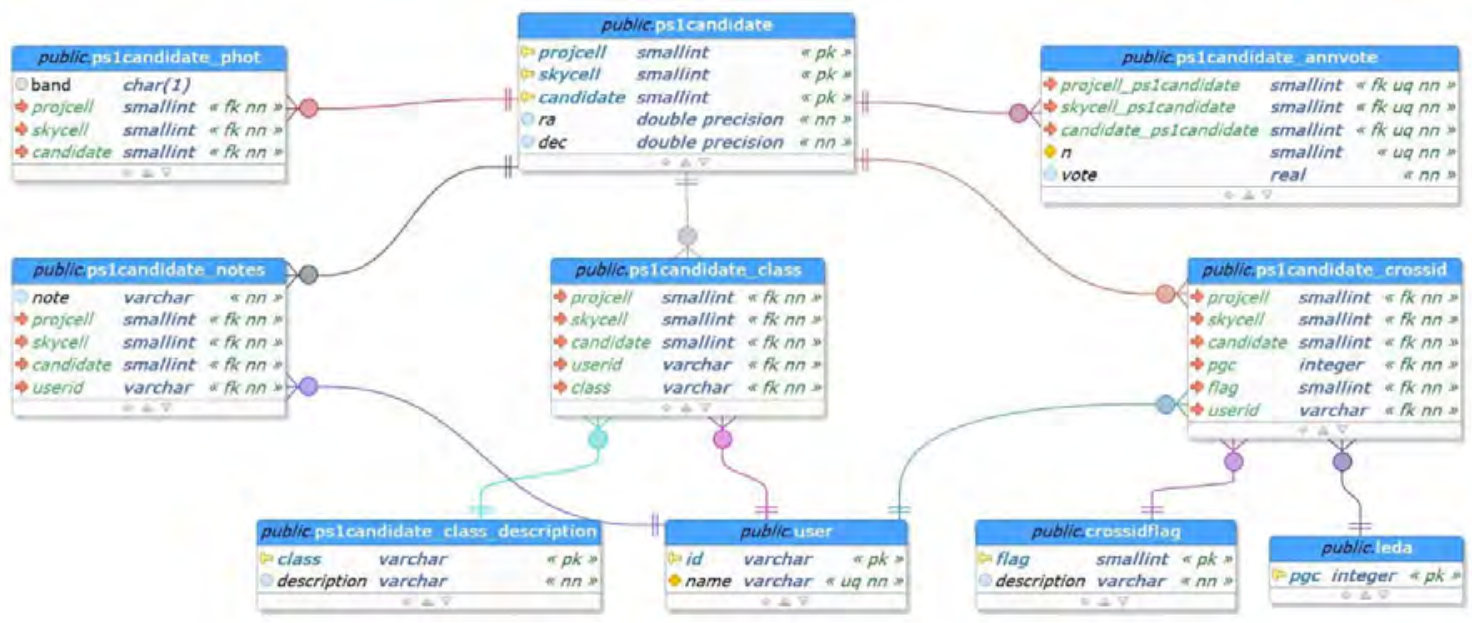

Figure 2: Database structure diagram.

ps1candidate_annvote — the classification performed using artificial neural networks;

ps1candidate class - the results of visual inspection of candidates;

ps1candidate_phot — the automatic photometry performed by SExtractor ${ }^{\mathrm{g}}$;

ps1candidate_crossid - the cross-identification with the galaxies from the HyperLeda database [4];

ps1candidate_notes - the various notes made while working with galaxies.

\section{Conclusion}

We present the first stage of development of our information system about edge-on galaxies. In the near future, a catalog of photometric parameters will be added from massive image analysis from various sky surveys such as SDSS, Pan-STARRS and Legacy Survey. At the moment, a rich observational material has been accumulated on the kinematics of gas in flat galaxies, which will also be available in the database soon. Also, the database will be replenished with data from literary sources.

\section{Acknowledgments}

This paper describes the structure of The Edge-on Galaxy Database. The research was carried out with the financial support of the Russian Foundation for Basic Research within the framework of the scientific project No. 19-32-90244.

\section{References}

1. I. D. Karachentsev, V. E. Karachentseva, Y. N. Kudrya, M. E. Sharina, and S. L. Parnovskij, Bulletin of the Special Astrophysics Observatory, 47, 5, 1999.

2. D. V. Bizyaev, S. J. Kautsch, A. V. Mosenkov, V. P. Reshetnikov, N. Y. Sotnikova, N. V. Yablokova, and R. W. Hillyer, ApJ, 787, 24, 2014.

ghttps://sextractor.readthedocs.io/ 
3. S. N. Mitronova, I. D. Karachentsev, V. E. Karachentseva, T. H. Jarrett, and Y. N. Kudrya, Bulletin of the Special Astrophysics Observatory, 57, 5, 2004.

4. D. Makarov, P. Prugniel, N. Terekhova, H. Courtois, and I. Vauglin, A\&A, 570, A13, 2014. 


\title{
Bulge-disk decomposition of ultrathin galaxies
}

\author{
A.V. Antipova ${ }^{1}$, A.V. Mosenkov ${ }^{2,3}$, D.I. Makarov ${ }^{1}$, V.P. Reshetnikov ${ }^{4} 1$ \\ ${ }^{1}$ Special Astrophysical Observatory, Russian Academy of Sciences, Nizhnii Arkhyz, 369167, Russia \\ ${ }^{2}$ Department of Physics and Astronomy, N283 ESC, Brigham Young University,Provo, UT 84602, USA \\ ${ }^{3}$ Pulkovo Observatory, Russian Academy of Sciences, St. Petersburg, 196140, Russia \\ ${ }^{4}$ Saint Petersburg State University, Universitetskii pr. 28, St. Petersburg, 198504, Russia
}

\section{DOI: 10.51194 /VAK2021.2022.1.1.139}

Galaxies visible at an angle of $\sim 90^{\circ}$ are the only objects outside the Milky Way in which it is possible to study the vertical distribution of matter in the disks. This opens up the possibility of studying the three-dimensional distribution of matter in galaxies. The edge-on galaxies have been actively studied by various groups of scientists, but only a small fraction of these galaxies have good quality photometry. Although, together with data on kinematics, reliable photometry of galaxies could be an excellent basis for studying the distribution of luminous and dark matter in these galaxies. Our sample is based on the RFGC catalog of thin galaxies [1] and includes 150 galaxies with an observed ratio of the $a / b>10$ in the $B$ filter and $a / b>8.5$ in the $R$ filter. The decomposition of two-dimensional images of ultrathin galaxies was carried out using the DECA software package [2]. The decomposition used disk and bulge models (a description of the models can be found in [2]. The work was carried out using data obtained from the Pan-STARRS1 (DR2) sky survey. We obtained the average surface brightness of our sample for $g$ filtr of galaxies equal to $20.86 \pm 0.04 \mathrm{mag}_{\mathrm{arcsec}}{ }^{2}$. It corresponds to $B$ - band face-on surface brightness of $24.08 \pm 0.05 \mathrm{mag} / \mathrm{arcsec}^{2}$. This result suggests that our sample consists of galaxies of low surface brightness. We find no significant correlation between surface brightness and scale ratio (Pearson's coefficient $=$ $-0.25)$. Note that in this case we did not recalculate the surface brightness values for the fase-on position. We found that Sd galaxies have a lower surface brightness and they are slightly thinner in comparison with galaxies of Sc type. The mean scale ration of Sc galaxies is $z_{0} / h=0.236 \pm 0.004$, while it is $z_{0} / h=0.200 \pm 0.004$ for Sd-type galaxies.

\section{Acknowledgments}

The reported study was funded by RFBR according to the research project 19-32-50129 19 .

\section{References}

1. I. D. Karachentsev, V. E. Karachentseva, Y. N. Kudrya, M. E. Sharina, and S. L. Parnovskij, Bulletin of the Special Astrophysics Observatory, 47, 5, 1999.

2. A. V. Mosenkov, Astrophysical Bulletin, 69, 99, 2014. 


\section{Peculiar velocities of SNe la in clusters of galaxies}

E. Balakina ${ }^{1}$, M. Pruzhinskaya ${ }^{2}$

elena@balakin.ru

${ }^{1}$ Lomonosov Moscow State University, Faculty of Physics, Leninskie Gory, 1-2, Moscow, 119991, Russia

${ }^{2}$ Lomonosov Moscow State University, Sternberg Astronomical Institute, Universitetsky pr. 13, Moscow, 119234, Russia

The study of the accelerating expansion of the Universe is possible because of the standardization procedure of Type Ia Supernovae (SNe). However, we still observe the residual dispersion on the Hubble diagram that could be related to environmental effects and unknown peculiar velocities of SNe Ia. In this analysis, we continue to study the peculiar velocities of SNe inside galaxy clusters and their influence on distance modulus measurements. We perform the fit for a low redshift $z \leq 0.15$ sample with a fixed value of $\Omega_{m}$ for C11 and G10 models and calculate $w R M S$ value to estimate the impact of peculiar velocity effect. We get the $w R M S=0.1643$ for the C11 sample with SNe Ia in clusters if we use the galaxy cluster $z_{\mathrm{cl}}$ instead of the host galaxy $z_{\mathrm{host}}, w R M S=0.1696$ in the original data (for G10 $w R M S=0.1617$ and $w R M S=$ 0.1643 , respectively). The results show the decreasing of residual dispersion on the Hubble diagram. Therefore, this effect is considerable for supernovae in clusters and should be taken into account in future cosmological analyses.

Keywords: type Ia Supernovae, observational cosmology, peculiar velocities

DOI: $10.51194 /$ VAK2021.2022.1.1.140

\section{Introduction}

Type Ia Supernova (SN) standardization procedure compensates for the scattering in the magnitude values of SNe and allows us to carry out cosmological calculations, including the measurement of Hubble constant. Nevertheless, even after applying the standardization procedure there is a dispersion on the Hubble diagram — distance modulus vs. redshift that could partly arise from the effects of environment $[1,2,3]$. In addition, there are some effects that affect another part of the Hubble diagram related to the redshift determination. One of them is the peculiar velocities of SNe Ia. This effect seems to be significant in galaxy clusters where peculiar velocities can reach thousands of $\mathrm{km} \mathrm{s}^{-1}, \sigma_{v}=1040 \mathrm{~km} \mathrm{~s}$ for the Perseus cluster [4] and $\sigma_{v}=1545 \mathrm{~km} \mathrm{~s}^{-1}$ for ACO 2319 [5] as an example.

Léget et al. [6] considered peculiar velocities of SNe Ia that exploded in galaxy clusters using 145 SNe Ia from the Nearby Supernova Factory ${ }^{\mathrm{a}}$. They used the galaxy cluster redshift $z_{\mathrm{cl}}$ instead of the host galaxy redshift $z_{\text {host }}$ to decrease the influence of peculiar velocities of host galaxies on redshift measurements (hereafter, peculiar velocity correction). Balakina and Pruzhinskaya [7] applied the same kind of analysis to the PANTHEON cosmological sample of SNe Ia [8]. Performing the cross-matching between PANTHeON SN Ia sample and several galaxy clusters catalogues Balakina and Pruzhinskaya [7] found 14 supernovae hosted by clusters of galaxies. It was shown that the supernovae positions on the Hubble diagram do change after applying peculiar velocity correction.

In the current study, we are going to perform the Hubble diagram fit of PANTHEON SNe Ia before and after peculiar velocity correction to evaluate its impact on the Hubble diagram residuals.

\section{Hubble diagram fit}

Since the effect of peculiar velocities decreases with redshift [9], we fix the cosmological parameter $\Omega_{m}$ and, then, perform the Hubble diagram fit only for supernovae with $z \leq 0.15$.

First, the global fit for the whole Pantheon sample is done to find $\Omega_{m}$ value. Theoretical distance modulus $\mu_{\mathrm{th}}$ is given by the equations:

$$
\begin{gathered}
\mu_{\mathrm{th}}=5 \log _{10} d_{L}-5 \\
d_{L}=(1+z) \frac{c}{H_{0}} \int_{0}^{z} \frac{d z^{\prime}}{\sqrt{\Omega_{m}}\left(1+z^{\prime}\right)^{3}+\Omega_{\Lambda}},
\end{gathered}
$$

where $d_{L}$ is the luminosity distance in parsecs.

Following [8] we calculate the observation distance modulus $\mu_{\mathrm{obs}}$ as

$$
\mu_{\mathrm{obs}}=m_{B}-M_{B}+\alpha x_{1}-\beta c+\Delta_{M}-\Delta_{B}
$$

where $M_{B}$ is the absolute $B$-band magnitude of a fiducial SN Ia which is characterized by stretch-value $x_{1}=0$ and colorvalue $c=0$. Parameter $\alpha$ is the coefficient of the relation between luminosity and stretch, and $\beta$ is the coefficient of the relation between luminosity and color. Finally, $\Delta_{M}$ is the distance correction based on the host galaxy mass of the SN, and $\Delta_{B}$ is a distance correction based on predicted biases from simulations. The error of distance determination could be described as $C=D_{\text {stat }}+C_{\text {sys }}$, where $C_{\text {sys }}$ is the systematic covariance, and the statistical matrix $D_{\text {stat }}$ components are

$$
\sigma^{2}=\sigma_{\text {phot }}^{2}+\sigma_{z}^{2}+\sigma_{\text {lens }}^{2}+\sigma_{\text {int }}^{2}+\sigma_{\text {bias }}^{2}
$$

only diagonal elements. $\sigma_{\text {phot }}^{2}$ - photometric error of the distance, $\sigma_{z}^{2}$ is the uncertainty conditioned by redshift measurements and peculiar velocity uncertainties, $\sigma_{\text {lens }}^{2}$ is the uncertainty from stochastic gravitational lensing, $\sigma_{\text {int }}^{2}$ is the intrinsic scatter, and $\sigma_{\text {bias }}^{2}$ expresses the distance bias correction uncertainty [8].

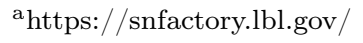


Table 1: The results of the Hubble diagram fit for the corrected and original PANTHEON SNe Ia sample up to $z=0.15$ for two scatter models C11 and G10.

\begin{tabular}{|c|c|c|c|c|c|c|c|}
\hline Model & $\Omega_{m}$ & Sample & $M_{B}$ & $\alpha$ & $\beta$ & $\gamma$ & $w R M S$ \\
\hline C11 & 0.297 & $\begin{array}{l}\text { original } \\
\text { corrected }\end{array}$ & $\begin{array}{c}-19.174 \\
\pm 0.016 \\
-19.175 \\
\pm 0.016\end{array}$ & $\begin{array}{c}0.1544 \\
\pm 0.0081 \\
0.1544 \\
\pm 0.0082\end{array}$ & $\begin{array}{c}3.5802 \\
\pm 0.1274 \\
3.5786 \\
\pm 0.1278\end{array}$ & $\begin{array}{c}0.0775 \\
\pm 0.0181 \\
0.0755 \\
\pm 0.0181\end{array}$ & $\begin{array}{l}0.1696 \\
0.1643\end{array}$ \\
\hline G10 & 0.306 & $\begin{array}{l}\text { original } \\
\text { corrected }\end{array}$ & $\begin{array}{c}-19.171 \\
\pm 0.014 \\
-19.171 \\
\pm 0.014\end{array}$ & $\begin{array}{c}0.1544 \\
\pm 0.0072 \\
0.1547 \\
\pm 0.0073\end{array}$ & $\begin{array}{c}3.0650 \\
\pm 0.0938 \\
3.0609 \\
\pm 0.0941\end{array}$ & $\begin{array}{c}0.0710 \\
\pm 0.0158 \\
0.0688 \\
\pm 0.0159\end{array}$ & $\begin{array}{l}0.1643 \\
0.1617\end{array}$ \\
\hline
\end{tabular}

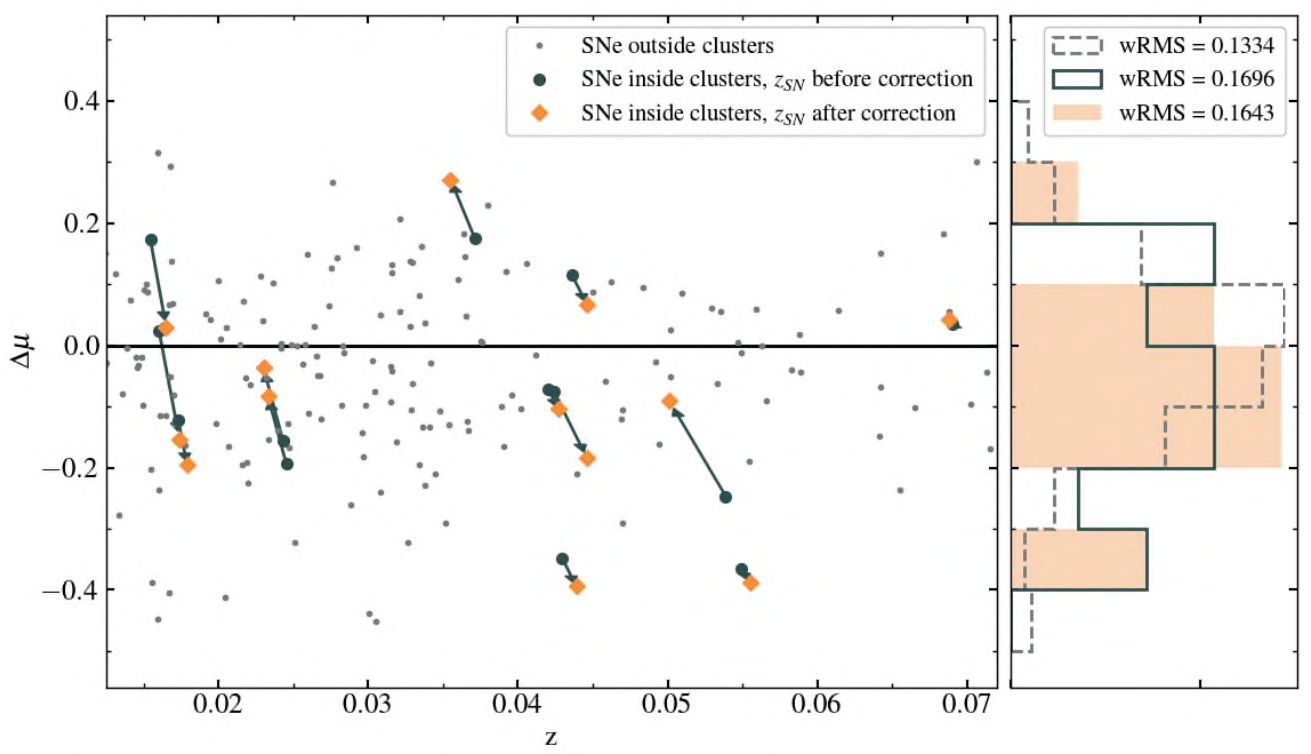

Figure 1: Distance modulus residuals on the Hubble diagram for C11 model before peculiar velocity correction (dark green circles) and after it (orange squares). Hubble-Lemaitre law is plotted by the black solid line based on the parameter values from the global fit.

Following [8], we performed the global fit for two different scatter models described in [10] (C11) and [11] (G10). Thus, we obtained $\Omega_{m}=0.297 \pm 0.022(\mathrm{C} 11)$ and $\Omega_{m}=0.306 \pm 0.021$ (G10).

Then, we make a 4-parameters fit $\left(M_{B}, \alpha, \beta, \gamma\right)$ of PANTheON SNe Ia with $z \leq 0.15$ considering 2 scatter models, C11 and G10, for two cases:

- original sample, $z=z_{\text {host }}$

- corrected sample, $z=z_{\mathrm{cl}}$, if SN inside a galaxy cluster

The fit results are presented in Table 1 .

To estimate the influence of peculiar velocities correction, we plot the Hubble diagram for low redshift supernovae, see Fig. 1, and compare the $w R M S$-value before and after peculiar correction. $w R M S$ is calculated as

$$
w R M S^{2}=\left(\sum_{s=1}^{N} w_{s}^{2}\right)^{-1} \sum_{s=1}^{N} w_{s}^{2} \Delta \mu_{s}^{2}, \quad w_{s}=\frac{1}{\sigma_{s}},
$$

where $N$ is the total number of SNe in the sample, $\sigma_{s}$ is calculated by equation 4 .

As we can see from Table 1, for both scatter models $w R M S$ is smaller after peculiar velocity correction, e.g. for C11 $w R M S$ equals 0.1606 if the fit is performed on the original not-corrected data, and $w R M S=0.1550$ if the galaxy cluster redshift is used instead of the host galaxy redshift for 14 supernovae in galaxy clusters.

\section{Conclusion}

We considered the influence of peculiar velocity of SN Ia from the PANTHEON sample on redshift measurement if a supernova exploded inside galaxy cluster. We carried out a global cosmological fit of the PANTHEON data and also performed two 
separate fits of the low-redshift supernovae before and after peculiar velocity correction. As assumed, the supernova positions on the Hubble diagram do change and that caused the decrease of the $w R M S$ value for the supernova sample with $z \leq 0.15$.

Finally, the decreasing of $w R M S$ allows us to conclude that the peculiar velocity effect is observable, and therefore, should be taken into account in the data analysis from the next generation of large-scale surveys like the Vera Rubin Observatory Legacy Survey of Space and Time [12].

\section{Acknowledgements}

The reported study was funded by RFBR and CNRS according to the research project No21-52-15024. E.A.B acknowledges support from a Theoretical Physics and Mathematics Advancement Foundation "BASIS" grant No20-2-1-34-1.

\section{References}

1. M. V. Pruzhinskaya, A. K. Novinskaya, N. Pauna, and P. Rosnet, MNRAS, 499, 5121, 2020.

2. M. Rigault, Y. Copin, G. Aldering, P. Antilogus, et al., A $6 A$, 560, A66, 2013.

3. F. X. Timmes, E. F. Brown, and J. W. Truran, ApJL, 590, L83, 2003.

4. J. A. L. Aguerri, M. Girardi, I. Agulli, A. Negri, C. Dalla Vecchia, and L. Domínguez Palmero, MNRAS, 494, 1681, 2020.

5. D. Fadda, M. Girardi, G. Giuricin, F. Mardirossian, and M. Mezzetti, ApJ, 473, 670, 1996.

6. P. F. Léget, M. V. Pruzhinskaya, A. Ciulli, E. Gangler, et al., AछA, 615, A162, 2018.

7. E. A. Balakina and M. V. Pruzhinskaya, Astronomy Reports, 65, 897-901, 2021.

8. D. M. Scolnic, D. O. Jones, A. Rest, Y. C. Pan, et al., ApJ, 859, 101, 2018.

9. F. Habibi, S. Baghram, and S. Tavasoli, International Journal of Modern Physics D, 27, 1850019, 2018.

10. N. Chotard, E. Gangler, G. Aldering, P. Antilogus, et al., A\&A, 529, L4, 2011.

11. J. Guy, M. Sullivan, A. Conley, N. Regnault, et al., A\&A, 523, A7, 2010.

12. LSST Science Collaboration, P. A. Abell, J. Allison, S. F. Anderson, et al., arXiv e-prints, arXiv:0912.0201, 2009. 


\title{
Sources of the survey on the declination of microquasar GRS $1915+105$
}

\author{
N. Bursov ${ }^{1}$, S. Trushkin ${ }^{1,2}$, A. Kudryashova ${ }^{1}$, P. Tsybulev ${ }^{1}$, A. Borisov ${ }^{1}$, M. Khabibullina ${ }^{1}$, D. Kuandykova $^{1}$ \\ nnb@sao.ru \\ ${ }^{1}$ Special astrophysical Observatory of RAS, Nizhny Arkhyz, Karachaevo-Cherkassia, 369167, Russia \\ ${ }^{2}$ Kazan Federal University, Kazan, Republic of Tatarstan, Russia
}

With the RATAN-600 radio telescope, we have detected about 1300 radio sources at $4.7 \mathrm{GHz}$ frequency, identified with sources from other catalogs, mostly from the NVSS catalog at $1.4 \mathrm{GHz}$. From 6 June 2020 to 28 May 2021, the strip (width $\sim 35^{\prime}$ ) of the sky on the microquasar GRS $1915+105$ declination (Dec $=+11^{\circ} 56^{\prime} 44^{\prime \prime}$ ) was observed with a four-beam complex of four-channel sensitive radiometers, established in the focal line of the 'Western sector' antenna of the RATAN600 . We summarized the data received in narrow channels to obtain maximum sensitivity of the flux $(\sim 3 \mathrm{mJy} /$ beam $)$ in the $600 \mathrm{MHz}$ frequency band at the center $4.7 \mathrm{GHz}$ frequency. This value is constrained only by the effect of confusion for an antenna beam with dimensions about $1^{\prime} \times 35^{\prime}$. We obtained about $20-25$ high-quality day records divided by hours every month and 12 average hour drift scans for a year. We plotted and analyzed the light curves for bright variable sources for all 365 days. We identified the detected sources with the objects from the CATS database to plot compiled radio spectra of the sources. Most sources were identified as galaxies and quasars. Some supernova remnants were detected in cross-sections of the Galactic plane.

Keywords: general: radio astronomy - survey - extra-galactic sources

DOI: 10.51194 /VAK2021.2022.1.1.141

\section{Introduction}

Blind sky radio surveys are the traditional method of the studies from the pioneer (3C, 4C, Parkes, GB6) to modern low-frequency surveys: TGSS [1] and GLEAM [2]. With the RATAN-600 radio telescope, some large surveys were carried out: the Zelenchuck survey in the region $0^{\circ}<\operatorname{Dec}<14^{\circ}$ [3], or so-called the "Cold" deep survey at 3.9 GHz [4, 5]. For many years, the RZF survey of the 2 -degree strip of sky at the declination of the source $3 \mathrm{C} 84$ was conducted $\left(\mathrm{R} . \mathrm{A} .=24^{\mathrm{h}}\right.$, Dec $\left.=+41^{\circ} .5 \pm 1^{\circ}[6]\right)$.

During the 3-year sky survey with the Western sector of RATAN-600 on a three-beam four-channel complex of radiometers at 4.3-4.9 GHz, we were looking for short-time bright pulses, called fast radio bursts (FRB). Such pulses must be affected by the cosmic dispersion in the ionized interstellar and intergalactic medium [7]. The sky survey was carried out on the 24-hour strip at the declination of $+11^{\circ}$ of the microquasar GRS1911 +105 on a four-beam complex of radiometers from June 9, 2020, to May 26, 2021.

We divided the total annual volume of data into 12 months, and data processing was carried out for each one: coordinates (right ascension) and flux densities adjustment, using the most bright sources, averaging the signals for each

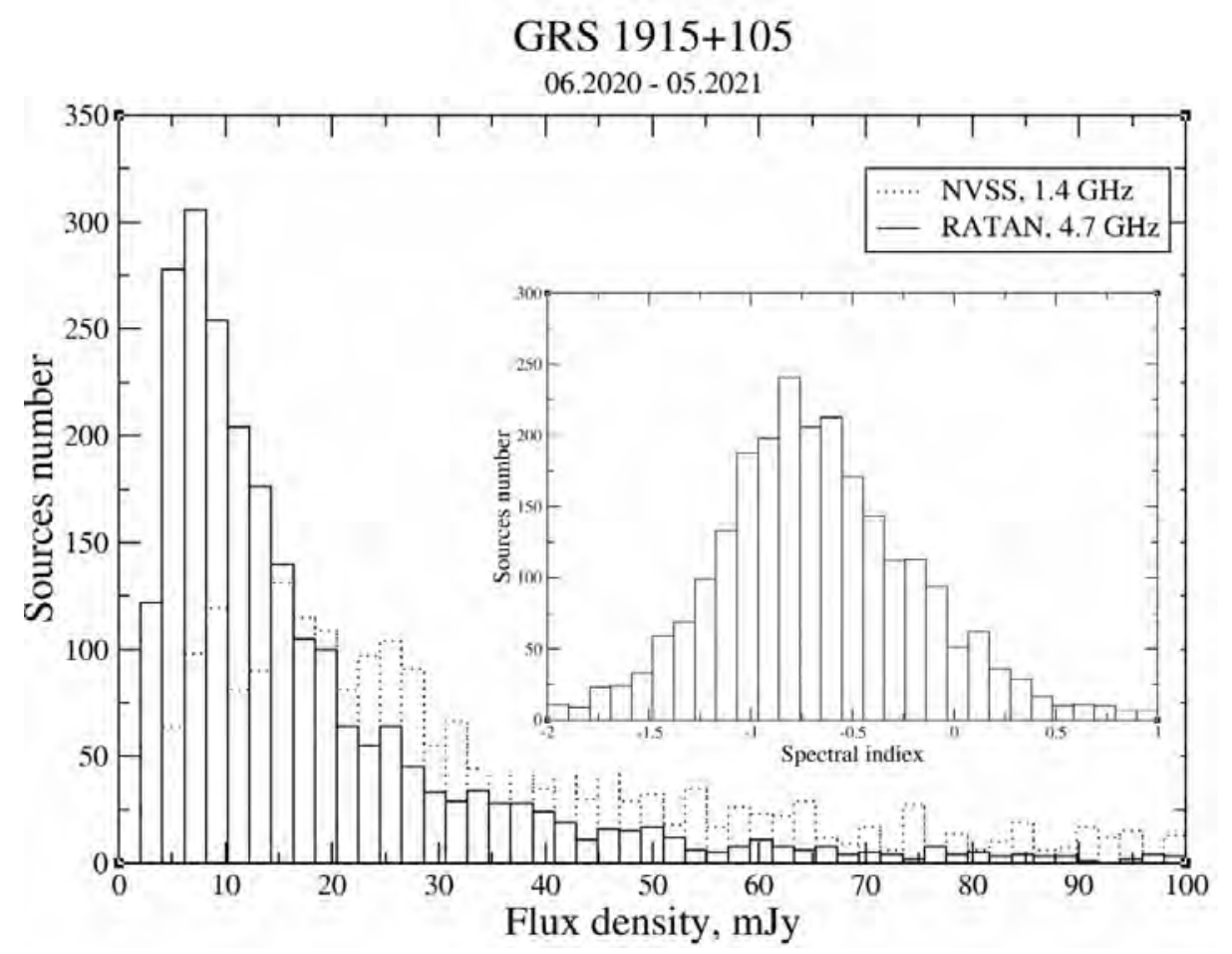

Figure 1: The flux density distribution for detected sources from this survey and the NVSS survey; inside: Distribution of the two-frequency spectral indices 

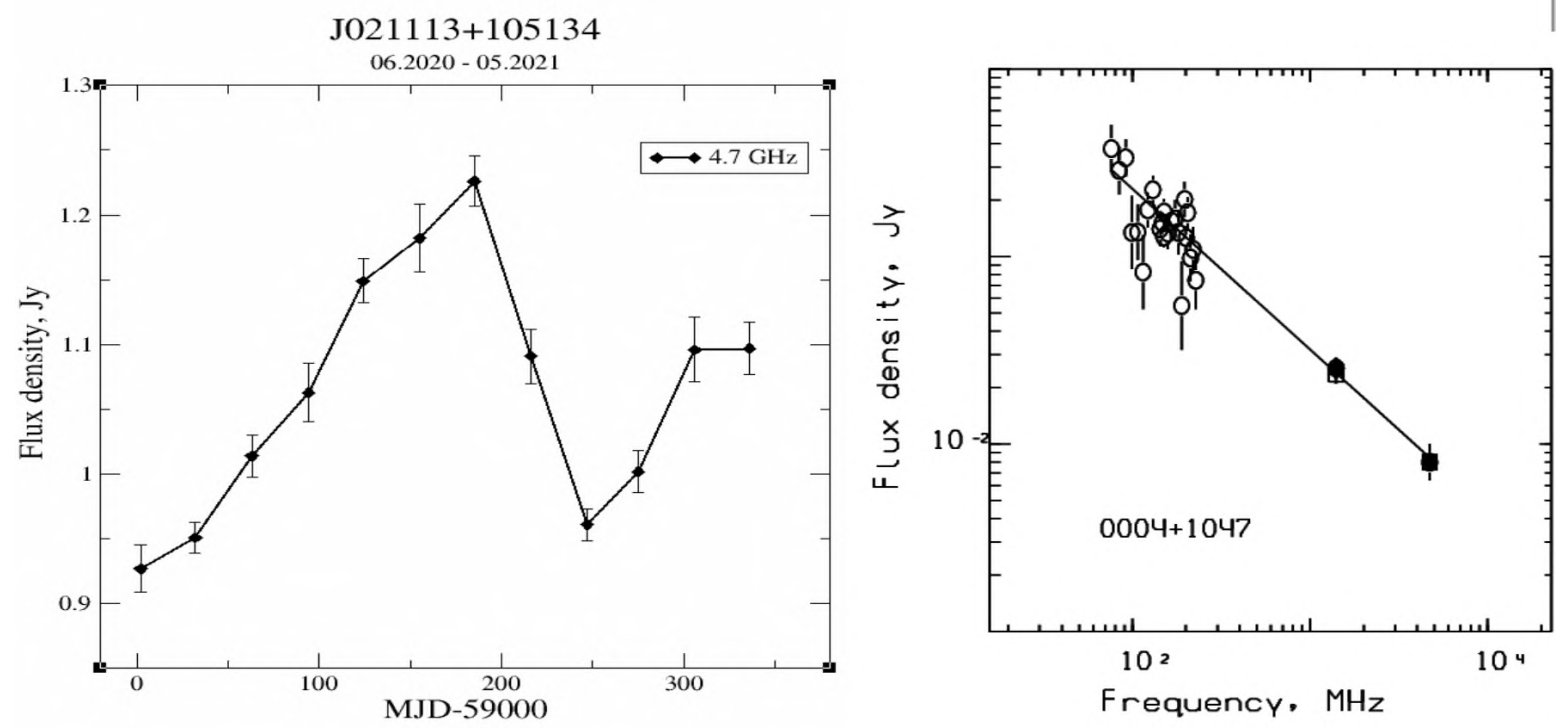

Figure 2: The light curve of NVSS J02113+105134 during the survey time (left) and the spectrum of the radio source NVSS J000436+104717 (RATAN-600 data is shown as a square) (right)

20-30 days. We obtained the calibration curve, produced convolution with the calculated beam, and averaged data for each R.A.-hour of the survey. Since the survey was conducted with a "Western sector" antenna directed exactly to the East, the sources have the oblique passage of the sky through the fixed diagram of the radio telescope. Data processing was done by the FADPS software [8]. As a result, the list of 1300 identified radio sources was obtained with coordinates from the NVSS catalog at $1.4 \mathrm{GHz}$ [9]. The flux density distribution of these sources (up to $100 \mathrm{mJy}$ ) is shown in Fig. 1 in comparison with the NVSS detected sources.

\section{Results}

The sample of bright sources was analysed to see if there were variable sources. Several sources show their variability. The light curve of one of them is presented on the left side of Fig. 2. The right side of this figure shows a supplement in the range of high-frequency continuum spectra of sources as an example (square point). For all detected sources, the spectra are plotted. For $26 \%$ of the sources, spectra are constructed for the first time, $34 \%$ of spectra are refined, and the rest are supplemented. The distribution of spectral indices is shown in Fig. 1 (inner figure) at two frequencies: 1.4 and $4.7 \mathrm{GHz}$. At $4.7 \mathrm{GHz}$, you can see an excess of sources with steep spectra in accordance with the general spectral distribution of extragalactic sources [10]. The plotted distribution has a significant fraction on the right side (positive spectral indices), which could be explained by the selection effect of our higher frequency survey. Also, it is possible that the extended NVSS-sources within the Galactic plane contribute to the distribution.

For all survey sources, optical identification was carried out. $60 \%$ of sources are found in the SDSS catalog. $80 \%$ of them are identified with galaxies, $20 \%$ - with quasars.

\section{References}

1. H. T. Intema, P. Jagannathan, K. P. Mooley, and D. A. Frail, A\&A, 598, A78, 2017.

2. N. Hurley-Walker, J. R. Callingham, P. J. Hancock, T. M. O. Franzen, et al., MNRAS, 464, 1146, 2017.

3. V. R. Amirkhanyan, A. G. Gorshkov, A. A. Kapustkin, V. K. Konnikova, et al., Soobshcheniya Spetsial'noj Astrofizicheskoj Observatorii, 47, 5, 1985.

4. Y. N. Parijskij, N. N. Bursov, R. Wielebinski, V. V. Vitkovskij, et al., Soviet Astronomy Letters, 13, $350,1987$.

5. I. N. Pariiski, N. N. Bursov, N. M. Lipovka, N. S. Soboleva, and A. V. Temirova, A\& A Sup., 87, 1, 1991.

6. N. N. Bursov, Y. N. Pariiskii, E. K. Maiorova, M. G. Mingaliev, A. B. Berlin, N. A. Nizhel'Skii, I. A. Glushkova, and T. A. Semenova, Astronomy Reports, 51, 197, 2007.

7. S. A. Trushkin, S. N. Fabrika, P. G. Tsybulev, and N. A. Nizhelskij, in SN 1987A, Quark Phase Transition in Compact Objects and Multimessenger Astronomy, 211-216 (2018).

8. O. V. Verkhodanov, S. A. Trushkin, H. Andernach, and V. N. Chernenkov, Bulletin of the Special Astrophysics Observatory, 58, 118, 2005.

9. J. J. Condon, W. D. Cotton, E. W. Greisen, Q. F. Yin, R. A. Perley, G. B. Taylor, and J. J. Broderick, AJ, 115, 1693, 1998.

10. P. Tiwari, Research in Astronomy and Astrophysics, 19, 096, 2019. 


\section{Nonlinear cosmogony of the spiral galaxy bulges}

F.U. Botirov, S.N. Nuritdinov

nur200848@mail.ru

National University of Uzbekistan, Tashkent, 100174, Uzbekistan

An early idea of one of the authors of this work is being developed, where the mechanism of instability of the warp perturbation mode against the background of an non-stationary disk was proposed for the first time in [1]. For this purpose, a model of a nonlinearly non-stationary self-gravitating disk with an anisotropic velocity diagram was first constructed. The model has a composite nature, or rather, it is a superposition of isotropic and anisotropic states of the disk. A nonstationary analogue of the dispersion equation of this composite model in the general case is obtained. The behavior of the domed disturbance mode, the instability of which leads to the formation of a classical bulge in the central region of the disk, is investigated. Critical diagrams of the dependence of the virial ratio on the degree of rotation of the system for various values of the superposition parameter and the corresponding diagrams for the increments of instability are constructed.

Keywords: nonlinear model; non-stationary self-gravitating disk; bulge of the Galaxy; dome perturbation mode

DOI: $10.51194 /$ VAK2021.2022.1.1.142

\section{Introduction}

The origins of galaxy bulges are poorly understood. Most authors (see, e.g., [2, 3] for a review) believe that bulges are well described within the framework of galaxy mergers. The bulges of disk-shaped galaxies are very heterogeneous. Despite this, today, basically, three types of bulges can be distinguished: classical bulges; box/peanut bulges; pseudo (disk-like) bulges. Obviously, the bulge cannot form against the background of a stationary model. In reality, its formation can occur during the nonlinearly non-stationary stage of galaxy evolution, for example, due to the gravitational instability of vertical oscillations of the central region of the disk. This idea was first put forward by one of the authors of this article in [1]. Below we develop this idea by first constructing an unsteady disk model with an anisotropic velocity diagram.

\section{Disk model non-stationary in the radial direction}

Among the various possible nonstationarities of the disk subsystem of galaxies, a special place is occupied by its radial oscillations. In reality, they may have not very large amplitudes, but theoretically it is easier to construct radially pulsating disks by generalizing some well-known equilibrium models to this case. Earlier, in the work of [4], a pulsating model of a self-gravitating disk was constructed by generalizing the equilibrium model of [5].

The issues of instability of a pulsating model with an isotropic velocity diagram [4] have been studied in detail in a number of works (see, e.g., $[6,7])$ and critical diagrams of the connection between $\Omega$ and $(2 T /|U|)_{0}$ have been determined. However, the isotropy of the velocity diagram is generally not entirely realistic and anisotropic models are required.

In order to cover a wide class of pulsating disks with an anisotropic velocity diagram, we found a composite version of the disk model with a phase distribution function in the form

$$
\begin{aligned}
\Psi=\frac{\nu \cdot \sigma_{0}}{2 \pi \Pi \sqrt{1-\Omega^{2}}} & {\left[\frac{1-\Omega^{2}}{\Pi^{2}}\left(1-\frac{r^{2}}{\Pi^{2}}\right)-\left(v_{r}-v_{a}\right)^{2}-\left(v_{\perp}-v_{b}\right)^{2}\right]^{-\frac{1}{2}} \cdot \chi(R-r)+} \\
+ & (1-\nu) \cdot \frac{8 \sigma_{0}}{\pi}\left[r^{2} v_{\perp}^{2}-r^{4} v_{\perp}^{4}+\left(\frac{1}{2}-3 r^{2} v_{\perp}^{2}\right) D-\frac{3}{8} D^{2}\right] \chi(D),
\end{aligned}
$$

where $\nu$ is the superposition parameter, which takes values from the interval $[0 ; 1], \Pi(t)=\frac{1+\lambda \cos \psi}{1-\lambda^{2}}, t=\frac{\psi+\lambda \sin \psi}{\left(1-\lambda^{2}\right)^{3 / 2}}$,

$$
D=\left(1-\frac{r^{2}}{\Pi^{2}}\right)\left(1-\Pi^{2} v_{\perp}^{2}\right)-\Pi^{2}\left(v_{r}-v_{a}\right)^{2} \geq 0
$$

In (1), $\Omega$ is a dimensionless parameter characterizing the degree of solid rotation of the disk $(0 \leq \Omega \leq 1)$,

$$
v_{a}=-\lambda \frac{r \sin \psi}{\sqrt{1-\lambda^{2}} \Pi^{2}}, v_{b}=\frac{\Omega r}{\Pi^{2}}
$$

$\lambda$ - radial pulsation amplitude, which is uniquely related to the virial parameter in the form $\lambda=1-(2 T /|U|)_{0}, \chi-$ Heaviside function, $\sigma_{0}$ - the value of the surface density of the disc at its center.

\section{Warp perturbations against the background of a pulsating disk}

In the general case, the perturbation in the form of a vertical bend has the form [8]

$$
H(\mathbf{r}, t)=a(t) \frac{1}{\xi} P_{N}^{m}(\xi) e^{i m \varphi}, \xi=\sqrt{1-\frac{r^{2}}{\Pi^{2}}},
$$

where the value of the function characterizes the amplitude of the disturbance, $N$ - radial wave number, $m$ - azimuthal wave number, $P_{N}^{m}(\xi)$ - attached Legendre polynomial, and $(N-m)$ must be odd. In this work, we are interested in the case when the radial wave number $N=3$, and the azimuthal wave number $m=0$. As will be seen below, a disturbance against a nonstationary background leads to a very interesting, more real picture of instability than against a stationary background. 

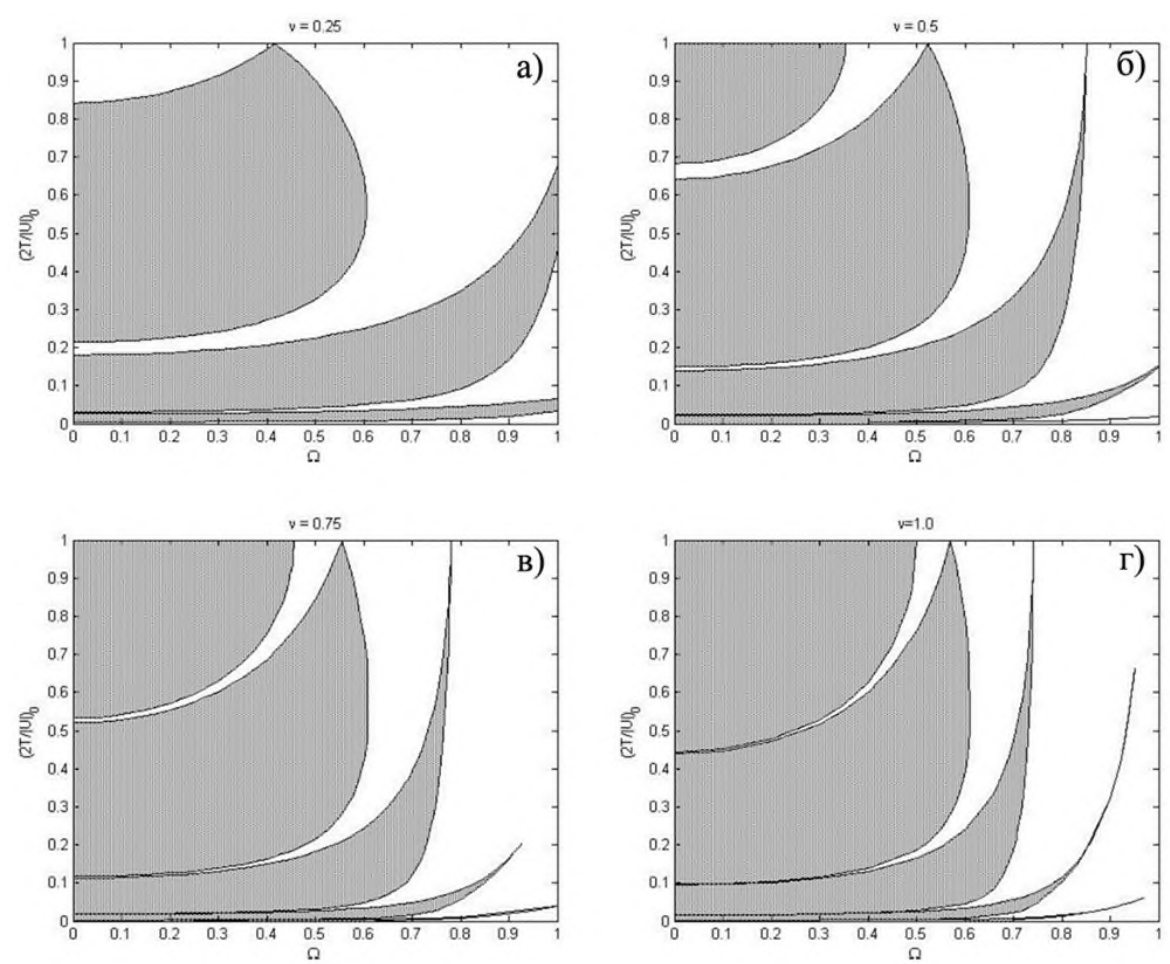

Figure 1: Critical dependence of the virial ratio on the rotation parameter for the perturbation mode $m=0$, $N=3$ : at $\nu=0.25(\mathrm{a}), 0.5(\mathrm{~b}), 0.75(\mathrm{v})$, and $1.0(\mathrm{~g})$. The dark areas correspond to unstable areas.

In (1), the first part of the distribution function corresponds to the case of a pulsating disk with an isotropic velocity diagram, and the second part is anisotropic, which can be obtained from the first by applying the weight function in the form $\rho(\Omega)=\frac{16}{\pi} \Omega^{2}\left(1-\Omega^{2}\right)^{3 / 2}$.

Then it is easy to derive the non-stationary dispersion equation (NDE) based on the corresponding results for the isotropic model using the weight function technique. This is how we found the following NDE for the domed disturbance mode against the background of a radially pulsating disk (1)

$$
\begin{array}{r}
(1+\lambda \cos \psi) \frac{d^{2} B}{d \psi^{2}}+\lambda \sin \psi \frac{d B}{d \psi}+ \\
+\left[\frac{5}{2}-\frac{10}{3} \nu\left(1-\Omega^{2}\right)-\frac{25\left(1-\lambda^{2}\right)}{12(1+\lambda \cos \psi)}(1-\nu)\right] \\
B(\psi)=0
\end{array}
$$

As you can see, NDE (5) depends on three parameters: $\lambda, \nu$ and $\Omega$. Obviously, a purely analytical study of it is impossible here, and therefore we perform numerical integration (5) for specific values of physical parameters. To construct the critical dependence of $\lambda=1-(2 T /|U|)_{0}$ on the rotation parameter at given values, we apply the well-known method of stability [9] of parametric resonance. For this purpose, we will consider the cases when the superposition parameter $\nu$ is equal to $0.25 ; 0.5 ; 0.75$ and 1.0 . The calculation results are shown in Fig. 1.

As can be seen from Fig. 1, at small and moderate values of the rotation parameter, the model becomes the most unstable. Only with an increase in rotation do the narrow channels of stability begin to expand. But with an increase in the superposition parameter, the stability channels narrow and the number of instability "petals" increases.

\section{References}

1. S. N. Nuritdinov, Symposium of the International Astronomical Union, Belgium, 153, 403, 1992.

2. M. Mollá, F. Ferrini, and G. Gozzi, MNRAS, 316, 345, 2000.

3. R. Zinn, ApJ, 293, 424, 1985.

4. S. N. Nuritdinov, Astronomicheskij Tsirkulyar, 1553, 9, 1992.

5. G. S. Bisnovatyi-Kogan and Y. B. Zel'dovich, Astrophysics, 6, 207, 1970.

6. S. N. Nuritdinov, K. T. Mirtadjieva, and M. Sultana, Astrophysics, 51, 410, 2008.

7. S. N. Nuritdinov, K. T. Mirtadjieva, I. Ahmad, and J. K. Ruzibaev, Astrophysics, 52, 584, 2009.

8. C. Hunter and A. Toomre, ApJ, 155, 747, 1969.

9. E. A. Malkov, Astrophysics, 24, 221, 1986. 


\section{Relic black holes and the nature of the dark matter of the Universe}

V.M. Charugin

charugin2010@mail.ru

Moscow Pedagogical State University, $1 / 1$ Malaya Pirogovskaya Str., Moscow 119991, Russia

DOI: 10.51194 /VAK2021.2022.1.1.143

The theory of evolution of the early universe predicts the formation of black holes of different masses. Depending on the physical processes, black holes with masses up to $10^{27} \mathrm{~g}$ can form. Due to the Hawking effect of quantum evaporation of black holes, black holes with masses of more than $10^{15} \mathrm{~g}$ have survived till the present time, and their radiation falls within the energy range of gamma quanta over tens of $\mathrm{MeV}$. Calculations have shown that if the mass function of primary black holes has a power form $N(M)=K \cdot M^{-\gamma}$, the intensity of background Hawking gamma-radiation from them $I(\nu) \sim \nu^{\gamma}$. Since the observed gamma background of the Universe in the energy range of quanta of 10-100 $\mathrm{MeV}$ has the form $I(\nu) \sim \nu^{-1.3}$ $\mathrm{MeV} \mathrm{cm}{ }^{-2} \mathrm{~s}^{-1} \operatorname{str}^{-1}$, the mass function of the relic black holes would be

$$
N(M)=1.3 \cdot 10^{-94} M^{1.3} \mathrm{~g}^{-1} \mathrm{~cm}^{-3},
$$

over the range of $1.6 \cdot 10^{14}<M<1.6 \cdot 10^{16} \mathrm{~g}$. If we assume that the distribution of relic black holes obtained from observations of the gamma background stretches to large masses, for example, to masses of $5 \cdot 10^{21} \mathrm{~g}$, then the average density of their mass will be about $0.25 \cdot 10^{-29} \mathrm{~g} \mathrm{~cm}^{-3}$ which is comparable to the estimate of the mass density of the dark matter in thenUniverse. At the same time, the main number and mass of black holes is concentrated toward large masses. In this case, the concentration of black holes will be about $2.2 \cdot 10^{41} \mathrm{~cm}^{-3}$. The average distance between relic black holes with masses of about $5 \cdot 10^{21} \mathrm{~g}$ is only one astronomical unit and in the volume of the sphere with the radius of the Jupiter's orbit there will be about fifty black holes. Natural questions arise: what physical processes in the early Universe led to such a distribution of the black holes by mass and why they abruptly finished at masses above $5 \cdot 10^{21} \mathrm{~g}$ ? And, of course, it would be interesting to try to find them. 


\section{Optical light curves of light-weight supermassive black holes produced by the Zwicky Transient Facility Forced Photometry Service}

M. Demianenko ${ }^{1,2,3}$, K. Grishin ${ }^{2}$, V. Toptun ${ }^{2,5}$, I. Chilingarian ${ }^{4,2}$, I. Katkov ${ }^{6,7,2}$, V. Goradzhanov ${ }^{2,5}$,

I. Kuzmin 2,5

demyanenko.mv@phystech.edu

${ }^{1}$ Moscow Institute of Physics and Technology (National Research University), Dolgoprudny, Russia

${ }^{2}$ Sternberg Astronomical Institute, M.V. Lomonosov Moscow State University, Moscow, Russia

${ }^{3}$ HSE University, Moscow, Russia

${ }^{4}$ Center for Astrophysics - Harvard and Smithsonian, Cambridge, USA

${ }^{5}$ Department of Physics, M.V. Lomonosov Moscow State University, Moscow, Russia

${ }^{6}$ New York University, Abu Dhabi, UAE

${ }^{7}$ Center for Astro, Particle, and Planetary Physics, New York University, Abu Dhabi, UAE

In this paper, we present an algorithm to correct optical light curves obtained using The Zwicky Transient Facility Forced Photometry Service and its application to the analysis of optical variability of 136 actvie galactic nuclei (AGN) powered by "light-weight" supermassive black holes (SMBH; $M_{\mathrm{BH}}<2 \cdot 10^{6} M_{\odot}$ ) including 24 intermediate-mass black holes (IMBH; $\left.M_{\mathrm{BH}}<2 \cdot 10^{5} M_{\odot}\right)$. We detected variability in nearly all sources and also analyzed its dependence on the X-ray luminosity for 101 objects. We also identified a previously unknown candidate tidal disruption event (TDE) in SDSS J112637.74+513423.0.

Keywords:active galactic nucleus, intermediate-mass black holes

DOI: $10.51194 /$ VAK2021.2022.1.1.144

\section{Introduction}

The presence of optical variability in a galaxy center is one of the most reliable diagnostics of an AGN to date. Optical light curves are used to search for a statistically significant variability of emission. The size of a region illuminated by an AGN in optical wavelengths defines the stochastic variability time scale in the light curves of such objects according to e.g. [1]. This allows one to use optical light curves to search and confirm IMBHs [2] because $M_{\mathrm{BH}}$ limits the AGN luminosity. $M_{\mathrm{BH}}$ can be measured from broad optical emission line profiles in AGN spectra [3, 4, 5].
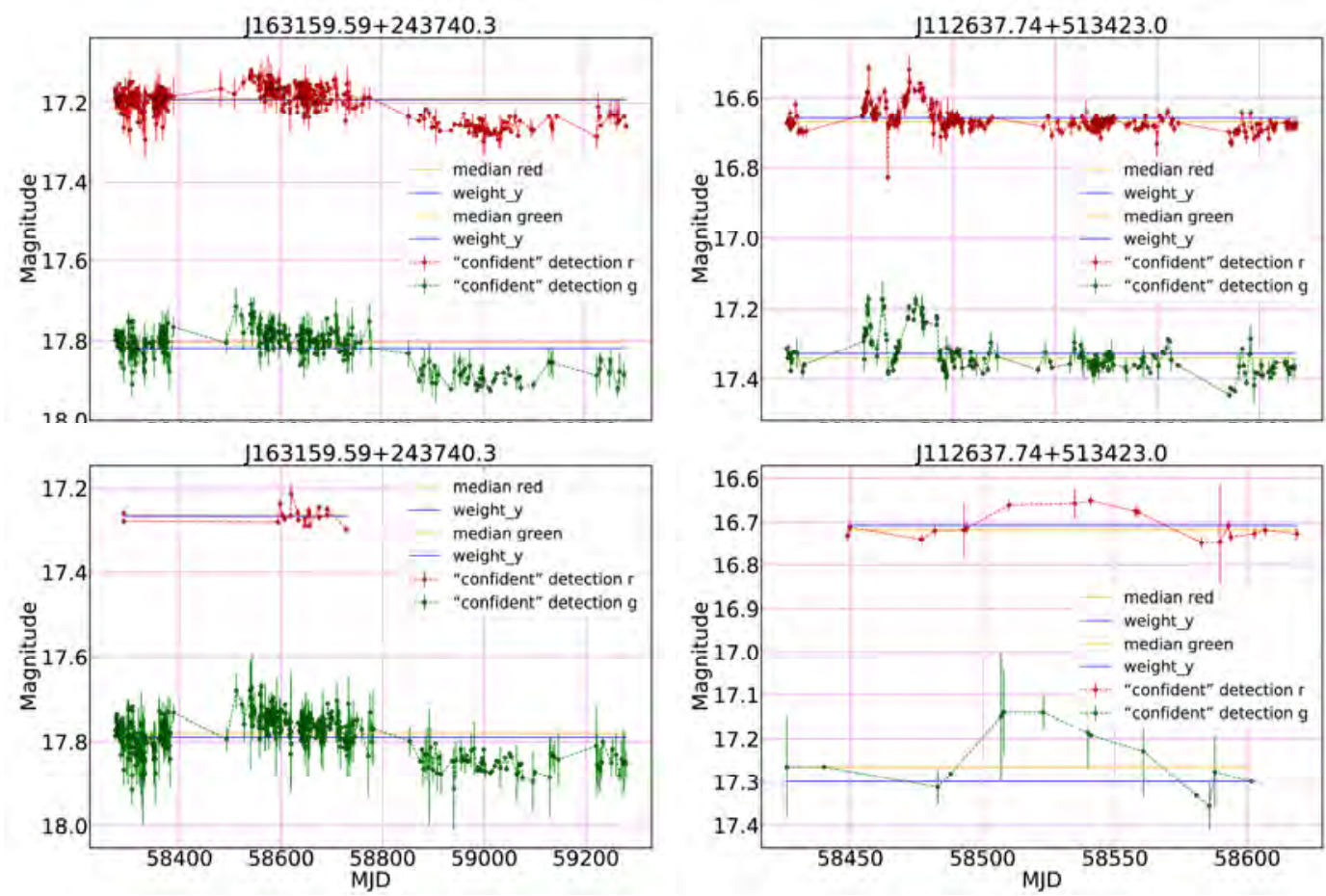

Figure 1: Top left: The light curve J163159.59+243740.3 obtained using ZTF Forced Photometry Service. Top right: The light curve of the candidate TDE before correction. Bottom left: The light curve of J163159.59+243740.3 after correction, points in one field of one quadrant of one CCD are visualized. Bottom right: The light curve of the candidate TDE after correction. The points in the most occurring quadrant of the CCD are shown. 


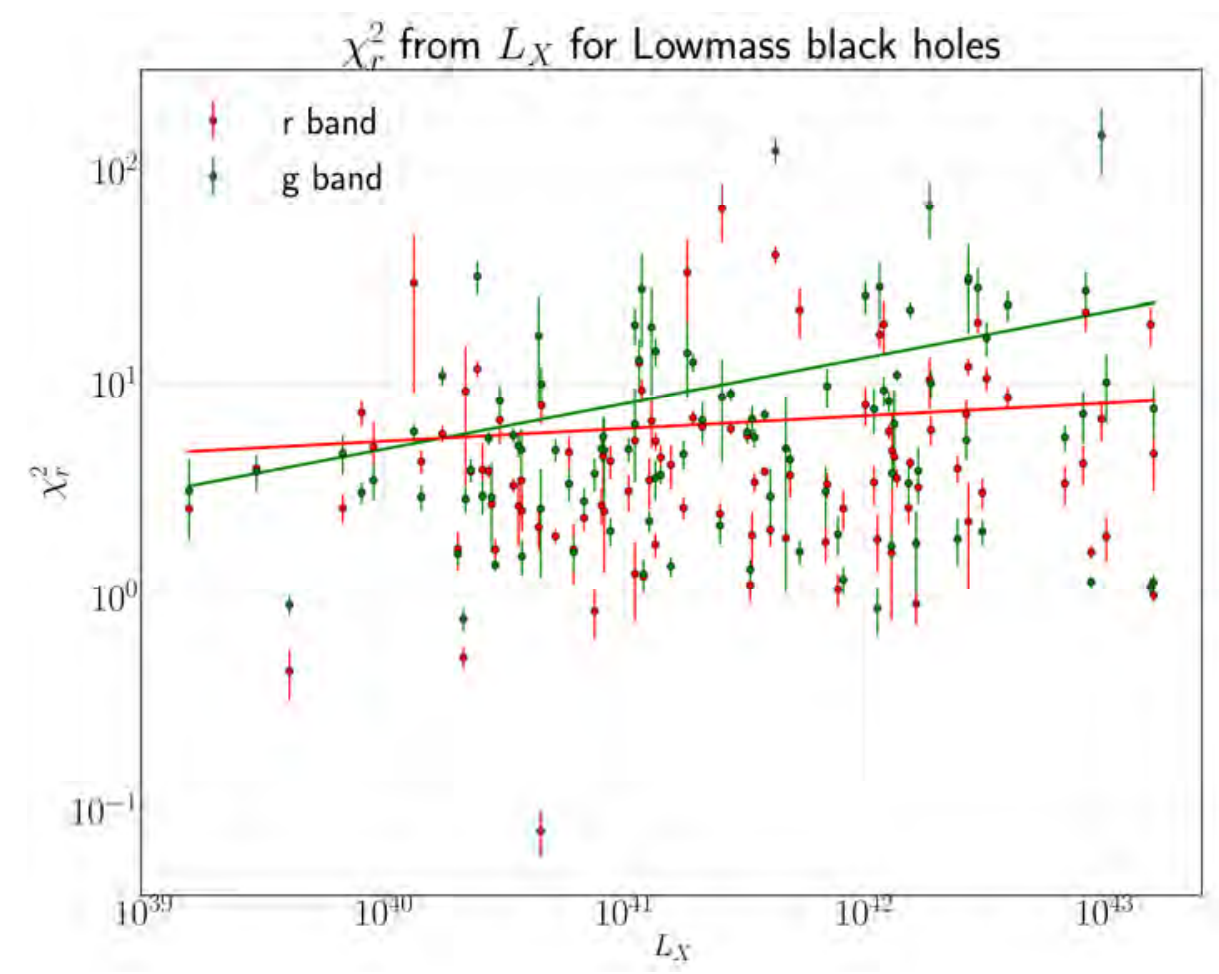

Figure 2: Dependence of the the optical light curve variability amplitude on the X-ray luminosity the two ZTF photometric bands, 'ZTF g' (green) and 'ZTF r' (red).

\section{Data}

Authors of [5] defined a sample of IMBHs from the analysis of optical spectra with the NBuRsTs full spectral fitting [6, 7] in the RCSED catalog [8], with $M_{\mathrm{BH}}$ estimated between $3 \cdot 10^{4} M_{\odot}$ and $2 \cdot 10^{5} M_{\odot} \cdot 10$ of these objects were confirmed in X-ray in [5], and another 14 were confirmed later from new and archival X-ray data. We define light-weight SMBHs as SMBHs having $M_{\mathrm{BH}}<2 \cdot 10^{6} M_{\odot} .112$ objects from the expanded sample of candidates light-weight SMBHs were confirmed by archival X-ray data. In total, we studies 136 optical light curves for 24 bona fide IMBHs and 112 bona fide light-weight SMBHs. Out of 136 objects, 101 had photometric points in two filters, 'ZTF r' and 'ZTF g'.

\section{Analysis of optical variability in light-weight SMBHs and IMBHs}

To estimate the optical variability amplitude and its timescale, we need to construct calibrated light curves without systematic variations exceeding 10-15\% of the host galaxy flux. We use differential images, which are obtained by subtracting the reference image from a current science image matching the point-spread-function (PSF). The reference image is a weighted average of all images for a given field, degraded to the same resolution using a Gaussian filter. Then, PSF photometry is performed without restrictions on the flux positivity. This approach is implemented in the ZTF Forced Photometry Service.

The resulting points do not always have the same zero point, because they were observed in different quadrants of different CCDs in the focal plane of the telescope. And also, color corrections are performed using zero color from the PanSTARRS photometric system. This correction is well defined only for those objects for which zero color measurement is available, otherwise the correction is estimated by the interpolation of PanSTARRS values. For each quadrant of the CCD and the field, the higher and lower flux limits of that sensor are defined, and points with flux measurements outside these limit derived from a specific observation should be excluded [9]. Here, we set the threshold signal-to-noise ratio (SNR) to 3 . The distance to the nearest reference was set to $<1$ arcsec, pixel quality indicators helped to clear outliers related to uncorrected cosmic ray hits and hot pixels. The zero-point correction was made for each observation, based on the CCD quadrant, and the average airmass during an observation. Then we defined the level of significance according to the $\chi^{2}$ criterion at which the light curve is not consistent with white noise on top of a constant. Many of the studied objects showed statistically significant variability on timescales from days to months.

Two examples of uncorrected ZTF Forced Photometry light curves are shown in Fig. 1 (top row). The bottom row of Fig. 1 shows the light curves after correction. In Fig.2 we show the dependence of the amplitude of optical variability (errors calculated using bootstrap) from the X-ray luminosity for 101 objects from our sample.

Also, a strong flare with an amplitude of 0.25 mag was observed in the object J112637.74+513423.0 for about 3 months, which can be either a heavily dust obscured supernova explosion or a star ruptured by tidal forces in the SMBH vicinity (TDE). We show a ZTF light curve of the TDE candidate in Fig 1 (top right).A more reliable light curve after the correction is shown in Fig 1 (bottom right): the "hump" is reliably traced by data points even in one CCD quadrant hence clearly confirming that the flare is real. 
The bottleneck that limits the scalability of our analysis and makes it yet impossible to study thousands of AGN is that the ZTF Forced Photometry Service can process up to 100 objects at a time and it takes hours to weeks to process every request.

\section{Conclusions}

The use of optical variability confirmed the high quality of the selection of IMBH and light-weight SMBHs candidates using a broad component $H \alpha$ and, in the future, will allow us to exclude candidates where broad lines have a transient or star formation related nature. Also, light curve analysis can be used for independent confirmation or selection of IMBH and light-weight SMBH candidates, because there are strong correlations of $M_{\mathrm{BH}}$ and the optical timescale according to [1].

MD is supported by the RSF project 17-72-20119.

\section{References}

1. C. J. Burke, Y. Shen, O. Blaes, C. F. Gammie, et al., Science, 373, 789, 2021.

2. J. Martínez-Palomera, P. Lira, I. Bhalla-Ladd, F. Förster, and R. M. Plotkin, ApJ, 889, 113, 2020.

3. J. E. Greene and L. C. Ho, ApJ, 610, 722, 2004.

4. E. Bon, P. Marziani, P. Jovanović, and N. Bon, Atoms, 7, 2019, URL https://www.mdpi.com/2218-2004/7/1/26.

5. I. V. Chilingarian, I. Y. Katkov, I. Y. Zolotukhin, K. A. Grishin, Y. Beletsky, K. Boutsia, and D. J. Osip, ApJ, 863, 1, 2018.

6. I. Chilingarian, P. Prugniel, O. Sil'Chenko, and M. Koleva, in A. r. Vazdekis and R. Peletier, eds., Stellar Populations as Building Blocks of Galaxies, volume 241, 175-176 (2007).

7. I. V. Chilingarian, P. Prugniel, O. K. Sil'Chenko, and V. L. Afanasiev, MNRAS, 376, 1033, 2007.

8. I. V. Chilingarian, I. Y. Zolotukhin, I. Y. Katkov, A.-L. Melchior, E. V. Rubtsov, and K. A. Grishin, ApJS, 228, 14, 2017.

9. F. J. Masci, R. R. Laher, B. Rusholme, D. L. Shupe, et al., PASP, 131, 018003, 2019. 


\title{
Contribution to the confusion noise from various sources
}

\author{
A.A. Ermash, S.V. Pilipenko, E.V. Mikheeva, V.N. Lukash \\ aermash@asc.rssi.ru \\ Lebedev Physical Institute, Russian Academy of Sciences, Leninskii pr. 53, Moscow, 119991 Russia
}

\begin{abstract}
The maximum possible depth of future far infrared and submillimetre telescopes will be limited by the confusion noise. Here we use the definition of the confusion noise as the width of the left side of the pixel histogram. In this paper we investigate the influence of the large-scale structure of the Universe and gravitational lensing on the confusion noise estimates. We also estimate the redshift and luminosity intervals of objects that give maximum contribution. The dependence on the diameter of the main mirror is also discussed.
\end{abstract}

Keywords: far infrared, evolution of galaxies

DOI: 10.51194 /VAK2021.2022.1.1.145

\section{Extragalactic Background Light model}

Recently we have created an EBL model that is used to estimate the parameters of the confusion noise for the Millimetron mission [1]. It was based on the eGALICS simulation (see. [2] and [3]). We used the SED library created by the GRASIL code [4] for a grid of parameters of galaxies. For active galactic nuclei (AGN) spectra we used the AGN type 1 SED by [5]. Gravitational lensing was also taken into account. In order to deal with the effect of "perspective" in the model cone the approach proposed by [6] was used. Random transformation was applied to each of the cubes: shift along each axis, mirroring and rotation of the cube to $-\pi / 2,+\pi / 2$ or $\pi$.

The Millimetron Space telescope will have $10 \mathrm{~m}$ diameter main mirror that will be actively cooled to $4.5 \mathrm{~K}$ and will carry two matrix detectors among other scientific payload: SACS (Shortwave Array Camera Spectrometer) and LACS (Longwave Array Camera Spectrometer). In total they will have eight bands: 70, 110, 250, 350 $\mu$ m (SACS) and 650, 850, 1100, $2000 \mu \mathrm{m}$ (LACS), respectively. In our previous paper we created model catalogs and maps for these eight bands and showed that observed number counts are reproduced reasonably well. The difference between number counts derived from model catalogs and from generated maps was also shown.

\section{Various effects that influence the confusion noise}

The first question we wanted to answer is how the presence of Large Scale Structure (LSS) of the Universe influences the confusion noise estimations. Intuitive answer to the question is that confusion, averaged over large angular area should not depend on the LSS, but estimations on different small maps should vary significantly. In order to do so we split our model area into smaller rectangles and estimate the confusion noise in them and find the error. The errors do not depend significantly on area of observations for wavebands from $650 \mu \mathrm{m}$ and larger. But for four SACS wavebands and size of maps about 1 angular minute LSS creates significant variations for different parts of the main model cone. The simple explanation of this effect is that limited angular resolution of Millimetron effectively smooths out the LSS.

We have created another model cone, where within each cube coordinates of objects were set randomly thus eliminating the LSS. The same splitting procedure as described above was then performed. Variations of the confusion noise between different parts of model maps are significantly lower. It implies that in order to create model maps LSS must be taken into account.

Gravitational lensing in general has little effect on the background. It significantly influences number counts curves only if they are very steep. In order to check its influence on the confusion noise we created model maps where gravitational lensing was switched off. The difference we obtained is well below the error values.

Another important question is which objects do really contribute to the confusion noise. We create a series of maps that contain only objects with redshifts from $z_{\min }$ to $z_{\max }$. Values of $z_{\min }$ and $z_{\max }$ were chosen on grid from 0 to 6 with a step of 0.1. On each map we estimated the confusion noise and created a two-dimensional plot that shows values of confusion for all these redshift intervals. The values of $z_{\min }$ and $z_{\max }$ for all considered wavebands are given in Table 1 . The result is quite unexpected. Minimum redshift value remains almost constant for all eight bands, while $z_{\text {max }}$ gradually decreases from $\sim 4$ to $\sim 3$ with increase of wavelength from $70 \mu \mathrm{m}$ to $2000 \mu \mathrm{m}$.

In the model cone we used maximum and minimum luminosities were $L_{\min }=10^{3} L_{\odot}$ and $L_{\max }=10^{13} L_{\odot}$, respectively. We create $2 \mathrm{~d}$ plots analogous to the ones we created for redshifts, but for $L_{\min }$ and $L_{\max }$. The resulting values are given in Table 1. The following conclusions can be made. Values of $L_{\min }$ and $L_{\max }$ depend on wavelength. Galaxies with luminosities between $10^{7} L_{\odot}$ and $10^{9} L_{\odot}$ create the confusion at $70 \mu \mathrm{m}$, while at large wavelengths mainly bright objects contribute to the confusion noise, $L>10^{11} L \odot$. It can be explained by the fact that angular resolution decreases with increase of wavelength and more bright objects begin to blend together.

For eight wavebands of Millimetron's detectors six colors can be defined as difference in flux between adjacent bands. We add two more bands - one shortwave $(50 \mu \mathrm{m})$ and one longwave $(3000 \mu \mathrm{m})$ in order to get two colors for each band of Millimetron. For example, for $250 \mu \mathrm{m}$ two colors can be derived: $C_{(350-250)}=\log _{10} S_{350}-\log _{10} S_{250}$ and $C_{(250-110)}=\log _{10} S_{250}-\log _{10} S_{110}$. So, for each of these two colors we can create a series of maps, containing only objects with $C_{(350-250) \text {,min }}<C_{(350-250)}<C_{(350-250) \text {, max }}$ and $C_{(250-110), \text { min }}<C_{(250-110)}<C_{(250-110), \text { max }}$, respectively. Performing this for each band we can derive color values of objects that contribute to the confusion noise. The results are given in Table 2. From the table the following conclusions can be derived. Objects that create the confusion noise at $70 \mu m$ have significant positive color coefficient with respect to the closest longer waveband. It means that they significantly contribute 
Table 1: Redshift and luminosity intervals of objects that create $90 \%$ of the confusion noise

\begin{tabular}{c|cccc|cccc}
\hline$\lambda[\mu m]$ & 70 & 110 & 250 & 350 & 650 & 850 & 1100 & 2000 \\
\hline$z_{\min }$ & 0.62 & 0.60 & 0.49 & 0.47 & 0.47 & 0.52 & 0.59 & 0.63 \\
$z_{\max }$ & 4.11 & 3.65 & 3.06 & 3.27 & 3.45 & 3.39 & 3.34 & 3.37 \\
\hline$L_{\min }$ & 6.9 & 7.1 & 8.1 & 9.3 & 10.7 & 11.1 & 11.2 & 10.4 \\
$L_{\max }$ & 9.1 & 9.9 & 11.2 & 12.0 & 11.6 & 11.6 & 12.2 & 11.7 \\
\hline
\end{tabular}

Table 2: Color of objects that contribute $90 \%$ to the confusion noise in each waveband of Millimetron. Colors are defined as difference in logarithmic flux in adjacent bands. The value of $C$ is defined as $\log _{10}\left(S_{\lambda_{1}}\right)-\log _{10}\left(S_{\lambda_{0}}\right)$. All wavelengths are in micrometers.

\begin{tabular}{|c|c|c|c|c|c|c|c|c|c|c|c|c|c|c|c|c|}
\hline$\overline{\lambda_{r}}$ & \multicolumn{2}{|c|}{70} & \multicolumn{2}{|c|}{110} & \multicolumn{2}{|c|}{250} & \multicolumn{2}{|c|}{350} & \multicolumn{2}{|c|}{650} & \multicolumn{2}{|c|}{850} & \multicolumn{2}{|c|}{1100} & \multicolumn{2}{|c|}{2000} \\
\hline$\lambda_{0}$ & 70 & 50 & 110 & 70 & 250 & 110 & 350 & 250 & 650 & 350 & 850 & 650 & 1100 & 850 & 2000 & 1100 \\
\hline$\lambda_{1}$ & 110 & 70 & 250 & 110 & 350 & 250 & 650 & 350 & 850 & 650 & 1100 & 850 & 2000 & 1100 & 3000 & 2000 \\
\hline$C_{\min }$ & $\overline{0.14}$ & 0.11 & $\overline{0.16}$ & 0.18 & -0.19 & 0.19 & -0.63 & -0.21 & -0.37 & -0.68 & -0.37 & -0.36 & -0.89 & -0.37 & -0.61 & -0.89 \\
\hline$C_{\max }$ & 0.38 & 0.35 & 0.68 & 0.39 & 0.17 & 0.75 & -0.10 & 0.19 & -0.20 & -0.08 & -0.26 & -0.20 & -0.75 & -0.26 & -0.56 & -0.75 \\
\hline
\end{tabular}

to the map at $110 \mu \mathrm{m}$, while at $50 \mu \mathrm{m}$ the relative contribution of these objects is lower. The situation gradually turns into opposite toward longer wavebands. It is generally accepted that in order to create algorithm that allows to negate the confusion to some degree observations at short wavelengths are used as strong priors on position, while observations on longer wavelengths are used as relatively weak priors on flux (see, e.g. [7]). It seems, that for shortest Millimetron wavebands information from observations on longer wavebands is also crucial.

One of the best ways to beat the confusion limit is to increase the angular resolution. But another factor that greatly influences the confusion is a shape of the number counts curve. On figure 1 we plot the dependence of the confusion noise on the diameter of the main mirror for eight wavebands. As can be seen, at short wavebands, as resolution increases, the confusion rapidly goes down and stops being an issue. At the same time, at four longest wavebands the $\sigma$ decreases gradually and even does not depend on wavelengths if $d>60 \mathrm{~m}$. It means that in order to beat the confusion limit we need not only higher resolution, but more complicated algorithms.

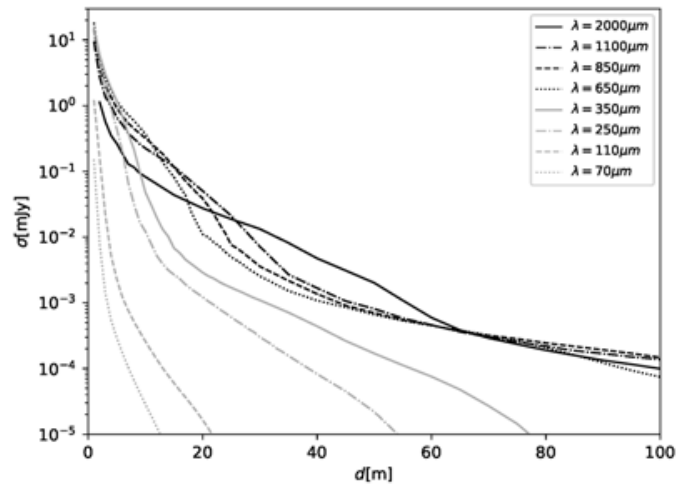

Figure 1: Confusion noise vs diameter of the main mirror. Eight wavebands of the Millimetron are plotted. Grey group of lines correspond to SACS, black group of lines corresponds to LACS.

\section{References}

1. A. A. Ermash, S. V. Pilipenko, and V. N. Lukash, Astronomy Letters, 46, 298, 2020.

2. M. Cousin, G. Lagache, M. Bethermin, J. Blaizot, and B. Guiderdoni, A\& A, 575, A32, 2015.

3. M. Cousin, G. Lagache, M. Bethermin, and B. Guiderdoni, A\& A, 575, A33, 2015.

4. L. Silva, G. L. Granato, A. Bressan, and L. Danese, ApJ, 509, 103, 1998.

5. J. Lyu and G. H. Rieke, ApJ, 841, 76, 2017.

6. J. Blaizot, Y. Wadadekar, B. Guiderdoni, S. T. Colombi, E. Bertin, F. R. Bouchet, J. E. G. Devriendt, and S. Hatton, MNRAS, 360, 159, 2005.

7. D. Liu, E. Daddi, M. Dickinson, F. Owen, et al., ApJ, 853, 172, 2018. 


\title{
Investigation of fast radio bursts on the BSA radio telescope of the Lebedev Physical Institute
}

\author{
V. Fedorova, A. Rodin
}

fedorova@prao.ru; rodin@prao.ru

PRAO ASC LPI, Pushchino, 142290, Russia

A comparative analysis of the observational characteristics of fast radio bursts at the frequencies of 111 and $1400 \mathrm{MHz}$ is carried out. Distributions are constructed with respect to the dispersion measure of radio bursts, which at both frequencies are described by a lognormal distribution with parameters $\mu=6.2, \sigma=0.7$. The dependence of the scattering value $\tau_{\mathrm{sc}}(D M)$ on the dispersion measure at 111 and $1400 \mathrm{MHz}$ is also constructed, which is fundamentally different from the dependence for pulsars. Comparative analysis of the relationship $\tau_{\mathrm{sc}}(D M)$ at $1400 \mathrm{MHz}$ and $111 \mathrm{MHz}$ showed that the dependence for both frequencies has the form $\tau_{\mathrm{sc}}(D M) \sim D M^{k}$, where $k=0.49 \pm 0.18$ and $k=0.43 \pm 0.15$ for the frequencies 111 and 1400 $\mathrm{MHz}$, respectively. The obtained dependence is explained by the theory of the extragalactic occurrence of fast radio bursts and the negligible contribution of intergalactic media to their scattering. From the dependency $\tau_{\mathrm{sc}}(D M)$ a total estimate of the contribution to the DM of the matter of the halo of our and the parent galaxy is obtained $D M_{\text {halo }}+\frac{D M_{\text {host }}}{1+z} \approx 60 \mathrm{pc} / \mathrm{cm}^{3}$. Based on the $\log \mathrm{N}-\log \mathrm{S}$ dependence, the average spectral index of radio bursts $\alpha=-0.63$ is derived.

Keywords: fast radio burst, BSA LPI, scattering, dispersion measure, host galaxy

DOI: 10.51194 /VAK2021.2022.1.1.146

\section{Dependence $\tau_{\mathrm{sc}}(D M)$ for fast radio bursts}

At the moment, the study of fast radio bursts is one of the most relevant areas in astrophysics. Radio bursts were first detected by [1]. At the moment, more than 600 pulses have been registered in the frequency range from $111 \mathrm{MHz}$ to 8 GHz. Since sufficient statistics were collected, we decided to analyze the observational properties of two groups of fast radio bursts. The first group included 63 pulses detected at a frequency of $111 \mathrm{MHz}$ from [2, 3, 4], the second - 61 pulses registered at a frequency of $1.4 \mathrm{GHz}$ from [5].

In the article by [6], the dependence of pulsar scattering on the dispersion measure $\tau_{\mathrm{sc}}(D M)$ was analyzed. As a result, it was obtained that the studied dependence has the form $\tau_{\mathrm{sc}}(D M)=60(D M / 100)^{2.2 \pm 0.1}$. This result was used as a basis for constructing a similar dependence for fast radio bursts. Thus, the results shown in Fig.1 were obtained:

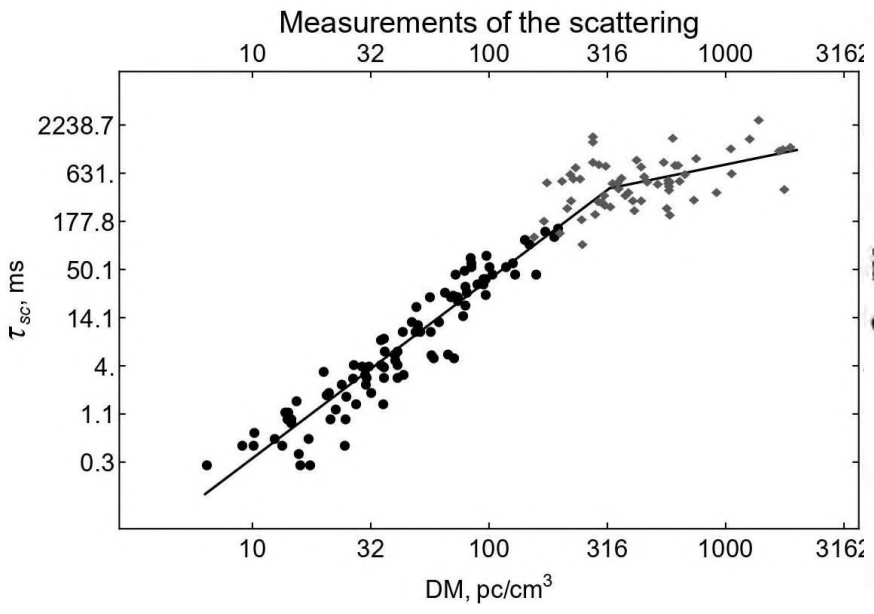

a)

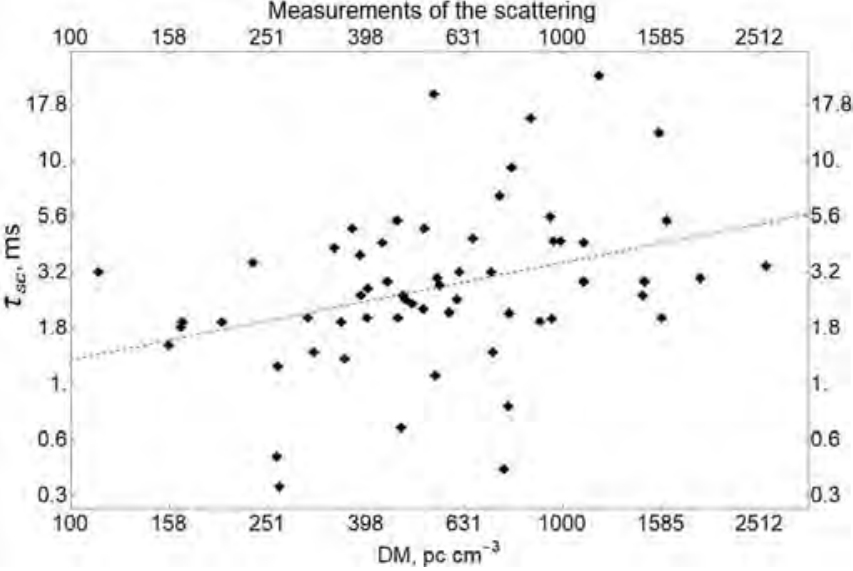

b)

Figure 1: Dependence $\tau_{\mathrm{sc}}(D M)$ for fast radio bursts detected at the frequency: a) $111 \mathrm{MHz}$, exponent $k=0.49 \pm$ 0.18 ; b) $1.4 \mathrm{GHz}$, exponent $k=0.43 \pm 0.15$. Within the margin of error, the slope coefficients coincide with each other.

The dependence of scattering on frequency is described by the law $\tau_{\mathrm{sc}}(f) \sim f^{-1.9 \pm 0.7}$ and is fundamentally different from the dependence $f^{-4}$ previously obtained for pulsars. This result can be interpreted under the assumption that the turbulence spectrum of the extragalactic medium differs from the Kolmogorov one and that the contribution to the scattering of the intergalactic medium is negligible. In our opinion, the main scattering of the signal occurs in galaxies, and the intergalactic environment practically does not affect this parameter. Based on this assumption, we obtained a total estimate of the contribution to the $D M$ of the matter of the halo of our and the host galaxy:

$$
D M_{\text {halo }}+\frac{D M_{\text {host }}}{1+z} \approx 60 \mathrm{pc} / \mathrm{cm}^{3}
$$




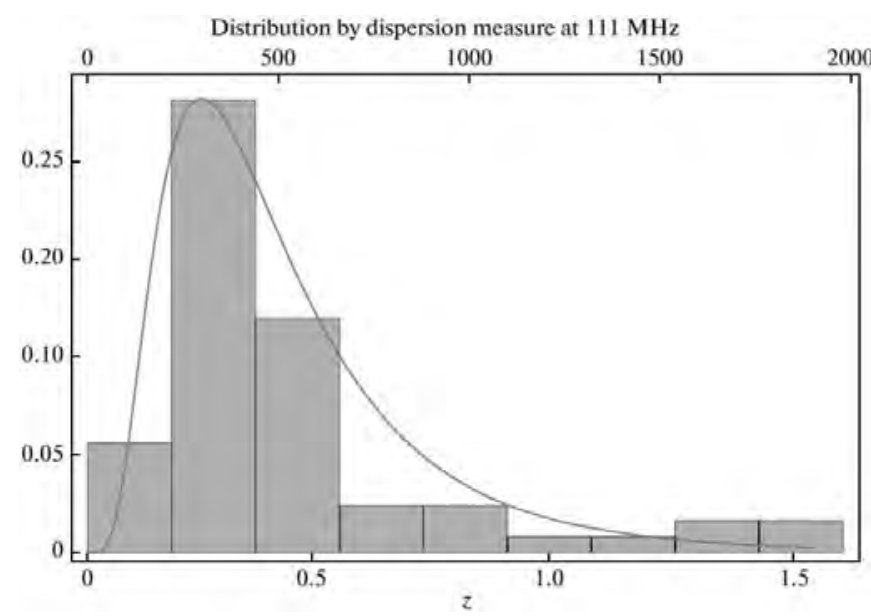

a)

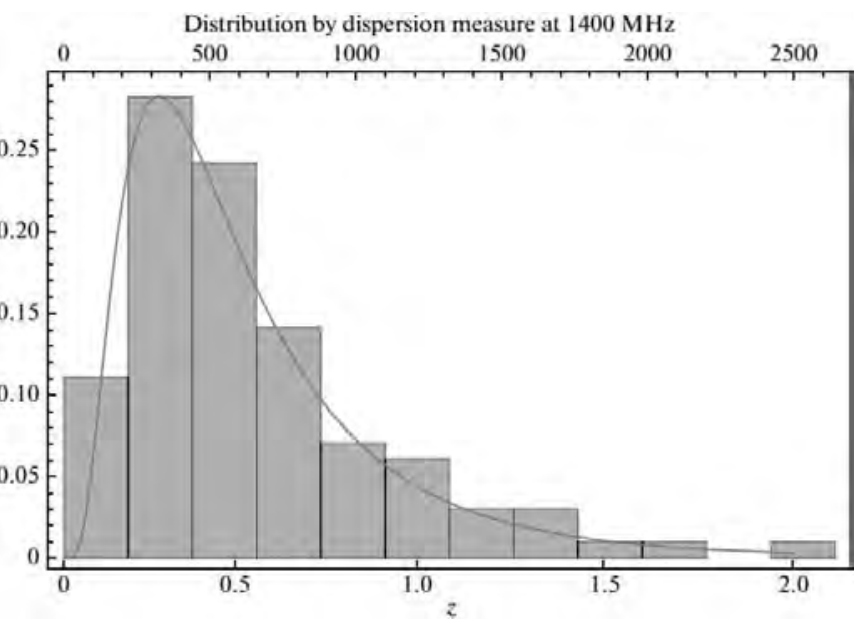

b)

Figure 2: Distribution of fast radio bursts at a) $111 \mathrm{MHz}$, b) $1.4 \mathrm{GHz}$.

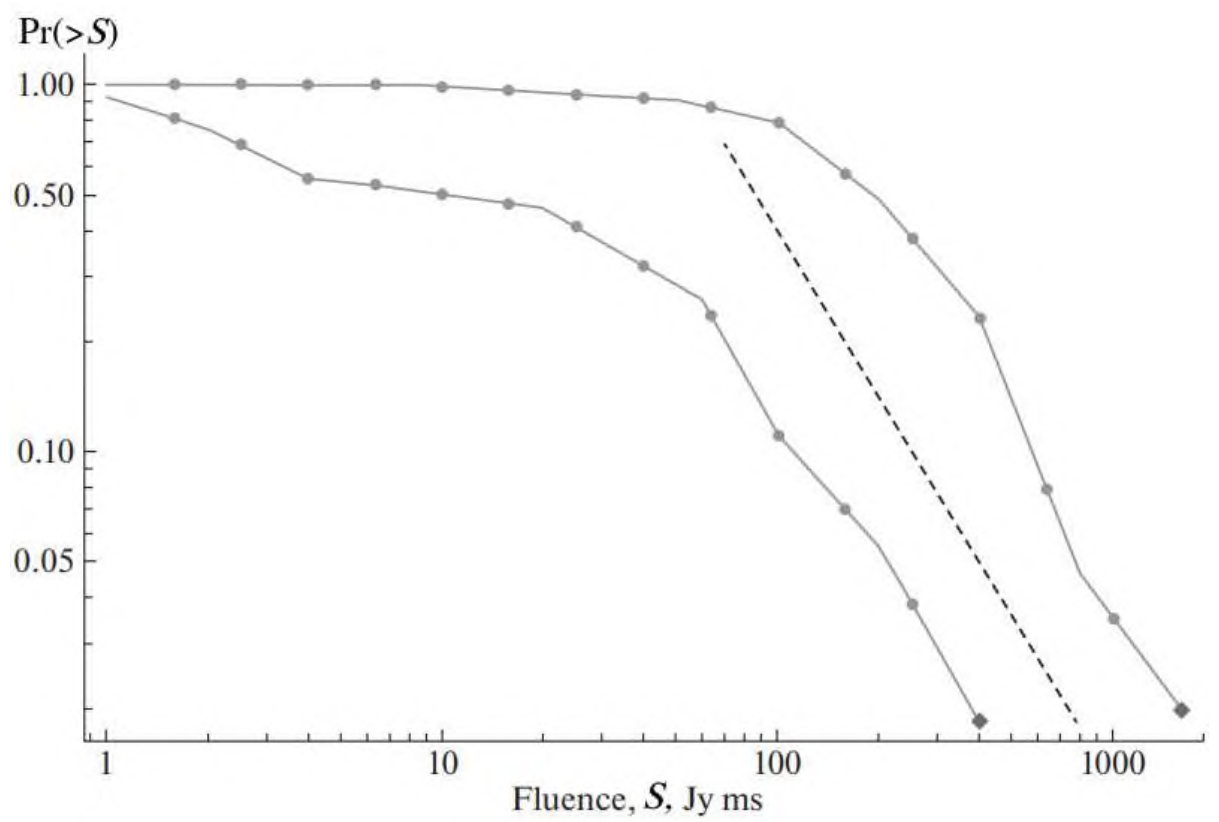

Figure 3: A graph of the $\log \mathrm{N}$ - Log S dependence for fast radio bursts detected at the frequencies of 111 and $1400 \mathrm{MHz}$ (upper and lower curves, respectively). The dotted line between the curves corresponds to the slope $-3 / 2$.

\section{Distribution of fast radio bursts by the dispersion measure}

In the course of the study, we constructed distributions of two groups of fast radio bursts by the dispersion measure. The result is shown in Fig. 2.

It can be seen from both distributions that they are well described by the lognormal distribution:

$$
P(x)=\frac{e^{-\frac{(\mu+\ln x)^{2}}{2 \sigma^{2}}}}{x \sqrt{2 \pi} \sigma}
$$

with the parameters $\mu=6.2$ and $\sigma=0.7$. Thus, the distribution data at a statistically significant level coincide and correspond to a characteristic range of dispersion measures $D M \approx 250 \div 950 \mathrm{pc} / \mathrm{cm}^{3}$.

\section{Dependence $\log N-\log S$}

In this paper, the dependence of $\log \mathrm{N}-\log \mathrm{S}$ on two groups of fast radio bursts was also analyzed (Fig.3). 
We made an assumption about the equality of the statistical properties of the pulses registered at the frequencies of $111 \mathrm{MHz}$ and $1.4 \mathrm{GHz}$. In this case, the average value of the spectral index can be determined $\alpha$ :

$$
\alpha=-0.63 \pm 0.20
$$

In other works, the evaluation of this parameter was given in the range of -1.9 to -0.6 .

In addition, analysis of the dependency $\log \mathrm{N}-\log \mathrm{S}$ at the frequency $111 \mathrm{MHz}$ and 1400 shows that they both follow the law $S^{-3 / 2}$ for $S_{111 \mathrm{MHz}}>200 \mathrm{Jy} \cdot \mathrm{ms}$ and $S_{1.4 \mathrm{GHz}}>50 \mathrm{Jy} \cdot \mathrm{ms}$.

\section{Conclusion}

The paper presents the result of the analysis of fast radio bursts detected at the frequencies of 111 and $1400 \mathrm{MHz}$ (for more details, see [4]). One of the important observational results of this study is the dependence $\tau_{\mathrm{sc}}(f) \sim f^{-1.9 \pm 0.7}$, which is fundamentally different from the dependence obtained for pulsars.

\section{References}

1. D. R. Lorimer, M. Bailes, M. A. McLaughlin, D. J. Narkevic, and F. Crawford, Science, 318, 777, 2007.

2. V. A. Fedorova and A. E. Rodin, Astronomy Reports, 63, 39, 2019.

3. V. A. Fedorova and A. E. Rodin, Astronomy Reports, 63, 877, 2019.

4. V. A. Fedorova and A. E. Rodin, Astronomy Reports, 65, 776, 2021.

5. E. Petroff, E. D. Barr, A. Jameson, E. F. Keane, et al., PASA, 33, e045, 2016.

6. A. D. Kuz'min, B. Y. Losovskii, and K. A. Lapaev, Astronomy Reports, 51, 615, 2007. 


\title{
Optical spectroscopic observations of intermediate-mass black holes and their host galaxies: the $M_{\mathrm{BH}}-\sigma_{*}$ relation
}

\author{
V. Goradzhanov ${ }^{1,3}$, I. Chilingarian ${ }^{1,2}$, I. Katkov ${ }^{1,4,5}$, K. Grishin ${ }^{1}$, V. Toptun ${ }^{1,3}$, I. Kuzmin ${ }^{1,3}$, M. \\ Demianenko ${ }^{1,6,7}$ \\ ${ }^{1}$ Sternberg Astronomical Institute, M.V. Lomonosov Moscow State University, Moscow, Russia \\ ${ }^{2}$ Center for Astrophysics - Harvard and Smithsonian (USA), \\ ${ }^{3}$ Department of Physics, M.V. Lomonosov Moscow State University, Moscow, Russia \\ ${ }^{4}$ New York University, Abu Dhabi, UAE \\ ${ }^{5}$ Center for Astro, Particle, and Planetary Physics, New York University, Abu Dhabi, UAE \\ ${ }^{6}$ Moscow Institute of Physics and Technology (National Research University), Dolgoprudny, Russia \\ ${ }^{7}$ HSE University, Moscow, Russia
}

Intermediate-mass black holes (IMBHs; $M_{\mathrm{BH}}<2 \cdot 10^{5} M_{\odot}$ ) in galaxy centers are cruciel for painting a coherent picture of the formation and growth of supermassive black holes (SMBHs). Using Big Data analysis, we identified 305 IMBH candidates for IMBH and 1623 candidates of 'light-weight' SMBHs $\left(2 \cdot 10^{5} M_{\text {odot }}<M_{\mathrm{BH}}<10^{6} M_{\odot}\right)$. For 35 host galaxies from this combined sample with the X-ray-confirmed active galactic nuclei (AGN) we collected and analyzed optical spectroscopic observations. These data show that bulge stellar velocity dispersions $\left(\sigma_{*}\right)$ lie in the range of $24 \ldots 118 \mathrm{~km} / \mathrm{s}$ and do not follow the correlation with $M_{\mathrm{BH}}$ established by larger SMBHs indicating that in the $10^{5}-10^{6} M_{\odot}$ range the accretion is the prevailing BH growth channel.

Keywords: cosmology: observations - early universe - galaxies: active - galaxies: nuclei - galaxies: Seyfert - quasars: supermassive black holes

DOI: $10.51194 /$ VAK2021.2022.1.1.147

\section{Introduction}

Currently observed black holes are categorized by mass into three types: stellar mass black holes $\left(4<M_{\mathrm{BH}}<100 M_{\odot}\right)$, supermassive black holes (SMBH; $M_{\mathrm{BH}}>10^{5} M_{\odot}$ ), and intermediate mass black holes (IMBH; $100<M_{\mathrm{BH}}<10^{5} M_{\odot}$ ). The growth of central SMBHs and the way they affect their host galaxies remains a highly controversial issue in modern astrophysics. From theoretical perspectives, a black hole seed with a starting mass of $50 M_{\odot}$ cannot grow to a billion solar mass SMBH via accretion in $\sim 1$ Gyr (or $z>7$ corresponding to record holding high-redshift quasars), because the accretion rate is limited by the Eddington luminosity $\left(L_{E d d}\right)$. If this is indeed the main SMBH growth channel, then 'undergrown' seeds will form a population of IMBHs.

Here we adopt the limit $M_{\mathrm{BH}}<2 \cdot 10^{5} M_{\odot}$ as the threshold mass for what we call an IMBH, because it corresponds to $+1 \sigma$ systematic uncertainty in virial mass estimate $[1,2]$ from broad $H \alpha$.

Central massive black holes in both galaxies with active and inactive nuclei follow rather tight correlations with the stellar velocity dispersion $\sigma_{*}$ of the bulge and the total stellar mass of the bulge $M_{\text {bulge }}[3,4]$, which is interpreted as a co-evolution of the bulge and SMBH via galaxy mergers. At the same time, SMBHs can gain mass via accretion [5] at any moment during the cosmic time. It is not yet fully known whether these relations hold for IMBH because of observational difficulties and small samples of known IMBHs.

In 2018, the largest to-date sample of 305 IMBH candidates was identified by [2] using data mining in the Reference Catalog of Spectral Energy Distribution [6] database of almost 1 million spectra of galaxies from the SDSS Survey [7]. Some of them were later observed with intermediate-resolution optical spectrographs MagE (6.5-m Magellan telescope) and RSS (10-m Southern African Large Telescope) to refine stellar velocity dispersions and improve $M_{\mathrm{BH}}$ measurements. Here we report preliminary results of this project and also present $\sigma_{*}$ measurements for a sample of 'light-weight' SMBHs $\left(<10^{6} M_{\odot}\right)$ populating the bottom part of the $M_{\mathrm{BH}}-\sigma_{*}$ relation.

\section{Spectral data analysis}

We obtained 30 spectra for 26 'light-weight' SMBHs using MagE and additional 9 spectra using RSS and reduced them with standard data processing pipelines. We fitted the reduced spectra with the NBursts technique [9, 10] to determine the stellar continuum and measure stellar velocity dispersions as low as $20 \mathrm{~km} / \mathrm{s} \mathrm{[11].} \mathrm{Then} \mathrm{we} \mathrm{subtracted} \mathrm{the} \mathrm{best-fitting} \mathrm{stellar}$ population templates from the spectra and applied a novel method of a two-dimensional nonparametric reconstruction of the $H \alpha$ profile of emission spectra (see Fig. 1). The method yields a non-parametric narrow-line profile featuring narrow lines from the AGN and a position-velocity diagram for narrow lines originating from a star-forming disk of its host galaxy. It also returns the parameters of the broad component of $H \alpha$ and $H \beta$ formed in the broad-line region, as well as corresponding fluxes. The broad $H \alpha$ flux and width are then used for the virial $M_{\mathrm{BH}}$ estimate using the calibration from [1]: $M_{\mathrm{BH}}=3.72 \cdot 10^{6}\left(\mathrm{FWHM}_{H \alpha} / 10^{3} \mathrm{~km} / \mathrm{s}\right)^{2.06} \cdot\left(L_{H \alpha} / 10^{42} \mathrm{erg} / \mathrm{s}\right)^{0.47}$. We rejected 7 galaxies from the MagE sample where broad components were not confidently detected. We also took into account slit losses assuming that the broad H $\alpha$ component comes from a point source and knowing the slit width.

\section{The $M_{\mathrm{BH}}-\sigma$ scaling relation}

The $M_{\mathrm{BH}}-\sigma_{\text {buldge }}$ relation constructed from the measured values of $\sigma_{*}$ and $M_{\mathrm{BH}}$ derived from MagE and SALT data is shown in Fig. 2. We can make a preliminary conclusion that light-weight SMBHs and IMBHs do not follow the relation 

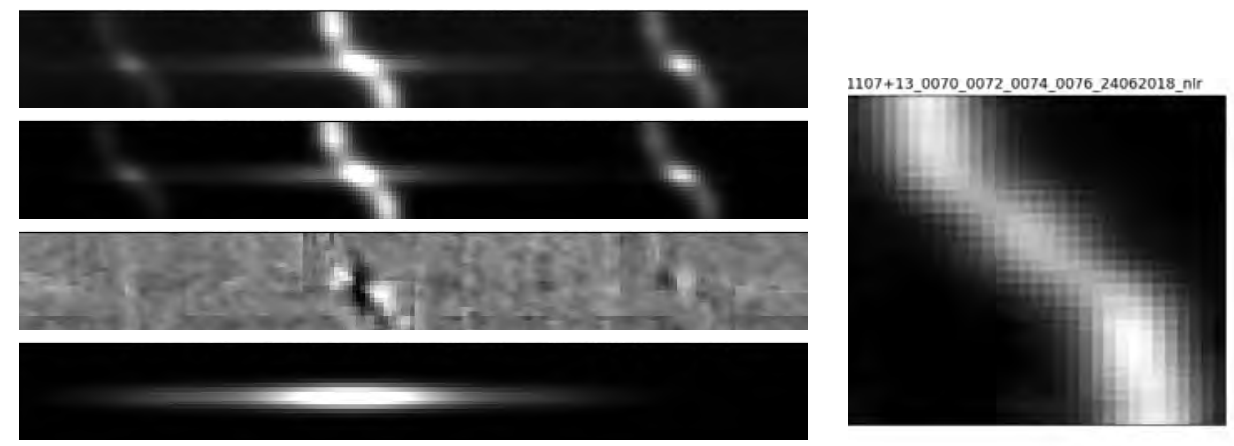

Figure 1: Left panels top to bottom: the original 2d emission spectrum of the galaxy J110731.22 +134712.9 in the $H \alpha$ region; the result of the fitting of emission lines; fitting residuals; the reconstructed broad line profile. Right panel: a $2 \mathrm{D}$ narrow-line profile of the $H \alpha$.

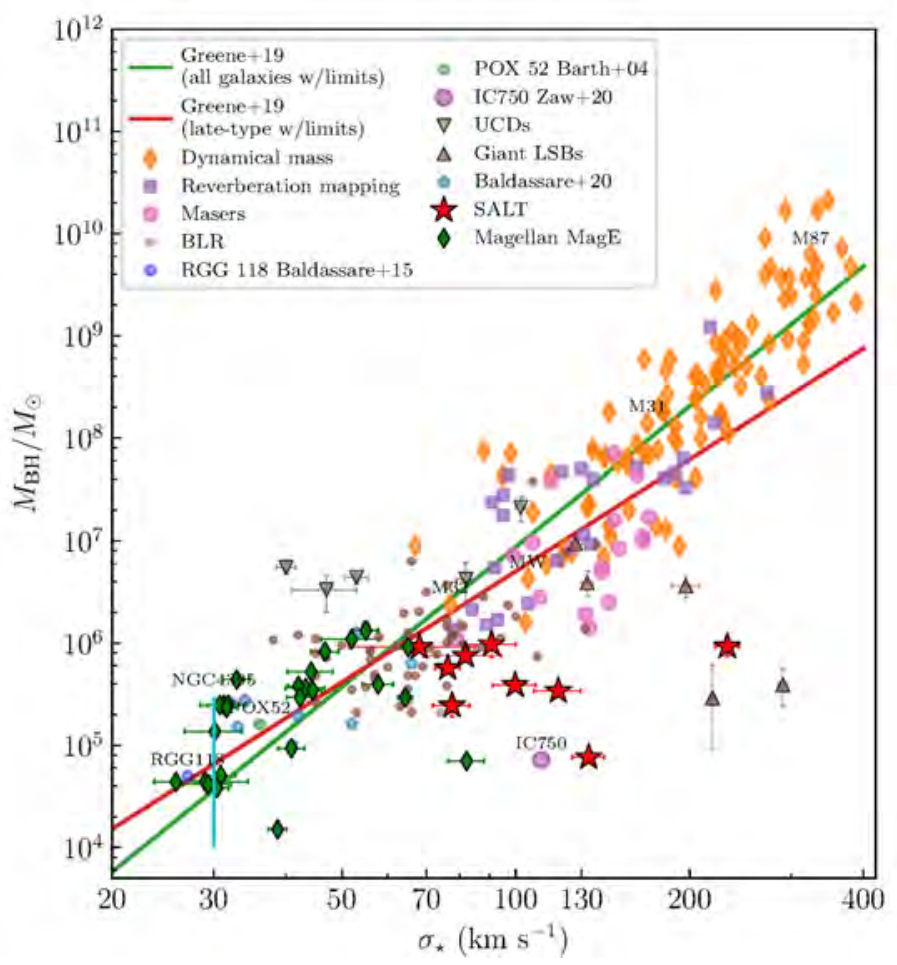

Figure 2: The $M_{\mathrm{BH}}-\sigma_{*}$ relation featuring IMBHs and light-weight SMBHs. Only one IMBH has a mass estimate based on masers [8]; the rest of the data points are virial estimates from $H \alpha$. New data from MagE and SALT are shown by green diamonds and red stars respectively.

established by more massive SMBHs and, hence, do not co-evolve with bulges of their host galaxies. Such situation can arise if the black hole mass growth in the low-mass regime is dominated by accretion rather than mergers. This result might have serious implications on the detectability of gravitational wave signals from merging IMBHs by the fortcoming LISA mission.

Acknowledgements. This project is supported by the RScF Grant 17-72-20119.

\section{References}

1. A. E. Reines, J. E. Greene, and M. Geha, $A p J$, 775, 116, 2013.

2. I. V. Chilingarian, I. Y. Katkov, I. Y. Zolotukhin, K. A. Grishin, Y. Beletsky, K. Boutsia, and D. J. Osip, ApJ, 863, $1,2018$.

3. L. Ferrarese and D. Merritt, $A p J L, \mathbf{5 3 9}$, L9, 2000.

4. R. C. E. van den Bosch, ApJ, 831, 134, 2016.

5. M. Volonteri, Science, 337, 544, 2012.

6. I. V. Chilingarian, I. Y. Zolotukhin, I. Y. Katkov, A.-L. Melchior, E. V. Rubtsov, and K. A. Grishin, ApJS, 228, 14, 2017. 
7. K. N. Abazajian, J. K. Adelman-McCarthy, M. A. Agüeros, S. S. Allam, et al., ApJS, 182, 543-558, 2009.

8. I. Zaw, M. J. Rosenthal, I. Y. Katkov, J. D. Gelfand, Y.-P. Chen, L. J. Greenhill, W. Brisken, and H. A. Noori, The Astrophysical Journal, 897, 111, 2020, URL https://doi.org/10.3847/1538-4357/ab9944.

9. I. V. Chilingarian, P. Prugniel, O. K. Sil'Chenko, and V. L. Afanasiev, MNRAS, 376, 1033, 2007.

10. I. Chilingarian, P. Prugniel, O. Sil'chenko, and M. Koleva, in A. Vazdekis and R. R. Peletier, eds., Stellar Populations as Building Blocks of Galaxies, Cambridge University Press, IAU Symposium, volume 241, 175-176 (2007).

11. I. V. Chilingarian and K. A. Grishin, PASP, 132, 064503, 2020. 


\section{Study of groups/clusters of galaxies with the SDSS}

F. Kopylova, A. Kopylov

flera@sao.ru,

WWW home page: http://wWw.sao.ru/hq/dolly/ntik_group.html

Special Astrophysical Observatory RAS, Nizhnij Arkhyz, 369167, Russia

For a large sample of groups/clusters of galaxies $(N=185)$, we obtained the scaling relations among of their photometrical and dynamical parameters. We find:

1. that in the virialized regions of the galaxy systems the total luminosity increase with mass $L_{K} \propto M_{200}^{0.77 \pm 0.01}\left(M_{K}<\right.$ $\left.-21 .^{m} 0\right)$

2. that the new halo boundary of the galaxy systems corresponds to the splashback radius $R_{s p}$. These radius is defined by the observed intergrated distribution of the number of galaxies as a function of the angular distance from the group/cluster center squared;

3. that the fraction of galaxies with quenched star formation is maximal in the central regions of the galaxy systems, and it decreases to the radius $R_{s p}$, but remains higher than in the field on $\sim 27 \%$.

Keywords: galaxies: groups and clusters: general — large-scale structure of Universe

DOI: 10.51194 /VAK2021.2022.1.1.148

Galaxy clusters, the largest gravitationally bound objects, which grow by accreting dark matter and luminous matter (groups of galaxies and galaxies) along filaments from the surrounding space (see, e.g., paper of [1]). They demonstrate a wide range of spatial density of galaxies: high-density areas in the center and low-density, almost field, on the periphery.

In this study, we used a sample of 157 groups/clusters of galaxies of the local Universe $(0.013<z<0.100)$ with line-of-sight velocity dispersions $200 \mathrm{~km} \mathrm{~s}^{-1}<\sigma<1100 \mathrm{~km} \mathrm{~s}^{-1}$. In total, in our sample there are of 185 groups/clusters of

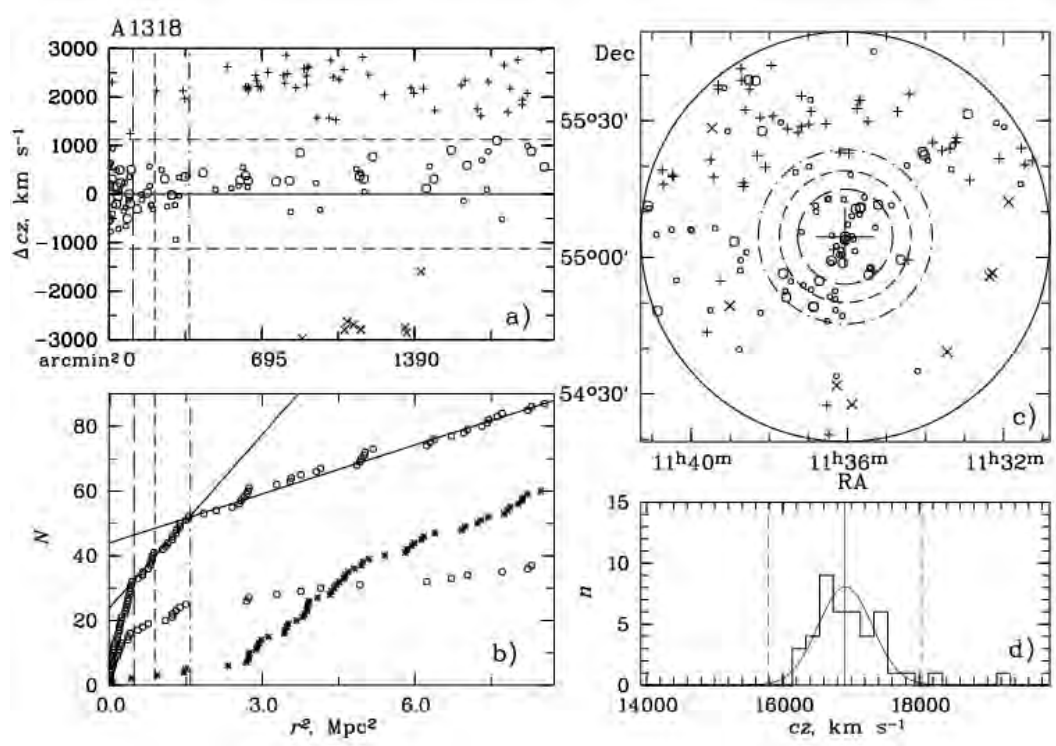

Figure 1: Distribution of galaxies in the cluster A 1318. The panel a) shows the deviation of galaxy radial velocities from the average radial velocity of the cluster, determined from the galaxies within the radius $R_{200}$. The horizontal dashed lines correspond to deviations $\pm 2.7 \sigma$, and the vertical short-dashed line shows the radius $R_{200}$, the longdashed line $-R_{c}$ radius, and the dot-dashed line $-R_{s p}$ radius. The larger circles denote galaxies brighter than $M_{K}^{*}=-24 \cdot^{m} 0$, the straight crosses mark the background galaxies, the diagonal crosses show the foreground galaxies. The panel b) presents the integrated distribution of the total number of galaxies (upper curve) as a function of squared distance from the cluster center. The lower points correspond to early-type galaxies brighter than $M_{K}<-21 .^{m} 0$ The circles correspond to the galaxies marked by the circles in panel (a), and the stars show the background galaxies. The solid line shows the linear sections of the profile. The panel c) shows the sky distribution in equatorial coordinates of galaxies presented in panel (a) (the designations are the same). The circles mark regions with the radii $R_{c}, R_{200}$ and $R_{s p}$. The region studied is bounded by a circle with the radius equal to $3.5 R_{200}$ (solid line). The center of the cluster is marked by a large cross. The panel $\mathbf{d}$ ) presents the distribution of radial velocities of all galaxies within the radius $R_{200}$ (the solid line shows the Gaussian for the cluster members). The solid vertical line shows the average radial velocity of the cluster, and the dashed lines correspond to $\pm 2.7 \sigma$ deviations. 
galaxies. These systems are located in the regions of big superclusters of galaxies Leo, Hercules, Ursa Major, Corona Borealis, Bootes, A 1656/ A1367 and in the regions other smaller superclusters and in the field. In our study, we used data from the SDSS (mainly), 2MASX and NED catalogs. For groups/clusters of galaxies from the observed profile (the intergrated distribution of the number of galaxies depending on the squared distance from the center) we have determined the radius $R_{s p}$ (splachback radius) [2] and $R_{c}$ (core radius) and the dependences of the radii from other characteristics of systems of galaxies. The dynamical masses of groups/clusters were determined from the dispersions of line-of-sight velocities of galaxies [3] by assuming the systems to be in virial equilibrium. In this case, the group/cluster radius within which the density the critical one by a factor of 200 is close to virial one and it can be estimated from the formula $R_{200}=\sqrt{3} \sigma /(10 H(z)) \mathrm{Mpc}$. The mass within $R_{200}$ is equal to $M_{200}=3 G^{-1} R_{200} \sigma_{200}^{2}$. We consider the galaxies with radial velocities greater than $2.7 \sigma$ to be background objects. In Figure we present a set of diagrams that describes in detail the structure and kinematics of the groups/clusters of galaxies, e.g., A 1318 as an example.

The parameters of the baryonic component of systems of galaxies (stars) can be measured in the process of observations. Infrared (IR) radiation of stars is not affected significantly by either starbursts in a galaxy or dust, because the central regions of clusters of galaxies are mostly occupied by early-type galaxies with old stellar population. IR radiation is therefore a more accurate tracer of mass of the stellar population in clusters of galaxies, and it is often used for this (see, e.g., [4, 5]). In this study for measuring the total $L_{K}$ - luminosity of galaxies in groups/clusters, we applied the method described by us in [6]. Because the range of the radial velocities of our sample is large, to determine the $L_{K}$-luminosity of systems of galaxies to the adopted limit $M_{K}=-21 .^{m} 0$ we used the parameters of the luminosity function obtained for superclusters of galaxies Leo and Hercules $(z<0.045)$ [7]. Eventually, we obtained scaling relations between the observed parameters of the groups/clusters and led them in the Table. The Table shows the slopes, normalizations, and scatters of the relations.

The specific star formation rate $(s S F R)$ in a galaxy is determined by the integral star formation rate divided by its stellar mass: $s S F R=S F R / M_{*}$. In the distribution of galaxies by the specific star formation rate the minimum is usually found, which separates the galaxies actively forming stars from galaxies that have quenched star formation and galaxies without star formation. Our results show that the fraction of galaxies with quenched star formation $\left(\log s S F R\left[\mathrm{Gyr}^{-1}\right]<\right.$ $-1.75)$ is maximal in the central regions of the galaxy groups/clusters and equals, on average, $0.81 \pm 0.02$; it decreases to $0.44 \pm 0.02$ outside of the radius $R_{s p}$, but still remains higher than that in the field by $27 \%\left(M_{K}<-21 .^{m} 5, N=40\right.$ groups/clusters) [8].

Table 1: Best fit parameters of the relations

\begin{tabular}{lccc}
\hline Relation & Slope & Normalization & Scatter \\
\hline $\mathrm{N}=185, M_{K}<-21^{m}$ & & & \\
$\log \left(L_{K, 200} / L_{\odot}\right)-\log \left(M_{200} / M_{\odot}\right)$ & $0.77 \pm 0.01$ & $+1.55 \pm 0.02$ & 0.146 \\
$\log \left(M_{200} / L_{K, 200}\right)-\log \left(M_{200} / M_{\odot}\right)$ & $0.29 \pm 0.01$ & $-2.34 \pm 0.02$ & 0.138 \\
$\log N_{200}-\log \left(M_{200} / M_{\odot}\right)$ & $0.82 \pm 0.01$ & $-9.89 \pm 0.02$ & 0.149 \\
\hline $\mathrm{N}=157$ & & & \\
$\log R_{s p}-\log L_{X}$ & $0.24 \pm 0.03$ & $-7.39 \pm 0.33$ & 0.092 \\
$\log R_{200}-\log L_{X}$ & $0.25 \pm 0.04$ & $-7.60 \pm 0.34$ & 0.097 \\
$\log R_{s p}-\log \left(M_{200} / M_{\odot}\right)$ & $0.32 \pm 0.02$ & $-1.42 \pm 0.13$ & 0.066 \\
$\log R_{s p}-\log R_{200}$ & $1.00 \pm 0.04$ & $+0.17 \pm 0.11$ & 0.064 \\
$\log R_{s p}-\log R_{c}$ & $0.95 \pm 0.05$ & $+0.46 \pm 0.14$ & 0.088 \\
\hline
\end{tabular}

\section{References}

1. J. M. Colberg, S. D. M. White, A. Jenkins, and F. R. Pearce, Mon. Not. R. Astron. Soc., 308, 593598, 1999.

2. B. Diemer and A. V. Kravtsov, Astrophys. Journal, 789, 118, 2014.

3. A. I. Kopylov and F. G. Kopylova, Astrophysical Bulletin, 70, 243256, 2015.

4. Y.-T. Lin, J. J. Mohr, and S. A. Stanford, Astrophys. Journal, 610, 745761, 2004.

5. M. Ramella, W. Boschin, M. J. Geller, A. Mahdavi, and K. Rines, Astronom. Journal, 128, 20222036, 2004.

6. F. G. Kopylova and A. I. Kopylov, Astrophysical Bulletin, 64, 123, 2009.

7. F. G. Kopylova and A. I. Kopylov, Astronomy Letters, 39, 116, 2013.

8. F. G. Kopylova and A. I. Kopylov, Astrophysical Bulletin, 74, 365378, 2019. 


\section{The fundamental plane of clusters and groups of galaxies}

F. Kopylova, A. Kopylov

flera@sao.ru

Special Astrophysical Observatory RAS, Nizhnij Arkhyz, 369167, Russia

DOI: 10.51194 /VAK2021.2022.1.1.149

Gravitationally bound systems such as globular clusters, early-type galaxies, and clusters of galaxies lie on the Fundamental Plane (FP), binding their size (effective radius), luminosity (average surface brightness) and radial velocity dispersion. To determine these parameters for 172 clusters and groups of galaxies we constructed the intergrated distribution of the total number of galaxies as a function of squared clustercentric distance. Such profiles of clusters or groups of galaxies [1, 2] allowed us to identify the average of the apocenters of orbits of galaxies - splashback radius $R_{s p}$. Also we determined the total luminosity or the total number of galaxies of clusters after background subtraction and measured the effective radius within which half of the total luminosity or half of the total number of galaxies are contained. The work is done on archival data SDSS, 2MASX, NED. Obtained by the method of least squares the FP of clusters and groups of galaxies in the $K$-band along the axis $\log L_{K}$ is: $L_{K} \propto R_{e}^{0.73} \sigma^{1.55}$.

In X-ray, the FP is: $L_{K} \propto R_{e}^{0.73} L_{X}^{0.28}$.

The slopes of FPs of early-type galaxies and clusters of galaxies are in good agreement with each other, and the zero-points are different. A comparison with the FP of another clusters of galaxies [3] showed that the Fundamental Plane parameters within errors are consistent. The existance of the FP is an indication that globular clusters, galaxies and galaxy clusters have similar formation processes (in the context of the chierarchical structure formation scenario). Also trend of cluster mass-to-light ratio must be taken into account for a proper interpretation of the observed scaling relations. Other scaling relations obtained by us for clusters and groups are:

$L_{K} \propto R_{e}^{0.74} \sigma^{1.69}\left(M_{X} / L_{K}\right)^{0.28}$

$L_{K} \propto \sigma^{2.13 \pm 0.13}$, the Faber-Jackson relation;

$L_{K} \propto R_{e}^{1.41 \pm 0.12}$, the Kormendy relation.

\section{References}

1. A. I. Kopylov and F. G. Kopylova, Astrophysical Bulletin, 70, 243, 2015.

2. F. G. Kopylova and A. I. Kopylov, Astrophysical Bulletin, 71, 257, 2016.

3. M. D'Onofrio, D. Bettoni, D. Bindoni, A. Cava, et al., Astronomische Nachrichten, 334, 373, 2013. 


\title{
The influence of systematic effects on the determination of the primordial helium abundance
}

\author{
O. Kurichin, P. Kislitsyn, A. Ivanchik \\ o.chinkuir@gmail.com \\ Ioffe Institute, Polytechnicheskaya 26, 194021, Saint-Petersburg, Russia
}

Various tests of the Primordial Nucleosynthesis $(\mathrm{PN})$ theory and Early Universe cosmology require an accurate determination of the primordial helium abundance $\left(Y_{p}\right)$. Metal deficient HII regions located in blue compact dwarf (BCD) galaxies are commonly used to determine $Y_{p}$. Since the measured fluxes of He and H lines emitted from such objects often deviate from their pure recombination values due to the influence of several systematic effects, one has to correctly account for these effects to determine $Y_{p}$ with high accuracy. In this paper, we present an updated estimate of $Y_{p}$ based on analyses of a large sample of SDSS objects and objects from HeBCD+NIR database. Using improved treatment of the HII region systematics we obtain $Y_{p}=0.2466 \pm 0.0019$, which is the most accurate estimate up to date, and is in good consistency with other independent estimates as well as the PN+Planck prediction of $Y_{p}=0.2470 \pm 0.0002$.

Keywords: Primordial Nucleosynthesis, Primordial Helium, HII regions

DOI: 10.51194 /VAK2021.2022.1.1.150

\section{Introduction}

Precise observational estimate of the primordial helium abundance $\left(Y_{p}\right)$ is a strong probe of self-consistency of the $\Lambda$ CDM cosmological model as well as it can be used to tight constraints on any non-standard Physics during PN era. The most used method to estimate $Y_{p}$ is the spectroscopic study of metal deficient HII regions located in blue compact dwarf galaxies (BCD), which interstellar medium has its elemental composition close to the primordial one. We can expect a correlation between the observed ${ }^{4} \mathrm{He}$ abundance $Y$ and metallicity $Z$, since both of this quantities are produced over time due to stellar nucleosynthesis. Thus the primordial ${ }^{4} \mathrm{He}$ abundance $Y_{p}$ can be estimated by extrapolating of the $Y-Z$ dependence to zero metallicity. This technique was firstly proposed in [1] and is still in use (e.g., see [2] and refs. therein).

The abundances in HII regions can be estimated using the ratio of intensities of recombination lines. However, there are numerous systematic effects which shift measured intensities of the lines from their pure recombination values. They include interstellar reddening, underlying stellar absorption, collisional excitation, self-absorption etc. In order to account for the systematics the photoionization models of HII regions are used. In our previous paper [2]) we used the photoionization model described in [3] to estimate current helium abundances $Y$. Recently, [4] proposed an update to their model, which revisits how the underlying absorption, collision excitation of HI and self-absorption in He lines are treated. In addition, we propose a modified method for the estimation of $Z$ of an object, as well as a new selection criterion associated with it.

In this paper we update our photoionization model according to the proposed changes and apply it to our spectroscopic database, described in [2], and discuss how the changes affect the estimate of $Y_{p}$.

\section{Photoionization model improvements}

\subsection{Model improvements}

In recent paper [4] presented updated scaling coefficients for underlying absorption in $\mathrm{H}$ and He lines, recalculated contribution of collisionally excited transitions in HI to Balmer series intensities, and proposed a new method of treating of the blended $\mathrm{H} 8+\mathrm{He} \lambda 3889$ line. Besides, authors proposed to include additional weak $\mathrm{H}$ and He lines to the analyses. In this paper we focus on first three suggested changes due to the following reason. Our spectroscopic database (while being larger compared to other ones from the literature) mostly consists of SDSS objects with medium to low quality of spectra, where such weak lines are almost never confidently detected.

\subsection{New OH estimation}

The oxygen abundance $\mathrm{O} / \mathrm{H}$, which is commonly used as the metallicity tracer of an HII region, is defined as sum of abundances of the two most relevant ionization states: $\mathrm{O} / \mathrm{H}=\mathrm{OII} / \mathrm{H}+\mathrm{OIII} / \mathrm{H}$. These abundances can be estimated using the observed fluxes of forbidden lines of OII and OIII and the temperatures of the corresponding ionization zones. According to the two-zone temperature model of an HII region, OIII is distributed in the high-ionization zone characterized by temperature $T_{e}(\mathrm{OIII})$. OII is distributed in the low-ionization zone characterized by temperature $T_{e}(\mathrm{OII})$. In other surveys, authors often estimate $T_{e}(\mathrm{OII})$ using model relations $T_{e}(\mathrm{OII})=f\left(T_{e}(\mathrm{OIII})\right)$, and $T_{e}(\mathrm{OIII})$ is estimated directly from [OIII] lines ration $(\lambda 4959+\lambda 5007) / \lambda 4363$. We propose to estimate both of these temperatures directly using [OII] lines ratio $(\lambda 3727) /(\lambda 7320+\lambda 7330)$ and $[\mathrm{OIII}]$ lines ration mentioned above. We find out that usage of the relations $T_{e}(\mathrm{OII})=$ $f\left(T_{e}(\mathrm{OIII})\right)$ can significantly shift the estimated $\mathrm{OII} / \mathrm{H}$ as well as lead to underestimation of $\mathrm{O} / \mathrm{H}$ uncertainty.

\subsection{Final database}

The final regression database is formed as follows. Firstly, from 588 objects, which were manually picked up from the SDSS catalog [2], and 83 high-quality spectra from HeBCD+NIR database [5, 6], we select objects whose spectrum allows a direct 
estimation of $T_{e}(\mathrm{OII})$ and $T_{e}(\mathrm{OIII})$. Secondly, from these objects we select ones where all lines required for the analyses with photoionization model are detectable on a CL of $\geq 3 \sigma$. This cuts leave 133 objects to analyse.

In order to check how each individual change affects the final $Y_{p}$ estimation we separately insert each one into the "original" photoionization model and get a separate sample for each change. We determine physical properties, current oxygen abundance $\mathrm{O} / \mathrm{H}$ and current helium to hydrogen density ratio $y$ using photoionization model described above. For the regression analyses we select objects using "goodness of fit" statistical $\chi^{2}$ criteria (95\% CL for a model with one degree of freedom corresponds to $\chi 2 \leq 4$ ). Thus we get four independent samples to analyse: sample $\mathrm{S}_{\mathrm{UA}}$ (obtained with new UA scaling), sample $\mathrm{S}_{\mathrm{CR}}$ (obtained with new CR coefficients), sample $\mathrm{S}_{3889}$ (obtained with new deblending method), and sample $S_{\text {tot }}$ (obtained with all changes incorporated). They consist of 68 objects for the sample $\mathrm{S}_{\mathrm{UA}}, 67$ objects for the sample $\mathrm{S}_{\mathrm{CR}}, 52$ objects for the sample $\mathrm{S}_{3889}$, and 65 for the sample $\mathrm{S}_{\text {tot }}$.

\section{Results and conclusion}

We perform a regression analyses of $y$ versus $\mathrm{O} / \mathrm{H}$ using the following expression:

$$
y=y_{\mathrm{p}}+\frac{d y}{d(\mathrm{O} / \mathrm{H})} \times \mathrm{O} / \mathrm{H}
$$

Here $y_{\mathrm{p}}$ denotes the primordial helium to hydrogen density ratio and is connected to the primordial helium abundance $Y_{p}$ via the relation:

$$
Y_{\mathrm{p}}=\frac{4 y_{\mathrm{p}}}{1+4 y_{\mathrm{p}}}
$$

We estimate $y_{\mathrm{p}}$ and slope $d y / d(\mathrm{O} / \mathrm{H})$ using $\mathrm{MCMC}$ routine for all samples $\mathrm{S}_{\mathrm{UA}}, \mathrm{S}_{\mathrm{CR}}, \mathrm{S}_{3889}$ and $\mathrm{S}_{\text {tot }}$. The results are summarized in Table 1. As can be seen the results for all studied samples are consistent with each other within margin of errors, while there is a noticeable scatter between their nominal values (with the largest shift corresponding to the new H8 + He $\lambda 3889$ deblending method). This shift may be due to the fact that the method does not work quite correctly on the SDSS spectra of medium and low quality, since, as shown in [4], for spectra of high quality the method work correctly. Thus the deblending method should be refined to be used for processing of the SDSS spectra.

Table 1: Estimates of $Y_{p}$ and $d y / d(\mathrm{O} / \mathrm{H})$ for the samples described in Sec. 2.3.

\begin{tabular}{ccc}
\hline Sample & $Y_{p}$ & $d y / d(\mathrm{O} / \mathrm{H})$ \\
\hline $\mathrm{S}_{\mathrm{UA}}$ & $0.2464 \pm 0.0020$ & $27 \pm 7$ \\
$\mathrm{~S}_{\mathrm{CR}}$ & $0.2457 \pm 0.0020$ & $29 \pm 7$ \\
$\mathrm{~S}_{3889}$ & $0.2506 \pm 0.0023$ & $18 \pm 7$ \\
$\mathrm{~S}_{\text {tot }}$ & $0.2504 \pm 0.0021$ & $18 \pm 7$ \\
\hline
\end{tabular}

According to this, we insert only the updates in UA scaling and updated HI collisional excitation rates into the photoionization model, and, after preforming the regression analyses as described above, we get the following estimate of $Y_{p}$ :

$$
Y_{p}=0.2466 \pm 0.0019
$$

It is the most precise $Y_{p}$ estimation up to date, and is in a good consistency with Planck's results $Y_{p}=0.2470 \pm 0.0002$ [7] and other independent estimation (see [2] and refs. therein).

\section{Acknowledgement}

The work was supported by the Russian Science Foundation (grant 18-12-00301).

\section{References}

1. M. Peimbert and S. Torres-Peimbert, ApJ, 193, 327, 1974.

2. O. A. Kurichin, P. A. Kislitsyn, V. V. Klimenko, S. A. Balashev, and A. V. Ivanchik, MNRAS, 502, $3045,2021$.

3. E. Aver, K. A. Olive, and E. D. Skillman, J. of Cosmology and Astroparticle Phys., 2015, 011, 2015.

4. E. Aver, D. A. Berg, K. A. Olive, R. W. Pogge, J. J. Salzer, and E. D. Skillman, J. of Cosmology and Astroparticle Phys., 2021, 027, 2021.

5. Y. I. Izotov, T. X. Thuan, and G. Stasińska, ApJ, 662, 15, 2007.

6. Y. I. Izotov, T. X. Thuan, and N. G. Guseva, $M N R A S, \mathbf{4 4 5}, 778,2014$.

7. Planck Collaboration, N. Aghanim, Y. Akrami, M. Ashdown, et al., A\&GA, 641, A6, 2020. 


\section{Catalog of edge-on galaxies using the Pan-STARRS1 survey data}

D. Makarov ${ }^{1}$, S. Savchenko 2,1 , A. Mosenkov ${ }^{3,4}$, D. Bizyaev ${ }^{5,6,1}$, V. Reshetnikov ${ }^{2,1}$, A. Antipova ${ }^{1}$, I. Tikhonenko $^{2}$, P. Usachev ${ }^{1,2}$, S. Borisov ${ }^{7,6,1}$, L. Makarova ${ }^{1}$, S. Kautsch ${ }^{8}$, A. Marchuk ${ }^{2,4}$, E. Rubtsov ${ }^{6}$, M. Edukov $^{2}$, M. Skryabina ${ }^{2}$, P. Smirnova ${ }^{2}$

${ }^{1}$ Special Astrophysical Observatory, Russian Academy of Sciences, Nizhnii Arkhyz, 369167 Russia

${ }^{2}$ Saint Petersburg State University, Universitetskii pr. 28, St. Petersburg, 198504 Russia

${ }^{3}$ Department of Physics and Astronomy, N283 ESC, Brigham Young University, Provo, UT 84602, USA

${ }^{4}$ Pulkovo Observatory, Russian Academy of Sciences, St. Petersburg, 196140 Russia

${ }^{5}$ Apache Point Observatory, New Mexico State University, Sunspot, New Mexico, USA

${ }^{6}$ Sternberg Astronomical Institute, M.V. Lomonosov Moscow State University, Universitetsky prospect 13, Moscow, 119234, Russia

${ }^{7}$ Department of Astronomy, University of Geneva, Chemin Pegasi 51, 1290 Versoix, Switzerland

${ }^{8}$ Nova Southeastern University, Fort Lauderdale, FL 33314, USA

We have created a catalog of edge-on galaxies based on the publicly available DR2 data from the Pan-STARRS survey. The intensive use of an artificial neural network has significantly improved the quality of candidate selection. The catalog provides homogeneous information on astrometry, photometry, and non-parametric morphological statistics for 16551 edgeon galaxies. Our catalog is intended for studying the three-dimensional structure of galaxies with different morphologies and the scaling relations for disks and bulges.

Keywords: astronomical data bases, catalogues, galaxy statistics, galaxy photometry

DOI: 10.51194 /VAK2021.2022.1.1.151

\section{Introduction}

The knowledge and information of the three-dimensional distribution of matter is extremely important to understand the dynamics and evolution of galaxies. Unfortunately, in extragalactic astronomy, projection effects obscure the "depth" of objects. As a result, we know the radial distribution of light in galaxies very well, but the vertical distribution of matter in the disks has been explored for only a small number of edge-on galaxies.

Only few catalogs are dedicated to edge-on galaxies. The updated version of the classic Flat Galaxies Catalogue [1], the Revised Flat Galaxy Catalogue (RFGC, [2]) contains 4236 thin spiral galaxies. These flat galaxies were selected during a systematic visual inspection of all blue end red prints of the Palomar Observatory Sky Survey (POSS-I) and the ESO/SERC sky survey. Using the modern Sloan Digital Sky Survey (SDSS), [3, 4] identified a number of edge-on galaxies, which were automatically classified into various Hubble types. Finally, the catalog of genuine edge-on disk galaxies (EGIS, [5]) containing 5747 objects was generated by automatic candidate selection from SDSS DR7 and their subsequent visual inspection.

Here we introduce a new catalog of 16551 Edge-on Galaxies found In the Pan-STARRS survey (EGIPS).

\section{Candidate selection}

We searched for edge-on galaxies using the public archive of the Panoramic Survey Telescope and Rapid Response System (Pan-STARRS, [6]) survey. The survey covers three quarters of the sky down to $\delta=-30^{\circ}$ in five $(g, r, i, z, y)$ broadband filters using the 1.8-meter telescope of the Haleakala Observatory (Hawaii, USA).

For accurate selection of candidates, we took advantage of the Artificial Neural Network (ANN) technology using the TENSORFLOW ${ }^{\mathrm{a}}$ package. The convolutional neural network (CNN) is commonly used to classify images. Our CNN was trained on a sample of 5747 edge-on galaxies from the EGIS catalog [5] in the three $g, r, i$ bands. The final formal classification accuracy is better than $99 \%$. The comparison with an independent sample of RFGC [2] galaxies showed that only 20 out of 3027 galaxies were misclassified. This translates into an error rate better than $1 \%$.

A typical candidate search consists of detecting objects in a $r$-band image using the detect_source function of the Pнотutils library [7]. Then the object image is cropped out of the full frame and scaled to $48 \times 48$ pixels. The stacked array of the $g, r$ and $i$ images is fed to our neural network. We consider an object as a good candidate for an edge-on galaxy if at least three of our five CNN models give a better than 0.5 probability for the edge-on class. Finally, after processing all $\sim$ 200,000 Pan-STARRS skycell fields, we selected 26719 candidates.

All candidates were visually inspected by a dozen professional astronomers to eliminate asterisms, image defects, misclassifications, as well as non-edge-on galaxies. To reduce subjectivity, each object was independently examined by at least three different people.

Finally, the catalogue contains 16551 objects. To date, it is the largest sample of edge-on galaxies. The public access to the EGIPS catalogue is supported by the Edge-on Galaxy Database ${ }^{\mathrm{b}}$ [8].

\section{Photometry and non-parametric morphology}

As part of the work on the catalog, we performed SExTRACTOR [9] photometry and non-parametric morphology estimation using the STATMORPH [10] package. Comparison of our Petrosian magnitudes obtained using Pan-STARRS1 survey data with independent aperture photometry based on SDSS images [5] shows excellent agreement, illustrated in Fig. 1. The scatter is

\footnotetext{
ahttps://www.tensorflow.org/

b https://www.sao.ru/edgeon/
} 


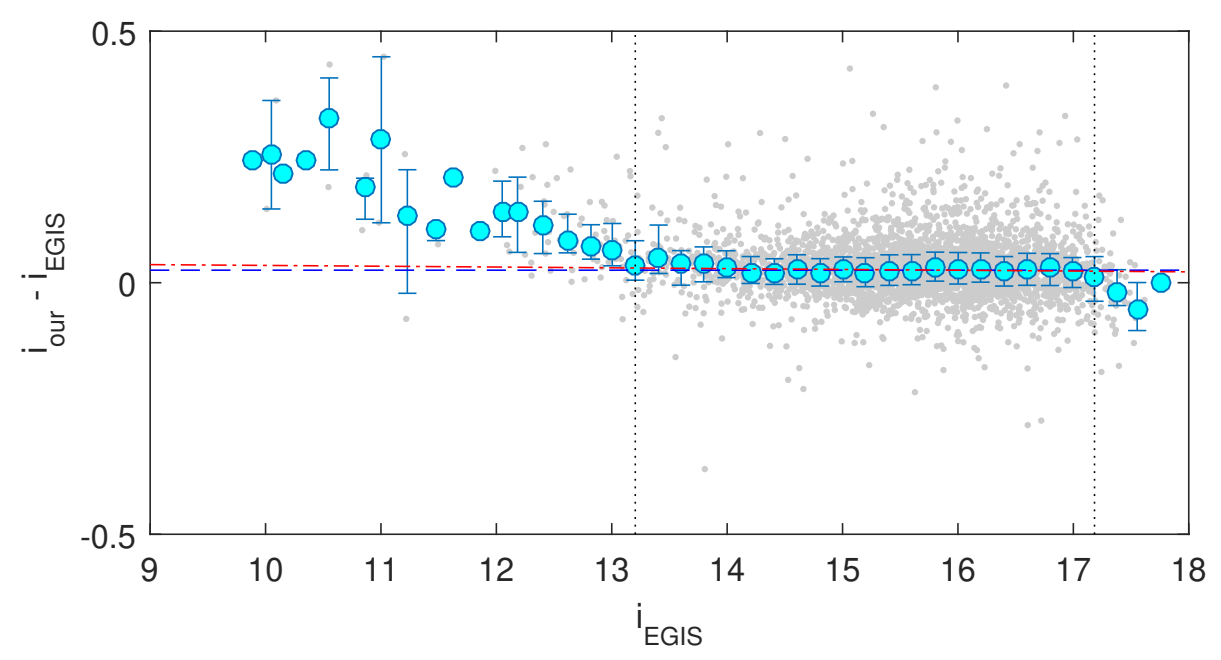

Figure 1: The difference between our Petrosian and EGIS aperture magnitudes in the $i$ band is shown by gray dots. The running median with a 0.2 mag window and corresponding 25 and $75 \%$ quartiles are indicated by cyan dots with error bars. The black dotted vertical lines represent the accordance range. The red dash-dotted line is a robust linear fitting inside the accordance range. The blue dashed line corresponds to the median difference between the photometries.

less than $0.05 \mathrm{mag}$ in the $r$ and $i$ bands and about $0.07 \mathrm{mag}$ in the $g$ band, despite the difference between the Pan-STARRS and SDSS photometric systems, and the difference in the photometry methodologies used. For the big galaxies brighter than $i \approx 13.2 \mathrm{mag}$ the total flux is systematically underestimated due to the specifics of the sky subtraction procedure in Pan-STARRS.

\section{Acknowledgements}

This research was supported by the Russian Science Foundation grant no. 19-12-00145. The work on the Edge-on Galaxy Database was supported by the Russian Foundation for Basic Research grant no. 19-32-90244.

\section{References}

1. I. D. Karachentsev, V. E. Karachentseva, and S. L. Parnovskij, Astronomische Nachrichten, 314, $97,1993$.

2. I. D. Karachentsev, V. E. Karachentseva, Y. N. Kudrya, M. E. Sharina, and S. L. Parnovskij, Bulletin of the Special Astrophysics Observatory, 47, 5, 1999.

3. S. J. Kautsch, E. K. Grebel, F. D. Barazza, and I. Gallagher, J. S., A\&A, 445, 765, 2006.

4. S. J. Kautsch, Astronomische Nachrichten, 330, 100, 2009.

5. D. V. Bizyaev, S. J. Kautsch, A. V. Mosenkov, V. P. Reshetnikov, N. Y. Sotnikova, N. V. Yablokova, and R. W. Hillyer, ApJ, 787, 24, 2014.

6. K. C. Chambers, E. A. Magnier, N. Metcalfe, H. A. Flewelling, et al., arXiv e-prints, arXiv:1612.05560, 2016.

7. L. Bradley, B. Sipöcz, T. Robitaille, E. Tollerud, et al., astropy/photutils: 1.0.0, 2020.

8. D. I. Makarov and A. V. Antipova, Astrophysical Bulletin, 76, 218, 2021.

9. E. Bertin and S. Arnouts, A\&SA Sup., 117, 393, 1996.

10. V. Rodriguez-Gomez, G. F. Snyder, J. M. Lotz, D. Nelson, et al., MNRAS, 483, 4140, 2019. 


\title{
A catalog of Supermassive Black Holes for observations of black holes shadows with the Millimetron space observatory
}

\author{
A. Malinovsky, E. Mikheeva, V. Lukash, S. Repin \\ amalin@asc.rssi.ru \\ Astro Space Center, P.N. Lebedev Physical Institute, Russian Academy of Sciences, Moscow, 117997 Russia
}

The paper presents a catalog of supermassive black holes (SMBHs) sharpened for interferometric observations at millimeter and submillimeter wavelengths, based on open sources. The catalog includes the object's name, coordinates, angular size, and mass, the angular size of the gravitational radius of the SMBH, and the integrated radio flux associated with the radiosource concluding the $\mathrm{SMBH}$ at $20-900 \mathrm{GHz}$, planned for the Event Horizon Telescope, the future space mission Millimetron, and others. The catalog is intended for use during planning of interferometric observations of SMBH shadows.

Keywords: interferometry, millimeter and submillimeter wavelengths, black holes, supermassive black hole

DOI: $10.51194 /$ VAK2021.2022.1.1.152

\section{Introduction}

A task that is currently of utmost interest for modern astrophysics is to develop and implement observational programs to explore accreting supermassive black holes (SMBHs) in the millimeter wave band using the method of very long baseline interferometry (VLBI). A specific feature of this wave band is that plasma near the event horizon can be optically thin in many sources, allowing one to hope that the image of the black hole (or its "shadow") can be directly discovered using VLBI methods.

The most ambitious observational projects in this area are the Event Horizon Telescope (EHT) and the Millimetron, the Russian space mission that is currently under development [1].

The Millimetron project, when operating in the VLBI observation mode, is a ground-space system. In comparison with the EHT, which is an interferometric array of ground-based observatories, the Millimetron is similar to it in the frequency range it uses and its sensitivity. However, its resolving power will be higher by a factor of approximately 50. Owing to this, it will be possible to both resolve a significantly larger number of space sources radiating in the millimeter waveband and observe sources resolvable by the EHT but in much greater detail. associated with gas accretion and jet formation.

Several catalogs of SMBHs are currently available, the main ones being $[2,3,4,5]$. However, the rate at which new data appear is high, and the continuous updating of SMBH catalogs is highly desirable. The content and format of the catalogs have been determined by the tasks and requirements of researchers studying SMBHs. The original purpose of our catalog was to select SMBHs whose shadows could be resolved by the Millimetron space observatory. This imposes restrictions not only on the angular size of a SMBH, but also on its celestial coordinates and flux densities in the Millimetron frequency channels. A table with the 20 best candidates for such observations is presented in [6].

It is planned that the Millimetron observatory will operate in wide frequency bands centered at 22, 43, 100, 240, and $350 \mathrm{GHz}$, and possibly also at 600 and $800 \mathrm{GHz}$. Work with published data demonstrates that, for a large number of SMBHs, the total fluxes in these bands are unknown. For some sources, the nearest measured points are several orders of magnitude in frequency away. For this reason, and also due to a possible change in the Millimetron orbit, we felt the need to compile as complete a catalog of SMBHs as possible.

\section{Structure of the SMBH catalog}

The catalog is organized as follows.

The first column shows the source name. Objects sometimes have one or more synonyms, which are also presented. This can help find an object in astronomical databases. As can be seen from the names of the SMBHs, some are in nuclei of nearby galaxies and some are active galactic nuclei (quasars and Seyfert galaxies).

The second column contains the mass of the SMBH in units of $10^{8} M_{\odot}$. We call this quantity the "mass parameter". The most massive single SMBH has a mass parameter of more than 100, while the lower mass boundary of the SMBHs is, by definition, $10^{-4}$. Several mass measurements are available for a number of the SMBHs, which can differ by a factor of five.

The third column of the catalog shows the distance to the object in megaparsecs. Several types of distance are used in cosmology, including the metric distance, luminosity distance, angular-size distance, and redshift distance. Our catalog is oriented at observations of black hole shadows, so we therefore prefer the angular-size distance, since this is the distance used to evaluate the angular scale of the shadow.

The fourth column indicates the angle at which the gravitational radius of the black hole should be observed in $\mu$ as. We emphasize that this is the gravitational radius, and the angular size of the shadow is about a factor of 10 larger.

The fifth and sixth columns present the coordinates of the SMBHs in equatorial coordinates for epoch J2000. For most sources, we limited the coordinate accuracy to one arcsecond. This is more than enough to determine the projection of the baseline when planning interferometric observations of the shadows.

The seventh column shows the central frequencies of the channels in which the SMBHs were observed in GHz. Since our catalog is focused on interferometric observations of shadows at millimeter and submillimeter wavelengths, where no significant self-absorption of the radiation is expected, we tried to restrict our consideration to frequencies of 22-800 GHz. 
However, for some sources, there were few observation points in this range, in which case we included data for other nearby frequencies in the catalog.

The eighth column contains the radiation fluxes at the corresponding frequencies in Jy. If a number of observations (at different times and with different receiving equipment) were carried out for a frequency channel of interest, we indicate the range of the observed quantities. Note that these fluxes do not characterize the SMBH itself, but instead a much larger area. The reason for this is obvious: until now, only a few objects have been observed in the required frequency range with very high angular resolution, comparable to the size of the gravitational radius of the SMBH. Therefore, the actual flux will, of course, be lower than the values indicated in the catalog.

The last column of the table contains references to source catalogs and/or published articles.

\section{Conclusion}

The catalog of SMBH presented here is includes 353 objects and intended for use in planning interferometric observations of SMBH shadows in the submillimeter and millimeter ranges. It is available at the website of the Astro Space Center of the P. N. Lebedev Physical Institute ${ }^{\mathrm{a}}$.

\section{References}

1. N. S. Kardashev, I. D. Novikov, V. N. Lukash, S. V. Pilipenko, et al., Phys. Usp., 57, 1199, 2014.

2. K. Gültekin, D. O. Richstone, K. Gebhardt, T. R. Lauer, et al., Astrophys. J., 698, 198, 2009.

3. N. J. McConnel, PhD Thesis, Univ. California, Berkeley (2012).

4. R. P. Saglia, M. Opitsch, P. Erwin, J. Thomas, et al., Astrophys. J., 818, 47, 2016.

5. R. C. E. van den Bosch, Astrophys. J., 831, 134, 2016.

6. P. B. Ivanov, E. V. Mikheeva, V. N. Lukash, A. M. Malinovsky, et al., Phys. Usp., 62, 423, 2019.

${ }^{\mathrm{a}} \mathrm{http}: / /$ millimetron.ru/index.php/en/scientific-program/the-catalog-of-supermassive-black-holes 


\title{
Statistical analysis of observational data for spiral galaxies with known halo parameters
}

\author{
K.A. Mannapova ${ }^{1}$, K.T. Mirtadjieva ${ }^{2,1}$ \\ mannapova.kamola@mail.ru \\ ${ }^{1}$ National University of Uzbekistan, Tashkent, Uzbekistan, \\ ${ }^{2}$ Ulugh Beg Astronomical Institute of the Uzbekistan Academy of Sciences, Uzbekistan
}

DOI: 10.51194 /VAK2021.2022.1.1.153

As known, spiral galaxies differ in both external and internal physical parameters. There are many catalogs of spiral galaxies, in which various parameters are collected, such as radial velocities, absolute magnitudes, redshifts, stellar masses, etc. It should be noted that until now a catalog has not been compiled that would contain parameters related to their halos. In this work, we have studied many contributions of various authors (here there are some of them $[1,2,3,4,5,6,7]$ and with the help of these data we have compiled for the first time a consolidated catalog of spiral galaxies, that contains physical parameters of halos for 207 objects. The catalog contains parameters such as the names of galaxies, morphological type of galaxies, their right ascension and declination, the value of the type code according to LEDA, radial velocity, redshift, semimajor and minor axis, distance to galaxies, absolute magnitude, halo mass, gas density in the halo, the surface brightness of the halo, the number of globular clusters in the halo, the mass of the globular clusters, the metallicity of the halo, the temperature of the gas in the halo, etc. The statistical analysis of the catalog was carried out, and the corresponding histograms were built.

\section{References}

1. S. Díaz-García, H. Salo, E. Laurikainen, and M. Herrera-Endoqui, A\&A, 587, A160, 2016.

2. S. Liu, C. Du, H. J. Newberg, Y. Chen, et al., ApJ, 862, 163, 2018.

3. C. J. Conselice, J. W. Twite, D. P. Palamara, and W. Hartley, ApJ, 863, 42, 2018.

4. M. Mouhcine, R. M. Rich, H. C. Ferguson, T. M. Brown, and T. E. Smith, ApJ, 633, 828, 2005.

5. S. Huang, A. Leauthaud, J. Greene, K. Bundy, et al., MNRAS, 480, 521, 2018.

6. G. Iorio and V. Belokurov, MNRAS, 482, 3868, 2019.

7. J.-T. Li, J. N. Bregman, Q. D. Wang, R. A. Crain, M. E. Anderson, and S. Zhang, ApJS, 233, $20,2017$. 


\title{
An analysis of methods for determining corotation radii in galaxies
}

\author{
A.A. Marchuk, A.V. Mosenkov, S.S. Savchenko \\ a.marchuk@spbu.ru \\ Central (Pulkovo) Astronomical Observatory, Russian Academy of Sciences, Pulkovskoye chaussee 65/1, St. Petersburg \\ 196140, Russia
}

In this paper, for the first time, the pairwise agreement of various methods for determining the corotation radii in the discs of galaxies is statistically analysed. General conclusions are drawn about the poor agreement of the methods used in the literature. We create a sample of galaxies with corotation radii, for which different methods provide consistent results. Based on this sample, in the case of more than one corotation radius we verify the predictions for the coupling of different parts in the spiral structure and make conclusions about its lifetime.

\section{Keywords: spiral galaxies, dynamics of galaxies}

DOI: $10.51194 /$ VAK2021.2022.1.1.154

Spirals are important for our understanding of the internal structure and evolution of galaxies, but the mechanisms and details of their formation remain debatable. Specifically, in numerical models transient spirals are obtained more often, where the angular velocity of rotation coincides with the rotation of the disc, i.e. $\Omega_{p}=\Omega_{d}$ (see [1]). At the same time, there is strong observational evidence in favour of the density wave theory $\Omega_{p}=$ const e.g. radial metallicity gradients or offsets between the spirals in different colours.

The most common way to find the speed of a spiral pattern is to determine the so-called corotation radius (CR), where the spirals rotate with the same angular speed as the disc. Being a 1:1 resonance, this corotation manifests itself in a variety of ways inside the dynamic structure of the galaxy. Although a variety of independent methods for estimating the CR have been proposed, the results of their application have not been systematically compared. The picture is further complicated by the fact that there can be several such radii in a galaxy.

In this study we attempt to collect the results of applying widely used methods for determining the CR in spiral galaxies. Our sample includes 655 spiral galaxies with estimated CR by the direct [2] method (T-W), from radial motions in the ISM velocity field (F-B, [3]), morphologically (morph, [4]), from numerical models (model, [5]), from the shift between galaxy potential and density (potential-density, [6]), from the radial gradient of metallicity (metallicity, [7]), from the offset between photometric bands (offset, [8]), from the widths of the spirals (spirals, [9]), and others (e.g. P-D, [10]).

Our final sample includes 1665 corotation radii. In total, CR are determined by at least two different methods for 185 galaxies. On average, about 2.5 measurements of the CR per galaxy are obtained. The average relative measurement error for CR in the sample equals $13-17 \%$, and the median is $6-9 \%$. The number of galaxies in the pairwise intersection is shown in the lower half of the matrix in Fig. 1. The methods are arranged in the descending order of the number of objects and their labels correspond to those in the text. It can be seen that the comparison is made for about 10 objects, on average.

It is rather difficult to come up with a correct way to compare the CR. For example, if we try to find the closest one CR to data from another method within the errors and count the number of matches, then methods with large error bars (like T-W) or those which predict a larger number of radii (like F-B method) will better match the others. We compare 3 different pairwise metrics, namely Jaccard similarity (JS, the number of overlapping CR within the errors divided by the total number), the proportion of galaxies which are completely consistent in an intersection, and MAE (the mean absolute error, for each CR using one method we find the nearest by its distance to the other, and vice versa).

In Fig. 1, the upper half shows the values for the second metric, i.e. the fraction of fully matching galaxies. It can be seen that, with the exception of a few outliers in the case of a small intersection size, the agreement is very poor. The large number of zeros is explained by the fact that in those cases two methods predict a different number of CR for the galaxy. For JS metric can reaches up to 0.7 , but this is primarily due to the fact that, as mentioned earlier, such pairs have very large error bars and therefore cannot be considered consistent. The average MAE value exceeds $0.2 r_{25}$, which also indicates a significant misplacement. In addition, we notice that the CR from the numerical models are systematically smaller than CR from the T-W method, and the P-D method always gives higher values than follows from the morphological method.

Despite the poor agreement of the methods, one can try to find individual galaxies with consistent information about the CR. This task is done visually according to the criteria that several predicted positions of the CR (better for 3-4 independent methods, rarely for 2) intersect in one area within the error bars, and this area does not exceed $0.15 r_{25}$. In total, 37 galaxies with the CR of varying reliability were selected using this criteria. Out of the selected galaxies, 16 have more than one single pattern, which are of particular interest. We have found the information about rotation curves for half of these 16 galaxies using WHISP, THINGS, and SPARC catalogues. Such information makes it possible to determine the internal and external Lindblad resonances (ILR and OLR, accordingly) and check the validity of predictions about the coupling of different pattern parts. In 5 galaxies out of 8 (namely, NGC5676, NGC3893, NGC3344, M74, and NGC5371) with high precision we find an expected coupling in the form $\operatorname{OLR}($ in $)=\operatorname{CR}($ out $)+\operatorname{CR}($ in $)=4: 1($ out $)$. This is not surprising since some of those galaxies include CR data from [3], who first revealed this dependence. However, an additional confirmation of this dependence and the reliability of the determined CR can be found. For example, for NGC5371 we find a map of the radial velocities for atomic hydrogen in [11], which, after applying the F-B method, confirms the position of the two distinguished patterns. For another galaxy, NGC5676, the F-B method does not help identify the last 1:1 resonance, which we find in other studies and which also perfectly fits into the mentioned coupling scheme.

Therefore, we see the confirmation of the reliability for determining the CR based on kinematic data. Interestingly, for such galaxies it is easy to estimate the lifetime of the spiral pattern due to the difference in the angular velocity of its individual parts. We do this using the formula $\tau=2 \pi /\left(\Omega_{p}^{\max }-\Omega_{p}^{\min }\right)$ which estimates the winding time of the spirals. This 


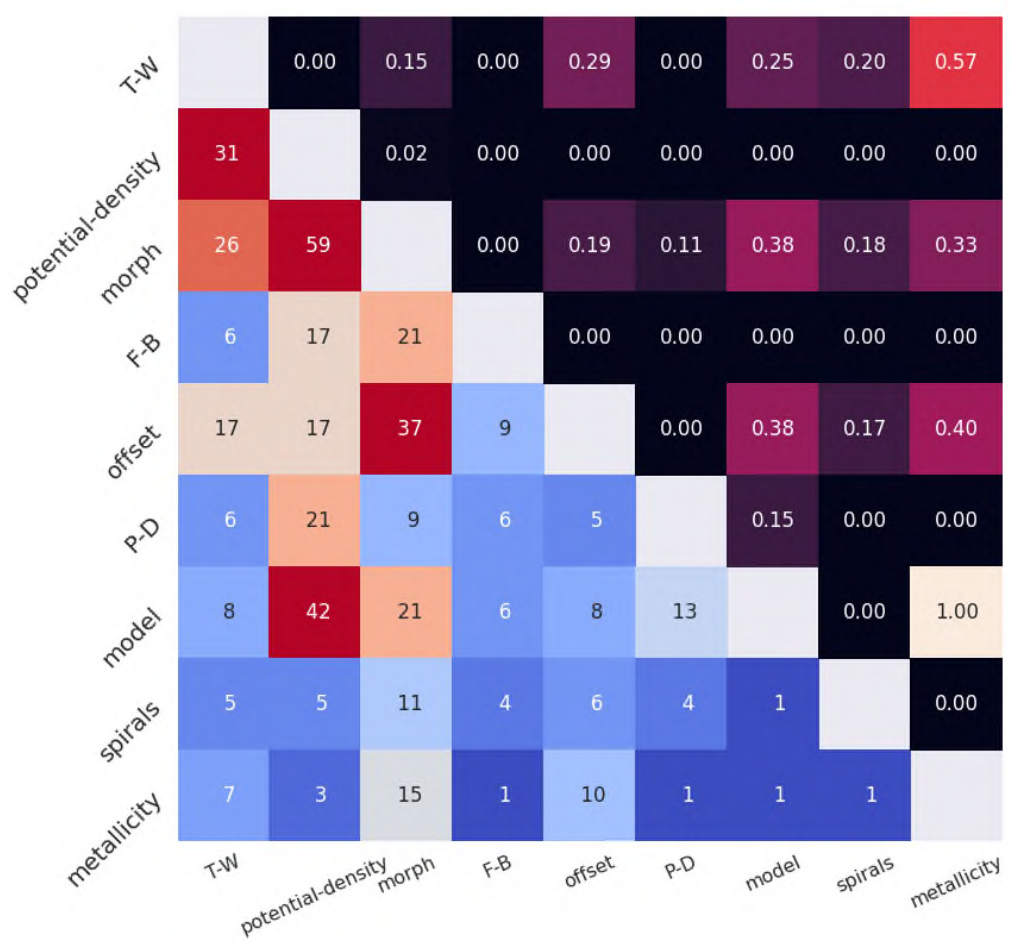

Figure 1: Size of the intersection and fraction of fully matching galaxies between methods.

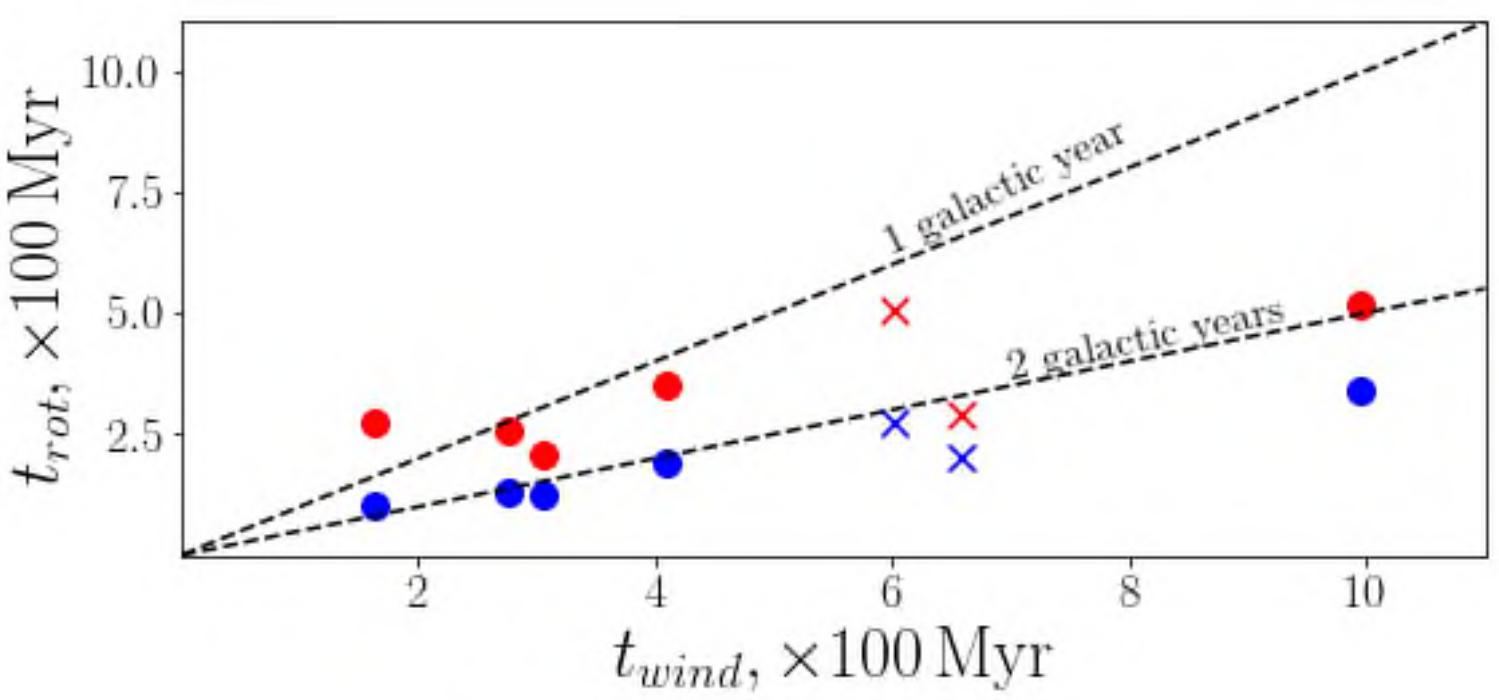

Figure 2: Spiral winding time versus rotation time.

time for 7 galaxies with the reliable CR is shown in Fig. 2. The vertical axis represents the time of one galactic year for each corresponding CR. It can be seen that in all cases the galactic spirals are short-lived structures with a lifetime of 1-2 galactic rotations. This result is consistent with the analysis performed in [12], [13] and others.

\section{References}

1. J. A. Sellwood and R. G. Carlberg, ApJ, 785, 137, 2014.

2. S. Tremaine and M. D. Weinberg, ApJL, 282, L5, 1984.

3. J. Font, J. E. Beckman, M. Querejeta, B. Epinat, P. A. James, J. Blasco-herrera, S. Erroz-Ferrer, and I. Pérez, ApJS, 210, 2, 2014. 
4. D. M. Elmegreen and B. G. Elmegreen, ApJ, 445, 591, 1995.

5. P. Rautiainen, H. Salo, and E. Laurikainen, MNRAS, 388, 1803, 2008.

6. R. J. Buta and X. Zhang, ApJS, 182, 559, 2009.

7. S. Scarano and J. R. D. Lépine, MNRAS, 428, 625, 2013.

8. M. S. del Rio and J. Cepa, A\&SA, 340, 1, 1998.

9. S. Savchenko, A. Marchuk, A. Mosenkov, K. Grishunin, P. placeholder, and P. placeholder2, MNRAS, 493, 390, 2020.

10. J. A. L. Aguerri, J. E. Beckman, and M. Prieto, AJ, 116, 2136, 1998.

11. K. G. Begeman, HI rotation curves of spiral galaxies, Ph.D. thesis, -, 1987.

12. M. R. Merrifield, R. J. Rand, and S. E. Meidt, MNRAS, 366, L17, 2006.

13. S. E. Meidt, R. J. Rand, and M. R. Merrifield, ApJ, 702, 277, 2009. 


\title{
Multifrequency study of FR0 radio galaxies with RATAN-600
}

\author{
A. Mikhailov, Yu. Sotnikova \\ mag10629@yandex.ru \\ Special Astrophysical Observatory of RAS, Nizhny Arkhyz 369167, Russia
}

The program for the study of FR0 radio galaxies has been carried out with RATAN-600 since February 2020. The quasisimultaneous radio spectra of 34 FR0 objects were measured from 3 to 10 times over 1.5 years of observations. FR0 radio galaxies are characterized by a flat spectrum with contribution of several compact components. Analysis of the RATAN-600 quasi-simultaneous radio spectra together with available literature data showed that $\sim 10 \%$ FR0s can be considered as lowpower GPS sources. Some of the FR0 objects can be relatively low power blazars. The further multi frequency investigation is necessary for understanding the nature of FR0 radio sources.

Keywords: galaxies: active, radio galaxies - blazars: general - radio continuum

DOI: $10.51194 /$ VAK2021.2022.1.1.155

\section{Introduction}

FR0 radio galaxies are dominant population among radio loud AGN in the local Universe [1]. Their characteristic feature is the deficit of extended radio emission. VLA observations at 1.5, 4.5 and $7.5 \mathrm{GHz}$ with the angular resolution up to 0.3 arcsec have shown that FR0s are still unresolved at the scales of $100-300 \mathrm{pc}$ in the most cases [2]. The features of extended emission were found only for 4 out of 18 sources. [3] studied the low-frequency properties of FR0s based on $150 \mathrm{MHz}$ LOFAR data and found an extended radio emission at scales $15-40 \mathrm{kpc}$ for 12 out of 66 objects. Parsec scale structures of FR0s were studied in $[4,5]$. Authors showed that FR0s usually have the radio morphology with jet-like structures in a combination with a bright radio core on the projected physical sizes of $0.3-10 \mathrm{pc}$. [4,5] have studied the jet kinematics of 8 FR0s and they found that the apparent speeds of most jet components are approximately $0.3 \mathrm{c}$ meaning the mildly relativistic jets.

The issue of the nature of FR0 radio galaxies is opened to discussion and requires multifrequency investigation. In [1], the following scenarios were considered: (1) FR0s are young objects during their life evolving into FRI radio galaxies; (2) FR0s might be recurrent objects in which formation of large-scale radio structures does not occur due to the relatively short time phases of activity; (3) they are objects with mildly relativistic jets. Collaborating VLBI and observations at sub arcsec angular resolution, and continuum radio spectra measurements are necessary for progress in understanding of FR0s nature and their relationship with classes of compact and extended radio sources.

\section{RATAN-600 observations}

Due to the scarcity of broad band radio data and lack of quasi-simultaneous measurements in the centimeter wavelength range we started the observations of FR0s with the RATAN-600 radio telescope in 2020. The advantage of the RATAN-600 measurements is an instantaneous radio spectra at the frequencies $1.2-22.3 \mathrm{GHz}$ which can be obtained at time scale $3-5$ minutes. A detailed description of data processing methods, the receivers and antenna parameters are presented in [6, 7]. The sample contains 34 objects from FR0CAT [1] with flux densities $S_{1.4 \mathrm{GHz}}>30$ mJy at declinations $-08^{\circ}<$ Dec $<+47^{\circ}$. The quasi-simultaneous radio spectra of 34 FR0 objects were measured from 3 to 10 times over 1.5 years of observations.

\section{Results}

The RATAN-600 data have allowed us to study FR0 radio galaxies at the centimeter range. The obtained radio properties of the objects are described in detail in [8]. The analysis of quasi simultaneous data has shown that half of the FR0 spectra at centimeter range can be considered as convex with decreasing the spectral index $\left(S \sim \nu^{\alpha}\right)$ with frequency increasing. This can be explained by the combined effects of self-absorption and the presence of compact components with different brightness temperatures. This interpretation can be tested using a high resolution radio imaging of various emitting components [9]. The average spectrum of FR0s was constructed using RATAN-600 data based on the method of approximation by polynomial functions of the spectrum of each object [10]. The obtained average spectrum can be considered as superposition of two spectral components: a decreasing component at the low-frequency band and a peaked one at the high-frequency band (Fig. 1). We investigated issue about the relationship between FR0s and GPS sources and estimated a fraction of GPS radio sources among FR0s as approximately $10 \%$ ([11], submitted). However, FR0s have spectra with less spectral curvature than canonical GPS sources. Some objects are quite variable and they can be blazars. We conclude that the original catalogue of FR0 radio galaxies [1] is heterogeneous and can contain low-power GPS objects, blazars and other classes of extragalactic radio sources. Further investigation of FR0s properties and their relationship with different AGNs types is necessary to better understand the nature of these objects.

\section{Future work}

We assume the further investigation of FR0 variability properties at longer time scales. We plan the complex studying FR0 radio galaxies including continuum measurements with RATAN-600 and imaging with high angular resolution with EVN and e-MERLIN. These observations will help to explore the structure of FR0s at scales from parsec to hundreds pc and to reveal features of possible extended radio components. 


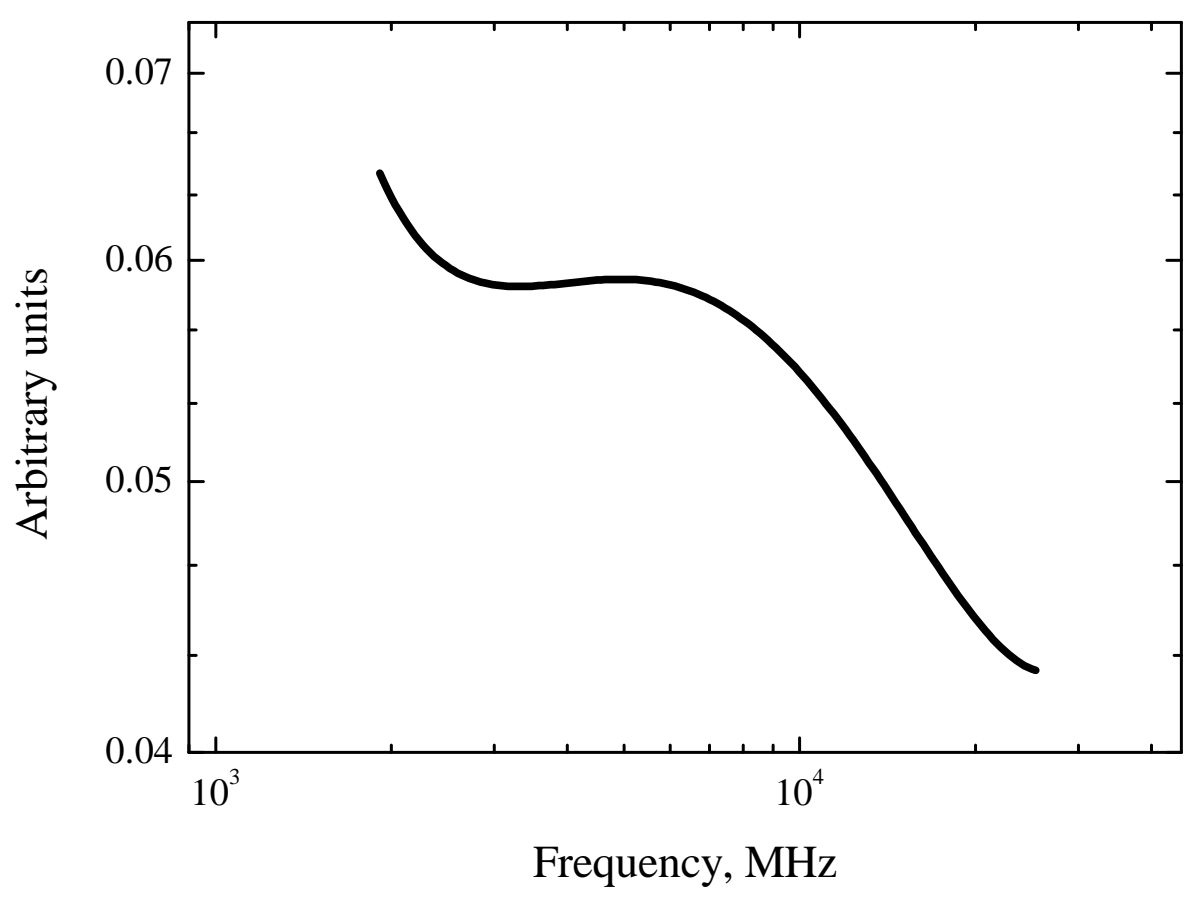

Figure 1: The average spectrum of FR0 radio galaxies according to RATAN-600 data [8].

\section{Acknowledgments}

The observations were carried out with the RATAN-600 scientific facility. Observations with RATAN-600 are supported by the Ministry of Science and Higher Education of the Russian Federation.

\section{References}

1. R. D. Baldi, A. Capetti, and F. Massaro, $A \mathscr{E} A$, 609, A1, 2018.

2. R. D. Baldi, A. Capetti, and G. Giovannini, MNRAS, 482, 2294, 2019.

3. A. Capetti, M. Brienza, R. D. Baldi, G. Giovannini, et al., A 6 A, 642, A107, 2020.

4. X. P. Cheng and T. An, ApJ, 863, 155, 2018.

5. X. Cheng, T. An, B. W. Sohn, X. Hong, and A. Wang, MNRAS, 506, 1609, 2021.

6. R. Y. Udovitskiy, Y. V. Sotnikova, M. G. Mingaliev, P. G. Tsybulev, G. V. Zhekanis, and N. A. Nizhelskij, Astrophysical Bulletin, 71, 496, 2016.

7. Y. V. Sotnikova, T. V. Mufakharov, E. K. Majorova, M. G. Mingaliev, R. Y. Udovitskii, N. N. Bursov, and T. A. Semenova, Astrophysical Bulletin, 74, 348, 2019.

8. A. G. Mikhailov and Y. V. Sotnikova, Astronomy Reports, 65, 233, 2021.

9. A. Capetti, R. D. Baldi, M. Brienza, R. Morganti, and G. Giovannini, A\&GA, 631, A176, 2019.

10. O. V. Verkhodanov, D. D. Kozlova, and Y. V. Sotnikova, Astrophysical Bulletin, 73, 393, 2018.

11. A. G. Mikhailov and Y. V. Sotnikova, Astronomische Nachrichten, xxx, xxx, 2021. 


\title{
$\mathrm{OH}$ megamaser galaxies observations with RATAN-600
}

\author{
A. Mikhailov ${ }^{1}$, Yu. Sotnikova ${ }^{1}$, T. Mufakharov ${ }^{1,2,3}$, M. Mingaliev ${ }^{1,2}$, N. Bursov ${ }^{1}$, T. Semenova ${ }^{1}$, \\ V. Stolyarov ${ }^{1,2,4}, \mathrm{Zh}$. $\mathrm{Wu}^{5}$ \\ mag10629@yandex.ru \\ ${ }^{1}$ Special Astrophysical Observatory of RAS, Nizhny Arkhyz 369167, Russia, \\ ${ }^{2}$ Kazan Federal University, 18 Kremlyovskaya St, Kazan 420008, Russia, \\ ${ }^{3}$ Shanghai Astronomical Observatory, Chinese Academy of Sciences, Shanghai 200030, China, \\ ${ }^{4}$ Astrophysics Group, Cavendish Laboratory, University of Cambridge, J J Thomson Avenue, Cambridge CB3 OHE, UK \\ ${ }^{5}$ College of Physics, Guizhou University, Guiyang 550025, China
}

We present the results of pilot observations of the $\mathrm{OH}$ megamaser (OHM) sample carried out with the radio telescope RATAN-600 at $1.2,2.3,4.7,8.2,11.2$, and $22.3 \mathrm{GHz}$ in 2019-2021. When comparing the radio continuum properties of the OHM sample and the control sample of galaxies without detected maser emission, we determine the median spectral index at $4.7 \mathrm{GHz} \alpha_{4.7}=-0.59$ for the OHM sample, and $\alpha_{4.7}=-0.71$ for the non-OHM galaxies, and steep radio spectra prevail in both samples. Subsamples of OHMs powered by active galactic nuclei (AGN) and star formation have no significant differences in their radio properties. AGN-powered galaxies exhibit larger scatter in a range of parameters and their standard deviations.

Keywords: galaxies: active, star formation - quasars: general - radio continuum

DOI: $10.51194 /$ VAK2021.2022.1.1.156

\section{Introduction}

The galaxies hosting $\mathrm{OH}$ megamaser (OHM) emission are usually associated with the luminous or ultraluminous infrared galaxies (U/LIRGs), containing a large amount of molecular gas in their centers [1,2]. The star formation processes or active galactic nuclei (AGNs) are usually responsible for their high luminosities. The radio continuum observations are important in determining the nature of the non-thermal radio emission and studying the mechanism for inducing maser radiation in OHMs, since the background continuum radio emission stimulates the maser emission in the galaxies [3].

\section{Sample and observations}

We used the sample compiled by [4] from the maser galaxies available in the literature (OHM sample) and the sources with no detection of maser emission (control sample) in the Arecibo survey sample [5]. We selected 74 OHM galaxies and 129 non-detections from [4] with $1.4 \mathrm{GHz}$ flux densities $S_{1.4}>5 \mathrm{mJy}$ for observations with RATAN.

The observations of the samples were conducted in 2019-2021 with the radio telescope RATAN-600 [6] operating in the transit mode and providing the $1-22 \mathrm{GHz}$ broad-band simultaneous measurements. We observed the sources from 3 to 40 times for each observing epoch to improve the signal-to-noise ratio. The observations were carried out with the radiometric array at six frequencies: $1.25,2.25,4.7,8.2,11.2$, and $22.3 \mathrm{GHz}$. The measurements were processed using the automated data reduction system [7, 8, 9] and the Flexible Astronomical Data Processing System (FADPS) standard package modules [10] for the broad-band RATAN continuum radiometers.

Using the Astrophysical CATalogs support System (CATS) database [11, 12], we complemented the compiled radio spectra in the samples with the following additional radio data: the Green Bank 6-cm survey (GB6, [13]), the GaLactic and Extragalactic All-sky Murchison Widefield Array (GLEAM) survey at 72-231 MHz (2013-2014, [14]), the Giant Metrewave Radio Telescope Sky Survey (TGSS) at $150 \mathrm{MHz}$ (2015, [15]), and the VLA measurements [16]. To find the counterparts of target objects in a large number of the radio catalogues, the astroquery Python package was used [17]. Radio fluxes for the OHMs and control sample objects were extracted from NED and VizieR Information System ${ }^{\mathrm{a}}$ [18].

\section{Results}

We revealed a low detection rate (4-75\%) at the RATAN frequencies, with the detection rate is being higher for the OHMs than for the control sample. We measured the radio continuum spectra for $57 \%$ of objects from both samples at frequencies higher than $4.7 \mathrm{GHz}$ for the first time. The samples stand out statistically by the flat-spectrum fraction. The OHM sample has $32 \%$ sources with flat spectra, while the control sample has $18 \%$. Steep radio spectra prevail in the samples: $53 \%$ and $61 \%$ for the OHM and control samples respectively. We determined the median spectral indices ${ }^{\mathrm{b}}$ at $1.4 \mathrm{GHz}\left(\alpha_{1.4}\right)$ as -0.55 and -0.69 , and at $4.7 \mathrm{GHz}\left(\alpha_{4.7}\right)$ as -0.59 and -0.71 for the OHM and control lists, respectively.

The median values of radio luminosity calculated at $1.4 \mathrm{GHz}$ are $\sim 2 \times 10^{23}$ and $\sim 5 \times 10^{23} \mathrm{~W} \mathrm{~Hz}^{-1}$ for the $\mathrm{OHM}$ and control sample, and the median values of radio loudness are 2.7 and 1.9 for the OHM and control sample, respectively. When adopting the radio loudness criteria similar to the commonly used for quasars by [19], all except one OHM and all except one source in the control sample are radio-loud. The distributions of radio parameters such as the spectral index, radio loudness, and radio luminosity are different at a significance level of 0.05 according to the Kolmogorov-Smirnov test for the control and OHM samples.

A significant part of both samples is galaxies classified as AGN: 35 of 74 in the OHM sample and 38 of 129 in the control sample. For the additional analysis of the results, we considered subsamples of galaxies containing AGN and without

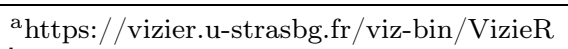

${ }^{\mathrm{b}}$ spectral index defined from the power-law $S_{\nu} \sim \nu^{\alpha}$, where $S_{\nu}$ is a flux density at the frequency $\nu$, and $\alpha$ is a spectral index 
them (non AGN). There is no significant difference in the radio properties for OHMs powered by AGN activity or star formation. On the other hand, for the galaxies without $\mathrm{OH}$ emission, AGN-powered galaxies have steeper radio spectra and they are brighter in the radio band than galaxies without AGNs. Our obtained radio parameters are summarised in Table 1.

Table 1: Mean values with their standard deviations in parentheses and median values of the spectral indices $\left(\alpha_{1.4}\right.$ and $\left.\alpha_{4.7}\right)$, radio loudness (log $R$ ), and radio luminosity $\left(\log P_{1.4}\right)$ are presented for the subsamples containing AGNs and without them (non AGN).

\begin{tabular}{|c|c|c|c|c|c|c|c|c|c|c|}
\hline sample & subsample & $\mathrm{N}$ & \multicolumn{2}{|l|}{$\alpha_{1.4}$} & \multicolumn{2}{|l|}{$\alpha_{4.7}$} & \multicolumn{2}{|l|}{$\log R$} & \multicolumn{2}{|c|}{$\begin{array}{l}\log P_{1.4}, \\
\mathrm{~W} \mathrm{~Hz}^{-1}\end{array}$} \\
\hline \multirow{3}{*}{$\mathrm{OHM}$} & AGN & 35 & $-0.45(0.39)$ & -0.49 & $-0.52(0.42)$ & -0.66 & $2.90(1.01)$ & 2.88 & $23.29(0.94)$ & 23.24 \\
\hline & non AGN & 39 & $-0.47(0.35)$ & -0.59 & $-0.54(0.35)$ & -0.54 & $2.40(0.66)$ & 2.46 & $23.24(0.59)$ & 23.39 \\
\hline & all & 74 & $-0.46(0.37)$ & -0.55 & $-0.54(0.38)$ & -0.59 & $2.64(0.87)$ & 2.70 & $23.26(0.77)$ & 23.3 \\
\hline \multirow{3}{*}{ control } & AGN & 38 & $-0.65(0.46)$ & -0.70 & $-0.79(0.44)$ & -0.81 & $2.31(1.11)$ & 2.08 & $24.37(1.10)$ & 24.09 \\
\hline & non AGN & 91 & $-0.63(0.52)$ & -0.69 & $-0.69(0.44)$ & -0.70 & $2.07(0.79)$ & 1.87 & $23.62(0.35)$ & 23.57 \\
\hline & all & 129 & $-0.64(0.50)$ & -0.69 & $-0.72(0.44)$ & -0.71 & $2.14(0.89)$ & 1.91 & $23.84(0.74)$ & 23.7 \\
\hline
\end{tabular}

\section{Acknowledgements}

The reported study was funded by the RFBR, project number 21-52-53035 "The Radio Properties and Structure of OH Megamaser Galaxies". Observations with RATAN-600 are supported by the Ministry of Science and Higher Education of the Russian Federation.

\section{References}

1. K. Y. Lo, ARA\&A, 43, 625, 2005.

2. D. A. Sales, A. Robinson, R. A. Riffel, T. Storchi-Bergmann, et al., MNRAS, 486, 3350, 2019.

3. W. A. Baan and H. R. Klöckner, A\&A, 449, 559, 2006.

4. J. S. Zhang, J. Z. Wang, G. X. Di, Q. F. Zhu, Q. Guo, and J. Wang, A\&SA, 570, A110, 2014.

5. J. Darling and R. Giovanelli, AJ, 124, 100, 2002.

6. Y. N. Parijskij, IEEE Antennas and Propagation Magazine, 35, 7, 1993.

7. P. G. Tsybulev, Astrophysical Bulletin, 66, 109, 2011.

8. P. G. Tsybulev, N. A. Nizhelskii, M. V. Dugin, A. N. Borisov, D. V. Kratov, and R. Y. Udovitskii, Astrophysical Bulletin, 73, 494, 2018.

9. R. Y. Udovitskiy, Y. V. Sotnikova, M. G. Mingaliev, P. G. Tsybulev, G. V. Zhekanis, and N. A. Nizhelskij, Astrophysical Bulletin, 71, 496, 2016.

10. O. V. Verkhodanov, Astronomical Data Analysis Software and Systems VI, A.S.P. Conference Series, $125,46,1997$.

11. O. V. Verkhodanov, S. A. Trushkin, and V. N. Chernenkov, Baltic Astronomy, 6, 275, 1997.

12. O. V. Verkhodanov, S. A. Trushkin, H. Andernach, and V. N. Chernenkov, Bulletin of the Special Astrophysics Observatory, 58, 118, 2005.

13. P. C. Gregory, W. K. Scott, K. Douglas, and J. J. Condon, ApJS, 103, 427, 1996.

14. N. Hurley-Walker, J. R. Callingham, P. J. Hancock, T. M. O. Franzen, et al., MNRAS, 464, 1146, 2017.

15. H. T. Intema, P. Jagannathan, K. P. Mooley, and D. A. Frail, A\& A, 598, A78, 2017.

16. S. E. Healey, R. W. Romani, G. B. Taylor, E. M. Sadler, R. Ricci, T. Murphy, J. S. Ulvestad, and J. N. Winn, ApJS, 171, 61, 2007.

17. A. Ginsburg, B. M. Sipő́cz, C. E. Brasseur, P. S. Cowperthwaite, et al., AJ, 157, 98, 2019.

18. F. Ochsenbein, P. Bauer, and J. Marcout, A\&A Sup., 143, 23, 2000.

19. K. I. Kellermann, R. Sramek, M. Schmidt, D. B. Shaffer, and R. Green, AJ, 98, 1195, 1989. 


\title{
Analysis of objects with ultra steep spectra in the central section of RATAN Zenith Field (RZF) survey
}

\author{
Yu.N. Pariiskii $^{1}$, T.A. Semenova ${ }^{2}$, A.V. Temirova ${ }^{1}$, N.N. Bursov ${ }^{2}$ \\ adelina_temirova@mail.ru \\ ${ }^{1}$ St. Petersburg Branch of SAO RAS, St. Petersburg, Russia \\ ${ }^{2}$ SAO RAS, Nizhniy Arkhys, Russia
}

DOI: 10.51194 /VAK2021.2022.1.1.157

Objects with USS (ultra steep spectra), which are the main indicator for finding possible candidates for distant galaxies, obtained as a result of a deep survey of the sky in the range $0^{h} \leq R . A . \leq 24^{h}, 40.5^{\circ} \leq D E C \leq 42.5^{\circ}$ on a radio telescope RATAN-600 at a wavelength $\lambda=7.6 \mathrm{~cm}$. In the central section of the scan $\pm 2^{\prime}$ out of 448 detected objects, 69 sources turned out to be ultra steep spectra $\alpha<-1.1, S \propto \nu^{\alpha}$. This sample includes relatively low-density radio sources $S_{3940 M H z, \text { median }}=5.8 \mathrm{mJy}$ and $S_{1400 \mathrm{MHz} \text {,median }}=20.8 \mathrm{mJy}$. Nine of sources have Megahertz - Peaked Spectra (MGP), suggesting which that may be High-Z Radio Galaxies ( HZRG). Found 2 GPS (Gigahertz Peaked Spectrum) objects (J070010+412930, J224408+412926). For 27 objects with USS spectra for which stellar values in different filters are known (SDSS), photometric redshifts and their radio luminosity at frequencies of 1400 and $3940 \mathrm{MHz}$ are determined. According to the SDSS (DR12) digital optical survey, using the NVSS radio maps and FIRST catalogs, optical identification of objects was carried out. Our sample of 19 galaxies and 8 star-like objects mainly belongs to the category of nearby galaxies $(\mathrm{N}=$ 12) with $z_{p h, \text { gal,mean }}=0.27 \pm 0.01$, found in the centimeter wavelengths $\lambda=7.6 \mathrm{~cm}$. These radio sources are either located in the dense intergalactic media of rich clusters of galaxies or are confined within their host galaxies. 10 galaxies with radio luminosities $L_{1400 M H z} \geq 10^{26} \mathrm{~W} / \mathrm{Hz}$ can be attributed to FR type II, of which 4 sources with $z_{p h, g a l}>0.5$ can be considered as possible candidates for radio-high active nuclei galaxies (AGNs). Only one galaxy with a USS spectrum turned out to be a rare nearby galaxy with a relatively low radio illumination with $L_{1400 M H z}=1,51 \times 10^{24} \mathrm{~W} / \mathrm{Hz}, z_{p h, g a l}=0.08$. The remaining 8 galaxies of intermediate luminosity are objects of the mixed type FRI - FRII. 


\title{
Determination of the magnetic field strength and geometry in the accretion disks of AGNs
}

\author{
M. Piotrovich ${ }^{1,2}$, S. Buliga ${ }^{1,2}$, T. Natsvlishvili ${ }^{1}$ \\ mpiotrovich@mail.ru \\ ${ }^{1}$ Central astronomical observatory at Pulkovo, 196140 Saint-Petersburg, Russia \\ ${ }^{2}$ Special Astrophysical Observatory, 369167 Nizhnij Arkhyz, Russia
}

Based on the spectropolarimetric data of 33 Seyfert type 1 galaxies observed with the BTA-6m telescope of the Special Astrophysical Observatory, we estimated the magnetic field values at the event horizon of the supermassive black hole $B_{\mathrm{H}}$ and the exponents of the power-law dependence $s$ of the magnetic field on the radius. We used the model of Shakura-Sunyaev accretion disk. The average value of $\log B_{\mathrm{H}}[\mathrm{G}]$ was found to be $\sim 4$. The average value of $s$ is $\approx 1.7$, and its distribution maximum span is in the range of $1.85<s<2.0$. This is a rather interesting result, since $s=5 / 4$ is usually adopted in calculations for Shakura-Sunyaev accretion disks. For two objects PG $1545+210$ and 2MASX J06021107+2828382, the measured degree of polarization is greater than the maximum possible value at the angle between the line of sight and the axis of the accretion disk $i=45^{\circ}$. It was concluded that for these objects the angle should be closer to $i=60^{\circ}$.

Keywords: accretion disks; magnetic fields; polarization; active galactic nuclei; supermassive black holes

DOI: 10.51194 /VAK2021.2022.1.1.158

According to modern concepts, accretion disks of active galactic nuclei (AGNs) should have an intense magnetic field. It is assumed that the magnetic field is formed as a result of the interaction of accreting matter with a rotating supermassive black hole (SMBH). The presence of a magnetic field should have a noticeable effect on the spectropolarimetric characteristics of the accretion disk radiation. The polarimetric observations demonstrate that AGNs have polarized radiation in different wavelength ranges, from ultraviolet to radio waves. In this work, we assume that for our sample of objects (Seyfert type 1) accretion disk is the main source of polarized radiation in optical range and we use Shakura-Sunyaev disk model.

When considering radiation from an axially symmetric accretion disk with a magnetic field, its integral Stokes parameters can be written in the following form [1]:

$$
\begin{aligned}
& \langle Q\rangle=Q(0, \mu) \frac{2}{\pi} \int_{0}^{\pi / 2} d \Phi \frac{1+a^{2}+b^{2} \cos ^{2} \Phi}{\left(1+a^{2}+b^{2} \cos ^{2} \Phi\right)^{2}-(2 a b \cos \Phi)^{2}}, \\
& \langle U\rangle=a Q(0, \mu) \frac{2}{\pi} \int_{0}^{\pi / 2} d \Phi \frac{1+a^{2}-b^{2} \cos ^{2} \Phi}{\left(1+a^{2}+b^{2} \cos ^{2} \Phi\right)^{2}-(2 a b \cos \Phi)^{2}},
\end{aligned}
$$

where $\mu=\cos i$, where $i$ is the angle between the line of sight and the axis of the disk, $Q(0, \mu)$ is the value of the Stokes parameter without a magnetic field. The parameters $a$ and $b$ are expressed, in turn, as follows:

$$
\begin{aligned}
& a=0.8 \mu \lambda^{2}(\mu m) B_{\|}(G), \\
& b=0.8 \sqrt{1-\mu^{2}} \lambda^{2}(\mu m) B_{\perp}(G),
\end{aligned}
$$

where $\lambda$ is the wavelength, $B_{\|}$and $B_{\perp}$ are, respectively, the component of the magnetic field in the disk parallel and perpendicular to the disk axis (see Fig.1 from [1]). Taking into account that in the Milne problem without a magnetic field, the Stokes parameter $U(0, \mu) \equiv 0$, we obtained the following value of the relative polarization and positional angle $\chi$ :

$$
\begin{aligned}
& P_{\text {rel }}=P(B, \mu) / P(0, \mu)=\sqrt{\langle Q\rangle^{2}+\langle U\rangle^{2}} / Q(0, \mu), \\
& \chi=0.5 \arctan (\langle U\rangle /\langle Q\rangle) .
\end{aligned}
$$

The polarization value $P(0, \mu)$ without a magnetic field was previously calculated by us numerically using the SobolevChandrasekhar model and is tabulated in [2]. Thus, we have the opportunity to accurately calculate the polarization value $P=P_{\text {rel }} P(0, \mu)$, using numerical integration for the parameter $P_{\text {rel }}$ and tabular values for $P(0, \mu)$. When considering the magnetic field in the accretion disk, it is usually assumed that its dependence on the radius has a power-law form:

$$
B(R)=B_{\mathrm{H}}\left(R_{\mathrm{H}} / R\right)^{s},
$$

where $B_{\mathrm{H}}$ is the value of the magnetic field intensity at the event horizon of SMBH in AGN, $R_{\mathrm{H}}=G M_{\mathrm{BH}}\left(1+\sqrt{1-a_{*}^{2}}\right) / c^{2}$ is the radius of the event horizon, $M_{\mathrm{BH}}$ is the mass of the SMBH, $a_{*}=c J / G M_{\mathrm{BH}}^{2}$ is the dimensionless spin of the $\mathrm{SMBH}$, $J$ is the angular momentum of the SMBH rotation. In our work, we decided to investigate the influence of parameter $s$ on the model and therefore we tried a number of values within $0.5<s<2$ range. For Shakura-Sunyaev accretion disk we have [3]:

$$
R_{\lambda}(\mathrm{cm})=0.97 \times 10^{10} \lambda[\mu m]^{4 / 3}\left(\frac{M_{\mathrm{BH}}}{M_{\odot}}\right)^{2 / 3}\left(\frac{l_{\mathrm{E}}}{\varepsilon}\right)^{1 / 3} .
$$

where $R_{\lambda}$ is the distance in the accretion disk, which corresponds to wavelength $\lambda, l_{\mathrm{E}}$ is the Eddington ratio, $\varepsilon$ is the radiative efficiency.

In our work, we used the already published data of spectropolarimetric observations of a sample of 33 AGN in type 1 Seyfert galaxies, carried out on the BTA-6m telescope with the participation of the authors. The observations were carried out with the SCORPIO and SCORPIO-2 focal reducers in the spectropolarimetric mode mounted at the prime focus. For each object, the dependence of the polarization and the gradient of the positional angle on $B_{\mathrm{H}}$ and $s$ was constructed. Then this dependence was compared with observational data. After that, the $B_{\mathrm{H}}$ values were additionally subject to the 
Table 1: Results of determination of $B_{\mathrm{H}}$ and parameter $s$ for our objects.

\begin{tabular}{|c|c|c|c|c|c|c|c|}
\hline Object & $P_{o b s}$ & $\log \left(\frac{M_{\mathrm{BH}}}{M_{\odot}}\right)$ & $a_{*}$ & $l_{\mathrm{E}}$ & $\log \left(B_{\mathrm{H}, \mathrm{pr}}[\mathrm{G}]\right)$ & $\log \left(B_{\mathrm{H}}[\mathrm{G}]\right)$ & $s$ \\
\hline 2MASS J02093740+5226396 & $1.47 \pm 0.46$ & 8.53 & 0.970 & 0.045 & $4.00_{-1.00}^{+1.00}$ & $3.33_{-0.30}^{+0.17}$ & $1.81 \pm 0.15$ \\
\hline 2MASX J02421465+0530361 & $0.89 \pm 0.43$ & 8.33 & 0.930 & 0.100 & $4.00_{-1.00}^{+1.00}$ & $3.84_{-0.84}^{+0.29}$ & $1.57 \pm 0.27$ \\
\hline 2MASX J06021107+2828382 & $2.34 \pm 0.40$ & 8.15 & 0.960 & 0.009 & $4.00_{-1.00}^{+1.00}$ & $3.39_{-0.28}^{+0.84}$ & $1.73 \pm 0.17$ \\
\hline $3 \mathrm{C} 390.3$ & $0.65 \pm 0.36$ & 9.12 & 0.998 & 0.004 & $3.41_{-0.37}^{+0.20}$ & $3.18_{-0.10}^{+0.28}$ & $1.87 \pm 0.11$ \\
\hline MCG 08-11-011 & $1.46 \pm 0.90$ & 8.12 & 0.950 & 0.060 & $4.00_{-1.00}^{+1.00}$ & $3.67_{-0.67}^{+0.10}$ & $1.68 \pm 0.24$ \\
\hline Mrk 79 & $1.31 \pm 0.38$ & 7.69 & 0.995 & 0.040 & $3.87_{-0.20}^{+0.15}$ & $3.86_{-0.12}^{+0.09}$ & $1.81 \pm 0.11$ \\
\hline Mrk 352 & $1.05 \pm 0.44$ & 7.19 & 0.750 & 0.033 & $4.00_{-1.00}^{+1.00}$ & $4.04_{-1.04}^{+0.30}$ & $1.55 \pm 0.28$ \\
\hline Mrk 509 & $1.21 \pm 0.43$ & 8.16 & 0.840 & 0.160 & $4.69_{-0.30}^{+0.30}$ & $4.44_{-0.04}^{+0.03}$ & $1.99 \pm 0.02$ \\
\hline Mrk 590 & $0.61 \pm 0.25$ & 7.20 & 0.815 & 0.214 & $5.05_{-0.30}^{+0.30}$ & $4.89_{-0.08}^{+0.07}$ & $1.72 \pm 0.08$ \\
\hline Mrk 1095 & $0.48 \pm 0.24$ & 8.27 & 0.930 & 0.069 & $3.74_{-0.19}^{+0.26}$ & $3.80_{-0.16}^{+0.12}$ & $1.46 \pm 0.15$ \\
\hline Mrk 1146 & $0.59 \pm 0.40$ & 7.41 & 0.997 & 0.130 & $4.60_{-0.30}^{+0.21}$ & $4.58_{-0.17}^{+0.12}$ & $1.59 \pm 0.16$ \\
\hline Mrk 1506 & $1.06 \pm 0.41$ & 7.74 & 0.950 & 0.050 & $4.43_{-0.11}^{+0.18}$ & $4.43_{-0.08}^{+0.18}$ & $1.96 \pm 0.05$ \\
\hline NGC 3227 & $1.18 \pm 0.31$ & 7.22 & 0.998 & 0.040 & $4.47_{-0.30}^{+0.11}$ & $4.42_{-0.19}^{+0.08}$ & $1.89 \pm 0.08$ \\
\hline NGC 4051 & $0.55 \pm 0.54$ & 6.77 & 0.970 & 0.209 & $5.08_{-0.17}^{+0.18}$ & $4.96_{-0.03}^{+0.03}$ & $1.28 \pm 0.31$ \\
\hline NGC 4593 & $1.06 \pm 0.50$ & 6.73 & 0.905 & 0.120 & $4.00_{-1.00}^{+1.00}$ & $4.34_{-1.34}^{+0.30}$ & $1.39 \pm 0.30$ \\
\hline NGC 5548 & $0.55 \pm 0.23$ & 7.68 & 0.970 & 0.050 & $4.48_{-0}^{+0 .}$ & $4.50_{-0.21}^{+0.34}$ & $1.80 \pm 0.12$ \\
\hline PG $0003+199$ & $0.52 \pm 0.35$ & 7.42 & 0.990 & 0.308 & $4.68_{-0.17}^{+0.17}$ & $4.69_{-0.11}^{+0.09}$ & $1.52 \pm 0.13$ \\
\hline PG $0007+106$ & $0.48 \pm 0.30$ & 8.14 & 0.993 & 0.100 & $4.21_{-0.12}^{+0.12}$ & $4.22_{-0.08}^{+0.06}$ & $1.60 \pm 0.14$ \\
\hline PG $0026+129$ & $0.71 \pm 0.23$ & 8.09 & 0.806 & 0.311 & $4.42_{-0.24}^{+0.29}$ & $4.48_{-0.18}^{+0.08}$ & $1.78 \pm 0.11$ \\
\hline PG $0049+171$ & $0.81 \pm 0.27$ & 8.35 & 0.998 & 0.025 & $3.92_{-0.18}^{+0.24}$ & $3.88^{+0.18}+0.10$ & $1.91 \pm 0.07$ \\
\hline PG $0050+124$ & $1.95 \pm 1.70$ & 7.44 & 0.997 & 0.155 & $4.00_{-1.00}^{+1.00}$ & $4.15_{-1.15}^{+0.30}$ & $1.52 \pm 0.34$ \\
\hline PG $0054+144$ & $1.15 \pm 0.53$ & 8.97 & 0.996 & 0.017 & $3.64_{-0.29}^{+0.22}$ & $3.42_{-0.05}^{+0.05}$ & $1.96 \pm 0.05$ \\
\hline PG $0804+761$ & $0.27 \pm 0.18$ & 8.22 & 0.912 & 0.157 & $4.25_{-0.20}^{+0.22}$ & $4.28_{-0.14}^{+0.11}$ & $1.42 \pm 0.16$ \\
\hline PG $0923+129$ & $0.21 \pm 0.11$ & 7.25 & 0.998 & 0.220 & $4.67_{-0.18}^{+0.15}$ & $4.67_{-0.11}^{+0.14}$ & $1.31 \pm 0.10$ \\
\hline PG $0923+201$ & $0.74 \pm 0.47$ & 9.02 & 0.996 & 0.027 & $3.66_{-0}^{+0}$. & $3.61_{-0.13}^{+0.10}$ & $1.81 \pm 0.14$ \\
\hline PG $1022+519$ & $0.50 \pm 0.43$ & 7.15 & 0.650 & 0.275 & $4.98_{-0.20}^{+0.24}$ & $4.91_{-0.06}^{+0.06}$ & $1.51 \pm 0.19$ \\
\hline PG $1309+355$ & $1.41 \pm 0.73$ & 9.06 & 0.991 & 0.024 & $3.63_{-0.36}^{+0.30}$ & $3.36_{-0.07}^{+0.06}$ & $1.94 \pm 0.06$ \\
\hline PG $1501+106$ & $0.93 \pm 0.73$ & 8.53 & 0.998 & 0.025 & $3.88_{-0.25}^{+0.36}$ & $3.83_{-0.14}^{+0.01}$ & $1.74 \pm 0.17$ \\
\hline PG $1545+210$ & $2.03 \pm 0.44$ & 9.32 & 0.916 & 0.021 & $3.52_{-0.31}^{+0.25}$ & $3.27_{-0.04}^{+0.04}$ & $1.96 \pm 0.05$ \\
\hline PG $1613+658$ & $0.75 \pm 0.43$ & 9.18 & 0.998 & 0.006 & $3.40_{-0.13}^{+0.10}$ & $3.32_{-0.04}^{+0.04}$ & $1.96 \pm 0.05$ \\
\hline PG $2112+059$ & $0.72 \pm 0.20$ & 8.69 & 0.583 & 0.299 & $4.31_{-0.42}^{+1.13}$ & $4.19_{-0.22}^{+0.04}$ & $1.84 \pm 0.11$ \\
\hline PG $2214+139$ & $1.23 \pm 0.24$ & 8.55 & 0.988 & 0.055 & $3.96_{-0.26}^{+0.42}$ & $3.77_{-0.05}^{+0.04}$ & $1.98 \pm 0.03$ \\
\hline PG $2233+134$ & $0.55 \pm 0.40$ & 8.04 & 0.500 & 0.270 & $4.00_{-1.00}^{+1.00}$ & $4.30_{-1.30}^{+0.30}$ & $1.34 \pm 0.36$ \\
\hline
\end{tabular}

condition that they must fall within the limits obtained for these objects by independent methods. Our results are presented in Table 1.

The average value of $\log B_{\mathrm{H}}[\mathrm{G}]$ was found to be $\sim 4$, which is in good agreement with the results obtained by other methods [4,5], in which the magnetic field strength was estimated using the physical parameters of the relativistic jets. It was possible to reveal the dependence of the magnetic field on the SMBHs mass and the Eddington ratio of the form $\log B_{\mathrm{H}}[\mathrm{G}] \approx(-0.69 \pm 0.04) \log \left(M_{\mathrm{BH}} / M_{\odot}\right)+(9.76 \pm 0.35)$ and $\log B_{\mathrm{H}}[\mathrm{G}] \approx(1.05 \pm 0.09) \log l_{\mathrm{E}}+(5.38 \pm 0.11)$, which agree well with the results of [5]. The average value of $s$ is $s \approx 1.7$, and the maximum distribution over $s$ is within $1.85<s<2.0$. This is a rather interesting result, since $s=5 / 4$ is usually taken in calculations for accretion disks in type 1 Seyfert nuclei. We also managed to estimate the dependence of $s$ on the SMBHs mass and the Eddington ratio of the form $s \approx(0.19 \pm 0.04) \log M_{\mathrm{BH}} / M_{\odot}+(0.18 \pm 0.34)$ and $s \approx(-0.25 \pm 0.06) \log l_{\mathrm{E}}+(1.41 \pm 0.08)$. And although these approximations have a larger error than in the case of $B_{\mathrm{H}}$, they are still of interest. In particular, it may indicate that the more complex disk models are required than the Shakura-Sunyaev model. And this problem undoubtedly requires further study. In addition, for two objects PG $1545+210$ and 2MASX J06021107+2828382, the measured polarization value was found to be greater than the maximum possible value at the inclination angle between the line of sight and the axis of the accretion disk $i=45^{\circ}$. Since the polarization increases with the angle, it was concluded that for these objects the angle should be closer to $i=60^{\circ}$. A more detailed description of our results can be found in [6].

This research was supported by the grant of Russian Science Foundation project number 20-12-00030 "Investigation of geometry and kinematics of ionized gas in active galactic nuclei by polarimetry methods". Observations with the SAO RAS telescope are supported by the Ministry of Science and Higher Education of the Russian Federation (including agreement No.05.619.21.0016, project IDRFMEFI61919X0016). 


\section{References}

1. N. A. Silant'ev, M. Y. Piotrovich, Y. N. Gnedin, and T. M. Natsvlishvili, $A \& A$ A, 507, 171, 2009.

2. Y. N. Gnedin, M. Y. Piotrovich, N. A. Silant'ev, T. M. Natsvlishvili, and S. D. Buliga, Astrophysics, 58, $443,2015$.

3. S. Poindexter, N. Morgan, and C. S. Kochanek, ApJ, 673, 34-38, 2008.

4. R. A. Daly, ApJ, 886, 37, 2019.

5. M. Y. Piotrovich, A. G. Mikhailov, S. D. Buliga, and T. M. Natsvlishvili, MNRAS, 495, 614, 2020.

6. M. Piotrovich, S. Buliga, and T. Natsvlishvili, Universe, 7, 202, 2021. 


\section{Absorption of gamma quanta from distant sources by thermal bremsstrahlung photons of hot gas in galaxy clusters}

A.N. Popov, D.P. Barsukov, A.V. Ivanchik, S.V. Bobashev alexander.popov@mail.ioffe.ru

Ioffe Institute, Saint Petersburg, Russia

DOI: 10.51194 /VAK2021.2022.1.1.159

The high energy gamma quanta from cosmological sources like blazar and active galactic nuclei interact with cosmological photon background [1]. At gamma quantum energy range $E \sim 100 \mathrm{TeV}-10^{7} \mathrm{TeV}$ the interaction with Cosmic Microwave Background (CMB) has the greatest impact on the spectra of distant sources [2]. At energy $E \sim 100 \mathrm{GeV}-100 \mathrm{TeV}$ the interaction to optical and infrared photons of Extragalactic Background Light (EBL) is dominated [3]. At smaller energies $E \sim 100 \mathrm{MeV}-10 \mathrm{GeV}$ the interaction to Cosmic Xray Background (CXB) photons [4] and Cosmic Ultraviolet (CUB) photons [5] may become important. The interaction of gamma quantum to thermal bremsstrahlung photons of hot intracluster gas with producing electron-positron pair in case of galaxy clusters was considered in [6]. In this paper we consider the interaction with bremsstrahlung photons of hot intracluster gas of some clusters. It is supposed that clusters are isothermal and electron number density has spherical distribution.

We receive that optical depth due to interaction with bremsstrahlung photons may be comparable to optical depth due to interaction with $\mathrm{CXB}$ and may sometimes exceed the last in $E \sim 1-10 \mathrm{GeV}$ range.

\section{References}

1. R. Ruffini, G. V. Vereshchagin, and S. S. Xue, ApछSS, 361, 82, 2016.

2. R. J. Gould and G. P. Schreder, Phys. Rev., 155, 1408, 1967.

3. A. Franceschini, G. Rodighiero, and M. Vaccari, A\&A, 487, 837, 2008.

4. M. Ajello, J. Greiner, G. Sato, D. R. Willis, et al., ApJ, 689, 666, 2008.

5. R. Hill, K. W. Masui, and D. Scott, ApSpe, 72, 663, 2018.

6. A. N. Popov, D. P. Barsukov, and A. V. Ivanchik, Astron. Lett., 44, 579, 2018. 


\title{
Superluminous quasars and mesolensing
}

\author{
A. Raikov ${ }^{1,2}$, N. Lovyagin ${ }^{3}$, V. Yershov ${ }^{4}$ \\ n.lovyagin@spbu.ru \\ ${ }^{1}$ Special Astrophysical Observatory, Russian Academy of Sciences, Niznii Arkhyz, 369167 Russia \\ ${ }^{2}$ Pulkovo Observatory, 65(1) Pulkovskoye shosse, St. Petersburg, 196140, Russia \\ ${ }^{3}$ Saint Petersburg State University, 7-9 Universitetskaya emb., St. Petersburg, 199034, Russia \\ ${ }^{4}$ Mullard Space Scince Laboratory, Holmbury St.Mary, RH5 6NT, United Kingdom
}

Observed magnitudes of many quasars with redshifts exceeding $z=5$ correspond to luminosities $L_{\mathrm{bol}}>10^{14} L_{\odot}$. The standard mechanism of quasar energy release by accretion suggests that masses of superluminous quasars should exceed $10^{10} M_{\odot}$. On the other hand, the age of these objects in the standard cosmological model is below one billion years, which is too short to explain their formation in the early Universe. Many quasars are known to be gravitationally lensed; showing multiple images of the same object. In the case of remote quasars with no multiple images, it is still possible that they are also gravitationally lensed by foreground objects of intermediate masses, such as globular clusters or dwarf galaxies. Such mesolensing would result in essential amplification of quasar brightnesses, subject to geometrical configuration between the lens and the lensed object. Here we estimate the fraction of quasars whose brightness might have been amplified by gravitational lensing.

Keywords: quasars, luminosity function, gravitational lensing

DOI: 10.51194 /VAK2021.2022.1.1.160

Since [1], it has been known that there is a statistically significant over-density of quasars within areas of the sky near foreground galaxies. One of the possible explanations for this phenomenon was suggested by [2,3] in terms of gravitational lensing of remote quasars by intermediate-mass objects $\left(10^{6}-10^{7} \mathrm{M}_{\odot}\right)$ located in the vicinities of foreground galaxies. These could be globular clusters or satellite dwarf galaxies. Gravitational lensing by intermediate-mass objects (mesolensing) differs from microlensing [4] by much longer duration of the effect. It is also different from lensing by large-mass foreground objects which usually splits the lensed object image into multiple images, whereas mesolensing does not split images and only results in brightness amplification.

Then [5] suggested that the same mesolensing effect might be responsible for the brightness amplification of some superluminous quasars at high redshifts. The problem is that within the standard cosmological model some of them have luminosities $L_{\mathrm{bol}}>10^{14} L_{\odot}$. By assuming that quasar energy is emitted via the mechanism of accretion onto a supermassive blackhole, the corresponding mass of such a blackhole should exceed $10^{10} M_{\odot}$. On the other hand, the age of high-redshift objects (less than $0.7 \mathrm{Gyr}$ ) is too small to explain their formation in the early Universe.

In the last few years, the number of catalogued high-redshift quasars has dramatically increased [6, 7, 8, 9], and so has the number of known superluminous quasars at high redhshifts. In X-rays, the all-sky survey by the Spektr-RG (SRG) space observatory also produces a growing number of high-redshift quasars with X-ray luminosities exceeding $10^{46} \mathrm{erg} / \mathrm{s}$ and masses of their blackholes exceeding $10^{9} M_{\odot}[10]$.

Most of the high-redshift superluminous quasars are seen as single sources, but we know [11] that their brightnesses could be enhanced by mesolensing without splitting source images, provided that the mass profile in the gravitational lens is of the King-type [12]. There are numerous objects with such mass profiles in the vicinities of foreground galaxies, so one would expect a large fraction of quasars to be lensed.

This fraction can be estimated by exploring statistical properties of available quasar catalogues, the most convenient one being the large astrometric catalogue of quasars LQAC-4 [7] because it includes absolute magnitudes for different wavelength bands.

We have converted these absolute magnitudes to bolometric luminosities by using the average spectral energy distribution of quasars published by [13]. The left panel of Fig. 1 shows the redshift distribution of these luminosities based on the infrared band $\mathrm{J}$ from LQAC-4.

The infrared band $\mathrm{J}$ is likely to be less affected by the observational selection effect than the other wavelength bands. However, even in this case, an observational selection effect takes place for redshifts $z>3$, because one can see that for these redshifts the quasar luminosity-evolution stripe narrows at its low-luminosity fringe, as shown in Fig. 1 (left). By contrast,
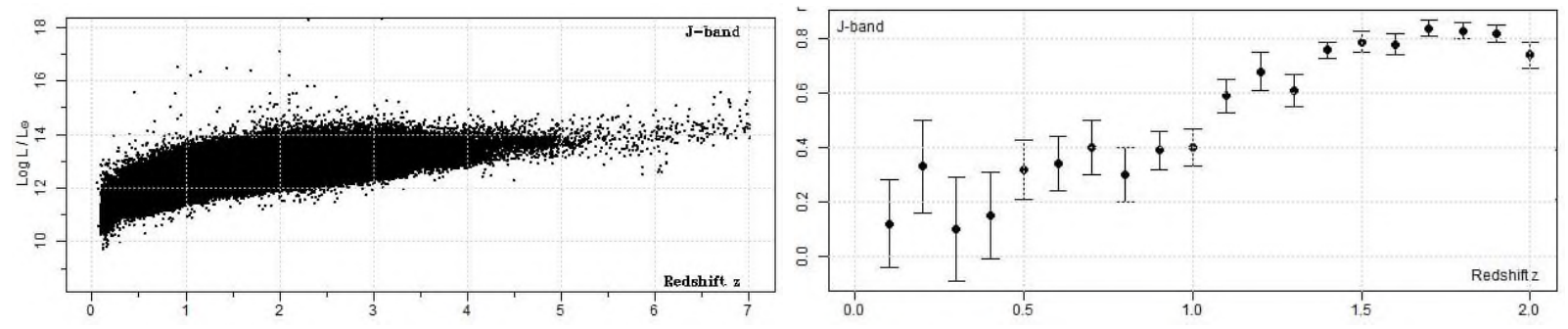

Figure 1: Left: redshift distribution of quasar luminosities from the LQAC-4 catalogue for the infrared band J; Right: fraction $r=\left(n_{\text {bright }}-n_{\text {faint }}\right) /\left(n_{\text {bright }}+n_{\text {faint }}\right)$ indicating the excess of bright quasars over the faint ones in the luminosity histograms for redshift slices within $0.1<z<2$ and with $\Delta z=0.1$ for each slice (two examples of these histograms are shown in Fig. 2 for $z=0.2$ and $z=0.8$ ). 

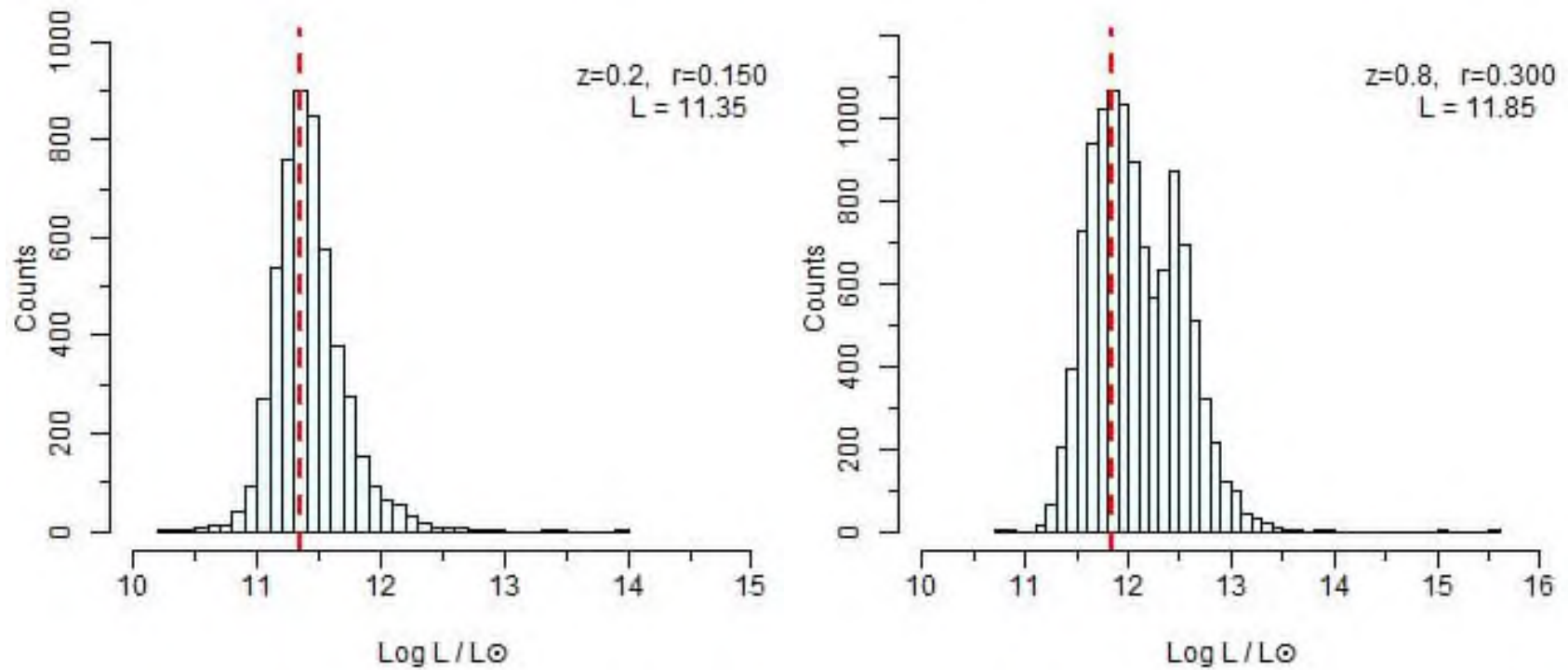

Figure 2: Two examples of quasar luminosity histograms for redshift slices at $z=0.2$ and $z=0.8$, with the slice width $\Delta z=0.1$, used for calculating the histogram asymmetries $r=0.15$ and $r=0.30$, respectively. The positions of the luminosity peaks, $L$, are indicated at the upper-right corners of each plot and also by the vertical dashed lines.

the width of this stripe at $0.5<z<2.0$ remains approximately constant, which indicates that the observational selection effect for low-redshift quasars is minimal or absent (indeed, one can expect that quasars, by being the brightest objects in the Universe, are all captured by modern telescopes at low redshifts).

We have built luminosity histograms for the quasar redshifts ranging from $z=0.1$ to $z=2.0$, using a redshift interval 0.1 and the same width for each redshift slice $\Delta z=0.1$. By counting the number of bright and faint objects, $n_{\text {bright }}$ and $n_{\text {faint }}$, above and below the luminosity $L$ corresponding to the histogram maximum, we can estimate the asymmetry - the excess of the number of bright quasars over the faint ones, $r=\left(n_{\text {bright }}-n_{\text {faint }}\right) /\left(n_{\text {bright }}+n_{\text {faint }}\right)$, which gives the fraction of gravitationally lensed quasars, assuming that the natural luminosity distribution of quasars at each redshift slice must be symmetrical with respect to the average luminosity.

As we can see from the right panel of Fig. 1, the number of gravitationally lensed quasars is growing with redshift, which is expected, as the number of foreground galaxies and their surrounding mesolenses (globular clusters) increases proportionally to the foreground volume. The negligibly small fraction of gravitationally lensed quasars at small redshifts reaches $80 \%$ at the redshift $z=1.5$. Beyond this point, the linear growth of $r$ breaks down and the fraction of lensed quasars becomes underestimated, which is likely to be caused by the observational selection effect.

The calculations for $z>2$ give us an underestimated fraction $r \approx 30 \%$. We can thus conclude (with caution) that the fraction of gravitationally lensed quasars is, at least, 30\%. However, our calculations suggest a more radical conclusion that practically all of the high-redshift quasars (say, for $z>5$ ) are gravitationally lensed, and that for some of them the geometrical configuration between the lensed quasars, the gravitational lens and the observer is such that the lensed quasars appear as superluminous objects, with their luminosities amplified by a factor of hundreds to thousands.

Therefore, the masses of supermassive blackholes associated with these quasars could actually be much smaller, which would resolve the conflict between the age of the Universe corresponding to the redshifts of superluminous quasars and the time needed for the formation of their supermassive blackholes.

The time scales of the quasar brightness variability due to their gravitational lensing on globular clusters can be from years to a few thousand years [11], depending on the source-to-lens geometrical configurations. Some smaller-time-scale variability can be expected due to microlensing on individual stars belonging to globular clusters.

As an example, the superluminous X-ray quasar SRGE J170245.3+130104 at redshift $z=5.5$ has reportedly reduced its brightness by half between the first and second all-sky surveys by the SRG space observatory [10]. This might be caused by either internal change in the quasar accretion process or by the change in the quasar-to-lens geometrical configuration. In the latter case, one would expect some further reduction of its observed brightness during the next forthcoming all-sky surveys by the SRG.

Foreground objects acting as mesolenses are unlikely to be observed directly or spectrally by having luminosities much smaller than those of quasars. However, since the lensing effect is the same for all wavelengths, comparing light curves in different spectral bands could be regarded as an observational test for mesolensing.

\section{Acknowledgement}

This research has been partly supported by the St. Petersburg State University's project \# 73555239. 


\section{References}

1. H. Arp, Quasars, redshifts, and controversies (1987).

2. Y. Baryshev, A. A. Raikov, and A. V. Yushchenco, in J. Surdej, D. Fraipont-Caro, E. Gosset, S. Refsdal, and M. Remy, eds., Liege International Astrophysical Colloquia, Liege International Astrophysical Colloquia, volume 31, 307 (1993).

3. A. V. Yushchenko, Y. V. Baryshev, and A. A. Raikov, Astronomical and Astrophysical Transactions, 17, 9, 1998.

4. E. V. Linder and P. Schneider, A\&A, 204, L8, 1988.

5. A. A. Raikov and V. V. Orlov, Astrophysical Bulletin, 71, 151, 2016.

6. E. W. Flesch, PASA, 32, e010, 2015.

7. C. Gattano, A. H. Andrei, B. Coelho, J. Souchay, C. Barache, and F. Taris, A\& A, 614, A140, 2018.

8. N. P. Ross and N. J. G. Cross, MNRAS, 494, 789, 2020.

9. E. W. Flesch, arXiv e-prints, arXiv:2105.12985, 2021.

10. G. A. Khorunzhev, A. V. Meshcheryakov, P. S. Medvedev, V. D. Borisov, et al., Astronomy Letters, 47, $123,2021$.

11. Y. V. Baryshev and Y. L. Ezova, Astron. Zhurn., 74, 497, 1997.

12. I. King, $A J, \mathbf{6 7}, 471,1962$.

13. C. M. Krawczyk, G. T. Richards, S. S. Mehta, M. S. Vogeley, S. C. Gallagher, K. M. Leighly, N. P. Ross, and D. P. Schneider, ApJS, 206, 4, 2013. 


\title{
Observational insights on the formation scenarios of giant low surface brightness galaxies
}

\author{
A. Saburova ${ }^{1,2}$, I. Chilingarian ${ }^{3,1}$, A. Kasparova ${ }^{1}$, O. Sil'chenko ${ }^{1}$, I. Katkov ${ }^{4,5,1}$, K. Grishin ${ }^{1}$, R. Uklein ${ }^{6}$ \\ saburovaann@gmail.com \\ ${ }^{1}$ Sternberg Astronomical Institute, M.V. Lomonosov Moscow State University, Universitetsky pr., 13, Moscow 119234, \\ Russia, \\ ${ }^{2}$ Institute of Astronomy, Russian Academy of Sciences, Pyatnitskaya st., 48, Moscow 119017, Russia, \\ ${ }^{3}$ Center for Astrophysics - Harvard and Smithsonian, 60 Garden Street MS09, Cambridge, MA 02138, USA, \\ ${ }^{4}$ New York University Abu Dhabi, PO Box 129188 Abu Dhabi, UAE, \\ ${ }^{5}$ Center for Astro, Particle, and Planetary Physics, NYU Abu Dhabi, PO Box 129188, Abu Dhabi, UAE, \\ ${ }^{6}$ Special Astrophysical Observatory, Russian Academy of Sciences, Nizhniy Arkhyz, Karachai-Cherkessian Republic \\ 357147, Russia
}

Giant low surface brightness galaxies (gLSBGs) with the disk radii of up to $130 \mathrm{kpc}$ represent a challenge for currently accepted theories of galaxy formation and evolution, because it is difficult to build-up such large dynamically cold systems via mergers preserving extended disks. We summarize the in-depth study of the sample of 7 gLSBGs based on the results of the performed spectral long-slit observations at BTA SAO RAS, surface photometry and Hi data available in literature. Our study revealed that most gLSBGs do not deviate from the Tully-Fisher relation. We discovered compact elliptical (cE) satellites in 2 out of these 7 galaxies. Provided the low statistical frequencies of gLSBGs and cEs, the chance alignment is improbable, so it can indicate that gLSBGs and $\mathrm{cE}$ are evolutionary connected and gives evidence in favor of the major merger formation scenario. Other formation paths of gLSBGs are also discussed.

Keywords: galaxy evolution, low surface brightness galaxies

DOI: 10.51194 /VAK2021.2022.1.1.161

\section{Introduction}

Giant low surface brightness galaxies (GLSBGs) deserve special interest since they harbor the largest rotating disks in the Universe. These galaxies have baryonic masses reaching $10^{11} \mathrm{M}_{\odot}$ and dynamical masses $\sim 10^{12} \mathrm{M}_{\odot}$. Currently accepted galaxy formation scenarios have hard time explaining their properties because for the mass assembly at such scale one typically needs dozens of minor and major mergers, which would likely destroy the disk, zero out the total angular momentum and turn a galaxy into a giant elliptical. Only a couple of dozens gLSBGs were found since the discovery of the prototypical galaxy Malin 1 in the 80s by [1] so they are considered extremely rare. The question of the gLSBGs formation remains open $[2,3,4,5,6,7]$ despite their importance as a "stress test" for the $\Lambda$ CDM cosmology. An in-plane major merger of two giant spiral galaxies with fine-tuned orbital parameters can end-up in a system resembling a gLSBG [6]. So does a triple major merger with two gas-rich galaxies when a host's hot halo gas cooling is triggered by the cold gas supply from the infalling systems and later forms a giant extended disc [8]. The analysis of the results of EAGLE simulations shows that the most extended LSB disks are formed by mergers [9]. Accretion of cold gas from a cosmic filament or gas-rich satellites [10] on-to a pre-existing high-surface brightness galaxy [11] can also foster a giant disk. The unusually sparse dark matter halo can also lead to the formation of giant LSB disc [2]. In this paper we briefly summarize the efforts that we made to understand the formation scenario of gLSBGs by observing a sample of 7 gLSBGs: Malin 1, Malin 2, NGC 7589, UGC 1378, UGC 1382, UGC 1922, and UGC 6614 .

\section{The results of long-slit spectral observations}

We performed spectral long-slit observations at 6m BTA telescope of SAO RAS using the long-slit regime of SCORPIO and SCORPIO-2 spectrographs $[12,13]$. The details of the data reduction and analysis could be found in [6, 11, 7]. The most prominent result of these observations is the discovered kinematically decoupled components in UGC 1922 and UGC 1382. In UGC 1382, the global gaseous disk counter-rotates with respect to stars, and this suggests the external origin of gas in the extended disk. Stellar populations in the central parts of 5 of the 7 gLSBGs are old and metal-rich supporting the scenario of accretion of gas onto a pre-existing high surface brightness galaxy.

\section{The discovery of the $\mathrm{cE}$ satellites}

The inspection of the archival HST images of the class prototype Malin 1 allowed us to make an important step towards understanding of the nature of gLSBGs. Malin 1 appeared to have two satellites which reminded the low-mass dense compact elliptical galaxies (cE) (see Fig. 1$)$. The cEs are rare low-mass systems $(\sim 0.5 \%$ of dwarf galaxy population) with small sizes $\left(r_{e}<1 \mathrm{kpc}\right)$ and high densities typically hosting old metal-rich stars [15, 16]. They likely originate from tidal stripping [17] of massive disky progenitors losing 90-95 percent of stellar mass [15, 18, 19, 20, 16]. The structural analysis of the WFPC2 data using GALFIT confirmed the cE classification, and the re-analysis of published optical spectra from the Russian 6-m telescope confirmed that these $\mathrm{cE}$ were indeed the satellites of Malin 1. Further visual inspection of high-quality optical image of UGC 1382 from Subaru Hyper-SuprimeCam Strategic survey revealed additional cE-satellite of gLSBG, the SDSS spectral data confirmed the physical association. As the next step we significantly extended our sample of gLSBGs by visual inspection of HSC and DECaLS data (Saburova et al. in prep.) and discovered more cE + gLSBGs systems (Chilingarian et al. in prep.). Given the statistical frequencies of gLSBGs and cEs, the chance of alignment is improbable thus $\mathrm{cE}$ and 

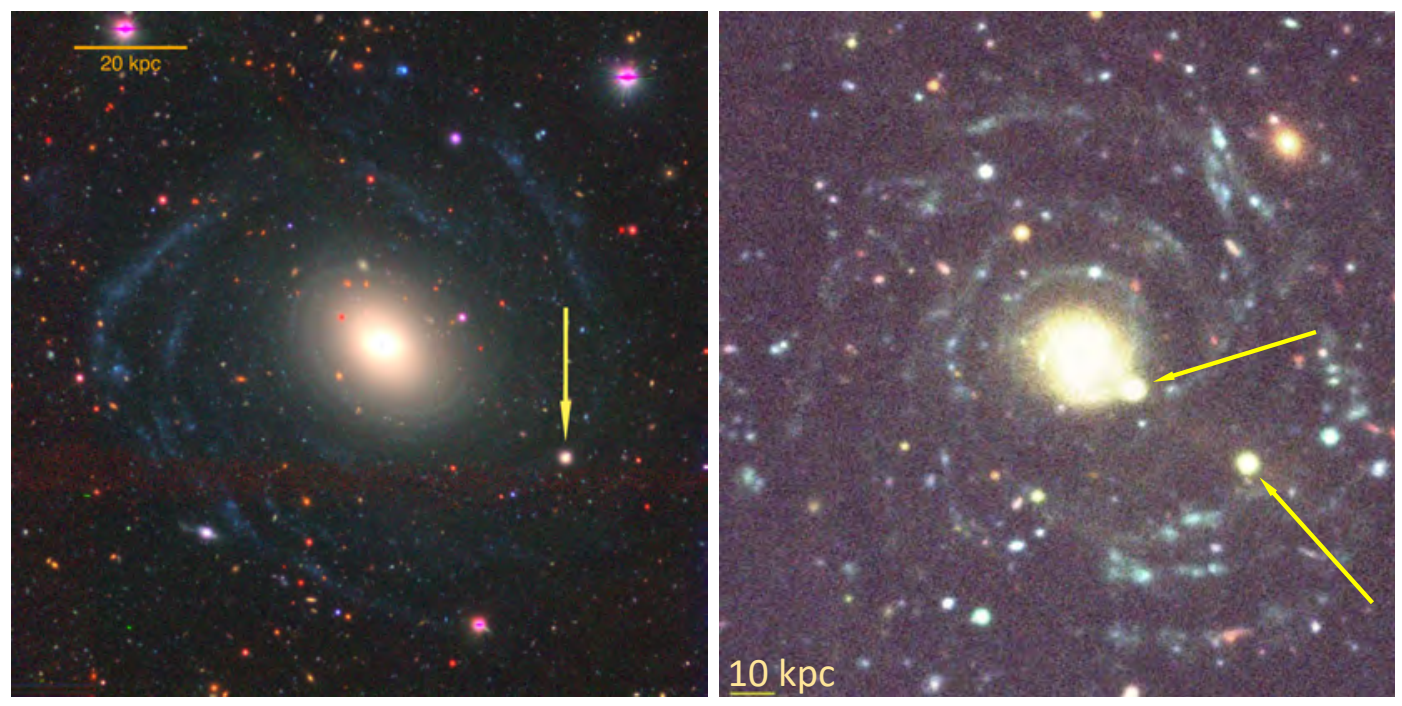

Figure 1: The colour composite images of UGC 1382 from HSC (left-hand panel) and Malin 1 from the CFHTMegacam Next Generation Virgo cluster Survey in u,g and i-bands taken from [14]. Arrows demonstrate the position of $\mathrm{cE}$ satellites

gLSBGs must be evolutionary connected which gives more arguments in favor of merger scenario at least for some of gLSBGs.

\section{The baryonic Tully-Fisher relation}

BTFR [21, 22] between gas+stellar mass and rotation velocity of galaxies is a useful tool for the diagnostic of the formation paths of galaxies. We found out that 6 out of the 7 gLSBGs lie on the high-mass extension of the BTFR which is also the case for the most luminous spiral galaxies [23]. This suggests that gLSBGs are scaled-up versions of less massive disks.

\section{The parameters of dark matter halos}

The parameters of dark matter halos of gLSBGs that we were able to derive from $\mathrm{HI}+$ optical rotation curves show dichotomy. Some gLSBGs have high radial scales of the dark halo density profile, while some others do not. Hence, the gLSBG class is in fact inhomogeneous, and there might exist different formation channels. At the same time according to our findings [7], most gLSBGs have external origin of the material for their extended disks (merger and accretion scenarios).

The mass modelling of the rotation curves was done with the support of the Russian Science Foundation (RScF) grant No. 19-72-20089.

\section{References}

1. G. D. Bothun, C. D. Impey, D. F. Malin, and J. R. Mould, AJ, 94, 23, 1987.

2. A. V. Kasparova, A. S. Saburova, I. Y. Katkov, I. V. Chilingarian, and D. V. Bizyaev, MNRAS, 437, $3072,2014$.

3. G. Galaz, C. Milovic, V. Suc, L. Busta, G. Lizana, L. Infante, and S. Royo, ApJL, 815, L29, 2015.

4. L. M. Z. Hagen, M. Seibert, A. Hagen, K. Nyland, et al., ApJ, 826, 210, 2016.

5. S. Boissier, A. Boselli, L. Ferrarese, P. Côté, et al., A $\& A, \mathbf{5 9 3}$, A126, 2016.

6. A. S. Saburova, MNRAS, 473, 3796, 2018.

7. A. S. Saburova, I. V. Chilingarian, A. V. Kasparova, O. K. Sil'chenko, K. A. Grishin, I. Y. Katkov, and R. I. Uklein, MNRAS, 503, 830, 2021.

8. Q. Zhu, D. Xu, M. Gaspari, V. Rodriguez-Gomez, et al., MNRAS, 480, L18, 2018.

9. A. Kulier, G. Galaz, N. D. Padilla, and J. W. Trayford, MNRAS, 496, 3996, 2020.

10. J. Peñarrubia, A. McConnachie, and A. Babul, ApJL, 650, L33, 2006.

11. A. Saburova, I. Chilingarian, A. Kasparova, I. Katkov, D. Fabricant, and R. Uklein, arXiv e-prints, arXiv:1908.11383, 2019.

12. V. L. Afanasiev and A. V. Moiseev, Astronomy Letters, 31, 194, 2005.

13. V. L. Afanasiev and A. V. Moiseev, Baltic Astronomy, 20, 363, 2011.

14. Junais and S. Boissier, in P. Di Matteo, O. Creevey, A. Crida, G. Kordopatis, J. Malzac, J. B. Marquette, M. N'Diaye, and O. Venot, eds., SF2A-2019: Proceedings of the Annual meeting of the French Society of Astronomy and Astrophysics, Di (2019).

15. I. Chilingarian, V. Cayatte, Y. Revaz, S. Dodonov, D. Durand, F. Durret, A. Micol, and E. Slezak, Science, 326, 1379, 2009.

16. A. Ferre-Mateu, M. Durre, D. A. Forbes, A. J. Romanowsky, A. Alabi, J. P. Brodie, and R. M. McDermid, arXiv e-prints, arXiv:2103.09241, 2021.

17. K. Bekki, W. J. Couch, and M. J. Drinkwater, ApJL, 552, L105, 2001. 
18. J. Price, S. Phillipps, A. Huxor, N. Trentham, et al., MNRAS, 397, 1816, 2009.

19. M. A. Norris, S. J. Kannappan, D. A. Forbes, A. J. Romanowsky, et al., MNRAS, 443, 1151, 2014.

20. I. Chilingarian and I. Zolotukhin, Science, 348, 418, 2015.

21. D. Sprayberry, G. M. Bernstein, C. D. Impey, and G. D. Bothun, ApJ, 438, 72, 1995.

22. S. S. McGaugh and J. M. Schombert, $A p J, \mathbf{8 0 2}, 18,2015$.

23. E. M. Di Teodoro, L. Posti, P. M. Ogle, S. M. Fall, and T. Jarrett, MNRAS, 507, 5820, 2021. 


\section{Spectral study of a candidate to polar-ring galaxies UGC 4261}

L. Shalyapina, O. Merkulova, G. Karataeva, I. Chugunov

liliys@yahoo.com

Saint-Petersburg University, 28 University pr., Petergof, St Petersburg, 198504, Russia

DOI: $10.51194 /$ VAK2021.2022.1.1.162

\section{Introduction}

Galaxy UGC 4261 has a peculiar morphology: the extended structure crossing its irregular body looks like an open ring. The existence of two kinematic almost orthogonal subsystems was obtained in [1] and [2]. We present the detailed spectral study based on the results of observations carried out at the 6-m telescope of the SAO RAS ${ }^{\mathrm{a}}$ with the SCORPIO focal reducer in the modes of FPI and slit spectroscopy, as well as using the MPFS spectrograph.

\section{Results}

Three subsystems in UGC 4261 were revealed: two subsystems are associated with two central bright knots and the third one - with external structures (the NE-loop, south- western tail and outer shell).

The study discovered different patterns of motion of stars and values of their velocity dispersions in the knots, as well as differences in the age and metallicity of the older stellar population in them (the first knot with the age of $8 \pm 2$ Gyr and metallicity $[\mathrm{Fe} / \mathrm{H}]=-1.5 \pm 0.1 \mathrm{dex}$, and the second knot of $12 \pm 3 \mathrm{Gyr}$ and $[\mathrm{Fe} / \mathrm{H}]=-2 . \pm 0.1$ dex). We suppose these knots are most likely the remnants of interacting galaxies. Both nuclei contain a young population of $100 \mathrm{Myr}$ with the metallicity of $0.17 \pm 0.05$ dex.

Tidal structures have similar stellar spectra with deep absorption lines of the Balmer series, the K CaII line. The kinematics of the stellar and gaseous components in these regions coincide. The oxygen abundance obtained from the red spectra is solar and higher than solar.

Based on the length of tidal structures (more than $10 \mathrm{kpc}$ ) [3],[4] and the type of stellar population, the interaction of galaxies took place on the order of several billion years ago. Galaxies of approximately the same mass and presumably of types $\mathrm{Sb} / \mathrm{dSb}$ and $\mathrm{Sd} / \mathrm{dSd}$ rotate around a common dynamic center; they have probably already had one turn. The star formation continues in nuclear regions of galaxies and in tidal structures.

\section{References}

1. V. P. Reshetnikov and F. Combes, $A \& A, \mathbf{2 9 1}, 57,1994$.

2. V. P. Reshetnikov, V. A. Hagen-Thorn, and V. A. Yakovleva, Astronomy Reports, 42, 439, 1998.

3. A. Toomre and J. Toomre, Violent Tides Between Galaxies, 271 (1977).

4. J. E. Barnes, MNRAS, 455, 1957, 2016.

${ }^{\mathrm{a} h t t p s: / / w w w . s a o . r u ~}$ 


\section{Studying the characteristics of spiral galaxies}

P. Smirnova ${ }^{1}$, A. Mosenkov ${ }^{2}$, A. Marchuk ${ }^{1}$

${ }^{1}$ Saint Petersburg State University, Department of Astrophysics, St. Petersburg, 198504 Russia,

${ }^{2}$ Department of Physics and Astronomy, N283 ESC, Brigham Young University, Provo, UT 84602, USA

DOI: $10.51194 /$ VAK2021.2022.1.1.163

All spirals in galaxies can be divided into three general types: grand design, multi-armed, and flocculent (see [1] and references therein). In this study, we explore the difference in the properties of the spiral structure in a different spatial environment: in isolated galaxies and in galaxies located in CGs. We used the HCG [2] and SDSSGA [3]) catalogs for CGs and catalogs of isolated galaxies [4]. Our samples comprise only those spiral galaxies, the inclination, and distance to which allow us to determine the type of the spiral pattern. We visually examine the spiral structure in all galaxies under consideration. Each galaxy is classified as grand-designed (G), multi-armed (M), or flocculent (F) if at least two out of three observers agreed on its type. Using the NED database, we extract their color value, apparent magnitude, and redshift. As a result, we are able to make a comparison of the spiral structure between the isolated galaxies and galaxies in groups. We determine the $M_{r}$ absolute magnitude and $g-r$ color from the retrieved redshifts in SDSS DR16 [5] and their apparent magnitudes taking into account the interstellar extinction and K-correction. From figure 1a, we can see that the galaxies in CGs are slightly brighter in the $r$ band than the isolated galaxies. From figure 1b,c, we can see that the maximum of the $g-r$ color for galaxies in CGs for both the HCG and SDSSGA lies slightly to the right, which means that the isolated galaxies turn out to be bluer than the CGs galaxies.
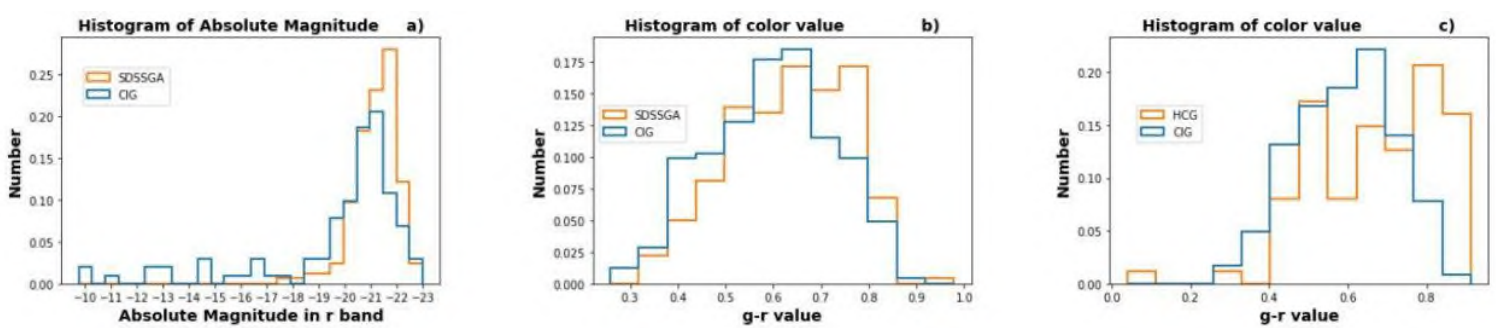

Figure 1: a) Distribution by $\left.\left.M_{r}, b\right), c\right) g-r$ distribution for SDSSGA, CIG and HCG, CIG.

We found that $73 \%$ of galaxies in CGs under study have G spirals versus $41 \%$ for isolated galaxies of the same type. In addition to that, the integral characteristics of the $\mathrm{G}$ spirals in isolation and tight environment are quantitatively slightly different which can be explained by their somewhat different evolutionary paths. This also explains the difference in the type of the spiral pattern: tidal interactions in compact groups induce the formation of $\mathrm{G}$ spirals.

\section{References}

1. S. Savchenko, A. Marchuk, A. Mosenkov, and K. Grishunin, MNRAS, 493, 390, 2020.

2. P. Hickson, ApJ, 255, 382, 1982.

3. J. Sohn, H. S. Hwang, M. J. Geller, A. Diaferio, K. J. Rines, M. G. Lee, and G.-H. Lee, Journal of Korean Astronomical Society, 48, 381, 2015.

4. M. Argudo-Fernández, S. Verley, G. Bergond, S. Duarte Puertas, et al., A\&A, 578, A110, 2015.

5. R. Ahumada, C. A. Prieto, A. Almeida, F. Anders, et al., ApJS, 249, 3, 2020. 


\section{Radio properties of quasars at $z \geq 3$}

Yu. Sotnikova ${ }^{1}$, T. Mufakharov ${ }^{1,2,3}$, A. Mikhailov ${ }^{1}$, M. Mingaliev ${ }^{1,2}$, Yu. Kovalev ${ }^{4}$

lacertae999@gmail.com

${ }^{1}$ Special Astrophysical Observatory of RAS, Nizhny Arkhyz 369167, Russia

${ }^{2}$ Kazan Federal University, Kazan 420008, Russia

${ }^{3}$ Shanghai Astronomical Observatory, Chinese Academy of Sciences, Shanghai 200030, China

${ }^{4}$ Astro Space Center of Lebedev Physical Institute, Moscow 117997, Russia

We studied the radio properties of optically selected quasars with $z \geq 3$. The sample constructed with quasars having the flux densities $S_{1.4 \mathrm{GHz}} \geq 100 \mathrm{mJy}$ at $z \geq 3$ and $S_{1.4 \mathrm{GHz}} \geq 20 \mathrm{mJy}$ at $z \geq 4$. The observations were conducted in $2017-2021$ with the radio telescope RATAN-600. Using the multifrequency quasi-simultaneous data we revealed that a peaked spectral shape is a common feature for $50 \%$ of bright high-redshift quasars, the majority of them are radio-loud with log $R>2.5$. Our data did not reveal any significant correlation between the redshift and spectral index. The average flux density variability of quasars are about $\operatorname{Var}_{S}=0.2-1.0$ at $4.7-11.2 \mathrm{GHz}$.

Keywords: galaxies: active, high-redshift - quasars: general - radio continuum

DOI: 10.51194/VAK2021.2022.1.1.164

\section{Introduction}

At high redshifts most of the detected radio-loud objects are expected to be blazars, a subclass of active galactic nuclei (AGNs) with a relativistic jet pointing toward the observer [1]. Blazars are known to typically have a flat spectrum, but at the same time we known the steep-spectrum [2,3,4] and the gigahertz-peaked spectrum (GPS) population of high-redshift radio-loud quasars [5, 4]. Currently, only a few high-redshift radio-loud quasars are found at $z \geq 6$ (e.g. [6, 7, 8, 9, 10]). They are detected at X-and $\gamma$-rays and present the most powerful supermassive black holes in the Universe. However, radio emission was not detected from quasars at the redshifts $z>7$. It is commonly believed not only due to radio telescopes sensitivity but also because they are radio quiet loud, or a relativistic jet has not formed yet in compact objects at these redshifts. Radio continuum spectrum and radio variability reflect a relativistic jet activity in radio sources. Their features can provide information of the AGNs at earlier stages of evolution. For most quasars at $z>3$ observed radio continuum data are available at very few frequencies. In this study we present new RATAN-600 results for high-redshift quasars obtained in 2017-2021 quasi-simultaneously at 1.2, 2.3, 4.7, 8.2, 11.2, and $22 \mathrm{GHz}$.

\section{Sample and observations}

The sample of $z \geq 3$ bright quasars was selected with NVSS ${ }^{\mathrm{a}} 1.4 \mathrm{GHz}$ flux density limit $S_{1.4 \mathrm{GHz}} \geq 100 \mathrm{mJy}$ and it consists of 102 objects observed in 2017-2020 [12]. The sample of $z \geq 4$ quasars, containing 36 sources, was selected with $S_{1.4 \mathrm{GHz}} \geq 20$ mJy and observed in 2020-2021. 11 of 36 quasars from the $z \geq 4$ sample are also included to the $102 z \geq 3$ sample.

The observations of the samples were conducted with the radio telescope RATAN-600 [13, 14] at six frequencies $(1.25,2.25,4.7,8.2,11.2$, and $22.3 \mathrm{GHz})$ simultaneously. Sources were observed from 3 to 15 times for each observing epoch to improve the signal-to-noise $(\mathrm{S} / \mathrm{N})$ ratio. The measurements were processed using the automated data reduction system [15, 16, 17] and the Flexible Astronomical Data Processing System (FADPS) standard package modules [18] for the broad-band RATAN continuum radiometers.

\section{Results}

We defined the spectral index from the power-law $S_{\nu} \propto \nu^{\alpha}\left(S_{\nu}\right.$ is a flux density at the frequency $\nu$, and $\alpha$ is a spectral index). The variability index $\operatorname{Var}_{S}$ was calculated using the standard formula by [19]. Analysis of averaged radio spectra revealed $\sim 50 \%$ of bright distant quasars at $z \geq 3$ have peaked radio spectra [20,12]. About $25 \%$ and $15 \%$ quasars have flat $(-0.5 \leq \alpha \leq 0)$ and steep $(-1.1<\alpha<-0.5)$ radio spectra, respectively. We did not find quasars with ultra-steep radio spectra $(\alpha \leq-1.1)$ in the both samples. For $15 \%$ quasars the radio spectra are well described by a sum of two spectral components (Figure 1) at low and high frequencies [21, 22]. The obtained spectral classification indicates the dominance of bright compact core emission and insignificant contribution of extended optically thin kpc-scale components in observed radio spectra. There is no any significant correlation between the redshifts and spectral indices. The radio luminosity $L_{4.7}$ was calculated with a median value of $\sim 2 \times 10^{44} \mathrm{erg} \mathrm{s}^{-1}$ for the samples. We estimated the radio loudness log $R$ with a mean value of 3.5 , its values span from 2.1 to 5.4. The majority of the quasars are highly radio-loud with $\log R>2.5$. The flux density variability of quasars is comparable to blazars and $\operatorname{Var}_{S}=0.2-1.0$ at $4.7-11.2 \mathrm{GHz}$.

\section{Acknowledgements}

This work was supported in the framework of the national project "Science" by the Ministry of Science and Higher Education of the Russian Federation under the contract 075-15-2020-778.

\footnotetext{
${ }^{a}$ National Radio Astronomy Observatory (NRAO) VLA Sky Survey [11]
} 


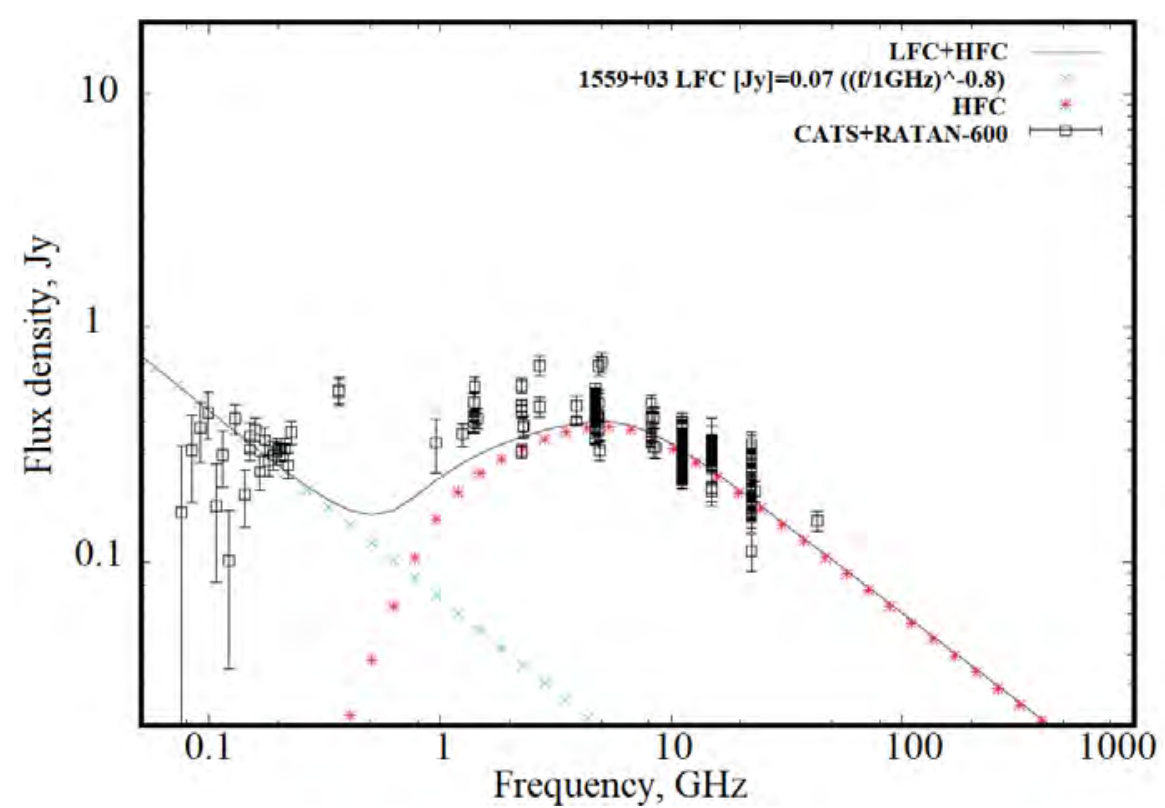

Figure 1: Broadband radio spectrum of the quasar J1559+03 can be explained by the presented model approximation. The blue dotted line represents the linear fitting optically thin model synchrotron emission of extended structures up to kpc scales. The red dotted line represents high frequency model component associated with the parsec scale jet and dominates at several $\mathrm{GHz}$ and above. The black line is the sum of the blue and red lines. In the spectra the RATAN-600 and literature measurements from the CATS database [23] are summarized.

\section{References}

1. C. M. Urry and P. Padovani, PASP, 107, 803, 1995.

2. E. Momjian, C. L. Carilli, and I. D. McGreer, AJ, 136, 344, 2008.

3. S. Frey, L. I. Gurvits, Z. Paragi, and K. É. Gabányi, A\&A, 484, L39, 2008.

4. S. Frey, Z. Paragi, L. I. Gurvits, D. Cseh, and K. É. Gabányi, A\&A, 524, A83, 2010.

5. C. P. O'Dea, MNRAS, 245, 20, 1990.

6. I. D. McGreer, R. H. Becker, D. J. Helfand, and R. L. White, ApJ, 652, 157, 2006.

7. S. Belladitta, A. Moretti, A. Caccianiga, C. Spingola, et al., $A \mathscr{E} A$, 635, L7, 2020.

8. P. Medvedev, S. Sazonov, M. Gilfanov, R. Burenin, et al., MNRAS, 497, 1842, 2020.

9. E. Bañados, C. Mazzucchelli, E. Momjian, A.-C. Eilers, et al., ApJ, 909, 80, 2021.

10. L. Ighina, S. Belladitta, A. Caccianiga, J. W. Broderick, G. Drouart, A. Moretti, and N. Seymour, A\&A, 647, L11, 2021.

11. J. J. Condon, W. D. Cotton, E. W. Greisen, Q. F. Yin, R. A. Perley, G. B. Taylor, and J. J. Broderick, AJ, 115, 1693, 1998.

12. Y. Sotnikova, A. Mikhailov, T. Mufakharov, M. Mingaliev, et al., arXiv e-prints, arXiv:2109.14029, 2021.

13. Y. N. Parijskij, IEEE Antennas and Propagation Magazine, 35, 7, 1993.

14. Y. V. Sotnikova, in I. I. Romanyuk, I. A. Yakunin, A. F. Valeev, and D. O. Kudryavtsev, eds., Ground-Based Astronomy in Russia. 21st Century, 32-40 (2020).

15. P. G. Tsybulev, Astrophysical Bulletin, 66, 109, 2011.

16. P. G. Tsybulev, N. A. Nizhelskii, M. V. Dugin, A. N. Borisov, D. V. Kratov, and R. Y. Udovitskii, Astrophysical Bulletin, 73, 494, 2018.

17. R. Y. Udovitskiy, Y. V. Sotnikova, M. G. Mingaliev, P. G. Tsybulev, G. V. Zhekanis, and N. A. Nizhelskij, Astrophysical Bulletin, 71, 496, 2016.

18. O. V. Verkhodanov, Astronomical Data Analysis Software and Systems VI, A.S.P. Conference Series, $125,46,1997$.

19. M. F. Aller, H. D. Aller, and P. A. Hughes, ApJ, 399, 16, 1992.

20. T. Mufakharov, A. Mikhailov, Y. Sotnikova, M. Mingaliev, V. Stolyarov, A. Erkenov, N. Nizhelskij, and P. Tsybulev, MNRAS, 503, 4662, 2021.

21. Y. Y. Kovalev, Y. A. Kovalev, N. A. Nizhelsky, and A. B. Bogdantsov, PASA, 19, 83, 2002.

22. Y. A. Kovalev, N. S. Kardashev, Y. Y. Kovalev, K. V. Sokolovsky, et al., Advances in Space Research, 65, 745, 2020.

23. O. V. Verkhodanov, S. A. Trushkin, H. Andernach, and V. N. Chernenkov, Bulletin of the Special Astrophysics Observatory, 58, 118, 2005. 


\title{
Vertical structure of barlenses in $\mathrm{N}$-body models
}

\author{
I.S. Tikhonenko ${ }^{1}$, A.A. Smirnov ${ }^{1,2}$, N.Ya. Sotnikova ${ }^{1}$ \\ n.sotnikova@spbu.ru \\ ${ }^{1}$ St. Petersburg State University, Universitetskij pr. 28, 198504 St. Petersburg, Stary Peterhof, Russia, \\ ${ }^{2}$ Central (Pulkovo) Astronomical Observatory of RAS, Pulkovskoye Chaussee 65/1, 196140 St. Petersburg, Russia
}

\begin{abstract}
Bars are common features of most disk galaxies in the local Universe. When viewed face-on, the bars can be classified into so-called face-on peanuts and bars with barlenses based on the shape of the central region. The dynamical origins of the latter type are still disputed in the literature. In this work, we conduct a detailed orbital analysis of the bars in several N-body models and study the vertical structure of the orbital groups comprising them. In particular, all orbital groups that are responsible for the barlens morphology have been identified. We find that a typical barlens is assembled almost exclusively from nearly flat rosette-like orbits trapped around well-known $x_{2}$ and $x_{4}$ families. Our result contradicts the widespread point of view that the barlenses and B/PS bulges (thick part of the bars viewed edge-on) are two manifestations of the same structure observed at different inclination angles.
\end{abstract}

Keywords: galaxies, bars, barlenses

DOI: $10.51194 /$ VAK2021.2022.1.1.165

Stellar bars play an important role in the dynamics of galaxies. In the edge-on view, the bars manifest themselves as the so-called boxy/peanut-shaped (B/PS) bulges, which are the vertically thick inner parts of the corresponding bars (see [1] for a review, $[2,3]$ for statistics).

The term "barlens" is relatively new. It was introduced in [4] to describe the fairly round central parts of bars in some galaxies. A typical barlens covers approximately one half of the bar length [4]. Its outline is circular-like, while its azimuthally averaged profile is exponential, which distinguishes it from the classical bulge [5, 6]. The barlenses are about four times larger than the nuclear disks and are also distinct from standard lenses [7]. In a typical face-on barred galaxy with a barlens, the bar shows almost round isophotes in its inner part (for example, NGC 4314). However, some galaxies have an almost opposite bar morphology, featuring box-like or peanut-like isophotes in the central parts of their bars (for example, IC 5240).

The dynamical structure and origin of the barlens component are still quite uncertain. The most prominent point of view is that the barlenses are simply B/PS bulges viewed face-on. This result is based on the similarity of their sizes and stellar populations $[6,5]$. In this work, we test this finding using self-consistent $N$-body simulations and methods of spectral dynamics.

Our models are described in detail in [8], here we briefly repeat the most important points. Each of the models consists of an exponential and dynamically cold disc ( $4 k k$ particles) isothermal in the vertical direction, a halo with NFW-like profile $(4.5 k k)$, and (in three of four models) a compact classical bulge $(0.4 k k)$. All models are initially set in the equilibrium state. We trace the evolution of each model with gyrfalcON [9] integrator from NEMO bundle [10] for approximately 8 Gyr with a high time resolution $\delta t \approx 1.7 \mathrm{Myr}$. At $t=450(\approx 6 \mathrm{Gyr})$, all models have a prominent $\mathrm{B} / \mathrm{PS}$ bulge in the edge-on view. In the face-on view, with an increase of the initial classical bulge concentration, the bar morphology smoothly changes from a face-on peanut to a bar with a barlens.

To classify the orbits in all models, we use the ratios of dynamical frequencies $\left(f_{x}, f_{y}, f_{z}, f_{R}\right)$ calculated in a coordinate system aligned with the bar (see [11, 8] for details, $[12,13,14]$ for a description of the method). We identify the following orbital groups: a "classic" bar with $f_{R} / f_{x}=2.0 \pm 0.1$, consisting of loop-like orbits ("x1-like") with $f_{y} / f_{x}=1 \pm 0.1$ and 'boxy bar' with $f_{y} / f_{x}>1.1$, as well as two non-classical groups: $\mathrm{bl}_{\mathrm{u}}$ and bl $\mathrm{o}_{\mathrm{o}}$ with $f_{R} / f_{x}>2$ and $f_{R} / f_{x}<2$, respectively. The sum of the last two groups satisfies all barlens properties (for example, it has an exponential surface brightness face-on profile, see the left panel of Fig. 1), while all other groups do not contribute to this morphological feature [8].

Using the described classification scheme, we directly compare edge-on views of all orbital groups in the model with a barlens. We found that the $\mathrm{bl}_{\mathrm{o}}$ and $\mathrm{bl}_{\mathrm{u}}$ groups make little contribution to the $\mathrm{B} / \mathrm{PS}$ bulge. The bl $\mathrm{l}_{\mathrm{u}}$ group consists of rather flat rosette-shaped orbits trapped around $x_{2}$ and $x_{4}$ families, while the B/PS bulge is dominated by $x_{1}$-like orbits [15].

To test the reasoning in [6] and [5] about the similarity of barlens and B/PS bulge sizes, we compare their respective sizes in our models using the method suggested in [16]. We consider several photometric cuts of edge-on galaxy image produced from the model with a barlens morphology at different distances from the disc plane (right panel of Fig.1). The $\mathrm{B} / \mathrm{PS}$ bulge size is then estimated based on the width of the plateau appearing on the furthest profile. Applying this procedure to all particles in the selected model and to the barlens component only, we obtain the barlens size, which turns out twice smaller than the size of the B/PS bulge.

All this evidence contradicts the statement about the common origin of barlenses and B/PS bulges. To understand the nature of barlenses, a detailed kinematic study is required. Such study is currently underway in our group, see also a report on the barlens kinematics prepared by our team in this volume.

This research was supported by the Russian Foundation for Basic Researches grant number 19-02-00249.

\section{References}

1. E. Athanassoula, in Secular Evolution of Galaxies, Cambridge University Press, 305-352 (2013).

2. R. Lütticke, R.-J. Dettmar, and M. Pohlen, A\&A Supplement Series, 145, 405, 2000.

3. A. Yoshino and C. Yamauchi, MNRAS, 446, 3749, 2015.

4. E. Laurikainen, H. Salo, R. J. Buta, and J. H. Knapen, MNRAS, 418, 1452, 2011. 

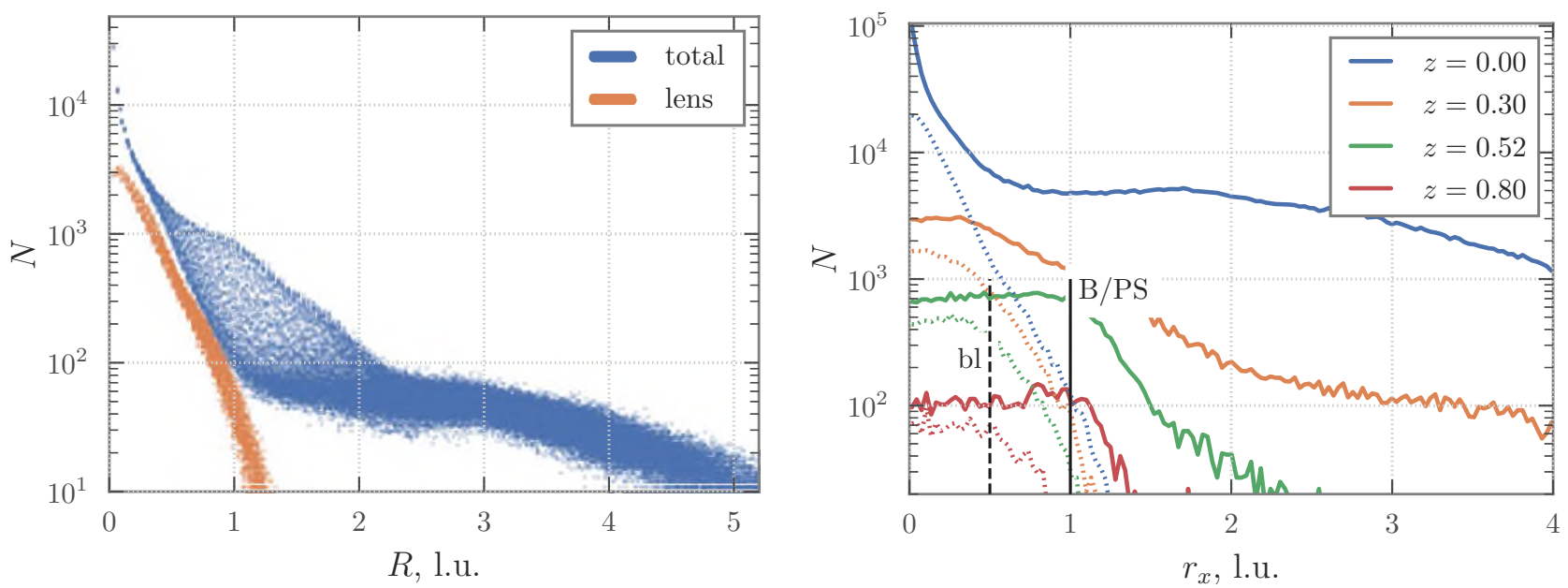

Figure 1: Left panel: face-on profiles for all particles in a model (blue color) and for a barlens component only (brown color). The barlens has an exponential profile. Right panel: photometric cuts for various distances from the disc plane for the total model (solid lines) and for the barlens only (dashed lines). Black lines indicate the measured size.

5. E. Laurikainen and H. Salo, A\& A, 598, A10, 2017.

6. E. Athanassoula, E. Laurikainen, H. Salo, and A. Bosma, MNRAS, 454, 3843, 2015, URL http://adsabs.harvard. edu/abs/2015MNRAS . 454.3843A.

7. J. Kormendy, ApJ, 227, 714, 1979 .

8. A. A. Smirnov, I. S. Tikhonenko, and N. Y. Sotnikova, MNRAS, 2021.

9. W. Dehnen, J. Comput. Phys., 179, 27, 2002.

10. P. Teuben, in Astronomical Data Analysis Software and Systems IV, volume 77, 398 (1995).

11. H. D. Parul, A. A. Smirnov, and N. Y. Sotnikova, ApJ, 895, 12, 2020.

12. J. Binney and D. Spergel, ApJ, 252, 308, 1982.

13. G. Gajda, E. L. Łokas, and E. Athanassoula, ApJ, 830, 108, 2016.

14. M. Valluri, J. Shen, C. Abbott, and V. P. Debattista, ApJ, 818, 141, 2016.

15. I. S. Tikhonenko, A. A. Smirnov, and N. Y. Sotnikova, A\&A, 648, L4, 2021.

16. R. Lütticke, R.-J. Dettmar, and M. Pohlen, A\&A, 362, 435, 2000. 


\title{
On the correlation between optical and $\gamma$-ray emission of the blazar 3C 454.3 during the multi-wavelength flare from 2012 to 2017
}

L. Ugol'kova ${ }^{1}$, E. Shimanovskaya ${ }^{1}$, V. Larionov ${ }^{2}$, S. Savchenko ${ }^{2}$, T. Grishina ${ }^{2}$, O. Ezhkova ${ }^{1}$, E. Kopatskaya ${ }^{2}$, E.

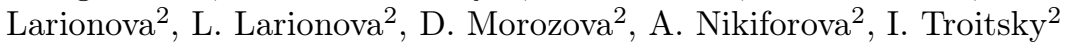
lsu1@mail.ru

\author{
${ }^{1}$ Sternberg Astronomical Institute (SAI), Lomonosov Moscow State University, University Av. 13, 119991, Russia \\ ${ }^{2}$ Astronomical Institute, St. Petersburg State University, 198504, Russia
}

The correlation of optical and $\gamma$-ray emission of the blazar 3C 454.3 during the multi-wavelength flare from 2012 to 2017 is analyzed. The light curves have a complex structure, especially during the period of maximum radiation in 2014, when the activity in the form of a series of 4-5 small flares is observed. The cross-correlation functions exhibit almost zero lag between BVR and $\gamma$-ray emission at different periods of the long-term flares. This suggests that optical and $\gamma$-ray emission come from approximately the same location (probably the base of the jet) and are physically connected. In general, optical emission lags behind gamma radiation except a few cases. It is assumed that both synchrotron radiation and inverse Compton scattering by relativistic electrons and protons, which is typical for strong flares, are present. The rotation of $\gamma$-ray radiation region relative to optical one is supposed.

Keywords: galaxies:active - quasars: individual: $3 C 454.3$

DOI: $10.51194 /$ VAK2021.2022.1.1.166

\section{Introduction}

The active nucleus of the galaxy 3C454.3 $(z=0.859)$ is one of the brightest blazars, which has been studied for many decades at different frequencies of the electromagnetic spectrum. It is an optically violent, flat spectrum radio quasar noted for being among the brightest $\gamma$-ray sources in the sky.

In this work, we investigate properties of the flare activity of the blazar 3C 454.3 observed from the end of 2012 till 2017 in the optical and gamma ranges. The correlation of optical and gamma-ray light curves at different periods of the source activity during the long-duration flare is analyzed.

\section{Observations}

The optical and $\gamma$-ray lightcurves for 3C 454.3 are presented in Fig. 1. The $\gamma$-ray data are obtained on the LAT instrument as part of the Fermi mission ${ }^{\mathrm{a}}$. Every point of the light curve corresponds to the daily-averaged photon flux, integrated in the range of energies from $100 \mathrm{MeV}$ to $300 \mathrm{GeV}$. The optical light curves are derived from the SMARTS project ${ }^{\mathrm{b}}$ and complemented with data, obtained on CrAO AZT-8 and Pulkovo LX-200 telescopes by Saint-Petersburg University team and on the AZT-5 telescope of the Crimean Station of the Sternberg Astronomical Institute of the Moscow State University.
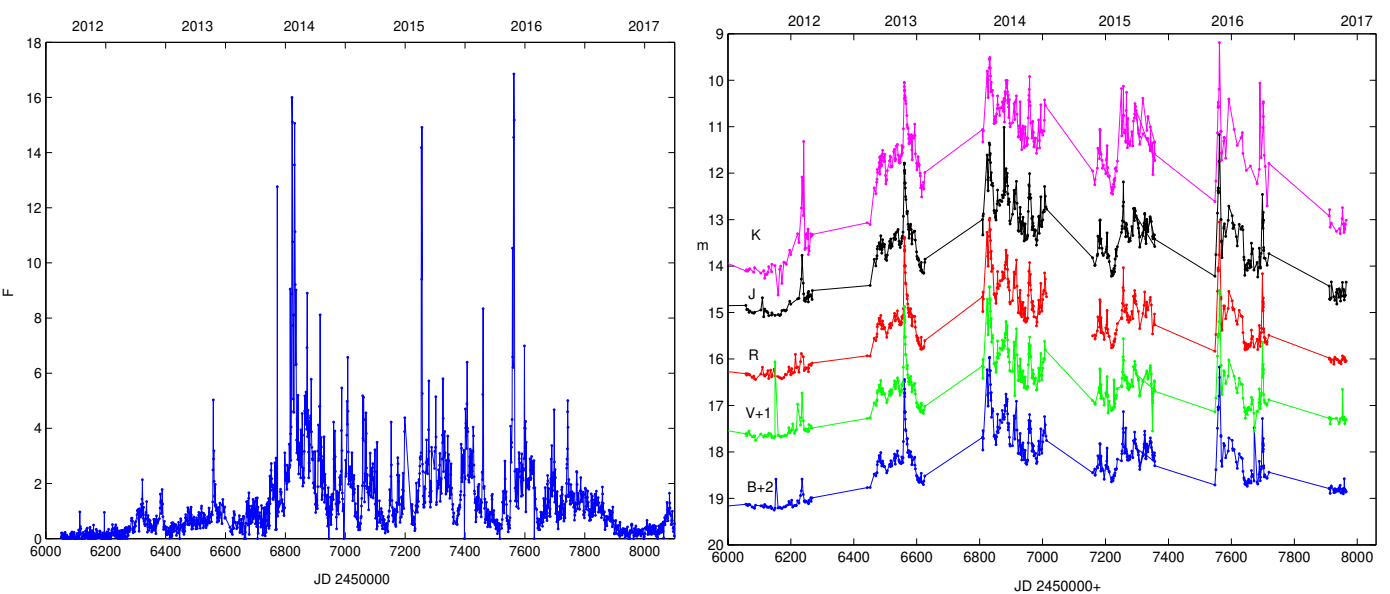

Figure 1: Left panel: Fermi observations of 3C454.3 from 2012 to 2017 in flux units $-F \times 10^{-6} \mathrm{ph} \mathrm{s}^{-1} \mathrm{~cm}^{-2}$. Right panel: SMARTS light curves of 3C454.3 in BVRJK bands.

In the optical and $\gamma$ ranges, the blazar activity in each year is expressed as several strong flares (from 2 to 5 ). The minimum of activity corresponds to JD 2456000-2456270 in 2012 and JD 2457870-2458060 in 2017. The maximum of radiation is in 2014. Optical and gamma-ray light curves of strong flares in 2013, 2014, 2015 and 2016 are shown in Fig. 2,

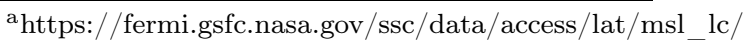

b http://www.astro.yale.edu/smarts/glast/home.php 

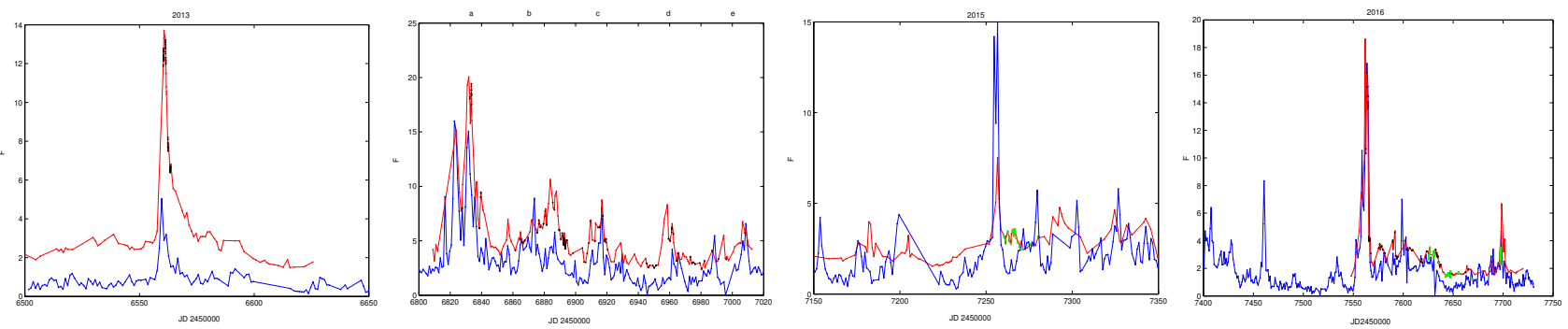

Figure 2: Optical (R band) and $\gamma$-ray light curves of strong flares in 2013, 2014, 2015 and 2016.

where red lines and dots are SMARTS data, black ones are Saint-Petersburg University team data, green dots are SAI data. The flux units are mJy. Blue lines and points are gamma-ray data in flux units $-F \times 10^{-6} \mathrm{phs}^{-1} \mathrm{~cm}^{-2}$. All sharp peaks of $\gamma$-ray radiation are mainly accompanied by optical flares.

\section{Cross-correlation of optical and $\gamma$-ray emissions of $3 \mathrm{C} 454.3$ at different periods of activity}

Using the modified method of Gaskel-Peterson-Spark [1, 2] of calculating the cross-correlation function (CCF), we calculate the correlation coefficient $\mathrm{K}$ and the time lag $\mathrm{D}$ (measured using the CCF maximum and the centroid) between the optical radiation and the $\gamma$-ray one. These values for different periods of the blazar activity are presented in the Table 1 . Plots of overlaid light curves and CCFs for each flare are presented in Fig. 3.

Table 1: Cross-correlation results

\begin{tabular}{cccccccccc}
\hline \hline & 2013 & $2014 \mathrm{a}$ & $2014 \mathrm{~b}$ & $2014 \mathrm{c}$ & $2014 \mathrm{~d}$ & $2014 \mathrm{e}$ & 2015 & $2016 \mathrm{a}$ & $2016 \mathrm{~b}$ \\
\hline $\mathrm{K}$ & 0.97 & 0.96 & 0.42 & 0.88 & 0.43 & 0.85 & 0.77 & 0.94 & 0.65 \\
$D_{\max }$ & +0.7 & +0.3 & +0.8 & +0.8 & -2.3 & 0.0 & 0.0 & -0.6 & +0.4 \\
$D_{\text {centroid }}$ & +0.7 & +0.3 & +0.8 & +0.8 & -2.3 & +0.2 & +0.3 & -0.6 & +0.8 \\
\hline
\end{tabular}
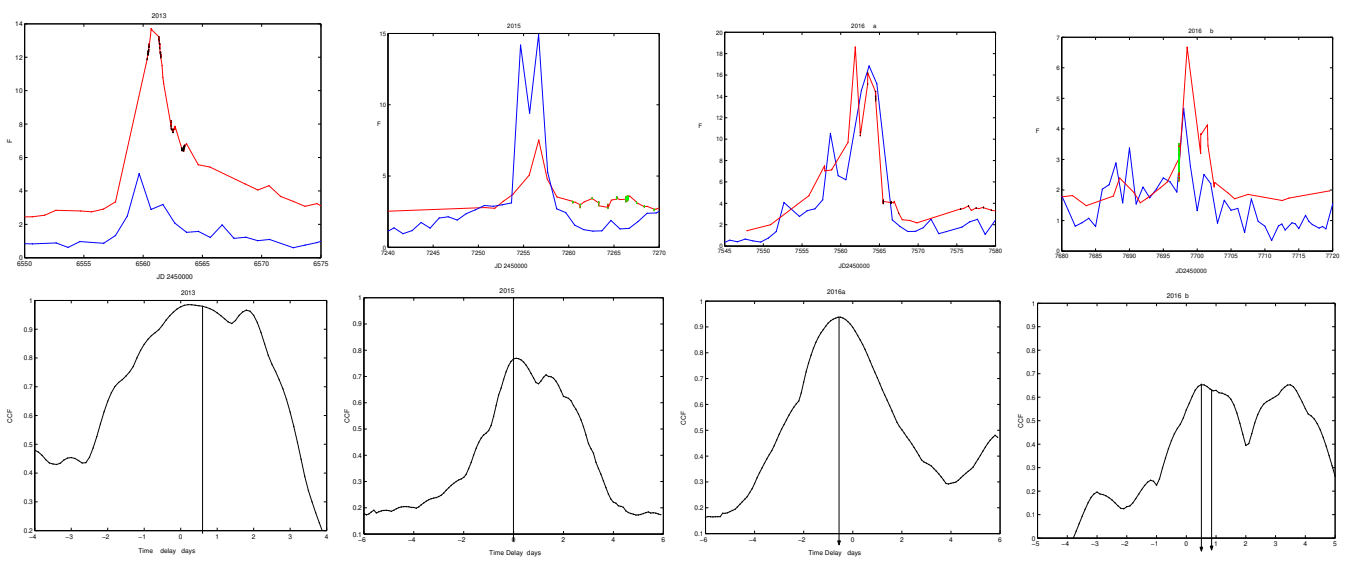

Figure 3: Light curves and CCFs in 2013, 2015, 2016a, 2016b.

For strong flares of every year, mainly the correlation coefficient $K=0.77-0.97$. For $2014 \mathrm{~b}, \mathrm{~d}$ flares, $K \simeq 0.42$. The delay $\mathrm{D}$ for different flares varies from 0 to 2.3 days. The optical radiation is delayed relative to $\gamma$-ray radiation, except for 2016a flare. Since basically there is a high degree of correlation between optical radiation and $\gamma$ radiation with almost zero lag, both optical and $\gamma$ emissions appear from approximately the same region, namely, at the base of the radio jet, and they are physically connected.

Analysis of the variability features during the period of maximum activity (2014, see Fig. 4) reveals that the observed activity is presented as a series of 5 small flares, following each other after 48-50 days, with the repetition of both the shape of light curves and the periodicity of calculated parameters of CCF. Along with the results of recent works, studying jets in active nuclei and the physics of processes associated with the twisted magnetic field both in the accretion disk and in the jet, it substantiate the rotation of the $\gamma$-ray radiation region relative to the optical one. One can assume the existence of a dense plasma bunch, possibly at the base of a relativistic jet, emitting in the gamma range and moving along a helical line $[3,4]$. This is supported by studies of jets in radio range using both ground-based telescopes (VLA, MERLIN, and VLBI) and space instruments such as RadioAstron [5, 6, 7]. According to [7], the flare activity of a blazar can be associated 

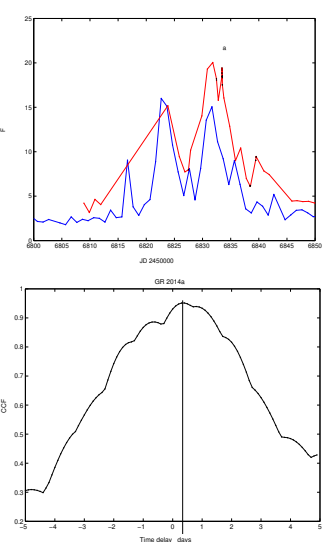

a)
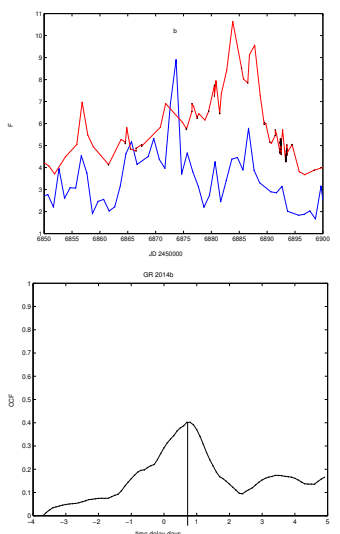

b)
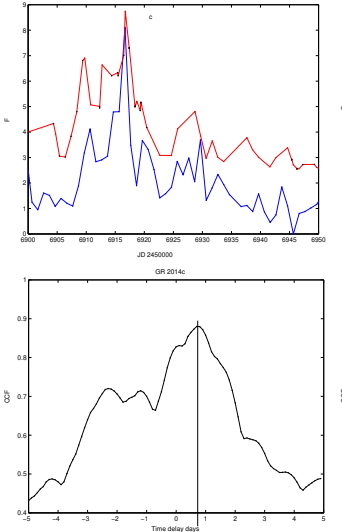

c)
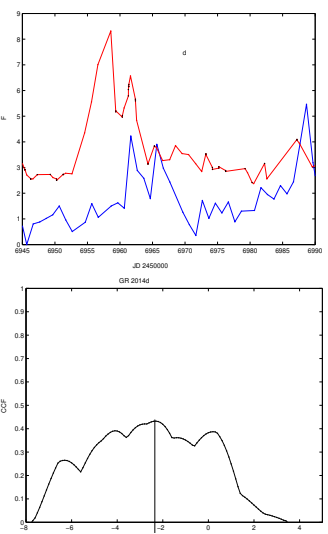

d)
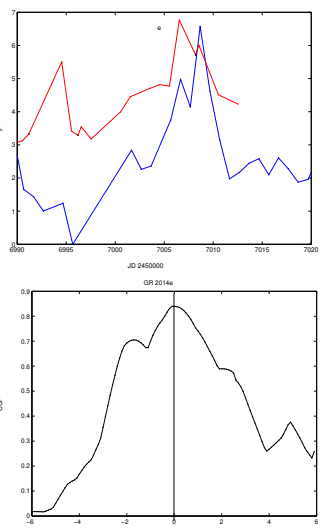

e)

Figure 4: Light curves and CCFs in 2014.

with a jet carrying spiral magnetic fields and propagating in an alternating direction. Likewise, [8] concluded that the most likely scenario involves a region of enhanced emission moving helically inside a curved jet. The helical motion gives rise to quasi-periodic oscillations in a light curve.

\section{References}

1. C. M. Gaskell and B. M. Peterson, ApJS, 65, 1, 1987.

2. V. L. Oknyanskii, Astronomy Letters, 19, 416, 1993.

3. V. M. Larionov, M. Villata, C. M. Raiteri, S. G. Jorstad, et al., MNRAS, 461, 3047, 2016.

4. S. G. Jorstad, A. P. Marscher, D. A. Morozova, I. S. Troitsky, et al., ApJ, 846, 98, 2017.

5. Y. Y. Kovalev, D. I. Zobnina, A. V. Plavin, and D. Blinov, MNRAS, 493, L54, 2020.

6. E. V. Kravchenko, Y. Y. Kovalev, and K. V. Sokolovsky, MNRAS, 467, 83, 2017.

7. M. Lyutikov and E. V. Kravchenko, MNRAS, 467, 3876, 2017.

8. A. Sarkar, A. C. Gupta, V. R. Chitnis, and P. J. Wiita, MNRAS, 501, 50, 2021. 


\section{Edge-on galaxies in the HST COSMOS field}

P. Usachev ${ }^{1,2,3}$, V. Reshetnikov ${ }^{1,2}$, S. Savchenko ${ }^{1,2,3}$

usachev.pavel31@gmail.com

${ }^{1}$ Saint Petersburg State University, St. Petersburg 198504, Russia,

${ }^{2}$ Special Astrophysical Observatory, Russian Academy of Sciences, 369167 Nizhnij Arkhyz, Russia,

${ }^{3}$ Central (Pulkovo) Astronomical Observatory, Russian Academy of Sciences, St. Petersburg 196140, Russia

\section{DOI: $10.51194 /$ VAK2021.2022.1.1.167}

Distant galaxies observed at large $z$ are younger objects compared to the galaxies in the local Universe. This makes it possible to study the evolution of galaxy characteristics with time. Analyzing the disk sizes and thicknesses of distant spiral galaxies comparing with nearby galaxies is part of this research.

This is possible with Hubble Space Telescope (HST) angular resolution [1]. We took the HST COSMOS deep field [2] which covers $1.3^{\circ} \times 1.3^{\circ}$ and contains more than 2 million galaxies. Some of them are extremely distant with $z>5$.

The selection of edge-on galaxies among all galaxies visible at a random angle to the line of sight is a difficult task. Due to limited angular resolution, many structural features of edge-on galaxies are poorly distinguishable. For this reason, the selection is usually done manually by viewing each individual galaxy.

In this work, we used a neural network developed and trained by researchers from St. Petersburg State University and Special Astrophysical Observatory (Savchenko et al., in prep.) to search edge-on galaxies. The processed images of 26113 galaxies from Mandelbaum et al. (2012) [3] were taken as the initial data.

The final sample of galaxies was compiled based on the results of the neural network operation and subsequent visual inspection. As a result, a sample of 950 candidates for edge-on galaxies in the HST COSMOS field was constructed with the average redshift $\langle z\rangle=0.41 \pm 0.20$ [4]. This sample will be used to study in detail the characteristics of the disks of distant galaxies.

This research was supported by the Russian Science Foundation grant 19-12-00145.

\section{References}

1. V. P. Reshetnikov, P. A. Usachev, and S. S. Savchenko, Astronomy Letters, 45, 565, 2019.

2. A. M. Koekemoer, H. Aussel, D. Calzetti, P. Capak, et al., ApJS, 172, 196, 2007.

3. R. Mandelbaum, C. M. Hirata, A. Leauthaud, R. J. Massey, and J. Rhodes, MNRAS, 420, 1518, 2012.

4. O. Ilbert, P. Capak, M. Salvato, H. Aussel, et al., ApJ, 690, 1236, 2009. 


\section{Dust discs in edge-on galaxies: case of NGC 4437}

P. Usachev ${ }^{1,2,3}$, A. Mosenkov ${ }^{4,1}$, S. Savchenko ${ }^{1,2,3}$, G. Gontcharov ${ }^{1}$, V. Il'in ${ }^{1,2,5}$, A. Marchuk ${ }^{1,2}$, A. Smirnov $^{1,2}$ usachev.pavel31@gmail.com

${ }^{1}$ Central (Pulkovo) Astronomical Observatory, Russian Academy of Sciences, Pulkovskoye chaussee 65/1, St. Petersburg 196140, Russia

${ }^{2}$ Saint Petersburg State University, Universitetskij pr. 28, St. Petersburg 198504, Russia

${ }^{3}$ Special Astrophysical Observatory, Russian Academy of Sciences, 369167 Nizhnij Arkhyz, Russia

${ }^{4}$ Department of Physics and Astronomy, N283 ESC, Brigham Young University, Provo, UT 84602, USA

${ }^{5}$ Saint Petersburg University of Aerospace Instrumentation, Bol. Morskaya ul. 67A, St. Petersburg 190000, Russia

We present a study of the distribution of dust in the nearby edge-on galaxy NGC 4437 by modelling its surface brightness distribution based on the Herschel data at $250 \mu \mathrm{m}$. Our model utilizes a 3D exponential disk function with two exponential scales and a break radius which is suitable to describe the observed galaxy profile in great detail. The model is improved by masking background and foreground galaxies and by analysing the brightness distribution of separate bright sources inside the galaxy. As a result, we found that this galaxy has a second dust disc. We also discuss the possibility of detecting a second dust disc component in other edge-on galaxies.

Keywords: edge-on galaxy, dust disc, galaxy dust

DOI: $10.51194 /$ VAK2021.2022.1.1.168

\section{Introduction}

One of the primary constituents of spiral galaxies are gas and dust which serve a source of star formation in galaxies. Dust is particularly interesting for studying in the context of the distribution of matter in galaxies. Also, it plays a crucial role for computational modelling the radiative transfer in the interstellar medium of galaxies.

One of the main goals for creating such programs is to produce images of galaxies in a wide range of spectrum, taking into account absorption, scattering, and re-emission. Dust effectively absorbs light in the visible range and re-emits it in the infrared. Therefore, edge-on galaxies often demonstrate a dark dust line against the background of a thicker bright stellar disc and bulge. Artificial images can be directly or for each component compared with the real galaxies.

It is well-known that general stellar discs in galaxies are typically divided into two discs components based on their kinematic, chemical and photometric properties namely thin and thick disks. In the same time, some recent studies show that dust components in galaxies can also have a complex structure, often revealing a second, thick dust disc or halo [1]. The aim of our study is to explore the dust structure in edge-on galaxies based on far-infrared (FIR) data. In this short paper, we pay our special attention to one of such galaxies, NGC 4437, to describe our fitting method and determine the characteristics of its dust structure.

\section{Data and methods}

In this study, we exploit FIR data obtained with the Herschel Space Observatory [2] for the edge-on galaxy NGC 4437. Specifically, for our analysis we use an image in the SPIRE250 $\mu \mathrm{m}$ from the DustPedia data archive [3].

The advantage of studying edge-on galaxies is that for them we can directly measure the thickness of the disc and identify deviations from a single model component in their surface brightness profiles that may indicate the presence of the second component. Mosenkov et al. (2019) [4] found that a simple exponential model for the dust disc is not quite accurate for describing the dust density distribution in many spiral galaxies.

Many functional approximations can be used to describe the surface brightness distribution in galaxies. In most cases, a simple exponential model for a disc is accurate enough. In the case of NGC 4437 we found the most accurate model by using three-dimensional broken exponential disk. In this model, the galaxy disk is inclined at a certain angle to the line of sight and has two different exponential slopes for the inner and outer parts of the galaxy with the break radius in the transition zone between them. The best-fit model for this galaxy is obtained using the IMFIT code [5].

Three-dimensional broken exponential disk function has the central brightness, both radial exponential scales, the break sharpness, perpendicular scale, and the inclination as free parameters [6]. For edge-on galaxies, the inclination parameter is exactly 90 degrees. Since the galaxy NGC 4437 is not ideally edge-on, the inclination is significant. We fixed the inclination parameter by assigning the value $86^{\circ}$ taken from Mosenkov et al. (2020) [7].

For the best modeling result, some technical procedures have been performed with the initial image taken from the DustPedia archive. These are subtraction of the background, rotation, centering, and masking of bright features which cannot be fitted with a simple smooth disc model.

\section{Results}

We obtained best-fit models for NGC 4437 for two cases: with a single dust disc and with two (thin and thick) dust discs which have different height scales. The fitting results are presented in Table below. Some parameters are fixed. One-dimensional profiles which represent cumulative profiles along radial and vertical axes of the galaxy and the model are shown in Fig. 1. The cumulative profile of the Point Spread Function (PSF) is shown to prove that the galaxy is resolved in both the radial and vertical directions. 


\begin{tabular}{|l|c||c|c|}
\hline \multicolumn{3}{|c|}{ IMFIT function } & \multicolumn{3}{|c|}{ BrokenExponentialDisk3D } \\
\hline parameter & Single disc & \multicolumn{2}{c|}{ Thin and thick discs } \\
\hline inc, inclination from [7], ${ }^{\circ}$ & 86 & 86 & 86 \\
J_0, normalized central surface brightness & $1 \pm 0.0005$ & $1.511 \pm 0.003$ & $0.0381 \pm 0.0004$ \\
h1, inner exponential scale, " & $126.18 \pm 0.06$ & $107.01 \pm 0.09$ & $1310 \pm 60$ \\
h2, outer exponential scale, " & $19.11 \pm 0.05$ & $3.77 \pm 0.05$ & $26.98 \pm 0.09$ \\
r_break, radius of the transition zone, " & $224.76 \pm 0.08$ & 231 [fixed] & 231 [fixed] \\
alpha, break sharpness parameter & 50 & 50 & 50 \\
n, vertical slope function parameter & 100 & 100 & 100 \\
z_0, vertical scale, " & $6.417 \pm 0.002$ & $3.759 \pm 0.009$ & $16.83 \pm 0.04$ \\
\hline
\end{tabular}
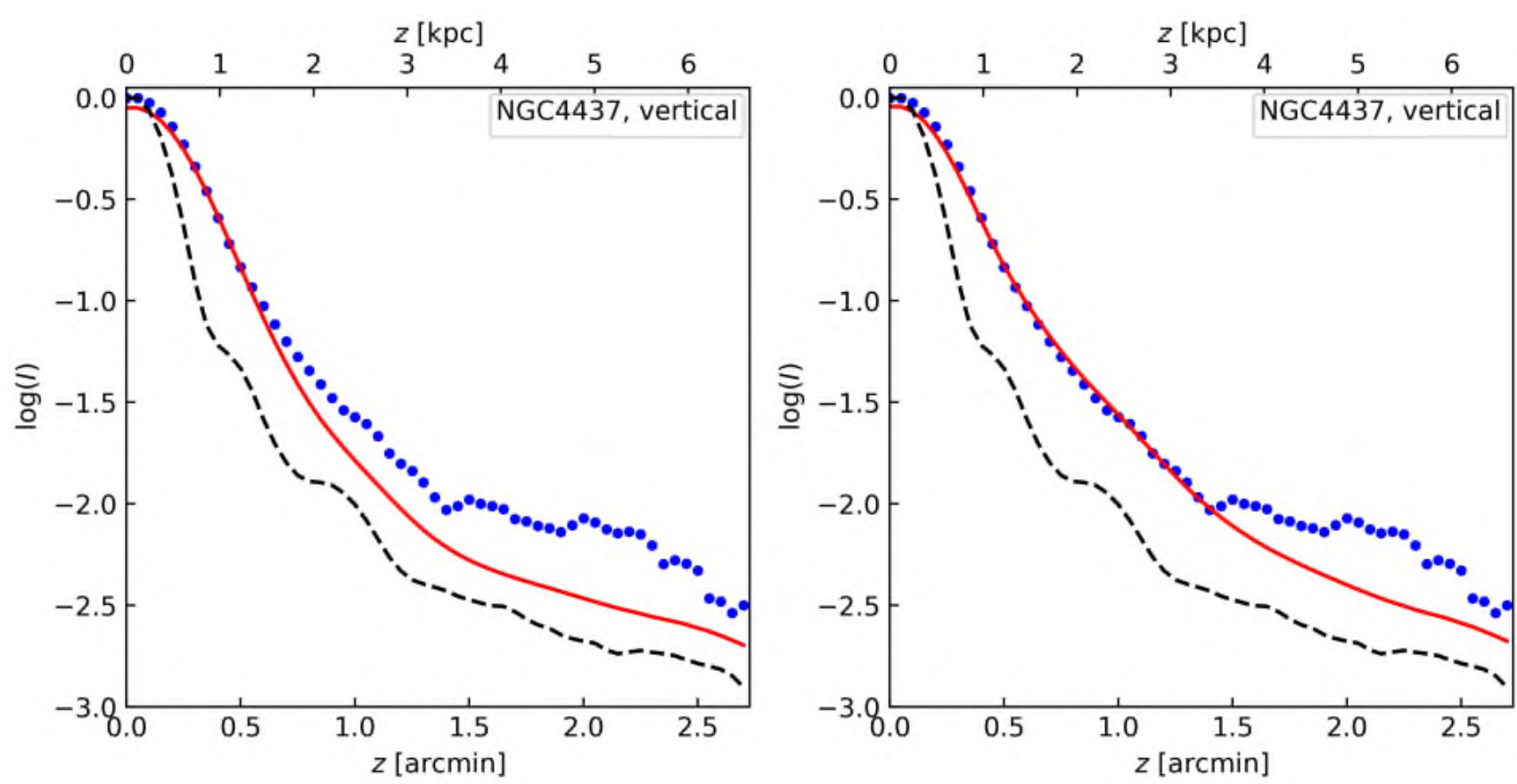

Figure 1: Cumulative median-averaged profiles of the galaxy surface brightness distribution along the direction perpendicular to the disc. The left panel represents the single disc model, and the right panel - the dual disc model. The luminosity units are normalized to the maximum. Blue dots are summarized intensity in a pixel column of the galaxy image perpendicular to the disc with fixed radial distance from the galaxy center. The solid red line is the same for model image. And the black dashed line is the same for the point source.

The result for the single-disk model indicates that there is a significant amount of residual light in the vertical (perpendicular to the disk) direction beyond $1.5 \mathrm{kpc}$. On the other hand, the model with two dust discs is more reliable and adequately describe the visible vertical profile up to $z=3.5 \mathrm{kpc}$.

The method of fitting we used in this study can be successfully applied to other edge-on galaxies for which Herschel data are available.

\section{Conclusion}

We explored the dust structure in the edge-on galaxy NGC 4437 by modelling its surface brightness distribution at $250 \mu \mathrm{m}$. We compared two models for this galaxy: a single-disk model and a model consisting of two (thin and thick) dust disks. The model with two dust disks is proved to be more accurate for this galaxy. This result is a direct evidence that the dust structure of galaxies is more complex than previously thought.

\section{Acknowledgements}

We acknowledge financial support from the Russian Science Foundation (grant no. 20-72-10052).

\section{References}

1. M. Bocchio, S. Bianchi, L. K. Hunt, and R. Schneider, $A \& A$, 586, A8, 2016.

2. G. L. Pilbratt, J. R. Riedinger, T. Passvogel, G. Crone, et al., A\&̊A, 518, L1, 2010. 
3. J. I. Davies, M. Baes, S. Bianchi, A. Jones, et al., PASP, 129, 044102, 2017.

4. A. V. Mosenkov, M. Baes, S. Bianchi, V. Casasola, et al., A $6 A$, 622, A132, 2019.

5. P. Erwin, ApJ, 799, 226, 2015.

6. P. C. van der Kruit and L. Searle, $A \& A$, 95, 105, 1981.

7. A. Mosenkov, R. M. Rich, A. Koch, N. Brosch, et al., MNRAS, 494, 1751, 2020. 


\title{
Grey intergalactic dust could explain the Hubble constant tension
}

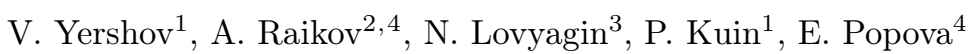 \\ vyershov@list.ru \\ ${ }^{1}$ Mullard Space Scince Laboratory, Holmbury St.Mary, RH5 6NT, UK \\ ${ }^{2}$ Special Astrophysical Observatory, Russian Academy of Sciences, Niznii Arkhyz, 369167 Russia \\ ${ }^{3}$ Saint Petersburg State University, 7-9 Universitetskaya emb., St. Petersburg, 199034, Russia \\ ${ }^{4}$ Pulkovo Observatory, 65(1) Pulkovskoye shosse, St. Petersburg, 196140, Russia
}

It is possible to explain the discrepancy (tension) between the local measurement of the cosmological parameter $H_{0}$ (the Hubble constant) and its value derived from the Planck-mission measurements of the Cosmic Microwave Background (CMB) by considering contamination of the CMB by emission from some medium surrounding distant extragalactic sources, such as extremely cold coarse-grain (grey) dust. Though being distant, such a medium would still be in the foreground with respect to the CMB, and, as any other foreground, it would alter the CMB power spectrum. This could contribute to the dispersion of CMB temperature fluctuations. By generating random samples of CMB with different dispersions, we have checked that the increased dispersion leads to a smaller estimated value of $H_{0}$, the rest of the cosmological model parameters remaining fixed. This might explain the reduced value of the Planck-derived parameter $H_{0}$ with respect to the local measurements.

Keywords: cosmic background radiation, cosmological parameters, intergalactic medium, dust, extinction

DOI: $10.51194 /$ VAK2021.2022.1.1.169

The importance of the issue with the Hubble constant as measured by two different methods (the $H_{0}$ tension) can be appreciated from recent comprehensive reviews on the subject $[1,2]$ and by the fact of special international conferences discussing exclusively this particular issue ${ }^{\mathrm{a}}$.

The Planck space observatory revealed a statistically significant discrepancy between the cosmological parameter $H_{0}$ as calculated within the standard cosmological model by using the Cosmic Microwave Background (CMB) power spectrum, $H_{0}=(67.37 \pm 0.54) \mathrm{km} \mathrm{s}^{-1} \mathrm{Mpc}^{-1}[3]$, and the values of this parameter obtained by using other methods - mostly from direct local measurements [4]. One of these local measurements is based on optical and infrared (IR) observations of variable Cepheid stars, with the recent calculation of $H_{0}$ based on this method being $H_{0}=(73.48 \pm 1.66) \mathrm{km} \mathrm{s}^{-1} \mathrm{Mpc}^{-1}[5]$.

Both local and Planck-derived estimates of $H_{0}$ have passed a number of rigorous tests by considering many possible sources of systematic errors $[6,7,8,9,10]$, but the discrepancy still remains.

Discussing the possible origin of this discrepancy, most authors and reviewers focus primarily on observational biases related to the method of standard-candles, Cepheids and type-Ia supernovae (SN), and on proposals going far beyond the standard cosmological and particle physics models. By contrast, possible biases intrinsic to the CMB measurements by Planck are passed by almost without further thought.

A few years ago, based on the WMAP and Planck-mission results, we found that there exists a correlation between the distances (redshifts) to extragalactic SNe and CMB temperature fluctuations [11, 12], from which we concluded that CMB power spectrum might be distorted by emission from distant (extragalactic) foreground. By using different Planck frequency bands and computing the fractions $\eta_{\nu}(z)$ of blackbody radiation energy as observed in each frequency band $\nu$ for different redshifts $z$ we can estimate the regression line slopes for these bands. The functions $\eta_{\nu}(z)$ are shown in Fig. 1, as calculated for the blackbody temperature $T_{\mathrm{bb}}=5 \mathrm{~K}$ and five of the Planck frequency bands $\nu=\{70,100,143,217,353\} \mathrm{GHz}$. Note that the temperature of the medium in thermal equilibrium with the $\mathrm{CMB}$ must exceed or be equal to $5 \mathrm{~K}$ for the redshifts $z>0.835$, so for these redshifts the functions $\eta_{\nu}(z)$ appear horizontal.

We can calculate slopes $\xi_{\nu}^{c}$ of these functions for different $T_{\mathrm{bb}}$ and compare them with the observed slopes $\xi_{\nu}^{o}$ corresponding to different Planck frequency bands $\nu=\{70,100,143,217,353\} \mathrm{GHz}$ which, according to our previous work, are the following: $\xi_{70}^{o}=0.96 \pm 0.63, \xi_{143}^{o}=1.09 \pm 0.31, \xi_{217}^{o}=0.61 \pm 0.36$ and $\xi_{353}^{o}=-0.99 \pm 0.48$ [12]. The slope for the $100 \mathrm{GHz}$-band was used as a reference for normalisation, so $\xi_{100}^{o}=1.00 \pm 0.39$.

The averages of the calculated slopes of these functions for the temperatures $T_{\mathrm{bb}}=\{3,4,5,6\} \mathrm{K}$ are $\xi_{70}^{c}=$ $0.39 \pm 0.06, \xi_{143}^{c}=1.63 \pm 0.17, \xi_{217}^{c}=1.23 \pm 0.74$ and $\xi_{353}^{c}=-1.43 \pm 0.59$. They are within the $1-\sigma$ tolerance interval with respect to to the experimental values $\xi_{\nu}^{o}$, which indicates that the temperature of the CMB-contaminating ingredient of the intergalactic medium is very low, likely to be between $3 \mathrm{~K}$ and $6 \mathrm{~K}$. This can give clues as to the nature of the medium, which can be coarse-grain (grey) dust, and which for some time has been suspected to populate intergalactic space $[13,14,15,16]$.

This "grey" dust leaves little or no imprint on the spectral energy distribution of background sources. However, it creates the long-known excess of radiation from some extragalactic objects in the far IR at $\lambda \approx 500 \mu \mathrm{m}$, which extends up to centimetre wavelengths and can interfere with the CMB radiation. Such a $500 \mu$ m radiation excess was confirmed and measured by the Herschel space observatory [17]. In the 1990s, this excess was interpreted as an elevated spatial mass density of cold dust with temperatures of 4 to $7 \mathrm{~K}$ [18]. Here we confirm this interpretation from a completely different point of view.

ahttps://www.eso.org/sci/meetings/2020/HO.html 

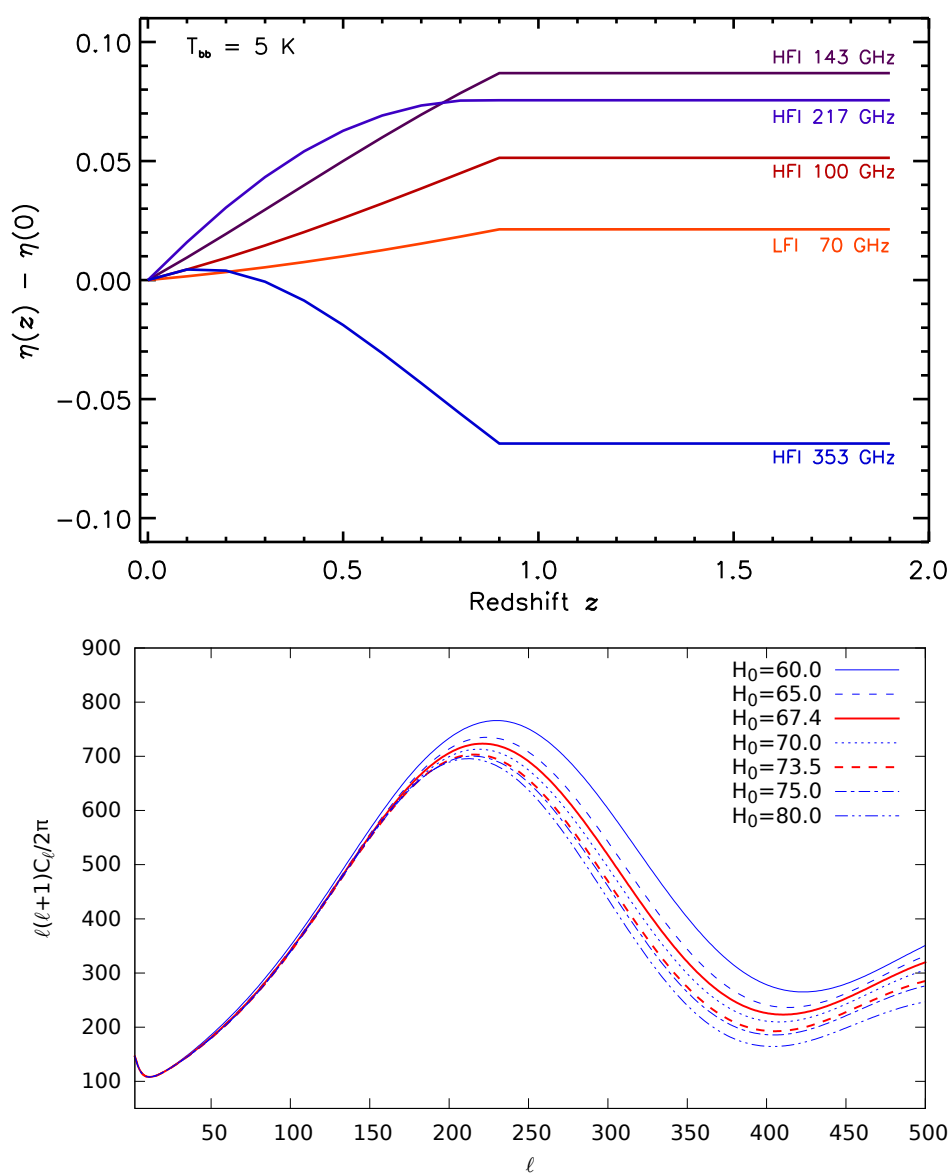

Figure 1: Top: Redshift dependences of the blackbody radiation energy fraction $\eta(z)$ as observed in five Planck frequency bands for $T_{\mathrm{bb}}=5 \mathrm{~K}$; the constant shifts of the curves with respect to each other have been normalised at $z=0$ by subtracting from them their individual values $\eta(0)$; Bottom: CMB power spectra (in the standard normalised presentation) generated by using the code for anisotropies in the microwave background (CAMB tool) for seven different values of $H_{0}$.

The angular sizes of the observed regions with the $500 \mu \mathrm{m}$ emission range from $0.02^{\circ}$ [19] to $0.5^{\circ}$ [17]. So this emission would effectively distort the CMB power spectrum at the multipole moments $\ell \approx 360$ and higher, which would change the estimated parameter $H_{0}$. In order to quantify these changes we have used the code for anisotropies in the microwave background (CAMB) created by [20], which allows the extraction of different cosmological parameters from theoretical CMB power spectra generated by the same code. The calculated changes are shown on the bottom panel of Fig. 1 for the first trough of the CMB power spectrum, where its effect on the calculated parameter $H_{0}$ is quite strong.

In this code, the coefficients $C_{\ell}$ of the CMB power spectrum are calculated as sums of the integrals $a_{\ell m}$, $|m| \leq \ell$, which include temperature fluctuations $\Delta T(x, \phi)$ over the celestial sphere, where $x \in[-1,1]$ is the cosine of the latitude and $\phi \in[0,2 \pi]$ is the longitude. Conversely, the functions $\Delta T(x, \phi)$ are calculated by summing up the integrals $a_{\ell m}$. For a given CMB power spectrum, we have calculated a set of corresponding values of $\Delta T(x, \phi)$ by using random $a_{\ell m}$ for $\ell=0,1, \ldots, \ell_{\max }$ with the restriction $\ell_{\max }=500$. We have taken five equal-spaced values of $H_{0}$, namely, 60, 65, 70, 75 and $80\left[\mathrm{~km} \mathrm{~s}^{-1} \mathrm{Mpc}^{-1}\right]$ plus the values $67.4\left[\mathrm{~km} \mathrm{~s}^{-1} \mathrm{Mpc}^{-1}\right]$ (solid red curve in Fig. 1) and $73.5\left[\mathrm{~km} \mathrm{~s}^{-1} \mathrm{Mpc}^{-1}\right]$ (dashed red curve) corresponding, respectively, to the Planck result and to the local measurements of $H_{0}$.

Additionally, for checking the consistency of our calculations we have taken a few sets of normally distributed randomised values of $a_{\ell m}^{i}, i=1,2, \ldots, 5$, so that for each of the selected values of $H_{0}$, we have obtained five samples of $a_{\ell m}^{H_{0}, i}$ and, correspondingly, five samples of values $\Delta T^{H_{0}, i}$. For each of them, we have calculated the average of the CMB temperature fluctuations $\overline{\Delta T}$ and its standard deviation $\sigma_{T}$.

Here we are mainly interested in the way the values $\sigma_{T}$ change when the parameter $H_{0}$ is varied. For each of these generated sequences, the trend of the calculated values $\sigma_{T}$ was practically the same. Namely, when the dispersion of the CMB temperature fluctuations increases, the value of the estimated $H_{0}$ decreases, the difference between the two discussed $H_{0}$ values 73.5 and $67.4\left[\mathrm{~km} \mathrm{~s}^{-1} \mathrm{Mpc}^{-1}\right]$ being related to $\Delta \sigma_{T}=-0.60 \pm 0.04 \mu \mathrm{K}$. This 
value is the measure of $\mathrm{CMB}$ contamination by photons from the medium surrounding remote clumps of matter and it can thus be used for estimating the amount of cold coarse-grain dust in the intergalactic medium.

\section{References}

1. E. Di Valentino, O. Mena, S. Pan, L. Visinelli, et al., Classical and Quantum Gravity, 38, $153001,2021$.

2. P. Shah, P. Lemos, and O. Lahav, arXiv e-prints, arXiv:2109.01161, 2021.

3. Planck Collaboration, N. Aghanim, Y. Akrami, M. Ashdown, et al., A\&SA, 641, A6, 2020.

4. A. G. Riess, Nature Reviews Physics, 2, 10, 2020.

5. A. G. Riess, S. Casertano, W. Yuan, L. Macri, et al., ApJ, 855, 136, 2018.

6. G. Efstathiou, MNRAS, 440, 1138, 2014.

7. B. R. Zhang, M. J. Childress, T. M. Davis, N. V. Karpenka, C. Lidman, B. P. Schmidt, and M. Smith, MNRAS, 471, 2254, 2017.

8. Planck Collaboration, N. Aghanim, Y. Akrami, M. Ashdown, et al., A 6 A, 607, A95, 2017.

9. S. M. Feeney, D. J. Mortlock, and N. Dalmasso, MNRAS, 476, 3861, 2018.

10. B. Follin and L. Knox, MNRAS, 477, 4534, 2018.

11. V. N. Yershov, V. V. Orlov, and A. A. Raikov, MNRAS, 423, 2147, 2012.

12. V. N. Yershov, V. V. Orlov, and A. A. Raikov, MNRAS, 445, 2440, 2014.

13. M. S. Eigenson, Azh, 26, 278, 1949.

14. F. Zwicky, Morphological astronomy (1957).

15. R. A. González, R. J. Allen, B. Dirsch, H. C. Ferguson, D. Calzetti, and N. Panagia, ApJ, 506, $152,1998$.

16. P. B. Alton, S. Bianchi, and J. Davies, Ap\&SS, 276, 949, 2001.

17. F. Galliano, S. Hony, J. P. Bernard, C. Bot, et al., A $₫ A$, 536, A88, 2011.

18. W. T. Reach, E. Dwek, D. J. Fixsen, T. Hewagama, et al., ApJ, 451, 188, 1995.

19. U. Lisenfeld, F. P. Israel, J. M. Stil, and A. Sievers, $A \mathscr{E} A, \mathbf{3 8 2}, 860,2002$.

20. A. Lewis, Phys. Rev. D, 87, 103529, 2013. 


\section{Relic neutrinos distribution function at low coordinate momentums}

V. Yurchenko, A. Ivanchik

yurchvlad@gmail.com

Ioffe Institute, St. Petersburg 194021, Russia

The effect of processes $p+e^{-} \rightarrow n+\nu_{e}$ and $\mu^{+} \rightarrow e^{+}+\tilde{\nu}_{\mu}+\nu_{e}$ on relic neutrino distribution function in the region of small coordinate momentums is studied. The Boltzmann kinetic equation on the electron neutrino distribution function is solved in this region at temperatures above $10 \mathrm{MeV}$ taking into account generation of electron neutrinos due to the above processes in the assumption that at these temperatures neutrinos of larger momentums are thermalized instantaneously. The effect of thermalization of neutrinos due to their scattering on electrons and positrons on the distribution function in this region at temperatures lesser than $10 \mathrm{MeV}$ is examined.

Keywords: relic neutrinos, kinetic equation, distribution function

DOI: $10.51194 /$ VAK2021.2022.1.1.170

\section{Relic neutrinos}

Relic neutrinos are neutrinos appeared in the Universe shortly after the Big Bang and forming in the modern Universe a background similar to the cosmic microwave background (CMB) of relic photons. Relic neutrinos are described by the Fermi-Dirac distribution function with the temperature $T_{\nu} \approx 1.945 \mathrm{~K}$, that is somewhat lesser in comparison with the photon temperature $T_{\gamma} \approx 2.7255 \mathrm{~K}$.

Neutrinos are weakly interacting particles and therefore relic neutrinos stop interacting between themselves and with other particles at about one second after the Big Bang (see, e.g., [1, 2] for details). Around the same time the epoch of electron-positron annihilation begins and almost all entropy of electrons and positrons is transferred to photons resulting in increase of the photon temperature relative to the neutrino temperature. Nevertheless, a small part of entropy of electrons and positrons is still transferred to neutrinos due to residual processes of scattering of neutrinos and antineutrinos with each other and on electrons and positrons and it results in appearing of nonthermal distortion in neutrino distribution function which can be calculated by means of solving the Boltzmann equation (see, e.g., [3]):

$$
H x \frac{\partial f_{\nu}\left(y_{\nu}\right)}{\partial x}=I_{C o l l}\left(y_{\nu}\right)
$$

where $H$ is the Hubble parameter, $x=m_{0} \cdot a$ is a dimensionless scale factor of the Universe, $m_{0}$ is an arbitrary scale of mass, $a$ is the scale factor of the Universe normalized by the condition $a T_{\gamma}=a T_{\nu}=1$ at temperatures of photons and neutrinos striving for infinity; $y_{\nu}=a \cdot p_{\nu}$ is a dimensionless coordinate momentum of neutrino and $p_{\nu}$ is a physical momentum of neutrino. $I_{C o l l}$ is the collision integral describing all processes listed above.

\section{Relic neutrinos of small momentums}

The described distortion of relic neutrino distribution function is dependent on neutrino momentum. Namely, the quantity $\left(f_{\nu}-f_{0}\right) / f_{0}$ (where $f_{0}=1 /(\exp \varepsilon / T+1)$ is the equilibrium distribution function of neutrinos) increases quadratically with increasing neutrino momentum. Also this distortion differs only slightly from zero in the region of small momentums. And the smaller neutrino momentum, the lesser the distortion. One can find a range of coordinate momentums where the distortion is arbitrary small. It means, that at all temperatures there are neutrino particles in the Universe that are not thermalized. The simple idea comes, that if there existed some processes generating neutrinos in this range of coordinate momentums, these neutrinos would not subsequently thermalize. At some temperature the lowest momentum of neutrinos which thermalize can be estimated from the relation

$$
p^{\prime} \approx \sqrt{\frac{H}{G_{F}^{2} \gamma_{e}^{2} T_{\gamma}^{3}}}, \quad \gamma_{e} \approx \frac{T_{\gamma}}{m_{e}},
$$

where $G_{F}$ is the Fermi coupling constant. In the present work we considered the effect of the processes $p+e^{-} \rightarrow$ $n+\nu_{e}$ and $\mu^{+} \rightarrow e^{+}+\tilde{\nu}_{\mu}+\nu_{e}$ that can generate electron neutrinos of arbitrary small momentums on the electron neutrino distribution function in the range of momentums $<p^{\prime}$ at temperatures from $10 \mathrm{MeV}$ to the temperature defined by eq. (2), given the value of $p^{\prime}$.

\section{Generation of neutrinos of small momentums}

The two processes are considered in this paper that can generate neutrinos of arbitrary small momentum. The first one is electron capture on proton $p+e^{-} \rightarrow n+\nu_{e}$. It is threshold process with the required total energy of 


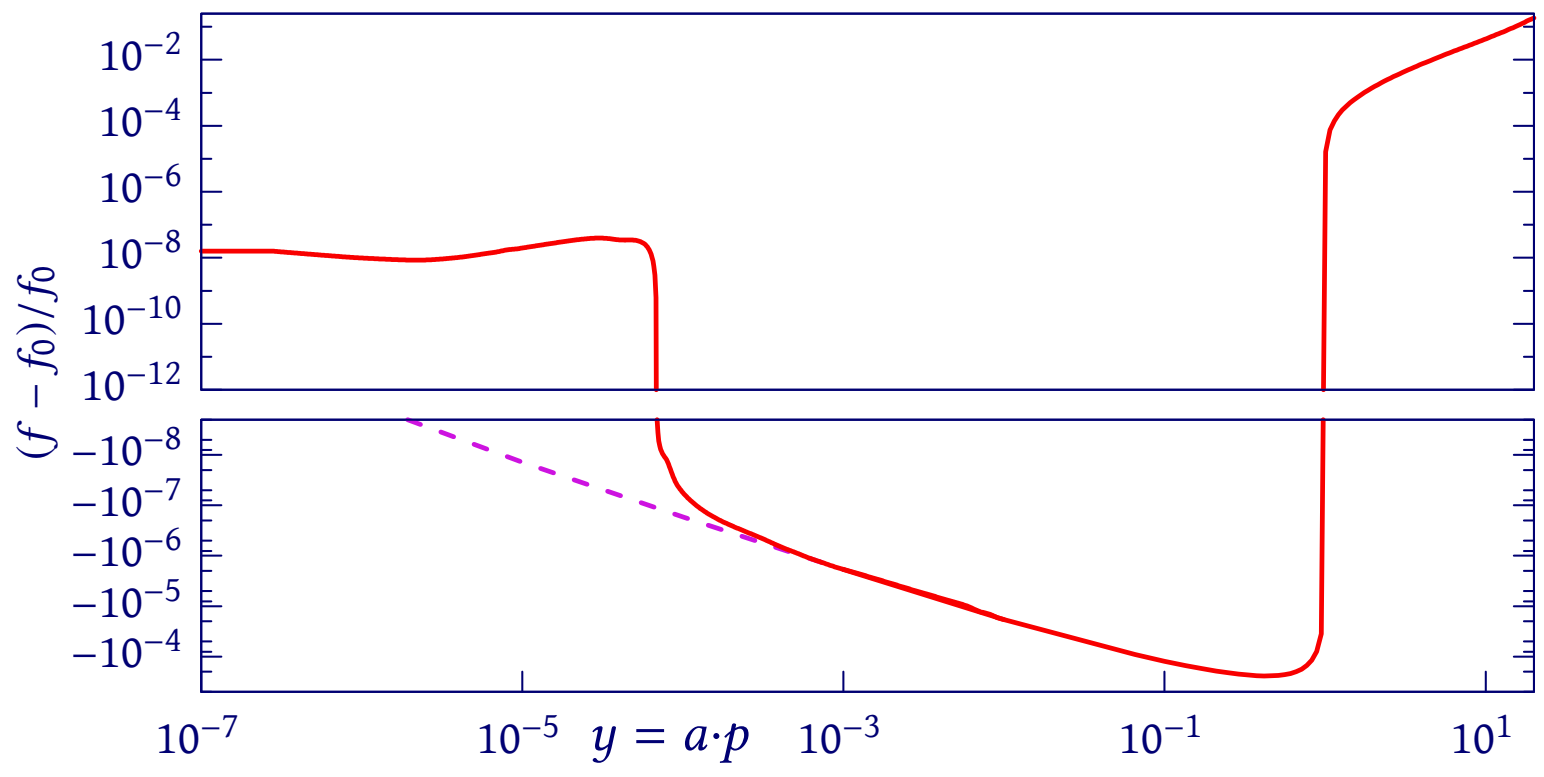

Figure 1: The resulting distortion of electron neutrino distribution due to processes generating neutrinos taking into account thermalization at temperatures $<10 \mathrm{MeV}$. The effect of thermalization dominates at momentums $>10^{-3}$. The effect of generation of neutrinos due to processes under consideration dominates at momentums $<10^{-4}$. The distortion without the processes generating neutrinos at momentums $\lesssim 10^{-3}$ is shown with dashed magenta line.

collision equal $m_{n}-m_{p}-m_{e} \equiv \Delta-m_{e} \approx 0.782 \mathrm{MeV}$. The second one is muon or antimuon decay for the time $\tau_{\mu} \approx 2.2 \cdot 10^{-6}$ to form electron antineutrino or neutrino respectively that possesses continuous spectrum.

The effect of these processes on neutrino distribution in the range of low momentums can be found by means of solving eq. (1) with appropriate rhs.:

$$
I_{\text {Coll }}^{p+e}=\frac{2 \pi^{2}}{\tau_{n} \lambda_{0}} n_{p}(x) \sqrt{\left(\Delta+p_{\nu}\right)^{2}-m_{e}^{2}}\left(\Delta+p_{\nu}\right) f_{e}\left(\Delta+p_{\nu}\right)\left(1-f_{\nu}\left(\varepsilon_{e}\right)\right)
$$

for the process of electron capture and

$$
I_{\text {Coll }}^{\mu}=\frac{1}{\tau_{\mu}} \frac{n_{\mu}}{n_{\nu_{e}}} w^{2}(1-w)\left(1-f_{\nu}\left(\varepsilon_{e}\right)\right), \quad w=2 \frac{p_{\nu}}{m_{\mu}}
$$

for the process of muon/antimuon decay. In these expressions $\tau_{n}$ is neutron lifetime, $\lambda_{0}$ is some normalization constant of dimension $\left[\mathrm{MeV}^{5}\right], n_{p}$ is the concentration of protons in the matter of the Universe, $f_{e, \nu}$ are distribution functions of electrons and neutrinos; $n_{\mu}$ and $n_{\nu_{e}}$ are concentrations of muons and electron neutrinos respectively, $m_{\mu}$ is the muon mass. In these formulas $p_{\nu}$ should be expressed through $y_{\nu}$. The electron neutrino spectrum from muon decay is taken in [4].

The resulting distortion of electron neutrino distribution function due to net effect of these processes in the considered range is obtained as

$$
\left.\Delta f_{\nu}\right|_{y_{\nu}}=\int_{x_{0}}^{x_{1}} \frac{\mathrm{d} x}{H x}\left(I_{\text {Coll }}^{p+e}\left(y_{\nu}\right)+I_{\text {Coll }}^{\mu}\left(y_{\nu}\right)\right)
$$

with $x_{0}$ corresponding the maximum temperature in the Universe when neutrinos of momentum $y_{\nu}$ were nonthermalizing and $x_{1}$ corresponding $T_{\gamma}=10 \mathrm{MeV}$.

After this distortion was calculated the effect of thermalization processes on the obtained distorted distribution function at temperatures $<10 \mathrm{MeV}$ was examined. The final result for the quantity $\left(f_{\nu}-f_{0}\right) / f_{0}$ is presented in Fig. 1.

\section{Results}

In the present paper the distortion of electron relic neutrinos distribution function due to processes $p+e^{-} \rightarrow n+\nu_{e}$ and $\mu^{+} \rightarrow e^{+}+\tilde{\nu}_{\mu}+\nu_{e}$ in the range of low coordinate momentums was calculated. At temperatures $<10 \mathrm{MeV}$ the distorted distribution function obtained was summarized with the effect of neutrino thermalization. It turned out, 
that in the range of coordinate momentums $\lesssim 10^{-4}$ an positive addition to neutrino distribution function appears due to considered processes, while without these processes this addition would be negative.

Thus, a numerical scheme was tried out that allows to test the effect of various processes generating neutrinos in the range of low momentums on relic neutrino distribution function with subsequent study of thermalization process on obtained distribution function.

\section{References}

1. S. Weinberg, Cosmology (2008).

2. D. S. Gorbunov and V. A. Rubakov, Introduction to the Theory of the Early Universe: Hot Big Bang Theory (2011).

3. A. D. Dolgov, S. H. Hansen, and D. V. Semikoz, Nuclear Physics B, 503, 426, 1997.

4. P.A. Zyla et al. (Particle Data Group), Progress of Theoretical and Experimental Physics, 2020, 2020, URL https://doi.org/10.1093/ptep/ptaa104,083C01. 


\title{
Kinematic analysis of $N$-body galaxy models with barlenses
}

\author{
D.A. Zakharova ${ }^{1}$, I.S. Tikhonenko ${ }^{1}$, A.A. Smirnov ${ }^{1,2}$, N.Ya. Sotnikova ${ }^{1}$ \\ n.sotnikova@spbu.ru \\ ${ }^{1}$ St. Petersburg State University, Universitetskij pr. 28, 198504 St. Petersburg, Stary Peterhof, Russia, \\ ${ }^{2}$ Central (Pulkovo) Astronomical Observatory of RAS, Pulkovskoye Chaussee 65/1, 196140 St. Petersburg, Russia
}

\begin{abstract}
The relationship between the three-dimensional structure of bars and their morphology in a face-on projection is not studied in sufficient detail. In [1] and [2], we distinguished different orbital components of bars with barlenses in several numerical models. In this work, we studied the impact of these orbital components on $h_{3}$ and $h_{4}$ parameters of the line-of-sight velocity distribution Hermitian series expansion. For a typical model with a barlens, we found that $h_{4}$ minima lie outside the barlens region. For individual orbital groups, we obtained that a more extended $b_{\circ}$ family contributes to the negative regions of $h_{4}$ profile, while a more compact $\mathrm{bl}_{\mathrm{u}}$ group does not have any connection with these regions and exhibits fast rotation. We conclude that the barlens orbital families do not contribute to the $h_{4}$ minima, which is the main kinematic signature of the $\mathrm{B} / \mathrm{PS}$ bulges if the galaxy is viewed face-on.
\end{abstract}

Keywords: galaxies, kinematics, bars, barlenses

DOI: 10.51194 /VAK2021.2022.1.1.171

\section{Bars and barlenses}

In disc galaxies, viewed face-on, one can highlight two distinct types of bars depending on the shape of the isophotes in the area occupied by the bar. The first type is the one in which the isophotes have a peanut-like profile ("peanutshaped bar"), while the prominent signature of the second type is a fairly round central part, sometimes also called "barlens" [3]. In edge-on galaxies, B/PS bulges can be found. They are the vertical extensions of the bars and are indicators of the bars three-dimensional structure [4]. [5] and [6] found that the linear sizes and stellar population of barlenses and B/PS bulges are nearly the same. Therefore, these authors suggested that the barlenses are simply $\mathrm{B} / \mathrm{PS}$ bulges viewed face-on.

In our previous works $[7,1,2]$, we studied the orbital composition of the bars in several self-consistent $N$-body models using the methods of spectral dynamics Specifically, we characterised each orbit, captured by the bar, in terms of its main frequencies: $f_{x}, f_{y}$, and $f_{\mathrm{R}}$, where $R$ is a cylindrical radius. Based on the respective resonance ratios, $f_{\mathrm{R}} / f_{x}$ and $f_{x} / f_{y}$, we distinguished several orbital groups that build the bar and the barlens in the considered models: a 'classic' bar with $f_{R} / f_{x}=2.0 \pm 0.1$, consisting of loop-like orbits ('x1-like') with $f_{y} / f_{x}=1 \pm 0.1$, a 'boxy bar' with $f_{y} / f_{x}>1.1$, and two non-classical groups: $\mathrm{bl}_{\mathrm{u}}$ and $\mathrm{bl}_{\mathrm{o}}$ with $f_{R} / f_{x}>0$ and $f_{R} / f_{x}<0$, respectively. In [1], it was found that a typical barlens is assembled from $b_{\mathrm{u}}$ and $\mathrm{bl}_{\mathrm{o}}$ orbital fimilies. Studying the vertical profiles of these orbital groups, [2] came to conclusion that the barlenses and the B/PS bulges are dynamically distinct systems supported by different types of orbits.

In this work, we study the kinematic properties of the individual orbital groups distinguished in [1] and [2] aiming to provide more arguments to separate the barlenses and B/PS bulges in observational studies.

To study the kinematic properties of a barlens component in our models, we construct synthetic data cubes for different inclinations of the disc and bar position angles. Then, we calculate the coefficients of the line-ofsight velocity distribution (LOSVD) Hermitian series expansion up to the fourth order. The first two non-trivial coefficients $h_{3}$ and $h_{4}$ are responsible for asymmetric and symmetric deviations from the Gaussian, respectively [8]. These parameters are important kinematic indicators, because B/PS bulges are proven to be associated with the minima of $h_{4}$ parameter values $[9,10]$.

We prepared the data cubes for all models (X, Xb, Blx, BL) for different inclinations $\left(i=20^{\circ}, 40^{\circ}, 60^{\circ}\right)$ and position angles $\left(\mathrm{PA}=0^{\circ}, 45^{\circ}, 90^{\circ}\right)$. The region of $h_{4}$ negative values (Fig. 1, left), roughly corresponds to the area occupied by the $\mathrm{B} / \mathrm{PS}$ bulge.

To determine the impact of different orbital families on the $h_{4}$ profile, we used the following technique. We cut out all the orbits of some specific family from the whole model cube and assessed the respective velocity profile changes (Fig. 1, middle panel). This procedure was repeated separately for each of the families that constitute the bar and the barlens. We applied this analysis to each pixel in the cube and thus highlighted the areas dominated by different orbital families (Fig. 1, right panel). Using this method, we localize the areas where the barlens turns out to be the main contributor of the $h_{4}$ profile. Fig. 2 shows these areas by colors for the whole barlens (left panel) and only $\mathrm{bl}_{\mathrm{o}}$ (middle panel) and $\mathrm{bl}_{\mathrm{u}}$ (right panel) parts. From the figure, it can be seen that both of these families cannot be associated with the minima of $h_{4}$. Thus, we can conclude that the barlens orbital families do not contribute to the main kinematic signature of the B/PS bulges. Moreover, if we consider the linear size of the barlens, it is obvious that the minima of $h_{4}$ lies outside the barlens region.

For all models with different face-on bar morphology we obtained the LOSVD moment maps, which can be directly compared with observational data.Using these maps, we separately studied the kinematic properties of previously identified orbital groups that build the bar and the barlens in galaxy models. We found that a more extended $\mathrm{bl}_{\mathrm{o}}$ family contributes to the negative regions of $h_{4}$ profile, while a more compact bl $\mathrm{l}_{\mathrm{u}}$ group does not 

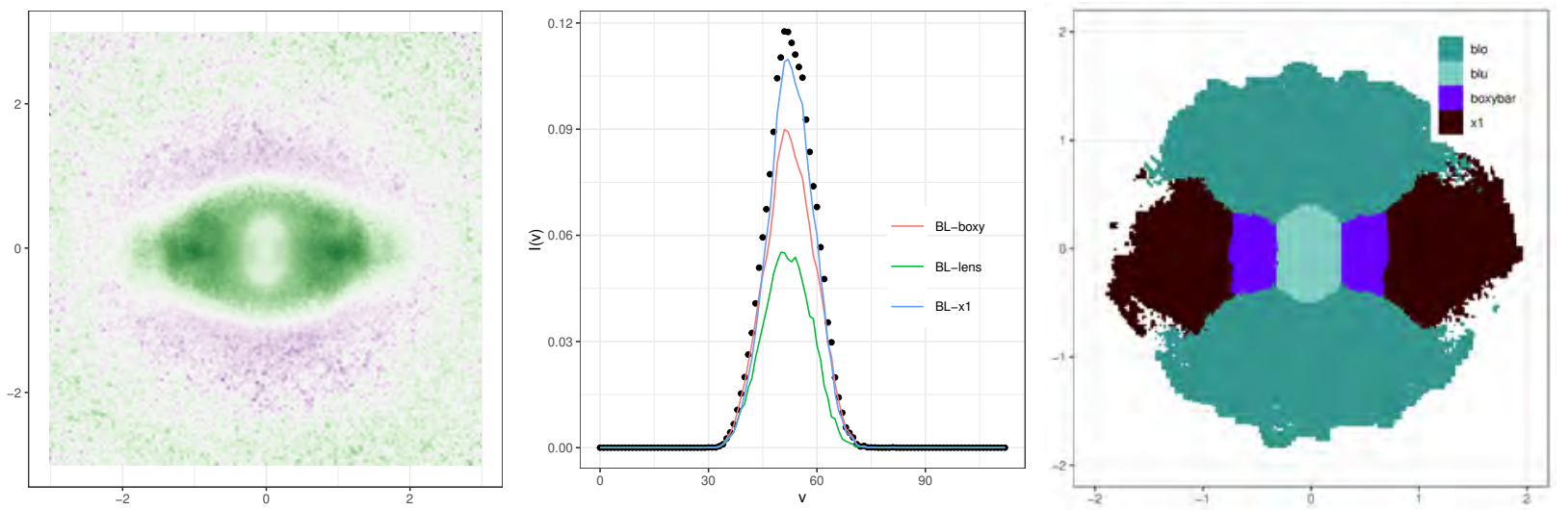

Figure 1: Left panel: the map of $h_{4}$ parameter for the model with the barlens, $i=20^{\circ}, \mathrm{PA}=0^{\circ}$. The negative values are indicated by green color, while the positive ones are in violet. Middle panel: the LOSVD's in pixel $(165,134)$. The dotted black line is a total distribution, the colored lines depict the distributions with a specific orbital group removed (the group name is given in the legend). Right panel: the map, indicating the dominant orbital group in each pixel.
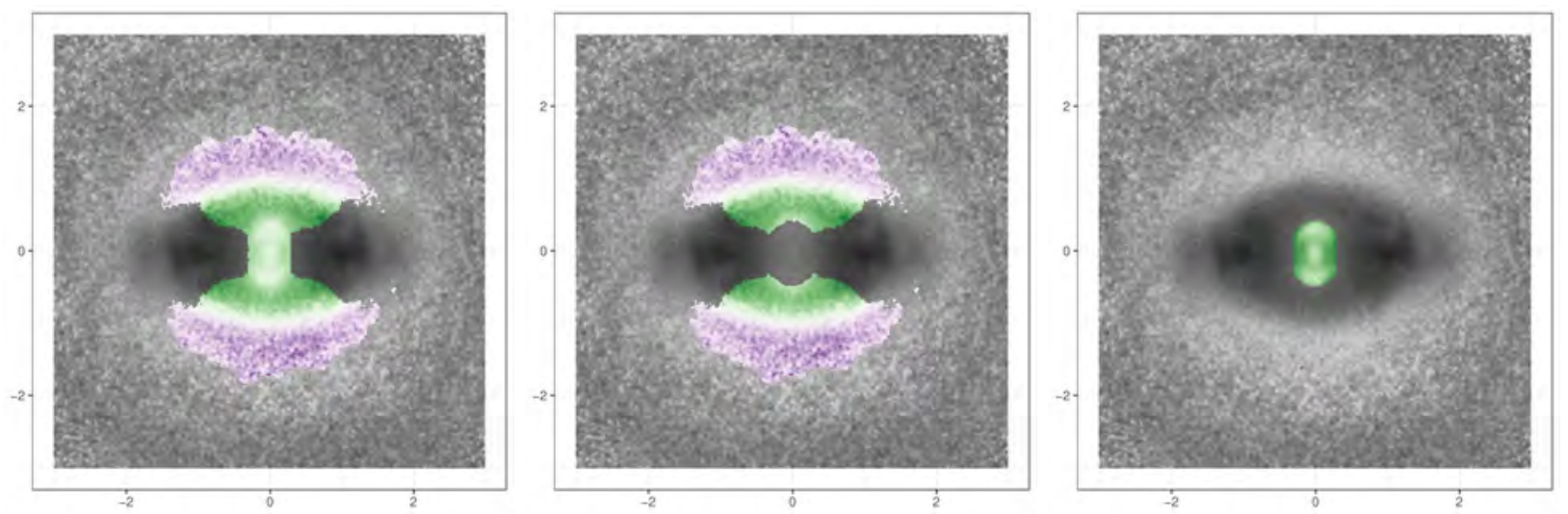

Figure 2: From left to right: the areas of the main contribution of the barlens (the sum of $\mathrm{bl}_{\mathrm{u}}$ and $\mathrm{bl}_{\mathrm{O}} \mathrm{groups}_{\text {) and }}$ separately the $\mathrm{bl}_{\mathrm{o}}$ and $\mathrm{bl}_{\mathrm{u}}$ groups are colored on the $h_{4}$ maps for the model with barlens $\left(i=20^{\circ}, \mathrm{PA}^{\circ} 0^{\circ}\right)$.

have any connection with these regions and exhibits fast rotation. Our data reproduces the well-established face-on kinematic signature of B/PS bulges - the symmetric minima on $h_{4}$ maps. We associate these minima with $x_{1}$ orbital group.

This research was supported by the Russian Foundation for Basic Researches grant number 19-02-00249.

\section{References}

1. A. A. Smirnov, I. S. Tikhonenko, and N. Y. Sotnikova, MNRAS, 2021.

2. I. S. Tikhonenko, A. A. Smirnov, and N. Y. Sotnikova, $A \& A$, 648, L4, 2021.

3. E. Laurikainen, H. Salo, R. J. Buta, and J. H. Knapen, MNRAS, 418, 1452, 2011.

4. F. Combes and R. H. Sanders, $A \mathscr{E} A$ A, 96, 164, 1981.

5. E. Athanassoula, E. Laurikainen, H. Salo, and A. Bosma, MNRAS, 454, 3843, 2015.

6. E. Laurikainen and H. Salo, A\&A, 598, A10, 2017.

7. H. D. Parul, A. A. Smirnov, and N. Y. Sotnikova, ApJ, 895, 12, 2020.

8. R. P. van der Marel and M. Franx, ApJ, 407, 525, 1993.

9. V. P. Debattista, C. M. Carollo, L. Mayer, and B. Moore, ApJ, 628, 678, 2005.

10. F. Iannuzzi and E. Athanassoula, MNRAS, 450, 2514, 2015. 


\title{
Sites of star formation in tidal structures
}

\author{
A. $Z_{\text {Zasov }}^{1,2}$, A. Saburova ${ }^{1}$, O. Egorov ${ }^{1}$ \\ zasov@sai.msu.ru \\ ${ }^{1}$ Sternberg Astronomical Institute, Moscow M.V. Lomonosov State University, Universitetskij pr., 13, Moscow, 119234, \\ Russia \\ ${ }^{2}$ Faculty of Physics, Moscow M.V. Lomonosov State University, Leninskie gory 1, Moscow, 119991, Russia
}

Regions of young stars and emission gas in tidal structures of interacting galaxies are studied on the basis of observations carried out (jointly with SAO RAS) at BTA telescope. Estimates of the line-of-sight velocities of gas and stars, the oxygen abundance of gas, and the age of stellar population were obtained. On the example of three systems (Arp 305, Arp 194, NGC 90) we demonstrate the variety of star formation conditions and properties of emission regions outside the stellar disks. In the first two systems, tidal dwarf galaxies were formed. In NGC 90 we found HII regions projecting onto the galaxy, which differ in velocity by more than $300 \mathrm{~km} \mathrm{~s}^{-1}$ from the galaxy, and, apparently, emerged in the gas ejected from the disk as a result of ram pressure of intergalactic gas.

DOI: 10.51194 /VAK2021.2022.1.1.172

Local emission regions related to young stars are often observed in a far periphery of galactic disks and in tidal debris of galaxies, where a formation of gravitationally bound tidal dwarf galaxies may take place under favourable conditions see the surveys by $[1,2]$. Dynamical properties of gas, its volume density and a chemical abundance of the outer star forming islands may differ significantly from those observed in spiral arms of galaxies. The mechanism of their formation is an open question. In general case, star formation outside optical disks is the result of a highly inhomogeneous gas structure, emerged as the result of collisions of gas streams or the local gravitational instability of gas. A study of emission regions in tidal structures is important for understanding how stars are formed under condition of low mean density of gas.

A program of spectral observations of tidal structures is carried out at $6 \mathrm{~m}$ BTA telescope of SAO RAS using the long-slit mode of SCORPIO-2 spectrograph [3] for a description of data processing and the main results we refer to $[4,5,6,7,8,9]$.

As an example, here we shortly describe three very different systems we observed: Arp 305, Arp 194 and NGC 90 (belonging to the system Arp 65, see Fig. 1). In Fig. 2 we demonstrate the image with overplotted position of the slit (a), the distributions of LOS velocities (b) and oxygen abundance (c) along the slit for all three considered systems. For Arp 194 we also give the distribution of the velocity dispersion along the slit.

Arp 305 is a small group of galaxies dominated by the wide pair of interacting spiral galaxies of moderate luminosity: NGC 4016 and NGC 4017, having very close systemic velocities (about 3500 kms). The projected separation of galaxies is about $86 \mathrm{kpc}$. Radio-observations revealed the concentration of HI in the bridge about halfway between galaxies which coincides with the optical chain of blue knots immersed in a faint haze, clearly visible in UV. It stretches along the line connecting the interacting galaxies at least at $7 \mathrm{kpc}$ [10]. This collection of young stellar objects is considered as the tidal dwarf galaxy (hereafter TDG) which luminosity is about $M_{g} \approx-17$. We observe neither noticeable rotation nor expansion of TDG: the mean velocities of its two brightest clumps differ by no more than $20 \mathrm{kms}$. The average weighted velocity dispersion within TDG after taking into account the uncertainties of measurements is about $17 \mathrm{kms}$.

A position of the regions crossed by the slit on a classical BPT diagnostic diagram is shown in Fig. 3. The diagram evidences a photo- ionizing mechanism of HII emission. The oxygen abundance of TDG we measured exceeds the value expected for its luminosity. However it should be taken into account that the mass of HI in TDG is greater than its stellar mass significantly, so one would expect a much lower gas enrichment for this dwarf in comparison with the "normal" dIrr galaxies with long-lasting star formation.
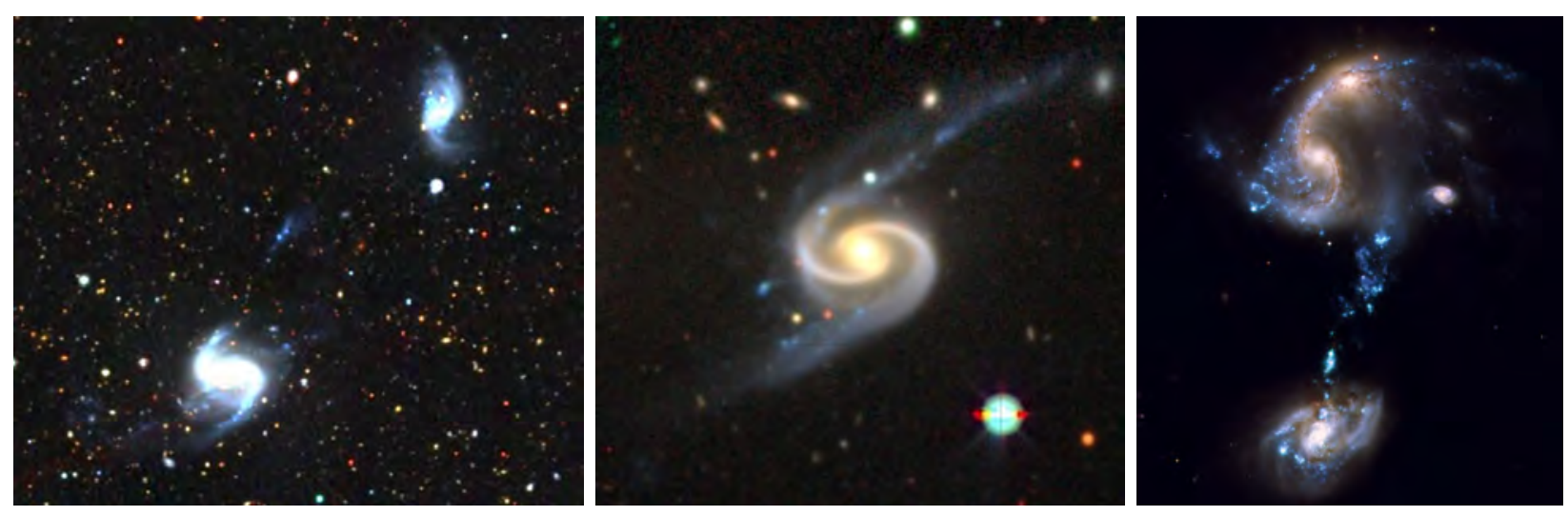

Figure 1: The colour composite images of Arp 305 (DECaLS), NGC 90 (DECaLS) and Arp 194 (HST). 

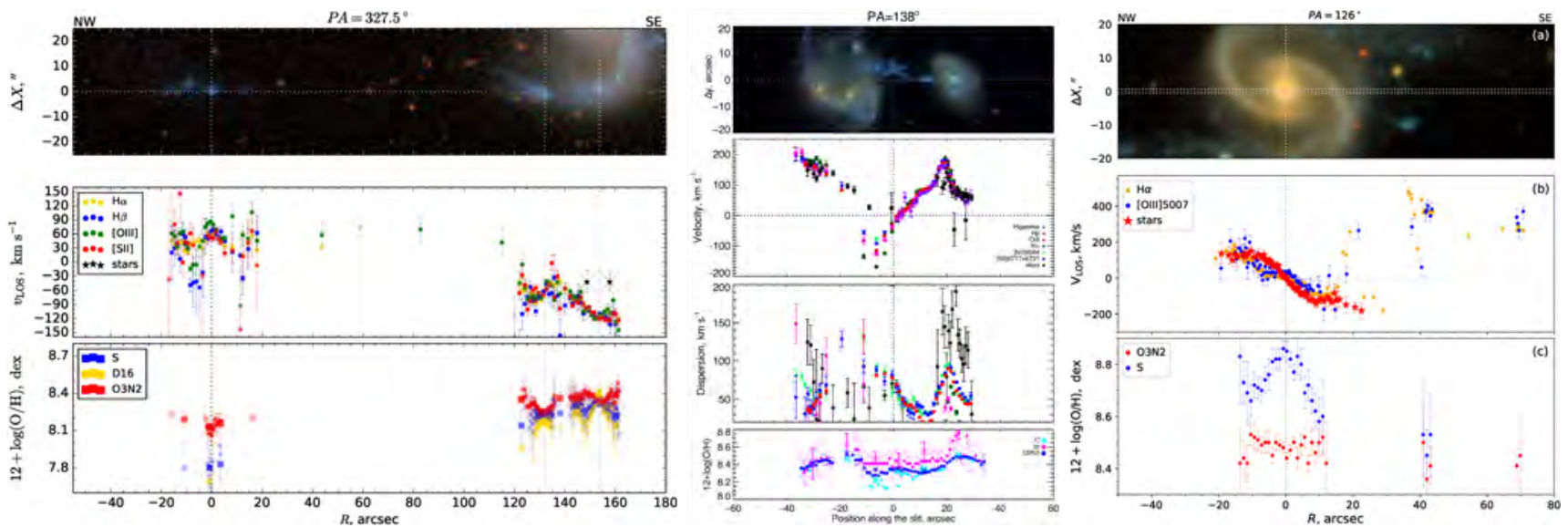

Figure 2: Radial variation of the parameters estimated from the fitting of the spectra (from left to right) of Arp 305, Arp 194 and NGC 90 (see the text).
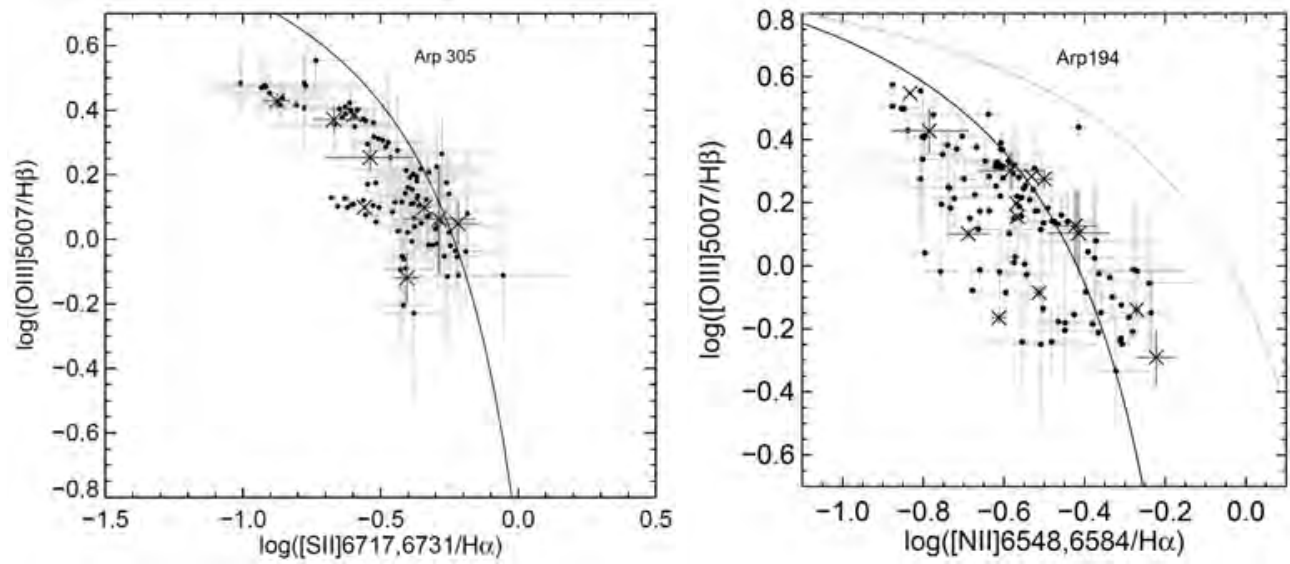

Figure 3: BPT-diagrams plotted for Arp 305 and Arp 194. Solid lines separate regions of pure photoionization excitation (below the black line), shock excitation (above both lines) and combined contribution of both mechanisms (between grey and black lines).

The virial mass $M_{d y n}$ of TDG exceeds significantly a mass of stellar population and is comparable with the observed mass of gas connected with TDG (about $7 \cdot 10^{8} M_{\odot}$ ). It confirms that TDG is at least close to be gravitationally bound, so it is naturally to expect a current star formation in the most dense regions near the tidal tail axis, where gas pressure is maximal. A rough estimate of Jeans mass following from the velocity dispersion of gas is compatible with the total (mostly gaseous) mass of TDG, as it is required for gravitational bounding. The colours of several bright stellar knots correspond to the models of stellar clusters where star formation begun 4-25 Myr ago.

Our data allow to conclude that most probably we observe TDG in a process of formation or rejuvenation. It agrees with the location of TDG, which is in the region of visible intersection of gas flow stretching toward the galaxy-companion with the diffuse extension of NW HI tidal tail (see the HI map in [10]).

Arp 194 is a bright example of recently collided spiral galaxies which gave birth to a chain of numerous small islands of current star formation forming a bridge between galaxies. The southern galaxy (S) evidently passed through a gaseous disk of the northern galaxy $(\mathrm{N})$ which in turn consists of two close merging components. Most regions of star formation beyond galaxies are very loose, however a large tidal dwarf is clearly visible near the (S)-component, which stands out by its size (about $5 \mathrm{kpc}$ ) and brightness.

We obtained several spectral cuts along the system, two of them crossing the tidal dwarf. This TDG evidently presents a gravitationally bound system. This is evidenced by the lowest velocity dispersion of its gas (that is of a set of HII regions at the line-of-sight), found from the emission lines linewidths. For comparison, the diffuse emission regions between galaxies are characterized by the enhanced dispersion of line-of-sight velocities reaching $50-70 \mathrm{~km} \mathrm{~s}^{-1}$. At the BPT diagram this rarified gas mostly lay beyond the line restricting the position of normal HII regions (see Fig. 3). 


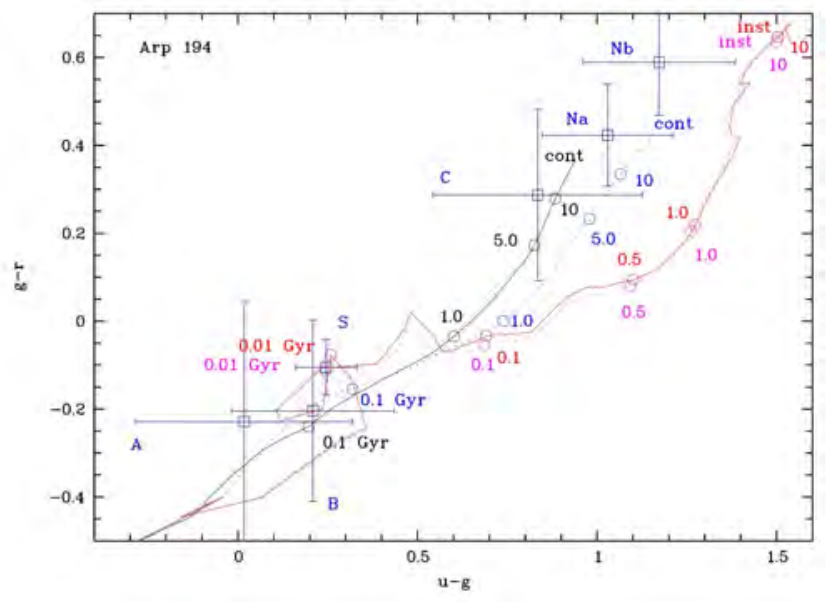

Figure 4: The $g-r$ versus $u-g$ diagram for Arp 194 (symbols with error-bars). The straight and dotted lines show the models for Salpeter and Kroupa IMFs correspondingly. We considered models for continuous star-formation (labeled as cont) and the models of instantaneous burst of star-formation (labeled as inst).

The gas in the bridge is only partially mixed chemically and spatially: we observe the $\mathrm{O} / \mathrm{H}$ gradient with the galactocentric distances both from $(\mathrm{S})$ and $(\mathrm{N})$ galaxies and the unusually high dispersion of $\mathrm{O} / \mathrm{H}$ in the outskirts of $(\mathrm{N})$-galaxy. We confirm that the age of stellar population of the tidal dwarf is low $\left(10^{7}-10^{8} \mathrm{yr}\right.$, see Fig. 4$)$. There is no evidence of the significant amount of dark matter in this dwarf galaxy. A proximity of TDG to the (S)-galaxy and a small difference of their velocities (about $60 \mathrm{kms}$ ) indicates that we observe it at the last stage of its fall onto a disk of parent galaxy.

NGC 90 is a galaxy with the unusually smooth inner spiral arms and a member of a rich group. Its neighbour spiral galaxy - NGC 93 - is only $64 \mathrm{kpc}$ away (in projection), however the velocities of galaxies differ by about $400 \mathrm{~km} \mathrm{~s}^{-1}$, so they hardly form a bound system. The most intriguing peculiarity of the galaxy is the presence of the huge HI "cloud" containing about half of total mass of a galaxy gas [11], which is strongly displaced outward the main body of galaxy. A cloud velocity differs by more than $300 \mathrm{kms}$ from the adjacent part of the galaxy, and therefore the "cloud" is loosely connected with the latter. Its nature is puzzling.

We fulfilled two spectral cuts of NGC 90 (through the centre and along the tidally stretched spiral arm) which allowed us to get the distribution of the velocity and oxygen abundances of emission gas $(\mathrm{O} / \mathrm{H})$ along the slits. In the central part of the galaxy, including inner spiral arms, a significant role belongs to a non-photoionization mechanism of gas excitation probably caused by shocks due to LINER-like activity of the nucleus. The $\mathrm{O} / \mathrm{H}$ is slightly underabundant in the central region (O3N2 method) and has a shallow abundance gradient, typical for interacting galaxies. The most interesting is, that we found the traces of current star formation in the abnormal HI "cloud" with the same LOS velocities. We argue that what we see as a "cloud" containing local knots of star formation is in reality a flow of gas sweeping by ram pressure and elongated nearly along the line of sight.

\section{References}

1. P. M. Weilbacher, On the Formation and Evolution of Dwarf Galaxies in Tidal Tails, Ph.D. thesis, -, 2002.

2. P.-A. Duc and F. Renaud, Tides in Colliding Galaxies, volume 861, 327 (2013).

3. V. L. Afanasiev and A. V. Moiseev, Baltic Astronomy, 20, 363, 2011.

4. A. Zasov, A. Saburova, I. Katkov, O. Egorov, and V. Afanasiev, MNRAS, 449, 1605, 2015.

5. A. V. Zasov, A. S. Saburova, O. V. Egorov, and V. L. Afanasiev, MNRAS, 462, 3419, 2016.

6. A. V. Zasov, A. S. Saburova, O. V. Egorov, and R. I. Uklein, MNRAS, 469, 4370, 2017.

7. A. V. Zasov, A. S. Saburova, O. V. Egorov, and V. L. Afanasiev, MNRAS, 477, 4908, 2018.

8. A. V. Zasov, A. S. Saburova, O. V. Egorov, and S. N. Dodonov, MNRAS, 486, 2604, 2019.

9. A. V. Zasov, A. S. Saburova, O. V. Egorov, and A. V. Moiseev, MNRAS, 498, 101, 2020.

10. C. Sengupta, T. C. Scott, S. Paudel, K. S. Dwarakanath, D. J. Saikia, and B. W. Sohn, MNRAS, 469, 3629, 2017.

11. C. Sengupta, T. C. Scott, S. Paudel, D. J. Saikia, K. S. Dwarakanath, and B. W. Sohn, A\&A, 584, A114, 2015. 


\title{
When dwarf galaxies turn to be spiral?
}

\author{
A. Zasov ${ }^{1,2}$, A. Khoperskov ${ }^{3}$, N. Zaitseva ${ }^{1}$, S. Khrapov $^{3}$ \\ zasov@sai.msu.ru \\ ${ }^{1}$ Sternberg Astronomical Institute, Moscow M.V. Lomonosov State University, Universitetskij pr., 13, Moscow 119234, \\ Russia, \\ ${ }^{2}$ Faculty of Physics, Moscow M.V. Lomonosov State University, Leninskie gory 1, Moscow 119991, Russia, \\ ${ }^{3}$ Volgograd State University, Universitetsky pr., 100, Volgograd 400062, Russia
}

Spiral structure is rarely observed in dwarf galaxies because the formation of spiral arms requires special conditions. We analyze the sample of about $40 \mathrm{dS}$-galaxies found among the bright late-type galaxies with $M_{B}>-18^{m}$ and photometric diameter $D_{25}<12 \mathrm{kpc}$. These galaxies do not differ statistically from non-dS galaxies apart from the lower average gas (HI) fraction. To check the conditions of formation of spiral structure we carried out a series of direct N-body/hydrodynamic simulations of dynamic evolution of low-mass stellar/gaseous discs varying the initial parameters of galaxies. We came to conclusion that the gravitational mechanism of formation of spiral structure is effective only for thin stellar/gaseous discs, non-typical for dwarf galaxies. The thicker stellar disc, the more gas is required for the spiral structure to form. The reduced gas content in most dS-galaxies may be a result of more efficient star formation in their relatively thin discs.

Keywords: galaxy: dwarfs, galaxy: spiral structure, modeling: $N$-body simulations

DOI: $10.51194 /$ VAK2021.2022.1.1.173

\section{Introduction}

Usually a spiral structure is not observed in low-luminous galaxies. However, a small fraction of dwarf galaxies still possesses a well distinguishable spiral pattern either of flocculent or (rare) a Grand Design type, and may be ascribed to dS-type.

A general idea of our investigation was to compare dS galaxies with the reference sample of non-spiral dwarf discy galaxies by their observed properties and to clarify the conditions for the formation and existence of long-lived spiral pattern in low-mass galaxies, using numerical self-consistent 3D models of dwarf galaxies.

\section{The sample of dwarf spiral galaxies}

In this work we consider low-luminous late-type galaxies with an absolute magnitude $M_{B}>-18^{m}$, and a diameter $D_{25}<12 \mathrm{kpc}$.

To create the sample of dS-galaxies we inspected visually the images of late-type (mostly dIr or dSm) galaxies from Hyperleda database brighter than $m_{B}=15$ which satisfy the aforementioned restrictions. As the result, the sample of about $40 \mathrm{dS}$-galaxies was created which we compared with the non-spiral dwarfs of the general sample. A comparison of the samples of spiral (dS) and non-spiral (dSm and dIrr) galaxies by their luminosities, diameters, total masses, velocities of rotation, led to conclusion that they share similar properties, although there are practically no dS galaxies with rotation velocities below $50-60 \mathrm{~km} \mathrm{sec}^{-1}$. In terms of isolation, or possessing of a bar dS-galaxies also do not stand out from other dwarf systems.

The only statistically significant difference we found between dS and non-dS dwarf galaxies of similar mass, luminosity or velocity of rotation is the reduced (in the mean) value of gas content in spiral dwarfs. As an example, Fig. 1 gives the relationship between specific angular momentum $[\mathrm{km} / \mathrm{sec} \cdot \mathrm{kpc}]$ and total mass of $\mathrm{HI}$ in dS (blue symbols) and non-dS dwarfs (red and green symbols). The straight lines mark the linear relationship $( \pm 1 \sigma)$ obtained earlier for isolated spiral galaxies [1], extrapolated to the low luminosity region. A mass of HI in dS galaxies is found to be systemically lower (2-3 times in the mean) than in the other dwarf galaxies with similar specific angular momentum. However, most dS-galaxies remain to be rather rich of gas, if to compare their mass of HI with stellar mass or luminosity.

\section{Numerical simulation of spiral arms formation in dwarf galaxies}

To clarify the conditions of formation of spiral arms in low mass galaxies we used the numerical simulations of dynamical evolution of stellar/gaseous discs. We proceeded from the natural assumption that the existence of spiral structure is associated with a self-gravity of a disc. In the models we constructed we try to find the parameters of slowly rotating discs which lead to the development of a long-lived global spiral pattern. We used 3-component models of initially equilibrium bulgeless galaxies, consisting of stellar and gaseous discs immersed in a rigid massive spherical pseudo-isothermic halo.

For all models we assume that initially a turbulent velocity dispersion of gas $c_{g}$ is $\approx 10 \mathrm{~km} \mathrm{sec}^{-1}$ allover a disc. To limit our models to dwarf galaxies, we accept the ratio of maximal circular velocity of a disc to a gas velocity dispersion $V_{g}^{\max } / c_{g} \leq 10$. We also take into account that stellar velocity dispersion is anisotropic in the radial $\left(c_{r}\right)$, azimuthal $\left(c_{\varphi}\right)$ and vertical $\left(c_{z}\right)$ direction. 


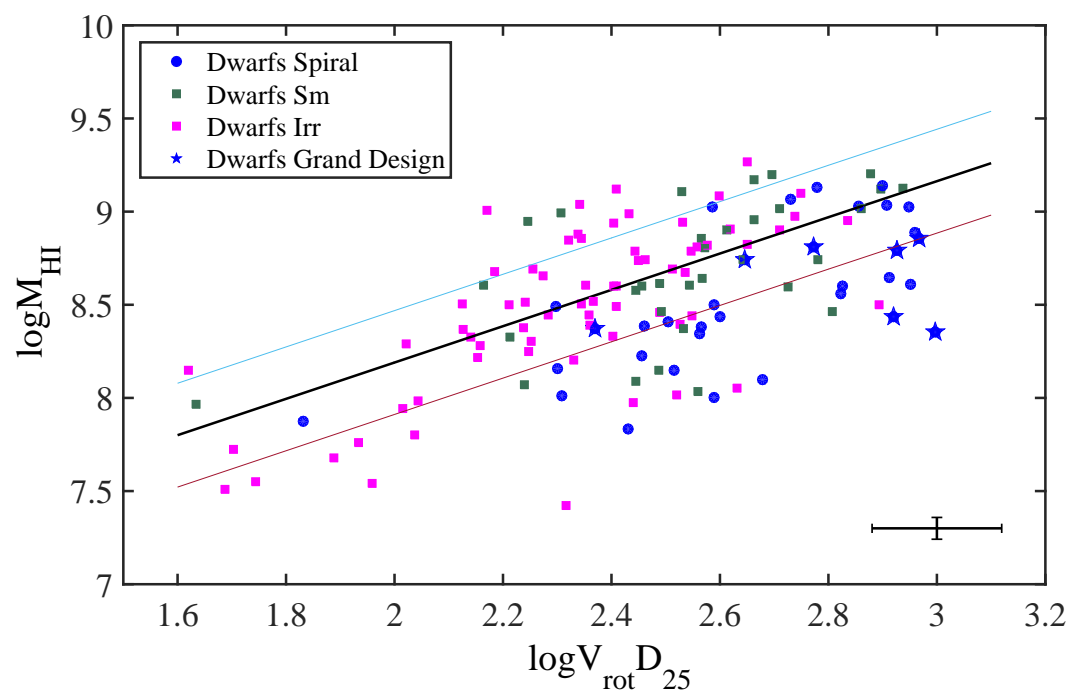

Figure 1: A relationship between total mass of HI and specific angular momentum of discs of dwarf galaxies.

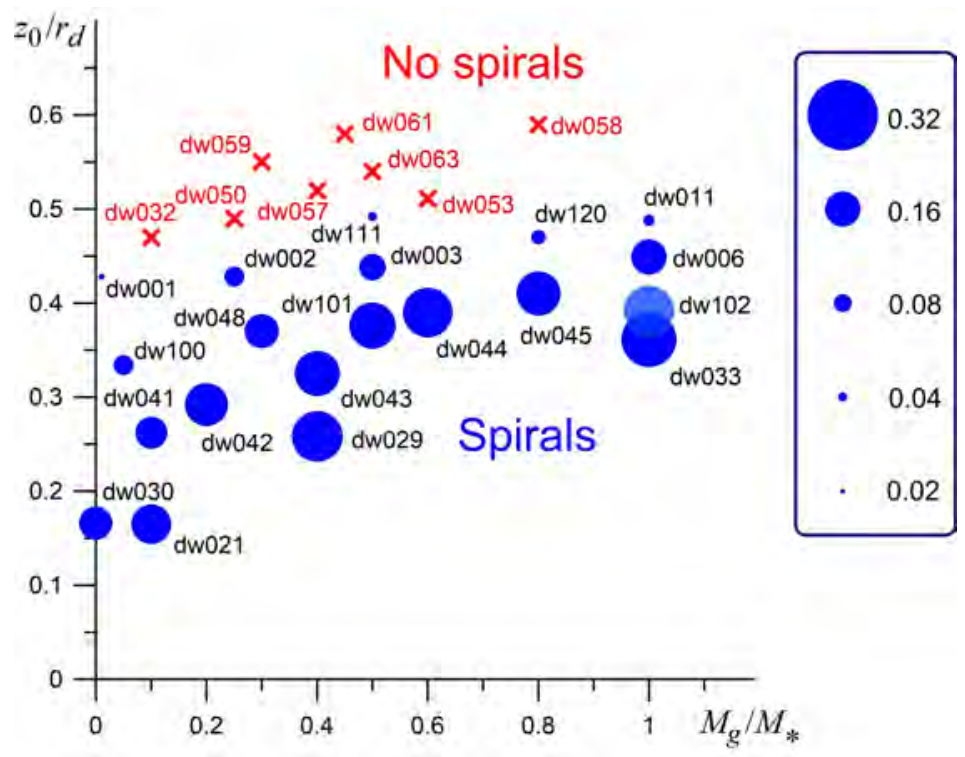

Figure 2: Relative mass of gas $\left(M_{g} / M_{*}\right)$ vs disc thickness $z_{0} / r_{d}$ for model galaxies with (circles) and without (crosses) spiral structure after several periods of rotation (see the text).

Our numerical models are based on the direct N-body simulations for stellar disc components and on the Lagrangian approach using the SPH-method for the gas components of galaxies. The gravitational force is calculated by the direct Particle-Particle method of summing the gravitational interaction of each particle with all the other stellar and gaseous particles. A self-consistent modeling of dynamics of collisionless and gaseous particles using a parallel CUDS algorithm "N-body $+\mathrm{SPH}$ " was described by [2]. A series of test numerical experiments with different total number of particles in the range $2^{19} \div 2^{23}$ showed a satisfactory convergence of solutions for $N_{\text {sum }} \geq 2^{21} \simeq 4 \cdot 10^{6}$. The equilibrium structure of stellar disc with a scale height $z_{0}(r)$ was achieved using the iteration procedure (see [3] for details).

All numerical models are self-consistent and begun their evolution from a quasi-equilibrium state. The evolution of the discs was traced during 5-15 periods of a disc rotation (depending on the timescale of perturbations growth). To reproduce dS-galaxies, we choose models where well-defined quasi-stationary spirals, more pronounced in gaseous components, developed after 1-2 periods of revolution. Gaseous spiral arms may either look as the Grand Design type, or possess a less ordered structure, more typical for flocculent galaxies. 


\section{Discussion and conclusion}

The most favorite conditions for the development of gravitational instability and the formation of extended spiral structure exist in the inner regions of a dynamically cold and relatively thin stellar disc which velocity of rotation does not exceed $6 c_{g}(\approx 60 \mathrm{~km} / \mathrm{s})$.

Diagram in Fig. 2 illustrates a role that the disc thickness and the mass of gas plays in the formation of spiral structure. The ratio $z_{0} / r_{d}$ at radial distance $r \sim 2 r_{d}$ is compared there with the ratio of gas-to-stellar mass for different models. Circles relate to those numerical models where the density waves of different amplitudes had formed and survived. A size of circles corresponds to the values of maximal amplitude of Fourier harmonics averaged over a disc of model galaxy. Crosses mark the models where a spiral structure has not formed, so these discs preserve the axisymmetric distribution of all parameters throughout evolution.

As it follows from the diagram, spirals may appear even in gas-poor discs, if they are thin enough. The thicker stellar disc, the more gas is required for the spiral structure to form. All disc models with $z_{0} / r_{d}>0.45-0.50$, which is quite typical for the observed dIrr galaxies, remained structureless during the experiments even when galaxies are rich of dynamically cold gas.

Thin stellar discs are more unstable to gravitational perturbations, which promote the formation of a spiral structure. This may explain the lower (in the mean) gas content in dS-galaxies in comparison with non-spiral dwarfs of similar size, luminosity or dynamic properties. Indeed, a volume density of gas plays a key role in the efficiency of star formation (SFE): the lower a density, the lower is SFE (see for example [4], [5]). Hence SFE tends to be higher in those galaxies where stellar discs are thinner and denser, since it is a stellar density which determines a thickness of the equilibrium gas layer in a disc.

To conclude, we demonstrate that only for galaxies with the most thin discs the conditions for the formation of spirals are realized. A longevity of spiral pattern is provided by the presence of cold gas (some role may also play a bar, formed during the evolution). From this point of view, the observed dS-galaxies are mostly those dwarf systems containing a cold gas, which stellar discs are thin enough for a spiral pattern to be formed.

\section{References}

1. A. V. Zasov and N. A. Zaitseva, Astronomy Letters, 43, 439, 2017.

2. S. Khrapov, A. Khoperskov, and V. Korchagin, Bulletin of the South Ural State University Series-Mathematical Modelling Programming \& Computer Softwar, 12, 123, 2019.

3. A. Khoperskov, D. Bizyaev, N. Tiurina, and M. Butenko, Astronomische Nachrichten, 331, $731,2010$.

4. O. V. Abramova and A. V. Zasov, Astronomy Letters, 38, 755, 2012.

5. C. Bacchini, F. Fraternali, G. Pezzulli, A. Marasco, G. Iorio, and C. Nipoti, AEA, 632, A127, 2019. 


\title{
Mass search of the RCR catalog sources at different bands of electromagnetic spectra
}

\author{
O.P. Zhelenkova ${ }^{1}$, E.K. Majorova ${ }^{1}$, A.V. Temirova ${ }^{2}$, N.N. Bursov ${ }^{1}$ \\ zhe@sao.ru \\ ${ }^{1}$ SAO RAS, Nizhniy Arkhys, Russia, \\ ${ }^{2}$ St. Petersburg Branch of SAO RAS, St. Petersburg, Russia
}

DOI: 10.51194 /VAK2021.2022.1.1.174

Study in the optical range of Radio Sources of the "Cold" Experiment (RC catalog) carried out at the radio telescope RATAN-600 in 1980-1999, immediately after receiving the first results were obtained. Program to search for distant radio galaxies "Big Trio" (RATAN-600, VLA, 6m BTA) is completed in 1993-2008 during which the most powerful radio galaxy on $\mathrm{z}=4.51$ was discovered. On the basis of modern surveys in optical, infrared, millimeter and radio ranges we carried out a study of the RATAN Cold Refined (RCR) catalog radio sources. We used next surveys-radio: VLSS, TXS, GLEAM, NVSS, FIRST; GB6; optics: DPOSS, SDSS DR7, Pan-STARRS, DES, archive NOAO; IR: LAS UKID, GPS UKIDSS, WISE. Depth of surveys was sufficient for host objects discovery for $95 \%$ objects. On radio luminosity estimates only $3 \%$ of RCR sources are of the FRI type, and the others are powerful radio galaxies of the FRII type. The absolute magnitude in the $r$ band to radio luminosity ratio were compared at $1.4 \mathrm{GHz}$ for RCR sources. The value of this ratio for quasars is not depends on radio luminosity, and for galaxies - the ratio has a minimum value for objects with maximum luminosity and increases with reduced radio of luminosity to a level characteristic of quasars. These sources were divided into groups based on value of radio luminosity, the number of quasars and galaxies in each group was calculated. It turned out that in such groups with increasing radio luminosity, the number of quasars increases while the number of galaxies decreases. Such behavior can be explained that screening properties of torus depend on power the radio source (AGN). The averaged scans of the Cold Experiment were searched for flux variability of the RCR sources. Found 3 candidates for radio transients. On maps of Planck missions at less than 5 sigma a positive signal $70 \%$ RCR sources is detected. Spectral studies confirm the association of positive spots with radio sources. The properties of "hot" and "cold" spots near RCR sources are investigated to search the Syunyaev-Zeldovich (SD) effect. Of the 830 objects, 135 SD candidates were detected less than 5 sigma. The fifth part of these objects has confirmation in clusters catalogs. 


\section{The jet interaction manifestations of the $\mathrm{RC} J 0311+0507$ radio galaxy at $z=4.51$ with the galactic environment}

O.P. Zhelenkova

zhe@sao.ru

Special Astrophysical Observatory, Nizhnij Arkhyz

\section{DOI: $10.51194 /$ VAK2021.2022.1.1.175}

The most distant object RC J0311 $+0507(4 \mathrm{C}+04.11)$ with $\mathrm{z}=4.514$ [1], discovered in the program of the search for distant radio galaxies $[2,3,4]$ has extreme luminosity about $3 \times 10^{29} \mathrm{~W} / \mathrm{Hz}$ at $0.5 \mathrm{GHz}$. High angular resolution maps $\left(0.035^{\prime \prime}\right)$ obtained with radio interferometers MERLIN and EVN [5] demonstrate the complex structure of the radio source - 8 small-sized details at the total angular size of the object $2.8^{\prime \prime}$ (or $19 \mathrm{kpc}$ ). The jet is strongly curved in the direction from the core to the southern hotspot, probably due to motion through a dense medium. For the radio galaxy there are observational data in the optical and infrared ranges. Angular sizes, positions and shape of the galaxy change from filter to filter. Note that in a narrow filter NL671, which exactly falls on the $L y \alpha$ emission at $z=4.514$, the size of the $L y \alpha$-envelope of the host galaxy is $60 \mathrm{kpc}$.

For detailed analysis, we performed astrometric re-calibration of image cutouts with size $5^{\prime} \times 5^{\prime}$ centered on the host galaxy position for BTA (R and SED665 filters), Subaru (R, NL671), UKIRT (K), Spitzer (3.6 and $4.5 \mathrm{~m} \mu$ bands) telescopes. SDSS objects with minimal displacement and coordinate errors were selected as reference ones. The spread of coordinate errors of reference objects on these frames ranged from $0.060^{\prime \prime}$ to $0.096^{\prime \prime}$. We then measured the position of the galaxy. The coordinates of the host in the $\mathrm{K}$ filter and in the 3.6 and $4.5 \mathrm{~m} \mu$ bands plotted on the EVN radio map are located near the radio core, while in other filters they are located along the jet and farther from the nucleus. The distance between the end positions is $0.89^{\prime \prime}$, which is higher than the $3 \sigma$ confidence level for coordinate errors. The displacement of the coordinates of the host galaxy in the filters (R, SED665, NL671), where Ly $\alpha$-emission dominates, relative to the position in the frames (K, 3.6 and $4.5 \mathrm{~m} \mu$ bands), where the main contribution to the emission is made by the stellar population, can be explained by the presence of a cloud of ionized gas in the southern hot spot, where the jet stopped propagating, as well as by the UV contribution of the jet with the boosting effect, since it is directed sufficiently close to the line of sight [6, 7].

\section{References}

1. A. I. Kopylov, W. M. Goss, Y. N. Parï̌skï, N. S. Soboleva, O. V. Verkhodanov, A. V. Temirova, and O. P. Zhelenkova, Astronomy Letters, 32, 433, 2006.

2. Y. N. Parijskij, W. M. Goss, A. I. Kopylov, N. S. Soboleva, N. S. Temirova, O. V. Verkhodanov, O. P. Zhelenkova, and M. N. Naugolnaya, Bulletin of the Special Astrophysics Observatory, 40, 5, 1996.

3. Y. N. Parijskij, N. S. Soboleva, W. M. Goss, A. I. Kopylov, O. V. Verkhodanov, A. V. Temirova, and O. P. Zhelenkova, in R. D. Ekers, C. Fanti, and L. Padrielli, eds., Extragalactic Radio Sources, volume 175, 591 (1996).

4. Y. N. Parijskij, W. M. Goss, A. I. Kopylov, N. S. Soboleva, A. V. Temirova, O. V. Verkhodanov, and O. P. Zhelenkova, in J. D. Hadjidemetrioy and J. H. Seiradakis, eds., Joint European and National Astronomical Meeting, 202 (1997).

5. Y. N. Parijskij, P. Thomasson, A. I. Kopylov, O. P. Zhelenkova, et al., MNRAS, 439, 2314, 2014.

6. Gopal-Krishna, P. Subramanian, P. J. Wiita, and P. A. Becker, A\&A, 377, 827, 2001.

7. M. G. Allen, W. B. Sparks, A. Koekemoer, A. R. Martel, et al., ApJS, 139, 411, 2002. 


\section{Unindentified sources of the 3CR catalogue}

O.P. Zhelenkova

zhe@sao.ru

Special Astrophysical Observatory, Nizhnij Arkhyz

DOI: 10.51194 /VAK2021.2022.1.1.176

The objects of the Cambridge survey [1,2] are one of the most studied samples of radio sources. The redshifts were determined for $92 \%$ of 298 objects included in the latest third release of 3CR [3]. Most of the objects in the $3 \mathrm{CR}$ catalogue belong to powerful FRII-type radio sources, of which $73 \%$ are radio galaxies and $19 \%$ are quasars, $1 \%$ are BLLac. The remaining opticaly unidentified sources were assigned to galaxies with hidden active nuclei. We searched for host galaxies for radio sources from the 3CR catalog without optical counterparts in the lists from [4] and [5]. We used SDSS, PanSTARRS, DES, LAS UKIRT, GLIMPSE and WISE surveys, archival data from the NOAO, NRAO, WSA, HST, Spitzer and Vizier, NED, Simbad databases. We managed to sort out the sources 3C 14.1 and 3C 22.1, for which no one-to-one correspondence was found in the NVSS survey [5]. So, two sources contribute to the source 3C 14.1: (A) 4C+58.02 (with known optical counterpart) and (B) 4C +60.02 . Three objects contribute to the 3C 22.1 source: (A) 4C+67.02, (B) GB6 B0041+6807, (C) TGSS J004647.0+681629. In total, we inspected 31 sources. The table shows the results for 10 sources for which we first found optical counterparts.

\begin{tabular}{|l|c|c|c|c|l|}
\hline Name & $R . A_{\text {opt }}, 2000$ & Dec $_{\text {opt }}, 2000$ & Magnitude & Filter & Survey \\
\hline 3CR 11.1 & $00: 29: 44.69$ & $+63: 58: 42.1$ & 16.12 & $\mathrm{~W} 1$ & GLIMPSE \\
3C 14.1 (B) & $00: 37: 24.65$ & $+60: 29: 41.2$ & 20.85 & $\mathrm{r}$ & PanSTARRS \\
3C 21.1 (A) & $00: 44: 39.38$ & $+68: 24: 05.5$ & 21.37 & $\mathrm{i}$ & PanSTARRS \\
3C 21.1 (B) & $00: 45: 41.21$ & $+67: 33: 21.2$ & 21.42 & $\mathrm{r}$ & PanSTARRS \\
3C 21.1 (C) & $00: 46: 46.65$ & $+68: 16: 29.5$ & 14.81 & $\mathrm{~W} 1$ & WISE \\
3CR 33.2 & $01: 08: 36.83$ & $+69: 22: 34.1$ & $>21.8$ & $\mathrm{r}$ & PanSTARRS \\
3CR 152.0 & $06: 04: 28.62$ & $+20: 21: 21.4$ & $>19.8$ & $\mathrm{y}$ & PanSTARRS \\
3CR 250.0 & $11: 08: 52.12$ & $+25: 00: 54.6$ & 23.50 & $\mathrm{r}$ & DECaLS \\
3CR 431.0 & $21: 18: 52.33$ & $+49: 36: 59.6$ & 17.15 & $\mathrm{~W} 1$ & GLIMPSE \\
3CR 454.2 & $22: 52: 05.54$ & $+64: 40: 11.4$ & 23.70 & $\mathrm{r}$ & SDSS \\
\hline
\end{tabular}

\section{References}

1. D. O. Edge, J. R. Shakeshaft, W. B. McAdam, J. E. Baldwin, and S. Archer, Mem. R. Astron. Soc., 68, 37, 1959 .

2. A. S. Bennett, Mem. R. Astron. Soc., 68, 163, 1962.

3. H. Spinrad, S. Djorgovski, J. Marr, and L. Aguilar, PASP, 97, 932, 1985.

4. A. R. Martel, J. A. Biretta, M. McMaster, W. B. Sparks, S. A. Baum, and P. J. McCarthy, in American Astronomical Society Meeting Abstracts, American Astronomical Society Meeting Abstracts, volume 193, 05.12 (1998).

5. A. Maselli, F. Massaro, G. Cusumano, V. La Parola, et al., MNRAS, 460, 3829, 2016. 


\title{
Search for radio sources - close neighbors according to modern radio and optical surveys
}

\author{
O.P. Zhelenkova \\ zhe@sao.ru \\ Special Astrophysical Observatory, Nizhnij Arkhyz
}

\section{DOI: 10.51194 /VAK2021.2022.1.1.177}

Why are not all galaxies active in the radio range? It is possible that studies of close pairs, where each galaxy or one in the pair is a radio source, will allow us to come closer to clarifying this phenomenon. The search for such objects was carried out in the work [1] on the basis of SDSS data. We decided to look for pairs of close radio sources among multicomponent sources. In [2], a morphological classification of NVSS survey sources was carried out using neural network algorithms, and a sample of candidates for giant radio galaxies (GRG) was prepared. With radio surveys: VLSSr, TGSS, GLEAM, VLASS, and the NRAO archive (to clarify the structure of radio sources), optical and infrared surveys: SDSS, PanSTARRS, PanSTARRS, WISE, DES (search for the host galaxy) and databases: Vizier, NED and SDSS (information about redshifts) we checked whether the objects in this list belong to GRG.

Of the 148 candidates considered, $58 \%$ can be attributed to GRG, for the rest of the objects either the size is less than $0.7 \mathrm{Mpc}$, or the candidate is unrelated objects that fall on the line of sight, which were combined by the algorithm into one radio source. Among these 148 candidates, we found 5 radio sources, which are close pairs of radio sources - the spectroscopic redshift of the host galaxies coincides. These are the following objects:

NVGRG J001117.5 +523204 - a pair of radio galaxies in the merging galaxy cluster ZwCl0008.8 +5215 ;

NVGRG J002011.3+000443 - four radio galaxies in the cluster;

NVGRG J012600.6-012052 - 3CR 40 in the cluster Abell 194 [3] with Minkowski's Object [4, 5];

NVGRG J013419.2+143014 — a pair of radio galaxies;

NVGRG J015622.1+053739 — two radio galaxies [6].

And 19 more radio sources in which the only one is a radio source in a pair of galaxies. For these 24 objects, the median magnitude $r=17.4^{\mathrm{m}}$. The redshift range is $0.018-0.436$, the median redshift $z=0.11$.

We plan to continue our work and expand the sample of pairs of radio sources.

\section{References}

1. I. N. Pashchenko and V. M. Vitrishchak, Astronomy Reports, 54, 97, 2010.

2. D. D. Proctor, ApJS, 224, 18, 2016.

3. I. Sakelliou, M. J. Hardcastle, and N. N. Jetha, MNRAS, 384, 87, 2008.

4. S. Croft, W. van Breugel, W. de Vries, M. Dopita, et al., ApJ, 647, 1040, 2006.

5. M. Lacy, S. Croft, C. Fragile, S. Wood, and K. Nyland, ApJ, 838, 146, 2017.

6. G. Schellenberger, J. M. Vrtilek, L. David, E. O'Sullivan, S. Giacintucci, M. Johnston-Hollitt, S. W. Duchesne, and S. Raychaudhury, ApJ, 845, 84, 2017. 
Modern Stellar Astronomy 


\section{On two forms of interstellar gas accretion in the formation of single stars}

T. Abdulmyanov

abdulmyanov.tagir@yandex.ru

Kazan State Power Engineering University, Kazan, Russia

In this paper, the mechanisms of star formation and the formation of the equatorial gas and dust disk of protostars are considered. The viscous dynamics of the interstellar matter of gas and dust disks is mainly determined by perturbations of the matter density during gas accretion onto the equilibrium core of the protostar. Using the model of pulsating perturbations of the density of the gas-dust envelope of the protostar and the Navier-Stokes equations, the formulas for the dynamic viscosity of Keplerian and almost Keplerian disks are obtained. It is shown that in the regime of unstable equilibrium of the envelope, accretion of gas onto the core of the protostar begins. In the regime of stable equilibrium, the fragmentation of the gas-dust envelope and the equatorial disk of the protostar occurs. In the ring-shaped fragments of the disk, the process of formation of "embryos" of planets begins and accretion on the "embryos" of the planet also begins.

Keywords: dynamic viscosity, protoplanetary disks, density wave disturbances

DOI: $10.51194 /$ VAK2021.2022.1.1.178

\section{Basic equations of the model of viscous dynamics of gas and dust particles in the equatorial disks of young stars}

Observations of dusty disks around young stars show that the large-scale structures of gas and dust disks fall into two distinct groups. Gas and dust disks around the stars TW Hya, HD 163296, HD 169142, HD 97048 [1, 2, 3] have ring-shaped fragmentation. The formation of such a disk structure is explained by the extreme deceleration of accretion onto the core of the protostar, which in the model [4] is presented as a stable equilibrium of the gas and dust envelope of the protostar. Gas and dust disks around the stars MWC 758, HD 100453, HD 135344B $[5,6,7]$ have a spiral structure. The appearance of such structures in disks can be a consequence of a change in the stability condition and a change in the envelope equilibrium mode to the fast protostar compression mode [4]. What mechanisms lead to the formation of such structures? These mechanisms could play a key role at the initial moment of the formation of planetisimals.

Modeling the compression of the protostellar cloud and dynamic processes in the accretion disks of young stars [8] shows that as a result of the migration and fall of massive gaseous clumps onto the protostar, irregular outbursts of luminosity of young stars can occur [9]. The fall of gas condensations (gas clamps) onto the protostar will form wave disturbances of the density of the protostar (density waves) [10]. A gas-dust disk will form on the equatorial plane of the rotating cloud. Equatorial gas and dust disks disintegrate over time from 5 milliom to 20 million years as a result of the action of the stellar wind and other reasons. The further evolution of the protoplanetary disk, the formation and growth of masses of small bodies will depend on the forms of viscous dynamics in the gas and dust disks and the interaction between the forming bodies and the gas. Let us consider a protostellar cloud with differential rotation and represent it as a union of thin axisymmetric disks, in which the motion of interstellar gas and dust occurs at the initial stage without internal friction $(\eta=0)$. With the compression of the protostar and significant changes in its density, the viscosity $\eta$ will be significant and it becomes necessary to take it into account $(\eta \neq 0)$. The equilibrium state of the dust shell was considered in the article [11] using the well-known equations of hydrodynamics $[12,13]$ :

$$
\begin{array}{r}
\frac{\partial \rho}{\partial t}+\mathbf{V} \cdot \operatorname{grad}(\rho)=-\rho \cdot \operatorname{div} \mathbf{V} \\
\frac{\partial \mathbf{V}}{\partial t}+(\mathbf{V} \nabla) \cdot \mathbf{V}=-\frac{1}{\rho} \nabla P-\nabla \Phi-2(\mathbf{\Omega} \times \mathbf{V})+ \\
+\Omega^{2}\left(x e_{x}+y e_{y}\right)+\frac{\mathbf{N}}{\rho}, \\
P=\frac{R}{\mu} \rho T, \nabla^{2} \Phi=4 \pi g \rho .
\end{array}
$$

The vector $\mathbf{N}=\left(N_{x}, N_{y}, N_{z}\right)$ in equation (2) is defined as follows:

$$
\begin{aligned}
& N_{x}=2 \frac{\partial}{\partial x}\left(\eta \frac{\partial V_{x}}{\partial x}\right)+\frac{\partial}{\partial y}\left[\eta\left(\frac{\partial V_{x}}{\partial y}+\frac{\partial V_{y}}{\partial x}\right)\right]+\frac{\partial}{\partial z}\left[\eta\left(\frac{\partial V_{x}}{\partial z}+\frac{\partial V_{z}}{\partial x}\right)\right]-S_{1}, \\
& N_{y}=2 \frac{\partial}{\partial y}\left(\eta \frac{\partial V_{y}}{\partial y}\right)+\frac{\partial}{\partial z}\left[\eta\left(\frac{\partial V_{y}}{\partial z}+\frac{\partial V_{z}}{\partial y}\right)\right]+\frac{\partial}{\partial x}\left[\eta\left(\frac{\partial V_{y}}{\partial x}+\frac{\partial V_{x}}{\partial y}\right)\right]-S_{2},
\end{aligned}
$$




$$
\begin{gathered}
N_{z}=2 \frac{\partial}{\partial z}\left(\eta \frac{\partial V_{z}}{\partial z}\right)+\frac{\partial}{\partial x}\left[\eta\left(\frac{\partial V_{z}}{\partial x}+\frac{\partial V_{x}}{\partial z}\right)\right]+\frac{\partial}{\partial y}\left[\eta\left(\frac{\partial V_{z}}{\partial y}+\frac{\partial V_{y}}{\partial z}\right)\right]-S_{3}, \\
S_{1}=\frac{2}{3} \frac{\partial}{\partial x}(\eta \cdot \operatorname{div} \mathbf{V}), S_{2}=\frac{2}{3} \frac{\partial}{\partial y}(\eta \cdot \operatorname{div} \mathbf{V}), S_{3}=\frac{2}{3} \frac{\partial}{\partial z}(\eta \cdot \operatorname{div} \mathbf{V}) .
\end{gathered}
$$

The dynamic viscosity $\eta$ in the vector $\mathbf{N}$ is a function of the coordinates $x, y, z: \eta(x, y, z)$. For the case of zero viscosity $\eta$, the solution to system (1-3) was obtained as the sum $\rho=\rho_{0}+\rho_{1}, P=P_{0}+P_{1}, \Phi=\Phi_{0}+\Phi_{1}, V=V_{0}+V_{1}$, where $\rho_{0}, P_{0}, \Phi_{0}, V_{0}$ is an unperturbed solution, $\rho_{1}, P_{1}, \Phi_{1}, V_{1}$ are small perturbations [11]. In the case of nonzero viscosity $\eta$, the equation for the density perturbations $\rho_{1}$ will have the following form:

$$
\frac{\partial^{2} \rho_{1}}{\partial t^{2}}=c^{2} \nabla^{2} \rho_{1}+4 \pi g \rho_{0} \rho_{1}+2 \rho_{0} \cdot \operatorname{div}(\boldsymbol{\Omega} \times \mathbf{V})+\rho_{0} \eta \cdot \operatorname{div}\left(\frac{\mathbf{N}}{\rho}\right)
$$

where $c$ is the speed of sound. The solution of the system of equations (1-3) can be obtained by numerical methods if the dynamic viscosity $\eta$ is known. If the viscosity $\eta$ is an unknown function, then the viscosity $\eta$ can be determined from the Navier-Stokes equations under additional assumptions. For example, for thin and Keplerian disks, the viscosity $\eta$ can be determined using the Maple computerized analytical computation system. Effective viscosity $\eta_{\mathrm{eff}}$ is equal to the sum of some constant component $\eta_{0}$, viscosity $\eta$ from pulsation disturbances and others. The effective viscosity components associated with other mechanisms of evolution of the equatorial disks of protostars were not taken into account in this model. The solution to the boundary value problem for equation (4), which takes into account the rotation of the protostellar cloud, will have the following form [11]:

$$
\begin{array}{r}
\rho_{1}(r, z, t)+\rho_{d}(r, t)=\sum_{k=0}^{n} d_{k}(t) J_{0}\left(\lambda_{k} r / R_{0}\right)+\rho\left(z_{1}\right) \exp (-\gamma z) a /(n+1) . \\
\cdot \sum_{k=0}^{n} \cos \left(t c \sqrt{\left(\lambda_{k} / R_{0}\right)^{2}-4 \pi g \rho_{0} / c^{2}-\gamma^{2} / c^{2}} J_{0}\left(\lambda_{k} r / R_{0}\right) .\right.
\end{array}
$$

The functions $d_{k}(t)$ are determined from the condition $\rho_{1}\left(r, R_{0}, 0\right)+\rho_{d}(r, 0)=0$ of the boundary value problem for equation (4), where $\rho_{0}$ is the average density of the protostar (disk), $\gamma=\left[1 /\left(z_{1}{ }^{\smile} z_{0}\right)\right] \ln \left(\rho\left(z_{0}\right) / \rho\left(z_{1}\right)\right)$, $z_{0}, z_{1}$ are the initial and final radius of the protostar, $\lambda_{k}$ are the zeros of the Bessel function $J_{0}(r), g$ is the gravitational constant, $c$ is the speed of sound.

\section{Acoustic experiments with a prototype accretion model and their results}

The idea of conducting new acoustic experiments appeared as a result of studying the works of [14, 15, 16]. In the work of [17], Chladni figures were obtained for the calculated values of natural frequencies:

$$
\Omega_{k}=\mu_{k}^{(0)} / R \sqrt{F / \rho}=2 \mu_{k}^{(0)} \omega_{0} / \pi
$$

where $F$ is the membrane tension force, $\rho$ is the material density, $\omega_{0}$ is the natural vibration frequency of the membrane, $\mu_{k}$ are the zeros of the zero-order Bessel function, $R$ is the disk radius. For the calculations, the following parameter values were used [17]: $F=E=195 \mathrm{GPa}$, where $E$ is the intrinsic elasticity of the membrane material $\rho=7.87 \cdot 10^{3} \mathrm{~kg} / \mathrm{m}^{3}, \omega_{0}=1.58 \mathrm{MHz}$ (iron). The aim of a new experiment with a disc (prototype) is to study the effect of total central disturbances on a complex of small stones distributed along the perimeter of a circular disc. Initial data of the experiment: 1 . A round iron disk, $9.5 \mathrm{~cm}$ in radius, not fixed around the perimeter. 2. The disc is fixed in the center on a metal $\operatorname{rod}(20 \mathrm{~cm})$. Central perturbations are transmitted through this rod to the disk. Oscillation frequency $50 \mathrm{~Hz} \pm \delta(\delta$ - small value). 3. At the initial moment of time, small stones (ASG) of various sizes from $0.5 \mathrm{~mm}$ to $3 \mathrm{~mm}$ are distributed along the perimeter of the disk. 4 . Duration of the experiment and the action of central disturbances: $3.8 \mathrm{~min}$.

\section{Comparison of the prototype and the accretion model}

Table 1 shows the eigenfrequencies of the prototype: $\Omega_{k}(M H z)$ and, for comparison, the eigenfrequencies $\omega_{k}(n H z)$ of the accretion model, as well as the return periods of disturbance waves $1 / \omega_{k}$, expressed in seconds in year. The natural frequency $\omega_{k}$ of the accretion model was calculated according to formula (5): $\omega_{k}=1 / T_{k}$. The period $T_{k}$ is determined from the following equation: $c R_{0}^{-3 / 4} \lambda_{k} T_{k} / R_{0}=2 \pi, c=1286 \mathrm{~m} / \mathrm{s}$ - the speed of sound for hydrogen at $\left.R_{0}=1 \mathrm{AU}\right)$. In contrast to the experiments presented in papers [15, 16, 17], the round disk was not perfectly flat. As a result, the bulk of stones, large and small, rushed to the center of the disc and formed a circle in the center, slowly rotating counterclockwise. Simultaneously with this, in small depressions of the disk along the perimeter, the enlargement of some collections and the exhaustion of others took place. The size of the stones did not matter. The collection formation process slowed down and accelerated for unknown reasons. The course of the experiment 
Table 1: Fundamental frequencies of the prototype $\left(\Omega_{k}\right)$ and the accretion model $\left(\omega_{k}\right)$.

\begin{tabular}{|ccccl|}
\hline$k$ & $\lambda_{k}$ & $\Omega_{k}(\mathrm{MHz})$ & $\omega_{k}(\mathrm{nHz})$ & $1 / \omega_{k}$ (year) \\
\hline 0 & 2.40 & 25.48 & 0.197 & 161.36 \\
1 & 5.52 & 58.48 & 0.452 & 70.31 \\
2 & 8.65 & 91.67 & 0.709 & 44.85 \\
3 & 11.79 & 125 & 0.965 & 32.91 \\
4 & 14.93 & 158 & 1.22 & 25.99 \\
5 & 18.07 & 191 & 1.22 & 25.99 \\
6 & 21.21 & 225 & 1.40 & 21.47 \\
7 & 24.35 & 258 & 1.99 & 15.94 \\
8 & 27.49 & 291 & 2.25 & 14.12 \\
9 & 30.63 & 325 & 2.51 & 12.67 \\
\hline
\end{tabular}

was filmed and presented in the form of a microfilm. There is no metal disk in the accretion model. Instead, it is the central equatorial disk of gas and dust in gravitational equilibrium. Perturbations of the near-Keplerian gravitational field generate acoustic waves similar to those formed in the prototype disk. The processes, in this case, will occur in the same way, since they have the same physical basis.

\section{Conclusions}

The results of the experiment with the prototype (6) of the accretion model (5) show: 1) accretion onto the core of the protostar and accretion onto the "embryo" of the planet can occur simultaneously at certain frequencies of central disturbances; 2) the prototype (6) and the accretion model (5) have a single physical (acoustic) basis and the results of the experiment with the prototype prove the adequacy of the accretion model $(5) ; 3)$ the accretion of gas and dust onto the core of the protostar occurs under the action of the force of the gravitational field of the gas-dust cloud. Accretion on the "embryo" of the planet occurs due, first of all, to the action of central disturbances of the gravitational field in the form of ultrasonic waves (density waves).

\section{References}

1. S. Wolf, F. Gueth, T. Henning, and W. Kley, ApJL, 566, L97, 2002.

2. N. Calvet, P. D'Alessio, L. Hartmann, D. Wilner, A. Walsh, and M. Sitko, ApJ, 568, $1008,2002$.

3. G. H. M. Bertrang, 2017.

4. T. R. Abdulmyanov, in Modern Star Astronomy, volume 1, 69-72 (2018).

5. A. Garufi, G. Meeus, M. Benisty, S. P. Quanz, et al., AछA, 603, A21, 2017.

6. A. Garufi, 2017.

7. F. Meru, 2017.

8. V. N. Snytnikov and O. P. Stoyanovskaya, Episodic Accretion and Outbursts of Young Stars at an Early Stage of their Evolution, in Life and Universe, 43-52 (2017).

9. T. R. Abdulmyanov, Astrophysical Bulletin, 75, 117, 2020.

10. K. Rohlfs, Lectures on the density wave theory. (1980).

11. T. R. Abdulmyanov, Moscow University Physics Bulletin, 74, 309, 2019.

12. L. D. Landau and E. M. Lifshits, Theoretical Physics. Hydrodynamics, volume VI (1986).

13. G. V. Lipunova, K. L. Malanchev, and N. I. Shakura, Accretion processes in astrophysics (in Russian), Phys.mat. lit. (2016).

14. J. V. Strutt, Sound theory, Moscow, GITTL Publ., volume 1 (1955).

15. V. V. Meleshko and S. O. Papkov, 12, 34, 2009.

16. O. A. Zhuravlev, K. S. Yu., and M. N. Ye., Mechanics and mechanical engineering, 5, 1, 2003.

17. V. S. Korneev, Bulletin of SGUGiT. Optics, optoelectronic devices and complexes, 22, 173, 2017. 


\title{
Determination of interstellar extinction parameters at high galactic latitudes
}

\author{
A. Avdeeva ${ }^{1,2}$, D. Kovaleva ${ }^{1}$, O. Malkov ${ }^{1}$, A. Nekrasov ${ }^{3}$ \\ ${ }^{1}$ Institute of Astronomy, 48 Pyatnitskaya St., Moscow 119017, Russia, \\ ${ }^{2}$ HSE University, 20 Myasnitskaya Ulitsa, Moscow 101000, Russia, \\ ${ }^{3}$ Moscow State University, Moscow, Russia
}

The distribution of visual interstellar extinction $A_{V}$ has been mapped in selected areas over the Southern sky, using RAVE DR6 and Gaia EDR3 data. $A_{V}$ was modelled as a barometric function of galactic latitude and distance. The function parameters were then approximated by spherical harmonics. The resulting analytical 3D model of the interstellar extinction can be used to predict $A_{V}$ values for stars with known parallaxes, as well as the total Galactic extinction in a given location in the sky.

Keywords: interstellar extinction, surveys

DOI: $10.51194 /$ VAK2021.2022.1.1.179

\section{Introduction}

Estimation of interstellar extinction is an important step in solving problems associated with determining the astrophysical parameters of galactic and extragalactic objects. For this reason, three-dimensional extinction maps are popular. They are constructed, for example, based on spectral and photometric data on stars, star clusters and models of dust distribution in the Galaxy.

The standard approach for building 3D maps is to divide the celestial sphere into cells with boundaries defined in galactic coordinates $l, b$ and then express the absorption (e.g. visual) $A_{V}(l, b)$ in a fixed direction as a function of the distance $A_{V}(l, b, d)$. The output is a set of discrete values for galactic coordinates and distance, which is not very convenient for use in large-scale studies.

The classical model of a homogeneous absorbing layer with a density that is exponentially distributed over height can be expressed by the cosecant law, firstly introduced by [1]:

$$
A_{V}=a_{0} \cdot \beta / \sin |b| \cdot(1-\exp (-d \cdot \sin |b| / \beta))
$$

Here $d$ is a distance from the Sun to the star. This dependence has two parameters: $a 0$, which shows the absorption per unit distance in the galactic plane, and $\beta$, which expresses the scale of heights.

[2] calculated visual extinctions for stars in 40 areas of the northern sky based on the LAMOST and GAIA EDR3 data. They approximated these data by the cosecant law (1) and obtained pairs of parameters $a 0$ and $\beta$ for these 40 areas. Then the parameter values were approximated by spherical functions over the entire sky. Thus, they have got not a separate set of visual extinction values, but a mathematical dependence of extinction on latitude, longitude and distance from the Sun.

The goal of this work is to use the data of the spectroscopic survey of the southern sky RAVE DR6 and the all-sky GAIA EDR3 in order to calculate the interstellar extinction in the selected areas of the southern sky, obtain the parameters $a 0$ and the $\beta$ cosecant law for these areas, and then approximate them by spherical harmonics over the entire sky.

\section{Data and calculating the visual extinction}

The work made use of data from GAIA EDR3 and RAVE DR6. Objects were selected from 40 areas in the coverage area of the RAVE survey in high latitudes $\left(|b|>20^{\circ}\right)$. We have made cross-identification of these objects with the objects of the GAIA EDR3 catalogue. For each of the resulting objects, the distance was taken from the [3] catalogue, also based on GAIA EDR3 data.

Extinction in the $\mathrm{V}$ band for each object was calculated using the following formula:

$$
A_{V}=c 1 / c 2 \cdot\left((B P-R P)-(B P-R P)_{0}\right)
$$

which includes the colour index $(B P-R P)$ from the GAIA catalogue and the intrinsic colour index $(B P-R P)_{0}$ determined from the objects temperature according to the RAVE DR6 (BDASP) data from [4]. The coefficient $c 1$ is $A_{G}$ to $E(B P-R P)$ ratio and $c 2$ is an absorption coefficient, $A_{G}$ to $A_{V}$ ratio. $A_{G}$ is the interstellar extinction in G-band of Gaia. According to Bono et.al, $\mathrm{c} 2=0.84$ and $\mathrm{c} 1$ is found to be equal to 2.02.

To eliminate the stars with outrageously low extinctions at far distances, we set the following restrictions on the data used for the work: $1.6>B P-R P>0.8$ and $\log _{B D A S P}>3.5$. For objects with such parameters, the RAVE data are consistent with the cosecant law and can be used to conduct this study. 


\section{Approximation for interstellar extinction in the selected areas}

The extinctions calculated in the areas were approximated by the cosecant law by minimizing the following $\chi^{2}$ functional:

$$
\chi^{2}=\sum_{n=1}^{N}\left(\frac{A_{V}\left(d_{n}\right)-A_{V, n}}{\epsilon_{n}\left(d_{n}, A_{V, n}\right)}\right)^{2}
$$

$A_{V}\left(d_{n}\right)$ - values, predicted by the cosecant law (1) for a distance $d_{n} ; \epsilon_{n}$ - uncertainty of extinction and distances, $\mathrm{N}$ is the total number of objects in the area.

The illustrations of this approximation are presented in Fig. 1 The figure shows data sets in four sites and the result of the approximation is a green curve. For 36 sites the approximation turned out to be successful.
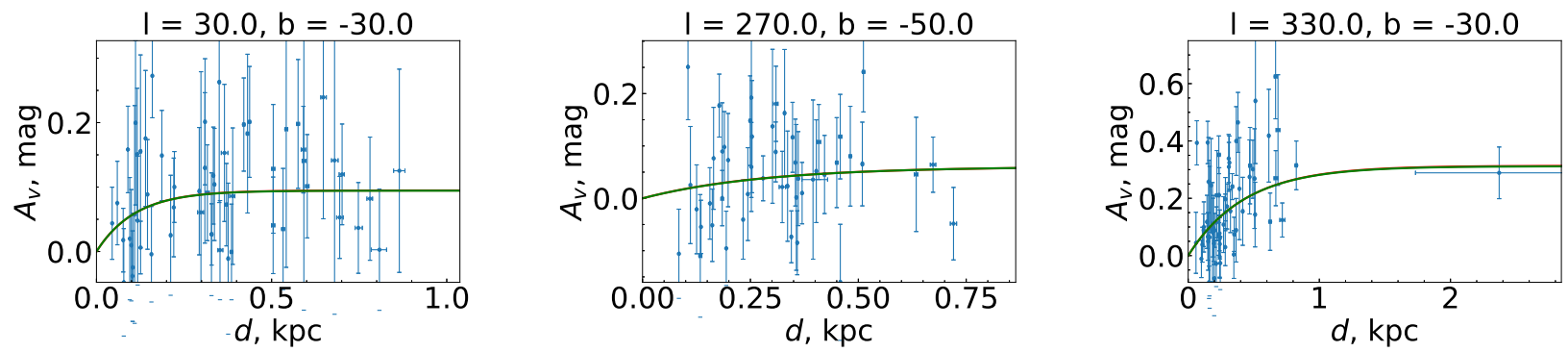

Figure 1: Examples of best-fit $\chi^{2}$ minimization. Galactic coordinates of the center of the region are on top of each graph. Green line is the result of approximation.

\section{Approximation for $a 0, \beta$ parameters over the entire sky}

Here is the result of the approximation of the parameters $a 0, \beta$, as well as the total Galactic extinction $A_{G a l}$ by a polynomial composed of spherical harmonics of degree and order 2 :

$$
\begin{aligned}
f(l, b)= & A_{00} Y_{0}^{0}\left(l, \frac{\pi}{2}-b\right)+A_{10} Y_{1}^{0}\left(l, \frac{\pi}{2}-b\right)+A_{11} Y_{1}^{1}\left(l, \frac{\pi}{2}-b\right)+ \\
& +A_{20} Y_{2}^{0}\left(l, \frac{\pi}{2}-b\right)+A_{21} Y_{2}^{1}\left(l, \frac{\pi}{2}-b\right)+A_{22} Y_{2}^{2}\left(l, \frac{\pi}{2}-b\right)
\end{aligned}
$$

For the approximation, we took the data obtained both in this work and from the work of [2]. The coefficient of the polynomial for $a 0, \beta$ and $A_{G a l}$ approximation are presented in Tab.1.

If we substitute the dependences obtained for $a 0$ and $\beta$ into the cosecant law, we obtain a formula that can be used to estimate the absorption at high galactic latitudes for a given direction and distance.

Table 1: The coefficients in the approximation of spherical harmonics

\begin{tabular}{c|ccc}
\hline$f(l, b)$ & $a_{0}$ & $\beta$ & $A_{\text {Gal }}$ \\
\hline$A_{00}$ & $10.0 \pm 1.5$ & $811.4 \pm 210.2$ & $0.967 \pm 0.113$ \\
$A_{10}$ & $-0.9 \pm 1.5$ & $495.3 \pm 212.4$ & $-0.148 \pm 0.114$ \\
$A_{11}$ & $-6.64 \pm 2.18$ & $715.7 \pm 298.9$ & $0.02 \pm 0.16$ \\
$A_{20}$ & $-4.0 \pm 1.8$ & $336.3 \pm 250.6$ & $-0.51 \pm 0.13$ \\
$A_{21}$ & $-2.6 \pm 1.9$ & $520.2 \pm 266.8$ & $-0.43 \pm 0.14$ \\
$A_{22}$ & $0.77 \pm 2.19$ & $32.4 \pm 300.5$ & $0.56 \pm 0.16$ \\
\hline
\end{tabular}

\section{Summary}

In this work, we have calculated the interstellar extinction $A_{V}$ for objects in 36 areas of the southern sky. We have found discrepancies in the LAMOST DR5 and RAVE DR6 data and determined the region in the parameter space in which the RAVE DR6 data agree with the cosecant law. We have approximated the parameters $a 0$ and $\beta$ both obtained in this work and in [2] on the entire celestial sphere by the spherical harmonics. As a result, we have obtained an approximate analytical formula for estimating the interstellar extinction at high galactic latitudes. 
Acknowledgment: Authors thank George Gontcharov for valuable comments and suggestions. We thank Kirill Grishin for fruitful discussion and help with approximation methods. This work was partly supported by the RFBR grant 20-52-53009.

\section{References}

1. P. P. Parenago, Astron. Zh., 13, 3, 1940.

2. A. Nekrasov, K. Grishin, D. Kovaleva, and O. Malkov, European Physical Journal Special Topics, 230, 2193, 2021.

3. C. A. L. Bailer-Jones, J. Rybizki, M. Fouesneau, M. Demleitner, and R. Andrae, AJ, 161, 147, 2021.

4. M. J. Pecaut and E. E. Mamajek, ApJS, 208, 9, 2013. 


\title{
Small-scale sectorial modes of perturbations against the background of a pulsating model of disk-like self-gravitating systems
}

\author{
J.M. Ganiev, S.N. Nuritdinov
}

ganiev_jakhongir@mail.ru

National University of Uzbekistan, Tashkent 100174, Uzbekistan

\section{DOI: 10.51194 /VAK2021.2022.1.1.180}

It is known that a large-scale and various small-scale structural formations in disk-like galaxies can be formed due to gravitational instabilities. The observed structural formations had been appeared during the non-stationary stage of the evolution of galaxies. Therefore, we study the gravitational instability of small-scale perturbations on the background of a radially pulsating disk.

In this paper we considered sectorial small-scale perturbation modes on the background of a pulsating disk model with an anisotropic velocity diagram for a set of values of wave numbers $m ; N:(m=N)=$ $(10 ; 10),(11 ; 11),(12 ; 12),(13 ; 13),(14 ; 14),(15 ; 15)$. A non-stationary dispersion equation on the background of a nonlinear non-stationary model is obtained, according to the general principle in the form:

$$
a_{\text {Aniz }}(\psi)=\frac{4}{N\left(N^{2}-1\right)^{3}} \gamma_{N m}(N-1)\left(N^{2}+N-m^{2}\right) \overline{D_{N}}+i \Omega m\left(N^{2}+N-m^{2}\right) d_{N} .
$$

Here $N$ is the radial wave number; $m$ is the azimuthal wave number. For the remaining designations, see [1]). The calculation results show that the instability in the case of $m ; N=10 ; 10$ begins with the value of the virial parameter's $(2 T / U)_{0}=0.056$ and reaches 0.325 (see Table 1 ). The critical values of the virial parameter's for the remaining cases is given in Table 1 . In addition, we have calculated and compared the values of instability increments for each mode.

Table 1: Critical values of the virial parameter's.

\begin{tabular}{ccccccc}
\hline$m ; N$ & $10 ; 10$ & $11 ; 11$ & $12 ; 12$ & $13 ; 13$ & $14 ; 14$ & $15 ; 15$ \\
\hline$(2 T / U)_{0}$ & $0.056-0.325$ & $0.049-0.282$ & $0.044-0.248$ & $0.039-0.221$ & $0.035-0.199$ & $0.032-0.180$ \\
\hline
\end{tabular}

\section{References}

1. J. M. Ganiev and I. U. Tadjibaev, in Modern Star Astronomy, volume 1, 104-106 (2018). 


\section{Interstellar extinction at high Galactic latitudes across the whole dust layer in the Galaxy}

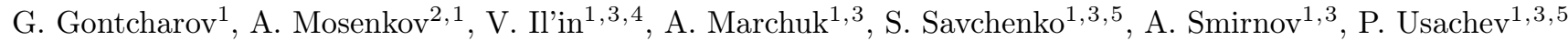
georgegontcharov@yahoo.com

${ }^{1}$ Central (Pulkovo) Astronomical Observatory, Russian Academy of Sciences, Pulkovskoye chaussee 65/1, St. Petersburg 196140, Russia,

${ }^{2}$ Department of Physics and Astronomy, N283 ESC, Brigham Young University, Provo, UT 84602, USA,

${ }^{3}$ Saint Petersburg State University, Universitetskij pr. 28, St. Petersburg 198504, Russia,

${ }^{4}$ Saint Petersburg University of Aerospace Instrumentation, Bol. Morskaya ul. 67A, St. Petersburg 190000, Russia,

${ }^{5}$ Special Astrophysical Observatory, Russian Academy of Sciences, 369167 Nizhnij Arkhyz, Russia

By use of various methods we estimate interstellar extinction at high Galactic latitudes across the whole dust layer in the Galaxy in a variety of photometric filters. First, we compare reddening estimates from the 3D map of Green-2019 (i) inside and (ii) around 23 Galactic globular clusters, i.e. where (i) the estimates are obtained from the Pan-STARRS photometry of the cluster stars and (ii) estimates from the Schlegel-1998 (SFD98) map are used due to the lack of the stars in Pan-STARRS. The estimates (i) turn out to be systematically higher than (ii) by $E(B-V)=0.04$ mag. This means that SFD98 underestimates low reddenings by the value $E(B-V)=0.04$ mag. Second, we estimate extinction from comparison of photometric observations and theoretical isochrones for 43 Galactic globular clusters. The lowest extinction is $A_{\mathrm{V}}=0.1 \mathrm{mag}$. Third, we analyze the spatial variations of the observed colours for an unprecedentedly large and complete sample of 101810 clump giants with precise parallaxes and photometry from Gaia DR2 and WISE. This sample is selected by us in the space cylinder with a radius of $700 \mathrm{pc}$ around the Sun and a height of $3600 \mathrm{pc}$ across the whole Galactic dust layer. Until now, complete samples of stars with precise distances and photometry have not been considered at such a large distance from the Galactic plane. This explains our unexpected results: the Galactic dust layer appears thicker than previously thought - the extinction continues to increase even at a distance of $|Z|>400$ pc from the plane of the Galaxy; the mean extinction across the whole half-layer below or above the Sun is $A_{\mathrm{V}}=0.2 \mathrm{mag}$. Fourth, by use of the 3D reddening maps of Gontcharov-2017, Lallement-2019, and Green-2019 we calculate the most probable parameters for a new version of our 3D model of the dust spatial distribution in a wide Galactic neighbourhoods of the Sun. Comparing with other models, our model provides the most accurate predictions of the observed colours of the above mentioned sample of $101810 \mathrm{clump}$ giants. This comparison predicts the median reddening $E(B-V)=0.06$ mag for high latitudes $|b|>50$ deg, and the dust container of the Gould Belt provides up to a half of this reddening.

Keywords: interstellar extinction, local interstellar matter, Solar neighbourhood, globular clusters: general

DOI: $10.51194 /$ VAK2021.2022.1.1.181

\section{Reddening inside and around Galactic globular clusters}

The 3D reddening map of GSZ19 [1] is the first map with accurate reddening estimates far beyond the dust layer at high-latitudes. It is based on Gaia DR2 parallaxes and a deep multiband photometry [2]. GSZ19 use thousands stars from Galactic globular clusters (GCs) in a typical voxel (space cell) within a GC field to derive reddening, distance modulus, absolute magnitude, and metallicity of this GC. These results agree with those for the same GCs obtained by other authors and using different methods. In contrast, GSZ19 use only a hundred of stars in a typical voxel outside the GC fields, due to a sparse distribution of the stars far from the Galactic mid-plane. Therefore, the GSZ19 reddening estimates in the GC voxels seem to be more reliable than those in the surrounding non-GC voxels. For $23 \mathrm{GCs}$ at the middle and high Galactic latitudes we discover that the former estimates are systematically higher by $\Delta E(B-V)=0.038$ than the latter. Given the mean reddening $E(B-V)=0.015 \mathrm{mag}$ from GSZ19 in all non-GC voxels near the Galactic poles, we estimate the mean reddening across the whole dust half-layer below or above the Sun, from the GSZ19 map as $E(B-V)=0.038+0.015=0.053$ mag.

\section{Isochrone fitting for 43 Galactic globular clusters}

We provide isochrone fits to colour-magnitude diagrams of 43 GCs at middle and high Galactic latitudes by use of photometry in various ultraviolet, optical and infrared (IR) filters in combination with theoretical isochrones from various models. To select the GC members, we use the Gaia EDR3 parallaxes and proper motions [3]. In order to estimate the extinction $A_{\mathrm{V}}$ from the measured reddening $E(V-I R)$ and a very low IR extinction $A_{\mathrm{IR}}$, we use the relation:

$$
A_{\mathrm{V}}=\left(A_{\mathrm{V}}-A_{\mathrm{IR}}\right)+A_{\mathrm{IR}}=E(V-I R)+A_{\mathrm{IR}}
$$

as well as similar relations for other filters. As a result, we find no GCs with $A_{\mathrm{V}}<0.1$. Our results for two GCs are presented in [4] and [5].

\section{Reddening of the clump giants}

We create and analyze a complete sample of 101810 giants with Gaia DR2 parallaxes within the red clump domain of the Hertzsprung-Russell diagram in the space cylinder with a radius of $700 \mathrm{pc}$ around the Sun and a height of 
$|Z|=1800$ pc. We use photometry from Gaia DR2 and WISE [6]. We describe the spatial variations of the modes of the observed colours and magnitudes by extinction and reddening in combination with linear vertical gradients of the intrinsic colours and absolute magnitudes of the clump red giants. We confirm the derived characteristics of the clump giants by comparing them with the theoretical predictions from various models. The derived extinctions and reddenings across the whole dust half-layer below or above the Sun converge to the median extinction $A_{\mathrm{V}}=0.2$ $\operatorname{mag}[7]$.

\section{3D dust distribution model}

We use the new version GM21 of our 3D dust distribution model [8], first introduced in 2009 [9]. GM21 proposes two intersecting dust layers, along the Galactic mid-plane and in the Gould Belt, with exponential vertical and sinusoidal longitudinal variations of the dust spatial density in each layer. The Belt layer is an ellipse, oriented nearly between the centre and anticentre of the Galaxy, and with a semi-major and semi-minor axes of 600 and 146 pc, respectively. The parameters of GM21 are calculated by use of the above mentioned sample of 101810 Gaia DR2 giants and, alternatively, by use of the 3D reddening maps of G17 [10], LBV19 [11], or GSZ19. The different data provide similar geometry of the model, but different thickness of the layers and different vertical distribution of dust. The data for the giants, with the most homogeneous covering of the vertical space column across the whole dust layers, provide for GM21 the median reddening $E(B-V)=0.06$ mag for $|b|>50$ degs.

Acknowledgement. We acknowledge financial support from the Russian Science Foundation (grant no. 20-7210052).

\section{References}

1. G. M. Green, E. Schlafly, C. Zucker, J. S. Speagle, and D. Finkbeiner, ApJ, 887, 93, 2019.

2. D. W. Evans, M. Riello, F. De Angeli, J. M. Carrasco, et al., A $\& A$, 616, A4, 2018.

3. M. Riello, F. De Angeli, D. W. Evans, P. Montegriffo, et al., A $6 A$, 649, A3, 2021.

4. G. A. Gontcharov, A. V. Mosenkov, and M. Y. Khovritchev, MNRAS, 483, 4949, 2019.

5. G. A. Gontcharov, M. Y. Khovritchev, and A. V. Mosenkov, MNRAS, 497, 3674, 2020.

6. E. L. Wright, P. R. M. Eisenhardt, A. K. Mainzer, M. E. Ressler, et al., AJ, 140, 1868, 2010.

7. G. A. Gontcharov and A. V. Mosenkov, MNRAS, 500, 2590, 2021.

8. G. A. Gontcharov and A. V. Mosenkov, MNRAS, 500, 2607, 2021.

9. G. A. Gontcharov, Astronomy Letters, 35, 780, 2009.

10. G. A. Gontcharov, Astronomy Letters, 43, 472, 2017.

11. R. Lallement, C. Babusiaux, J. L. Vergely, D. Katz, F. Arenou, B. Valette, C. Hottier, and L. Capitanio, A\& A, 625, A135, 2019. 


\title{
The properties of Galactic globular clusters from Gaia EDR3 and other data compared with theoretical isochrones
}

\author{
G. Gontcharov ${ }^{1}$, A. Mosenkov ${ }^{2,1}$, M. Khovritchev ${ }^{1,3}$, V. Il'in ${ }^{1,3,4}$, A. Marchuk ${ }^{1,3}$, S. Savchenko ${ }^{1,3,5}$, A. \\ Smirnov $^{1,3}$, P. Usachev ${ }^{1,3,5}$ \\ georgegontcharov@yahoo.com \\ ${ }^{1}$ Central (Pulkovo) Astronomical Observatory, Russian Academy of Sciences, Pulkovskoye chaussee 65/1, St. Petersburg \\ 196140, Russia \\ ${ }^{2}$ Department of Physics and Astronomy, N283 ESC, Brigham Young University, Provo, UT 84602, USA \\ ${ }^{3}$ Saint Petersburg State University, Universitetskij pr. 28, St. Petersburg 198504, Russia \\ ${ }^{4}$ Saint Petersburg University of Aerospace Instrumentation, Bol. Morskaya ul. 67A, St. Petersburg 190000, Russia \\ ${ }^{5}$ Special Astrophysical Observatory, Russian Academy of Sciences, 369167 Nizhnij Arkhyz, Russia
}

\begin{abstract}
We fit theoretical isochrones from different models of internal structure and evolution of stars to photometric data for the stars in globular clusters of our Galaxy. To select cluster members, determine cluster sizes and calculate systemic proper motions, we use parallaxes and proper motions from Gaia EDR3. To calculate the most probable distance, age, interstellar extinction in a variety of filters and differential reddening in cluster fields, we use photometry in more than 26 filters between the ultraviolet and mid-infrared waverange from HST, Gaia EDR3, Pan-STARRS DR1, DES, SDSS, unWISE, SAGE and other datasets in combination with the PARSEC, MIST, DSEP, and BaSTI-IAC isochrones, as for the solar metallicity scale as for alpha- and helium-enriched scales. The metallicity and enrichment of the clusters is taken from spectroscopic observations and tested for compliance with the photometric results. We carry out a thorough analysis of random and systematic uncertainties of the obtained results. The derived extinctions in many filters allow us to estimate an empirical extinction law for each cluster. A complete analysis has been performed for five clusters (NGC288, NGC362, NGC5904, NGC6205, and NGC6218), a preliminary analysis, based on the Gaia EDR3 astrometry and photometry only, has been done for 38 more clusters. The main conclusions are as follows. First, unprecedentedly accurate astrometry of Gaia EDR3 allows us to segregate the cluster members from fore- and background stars and to indicate that the size of many clusters is much larger than previously thought. Second, the distances, derived by us from the photometry-to-isochrone fitting, are still more precise than distances from the Gaia EDR3 parallaxes. Third, contrary to the popular 2D reddening maps of Schlegel-1998 and Planck, we found no clusters at high Galactic latitudes with an extinction $A_{\mathrm{V}}<0.1$. Fourth, for the horizontal branch second parameter quartet NGC 288-NGC 362-NGC 5904-NGC 6218, the age is undoubtedly the second parameter.
\end{abstract}

DOI: 10.51194/VAK2021.2022.1.1.182

Recently, all ingredients of successful isochrone-to-data fitting for Galactic globular clusters (GCs) have been improved considerably. Namely, very accurate parallaxes and proper motions from Gaia EDR3 [1] allow one to segregate cluster members from contaminating stars. Moreover, very precise and extended datasets have been obtained, as from space, such as those from Hubble Space Telescope (HST) WFC3 and ACS cameras [2], from Gaia EDR3, and from Wide-field Infrared Survey Explorer (WISE)'s unWISE catalogue [3], as from the ground, such as a compilation of data by Stetson [4]. Finally, reliable alpha-enriched isochrones have been presented by the Dartmouth Stellar Evolution Program (DSEP) [5] and a Bag of Stellar Tracks and Isochrones (BaSTI-IAC) [6]. The solar-scaled models from the PAdova and TRieste Stellar Evolution Code (PARSEC) [7] and the MESA Isochrones and Stellar Tracks (MIST) [8] cannot substitute $\alpha$-enhanced ones.

We provide isochrone fits to CMDs with 29, 34, 30, 26, and 26 photometric filters for NGC 5904, NGC 6205 (M13), NGC 288, NGC 362, and NGC6218 (M12), respectively. Filters between the HST/WFC3 F275W at 285 $\mathrm{nm}$ and the WISE $W 1$ at $3317 \mathrm{~nm}$ are considered, i.e. from the ultraviolet to the mid-infrared range. Our results for these GCs are presented in [9], [10], and [11]. All the derived parameters agree with the previous estimates from [12] and other studies. The derived extinction law is close to the common law of Cardelli-1989 [13] for low-latitude GCs, while for high-latitude GCs we find a higher extinction-to-reddening ratio $R_{\mathrm{V}} \equiv A_{\mathrm{V}} / E(B-V)$, in agreement with the finding of [14]. Consequently, for high-latitude GCs the derived $E(B-V)$ estimates are comparable or even lower than those from the 2D dust emission maps [15], [16], and [17], while the derived $A_{\mathrm{V}}$ estimates are significanly higher than those from these maps. This suggests that for extragalactic sources we should use the available reddening and extinction maps with caution.

Acknowledgement. We acknowledge financial support from the Russian Science Foundation (grant no. 20-7210052).

\section{References}

1. M. Riello, F. De Angeli, D. W. Evans, P. Montegriffo, et al., A $\& A, 649$, A3, 2021.

2. D. Nardiello, M. Libralato, G. Piotto, J. Anderson, et al., MNRAS, 481, 3382, 2018.

3. E. F. Schlafly, A. M. Meisner, and G. M. Green, ApJS, 240, 30, 2019.

4. P. B. Stetson, E. Pancino, A. Zocchi, N. Sanna, and M. Monelli, MNRAS, 485, 3042, 2019.

5. A. Dotter, B. Chaboyer, D. Jevremović, V. Kostov, E. Baron, and J. W. Ferguson, ApJS, 178, 89, 2008. 
6. A. Pietrinferni, S. Hidalgo, S. Cassisi, M. Salaris, et al., ApJ, 908, 102, 2021.

7. A. Bressan, P. Marigo, L. Girardi, B. Salasnich, C. Dal Cero, S. Rubele, and A. Nanni, MNRAS, 427, 127, 2012.

8. J. Choi, A. Dotter, C. Conroy, M. Cantiello, B. Paxton, and B. D. Johnson, ApJ, 823, $102,2016$.

9. G. A. Gontcharov, A. V. Mosenkov, and M. Y. Khovritchev, MNRAS, 483, 4949, 2019.

10. G. A. Gontcharov, M. Y. Khovritchev, and A. V. Mosenkov, MNRAS, 497, 3674, 2020.

11. G. A. Gontcharov, M. Y. Khovritchev, A. V. Mosenkov, V. B. Il'in, et al., MNRAS, 508, $2688,2021$.

12. W. E. Harris, AJ, 112, 1487, 1996.

13. J. A. Cardelli, G. C. Clayton, and J. S. Mathis, ApJ, 345, 245, 1989.

14. G. A. Gontcharov, Astronomy Letters, 38, 12, 2012.

15. D. J. Schlegel, D. P. Finkbeiner, and M. Davis, ApJ, 500, 525, 1998.

16. E. F. Schlafly and D. P. Finkbeiner, ApJ, 737, 103, 2011.

17. A. M. Meisner and D. P. Finkbeiner, ApJ, 798, 88, 2015. 


\section{A Study of Double- and Multi-Mode High-Amplitude Delta Scuti Variables}

A.V. Khruslov

khruslov@bk.ru

Sternberg Astronomical Institute, Moscow State University,

Universitetskij pr. 13, Moscow 119992, Russia

\section{DOI: 10.51194 /VAK2021.2022.1.1.183}

I searched for double- and multi-mode high-amplitude $\delta$ Scuti variables using data of the ASAS-SN survey. To select candidates, I used the ASAS-SN Database ${ }^{a}$. I searched for stars with scattered light curves and then analyzed data using Deeming's method implemented in the WinEfk code developed by V.P. Goranskij ${ }^{\text {b }}$. I detected $\sim 300$ double-mode stars pulsating in the fundamental and first-overtone modes, $\sim 20$ stars pulsating in the firstand second-overtone modes, four three-modal stars pulsating in the fundamental, first- and second-overtone modes, and several candidate HADS variables pulsating in higher overtones. Detected variables are shown in the Petersen diagram (Fig. 1). Designations: $P_{L}$ : longer period; $P_{S}$ : shorter period; open symbols: known Galactic HADS(B) stars; filled symbols: new double- and multi-mode variables. Circles, triangles and squares: double- , triple- and quadruple-mode variables.

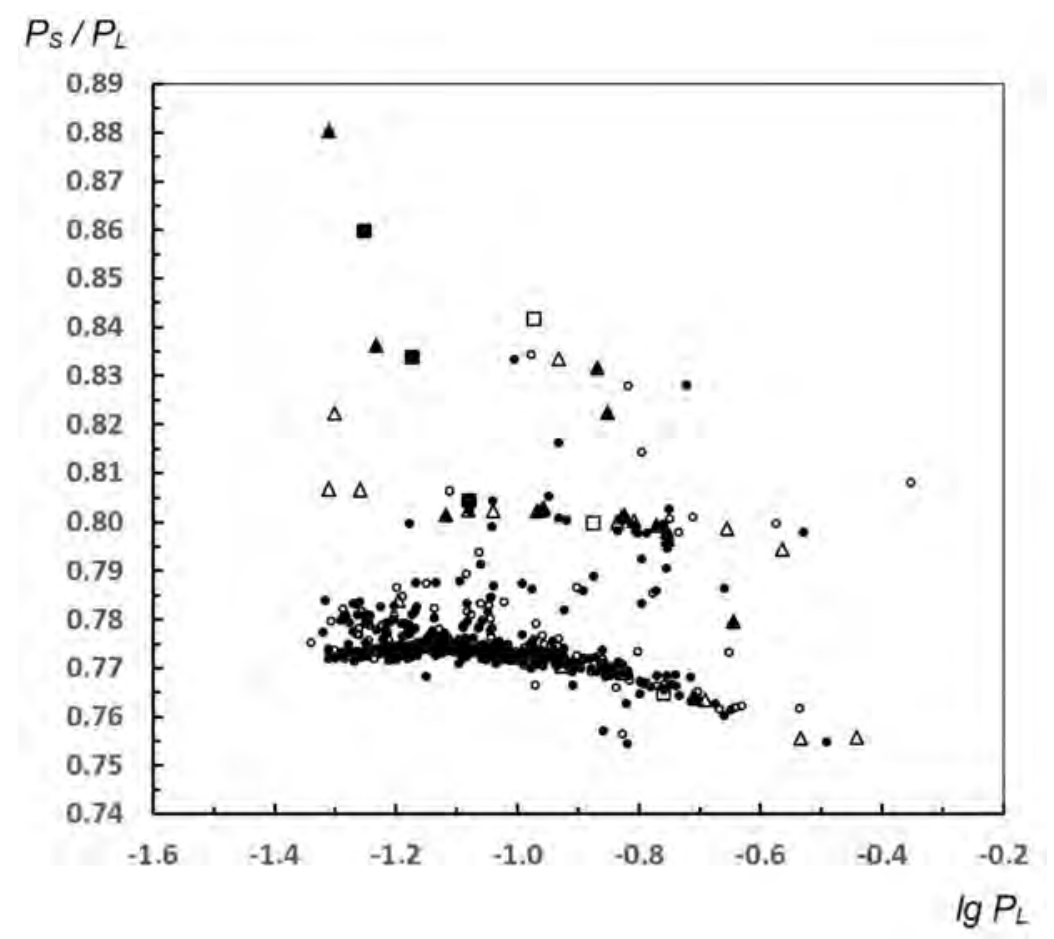

Figure 1: The Petersen diagram for the $\operatorname{HADS}(\mathrm{B})$ stars.

ahttps://asas-sn.osu.edu/variables

b http://www.vgoranskij.net/software/ 


\section{Possible companions of the visual double star ADS 9346=WDS $14410=+5757$}

O.V. Kiyaeva, M.Yu. Khovritchev

Central Astronomical Observatory (Pulkovo) RAN

DOI: 10.51194 /VAK2021.2022.1.1.184

The visual double star ADS 9346 is known as a wide pair with a period more than 2000 years. We give the reasons to assume that there are satellites near the main stars.

1. There are perturbations in the relative motion according to the Pulkovo 26-inch refractor photo and CCD observations 1979-2019.

2. According to a radial velocity observations 2000-2008 with CORAVEL by A. Tokovinin [1, 2] a more massive component A is moving faster than B $\left(\dot{V}_{r A}=-0.160 \pm 0.060, \dot{V}_{r B}=-0.047 \pm 0.061\right) \mathrm{km} / \mathrm{s} / \mathrm{yr}$. Hence, a lowmassive companion can be near A.

3. The analysis of the spectrum energy distribution show us that there is an IR-excess for the A-star at 100 $\mu \mathrm{m}$.

4. There are two radial velocity observations with high accuracy for component A: in 2006.03 [3] and in 2015.5 (Gaia DR2), but there are no radial velocity observations for B in these reviews.

5. Gaia DR2 contains the parallaxes of both components with the same accuracy (0.03 mas). In the Gaia EDR3 the error of the parallax of the component B is measured with a lower accuracy: 0.016 mas for the A-star and 0.043 mas for the B-star.

Hence, there are indirect evidences to assume that the star B may have also short-period companion, but we cannot consider this question now.

The orbit of the A component photocentre is following: $P=15.0 \pm 0.5 y r, T=2009.5 \pm 0.1, e=0.86 \pm 0.01, a=$ $11.6 \pm 2.5$ mas $, i=111 \pm 22^{\circ}, \Omega=266 \pm 21^{\circ}, \omega=315 \pm 32^{\circ}$.

We used Pulkovo CCD observations, Hipparcos, Gaia DR2 and Gaia EDR3 positions. Since $i \approx 90^{\circ}$, we can observe the moving along the node line in the sky plane. Agreement with high accuracy radial velocity observations $\left(\mathrm{t}=2006.03, V_{r A}=-14.632 \pm 0.027 \mathrm{~km} / \mathrm{s} ; \mathrm{t}=2015.5, V_{r A}=-14.15 \pm 0.12 \mathrm{~km} / \mathrm{s}\right)$ is independent control of our solution, but to confirm our orbit it is necessary to observe radial velocity near the periastron passage which we expect soon, in 2024 .

The detail investigation is presented in [4].

This work was supported by RFBR grants 19-02-00843 A and 20-02-00563 A and with the grant 075-15-2020780 of the Government of the Russian Federation and the Ministry of Higher Education and Science.

\section{References}

1. A. A. Tokovinian, Astron. Zhurn., 64, 196, 1987.

2. O. V. Kiyaeva, N. A. Gorynya, and I. S. Izmailov, Astronomy Letters, 36, 204, 2010.

3. B. Deka-Szymankiewicz, A. Niedzielski, M. Adamczyk, M. Adamów, G. Nowak, and A. Wolszczan, AËA, 615, A31, 2018.

4. O. V. Kiyaeva, M. Y. Khovritchev, A. M. Kulikova, N. V. Narizhnaya, T. A. Vasilyeva, A. A. Apetyan, and ., arXiv e-prints, arXiv:2108.11841, 2021. 


\section{Candidates to visual binaries with non-coeval components}

O. Malkov ${ }^{1}$, A. Kniazev ${ }^{2,3,4}$

malkov@inasan.ru

${ }^{1}$ Institute of Astronomy RAS, Pyatnitskaya St. 48, Moscow, Russia,

${ }^{2}$ South African Astronomical Observatory, Cape Town, 7935, South Africa,

${ }^{3}$ Southern African Large Telescope, Cape Town, 7935, South Africa,

${ }^{4}$ Sternberg State Astronomical Institute, Moscow, Universitetsky ave., 13, Russia

All possible scenarios of binary star formation lead to construct a pair with components of the same age. The only exception is the capture, a phenomenon thought to be rather rare. However, in some regions (e.g., in the densest stellar clusters or in the central part of the Galaxy) the probability of the capture increases. In the paper we study several candidates to binary systems with different ages of components. Their evolutionary status requires further investigation.

Keywords: binary stars, stellar mass

DOI: $10.51194 /$ VAK2021.2022.1.1.185

\section{Introduction}

The formation of binary stars basically follows two scenarios: fission of rotating molecular gas clouds during gravitational collapse, and inelastic collisions of stars during the formation of young star clusters [3]. A capture of
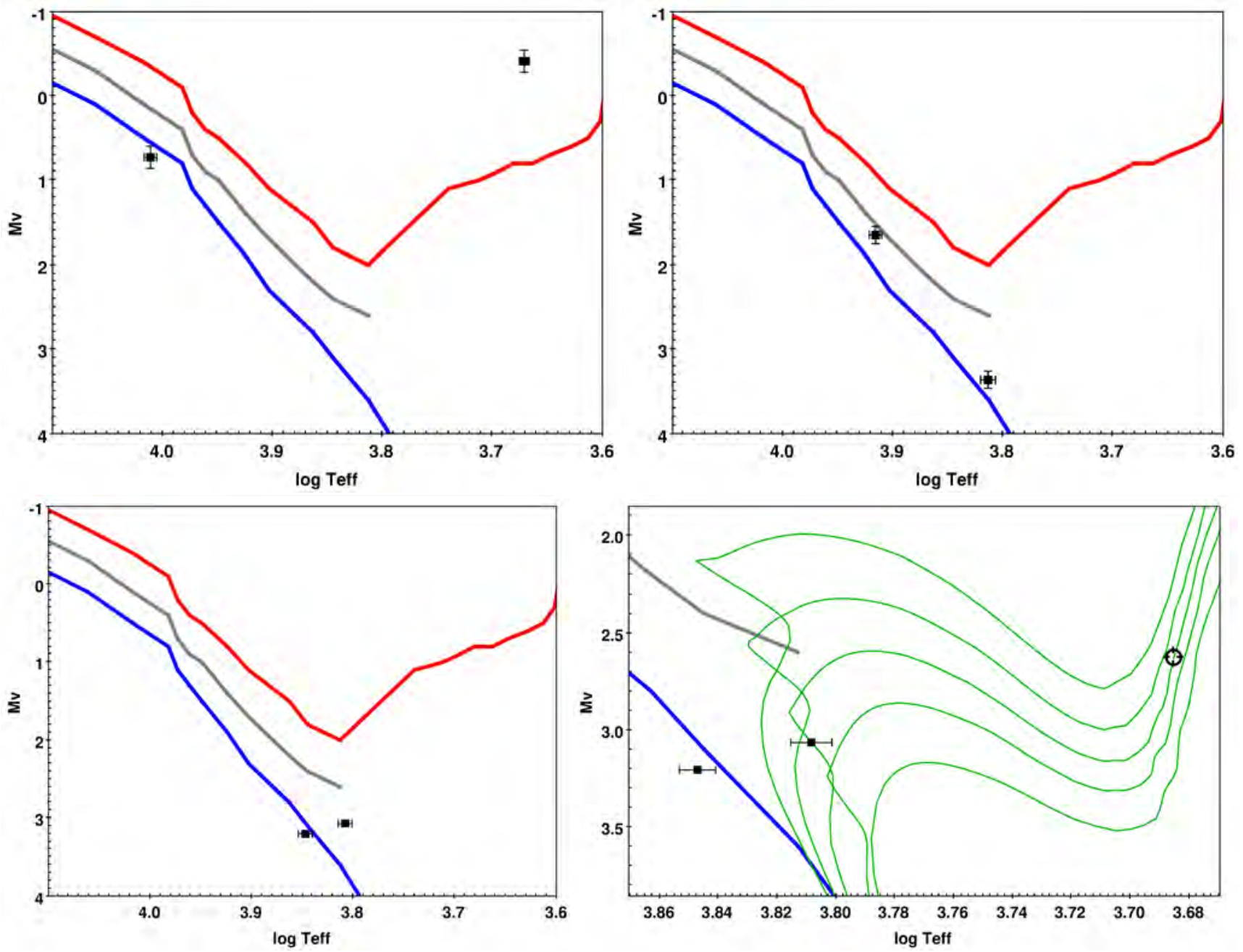

Figure 1: Studied systems on the HRD. Upper row: HD 101379 (left) and HD 160928 (right), lower row: HD 156331, at different scales. Solid curves represent main sequence, subgiant and giant sequences (blue, grey and red, respectively) from [1]. Thin green curves are isochrones from [2] for ages (from top to bottom) $\log \tau=9.3,9.4,9.5,9.6,9.7$, "no rotation" case, initial metallicity $\mathrm{Z}=0.014$. Black squares represent components of the binaries, with uncertainty bars. Black circle (in the right bottom panel) indicates a place where the HD 156331 primary would have been if it had been the same age as the HD 156331 secondary. 
a component from the field stars is not ruled out in principle either, although it should be relatively rare. Capture occurs when two stars pass close to each other in the presence of a scattering medium that can take in excess kinetic energy, to leave the two stars bound. This medium could be a third star, a circumstellar disk, or the stars themselves, if the collision is close enough to cause the tides to rise and fall. Capture in the presence of a stellar disk may play some role in the formation of wide systems, because the capture cross section has to be on the order of the size of the disk and thus lead to the formation of systems with large semi-axes about 100 AU. Capture in the presence of a third body and "tidal capture" requires a high stellar density, which is atypical for field stars.

An indicator of the capture could be the difference in the ages of the components. It is evident, in particular, that in evolutionary wide systems (i.e., systems with no matter transfer between components today or in the past) with components of the same age, a less massive component cannot appear to be more evolved.

The aim of this study is to find non-coeval pairs among visual binaries. For indication of non-coevality we compared spectral classes and masses of the components, estimated from the spectral classification. Applying this simple method to the Sixth Catalog of Orbits of Visual Binary Stars, ORB6 [4], we found thirteen systems where less massive component looks more evolved, and, consequently, the components are probably non-coeval [5].

We have made a search for additional data on these thirteen systems in ORB6 [4], Catalogue of Stellar Spectral Classifications [6], Multiple star catalogue, MSC [7], as well as in the SIMBAD database.

Three of these thirteen systems have been observed with èchelle spectrograph HRS at the Southern African Large Telescope (SALT). All our observations were taken with MR mode $(\mathrm{R}=36500-39000)$ and the standard HRS pipeline was used for its reduction [8]. Totally reduced èchelle spectra were split into two components with use of the software package fbs (Fitting Binary Stars; [9]). Obtained parameters of observed systems are analyzed in the next Section.

\section{Parameters of the studied systems}

The parameters of three systems observed with SALT are shown in Fig. 1 and presented in Tables 1 and 2.

Table 1: Systems under study. Pairs

\begin{tabular}{rrrrrl}
\hline \hline Name & V, mag & $\varpi$, mas & $\sigma_{\varpi}$ & Weight & $E_{B-V}$ \\
\hline HD 101379 & 5.095 & 8.397 & 0.507 & 0.741 & 0.144 \\
HD 156331 & 6.267 & 16.703 & 0.048 & 0.532 & 0.000 \\
HD 160928 & 5.871 & 13.053 & 0.599 & 0.829 & 0.0 \\
\hline
\end{tabular}

Weight $(=\mathrm{L} 2 / \mathrm{L} 1)$ and $E_{B-V}$ are determined from SALT observations, parallax $\varpi$ and visual brightness $V$ are taken from Gaia EDR3 and SIMBAD, respectively.

Table 2: Systems under study. Components

\begin{tabular}{rrrrrrr}
\hline \hline Name & & SpT & $M_{V}$ & $\sigma_{M v}$ & $\log$ Teff & $\sigma_{\log T}$ \\
\hline HD 101379 & A & K3 & -0.405 & 0.131 & 3.6709 & 0.0047 \\
HD 101379 & B & B9 & 0.736 & 0.131 & 4.0106 & 0.0058 \\
HD 156331 & A & F5 & 3.066 & 0.006 & 3.8084 & 0.0068 \\
HD 156331 & B & F2 & 3.205 & 0.006 & 3.8470 & 0.0062 \\
HD 160928 & A & A5 & 1.653 & 0.100 & 3.9152 & 0.0053 \\
HD 160928 & B & F5 & 3.367 & 0.100 & 3.8136 & 0.0067 \\
\hline
\end{tabular}

We can see from Fig. 1 that more massive (and more luminous) components of HD 101379 and HD 160928 look more evolved than the less massive ones. Contrary, the secondary (less massive) component of HD 156331 is more evolved than the primary. It is an indication of the non-coevality of the components and, consequently, we can assume that this system was formed by a capture. To prove the non-coevality one needs a detailed investigation of the candidates.

\section{Acknowledgments}

The work was partly supported by the Russian Foundation for Basic Researches (project 19-07-01198). A. K. acknowledges support from the National Research Foundation (NRF) of South Africa. This research has made 
use of NASA's Astrophysics Data System, of the SIMBAD database, operated at CDS (Strasbourg, France), of TOPCAT, an interactive graphical viewer and editor for tabular data [10].

\section{References}

1. V. Straižys, Multicolor stellar photometry, Tucson : Pachart Pub. House (1992).

2. S. Ekström, C. Georgy, P. Eggenberger, G. Meynet, et al., A\&̋A, 537, A146, 2012.

3. A. V. Tutukov and A. M. Cherepashchuk, Physics Uspekhi, 63, 209, 2020.

4. W. I. Hartkopf, B. D. Mason, and C. E. Worley, AJ, 122, 3472, 2001.

5. O. Y. Malkov, INASAN Science Reports, 5, 341, 2020.

6. B. A. Skiff, VizieR Online Data Catalog, B/mk, 2014.

7. A. Tokovinin, ApJS, 235, 6, 2018.

8. A. Y. Kniazev, I. A. Usenko, V. V. Kovtyukh, and L. N. Berdnikov, Astrophysical Bulletin, 74, $208,2019$.

9. A. Y. Kniazev, O. Y. Malkov, I. Y. Katkov, and L. N. Berdnikov, Research in Astronomy and Astrophysics, 20, 119, 2020.

10. M. B. Taylor, in P. Shopbell, M. Britton, and R. Ebert, eds., Astronomical Data Analysis Software and Systems XIV, Astronomical Society of the Pacific Conference Series, volume 347, 29 (2005). 


\section{Chemical composition and ages of four globular clusters in M31}

M. Maricheva, M. Sharina

marichevar@gmail.com

Special Astrophysical Observatory, Russian Academy of Sciences, Nizhnii Arkhyz, 369167 Russia

DOI: $10.51194 /$ VAK2021.2022.1.1.186

We determined ages, specific helium abundances (Y), and the abundances of Fe, C, N, Mg, Ca, Mn, Ti, and Cr for four globular clusters (GCs) in M31: Bol6, Bol20, Bol45, and Bol50 using the method by [1]. The observed medium-resolution integrated-light (IL) spectra of GCs were approximated by the synthetic ones, calculated on the basis of plane-parallel hydrostatic models of stellar atmospheres [2] with the parameters set by theoretical isochrones of stellar evolution. The synthetic stellar spectra were summed according to the stellar mass function by [3]. The spectra of GCs were obtained in 2020 at the 6 -m SAO RAS telescope with the SCORPIO-1 multimode focal reducer [4] in the long slit mode. We derived the abundances of the chemical elements for Bol20, Bol50 for the first time. For all four objects, Y was determined for the first time. All the studied clusters turned out to be older than 10 Gyr with the metalicities in the range: from -0.75 to -1.1 dex. A reasonable agreement was achieved between the obtained chemical abundances for Bol6 and Bol45 and the corresponding literature data of highresolution IL spectroscopy [5], [6]. We compared our results with the available literature data of IL spectroscopy of GCs with similar chemical abundances in our Galaxy and M31 ([1, 7] and references therein). We did not find complete analogues for the studied clusters in terms of age, helium content, and chemical composition. The closest analogue for Bol45 appeared to be the Galactic GC NGC6637. Slightly shallower hydrogen lines in the spectrum of NGC6637 can be explained by the lower helium content. The alpha-element abundances in the four clusters are about 0.2 dex higher than the average for stars in the Galactic field at $[\mathrm{Fe} / \mathrm{H}] \sim 1$ dex [8]. The obtained alpha-process elemental abundances in four clusters turned out to correspond to the average value for stars in the M31 inner halo at a given distance from the centre of M31 with the metallicity of the studied clusters to be lower than the average metallicity of red giants in the M31 halo [9].

\section{References}

1. M. E. Sharina, V. V. Shimansky, and N. N. Shimanskaya, Astrophysical Bulletin, 75, 247, 2020.

2. F. Castelli and R. L. Kurucz, IAU Symp., 210, A20, 2003.

3. G. Chabrier, The Initial Mass Function 50 years later, Astrophysics and Space Science Library, 327, $41,2005$.

4. V. L. Afanasiev and A. V. Moiseev, Astronomy Letters, 31, 194, 2005.

5. J. E. Colucci, R. A. Bernstein, and J. G. Cohen, ApJ, 797, 116, 2014.

6. C. M. Sakari, M. D. Shetrone, R. P. Schiavon, D. Bizyaev, et al., ApJ, 829, 116, 2016.

7. M. E. Sharina, V. V. Shimansky, and D. A. Khamidullina, Astrophysical Bulletin, 73, 318, 2018.

8. K. A. Venn, M. Irwin, M. D. Shetrone, C. A. Tout, V. Hill, and E. Tolstoy, AJ, 128, 1177, 2004.

9. K. M. Gilbert, J. Wojno, E. N. Kirby, I. Escala, R. L. Beaton, P. Guhathakurta, and S. R. Majewski, $A J$, 160, 41, 2020. 


\title{
Confirmation of the symbiotic nature of CSS 1102 on the basis of spectral and photometric observations carried out at the CMO SAI MSU
}

\author{
N. Maslennikova ${ }^{1,2}$, A. Tatarnikova ${ }^{1}$, A. Tatarnikov ${ }^{1}$, N. Ikonnikova ${ }^{1}$, A. Dodin ${ }^{1}$ \\ maslennikova.na16@physics.msu.ru \\ ${ }^{1}$ Lomonosov Moscow State University, Sternberg Astronomical Institute, 13 Universitetskij prospekt, Moscow 119234, \\ Russia, \\ ${ }^{2}$ Faculty of Physics, Moscow M.V. Lomonosov State University, Leninskie gory 1, Moscow, 119991, Russia
}

The results of new spectral and photometric observations of the poorly studied S-star CSS 1102 are presented. According to the analysis of spectra, CSS 1102 was classified as a symbiotic star. We observed HI, HeI and [NeIII] emission lines in both spectra of the star. The absorption spectrum demonstrates the prominent molecular bands of $\mathrm{TiO}$, ZrO and YO. The system consists of a cold component that is a zirconium star of spectral type S4.5/2, a hot component with $40000 K<T_{\text {hot }}<55000 K$, and a nebula. Photometric monitorings in the B band showed the existence of a flickering effect with different periods in CSS 1102. This variability may be associated with emission of an accretion disk in CSS 1102 system.

Keywords: symbiotic star, flicker effect, CSS 1102

DOI: 10.51194 /VAK2021.2022.1.1.187

\section{Introduction}

CSS 1102 is a poorly studied object included in the general catalog of galactic S-stars [1]. According to the AAVSO data, the apparent brightness of the star varies in the range $12.6-13^{m}$ in the $\mathrm{V}$ band. The distance to CSS 1102 is not less than a few kiloparsecs. CSS 1102 has been considered as a suspected symbiotic star since June 2020 [2].

CSS 1102 was assumed to have flicker effect [2]. The flickering (fast brightness variability) is a very rare phenomenon in symbiotic stars. Less than 20 objects have demonstrated such type of variability so far. The symbiotic stars with flickering can be divided into two subclasses with different characteristics of variability and physical mechanisms causing this variability: 1) Small amplitudes and narrow peaks on power spectra are characteristic of intermediate polars' type fast brightness variability (for example, Z And $P=28$ min, $\Delta m_{B}=2 m m a g$ ); 2 ) Larger amplitudes and a wide range of frequencies on power spectra indicate variability associated with an existence of accretion disks in these systems (for example, T CrB, RS Oph, CH Cyg).

The main goal of our work is to classify CSS 1102 and determine the flicker effect parameters.

\section{Observations}

Photometric observations of CSS 1102 were carried out on 1.09.2020, 27.10.2020 and 10.07.2021 with 60-cm telescope at the CMO SAI MSU [3] in BVRcIc. In addition to multicolor brightness estimates, the star was monitored

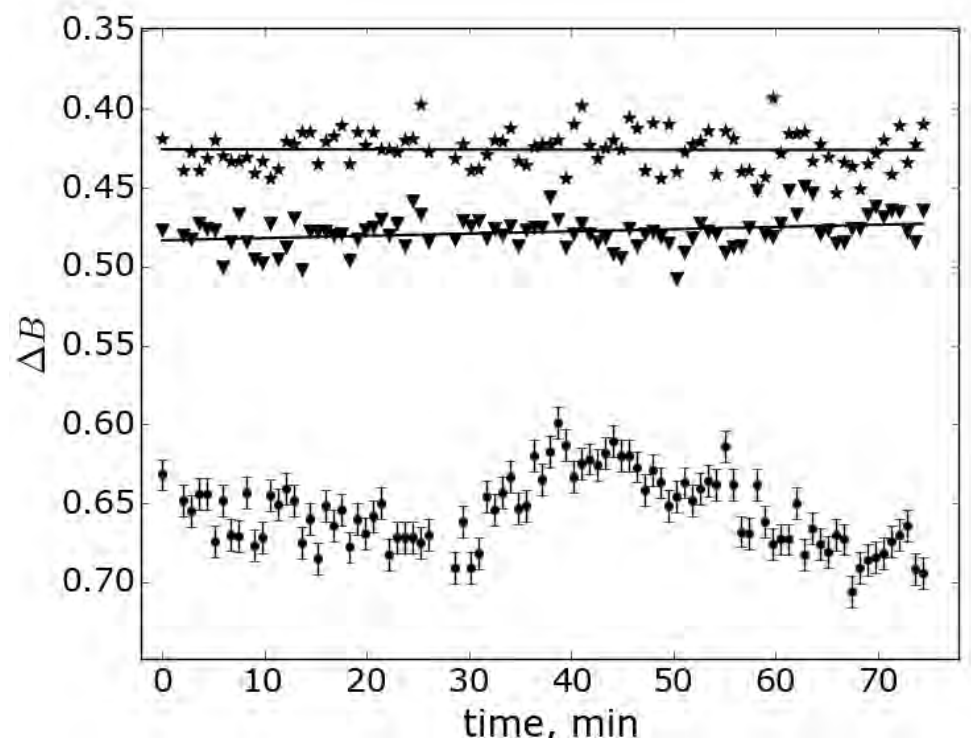

Figure 1: B band light curve of CSS 1102 (dots) obtained 1.09.2020. Asterisks and triangles are control stars. 
for 75 and 125 minutes in the B band with a frame exposure of 40 seconds to search for flicker effect on 1.09 .2020 and 10.07.2021. This band was chosen because the flickering is associated with a hot component or an accretion disk, and the maximum effect of their variability can be expected in the UV (or blue) spectral region. CSS 1102 is a southern sky object, so it culminates at 23 degrees in the CMO, and a large air mass hinders UV observation.

Spectral observations of CSS 1102 were carried out with the Transient Double-beam Spectrograph mounted on the 2.5-meter telescope at the CMO SAI MSU on 31.08.2020 and 28.10.2020 with 1 arcsec slit (with an exposure of $1200 \mathrm{sec})$ in the wavelength range $3600-7500 \AA$ with a resolution power in the short-wave channel (3600-5770 $\AA$ ) $\mathrm{R}=1300$ and in the long-wave channel $(5670-7500 \AA) \mathrm{R}=2500$ [4]. The spectrum of the S-star HD64332 was also observed to simulate the cold component of CSS 1102. All spectra were converted to the Solar System barycenter and corrected for interstellar extinction.

\section{Analysis of observations}

Our estimates have shown that CSS 1102 changed the brightness between 01.09.2020 and 27.10.2020 in the B, V, Rc bands by about $0.2^{m}$, in the Ic band by $0.08^{m}$. The determination of the characteristic time of the flicker effect from the monitoring data on 01.09.2020 (the light curves of CSS 1102 and control stars are shown in Fig.1) and 10.07.2021 were carried out using Fourier analysis and wavelet analysis (its apparatus was developed by [5]). A characteristic time of a brightness variations was 35-40 minutes on 1.09.2020. A characteristic amplitude of the flicker effect was about $0.04^{m}-0.05^{m}$ in the B band (photometry errors are $0.010^{m}$ estimated from a standard deviation of the control stars brightness from the mean value). On 10.07.2021, the characteristic times of flicker effect were 48 minutes and 10 minutes with amplitudes were about $0.04^{m}$ and $0.02^{m}$ (photometry errors are $0.011^{m}$ ), respectively. Thus, sufficiently large amplitudes and a wide power range in the frequency band showed that flickering is associated with an accretion disk.

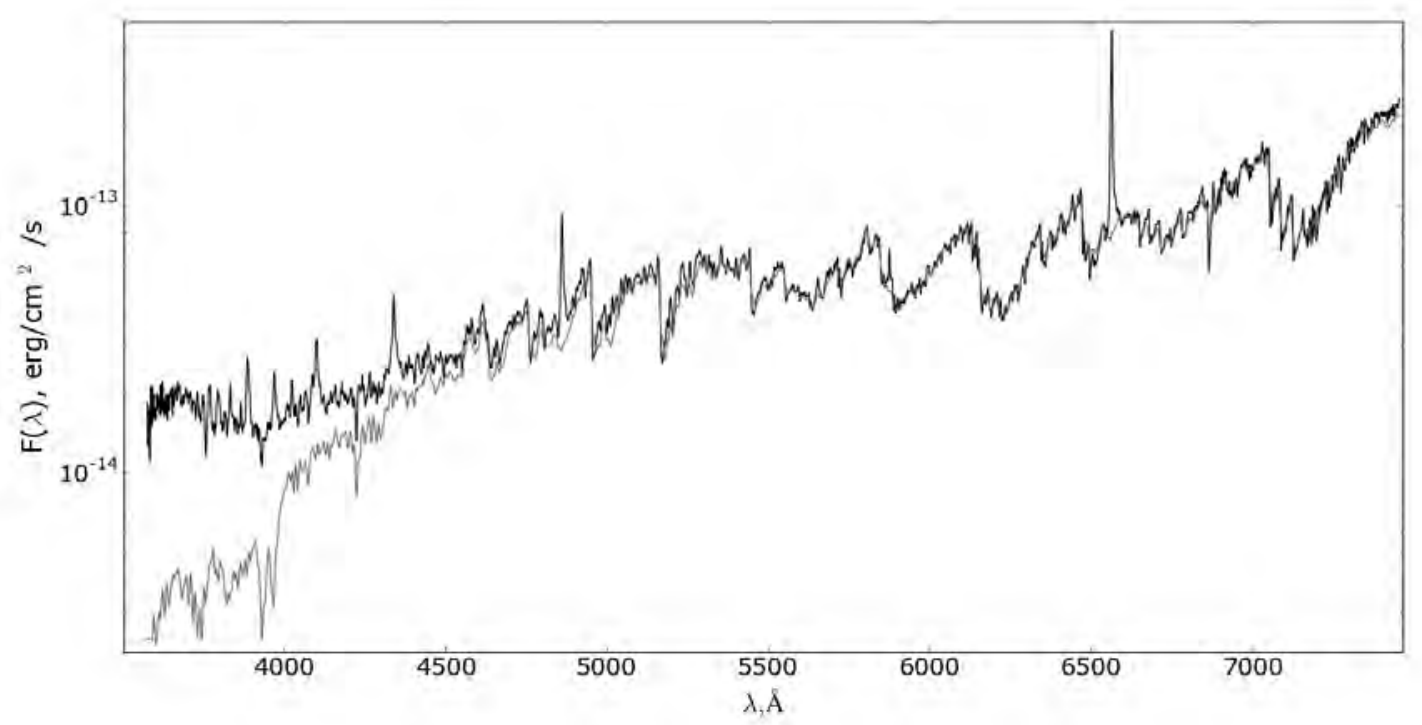

Figure 2: Spectra of CSS 1102 (black line) and HD64332 (grey line) converted to the Solar System barycenter and corrected for interstellar extinction

According to comparison of the spectral energy distributions of CSS 1102 and HD64332 (Fig. 2), the cold component of CSS 1102 is a zirconium star of spectral type S4.5/2 ( $\left.T_{\text {eff }} \approx 3400 K\right)$. In addition to molecular bands of $\mathrm{ZrO}$, the absorption spectrum contains bright $\mathrm{TiO}$ bands, which are used as a temperature criteria in early subtypes of S stars. The YO band $(6132 \AA)$ is also well observed. Such the spectrum indicates that a red giant is at the stage of enrichment its atmosphere with s-process elements. It leads to a change in the spectral type from the initial M through the intermediate MS-type to the S-type (and then to a carbon star of spectral type C).

The CSS 1102 emission spectrum contains few lines. The lines of the Balmer series of hydrogen dominate. Balmer's decrement $\left(\frac{H_{\gamma}}{H_{\beta}}=0.42 \pm 0.08, \frac{H_{\delta}}{H_{\beta}}=0.29 \pm 0.09\right)$ assumes nebular origin of hydrogen lines. Both spectra also contains HeI lines, and weak forbidden lines [NeIII] confirming the assumption about the symbiotic nature of CSS 1102.

Unfortunately, there are not lines of ions with high ionization potentials in spectrum (for example, HeII $4686 \AA$ ), so the temperature of the hot component is not determined. We can only give a rough estimate: $40000 \mathrm{~K}<$ $T_{\text {hot }}<55000 \mathrm{~K}[6]$. 


\section{Conclusions}

According to our spectral observations of CSS 1102 the object was classified as a symbiotic star. The system consists of a cool component that is a zirconium star of spectral type S4.5/2, a hot component with $40000 K<$ $T_{\text {hot }}<55000 \mathrm{~K}$, a nebula and (may be) an accretion disk.

Our photometric monitoring revealed fast brightness variability during both sets of observations. Flickering with a period of $40 \mathrm{~min}$ and an amplitude is about $0.04^{\mathrm{m}}$ was detected both on 1.09.2020 and 10.07.2021 light curves. But 10.07.2021 the power analysis showed an additional peak with period and amplitude are about 10 min and $0.02^{m}$, respectively. Flickering in CSS 1102 is associated with an accretion disk.

\section{References}

1. C. B. Stephenson, Publications of the Warner 85 Swasey Observatory, 1984.

2. AAVSO Alert Notice 719, 2020.

3. L. Berdnikov, A. Belinskij, N. Shatskij, M. A. Burlak, N. P. Ikonnikova, E. O. Mishin, D. V. Cheryasov, and S. V. Zhuiko, Astr. Rep., 64, 310, 2020.

4. S. A. Potanin, A. A. Belinski, A. Dodin, S. G. Zheltoukhov, et al., Astr. Let., 46, 836, 2020.

5. A. Grossmann and J. Morlet, SIAM Journal on Math. Analysis, 15, 723, 1984.

6. U. Murset and H. Nussbaumer, Astronomy and Astrophysics, 282, 586, 1994. 


\title{
Influence of the bar on the kinematics of the Galactic disk near the Outer Lindblad Resonance. Comparison with Gaia EDR3 data
}

\author{
A.M. Melnik ${ }^{1}$, A.K. Dambis ${ }^{1}$, E.N. Podzolkova ${ }^{2}$, L.N. Berdnikov ${ }^{1}$ \\ anna@sai.msu.ru \\ ${ }^{1}$ Sternberg Astronomical Institute, Lomonosov Moscow State University, Universitetskii pr. 13, Moscow, 119991, Russia, \\ ${ }^{2}$ Faculty of Physics, Lomonosov Moscow State University, Leninskie Gory 1-2, Moscow, 119991, Russia
}

A model of the Galaxy with the outer ring $R_{1} R_{2}$ can reproduce the observed distribution of the radial, $V_{R}$, and azimuthal, $V_{T}$, velocity components along the Galactocentric distance, $R$, derived from the Gaia EDR3 data. The best agreement between the model and observed velocities corresponds to the time $1.8 \pm 0.5 \mathrm{Gyr}$ after the start of the simulation. The angular velocity of the bar of $\Omega_{b}=55 \pm 3 \mathrm{~km} \mathrm{~s}^{-1} \mathrm{kpc}^{-1}$ and the position angle of the bar of $\theta_{b}=45 \pm 15^{\circ}$ provide the best agreement between the model and observed velocities.

Keywords: the Galaxy, kinematics and dynamics, Gaia EDR3

DOI: 10.51194 /VAK2021.2022.1.1.188

\section{Introduction}

There is extensive evidence that our Galaxy includes a bar $[1,2]$. However, the angular velocity, $\Omega_{b}$, position angle, $\theta_{b}$, as well as the age of the bar remain the subject of debate.

The resonance between the orbital rotation with respect to the bar and the epicyclic motion causes the formation of elliptic resonance rings. The outer resonance rings are located near the Outer Lindblad Resonance (OLR): the rings $R_{1}$ or pseudorings $R_{1}^{\prime}$ (broken rings) lie a bit closer to the galactic center and are stretched perpendicular to the bar while the rings $R_{2}$ or pseudorings $R_{2}^{\prime}$ are located a bit farther away from the center and are stretched parallel to the bar [3]. The fraction of galaxies with outer rings is as high as $20-30$ per cent among galaxies with strong and moderate bars [4].

In this paper we study the kinematics of disk stars with Gaia EDR3 data [5, 6] and build a model of the Galaxy which reproduces the observed distributions of the radial and azimuthal velocities along the Galactocentric distance, $R$.

\section{Results and conclusions}

We selected Gaia EDR3 stars located near the Galactic plane, $|z|<200$ pc, and in the sector of the Galactocentric angles $|\theta|<15^{\circ}$ that have reliable parallaxes, $\varpi\left(\varpi / \varepsilon_{\varpi}>5\right)$, and line-of-sight velocities measured by the Gaia spectrometer. We determined the radial, $V_{R}$, and azimuthal, $V_{T}$, components of stellar velocities directed correspondingly along the Galactic radius-vector and along the Galactic circular rotation, and calculated the median values of the velocities $V_{R}$ and $V_{T}$ in $\Delta R=250$-pc wide bins along Galactocentric distance $R$.

The distribution of observed velocities appears to have some specific features: the radial velocity $V_{R}$ demonstrates a smooth fall from $+5 \mathrm{~km} \mathrm{~s}^{-1}$ at Galactocentric distance of $R \approx R_{0}-1.5 \mathrm{kpc}$ to $-3 \mathrm{~km} \mathrm{~s}^{-1}$ at $R \approx R_{0}+1.0$ kpc while the azimuthal velocity $V_{T}$ shows a sharp drop by $7 \mathrm{~km} \mathrm{~s}^{-1}$ in the distance interval $R_{0}<R<R_{0}+1.0$ kpc, where $R_{0}$ is the solar Galactocentric distance.

We built a model of the Galaxy which includes bulge, bar, disc and halo components. The bar was modelled as a Ferrers ellipsoid with the semi-axes of $a=3.5$ and $b=1.35 \mathrm{kpc}$, respectively. A more detailed description of the construction method for each Galactic subsystem can be found in [7]. The initial distribution of model velocities were determined from the solution of the Jeans equation [8].

To compare the model and observed velocity distributions we calculated statistics $\chi_{R}^{2}$ and $\chi_{T}^{2}$, which are the sum of squared differences between the model and observed velocities obtained for the radial and azimuthal velocity profiles, respectively. Figure 1 (a) shows the variations of the statistic $\chi^{2}=\chi_{R}^{2}+\chi_{T}^{2}$ with time. We can see the presence of a shallow minimum at $t=1.8 \pm 0.5$ Gyr which determines the epoch when model and observed velocities demonstrate the best agreement.

Figure 1 (b) shows variations in the $\chi^{2}$ values averaged over the time periods $t=1.0-1.5,1.5-2.0$, and 2.5-3.0 Gyr as a function of the bar angular velocity $\Omega_{b}$. We can see that the $\chi^{2}$ functions achieve minima at $\Omega_{b}=55 \pm 3$ $\mathrm{km} \mathrm{s}^{-1} \mathrm{kpc}^{-1}$. The function $\chi^{2}$ computed for the time period 1.5-2.0 Gyr reaches smaller values than $\chi^{2}$ obtained for other periods but the difference is close to $1 \sigma$.

Fig. 1 (c) shows changes in the $\chi^{2}$ values averaged over the three time periods with the position angle $\theta_{b}$, which is the angle between the direction of the bar major axis and the Sun-Galactic center line. We can see that the $\chi^{2}$ function built for the time period 1.5-2.0 Gyr demonstrates a sharp drop in the interval $0-30^{\circ}$ followed by a plateau with a shallow minimum at $\sim 45^{\circ}$.

Thus, we provide evidence in support of the idea that the Galactic bar is relatively young (for example, [9]). Our simulations show that the age of the bar must be $t=1.8 \pm 0.5$ Gyr. The model of the Galaxy with the bar 

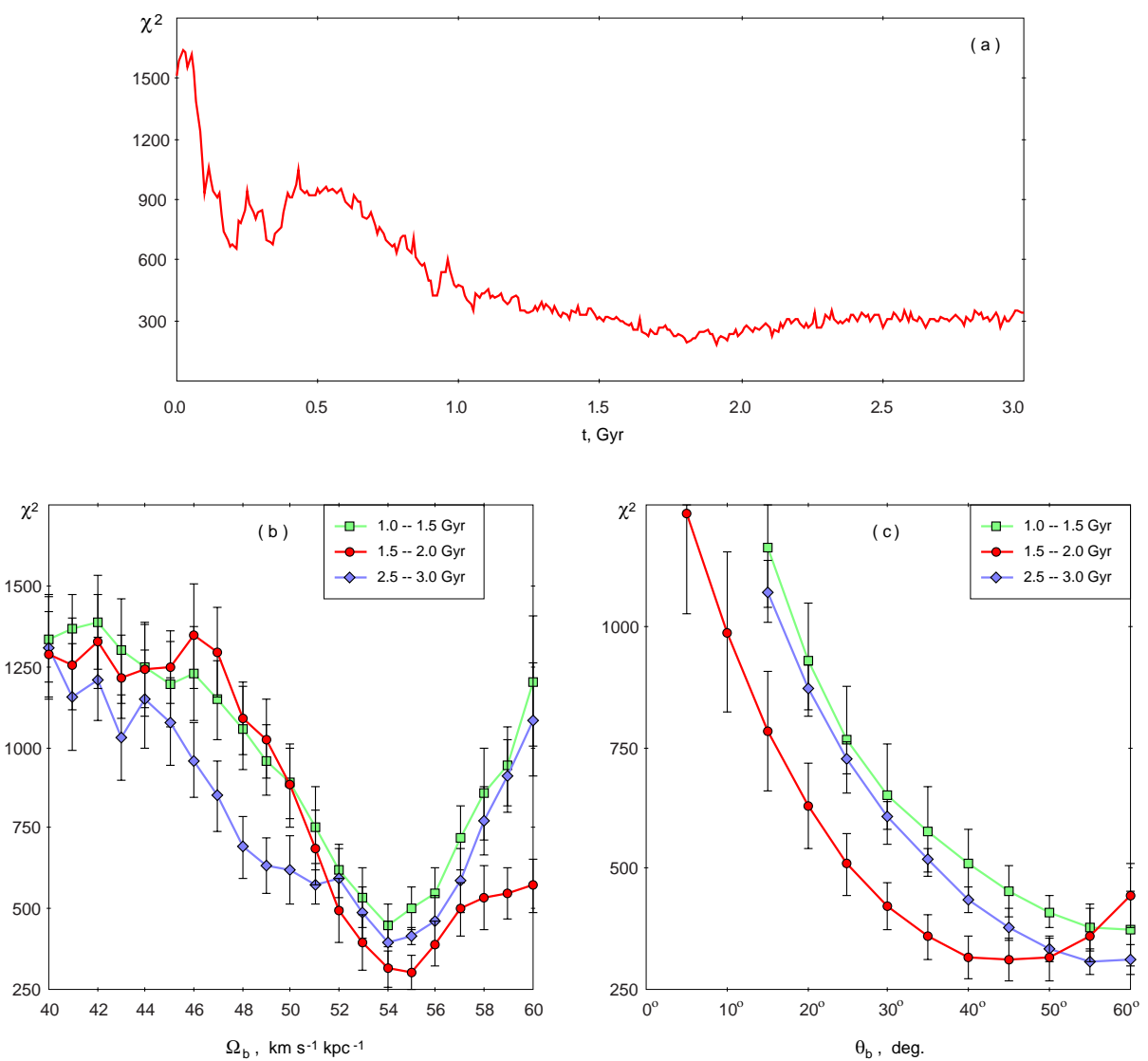

Figure 1: (a) The dependence of the statistics $\chi^{2}=\chi_{R}^{2}+\chi_{T}^{2}$ on time. We can see that $\chi^{2}$ reaches a minimum at the time $1.8 \pm 0.5$ Gyr. (b) Variations in the $\chi^{2}$ values averaged over the time periods $t=1.0-1.5,1.5-2.0$ and 2.5-3.0 Gyr along the angular velocity of the bar, $\Omega_{b}$. The minima of the $\chi^{2}$ functions correspond to the value of $\Omega_{b}=55 \mathrm{~km} \mathrm{~s}^{-1} \mathrm{kpc}^{-1}$. (c) Variations in the $\chi^{2}$ values averaged over three time periods along the position angle of the bar, $\theta_{b}$. The $\chi^{2}$ function built for the time period 1.5-2.0 Gyr demonstrates a sharp drop at the interval $0-30^{\circ}$ followed by a plateau with a shallow minimum at $\sim 45^{\circ}$. The vertical lines indicate the dispersions of the $\chi^{2}$ values at the corresponding time periods.

rotating at the angular velocity of $\Omega_{b}=55 \mathrm{~km} \mathrm{~s}^{-1} \mathrm{kpc}^{-1}$ provides the best agreement between the model and observed velocity profiles. This angular velocity sets the OLR of the bar at the Galactocentric distance of $R_{0}-0.5$ kpc. The optimum value of the position angle of the bar is $\theta_{b}=45 \pm 15^{\circ}$ (for more details see [8]).

\section{Acknowledgements}

This work has made use of data from the European Space Agency (ESA) mission Gaia (https://www.cosmos.esa.int/gaia), processed by the Gaia Data Processing and Analysis Consortium (DPAC, https://www.cosmos.esa.int/web/gaia/dpac/consortium). Funding for the DPAC has been provided by national institutions, in particular the institutions participating in the Gaia Multilateral Agreement.

\section{References}

1. R. A. Benjamin, E. Churchwell, B. L. Babler, R. Indebetouw, et al., ApJL, 630, L149, 2005.

2. M. Ness and D. Lang, AJ, 152, 14, 2016.

3. R. Buta and F. Combes, Fundam. Cosmic Phys., 17, 95, 1996.

4. S. Comerón, H. Salo, E. Laurikainen, J. H. Knapen, et al., A $\& A$, 562, A121, 2014.

5. Gaia Collaboration, T. Prusti, J. H. J. de Bruijne, A. G. A. Brown, et al., A\&A, 595, A1, 2016.

6. Gaia Collaboration, A. G. A. Brown, A. Vallenari, T. Prusti, et al., A\&A, 649, A1, 2021.

7. A. M. Melnik, MNRAS, 485, 2106, 2019.

8. A. M. Melnik, A. K. Dambis, E. N. Podzolkova, and L. N. Berdnikov, MNRAS, 507, 4409, 2021.

9. T. Bensby, S. Feltzing, A. Gould, J. C. Yee, et al., A\&A, 605, A89, 2017. 


\section{Discovery and study of new white dwarfs by means of Gaia EDR3 accurate astrometry}

A.M. Mickaelian, G.A. Mikayelyan, H.V. Abrahamyan, G.M. Paronyan

aregmick@yahoo.com

NAS RA V. Ambartsumian Byurakan Astrophysical Observatory (BAO)

The discovery and study of new White Dwarfs (WDs) is extremely important for understanding the kinematics and dynamics of the local stellar population. They are evolutionary signatures of the Milky Way, as most of stars pass through this evolutionary stage. We have carried out a statistical analysis of the White Dwarfs Catalogue to establish their average physical properties. Based on these data, we have searched for new WDs using Gaia EDR3 accurate astrometry. It provides huge amount of data to confirm WDs and reveal many more such objects. Gaia's astrometric accuracy allows more detailed studies of WDs and many other stars. Using Gaia accurate data in combination with SDSS and DFBS low-dispersion spectra and additional multiwavelength data, we have revealed many new WDs. Many of them are subject for studies on binarity and variability. WDs among FBS blue stellar objects at high galactic latitudes have been searched and revealed. Search for high galactic latitude WDs in DFBS have also been carried out. Using the revealed and calculated characteristics, a statistical study of WD properties is being done.

DOI: $10.51194 /$ VAK2021.2022.1.1.189

\section{Statistical Analysis of the Catalogue of White Dwarfs}

We have analyzed The Catalogue of Spectroscopically Identified White Dwarfs (Version April 2014) by G. P. McCook and E. M. Sion [1] to summarize the statistical properties of WDs. The total number of WDs in this version is 14,294 . Among them there are:

- 368 WDs having parallaxes, in the range 0.0001-0.575, the average is 0.046;

- 10,871 WDs having total proper motion (PM) in the range of 0.0010-4.0800 arcsec/year;

- only 345 have adopted apparent velocities (Adp-V) in the range of $0.058-97.190 \mathrm{~km} / \mathrm{s}$, among them 328 (95.1\%) have velocities less than $75 \mathrm{~km} / \mathrm{s}$;

- 1937 WDs have absolute magnitudes in the range of $-0.11 \ldots 18.10$; the distribution by absolute magnitudes shows that we can consider as the limit for WD $7.5^{\mathrm{m}}$ (brighter WDs are extremely rare);

- 1919 WDs (out of 1937, 99.1\%) are fainter than $7.5^{m}$ or even $9.5^{m}$ (1792 out of 1937, 92.5\%).

Let us note that the WD sample is incomplete after $11.5^{m}$ (the distribution graph goes down).

In Figure 1 we give the distribution of absolute magnitudes (left panel) and adopted apparent velocities (right panel) for McCook \& Sion Catalogue WDs allowing estimate the limit of $M_{a b s}$ for WDs (two breaks at $7.5^{m}$ and $9.5^{\mathrm{m}}$ ) and the limit of the apparent velocity (the break at $75 \mathrm{~km} / \mathrm{s}$ ). Adopting these values, we could distinguish WDs from the other stars.

In Figure 2 we give the distribution of the parallaxes vs. proper motions for McCook \& Sion WDs. The dependence is obvious and one can roughly estimate the parallax based on the measured proper motion, hence have understanding on the distance. The absolute magnitude can be derived accordingly.
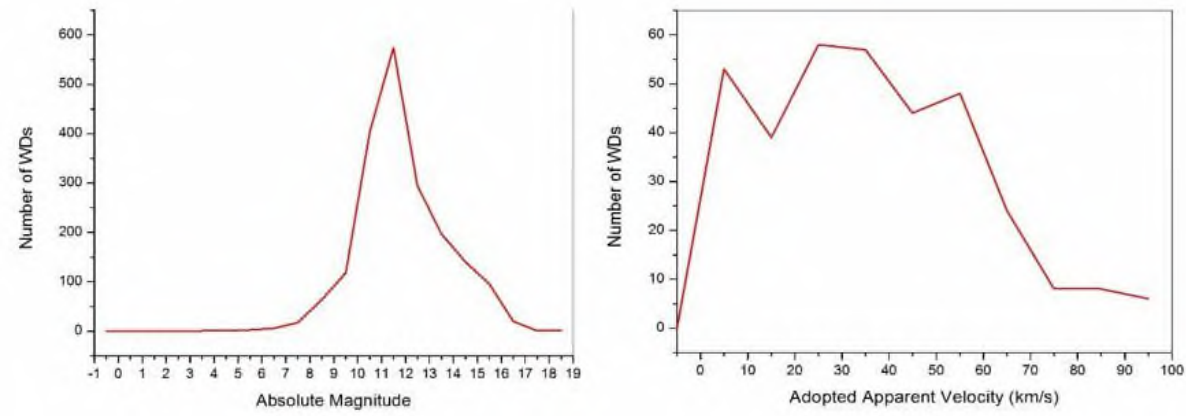

Figure 1: Distribution of absolute magnitudes and adopted apparent velocities for McCook \& Sion Catalogue WDs. 


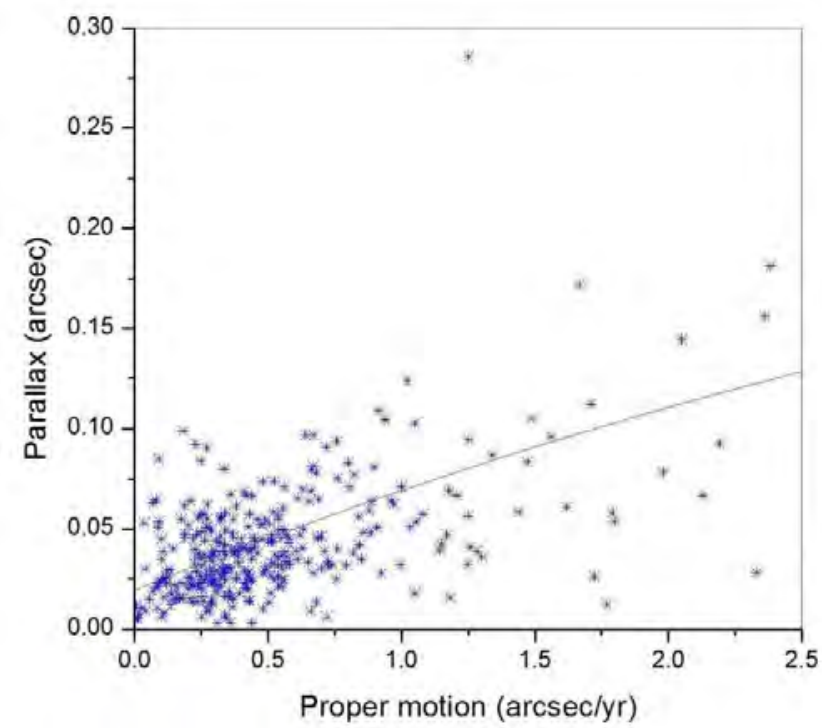

Figure 2: Distribution of parallaxes vs. proper motions for McCook \& Sion Catalogue WDs.

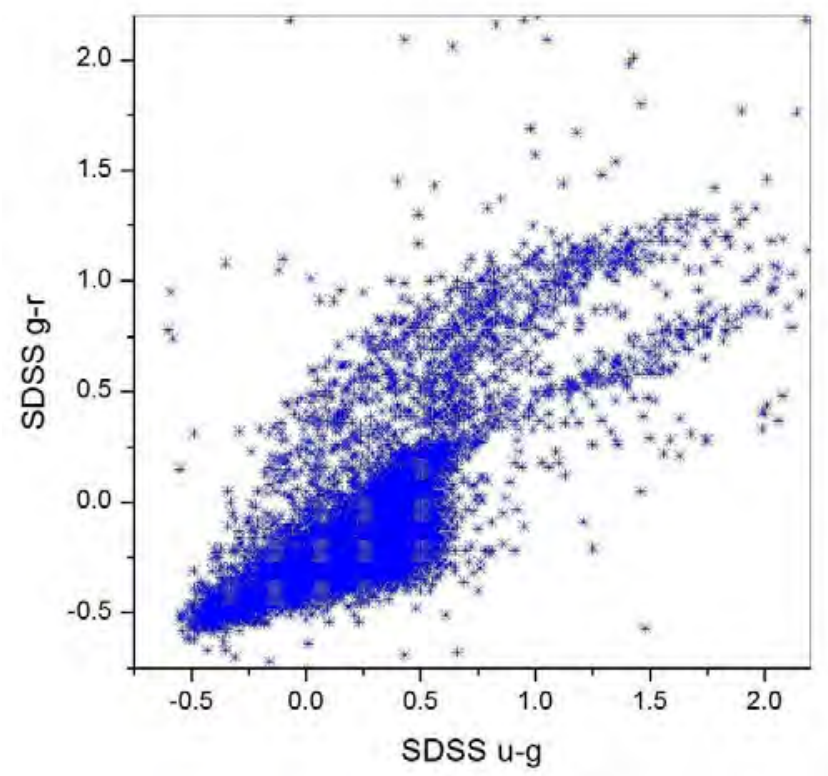

Figure 3: SDSS color-color diagram for McCook \& Sion Catalogue 4468 WDs.

\section{Gaia EDR3 data and SDSS photometry and spectra}

We have carried out cross-correlations of WDs with Gaia EDR3 [2] for parallaxes, PM, distances and $\mathrm{T}_{\mathrm{eff}}$, as well as cross-correlations with SDSS DR16 [3] for photometry (magnitudes and colors) and spectra. The search radius was 5 arcsec in both cases. For distances, Gaia EDR3 catalogue [4] was used giving data on 1.47 billion stars. Here are the numbers of associations:

- Gaia EDR3: 4338 associations,

- Gaia distances: 4233 associations,

- SDSS DR16: 4468 associations.

In Figure 3, we give the SDSS color-color diagram for McCook \& Sion Catalogue 4468 WDs, where most of the objects are concentrated in the lower left region (relatively bluer objects, typical of WDs).

\section{FBS Blue Stellar Objects, their proper motions and white dwarfs among them}

The First Byurakan Survey (FBS) Blue Stellar Objects (BSOs) are the result of the search of stellar objects with the same Markarian criteria in the FBS plates. Later on, the Digitized FBS (DFBS) database was used. 185 WDs have been revealed, including by using the astrometry first from APM, USNO, MAPS and GSC databases and then using the accurate astrometry from Gaia. The color-magnitude diagram is given in Figure 4.

Two examples of WDs low-dispersion spectra (both 2D and 1D extraction) are given in Figure 5. The extraction is done using the dedicated software bSpec [5].

At present search for WDs in DFBS has been conducted.

\section{Results and Summary}

One can summarize our results as follows:

- we have carried out statistical analysis of WD Catalogue: we have established the range and average numbers for absolute magnitudes, colors, proper motions, $T_{\text {eff }}$, etc.; the limits of adopted tangential velocities for calculation of the absent proper motions were derived;

- revealing thousands of new WD candidates from Gaia EDR3 accurate astrometry;

- revealing thousands of new spectroscopically identified WDs at high Galactic latitudes from SDSS;

- identifying all WDs among the FBS blue stellar objects, 185 in total; 


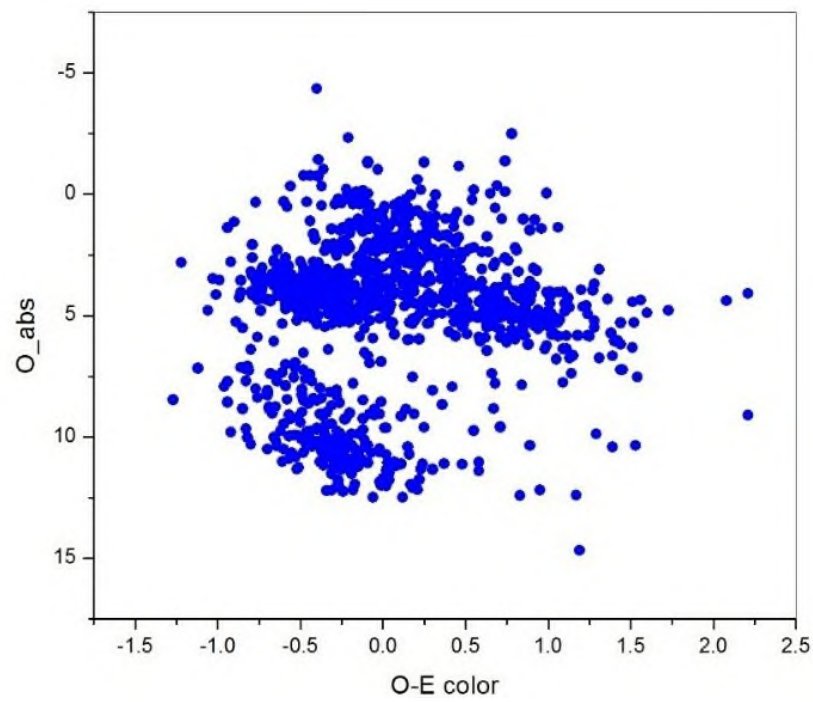

Figure 4: MAPS color-magnitude diagram for FBS BSOs. WDs are easily distinguishable on the lower left part of the diagram, as well as hot subdwarfs, somewhat upper from this locus.

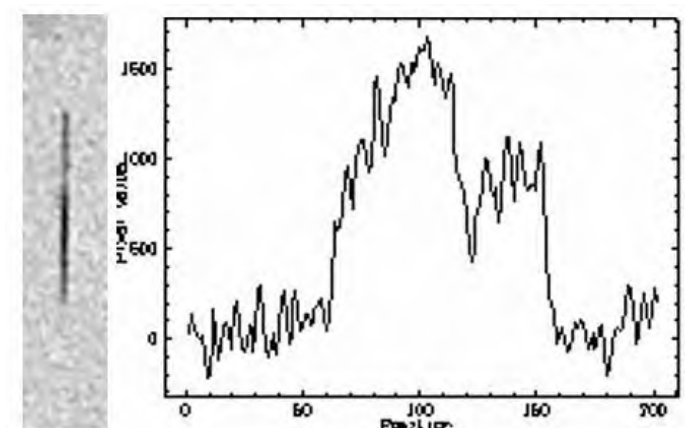

PG 0109+111 (DO)

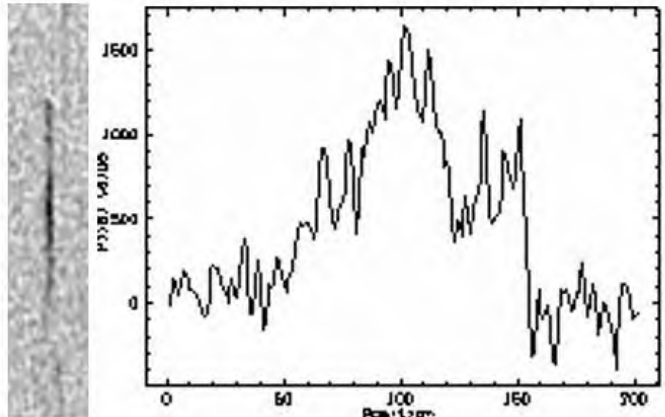

PG $1449+168$ (DA3)

Figure 5: DFBS low-dispersion spectra for two genuine WDs showing the shape of their spectra. Both 2D and 1D extractions are given.

- revealing thousands of new high Galactic latitude WD candidates in the DFBS.

Acknowledgement. The authors are grateful to ANSEF foundation for the grant PS-astroex-2512 "Discovery and study of new white dwarfs using Gaia accurate astrometry" (2021-2022). SDSS and Gaia teams are acknowledged as well.

\section{References}

1. G. P. McCook and E. M. Sion, VizieR Online Data Catalog, B/wd, 2014.

2. Gaia Collaboration, A. G. A. Brown, A. Vallenari, T. Prusti, et al., A $\& A, 650$, C3, 2021.

3. R. Ahumada, C. A. Prieto, A. Almeida, F. Anders, et al., ApJS, 249, 3, 2020.

4. C. A. L. Bailer-Jones, J. Rybizki, M. Fouesneau, M. Demleitner, and R. Andrae, VizieR Online Data Catalog, $\mathrm{I} / 352,2021$.

5. A. M. Mickaelian, L. A. Sargsyan, R. Nesci, G. Cirimele, and A. Sarkissian, in Y. Mizumoto, K. I. Morita, and M. Ohishi, eds., Astronomical Data Analysis Software and Systems XIX, Astronomical Society of the Pacific Conference Series, volume 434, 325 (2010). 


\section{Biermann battery as a source of cosmic magnetic fields}

E. Mikhailov ${ }^{1,2}$, R. Andreasyan ${ }^{3}$

ea.mikhajlov@physics.msu.ru; e.mikhajlov@lebedev.ru; randreasyan@gmail.com

${ }^{1}$ M.V.Lomonosov Moscow State University, Moscow 119991, Russia

${ }^{2}$ P.N.Lebedev Physical Institute of Russian Academy of Sciences, Moscow 119333, Russia

${ }^{3}$ V.A.Ambartsumian Byrakan Astrophysical Observatory of National Academy of Sciences of Republic of Armenia,

Byurakan 0213, Armenia

Nowadays it is well-known that some spiral galaxies, accretion discs, stars etc. have large scale magnetic fields. Their existence is supported by Faraday rotation measurements and another observational data. As for theoretical models, the magnetic field generation is usually described by the dynamo mechanism. It explains the exponential growth of the field. However, this model does not describe the formation of initial magnetic field, which should be considered using another approaches. One of the most reasonable methods is connected by the Biermann battery mechanism. It uses the fact that particles (protons and electrons) have the same charge and significantly different masses. This leads to the emergence of circular currents which produce vertical magnetic fields. Their structure is described by the integral equation which can be solved using regularization methods.

Keywords: dynamo theory, Biermann battery, integral equation

DOI: $10.51194 /$ VAK2021.2022.1.1.190

\section{Origin of cosmic magnetic fields}

Today we have information about magnetic fields of different astrophysical objects, such as the Sun, another stars, galaxies, accretion discs and another celestial bodies have large-scale magnetic field [1]. There are different observational methods which give us opportunities to measure them: Zeeman effect, spectra of the synchrotron emission and Faraday rotation measurements of polarization plane of radiowaves which is now is one of the most important instruments to study cosmic magnetic fields.

Theoretically, their generation can be explained using approaches of the dynamo theory [2]. Usually it is based on two main effects. Firstly, it is the $\alpha$-effect, which is connected with helicity of turbulent motions and transforms the toroidal magnetic field to poloidal one. After that, we should take into account the differential rotation $(\Omega-$ effect), which described the non-solid body movement of the object and transforms the poliodal magnetic field to the toroidal one. They compete with the turbulent diffusivity, and if the $\alpha \Omega$-mechanism is strong enough, the field will grow according to the exponential law. However, the main problem is connected with the existence of initial field, which cannot be described by the dynamo mechanism and should be supported with another theoretical models.

There are different ideas which can describe the origin of the magnetic field, for example Weibel instability and cosmological effects. From our point of view, the Biermann battery effect could be most possible mechanism of formation the magnetic field in galaxies and accretion discs [3]. It is based on emission of protons and electrons from the central object to the disc. They have the same charge, and different masses [4]. They interact with the rotating medium with different typical times. The electrons move nearly sticked with the medium, and the protons have a lag. Such processes produce circular currents. They are connected with vertical magnetic fields. They can be found using integral equation, which is solved using regularization methods [5].

\section{Integral equation for the magnetic field and its solution}

The magnetic field evolution in the simplest case can be described by the integral equation (all physical quantities are measured in dimensionless units connected with their typical values) [5]:

$$
B(r)=\lambda \int_{a}^{1} K(r, R) B(R) d R+f(r) ;
$$

where $a$ is the inner radius of the disc, $B$ is the magnetic field, $\lambda$ is the parameter characterizing the process, and for the used functions we have the formulaes [5]:

$$
\begin{gathered}
f(r)=-2 \int_{a}^{1} \frac{d R}{R^{2}} \int_{0}^{\pi} \frac{\left(1-\frac{r}{R} \cos \varphi\right) d \varphi}{\left(1+\frac{r^{2}}{R^{2}}-2 \frac{r}{R} \cos \varphi\right)^{3 / 2}} . \\
K(r, R)=-\frac{2}{R^{2}} \int_{0}^{\pi} \frac{\left(1-\frac{r}{R} \cos \varphi\right) d \varphi}{\left(1+\frac{r^{2}}{R^{2}}-2 \frac{r}{R} \cos \varphi\right)^{3 / 2}} .
\end{gathered}
$$

We have $\lambda \gg 1$, so it is important to use regularization methods, such as Tikhonov functional [6]. The result is shown on fig.1. 


\section{Conclusion}

We have described the structure of the initial magnetic field, which is produced by the Biermann battery mechanism in the simplest case. The produced magnetic field is enough to run the typical magnetohydrodynamical processes. It is necessary to emphasize that it is can be important to take into account another effects such as the ionization of the rotating medium.

These results can be used for studying the magnetic fields in galaxies, accreting discs surrounding compact objects and in another binary systems [5].

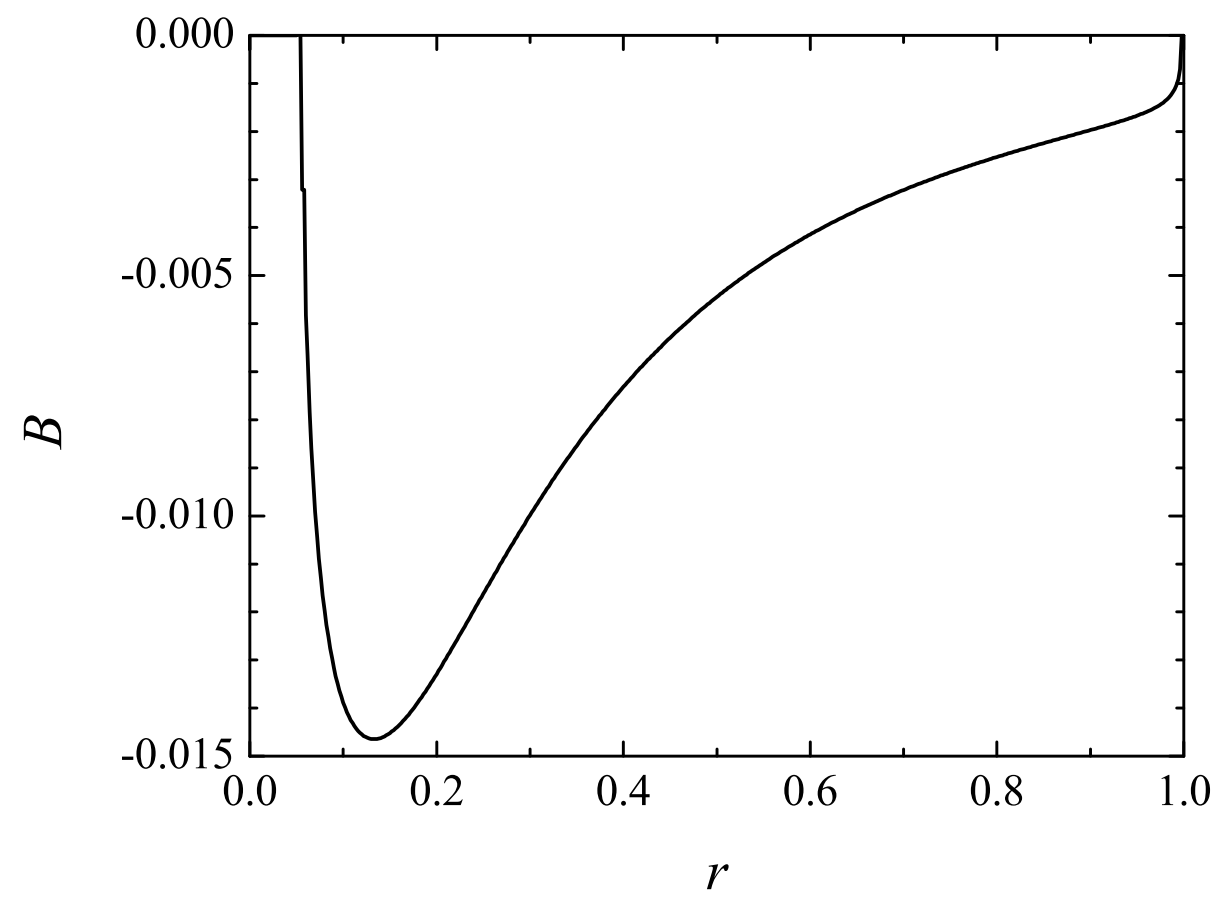

Figure 1: The solution for the magnetic field for $\lambda=50$.

\section{References}

1. I. B. Zeldovich, A. A. Ruzmaikin, and D. D. Sokolov, Nonlinear Waves: Self-Organization, 166-181 (1983).

2. D. D. Sokoloff, Physics Uspekhi, 58, 601-605, 2015.

3. L. Biermann and A. Schlüter, Physical Review, 82, 863, 1951.

4. R. R. Andreasyan, Astrophysics, 39, 58, 1996.

5. E. A. Mikhailov and R. R. Andreasyan, Astronomy Reports, 65, 715, 2021.

6. V. T. Volkov and A. G. Yagola, Integral equations. Calculus of variations, KDU (2007). 


\title{
Determination of the degree of star concentration in globular clusters based on space observation data
}

\author{
S. Nuritdinov ${ }^{1}$, A. Rastorguev ${ }^{2}$, I. Tadjibaev ${ }^{3}$ \\ nur200848@mail.ru \\ ${ }^{1}$ Ulugh-Beg National University, Tashkent, 100174 Uzbekistan, \\ ${ }^{2}$ Moscow State University, Moscow, 119991 Russia, \\ ${ }^{3}$ Chirchik State Pedagogical Institute, Chirchik, 111700, Uzbekistan
}

\begin{abstract}
Using the widely known simplex method of minimizing the square of the difference between the observed and theoretical densities, we have found the corresponding free parameters for globular clusters. As observational data we have taken mostly the density profiles constructed by [1] based on a combination of Hubble Space Telescope and ground-based data. One of the free parameters of our model characterizes the concentration of stars toward the globular cluster center. Errors for free parameters of the model are calculated.
\end{abstract}

Keywords: globular clusters, classification, concentration, apparent density profile

DOI: 10.51194 /VAK2021.2022.1.1.191

\section{Introduction}

So far there is no satisfactory solution of classification of globular clusters (GC) in astrophysics, although a number of interesting studies have been performed by various authors in this time (see $[2,3,4,5,6,7,8]$ and a very useful catalog has been compiled by [9].

It is important to note that the GC classification should be primarily easily applicable in practice and reflect at least one or two clearly observed properties, for example, the concentration of stars toward the cluster center, the cluster shape, sizes, structure, etc. and, at the same time, this classification must correlate with the main physical characteristics of these clusters deduced from observations. If a classification that satisfies these requirements can in no way be developed, then this implies the necessity of including an additional auxiliary parameter in the problem. Before us some authors proposed to introduce an auxiliary second parameter. For example, [10] introduced the concentration parameter c, with some of the GC catalogs still including this parameter. However, we now know that the parameter $\mathrm{c}$ does not correlate in any way whatsoever with either of the physical GC characteristics. Therefore, we decided to search for such a second parameter in other various ways.

The questions of GC classification were first considered by [11]. The authors proposed a GC classification based on the concentration of stars toward the cluster center and divided this entire material into 12 classes based on the apparent concentration of stars toward the cluster center. Now it is easy to verify that this classification, which consists of 12 groups of apparent concentrations, is obviously subjective. The results by [12, 2, 3, 13, 14, 6] are also of interest from the standpoint. So, all of this is very far from practical applications, and the correlations of these clusters with other observed physical parameters in the form of empirical relations were not discussed.

\section{Concentration of stars toward the GC center}

Among the main properties of GCs that should be used in solving the problem of their classification, the concentration of stars toward the cluster center nevertheless claims to rank first. Shapley and Sawyer correctly chose this GC property to develop their classification. However, their division into 12 classes is not completely unambiguous and, unfortunately, is somewhat subjective. This follows, for example, from a comparison of the distribution of Shapley-Sawyer concentration classes with the corresponding [10] parameter:

$$
c=\lg \left(r_{t} / r_{c}\right), \quad r_{t}=R\left(\frac{M}{2 M_{g}}\right)^{1 / 3}
$$

where $r_{t}$ is the tidal radius of the cluster, $r_{c}$ is the radius of its core, $R$ is the distance from the Galactic center to the GC, $M$ and $M_{g}$ are the masses of the cluster and the Galaxy, respectively. As can be seen, it is not easy to determine an accurate value of the parameter $c$ from observations, because the results depend on the accuracy of four quantities. Our calculations show that none of these physical characteristics of GCs has a correlation with the concentration parameter $c$. The highest value of this correlation is 0.34 (for $M_{V}$ ), while all the remaining values of the correlation coefficient are less than 0.12

Our calculations suggest that instead of the concentration parameter c we should search for a different auxiliary quantity that also characterizes the concentration of stars toward the GC center. For this purpose, we intercompared the apparent stellar surface density profiles of GCs by taking into account the [10] model, where $\sigma=\left(1+\frac{r^{2}}{r_{c}^{2}}\right)^{-1}$. As a result, we found that the bulk of the curve, except the peripheral region, could be described by the following 
function:

$$
\sigma\left(r, \gamma, r^{*}, \sigma_{0}\right)=\left[1+\left(\frac{r}{r^{*}}\right)^{2}\right]^{-\gamma} .
$$

Here, $\gamma, r^{*}$, and $\sigma_{0}$ are free parameters, with $\gamma$ characterizing the concentration of stars toward the GC center, $r^{*}$ being a quantity related to the cluster core radius $r_{c}$, and $\sigma_{0}$ being the apparent density at the center.

Note that the accuracy of finding the free parameters of the model $(2)$, especially the concentration $(\gamma)$ of stars toward the center, for specific GCs depends strongly on the accuracy of determining the observed density in them.

\section{Calculating the model parameters for GC}

To determine the above three free parameters in (2) for specific GCs, we need to minimize the function

$$
F\left(\gamma, r^{*}, \sigma_{0}\right)=\sum\left[\sigma\left(r_{k}, \gamma, r^{*}, \sigma_{0}\right)-\sigma_{o b s}^{(k)}\right]^{2},
$$

which describes the behavior of the sum of the squares of the difference between the theoretical $(\sigma)$ and observed $\left(\sigma_{o b s}^{(k)}\right)$ densities in annuli around the cluster center. It will suffice to minimize the function in (3) by the simplex method that has long been tested in many problems of astrophysics (see [15]).

The results of our minimization of the function in (3) in three parameters for each GC based on the combined observational data from [1] are presented in Table 1 in the work [8]. Unfortunately, in the simplex method, it is impossible to determine the errors of free parameters. Therefore, regardless of this method, we were forced to apply another, more complex method of determination, namely the $\chi^{2}$ method:

$$
\chi^{2}=\sum_{k} \frac{\left[\sigma\left(r_{k}, \gamma, r^{*}, \sigma_{0}\right)-\sigma_{o b s}^{(k)}\right]^{2}}{\sigma\left(r_{k}, \gamma, r^{*}, \sigma_{0}\right)} .
$$

Table 1: The results of calculating the free parameters of the model by the $\chi^{2}$ method

\begin{tabular}{|l|l|l|l|l|l|l|}
\hline Name GC & $\gamma$ & $\Delta \gamma$ & $r^{*}$ & $\Delta r^{*}$ & $\sigma_{0}$ & $\Delta \sigma_{0}$ \\
\hline NGC 104 & 0.73 & 0.24 & 18.68 & 8.52 & 7.69 & 0.96 \\
\hline NGC 288 & 1.38 & 0.96 & 93.75 & 63.18 & 0.17 & 0.16 \\
\hline AM 1 & 1.72 & 1.19 & 14.63 & 9.71 & 0.47 & 0.45 \\
\hline Eridanus & 1.51 & 0.60 & 19.87 & 7.86 & 0.86 & 0.15 \\
\hline NGC 1851 & 0.99 & 0.36 & 4.78 & 2.05 & 5.62 & 1.17 \\
\hline NGC 1904 & 0.91 & 0.27 & 7.42 & 3.32 & 5.70 & 1.34 \\
\hline NGC 2419 & 1.18 & 0.33 & 20.06 & 6.39 & 3.60 & 0.43 \\
\hline Palomar 3 & 2.11 & 0.91 & 48.45 & 18.21 & 0.04 & 0.01 \\
\hline Palomar 4 & 2.16 & 1.01 & 39.31 & 15.01 & 0.04 & 0.01 \\
\hline NGC 5024 & 1.00 & 0.24 & 22.08 & 7.41 & 2.96 & 0.40 \\
\hline NGC 5272 & 0.81 & 0.23 & 17.21 & 7.72 & 3.10 & 0.51 \\
\hline NGC 5466 & 1.48 & 0.60 & 102.88 & 38.95 & 0.10 & 0.01 \\
\hline NGC 5824 & 0.73 & 0.17 & 2.71 & 1.12 & 7.56 & 1.34 \\
\hline NGC 5904 & 0.68 & 0.14 & 14.64 & 5.31 & 4.07 & 0.53 \\
\hline Palomar 14 & 0.99 & 0.47 & 38.37 & 21.65 & 0.25 & 0.06 \\
\hline NGC 6121 & 0.81 & 0.35 & 47.43 & 30.39 & 0.26 & 0.09 \\
\hline NGC 6205 & 1.05 & 0.32 & 48.22 & 15.18 & 3.58 & 0.31 \\
\hline NGC 6229 & 1.02 & 0.34 & 8.27 & 3.28 & 5.26 & 0.78 \\
\hline NGC 6254 & 1.02 & 0.28 & 38.33 & 14.38 & 0.50 & 0.08 \\
\hline NGC 6266 & 0.86 & 0.21 & 12.44 & 4.17 & 10.4 & 1.21 \\
\hline NGC 6341 & 1.03 & 0.46 & 14.54 & 8.28 & 2.54 & 0.81 \\
\hline Terzan 5 & 1.31 & 0.45 & 10.28 & 3.87 & 7.17 & 1.06 \\
\hline NGC 6626 & 0.71 & 0.18 & 6.85 & 3.18 & 2.36 & 0.58 \\
\hline NGC 6809 & 0.85 & 0.35 & 69.07 & 40.75 & 0.20 & 0.06 \\
\hline NGC 6864 & 1.18 & 0.46 & 5.71 & 2.5 & 16.82 & 3.01 \\
\hline NGC 7089 & 0.89 & 0.29 & 12.43 & 5.8 & 3.13 & 0.71 \\
\hline
\end{tabular}


Thus, we have obtained the values of the free parameters, which almost coincide with the results found by the simplex method. The results of the chi-square method and the errors of the three free parameters are given by us in Table 1.

Among the three parameters of the model, almost complete agreement is well observed for the concentration $\gamma$. We can confidently assert that the values of the degree of concentration $\gamma$ lie in the interval (0.65; 2.15). Thus, this GC classification is simpler than the 12 classes according to [11]. In future it will be possible to refine these ranges in $\gamma$.

\section{Conclusions}

First of all, we would like to note that using the above method, we planned to also process the surface density observations from [16]. Unfortunately, in this case there is no sense to calculate $\gamma$ for two reasons. First, there are no observations for the central GC regions in [16] and second, the data in their paper cover mainly the peripheral part of GCs, which strongly affects the concentration $\gamma$ and $r^{*}$. Thus, in this paper we calculated the parameter $\gamma$ for GCs by theoretically modeling the apparent GC surface density (2) with three free parameters and minimizing the sum of the squares of the difference between this theoretical function and the observed density function.

\section{References}

1. P. Miocchi, B. Lanzoni, F. Ferraro, E. Dalessandro, et al., Astrophys. Journ., 774, 151, 2013.

2. F. D. Hartwick, Astrophys. Jour., 154, 475, 1968.

3. B. V. Kukarkin, Sov. Astron., 15, 89, 1971.

4. A. V. Mironov, A. S. Rastorguev, and N. N. Samus, Sov. Astron., 20, 656, 1976.

5. V. Straizys, Astrophys. Space Sci., 81, 179, 1982.

6. A. M. Eigenson and O. S. Yatsyk, Astron. Rep., 39, 569, 1995.

7. I. U. Tadjibaev and S. N. Nuritdinov, Ukr. J. Phys., 64, 271, 2019.

8. S. N. Nuritdinov, I. U. Tadjibaev, and A. S. Rastorguev, Astron. Letters, 43, 163, 2021.

9. W. E. Harris, arXiv:1012.3224H, 2010.

10. I. R. King, Astron. Journ., 67, 471, 1962.

11. H. Shapley and H. B. Sawyer, Harvard Observ. Bull., 849, 11, 1927.

12. A. G. Mowbray, Sov. Astron., 104, 47, 1946.

13. A. V. Mironov, Sov. Astron., 17, 16, 1973.

14. A. V. Mironov and N. N. Samus, Perem. Zvezdy, 19, 337, 1974.

15. A. E. Ashurov and S. N. Nuritdinov, ASP Conf. Ser., 228, 371, 2001.

16. d. T. J. L. Boer, M. Gieles, E. Balbinot, V. Henault-Brunet, A. Solima, I. I. Watkins, and I. Claydon, $M N R A S$, 485, 4906, 2019. 


\title{
Parametrization of long-period eclipsing binaries
}

\author{
P. Pakhomova ${ }^{1}$, L. Berdnikov ${ }^{2}$, A. Kniazev ${ }^{2,3,4}$, I. Katkov ${ }^{2,5}$, O. Malkov ${ }^{1,2}$ \\ ${ }^{1}$ Institute of Astronomy of RAS, Moscow, Pyatnitskaya str., 48, Russia, \\ ${ }^{2}$ Sternberg State Astronomical Institute, Lomonosov Moscow State University, \\ ${ }^{3}$ South African Astronomical Observatory, P.O. Box 9, Observatory, Cape Town, \\ ${ }^{4}$ Southern African Large Telescope, P.O. Box 9, Observatory, Cape Town, \\ ${ }^{5}$ Center for Astro, Particle, and Planetary Physics, NYU Abu Dhabi
}

One of the important sources for independent determination of stellar masses are eclipsing binaries with components on the Main Sequence, and with observable spectral lines of both components. The parameters of such stars are used to construct the mass-luminosity relation for stars of high and intermediate masses. Among them, the type of long-period eclipsing binaries stands out, the parameters of which are currently not fully determined, which is associated with the difficulties caused by the need for long-term observations. In this article we will review the currently available observational data for such objects and discuss the prospects for their use to determine stellar masses.

Keywords: stars: mass-luminosity relation, mass function — stars: binaries: spectroscopic - techniques: photometric, spectroscopic

DOI: $10.51194 /$ VAK2021.2022.1.1.192

\section{Introduction}

The mass of a star is one of the most important parameters that determine its evolution. However, for a single star, it is impossible to determine the mass directly, and indirect methods are used to estimate the masses, and the subsequent transition to masses is carried out using the mass-luminosity ratio (MLR). For its construction, orbital binaries are well suited and used, that is, visual binaries with known orbital parameters.

However, such systems have component masses less than $1.5 \mathrm{M} \odot$. Therefore, it is possible to construct a MLR using them only for small masses. For larger masses, double-lined eclipsing binaries (DLEB) are used. However, we must be sure that in this case we get a MLR which can really be legitimately used for single stars. Single young massive stars are fast rotators. And close eclipsing binaries with periods less than 15 days are almost all synchronized with orbital rotation, so they rotate more slowly. Accordingly, we cannot apply the mass-luminosity ratio built for slow rotators for single rapidly rotating stars. Hence, it is necessary to look for other objects.

Such objects are long-period DLEB. They are mostly not yet synchronized and are fast rotators, that is, their evolutionary path is similar to the evolution of single stars. Therefore, our goal is to construct MLR for stars of high and intermediate masses based on a sample of such systems.

\section{The sample}

We have selected eclipsing systems from the General Catalog of Variable Stars [1] and the Catalog of Eclipsing Variables [2] by such criteria:

- apparent magnitudes are brighter of 13.0 in the $\mathrm{V}$ band

- both components are on the main sequence

- spectral classes are no later than F

- periods are between 15 days and 1 year

Initially, 132 stars were selected, but after collecting and analyzing the available published data, the sample was reduced, and at the moment it contains 45 stars. In the future, it will also be reduced and, if necessary, replenished with new objects. Table 1 presents the list of 22 stars from our sample in the Northern hemisphere.

\section{Data selection and analysis}

\subsection{Photometry}

The first step of our work is to search and collect available photometric data on our stars from various surveys and databases. Among them are the ASAS and ASAS-SN surveys, the OMC-INTEGRAL database, the SuperWASP, TESS, KEPLER telescope databases, and the AAVSO database of the American Association of Variable Star Observers.

Then, based on these data, the light curves were constructed and analyzed in order, firstly, to carry out additional filtering of the sample by the shape of the curve (since from the shape of the light curve it may become clear that this is not an eclipsing system, but some other type of variable, for example). Secondly, in order to understand the quality of existed photometry. 
Table 1: List of stars in our sample — northern sky

\begin{tabular}{clrcccl}
\hline$\#$ & Name & $\mathrm{V}(\mathrm{mag})$ & $\mathrm{P}($ day $)$ & RA $(2000.0)$ & DEC $(2000.0)$ & Type \\
\hline 1 & SY And & 10.70 & 34.9085 & 3.31833 & 43.71139 & A0+K1 \\
2 & CD And & 9.90 & 34.4434 & 21.61833 & 44.35695 & F8 \\
3 & V436 Per & 5.49 & 25.9359 & 27.99708 & 55.14750 & B1.5V \\
4 & AN Cam & 10.40 & 20.9986 & 61.49000 & 76.88667 & F8 \\
5 & V454 Aur & 7.74 & 27.0270 & 95.51292 & 34.59750 & F8 \\
6 & TU Lyn & 11.40 & 38.9461 & 97.76125 & 61.24139 & F0: \\
7 & alf CrB & 2.21 & 17.3599 & 233.67208 & 26.71472 & B9.5IV $+\mathrm{G} 5 \mathrm{~V}$ \\
8 & V541 Cyg & 10.20 & 15.3378 & 295.62250 & 31.32778 & A0 \\
9 & IM Del & 11.83 & 34.3383 & 305.62500 & 18.56500 & - \\
10 & MP Del & 7.56 & 21.3387 & 307.11083 & 11.72083 & Am \\
11 & V1326 Cyg & 11.30 & 16.6817 & 308.62750 & 54.21028 & B7 \\
12 & OT And & 7.32 & 20.8529 & 350.00500 & 41.75500 & - \\
13 & V409 Cyg & 13.30 & 37.7260 & 317.75958 & 49.70111 & - \\
14 & V698 Cyg & 12.20 & 97.7732 & 299.9725 & 36.27778 & A7IV \\
15 & V1156 Cyg & 13.50 & 44.5647 & 297.65458 & 29.35472 & - \\
16 & EU Gem & 13.70 & 52.2665 & 99.925 & 17.19222 & - \\
17 & V340 Lac & 11.80 & 19.9433 & 333.18542 & 54.18583 & A8 \\
18 & CR Per & 12.40 & 22.6810 & 32.4675 & 57.90917 & - \\
19 & V355 Her & 13.20 & 17.0000 & 276.8375 & 13.16917 & - \\
20 & V413 And & 7.61 & 50.1150 & 358.51667 & 39.28250 & A7V+G0III \\
21 & V733 Per & 11.89 & 77.5300 & 52.95208 & 36.21250 & - \\
22 & LX Gem & 13.36 & 145.1000 & 100.02083 & 15.11111 & - \\
\hline & & & & & &
\end{tabular}

During the last two years we took photometric observations at the Las Cumbres (LCO) observatory. This observatory consists of thirteen $1-\mathrm{m}$ and ten $40-\mathrm{cm}$ robotic telescopes with remote access located at seven observational sites around the world. In addition, starting from this year we began to receive photometric data with the 60-cm telescope of Caucasian Mountain Observatory and with 1-m Zeiss telescope of Simeiz observatory (INASAN). Additionally, we plan to carry out photometric observations with small robotic telescopes of the Special Astrophysical Observatory (hereafter SAO RAN), telescopes of the Terskol Observatory of INASAN and two 1-meter telescopes of the South African Astrophysical Observatory.

\subsection{Spectroscopy}

We observe stars of the southern sky with the 11-meter SALT telescope using a high-resolution échelle spectrograph (HRS) there. These spectral observations started about 5 years ago and more than two hundred spectra were taken and parameters of some binary systems were obtained. Our first results of this work were presented in articles [3] and [4].

Since the work with the northern sky sample began much later, we can describe our current plans mainly. Currently, we receive spectra with HERMES spectrograph on the Canary Mercator 1.2-meter telescope. And we are planning to carry out spectral observations at the Kourovka Astronomical Observatory, at SAO RAN, at the Terskol Observatory using the MAESTRO high-resolution spectrograph and also at the Simeiz Observatory, where a spectrograph has recently appeared. In addition, we include the Caucasian Mountain Observatory in this list, despite the fact that the spectrograph has not yet been installed at the observatory, since our tasks involve long-term observations.

\section{Conclusions}

Thus, at the moment we can talk about such intermediate results of our work:

1. Over 5 years of spectral observations at SALT, we obtained more than two hundred échelle spectra for 43 stars (some of these stars were subsequently excluded from the sample based on the results of spectral analysis).

2. We have collected and analyzed photometric data from most of the available databases and surveys.

3. For 32 stars from our sample, we conduct observations and obtain photometry at the LCO.

4. At this year we received the first observational data for our project from KGO and Simeiz. 


\section{Acknowledgements}

This work was partially supported by RFBR grants 19-07-01198 and 20-52-53009.

\section{References}

1. N. N. Samus', O. V. Durlevich, E. V. Kazarovets, N. N. Kireeva, and E. N. Pastukhova, Astronomy Reports, 62, 1050, 2018.

2. E. A. Avvakumova, O. Y. Malkov, and A. Y. Kniazev, Astronomische Nachrichten, 334, 860, 2013.

3. A. Y. Kniazev, O. Y. Malkov, I. Y. Katkov, and L. N. Berdnikov, Research in Astronomy and Astrophysics, 20, 119, 2020.

4. A. Kniazev, Ap\&SS, 365, 169, 2020. 


\title{
Magnetic field of chemically peculiar stars in Orion OB1 association
}

\author{
I.I. Romanyuk ${ }^{1}$, E.A. Semenko ${ }^{1,2}$, A.V. Moiseeva ${ }^{1}$, I.A. Yakunin ${ }^{1,3}$, D.O. Kudryavtsev ${ }^{1}$ \\ roman@sao.ru, \\ WWW home page: https://www.sao.ru/hq/lizm/en/ \\ ${ }^{1}$ Special Astrophysical Observatory, Russian Academy of Sciences, 369167, Nizhnii Arkhyz, Russia, \\ ${ }^{2}$ National Astronomical Research Institute of Thailand, 50180, Chiangmai, Thailand, \\ ${ }^{3}$ St.-Petersburg State University, 199034, St. Petersburg, Russia
}

\begin{abstract}
We present results of magnetic field measurements for 57 chemically peculiar stars in the Orion OB1 association. We have found 32 magnetic stars in the association (or $56 \%$ of CP stars) which is more than twice larger than usual $25 \%$ for cooler Ap stars. We have found that the proportion of chemically peculiar stars relative to the normal ones strongly decreases with age in the interval from $2 \mathrm{Myr}$ to $10 \mathrm{Myr}$. The proportion of magnetic stars relative to all peculiar stars also strongly decreases (more than twice) with age in the same time interval as well as the magnetic field strength.
\end{abstract}

Keywords: chemically peculiar stars, magnetic field, evolution

DOI: 10.51194 /VAK2021.2022.1.1.193

\section{Introduction}

In the Orion constellation, at a distance of about $400 \mathrm{pc}$, there is one of the most famous groups of early-type stars, the Orion OB1 association. [1] made the first attempt to analyze the association structure and identified four subgroups: 1a, 1b, 1c, and 1d which differed in age and stellar composition. Subgroup 1a belongs to the northern region of the association, subgroup $1 \mathrm{~b}$ is the Orion's "belt", $1 \mathrm{c}$ is the region south of the Orion's belt, and 1d is a very compact area in the center of the association within subgroup $1 \mathrm{c}$.

\section{Chemically peculiar stars in the association}

Approximately 10-15\% of all A and B stars of the Main Sequence have anomalous chemical abundance: an increased abundance of chemical elements heavier than iron, hot B stars have an increased abundance of silicon and helium, and some of them have a smaller abundance of helium compared to that of the Sun. Such objects are called chemically peculiar stars (CP stars). Among these particular objects, [2] discovered stars with strong magnetic fields. Further research has shown that the magnetic field is found not in all but in about $25 \%$ of them. Possibly, all CP stars have magnetic fields, but they are weak and below a standard detection threshold of about $100 \mathrm{G}$.

One of the unsolved issues in astrophysics is the origin of strong large-scale magnetic fields in CP stars and their chemical abundance anomalies. Previous studies of open clusters of different ages indicated that the magnetic field of young CP stars is possibly higher than that of old [3, 4]. To study this problem with the 6-m BTA telescope, we have set up an extensive program of studies of CP stars in clusters of different ages. We started our work by studying the Orion OB1 association, the paper by [5] was taken as the basis for the stellar population of the association; using the catalog of chemically peculiar stars by [6], we identified 85 association objects: A and B stars with anomalous lines in the spectra [7]. Using the data from the HIPPARCOS and GAIA satellites, we have found that $23 \mathrm{~A}$ stars from the sample are not members of the association, they are foreground objects and are only projected onto it. Further studies have shown that among 62 objects, there are several non-magnetic or weakly magnetic $\mathrm{HgMn}$ stars and one $\mathrm{B}[\mathrm{e}]$ star. The remaining $57 \mathrm{CP}$ stars are potentially magnetic and have been included in the observation program.

\section{Magnetic CP stars in the association}

Most observations of peculiar stars in the Orion OB1 association were carried out between 2013 and 2019 at the Main Stellar Spectrograph (MSS) ${ }^{\mathrm{a}}$ of the BTA telescope [8] with a circular polarization analyzer [9]. The observation methods, the detailed analysis for each $\mathrm{CP}$ star of the association, and key findings of subgroups are published in the papers by $[10,11,12]$.

As a result, we have found that the root-mean-square magnetic field $\left\langle B_{\mathrm{e}}\right\rangle$ in subgroups $1 \mathrm{a}$ and $1 \mathrm{c}$ is on average the same; in subgroup 1c, it is significantly higher. Our results indicate a sharp drop of the proportion of magnetic $\mathrm{CP}$ stars and a simultaneous decrease in their magnetic fields with age. Let us also note that, contrary to our expectations, the strongest magnetic field for the stars in the Orion OB1 association is observed not in the center, where the star-forming regions are located, but on the periphery of the association. One way or another, we obtain the data about the reliable difference in the magnetic properties of stars inside and outside the Orion nebula. The proportion of magnetic stars in the nebula is four times smaller and the average magnetic field is two times smaller than those of objects outside it.

aThe web-page of the MSS instrument: https://www.sao.ru/hq/lizm/mss/en/index.html 


\section{Conclusion}

With the 6-m telescope, we have obtained the extensive observed data: more than 600 moderate-resolution spectra with a circular polarization analyzer. We have found that the total number of CP stars with the detected magnetic field is $56 \%$ of the total number in the association, which is twice as much as is usually assumed. A sharp drop of the proportion of chemically peculiar stars in the association was also found in the age range from 2 to 10 Myr. We also observe a sharp decrease in the proportion of magnetic stars in the Orion Nebula. Obviously, the formation of magnetic fields of stars occurs in a complex manner. The data obtained with the example of the Orion OB1 association generally support the theory of the fossil origin of magnetic fields of chemically peculiar stars; however, the process of the field formation itself can have a number of features that manifest themselves observationally.

\section{Acknowledgments}

The observations carried out with the 6-m BTA are supported by the Ministry of Science and Higher Education of the Russian Federation (including agreement No. 05.619.21.0016, project ID RFMEFI61919X0016). The renovation of the telescope equipment is currently provided within the "Science" national project. The authors are thankful to the Russian Science Foundation for financial support of this work (RSF grant No. 21-12-00147). The work of I. Yakunin was funded by RFBR No. 19-32-60007.

\section{References}

1. A. Blaauw, ARA\&A, 2, 213, 1964.

2. H. W. Babcock, ApJ, 105, 105, 1947.

3. E. F. Borra and J. D. Landstreet, ApJ, 228, 809, 1979.

4. D. A. Bohlender, D. N. Brown, J. D. Landstreet, and I. B. Thompson, ApJ, 323, 325, 1987.

5. A. G. A. Brown, E. J. de Geus, and P. T. de Zeeuw, A\&A, 289, 101, 1994.

6. P. Renson and J. Manfroid, A\&A, 498, 961, 2009.

7. I. I. Romanyuk, E. A. Semenko, I. A. Yakunin, and D. O. Kudryavtsev, Astrophysical Bulletin, 68, $300,2013$.

8. V. E. Panchuk, G. A. Chuntonov, and I. D. Naidenov, Astrophysical Bulletin, 69, 339, 2014.

9. G. A. Chountonov, in Magnetic Stars, 286-291 (2004).

10. I. I. Romanyuk, E. A. Semenko, A. V. Moiseeva, I. A. Yakunin, and D. O. Kudryavtsev, Astrophysical Bulletin, $\mathbf{7 4}, 55,2019$.

11. I. I. Romanyuk, E. A. Semenko, A. V. Moiseeva, I. A. Yakunin, and D. O. Kudryavtsev, Astrophysical Bulletin, 76, 39, 2021 .

12. I. I. Romanyuk, E. A. Semenko, A. V. Moiseeva, I. A. Yakunin, and D. O. Kudryavtsev, Astrophysical Bulletin, 76, 163, 2021. 


\title{
A search of unresolved binaries in open clusters by the photometry data in visible and infrared
}

\author{
A.F. Seleznev, A.A. Malofeeva \\ anton.seleznev@urfu.ru \\ Ural Federal University, Mira str. 19, Ekaterinburg 620002, Russia
}

The general idea is to find the photometric diagram where the unresolved binary stars are well detached from the single stars, even for small values of the component mass ratio $q$. We use the diagram (H-W2)-W1-W2-(BP-K) to estimate the binary and multiple star ratio and the distribution of $q$ for the Pleiades in the primary component mass interval between 0.5 and 1.8 solar masses. A large number of binaries with secondary components being brown dwarfs or stars with the remnants of protostellar discs providing slight infrared excess are among the low-mass stars with the mass of the primary component lower than 0.5 solar masses.

Keywords: binaries: general, brown dwarfs, open clusters and associations: individual: Pleiades, techniques: photometric

DOI: 10.51194 /VAK2021.2022.1.1.194

The present-day data on the binary and multiple star content in star clusters are rather fragmentary and often contradictory (see a review in [1] and [2]). We can use spectroscopy to look for the unresolved binaries, but it needs the large telescopes working in the monitoring regime, which is very low possible. Photometry is much more accessible. However, the binary systems with a low component mass ratio $q \leqslant 0.4 \div 0.5$ are difficult to detach from single stars on photometric diagrams in the visible wavelengths.

Compared to their primary components, the binary systems have a slight infrared excess, which becomes registerable in the near-infrared wavelengths. [3] have used this to determine the masses of the unresolved binary system components by approximating their spectral energy distribution.
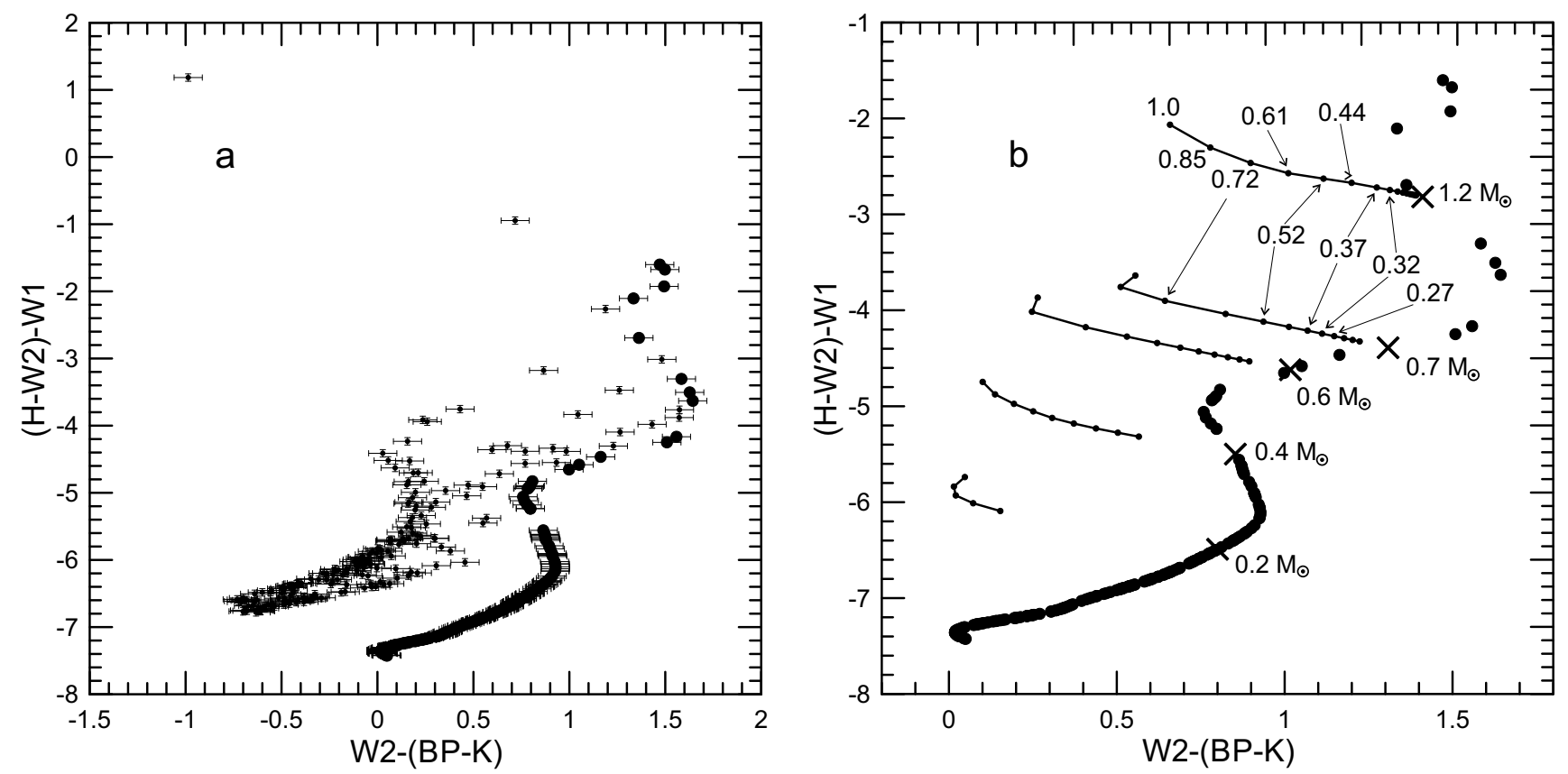

Figure 1: (a) Diagram W2-(BP-K)-(H-W2)-W1 for the cluster model. Big points — single stars, minor points binary stars. (b) A displacement of the unresolved binary star from the single star position depending on the $q$ ratio.

We decided to find the photometric diagram composed of stellar magnitudes and color indexes from visible and infrared passbands with the following property. The unresolved binary stars should be well detached from the single stars in this diagram, even for small values of q. With this goal, we composed the model of the open cluster consisting of 200 single and 200 binary stars. The masses of the single stars and the primary components of binaries were distributed following a Salpeter mass function in the mass interval from 0.1 to 6.8 solar masses. For secondary components of the binaries, we used the flat distribution in the interval of masses from 0.1 solar masses to the mass of the primary component. Then we compiled the photometric catalog for this model using stellar magnitudes for bands U, B, V, R, u, g, r, i, z, G, BP, RP, J, H, KS, W1, W2 from the tables of theoretical isochrones [4]. Two isochrones have been used for an age of $5 \cdot 10^{7}$ years (to search an acceptable diagram) and 
$\log t=8.1$ (to investigate the Pleiades sample) and the solar metallicity. We have looked for the diagram by going over the different combinations of the bands and color indexes.

Fig. 1 shows the diagram W2-(BP-K)-(H-W2)-W1 for the cluster model and the lines of the binary star displacement. We decided to use this diagram because binary stars with small $q$ values are well resolved, and the lines of the binary star displacement are close to the horizontal lines. There is a gap between the single and binary stars in the lower part of the diagram. The reason is an absence of the stellar magnitude data for stars with a mass lower than $0.1 M_{\odot}$ and the brown dwarfs.
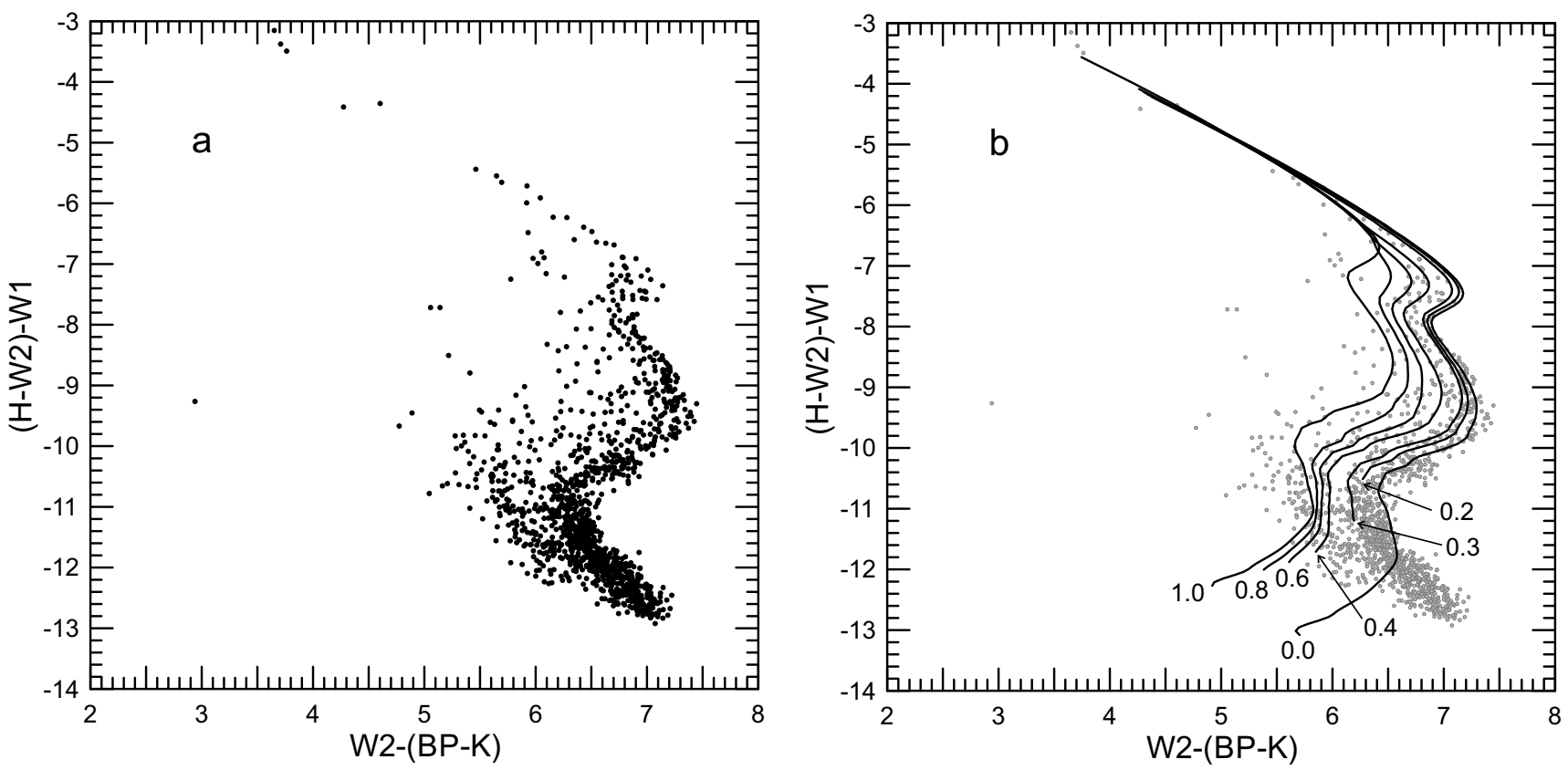

Figure 2: (a) Diagram W2-(BP-K)-(H-W2)-W1 for the Pleiades cluster. (b) Lines of constant $q$ values overlapped the Pleiades diagram.

Fig. 2a shows the diagram W2-(BP-K)-(H-W2)-W1 for the Pleiades cluster. Sample of 1293 stars is the intersection of the sample of [5] and the sample of [6] for the circle of 10.9 degrees around the cluster center and $G<18$. In Fig. 2b the line of constant $q$ values are overlapped the Pleiades diagram. In order to do this, we used the Pleiades parameters from the catalog of [7]. It is well seen that we cannot use this diagram for the binary stars search in the upper part of the diagram where the $q$-lines superimpose each other. In the lower part of the diagram we cannot use it because the theoretical isochrone (the line of single stars) does not coincide with the cluster sequence.

Nevertheless, the region to the left from the single stars line in the lower part of the Pleiades diagram is filled with stars in contrast to the diagram of the cluster model. It means that the Pleiades have many binary stars with very small-masses components, generally the brown dwarfs. Another possibility is many stars with the remnants of protostellar disks, which provide a slight infrared excess.

We use this diagram for counting the binary stars with the different $q$ values in the range $[0.5 ; 1.8] M_{\odot}$. We consider stars to the right side of the line $q=0$ as single ones in the first case. Stars to the right side of the line $q=0.2$ are considered as single ones in the second case. We suspect stars to the left side of the line $q=1$ as stars with a multiplicity of more than 2 . We have performed the star counts several times, displacing the position of stars accordingly to the errors of the photometry. In the first case, we have obtained the binary star ratio $\alpha=0.70 \pm 0.14$, the ratio of stars with the multiplicity more than $2 \beta=0.10 \pm 0.00$, and the exponent $\gamma=-0.53 \pm 0.10$ of the power law for the $q$ distribution $d N / d q \sim q^{\gamma}$. In the second case, we have obtained $\alpha=0.54 \pm 0.11, \beta=0.14 \pm 0.01$, and $\gamma=-0.63 \pm 0.22$.

We have obtained a much greater ratio of binary stars in the Pleiades than the previous investigators $[8,9,10]$ because with the diagram W2-(BP-K)-(H-W2)-W1, we can detect numerous binary stars with small $q$ values.

The work of A.F. Seleznev was supported by the Ministry of Science and Higher Education of the Russian Federation, FEUZ-2020-0030.

\section{References}

1. O. I. Borodina, A. F. Seleznev, G. Carraro, and V. M. Danilov, ApJ, 874, 127, 2019.

2. O. I. Borodina, G. Carraro, A. F. Seleznev, and V. M. Danilov, ApJ, 908, 60, 2021. 
3. B. A. Thompson, P. M. Frinchaboy, T. Spoo, and J. Donor, AJ, 161, 160, 2021.

4. A. Bressan, P. Marigo, L. Girardi, B. Salasnich, C. Dal Cero, S. Rubele, and A. Nanni, MNRAS, 427, 127, 2012.

5. V. M. Danilov and A. F. Seleznev, Astrophysical Bulletin, 75, 407, 2020.

6. N. Lodieu, A. Pérez-Garrido, R. L. Smart, and R. Silvotti, A\&̈A, 628, A66, 2019.

7. W. S. Dias, H. Monteiro, A. Moitinho, J. R. D. Lépine, G. Carraro, E. Paunzen, B. Alessi, and L. Villela, MNRAS, 504, 356, 2021 .

8. J. C. Mermilliod, J. M. Rosvick, A. Duquennoy, and M. Mayor, A\& A, 265, 513, 1992.

9. J. Bouvier, F. Rigaut, and D. Nadeau, A\&A, 323, 139, 1997.

10. V. M. Danilov, Astrophysical Bulletin, 76, 55, 2021. 


\title{
A special class of potentials admitting a local integral for rotating systems
}

\author{
F.T. Shamshiyev
}

shamshiyev61@gmail.com

National University of Uzbekistan Tashkent, Uzbekistan

The search for qualitative regularities in the motion of bodies obeying Newton's law has always been an important task in celestial mechanics. In stellar dynamics, the relevance of such searches remains, and they have become associated with a wider class of possible potentials due to the variety of gravitational fields of the stars and galaxies encountered. A special class of potentials admitting a local integral is described in [1], which can be used to study the motion of stars in stellar systems close to spherical symmetry. In the proposed article we will consider the same problem in the presence of rotation. The class of potentials are constructed.

Keywords: local integral, gravitational potential, trajectories

DOI: $10.51194 /$ VAK2021.2022.1.1.195

It is well known that exact integrals of motion being independent of the classical ones for motion in a potential field can be found in rare cases only, i.e., even in two-dimensional problems and move less often in three dimensional ones. Therefore, it is of interest in searching for such broader cases in the case in which the regularity of motion is manifested by the fact that the particle remains on some invariant manifold in the phase space. In this paper, in contrast to [1], we will consider cases when the system rotates with a constant angular velocity $\Omega$. We assume that the velocity field is represented as

$$
\left\{\begin{array}{l}
u=u_{0} \pm \alpha \sqrt{S+2 h}+\Omega y \\
v=v_{0} \pm \beta \sqrt{S+2 h}-\Omega y \\
w=w_{0} \pm \gamma \sqrt{S+2 h}
\end{array}\right.
$$

with $S, u_{0}, v_{0}, w_{0}, \alpha, \beta, \gamma$ are coordinate functions, $h$ is an arbitrary energy constant. Suppose that for the function $\alpha, \beta, \gamma$ the equality.

$$
\alpha^{2}+\beta^{2}+\gamma^{2}=1
$$

is valid. The Jacobi integral is known for such systems

$$
\frac{u^{2}+v^{2}+w^{2}}{2}-\frac{\Omega^{2}\left(x^{2}+y^{2}\right)}{2}-U(x, y, z)=h .
$$

The possibility of such a velocity field (1) is checked in the usual way by the condition that the expression

$$
u d x+v d y+w d z
$$

is a complete differential [2]. Let's check this condition. Since, in the presence of rotation, the impulses are $u-\Omega y$, $v+\Omega x, w$ this condition gives,

$$
\left(u_{0} \pm \alpha \sqrt{S+2 h}+\Omega y\right) d x+\left(v_{0} \pm \beta \sqrt{S+2 h}-\Omega x\right) d y+\left(w_{0} \pm \gamma \sqrt{S+2 h}\right) d z
$$

It is easy to see that this is true separately for the half of sum and half of difference of two expressions (5), which differ by the signs "+" and "-". In the first case

$$
u_{0}-\Omega y=\frac{\partial \Phi}{\partial x}, v_{0}+\Omega x=\frac{\partial \Phi}{\partial y}, w_{0}=\frac{\partial \Phi}{\partial z}
$$

that is, it turns out that the vortex of the vector $\left(u_{0}, v_{0}, w_{0}\right)$ vanishes and therefore this vector is the gradient of some function $\Phi(x, y, z)$. In the second case we are equating to zero, for example, the $z$-component gives

$$
(S+2 h) \frac{\partial \alpha}{\partial y}+\frac{\alpha}{2} \frac{\partial S}{\partial y}-(S+2 h) \frac{\partial \beta}{\partial x}-\frac{\beta}{2} \frac{\partial S}{\partial x}=0
$$

that is $\alpha, \beta, \gamma$ is the gradient of some function $L(x, y, z)$

$$
(\alpha, \beta, \gamma)=\nabla L(x, y, z)
$$

Also by analogy [1],

$$
\frac{\partial L}{\partial x} \frac{\partial S}{\partial y}-\frac{\partial L}{\partial y} \frac{\partial S}{\partial x}=0
$$




$$
S=U_{0}(L)
$$

So,

$$
\begin{gathered}
u=\frac{\partial \Phi}{\partial x} \pm \frac{\partial L}{\partial x} \sqrt{U_{0}(L)+2 h}+\Omega y \\
v=\frac{\partial \Phi}{\partial y} \pm \frac{\partial L}{\partial y} \sqrt{U_{0}(L)+2 h}-\Omega x \\
w=\frac{\partial \Phi}{\partial z} \pm \frac{\partial L}{\partial z} \sqrt{U_{0}(L)+2 h}
\end{gathered}
$$

The joint solution of (3) and (10) gives

$$
\begin{gathered}
2(U+h)=(\nabla \Phi)^{2}+2 \Omega\left(y \frac{\partial \Phi}{\partial x}-x \frac{\partial \Phi}{\partial y}\right) \times 2(\langle\nabla \Phi, \nabla L\rangle+ \\
\left.\Omega\left(y \frac{\partial L}{\partial x}-x \frac{\partial L}{\partial y}\right)\right)\left(U_{0}(L)+2 h\right)(\nabla L)^{2} .
\end{gathered}
$$

The two-signity " \pm " should obviously disappear, from which the condition follows

$$
\left(\langle\nabla \Phi, \nabla L\rangle+\Omega\left(y \frac{\partial L}{\partial x}-x \frac{\partial L}{\partial y}\right)=0\right.
$$

In addition, $L$ by virtue of (2), must satisfy the equation

$$
\left(\frac{\partial L}{\partial x}\right)^{2}+\left(\frac{\partial L}{\partial y}\right)^{2}+\left(\frac{\partial L}{\partial z}\right)^{2}=1
$$

which formally coincides with the eikonal equation well known in geometric optics [3]. Taking into account (12) and (13), we obtain from (11) the general form of the potential

$$
U=\frac{1}{2}\left[(\nabla \Phi)^{2}+U_{0}(L)+\Omega\left(y \frac{\partial L}{\partial x}-x \frac{\partial L}{\partial y}\right)\right] .
$$

Consider the equation (12). In the degenerate case of the rotational symmetry

$$
L=L(r, z)
$$

where

$$
r=\sqrt{x^{2}+y^{2}}
$$

Therefore,

$$
\langle\nabla \Phi, \nabla L\rangle=0
$$

so, the problem of determining the unknown functions $L$ and $\Phi$ is simplified. Then, equation (13) will have the form

$$
\left(\frac{\partial L}{\partial r}\right)^{2}+\left(\frac{\partial L}{\partial z}\right)^{2}=1
$$

It is easy to see this condition is fulfilled when

$$
\frac{\partial L}{\partial r}=\cos \Psi, \frac{\partial L}{\partial r}=\sin \Psi
$$

where $\Psi$ is some function of coordinates. Then from (17) follows the equation

$$
\sin \Psi \frac{\partial \Psi}{\partial z}+\cos \Psi \frac{\partial \Psi}{\partial r}=0
$$

which admits a solution

$$
r \sin \Psi-z \cos \Psi=f^{\prime}(\Psi)
$$

Now we can easily find the unknown function $L$

$$
L=\int(\cos \Psi d r+\sin \Psi d z)=r \cos \psi+z \sin \Psi+f(\Psi)
$$


Since rotational symmetry is assumed for $L$, then we have

$$
\frac{x}{r} \frac{\partial \Phi}{\partial x} \frac{\partial L}{\partial r}+\frac{y}{r} \frac{\partial \Phi}{\partial y} \frac{\partial L}{\partial r}+\frac{\partial \Phi}{\partial z} \frac{\partial L}{\partial z}=0 .
$$

It is also convenient for $\Phi$ to take the expression in cylindrical coordinates

$$
\Phi=\Phi(r, \theta, z), \theta=\arctan \frac{y}{x}
$$

In this case, from (16) we get

$$
\frac{\partial \Phi}{\partial r} \frac{\partial L}{\partial r}+\frac{\partial \Phi}{\partial z} \frac{\partial L}{\partial z}=0
$$

Taking into account (18), the equation is obtained

$$
\cos \Psi \frac{\partial \Phi}{\partial r}+\sin \Psi \frac{\partial \Phi}{\partial z}=0
$$

which admits a solution

$$
\Phi=F(\Psi, \theta)+C, C=\text { const }
$$

where $F(\Psi, \theta)$ is some arbitrary function.

Thus, this particular problem is completely solved. $\Psi$ is determined from $(20), L$ and $\Phi$, respectively, from (21) and (26). Then from (14) we can find a class of potentials admitting a velocity field (1), although not the most general. In principle, rotational symmetry is not required for $L$, but for such asymmetric $L(x, y, z)$ calculations are much more complicated. However, the considered case should be of some interest, since it preserves some dynamic features of rotationally symmetric systems. Our case gives, accordingly, a kind of generalization of such symmetric models. By definition, the local integral does not allow us to find the trajectory, but it is possible by solving a system of differential equations

$$
\frac{d x}{u}=\frac{d y}{v}=\frac{d z}{w}=d t,
$$

where $u, v, w$ as coordinate functions are taken from (1), which facilitates solutions of the original system of the 6 th order.

\section{References}

1. V. A. Antonov and F. T. Shamshiev, Sov. Astron., 36, 501, 1992.

2. F. R. Gantmacher, Lectures on analytical mechanics, M.: Fizmatlit. (2002).

3. L. D. Landau and E. M. Lifshitz, Theoritical physics. V.2 Theory of Fields, M.: Fizmatlit. (2018). 


\title{
Long-term multi-frequency studies of flaring activity from microquasars
}

\author{
S. Trushkin, A. Shevchenko, N. Bursov, P. Tsybulev, N. Nizhelsky \\ Special Astrophysical Observatory RAS, Nizhnij Arkhyz, 369167, Russian Federation
}

The long-term monitoring at RATAN-600 of studies of bright X-ray binary stars in various ranges of the electromagnetic spectrum, a search and detailed study of correlations between variable X-ray, radio and gamma radiation was carried out. It is a key point for understanding the formation of jet emissions from accreting matter onto a black hole (or neutron star). From April 2019 For a year, we began to use the multi-azimuth measurement mode on the Southern Sector antenna system with a flat reflector, when 31 measurements of flux densities at frequencies 4.7, 8.6, 15 and $30 \mathrm{GHz}$ of several giant Cygnus X-3 flares, SS433 [1] and GRS1915+105 bright flashes were carried out for 5-6 hours around the culmination of the source. In January 2020, Cyg X-3 switched to a hyper-soft X-ray state, the exit from which in early February led to the brightest the radio flash for the all history of its observations. The Cygnus X-3 flow density increased from 5 mJy to 20 Jy at a frequency of $4.7 \mathrm{GHz}$ and up to $22 \mathrm{Jy}$ at $2.3 \mathrm{GHz}$ in $2-3$ days. In multi-azimuthal observations in the beginning phase, we registered a linear law of increase in the flux at times from 1 to 5 hours. Comparing the data of the space Gamma-ray telescopes (Swift, AGILE and Fermi) and the MAXI and NICER X-ray monitors on board the ISS, we found that flaring events from the radio to the Gamma-rays are interrelated, which is a reflection of the causal relationship of physical processes in the accretion disk and in jet emissions. The spectral and time dependence of the evolution of flares allow us to model the synchrotron radiation of microquasars based on changes in the volume of jet emissions, the strength of their magnetic field and the mode of generation and absorption of radio radiation from relativistic electrons. Radio flares of the microquasar GRS1915+105, as a clear manifestation of a new jet activity, always have the character of a reaction to changes in the conditions for generating X-rays in the accretion disk (MAXI) and in the corona (Swift). We have studied in details periodic radio flares from the X-ray binary with bright Gamma-ray radiation LSI+61d303 for more than 60 orbital periods. Undoubtedly, the nature of these flares changes dramatically depending on the known super-orbital 4.6-year period.

Keywords: stars: evolution — binary systems: black holes : general - microquasars

DOI: $10.51194 /$ VAK2021.2022.1.1.196

\section{SS433}

In 2020 the microquasar SS433 was investigated at several frequencies. In general, these data are a continuation of the long-term almost daily monitoring of the microquasar since 2011 at the RATAN-600. The light curves of the microquasar were measured for more than 2500 days, and the spectral indices (SpI) of synchrotron radiation in the range from 1 to $22 \mathrm{GHz}$ were determined every day. As shown in joint (optics, X-ray, VLBI) programs, all the flare peaks in the light curves find their explanation in the non-stationary process of ejection of relativistic jet ejections of matter from the inner regions of the accretion disk around the black hole, which is probably compact object in the binary. In the recent multi-frequency monitoring of the Galactic microquasars with the RATAN-600 radio telescope we have detected some bright flares at $2.25-22.3 \mathrm{GHz}$.

During the active period, the flux of flares increased by 2-3 times, while in a quiet state, the source flux variations did not exceeded 10 percent. These flares were characterized by an optically thin synchrotron power-law spectrum with change of the SpI from -0.2 to -0.7 . Each outburst in SS433 is well described by a single short-term $(\sim$ a day) generation of relativistic electrons in a blobs of matter moving outward ( $0.26 \mathrm{c})$ from the object, followed by the decline of radio emission due to the adiabatic expansion during 10-15 days.
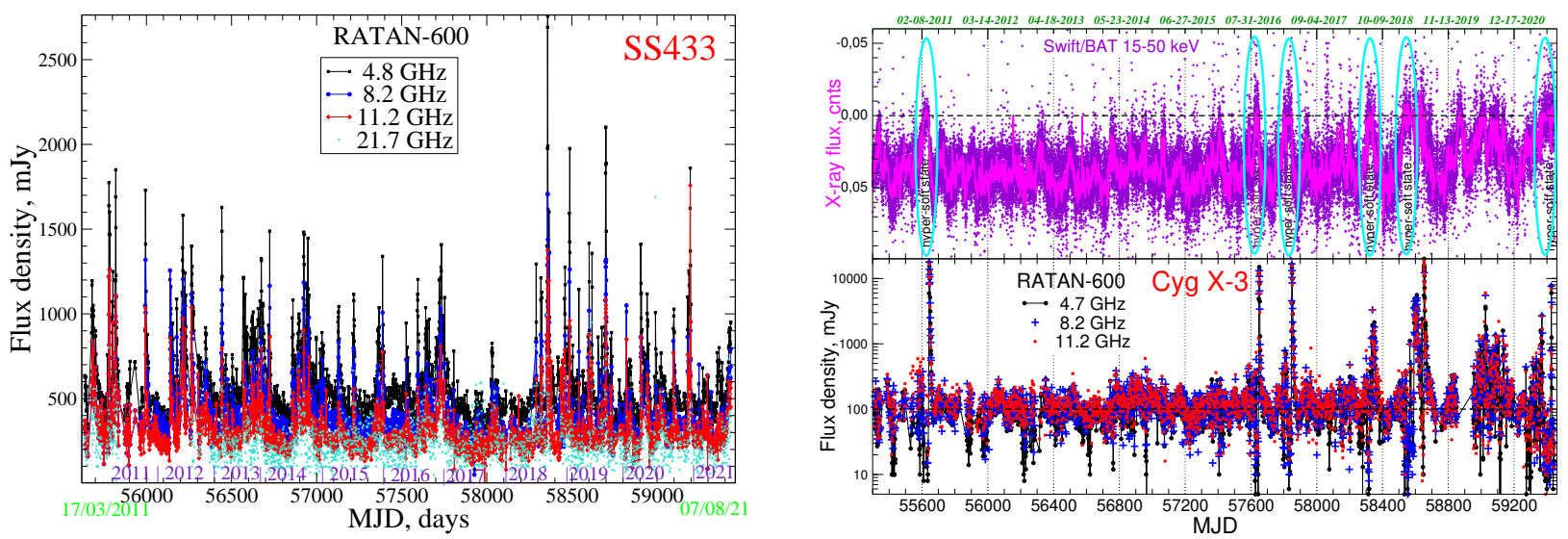

Figure 1: left: The light curves of SS433 during almost ten years. right:The light curves of Cyg X-3. Top panel: Swift/BAT data at 15-50 keV, bottom panel: RATAN-600 data at three frequencies. The 'hyper-soft' states are highlighted by ellipses. 

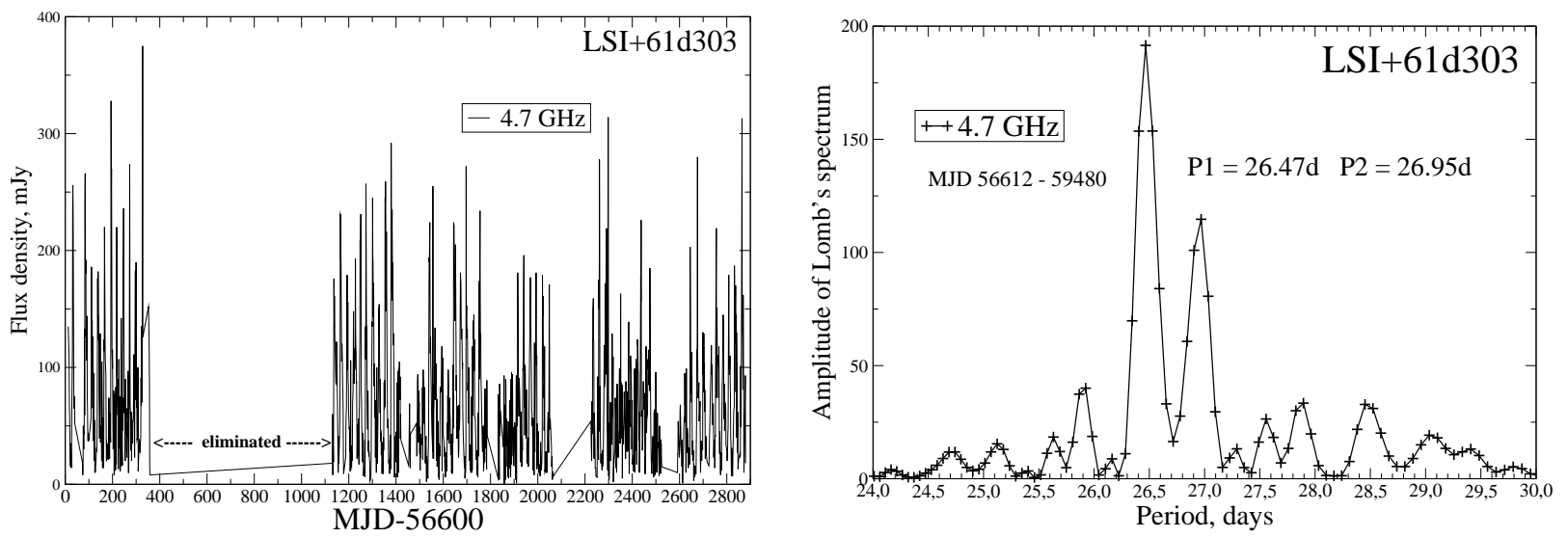

Figure 2: left: The light curve of LSI $+61 \mathrm{~d} 303$ at $4.7 \mathrm{GHz}$. Notice that some data was eliminated. right: The Lomb-Scargle periodogram of this light curve detected two detected periods: 26.47 and 26.95 days

\section{Flares of Cygnus X-3}

Multi-frequency daily observations of the Cygnus X-3 are continue with RATAN-600 during more than 10 years [2]. In 2000 we have measured light curves of their radio emission at 2-30 $\mathrm{GHz}$ and we have detected a giant flare in February just before so-called 'hyper-soft' X-ray state. The flux density at $4.7 \mathrm{GHz}$ increased to 10 Jy on MJD 58887.35 with the optically thin spectrum at frequencies higher $4.7 \mathrm{GHz}$, having the spectral index -0.28 . Before on MJD 58885.5 hard X-ray flux (Swift/BAT) increased. That means that the binary entered in a soft state and later in a hard state. The flaring radio activity of Cygnus X-3 went on, probably it is related with the gamma-ray flaring activity from Cygnus X-3. In the peak, the flux density were grow up to 19.1 Jy at 4.7 GHz (MJD 58990.4). Cygnus X-3 was in the beginning of jets ejection that is usually characterized by two regimes: an optically thick one at frequencies lower $4.7 \mathrm{GHz}$ and by an optically thin one at frequencies higher 8.2 GHz. The Swift/BAT data and the Fermi gamma-ray data well associated with two last radio flares detected with RATAN-600. In August 2021 we have detected the strong flare about 8 Jy again during a long-term 'hyper-soft' X-ray state.

\section{3. $\mathrm{LSI}+61 \mathrm{~d} 303$}

From 2013 we followed more then 100 periodical flares from LSI+61d303, well-known X-ray binary, X-ray and Gamma-Ray source, and probably a microquasar, e.i. sources with relativistic jets, flowing from accretion disk around a black hole or a neutron star. Its important problem of the binary is a existence of super-orbital period around 1650 days [3]. Is it a physical period of the some enigmatic process or it is a reflection of the beat of the close periods - orbital one (26.496d) and precessional one of the jets (26.95d). For temporal analysis on the radio light curves we studied the Lomb-Scargle periodograms for total 2875 days (almost 8 years) containing more then 2000 flux points at 4.7 and $11.2 \mathrm{GHz}$. We have detected only orbital period 26.5 days for total sample. But if we eliminate the data with obviously unclear periodicity from MJD 56860 to MJD 57730 (870 days of measurements) we detected two periods: 26.47 and 26.95 days in well accordance with [3] results. Our total light curves during almost two super-orbital 4.6 year-period have clear the long modulation of the maximum fluxes of the orbital flares and the shiftings of orbital phase of these maxima as was found [4].

\section{Discussion}

Our long-term multi-frequency daily monitoring programs of the small sample of the bright microquasars obtain good results for flaring variability of the radio emission, clearly connected the jets activity. We have studied the various correlations or associations radio emission, optical magnitudes (SS433), X-ray (GRS1915+105, Cyg X-3), gamma-ray (LSI+61d303, Cyg X-3) fluxes. The flaring radio flux is a good tracer of the common activity in the $\mathrm{X}$-ray binary systems and could be a base of ToO programs.

\section{References}

1. S. A. Trushkin, N. N. Bursov, and N. A. Nizhelskij, Bulletin of the Special Astrophysics Observatory, 56, 57, 2003.

2. S. Trushkin, M. McCollough, N. Nizhelskij, and P. Tsybulev, Galaxies, 5, 86, 2017.

3. M. Massi and G. Torricelli-Ciamponi, A\&A, 585, A123, 2016.

4. P. C. Gregory, ApJ, 575, 427, 2002. 


\title{
Fastly varying objects: fast radio bursts, magnetars, and pulsars with RATAN-600
}

\author{
S. Trushkin, P. Tsybulev, N. Nizhelsky, N. Bursov, A. Shevchenko \\ satr@sao.ru, \\ WWW home page: http://www.sao.ru/hq/lran/staff/satr/index.html.en \\ Special Astrophysical Observatory RAS, Nizhnij Arkhyz, 36916\%, Russian Federation
}

\begin{abstract}
With RATAN-600 radio telescope we can conduct studies of pulsed radiation of events associated with fast radio bursts (FRB), giant pulses (GP) of pulsars and flare activity of magnetars at high frequencies. Now the telescope has been equipped with full-power radiometers with the highest possible time resolution and band division at a frequency of $4.7 \mathrm{GHz}$ into four narrow channels $(150 \mathrm{MHz})$, which needed for detection of pulse shifts due to cosmic dispersion. As the main aim, the search for FRB in the blind 24h-surveys of sky regions has been conducted for more than four years on four radiometers (beams). In the sky regions at declinations of 33, 22, 5 and 11 degrees, no FRB with a flux greater than 5 Jy were detected. However, in the Crab Nebula, we registered seven GP from its 33-millisecond pulsar. The maximum pulse was above 100 Jy. Measurements of radio pulses from the X-ray magnetar XTE J1810-197 were carried out from 19 December 2018, when, after ten years on December 8, 2018 a bright X-ray flare occurred and weak radio pulses were detected with a pulsar rotation period of 5.54 seconds. Based on the arrival times of pulses in narrow channels, we confirmed the measure of dispersion $\mathrm{DM}=178 \mathrm{pc} / \mathrm{cm}^{3}$. The pulses themselves, with a total duration of about $200 \mathrm{~ms}$, included up to 15 sub-pulses with a width of 10 to $50 \mathrm{~ms}$ with a flux density of up to 5 Jy. During three months XTEJ1809-197 remained the brightest radio pulsar in the Milky Way.
\end{abstract}

Keywords: stars: evolution - neutron star: pulsars: general - magnetar

DOI: $10.51194 /$ VAK2021.2022.1.1.197

\section{Introduction}

Last ten years of observational radio astronomy lives in new revolution, observing a lot of fast varying objects from radio pulsars with unusual properties, extragalactic fast radio bursts (FRB), and first Galactic FRB (SGR $1935+2154)$.

During the 4-year sky survey with the Western sector of RATAN-600 on a four-beam four-channel array of radiometers at 4.3-4.9 GHz we are looking for short bright pulses from the fast radio bursts (FRB). Such pulses should be affected by cosmic dispersion in ionized interstellar and intergalactic medium. We have only detected the some giant pulses (GP) with fluxes of up to $100 \mathrm{Jy}$ from the Crab nebula pulsar. In 2017 we have detected the quite bright pulses from nearby pulsar PSR $0329+54$ on $2.3,4.7$ and $8.2 \mathrm{GHz}$ in the broad bands. Now GP from young pulsars, and pulsations from neutron star with extremely strong magnetic field, magnetars, considered to be the most probable sources of FRB.

The source XTE J1809-197 was detected by a bright X-ray flare in 2003 [1]. It showed periodic change brightness with the period of 5.5414 seconds [2]. Almost immediately it is attributed to a small sample of anomalous X-ray pulsars (AXP) or transient magnetars. Only 30 AXPs are known in the Milky way. The distance to the magnetar is $3.5 \mathrm{kpc}$ [3]. Extremely high luminosity was estimated, and the X-ray spectrum well fitted by two (warm and hot) black-body (bb) radiation or by bb $(0.6-07 \mathrm{keV})+$ a power law with $\Gamma=3$. Although AXP were not thought to emit pulsing radio emission, in 2005, XTE J1809-197 had been detected bright (tenths of Jy) variable radio emission with relatively small pulse width $(0.2 \mathrm{~s})$, inter-pulse and high variability of the mean profile [3].

Existence of the bright inter-pulse indicates that the slope of the magnetic axis to the axis of rotation can be close to 90 degrees. No connection with the known supernova remnant (SNR) has been found, although the estimated age of the magnetar is 15000 years. It is worth noting that the magnetar locates in a complex region of the Galactic plane with nearby bright HII regions, and it is quite difficult to distinguish a faint SNR. We searched for the non-thermal radiation from possible SNR in the maps of different surveys of the sky, but did not find any bright extended source around XTE J1810-197.

After the 50-fold decrease of the X-ray flux in 2007 XTE J1809-197 became undetectable at radio waves. Then from 2008 to October 2018 the 76-meter telescope Lovell have not detected radio emission. On 8 December pulses with a known period were again recorded at a signal-to-noise level of 400 at the frequency $1.4 \mathrm{GHz}$. On December 11 with a 100-meter telescope in Effelsberg, the flux was found at levels $22.5+/-0.2 \mathrm{mJy}$ at $4.74 \mathrm{GHz}$ and $14.9+/-0.2 \mathrm{mJy}$ at $8.35 \mathrm{GHz}$, that gave a relatively flat the spectral index is -0.7 .

Almost 100\% linear polarization were discovered with rapid change of positional angle and 10 per cent circular polarization of the pulses, but the inter-pulses are missing. Data from the MAXI monitor on the ISS confirmed a significant increase in X-ray flux from the magnetar just on December 82018 and later radiation was detected constantly. On December 12 the pulses at $835 \mathrm{MHz}$ were detected at the level of 9 mJy at the telescope UTMOST. Later the pulses of the magnetar were detected at 8.4 and $32 \mathrm{GHz}$ with the 34 -meter telescope in Canberra. 


\section{Discussion}

The properties of these pulses — peak flux density and substructure — are exceptionally strong and randomly changed from a pulse to a pulse. frequency at $4.7 \mathrm{GHz}$ with a total duration of about 200 ms consisted of $1-10$ different amplitude sub-pulses with a duration of 2-10 ms. And these parameters do not depend from the frequency range. According to the results of measurements with a fixed carriage with receiver horns average flux pulses were detected and thus we can plot a radio spectrum. It is well approximated by a power-law dependence with spectral index $-1.5 \pm 0.2$, that is, in general, very similar to the average spectrum radio pulsars. One of the brightest pulsation of XTEJ1809-149 25 March 2019 consisted of 10 narrow (<10 ms) sub-pulses, while often a main pulse had only two components. In general, records the light curves for 132 seconds at different frequencies are similar, although the numbers of brighter pulses at 2.3 and $4.7 \mathrm{GHz}$ are significantly higher than at $8.2 \mathrm{GHz}$. But for full confidence in this conclusion we need to get more meaningful statistics.

For recordings in early January at $4.7 \mathrm{GHz}$, we performed a Fourier analysis, and then added all the spectra to determine the position of the most accurate a bright harmonic of $0.180 \mathrm{~Hz}$, or a 5.54 -second pulsar period. The obtained position of the harmonic maximum corresponded to the period $5.56 \pm 0.02$ seconds that within errors corresponds to the value $5.541455 \mathrm{~s}$, obtained from data for 20-30 minutes.

In general, during observations on the RATAN-600 telescope [4] we have detected about 2000 pulses from the magnetar XTEJ1809-197 at $4.7 \mathrm{GHz}$ and tens at 2.3 and $8.2 \mathrm{GHz}$. Single pulses reached the values of several Jy, which is characteristic only for giant pulses from some radio pulsars, for example, as from the 33-ms pulsar in the Crab nebula. Average 25 pulses day by day had a flux from 0.5 to $4 \mathrm{Jy}$ at frequency $4.7 \mathrm{GHz}$. In contrast to the known data in 2005-2007 we could not to detect inter-pulse.

In 2019 the studies of this transient event from XTE J1809-197 were published by [5] about Parkes radio telescope wideband measurements, by [6] about MAXI and NuStar measurements, [7] about the Effelsberg and Lovell telescopes measurements and by [8] about the GMRT low frequencies measurements.

\section{References}

1. A. I. Ibrahim, C. B. Markwardt, J. H. Swank, S. Ransom, et al., ApJL, 609, L21, 2004.

2. F. Bernardini, G. L. Israel, S. Dall'Osso, L. Stella, et al., A\&\&A, 498, 195, 2009.

3. D. J. Helfand, S. Chatterjee, W. F. Brisken, F. Camilo, J. Reynolds, M. H. van Kerkwijk, J. P. Halpern, and S. M. Ransom, ApJ, 662, 1198, 2007.

4. S. A. Trushkin, N. N. Bursov, P. G. Tsybulev, N. A. Nizhelskij, and A. Erkenov, The Astronomer's Telegram, $\mathbf{1 2 3 7 2}, 1,2019$.

5. S. Dai, M. E. Lower, M. Bailes, F. Camilo, et al., ApJL, 874, L14, 2019.

6. E. V. Gotthelf, J. P. Halpern, J. A. J. Alford, T. Mihara, et al., ApJL, 874, L25, 2019.

7. L. Levin, A. G. Lyne, G. Desvignes, R. P. Eatough, et al., MNRAS, 488, 5251, 2019.

8. Y. Maan, B. C. Joshi, M. P. Surnis, M. Bagchi, and P. K. Manoharan, ApJL, 882, L9, 2019. 
Astronomical Education 


\title{
Estimation of asteroid-comet hazard according to open scientific data as a student practical work
}

\author{
I.V. Kuznetsova, M.E. Prokhorov \\ irinakuznetsova-64@mail.ru \\ Sternberg Astronomical Institute of Moscow State University, Univeritetsky prospect 13, 119991 Moscow, Russia
}

One of the forms of teaching physics and astronomy at a university can be practical work using modern open scientific data. Based on the proposed methodology, the student can make an interesting, modern, relevant research work. As an example of such practical work, it is proposed to estimate the asteroid-comet hazard - the frequency of asteroids and large meteorites falling to the Earth by counting craters on the surface of the Moon and Mercury.

Keywords: asteroid-comet hazard, impact craters, open data

DOI: 10.51194/VAK2021.2022.1.1.198

One of the forms of teaching physics at universities is laboratory works. They have a long-established format everywhere. We offer laboratory practical work in a new format. Works of this type should permit the students for the limited time allotted for their implementation:

- to get acquainted with the field of science or sciences that the laboratory work belongs to;

- to master the necessary part of the mathematical apparatus;

- independently process the initial data for laboratory work obtained from open sources;

- to bring the processing to the final conclusions and compare them with the results of similar professional scientific research presented in the literature [1].

The main difference of this work is the use of modern open scientific data, which makes it possible to make the work an interesting, modern, and multidisciplinary project. It is possible to directly compare the results of the project with published fundamental research and discuss the differences obtained.

Access to such data is facilitated by the policy of open access to data of international basic scientific research [2].

As for the works of the new format, instead of an extremely complex and expensive experimental working setup, the student is offered real "raw" or partially processed experimental data contained in scientific databases, and in practical work it is possible to carry out their full-fledged processing, get their own results, make theoretical estimates.

As an example of such work, we proposed to determine the frequency of asteroids and large meteorites (of the Tunguska class) falling to the Earth by counting craters on the surface of the Moon, and similar research for Mercury.

The practical work is based on the processing of photographic images of the Moon's surface [3, 4] and Mercury's surface [5] for determination of the frequency of asteroids falling on the Moon, Earth and Mercury, and then to assessment of asteroid-comet hazard.

The complexity of the methods in the proposed work is within the framework of the general physics course for natural science and engineering-technical specialties of universities.

The goals of the work are:

- training in the basics of research activities

- introduction to the concept of asteroid-comet hazard;

- investigation of possible mechanisms of crater formation and calculation of their sizes on Earth, on the Moon and on Mercury, depending on the parameters;

- estimation of the frequency of asteroids falling to Earth from photos of the Moon and Mercury.

The methodological description consists of four parts, and contains both the educational and theoretical material necessary for a sufficiently detailed acquaintance with the problem of research in the work, and the practical part for obtaining their own scientific results. The appendices contain all the necessary reference materials and the distributions of the authors of the practical work, obtained on the basis of the materials used.

The overview part offers a brief description of the current state of the problem area. In our case, this is information about the origin, properties and classification of small bodies of the Solar System, information about the geological structures of impact craters found on the Earth's surface. It also contains a description of the problem of asteroid-comet hazard. The problem of asteroid-comet hazard is currently an urgent area of research 
at the intersection of astronomy, physics of the solar system, geophysics, geology, space research and ensuring the safety of human life, including.

The speed of asteroid fall varies slightly - from 11 to several tens of $\mathrm{km} / \mathrm{s}$, but their masses can differ by many orders of magnitude.

Asteroid falls of more than 100-140 $\mathrm{m}$ in size are considered hazardous - the explosions caused by them can destroy a small city.

With the increase in the size of the asteroid, its hazard increases dramatically: thus, the fall of an asteroid with a diameter of $1 \mathrm{~km}$ will cause a regional catastrophe and lead to global climate consequences. The fall of an asteroid measuring ten kilometers in diameter will cause a global catastrophe and a "nuclear winter" lasting a decade. Smaller asteroids can also be hazardous, examples are the Tunguska meteorite, whose dimensions are estimated at $30 \mathrm{~m}$ in diameter, and the Chelyabinsky (Chebarkul) meteorite (18 $\mathrm{m}$ in diameter, respectively).

Only a few hundred impact craters are known on Earth today. The age of the oldest of them (at the same time they are also the largest) is hundreds of millions of years, which is much less than the age of the Earth. This is due to the processes of erosion and volcanism, which erase the traces of falling celestial bodies from the surface of the Earth.

The Earth and Moon are located so close to each other that they have a chance to collide with the same asteroids, i.e. they are exposed to the same of cosmic bodies. There is no atmosphere on the Moon and geological activity has practically ceased, which is why the craters that have appeared on the surface of the Moon remain forever. Counting craters on the surface of the Moon allows us to estimate the frequency of asteroids falling on the Earth.

The theoretical part describes the basic physical and mathematical relations that are important for performing calculations in this laboratory work.

A number of these relations can be proposed for the derivation of students, within the framework of simplified models.

The practical part is the actual laboratory work, containing

1. source data taken from open sources;

2. recommendations for their processing;

3. reference data required for all stages of processing the source data and obtaining own results of the work.

The methodological part is intended for teachers conducting laboratory work. It sets out the goals and recommended work plan, recommendations for the design of the results obtained and a list of possible control questions. The approximate time spent on carrying out laboratory work and its individual parts are presents. Reference materials are provided in the appendix.

In the practical part, students are offered:

- on the photo of a fragment of the surface of the Moon or Mercury given to him, to count the number of craters, determine their sizes and sort them by size

- to determine which of these craters are formed by asteroids exceeding the "hazardous size";

- to calculate the frequency of "hazardous asteroids" falling on the moon or Mercury as a whole;

- to get an estimate of the fall of "hazardous asteroids" to the Earth;

- to compare this estimate with the frequency of asteroid impacts on the Earth obtained by other methods (from reference data).

Also, at the beginning of the work, it is necessary to determine the scale of the images.

As a conclusion, we note the advantages of such works: The proposed laboratory work allows the student to independently conduct a small, but integral scientific research on an actual topic and compare own results with the results of modern scientific research.

In the work described above, the student gets acquainted and operates with the data of several sciences: physics, astronomy, geology.

This approach encourages the student to an earlier and deeper acquaintance with scientific activities [6].

The work is practical, while its implementation does not require practically any technical equipment, i.e. it can be carried out in any universities and also included in distance learning courses. It should also be noted the large potential variability of the considered work [7]. 


\section{References}

1. B. M. Shustov and L. V. Rykhlova, Asteroid-Comet Hazard: Yesterday, Today, Tomorrow (Moscow: Fizmatlit) (2010).

2. J. Willinsky, The Access Principle: The Case for Open Access to Research and Scholarship (Universities Space Research Association, Lunar and Planetary Institute) (2006).

3. Digital Lunar Orbiter Photographic Atlas of the Moon (Universities Space Research Association, Lunar and Planetary Institute) (2019), URL http://www. Ipi.usra.edu/resources/lunarorbiter/.

4. Interactive Map of Moon, Based on Lunar Reconnaissance Orbiter Data (Arizona State University, School of Earth and Space Exploration) (2001-2020), URL http://quickmap.Iroc.asu.edu/.

5. 1:5 Million-Scale Maps of Mercury (Arizona State University, Space Exploration Resources, Mercury Gazetteer) (2019), URL http://planetarynames.wr.usgs.gov/Page/mercuryQuadMap.

6. A. A. Astakhova, A. P. Degtyareva, O. V. Kolyasnikov, E. A. Mendeleeva, N. I. Morozova, M. G. Sergeeva, and A. S. Sigeev, Nauka i shkola, 135, 2017.

7. I. V. Kuznetsova and M. E. Prokhorov, Memoirs of the Faculty of Physics, Lomonosov Moscow State University, 1811001, 2018. 


\section{Contents}

Public Lecture

I.D. Novikov Breakthrough projects of the scientific program of the Millimetron Observatory

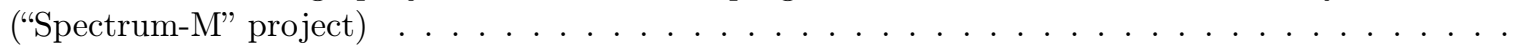

Plenary Talks

L.N. Berdnikov, N.N. Samus Period Variations of Cepheids and Stellar Evolution . . . . . . . . . . 9

B. Kondratyev, N. Emelyanov On some recent advances in celestial mechanics . . . . . . . . . . 15

S.A. Lamzin Young stars . . . . . . . . . . . . . . . . . . . . . . . . . . . . . 31

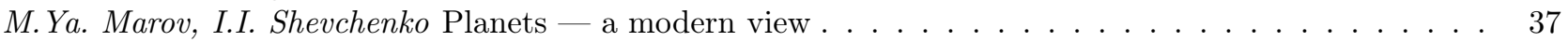

I. Zimovets, I. Sharykin A brief review on vertical electric currents in flaring active regions at the Sun . 42

\section{Ground-based and Space-based Tools and Methods of Astronomy}

M.Yu. Arkhipov, E.S. Golubev, A.O. Lyakhovets, A.V. Smirnov, S.D. Fedorchuk Adaptation system of

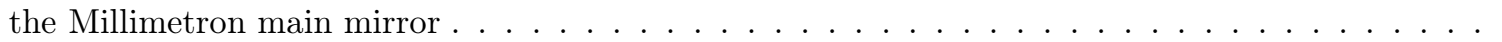

V.I. Ardilanov, V.A. Murzin, I.V. Afanasyeva, N.G. Ivaschenko, M.A. Pritychenko, S.N. Dodonov Highspeed camera based on a large-format low-noise CMOS imager . . . . . . . . . . . . .

G.S. Fedoseev, V.V. Krushinsky, M.G. Medvedev, K.A. Stepanova, A.I. Vasyunin Design of Cryogenic Ultra-High Vacuum Setup for Simulation of Chemical Processes in the Star and Planet Formation

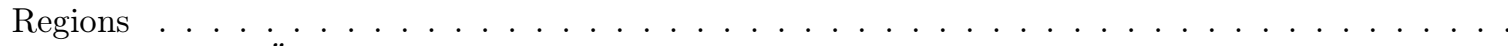

I. M. Khamitov, T. Özı̧ıı, S. Alis, I.F. Bikmaev, R.A. Burenin, M. Dindar, S. Dindar, N. Ertan, S. Fişek, M. Glushkov, T. Güver, Y. Kılıç, O. Okuyan, S. Süvari, A. Tat, F. K. Yelkenci Multiobject spectroscopy in the observational complex of the RTT150 . . . . . . . . . . . .

Yu.A. Kovalev, V. Vasil'kov, A. Ermakov, E. Vinyaikin, M. Lisakov, M. Popov, V. Soglasnov, M. Larionov, N. Nikolaev, E. Mironova, M. Burgin, Yu.Yu. Kovalev, P. Voitsik, A. Kutjkin, A. Alakoz, N. Shakhvorostova, K. Belousov, A. Kovalenko, N. Nizhelsky, G. Zhekanis,P. Tsubulev RadioAstron project: calibration of the space radio telescope in flight in 2011-2019 . . . . . . . . . . . .

A.A. Kudryashova, N.N. Bursov Method of processing data obtained during RATAN-600 West sector surveys with static antenna . . . . . . . . . . . . . . . . .

A.O. Lyakhovets, A.S. Andrianov, M.Yu. Arkhipov, E.S. Golubev AstroMagick: software for controlling and adapting the surface of the main mirror of the Millimetron space observatory . . . . . . . .

E. Mikhailov Corrections of the operational orbit of the "Spectr-RG" spacecraft in order to ensure the specified conditions of radio visibility from Russian ground stations . . . . . . . . . . . . .

M.E. Prokhorov, A.I. Zakharov, O.Yu. Stekolshchikov, M.S. Tuchin Project Lyra not on ISS _ . . . . 69

A. Shugarov, B. Shustov, V. Shmagin, A. Buslaeva System of Observation of Day-time Asteroids (SODA) 71

A. Shugarov, E. Vishnyakov, A. Shatohin, S. Kuzin, A. Kirichenko, A. Nikolenko Main qualification campaign results of WSO-UV mission UV CCD detectors . . . . . . . . . . . . . .

\section{Astrometry and Celestial Mechanics}

T. Abdulmyanov Comparison of the dynamics of Jupiter's coorbital asteroids and the dynamics of bodies

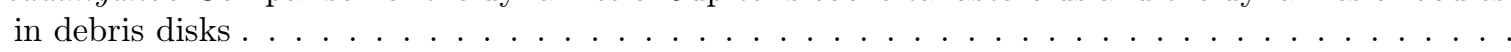

T. Abdulmyanov, M. Sokolova, V. Usanin On the possibility of expanding the Tisserand constant for Jupiter-family comets in a binomial series . . . . . . . . . . . . . . . . . . .

A. Burov, V. Nikonov Libration points of uniformly rotating celestial bodies with cavities . . . . . . .

A. Burov, E. Nikonova Generating function of Euler-Poinsot tensor's components for small celestial bodies . . . . . . . . . . . . . . . . . . . . . .

I. Chuvashov, P. Levkina Non-gravitational effects in the orbital motion of space debris objects . . . . .

M. Kan, D. Pavlov Dynamical estimation of masses of the Main asteroid belt and some individual asteroids within the EPM ephemeris using infrared data . . . . . . . . . . . . .

A.A. Kluykov, L.V. Rykhlova Multi-wave fundamental astrometry . . . . . . . . . . . . . . . .

G. Kokhirova, P. Levkina, N. Bakhtigaraev, U. Khamroev Facilities of astrophysical telescopes in space debris research . . . . . . . . . . . . . . . . . . . . . . . .

G. Kurbasova, A. Volvach, L. Volvach Analysis of corrections to deformations of the earth surface at VLBI station "SIMEIZ"

S. Pasynok, I. Bezmenov, I. Ignatenko, V. Ivanov, E. Tsyba, V. Zharov Current state and future plans of the Earth's orientation parameters evaluation activities in MMC SSTF . . . . . . . . . . .

S. Pavlov, Yu. Medvedev Determination of sunskirters' orbits on the example of comet 323P/SOHO . .

V. Safronova, E. Kuznetsov Estimation of the age of two young asteroid pairs with close orbits . . . . . 
T.N. Sannikova Comparison of the semi-major axis drift taking into account the Yarkovsky effect in two orbital reference frames . . . . . . . . . . . . . . . . . . . . . . . . . .

A. Shchepalova Metrics in The Space of Keplerian Orbits and in the Family of Its Quotient Spaces . . . 114

A. Tsaregorodtsev, D. Tuchin Ballistic design of flights to the planets of the Solar System . . . . . . . . 117

\section{Stars and Interstellar Medium}

M. Abubekerov, N. Gostev Possible Variations in the Limb-Darkening Coefficients of Eclipsed Stars at Short Time Intervals . . . . . . . . . . . . . . . . . . . . . . . . .

E. Antokhina, I. Antokhin, G. Lenoir-Craig, N. St.-Louis, A. Moffat Light-curve modelling in a Roche plus stellar wind model: the massive binary WR22 . . . . . . . . . . . . . . . . . . . .

O. Antoniuk, E. Pavlenko, K. Antoniuk, N. Pit, G. Kokhirova, F. Rakhmatullaeva, A. Sosnovskij ASASSN-19fy: The unusual dwarf nova in the period gap . . . . . . . . . . . . . . . . . .

S. Artemenko, E. Babina, K. Grankin, P. Petrov Accretion and wind modulation in T Tauri star RY Tau: a planet at 0.2 AU? . . . . . . . . . . . . . . . . . . . .

E.V. Babina, S.A. Artemenko, K.N. Grankin, P.P. Petrov Dynamics of wind as indicator of magnetic activity of T Tauri star RY Tau . . . . . . . . . . . . . . . . . . . . . . . .

A.M. Cherapashchuk, A.V.Dodin, K.A. Postnov, A.A. Belinski, M.A. Burlak, N.P. Ikonnikova, T.R. Irsmambetova Parameters of the microquasar SS433 based on the observations in SAI MSU . . . .

A.M. Cherepashchuk, T.S. Khruzina, A.I. Bogomazov Parameters of Sco X-1 . . . . . . . . . . . .

O. Eretnova, A. Dudorov The mass function of pre-main sequence stars . . . . . . . . . . . . . . . . . .

E.N. Fadeev, A.S. Andrianov, M.S. Burgin, M.V. Popov, A.G. Rudnitskii, T.V. Smirnova, V.A. Soglasnov, V.A. Zuga Anisotropic scattering of pulsars radio emission . . . . . . . . . . . . . . . . .

V.V. Grigoryev, T.V. Demidova Two-dimensional MHD model of gas flow dynamics near a young star with a jet and a protoplanetary disk

N. Ikonnikova, M. Burlak, S. Shugarov, A. Belinskii, A. Fedoteva, A. Tatarnikov, A. Dodin, S. Potanin, K. Atapin, S. Zheltoukhov IRAS 02143+5852: W Vir Cepheid with a dust shell . . . . . . . . . . . .

S. Kalenskii, P. Golysheva, P. Bergnan, H. Olofsson, and K. Degtyarev Spectral survey of the massive

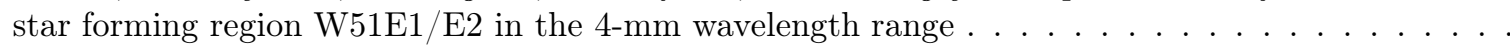

N. Kargaltseva, S. Khaibrakhmanov, A. Dudorov, S. Zamozdra, A. Zhilkin Influence of the magnetic field on the formation of protostellar disks . . . . . . . . . . . . . . . . . . . . . .

E. Karitskaya, N. Bochkarev Features of the Flow of Matter in the X-Ray Binary Her X-1 . . . . . . .

S. Karpov, G. Beskin Mini-MegaTORTORA photometric survey of the northern sky . . . . . . . . . . .

S. Khaibrakhmanov, A. Dudorov Dynamics of magnetic flux tubes in accretion disks of Herbig Ae/Be stars . . . . . . . . . . . . . . . . . . . . . . . . . .

A. Kholtygin, A. Valeev, A. Moiseeva, I. Yakunin, N. Ikonnikova, A. Dodin, A. Kostenkov, O. Tsiopa, V. Puzin, M. Kurdoyakova, I. Sokolov Fast and superfast variability of line profiles in spectra of early type stars . . . . . . . . . . . . . . . . . . . . . . . .

M. Kiskin, A. Vasyunin, V. Akimkin Modeling the formation of complex organic molecules in a protoplanetary disk . . . . . . . . . . . . . . . . . . . .

A. Kuzin, D. Lisitsin Photometry and kinematics of star forming complexes in external galaxies . . . .

D. Ladeyschikov, A. Sobolev Multiwavelength analysis of star-forming regions using the online database

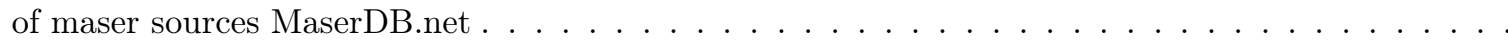

A.D. Lavrukhina, K.L. Malanchev Light curve features extraction from astronomical source . . . . . . .

B. Losovsky The specifics of pulsar radio emission . . . . . . . . . . . . . . . . . . . . . . .

T. Yu. Magakian, A. M. Tatarnikov, T. A. Movsessian, H. R. Andreasyan Near-infrared detection of $\mathrm{H}_{2}$ flows in the core of Mon R1 association

V.M. Malofeev, I.F. Malov, O.I. Malov, D.A. Teplykh On features of the pulsar B0950+08 radiation at the frequency of $111 \mathrm{MHz} \ldots \ldots \ldots \ldots \ldots \ldots \ldots \ldots$

I. Malov On giant pulses in radio pulsars . . . . . . . . . . . . . . . . . . . . . . . . .

I. Maslov, V. Shenavrin, B. Safonov The epsilon Aurigae Observation. Infrared Photometry and Specklepolarimetry

O.V. Maryeva, V.V. Gvaramadze, A.Y. Kniazev, L.N. Berdnikov Wray 15-906 low mass Luminous Blue Variable on a pre-supernova stage . . . . . . . . . . . . . . . . . . . . .

M.G. Medvedev, A.B. Ostrovskii, G.S. Fedoseev, A.I. Vasyunin Microscopic simulation of chemical pro-

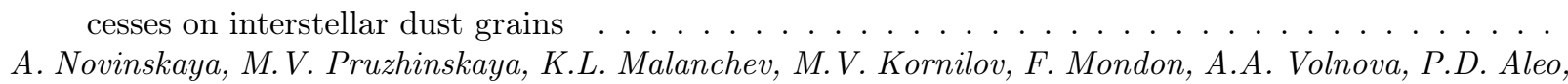
E.E.O. Ishida, V.S. Korolev, S. Sreejith Supernovae search in the Zwicky Transient Facility Data

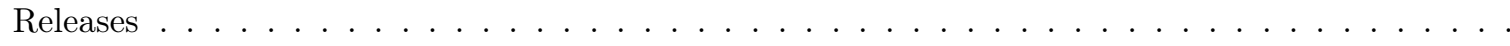


O.L. Ryabukhina, M.S. Kirsanova, D.S. Wiebe Astrochemical study of a dense molecular clump

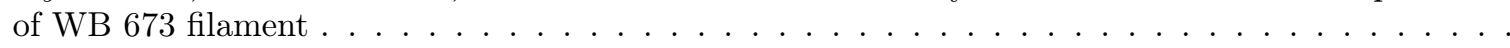

A. Sadovski, A. Struminsky Flares and coronal mass ejections for stars of different classes . . . . . . . . N.N. Samus, E.V. Kazarovets, O.V. Durlevich, N.N. Kireeva, E.N. Pastukhova, A.V. Khruslov New Name-lists of Variable Stars and Prospects of GCVS . . . . . . . . . . . . . . . . .

N.A. Satonkin, A.B. Ostrovsky, K. Kalnin, G.S. Fedoseev, A.I. Vasyunin Three-dimensional modeling of the formation of molecular hydrogen on the surface of an interstellar dust grain by the off-lattice Monte Carlo method . . . . . . . . . . . . . . . . . . . . . . . . . .

S. Savchenko, D. Polyakov, A. Mosenkov, A. Marchuk, V. Il'in, G. Gontcharov, A. Smirnov, P. Usachev Investigation of Galactic cirri based on SDSS Stripe 82 images . . . . . . . . . . . . . . .

T. Semenikhin, M. Kornilov, M. Pruzhinskaya Bolometric light curves and parameters of superluminous

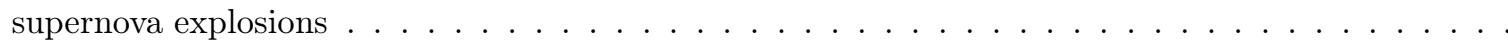

N. Shakht, D. Gorshanov, I. Izmailov Checking the possibility of determining the orbits of stars, orbiting the center of the Galaxy, and the estimate some relativistic effects . . . . . . . . . . . .

S. Shulman, V. Grinin Modeling of UX Ori Stars Eclipses . . . . . . . . . . . . . . . . . .

E. Sivkova, D. Wiebe, M. Murga Dust destruction at high Galactic altitude . . . . . . . . . . . . . . . .

A.A Sosnovskij, E.P. Pavlenko EZ Lyn: White Dwarf fast cooling after 2010 outburst . . . . . . . . . .

I.M. Sultanov, S.A. Khaibrakhmanov, A.E. Dudorov Angular momentum evolution of collapsing magnetic

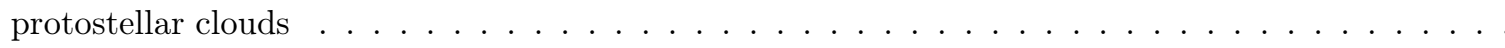

M. Timirkeeva, I. Malov, V. Malofeev, O. Malov Observations of the gamma-ray pulsar J1836+5925 at $111 \mathrm{MHz}$

A. Avtaeva, V. Shematovich Comparison of non-thermal atmospheric losses for hot exoplanets . . . . .

R.V. Baluev, E.N. Sokov, V.Sh. Shaidulin, and the EXPANSION team The impact of star spots and other photospheric structures on exoplanetary transit timings . . . . . . . . . . . . . . . . .

$V . V$. Busarev Active asteroids of the Main Belt as probable relics of the formation processes of giant

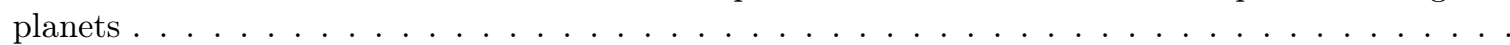

Yu.A. Chernetenko, V.B. Kuznetsov On the nongravitation effect in the motion

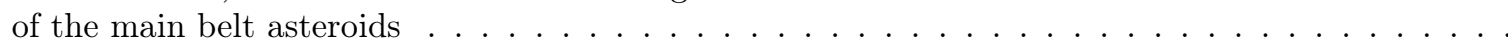

I. Dolgakov Ephemerides of the Neptune's satellites . . . . . . . . . . . . . . . . . . . .

$Y$ u. Eroshenko Astronomical constraints on primordial black holes in the Solar System . . . . . . . . . .

A.A. Fedotov, R.V. Baluev Search for deviations in exoplanetary infrared profiles, based on the secondary

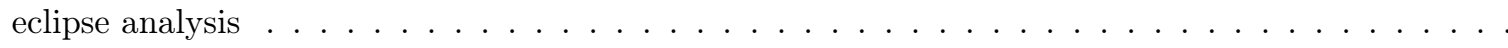

E.A. Feoktistova, Zh.F. Rodionova, A.Yu. Zharkova, A.A. Kokhanov, I.Yu. Zavyalov Analisys of mor-

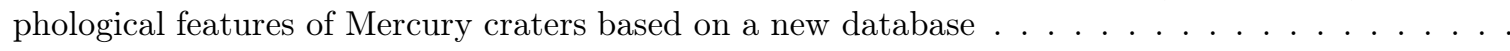

M.A. Ibrahimov Impact flare in exosystems: optical manifestation . . . . . . . . . . . . . . . . . . . . .

S.I. Ipatov Formation of the terrestrial planets and the Moon . . . . . . . . . . . . . . . . . . . . . .

S.I. Ipatov Migration of planetesimals to planets located in habitable zones in the Solar System and in the Proxima Centauri system . . . . . . . . . . . . . . . . . .

E. Kronrod, V. Kronrod, O. Kuskov Thermal evolution of the cores of the icy satellites of the giant planets . . . . . . . . . . . . . . . . . . . . . . . . .

E. Kuznetsov, O. Al-Shiblawi, V. Gusev, D. Ustinov Estimation of the age of pairs of trans-Neptunian objects in close orbits . . . . . . . . . . . . . . . . . . . . . . . . .

E. Kuznetsov, M. Vasileva, A. Rosaev, E. Plávalová Orbital evolution of young pairs of main belt asteroids in the vicinity of resonances and approaching planets . . . . . . . . . . . . .

I. Kuznetsov, I. Shashkova, A. Poroykov, A. Zakharov, S. Bednyakov, A. Bychkova, G. Dolnikov, A. Dubov, A. Kartasheva, A. Lyash, A. Shehovtsova Experimental evidence of the dust particles de-

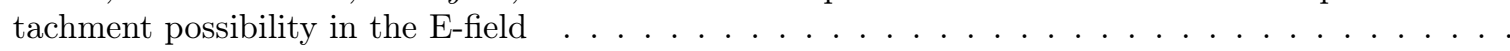

N. Lyapsina, G. Beskin, A. Biryukov, S. Bondar, A. Gutaev, E. Ivanov, S. Karpov, E. Katkova, A. Perkov, V. Sasyuk Investigation of meteors with sub-second temporal resolution in wide-field optical

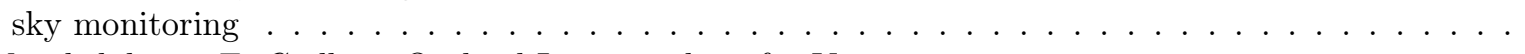

T. Menshchikova, T. Gudkova On load Love numbers for Venus . . . . . . . . . . . . . . . . .

M.V. Patsaeva, I.V. Khatuntsev, A.V. Turin, L.V. Zasova Variations of the wind speed at the top cloud layer in equatorial latitudes according to results of long-term observations of VMC (Venus Express) and UVI (Akatsuki) . . . . . . . . . . . . . . . . . . . . . . . .

S. Pugacheva, E. Feoktistova, V. Shevchenko Migration of water to the polar regions of the Moon . . .

A.A. Savelova, V.V. Busarev, M.P. Shcherbina Modeling of asteroids reflectance spectra and estimation of their taxonomic types and surface composition . . . . . . . . . . . . . . . . . 
M.P. Shcherbina, V.V. Busarev, and S.I. Barabanov New reflectance spectra of Main belt asteroids: clarification of taxonomic types and composition of matter . . . . . . . . . . . . . . .

M. Vasileva, E. Kuznetsov Yarkovsky effect: relationship between acceleration $A_{2}$ and semi-major axis drift velocity for Main Belt asteroids . . . . . . . . . . . . . . . . . . . . .

I. Vinogradov and Work team of the ISCRA-V experiment Investigation of sulphurous components of rarefied atmosphere of Venus: ISCRA-V experiment at the Venera-D lander . . . . . . . . . . . . 263

\section{Relativistic Astrophysics and Gravity Waves}

K. Atapin, A. Vinokurov, Yu. Solovyeva, S. Fabrika NGC 5474 X-1: the neutron star ULX in an old stellar cluster . . . . . . . . . . . . . . . . . . . . . . . .

D.P. Barsukov, A.A. Matevosyan, I.K. Morozov, A.N. Popov, M.V. Vorontsov The influence of small-

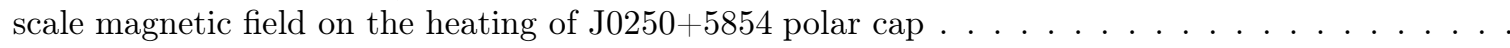

V. Beskin Radio pulsars - two old questions . . . . . . . . . . . . . . . . . . . . . .

M. Bugaev, I. Novikov, S. Repin, A. Shelkovnikova Building of zero geodesics in the wormhole field . .

M. Bugaev, I. Novikov, S. Repin, A. Shelkovnikova Shadows of black holes and wormholes: similarities

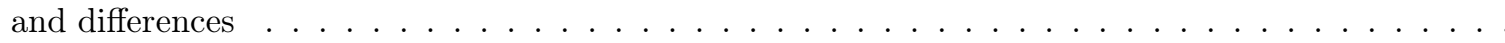

M.A. Buldakov, E.O. Vasiliev, A.S. Andrianov Rotation measure and vertical structure of accretion disks . . . . . . . . . . . . . . . . . . . . . . . . .

L. Chmyreva, G. Beskin Peculiar objects in the probable pulsar birthplaces - stellar-mass black hole

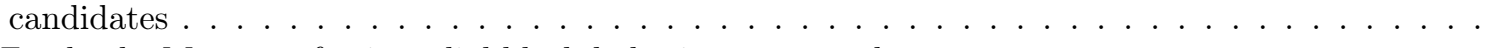

$Y$. Eroshenko Mergers of primordial black holes in compact clusters . . . . . . . . . . . . . . . . . .

A.S. Gorban, S.V. Molkov, S.S. Tsygankov, A.A. Lutovinov Study of the transient X-ray pulsar XTE $\mathrm{J} 1946+274$ observed with NuSTAR . . . . . . . . . . . . . . . . . .

A.D. Kaminker, D.G. Yakovlev, A.Y. Potekhin, P. Haensel Heat diffusion in outer crust and X-ray superbursts of accreting neutron stars . . . . . . . . . . . . . . . .

A. Khokhriakova, S. Popov Magneto-rotational evolution of neutron stars with hysteresis effect

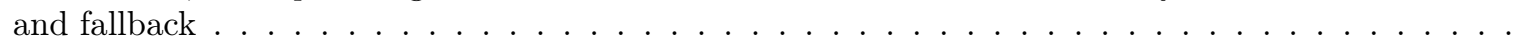

Yu.A. Kovalev, Yu.Yu. Kovalev, A. Popkov, Y. Sotnikova, S. Troitsky, A. Plavin, A. Erkenov RATAN-600 spectra as indicators of proton jets producing neutrino in blazars . . . . . . . . . .

K. Levenfish, G. Ponomaryov, A. Petrov X-ray morphology of pulsar wind nebulae: the effect of local medium motion . . . . . . . . . . . . . . . . . . . . . . . . . . . . . .

G. Lipunova, K. Malanchev, A. Avakyan, N. Shakura, S. Tsygankov, A. Tavleev, D. Kolesnikov Modeling outbursts of viscous accretion discs . . . . . . . . . . . . . . . . . . . . . . .

I.D. Markozov, A.A. Mushtukov, D.I. Nagirner Uncertainties in estimations of actual luminosity in bright X-Ray pulsars . . . . . . . . . . . . . . . . . . . . . . .

E. Mikheeva, S. Repin, V.N. Lukash Simulations of black hole observations with space-ground interfer-

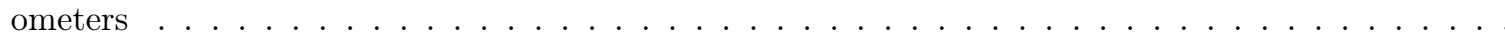

M.V. Pashentseva, E.A. Mikhailov Magnetic fields of accretion disks and their vertical structure . . . .

K. Postnov, A. Kuranov, L. Yungelson, M. Gilfanov X-ray luminosity function of accreting neutron stars

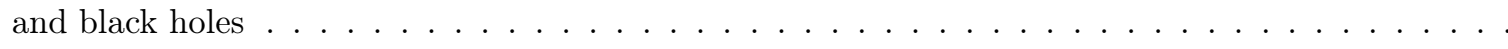

A. Tanashkin, G. Beskin, S. Karpov, V. Plokhotnichenko, Yu. Shibanov, D. Zyuzin Change of the structure and physical properties of optical pulsations from transitional millisecond

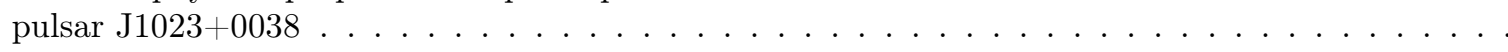

A.S. Tavleev, G.V. Lipunova, K.L. Malanchev Modelling of vertical structure of accretion discs around

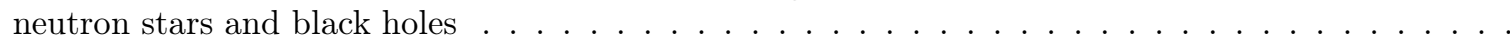

V. Toptun, I. Chilingarian, K. Grishin, I. Katkov, I. Zolotukhin, V. Goradzhanov, M. Demianenko, I. Kuzmun Confirmation of intermediate-mass black holes candidates with x-ray observations . . . . . .

The Sun

O. Andreeva, V. Malashchuk Observations at near-infrared wavelengths at the Tower Solar Telescope

TST-2 in Crimean Astrophysical Observatory . . . . . . . . . . . . . . . . . . . . .

A. Borisenko, I. Podgorny, A. Podgorny First flare M 1.9 AR 10365: comparing results real-scale time

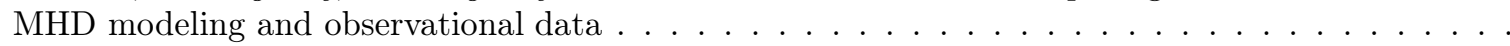

D. V. Erofeev On the polarization of velocity fluctuations in undisturbed solar wind . . . . . . . . . . .

O.A. Golubchina Temperature characteristics of radio emission of polar coronal holes on the Sun . . . .

V.A. Kotov Origin and length of solar cycle . . . . . . . . . . . . . . . . . . . .

Yu.A. Kupryakov, K.V. Bychkov, O.M. Belova, A.B. Gorshkov, P. Heinzel, P. Kotrč Observation of solar flare intensity curves and comparing them with stellar flares . . . . . . . . . . . . . . . . . 314

L. Ledentsov Thermal Trigger for Solar Flares . . . . . . . . . . . . . . . . . . . . . . . . . . . . . . . . . 317

I. Lopin MHD wave modes of axial symmetry in coronal flux tubes with magnetically twisted layer . . . 319 
S.G. Mozharovsky Visualization of the microturbulence distribution on the surface of the Sun . . . . . . 322

S.G. Mozharovsky Comparison of tree methods for estimating the magnetic field value obtained from the

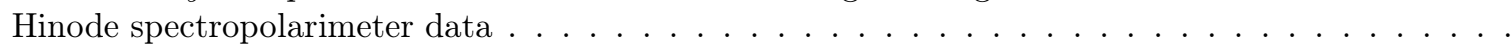

S. G. Mozharovsky Possibilities of velocity field analysis from Hinode SOT/SP data . . . . . . . . . . . . .

A.I. Podgorny, I.M. Podgorny, A.V. Borisenko, N.S. Meshalkina The choice of parameters and the appearance of a flare situation in MHD simulations above the active region in the real scale of time . . . . . . . . . . . . . . . . . . . . . . .

M. Ragulskaya, E. Tekutskaya Solar-terrestrial relations: solar activity and the COVID-19 pandemic . .

L. Starkova Conditions of generating a toroidal magnetic field during the period of decreasing magnetic

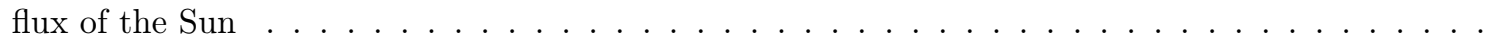

A. Struminsky, A. Sadovski, I. Grigorieva CME acceleration in impulsive (X6.9 09.08.2011) and gradual (M3.7 07.03.2011) solar flares

Yu. Tsap, A. Stepanov, Yu. Kopylova Flare energy release and avalanche ionization of plasma by runaway

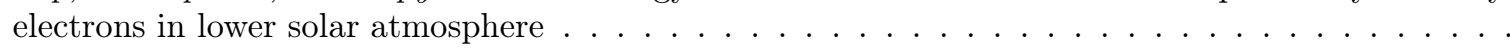

F.V. Vereshchagin Processing and imaging of a series of monochromatic images of regions of the Sun, obtained on the multichannel CCD spectroheliograph, in the programs ImageJ and ParaView . . .

E. Vernova, M. Tyasto, D. Baranov Cyclic variations of polarity of the weak photospheric

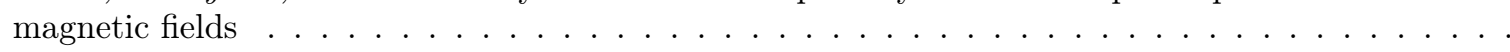

S. Yazev, V. Tomozov, E. Isaeva Activity Complexes on the Sun in Cycle 24 . . . . . . . . . . . .

D. Zhdanov First observations of the microwave fine structures with the $3-6 \mathrm{GHz}$ Siberian

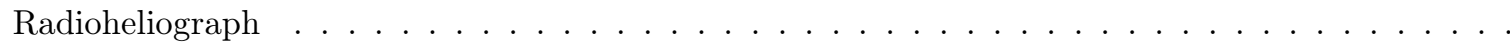

Physics of Galaxies and Cosmology

A.V. Antipova, D.I. Makarov, S.S. Savchenko The database for studying edge-on galaxies . . . . . . . .

A.V. Antipova, A.V. Mosenkov, D.I. Makarov, V.P. Reshetnikov Bulge-disk decomposition of ultrathin

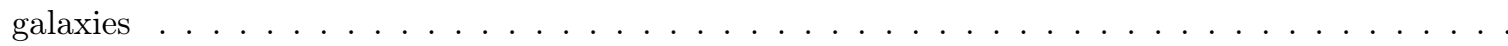

E. Balakina, M. Pruzhinskaya Peculiar velocities of SNe Ia in clusters of galaxies . . . . . . . . . . . . .

N. Bursov, S. Trushkin, A. Kudryashova, P. Tsybulev, A. Borisov, M. Khabibullina, D. Kuandykova Sources of the survey on the declination of microquasar GRS $1915+105$. . . . . . . . . . . . .

F.U. Botirov, S.N. Nuritdinov Nonlinear cosmogony of the spiral galaxy bulges . . . . . . . . . . . . . .

V.M. Charugin Relic black holes and the nature of the dark matter of the Universe . . . . . . . . . . . . .

M. Demianenko, K. Grishin, V. Toptun, I. Chilingarian, I. Katkov, V. Goradzhanov, I. Kuzmin Optical light curves of light-weight supermassive black holes produced by the Zwicky Transient Facility Forced Photometry Service

A.A. Ermash, S.V. Pilipenko, E.V. Mikheeva, V.N. Lukash Contribution to the confusion noise from

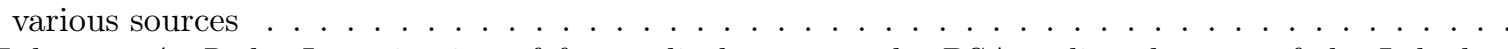

V. Fedorova, A. Rodin Investigation of fast radio bursts on the BSA radio telescope of the Lebedev

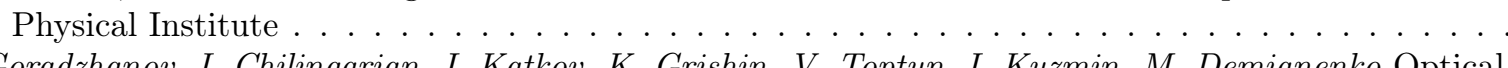

V. Goradzhanov, I. Chilingarian, I. Katkov, K. Grishin, V. Toptun, I. Kuzmin, M. Demianenko Optical spectroscopic observations of intermediate-mass black holes and their host galaxies: the $M_{\mathrm{BH}}-\sigma_{*}$

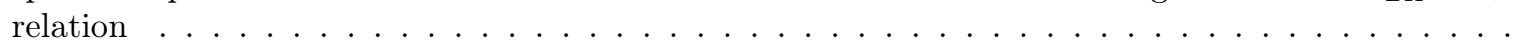

F. Kopylova, A. Kopylov Study of groups/clusters of galaxies with the SDSS . . . . . . . . . . . .

F. Kopylova, A. Kopylov The fundamental plane of clusters and groups of galaxies . . . . . . . . . . . .

O. Kurichin, P. Kislitsyn, A. Ivanchik The influence of systematic effects on the determination of the

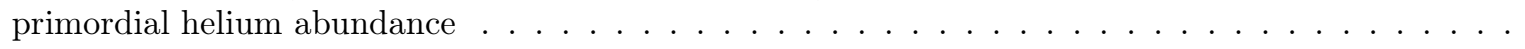

D. Makarov, S. Savchenko, A. Mosenkov, D. Bizyaev, V. Reshetnikov, A. Antipova, I. Tikhonenko, P. Usachev, S. Borisov, L. Makarova, S. Kautsch, A. Marchuk, E. Rubtsov, M. Edukov, M. Skryabina, P. Smirnova Catalog of edge-on galaxies using the Pan-STARRS1 survey data . . . . . . . . . . .

A. Malinovsky, E. Mikheeva, V. Lukash, S. Repin A catalog of Supermassive Black Holes for observations of black holes shadows with the Millimetron space observatory . . . . . . . . . . . . . .

K.A. Mannapova, K.T. Mirtadjieva Statistical analysis of observational data for spiral galaxies with known halo parameters . . . . . . . . . . . . . . . . . . . . .

A.A. Marchuk, A.V. Mosenkov, S.S. Savchenko An analysis of methods for determining corotation radii

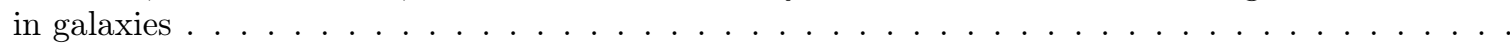

A. Mikhailov, Yu. Sotnikova Multifrequency study of FR0 radio galaxies with RATAN-600 . . . . . . .
A. Mikhailov, Yu. Sotnikova, T. Mufakharov, M. Mingaliev, N. Bursov, T. Semenova, V. Stolyarov, Zh. $W u \mathrm{OH}$ megamaser galaxies observations with RATAN-600

Yu.N. Pariiskii, T.A. Semenova, A.V. Temirova, N.N. Bursov Analysis of objects with ultra steep spectra in the central section of RATAN Zenith Field (RZF) survey . . . . . . . . . . . . . 
M. Piotrovich, S. Buliga, T. Natsvlishvili Determination of the magnetic field strength and geometry in the accretion disks of AGNs . . . . . . . . . . . . . . . . . . . . . . .

A.N. Popov, D.P. Barsukov, A.V. Ivanchik, S.V. Bobashev Absorption of gamma quanta from distant

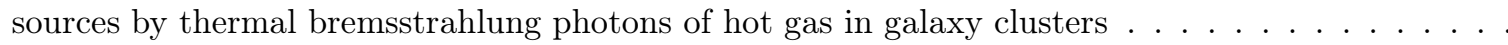

A. Raikov, N. Lovyagin, V. Yershov Superluminous quasars and mesolensing . . . . . . . . . . . . . . .

A. Saburova, I. Chilingarian, A. Kasparova, K. Grishin, O. Sil'chenko, I. Katkov, R. Uklein Observational insights on the formation scenarios of giant low surface brightness galaxies . . . . . . . . . . .

L. Shalyapina, O. Merkulova, G. Karataeva, I. Chugunov Spectral study of a candidate to polar-ring galaxies UGC $4261 \ldots \ldots \ldots \ldots \ldots \ldots \ldots$

P. Smirnova, A. Mosenkov, A. Marchuk Studying the characteristics of spiral galaxies . . . . . . . . . .

Yu. Sotnikova, T. Mufakharov, A. Mikhailov, M. Mingaliev, Yu. Kovalev Radio properties of quasars at

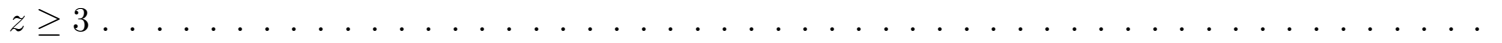

I.S. Tikhonenko, A.A. Smirnov, N.Ya. Sotnikova Vertical structure of barlenses in N-body models . . .

L. Ugol'kova, E. Shimanovskaya, V. Larionov, S. Savchenko, T. Grishina, O. Ezhkova, E. Kopatskaya, E. Larionova, L. Larionova, D. Morozova, A. Nikiforova, I. Troitsky On the correlation between optical and $\gamma$-ray emission of the blazar 3C 454.3 during the multi-wavelength flare

from 2012 to $2017 \ldots \ldots \ldots \ldots \ldots \ldots \ldots \ldots \ldots \ldots$

P. Usachev, V. Reshetnikov, S. Savchenko Edge-on galaxies in the HST COSMOS field . . . . . . . . .

P. Usachev, A. Mosenkov, S. Savchenko, G. Gontcharov, V. Il'in, A. Marchuk, A. Smirnov Dust discs in edge-on galaxies: case of NGC 4437

V. Yershov, A. Raikov, N. Lovyagin, P. Kuin, E. Popova Grey intergalactic dust could explain the Hubble constant tension . . . . . . . . . . . . . . . . . . . . .

V. Yurchenko, A. Ivanchik Relic neutrinos distribution function at low coordinate momentums . . . . .

D.A. Zakharova, I.S. Tikhonenko, A.A. Smirnov, N.Ya. Sotnikova Kinematic analysis of N-body galaxy

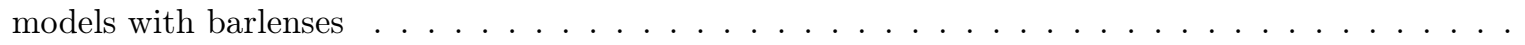

A. Zasov, A. Saburova, O. Egorov Sites of star formation in tidal structures . . . . . . . . . . . .

A. Zasov, A. Khoperskov, N. Zaitseva, S. Khrapov When dwarf galaxies turn to be spiral? . . . . . . . .

O.P. Zhelenkova, E.K. Majorova, A.V. Temirova, N.N. Bursov Mass search of the RCR catalog sources at different bands of electromagnetic spectra . . . . . . . . . . . . . . . . .

O.P. Zhelenkova The jet interaction manifestations of the RC J0311+0507 radio galaxy at $z=4.51$ with

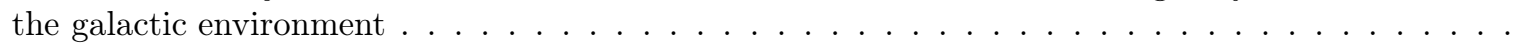

O.P. Zhelenkova Unindentified sources of the 3CR catalogue . . . . . . . . . . . . . . .

O.P. Zhelenkova Search for radio sources - close neighbors according to modern radio and optical surveys . . . . . . . . . . . . . . . . . . . . . . . .

T. Abdulmyanov On two forms of interstellar gas accretion in the formation of single stars . . . . . . . 430

A. Avdeeva, D. Kovaleva, O. Malkov, A. Nekrasov Determination of interstellar extinction parameters at high galactic latitudes . . . . . . . . . . . . . . . . . . . . . . . . .

J.M. Ganiev, S.N. Nuritdinov Small-scale sectorial modes of perturbations against the background of a pulsating model of disk-like self-gravitating systems . . . . . . . . . . . . . . .

G. Gontcharov, A. Mosenkov, V. Il'in, A. Marchuk, S. Savchenko, A. Smirnov, P. Usachev Interstellar extinction at high Galactic latitudes across the whole dust layer in the Galaxy . . . . . . . . . .

G. Gontcharov, A. Mosenkov, M. Khovritchev, V. Il'in, A. Marchuk, S. Savchenko, A. Smirnov, P. Usachev The properties of Galactic globular clusters from Gaia EDR3 and other data compared

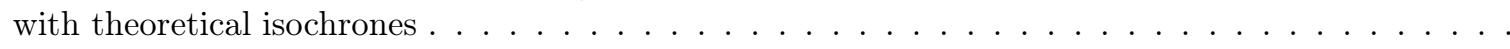

A.V. Khruslov A Study of Double- and Multi-Mode High-Amplitude Delta Scuti Variables . . . . . . . .

O.V. Kiyaeva, M.Yu. Khovritchev Possible companions of the visual double star ADS 9346=WDS

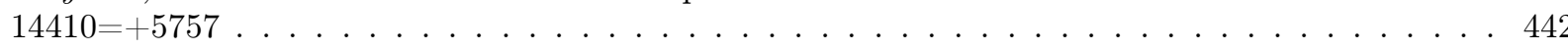

O. Malkov, A. Kniazev Candidates to visual binaries with non-coeval components . . . . . . . . . . . 443

M. Maricheva, M. Sharina Chemical composition and ages of four globular clusters in M31 . . . . . . . 446

N. Maslennikova, A. Tatarnikova, A. Tatarnikov, N. Ikonnikova, A. Dodin Confirmation of the symbiotic nature of CSS 1102 on the basis of spectral and photometric observations carried out at the CMO SAI MSU

A.M. Melnik, A.K. Dambis, E.N. Podzolkova, L.N. Berdnikov Influence of the bar on the kinematics of the Galactic disk near the Outer Lindblad Resonance. Comparison with Gaia EDR3 data . . . . .

A.M. Mickaelian, G.A. Mikayelyan, H.V. Abrahamyan, G.M. Paronyan Discovery and study of new

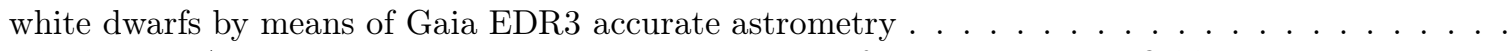

E. Mikhailov, R. Andreasyan Biermann battery as a source of cosmic magnetic fields . . . . . . . . . 
S. Nuritdinov, A. Rastorguev, I. Tadjibaev Determination of the degree of star concentration in globular clusters based on space observation data . . . . . . . . . . . . . . . . . . . . 457

P. Pakhomova, L. Berdnikov, A. Kniazev, I. Katkov, O. Malkov Parametrization of long-period eclipsing

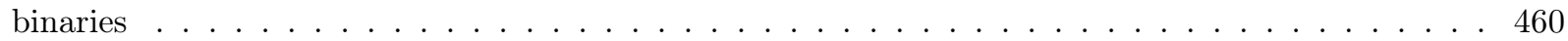

I. Romanyuk, E. Semenko, A. Moiseeva, I. Yakunin, D. Kudryavtsev Magnetic field of chemically peculiar stars in Orion OB1 association . . . . . . . . . . . . . . . . . . . . . . . . . . 463

A.F. Seleznev, A.A. Malofeeva A search of unresolved binaries in open clusters by the photometry data in visible and infrared . . . . . . . . . . . . . . . . . . . . . . . . . . 465

F.T. Shamshiyev A special class of potentials admitting a local integral for rotating systems . . . . . . . 468

S. Trushkin, A. Shevchenko, N. Bursov, P. Tsybulev, N. Nizhelsky Long-term multi-frequency studies of flaring activity from microquasars . . . . . . . . . . . . . . . . . . . . 471

S. Trushkin, P. Tsybulev, N. Nizhelsky, N. Bursov, A. Shevchenko Fastly varying objects: fast radio bursts, magnetars, and pulsars with RATAN-600 . . . . . . . . . . . . . . . . . . . 473

\section{Astronomical Education}

I. V. Kuznetsova, M.E. Prokhorov Estimation of asteroid-comet hazard according to open scientific data as a student practical work . . . . . . . . . . . . . . . . . . . . . . . . 476 
Astronomy at the epoch of multimessenger studies.

Proceedings of the VAK-2021 conference, Aug 23-28, 2021

Сдано в набор 21.12.2021. Подписано в печать 22.12.2021

Формат 60x90/8. Бумага офсетная.

Уч.-изд. п.л. 60,75. Физ. п.л. 60,75. Тираж 100. Заказ № 2354

Издательство «Янус-К»

127411, Москва, Учинская ул., д. 1

Отпечатано в ООО «ИНФОРМ-СОФТ»

119034, Москва, Еропкинский пер., д. 16

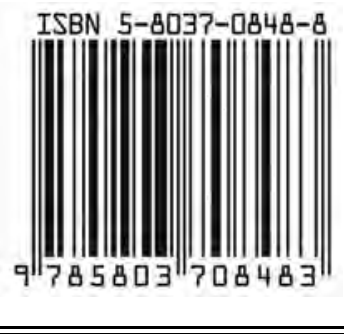

